January 2000 • NREL/SR-540-26935

\title{
Mack LNG Vehicle Development
}

Southwest Research Institute

San Antonio, Texas

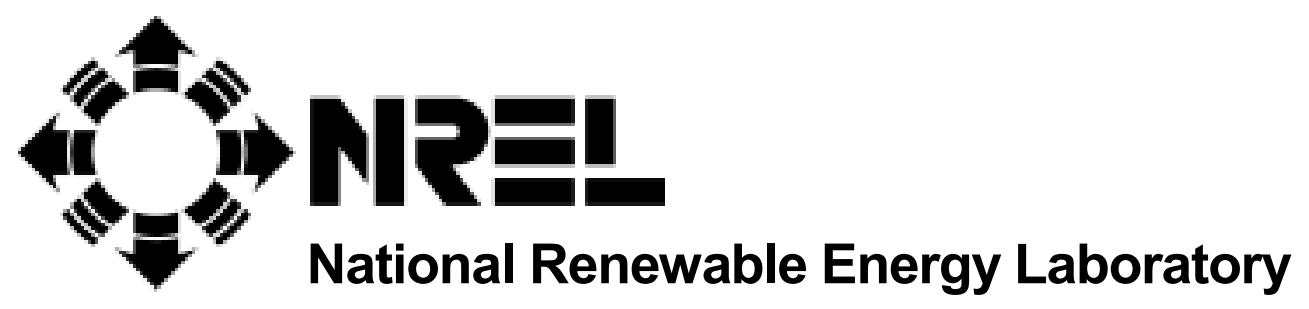

1617 Cole Boulevard

Golden, Colorado 80401-3393

NREL is a U.S. Department of Energy Laboratory

Operated by Midwest Research Institute • Battelle • Bechtel

Contract No. DE-AC36-99-G010337 


\section{Mack LNG Vehicle Development}

Southwest Research Institute

San Antonio, Texas

NREL Technical Monitor: Mike Frailey

Prepared under Subcontract No. AAC-5-15249-01

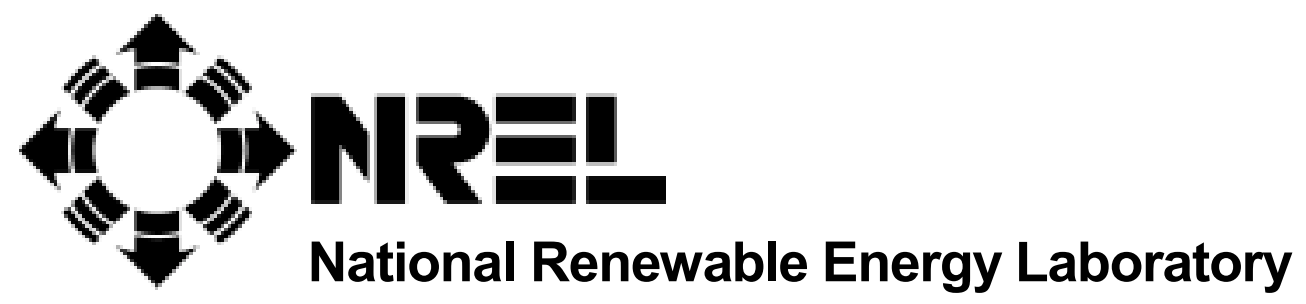

1617 Cole Boulevard

Golden, Colorado 80401-3393

NREL is a U.S. Department of Energy Laboratory

Operated by Midwest Research Institute • Battelle • Bechtel

Contract No. DE-AC36-99-G010337 


\section{NOTICE}

This report was prepared as an account of work sponsored by an agency of the United States government. Neither the United States government nor any agency thereof, nor any of their employees, makes any warranty, express or implied, or assumes any legal liability or responsibility for the accuracy, completeness, or usefulness of any information, apparatus, product, or process disclosed, or represents that its use would not infringe privately owned rights. Reference herein to any specific commercial product, process, or service by trade name, trademark, manufacturer, or otherwise does not necessarily constitute or imply its endorsement, recommendation, or favoring by the United States government or any agency thereof. The views and opinions of authors expressed herein do not necessarily state or reflect those of the United States government or any agency thereof.

Available electronically at http://www.doe.gov/bridge

Available for a processing fee to U.S. Department of Energy

and its contractors, in paper, from:

U.S. Department of Energy

Office of Scientific and Technical Information

P.O. Box 62

Oak Ridge, TN 37831-0062

phone: 865.576.8401

fax: 865.576.5728

email: reports@adonis.osti.gov

Available for sale to the public, in paper, from:

U.S. Department of Commerce

National Technical Information Service

5285 Port Royal Road

Springfield, VA 22161

phone: 800.553.6847

fax: 703.605.6900

email: orders@ntis.fedworld.gov

online ordering: http://www.ntis.gov/ordering.htm

Printed on paper containing at least $50 \%$ wastepaper, including $20 \%$ postconsumer waste 


\section{Mack LNG Vehicle Development}

Prepared by James P. Chiu for:

Mack Trucks, Inc.

13302 Pennsylvania Ave.

Hagerstown, MD 21742

Submitted to:

National Renewable Energy Laboratory

1617 Cole Boulevard

Golden, CO 80401-3393

Submitted by:

Southwest Research Institute

6220 Culebra Road

San Antonio, TX 78228-0510

(210) 684-5111

Note: This document is an abbreviated version of a larger report that contains the following appendices:
A. Recorded Data for Lean Limit Tests
B. Recorded Data for Steady-State Tests
C. Final Calibration, 325 bhp
D. Final Report for Task 2 - 1000-Hour Super Thermal Cycle Test, SwRI Project No. 03-7501-002
E. $\quad$ Final Report for 1000-Hour Super Thermal Cycle Test on a Mack E-7 Natural Gas Fueled Engine, SwRI Project No. 03-1248
F. Histograms from the Data Logger
G. Final Report for Transient Emissions Testing on a Mack E\&G Engine, SwRi Project No. 03-2377
H. Final Calibration, 350 bhp

If you would like a copy of the appendices, please contact Mike Frailey at the National Renewable Energy Laboratory at 303-275-3607 or mike_frailey@nrel.gov. 


\section{Executive Summary}

The goal of this project was to install a production-ready, state-of-the-art engine control system on the Mack E7G natural gas engine to improve efficiency and lower exhaust emissions. In addition, the power rating was increased from 300 brake horsepower (bhp) to $325 \mathrm{bhp}$. The emissions targets were oxides of nitrogen plus nonmethane hydrocarbons $\left(\mathrm{NO}_{\mathrm{x}}+\mathrm{NMHC}\right.$ ) of less than $2.5 \mathrm{~g} / \mathrm{bhp}-\mathrm{hr}$ (with a mandatory $\mathrm{NO}_{\mathrm{x}}$ of less than $2.5 \mathrm{~g} / \mathrm{bhp}-\mathrm{hr}$ ) and particulate matter of less than $0.05 \mathrm{~g} / \mathrm{bhp}-\mathrm{hr}$ on $99 \%$ methane. Vehicle durability and field tests were also conducted.

A Woodward $\mathrm{OH}-1$ state-of-the-art engine controller was successfully installed on the Mack E7G engine and a calibration for the Mack engine was developed. Knock tolerance testing and misfire limit testing were conducted to aid in the development of the calibration. The first 1000 -hour durability test was conducted with partial funding from this project, and a second 1000-hour durability test to address the concerns from the first test was successfully completed. A data logger was installed on an in-service refuse hauler and engine and vehicle operation was successfully recorded.

The brake-specific fuel consumption over the transient emissions test cycle was decreased from 0.474 $\mathrm{lb} / \mathrm{bhp}$-hr on the previous version of this engine, to $0.400 \mathrm{lb} / \mathrm{bhp}-\mathrm{hr}$ on the current version. The $\mathrm{NO}_{\mathrm{x}}$ emissions over the transient emissions test cycle were decreased from $2.95 \mathrm{~g} / \mathrm{bhp}$-hr to $2.20 \mathrm{~g} / \mathrm{bhp}$-hr. The $\mathrm{NO}_{\mathrm{x}}+\mathrm{NMHC}$ emissions were $2.53 \mathrm{~g} / \mathrm{bhp}-\mathrm{hr}$ and particulate matter was $0.03 \mathrm{~g} / \mathrm{bhp}$-hr on the current version of this engine. Emissions for the current version of this engine include the deterioration factor, whereas emissions from the previous version do not include the deterioration factor. California Air Resources Board (CARB) certification gas was used for the transient emissions testing on the current version of this engine. $\mathrm{NO}_{\mathrm{x}}+\mathrm{NMHC}$ emissions are expected to decrease on a $99 \%$ methane fuel. Actual driving cycle fuel economy comparisons are not readily available because the previous version of this engine was installed in a refuse hauler, which was at a different location. Further development of this engine should include efficiency improvements and $\mathrm{NO}_{x}$ reductions. 


\section{Contents}

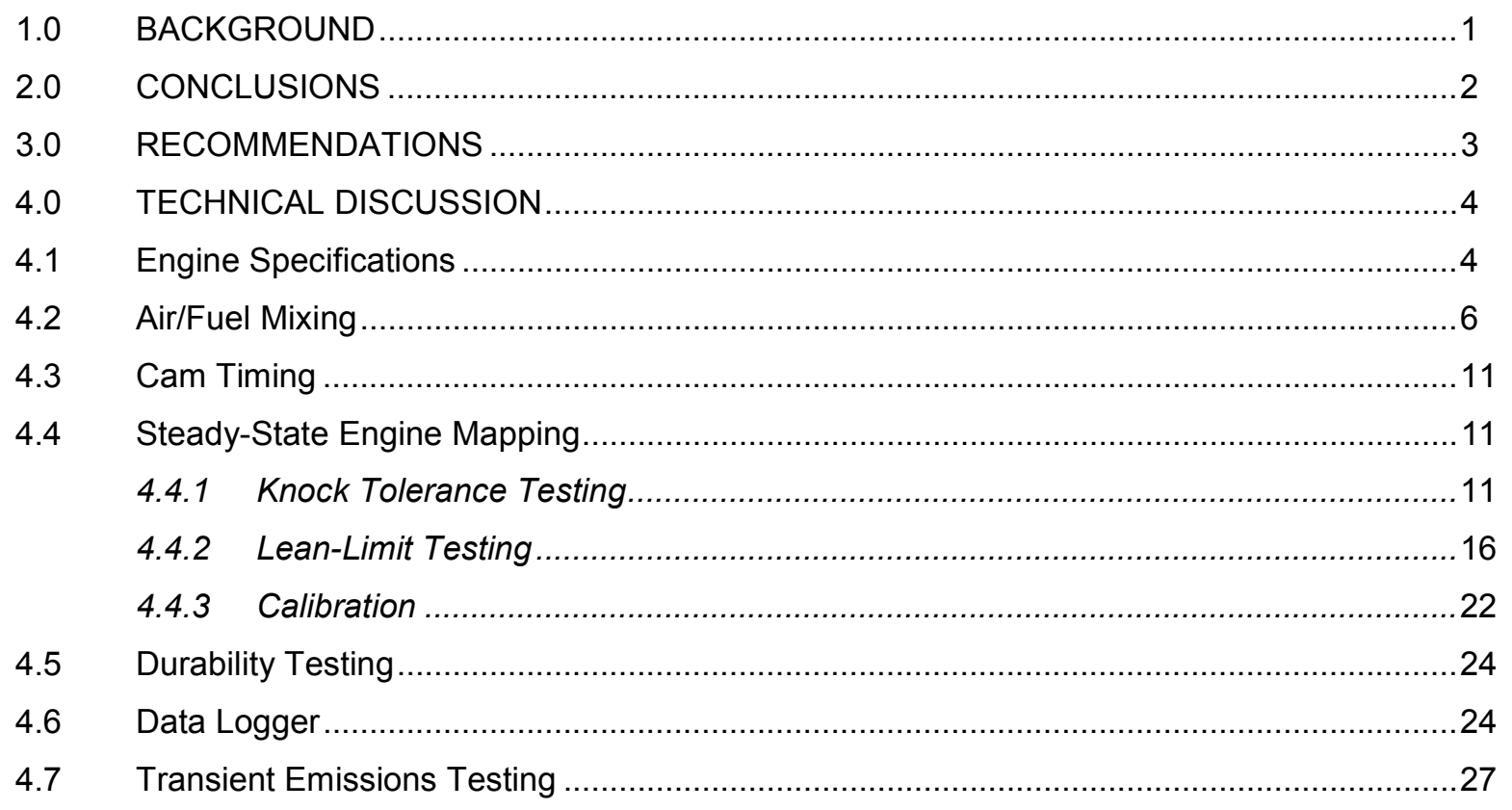




\section{List Of Figures}

1. Average Equivalence Ratios (AERs) and the Standard Error (SE)

for the Six Cylinders Using Mixing Elbow Number 1

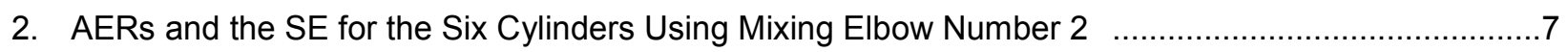

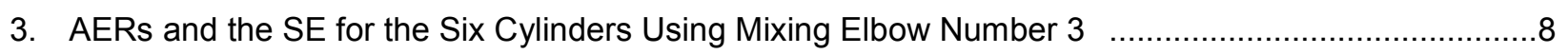

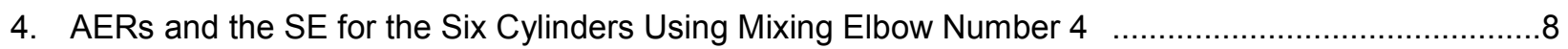

5. AERs and the SE for the Six Cylinders Using Mixing Elbow Number 5 ...........................................

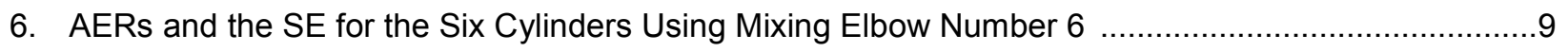

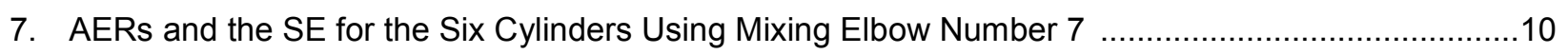

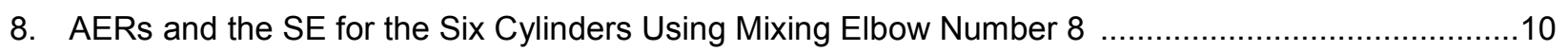

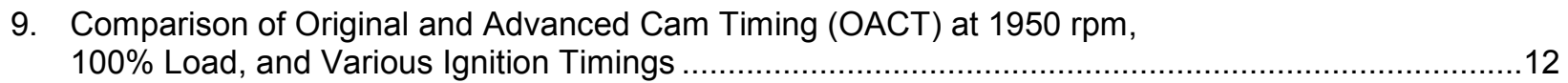

10. Comparison of OACT at $1950 \mathrm{rpm}, 75 \%$ Load, and Various Ignition Timings .................................12

11. Comparison of OACT at $1950 \mathrm{rpm}, 50 \%$ Load, and Various Ignition Timings ..................................12

12. Comparison of OACT at $1500 \mathrm{rpm}, 100 \%$ Load, and Various Ignition Timings ................................13

13. Comparison of OACT at $1500 \mathrm{rpm}, 75 \%$ Load, and Various Ignition Timings ..................................13

14. Comparison of OACT at $1500 \mathrm{rpm}, 50 \%$ Load, and Various Ignition Timings .................................13

15. Comparison of OACT at $1250 \mathrm{rpm}, 100 \%$ Load, and Various Ignition Timings .................................14

16. Comparison of OACT at $1250 \mathrm{rpm}, 75 \%$ Load, and Various Ignition Timings ..................................14

17. Comparison of OACT at $1250 \mathrm{rpm}, 50 \%$ Load, and Various Ignition Timings ....................................14

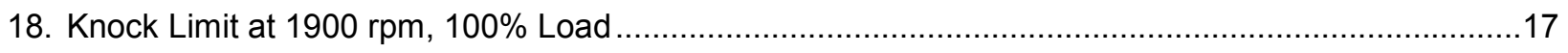

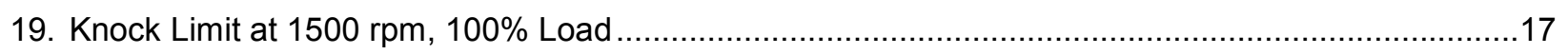

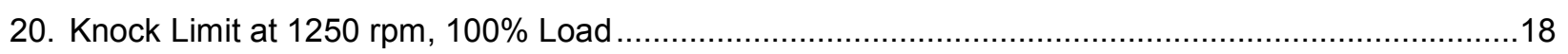

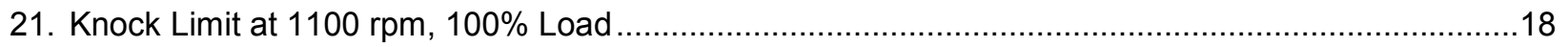

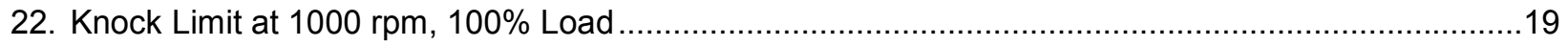

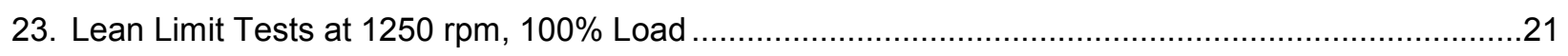

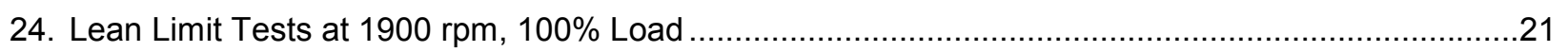

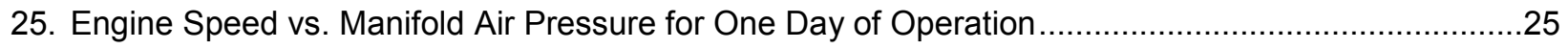

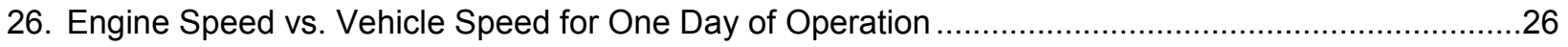

27. Manifold Air Pressure vs. Vehicle Speed for One Day of Operation .................................................27 


\section{List of Tables}

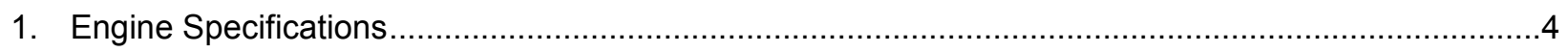

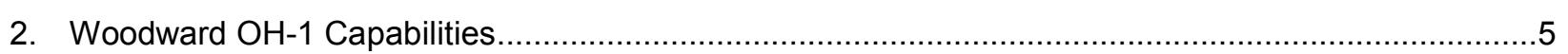

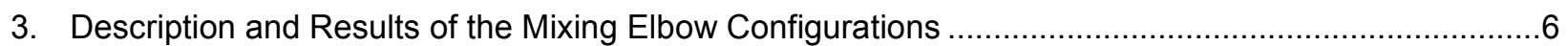

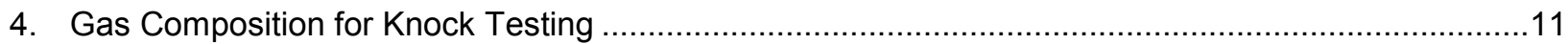

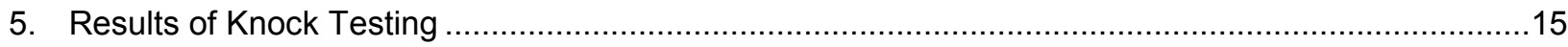

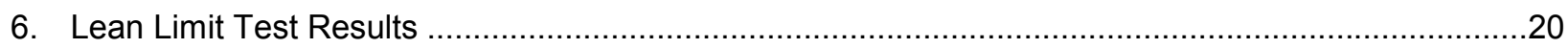

7. Test Points for the 12-Mode Steady-State Prediction for Transient Emissions..................................23

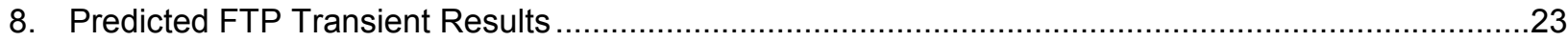

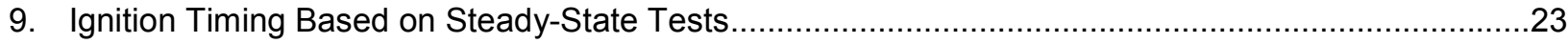

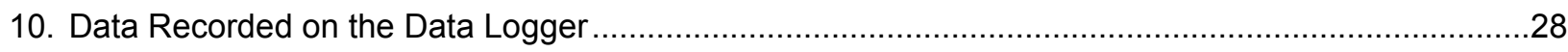

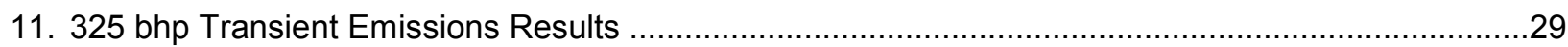

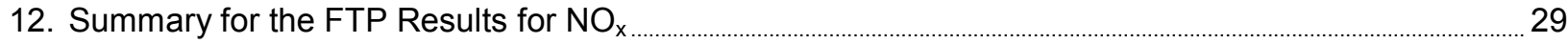

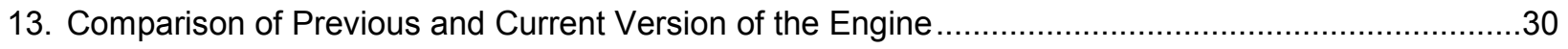

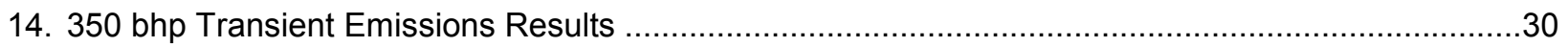




\section{Acronyms}

\begin{tabular}{|c|c|}
\hline AER & average equivalence ratios \\
\hline bhp & brake horsepower \\
\hline BSCO & brake specific carbon monoxide \\
\hline $\mathrm{BSNO}_{\mathrm{x}}$ & brake specific nitrogen oxides \\
\hline BSTHC & brake specific total hydrocarbon \\
\hline BTDC & before top dead center \\
\hline BTE & brake thermal efficiency \\
\hline CARB & California Air Resources Board \\
\hline CBD & central business district \\
\hline CNG & compressed natural gas \\
\hline $\mathrm{COV}_{\text {imep }}$ & $\begin{array}{l}\text { coefficient of variation of the indicated mean effective pressure } \\
\text { degree }\end{array}$ \\
\hline $\begin{array}{l}\text { deg } \\
{ }^{\circ} \mathrm{F}\end{array}$ & $\begin{array}{l}\text { degree } \\
\text { degree Fahrenheit }\end{array}$ \\
\hline EPA & Environmental Protection Agency \\
\hline $\mathrm{ft}-\mathrm{lb}$ & foot-pound \\
\hline FTP & Federal Test Procedure \\
\hline g/bhp-hr & grams/brake horsepower per hour \\
\hline GEM & Gas Engine Management \\
\hline $\mathrm{HC}$ & hydrocarbons \\
\hline HC/OMHCE & hydrocarbon/organic material hydrocarbon equivalent \\
\hline imep & indicated mean effective pressure \\
\hline LNG & liquefied natural gas \\
\hline MAP & manifold absolute pressure \\
\hline MBT & minimum for best torque \\
\hline mpg & miles per gallon \\
\hline mph & miles per hour \\
\hline $\mathrm{ms}$ & millisecond \\
\hline MS & mass spectrometer ** \\
\hline NMHC & nonmethane hydrocarbon \\
\hline OMNMHC & organic material non-methane hydrocarbon \\
\hline $\mathrm{NO}_{\mathrm{x}}$ & oxides of nitrogen \\
\hline OACT & original and advanced cam timing \\
\hline $\mathrm{OH}-1$ & on highway-1 \\
\hline PM & particulate matter \\
\hline ppm & parts per million \\
\hline psia & pounds per square inch absolute \\
\hline psig & pounds per square inch gauge \\
\hline PWM & pulse-width modulated \\
\hline $\mathrm{rpm}$ & revolutions per minute \\
\hline SE & standard error \\
\hline SwRI & Southwest Research Institute \\
\hline $\mathrm{THC}$ & total hydrocarbons \\
\hline UEGO & universal exhaust gas oxygen \\
\hline VE & volumetric efficiency \\
\hline WOT & wide open throttle \\
\hline
\end{tabular}




\subsection{Background}

This project was a continuation of the development of a natural-gas refuse hauler. A compressed natural gas (CNG) Mack MR demonstration vehicle was operated for nearly a year in revenue service on a previous project. Engine performance, emissions, driveability, and durability were acceptable for a demonstration vehicle; however, several areas were identified for improvement. These included vehicle range, refueling time, driving cycle fuel economy, and installation of improved and production-ready natural gas specific engine control hardware.

Vehicle range and refueling time can be improved by operating the vehicle on liquefied natural gas (LNG). Project efforts focused on minimizing throttling losses and increasing the $\mathrm{NO}_{x}$ efficiency tradeoff by using a state-of-the-art engine controller.

The original objectives of this project were to:

1. Improve the driving cycle thermal efficiency of the engine

2. Install and aid in the development of a state-of-the-art engine control system

3. Assess control system and engine integrity through a 480-hour durability test

4. Calibrate the engine to exactly meet the diesel torque curve of the $300 \mathrm{bhp}$ engine

5. Conduct transient emissions testing to verify that exhaust emissions are lower than the 1998 Environmental Protection Agency (EPA) emissions targets with $\mathrm{NO}_{\mathrm{x}}$ emissions being less than $2.0 \mathrm{~g} / \mathrm{bhp}-\mathrm{hr}$

6. Monitor the vehicle through a 3-month field test using an on-board data logger equipped with a cellular telephone

7. Provide detailed drawings, manuals, and training to Mack personnel so that Mack can develop a full understanding of the engine control system and be capable of producing six identical vehicles.

Several project components were revised as follows:

- A 1000-hour durability test was performed. This project funded up to the amount provided in the contract, and Mack Trucks funded the remainder.

- The engine was calibrated to a $325 \mathrm{bhp}$ at 1950 revolutions per minute (rpm) and $1180 \mathrm{ft}-\mathrm{lb}$ at $1250 \mathrm{rpm}$ torque curve.

- The PM target had been revised to $0.05 \mathrm{~g} / \mathrm{bhp}$-hr, and there was an additional target for $\mathrm{NO}_{\mathrm{x}}+\mathrm{NMHC}$ of less than $2.5 \mathrm{~g} / \mathrm{bhp}-\mathrm{hr}$. 


\subsection{Conclusions}

The successful completion of the project resulted in several conclusions regarding the technical content of the program, described below.

In comparing the previous demonstration engine to the current engine developed in this project:

- Peak power was increased from $300 \mathrm{bhp}$ to $325 \mathrm{bhp}$ to match the 325-bhp diesel torque curve.

- Peak torque was increased from $1100 \mathrm{ft}-\mathrm{lb}$ to $1180 \mathrm{ft}-\mathrm{lb}$ to match the $325 \mathrm{bhp}$ diesel torque curve.

- The $\mathrm{NO}_{\mathrm{x}}$ emissions over the transient emissions test cycle decreased from $2.95 \mathrm{~g} / \mathrm{bhp}$-hr to 2.20 g/bhp-hr.

- The brake specific fuel consumption over the transient emissions test cycle decreased from 0.474 $\mathrm{lb} / \mathrm{bhp}$-hr to $0.400 \mathrm{lb} / \mathrm{bhp}$-hr, increasing efficiency.

- A comparison of in-use fuel economy was not possible due to the different driving cycles of the previous demonstration engine and the current engine.

A state-of-the-art engine control system was successfully integrated onto the engine. Control system and engine integrity were assessed through a 1000-hour durability test, which indicated a problem with the piston. A subsequent 1000 -hour durability test was conducted on a separate project at the Southwest Research Institute (SwRI) with revised pistons. This test confirmed that the problem was solved.

The $\mathrm{NO}_{\mathrm{x}}+\mathrm{NMHC}$ emissions were $2.53 \mathrm{~g} / \mathrm{bhp}-\mathrm{hr}$ with the deterioration factor and $2.40 \mathrm{~g} / \mathrm{bhp}-\mathrm{hr}$ without the deterioration factor on CARB gas. Emissions on $99 \%$ methane were not conducted with the final calibration, but were expected to be below the $2.5 \mathrm{~g} / \mathrm{bhp}$-hr target, even with the deterioration factor.

A vehicle was successfully monitored using an on-board data logger.

Mack has produced six identical engines with this state-of-the-art control system and all associated engine and vehicle modifications.

Durability of the cylinder heads, including valve recession, was greatly improved from the previous engine (one of the problem areas from the final report for the previous engine). Funding to test head durability was provided by Mack Trucks. 


\subsection{Recommendations}

Following are recommendations for further development of the engine.

1. Lower $\mathrm{NO}_{\mathrm{x}}$ emissions are possible on this engine with:

- Humidity compensation to allow for a calibration with lower $\mathrm{NO}_{\mathrm{x}}$ emissions on the transient test cycle (which has been demonstrated on this engine) without producing driveability problems in the field.

- Decreased equivalence ratio at the lower engine speeds and full load to reduce $\mathrm{NO}_{\mathrm{x}}$ emissions during transients from idle along with alternative turbo lag compensation methods to retain driveability.

2. Higher thermal efficiency for this engine is possible with:

- Humidity compensation to allow for equivalence ratios closer to the lean-limit.

- Turbo lag compensation methods to reduce transient $\mathrm{NO}_{\mathrm{x}}$, which can allow for higher $\mathrm{NO}_{\mathrm{x}}$, and therefore higher efficiency over the driving cycle of the engine.

3. Investigation of other methods to improve the $\mathrm{NO}_{x}$ efficiency trade-off of this engine.

Higher thermal efficiency and improved $\mathrm{NO}_{x}$ efficiency trade-offs are being investigated and developed under the current Efficiency Enhancements for the Mack E7G Natural Gas-Fueled Engine program. 


\subsection{Technical Discussion}

This section describes the results of the project.

\subsection{Engine Specifications}

The Mack E7 engine (S/N 1P0144) was used for calibration development on this project. This engine was used on the previous Mack natural gas engine project, but was rebuilt with new cylinder liners, new pistons with an 11.5:1 compression ratio (replaces pistons with a 10.5:1 compression ratio), new piston rings, new cylinder heads with higher swirl, and a new camshaft. Engine specifications are shown in Table 1.

Table 1. Engine Specifications

\begin{tabular}{||l|l||}
\hline \hline Engine Model & E7G-325 \\
\hline Engine Serial Number & $1 \mathrm{P} 1044$ \\
\hline Engine Displacement (in ${ }^{3} /$ liter) & $728 / 12$ \\
\hline Bore and Stroke (in/mm) & $4.875 / 123.8 \times 6.5 / 165.1$ \\
\hline Ignition System Controller Supplier & MESA/Woodward Governor Company \\
\hline Ignition System Coil Supplier & Nippondenso \\
\hline Ignition System Type & Inductive, Coil on Plug (non-waste spark) \\
\hline Ignition System Timing Sensor & Electro, K Coil (originally), Bosch (currently) \\
\hline Spark Plug Supplier & Champion \\
\hline Spark Plug Model & RX501PYP/2H6 \\
\hline Fuel Metering System Supplier & MESA/Woodward Governor Company \\
\hline Fuel Injector Type & Servojet SP021 \\
\hline Number of Injectors & 8 \\
\hline Throttle Supplier & Woodward Governor Company \\
\hline Throttle Model & FloTech, 68mm \\
\hline Throttle Type & Electronic, Analog \\
\hline Universal exhaust gas oxygen (UEGO) sensor & Honda version of the NGK \\
\hline Turbocharger & $\begin{array}{l}\text { Mack 631GCX5146M, Garrett GT40-88 } \\
\text { Compressor Trim, A/R = 52/88mm, 0.58 } \\
\text { Turbine Trim, A/R = 78/77mm, 1.05 }\end{array}$ \\
\hline \hline
\end{tabular}


A Mesa gas engine management (GEM) controller was selected for this project. The Mesa heavyduty natural gas business was bought by Woodward Governor Company, and the controller is now called the On Highway-1 $(\mathrm{OH}-1)$. The $\mathrm{OH}-1$ uses a state-of-the-art speed-density control strategy that provides excellent driveability and response without the aid of a mass air flow sensor. Table 2 shows the capabilities of the $\mathrm{OH}-1$. It is a closed-loop lean-burn strategy with adaptive learn controller. It includes advanced turbo lag compensation, electronic wastegate control, an electronic throttle, highenergy inductive ignition system, full diagnostics, and reduced power engine protection. Fuel is inducted upstream of the throttle. Pseudo-continuous fuel metering is accomplished through single point injection using eight pulse-width modulated (PWM) fuel injectors that supply a common manifold. The injectors are fired sequentially in pairs and fuel flow is governed by modulating the pulse width of the fuel injector drive signals. A drive-by-wire throttle is utilized for speed governing, overboost protection, and driveability enhancements.

Table 2. Woodward OH-1 Capabilities

\begin{tabular}{||l|l||}
\hline Capability & Description \\
\hline Diagnostics & $\begin{array}{l}\text { Advanced diagnostics for easy debugging with limp } \\
\text { home mode for component failure }\end{array}$ \\
\hline Drive-By-Wire & $\begin{array}{l}\text { Idle speed control, packing speed control, speed } \\
\text { governing, load limiting during component failure, } \\
\text { throttle opening during deceleration for reduced oil } \\
\text { consumption }\end{array}$ \\
\hline Cold Start & $\begin{array}{l}\text { Fuel enrichment and ignition timing advance to } \\
\text { avoid lean misfire during cold engine operation with } \\
\text { boost limits }\end{array}$ \\
\hline Equivalence Ratio Control & $\begin{array}{l}\text { A volumetric efficiency table with adaptive learn to } \\
\text { determine fueling rate with an UEGO sensor for } \\
\text { closed-loop control }\end{array}$ \\
\hline Manifold Filling Calibration & $\begin{array}{l}\text { Predictions for air flow during transients for more } \\
\text { accurate equivalence ratio control }\end{array}$ \\
\hline Turbo Lag Compensation & $\begin{array}{l}\text { Fuel enrichment and ignition timing retard to reduce } \\
\text { turbo lag without increasing NO }{ }_{x} \text { emissions }\end{array}$ \\
\hline Boost Control Calibration & $\begin{array}{l}\text { Controls turbocharger wastegate to determine } \\
\text { power for a given throttle position, also used to } \\
\text { reduce throttling losses by using the wastegate for } \\
\text { load control with an open throttle }\end{array}$ \\
\hline Ignition Timing Calibration & $\begin{array}{l}\text { Table to determine ignition timing for various } \\
\text { speeds and loads }\end{array}$ \\
\hline Equivalence Ratio Calibration & $\begin{array}{l}\text { Table to determine equivalence ratio for various } \\
\text { speeds and loads }\end{array}$ \\
\hline
\end{tabular}

Part-load fuel economy was increased by the use of a "delta-P" load control strategy. With this strategy, the throttle on the engine is kept as wide open as possible, and load is modulated by wastegate control. Foot pedal position is used to determine load. The pressure differential (delta-P) across the throttle is minimized at part loads, thereby reducing pumping losses and increasing efficiency. The fuel is shut off during deceleration and braking conditions to provide additional improvements in fuel economy. Under these conditions, the throttle is opened, thereby increasing manifold air pressure and reducing oil consumption. 


\subsection{Air/Fuel Mixing}

A study was conducted to look at the effects of the mixing ring and elbow (mixing elbow) on lean-limit and cylinder-to-cylinder equivalence ratios. The mixing ring is an annular manifold around the inlet pipe to the engine. It is located after the intercooler and before the throttle. To aid in the air/fuel mixing, a $90^{\circ}$ elbow is connected between the mixing ring and the throttle and eight configurations were tested as shown in Table 3. The seven engine operating conditions were $100 \%, 50 \%$, and $25 \%$ load at both $1250 \mathrm{rpm}$ and $1950 \mathrm{rpm}$, and an idle condition. Equivalence ratio was measured for each individual cylinder with an UEGO sensor. The equivalence ratios for all six cylinders were averaged and a standard error was calculated.The results are shown in Table 3 for the leanest equivalence ratio without audible misfire at $1950 \mathrm{rpm}$ and $100 \%$ load, and the average of the seven standard errors for the seven engine operating conditions. Results for each load condition are shown in Figures 1 through 8. Elbow number 3 was selected for this engine because of its lowest lean-limit and low average standard error.

Table 3. Description and Results of the Mixing Elbow Configurations

\begin{tabular}{||c|c|c|c|c|c|c|c|c||}
\hline $\begin{array}{c}\text { ELBOW } \\
\text { NUMBER }\end{array}$ & $\mathbf{1}$ & $\mathbf{2}$ & $\mathbf{3}$ & $\mathbf{4}$ & $\mathbf{5}$ & $\mathbf{6}$ & $\mathbf{7}$ & $\mathbf{8}$ \\
\hline $\begin{array}{l}64 \mathrm{~mm} \text { spacer } \\
\text { between } \\
\text { elbow and } \\
\text { throttle }\end{array}$ & No & No & Yes & Yes & Yes & Yes & No & No \\
\hline $\begin{array}{l}152 \mathrm{~mm} \\
\text { spacer } \\
\text { between } \\
\text { elbow and } \\
\text { mixing ring }\end{array}$ & No & No & No & No & Yes & Yes & Yes & Yes \\
\hline $\begin{array}{l}\text { Number of } \\
\text { holes in } \\
\text { mixing ring }\end{array}$ & 18 & 9 & 9 & 18 & 18 & 9 & 9 & 18 \\
\hline $\begin{array}{l}\text { Equivalence } \\
\text { ratio limit w/o } \\
\text { audible misfire } \\
\text { at 1950 rpm, } \\
\text { 100\% load }\end{array}$ & 0.62 & 0.62 & 0.59 & 0.62 & 0.62 & 0.61 & 0.60 & 0.63 \\
\hline $\begin{array}{l}\text { Average of the } \\
\text { standard error } \\
\text { of the seven } \\
\text { load } \\
\text { conditions }\end{array}$ & 0.0127 & 0.0041 & 0.0037 & 0.0066 & 0.0129 & 0.0044 & 0.0032 & 0.0053 \\
\hline
\end{tabular}




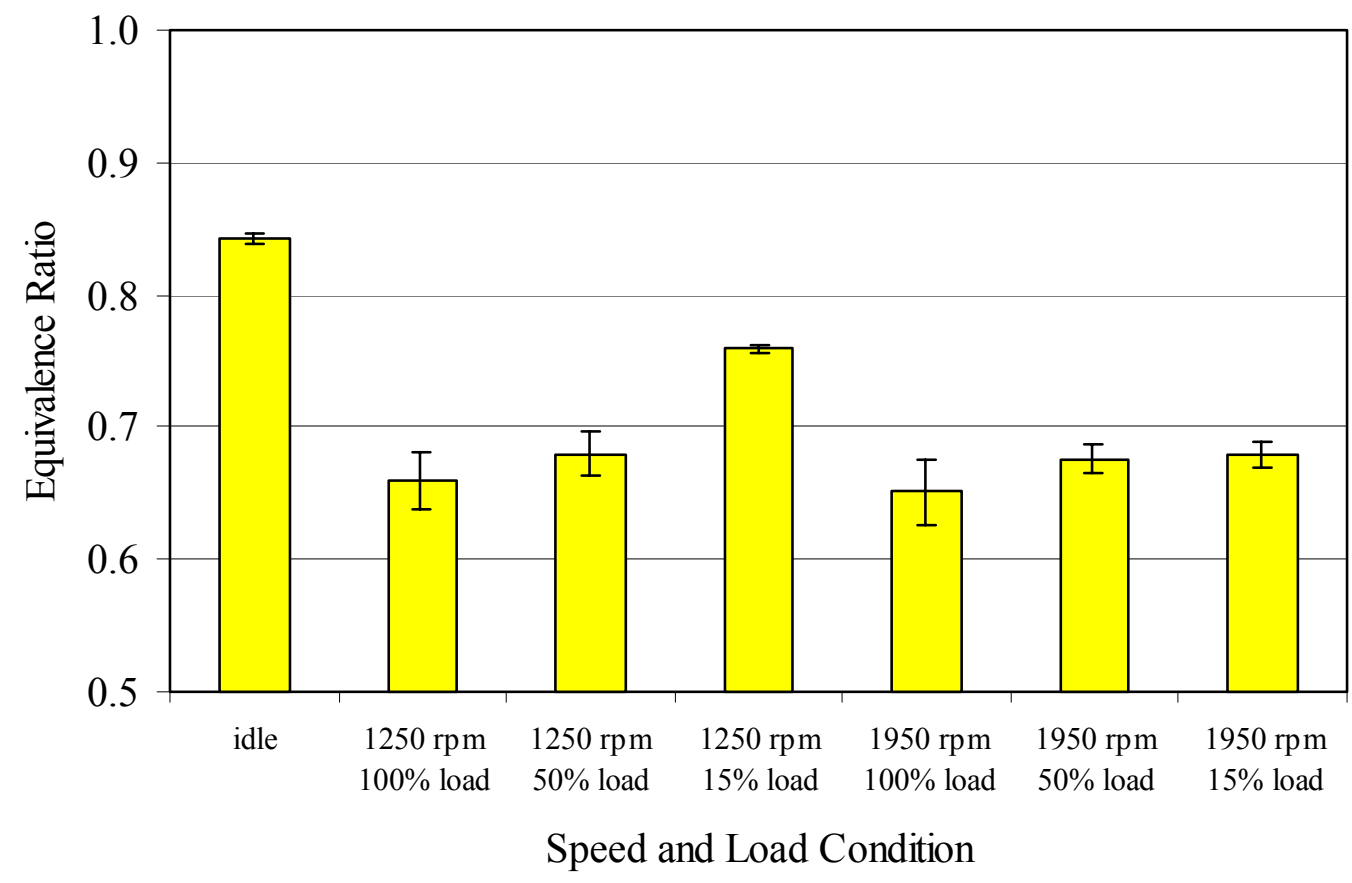

Figure 1. Average Equivalence Ratios and the Standard Error For the Six Cylinders using Mixing Elbow Number 1

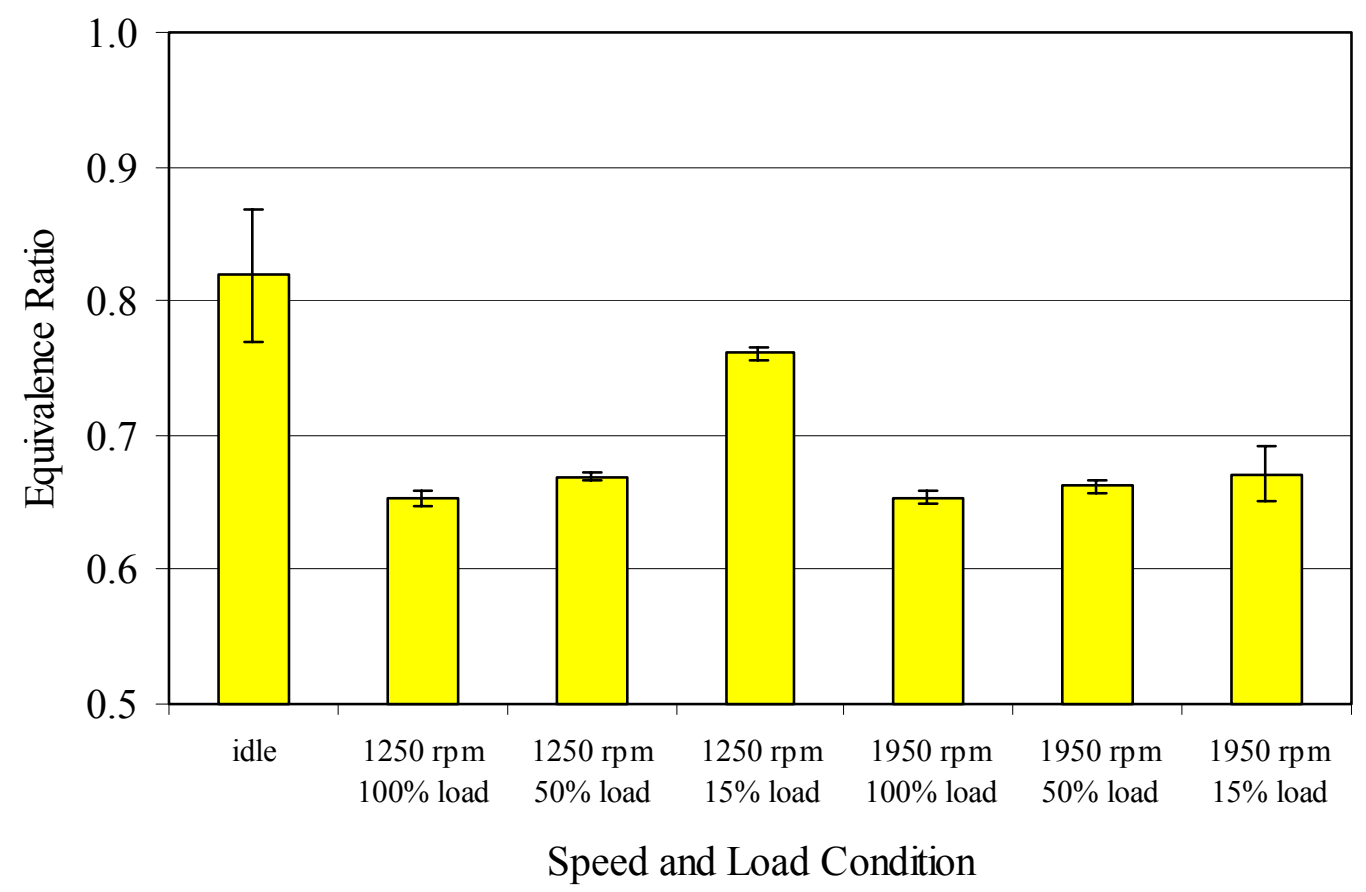

Figure 2. Average Equivalence Ratios and the Standard Error For the Six Cylinders using Mixing Elbow Number 2 


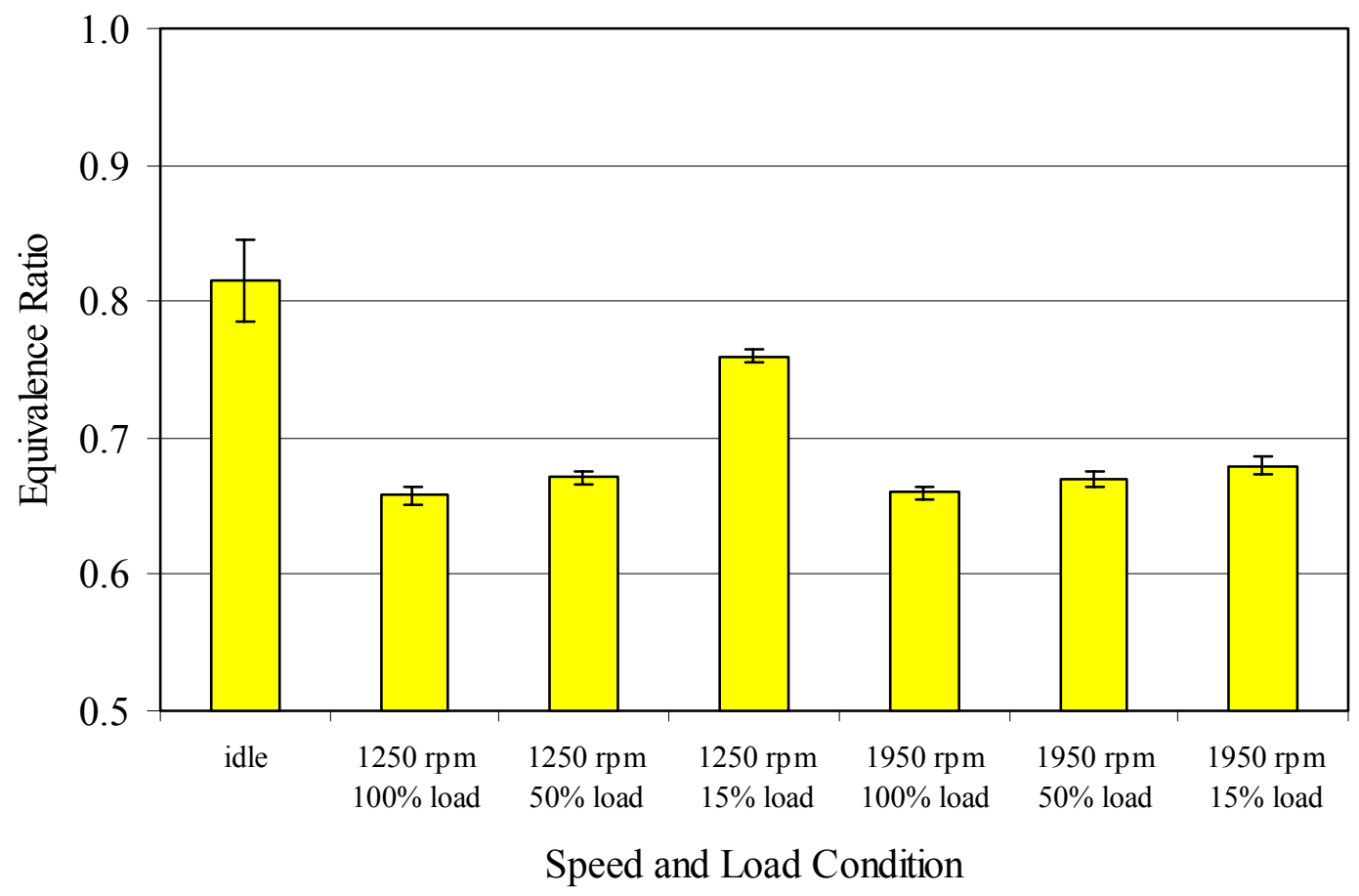

Figure 3. Average Equivalence Ratios and the Standard Error For the Six Cylinders using Mixing Elbow Number 3

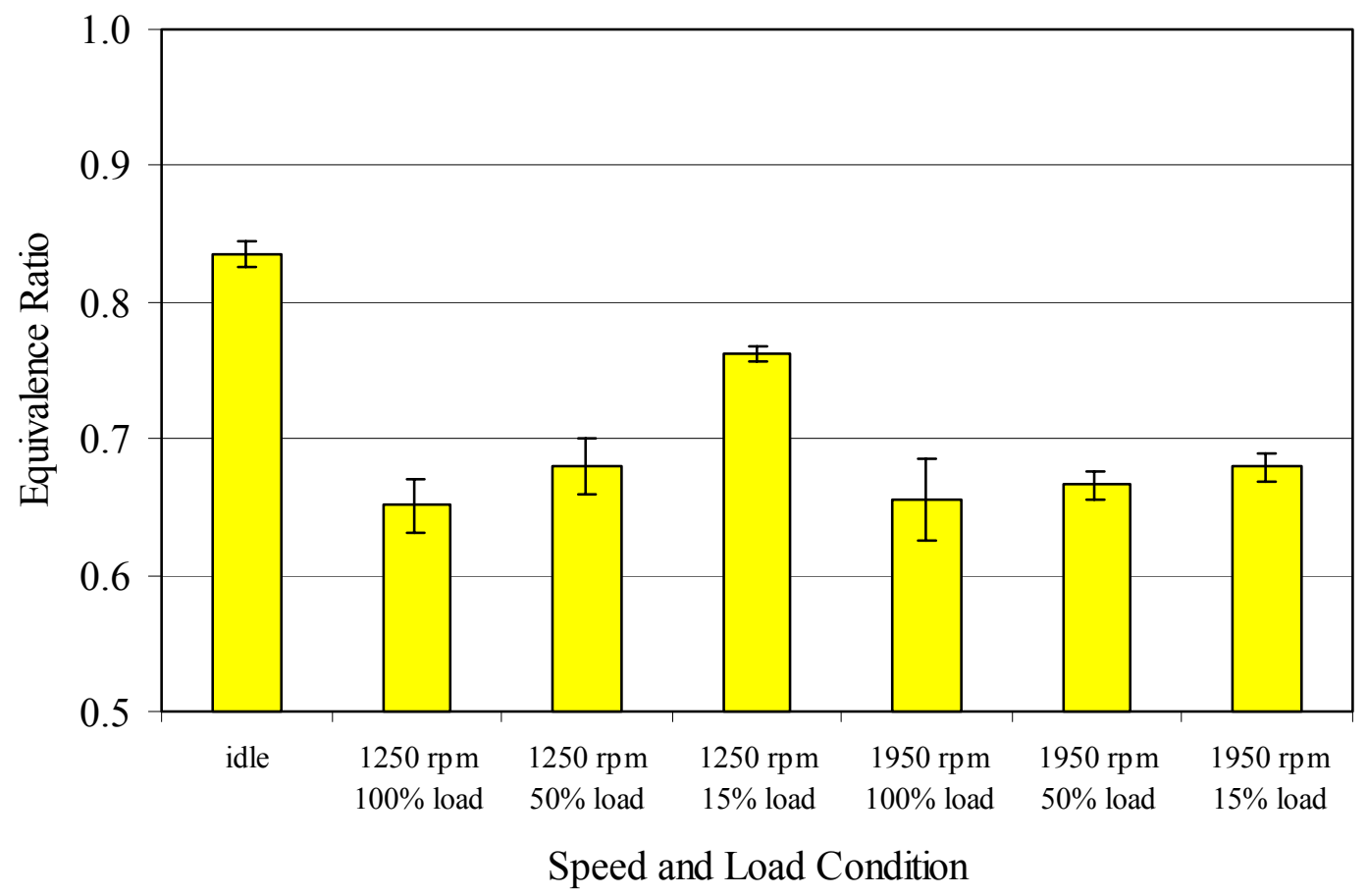

Figure 4. Average Equivalence Ratios and the Standard Error For the Six Cylinders using Mixing Elbow Number 4 


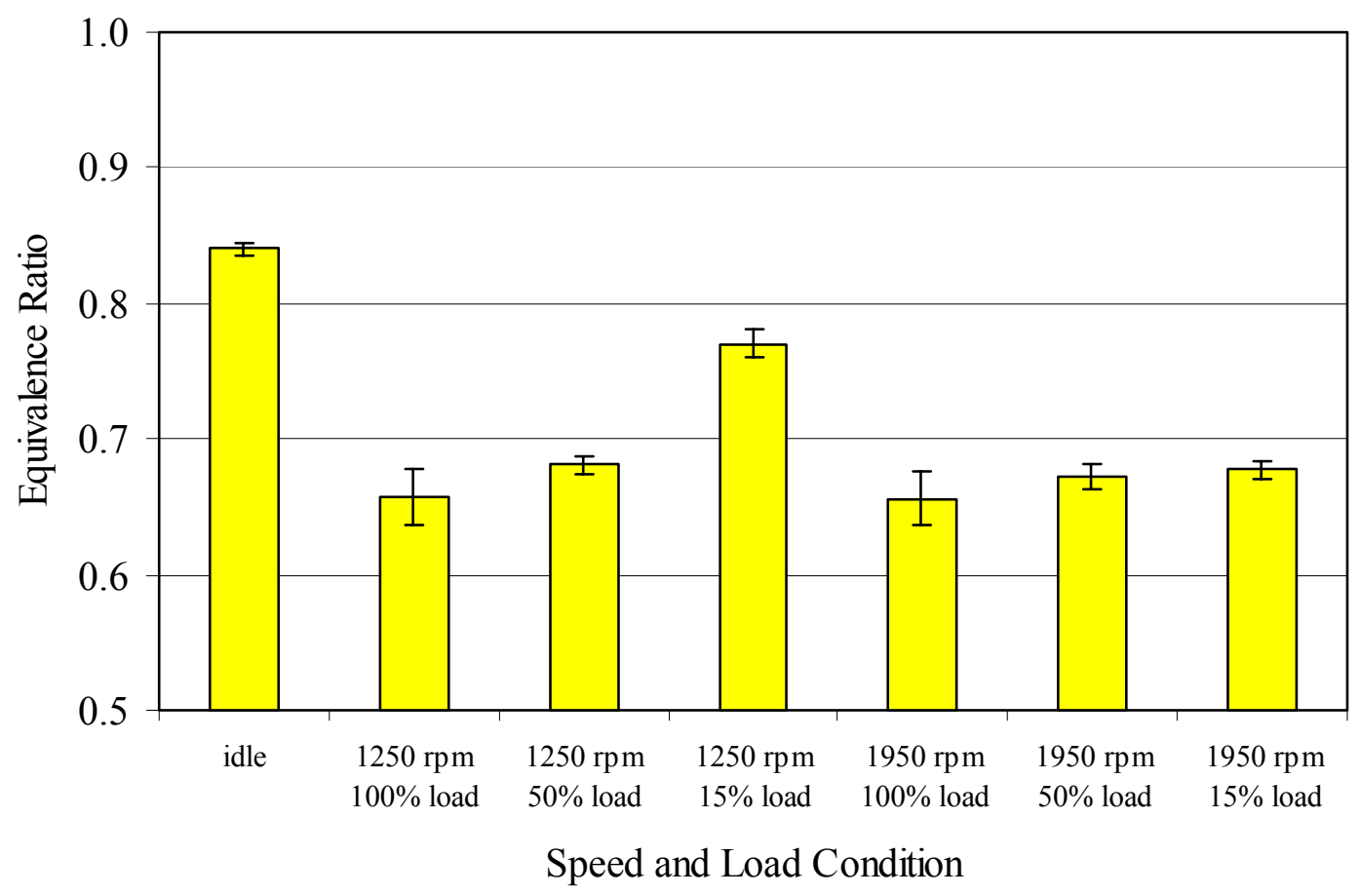

Figure 5. Average Equivalence Ratios and the Standard Error For the Six Cylinders using Mixing Elbow Number 5

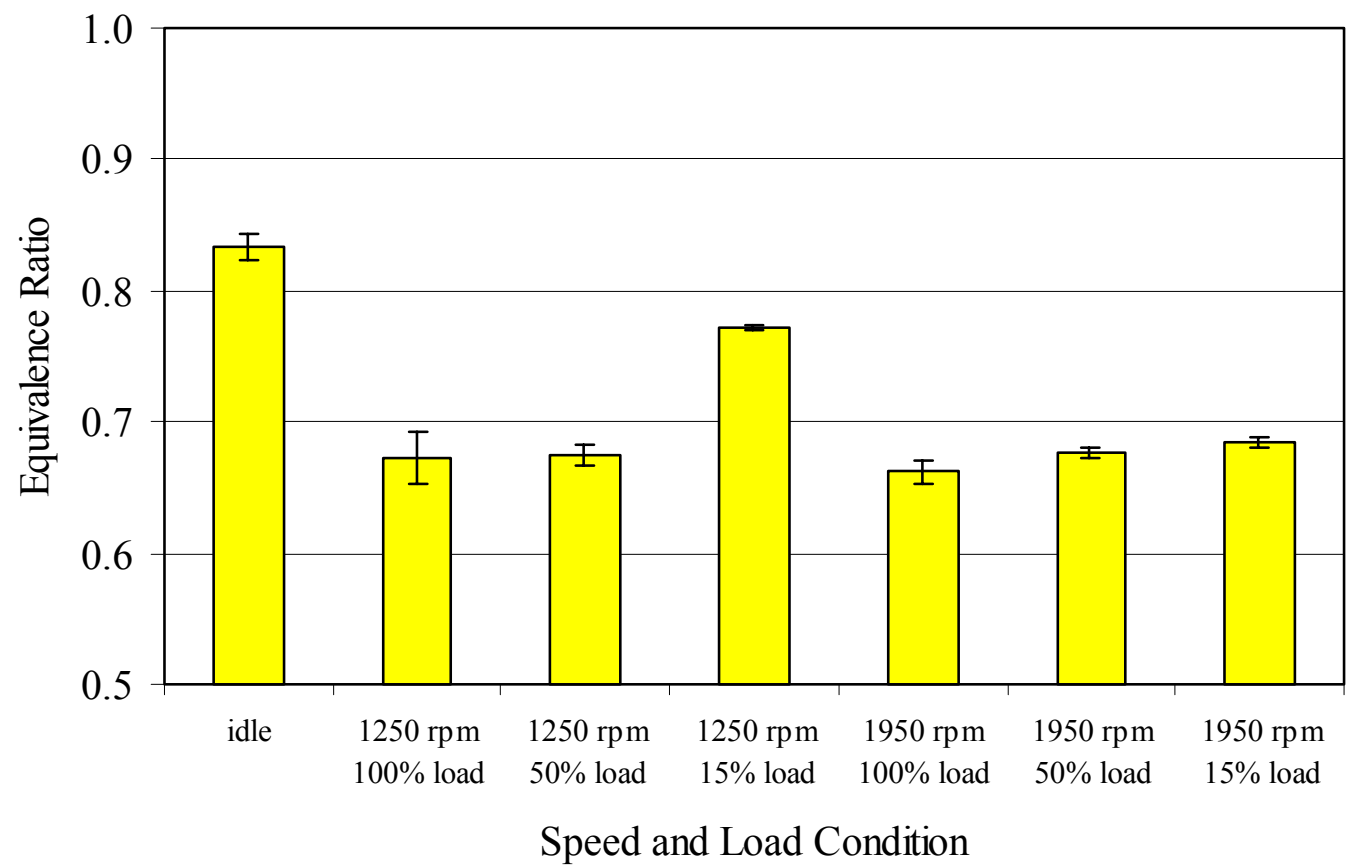

Figure 6. Average Equivalence Ratios and the Standard Error For the Six Cylinders using Mixing Elbow Number 6 


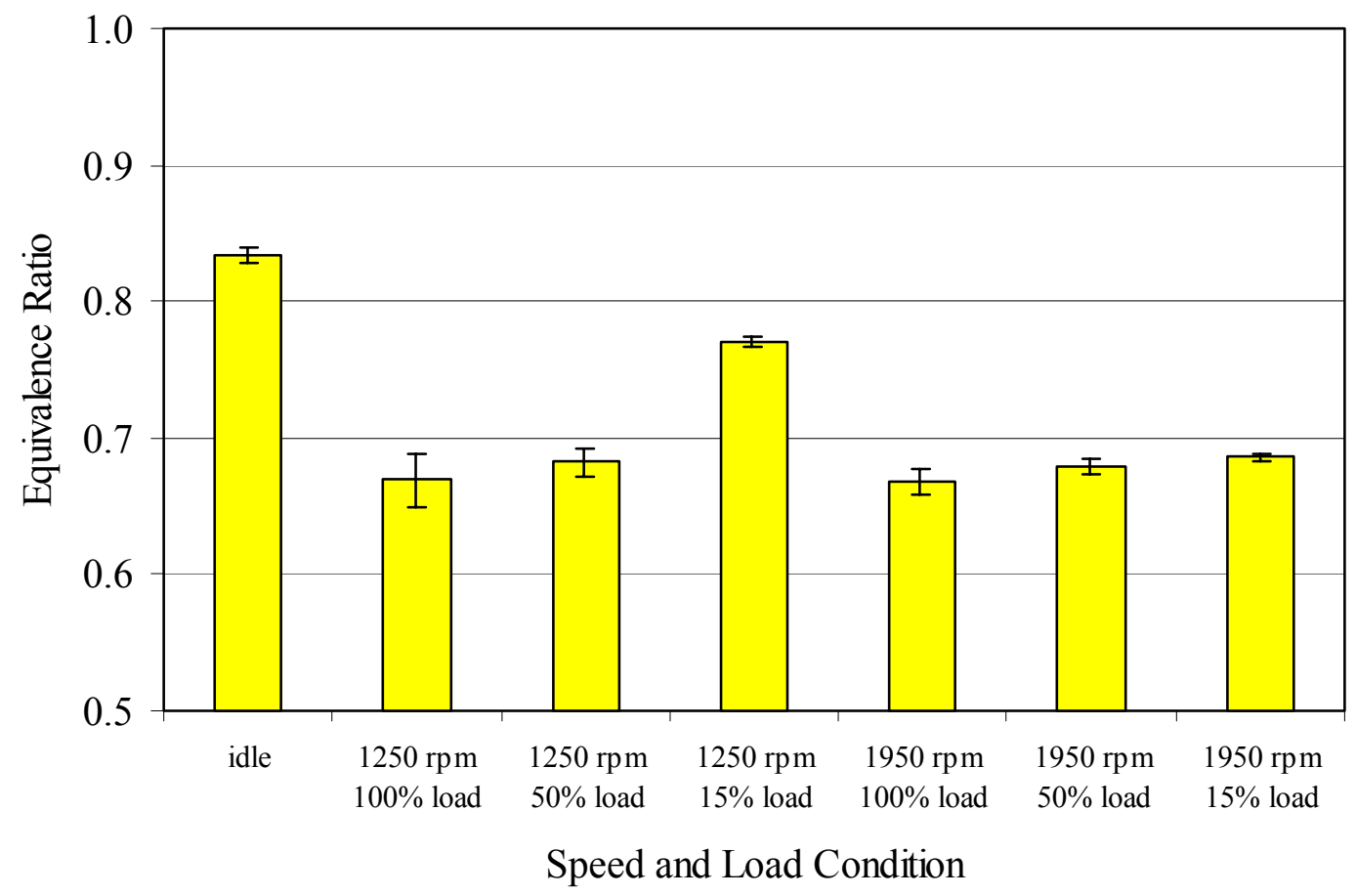

Figure 7. Average Equivalence Ratios and the Standard Error For the Six Cylinders using Mixing Elbow Number 7

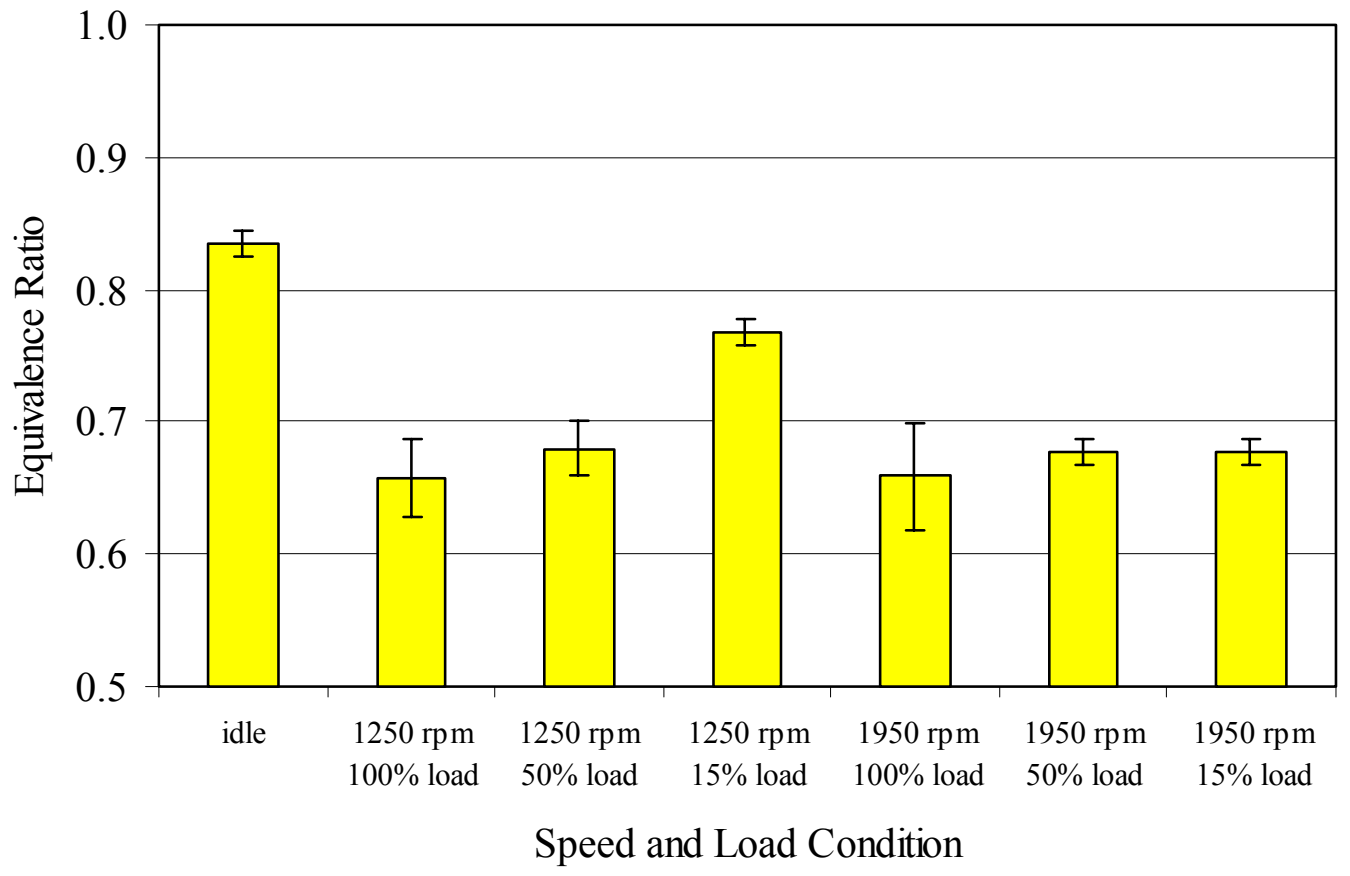

Figure 8. Average Equivalence Ratios and the Standard Error For the Six Cylinders using Mixing Elbow Number 8 


\subsection{Cam Timing}

Another project, funded by Mack Trucks, was conducted concurrently with the Mack E7G project. A separate engine was used for each project, and one engine showed higher torque at low engine speeds than the other engine. Mack Trucks had mis-machined a cam gear such that it advanced the cam by one tooth. Therefore, a study was conducted to determine the optimal cam timing for this engine; the cam profile was the same as the diesel engine it was derived from. The cam timing was tested at the original diesel cam timing and advanced one tooth on the cam gear. One tooth advanced is 8.57 crank angle degrees in an 84-tooth cam gear. Figures 9 through 17 show the results for 1950, 1500, and $1250 \mathrm{rpm}$ at $100 \%, 75 \%$, and $50 \%$ load. For either cam timing, the efficiencies are about the same for a given $\mathrm{NO}_{\mathrm{x}}$ level, whereas the $\mathrm{HC}$ emissions are lower with the original cam timing. With the advanced cam timing, higher torque at $1100 \mathrm{rpm}$ at WOT were noted. The advanced cam timing was selected for this engine at the higher low-speed torque capability.

\subsection{Steady-State Engine Mapping}

Engine mapping included knock tolerance testing, lean-limit testing, and calibration of the engine controller tables.

\subsubsection{Knock Tolerance Testing}

Knock tolerance testing for the Mack E7G engine was presented in the final report for the Mack LNG Engine Development for Long-Haul Truck, SwRI Project No. 03-7816, and is presented again here. Results are based on an engine rated at $350 \mathrm{bhp}$ at $1900 \mathrm{rpm}$.

Knock testing was performed to determine the highest intake manifold air temperature, highest equivalence ratio, and the most advance ignition timing possible without the engine knocking. The testing was conducted at various equivalence ratios, 105 and $150 \operatorname{deg} \mathrm{F}$ air temperature after the intercooler, and 200 deg $\mathrm{F}$ coolant out temperature. Ignition timing was advanced until the pressure signal from the number two cylinder showed presence of knock. The composition of the natural gas fuel used for knock testing is shown in Table 4.

Table 4. Gas Composition for Knock Testing

\begin{tabular}{||l|l||}
\hline Gas & Volume (\%) \\
\hline \hline Methane & 85.1 \\
\hline Ethane & 9.0 \\
\hline Propane & 2.4 \\
\hline Nitrogen & 3.5 \\
\hline
\end{tabular}




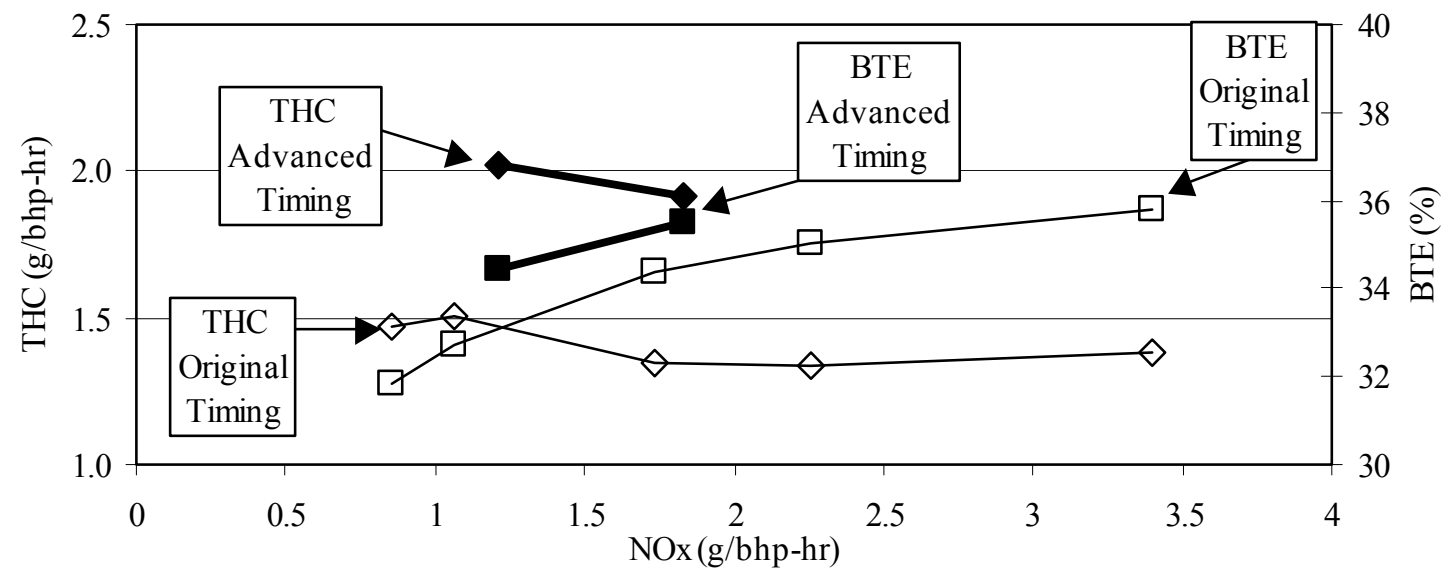

Figure 9. Comparison of Original and Advanced Cam Timing

At $1950 \mathrm{rpm}, 100 \%$ Load, and Various Ignition Timings

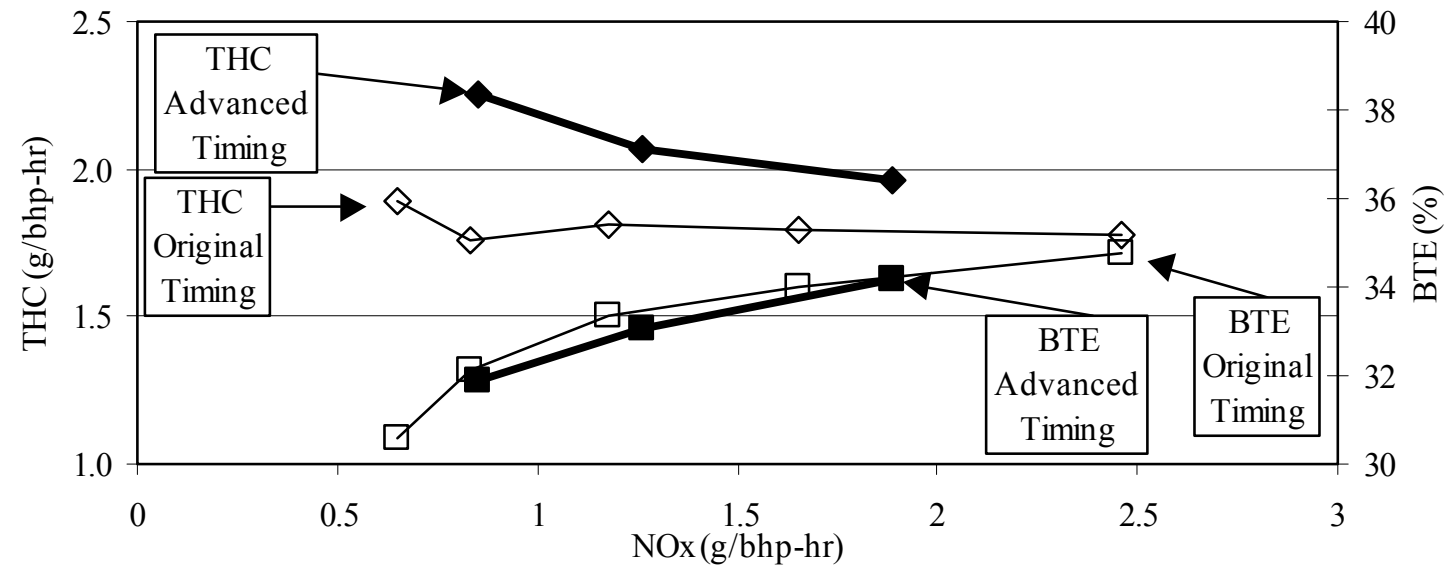

Figure 10. Comparison of Original and Advanced Cam Timing At $1950 \mathrm{rpm}, \mathbf{7 5 \%}$ Load, and Various Ignition Timings

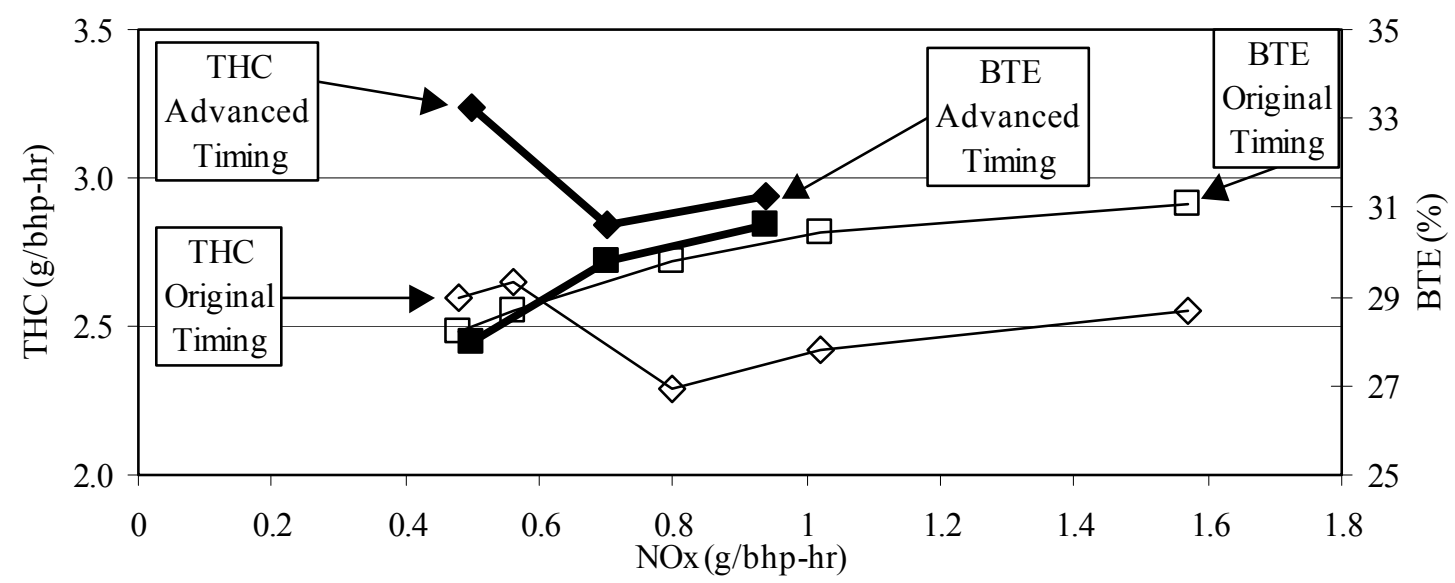

Figure 11. Comparison of Original and Advanced Cam Timing At $1950 \mathrm{rpm}, \mathbf{5 0 \%}$ Load, and Various Ignition Timings 


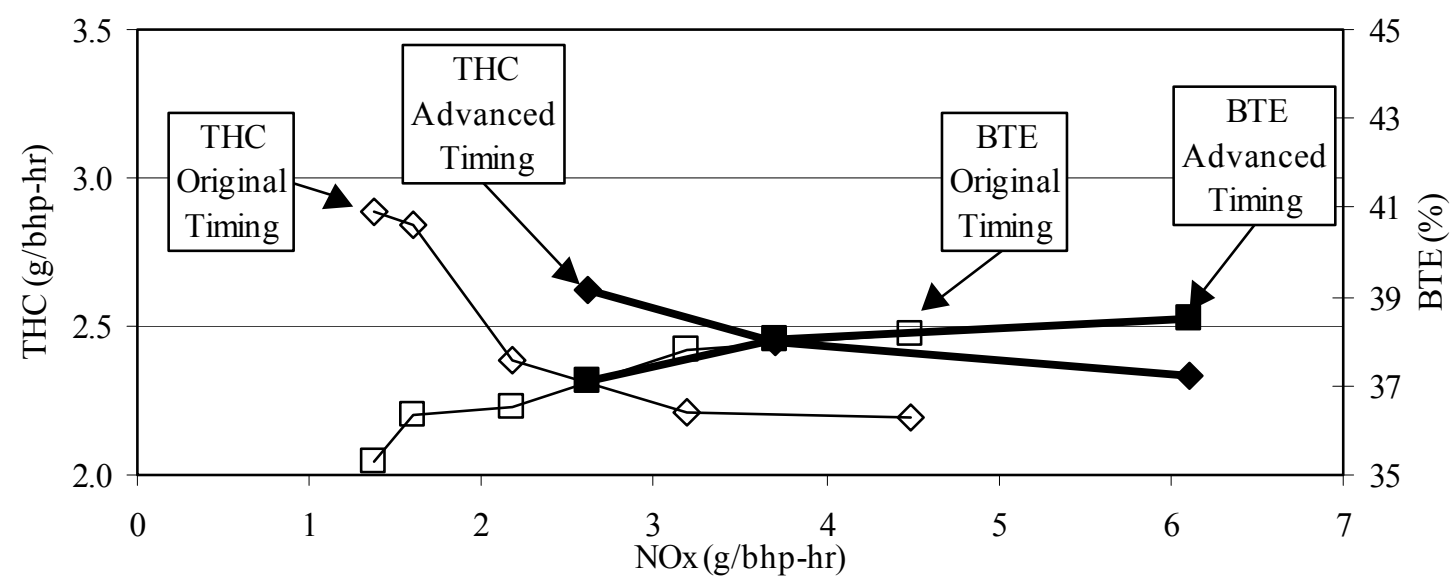

Figure 12. Comparison of Original and Advanced Cam Timing At 1500 rpm, 100\% Load, and Various Ignition Timings

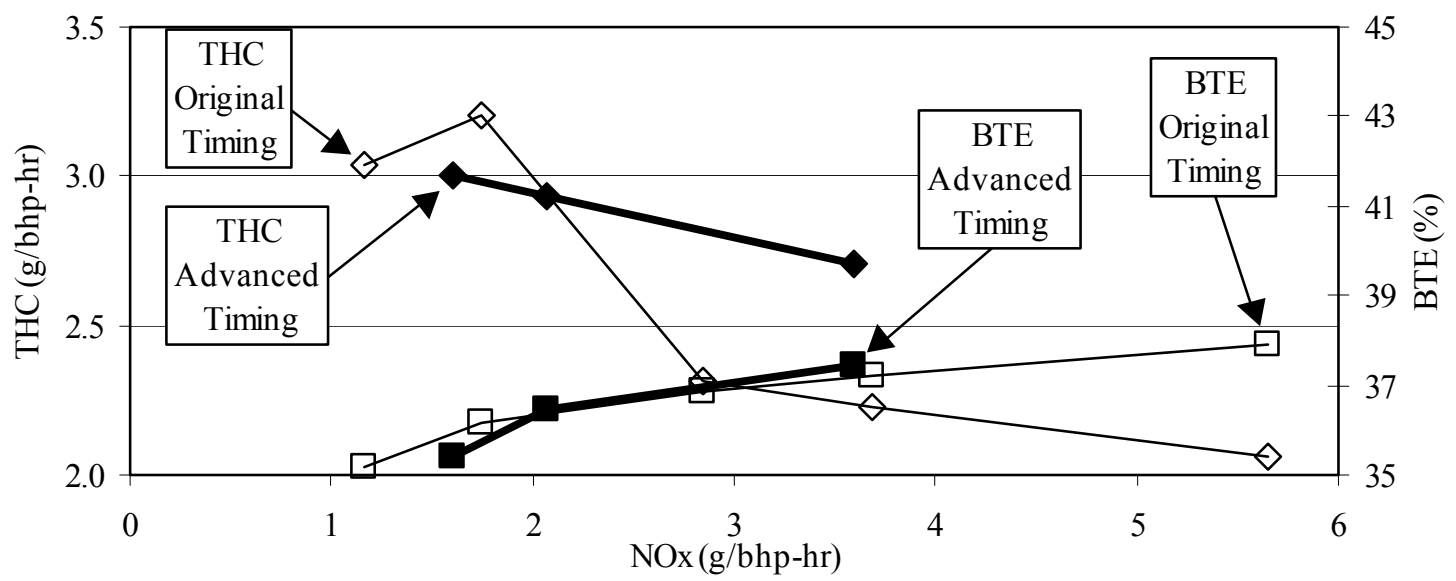

Figure 13. Comparison of Original and Advanced Cam Timing At $1500 \mathrm{rpm}, \mathbf{7 5 \%}$ Load, and Various Ignition Timings

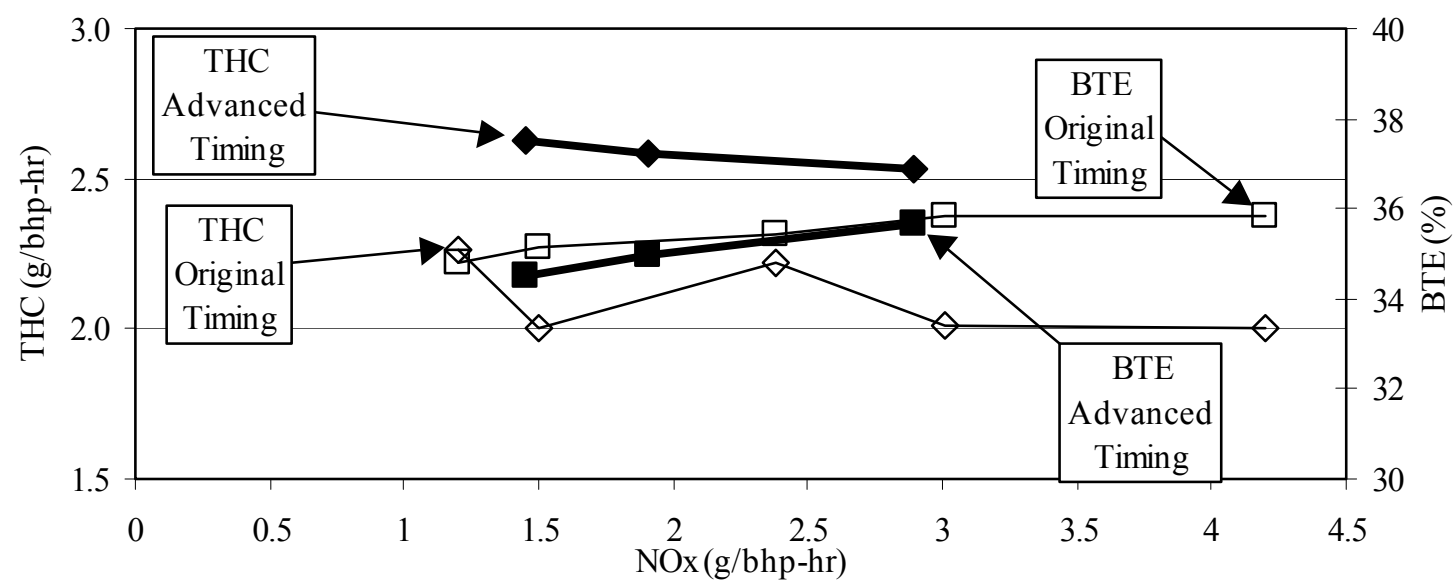

Figure 14. Comparison of Original and Advanced Cam Timing At $1500 \mathrm{rpm}, \mathbf{5 0 \%}$ Load, and Various Ignition Timings 


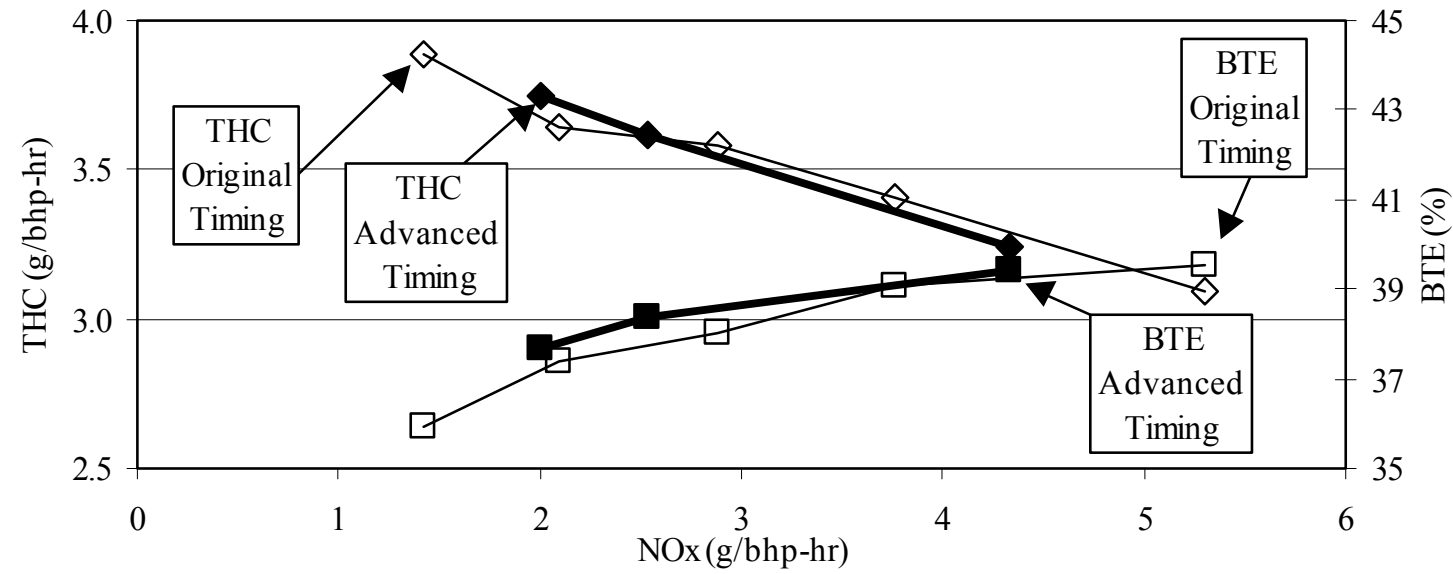

Figure 15. Comparison of Original and Advanced Cam Timing At $1250 \mathrm{rpm}, \mathbf{1 0 0 \%}$ Load, and Various Ignition Timings

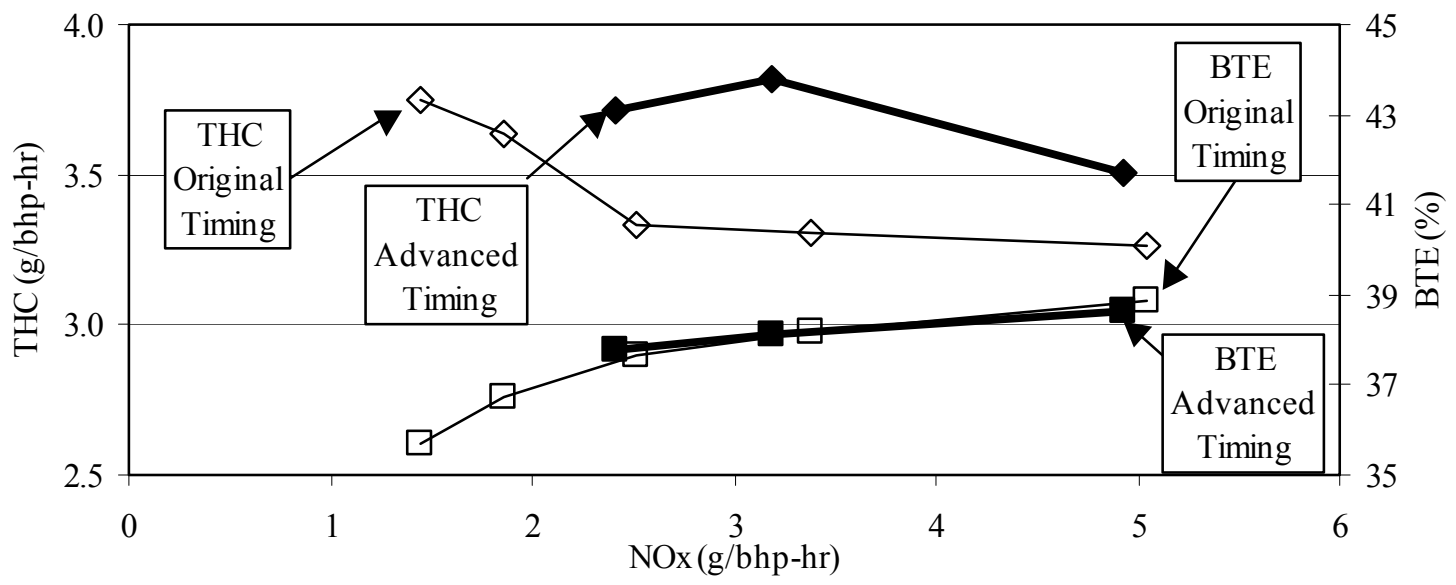

Figure 16. Comparison of Original and Advanced Cam Timing At $1250 \mathrm{rpm}, \mathbf{7 5 \%}$ Load, and Various Ignition Timings

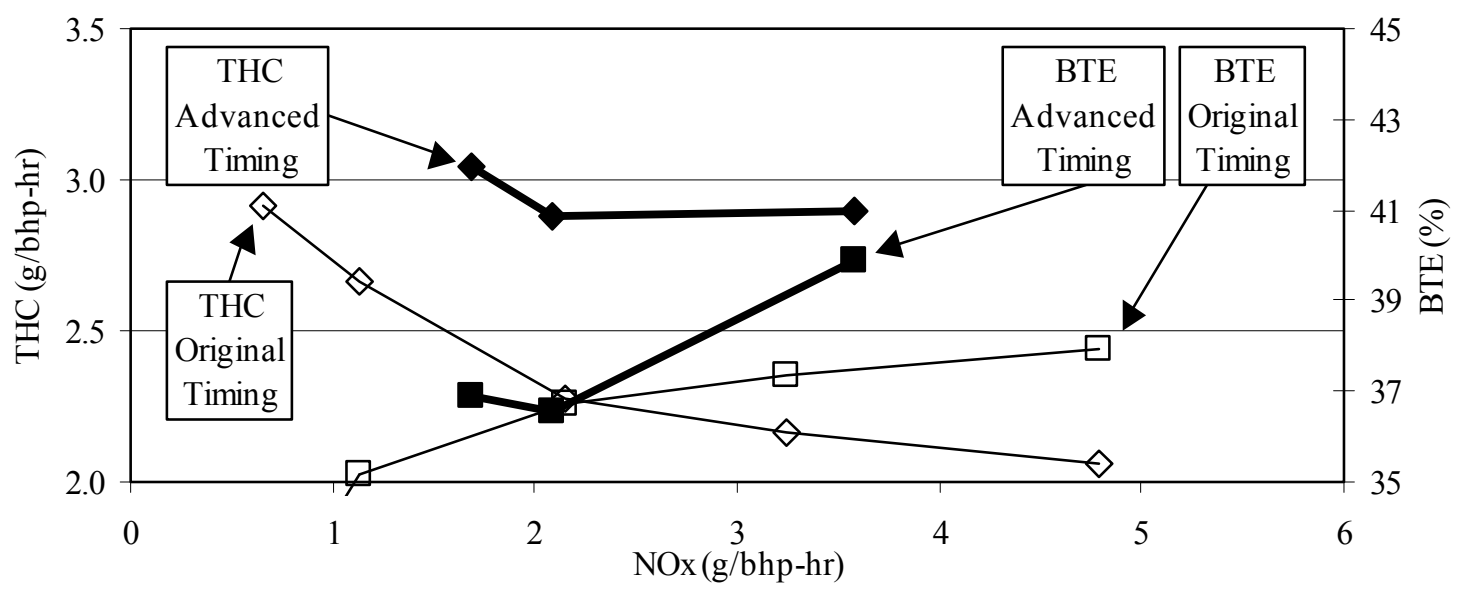

Figure 17. Comparison of Original and Advanced Cam Timing At 1250 rpm, 50\% Load, and Various Ignition Timings. 
The results of the knock testing are shown in Table 5 and in Figures 18 through 22. Figures 18 through 20 show tests at equivalence ratios between 0.65 and 0.75 for engine speed of 1900,1500 , and 1250 rpm. Also shown on the graphs as reference are the points of MBT and MBT-6 ignition timings at an equivalence ratio of 0.65 . Figures 21 and 22 show tests at equivalence ratios between 0.80 and 0.85 for engine speeds of 1100 and $1000 \mathrm{rpm}$. There are no ignition timing points shown on these graphs since timing are selected to provide sufficient boost for low speed torque.

Table 5. Results of Knock Testing

\begin{tabular}{|c|c|c|c|}
\hline $\begin{array}{c}\text { Engine Speed } \\
\text { (rpm) }\end{array}$ & $\begin{array}{c}\text { Equivalence } \\
\text { Ratio }\end{array}$ & $\begin{array}{l}\text { Ignition Timing at } \\
\text { Presence of Knock } \\
\text { for } 105 \text { deg F After } \\
\text { Intercooler } \\
\text { Temperature } \\
\text { (deg BTDC) }\end{array}$ & $\begin{array}{l}\text { Ignition Timing at } \\
\text { Presence of Knock } \\
\text { for } 150 \text { deg F After } \\
\text { Intercooler } \\
\text { Temperature } \\
\text { (deg BTDC) }\end{array}$ \\
\hline 1900 & 0.65 & 30 & 23 \\
\hline 1900 & 0.70 & 23 & 19 \\
\hline 1900 & 0.75 & 19 & 15 \\
\hline 1500 & 0.65 & 25 & 20 \\
\hline 1500 & 0.70 & 22 & 17 \\
\hline 1500 & 0.75 & 18 & 16 \\
\hline 1250 & 0.67 & 23 & 18 \\
\hline 1250 & 0.70 & 22 & 17 \\
\hline 1250 & 0.75 & 19 & 15 \\
\hline 1100 & 0.80 & 18 & 15 \\
\hline 1100 & 0.85 & 14 & 10 \\
\hline 1000 & 0.80 & 19 & 12 \\
\hline 1000 & 0.85 & 17 & 9 \\
\hline
\end{tabular}




\subsubsection{Lean-Limit Testing}

Lean-limit testing for the Mack E7G engine was presented in the final report Mack LNG Engine Development for Long-Haul Truck, SwRI Project No. 03-7816, and is presented again here. Results are based on an engine rated at $350 \mathrm{bhp}$ at $1900 \mathrm{rpm}$.

Lean-limit tests were conducted at engine speeds of 1250 and $1900 \mathrm{rpm}$, and $100 \%$ load. Cylinder pressure data were recorded from the \#2 cylinder, and the $\mathrm{COV}_{\text {imep }}$ was calculated over 50 engine cycles. The lean limit is defined as the equivalence ratio where the $\mathrm{COV}_{\text {imep }}$ exceeds $5 \%$. Tests were performed at three ignition timings: MBT, MBT-3, and MBT-6, and the equivalence ratio was lowered until audible misfire was detected. Figure 23 shows the results for $1250 \mathrm{rpm}$. At an ignition timing of 16 degrees BTDC, the lean limit is at an equivalence ratio of approximately 0.58 . Figure 24 shows the results for $1900 \mathrm{rpm}$. At an ignition timing of $21 \mathrm{deg}$ BTDC, the lean limit is at an equivalence ratio of approximately 0.60 . Table 6 shows the results of the lean-limit tests. The run numbers refer to the recorded data included in Appendix A. 


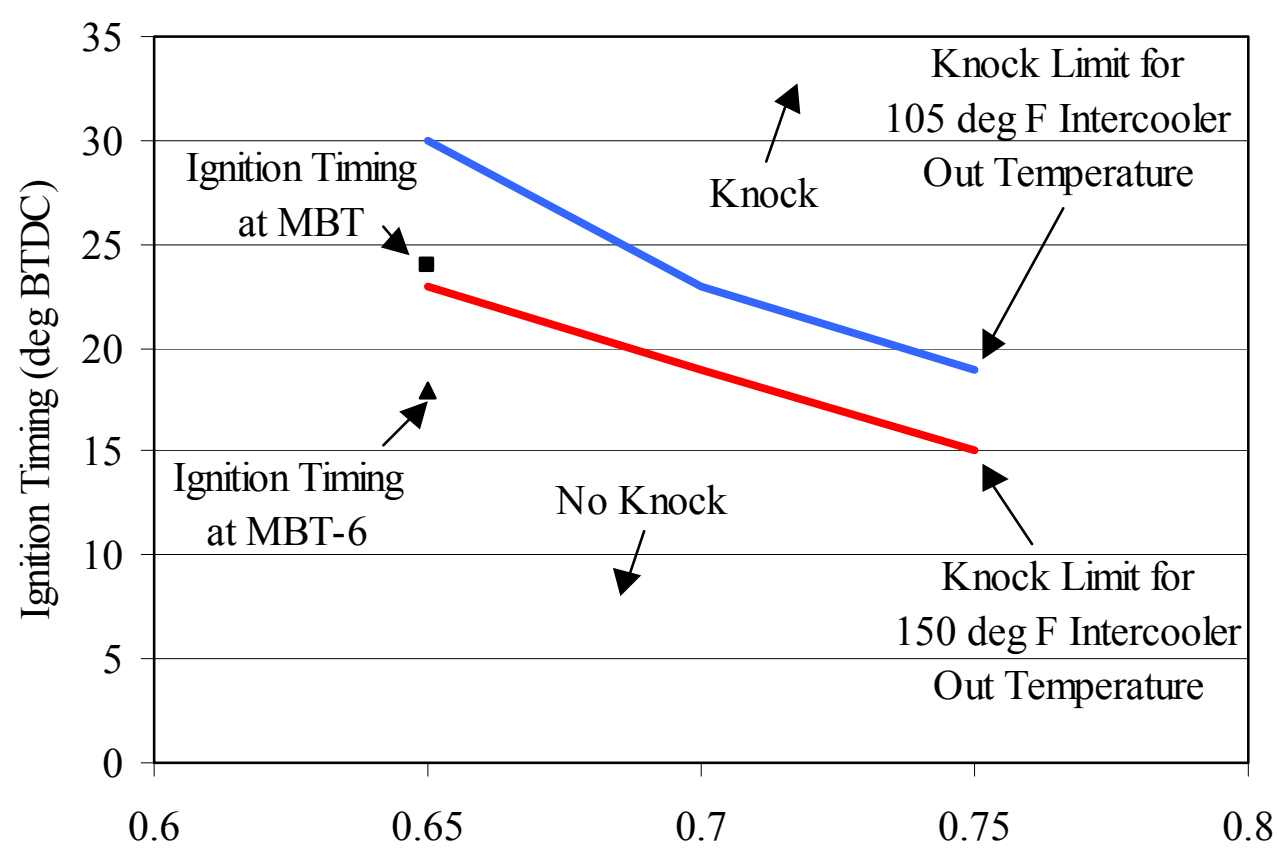

Equivalence Ratio

Figure 18. Knock Limit at $1900 \mathrm{rpm}, 967 \mathrm{ft}-\mathrm{lb}$.

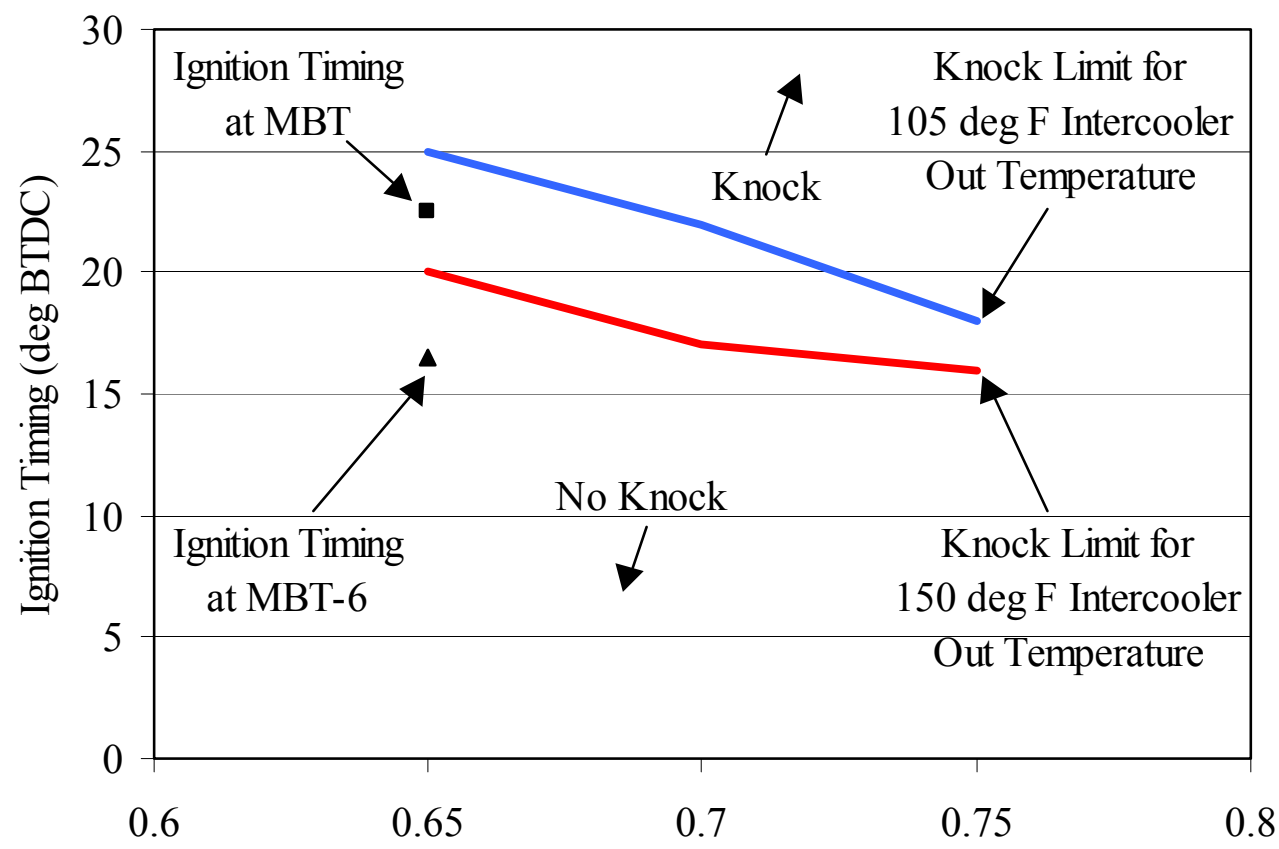

Equivalence Ratio

Figure 19. Knock Limit at 1500 rpm, 1175 ft-lb. 


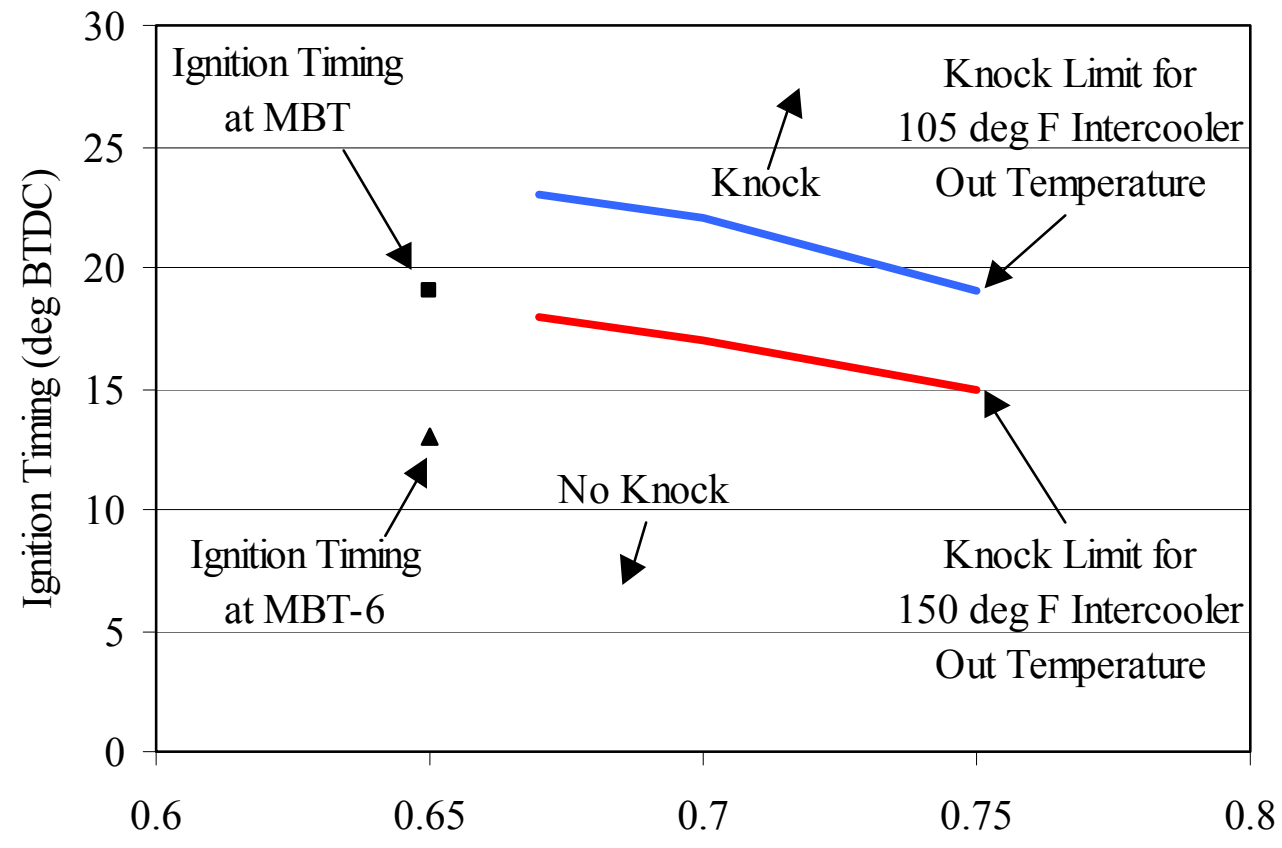

Equivalence Ratio

Figure 20. Knock Limit at 1250 rpm, $1250 \mathrm{ft}-\mathrm{lb}$.

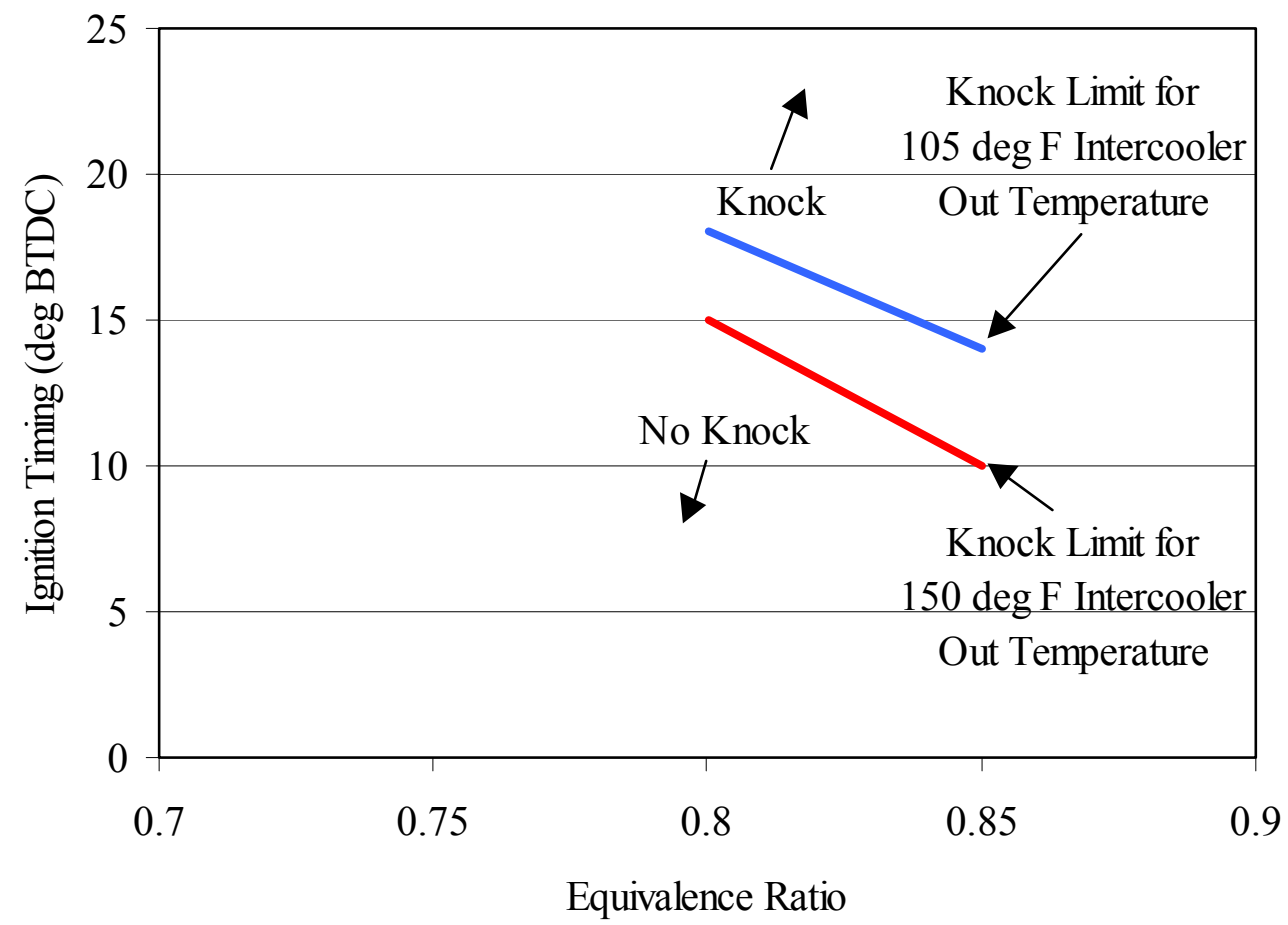

Figure 21. Knock Limit at $1100 \mathrm{rpm}, 1230 \mathrm{ft}-\mathrm{lb}$. 


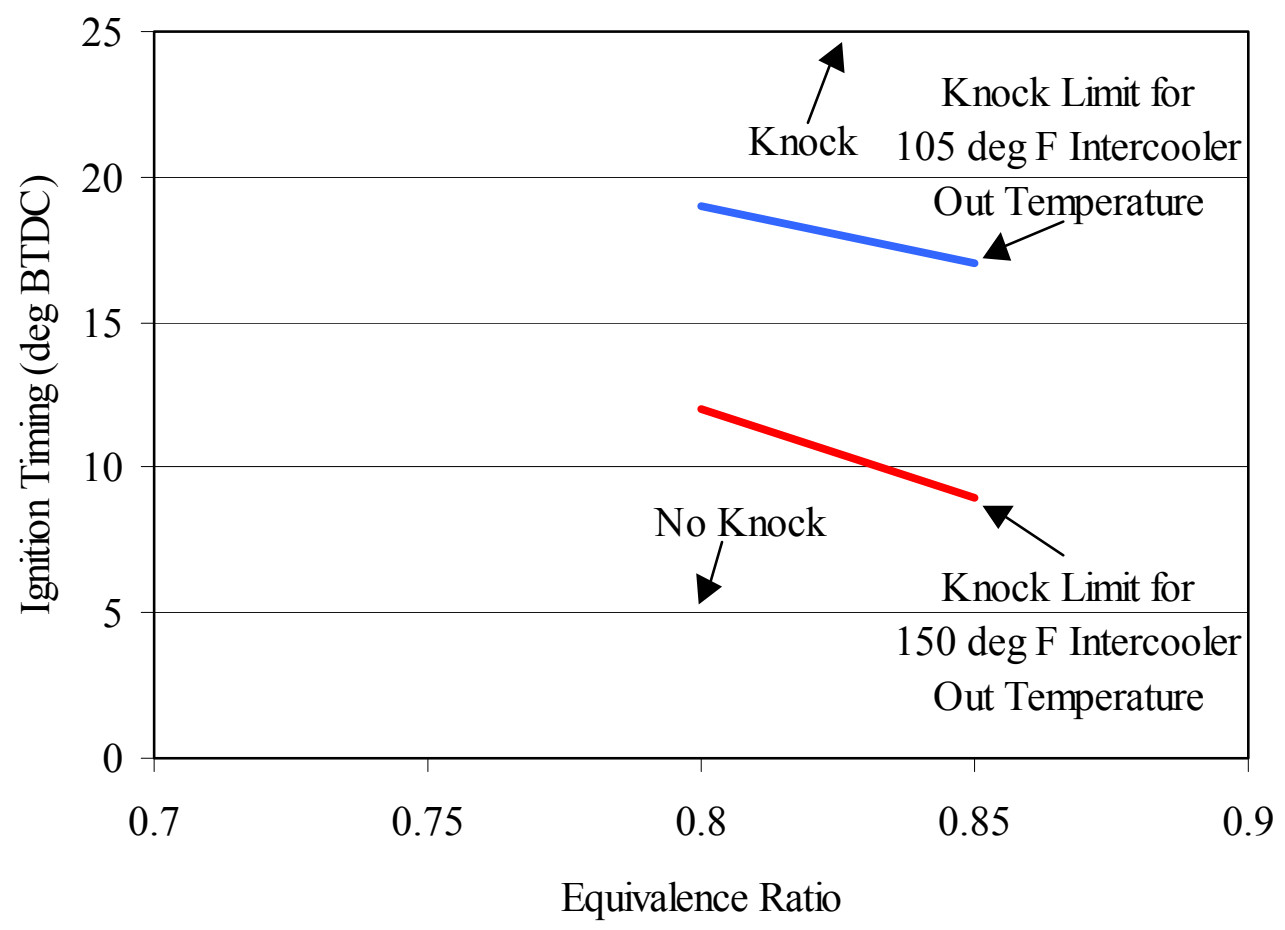

Figure 22. Knock Limit at 1000 rpm, 1115 ft-lb. 
Table 6. Lean Limit Test Results

\begin{tabular}{|c|c|c|c|c|c|c|c|}
\hline \multicolumn{4}{|c|}{1250 RPM } & \multicolumn{4}{|c|}{1900 RPM } \\
\hline $\begin{array}{c}\text { Run } \\
\text { Number }\end{array}$ & $\begin{array}{c}\text { Ignition } \\
\text { Timing } \\
\text { ( }{ }^{\circ} \text { BTDC) }\end{array}$ & $\begin{array}{l}\text { Equiv. } \\
\text { Ratio }\end{array}$ & $\begin{array}{c}\mathrm{COV}_{\text {imep }} \\
(\%)\end{array}$ & $\begin{array}{c}\text { Run } \\
\text { Number }\end{array}$ & $\begin{array}{c}\text { Ignition } \\
\text { Timing } \\
\text { ( }{ }^{\circ} \text { BTDC) }\end{array}$ & $\begin{array}{l}\text { Equiv. } \\
\text { Ratio }\end{array}$ & $\begin{array}{c}\mathrm{COV}_{\text {imep }} \\
(\%)\end{array}$ \\
\hline 866 & 19 & 0.65 & 1.24 & 844 & 24 & 0.65 & 1.62 \\
\hline 867 & 16 & 0.65 & 1.93 & 845 & 21 & 0.65 & 2.06 \\
\hline 868 & 13 & 0.65 & 3.58 & 846 & 18 & 0.65 & 2.93 \\
\hline 869 & 19 & 0.67 & 1.24 & 847 & 24 & 0.67 & 1.21 \\
\hline 870 & 16 & 0.67 & 1.77 & 848 & 21 & 0.67 & 2.00 \\
\hline 871 & 13 & 0.67 & 2.23 & 849 & 18 & 0.67 & 2.22 \\
\hline 872 & 19 & 0.64 & 1.55 & 850 & 24 & 0.64 & 1.99 \\
\hline 873 & 16 & 0.64 & 2.31 & 851 & 21 & 0.64 & 2.95 \\
\hline 874 & 13 & 0.64 & 4.82 & 852 & 18 & 0.64 & 3.53 \\
\hline 875 & 19 & 0.63 & 1.69 & 853 & 24 & 0.63 & 2.11 \\
\hline 876 & 16 & 0.63 & 2.09 & 854 & 21 & 0.63 & 2.49 \\
\hline 877 & 13 & 0.63 & 4.11 & 855 & 18 & 0.63 & 3.88 \\
\hline 878 & 19 & 0.62 & 1.96 & 856 & 24 & 0.62 & 1.80 \\
\hline 879 & 16 & 0.62 & 4.21 & 857 & 21 & 0.62 & 3.36 \\
\hline 880 & 13 & 0.62 & 4.27 & 858 & 18 & 0.62 & 4.53 \\
\hline 881 & 19 & 0.61 & 1.98 & 859 & 24 & 0.61 & 2.79 \\
\hline 882 & 16 & 0.61 & 3.51 & 860 & 21 & 0.61 & 3.88 \\
\hline 883 & 13 & 0.61 & 5.55 & 861 & 18 & 0.61 & 5.31 \\
\hline 884 & 19 & 0.60 & 3.08 & 862 & 24 & 0.60 & 3.36 \\
\hline 885 & 16 & 0.60 & 3.45 & 863 & 21 & 0.60 & 4.74 \\
\hline 886 & 13 & 0.60 & 7.32 & 864 & 18 & 0.60 & 6.32 \\
\hline 887 & 19 & 0.59 & 2.51 & 865 & 24 & 0.59 & 4.89 \\
\hline 888 & 16 & 0.59 & 3.76 & & & & \\
\hline 889 & 13 & 0.59 & 5.38 & & & & \\
\hline 890 & 19 & 0.58 & 4.06 & & & & \\
\hline 891 & 16 & 0.58 & 5.53 & & & & \\
\hline
\end{tabular}




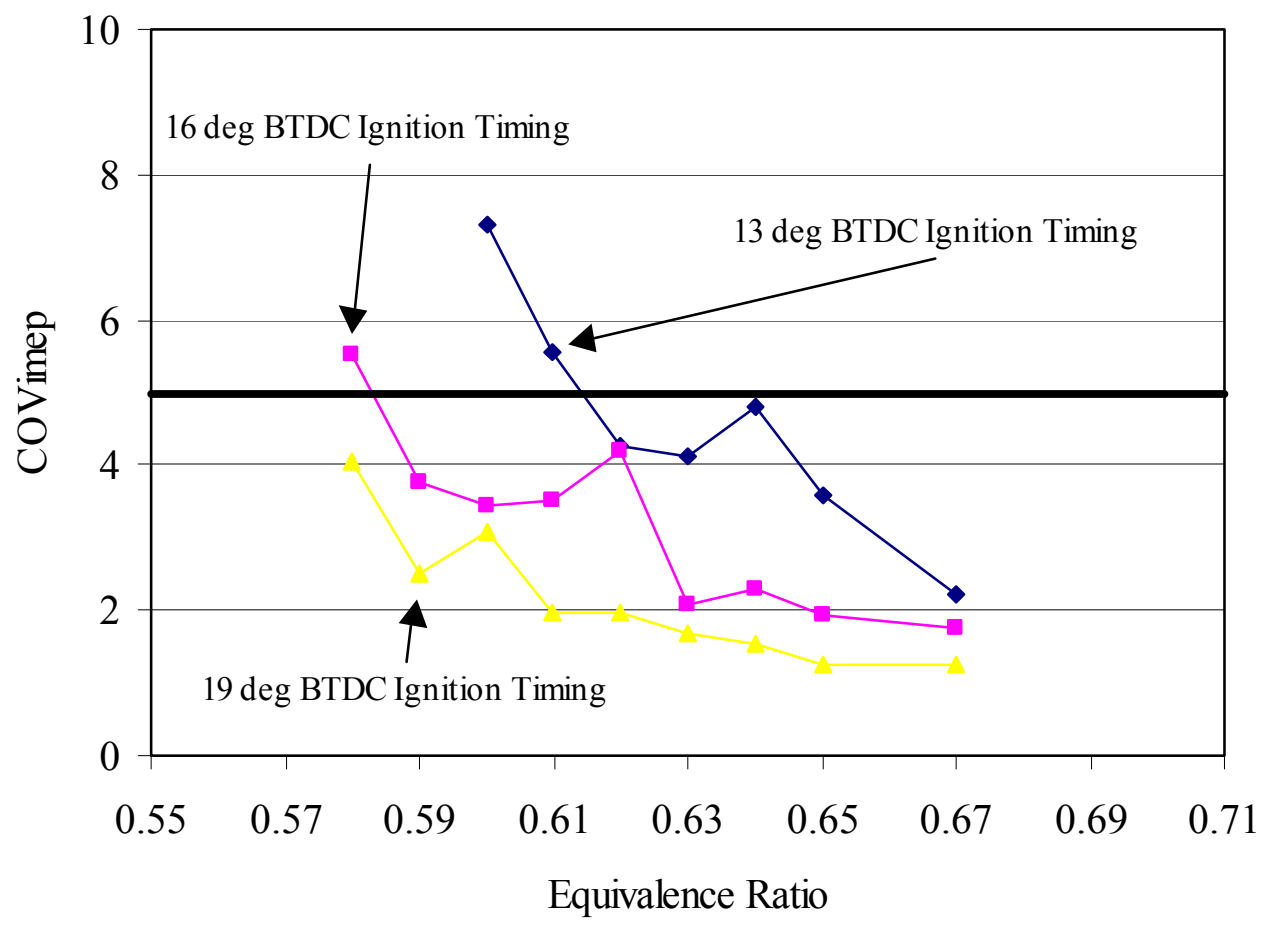

Figure 23. Lean Limit Tests at $1250 \mathrm{rpm}, 1250 \mathrm{ft}-\mathrm{lb}$

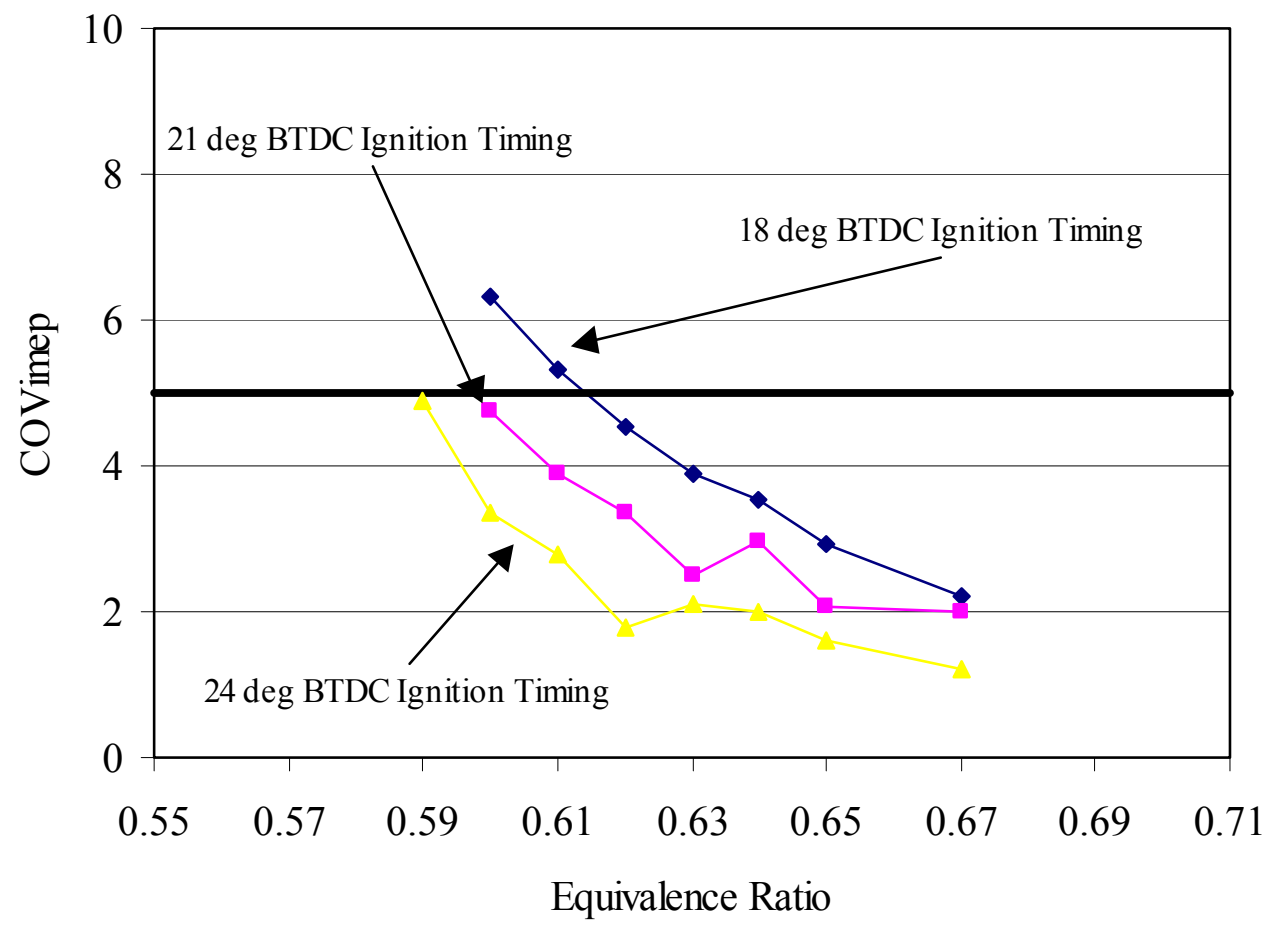

Figure 24. Lean Limit Tests at 1900 rpm, $967 \mathrm{ft}-\mathrm{lb}$ 


\subsubsection{Calibration}

Steady-state tests of the Mack E7G engine included calibration of boost control, volumetric efficiency, equivalence ratio, and ignition timing. Transient tests included further calibration of equivalence ratio and ignition timing, calibration of boost control to rematch the 325 bhp torque curve with the change in ignition timing at WOT, and calibration of the turbo lag compensation.

Turbocharger boost determines the power output of the engine for a given throttle position. It is controlled through a pneumatic wastegate controller. Since Mack Trucks builds the engines and vehicles, a source of pressurized air can be made available on the truck for the engine controller to use under any operating condition. In the test stand, shop air was used to simulate the constant source of pressurized air. The pressurized air to the wastegate actuator is controlled through a pulse width modulated solenoid valve. The engine controller determines the amount of turbocharger boost based on throttle position and engine speed through a lookup table. An electronic throttle is used on this engine with a throttle command of 0.5 to 4.5 volts.

At throttle positions of less than 2.0 volts, the wastegate is fully opened to minimize throttling losses. At positions of 2.0 to about 4.0 volts, the turbocharger boost is set to minimize the pressure drop across the throttle. At throttle positions over 4.0 volts, the turbocharger boost is set to match the 325 bhp torque curve.

Volumetric efficiency (VE) is used to determine the open-loop fueling rate to the engine for the desired equivalence ratio. Corrections to the volumetric efficiency (VE) are accomplished through closed-loop control based on feedback from the universal exhaust gas oxygen (UEGO) sensor. A closed-loop multiplier performs immediate corrections to the VE to maintain the correct equivalence ratio. An adaptive loop multiplier, which is saved in a table, performs longer term corrections. The engine was operated at steady-state conditions with the adaptive loop multiplier set to zero, and the volumetric efficiency table adjusted to maintain a closed-loop multiplier of less than $1.00 \%$.

The equivalence ratio table is used for air/fuel ratio control of the lean burn engine. As equivalence ratio is lowered from stoichiometric, the efficiency increases and the $\mathrm{NO}_{\mathrm{x}}$ emissions decrease. If the equivalence ratio is too low, the hydrocarbon emissions increase, efficiency decreases, and misfires are possible. Equivalence ratio was set for lean operation with a margin above the misfire limit for the higher engine speeds where turbo boost was sufficient to run lean, and was set for power and driveability at lower engine speeds where turbo boost was not sufficient to run lean (particularly at WOT). Further refinements to the equivalence ratio table were performed during transient emissions testing. These included a lower equivalence ratio at idle to reduce $\mathrm{NO}_{x}$ emissions on the transient emissions test cycle, and lower equivalence ratios at low speeds, including low to mid loads to reduce $\mathrm{NO}_{\mathrm{x}}$ emissions for the West Virginia University central business district (CBD) cycle on a chassis dyno.

Ignition timing is set as a compromise between efficiency and $\mathrm{NO}_{x}$ emissions. The best efficiency is at MBT timing, but to meet the $\mathrm{NO}_{x}$ emissions goals, retarded timings are required. A 12-mode steady-state prediction for $\mathrm{NO}_{x}$ emissions was used to determine the initial ignition-timing table for transient emissions testing. The 12-mode test is shown in Table 7. Results of the 12-mode test for ignition timing retarded the same amount at all 12 test points and are shown in Table 8. The 12-mode results for the initial ignition timing table for transient emissions testing is shown in Table 9. The transient emissions test with the initial ignition timing table was $2.86 \mathrm{~g} / \mathrm{bhp}-\mathrm{hr}$. Ignition timing was modified to lower the $\mathrm{NO}_{\mathrm{x}}$ emissions on the transient test to $2.15 \mathrm{~g} / \mathrm{bhp}-\mathrm{hr}$. 
Table 7. Test Points for the 12-Mode Steady-State Prediction for Transient Emissions

\begin{tabular}{|c|c|c|c|c|c|c|}
\hline Mode & $\begin{array}{c}\text { Engine Speed } \\
(\mathbf{\%})\end{array}$ & $\begin{array}{c}\text { Load } \\
\mathbf{( \% )}\end{array}$ & $\begin{array}{c}\text { Weight } \\
\mathbf{( \% )}\end{array}$ & $\begin{array}{c}\text { Engine Speed } \\
(\mathbf{r p m})\end{array}$ & $\begin{array}{c}\text { Torque } \\
\text { (ft-lb) }\end{array}$ & $\begin{array}{c}\text { MBT } \\
\text { ('BTDC) }\end{array}$ \\
\hline \hline 1 & 0 & CITT & 8.8 & 600 & 160 & 20 \\
\hline 2 & 50 & 83 & 2.6 & 1300 & 963 & 22 \\
\hline 3 & 50 & 100 & 2.1 & 1300 & 1160 & 21 \\
\hline 4 & 67 & 73 & 3.4 & 1520 & 896 & 26 \\
\hline 5 & 83 & 50 & 7.0 & 1730 & 485 & 28 \\
\hline 6 & 83 & 73 & 5.5 & 1730 & 805 & 27 \\
\hline 7 & 83 & 100 & 11.9 & 1730 & 970 & 23 \\
\hline 8 & 100 & 33 & 5.1 & 1950 & 287 & 30 \\
\hline 9 & 100 & 50 & 9.6 & 1950 & 435 & 27 \\
\hline 10 & 100 & 67 & 7.2 & 1950 & 583 & 26 \\
\hline 11 & 100 & 83 & 16.8 & 1950 & 722 & 26 \\
\hline 12 & 100 & 100 & 20.0 & 1950 & 870 & 23 \\
\hline
\end{tabular}

Table 8. Predicted FTP Transient Results

\begin{tabular}{|c|c|c|c|c|}
\hline $\begin{array}{c}\text { Ignition Timing } \\
\text { ('BTDC) }\end{array}$ & $\begin{array}{c}\text { BSNO }_{\mathbf{x}} \\
\text { (g/bhp-hr) }\end{array}$ & $\begin{array}{c}\text { BSTHC } \\
\text { (g/bhp-hr) }\end{array}$ & $\begin{array}{c}\text { BSCO } \\
\text { (g/bhp-hr) }\end{array}$ & $\begin{array}{c}\text { BTE } \\
\text { (\%) }\end{array}$ \\
\hline \hline MBT & 4.18 & 1.75 & 1.15 & 35.1 \\
\hline MBT-3 & 2.93 & 1.77 & 1.14 & 34.6 \\
\hline MBT-5 & 2.12 & 1.85 & 1.14 & 34.2 \\
\hline MBT-7 & 1.58 & 1.79 & 1.12 & 33.5 \\
\hline
\end{tabular}

Table 9. Ignition Timing Based on Steady-State Tests

\begin{tabular}{|c|c|c|c|}
\hline Mode & Ignition Timing ('BTDC) & BSNO $_{\mathbf{x}}$ (g/bhp-hr) & BTE (\%) \\
\hline \hline 1 & MBT & 0.52 & 26.8 \\
\hline 2 & MBT-7 & 2.14 & 37.9 \\
\hline 3 & MBT-7 & 2.10 & 37.8 \\
\hline 4 & MBT-7 & 3.10 & 38.3 \\
\hline 5 & MBT-5 & 1.67 & 33.6 \\
\hline 6 & MBT-7 & 2.63 & 37 \\
\hline 7 & MBT-7 & 1.73 & 36.4 \\
\hline 8 & MBT & 3.00 & 30 \\
\hline 9 & MBT-5 & 1.34 & 31.5 \\
\hline 10 & MBT-5 & 1.75 & 34.4 \\
\hline 11 & MBT-5 & 2.09 & 34.3 \\
\hline 12 & MBT-5 & 1.45 & 34.1 \\
\hline Prediction & & 1.86 & \\
\hline
\end{tabular}

Recorded data for the steady-state tests are included in Appendix B. Tables with the final calibration to be used for certification are included in Appendix C. 


\subsection{Durability Testing}

The first durability test was reported in the final report for Task 2 - 1000-Hour Super Thermal Cycle Test, SwRI Project No. 03-7501-002, and is included in Appendix D. The second durability test was reported in the final report for the 1000-Hour Super Thermal Cycle Test on a Mack E-7 Natural Gas Fueled Engine, SwRI Project No. 03-1248, and is included in Appendix E.

\subsection{Data Logger}

A data logger was installed on a refuse truck for in service use. The data logger recorded information from the vehicle and the engine. A list of data recorded is shown in Table 10. Due to harsh environmental conditions, the fuel system pressure sensors added for data logging failed. The fuel pressure used by the engine controller was still operational. Data for engine speed, vehicle speed, and MAP for a typical day are shown in Figures 25-27. Most of the occurrences occur at idle (600 rpm, 0 $\mathrm{mph}, 8 \mathrm{psia})$, followed by packing (1200 rpm, $0 \mathrm{mph}, 11 \mathrm{psia})$. There were 395 total minutes of data logging time for this day, 31 minutes were used for packing and 122 minutes were used for idling.

Histograms for data recorded from 4/23/98 to 6/29/98 are included in Appendix $F$. In the period from 4/23/98 to 6/29/98 the data logger recorded 2909 miles with 2063 gallons of LNG used for a fuel economy of $1.41 \mathrm{mpg}$, which is a diesel equivalent fuel economy of $2.28 \mathrm{mpg}$. Of the 2063 gallons used, 227 gallons were used at idle and 236 gallons were used for packing, which represents $11.0 \%$ and $11.4 \%$ of the total fuel used, respectively. 


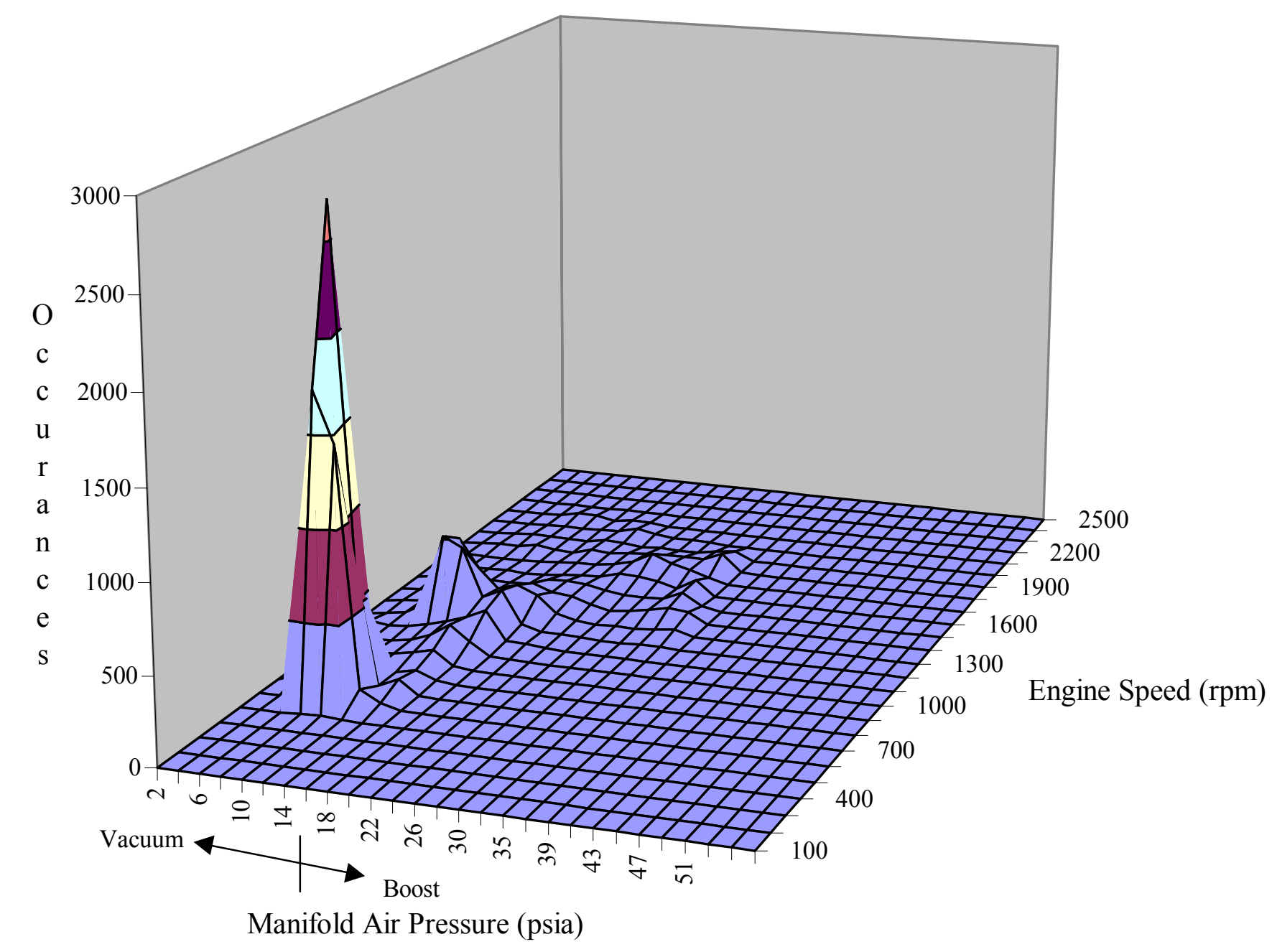

Figure 25. Engine Speed vs. Manifold Air Pressure for One Day of Operation 


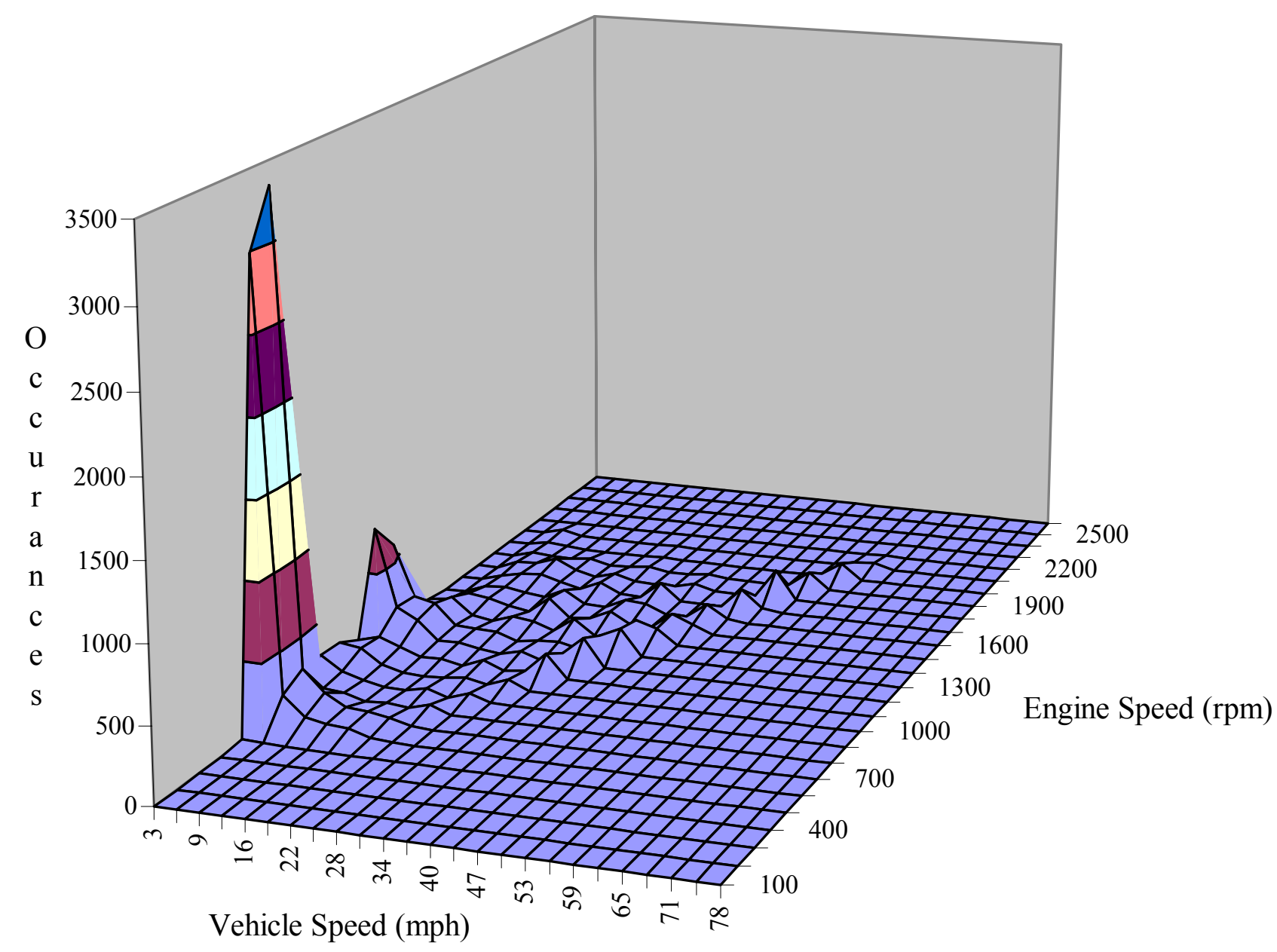

Figure 26. Engine Speed vs. Vehicle Speed for One Day of Operation 


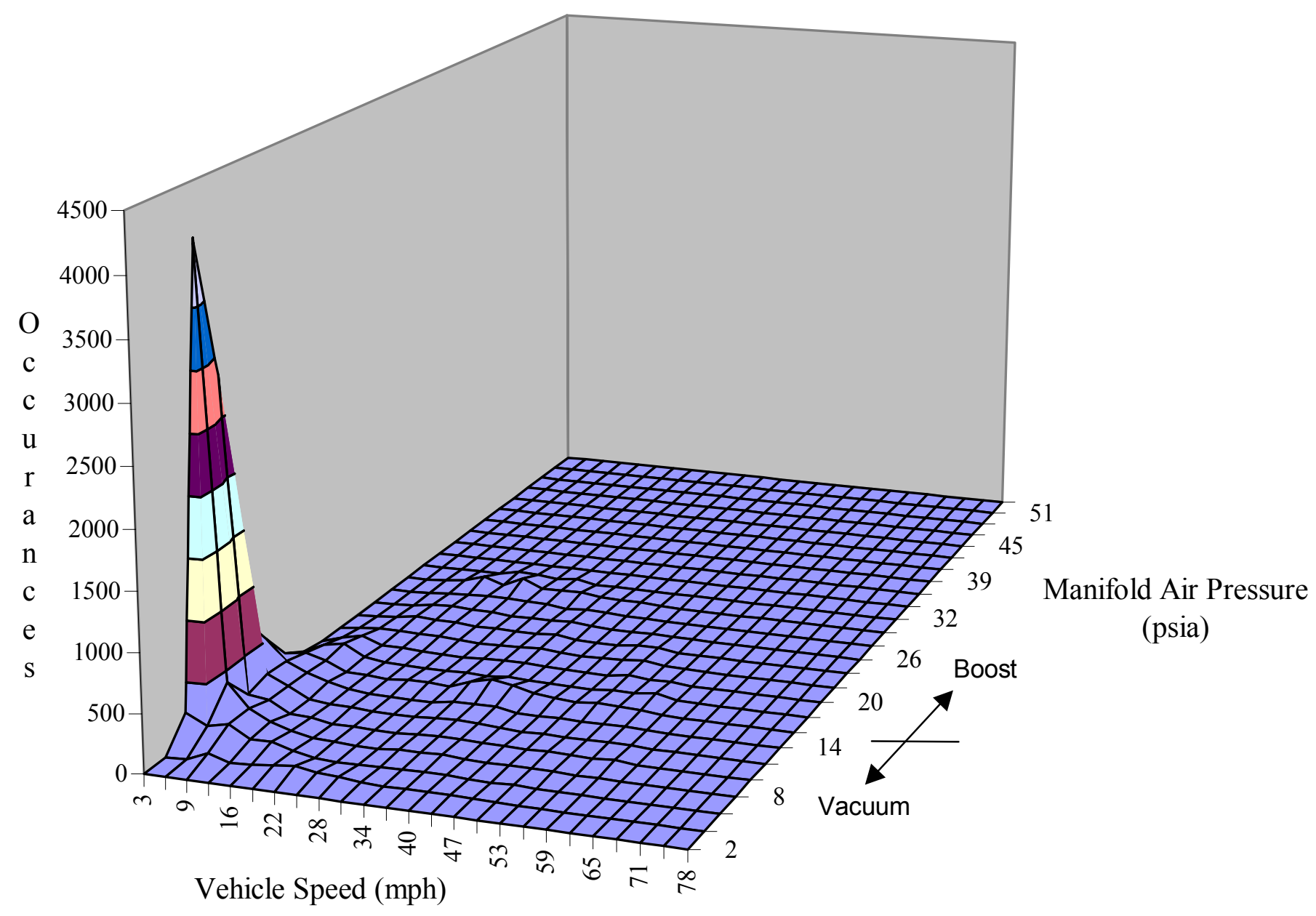

Figure 27. Manifold Air Pressure vs. Vehicle Speed for One Day of Operation 
This page left blank intentionally. 
Table 10. Data Recorded on the Data Logger

\begin{tabular}{|c|c|c|}
\hline Time & Battery (volts) & $\begin{array}{c}\text { Turbo Lag Compensation Enable } \\
(\%)\end{array}$ \\
\hline Date & Barometric Pressure (psia) & $\begin{array}{c}\text { Turbo Lag Compensation } \\
\text { Enrichment (\%) }\end{array}$ \\
\hline Odometer Reading (miles) & Equivalence Ratio Feedback & $\begin{array}{l}\text { Turbo Lag Compensation Retard } \\
\text { (deg) }\end{array}$ \\
\hline Vehicle Speed (mph) & Equivalence Ratio Command & Close Loop Multiplier (\%) \\
\hline Engine Speed (rpm) & Equivalence Ratio Modifier for ECT & Adaptive Loop Multiplier (\%) \\
\hline Fuel Flow (lb/hr): & \#1 Tank Pressure (psig) & Manifold Air Pressure Signal (volts) \\
\hline $\begin{array}{l}\text { Instantaneous Fuel Economy } \\
\text { (mpg) }\end{array}$ & \#2 Tank Pressure (psig) & Preturbine Pressure Signal (volts) \\
\hline $\begin{array}{l}\text { Cumulative Fuel Economy } \\
\text { (mpg) }\end{array}$ & $\begin{array}{l}\text { Fuel Pressure Before Vaporizer } \\
\text { (psig) }\end{array}$ & $\begin{array}{l}\text { Exhaust Back Pressure Signal } \\
\text { (volts) }\end{array}$ \\
\hline Manifold Air Pressure (psia) & $\begin{array}{l}\text { Fuel Pressure After Vaporizer } \\
\text { (psig) }\end{array}$ & Gas Pressure Signal (volts) \\
\hline Exhaust Back Pressure (psia) & Fuel Pressure at Regulator (psig) & Manifold Air Temp Signal (volts) \\
\hline Preturbine Pressure (psia) & Fuel Pressure at Filter (psig) & Engine Coolant Temp Signal (volts) \\
\hline Manifold Air Temp (deg F) & $\begin{array}{l}\text { Air Pressure Before Intercooler } \\
\text { (psia) }\end{array}$ & Gas Temperature Signal (volts) \\
\hline Engine Coolant Temp (deaf) & Air Pressure After Intercooler (psia) & UEGO Signal (volts) \\
\hline Gas Pressure (psia) & Relative Humidity (\%) & UEGO Pumping Signal (volts) \\
\hline Gas Temperature (deg F) & EMC Run Time (sec) & UEGO Heater Signal (volts) \\
\hline $\begin{array}{l}\text { Actual Foot Pedal Position } \\
\text { (volts) }\end{array}$ & Ignition Timing (deg BTDC) & UEGO Resistor Signal (volts) \\
\hline $\begin{array}{l}\text { Normalized Foot Pedal } \\
\text { Position (volts) }\end{array}$ & Injection Pulse Width (ms) & ECM Control Mode \\
\hline $\begin{array}{l}\text { Throttle Position Command } \\
\text { (volts) }\end{array}$ & Uncorrected Fuel Flow (mg/inj) & ECM Run Mode \\
\hline $\begin{array}{c}\text { Throttle Position Feedback } \\
\text { (volts) }\end{array}$ & Predicted Volumetric Efficiency (\%) & UEGO Mode \\
\hline Ambient Air Temp (deg F) & $\begin{array}{c}\text { Volumetric Efficiency Modification } \\
(\%)\end{array}$ & Fault Codes \\
\hline Oil Temp (deg F) & Actual Volumetric Efficiency (\%) & Cumulative Fuel Usage (gal) \\
\hline Exhaust Temp (deg F) & $\begin{array}{l}\text { Change in Manifold Air Pressure } \\
(\mathrm{psia/s})\end{array}$ & Idle Fuel Usage (gal) \\
\hline Data Logger Temp (deg F) & Boost Control PWM (\%) & Packing Fuel Usage (gal) \\
\hline
\end{tabular}

\subsection{Transient Emissions Testing}

Results from the transient emissions testing were reported in the final report for the Transient Emissions Testing on a Mack E7G Engine, SwRI Project No. 03-2377, and is included in Appendix G. The emissions certification levels for the final calibration, with a deterioration factor, are shown in Table 11. Screen shots from the engine controller for this calibration are included in Appendix C. CARB certification gas was used for these tests. The objectives of this project were $\mathrm{NO}_{\mathrm{x}}+\mathrm{NMHC}^{\mathrm{N}}$ emissions of less than 2.5 $\mathrm{g} / \mathrm{bhp}$-hr on a natural gas with $99 \%$ methane. The use of CARB certification gas increases both the $\mathrm{NO}_{\mathrm{x}}$ and $\mathrm{NMHC}$ emissions. CARB gas has $90 \%$ methane along with $4 \%$ ethane and $2 \%$ butane. Ethane and 
butane increase the flame speed and therefore increase $\mathrm{NO}_{x}$ emissions. Ethane and butane also increase the NMHC emissions since the unburned fuel has an increased amount of NMHCs. The final calibration was selected for $\mathrm{NO}_{\mathrm{x}}$ emissions below $2.5 \mathrm{~g} / \mathrm{bhp}-\mathrm{hr}$ with acceptable driveability. $\mathrm{NO}_{\mathrm{x}}$ emissions below $2.0 \mathrm{~g} / \mathrm{bhp}$-hr are possible on the transient emissions tests, but driveability problems were noted in the field due to the amount of timing retard required for the lower emissions combined with humidity effects. A summary of the $\mathrm{NO}_{\mathrm{x}}$ emissions and fuel economy over the transient test cycle is presented in Table 12. The calibration used for the results in Table 9 was the initial calibration for the transient emissions testing. The $\mathrm{NO}_{\mathrm{x}}$ emissions for this test was $2.86 \mathrm{~g} / \mathrm{bhp}-\mathrm{hr}$. Similar trends were noted at lower $\mathrm{NO}_{\mathrm{x}}$ emissions levels. Table 12 shows that the transient from idle uses $9 \%$ of the time in the cycle, yet accounts for $41 \%$ of the $\mathrm{NO}_{\mathrm{x}}$ emissions. This is due to the steep torque rise of this engine and the high equivalence ratios used during the WOT transient from idle. The high equivalence ratios are used to improve driveability. This calibration, where the high equivalence ratios are high, will not pass 2002 emissions for natural gas fueled engines due to high steady-state $\mathrm{NO}_{x}$ emissions. In 2002, natural gas engines will have the same limit on steady-state emissions over the entire operating range as diesel engines.

Table 11. 325 bhp Transient Emissions Results

\begin{tabular}{|c|c|}
\hline Emission & $\begin{array}{c}\text { Emission Level } \\
\text { (g/bhp-hr) }\end{array}$ \\
\hline $\mathrm{HC} / \mathrm{OMHCE}$ & 3.1 \\
\hline $\mathrm{NMHC/OMNMHC}$ & 0.324 \\
\hline NMHC+ $\mathrm{NO}_{\mathrm{x}}$ & 2.53 \\
\hline Carbon Monoxide & 1.4 \\
\hline Oxides of Nitrogen & 2.2 \\
\hline Particulates & 0.03 \\
\hline Formaldehyde & 0.136 \\
\hline
\end{tabular}

Table 12. Summary for the FTP Results for $\mathrm{NO}_{x}$ Emissions at $2.86 \mathrm{~g} / \mathrm{bhp}-\mathrm{hr}$

\begin{tabular}{||l|c|c|c|c|c||}
\hline \multicolumn{1}{|c|}{ Description } & Idle & $\begin{array}{c}\text { Mid-High } \\
\text { Speed } \\
\text { Mid Load }\end{array}$ & $\begin{array}{c}\text { High Speed } \\
\text { High Load }\end{array}$ & $\begin{array}{c}\text { Transient } \\
\text { from Idle }\end{array}$ & Remainder \\
\hline Occurrences & $42 \%$ & $25 \%$ & $10 \%$ & $9 \%$ & $14 \%$ \\
\hline $\begin{array}{l}\text { Total Contribution to } \\
\mathrm{NO}_{\mathrm{x}} \text { Emissions }\end{array}$ & $12 \%$ & $18 \%$ & $17 \%$ & $41 \%$ & $12 \%$ \\
\hline $\begin{array}{l}\text { Average Diluted } \\
\mathrm{NO}_{\mathrm{x}} \text { Emissions }\end{array}$ & $7.3 \mathrm{ppm}$ & $18.8 \mathrm{ppm}$ & $43.1 \mathrm{ppm}$ & $119.5 \mathrm{ppm}$ & $22.1 \mathrm{ppm}$ \\
\hline $\begin{array}{l}\text { Total Contribution to } \\
\text { Fuel Usage }\end{array}$ & $12 \%$ & $27 \%$ & $38 \%$ & $7 \%$ & $16 \%$ \\
\hline
\end{tabular}

The previous version of this engine was rated at $300 \mathrm{bhp}$ and had $\mathrm{NO}_{\mathrm{x}}$ emissions of $2.95 \mathrm{~g} / \mathrm{bhp}$-hr with no deterioration factor and a brake specific fuel consumption of $0.474 \mathrm{lb} / \mathrm{bhp}-\mathrm{hr}$. This final calibration for this engine has $\mathrm{NO}_{\mathrm{x}}$ emissions of $2.2 \mathrm{~g} / \mathrm{bhp}-\mathrm{hr}$ and a brake specific fuel consumption of $0.400 \mathrm{lb} / \mathrm{bhp}$-hr. Actual driving cycle fuel economy comparisons are not readily available since the previous version of this engine was installed in a refuse hauler which was at a different location as the current version. A summary of the comparison of the previous and current version of the engine is shown in Table 13. 
Table 13. Comparison of Previous and Current Version of the Engine

\begin{tabular}{|l|c|c|}
\hline & Previous Version & Current Version \\
\hline \hline Power (bhp) & 300 & 325 \\
\hline Torque (ft-lb) & 1100 & 1180 \\
\hline $\mathrm{NO}_{\mathrm{x}}(\mathrm{g} / \mathrm{bhp}-\mathrm{hr})$ & 2.95 & 2.20 \\
\hline $\mathrm{BSFC}(\mathrm{lb} / \mathrm{bhp}-\mathrm{hr})$ & 0.474 & 0.400 \\
\hline
\end{tabular}

A calibration with a 350-bhp rating was tested for transient emissions. The emissions certification levels for the final calibration, with a deterioration factor, are shown in Table 14. Screen shots from the engine controller for this calibration are included in Appendix H. CARB certification gas was used for these tests.

Table 14. 350 bhp Transient Emissions Results

\begin{tabular}{|l|l|}
\hline Emission & $\begin{array}{l}\text { Emission Level } \\
\text { (g/bhp-hr) }\end{array}$ \\
\hline \hline $\mathrm{HC} / \mathrm{OMHCE}$ & 2.9 \\
\hline $\mathrm{NMHC/OMNMHC}$ & 0.276 \\
\hline $\mathrm{NMHC}_{\mathrm{NO}}$ & 2.60 \\
\hline Carbon Monoxide & 1.3 \\
\hline Oxides of Nitrogen & 2.3 \\
\hline Particulates & 0.03 \\
\hline Formaldehyde & 0.116 \\
\hline
\end{tabular}


January 2000 • NREL/SR-540-26935

\section{Mack LNG Vehicle Development: Appendices}

Southwest Research Institute

San Antonio, Texas

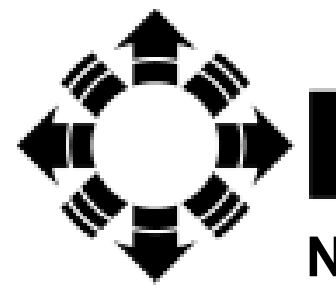

National Renewable Energy Laboratory

1617 Cole Boulevard

Golden, Colorado 80401-3393

NREL is a U.S. Department of Energy Laboratory

Operated by Midwest Research Institute $\bullet$ Battelle $\bullet$ Bechtel

Contract No. DE-AC36-99-G010337 


\section{Mack LNG Vehicle Development: Appendices}

Southwest Research Institute San Antonio, Texas

NREL Technical Monitor: Mike Frailey

Prepared under Subcontract No. AAC-5-15249-01

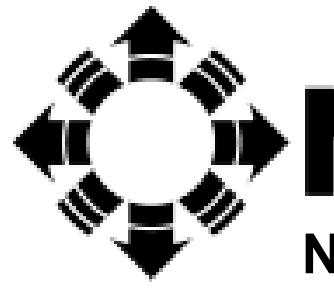

National Renewable Energy Laboratory

1617 Cole Boulevard

Golden, Colorado 80401-3393

NREL is a U.S. Department of Energy Laboratory

Operated by Midwest Research Institute • Battelle • Bechtel

Contract No. DE-AC36-99-G010337 


\section{NOTICE}

This report was prepared as an account of work sponsored by an agency of the United States government. Neither the United States government nor any agency thereof, nor any of their employees, makes any warranty, express or implied, or assumes any legal liability or responsibility for the accuracy, completeness, or usefulness of any information, apparatus, product, or process disclosed, or represents that its use would not infringe privately owned rights. Reference herein to any specific commercial product, process, or service by trade name, trademark, manufacturer, or otherwise does not necessarily constitute or imply its endorsement, recommendation, or favoring by the United States government or any agency thereof. The views and opinions of authors expressed herein do not necessarily state or reflect those of the United States government or any agency thereof.

Available electronically at http://www.doe.gov/bridge

Available for a processing fee to U.S. Department of Energy

and its contractors, in paper, from:

U.S. Department of Energy

Office of Scientific and Technical Information

P.O. Box 62

Oak Ridge, TN 37831-0062

phone: 865.576.8401

fax: 865.576.5728

email: reports@adonis.osti.gov

Available for sale to the public, in paper, from:

U.S. Department of Commerce

National Technical Information Service

5285 Port Royal Road

Springfield, VA 22161

phone: 800.553.6847

fax: 703.605.6900

email: orders@ntis.fedworld.gov

online ordering: http://www.ntis.gov/ordering.htm

Printed on paper containing at least $50 \%$ wastepaper, including $20 \%$ postconsumer waste 


\section{MACK LNG VEHICLE DEVELOPMENT}

\section{DRAFT AND CONFIDENTIAL FINAL REPORT}

SwRI Project No. 03-7276

Prepared for:

Mack Trucks, Inc.

13302 Pennsylvania Ave.

Hagerstown, MD 21742

Prepared by:

James P. Chiu

July 8, 1999

Approved:

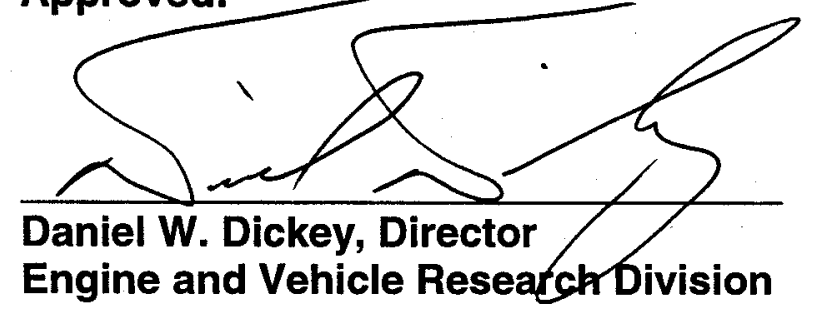

This report must be reproduced in full, unless SwRI approves a summary or abridgement 


\section{EXECUTIVE SUMMARY}

The goal of this project was to install a production ready, state-of-the-art engine control system on the Mack E7G natural gas engine to improve efficiency and lower exhaust emissions. In addition, the power rating was increased from $300 \mathrm{bhp}$ to $325 \mathrm{bhp}$. The emissions targets were $\mathrm{NO}_{\mathrm{x}}+\mathrm{NMHC}$ of less than $2.5 \mathrm{~g} / \mathrm{bhp}-\mathrm{hr}$ (with a mandatory $\mathrm{NO}_{\mathrm{x}}$ of less than $2.5 \mathrm{~g} / \mathrm{bhp}-\mathrm{hr}$ ) and particulate matter of less than $0.05 \mathrm{~g} / \mathrm{bhp}-\mathrm{hr}$ on $99 \%$ methane. Durability and field testing were also conducted.

A Woodward OH-1 state-of-the-art engine controller was successfully installed on the Mack E7G engine and a calibration for the Mack engine was developed. Knock tolerance testing and misfire limit testing were conducted to aid in the development of the calibration. The first 1000-hour durability test was conducted with partial funding from this project, and a second 1000-hour durability test to address the concerns from the first test was successfully completed. A data logger was installed on an in-service refuse hauler and engine and vehicle operation was successfully recorded.

The brake specific fuel consumption over the transient emissions test cycle was decreased from $0.474 \mathrm{lb} / \mathrm{bhp}-\mathrm{hr}$ on the previous version of this engine to $0.400 \mathrm{lb} / \mathrm{bhp}$-hr on the current version. The $\mathrm{NO}_{\mathrm{x}}$ emissions over the transient emissions test cycle were decreased from $2.95 \mathrm{~g} / \mathrm{bhp}-\mathrm{hr}$ to $2.20 \mathrm{~g} / \mathrm{bhp}-\mathrm{hr}$. The $\mathrm{NO}_{\mathrm{x}}+\mathrm{NMHC}$ emissions were $2.53 \mathrm{~g} / \mathrm{bhp}-\mathrm{hr}$ and particulate matter was $0.03 \mathrm{~g} / \mathrm{bhp}$-hr on the current version of this engine. Emissions for the current version of this engine include the deterioration factor, whereas emissions from the previous version are results without deterioration. CARB certification gas was used for the transient emissions testing on the current version of this engine. $\mathrm{NO}_{\mathrm{x}}+\mathrm{NMHC}$ emissions are expected to decrease on a $99 \%$ methane fuel. Actual driving cycle fuel economy comparisons are not readily available since the previous version of this engine was installed in a refuse hauler, which was at a different location. Further development of this engine should include efficiency improvements and $\mathrm{NO}_{\mathrm{x}}$ reductions. 
TABLE OF CONTENTS

$\underline{\text { Page }}$

LIST OF ILLUSTRATION ………………………................................................... iv

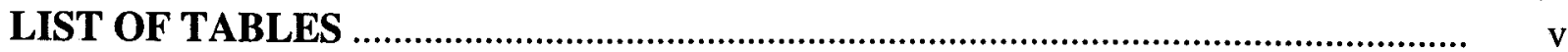

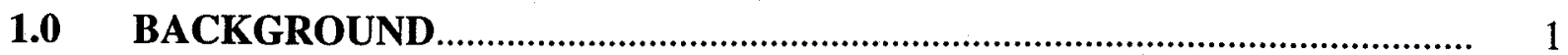

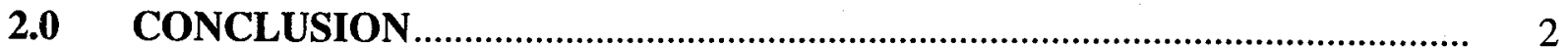

3.0 RECOMMENDATIONS ................................................................................ 3

4.0 TECHNICAL DISCUSSION ……………………............................................

4.1 Engine Specifications …………………………........................................... 4

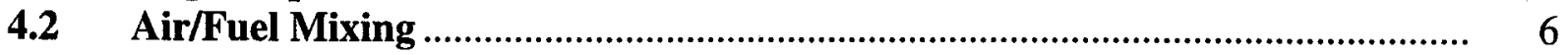

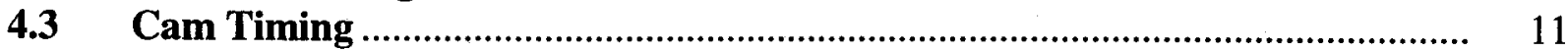

4.4 Steady-State Engine Mapping .......................................................................... 11

4.4.1 Knock Tolerance Testing .....................................................................

4.4.2 Lean-Limit Testing .................................................................. 15

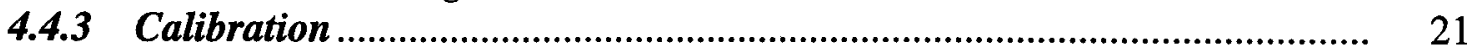

4.5 Durability Testing ............................................................................................. 23

4.6 Data Logger .................................................................................................. 23

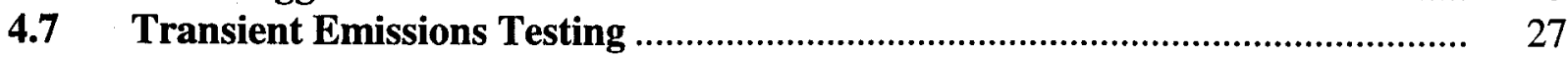

APPENDICES:

A - Recorded Data for Lean Limit Tests

B - Recorded Data for Steady-State Tests

C - Final Calibration, 325 bhp

D - Final Report for Task 2 - 1000-Hour Super Thermal Cycle Test, SwRI Project No. 03-7501-002

E - Final Report for 1000-Hour Super Thermal Cycle Test on a Mack E-7 Natural Gas Fueled Engine, SwRI Project No. 03-1248

F - Histograms from the Data Logger

G - Final Report for Transient Emissions Testing on a Mack E7G Engine, SwRI Project No. 03-2377

H - Final Calibration, 350 bhp 


\section{LIST OF ILLUSTRATIONS}

Figure

Page

1 Average Equivalence Ratios and the Standard Error for the

Six Cylinders Using Mixing Elbow Number 1

2 Average Equivalence Ratios and the Standard Error for the

Six Cylinders Using Mixing Elbow Number 2.

Average Equivalence Ratios and the Standard Error for the

Six Cylinders Using Mixing Elbow Number 3

Average Equivalence Ratios and the Standard Error for the

Six Cylinders Using Mixing Elbow Number 4.

Average Equivalence Ratios and the Standard Error for the

Six Cylinders Using Mixing Elbow Number 5.

Average Equivalence Ratios and the Standard Error for the

Six Cylinders Using Mixing Elbow Number 6

Average Equivalence Ratios and the Standard Error for the

Six Cylinders Using Mixing Elbow Number 7.

Average Equivalence Ratios and the Standard Error for the

Six Cylinders Using Mixing Elbow Number 8

Comparison of Original and Advanced Cam Timing at 1950 rpm,

$100 \%$ Load, and Various Ignition Timings

Comparison of Original and Advanced Cam Timing at $1950 \mathrm{rpm}$,

75\% Load, and Various Ignition Timings

Comparison of Original and Advanced Cam Timing at $1950 \mathrm{rpm}$,

50\% Load, and Various Ignition Timing.

Comparison of Original and Advanced Cam Timing at 1500 rpm, 100\% Load, and Various Ignition Timing

Comparison of Original and Advanced Cam Timing at 1500 rpm,

75\% Load, and Various Ignition Timing

Comparison of Original and Advanced Cam Timing at $1500 \mathrm{rpm}$, 50\% Load, and Various Ignition Timing.

Comparison of Original and Advanced Cam Timing at $1250 \mathrm{rpm}$, $100 \%$ Load, and Various Ignition Timing

Comparison of Original and Advanced Cam Timing at 1250 rpm, 75\% Load, and Various Ignition Timing

Comparison of Original and Advanced Cam Timing at 1250 rpm, 50\% Load, and Various Ignition Timing.

Knock Limit at 1900 rpm, $100 \%$ Load

Knock Limit at 1500 rpm, 100\% Load

Knock Limit at $1250 \mathrm{rpm}, 100 \%$ Load

Knock Limit at 1100 rpm, 100\% Load

Knock Limit at 1000 rpm, $100 \%$ Load

Lean Limit Tests at $1250 \mathrm{rpm}, 100 \%$ Load .

Lean Limit Tests at 1900 rpm, 100\% Load

Engine Speed vs. Manifold Air Pressure for One Day of Operation.

Engine Speed vs. Vehicle Speed for One Day of Operation 


\section{LIST OF TABLES}

Table

Page

$1 \quad$ Engine Specifications ............................................................................. 4

2 Woodward OH-1 Capabilities...................................................................... 5

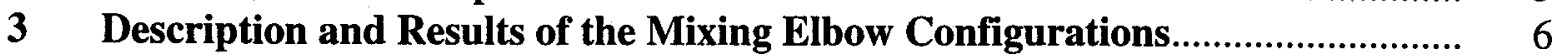

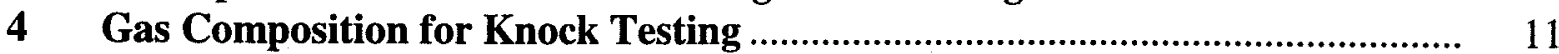

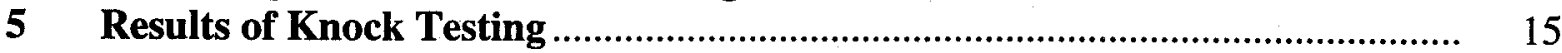

$6 \quad$ Lean Limit Test Results .................................................................................... 19

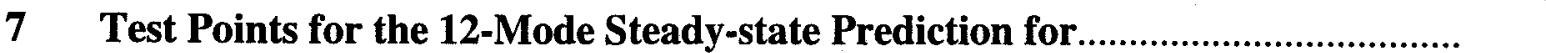

Transient Emissions .................................................................................. 22

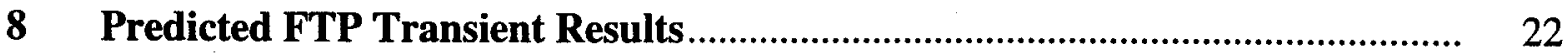

$9 \quad$ Ignition Timing Based on Steady-State Tests .................................................... 23

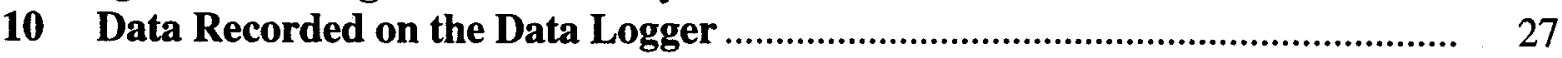

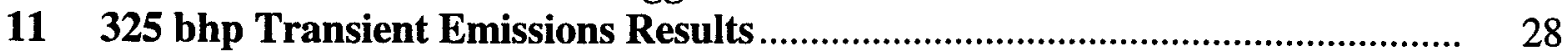

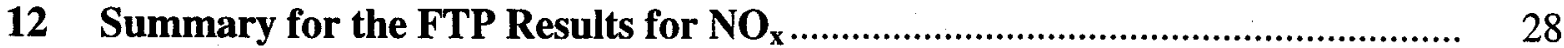

13 Comparison of Previous and Current Version of the Engine ........................... 29

14350 bhp Transient Emissions Results ............................................................. 29 


\subsection{BACKGROUND}

This project was a continuation of the development of a natural gas refuse hauler. A compressed natural gas (CNG) Mack MR demonstration vehicle was operated for nearly a year in revenue service on a previous project. Engine performance, emissions, driveability, and durability were acceptable for a demonstration vehicle; however, several areas of improvement were identified. These included vehicle range, refueling time, driving cycle fuel economy, and installation of improved and production-ready natural gas specific engine control hardware.

Vehicle range and refueling time can be improved by operating the vehicle on liquefied natural gas (LNG). Efforts on this project concentrated on minimizing throttling losses and increasing the $\mathrm{NO}_{\mathrm{x}}$ efficiency tradeoff by using a state-of-the-art engine controller.

The original objectives of this project were to:

1. Improve the driving cycle thermal efficiency of the engine,

2. Install and aid in the development of a state-of-the-art engine control system,

3. Assess control system and engine integrity through a 480-hour durability test,

4. Calibrate the engine to exactly meet the diesel torque curve of the $300 \mathrm{bhp}$ engine,

5. Conduct transient emissions testing to verify that exhaust emissions are lower than the 1998 EPA emissions targets with $\mathrm{NO}_{\mathrm{x}}$ emissions being less than $2.0 \mathrm{~g} / \mathrm{bhp}-\mathrm{hr}$,

6. Monitor the vehicle through a three-month field test using an on-board data logger equipped with a cellular telephone, and

7. Provide detailed drawings, manuals, and training to Mack personnel so that Mack can develop a full understanding of the engine control system and be capable of producing six identical vehicles.

The objectives had been revised as follows:

3. A 1000-hour durability test was performed. This project funded up to the amount provided in the contract, and the remainder was funded by Mack Trucks.

4. The engine was calibrated to a $325 \mathrm{bhp}$ at $1950 \mathrm{rpm}$ and $1180 \mathrm{ft}-\mathrm{lb}$ at $1250 \mathrm{rpm}$ torque curve.

5. The PM target had been revised to $0.05 \mathrm{~g} / \mathrm{hhp}-\mathrm{hr}$, and there was an additional target for $\mathrm{NO}_{\mathrm{x}}+\mathrm{NMHC}$ of less than $2.5 \mathrm{~g} / \mathrm{bhp}-\mathrm{hr}$. 


\subsection{CONCLUSIONS}

Following the successful completion of the project, several conclusions regarding the technical content of the program were made.

1. In comparing the previous demonstration engine to the current engine developed in this project:

a. Peak power was increased from $300 \mathrm{bhp}$ to $325 \mathrm{bhp}$ to match the $325 \mathrm{bhp}$ diesel torque curve.

b. Peak torque was increased from $1100 \mathrm{ft}-\mathrm{lb}$ to $1180 \mathrm{ft}-\mathrm{lb}$ to match the $325 \mathrm{bhp}$ diesel torque curve.

c. The $\mathrm{NO}_{\mathrm{x}}$ emissions over the transient emissions test cycle decreased from $2.95 \mathrm{~g} / \mathrm{bhp}-\mathrm{hr}$ to $2.20 \mathrm{~g} / \mathrm{bhp}-\mathrm{hr}$.

d. The brake specific fuel consumption over the transient emissions test cycle decreased from $0.474 \mathrm{lb} / \mathrm{bhp}-\mathrm{hr}$ to $0.400 \mathrm{lb} / \mathrm{bhp}-\mathrm{hr}$, which is an increase in efficiency.

e. A comparison of in-use fuel economy was not possible due to the different driving cycles of the previous demonstration engine and the current engine.

2. A state-of-the-art engine control system was successfully integrated onto the engine.

3. Control system and engine integrity were assessed through a 1000 -hour durability test which indicated a problem with the piston, and a subsequent 1000-hour durability test was conducted on a separate project at SwRI with revised pistons which confirmed the problem was solved.

4. The $\mathrm{NO}_{\mathrm{x}}+\mathrm{NMHC}$ emissions were $2.53 \mathrm{~g} / \mathrm{bhp}-\mathrm{hr}$ with the deterioration factor and $2.40 \mathrm{~g} / \mathrm{bhp}-\mathrm{hr}$ without the deterioration factor on CARB gas; emissions on $99 \%$ methane were not conducted with the final calibration, but were expected to be below the $2.5 \mathrm{~g} / \mathrm{bhp}-\mathrm{hr}$ target, even with the deterioration factor.

5. A vehicle was successfully monitored using an on-board data logger.

6. Mack has produced six identical engines with this state-of-the-art control system and all associated engine and vehicle modifications.

7. Durability of the cylinder heads, including valve recession, was greatly improved from the previous engine (one of the problem areas from the final report for the previous engine). Funding to test head durability was provided by Mack Trucks. 


\subsection{RECOMMENDATIONS}

Recommendations for further development of this engine are as follows:

1. Lower $\mathrm{NO}_{\mathrm{x}}$ emissions are possible on this engine with:

a. Humidity compensation to allow for a calibration with lower $\mathrm{NO}_{\mathrm{x}}$ emissions on the transient test cycle (which has been demonstrated on this engine) without producing driveability problems in the field.

b. Decreased equivalence ratio at the lower engine speeds and full load to reduce $\mathrm{NO}_{\mathrm{x}}$ emissions during transients from idle along with alternative turbo lag compensation methods to retain driveability.

2. Higher thermal efficiency for this engine is possible with:

a. Humidity compensation to allow for equivalence ratios closer to the lean-limit.

b. Turbo lag compensation methods to reduce transient $\mathrm{NO}_{\mathrm{x}}$, which can allow for higher $\mathrm{NO}_{\mathrm{x}}$, and therefore higher efficiency over the driving cycle of the engine.

3. Investigation of other methods to improve the $\mathrm{NO}_{\mathrm{x}}$ efficiency trade-off of this engine.

Higher thermal efficiency and improved $\mathrm{NO}_{\mathrm{x}}$ efficiency trade-offs are being investigated and developed under the current "Efficiency Enhancements for the Mack E7G Natural GasFueled Engine" program. 


\subsection{TECHNICAL DISCUSSION}

This section describes the results of the project.

\subsection{Engine Specifications}

The Mack E7 engine (S/N 1P0144) was used for calibration development on this project. This engine was used on the previous Mack natural gas engine project, but was rebuilt with new cylinder liners, new pistons with an 11.5:1 compression ratio (replaces pistons with a 10.5:1 compression ratio), new piston rings, new cylinder heads with higher swirl, and a new camshaft. Engine specifications are shown in Table 1.

Table 1. Engine Specifications

\begin{tabular}{|l|l|}
\hline Engine Model & E7G-325 \\
\hline Engine Serial Number & $1 \mathrm{P} 1044$ \\
\hline Engine Displacement (in 3 /liter) & $728 / 12$ \\
\hline Bore and Stroke (in/mm) & $4.875 / 123.8 \times 6.5 / 165.1$ \\
\hline Ignition System Contoller Supplier & MESA/Woodward Governor Company \\
\hline Ignition System Coil Supplier & Nippondenso \\
\hline Ignition System Type & Inductive, Coil on Plug (non-waste spark) \\
\hline Ignition System Timing Sensor & Electro, K Coil Originally. Bosch Currently \\
\hline Spark Plug Supplier & Champion \\
\hline Spark Plug Model & RX501PYP/2H6 \\
\hline Fuel Metering System Supplier & MESA/Woodward Governor Company \\
\hline Fuel Injector Type & Servojet SP021 \\
\hline Number of Injectors & 8 \\
\hline Throttle Supplier & Woodward Governor Company \\
\hline Throttle Model & FloTech, 68mm \\
\hline Throttle Type & Electronic, Analog \\
\hline UEGO Sensor & Honda version of the NGK \\
\hline Turbocharger & $\begin{array}{l}\text { Mack 631GCX5146M, Garrett GT40-88 } \\
\text { Compressor Trim, A/R = 52/88mm, 0.58 } \\
\text { Turbine Trim, A/R = 78/77mm, 1.05 }\end{array}$ \\
\hline
\end{tabular}


A Mesa Gas Engine Management (GEM) controller was selected for this project. The Mesa heavy-duty natural gas business was bought by Woodward Governor Company, and the controller is now called the On Highway-1 (OH-1). The OH-1 uses a state-of-the-art speeddensity control strategy that provides excellent driveability and response without the aid of a mass air flow sensor. Table 2 shows the capabilities of the OH-1. It is a closed-loop lean burn strategy with adaptive learn. It includes advanced turbo lag compensation, electronic wastegate control, an electronic throttle, high-energy inductive ignition system, full diagnostics, and reduced power engine protection. Fuel is inducted upstream of the throttle. Pseudo-continuous fuel metering is accomplished through single point injection using eight PWM fuel injectors that supply a common manifold. The injectors are fired sequentially in pairs and fuel flow is governed by modulating the pulse width of the fuel injector drive signals. A drive-by-wire throttle is utilized for speed governing, overboost protection, and driveability enhancements.

\section{Table 2. Woodward OH-1 Capabilities}

\begin{tabular}{|c|l|}
\hline Capability & \multicolumn{1}{|c|}{ Description } \\
\hline Diagnostics & $\begin{array}{l}\text { Advanced diagnostics for easy debugging with } \\
\text { limp home mode for component failure }\end{array}$ \\
\hline Drive-By-Wire & $\begin{array}{l}\text { Idle speed control, packing speed control, } \\
\text { speed governing, load limiting during } \\
\text { component failure, throttle opening during } \\
\text { deceleration for reduced oil consumption }\end{array}$ \\
\hline Cold Start & $\begin{array}{l}\text { Fuel enrichment and ignition timing advance to } \\
\text { avoid lean misfire during cold engine operation } \\
\text { with boost limits }\end{array}$ \\
\hline Equivalence Ratio Control & $\begin{array}{l}\text { A volumetric efficiency table with adaptive } \\
\text { learn to determine fueling rate with an UEGO } \\
\text { sensor for closed-loop control }\end{array}$ \\
\hline Turbo Lag Compensation & $\begin{array}{l}\text { Predictions for air flow during transients for } \\
\text { more accurate equivalence ratio control } \\
\text { reduce turbo lag without increasing NO } \\
\text { emissions }\end{array}$ \\
\hline Boost Control Calibration & $\begin{array}{l}\text { Controls turbocharger wastegate to determine } \\
\text { power for a given throttle position, also used to } \\
\text { reduce throttling losses by using the wastegate } \\
\text { for load control with an open throttle }\end{array}$ \\
\hline Ignition Timing Calibration & $\begin{array}{l}\text { Table to determine ignition timing for various } \\
\text { speeds and loads }\end{array}$ \\
\hline Equivalence Ratio Calibration & $\begin{array}{l}\text { Table to determine equivalence ratio for } \\
\text { various speeds and loads }\end{array}$ \\
\hline
\end{tabular}


Part-load fuel economy was increased by the use of a "delta-P" load control strategy. With this strategy, the throttle on the engine is kept as wide open as possible, and load is modulated by wastegate control. Foot pedal position is used to determine load. The pressure differential (delta-P) across the throttle is minimized at part loads, thereby reducing pumping losses and increasing efficiency. The fuel is shut off during deceleration and braking conditions to provide additional improvements in fuel economy. Under these conditions, the throttle is opened, thereby increasing manifold air pressure and reducing oil consumption.

\subsection{Air/Fuel Mixing}

A study was conducted to look at the effects of the mixing ring and elbow (mixing elbow) on lean-limit and cylinder to cylinder equivalence ratios. The mixing ring is an annular manifold around the inlet pipe to the engine. It is located after the intercooler and before the throttle. To aid in the air/fuel mixing, a $90^{\circ}$ elbow is connected between the mixing ring and the throttle. Eight configurations were tested as shown in Table 3. The seven engine operating conditions were 100,50 , and 25 percent load at both 1250 and $1950 \mathrm{rpm}$, and an idle condition. Equivalence ratio was measured for each individual cylinder with an UEGO sensor. The equivalence ratios for all six cylinders were averaged and a standard error was calculated. The results are shown in Table 3 for the leanest equivalence ratio without audible misfire at $1950 \mathrm{rpm}$ and $100 \%$ load, and the average of the seven standard errors for the seven engine operating conditions. Results for each load condition are shown in Figures 1-8. Elbow number 3 was selected for this engine because of its lowest lean-limit and low average standard error.

Table 3. Description and Results of the Mixing Elbow Configurations

\begin{tabular}{|l|c|c|c|c|c|c|c|c|}
\hline ELBOW NUMBER & $\mathbf{1}$ & $\mathbf{2}$ & $\mathbf{3}$ & $\mathbf{4}$ & $\mathbf{5}$ & $\mathbf{6}$ & $\mathbf{7}$ & $\mathbf{8}$ \\
\hline $\begin{array}{l}\text { 64 mm Spacer } \\
\text { Between Elbow and } \\
\text { Throttle }\end{array}$ & No & No & Yes & Yes & Yes & Yes & No & No \\
\hline $\begin{array}{l}\text { 152 mm Spacer } \\
\text { Between Elbow and } \\
\text { Mixing Ring }\end{array}$ & No & No & No & No & Yes & Yes & Yes & Yes \\
\hline $\begin{array}{l}\text { Number of Holes in } \\
\text { Mixing Ring }\end{array}$ & 18 & 9 & 9 & 18 & 18 & 9 & 9 & 18 \\
\hline $\begin{array}{l}\text { Equivalence Ratio } \\
\text { Limit w/o Audible } \\
\text { Misfire at 1950 rpm, } \\
100 \% \text { Load }\end{array}$ & 0.62 & 0.62 & 0.59 & 0.62 & 0.62 & 0.61 & 0.60 & 0.63 \\
\hline $\begin{array}{l}\text { Average of the } \\
\text { Standard Error of the } \\
\text { Seven Load } \\
\text { Conditions }\end{array}$ & 0.0127 & 0.0041 & 0.0037 & 0.0066 & 0.0129 & 0.0044 & 0.0032 & 0.0053 \\
\hline
\end{tabular}




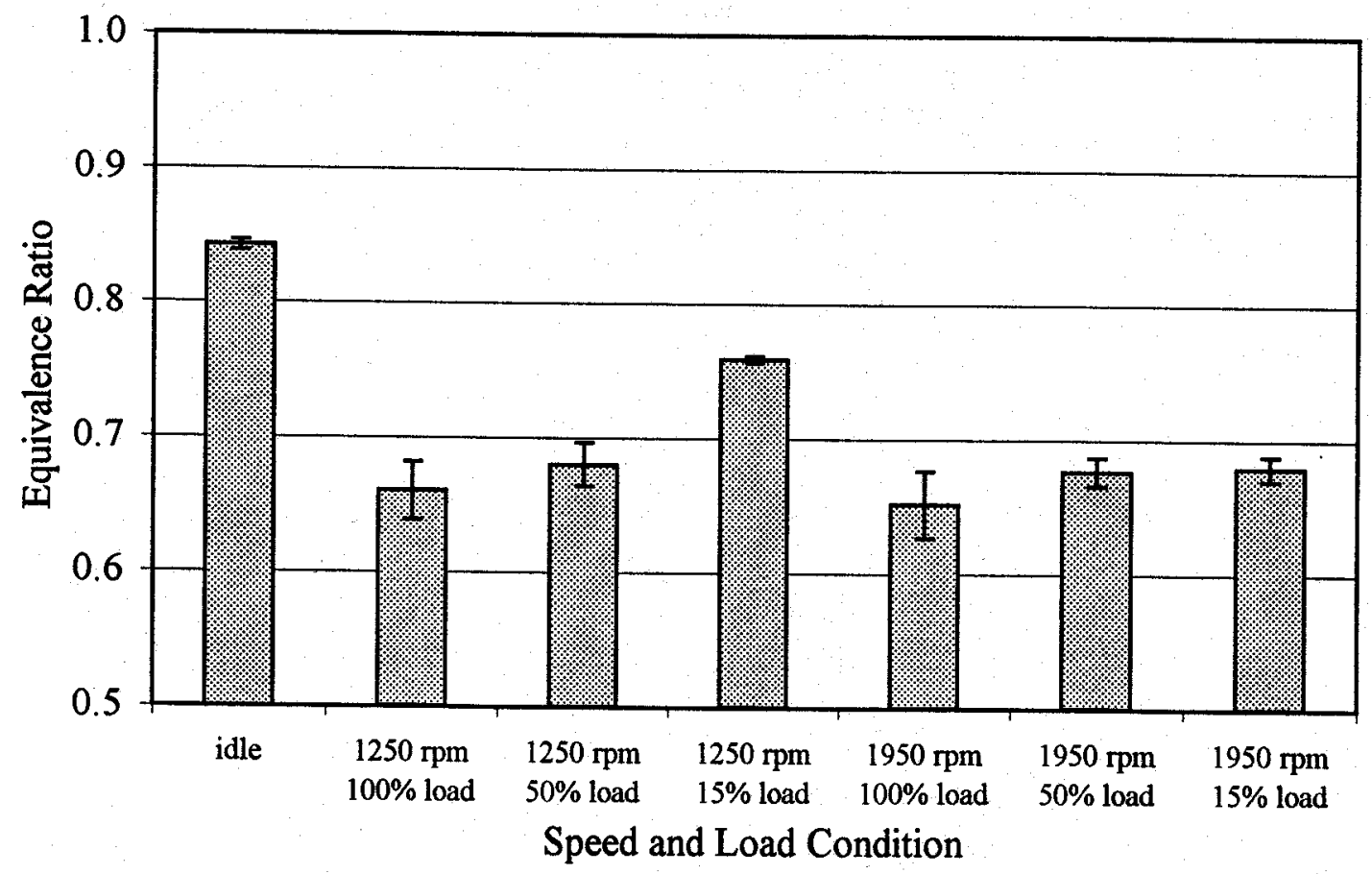

Figure 1. Average Equivalence Ratios and the Standard Error for the Six Cylinders using Mixing Elbow Number 1.

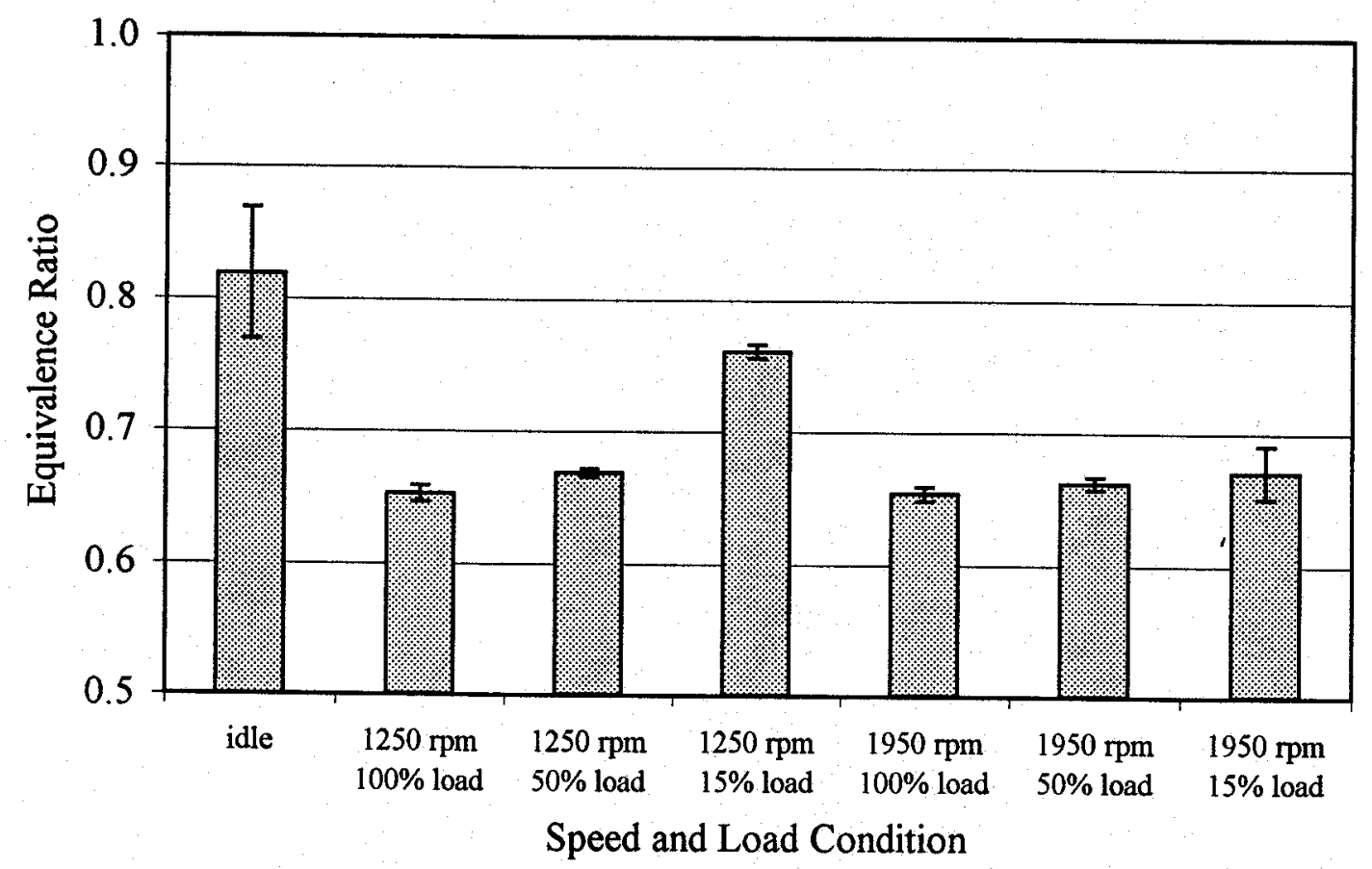

Figure 2. Average Equivalence Ratios and the Standard Error for the Six Cylinders using Mixing Elbow Number 2. 


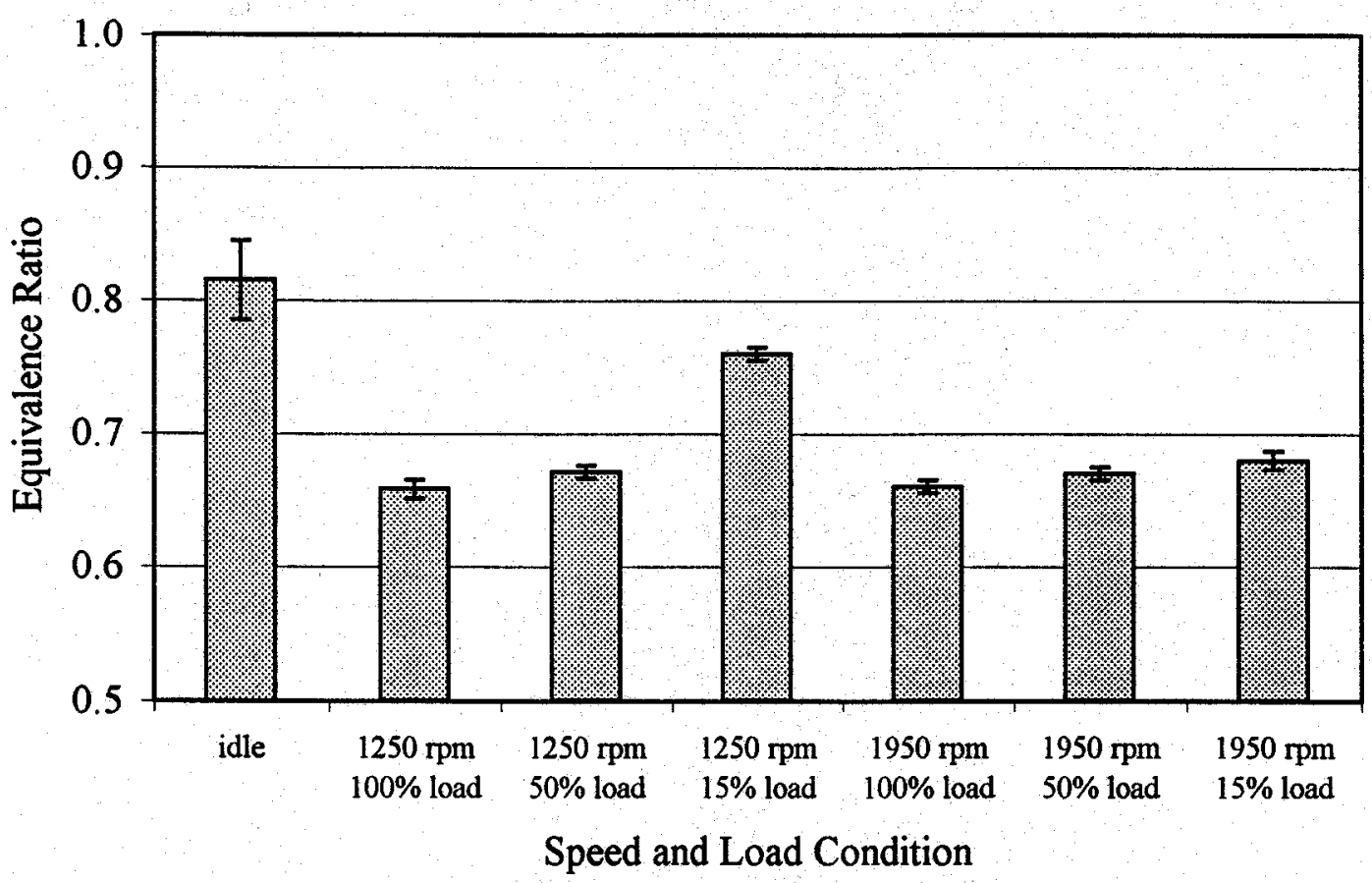

Figure 3. Average Equivalence Ratios and the Standard Error for the Six Cylinders using Mixing Elbow Number 3.

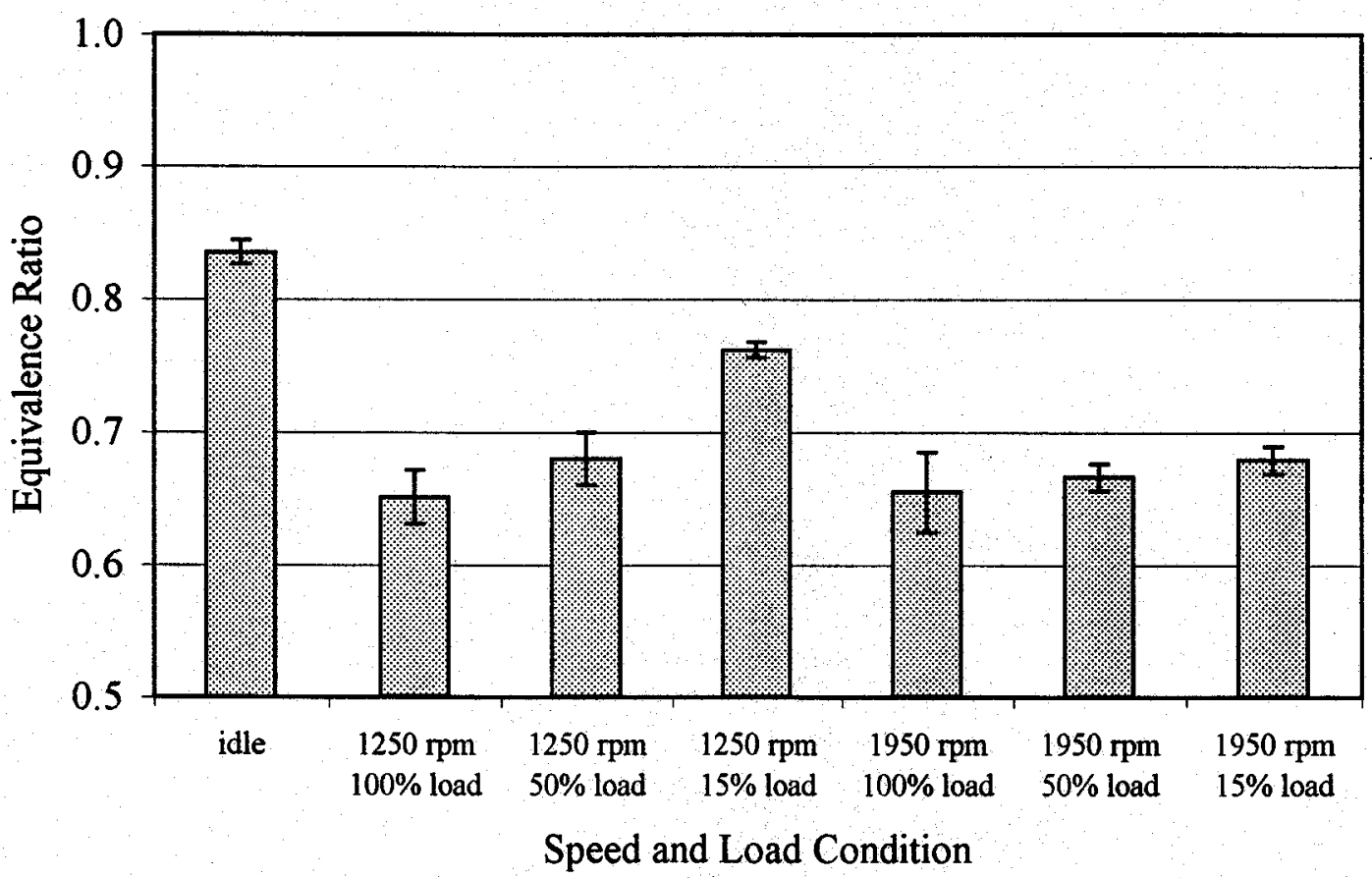

Figure 4. Average Equivalence Ratios and the Standard Error for the Six Cylinders using Mixing Elbow Number 4. 


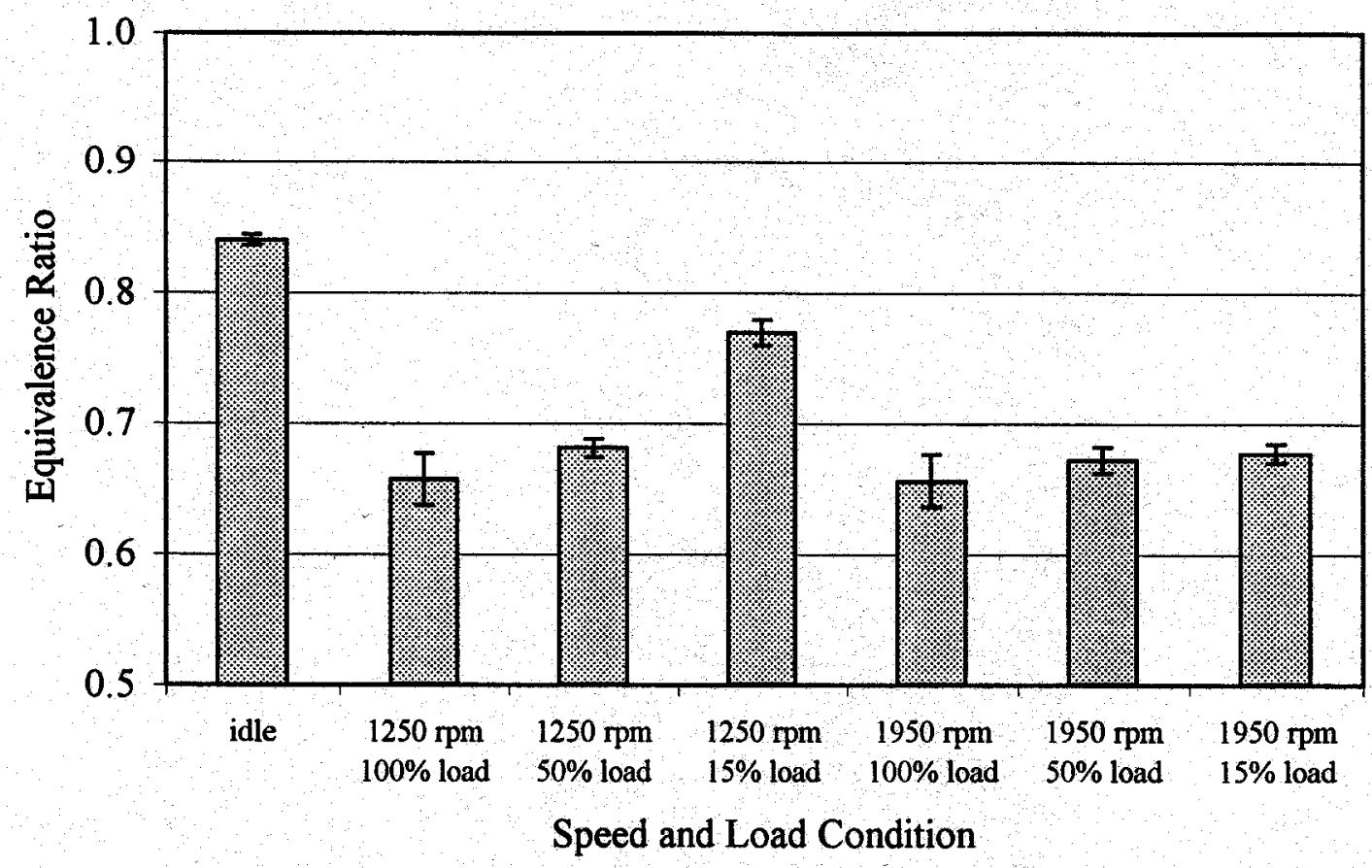

Figure 5. Average Equivalence Ratios and the Standard Error for the Six Cylinders using Mixing Elbow Number 5.

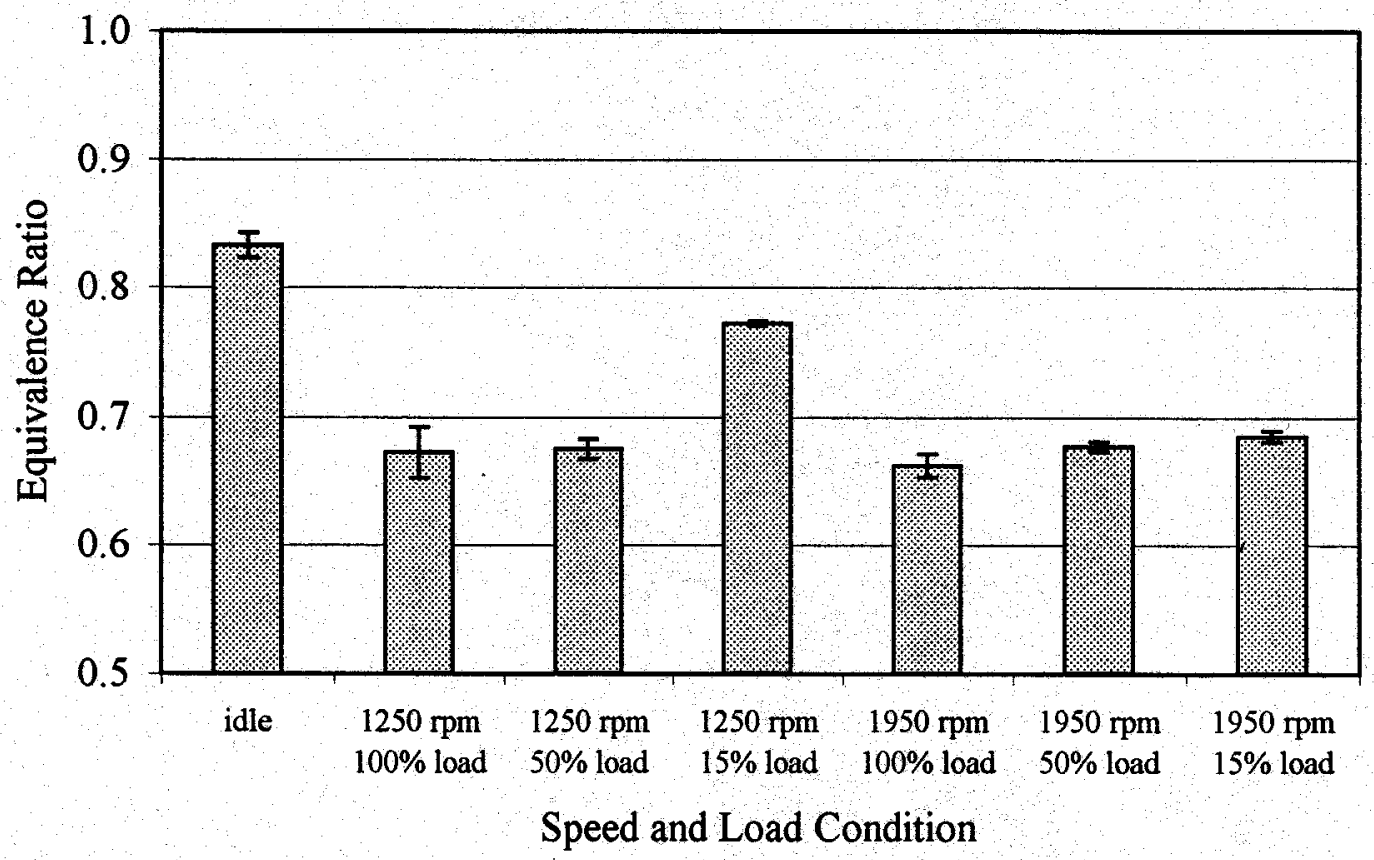

Figure 6. Average Equivalence Ratios and the Standard Error for the Six Cylinders using Mixing Elbow Number 6. 


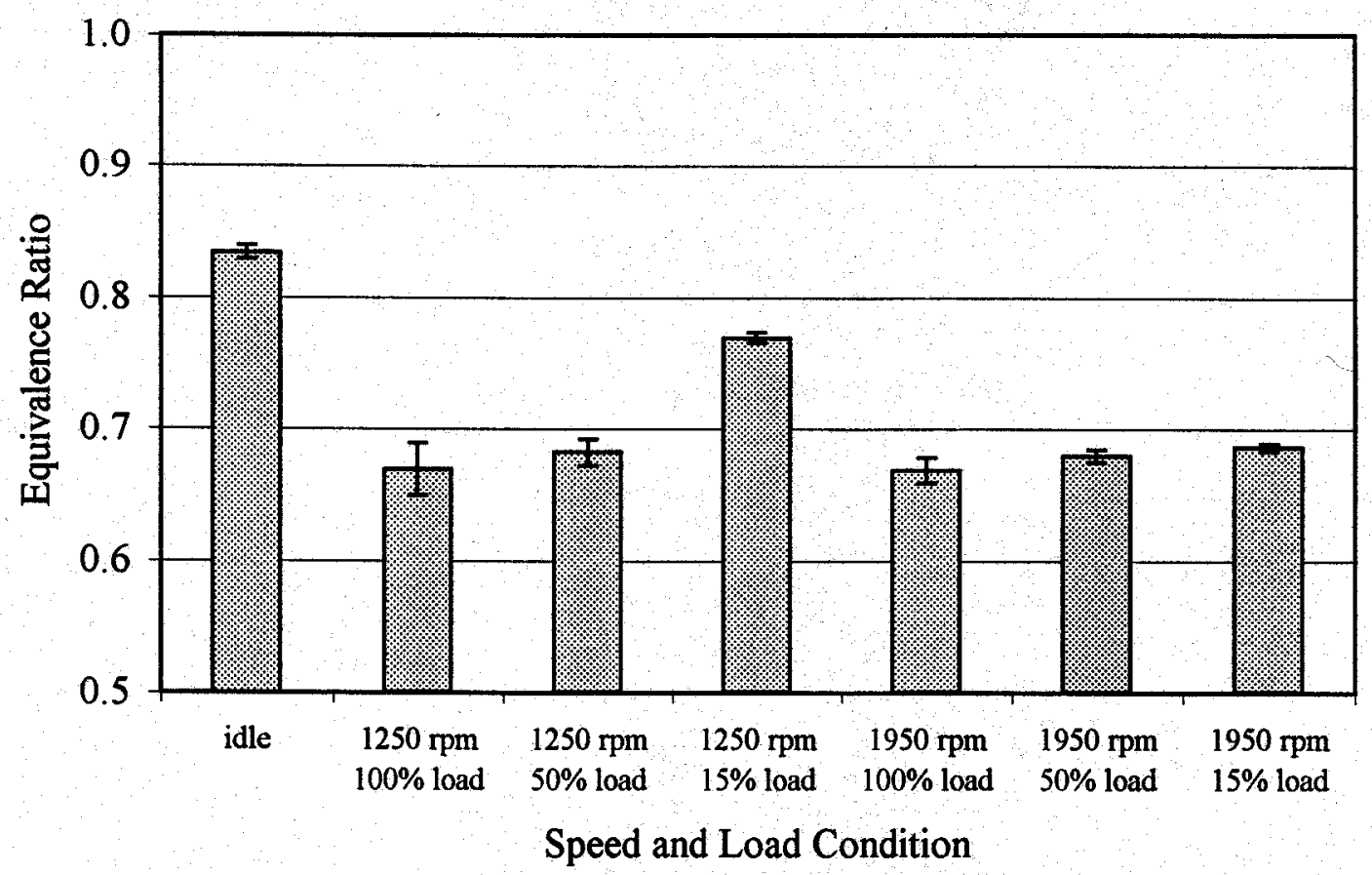

Figure 7. Average Equivalence Ratios and the Standard Error for the Six Cylinders using Mixing Elbow Number 7.

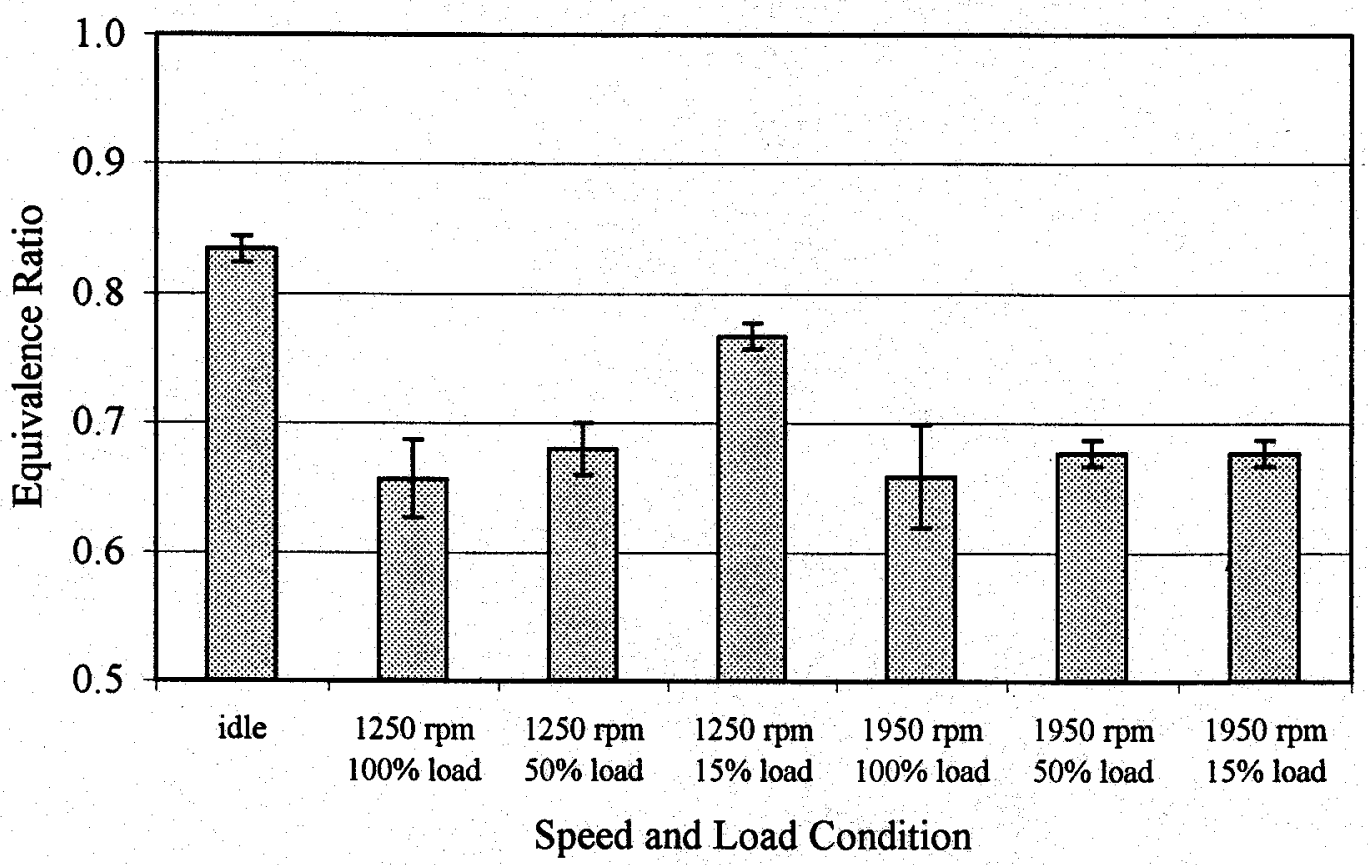

Figure 8. Average Equivalence Ratios and the Standard Error for the Six Cylinders using Mixing Elbow Number 8. 


\subsection{Cam Timing}

Another project, funded by Mack Trucks, was being conducted concurrently with this project. A separate engine was used for each project, and one engine showed higher torque at low engine speeds than the other engine. Mack Trucks had mis-machined a cam gear such that it advanced the cam by one tooth. Therefore, a study was conducted to determine the optimal cam timing for this engine. The cam profile for this engine is the same as the diesel engine it was derived from. The cam timing was tested at the original diesel cam timing and advanced one tooth on the cam gear. With an 84 tooth cam gear, one tooth advanced is 8.57 crank angle degrees. Figures 9-17 show the results for 1950, 1500, and $1250 \mathrm{rpm}$ at 100,75 , and $50 \%$ load. For either cam timing, the efficiencies are about the same for a given $\mathrm{NO}_{\mathrm{x}}$ level, whereas the $\mathrm{HC}$ emissions are lower with the original cam timing. With the advanced cam timing, higher torque at $1100 \mathrm{rpm}$ at WOT were noted. The advanced cam timing was selected for this engine for the higher low speed torque capability.

\subsection{Steady-State Engine Mapping}

Engine mapping included knock tolerance testing, lean-limit testing, and calibration of the engine controller tables.

\subsubsection{Knock Tolerance Testing}

Knock tolerance testing for the Mack E7G engine was presented in the final report for the Mack LNG Engine Development for Long-Haul Truck, SwRI Project No. 03-7816, and is presented again here. Results are based on an engine rated at $350 \mathrm{bhp}$ at $1900 \mathrm{rpm}$.

Knock testing was performed to determine the highest intake manifold air temperature, highest equivalence ratio and the most advance ignition timing possible without the engine knocking. The testing was conducted at various equivalence ratios, 105 and $150 \mathrm{deg} \mathrm{F}$ air temperature after the intercooler, and $200 \mathrm{deg} \mathrm{F}$ coolant out temperature. Ignition timing was advanced until the pressure signal from the $\# 2$ cylinder showed presence of knock. The composition of the natural gas fuel used for knock testing is shown in Table 4.

Table 4. Gas Composition for Knock Testing

\begin{tabular}{|c|c|}
\hline Gas & Volume (\%) \\
\hline \hline Methane & 85.1 \\
\hline Ethane & 9.0 \\
\hline Propane & 2.4 \\
\hline Nitrogen & 3.5 \\
\hline
\end{tabular}




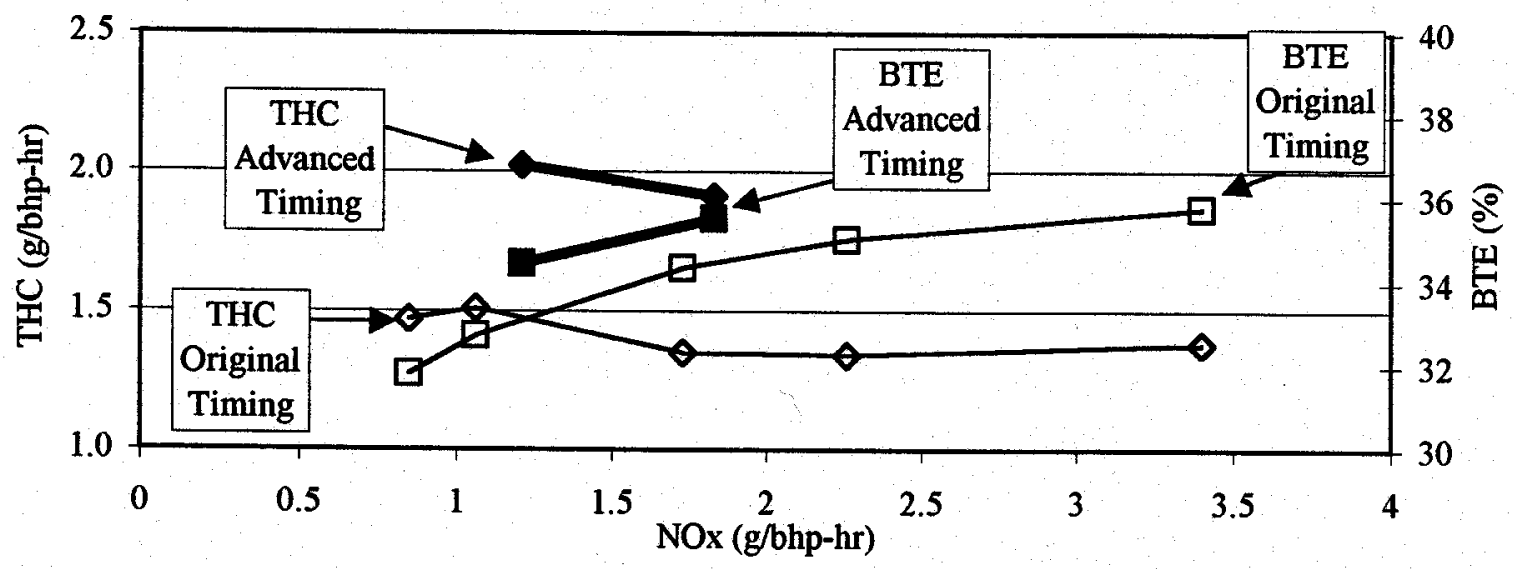

Figure 9. Comparison of Original and Advanced Cam Timing at $1950 \mathrm{rpm}, 100 \%$ Load, and Various Ignition Timings

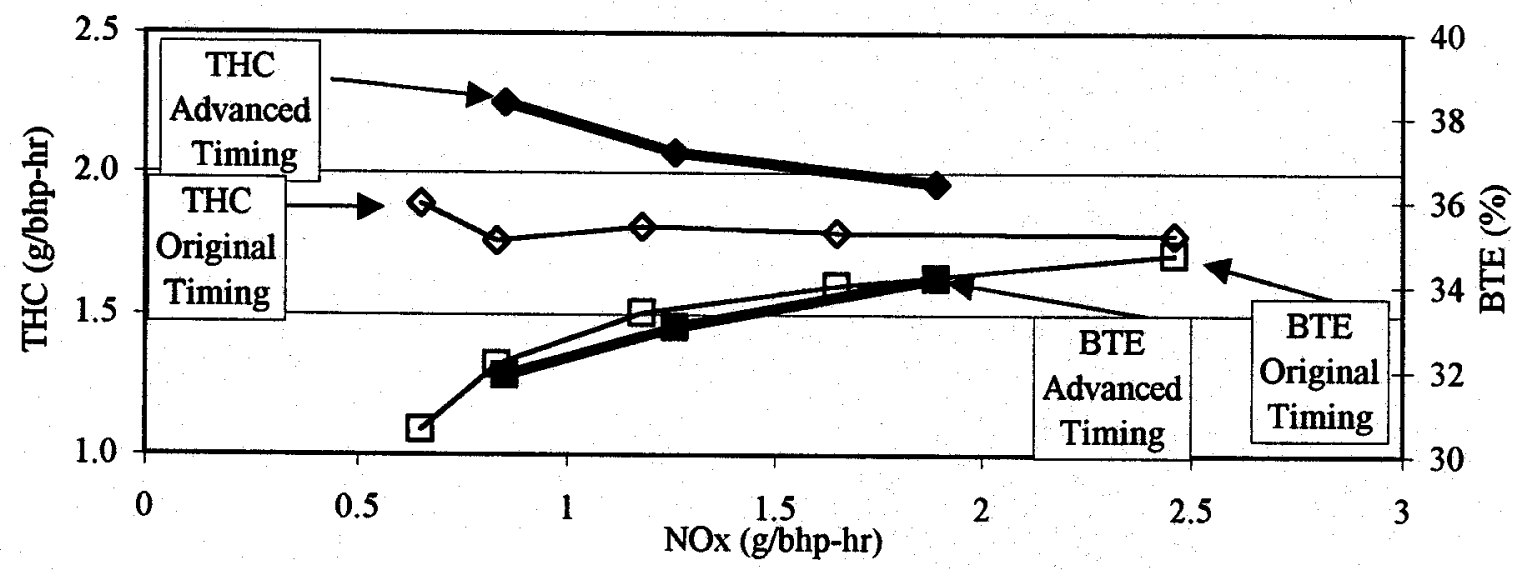

Figure 10. Comparison of Original and Advanced Cam Timing at $1950 \mathrm{rpm}, \mathbf{7 5} \%$ Load, and Various Ignition Timings

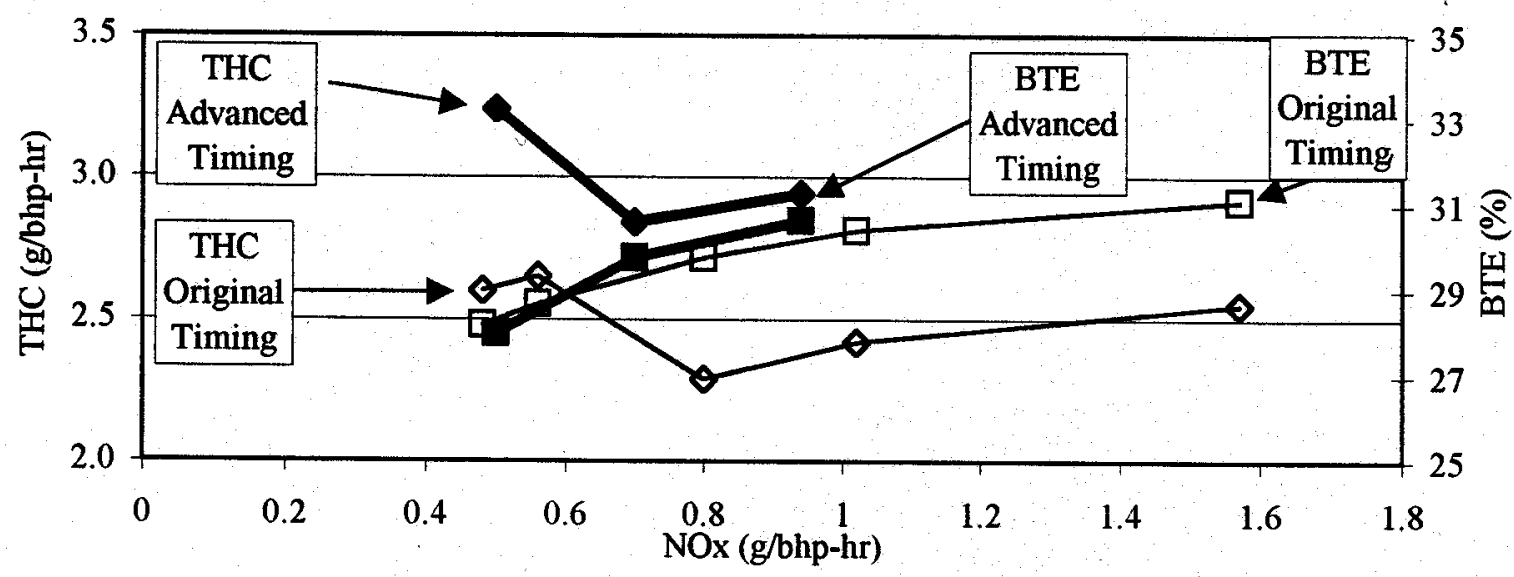

Figure 11. Comparison of Original and Advanced Cam Timing at $1950 \mathrm{rpm}, \mathbf{5 0 \%}$ Load, and Various Ignition Timings 


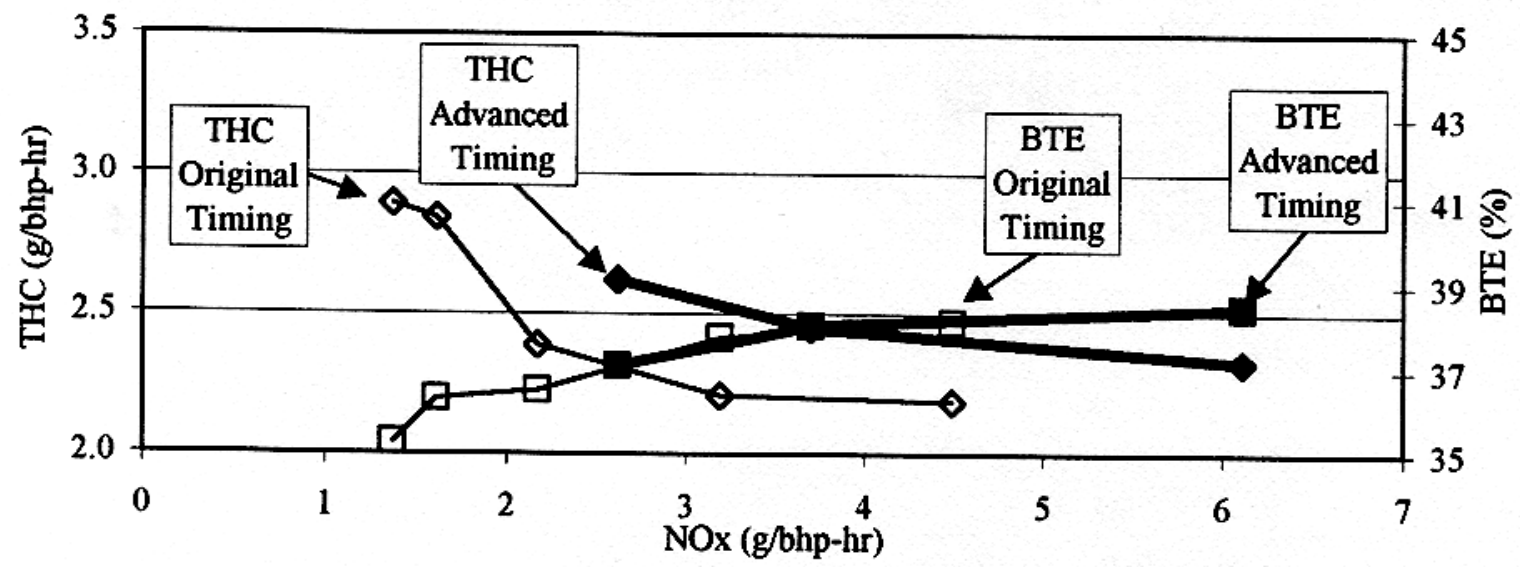

Figure 12. Comparison of Original and Advanced Cam Timing at $1500 \mathrm{rpm}, 100 \%$ Load, and Various Ignition Timings

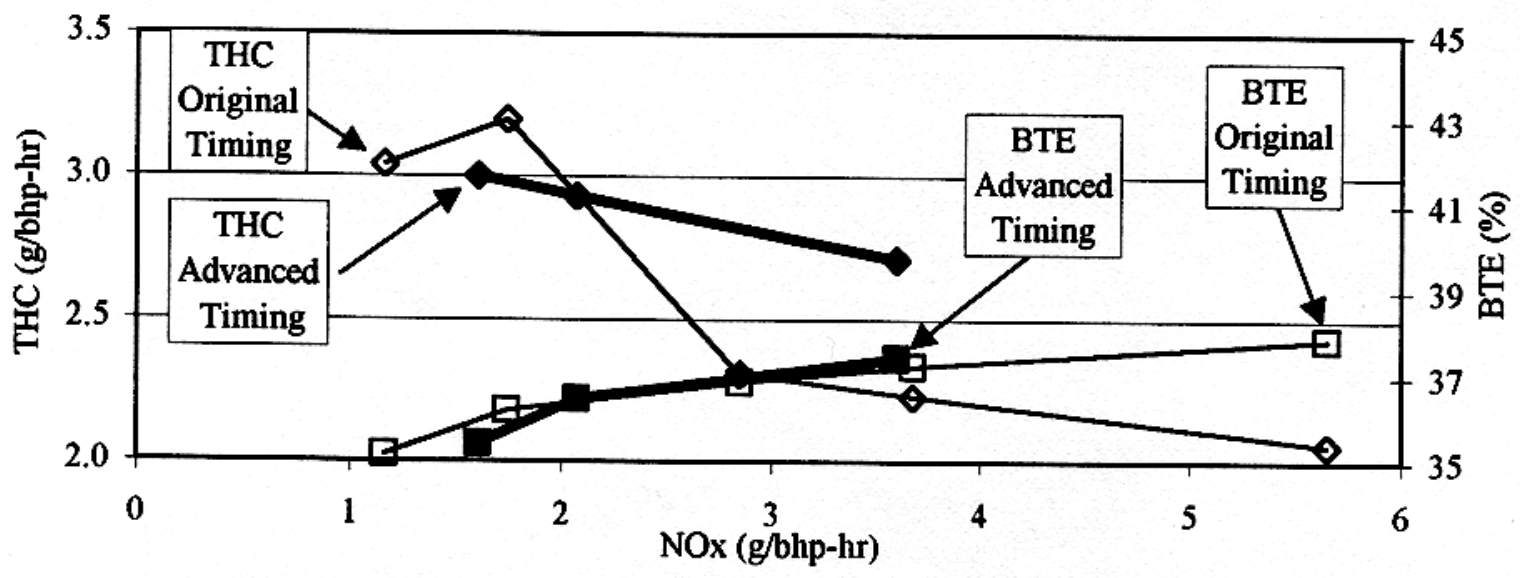

Figure 13. Comparison of Original and Advanced Cam Timing at $1500 \mathrm{rpm}, \mathbf{7 5 \%}$ Load, and Various Ignition Timings

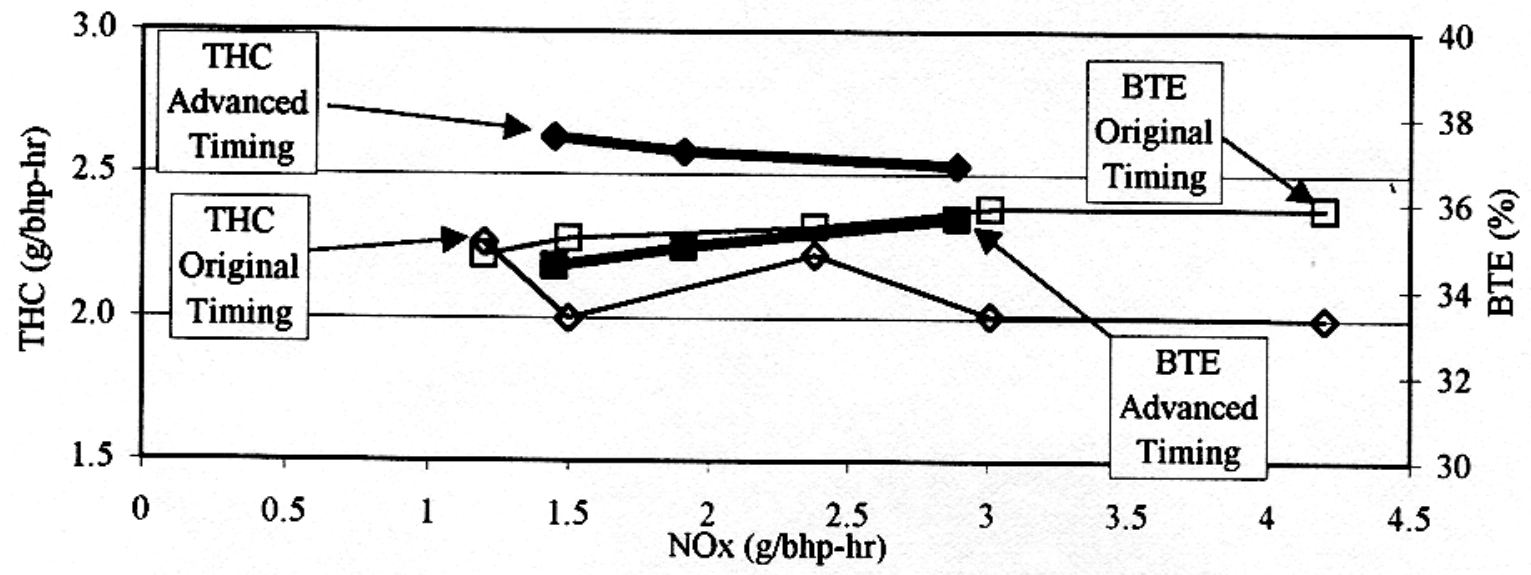

Figure 14. Comparison of Original and Advanced Cam Timing at $1500 \mathrm{rpm}, \mathbf{5 0} \% \mathrm{Load}$, and Various Ignition Timings 


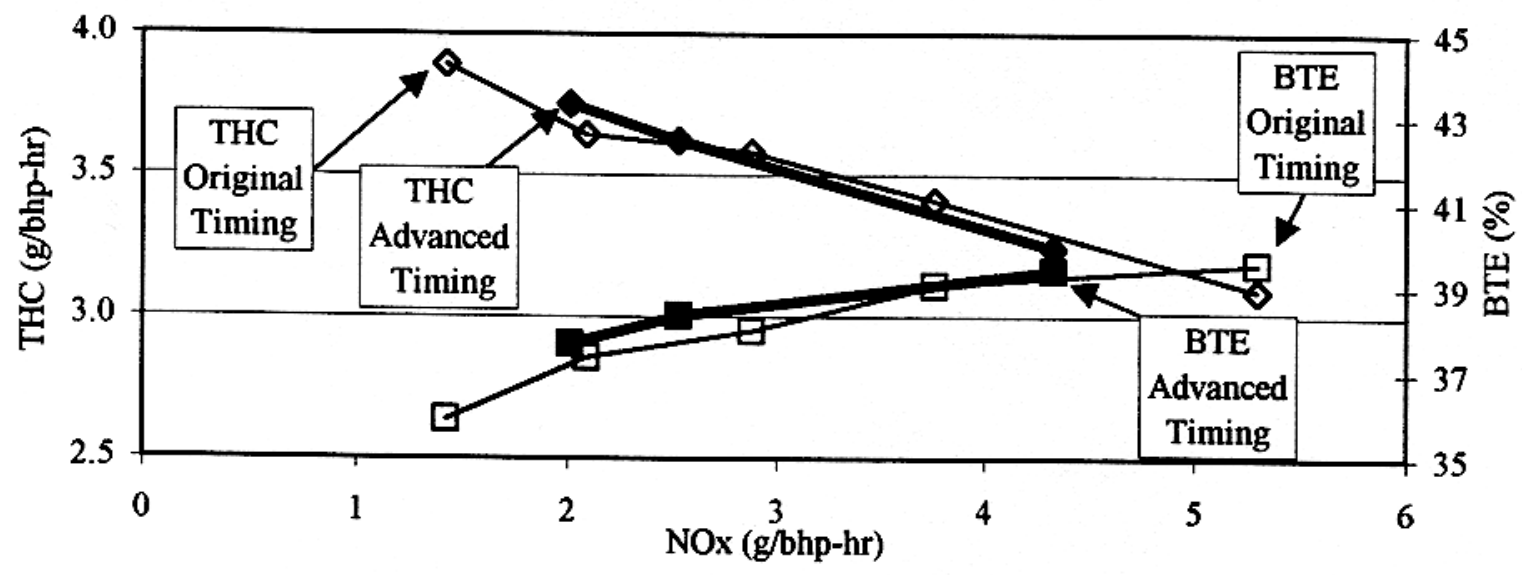

Figure 15. Comparison of Original and Advanced Cam Timing at $1250 \mathrm{rpm}, 100 \%$ Load, and Various Ignition Timings

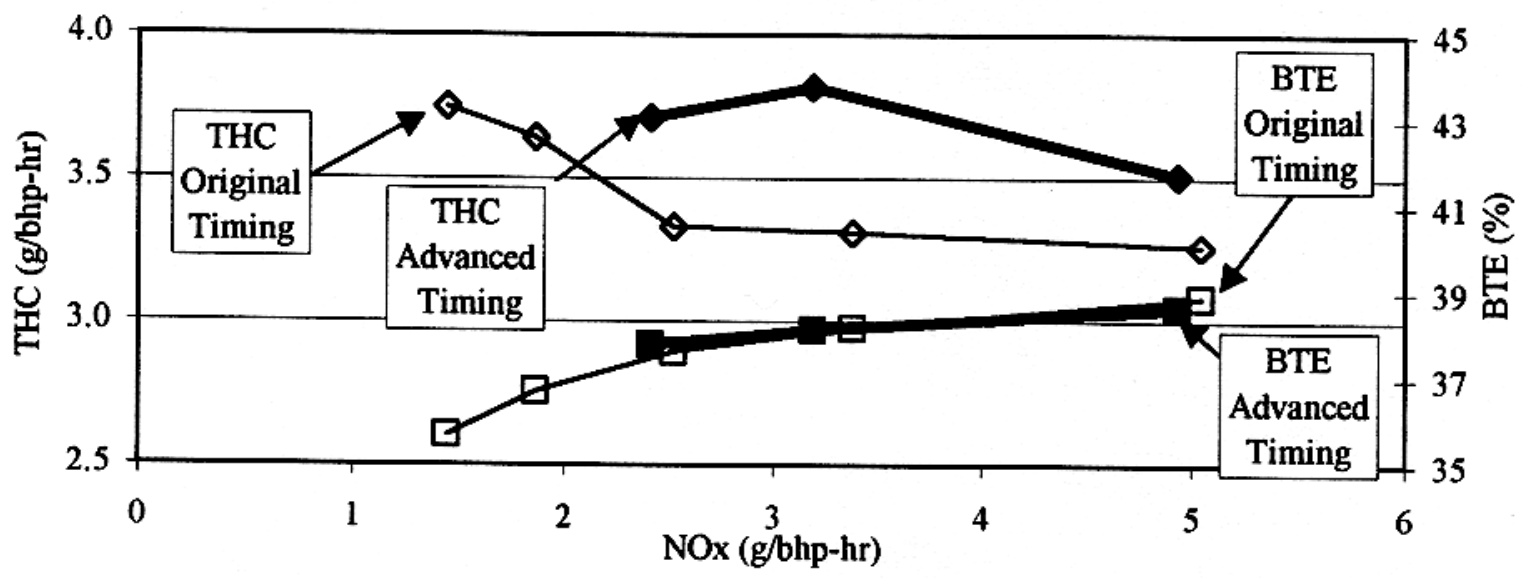

Figure 16. Comparison of Original and Advanced Cam Timing at $1250 \mathrm{rpm}, \mathbf{7 5} \%$ Load, and Various Ignition Timings

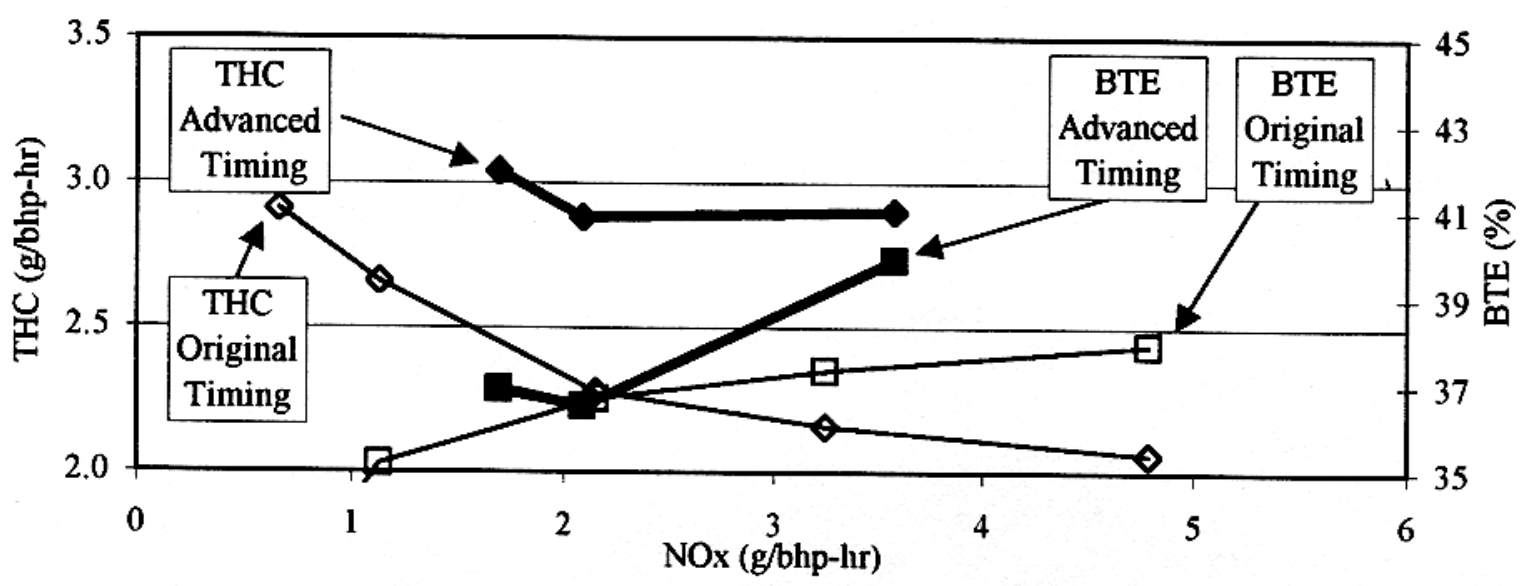

Figure 17. Comparison of Original and Advanced Cam Timing at $1250 \mathrm{rpm}, \mathbf{5 0} \%$ Load, and Various Ignition Timings 
The results of the knock testing are shown in Table 5 and in Figures 18-22. Figures 18-20 show tests at equivalence ratios between 0.65 and 0.75 for engine speed of 1900,1500 , and 1250 $\mathrm{rpm}$. Also shown on the graphs as reference are the points of MBT and MBT- 6 ignition timings at an equivalence ratio of 0.65 . Figures 21 and 22 show tests at equivalence ratios between 0.80 and 0.85 for engine speeds of 1100 and $1000 \mathrm{rpm}$. There are no ignition timing points shown on these graphs since timing are selected to provide sufficient boost for low speed torque.

Table 5. Results of Knock Testing

\begin{tabular}{|c|c|c|c|}
\hline $\begin{array}{c}\text { Engine Speed } \\
\text { (rpm) }\end{array}$ & $\begin{array}{c}\text { Equivalence } \\
\text { Ratio }\end{array}$ & $\begin{array}{c}\text { Ignition Timing at } \\
\text { Presence of Knock for } \\
\text { 105 deg F After } \\
\text { Intercooler } \\
\text { Temperature } \\
\text { (deg BTDC) }\end{array}$ & $\begin{array}{c}\text { Ignition Timing at } \\
\text { Presence of Knock for } \\
\text { 150 deg F After } \\
\text { Intercooler } \\
\text { Temperature } \\
\text { (deg BTDC) }\end{array}$ \\
\hline \hline 1900 & 0.65 & 30 & 23 \\
\hline 1900 & 0.70 & 23 & 19 \\
\hline 1900 & 0.75 & 19 & 15 \\
\hline 1500 & 0.65 & 25 & 20 \\
\hline 1500 & 0.70 & 22 & 17 \\
\hline 1500 & 0.75 & 18 & 16 \\
\hline 1250 & 0.67 & 23 & 18 \\
\hline 1250 & 0.70 & 22 & 17 \\
\hline 1250 & 0.75 & 19 & 15 \\
\hline 1100 & 0.80 & 18 & 10 \\
\hline 1100 & 0.85 & 14 & 12 \\
\hline 1000 & 0.80 & 19 & 9 \\
\hline 1000 & 0.85 & 17 & \\
\hline
\end{tabular}

\subsubsection{Lean-Limit Testing}

Lean-limit testing for the Mack E7G engine was presented in the final report for the Mack LNG Engine Development for Long-Haul Truck, SwRI Project No. 03-7816, and is presented again here. Results are based on an engine rated at $350 \mathrm{bhp}$ at $1900 \mathrm{rpm}$.

Lean limit tests were conducted at engine speeds of 1250 and $1900 \mathrm{rpm}$, and $100 \%$ load. Cylinder pressure data were recorded from the $\# 2$ cylinder, and the $\mathrm{COV}_{\text {imep }}$ was calculated over 50 engine cycles. The lean limit is defined as the equivalence ratio where the $\mathrm{COV}_{\text {imep }}$ exceeds 5\%. Tests were performed at three ignition timings: MBT, MBT-3, and MBT-6, and the equivalence ratio was lowered until audible misfire was detected. Figure 23 shows the results for $1250 \mathrm{rpm}$. At an ignition timing of 16 degrees BTDC, the lean limit is at an equivalence ratio of approximately 0.58. Figure 24 shows the results for $1900 \mathrm{rpm}$. At an ignition timing of 21 


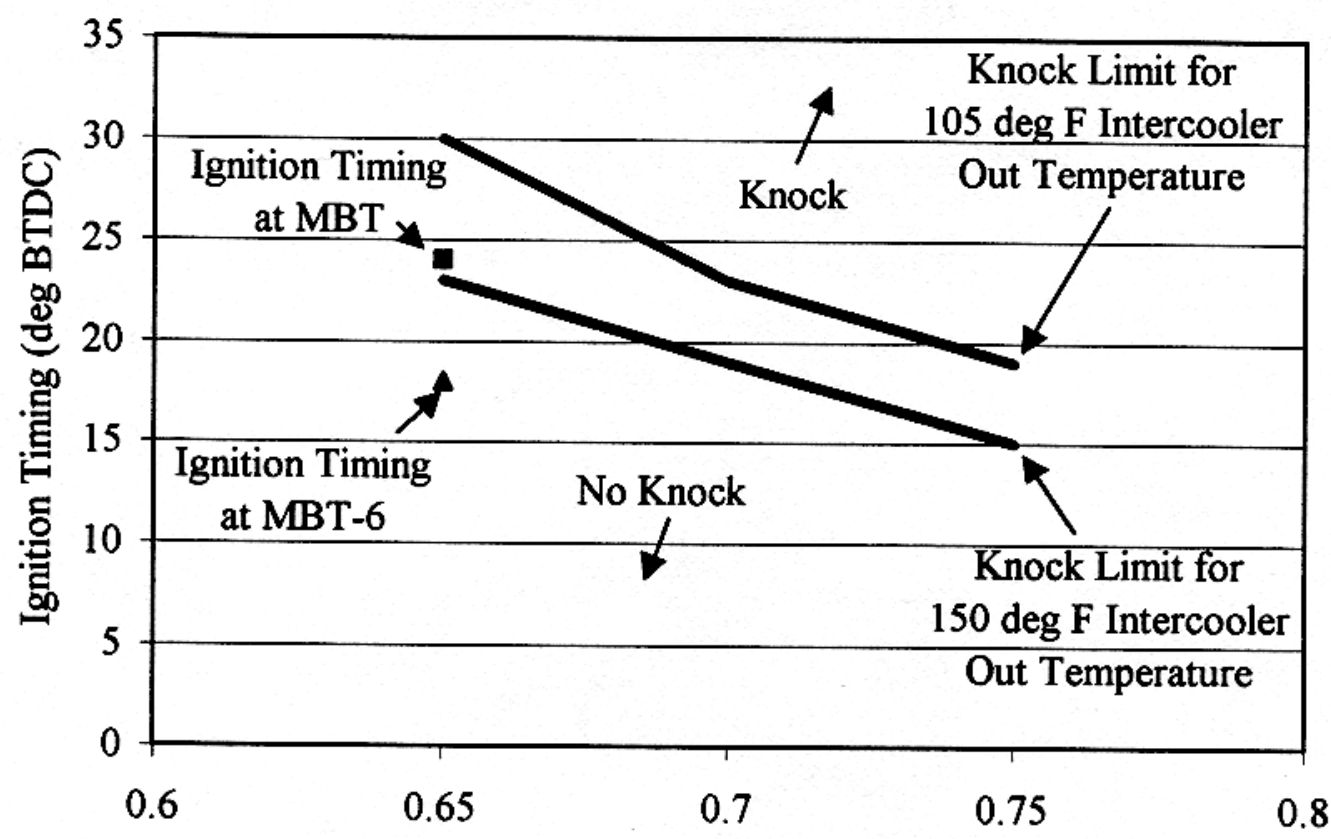

Equivalence Ratio

Figure 18. Knock Limit at 1900 rpm, $967 \mathrm{ft}-\mathrm{lb}$

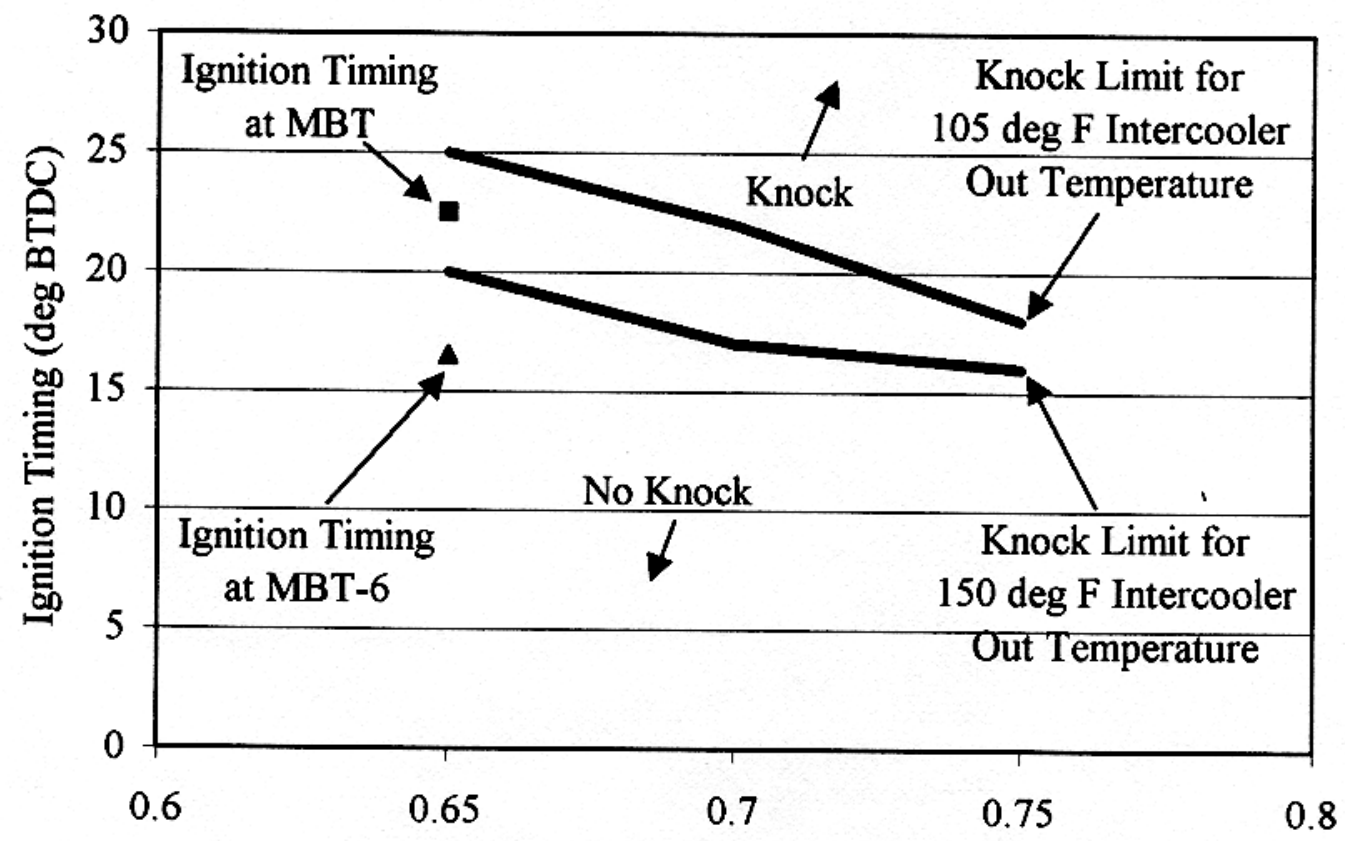

Equivalence Ratio

Figure 19. Knock Limit at $1500 \mathrm{rpm}, 1175 \mathrm{ft}-\mathrm{lb}$ 


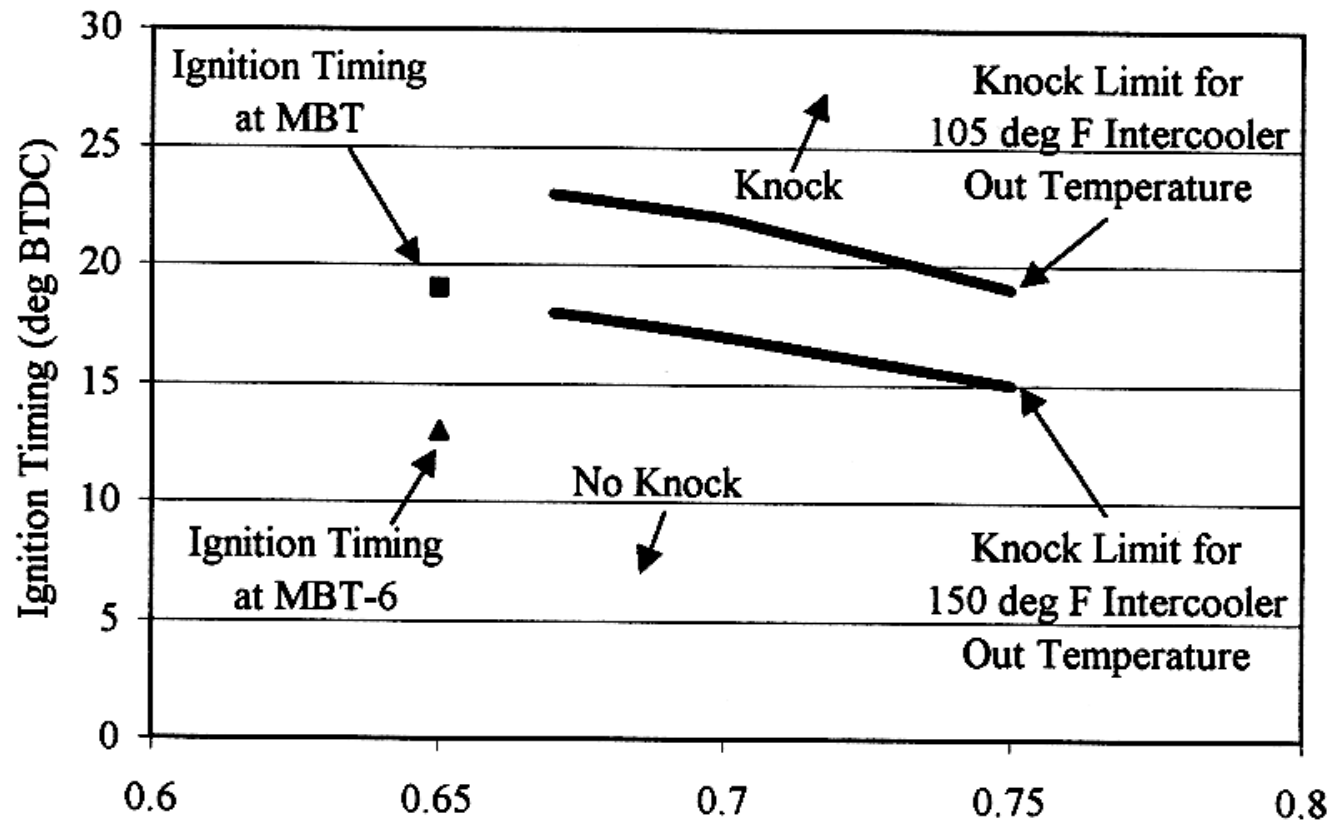

Equivalence Ratio

Figure 20. Knock Limit at $1250 \mathrm{rpm}, 1250 \mathrm{ft}-\mathrm{lb}$

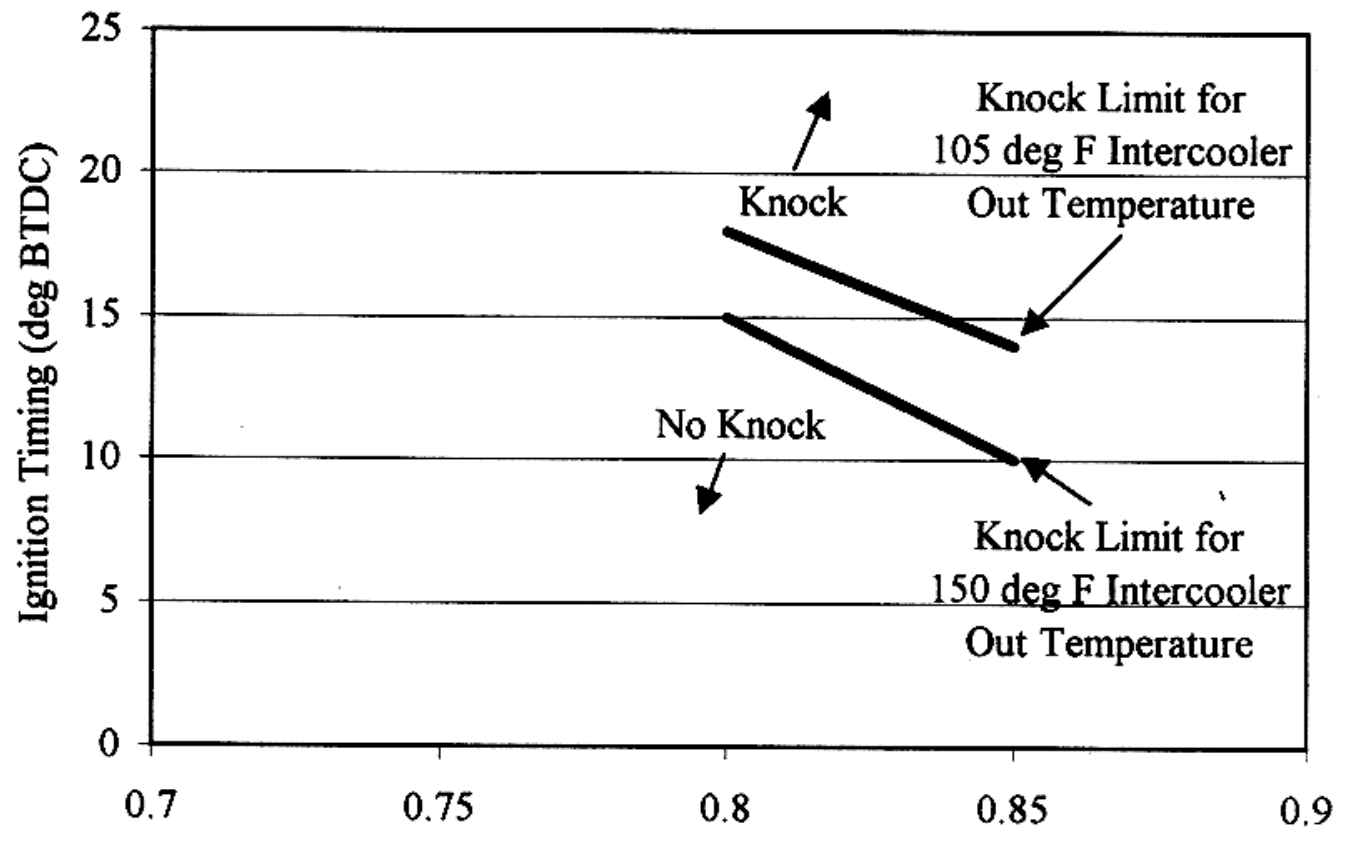

Equivalence Ratio

Figure 21. Knock Limit at 1100 rpm, $1230 \mathrm{ft}-\mathrm{lb}$ 


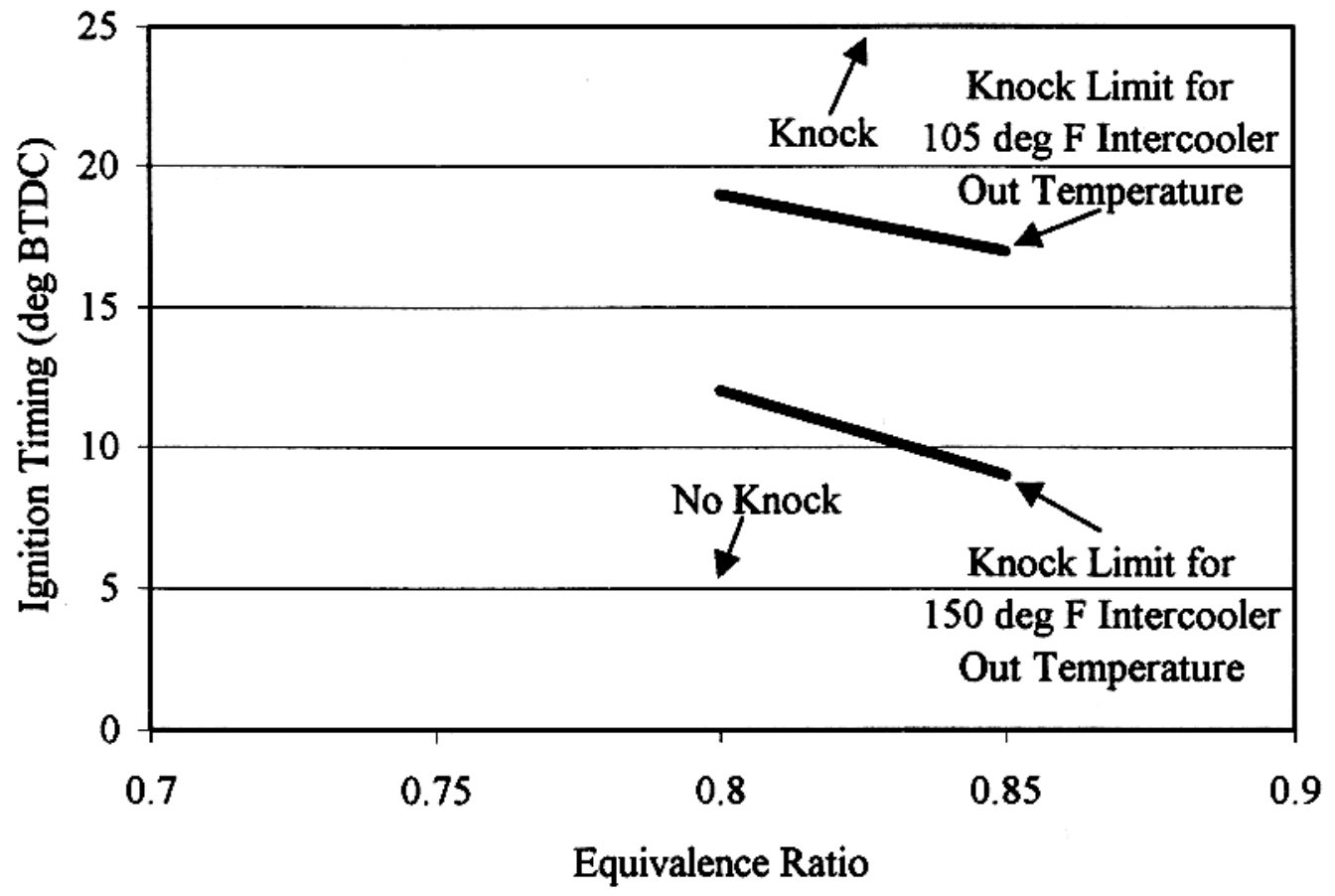

Figure 22. Knock Limit at $1000 \mathrm{rpm}, 1115 \mathrm{ft}-\mathrm{lb}$ 
degrees BTDC, the lean limit is at an equivalence ratio of approximately 0.60 . Table 6 shows the results of the lean limit tests. The run numbers refer to the recorded data included in Appendix A.

Table 6. Lean Limit Test Results

\begin{tabular}{|c|c|c|c|c|c|c|c|}
\hline \multicolumn{4}{|c|}{$1250 \mathrm{RPM}$} & \multicolumn{4}{|c|}{1900 RPM } \\
\hline $\begin{array}{c}\text { Run } \\
\text { Number }\end{array}$ & $\begin{array}{c}\text { Ignition } \\
\text { Timing } \\
\left({ }^{\circ} \mathrm{BTDC}\right)\end{array}$ & $\begin{array}{l}\text { Equiv. } \\
\text { Ratio }\end{array}$ & $\begin{array}{c}\mathrm{COV}_{\text {imep }} \\
(\%)\end{array}$ & $\begin{array}{c}\text { Run } \\
\text { Number }\end{array}$ & $\begin{array}{l}\text { Ignition } \\
\text { Timing } \\
\text { ('BTDC) }\end{array}$ & $\begin{array}{l}\text { Equiv. } \\
\text { Ratio }\end{array}$ & $\begin{array}{c}\mathrm{COV}_{\text {imep }} \\
(\%)\end{array}$ \\
\hline 866 & 19 & 0.65 & 1.24 & 844 & 24 & 0.65 & 1.62 \\
\hline 867 & 16 & 0.65 & 1.93 & 845 & 21 & 0.65 & 2.06 \\
\hline 868 & 13 & 0.65 & 3.58 & 846 & 18 & 0.65 & 2.93 \\
\hline 869 & 19 & 0.67 & 1.24 & 847 & 24 & 0.67 & 1.21 \\
\hline 870 & 16 & 0.67 & 1.77 & 848 & 21 & 0.67 & 2.00 \\
\hline 871 & 13 & 0.67 & 2.23 & 849 & 18 & 0.67 & 2.22 \\
\hline 872 & 19 & 0.64 & 1.55 & 850 & 24 & 0.64 & 1.99 \\
\hline 873 & 16 & 0.64 & 2.31 & 851 & 21 & 0.64 & 2.95 \\
\hline 874 & 13 & 0.64 & 4.82 & 852 & 18 & 0.64 & 3.53 \\
\hline 875 & 19 & 0.63 & 1.69 & 853 & 24 & 0.63 & 2.11 \\
\hline 876 & 16 & 0.63 & 2.09 & 854 & 21 & 0.63 & 2.49 \\
\hline 877 & 13 & 0.63 & 4.11 & 855 & 18 & 0.63 & 3.88 \\
\hline 878 & 19 & 0.62 & 1.96 & 856 & 24 & 0.62 & 1.80 \\
\hline 879 & 16 & 0.62 & 4.21 & 857 & 21 & 0.62 & 3.36 \\
\hline 880 & 13 & 0.62 & 4.27 & 858 & 18 & 0.62 & 4.53 \\
\hline 881 & 19 & 0.61 & 1.98 & 859 & 24 & 0.61 & 2.79 \\
\hline 882 & 16 & 0.61 & 3.51 & 860 & 21 & 0.61 & 3.88 \\
\hline 883 & 13 & 0.61 & 5.55 & 861 & 18 & 0.61 & 5.31 \\
\hline 884 & 19 & 0.60 & 3.08 & 862 & 24 & 0.60 & 3.36 \\
\hline 885 & 16 & 0.60 & 3.45 & 863 & 21 & 0.60 & 4.74 \\
\hline 886 & 13 & 0.60 & 7.32 & 864 & 18 & 0.60 & 6.32 \\
\hline 887 & 19 & 0.59 & $2.51^{\prime}$ & 865 & 24 & 0.59 & 4.89 \\
\hline 888 & 16 & 0.59 & 3.76 & & & & \\
\hline 889 & 13 & 0.59 & 5.38 & & & & \\
\hline 890 & 19 & 0.58 & 4.06 & & & & \\
\hline 891 & 16 & 0.58 & 5.53 & & & & \\
\hline
\end{tabular}




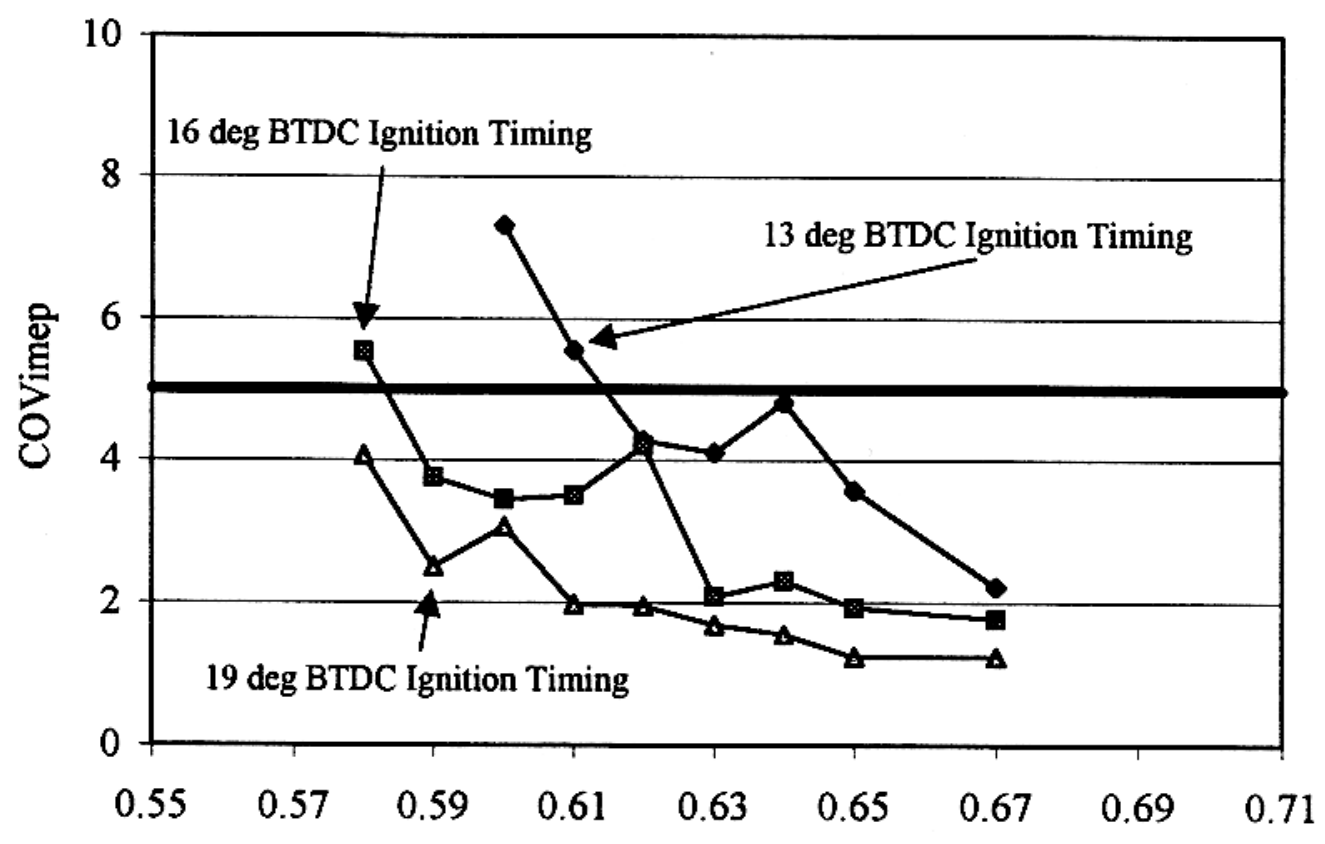

Equivalence Ratio

Figure 23. Lean Limit Tests at $1250 \mathrm{rpm}, 1250 \mathrm{ft}-\mathrm{lb}$

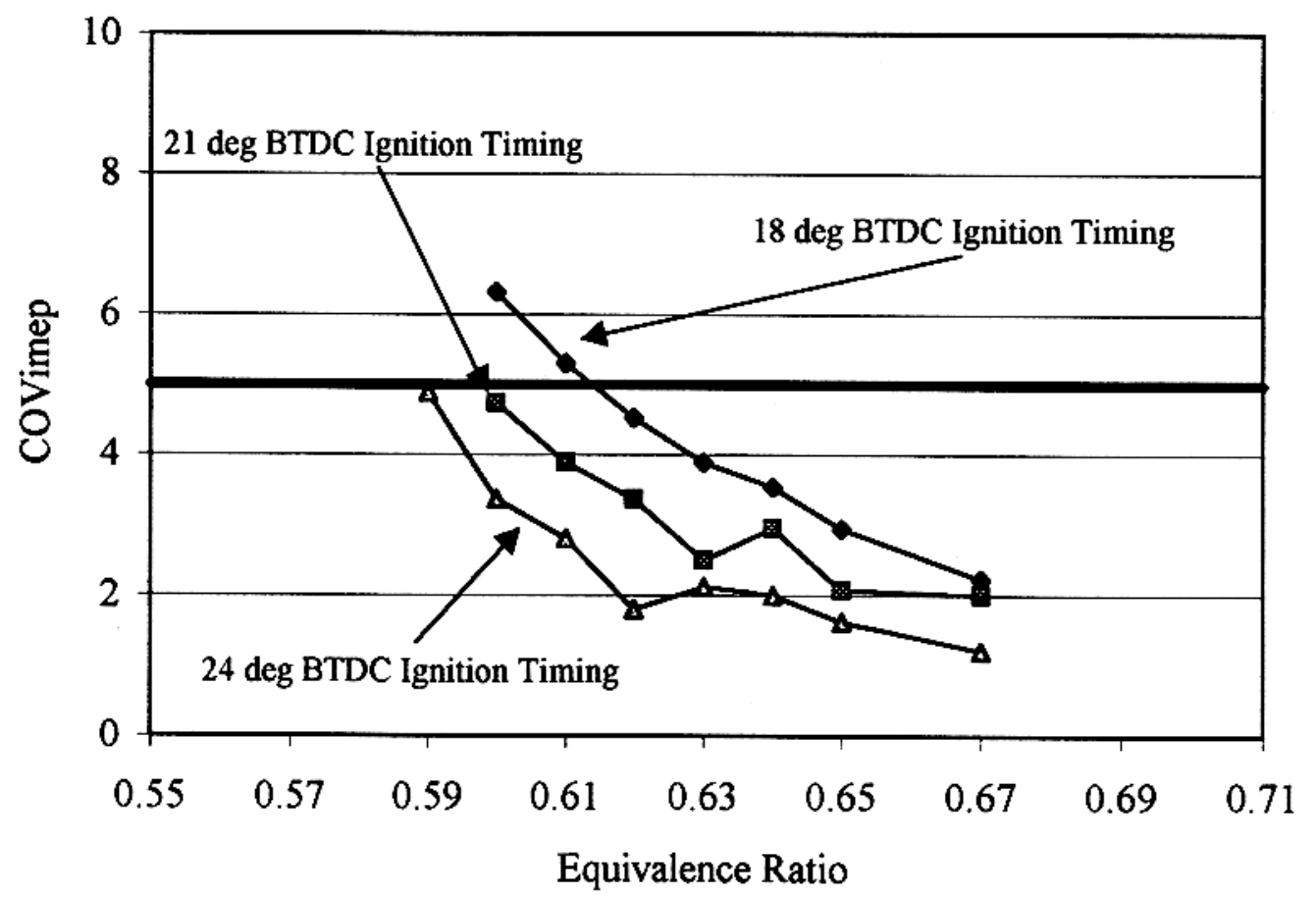

Figure 24. Lean Limit Tests at 1900 rpm, 967 ft-lb 


\subsubsection{Calibration}

Steady-state tests of the Mack E7G engine included calibration of boost control, volumetric efficiency, equivalence ratio, and ignition timing. Transient tests included further calibration of equivalence ratio and ignition timing, calibration of boost control to rematch the 325 bhp torque curve with the change in ignition timing at WOT, and calibration of the turbo lag compensation.

Turbocharger boost determines the power output of the engine for a given throttle position. It is controlled through a pneumatic wastegate controller. Since Mack Trucks builds the engine and the vehicle, a source of pressurized air can be made available on the truck for the engine controller to use under any operating condition. In the test stand, shop air was used to simulate the constant source of pressurized air. The pressurized air to the wastegate actuator is controlled through a pulse width modulated solenoid valve. The engine controller determines the amount of turbocharger boost based on throttle position and engine speed through a look up table. An electronic throttle is used on this engine with a throttle command of 0.5 to 4.5 volts.

At throttle positions of less than 2.0 volts, the wastegate is fully opened to minimize throttling losses. At positions of 2.0 to about 4.0 volts, the turbocharger boost is set to minimize the pressure drop across the throttle. At throttle positions over 4.0 volts, the turbocharger boost is set to match the 325 bhp torque curve.

Volumetric efficiency (VE) is used to determine the open-loop fueling rate to the engine for the desired equivalence ratio. Corrections to the VE are accomplished through closed-loop control based on feedback from the universal exhaust gas oxygen (UEGO) sensor. A closed-loop multiplier performs immediate corrections to the VE to maintain the correct equivalence ratio. An adaptive loop multiplier, which is saved in a table, performs longer term corrections. The engine was operated at steady-state conditions with the adaptive loop multiplier set to zero, and the volumetric efficiency table adjusted to maintain a closed-loop multiplier of less than $1.00 \%$.

The equivalence ratio table is used for air/fuel ratio control of the lean burn engine. As equivalence ratio is lowered from stoichiometric, the efficiency increases and the $\mathrm{NO}_{\mathrm{x}}$ emissions decrease. If the equivalence ratio is too low, the hydrocarbon emissions increase, efficiency decreases, and misfires are possible. Equivalence ratio was set for lean operation with a margin above the misfire limit for the higher engine speeds where turbo boost was sufficient to run lean, and was set for power and driveability at lower engine speeds where turbo boost was not sufficient to run lean (particularly at WOT). Further refinements to the equivalence ratio table were performed during transient emissions testing. These included a lower equivalence ratio at idle to reduce $\mathrm{NO}_{\mathrm{x}}$ emissions on the transient emissions test cycle, and lower equivalence ratios at low speeds, including low to mid loads to reduce $\mathrm{NO}_{\mathrm{x}}$ emissions for the West Virginia University CBD cycle on a chassis dyno.

Ignition timing is set as a compromise between efficiency and $\mathrm{NO}_{\mathrm{x}}$ emissions. The best efficiency is at MBT timing, but to meet the $\mathrm{NO}_{\mathrm{x}}$ emissions goals, retarded timings are required. A 12-mode steady-state prediction for $\mathrm{NO}_{\mathrm{x}}$ emissions was used to determine the initial ignitiontiming table for transient emissions testing. The 12-mode test is shown in Table 7. Results of 
the 12-mode test for ignition timing retarded the same amount at all 12 test points and are shown in Table 8. The 12-mode results for the initial ignition timing table for transient emissions testing is shown in Table 9. The transient emissions test with the initial ignition timing table was

$2.86 \mathrm{~g} / \mathrm{bhp}-\mathrm{hr}$. Ignition timing was modified to lower the $\mathrm{NO}_{\mathrm{x}}$ emissions on the transient test to $2.15 \mathrm{~g} / \mathrm{bhp}-\mathrm{hr}$.

Table 7. Test Points for the 12-Mode Steady-State Prediction for Transient Emissions

\begin{tabular}{|c|c|c|c|c|c|c|}
\hline Mode & $\begin{array}{c}\text { Engine Speed } \\
(\boldsymbol{\%})\end{array}$ & $\begin{array}{c}\text { Load } \\
(\boldsymbol{\%})\end{array}$ & $\begin{array}{c}\text { Weight } \\
(\boldsymbol{\%})\end{array}$ & $\begin{array}{c}\text { Engine } \\
\text { Speed (rpm) }\end{array}$ & $\begin{array}{c}\text { Torque } \\
(\mathbf{f t}-\mathbf{l b})\end{array}$ & $\begin{array}{c}\text { MBT } \\
\left({ }^{\circ} \mathbf{B T D C}\right)\end{array}$ \\
\hline 1 & 0 & CITT & 8.8 & 600 & 160 & 20 \\
\hline 2 & 50 & 83 & 2.6 & 1300 & 963 & 22 \\
\hline 3 & 50 & 100 & 2.1 & 1300 & 1160 & 21 \\
\hline 4 & 67 & 73 & 3.4 & 1520 & 896 & 26 \\
\hline 5 & 83 & 50 & 7.0 & 1730 & 485 & 28 \\
\hline 6 & 83 & 73 & 5.5 & 1730 & 805 & 27 \\
\hline 7 & 83 & 100 & 11.9 & 1730 & 970 & 23 \\
\hline 8 & 100 & 33 & 5.1 & 1950 & 287 & 30 \\
\hline 9 & 100 & 50 & 9.6 & 1950 & 435 & 27 \\
\hline 10 & 100 & 67 & 7.2 & 1950 & 583 & 26 \\
\hline 11 & 100 & 83 & 16.8 & 1950 & 722 & 26 \\
\hline 12 & 100 & 100 & 20.0 & 1950 & 870 & 23 \\
\hline
\end{tabular}

Table 8. Predicted FTP Transient Results

\begin{tabular}{|c|c|c|c|c|}
\hline $\begin{array}{c}\text { Ignition Timing } \\
\left({ }^{\circ} \text { BTDC) }\right.\end{array}$ & $\begin{array}{c}\text { BSNO } \\
\text { (g/bhp-hr) }\end{array}$ & $\begin{array}{c}\text { BSTHC } \\
(\mathbf{g} / \mathbf{b h p}-\mathbf{h r})\end{array}$ & $\begin{array}{c}\text { BSCO } \\
(\mathbf{g} / \mathbf{b h p}-\mathbf{h r})\end{array}$ & $\begin{array}{c}\text { BTE } \\
(\boldsymbol{\%})\end{array}$ \\
\hline MBT & 4.18 & 1.75 & 1.15 & 35.1 \\
\hline MBT-3 & 2.93 & 1.77 & 1.14 & 34.6 \\
\hline MBT-5 & 2.12 & 1.85 & 1.14 & 34.2 \\
\hline MBT-7 & 1.58 & 1.79 & 1.12 & 33.5 \\
\hline
\end{tabular}


Table 9. Ignition Timing Based on Steady-state Tests

\begin{tabular}{|c|c|c|c|}
\hline Mode & Ignition Timing ('BTDC) & BSNO $_{\mathbf{x}}$ (g/bhp-hr) & BTE (\%) \\
\hline 1 & MBT & 0.52 & 26.8 \\
\hline 2 & MBT-7 & 2.14 & 37.9 \\
\hline 3 & MBT-7 & 2.10 & 37.8 \\
\hline 4 & MBT-7 & 3.10 & 38.3 \\
\hline 5 & MBT-5 & 1.67 & 33.6 \\
\hline 6 & MBT-7 & 2.63 & 37 \\
\hline 7 & MBT-7 & 1.73 & 36.4 \\
\hline 8 & MBT & 3.00 & 30 \\
\hline 9 & MBT-5 & 1.34 & 31.5 \\
\hline 10 & MBT-5 & 1.75 & 34.4 \\
\hline 11 & MBT-5 & 2.09 & 35.3 \\
\hline 12 & MBT-5 & 1.45 & 34.9 \\
\hline Prediction & & 1.86 & 34.1 \\
\hline
\end{tabular}

Recorded data for the steady-state tests are included in Appendix B. Tables with the final calibration to be used for certification are included in Appendix C.

\subsection{Durability Testing}

The first durability test was reported in the final report for Task $2-1000$-Hour Super Thermal Cycle Test, SwRI Project No. 03-7501-002, and is included in Appendix D. The second durability test was reported in the final report for the 1000-Hour Super Thermal Cycle Test on a Mack E-7 Natural Gas Fueled Engine, SwRI Project No. 03-1248, and is included in Appendix E.

\subsection{Data Logger}

A data logger was installed on a refuse truck for in service use. The data logger recorded information from the vehicle and the engine. A list of data recorded is shown in Table 10. Due to harsh environmental conditions, the fuel system pressure sensors added for data logging failed. The fuel pressure used by the engine controller was still operational. Data for engine speed, vehicle speed, and MAP for a typical day are shown in Figures 25-27. Most of the occurrences occur at idle (600 rpm, $0 \mathrm{mph}, 8 \mathrm{psia})$, followed by packing ( $1200 \mathrm{rpm}, 0 \mathrm{mph}, 11$ psia). There were 395 total minutes of data logging time for this day, 31 minutes were used for packing and 122 minutes were used for idling.

Histograms for data recorded from 4/23/98 to 6/29/98 are included in Appendix F. In the period from 4/23/98 to 6/29/98 the data logger recorded 2909 miles with 2063 gallons of LNG used for a fuel economy of $1.41 \mathrm{mpg}$, which is a diesel equivalent fuel economy of $2.28 \mathrm{mpg}$. Of the 2063 gallons used, 227 gallons were used at idle and 236 gallons were used for packing, which represents $11.0 \%$ and $11.4 \%$ of the total fuel used, respectively. 


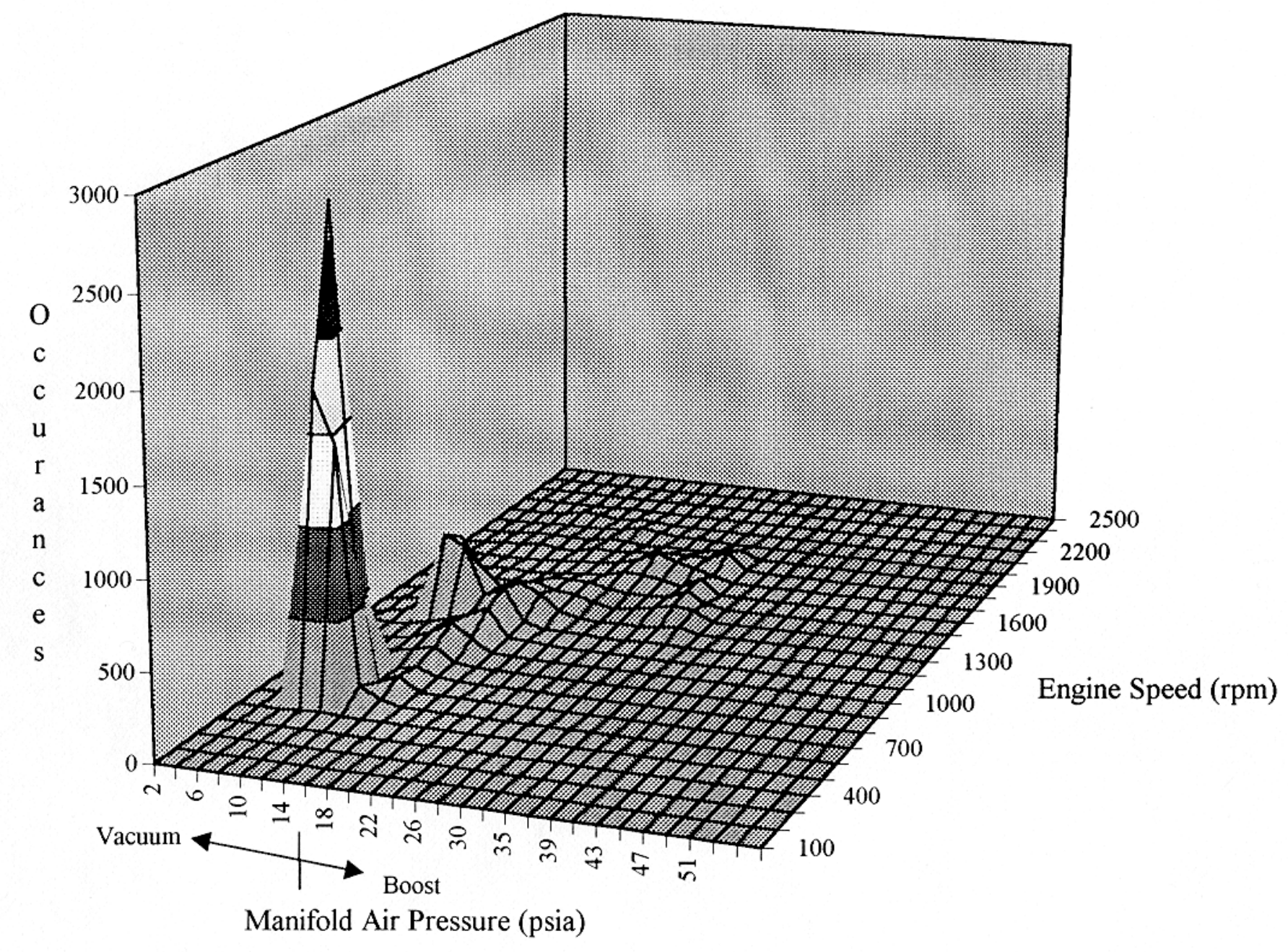

Figure 25. Engine Speed vs. Manifold Air Pressure for One Day of Operation 


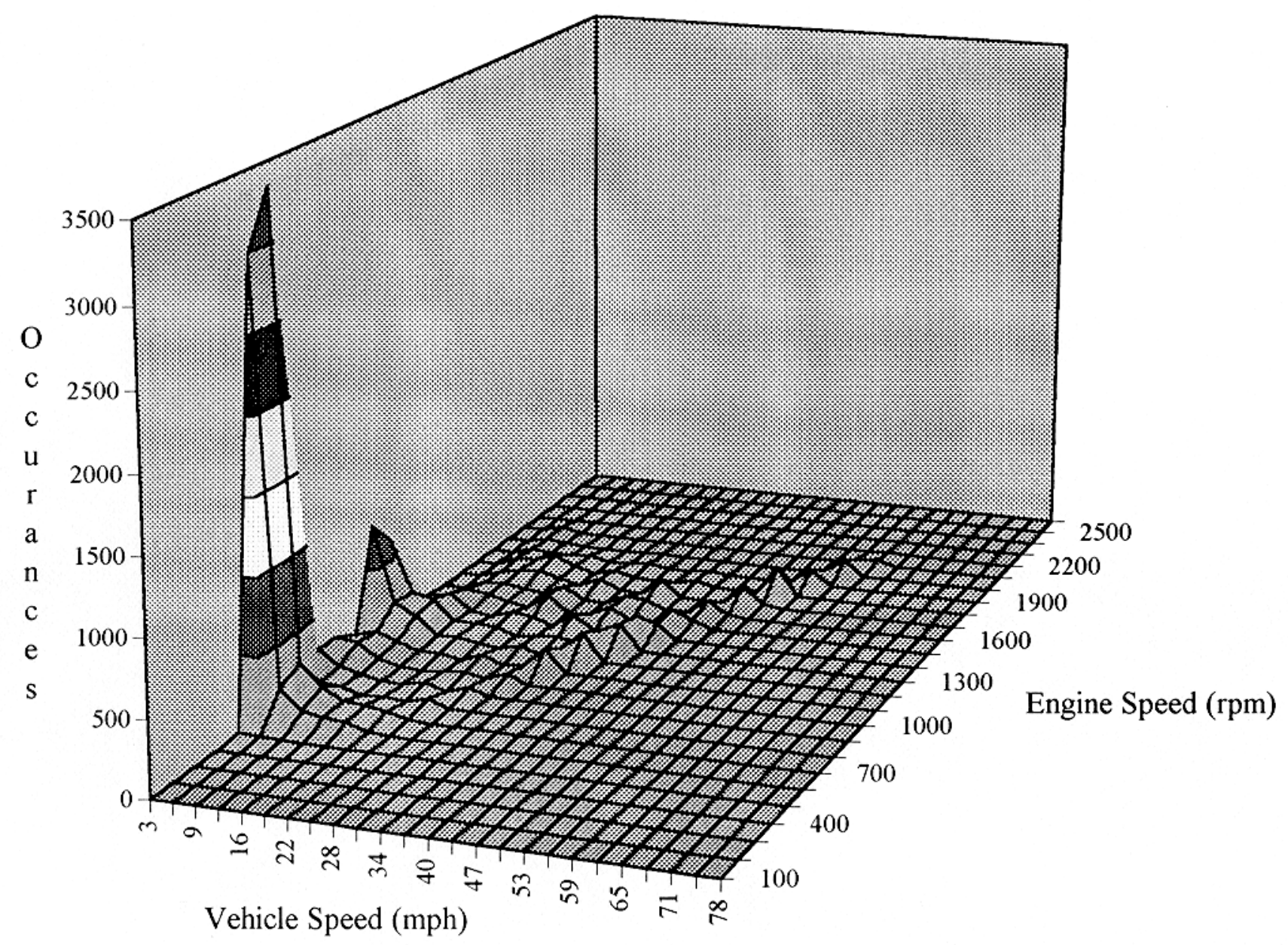

Figure 26. Engine Speed vs. Vehicle Speed for One Day of Operation 


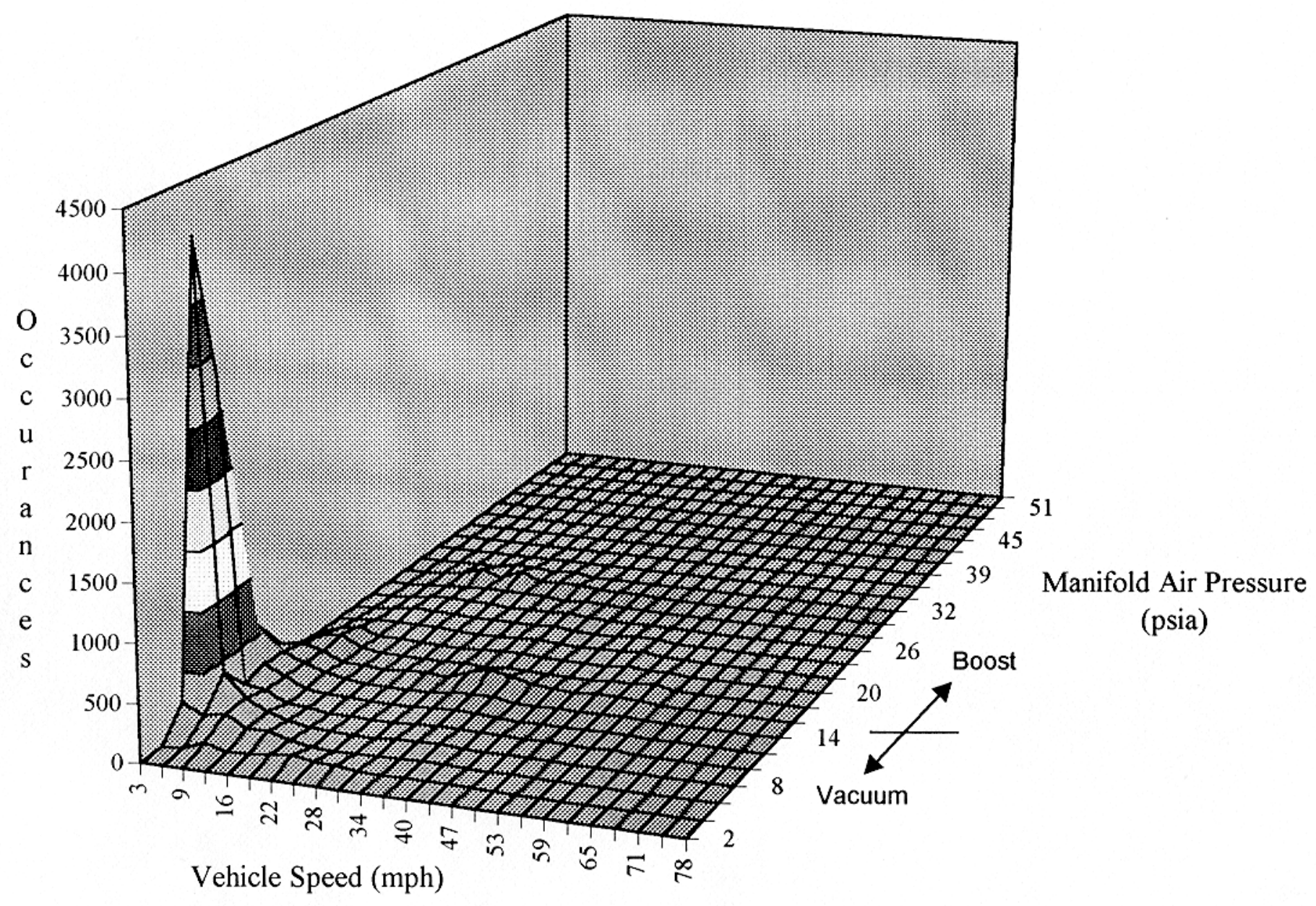

Figure 27. Manifold Air Pressure vs. Vehicle Speed for One Day of Operation 
Table 10. Data Recorded on the Data Logger

\begin{tabular}{|c|c|c|}
\hline Time & Battery (volts) & Turbo Lag Compensation Enable (\%) \\
\hline Date & Barometric Pressure (psia) & $\begin{array}{l}\text { Turbo Lag Compensation Enrichment } \\
\qquad(\%)\end{array}$ \\
\hline Odometer Reading (miles) & Equivalence Ratio Feedback & Turbo Lag Compensation Retard (deg) \\
\hline Vehicle Speed (mph) & Equivalence Ratio Command & Close Loop Multiplier (\%) \\
\hline Engine Speed (rpm) & Equivalence Ratio Modifier for ECT & Adaptive Loop Multiplier (\%) \\
\hline Fuel Flow (lb/hr): & \#1 Tank Pressure (psig) & Manifold Air Pressure Signal (volts) \\
\hline $\begin{array}{c}\text { Instantaneous Fuel Economy } \\
\text { (mpg) }\end{array}$ & \#2 Tank Pressure (psig) & Preturbine Pressure Signal (volts) \\
\hline Cumulative Fuel Economy (mpg) & Fuel Pressure Before Vaporizer (psig) & Exhaust Back Pressure Signal (volts) \\
\hline Manifold Air Pressure (psia) & Fuel Pressure After Vaporizer (psig) & Gas Pressure Signal (volts) \\
\hline Exhaust Back Pressure (psia) & Fuel Pressure at Regulator (psig) & Manifold Air Temp Signal (volts) \\
\hline Preturbine Pressure (psia) & Fuel Pressure at Filter (psig) & Engine Coolant Temp Signal (volts) \\
\hline Manifold Air Temp (degF) & Air Pressure Before Intercooler (psia) & Gas Temperature Signal (volts) \\
\hline Engine Coolant Temp (degF) & Air Pressure After Intercooler (psia) & UEGO Signal (volts) \\
\hline Gas Pressure (psia) & Relative Humidity (\%) & UEGO Pumping Signal (volts) \\
\hline Gas Temperature (degF) & EMC Run Time (sec) & UEGO Heater Signal (volts) \\
\hline Actual Foot Pedal Position (volts) & Ignition Timing (deg BTDC) & UEGO Resistor Signal (volts) \\
\hline \begin{tabular}{|c|}
$\begin{array}{c}\text { Normalized Foot Pedal Position } \\
\text { (volts) }\end{array}$ \\
\end{tabular} & Injection Pulse Width (ms) & ECM Control Mode \\
\hline $\begin{array}{l}\text { Throttle Position Command } \\
\text { (volts) }\end{array}$ & Uncorrected Fuel Flow (mg/inj) & ECM Run Mode \\
\hline Throttle Position Feedback (volts) & Predicted Volumetric Efficiency (\%) & UEGO Mode \\
\hline Ambient Air Temp (degF) & $\begin{array}{c}\text { Volumetric Efficiency Modification } \\
(\%)\end{array}$ & Fault Codes \\
\hline Oil Temp (degF) & Actual Volumetric Efficiency (\%) & Cumulative Fuel Usage (gal) \\
\hline Exhaust Temp (degF) & $\begin{array}{c}\text { Change in Manifold Air Pressure } \\
(\mathrm{psia} / \mathrm{s})\end{array}$ & Idle Fuel Usage (gal) \\
\hline Data Logger Temp (degF) & Boost Control PWM (\%) & Packing Fuel Usage (gal) \\
\hline
\end{tabular}

\subsection{Transient Emissions Testing}

Results from the transient emissions testing were reported in the final report for the Transient Emissions Testing on a Mack E7G Engine, SwRI Project No. 03-2377, and is included in Appendix G. The emissions certification levels for the final calibration, with a deterioration factor, are shown in Table 11. Screen shots from the engine controller for this calibration are included in Appendix C. CARB certification gas was used for these tests. The objectives of this project were $\mathrm{NO}_{\mathrm{x}}+\mathrm{NMHC}$ emissions of less than $2.5 \mathrm{~g} / \mathrm{bhp}-\mathrm{hr}$ on a natural gas with $99 \%$ methane. The use of CARB certification gas increases both the $\mathrm{NO}_{\mathrm{x}}$ and $\mathrm{NMHC}$ emissions. CARB gas has $90 \%$ methane along with $4 \%$ ethane and $2 \%$ butane. Ethane and butane increase the flame speed and therefore increase $\mathrm{NO}_{\mathrm{x}}$ emissions. Ethane and butane also increase the NMHC emissions since the unburned fuel has an increased amount of NMHCs. The final calibration was selected for $\mathrm{NO}_{\mathrm{x}}$ emissions below $2.5 \mathrm{~g} / \mathrm{bhp}$-hr with acceptable driveability. $\mathrm{NO}_{\mathrm{x}}$ 
emissions below $2.0 \mathrm{~g} / \mathrm{bhp}-\mathrm{hr}$ are possible on the transient emissions tests, but driveability problems were noted in the field due to the amount of timing retard required for the lower emissions combined with humidity effects. A summary of the $\mathrm{NO}_{\mathrm{x}}$ emissions and fuel economy over the transient test cycle is presented in Table 12. The calibration used for the results in Table 9 was the initial calibration for the transient emissions testing. The $\mathrm{NO}_{\mathrm{x}}$ emissions for this test was $2.86 \mathrm{~g} / \mathrm{bhp}-\mathrm{hr}$. Similar trends were noted at lower $\mathrm{NO}_{\mathrm{x}}$ emissions levels. Table 12 shows that the transient from idle uses $9 \%$ of the time in the cycle, yet accounts for $41 \%$ of the $\mathrm{NO}_{\mathrm{x}}$ emissions. This is due to the steep torque rise of this engine and the high equivalence ratios used during the WOT transient from idle. The high equivalence ratios are used to improve driveability. This calibration, where the high equivalence ratios are high, will not pass 2002 emissions for natural gas fueled engines due to high steady state $\mathrm{NO}_{\mathrm{x}}$ emissions. In 2002, natural gas engines will have the same limit on steady state emissions over the entire operating range as diesel engines.

Table 11. 325 bhp Transient Emissions Results.

\begin{tabular}{|l|c|}
\hline \multicolumn{1}{|c|}{ Emission } & $\begin{array}{c}\text { Emission Level } \\
(\mathbf{g} / \mathbf{b h p}-\mathbf{h r})\end{array}$ \\
\hline HC/OMHCE & 3.1 \\
\hline NMHC/OMNMHC & 0.324 \\
\hline NMHC+ NO $_{\mathrm{x}}$ & 2.53 \\
\hline Carbon Monoxide & 1.4 \\
\hline Oxides of Nitrogen & 2.2 \\
\hline Particulates & 0.03 \\
\hline Formaldehyde & 0.136 \\
\hline
\end{tabular}

Table 12. Summary for the FTP Results for $\mathrm{NO}_{\mathrm{x}}$ Emissions at $2.86 \mathrm{~g} / \mathrm{bhp}-\mathrm{hr}$

\begin{tabular}{|l|c|c|c|c|c|}
\hline \multicolumn{1}{|c|}{ Description } & Idle & $\begin{array}{c}\text { Mid-High } \\
\text { Speed } \\
\text { Mid Load }\end{array}$ & $\begin{array}{c}\text { High Speed } \\
\text { High Load }\end{array}$ & $\begin{array}{c}\text { Transient } \\
\text { from Idle }\end{array}$ & Remainder \\
\hline Occurrences & $42 \%$ & $25 \%$ & $10 \%$ & $9 \%$ & $14 \%$ \\
\hline $\begin{array}{l}\text { Total Contribution to } \\
\text { NO }{ }_{x} \text { Emissions }\end{array}$ & $12 \%$ & $18 \%$ & $17 \%$ & $41 \%$ & $12 \%$ \\
\hline $\begin{array}{l}\text { Average Diluted } \\
\text { NO } \mathrm{x}_{\mathrm{x}} \text { Emissions }\end{array}$ & $7.3 \mathrm{ppm}$ & $18.8 \mathrm{ppm}$ & $43.1 \mathrm{ppm}$ & $119.5 \mathrm{ppm}$ & $22.1 \mathrm{ppm}$ \\
\hline $\begin{array}{l}\text { Total Contribution to } \\
\text { Fuel Usage }\end{array}$ & $12 \%$ & $27 \%$ & $38 \%$ & $7 \%$ & $16 \%$ \\
\hline
\end{tabular}

The previous version of this engine was rated at $300 \mathrm{bhp}$ and had $\mathrm{NO}_{\mathrm{x}}$ emissions of 2.95 $\mathrm{g} / \mathrm{bhp}-\mathrm{hr}$ with no deterioration factor and a brake specific fuel consumption of $0.474 \mathrm{lb} / \mathrm{bhp}-\mathrm{hr}$. This final calibration for this engine has $\mathrm{NO}_{\mathrm{x}}$ emissions of $2.2 \mathrm{~g} / \mathrm{bhp}-\mathrm{hr}$ and a brake specific fuel consumption of $0.400 \mathrm{lb} / \mathrm{bhp}-\mathrm{hr}$. Actual driving cycle fuel economy comparisons are not readily available since the previous version of this engine was installed in a refuse hauler which was at a different location as the current version. A summary of the comparison of the previous and current version of the engine is shown in Table 13. 
Table 13. Comparison of Previous and Current Version of the Engine

\begin{tabular}{|l|c|c|}
\hline & $\begin{array}{c}\text { Previous } \\
\text { Version }\end{array}$ & $\begin{array}{c}\text { Current } \\
\text { Version }\end{array}$ \\
\hline Power (bhp) & 300 & 325 \\
\hline Torque (ft-lb) & 1100 & 1180 \\
\hline NO $_{\mathrm{x}}$ (g/bhp-hr) & 2.95 & 2.20 \\
\hline BSFC (lb/bhp-hr) & 0.474 & 0.400 \\
\hline
\end{tabular}

A calibration with a 350 bhp rating was tested for transient emissions. The emissions certification levels for the final calibration, with a deterioration factor, are shown in Table 14. Screen shots from the engine controller for this calibration are included in Appendix H. CARB certification gas was used for these tests.

Table 14. 350 bhp Transient Emissions Results.

\begin{tabular}{|l|c|}
\hline \multicolumn{1}{|c|}{ Emission } & $\begin{array}{c}\text { Emission Level } \\
\text { (g/bhp-hr) }\end{array}$ \\
\hline HC/OMHCE & 2.9 \\
\hline NMHC/OMNMHC & 0.276 \\
\hline NMHC+ NO $_{\mathrm{x}}$ & 2.60 \\
\hline Carbon Monoxide & 1.3 \\
\hline Oxides of Nitrogen & 2.3 \\
\hline Particulates & 0.03 \\
\hline Formaldehyde & 0.116 \\
\hline
\end{tabular}


APPENDIX A

RECORDED DATA FOR LEAN LIMIT TESTS 
= CelI 04 MAC2 Project Low-Speed Data =

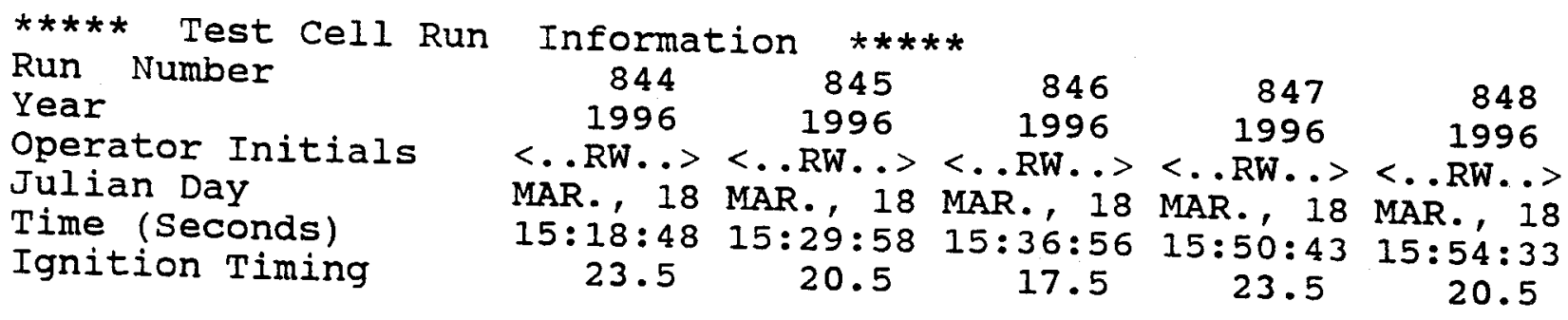

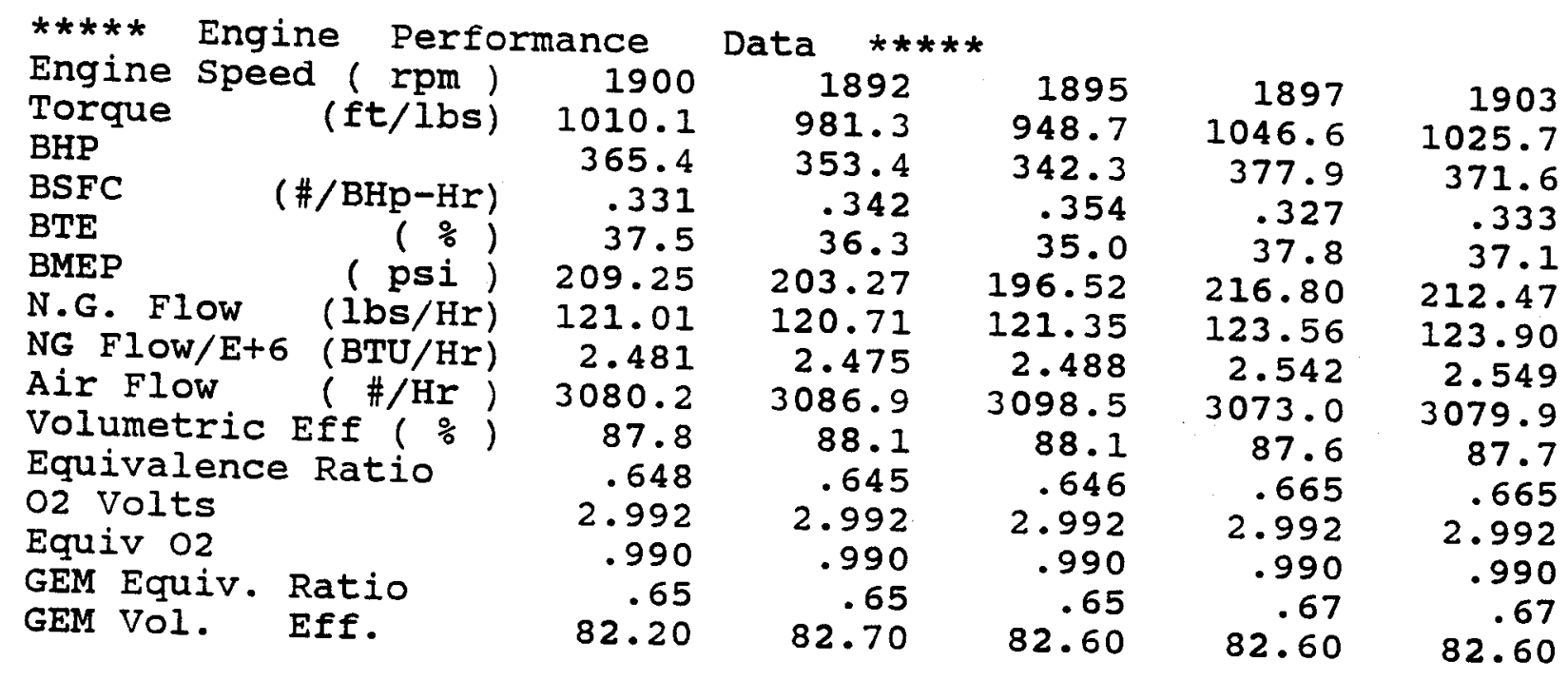

\begin{tabular}{|c|c|c|c|c|c|c|}
\hline Engine & Temper & are & Data & & & \\
\hline Water In & $(\operatorname{Deg} F)$ & 190 & 187 & 189 & 186 & 186 \\
\hline Water out & (Deg $F)$ & 199 & 197 & 198 & 195 & 196 \\
\hline Oil sump & (Deg F) & 238 & 238 & 238 & 241 & 240 \\
\hline $\begin{array}{l}\text { Intake @ LFE } \\
\text { Boost B4 IC }\end{array}$ & (Deg F) & 69 & 69 & 69 & 69 & 69 \\
\hline $\begin{array}{l}\text { Boost B4 IC } \\
\text { Boost AF IC }\end{array}$ & 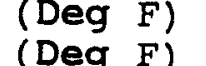 & $\begin{array}{l}277 \\
104\end{array}$ & 277 & 278 & 278 & 277 \\
\hline Pre-Turbine & (Deg F) & 1199 & $\begin{array}{r}102 \\
1226\end{array}$ & $\begin{array}{r}103 \\
1259\end{array}$ & 103 & 103 \\
\hline stack & (Deg F) & 1011 & 1038 & $\begin{array}{l}1259 \\
1068\end{array}$ & 1209 & 1232 \\
\hline Exhaust I & $(\operatorname{Deg} F)$ & 1145 & 1173 & $\begin{array}{l}1068 \\
1202\end{array}$ & 1023 & 1046 \\
\hline Exhaust 2 & $(\operatorname{Deg} F)$ & 1126 & 1152 & $\begin{array}{l}1202 \\
1183\end{array}$ & 1158 & 1181 \\
\hline Exhaust 3 & (Deg F) & 1121 & 1147 & 1183 & 1139 & 1161 \\
\hline Exhaust 4 & (Deg F) & 1121 & 1148 & 1178 & 1131 & 1155 \\
\hline Exhaust 5 & $(\operatorname{Deg} F)$ & 1101 & $\begin{array}{l}1148 \\
1126\end{array}$ & 1180 & 1132 & 1155 \\
\hline Exhaust 6 & $\left(\begin{array}{ll}\operatorname{Deg} & F\end{array}\right)$ & 1085 & $\begin{array}{l}1126 \\
1110\end{array}$ & 1157 & 1110 & 1131 \\
\hline Gas @ Meter & (Deg F) & 67 & $\begin{array}{r}1110 \\
64\end{array}$ & 1141 & 1097 & 1117 \\
\hline Dyno $\mathrm{H} 2 \mathrm{O}$ & (Deg F) & 108 & $\begin{array}{r}64 \\
108\end{array}$ & 65 & 65 & 64 \\
\hline$t+t$ & & & & & 112 & 112 \\
\hline$\star * \star \star \star *$ Engine & Pressu & & Data & & & \\
\hline $\begin{array}{l}\text { Main Oil Pres } \\
\text { Fuel Pressure }\end{array}$ & (psig) & 47.6 & 47.7 & 47.7 & 47.3 & 47.4 \\
\hline Compress out & $\begin{array}{l}(p s i g) \\
(\text { psig) }\end{array}$ & $\begin{array}{r}80.5 \\
20.53\end{array}$ & 80.6 & 80.3 & 80.3 & 80.3 \\
\hline Inlet Manifolc & $d$ (psig) & 18.36 & 20.45 & 20.62 & 20.49 & 20.47 \\
\hline Inlet Manifolc & $d(p s i a)$ & $\begin{array}{l}10.30 \\
32.72\end{array}$ & 18.29 & 18.46 & 18.36 & 18.32 \\
\hline Pre-Turbine & (psiq) & $\begin{array}{l}32.72 \\
19.15\end{array}$ & 32.64 & 32.81 & 32.71 & 32.67 \\
\hline Exhaust $\mathrm{Bk} \mathrm{Pr}$ & ("HG) & $\begin{array}{r}19.15 \\
4.54\end{array}$ & 19.08 & 19.21 & 19.06 & 18.97 \\
\hline LFE Filter Pr & ("H2O) & & 4.73 & 4.88 & 4.64 & 4.72 \\
\hline LFE Delta Pr & ("H2O) & $\begin{array}{l}1.35 \\
5.65\end{array}$ & 1.38 & 1.25 & 1.24 & 1.24 \\
\hline$(1 \mathrm{bH} 2 \mathrm{O}$ & / IbAir) & $\begin{array}{r}5.65 \\
.0026\end{array}$ & 5.66 & 5.68 & 5.64 & 5.65 \\
\hline $\mathrm{RH}$ (out) & $\div$ & $\begin{array}{l}.0026 \\
15.67\end{array}$ & .0026 & .0026 & .0025 & .0025 \\
\hline Barometer & "Hg ) & $\begin{array}{l}15 \cdot 67 \\
29.22\end{array}$ & 15.78 & 15.60 & 14.94 & 14.96 \\
\hline & & & 29.22 & 29.22 & 29.21 & 29.22 \\
\hline
\end{tabular}


= Cell 04 MAC2 Project Low-Speed Data =

\begin{tabular}{|c|c|c|c|c|c|c|c|}
\hline \multirow{2}{*}{$\begin{array}{l}\star \star \star \star \star \star \\
N G \% 1\end{array}$} & Natura & Gas & Composit & \multicolumn{2}{|c|}{ 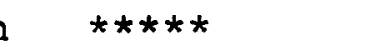 } & \multirow[b]{2}{*}{95.44} & \multirow[b]{2}{*}{95.44} \\
\hline & Methane & & 95.38 & 95.38 & 95.38 & & \\
\hline NG \% & Ethane & & 2.37 & 2.37 & 2.37 & 2.44 & 2.44 \\
\hline NG \% & Propane & & .28 & .28 & .28 & .32 & .32 \\
\hline NG \% & $\mathrm{CO} 2$ & & .98 & .98 & .98 & .98 & .98 \\
\hline NG \% 1 & Nitrogen & & .86 & .86 & .86 & .65 & .65 \\
\hline Butan & he Actual ( & ) & .12 & .12 & .12 & .16 & .16 \\
\hline MWT & & & 16.88 & 16.88 & 16.88 & 16.90 & 16.90 \\
\hline Fuel & LHV & & 20504 . & 20504 & 20504 . & 20573 . & 20573 \\
\hline
\end{tabular}


= Cell 04 MAC2 Project Low-Speed Data =

\begin{tabular}{|c|c|c|c|c|c|}
\hline ell Ru & Info & & & & \\
\hline $\begin{array}{l}\text { Run Number } \\
\text { Year }\end{array}$ & 849 & 850 & 85 & 852 & 853 \\
\hline $\begin{array}{l}\text { Year } \\
\text { Operator Initi }\end{array}$ & RW..> & 1 & & & 19 \\
\hline Tulian Day & MAR. & MAR., 18 & MAR. & MAR., 18 & MAR , 18 \\
\hline $\begin{array}{l}\text { Time (Sec } \\
\text { Ignition }\end{array}$ & $\begin{array}{l}57: 52 \\
17.5\end{array}$ & $\begin{array}{c}16: 01: 44 \\
23.5\end{array}$ & $\begin{array}{c}16: 04: 59 \\
20.5\end{array}$ & $\begin{array}{c}16: 08: 15 \\
17.5\end{array}$ & $\begin{array}{c}16: 12: 39 \\
23.5\end{array}$ \\
\hline
\end{tabular}

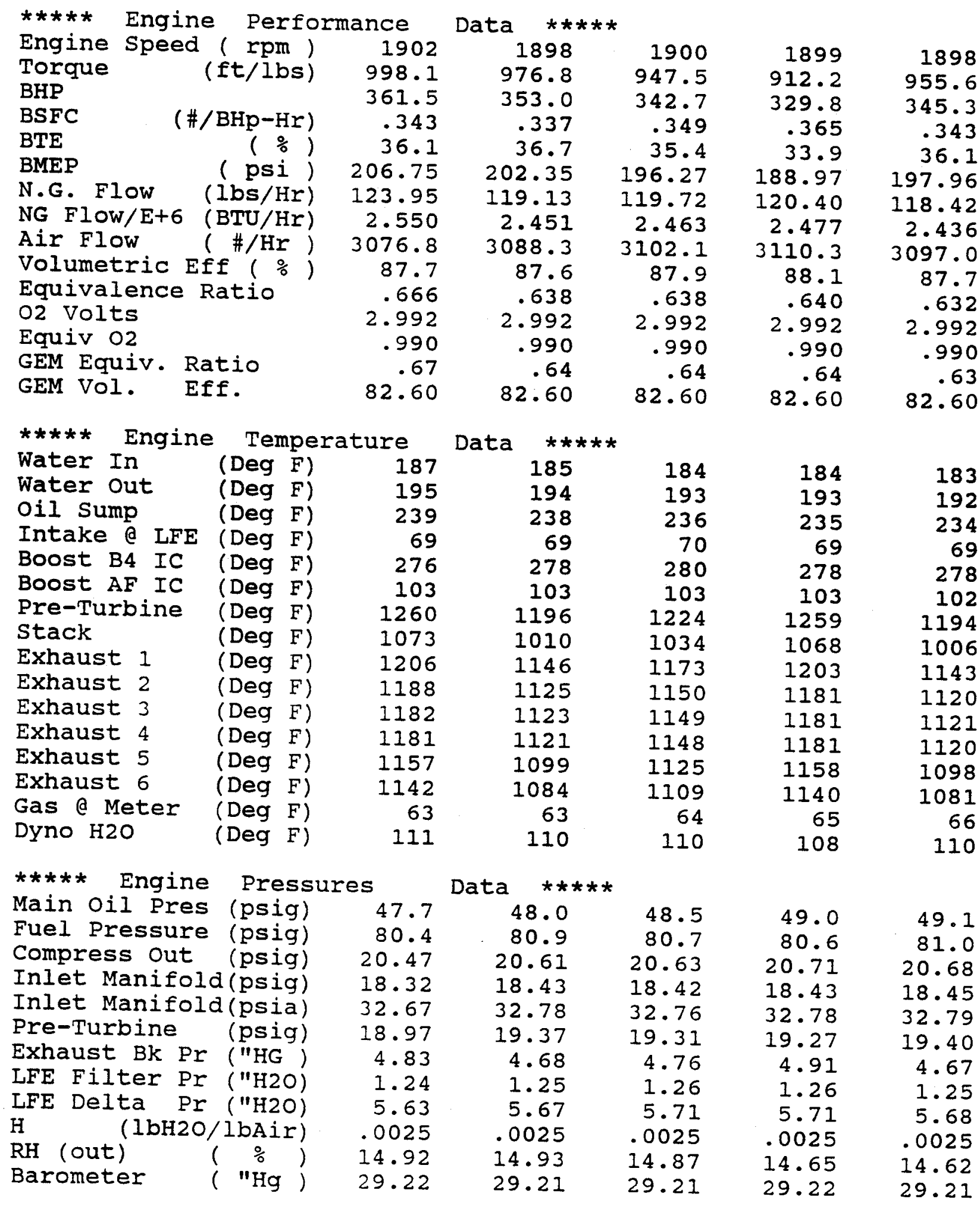


= Cell 04 MAC2 Project Low-Speed Data =

$\begin{array}{lrrrrr}* * * * * \text { Natural Gas Composition } * * * * * & & & \\ \text { NG \% Methane } & 95.44 & 95.44 & 95.44 & 95.44 & 95.44 \\ \text { NG \% Ethane } & 2.44 & 2.44 & 2.44 & 2.44 & 2.44 \\ \text { NG \% Propane } & .32 & .32 & .32 & .32 & .32 \\ \text { NG \% CO2 } & .98 & .98 & .98 & .98 & .98 \\ \text { NG \% Nitrogen } & .65 & .65 & .65 & .65 & .65 \\ \text { Butane Actual( } \% & .16 & .16 & .16 & .16 & .16 \\ \text { MWT } & 16.90 & 16.90 & 16.90 & 16.90 & 16.90 \\ \text { Fuel LHV } & 20573 . & 20573 . & 20573 . & 20573 . & 20573 .\end{array}$


= Cell 04 MAC2 Project Low-speed Data =

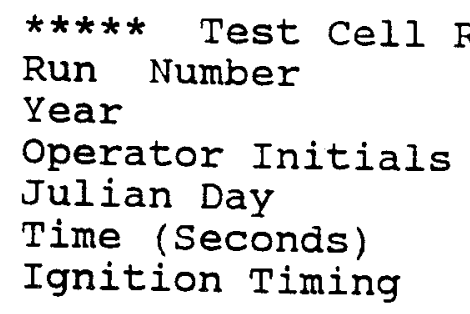

Information $* * * * *$

\begin{tabular}{|c|c|c|c|c|}
\hline $\begin{array}{c}854 \\
1996 \\
<\ldots \text { RW. } \\
\text { MAR. }, 18 \\
16: 15: 56 \\
20.5\end{array}$ & $\begin{array}{r}855 \\
1996 \\
<\ldots \text { RW.. } \\
\text { MAR. } 18 \\
16: 19: 57 \\
17.5\end{array}$ & $\begin{array}{r}856 \\
1996 \\
<\ldots \text { JM... } \\
\text { MAR. , } 18 \\
16: 23: 53 \\
23.5\end{array}$ & $\begin{array}{c}857 \\
1996 \\
<\ldots \text { RW.. } \\
\text { MAR., } 18 \\
16: 28: 36 \\
20.5\end{array}$ & $\begin{array}{r}858 \\
1996 \\
<\ldots \text { RW. . } \\
\text { MAR., } 18 \\
16: 31: 36 \\
17.5\end{array}$ \\
\hline
\end{tabular}

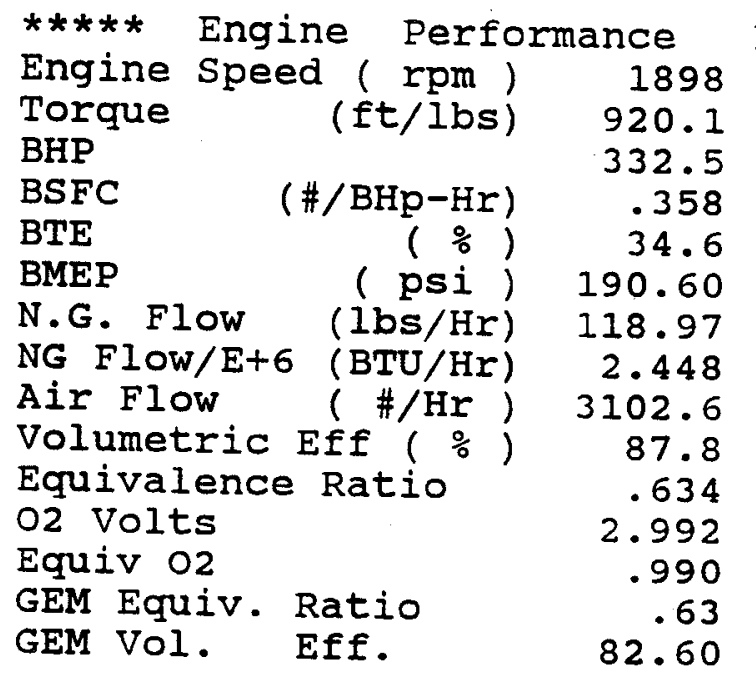

Data $* * * * *$

$\begin{array}{rr}1895 & 1900 \\ 884.0 & 933.1 \\ 319.0 & 337.5 \\ .372 & .347 \\ 33.3 & 35.6 \\ 183.12 & 193.29 \\ 118.62 & 117.25 \\ 2.440 & 2.412 \\ 3112.7 & 3105.3 \\ 88.3 & 87.8 \\ .630 & .624 \\ 2.992 & 2.992 \\ .990 & .990 \\ .63 & .62 \\ 82.60 & 82.60\end{array}$

$\begin{array}{rr}1899 & 1893 \\ 891.7 & 855.6 \\ 322.4 & 308.3 \\ .363 & .383 \\ 34.1 & 32.3 \\ 184.71 & 177.24 \\ 117.11 & 118.12 \\ 2.409 & 2.430 \\ 3130.8 & 3121.7 \\ 88.5 & 88.6 \\ .619 & .626 \\ 2.992 & 2.992 \\ .990 & .990 \\ .62 & .62 \\ 82.60 & 82.60\end{array}$

\begin{tabular}{llr}
$\star * * * *$ Engine & \multicolumn{2}{c}{ Temperature } \\
Water In & (Deg F) & 184 \\
Water Out & (Deg F) & 192 \\
Oil Sump & (Deg F) & 234 \\
Intake E LFE & (Deg F) & 69 \\
Boost B4 IC & (Deg F) & 278 \\
Boost AF IC & (Deg F) & 103 \\
Pre-Turbine & (Deg F) & 1226 \\
Stack & (Deg F) & 1034 \\
Exhaust 1 & (Deg F) & 1169 \\
Exhaust 2 & (Deg F) & 1148 \\
Exhaust 3 & (Deg F) & 1149 \\
Exhaust 4 & (Deg F) & 1150 \\
Exhaust 5 & (Deg F) & 1127 \\
Exhaust 6 & (Deg F) & 1110 \\
Gas a Meter & (Deg F) & 66 \\
Dyno H20 & (Deg F) & 108
\end{tabular}

Data $* * * * *$

$\begin{array}{rr}184 & 184 \\ 193 & 192 \\ 233 & 233 \\ 69 & 69 \\ 278 & 277 \\ 103 & 102 \\ 1264 & 1196 \\ 1073 & 1009 \\ 1205 & 1144 \\ 1182 & 1121 \\ 1184 & 1122 \\ 1187 & 1122 \\ 1162 & 1100 \\ 1142 & 1083 \\ 64 & 64 \\ 107 & 108\end{array}$

$\begin{array}{rr}183 & 184 \\ 192 & 192 \\ 233 & 232 \\ 69 & 69 \\ 279 & 279 \\ 103 & 103 \\ 1230 & 1266 \\ 1035 & 1072 \\ 1174 & 1209 \\ 1149 & 1184 \\ 1150 & 1182 \\ 1153 & 1186 \\ 1131 & 1165 \\ 1112 & 1143 \\ 63 & 64 \\ 107 & 105\end{array}$

***** Engine Pressures

Main Oil Pres (psig) 49.1

Fuel Pressure (psig) 80.9

Compress out (psig) 20.73

Inlet Manifold(psig) $\quad 18.48$

Inlet Manifold(psia) $\quad 32.83$

Pre-Turbine (psig) 19.42

Exhaust Bk Pr ("HG) 4.80

LFE Filter Pr ("H2O) 1.26

LFE Delta Pr ("H2O) 5.69

$\mathrm{H}$ (IbH2O/IbAir) .0025

RH (out) (\%) 14.53

Barometer ( $\mathrm{Hg}$ ) 29.22

Data $* * * * *$

$\begin{array}{rrrr}49.4 & 49.3 & 49.5 & 49.6 \\ 81.1 & 81.2 & 81.1 & 81.1 \\ 20.68 & 20.62 & 20.66 & 20.64 \\ 18.44 & 18.43 & 18.43 & 18.43 \\ 32.79 & 32.78 & 32.78 & 32.78 \\ 19.27 & 19.37 & 19.38 & 19.28 \\ 4.91 & 4.68 & 4.78 & 4.92 \\ 1.26 & 1.26 & 1.27 & 1.26 \\ 5.71 & 5.69 & 5.75 & 5.73 \\ .0025 & .0025 & .0024 & .0024 \\ 14.49 & 14.48 & 14.35 & 14.24 \\ 29.22 & 29.22 & 29.22 & 29.22\end{array}$


= Cell 04 MAC2 Project Low-Speed Data =

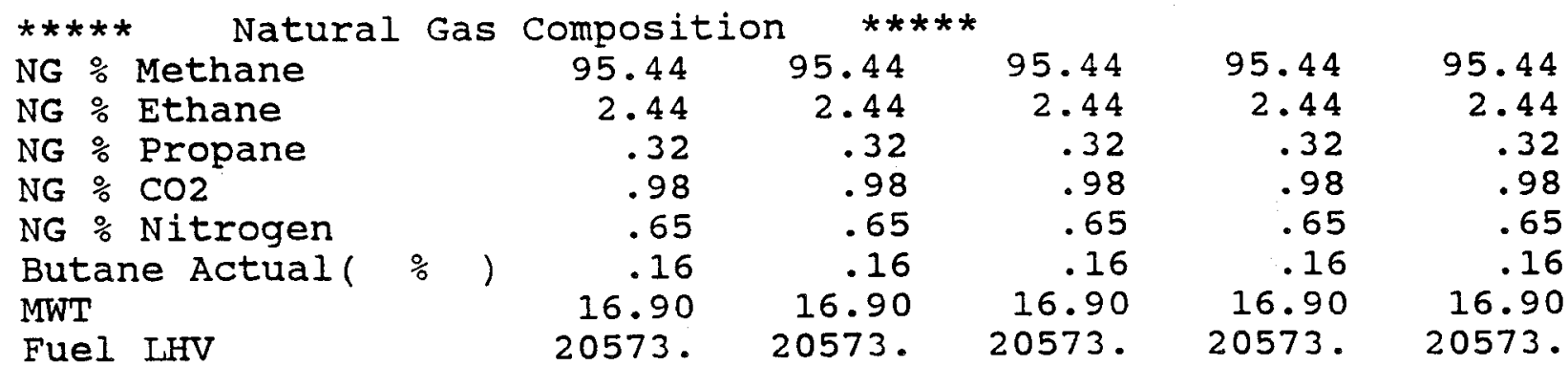


= Cell 04 MAC2 Project Low-Speed Data =

$\star * * * *$ Test Cell
Run Number
Year
Operator Initials
Julian Day
Time (Seconds)
Ignition Timing
Information $* * * * *$

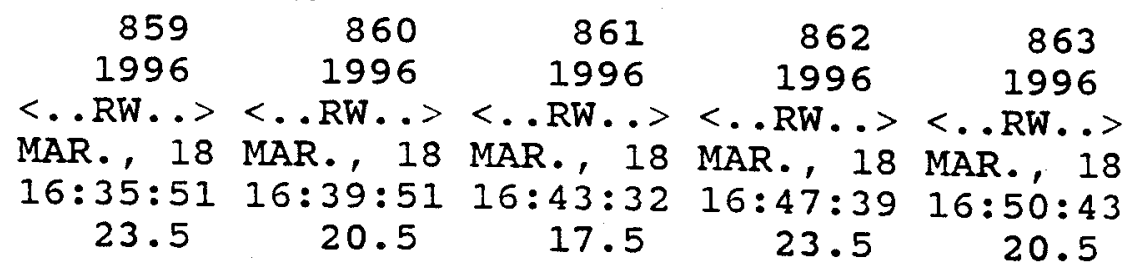

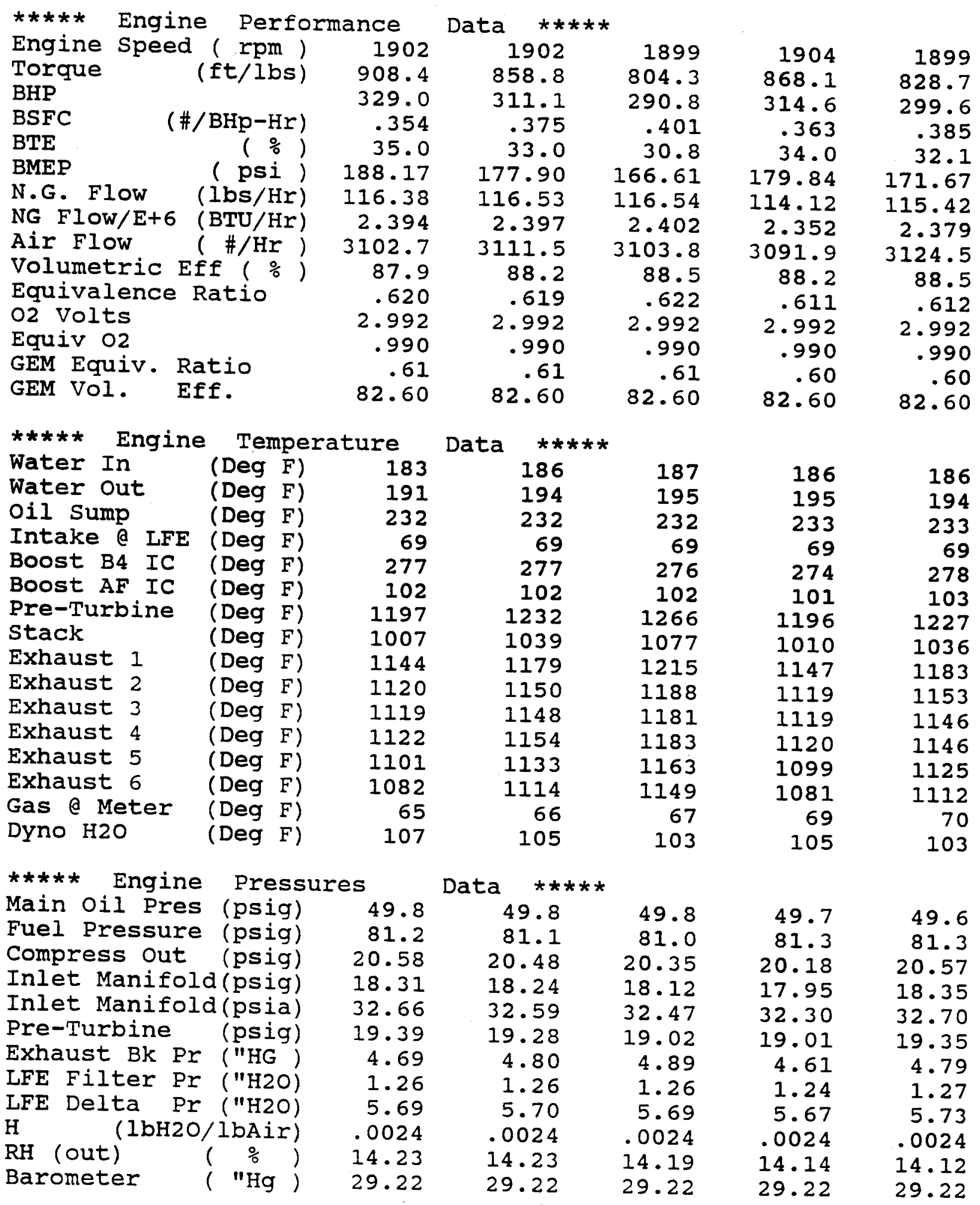


= CelI 04 MAC2 Project Low-Speed Data =

$\begin{array}{lrrrrr}* * * * * \quad \text { Natural } & \text { Gas Composition } * * * * * \\ \text { NG \% Methane } & 95.44 & 95.44 & 95.68 & 95.68 & 95.68 \\ \text { NG \% Ethane } & 2.44 & 2.44 & 2.35 & 2.35 & 2.35 \\ \text { NG \% Propane } & .32 & .32 & .27 & .27 & .27 \\ \text { NG \% CO2 } & .98 & .98 & .99 & .99 & .99 \\ \text { NG \% Nitrogen } & .65 & .65 & .54 & .54 & .54 \\ \text { Butane Actual }(\% & .16 & .16 & .17 & .17 & .17 \\ \text { MWT } & 16.90 & 16.90 & 16.86 & 16.86 & 16.86 \\ \text { Fuel LHV } & 20573 . & 20573 . & 20608 . & 20608 . & 20608 .\end{array}$


= Cell 04 MAC2 Project Low-Speed Data =

\begin{tabular}{|c|c|c|c|c|c|}
\hline$* * * * *$ Test Cell Run & Informa & a & & & \\
\hline Run Number & 864 & 865 & 866 & 867 & 868 \\
\hline Year & 1996 & 1996 & 1996 & 1996 & 1996 \\
\hline Operator Initials & $<\ldots R W \ldots>$ & $<\ldots$ JM.. $>$ & $<\ldots$ RW.. $>$ & $<\ldots$ RW .. > & $<\ldots$ RW.. $>$ \\
\hline Julian Day & MAR., 18 & MAR., 18 & MAR., 19 & MAR., 19 & MAR., 19 \\
\hline Time (Seconds) & $16: 53: 28$ & $16: 57: 19$ & $09: 06: 10$ & $09: 10: 42$ & $09: 16: 26$ \\
\hline Ignition Timing & 17.5 & 23.5 & 19.0 & 16.0 & \\
\hline Engine & mance & Data & & & \\
\hline Engine speed (rpm) & 1903 & 1902 & 1255 & 1232 & 1254 \\
\hline Torque (ft/Ibs) & 725.5 & 774.1 & 1275.4 & 1256.8 & 1213.8 \\
\hline BHP & 262.8 & 280.4 & 304.7 & 294.8 & 289.7 \\
\hline$(\# / \mathrm{BHp}-\mathrm{Hr} r)$ & .436 & .401 & .322 & .334 & .341 \\
\hline$\left(\frac{\circ}{0}\right)$ & 28.3 & 30.8 & 38.5 & 37.1 & 36.3 \\
\hline BMEP & 150.28 & 160.36 & 264.19 & 260.35 & 251.43 \\
\hline & 114.66 & 112.33 & 98.01 & 98.44 & 98.88 \\
\hline $\begin{array}{l}\text { NG Flow } / \mathrm{E}+6 \\
\text { Air Flow }\end{array}$ & 2.363 & 2.315 & 2.013 & 2.022 & 2.031 \\
\hline Volumetric Eff $\left(\frac{\%}{0}\right)$ & $\begin{array}{r}3133.6 \\
88.5\end{array}$ & 3130.4 & 2401.2 & 2420.2 & 2433.6 \\
\hline Equivalence Ratio & .000 & $\begin{array}{l}88.4 \\
.594\end{array}$ & $\begin{array}{r}93.0 \\
674\end{array}$ & 95.6 & 94.4 \\
\hline o2 Volts & 2.992 & 2.992 & 2.993 & $\begin{array}{r}.672 \\
2.993\end{array}$ & .671 \\
\hline Equiv 02 & .990 & .990 & .989 & $\begin{array}{r}2.993 \\
.989\end{array}$ & $\begin{array}{r}2.993 \\
.989\end{array}$ \\
\hline GEM Equiv. Ratio & .60 & .59 & .65 & .65 & .65 \\
\hline GEM Vol. Eff. & 82.60 & 82.60 & 86.20 & 86.20 & 86.20 \\
\hline Engine & :ature & $* * * *$ & & & \\
\hline Water In $(\operatorname{Deg} F)$ & 185 & 183 & 186 & 186 & 187 \\
\hline Water out & 194 & 191 & 196 & 196 & 197 \\
\hline Oil Sump & 232 & 230 & 229 & 229 & 228 \\
\hline Intake e LFE (Deg F) & 69 & 69 & 57 & 57 & 57 \\
\hline Boost B4 IC (Deg F) & 278 & 279 & 281 & 281 & 281 \\
\hline Boost AF IC & 103 & 103 & 103 & 103 & 102 \\
\hline Pre-Turbine & 1236 & 1166 & 1101 & 1124 & 1161 \\
\hline (Deg F) & 1056 & 989 & 912 & 934 & 973 \\
\hline Exhaust 1 & 1217 & 1151 & 1042 & 1062 & 1090 \\
\hline Exhaust 2 & 1186 & 1121 & 1040 & 1062 & 1094 \\
\hline Exhaust 3 & 1163 & 1103 & 1025 & 1050 & 1082 \\
\hline Exhaust 4 & 1137 & 1075 & 1024 & 1045 & 1080 \\
\hline Exhaust 5 & 1119 & 1063 & 999 & 1019 & 1053 \\
\hline Exhaust 6 & 1130 & 1070 & 967 & 984 & 1016 \\
\hline Gas a Meter & 69 & 67 & 55 & 55 & 57 \\
\hline Dyno $\mathrm{H} 2 \mathrm{O}$ & 100 & 101 & 109 & 108 & 107 \\
\hline$\star * * * *$ Engine & es & $\star \star * *$ & & & \\
\hline Main Oil Pres (psig) & 50.2 & 50.9 & 29.6 & 29.6 & 29.8 \\
\hline Fuel Pressure (psig) & 81.3 & 81.7 & 83.7 & 83.6 & 83.5 \\
\hline Compress out (psig) & 20.71 & 20.62 & 22.97 & 22.99 & 23.03 \\
\hline Inlet Manifold(psig) & 18.39 & $18 \cdot 34$ & 21.90 & 21.85 & 21.85 \\
\hline Inlet Manifold(psia) & 32.74 & 32.69 & 36.36 & 36.31 & 36.31 \\
\hline Pre-Turbine (psig) & 19.38 & 19.51 & 15.30 & 15.21 & 15.21 \\
\hline Exhaust Bk Pr ("HG) & 4.93 & 4.67 & 2.61 & 2.64 & 2.72 \\
\hline LFE Filter Pr ("H2O) & 1.27 & 1.27 & .78 & .78 & .79 \\
\hline LFE Delta Pr ("H2O) & 5.75 & 5.74 & 4.15 & 4.19 & 4.21 \\
\hline (IbH2O/IbAir) & .0024 & .0024 & .0021 & .0021 & .0020 \\
\hline RH (out) & 14.14 & 14.07 & 19.26 & 18.60 & 18.39 \\
\hline Barometer & 29.22 & 29.22 & 29.44 & 29.44 & 29.44 \\
\hline
\end{tabular}


= Cell 04 MAC2 Project Low-speed Data =

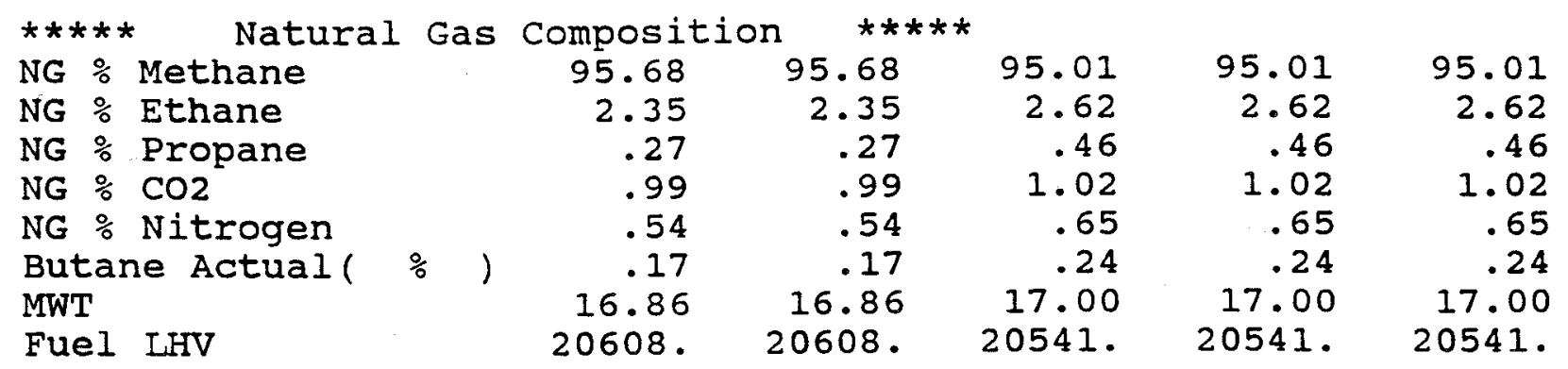


= Cell 04 MAC2 Project Low-Speed Data =

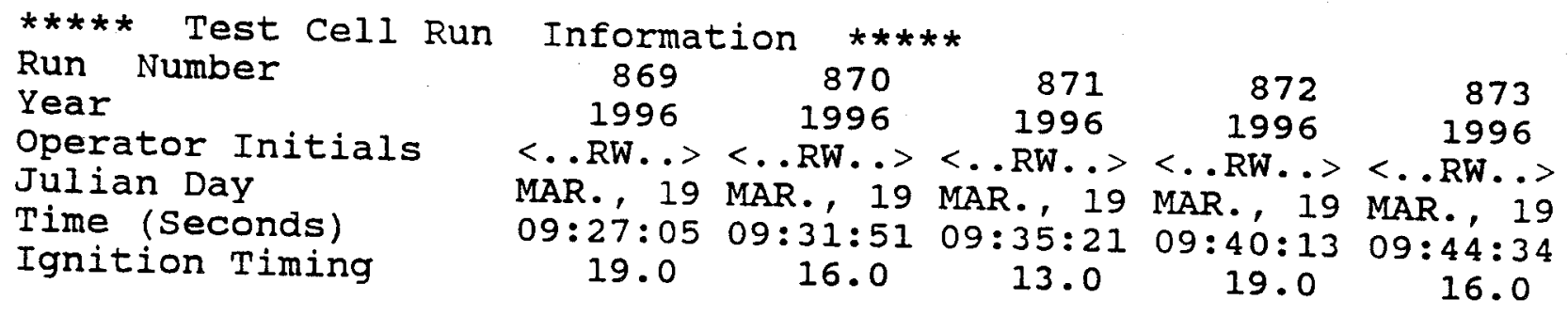

\begin{tabular}{|c|c|c|c|}
\hline ingine & ance & Data & \\
\hline ngine speed ( rpm) & 1253 & 1243 & 1254 \\
\hline$(\mathrm{ft} / \overline{1}$ & 133 & 1314.6 & 12 \\
\hline$\Gamma$ & 31 & 311. & \\
\hline$(\# / \mathrm{BHp}-\mathrm{Hr} r)$ & 3 & .323 & \\
\hline (psi) & 275.67 & $\begin{array}{r}38.3 \\
272.33\end{array}$ & 265.14 \\
\hline G. Flow & 100.18 & 100.63 & 101.13 \\
\hline $\begin{array}{ll}\text { G Flow/E+6 } & \text { (BTU/Hr) } \\
\text { ir Flow } & \text { ( } / \mathrm{Hr}\end{array}$ & 2.058 & 2.067 & 2.077 \\
\hline olumetric Eff ( & $\begin{array}{r}2395 \\
93\end{array}$ & $\begin{array}{r}2405.7 \\
94.3\end{array}$ & $\begin{array}{r}2423.2 \\
94.1\end{array}$ \\
\hline $\begin{array}{l}\text { Equivalence Ratio } \\
22 \text { Volts }\end{array}$ & .690 & .691 & .689 \\
\hline Equiv 02 & $2 \cdot 9$ & 2.5 & 2.993 \\
\hline EM Equiv & .8 & & .989 \\
\hline EM Vo] & 86.20 & 86.20 & 86.2 \\
\hline
\end{tabular}

$\begin{array}{rr}1243 & 1253 \\ 1264.6 & 1238.8 \\ 299.4 & 295.6 \\ .322 & .328 \\ 38.5 & 37.8 \\ 261.97 & 256.63 \\ 96.47 & 97.00 \\ 1.982 & 1.992 \\ 2418.0 & 2426.9 \\ 94.4 & 93.9 \\ .659 & .660 \\ 2.993 & 2.993 \\ .989 & .989 \\ .64 & .64 \\ 86.20 & 86.20\end{array}$

\section{***** Engine Temperature}

$\begin{array}{llr}\text { Water In } & \text { (Deg F) } & 187 \\ \text { Water Out } & \text { (Deg F) } & 197 \\ \text { Oil Sump } & \text { (Deg F) } & 231 \\ \text { Intake Q LFE } & \text { (Deg F) } & 57 \\ \text { Boost B4 IC } & \text { (Deg F) } & 281 \\ \text { Boost AF IC } & \text { (Deg F) } & 102 \\ \text { Pre-Turbine } & \text { (Deg F) } & 1115 \\ \text { Stack } & \text { (Deg F) } & 926 \\ \text { Exhaust I } & \text { (Deg F) } & 1051 \\ \text { Exhaust 2 } & \text { (Deg F) } & 1052 \\ \text { Exhaust 3 } & \text { (Deg F) } & 1040 \\ \text { Exhaust 4 } & \text { (Deg F) } & 1036 \\ \text { Exhaust 5 } & \text { (Deg F) } & 1011 \\ \text { Exhaust 6 } & \text { (Deg F) } & 979 \\ \text { Gas a Meter } & \text { (Deg F) } \\ \text { Dyno H20 } & \text { (Deg F) } & 109\end{array}$

Data $* * * * *$

$\begin{array}{rr}186 & 187 \\ 197 & 197 \\ 232 & 231 \\ 57 & 57 \\ 280 & 281 \\ 102 & 102 \\ 1135 & 1167 \\ 947 & 979 \\ 1068 & 1094 \\ 1071 & 1100 \\ 1061 & 1088 \\ 1055 & 1085 \\ 1029 & 1059 \\ 996 & 1021 \\ 55 & 55 \\ 109 & 108\end{array}$

Data $* * * * *$

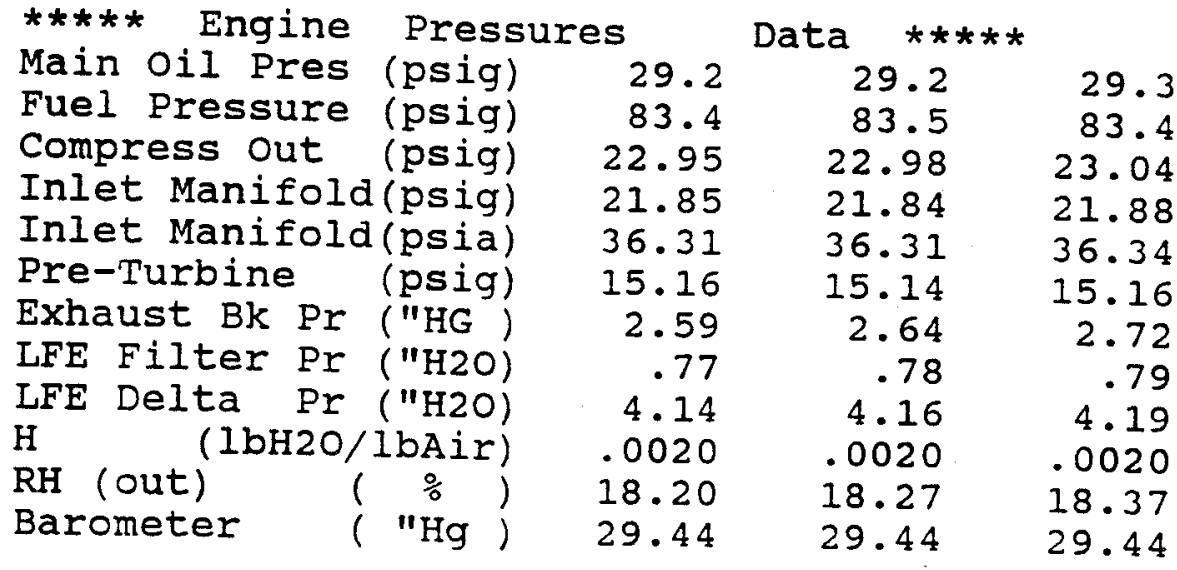

$\begin{array}{rr}185 & 184 \\ 195 & 194 \\ 230 & 228 \\ 57 & 57 \\ 281 & 280 \\ 102 & 102 \\ 1098 & 1118 \\ 909 & 928 \\ 1035 & 1055 \\ 1036 & 1055 \\ 1026 & 1045 \\ 1021 & 1040 \\ 998 & 1014 \\ 963 & 978 \\ 56 & 57 \\ 107 & 106\end{array}$

$\begin{array}{rr}29.5 & 29.9 \\ 84.0 & 83.8 \\ 23.02 & 23.01 \\ 21.86 & 21.89 \\ 36.32 & 36.35 \\ 15.32 & 15.33 \\ 2.60 & 2.65 \\ .78 & .80 \\ 4.18 & 4.20 \\ .0020 & .0020 \\ 18.47 & 18.24 \\ 29.44 & 29.44\end{array}$


= Cell 04 MAC2 Project Low-Speed Data =

$\begin{array}{lrrrrr}* * * * * \text { Natural Gas Composition } & * * * * * & & \\ \text { NG \% Methane } & 95.01 & 95.01 & 95.01 & 95.01 & 95.01 \\ \text { NG \% Ethane } & 2.62 & 2.62 & 2.62 & 2.62 & 2.62 \\ \text { NG \% Propane } & .46 & .46 & .46 & .46 & .46 \\ \text { NG \% CO2 } & 1.02 & 1.02 & 1.02 & 1.02 & 1.02 \\ \text { NG \% Nitrogen } & .65 & .65 & .65 & .65 & .65 \\ \text { Butane Actual( } \% & .24 & .24 & .24 & .24 & .24 \\ \text { MWT } & 17.00 & 17.00 & 17.00 & 17.00 & 17.00 \\ \text { Fuel LHV } & 20541 . & 20541 . & 20541 . & 20541 . & 20541 .\end{array}$


= Cell 04 MAC2 Project Low-Speed Data =

\begin{tabular}{|c|c|c|c|c|c|}
\hline$* * * * *$ Test Cell Run & Informa & $\star \star \star$ & & & \\
\hline $\begin{array}{l}\text { Run Number } \\
\text { Year }\end{array}$ & $\begin{array}{r}874 \\
1996\end{array}$ & 875 & 876 & 877 & 878 \\
\hline operator Initials & $\begin{array}{l}1996 \\
<. \text { RW.. > }\end{array}$ & $\begin{array}{l}1996 \\
. R W \ldots>\end{array}$ & $\begin{array}{l}1996 \\
<\ldots \text {. RW . }>\end{array}$ & 1996 & 1996 \\
\hline $\begin{array}{l}\text { Julian Day } \\
\text { Time (Seconds) }\end{array}$ & MAR , 19 & MAR , 19 & MAR., 19 & MAR., 19 & $\begin{array}{l}<. . R W . .> \\
\text { MAR., } 19\end{array}$ \\
\hline Ignition Timing & 13.0 & $\begin{array}{l}53: 27 \\
19.0\end{array}$ & $\begin{array}{c}09: 57: 49 \\
16.0\end{array}$ & $\begin{array}{c}10: 01: 33 \\
13.0\end{array}$ & $\begin{array}{c}10: 06: 40 \\
19.0\end{array}$ \\
\hline
\end{tabular}

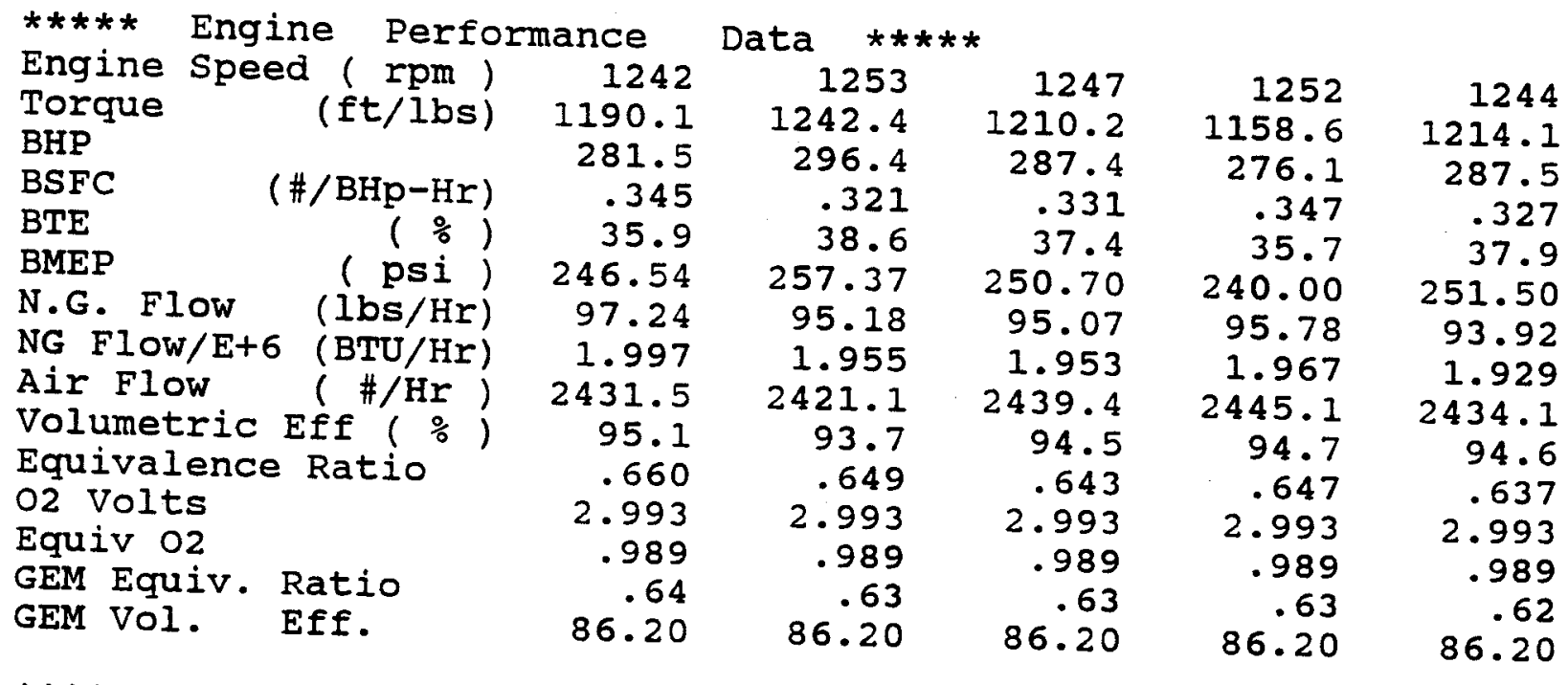

Data $* * * * *$

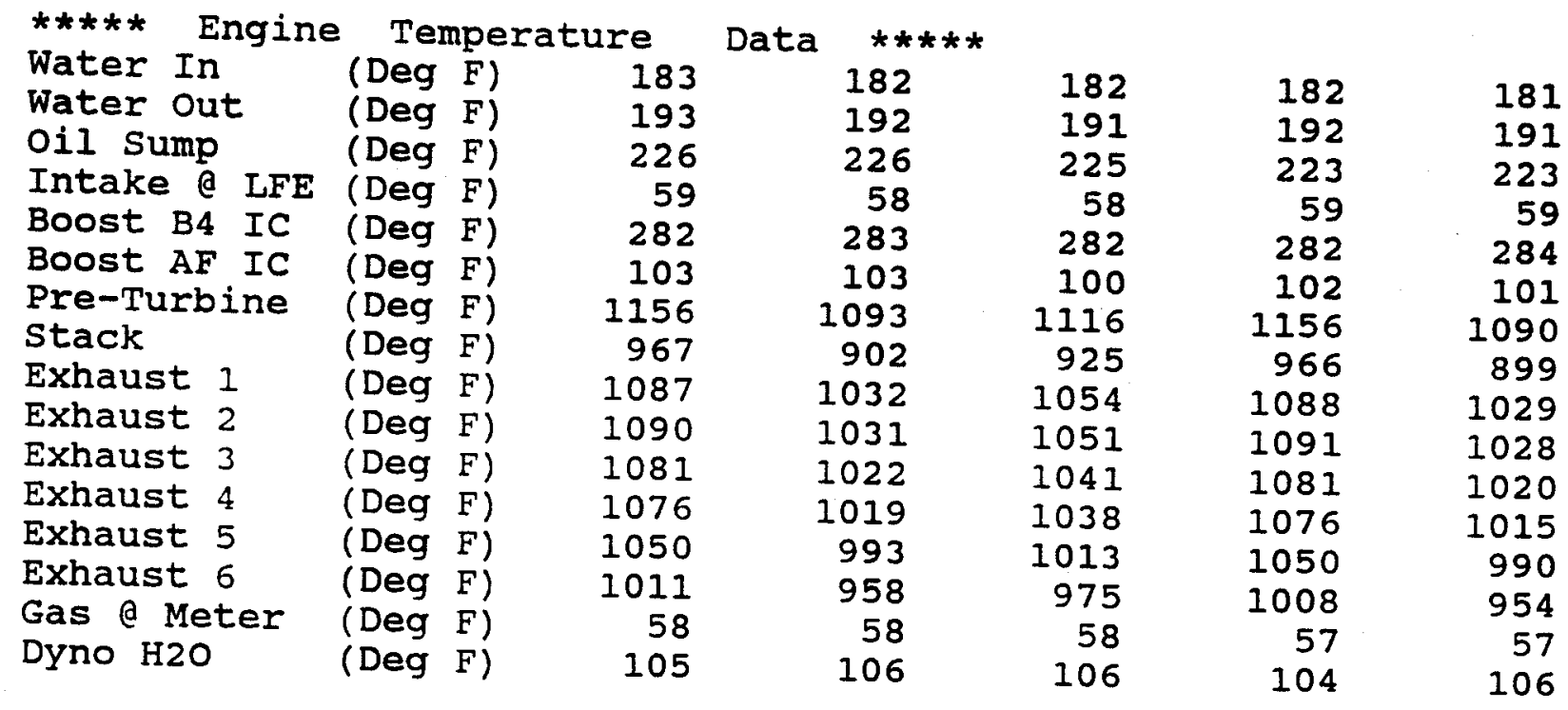

\begin{tabular}{|c|c|c|c|c|c|c|}
\hline Engine & Press & & Data & & & \\
\hline Main Oil Pres & (psig) & 30.2 & 30.3 & 30.5 & 30.7 & 30.7 \\
\hline Fuel Pressure & (psig) & 83.7 & 84.1 & 84.0 & 83.9 & 84.1 \\
\hline $\begin{array}{l}\text { Compress out } \\
\text { Inlet Man }\end{array}$ & (psig) & 23.04 & 23.07 & 23.02 & 22.93 & 23.02 \\
\hline $\begin{array}{l}\text { Inlet Manifold } \\
\text { Inlet Manifold }\end{array}$ & (psig) & 21.89 & 21.93 & 21.90 & 21.85 & 21.86 \\
\hline $\begin{array}{l}\text { Inlet Manifold } \\
\text { Pre-Turbine }\end{array}$ & $\begin{array}{l}\text { (psia) } \\
\text { (psig) }\end{array}$ & 36.36 & 36.39 & 36.36 & 36.32 & 36.32 \\
\hline Exhaust Bk Pr & $($ ("HG $)$ & $\begin{array}{r}15 \cdot 32 \\
2.74\end{array}$ & $\begin{array}{r}15.44 \\
2.61\end{array}$ & 15.41 & 15.32 & 15.47 \\
\hline LFE Filter Pr & ("H2O) & .80 & $\begin{array}{r}2.61 \\
.79\end{array}$ & 2.66 & 2.73 & 2.61 \\
\hline LFE Delta Pr & ("H2O) & 4.23 & 4.21 & .81 & .82 & .81 \\
\hline$(1 \mathrm{bH} 20 / 1$ & (bAir) & .0020 & .0021 & 4.24 & 4.25 & 4.24 \\
\hline RH (out) & $\% \quad$ ) & 18.26 & 18.10 & .0021 & .0021 & .0021 \\
\hline Barometer & "Hg ) & 29.44 & 29.44 & $\begin{array}{l}17.90 \\
29.44\end{array}$ & 17.79 & 17.60 \\
\hline
\end{tabular}


= Cell 04 MAC2 Project Low-Speed Data =

$\begin{array}{lrrrrr}* * * * * \text { Natural Gas Composition } * * * * * & & \\ \text { NG \% Methane } & 95.01 & 95.01 & 95.01 & 95.01 & 95.01 \\ \text { NG \% Ethane } & 2.62 & 2.62 & 2.62 & 2.62 & 2.62 \\ \text { NG \% Propane } & .46 & .46 & .46 & .46 & .46 \\ \text { NG \% CO2 } & 1.02 & 1.02 & 1.02 & 1.02 & 1.02 \\ \text { NG \% Nitrogen } & .65 & .65 & .65 & .65 & .65 \\ \text { Butane Actual }(\%) & .24 & .24 & .24 & .24 & .24 \\ \text { MWT } & 17.00 & 17.00 & 17.00 & 17.00 & 17.00 \\ \text { Fuel LHV } & 20541 . & 20541 . & 20541 . & 20541 . & 20541 .\end{array}$


= Cell 04 MAC2 Project Low-Speed Data =

\begin{tabular}{|c|c|c|c|c|c|}
\hline$* * * * *$ Test Cell Run & Informa & ion & & & \\
\hline $\begin{array}{l}\text { Run Number } \\
\text { Year }\end{array}$ & $\begin{array}{r}879 \\
1996\end{array}$ & 880 & 881 & 882 & 883 \\
\hline Operator Initials & $\begin{array}{c}1996 \\
<\ldots \text { RW.. }>\end{array}$ & $\begin{array}{l}1996 \\
<\ldots \text { RW.. }>\end{array}$ & $\begin{array}{c}1996 \\
<\ldots \text { RW. . > }\end{array}$ & $\begin{array}{l}1996 \\
<\ldots \text { RW . > }\end{array}$ & 1996 \\
\hline $\begin{array}{l}\text { Julian Day } \\
\text { Time (Seconds) }\end{array}$ & $\begin{array}{l}\text { MAR }, 19 \\
10: 11: 24\end{array}$ & MAR , 19 & MAR, 19 & MAR. , 19 & MAR., 19 \\
\hline Ignition Timing & 16.0 & $\begin{array}{c}10.16 .18 \\
13.0\end{array}$ & $\begin{array}{c}10: 21: 44 \\
19.0\end{array}$ & $\begin{array}{c}10: 25: 08 \\
16.0\end{array}$ & $\begin{aligned} 10: 29: 11 \\
13.0\end{aligned}$ \\
\hline
\end{tabular}

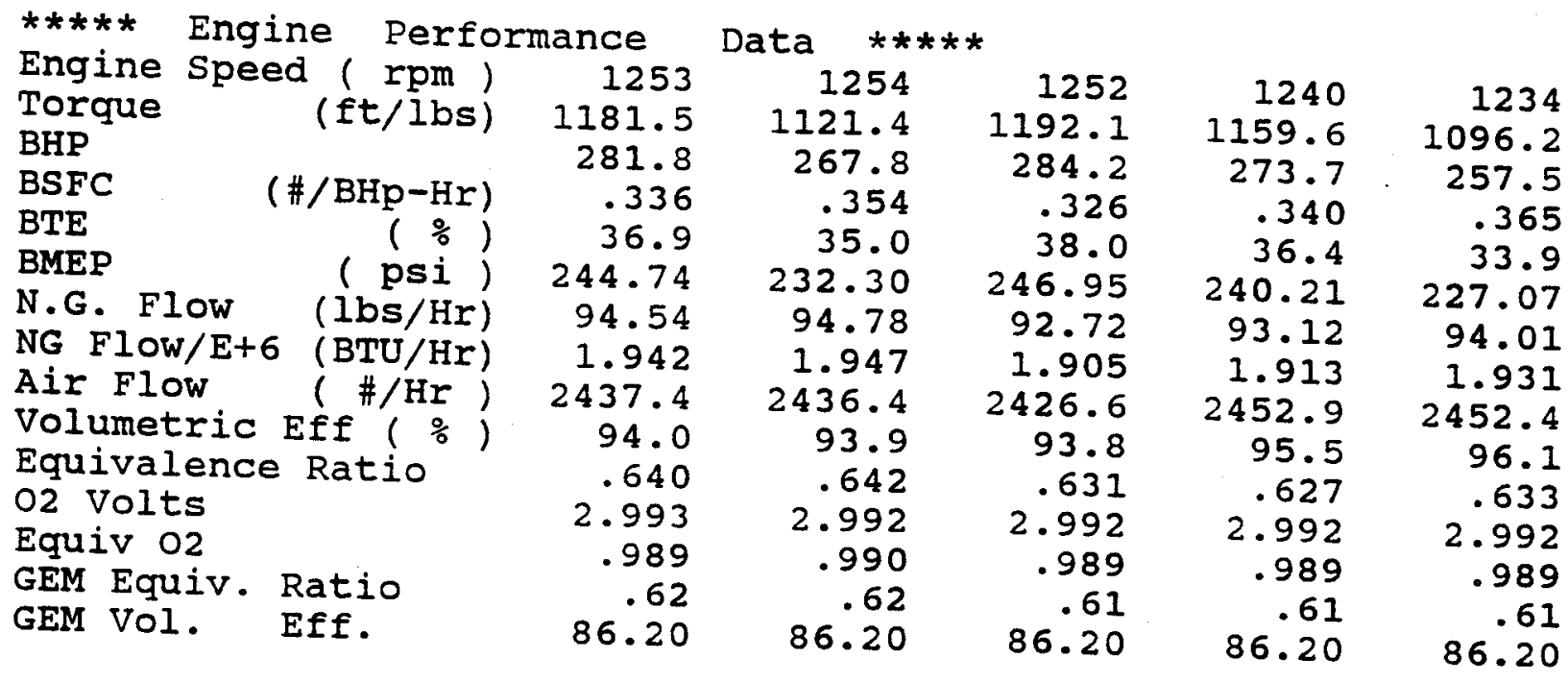

$\begin{array}{llrrrrr}* * * * * \text { Engine } & \text { Temperature } & \text { Data } & * * * * * & & & \\ \text { Water In } & \text { (Deg F) } & 181 & 182 & 181 & 181 & 182 \\ \text { Water Out } & \text { (Deg F) } & 191 & 192 & 191 & 191 & 192 \\ \text { Oil Sump } & \text { (Deg F) } & 222 & 222 & 222 & 222 & 221 \\ \text { Intake a LFE (Deg F) } & 59 & 60 & 60 & 60 & 60 \\ \text { Boost B4 IC (Deg F) } & 283 & 283 & 284 & 284 & 285 \\ \text { Boost AF IC (Deg F) } & 101 & 101 & 101 & 101 & 103 \\ \text { Pre-Turbine } & \text { (Deg F) } & 1115 & 1160 & 1088 & 1115 & 1161 \\ \text { Stack } & \text { (Deg F) } & 923 & 973 & 896 & 922 & 971 \\ \text { Exhaust 1 } & \text { (Deg F) } & 1053 & 1092 & 1028 & 1053 & 1096 \\ \text { Exhaust 2 } & \text { (Deg F) } & 1050 & 1093 & 1023 & 1050 & 1097 \\ \text { Exhaust 3 } & \text { (Deg F) } & 1041 & 1084 & 1015 & 1042 & 1088 \\ \text { Exhaust 4 } & \text { (Deg F) } & 1038 & 1081 & 1013 & 1038 & 1082 \\ \text { Exhaust 5 } & \text { (Deg F) } & 1014 & 1056 & 989 & 1013 & 1056 \\ \text { Exhaust 6 } & \text { (Deg F) } & 975 & 1011 & 952 & 972 & 1011 \\ \text { Gas a Meter } & \text { (Deg F) } & 58 & 59 & 60 & 60 & 59 \\ \text { Dyno H20 } & \text { (Deg F) } & 105 & 103 & 105 & 105 & 103\end{array}$

\begin{tabular}{|c|c|c|c|c|c|c|}
\hline Engine & ress & & Data & & & \\
\hline Main Oil Pres & (psig) & 30.9 & 30.9 & 30.9 & 31.0 & \\
\hline Fuel Pressure & (psig) & 84.0 & 83.9 & 84.1 & 84.1 & $\begin{array}{l}30.9 \\
84.0\end{array}$ \\
\hline Compress Out & (psig) & 23.06 & 23.06 & 22.96 & 23.03 & 23.07 \\
\hline $\begin{array}{l}\text { Inlet Manifold } \\
\text { Inlet Manifold }\end{array}$ & (psig) & 21.90 & 21.89 & 21.85 & 21.87 & 21.9 \\
\hline $\begin{array}{l}\text { Met Manitold } \\
\text { Pre-Turbine }\end{array}$ & $\begin{array}{l}\text { (psia) } \\
\text { (psiq) }\end{array}$ & 36.36 & 36.35 & 36.31 & 36.34 & 36.4 \\
\hline Exhaust Bk Pr & $($ ("Psig) & $\begin{array}{r}15.44 \\
2.66\end{array}$ & $\begin{array}{r}15.36 \\
2.75\end{array}$ & $\begin{array}{r}15.49 \\
2.60\end{array}$ & 15.45 & 15.42 \\
\hline LFE Filter $\operatorname{Pr}$ & ("H2O) & .81 & .82 & $\begin{array}{r}2.60 \\
.81\end{array}$ & 2.66 & 2.7 \\
\hline FE Delta Pr & ("H2O) & 4.25 & 4.25 & $\begin{array}{r}.81 \\
4.25\end{array}$ & $\begin{array}{r}.83 \\
29\end{array}$ & $\begin{array}{r}.8 \\
4.25\end{array}$ \\
\hline$(1 \mathrm{bH} 2 \mathrm{O})$ & (lbAir) & .0021 & .0021 & .0020 & $\begin{array}{r}4.29 \\
.0020\end{array}$ & $\begin{array}{r}4.2 \\
.002\end{array}$ \\
\hline RH (out) & $\% \quad 1$ & 17.16 & 16.97 & 16.75 & 16.62 & 16.5 \\
\hline ometer & "Hg ) & 29.44 & 29.44 & 29.44 & 29.45 & 29 \\
\hline
\end{tabular}


= Cell 04 MAC2 Project Low-Speed Data =

\begin{tabular}{|c|c|c|c|c|c|c|c|}
\hline 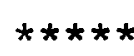 & Natura & Gas & Composit & $* *$ & & & \\
\hline NG \% & Methane & & 95.01 & 95.01 & 95.01 & 95.01 & 95.01 \\
\hline NG $\%$ & Ethane & & 2.62 & 2.62 & 2.62 & 2.62 & 2.62 \\
\hline NG \% & Propane & & .46 & .46 & .46 & .46 & .46 \\
\hline NG \% & $\mathrm{CO} 2$ & & 1.02 & 1.02 & 1.02 & 1.02 & 1.02 \\
\hline NG \% & Nitrogen & & .65 & .65 & .65 & .65 & .65 \\
\hline Butan & he Actual ( & $\%$ & .24 & .24 & .24 & .24 & .24 \\
\hline MWT & & & 17.00 & 17.00 & 17.00 & 17.00 & 17.00 \\
\hline Fuel & LHV & & 20541 . & 20541 & 20541 . & 20541 . & 20541 . \\
\hline
\end{tabular}


= Cell 04 MAC2 Project Low-speed Data =

\begin{abstract}
$* * * * *$ Test Cell
Run Number
Year
Operator Initials
Julian Day
Time (Seconds)

Ignition Timing
\end{abstract}

Information $* * * * *$

$$
\begin{aligned}
& \begin{array}{lrrrr}
884 & 885 & 886 & 887 & 888
\end{array} \\
& \begin{array}{lllll}
1996 & 1996 & 1996 & 1996 & 1996
\end{array}
\end{aligned}
$$

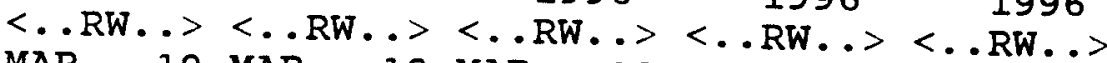

$$
\begin{aligned}
& \text { MAR., } 19 \text { MAR., } 19 \text { MAR., } 19 \text { MAR., } 19 \text { MAR., } 19 \\
& 10: 34: 26 \quad 10: 37: 50 \quad 10: 41: 39 \quad 10: 46: 19 \text { 10:49:49 } \\
& \begin{array}{rrrrr}
19.0 & 16.0 & 13.0 & 19.0 & 16.0
\end{array}
\end{aligned}
$$

\begin{tabular}{|c|c|c|c|c|c|c|}
\hline$* * * * *$ Engine & Press & & Data & & & \\
\hline Main Oil Pres & (psig) & 31.0 & 31.0 & 31.0 & 31.1 & 31.1 \\
\hline Fuel Pressure & (psig) & 84.3 & 84.2 & 84.0 & 84.2 & 84.0 \\
\hline Compress out & (psig) & 22.96 & 22.95 & 23.35 & 23.13 & 23.44 \\
\hline & (psig) & 21.80 & 21.85 & 22.23 & 21.98 & 22.27 \\
\hline $\begin{array}{l}\text { Inlet Manifold } \\
\text { Pre-Turbine }\end{array}$ & (psia) & 36.26 & 36.31 & 36.70 & 36.44 & 36.73 \\
\hline Exhaust BK Pr & ("HG) & $\begin{array}{r}15.44 \\
2.58\end{array}$ & $\begin{array}{r}15.42 \\
2.64\end{array}$ & 15.59 & 15.60 & 15.71 \\
\hline LFE Filter $\mathrm{Pr}$ & ("H2O) & .81 & $\begin{array}{r}2.64 \\
.82\end{array}$ & 2.83 & 2.62 & 2.73 \\
\hline LFE Delta Pr & ("H2O) & 4.24 & 4.28 & $\begin{array}{r}.86 \\
4.37\end{array}$ & .83 & .86 \\
\hline$(1 \mathrm{bH} 2 \mathrm{O} / \mathrm{l}$ & IbAir) & .0020 & .0020 & $\begin{array}{l}4.37 \\
0.37\end{array}$ & 4.28 & 4.33 \\
\hline $\mathrm{RH}$ (out) & j & 16.45 & 16.40 & .0021 & .0020 & .0021 \\
\hline arometer & "Hg ) & 29.45 & 29.45 & $\begin{array}{l}16.33 \\
29.45\end{array}$ & $\begin{array}{l}16.21 \\
29.45\end{array}$ & $\begin{array}{l}16.16 \\
29.45\end{array}$ \\
\hline
\end{tabular}

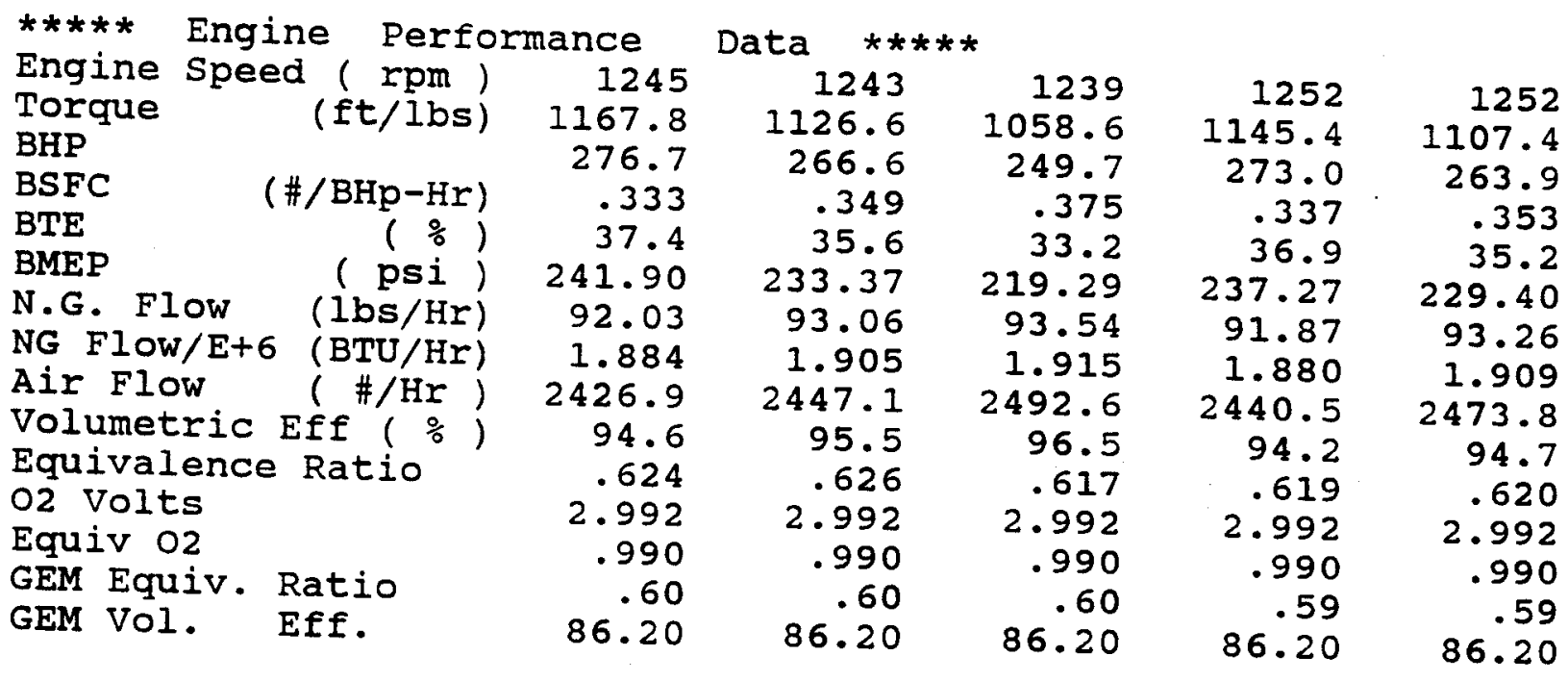

$\begin{array}{llrrrrr}* * * * * & \text { Engine } & \text { Temperature } & \text { Data } & * * * * * & & \\ \text { Water In } & \text { (Deg F) } & 181 & 182 & 182 & 182 & 182 \\ \text { Water Out } & \text { (Deg F) } & 191 & 191 & 192 & 192 & 192 \\ \text { Oil Sump } & \text { (Deg F) } & 222 & 221 & 221 & 221 & 221 \\ \text { Intake D LFE (Deg F) } & 60 & 60 & 61 & 61 & 61 \\ \text { Boost B4 IC } & \text { (Deg F) } & 284 & 284 & 287 & 286 & 287 \\ \text { Boost AF IC } & \text { (Deg F) } & 103 & 103 & 103 & 103 & 103 \\ \text { Pre-Turbine } & \text { (Deg F) } & 1089 & 1118 & 1172 & 1090 & 1123 \\ \text { Stack } & \text { (Deg F) } & 896 & 924 & 980 & 899 & 928 \\ \text { Exhaust 1 } & \text { (Deg F) } & 1026 & 1056 & 1107 & 1030 & 1062 \\ \text { Exhaust 2 } & \text { (Deg F) } & 1022 & 1052 & 1107 & 1024 & 1056 \\ \text { Exhaust 3 } & \text { (Deg F) } & 1015 & 1044 & 1098 & 1018 & 1048 \\ \text { Exhaust 4 } & \text { (Deg F) } & 1014 & 1042 & 1093 & 1017 & 1047 \\ \text { Exhaust 5 } & \text { (Deg F) } & 989 & 1017 & 1066 & 992 & 1021 \\ \text { Exhaust 6 } & \text { (Deg F) } & 951 & 974 & 1019 & 950 & 978 \\ \text { Gas D Meter } & \text { (Deg F) } & 60 & 60 & 60 & 61 & 62 \\ \text { Dyno H20 } & \text { (Deg F) } & 105 & 104 & 102 & 104 & 104\end{array}$


= Cell 04 MAC2 Project Low-Speed Data =

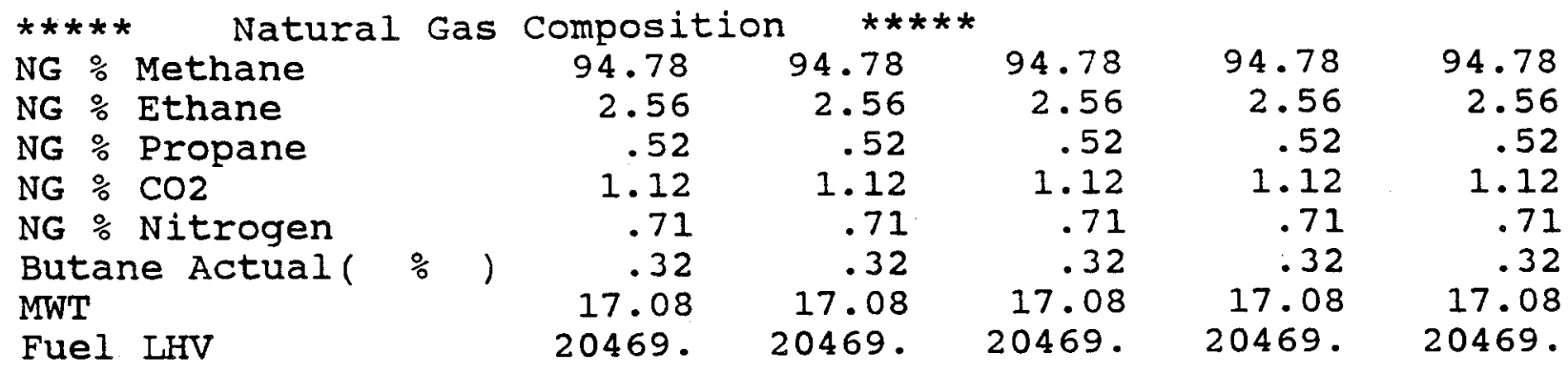


= Cell 04 MAC2 Project Low-speed Data =

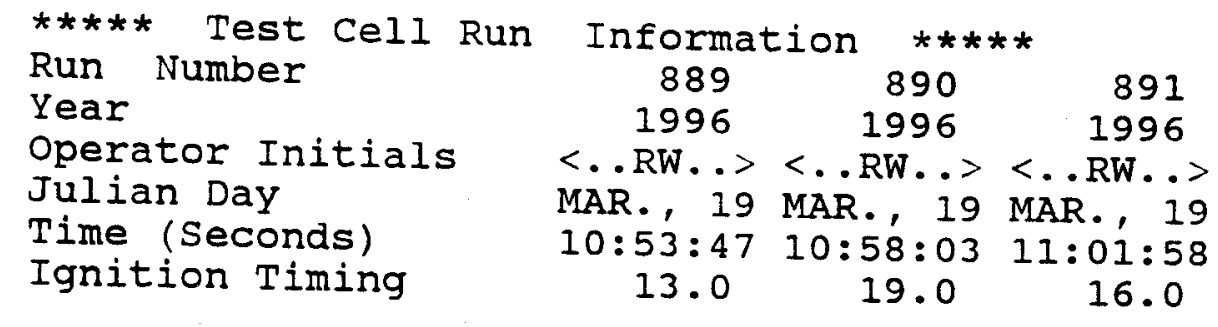

\begin{tabular}{|c|c|c|c|}
\hline Engine & nance & Data & \\
\hline gine Speed & 1237 & 1245 & 1261 \\
\hline$(\mathrm{ft} /$ & 10 & 1118 & 10 \\
\hline & & 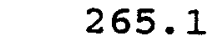 & \\
\hline (\#/BHp-Hr) & 390 & .3 & $.36]$ \\
\hline$\left(\frac{\circ}{6}\right)$ & 3 & 36 & 34.5 \\
\hline (psi) & 209.81 & 231.70 & 219 \\
\hline $\begin{array}{ll}\text { G G Flow } & (\mathrm{lbs} / \mathrm{Hr}) \\
\mathrm{G} \text { Flow } / \mathrm{E}+6 & (\mathrm{BTU} / \mathrm{Hr})\end{array}$ & 92 & 91. & 91 \\
\hline ir Flow $(\# / \mathrm{Hr})$ & 2477.3 & $\begin{array}{r}1.870 \\
2473.9\end{array}$ & $\begin{array}{r}1.880 \\
2492.2\end{array}$ \\
\hline olumetric Eff $(\%)$ & 96.2 & 95 & 94.8 \\
\hline $\begin{array}{l}\text { iquivalence Ratio } \\
2 \text { Volts }\end{array}$ & .617 & .6 & .60 \\
\hline $\begin{array}{l}\text { quiv } 02 \\
\text { quiv }\end{array}$ & $2 \cdot 9$ & 2.9 & 2.99 \\
\hline EM Equiv. Rati & & & .99 \\
\hline EM Vol & 86.20 & 86.2 & 86.28 \\
\hline
\end{tabular}

\begin{tabular}{llrrr}
$* * * * *$ Engine & \multicolumn{3}{c}{ Temperature } & Data \\
Water In & (Deg F) & 183 & 183 & 182 \\
Water Out & (Deg F) & 192 & 192 & 191 \\
Oil Sump & (Deg F) & 221 & 221 & 221 \\
Intake O LFE & (Deg F) & 62 & 61 & 62 \\
Boost B4 IC & (Deg F) & 289 & 288 & 288 \\
Boost AF IC & (Deg F) & 104 & 102 & 103 \\
Pre-Turbine & (Deg F) & 1178 & 1095 & 1128 \\
Stack & (Deg F) & 987 & 903 & 935 \\
Exhaust 1 & (Deg F) & 1114 & 1035 & 1070 \\
Exhaust 2 & (Deg F) & 1113 & 1029 & 1063 \\
Exhaust 3 & (Deg F) & 1103 & 1024 & 1053 \\
Exhaust 4 & (Deg F) & 1097 & 1021 & 1051 \\
Exhaust 5 & (Deg F) & 1071 & 996 & 1025 \\
Exhaust 6 & (Deg F) & 1026 & 955 & 982 \\
Gas a Meter & (Deg F) & 62 & 62 & 62 \\
Dyno H20 & (Deg F) & 101 & 103 & 102
\end{tabular}

\begin{tabular}{|c|c|c|c|c|}
\hline Engine & Press & & Data & \\
\hline Main Oil Pres & (psig) & 31.1 & 31.2 & 31 \\
\hline Fuel Pressure & (psig) & 84.1 & 84.3 & \\
\hline Compress Out & (psig) & 23.45 & 23.23 & 23 \\
\hline Inlet Manifold & (psig) & 24 & 22.07 & 22.1 \\
\hline Let Manif & ia) & 36 & 36.53 & 36.6 \\
\hline Pre-Turbine & (psig) & 15.62 & 15.72 & 15.7 \\
\hline Exhaust Bk Pr & ("HG ) & 2.83 & 2.66 & 2.7 \\
\hline LFE Filter Pr & ("H2O) & .84 & .85 & .8 \\
\hline LFE Delta & ("H2O) & 4.36 & 4.35 & 4.3 \\
\hline$(1 \mathrm{bl}$ & lbAir) & .0021 & .0021 & .002 \\
\hline $\mathrm{RH}$ (out) & $\% \quad)$ & 16.01 & 15.96 & 15.8 \\
\hline Barometer & "Hg ) & 29.45 & 29.45 & 29.4 \\
\hline
\end{tabular}




$$
\text { = Cell } 04 \text { MAC2 Project Low-Speed Data = }
$$

$\begin{array}{lrrr}* * * * * \text { Natural Gas Composition } * * * * * \\ \text { NG \% Methane } & 94.78 & 94.78 & 94.78 \\ \text { NG \% Ethane } & 2.56 & 2.56 & 2.56 \\ \text { NG \% Propane } & .52 & .52 & .52 \\ \text { NG \% CO2 } & 1.12 & 1.12 & 1.12 \\ \text { NG \% Nitrogen } & .71 & .71 & .71 \\ \text { Butane Actual }(\% & .32 & .32 & .32 \\ \text { MWT } & 17.08 & 17.08 & 17.08 \\ \text { Fuel LHV } & 20469 . & 20469 . & 20469 .\end{array}$


= Cell 04 MAC2 Project Low-Speed Data =

\begin{tabular}{|c|c|c|c|c|c|}
\hline Test Cell Run & Informa & cion & & & \\
\hline $\begin{array}{l}\text { Run Number } \\
\text { Year }\end{array}$ & 1213 & 1214 & 1215 & 1216 & 1217 \\
\hline Operator Initials & $\begin{aligned} & 1996 \\
&<. . \text { RW. . > }\end{aligned}$ & $\begin{array}{l}1996 \\
<\ldots \text { RW..> }\end{array}$ & $\begin{array}{l}1996 \\
<\end{array}$ & 1996 & 1996 \\
\hline $\begin{array}{l}\text { Julian Day } \\
\text { Time (Seconds) }\end{array}$ & APR., 02 & APR., 02 & APR., 02 & $\begin{array}{l}<\ldots R W . .> \\
\text { APR., } 022\end{array}$ & $\begin{array}{l}<\ldots \text { RW. } \\
\text { APR., } 02\end{array}$ \\
\hline $\begin{array}{l}\text { Ignition Timing } \\
\text { Ignition }\end{array}$ & $\begin{array}{c}14: 53: 52 \\
23.0\end{array}$ & $\begin{array}{c}15: 07: 36 \\
23.0\end{array}$ & $\begin{array}{c}15: 19: 11 \\
21.0\end{array}$ & $\begin{array}{c}15: 33: 03 \\
21.0\end{array}$ & $\begin{aligned} 15: 49: 17 \\
19.0\end{aligned}$ \\
\hline
\end{tabular}

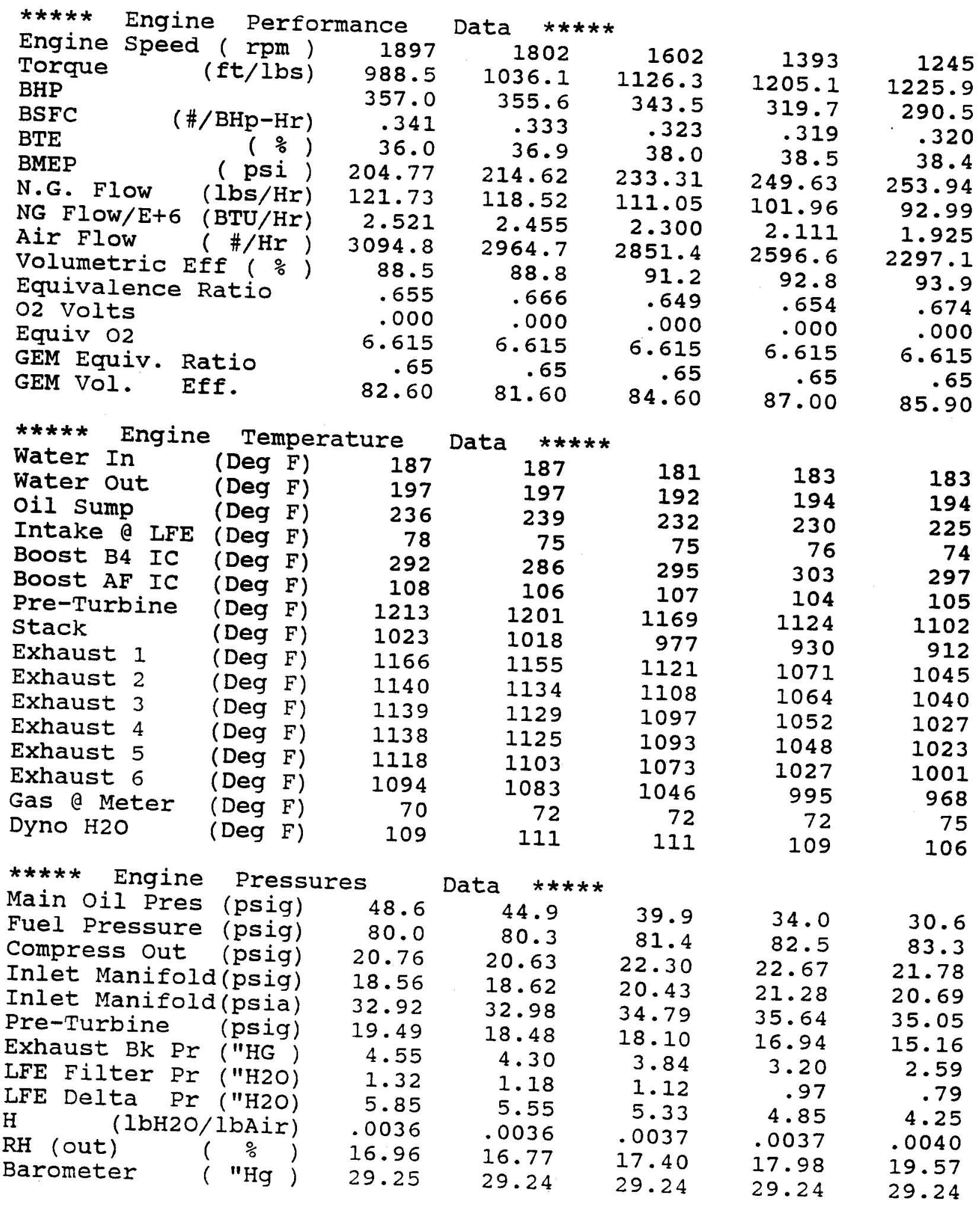




$$
\text { = Cell } 04 \text { MAC2 Project Low-Speed Data = }
$$

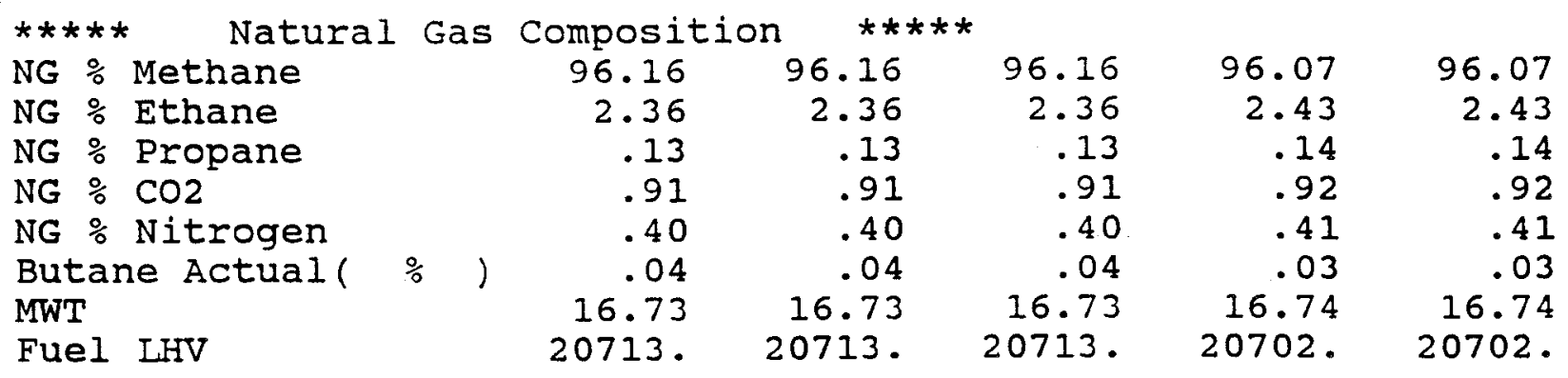




\section{= Cell 04 MAC2 Project Low-Speed Data =}

$\begin{array}{lcc}* * * * * \text { Test Cell Run } & \text { Information } * * * * * \\ \text { Run Number } & 1218 & 1219 \\ \text { Year } & 1996 & 1996 \\ \text { Operator Initials } & <\ldots \text { RW. }> & <. . \text { RW. } \\ \text { Julian Day } & \text { APR., O2 APR. O } 2 \\ \text { Time (Seconds) } & 16: 01: 27 & 16: 32: 24 \\ \text { Ignition Timing } & 13.0 & 10.0\end{array}$

\begin{tabular}{|c|c|c|}
\hline Engine & hc & Data \\
\hline Engine Speed ( $\mathrm{rpm}$ ) & 1105 & 1008 \\
\hline Torque & $1248 \cdot 6$ & 969.6 \\
\hline $\begin{array}{l}\text { BHP } \\
\text { BSFC }\end{array}$ & $\begin{array}{r}262.8 \\
323\end{array}$ & 186.2 \\
\hline $\begin{array}{r}(\pi / B H p-H r) \\
\left(\begin{array}{c}\% \\
0\end{array}\right)\end{array}$ & 38.1 & .330 \\
\hline psi & 258.65 & $\begin{array}{r}37.3 \\
200.86\end{array}$ \\
\hline N.G. Flow & 84.77 & 61.48 \\
\hline NG Flow/E+6 (BTU/Hr) & 1.755 & 1.272 \\
\hline Air Flow $(\# / \mathrm{Hr})$ & 1747.1 & 1247.3 \\
\hline Volumetric Eff ( $\left.\frac{\circ}{\circ}\right)$ & 92.6 & 90.8 \\
\hline $\begin{array}{l}\text { Equivalence Ratio } \\
\text { o2 Volts }\end{array}$ & .808 & .820 \\
\hline Equiv 02 & 6.615 & .000 \\
\hline GEM Equiv. Ratio & .78 & .78 \\
\hline GEM Vol. & 84.70 & 885.90 \\
\hline
\end{tabular}

\begin{tabular}{llrr}
$* * * * *$ Engine & \multicolumn{2}{c}{ Temperature } & Data \\
Water In & (Deg F) & 180 & 169 \\
Water Out & (Deg F) & 193 & 186 \\
Oil Sump & (Deg F) & 229 & 218 \\
Intake a LFE & (Deg F) & 73 & 75 \\
Boost B4 IC & (Deg F) & 264 & 209 \\
Boost AF IC & (Deg F) & 101 & 95 \\
Pre-Turbine & (Deg F) & 1170 & 1131 \\
Stack & (Deg F) & 1010 & 1011 \\
Exhaust 1 & (Deg F) & 1093 & 1049 \\
Exhaust 2 & (Deg F) & 1097 & 1044 \\
Exhaust 3 & (Deg F) & 1074 & 1028 \\
Exhaust 4 & (Deg F) & 1073 & 1026 \\
Exhaust 5 & (Deg F) & 1046 & 999 \\
Exhaust 6 & (Deg F) & 1013 & 970 \\
Gas a Meter & (Deg F) & 78 & 80 \\
Dyno H20 & (Deg F) & 104 & 95
\end{tabular}

$\begin{array}{lrr}* * * * * \text { Engine } & \text { Dressures } & \text { Data } * * * * * \\ \text { Main Oil Pres (psig) } & 25.9 & 25.4 \\ \text { Fuel Pressure (psig) } & 84.3 & 87.2 \\ \text { Compress Out (psig) } & 16.94 & 10.25 \\ \text { Inlet Manifold(psig) } & 16.20 & 9.83 \\ \text { Inlet Manifold(psia) } & 30.56 & 24.18 \\ \text { Pre-Turbine (psig) } & 10.67 & 6.44 \\ \text { Exhaust Bk Pr ("HG) } & 1.95 & 1.20 \\ \text { LFE Filter Pr ("H2O) } & .49 & .26 \\ \text { LFE Delta Pr ("H2O) } & 3.20 & 2.29 \\ \text { H (lbH2O/lbAir) } & .0037 & .0039 \\ \text { RH (out) ( } \% \text { ( } \% \text { ) } & 18.92 & 19.70 \\ \text { Barometer } \quad \text { ("Hg) } & 29.23 & 29.23\end{array}$


= Cell 04 MAC2 Project Low-Speed Data =

$\begin{array}{lrr}* * * * * \text { Natural Gas Composition } & * * * * * \\ \text { NG } \% \text { Methane } & 96.07 & 95.97 \\ \text { NG } \% \text { Ethane } & 2.43 & 2.46 \\ \text { NG \% Propane } & .14 & .16 \\ \text { NG \% CO2 } & .92 & .94 \\ \text { NG \% Nitrogen } & .41 & .42 \\ \text { Butane Actual ( } \% & .03 & .05 \\ \text { MWT } & 16.74 & 16.77 \\ \text { Fuel LHV } & 20702 . & 20686 .\end{array}$


APPENDIX B

RECORDED DATA FOR STEADY-STATE TESTS 
Recorded Data

\begin{tabular}{|c|c|c|c|c|c|c|c|c|c|c|c|c|}
\hline $\begin{array}{c}\begin{array}{c}\text { Run } \\
\text { Number }\end{array} \\
564\end{array}$ & $\begin{array}{c}\text { Methane } \\
(\%) \\
96.49\end{array}$ & $\begin{array}{c}\text { Ethane } \\
(\%)\end{array}$ & $\begin{array}{c}\text { Propane } \\
(\%)\end{array}$ & $\begin{array}{c}\text { Butane } \\
(\%)\end{array}$ & $\mathrm{CO} 2(\%)$ & N2 (\%) & $\begin{array}{c}\text { Air Flow } \\
\text { (lb/hr) }\end{array}$ & $\begin{array}{l}\text { Power } \\
\text { (bhp) }\end{array}$ & $\begin{array}{c}\text { Humidity } \\
\text { (Ib } \\
\text { H2O/Air) }\end{array}$ & $\begin{array}{c}\text { NG Flow } \\
\text { (lb/hr) }\end{array}$ & $\begin{array}{l}\text { Speed } \\
\text { (rpm) }\end{array}$ & $\begin{array}{c}\text { Torque (ft- } \\
\text { lb) }\end{array}$ \\
\hline$\frac{564}{565}$ & $\frac{96.49}{96.49}$ & $\frac{2.14}{2.14}$ & 0.11 & 0.02 & 0.77 & 0.47 & 471.58 & 12.6 & 0.011 & 20.17 & 1949 & 33.9 \\
\hline 567 & $\frac{96.49}{96.44}$ & 2.14 & 0.11 & 0.02 & 0.77 & 0.47 & 668.94 & 58.9 & 0.0109 & 28.99 & 1704 & 181.5 \\
\hline 568 & $\frac{96.44}{96.44}$ & 2.26 & 0.11 & 0.02 & 0.69 & 0.48 & 950.73 & 93.7 & 0.011 & 38.44 & 1701 & 289.3 \\
\hline 569 & $\begin{array}{r}96.44 \\
96.44\end{array}$ & 2.26 & 0.11 & 0.02 & 0.69 & 0.48 & 1163 & 119.1 & 0.011 & 46.69 & 1705 & 366.8 \\
\hline 570 & $\frac{96}{96} \cdot \frac{44}{44}$ & 2.26 & 0.11 & 0.02 & 0.69 & 0.48 & 1451.8 & 160 & 0.011 & 58.49 & 1703 & 493.4 \\
\hline 571 & 96.44 & 2.26 & 0.11 & 0.02 & 0.69 & 0.48 & 1771.1 & 207.4 & 0.0107 & 71.77 & 1700 & 640.9 \\
\hline 572 & $\frac{96.44}{96.44}$ & 2.26 & 0.11 & 0.02 & 0.69 & 0.48 & 2080.8 & 242.6 & 0.0106 & 81.4 & 1711 & 745 \\
\hline 573 & 96.44 & 2.26 & 0.11 & 0.02 & 0.69 & 0.48 & 2317.5 & 271.9 & $0.01 \overline{05}$ & 91.06 & 1698 & 840.9 \\
\hline 574 & $\frac{96.44}{96.42}$ & 2.26 & 0.11 & 0.02 & 0.69 & 0.48 & 2569.9 & 297.5 & 0.0102 & 100.04 & 1691 & 924.1 \\
\hline 575 & $\frac{96.42}{96.42}$ & 2.27 & 0.11 & 0.02 & 0.7 & 0.48 & 1644.2 & 167.8 & 0.0101 & 66.87 & 1940 & 454.4 \\
\hline $57 \frac{5}{57}$ & 96.42 & 2.27 & 0.11 & 0.02 & 0.7 & 0.48 & 1942.7 & 201.6 & 0.01 & 77.46 & 1955 & 541.4 \\
\hline & 96.42 & 2.27 & 0.11 & 0.02 & 0.7 & 0.48 & 2249.7 & 249.6 & 0.0101 & 89.11 & 1949 & 672.7 \\
\hline$\frac{577}{578}$ & 96.42 & 2.27 & 0.11 & 0.02 & 0.7 & 0.48 & 2532.8 & 281.9 & 0.0101 & 98.19 & 1951 & 758.9 \\
\hline 578 & 96.42 & 2.27 & 0.11 & 0.02 & 0.7 & 0.48 & 2871.2 & 327.6 & 0.0102 & 111.82 & 1948 & 883.1 \\
\hline 579 & 96.42 & 2.27 & 0.11 & 0.02 & 0.7 & 0.48 & 327.05 & 16 & 0.0102 & 14.34 & 1498 & 56.2 \\
\hline 580 & 96.42 & 2.27 & 0.11 & $0 . \overline{02}$ & 0.7 & 0.48 & 558.48 & 51.9 & 0.0103 & 24.14 & 1505 & 181.1 \\
\hline 581 & 96.42 & 2.27 & 0.11 & 0.02 & 0.7 & 0.48 & 815.75 & 82 & 0.0102 & 33.47 & 1505 & 286 \\
\hline 582 & 96.37 & 2.07 & 0.14 & 0.04 & 0.74 & 0.64 & 1030.9 & 110.7 & 0.0102 & 41.57 & 1497 & 388.3 \\
\hline 583 & 96.37 & 2.07 & 0.14 & 0.04 & 0.74 & 0.64 & 1310.2 & 150 & 0.0102 & 53.1 & 1500 & 525.3 \\
\hline 584 & 96.37 & 2.07 & 0.14 & 0.04 & 0.74 & 0.64 & 1586.2 & 186.5 & 0.01 & 63.6 & 1494 & 655.5 \\
\hline 585 & 96.37 & 2.07 & 0.14 & 0.04 & 0.74 & 0.64 & 263.47 & 14.1 & 0.0101 & 12.78 & 1255 & 59.1 \\
\hline 586 & 96.37 & 2.07 & 0.14 & 0.04 & 0.74 & 0.64 & 860.1 & 96.9 & 0.0101 & 34.88 & 1255 & 405.8 \\
\hline 587 & 96.37 & 2.07 & 0.14 & 0.04 & 0.74 & 0.64 & 1108.5 & 131.6 & 0.01 & 44.59 & 1252 & 551.7 \\
\hline 588 & 94.41 & 2.63 & 0.51 & 0.28 & 1.02 & 1.15 & 238.18 & 16.5 & 0.0016 & 12.25 & 1084 & 80.2 \\
\hline 589 & 94.41 & 2.63 & 0.51 & 0.28 & 1.02 & 1.15 & 788.62 & 103.6 & 0.0015 & 36.43 & 1104 & 493.1 \\
\hline 590 & 94.41 & 2.63 & 0.51 & 0.28 & 1.02 & 1.15 & 217.74 & 16.7 & 0.0016 & 10.64 & 1006 & 87 \\
\hline 591 & 94.36 & 2.68 & 0.51 & 0.28 & 1.01 & 1.16 & 725.61 & 93.1 & 0.0015 & 34.38 & 1001 & 488.5 \\
\hline 592 & 94.36 & 2.68 & 0.51 & 0.28 & 1.01 & $1.1 \overline{6}$ & 162.83 & 15.1 & 0.0015 & 8.32 & 798 & 99.2 \\
\hline 593 & 94.36 & 2.68 & 0.51 & 0.28 & 1.01 & 1.16 & 289.81 & 38.5 & 0.0015 & 14.91 & 805 & 251.5 \\
\hline 594 & 94.36 & 2.68 & 0.51 & 0.28 & 1.01 & 1.16 & 438.32 & 59.5 & 0.0015 & 21.81 & 795 & 393 \\
\hline 595 & 94.29 & 2.67 & 0.52 & 0.3 & 1.04 & 1.19 & 583.48 & 82.6 & 0.0016 & 30.46 & 800 & 542.5 \\
\hline 596 & 94.29 & 2.67 & 0.52 & 0.3 & 1.04 & 1.19 & 120.6 & 10.2 & 0.0015 & 6.7 & 650 & 82.8 \\
\hline 597 & 94.29 & 2.67 & 0.52 & 0.3 & 1.04 & 1.19 & 214.37 & 28.9 & 0.0015 & 11.57 & 642 & 236.4 \\
\hline
\end{tabular}


Recorded Data

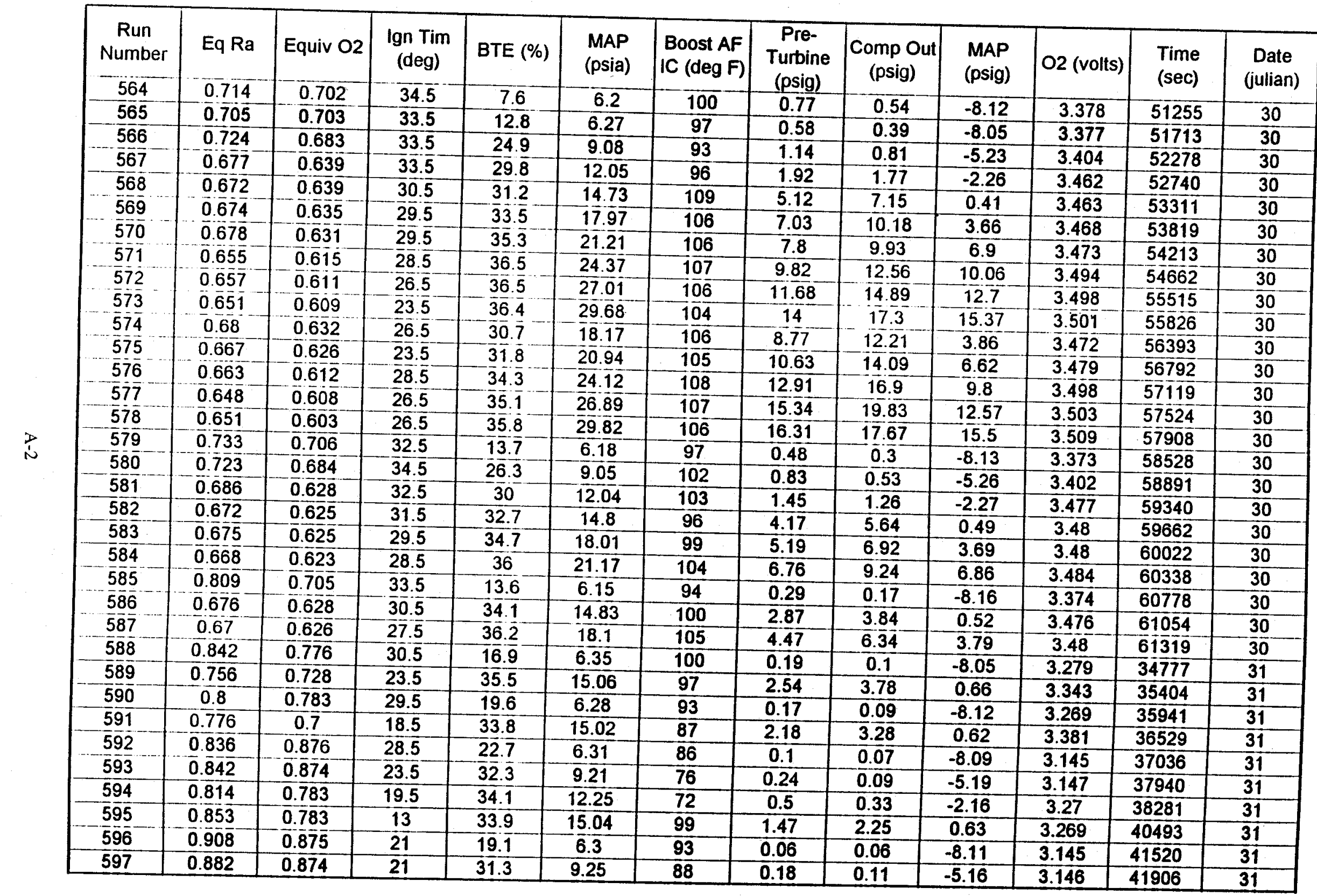




\begin{tabular}{|c|c|c|c|c|c|c|c|c|c|c|c|c|}
\hline $\begin{array}{c}\begin{array}{c}\text { Run } \\
\text { Number }\end{array} \\
\end{array}$ & Year & $\begin{array}{c}\text { BSFC } \\
(\# / \mathrm{bhp}-\mathrm{hr})\end{array}$ & $\begin{array}{c}\text { BMEP } \\
\text { (psi) }\end{array}$ & $\begin{array}{c}\text { Vol Eff } \\
(\%)\end{array}$ & $\begin{array}{c}\text { Intake Air } \\
\text { (acfm) }\end{array}$ & $\begin{array}{c}\text { Intake Air } \\
\text { (scfm) }\end{array}$ & $\begin{array}{c}\text { Boost B4 } \\
\text { IC (deg F) }\end{array}$ & $\begin{array}{c}\text { Stack } \\
\text { (deg F) }\end{array}$ & $\begin{array}{c}\text { Pre- } \\
\text { Turbine } \\
\text { (deg F) }\end{array}$ & $\begin{array}{l}\text { Fuel } \\
\text { Press } \\
\text { (psig) }\end{array}$ & EBP $(" \mathrm{Hg})$ & Baro $(" \mathrm{Hg})$ \\
\hline 564 & 1996 & 1.604 & 7.01 & 69.9 & 110 & 105 & 92 & 916 & 1061 & 90.2 & 0.08 & 29.15 \\
\hline 565 & 1996 & 0.96 & 11.61 & 69 & 96 & 92 & 90 & $86 \overline{6}$ & 1010 & 90.3 & 0.05 & 29.15 \\
\hline 566 & 1996 & 0.492 & 37.6 & 76.5 & 155 & 149 & 92 & 898 & 992 & 89.2 & 0.14 & 29.14 \\
\hline 567 & 1996 & 0.41 & 59.93 & 82.2 & 221 & 212 & 104 & 920 & 1002 & 88.2 & 0.26 & 29.14 \\
\hline 568 & 1996 & 0.392 & 75.98 & 83.9 & 271 & 259 & 167 & 921 & 1043 & 87.4 & 0.38 & 29.14 \\
\hline 569 & 1996 & 0.366 & $102 . \overline{2}$ & $8 \overline{5} . \overline{5}$ & 341 & 323 & 199 & 931 & 1070 & 86.2 & 0.6 & 29.14 \\
\hline 570 & 1996 & 0.346 & 132.77 & 88.5 & 416 & 394 & 192 & 955 & 1084 & 85 & 0.9 & 29.14 \\
\hline $57 \overline{1}$ & 1996 & 0.335 & 154.32 & 90 & 488 & 463 & 216 & 954 & 1100 & 84.1 & 1.25 & 29.13 \\
\hline 572 & 1996 & 0.335 & 174.19 & 90.9 & 549 & $5 \overline{16}$ & 240 & 958 & 1118 & 83.6 & 1.57 & 29.13 \\
\hline $57 \overline{3}$ & 1996 & 0.336 & 191.43 & 91.7 & 607 & 572 & 260 & 970 & 1144 & $82 . \overline{5}$ & 1.97 & 29.14 \\
\hline $57 \overline{4}$ & 1996 & 0.398 & 94.13 & 84.1 & 388 & 366 & 222 & 976 & 1125 & 86.8 & 0.79 & 29.14 \\
\hline$\overline{5} \overline{7} \overline{5}$ & 1996 & 0.384 & 112.16 & 85.3 & 461 & 432 & 236 & 1008 & 1166 & 85.6 & 1.12 & 29.14 \\
\hline$\overline{5} \overline{7} \overline{6}$ & 1996 & 0.357 & 139.34 & 86.4 & 536 & 501 & 261 & 964 & 1140 & 83.8 & $1.4 \overline{8}$ & 29.14 \\
\hline 577 & 1996 & 0.348 & 157.2 & 86.9 & 602 & 564 & 284 & 970 & 1166 & 82.6 & 1.9 & 29.14 \\
\hline 578 & $19 \overline{96}$ & 0.341 & 182.94 & 88.9 & 684 & 639 & 269 & 994 & 1172 & 81.4 & 2.53 & 29.14 \\
\hline $57 \overline{9}$ & 1996 & 0.895 & 11.63 & 63 & 78 & 73 & 104 & 840 & 992 & 90.9 & 0.03 & 29.14 \\
\hline 580 & 1996 & 0.465 & 37.51 & 73.6 & 133 & 124 & 97 & 853 & 946 & 90 & 0.09 & 29.14 \\
\hline 581 & 1996 & 0.408 & 59.24 & 80.7 & 193 & 182 & 103 & 882 & 964 & 89.3 & 0.19 & 29.14 \\
\hline 582 & 1996 & 0.375 & 80.43 & 82.3 & 244 & 229 & 154 & 879 & 990 & 88.6 & 0.3 & 29.14 \\
\hline 583 & 1996 & 0.354 & 108.81 & 86.4 & 310 & 292 & 167 & 902 & 1017 & 87.4 & 0.48 & 29.14 \\
\hline 584 & 1996 & 0.341 & 135.78 & 89.9 & 376 & 353 & 190 & 915 & 1041 & 85.7 & 0.69 & 29.14 \\
\hline 585 & 1996 & 0.905 & 12.24 & 61 & 62 & 59 & 102 & 776 & 929 & 90.8 & 0.03 & 29.14 \\
\hline 586 & 1996 & 0.36 & 84.07 & 82.3 & 203 & 191 & 132 & 833 & 936 & 89 & 0.24 & 29.14 \\
\hline 587 & 1996 & 0.339 & 114.29 & $87 . \overline{8}$ & 261 & 247 & 159 & 857 & 972 & 87.9 & 0.38 & 29.14 \\
\hline 588 & 1996 & 0.74 & 16.61 & 61.8 & 50 & 53 & 73 & 732 & 890 & 91.9 & 0.03 & 29.32 \\
\hline 589 & 1996 & 0.351 & 102.15 & 83.7 & 163 & 176 & 103 & 888 & 994 & 89.6 & 0.19 & 29.32 \\
\hline 590 & 1996 & 0.639 & 18.02 & 60.6 & 45 & 48 & 70 & 732 & 885 & 91.6 & 0.03 & 29.32 \\
\hline 591 & 1996 & 0.369 & 101.18 & 83.8 & 149 & 162 & 94 & 869 & 971 & 89.6 & 0.15 & 29.32 \\
\hline 592 & 1996 & 0.551 & 20.56 & 56.2 & 34 & 36 & 70 & 692 & 839 & 91.6 & 0.02 & 29.32 \\
\hline 593 & 1996 & 0.387 & 52.1 & 66.8 & 60 & 65 & 62 & $\overline{7} \overline{5} \overline{2}$ & 878 & 90.8 & 0.05 & 29.32 \\
\hline 594 & 1996 & 0.366 & 81.4 & 76.1 & 90 & 98 & 62 & 800 & 917 & 89.8 & 0.15 & 29.32 \\
\hline 595 & 1996 & 0.369 & 112.39 & 86.6 & 121 & 130 & 88 & 891 & 994 & 90.3 & 0.21 & 29.33 \\
\hline 596 & 1996 & 0.654 & 17.16 & 52.3 & 25 & 27 & 73 & 579 & 737 & 91.6 & 0 & 29.33 \\
\hline 597 & 1996 & 0.4 & 48.96 & 63.3 & 44 & 48 & 66 & 658 & 790 & 90.9 & 0.09 & 29.33 \\
\hline
\end{tabular}




\begin{tabular}{|c|c|c|c|c|c|c|c|c|c|c|c|c|}
\hline $\begin{array}{c}\text { Run } \\
\text { Number }\end{array}$ & RH (\%) & Density & Stioch A/F & $\begin{array}{c}\text { GEM Eq } \\
\mathrm{Ra}\end{array}$ & $\begin{array}{c}\text { GEM Vol } \\
\text { Eff }(\%)\end{array}$ & $\begin{array}{l}\text { Exh \#1 } \\
\text { (deg F) }\end{array}$ & $\begin{array}{l}\text { Exh \#2 } \\
(\operatorname{deg} F)\end{array}$ & $\begin{array}{l}\text { Exh \#3 } \\
(\operatorname{deg} F)\end{array}$ & $\begin{array}{l}\text { Exh \#4 } \\
\text { (deg F) }\end{array}$ & $\begin{array}{l}\text { Exh \#5 } \\
\text { (deg F) }\end{array}$ & $\begin{array}{l}\text { Exh \#6 } \\
(\operatorname{deg} F)\end{array}$ & $\begin{array}{c}\text { Water In } \\
\text { (deg F) }\end{array}$ \\
\hline 56 & $\frac{62.97}{62.68}$ & 0.3 & 16.7 & 0.72 & 60.3 & 1051 & 942 & 949 & 944 & 927 & 954 & 168 \\
\hline$\frac{565}{566}$ & $\frac{62.68}{61.63}$ & 0.299 & 16.7 & 0.72 & 61.3 & 996 & 898 & 899 & 891 & 878 & 915 & 163 \\
\hline $\begin{array}{l}566 \\
567\end{array}$ & 61.63 & 0.294 & 16.7 & 0.7 & 70.1 & 976 & 905 & 924 & 913 & 892 & 910 & 162 \\
\hline$\frac{567}{568}$ & 62.26 & 0.289 & 16.73 & 0.66 & 69.7 & 956 & 899 & 919 & 913 & 889 & 906 & 170 \\
\hline$\frac{568}{569}$ & 60.96 & 0.286 & 16.73 & 0.66 & 75.2 & 1001 & 942 & 957 & 953 & 936 & 943 & 178 \\
\hline $\begin{array}{l}569 \\
570\end{array}$ & 58.03 & 0.283 & 16.73 & 0.66 & 75.2 & 1015 & 970 & 983 & 979 & 962 & 962 & 186 \\
\hline $57 \overline{0}$ & 54.16 & 0.28 & 16.73 & 0.66 & 76.1 & 1009 & 983 & 990 & 994 & 977 & 965 & 177 \\
\hline 571 & 52.89 & 0.28 & 16.73 & 0.65 & 80.1 & 1026 & 1006 & 1009 & 1015 & 997 & 980 & 186 \\
\hline 572 & 49.52 & 0.282 & 16.73 & 0.65 & 82.1 & 1048 & 1031 & 1033 & 1037 & 1017 & 999 & 181 \\
\hline 573 & 48.29 & 0.28 & 16.73 & 0.65 & 81 & 1076 & 1066 & 1063 & 1064 & 1044 & 1024 & 181 \\
\hline 574 & 48.06 & 0.29 & 16.73 & 066 & 74.9 & 1081 & 1030 & 1045 & 1033 & 1021 & 1022 & 176 \\
\hline 575 & 48.65 & 0.285 & 16.73 & 0.66 & 81.3 & 1116 & 1073 & 1084 & 1076 & 1060 & 1055 & 181 \\
\hline $5 \overline{5} 6$ & 49 & 0.28 & 16.73 & 0.65 & 81.3 & 1082 & 1054 & 1061 & 1056 & 1041 & 1034 & 183 \\
\hline $57 \overline{7}$ & 48.15 & 0.278 & 16.73 & 0.65 & 80.4 & 1106 & 1082 & 1087 & 1084 & 1068 & 1058 & 187 \\
\hline 578 & 47.95 & 0.276 & 16.73 & 0.65 & 80.9 & 1113 & 1095 & 1096 & 1095 & 1076 & 1063 & 181 \\
\hline 579 & 47.43 & 0.302 & 16.73 & 0.72 & 60.9 & 959 & 880 & 897 & 881 & 853 & 901 & $1 \overline{6} 0$ \\
\hline 580 & 47.38 & 0.298 & 16.73 & 0.7 & 67.5 & 931 & 867 & 891 & 876 & 852 & 868 & 147 \\
\hline 581 & 46.02 & 0.294 & 16.73 & 0.66 & 67 & 915 & 869 & 888 & 882 & 855 & 864 & 158 \\
\hline 582 & 45.83 & 0.29 & 16.66 & 0.65 & 66.1 & 942 & 890 & 908 & 904 & 880 & 886 & 168 \\
\hline 583 & 45.61 & 0.286 & 16.66 & 0.65 & 77 & 951 & 916 & 929 & 929 & 905 & 899 & 178 \\
\hline 584 & 45.52 & 0.281 & 16.66 & 0.65 & 77.5 & 965 & 942 & 946 & 953 & 930 & 915 & 174 \\
\hline 585 & 45.99 & 0.296 & 16.66 & 0.72 & 65.1 & 914 & 836 & 832 & 858 & 824 & 851 & 140 \\
\hline 586 & 46.4 & 0.291 & 16.66 & 0.65 & 71.1 & 888 & 841 & 859 & 854 & 829 & 828 & 135 \\
\hline 587 & 46.75 & 0.287 & 16.66 & 0.65 & 79.5 & 906 & 873 & 885 & 885 & 860 & 850 & 156 \\
\hline 588 & 29.47 & 0.335 & 16.37 & 0.79 & 76.3 & 883 & 816 & 852 & 839 & 803 & 840 & 103 \\
\hline 589 & 29.99 & 0.321 & 16.37 & 0.75 & 81.3 & 937 & 887 & 909 & 901 & 873 & 872 & 130 \\
\hline 590 & 29.47 & 0.325 & 16.37 & 0.8 & 77.5 & 886 & 811 & 841 & 830 & 797 & 812 & 111 \\
\hline 591 & 29.81 & 0.317 & 16.37 & 0.72 & 81.6 & 917 & 870 & 892 & 882 & 853 & 848 & 107 \\
\hline 592 & 29.73 & 0.322 & 16.37 & 0.89 & 69.4 & 844 & 767 & 783 & 801 & 764 & 793 & 95 \\
\hline $59 \overline{3}$ & 29.85 & 0.319 & 16.37 & 0.89 & $6 \overline{8.7}$ & 863 & 802 & 822 & 820 & 796 & 826 & 89 \\
\hline 594 & 30.62 & 0.315 & 16.37 & 0.8 & 72.5 & 858 & 811 & 838 & 841 & 800 & 801 & 92 \\
\hline 595 & 29.59 & 0.316 & 16.35 & 0.8 & 76.8 & 909 & 870 & 898 & 897 & 864 & 851 & 127 \\
\hline 596 & 29.69 & 0.322 & 16.35 & 0.89 & 61.2 & 747 & 680 & 700 & 720 & 692 & 680 & 74 \\
\hline 597 & 29.92 & 0.321 & 16.35 & 0.89 & 65.6 & 792 & 733 & 770 & 764 & 729 & 720 & 75 \\
\hline
\end{tabular}




\begin{tabular}{|c|c|c|c|c|c|c|c|c|c|c|c|c|}
\hline $\begin{array}{c}\text { Run } \\
\text { Number }\end{array}$ & $\begin{array}{c}\text { Water Out } \\
\text { (deg F) }\end{array}$ & $\begin{array}{c}\text { Oil Sump } \\
\text { (deg F) }\end{array}$ & $\begin{array}{c}\text { Oil } \\
\text { Gallery } \\
\text { (deg F) }\end{array}$ & $\begin{array}{c}\text { Intake @ } \\
\text { LFE (deg } \\
\text { F) }\end{array}$ & $\begin{array}{c}\text { Oil Press } \\
\text { (psig) }\end{array}$ & $\begin{array}{c}\begin{array}{c}\text { Fuel Flow } \\
\text { (@ RT } \\
\text { Meter) }\end{array} \\
\end{array}$ & $\begin{array}{c}\text { Inlet Rest } \\
(" \mathrm{H} 2 \mathrm{O})\end{array}$ & $H C(p p m)$ & CO (ppm) & $\mathrm{CO} 2(\%)$ & $\begin{array}{l}\text { NOx } \\
\text { (ppm) }\end{array}$ & $\mathrm{O} 2(\%)$ \\
\hline 564 & 182 & 208 & 104 & 72 & 62.7 & 0.3568 & 1.17 & 2729 & 852.2 & 7.99 & 65.4 & 7.03 \\
\hline 565 & 180 & 204 & 103 & 71 & 55 & 0.3096 & 1.01 & 2261 & 655.1 & 8 & 100.1 & 6.93 \\
\hline 566 & 183 & 206 & 98 & 72 & 53.4 & 0.524 & 1.74 & 1334 & 413.5 & 7.84 & 454.8 & 7.12 \\
\hline 567 & 185 & 209 & 98 & 72 & 51.8 & 0.7056 & 2.61 & 1304 & 368.6 & 7.3 & 342.6 & 8.01 \\
\hline 568 & 187 & 214 & 110 & $7 \overline{3}$ & 49.7 & 0.8675 & 3.35 & 1136 & 351.7 & 7.31 & 362.2 & 7.98 \\
\hline $56 \overline{9}$ & 192 & 219 & 107 & 75 & 47.5 & 1.0994 & 4.48 & 986 & 340.8 & 7.33 & 531.1 & 7.96 \\
\hline 570 & 187 & 222 & 106 & $7 \overline{4}$ & 46.3 & 1.3614 & 5.85 & 821 & 346.5 & 7.32 & 915.2 & 7.96 \\
\hline 571 & 194 & 226 & 107 & 74 & 45 & 1.5441 & 7.25 & 872 & 334.9 & 7.15 & 726.3 & 8.22 \\
\hline 572 & 190 & 228 & 106 & 77 & 43.9 & 1.7157 & 8.5 & 867 & 326.8 & 7.13 & 635.2 & 8.27 \\
\hline 573 & 190 & 228 & 104 & 76 & 43.5 & 1.9015 & 9.95 & 909 & 318.7 & 7.11 & 498.5 & 8.29 \\
\hline 574 & 186 & 224 & 106 & 76 & 55 & 1.226 & 5.3 & 1009 & 352.6 & 7.28 & 2646 & 8.02 \\
\hline 575 & 190 & 225 & 105 & $7 \overline{8}$ & 54.6 & 1.4427 & 6.62 & 876 & 342.8 & 7.27 & 200 & 8.07 \\
\hline $57 \overline{6}$ & 191 & 228 & 107 & $7 \overline{9}$ & 52.8 & 1.6901 & 8.27 & 800 & 334.9 & 7.14 & 425.1 & 8.26 \\
\hline $57 \overline{7}$ & 195 & 233 & 107 & $7 \overline{8}$ & 50.9 & 1.8784 & 9.69 & $\overline{70} \overline{7}$ & 327.2 & 7.16 & 415.5 & 8.24 \\
\hline 578 & 191 & 233 & 106 & $\overline{79}$ & 50.8 & 2.1512 & 11.75 & 720 & 328 & 7.13 & 505.4 & 8.28 \\
\hline 579 & 179 & 207 & 107 & 78 & 47.7 & 0.252 & 0.84 & 2264 & 644 & 7.96 & 69.7 & 6.99 \\
\hline 580 & 181 & 202 & 106 & 78 & 47.7 & 0.4302 & 1.47 & 1361 & 411.2 & 7.79 & 404.5 & 7.21 \\
\hline 581 & 182 & 204 & 105 & 78 & 46.3 & 0.6056 & 2.25 & 1445 & 384.1 & 7.12 & 209.6 & 8.28 \\
\hline 582 & 185 & 207 & 101 & $\overline{7} \overline{7}$ & 44.4 & 0.761 & 2.97 & 1276 & 358.3 & 7.13 & 341.6 & 8.27 \\
\hline 583 & 189 & 212 & 102 & 77 & 42.5 & 0.9878 & 3.99 & 1085 & 343.4 & 7.17 & 559.5 & 8.21 \\
\hline $58 \overline{4}$ & 187 & 215 & 104 & 77 & 41.3 & 1.2021 & 5.09 & $107 \overline{5}$ & 347.2 & 7.16 & 776.7 & 8.23 \\
\hline 585 & 178 & 204 & 109 & 78 & 39.4 & 0.2293 & 0.68 & 3745 & 595.5 & 7.75 & 77 & 7.26 \\
\hline 586 & 181 & 201 & 103 & 77 & 37.6 & 0.6365 & 2.38 & 1236 & 356.4 & 7.16 & 394.7 & 8.23 \\
\hline $58 \overline{7}$ & 183 & 204 & 105 & 76 & 35.9 & 0.8249 & 3.23 & 1080 & 357.9 & 7.16 & 717.5 & 8.23 \\
\hline 588 & 177 & 198 & 110 & 45 & 33.6 & 0.1943 & 0.42 & 1497 & 559.1 & 9.14 & 304.7 & 5.15 \\
\hline 589 & 182 & 201 & 96 & 43 & 31.5 & 0.6026 & 1.78 & 518 & 242.2 & 8.68 & 3642.4 & 5.74 \\
\hline 590 & 178 & 198 & 106 & 41 & 30.4 & 0.1738 & 0.34 & 1894 & 561.8 & 9.2 & 463.7 & 5 \\
\hline 591 & 181 & 197 & 90 & 40 & 28.6 & 0.5769 & 1.62 & 598 & 280.1 & 8.32 & 1707.1 & 6.43 \\
\hline 592 & 178 & 195 & 108 & 41 & 23.1 & 0.1374 & 0.32 & 3453 & 580.4 & 10.17 & 889 & 3.27 \\
\hline 593 & 181 & 194 & 87 & 42 & 23 & 0.2483 & 0.43 & 905 & 398.8 & 10.31 & 3446 & 2.88 \\
\hline 594 & 181 & 195 & 80 & 42 & 22.3 & 0.3679 & 0.74 & 600 & 247 & 9.3 & 3444.8 & 4.67 \\
\hline 595 & 179 & 202 & 99 & 43 & 21 & 0.5116 & 1.18 & 548 & 163.4 & 9.32 & 4589.8 & 4.55 \\
\hline 596 & 179 & 188 & 123 & 43 & 18.5 & 0.1105 & 0.27 & 3347 & 582.6 & 9.99 & 208.4 & 3.51 \\
\hline 597 & 181 & 189 & 101 & 42 & 18 & 0.1917 & 0.5 & 844 & 299.1 & 10.31 & 2533.7 & 2.82 \\
\hline
\end{tabular}


Recorded Data

\begin{tabular}{|c|c|c|c|c|c|c|c|c|c|c|c|c|}
\hline $\begin{array}{c}\begin{array}{c}\text { Run } \\
\text { Number }\end{array} \\
\end{array}$ & $\begin{array}{c}\text { Methane } \\
(\%)\end{array}$ & $\begin{array}{c}\text { Ethane } \\
(\%)\end{array}$ & $\begin{array}{c}\text { Propane } \\
(\%)\end{array}$ & $\begin{array}{c}\text { Butane } \\
(\%)\end{array}$ & $\mathrm{CO} 2(\%)$ & N2 (\%) & $\begin{array}{c}\begin{array}{c}\text { Air Flow } \\
(\mathrm{lb} / \mathrm{hr})\end{array} \\
\end{array}$ & $\begin{array}{l}\text { Power } \\
\text { (bhp) }\end{array}$ & \begin{tabular}{|c|}
$\begin{array}{c}\text { Humidity } \\
\text { (Ib }\end{array}$ \\
H20/Air) \\
\end{tabular} & $\begin{array}{c}\text { NG Flow } \\
\text { (lb/hr) }\end{array}$ & $\begin{array}{c}\text { Speed } \\
(\mathrm{rpm})\end{array}$ & $\begin{array}{c}\text { Torque (ft- } \\
\text { Ib) }\end{array}$ \\
\hline 598 & 94.29 & 2.67 & 0.52 & 0.3 & 1.04 & 1.19 & 337.16 & 45.9 & 0.0015 & 16.73 & 650 & 370.5 \\
\hline 599 & $\overrightarrow{94} .2 \overline{9}$ & 2.67 & 0.52 & 0.3 & $1 . \overline{04}$ & 1.19 & 487.82 & 65.4 & 0.0015 & 23.52 & 649 & 529.4 \\
\hline 600 & 95.06 & 2.24 & 0.32 & 0.18 & 0.98 & 1.21 & 1701.4 & 168.9 & 0.0019 & 67.08 & 1945 & 456.1 \\
\hline 601 & 95.06 & 2.24 & 0.32 & 0.18 & 0.98 & 1.21 & 1702.7 & 159.6 & 0.0019 & 67.32 & 1944 & 431.2 \\
\hline 602 & 95.06 & 2.24 & 0.32 & 0.18 & 0.98 & 1.21 & 2016.8 & 208.6 & 0.002 & 78.73 & 1948 & 562.5 \\
\hline $60 \overline{3}$ & 95.06 & 2.24 & 0.32 & 0.18 & 0.98 & 1.21 & 2024.5 & 197.1 & 0.002 & 79.57 & 1948 & 531.5 \\
\hline 604 & 95.06 & 2.24 & 0.32 & 0.18 & 0.98 & 1.21 & 2306.4 & 247.3 & 0.0019 & 88.67 & 1944 & 668.3 \\
\hline 605 & 95.06 & $2 . \overline{24}$ & 0.32 & 0.18 & 0.98 & 1.21 & 2310.1 & 239.7 & 0.0019 & 90.09 & 1944 & 647.4 \\
\hline 606 & 95.05 & 2.26 & 0.34 & $0.1 \overline{9}$ & 1.01 & 1.17 & 2587.3 & 285.5 & 0.002 & 100.5 & 1946 & 770.5 \\
\hline 607 & 95.05 & 2.26 & 0.34 & 0.19 & 1.01 & 1.17 & 2605.6 & 269 & 0.0019 & $101: 17$ & 1942 & 727.7 \\
\hline 608 & 95.05 & 2.26 & 0.34 & 0.19 & 1.01 & 1.17 & 2965.5 & 335.7 & 0.002 & 114.37 & 1948 & 905.2 \\
\hline 609 & 95.05 & 2.26 & 0.34 & 0.19 & 1.01 & 1.17 & 2951.1 & 320.2 & 0.0021 & 114.99 & 1948 & 863.4 \\
\hline 610 & 95.05 & 2.26 & 0.34 & 0.19 & 1.01 & 1.17 & 397.98 & 15.4 & 0.0017 & 17.03 & 1726 & 46.7 \\
\hline 611 & 95.05 & 2.26 & 0.34 & 0.19 & 1.01 & 1.17 & 397.68 & 15.4 & 0.0017 & 17.4 & 1674 & 48.3 \\
\hline 612 & 95.15 & 2.2 & 0.33 & 0.18 & 1 & 1.14 & 682.76 & 59.6 & 0.0017 & 29.36 & 1708 & 183.3 \\
\hline 613 & 95.15 & 2.2 & 0.33 & 0.18 & 1 & 1.14 & 689.03 & 58 & 0.0017 & 29.62 & 1705 & 178.7 \\
\hline 614 & 95.15 & 2.2 & 0.33 & 0.18 & 1 & 1.14 & 980.34 & 97.4 & 0.0018 & 39.64 & 1694 & 302 \\
\hline 615 & 95.15 & 2.2 & 0.33 & 0.18 & 1 & 1.14 & 989.67 & 95.1 & 0.0018 & 39.7 & 1692 & 295.1 \\
\hline 616 & 94.18 & 2.83 & 0.55 & 0.33 & 1.28 & 0.83 & 1184.9 & 115.3 & 0.0027 & 46.39 & 1694 & 357.6 \\
\hline 617 & 94.18 & 2.83 & 0.55 & 0.33 & 1.28 & 0.83 & 1213.9 & 115.3 & 0.0028 & 47.68 & 1688 & 358.9 \\
\hline 618 & 94.18 & 2.83 & 0.55 & 0.33 & 1.28 & 0.83 & 1512.6 & 160.1 & 0.0028 & 59.93 & 1696 & 495.8 \\
\hline 619 & 94.18 & 2.83 & 0.55 & 0.33 & 1.28 & 0.83 & 1526.7 & 154.7 & 0.0026 & 60.32 & 1691 & 480.5 \\
\hline 620 & 95.53 & 2.14 & 0.3 & 0.19 & 1.47 & 0.37 & 1841 & 203.7 & 0.0027 & 71.22 & 1697 & 630.5 \\
\hline 621 & 95.53 & 2.14 & 0.3 & 0.19 & 1.47 & 0.37 & 1852.9 & 193.8 & 0.0027 & 71.61 & 1698 & 599.7 \\
\hline 622 & 95.53 & 2.14 & 0.3 & 0.19 & 1.47 & 0.37 & 2118.9 & 239.9 & 0.0028 & 81.42 & 1698 & 742.1 \\
\hline 623 & 95.53 & 2.14 & 0.3 & 0.19 & 1.47 & 0.37 & 2228.3 & 246.1 & 0.0028 & 85.76 & 1696 & 761.8 \\
\hline 624 & 95.53 & 2.14 & 0.3 & 0.19 & 1.47 & 0.37 & 2359.2 & 270.2 & 0.0028 & 90.36 & 1697 & 836.1 \\
\hline 625 & 95.53 & 2.14 & 0.3 & 0.19 & 1.47 & 0.37 & 2377.3 & 258.1 & 0.0028 & 90.83 & 1691 & 801.5 \\
\hline 626 & 95.53 & 2.14 & 0.3 & 0.19 & 1.47 & 0.37 & 2667.5 & 307.1 & 0.003 & 102.47 & 1703 & 947 \\
\hline $62 \overline{7}$ & 95.53 & 2.14 & 0.3 & 0.19 & 1.47 & 0.37 & 2669.9 & 293 & 0.003 & 103.75 & 1698 & 906.3 \\
\hline$\overline{628}$ & 95.48 & 2.14 & 0.26 & 0.16 & 1.54 & 0.43 & 346.28 & 16.6 & 0.0029 & 15.06 & 1504 & 58 \\
\hline 629 & 95.48 & 2.14 & 0.26 & 0.16 & 1.54 & 0.43 & 348.63 & 15.8 & 0.0029 & 15.18 & 1506 & 55 \\
\hline 630 & 95.48 & 2.14 & 0.26 & 0.16 & 1.54 & 0.43 & 604.3 & 56.2 & 0.0029 & 26.32 & 1508 & 195.6 \\
\hline 631 & 95.48 & 2.14 & 0.26 & 0.16 & 1.54 & 0.43 & 613.87 & 54.5 & 0.003 & 26.38 & 1511 & 189.4 \\
\hline
\end{tabular}


Recorded Data

\begin{tabular}{|c|c|c|c|c|c|c|c|c|c|c|c|c|}
\hline $\begin{array}{c}\text { Run } \\
\text { Number }\end{array}$ & Eq Ra & Equiv 02 & $\begin{array}{l}\text { Ign Tim } \\
\text { (deg) }\end{array}$ & BTE (\%) & $\begin{array}{l}\text { MAP } \\
\text { (psia) }\end{array}$ & $\begin{array}{l}\text { Boost AF } \\
\text { IC (deg F) }\end{array}$ & \begin{tabular}{|c|} 
Pre- \\
Turbine \\
(psig)
\end{tabular} & $\mid \begin{array}{c}\text { Comp Out } \\
\text { (psig) }\end{array}$ & $\begin{array}{l}\text { MAP } \\
\text { (psig) }\end{array}$ & $O 2$ (volts) & $\begin{array}{l}\text { Time } \\
\text { (sec) }\end{array}$ & $\begin{array}{c}\text { Date } \\
\text { (julian) }\end{array}$ \\
\hline 598 & 0.811 & 0.783 & 16 & 34.3 & 12.17 & 80 & 0.44 & 0.39 & -2.24 & 3.269 & 42262 & 31 \\
\hline 599 & 0.788 & 0.734 & 16 & 34.8 & 15.03 & 75 & 1.16 & 1.84 & 0.63 & 3.336 & 42731 & 31 \\
\hline 600 & 0.646 & 0.607 & 26 & 31.4 & 18.21 & 105 & 8.76 & 12.28 & 3.83 & 3.504 & 51060 & 31 \\
\hline 601 & 0.648 & 0.607 & 21 & 29.6 & 18.23 & 104 & 8.7 & 12.3 & 3.85 & 3.505 & $51 \overline{434}$ & 31 \\
\hline 602 & 0.64 & 0.602 & 24 & 33.1 & 21.3 & 105 & 10.69 & 14.29 & 6.93 & 3.511 & 52291 & 31 \\
\hline 603 & 0.644 & 0.602 & 19 & 30.9 & 21.32 & 105 & 10.64 & 14.32 & 6.94 & 3.51 & 52567 & 31 \\
\hline 604 & 0.63 & 0.599 & 24.5 & 34.8 & 24.03 & 104 & 12.76 & 16.71 & 9.66 & 3.514 & 53229 & 31 \\
\hline 605 & 0.639 & 0.598 & 19.5 & 33.2 & 24.21 & 105 & 12.76 & 16.76 & $9 . \overline{84}$ & 3.515 & 53692 & 31 \\
\hline 606 & 0.636 & 0.598 & 23 & $3 \overline{5} \overline{5}$ & 27.14 & 110 & 15.19 & 19.99 & 12.77 & 3.516 & 54316 & 31 \\
\hline $6 \overline{7}$ & 0.636 & 0.594 & 18 & 33.2 & 26.92 & 106 & 14.9 & 19.69 & 12.55 & 3.521 & 54793 & 31 \\
\hline 608 & 0.632 & 0.589 & 24 & 36.6 & 30.16 & 107 & 16.14 & 17.77 & 15.79 & 3.528 & 55868 & 31 \\
\hline 609 & 0.638 & 0.589 & 19 & $34 . \overline{8}$ & 30.19 & 108 & 16.15 & 17.84 & 15.82 & 3.528 & 56372 & 31 \\
\hline 610 & 0.701 & 0.677 & 30 & 11.3 & 6.27 & 94 & 0.58 & 0.47 & -8.1 & 3.411 & 57385 & 31 \\
\hline 611 & 0.717 & 0.671 & 25 & 11.1 & 6.38 & 91 & $0 . \overline{56}$ & $0.4 \overline{4}$ & -7.99 & 3.419 & 57655 & 31 \\
\hline 612 & 0.705 & 0.659 & 29 & 25.3 & 9.21 & 81 & 1.12 & 0.92 & -5.17 & 3.436 & 58207 & 31 \\
\hline 613 & 0.705 & $0 . \overline{661}$ & 24 & 24.4 & 9.24 & 77 & 1.14 & 0.99 & -5.14 & 3.433 & 58504 & 31 \\
\hline 614 & 0.663 & 0.626 & 30 & 30.7 & 12.2 & 79 & 1.97 & 2 & -2.18 & 3.48 & 58906 & 31 \\
\hline 615 & 0.658 & 0.623 & 25 & 29.9 & 12.29 & 81 & 2 & 2.11 & -2.09 & 3.483 & 59146 & 31 \\
\hline 616 & 0.64 & 0.612 & 29 & 31.1 & 14.79 & 105 & 5.12 & 7.22 & 0.42 & 3.497 & 32667 & 32 \\
\hline 617 & 0.642 & 0.612 & 24 & 30.3 & 15.19 & 104 & 5.42 & 7.84 & 0.83 & 3.497 & 32959 & 32 \\
\hline 618 & 0.647 & 0.609 & 26 & 33.4 & 18.38 & 105 & 6.96 & 9.64 & 4.02 & 3.501 & 33703 & 32 \\
\hline 619 & 0.646 & 0.608 & 21 & 32.1 & 18.52 & 106 & 7.02 & 9.81 & 4.16 & 3.503 & 33937 & 32 \\
\hline 620 & 0.635 & 0.603 & 24 & 35.7 & 21.51 & 105 & 7.89 & 9.86 & 7.15 & 3.509 & 34539 & 32 \\
\hline 621 & 0.634 & $0 . \overline{606}$ & 19 & 33.8 & 21.53 & 106 & 7.89 & 9.9 & 7.17 & 3.506 & 34924 & 32 \\
\hline 622 & 0.63 & $0 . \overline{601}$ & 24 & 36.7 & 24.43 & 108 & 9.7 & 12.13 & 10.07 & 3.512 & 35378 & 32 \\
\hline 623 & 0.631 & 0.6 & 19 & 35.8 & 25.42 & 106 & 10.37 & 13.07 & 11.06 & 3.513 & 35655 & 32 \\
\hline 624 & 0.628 & 0.598 & 23 & 37.3 & 27.13 & 107 & 11.53 & 14.53 & 12.76 & 3.516 & 36591 & 32 \\
\hline 625 & 0.627 & 0.598 & 18 & 35.4 & 27.14 & 106 & 11.48 & 14.62 & 12.78 & 3.516 & 36790 & 32 \\
\hline 626 & 0.63 & 0.594 & 23 & 37.4 & 30.29 & 109 & 14.16 & 17.65 & 15.92 & 3.521 & 37256 & 32 \\
\hline 627 & 0.638 & 0.592 & 18 & 35.2 & 30.42 & 111 & 14.2 & 17.81 & 16.05 & 3.523 & 37474 & 32 \\
\hline 628 & 0.712 & 0.674 & 30 & 13.8 & 6.37 & 92 & 0.41 & 0.36 & -8 & 3.416 & 39245 & 32 \\
\hline 629 & 0.713 & 0.675 & 25 & 13 & 6.36 & 86 & 0.41 & 0.36 & -8.01 & 3.414 & 39614 & 32 \\
\hline 630 & 0.713 & 0.66 & 29.5 & 26.7 & 9.36 & 80 & 0.81 & 0.71 & -5.01 & 3.434 & 40157 & 32 \\
\hline 631 & 0.703 & 0.659 & 24.5 & 25.8 & 9.35 & 78 & 0.82 & 0.74 & -5.01 & 3.435 & 40548 & 32 \\
\hline
\end{tabular}


Recorded Data

\begin{tabular}{|c|c|c|c|c|c|c|c|c|c|c|c|c|}
\hline $\begin{array}{c}\begin{array}{c}\text { Run } \\
\text { Number }\end{array} \\
\end{array}$ & Year & $\begin{array}{c}\text { BSFC } \\
(\# / b h p-h r)\end{array}$ & $\begin{array}{c}\text { BMEP } \\
\text { (psi) }\end{array}$ & $\begin{array}{l}\text { Vol Eff } \\
(\%)\end{array}$ & $\begin{array}{c}\text { Intake Air } \\
\text { (acfm) }\end{array}$ & $\begin{array}{c}\text { Intake Air } \\
\text { (scfm) }\end{array}$ & $\begin{array}{l}\text { Boost B4 } \\
\text { IC (deg F) }\end{array}$ & $\begin{array}{c}\text { Stack } \\
\text { (deg F) }\end{array}$ & $\begin{array}{c}\text { Pre- } \\
\text { Turbine } \\
\text { (deg F) }\end{array}$ & $\begin{array}{l}\text { Fuel } \\
\text { Press } \\
\text { (psig) }\end{array}$ & EBP $(" \mathrm{Hg})$ & Baro ("Hg) \\
\hline 598 & 1996 & 0.365 & 76.74 & 73.2 & 69 & 75 & 64 & 731 & 851 & 90.2 & 0.12 & 29.33 \\
\hline 599 & 1996 & 0.36 & 109.66 & 84.9 & 100 & 109 & 79 & 786 & 887 & 89.5 & 0.11 & 29.33 \\
\hline 600 & 1996 & 0.397 & 94.48 & 85.1 & 366 & 379 & 195 & 976 & 1124 & 87.3 & 0.81 & 29.27 \\
\hline 601 & 1996 & 0.422 & 89.32 & 85 & 365 & 379 & 194 & 1015 & 1163 & 87.2 & 0.84 & 29.27 \\
\hline 602 & 1996 & $0.37 \overline{7}$ & 116.51 & 86.1 & 440 & 449 & 212 & 998 & 1154 & 85.8 & 1.16 & 29.26 \\
\hline 603 & 1996 & 0.404 & 110.09 & 86.3 & 440 & 451 & 212 & 1039 & 1199 & 85.9 & 1.2 & 29.26 \\
\hline 604 & 1996 & 0.359 & 138.43 & 87.2 & 498 & 513 & 229 & 988 & 1160 & 84.9 & 1.5 & 29.26 \\
\hline 605 & 1996 & 0.376 & 134.1 & 86.9 & 504 & 514 & 233 & 1027 & 1203 & 84.8 & 1.59 & 29.26 \\
\hline 606 & 1996 & 0.352 & 159.62 & 87.4 & 566 & $57 \overline{6}$ & 257 & 997 & 1189 & 83.8 & 1.99 & 29.26 \\
\hline 607 & 1996 & 0.376 & 150.75 & 88.3 & 560 & 580 & 253 & 1047 & 1236 & 84 & 2.07 & 29.26 \\
\hline 608 & 1996 & 0.341 & 187.52 & 89.6 & 643 & 660 & 242 & 1013 & 1185 & 82.6 & 2.63 & 29.26 \\
\hline 609 & $19 \overline{96}$ & 0.359 & 178.86 & 89.3 & 650 & 657 & 245 & 1061 & 1230 & 82.9 & 2.77 & 29.25 \\
\hline 610 & 1996 & 1.109 & 9.68 & 64.2 & 85 & 89 & 76 & 879 & 1032 & 91.1 & 0.06 & 29.25 \\
\hline 611 & 1996 & 1.129 & 10.01 & 64.7 & 85 & 89 & 74 & 881 & 1035 & 91 & 0.06 & 29.26 \\
\hline 612 & 1996 & $0.49 \overline{3}$ & 37.96 & 74 & 145 & 152 & 75 & 912 & 1006 & 89.9 & 0.15 & 29.26 \\
\hline 613 & 1996 & 0.511 & 37.01 & 74.1 & 145 & $15 \overline{3}$ & $\overline{7} \overline{3}$ & 932 & 1030 & $89 . \overline{8}$ & 0.14 & 29.26 \\
\hline 614 & 1996 & 0.407 & 62.57 & 80.3 & 205 & 218 & 83 & 935 & 1017 & 89 & 0.27 & 29.26 \\
\hline 615 & 1996 & 0.417 & 61.13 & 80.9 & 207 & 220 & 84 & 953 & 1041 & 88.9 & 0.27 & 29.26 \\
\hline 616 & 1996 & 0.402 & 74.08 & 83.7 & 255 & 264 & 151 & 923 & 1042 & 88.7 & 0.37 & 29.24 \\
\hline 617 & 1996 & 0.413 & 74.36 & 83.8 & 263 & 270 & 158 & 939 & 1066 & 88.7 & 0.4 & 29.24 \\
\hline 618 & 1996 & 0.374 & 102.7 & 86.1 & 332 & 337 & 176 & 946 & 1077 & $8 \overline{6.9}$ & 0.63 & 29.24 \\
\hline 619 & 1996 & 0.39 & 99.53 & 86.5 & 334 & 340 & 178 & 976 & 1112 & 86.7 & 0.65 & 29.24 \\
\hline 620 & 1996 & 0.35 & 130.62 & 89.4 & 402 & 410 & 175 & 976 & 1102 & 85.8 & 0.94 & 29.25 \\
\hline 621 & $19 \overline{96}$ & 0.369 & 124.22 & 90 & 405 & 412 & 176 & 1015 & 1145 & 85.7 & 0.98 & 29.25 \\
\hline 622 & 1996 & 0.339 & 153.73 & 90.9 & 462 & 472 & 195 & 976 & 1115 & 84.9 & 1.27 & 29.25 \\
\hline 623 & 1996 & 0.349 & 157.82 & 91.6 & 485 & 496 & 198 & 1002 & 1146 & 84.6 & 1.42 & 29.25 \\
\hline $62 \overline{4}$ & 1996 & 0.334 & 173.2 & 91.1 & 516 & 525 & 216 & 985 & 1138 & 84.6 & 1.62 & 29.26 \\
\hline 625 & 1996 & 0.352 & 166.04 & 91.9 & 517 & 529 & 215 & 1021 & 1177 & 84.4 & 1.68 & 29.25 \\
\hline 626 & 1996 & 0.334 & 196.17 & 92.3 & 588 & 594 & 244 & 981 & $115 \overline{3}$ & 83.3 & 2.05 & 29.25 \\
\hline 627 & 1996 & 0.354 & 187.75 & 92.6 & 589 & 594 & 245 & 1021 & 1194 & 83.2 & 2.13 & 29.25 \\
\hline 628 & 1996 & 0.906 & 12.02 & 63 & 75 & 77 & 78 & 813 & 987 & 90.5 & 0.04 & 29.26 \\
\hline 629 & 1996 & 0.963 & 11.39 & 62.8 & 75 & 78 & 78 & 835 & 1007 & 90.3 & 0.03 & 29.26 \\
\hline 630 & 1996 & 0.469 & 40.52 & 73.1 & 130 & 135 & 78 & 871 & 967 & 89.1 & 0.11 & 29.25 \\
\hline 631 & 1996 & 0.484 & 39.23 & 73.9 & 134 & 137 & 80 & 884 & 985 & 89.1 & 0.11 & 29.25 \\
\hline
\end{tabular}


Recorded Data

\begin{tabular}{|c|c|c|c|c|c|c|c|c|c|c|c|c|}
\hline $\begin{array}{c}\text { Run } \\
\text { Number }\end{array}$ & $\mathrm{RH}(\%)$ & Density & Stioch A/F & $\underset{\mathrm{Ra}}{\operatorname{GEM} \mathrm{Eq}}$ & $\begin{array}{c}\text { GEM Vol } \\
\text { Eff }(\%)\end{array}$ & $\begin{array}{l}\text { Exh \#1 } \\
(\operatorname{deg} F)\end{array}$ & $\begin{array}{l}\text { Exh \#2 } \\
(\operatorname{deg} F)\end{array}$ & $\begin{array}{l}\text { Exh \#3 } \\
(\operatorname{deg} F)\end{array}$ & $\begin{array}{l}\text { Exh \#4 } \\
(\operatorname{deg} F)\end{array}$ & $\begin{array}{l}\text { Exh \#5 } \\
(\operatorname{deg} F)\end{array}$ & $\begin{array}{r}\text { Exh \#6 } \\
(\operatorname{deg} F)\end{array}$ & $\begin{array}{c}\text { Water In } \\
\text { (deg F) }\end{array}$ \\
\hline 598 & 30.03 & 0.318 & 16.35 & 0.8 & 70.5 & 817 & 772 & 800 & 800 & 754 & 744 & 79 \\
\hline 599 & 30.24 & 0.315 & 16.35 & 0.75 & 76.1 & 812 & 780 & 800 & 799 & 760 & 745 & 85 \\
\hline 600 & 25.12 & 0.304 & 16.39 & 0.64 & 74.4 & 1077 & 1023 & 1038 & 1026 & 1022 & 1019 & 179 \\
\hline 601 & 25.23 & 0.305 & 16.39 & 0.64 & 74.4 & 1123 & 1063 & 1076 & 1065 & 1055 & 1051 & 174 \\
\hline 602 & 25.36 & 0.306 & 16.39 & 0.64 & 82.1 & 1102 & 1060 & 1067 & 1063 & 1050 & 1043 & 171 \\
\hline 603 & 25.37 & 0.306 & 16.39 & 0.64 & 81.2 & 1145 & 1101 & 1108 & 1106 & 1092 & 1083 & 174 \\
\hline 604 & 24.6 & 0.307 & 16.39 & 0.64 & 81.6 & 1102 & 1070 & 1075 & $10 \overline{2}$ & $10 \overline{58}$ & 1052 & 177 \\
\hline 605 & 24.99 & 0.307 & 16.39 & 0.64 & 81.6 & 1146 & 1112 & $11 \overline{6}$ & 1115 & 1097 & 1088 & 179 \\
\hline 606 & 24.97 & 0.307 & 16.38 & 0.64 & 80.4 & 1128 & 1102 & 1105 & 1104 & 1091 & $107 \overline{7}$ & 185 \\
\hline $60 \overline{7}$ & 25.48 & 0.307 & 16.38 & 0.64 & 80.6 & 1178 & 1149 & $11 \overline{2}$ & 1152 & 1134 & 1117 & 183 \\
\hline 608 & 25.17 & 0.307 & 16.38 & 0.64 & 80.7 & 1126 & 1104 & 1105 & 1106 & 1086 & 1073 & $18 \overline{2}$ \\
\hline $60 \overline{9}$ & 25.14 & 0.309 & 16.38 & 0.64 & 80.7 & 1179 & 1154 & 1154 & 1149 & 1129 & 1114 & 175 \\
\hline 610 & 28.06 & 0.324 & 16.38 & 0.69 & 60.7 & 1021 & 915 & 923 & 908 & 894 & 927 & 117 \\
\hline 611 & 28.29 & 0.321 & 16.38 & 0.69 & 60.7 & 1038 & 926 & 923 & 906 & 902 & 933 & 116 \\
\hline 612 & 28.4 & 0.312 & 16.39 & 0.68 & 69.4 & 993 & 918 & 939 & 922 & 904 & 918 & 135 \\
\hline 613 & 28.02 & 0.31 & 16.39 & 0.68 & $6 \overline{9} .8$ & 1024 & 942 & 961 & 944 & 926 & 941 & 143 \\
\hline 614 & 28.33 & 0.305 & 16.39 & 0.65 & 69.5 & 971 & 912 & 931 & 927 & 903 & 915 & 155 \\
\hline 615 & 28.76 & 0.305 & 16.39 & 0.65 & 69.5 & 999 & 936 & 952 & 948 & 923 & 936 & $15 \overline{7}$ \\
\hline 616 & 50.85 & 0.309 & 16.34 & 0.64 & 74.2 & 1003 & 945 & 960 & 950 & 935 & 940 & 163 \\
\hline 617 & 51.13 & 0.309 & 16.34 & 0.64 & 75 & 1030 & 968 & 983 & 973 & 956 & 960 & 163 \\
\hline 618 & 49.48 & 0.304 & 16.34 & 0.64 & 75 & 1017 & 977 & 990 & 985 & 965 & 964 & 168 \\
\hline 619 & 48.03 & 0.304 & 16.34 & 0.64 & $7 \overline{5}$ & 1056 & 1010 & 1023 & 1020 & 996 & 993 & 170 \\
\hline 620 & 49.25 & 0.299 & 16.41 & 0.64 & 76.2 & 1030 & 1000 & 1007 & 1014 & 994 & 979 & 175 \\
\hline 621 & 49.07 & 0.301 & 16.41 & 0.64 & 76.2 & 1073 & 1038 & 1044 & 1058 & 1033 & 1011 & 175 \\
\hline 622 & 50.5 & 0.301 & 16.41 & 0.64 & 80 & 1043 & 1019 & 1022 & 1030 & 1011 & 991 & 163 \\
\hline 623 & 51.33 & 0.302 & 16.41 & 0.64 & 80 & 1074 & 1047 & 1050 & 1059 & 1039 & 1015 & 163 \\
\hline 624 & 51.85 & 0.307 & 16.41 & 0.64 & 82 & 1070 & 1047 & 1048 & 1057 & 1036 & 1014 & 167 \\
\hline 625 & 52.26 & 0.307 & 16.41 & 0.64 & 82 & 1112 & 1084 & 1086 & 1094 & 1070 & 1048 & 168 \\
\hline 626 & 53.14 & 0.304 & 16.41 & 0.64 & 81 & 1087 & 1073 & 1068 & 1072 & 1054 & 1036 & $17 \overline{3}$ \\
\hline 627 & 52.44 & 0.304 & 16.41 & 0.64 & 81 & 1136 & 1116 & 1113 & 1112 & 1093 & 1071 & 173 \\
\hline 628 & 56.06 & 0.316 & 16.36 & 0.69 & 60.9 & 960 & 866 & 862 & 874 & 849 & 879 & 89 \\
\hline 629 & 55.89 & 0.313 & 16.36 & 0.69 & 60.9 & 986 & 890 & 896 & 888 & 864 & 895 & 90 \\
\hline 630 & 54 & 0.306 & 16.36 & 0.69 & 69.1 & 944 & 884 & 904 & 893 & 868 & 883 & 101 \\
\hline 631 & 54.87 & 0.304 & 16.36 & 0.68 & 67.5 & 972 & 906 & 926 & 910 & 885 & 898 & 103 \\
\hline
\end{tabular}




\begin{tabular}{|c|c|c|c|c|c|c|c|c|c|c|c|c|}
\hline $\begin{array}{c}\text { Run } \\
\text { Number }\end{array}$ & $\mid \begin{array}{c}\text { Water Out } \\
\text { (deg F) }\end{array}$ & $\begin{array}{c}\text { Oil Sump } \\
\text { (deg F) }\end{array}$ & $\begin{array}{c}\text { Oil } \\
\text { Gallery } \\
\text { (deg } F \text { ) }\end{array}$ & $\begin{array}{c}\text { Intake @ } \\
\text { LFE (deg } \\
\text { F) } \\
\end{array}$ & $\begin{array}{l}\text { Oil Press } \\
\text { (psig) }\end{array}$ & \begin{tabular}{|c|} 
Fuel Flow \\
(@ RT \\
Meter) \\
\end{tabular} & $\begin{array}{c}\text { Inlet Rest } \\
(" \mathrm{H} 2 \mathrm{O})\end{array}$ & $\mathrm{HC}(\mathrm{ppm})$ & $\mathrm{CO}(\mathrm{ppm})$ & $\mathrm{CO} 2(\%)$ & $\begin{array}{c}\text { NOx } \\
(\mathrm{ppm})\end{array}$ & $\mathrm{O} 2(\%)$ \\
\hline 598 & 180 & 191 & 89 & 41 & 17.9 & 0.2793 & 0.61 & 668 & 255.9 & 9.27 & 2653.6 & 4.69 \\
\hline 599 & 181 & 194 & 83 & 41 & 17.3 & 0.3971 & 0.9 & 633 & 225.1 & 8.69 & 4106.7 & 5.68 \\
\hline 600 & 189 & 221 & 102 & 53 & 56.3 & 1.1728 & 5.06 & 1015 & 353.8 & 7.16 & 267.5 & 8.27 \\
\hline 601 & 185 & 222 & 102 & 52 & 56.6 & 1.173 & 5.1 & 1005 & 362.8 & 7.16 & 120.7 & 8.27 \\
\hline 602 & $18 \overline{5}$ & 223 & 102 & 56 & 55.8 & 1.3684 & 6.52 & 882 & 340.9 & 7.16 & 243.4 & 8.27 \\
\hline 603 & 185 & 224 & 102 & 55 & 55.7 & 1.3801 & 6.53 & 861 & 344.2 & 7.16 & 123.6 & 8.27 \\
\hline 604 & 188 & 227 & 101 & 53 & 54.1 & 1.534 & 7.8 & 755 & 329.6 & 7.16 & 360.3 & 8.27 \\
\hline 605 & 189 & 228 & 102 & 56 & 53.9 & 1.5592 & 8.02 & 745 & 322.4 & 7.18 & $18 \overline{2} .7$ & 8.24 \\
\hline 606 & 194 & 232 & 105 & 57 & 52 & 1.7388 & 9.6 & 636 & 319.2 & 7.18 & 383.8 & 8.24 \\
\hline 607 & 192 & 231 & 102 & 52 & 52.5 & 1.7495 & 9.45 & 656 & 308.8 & 7.18 & 171.3 & 8.25 \\
\hline 608 & 191 & 235 & 102 & $5 \overline{4}$ & 50.8 & 1.9796 & 11.57 & 671 & 314.5 & 7.15 & 580.3 & 8.28 \\
\hline 609 & 187 & 232 & 103 & 59 & 52.2 & 1.9791 & 11.7 & 694 & 308.6 & 7.17 & 245.7 & 8.32 \\
\hline 610 & 178 & 202 & 98 & 51 & 57.8 & 0.2792 & 0.91 & 1948 & 699.8 & 7.89 & 58.7 & 7.24 \\
\hline 611 & 178 & 201 & 96 & 50 & 56.1 & 0.2877 & 0.91 & 1910 & 706.8 & 7.8 & 43 & 7.38 \\
\hline 612 & 181 & 205 & 87 & 49 & 54.9 & 0.4999 & 1.62 & 1308 & 431.6 & 7.73 & 246.9 & 7.43 \\
\hline 613 & 181 & 206 & 84 & 47 & 54.6 & 0.5083 & 1.65 & 1251 & 417.8 & 7.73 & 130.5 & 7.42 \\
\hline 614 & 182 & 208 & 83 & 45 & 52.9 & 0.6897 & 2.46 & 1224 & 366.3 & 7.32 & 286.5 & 8.07 \\
\hline 615 & 182 & 209 & 84 & 45 & 52.5 & 0.6921 & 2.49 & 1220 & 372.3 & 7.31 & 149.3 & 8.09 \\
\hline 616 & 181 & 210 & 103 & 52 & 50.6 & 0.7989 & 3.22 & 1323 & 371.2 & 7.18 & 236.9 & 8.35 \\
\hline $61 \overline{7}$ & 182 & 210 & 103 & 5 & 50.4 & 0.8211 & 3.36 & 1291 & 378 & 7.17 & 129.9 & 8.36 \\
\hline 618 & 185 & 215 & 103 & 58 & 48.4 & 1.0466 & 4.51 & 1067 & 349.2 & 7.19 & 298 & 8.34 \\
\hline $61 \overline{9}$ & 185 & 215 & 103 & 57 & 48.3 & 1.0546 & 4.58 & 1038 & 349.6 & 7.19 & 143.5 & 8.33 \\
\hline 620 & 187 & 219 & 103 & 56 & 46.8 & 1.2637 & 5.83 & 933 & 338.3 & 7.18 & 380.2 & 8.34 \\
\hline 621 & 187 & 219 & 103 & 57 & 47 & 1.2629 & 5.91 & 928 & 333.2 & 7.19 & 166.6 & 8.35 \\
\hline 622 & 182 & 219 & 104 & 56 & 46.4 & 1.4359 & 7.1 & 929 & 333.4 & 7.17 & 487 & 8.37 \\
\hline 623 & 182 & 218 & 102 & 55 & 46.5 & 1.5103 & 7.63 & 949 & 322.1 & 7.17 & 212.2 & 8.37 \\
\hline 624 & 185 & 221 & 103 & 57 & 45.2 & 1.5643 & 8.31 & 943 & 323.1 & 7.17 & 452.7 & 8.37 \\
\hline 625 & 186 & 221 & 102 & $5 \overline{5}$ & 45.1 & 1.5729 & 8.38 & 1020 & 313.2 & 7.16 & 199.6 & 8.39 \\
\hline 626 & 187 & 224 & 104 & 59 & 44.2 & 1.7889 & 10.08 & 862 & 317.5 & 7.16 & 510.5 & 8.39 \\
\hline 627 & 187 & 225 & 106 & 59 & 44 & 1.8123 & 10.07 & 1055 & 306.3 & 7.15 & 229.7 & 8.41 \\
\hline 628 & 180 & 198 & 99 & 53 & 49.5 & 0.253 & 0.84 & 3096 & 694.5 & 7.63 & 43.2 & 7.67 \\
\hline 629 & 180 & 198 & 95 & 53 & 49.6 & 0.2574 & 0.85 & 2080 & 712.2 & 7.79 & 33 & 7.43 \\
\hline 630 & 181 & 201 & 87 & 53 & 47.8 & 0.4571 & 1.49 & 1391 & 427.8 & 7.71 & 260.1 & 7.45 \\
\hline 631 & 181 & 201 & 85 & 56 & 47.8 & 0.4609 & 1.52 & 1371 & 427.7 & 7.69 & 130.1 & 7.49 \\
\hline
\end{tabular}


Recorded Data

\begin{tabular}{|c|c|c|c|c|c|c|c|c|c|c|c|c|}
\hline $\begin{array}{c}\text { Run } \\
\text { Number }\end{array}$ & $\begin{array}{c}\text { Methane } \\
(\%)\end{array}$ & $\begin{array}{c}\text { Ethane } \\
(\%)\end{array}$ & $\begin{array}{c}\text { Propane } \\
(\%)\end{array}$ & $\begin{array}{c}\text { Butane } \\
(\%)\end{array}$ & $\mathrm{CO} 2(\%)$ & N2 (\%) & $\begin{array}{l}\text { Air Flow } \\
\text { (lb/hr) }\end{array}$ & $\begin{array}{c}\text { Power } \\
\text { (bhp) }\end{array}$ & $\begin{array}{c}\text { Humidity } \\
\text { (lb } \\
\text { H20/Air) } \\
\end{array}$ & $\begin{array}{c}\text { NG Flow } \\
\text { (lb/hr) }\end{array}$ & $\begin{array}{c}\text { Speed } \\
\text { (rpm) }\end{array}$ & $\begin{array}{c}\text { Torque (ft- } \\
\text { (b) }\end{array}$ \\
\hline 632 & 95.48 & 2.14 & 0.26 & 0.16 & 1.54 & 0.43 & 854.63 & 88.3 & 0.003 & 35.03 & $\overline{1494}$ & 310.3 \\
\hline 633 & 95.48 & 2.14 & 0.26 & 0.16 & 1.54 & 0.43 & 862.31 & 85.9 & 0.003 & 34.96 & 1492 & 302.5 \\
\hline 634 & 95.25 & 2.24 & 0.29 & 0.17 & 1.62 & 0.43 & 1052.9 & 108.4 & 0.0029 & 40.92 & 1504 & 378.5 \\
\hline 635 & 95.25 & 2.24 & 0.29 & 0.17 & 1.62 & 0.43 & 1082.2 & 107.6 & 0.0029 & 41.66 & $15 \overline{02}$ & 376.4 \\
\hline 636 & 95.25 & 2.24 & 0.29 & 0.17 & 1.62 & 0.43 & 1352.9 & 151.5 & 0.0028 & 53.06 & 1496 & 532 \\
\hline 637 & 95.25 & 2.24 & 0.29 & 0.17 & 1.62 & 0.43 & 1361 & 145.8 & 0.0029 & 53.21 & 1496 & 511.9 \\
\hline 638 & 95.13 & 2.32 & 0.33 & 0.19 & 1.6 & 0.43 & 1623.9 & 188 & 0.003 & 63.57 & 1499 & 658.5 \\
\hline 639 & 95.13 & 2.32 & 0.33 & 0.19 & 1.6 & 0.43 & 1636.5 & 180.8 & 0.003 & 63.67 & 1498 & 633.9 \\
\hline 640 & 95.13 & 2.32 & 0.33 & 0.19 & 1.6 & 0.43 & 2140.1 & 251.2 & 0.0031 & 80.71 & 1505 & 876.6 \\
\hline 641 & $9 \overline{5} .1 \overline{3}$ & 2.32 & 0.33 & 0.07 & 1.6 & 0.43 & 2130.6 & 241 & 0.0031 & 81.06 & $150 \overline{0}$ & 840.4 \\
\hline 642 & 94.85 & 2.56 & 0.36 & 0.21 & 1.61 & 0.41 & 2627.5 & 307.9 & 0.0031 & 100.7 & 1492 & 1083.7 \\
\hline 643 & 94.85 & 2.56 & 0.36 & 0.21 & 161 & 0.41 & 2638.4 & 287.6 & 0.0031 & 100.89 & $1 \overline{4} \overline{8} \overline{7}$ & 1015.7 \\
\hline 644 & $\overline{9} 4.85$ & 2.56 & 0.36 & $0 . \overline{2} i$ & $1 . \overline{6} 1$ & 0.41 & 262.31 & 12.8 & 0.0031 & 11.75 & 1250 & 53.6 \\
\hline 645 & 94.85 & 2.56 & $0 . \overline{36}$ & 0.21 & 1.61 & 0.41 & 257.85 & 12.7 & 0.0031 & 12.06 & 1239 & $5 \overline{3.7}$ \\
\hline 646 & 94.85 & 2.56 & 0.36 & 0.21 & 1.61 & 0.41 & 887.44 & 97.6 & 0.003 & 35.86 & 1248 & 410.7 \\
\hline 647 & 94.85 & 2.56 & 0.36 & 0.21 & 1.61 & 0.41 & 906.73 & $96 . \overline{6}$ & 0.003 & 36.42 & 1249 & 406.4 \\
\hline 648 & 94.85 & 2.56 & 0.36 & 0.21 & 1.61 & 0.41 & 1132.6 & 131.5 & 0.0031 & 44.87 & 1243 & $5 \overline{55.6}$ \\
\hline 649 & 94.85 & 2.56 & 0.36 & 0.21 & 1.61 & 0.41 & 1148.4 & 127.2 & 0.0031 & 45.06 & 1260 & 530.3 \\
\hline 650 & 94.91 & 2.53 & 0.36 & 0.2 & 1.56 & 0.45 & 230.55 & 18.8 & 0.003 & 12.97 & 1121 & 88.1 \\
\hline 651 & 94.91 & 2.53 & 0.36 & 0.2 & 1.56 & 0.45 & 230.97 & 18.6 & 0.0029 & 13.08 & 1122 & 87.1 \\
\hline 652 & 94.91 & 2.53 & 0.36 & $0 . \overline{2}$ & 1.56 & 0.45 & 774.06 & 97.2 & 0.0031 & 34.24 & 1108 & 461 \\
\hline 653 & 94.91 & 2.53 & 0.36 & 0.2 & 1.56 & 0.45 & 794.02 & 97.6 & 0.003 & 34.53 & 1109 & 461.9 \\
\hline 654 & 94.91 & 2.53 & 0.36 & 0.2 & 1.56 & 0.45 & 210.82 & 16.2 & 0.003 & 9.89 & 999 & 85.2 \\
\hline 655 & 94.91 & 2.53 & 0.36 & 0.2 & 1.56 & 0.45 & 211.23 & 15.9 & 0.003 & 9.86 & 991 & 84.3 \\
\hline 656 & 94.91 & 2.53 & 0.36 & 0.2 & 1.56 & 0.45 & 707.96 & 91.6 & 0.003 & 33.62 & 1008 & 477.6 \\
\hline 657 & 94.91 & 2.53 & 0.36 & 0.2 & 1.56 & 0.45 & 713.23 & 91.3 & 0.003 & 33.82 & 1007 & 476.4 \\
\hline 658 & 94.86 & 2.53 & 0.36 & 0.2 & 1.53 & 0.52 & 149.9 & 12.7 & 0.003 & 7.66 & 799 & 83.2 \\
\hline $65 \overline{9}$ & 94.86 & 2.53 & 0.36 & 0.2 & 1.53 & 0.52 & 149.32 & 12.6 & 0.003 & 7.64 & 797 & 83.3 \\
\hline 660 & 94.86 & 2.53 & 0.36 & 0.2 & 1.53 & 0.52 & 280.42 & 34.9 & 0.0029 & 13.5 & 794 & 230.6 \\
\hline 661 & 94.86 & 2.53 & 0.36 & 0.2 & 1.53 & 0.52 & 279.58 & 35.1 & 0.0029 & 13.55 & 796 & 231.7 \\
\hline 662 & 94.86 & 2.53 & 0.36 & 0.2 & 1.53 & 0.52 & 424.94 & 55.7 & 0.003 & 20.45 & 800 & 365.7 \\
\hline $66 \overline{3}$ & 94.86 & 2.53 & 0.36 & 0.2 & 1.53 & 0.52 & 429.01 & 55.9 & 0.003 & 20.51 & 800 & 366.8 \\
\hline 664 & 94.84 & 2.52 & 0.39 & 0.22 & $1 . \overline{45}$ & 0.58 & 581.5 & 75.7 & 0.003 & 27.93 & 799 & 498 \\
\hline 665 & 94.84 & $2.5 \overline{2}$ & 0.39 & 0.22 & 1.45 & 0.58 & 588.22 & 74.9 & 0.003 & 28.39 & 798 & 492.9 \\
\hline
\end{tabular}




\begin{tabular}{|c|c|c|c|c|c|c|c|c|c|c|c|c|}
\hline $\begin{array}{c}\text { Run } \\
\text { Number }\end{array}$ & Eq Ra & Equiv $\mathrm{O} 2$ & $\begin{array}{c}\text { Ign Tim } \\
\text { (deg) }\end{array}$ & BTE (\%) & $\begin{array}{r}\text { MAP } \\
\text { (psia) }\end{array}$ & $\begin{array}{l}\text { Boost AF } \\
\text { IC (deg F) }\end{array}$ & $\begin{array}{c}\text { Pre- } \\
\text { Turbine } \\
\text { (psig) }\end{array}$ & $\mid \begin{array}{c}\text { Comp Out } \\
\text { (psig) }\end{array}$ & $\begin{array}{l}\text { MAP } \\
\text { (psig) }\end{array}$ & $O 2$ (volts) & $\begin{array}{l}\text { Time } \\
\text { (sec) }\end{array}$ & $\begin{array}{c}\text { Date } \\
\text { (julian) }\end{array}$ \\
\hline 632 & 0.671 & 0.627 & 29.5 & 31.5 & 12.3 & 83 & 1.44 & 1.52 & -2.06 & 3.478 & 41024 & 32 \\
\hline 633 & 0.663 & 0.626 & 24.5 & 30.7 & 12.36 & 85 & 1.48 & 1.61 & -2.01 & 3.479 & 41254 & 32 \\
\hline 634 & 0.635 & 0.613 & 28 & 33.2 & 14.8 & 105 & 3.93 & 5.86 & 0.44 & 3.496 & 41901 & 32 \\
\hline 635 & 0.629 & 0.612 & 23 & 32.4 & 15.17 & 104 & 4.14 & 6.42 & 0.81 & 3.497 & 42167 & 32 \\
\hline 636 & 0.64 & 0.61 & 26 & 35.8 & 18.26 & 103 & 4.9 & 6.9 & 3.9 & 3.5 & 42584 & 32 \\
\hline 637 & 0.638 & 0.611 & 21 & 34.3 & 18.3 & 104 & 4.89 & 6.95 & 3.93 & 3.499 & 42791 & 32 \\
\hline 638 & 0.639 & 0.61 & 24 & 37 & 21.33 & 105 & 6.34 & 9.14 & 6.99 & 3.5 & 47124 & 32 \\
\hline 639 & 0636 & 0.609 & 19 & 35.6 & 21.36 & 105 & 6.36 & 9.19 & 7.01 & 3.502 & 47661 & 32 \\
\hline 640 & 0.616 & 0.601 & 23 & 39 & 27.27 & 108 & 9.86 & 14.36 & 12.92 & 3513 & 48167 & 32 \\
\hline 641 & 0621 & 0.602 & 23 & 37.2 & 27.1 & 107 & 9.72 & 14.21 & 12.75 & 3.51 & 48517 & 32 \\
\hline 642 & 0.626 & 0.595 & 20 & 38.3 & 33.42 & 110 & 14.32 & 20.56 & 19.08 & 3.52 & 49105 & 32 \\
\hline 643 & 0.624 & 0.594 & 15 & $3 \overline{5} . \overline{7}$ & 33.3 & 110 & 14.25 & 20.43 & 18.96 & 3.521 & 49255 & $3 \overline{2}$ \\
\hline 644 & 0.731 & 0.671 & 29.5 & 13.6 & 6.31 & 100 & 0.3 & 0.25 & -8.03 & 3.419 & $4977 \overline{1}$ & $3 \overline{2}$ \\
\hline 645 & 0.763 & 0.681 & 24.5 & 13.2 & 6.33 & 100 & 0.3 & 0.24 & -8.01 & 3.406 & 49969 & 32 \\
\hline 646 & 0.66 & 0.615 & 26 & 34.1 & 15.07 & 102 & 3 & 4.22 & 0.74 & 3.493 & 50835 & 32 \\
\hline 647 & 0.656 & 0.615 & 20.5 & 33.2 & 15.35 & 101 & 3.17 & 4.6 & 1.01 & 3.493 & 51003 & 32 \\
\hline 648 & 0.647 & 0.616 & 24 & 36.7 & 18.26 & 101 & 4.5 & 6.69 & 3.92 & 3.492 & 51292 & 32 \\
\hline 649 & 0.641 & 0.613 & 19 & 35.4 & 18.23 & 100 & 4.68 & 7.14 & 3.9 & 3.497 & 51604 & 32 \\
\hline 650 & 0.919 & 0.772 & 29.5 & 18.2 & 6.32 & 97 & 0.24 & 0.19 & -8.01 & 3.285 & 52562 & 32 \\
\hline 651 & 0.926 & 0.77 & 24.5 & 17.8 & 6.3 & 94 & 0.24 & 0.19 & -8.03 & 3.287 & 52832 & 32 \\
\hline 652 & 0.723 & 0.679 & 24.5 & 35.6 & 15 & 103 & 2.52 & 3.68 & 0.66 & 3.41 & 53675 & 32 \\
\hline 653 & 0.711 & 0.678 & 19.5 & 35.4 & 15.15 & 104 & 2.61 & 3.9 & 0.82 & 3.41 & 53960 & 32 \\
\hline $6 \overline{54}$ & 0.767 & 0.736 & 29.5 & 20.5 & 6.44 & 95 & 0.19 & 0.17 & -7.89 & 3.333 & 54519 & 32 \\
\hline 655 & 0.763 & 0.737 & 24.5 & 20.2 & 6.46 & 93 & 0.19 & 0.16 & -7.87 & 3.331 & 54814 & 32 \\
\hline 656 & 0.776 & 0.7 & 23.5 & 34.1 & 15 & 97 & 2.15 & 3.18 & 0.67 & 3.381 & 55645 & 32 \\
\hline 657 & 0.775 & 0.7 & 18.5 & 33.8 & 15.08 & 101 & 2.19 & 3.28 & 0.75 & 3.381 & 55908 & 32 \\
\hline 658 & 0.834 & 0.782 & 29 & 20.7 & 6.31 & 97 & 0.12 & 0.13 & -8.02 & 3.271 & 56633 & 32 \\
\hline 659 & 0.835 & 0.787 & 24 & 20.7 & 6.29 & 93 & 0.12 & 0.13 & -8.04 & 3.264 & 56946 & 32 \\
\hline 660 & 0.786 & 0.788 & 26 & 32.4 & 9.27 & 81 & 0.23 & 0.16 & -5.07 & 3.262 & 57939 & 32 \\
\hline 661 & 0.792 & 0.785 & 21 & 32.4 & 9.27 & 79 & 0.23 & 0.17 & -5.07 & 3.266 & 58195 & 32 \\
\hline 662 & 0.786 & 0.735 & 24 & 34.1 & 12.24 & 77 & 0.48 & 0.35 & -2.1 & 3.333 & 58656 & 32 \\
\hline 663 & 0.781 & 0.736 & 19 & 34.1 & 12.24 & 77 & 0.49 & 0.37 & -2.09 & 3.333 & 58942 & 32 \\
\hline 664 & 0.785 & 0.702 & 16 & 33.9 & 15.05 & 90 & 1.56 & 2.12 & 0.72 & 3.379 & 59686 & 32 \\
\hline 665 & 0.789 & 0.702 & 11 & 33 & 15.21 & 94 & 1.64 & 2.31 & 0.88 & 3.378 & 60009 & 32 \\
\hline
\end{tabular}




\begin{tabular}{|c|c|c|c|c|c|c|c|c|c|c|c|c|}
\hline $\begin{array}{c}\begin{array}{c}\text { Run } \\
\text { Number }\end{array} \\
\end{array}$ & Year & $\begin{array}{c}\text { BSFC } \\
(\# / b h p-h r)\end{array}$ & $\begin{array}{c}\text { BMEP } \\
\text { (psi) }\end{array}$ & $\begin{array}{c}\text { Vol Eff } \\
(\%)\end{array}$ & $\begin{array}{c}\text { Intake Air } \\
\text { (acfm) }\end{array}$ & $\begin{array}{c}\text { Intake Air } \\
\text { (scfm) }\end{array}$ & $\begin{array}{c}\text { Boost B4 } \\
\text { IC (deg F) }\end{array}$ & $\begin{array}{c}\text { Stack } \\
\text { (deg F) }\end{array}$ & $\begin{array}{c}\text { Pre- } \\
\text { Turbine } \\
\text { (deg F) }\end{array}$ & $\begin{array}{l}\text { Fuel } \\
\text { Press } \\
\text { (psig) }\end{array}$ & EBP $(" \mathrm{Hg})$ & Baro ("Hg) \\
\hline 632 & 1996 & 0.397 & 64.27 & 79.5 & 185 & 190 & 87 & 901 & 985 & 88.4 & 0.21 & 29.25 \\
\hline 633 & 1996 & 0.407 & 62.66 & 80.1 & 186 & 192 & 88 & 919 & 1008 & 88.4 & 0.21 & 29.25 \\
\hline 634 & 1996 & 0.377 & 78.41 & 83.8 & 228 & 234 & 140 & 899 & 1012 & 88.5 & 0.3 & 29.25 \\
\hline 635 & 1996 & 0.387 & 77.98 & 84 & 237 & 241 & 147 & 921 & 1042 & 88.3 & 0.32 & 29.25 \\
\hline 636 & 1996 & 0.35 & 110.2 & 87.5 & 294 & 301 & 147 & 926 & 1035 & 87.6 & 0.5 & 29.24 \\
\hline 637 & 1996 & 0.365 & 106.04 & 88 & 293 & 303 & 145 & 957 & 1074 & 87.6 & 0.51 & 29.24 \\
\hline 638 & 1996 & 0.338 & 136.4 & 90.1 & 353 & 361 & 168 & 943 & 1065 & 86.9 & 0.72 & 29.21 \\
\hline 639 & 1996 & 0.352 & 1313 & 90.7 & 359 & 364 & 171 & 978 & 1103 & 87 & 0.74 & 29.21 \\
\hline 640 & 1996 & 0.321 & 181.59 & 928 & 465 & 476 & 215 & 949 & 1102 & 85.5 & 1.26 & 29.21 \\
\hline $64 \overline{1}$ & 1996 & 0.336 & 174.09 & $92 . \overline{7}$ & 466 & 474 & 214 & $98 \overline{7}$ & 1139 & 85.6 & 1.3 & 29.21 \\
\hline 642 & 1996 & 0.327 & 224.48 & 94 & 576 & 585 & 267 & 955 & 1145 & 84 & 1.96 & 29.2 \\
\hline $64 \overline{3}$ & 1996 & 0.351 & 210.4 & 95.1 & 579 & 587 & 265 & 1000 & 1190 & 84.1 & 2.06 & 29.2 \\
\hline $64 \overline{4}$ & 1996 & 0.921 & 11.11 & 59 & 57 & 58 & 91 & 795 & 942 & 91.8 & 0.03 & 29.21 \\
\hline 645 & 1996 & 0.951 & 11.13 & 58.4 & 56 & 57 & 86 & 775 & 944 & 91.6 & 0.03 & 29.2 \\
\hline 646 & 1996 & 0.367 & 85.07 & 83.3 & 190 & 198 & 118 & $86 \overline{3}$ & 965 & 89.8 & 0.26 & 29.19 \\
\hline $64 \overline{7}$ & 1996 & 0.377 & 84.18 & 83.4 & 194 & 202 & 121 & 877 & 990 & 89.8 & 0.27 & 29.19 \\
\hline 648 & 1996 & 0.341 & 115.09 & 88 & 242 & 252 & 143 & 876 & 990 & 89.2 & 0.38 & 29.18 \\
\hline 649 & 1996 & 0.354 & 109.85 & 87.8 & 246 & 256 & 150 & 904 & 1026 & 89.2 & 0.4 & 29.18 \\
\hline 650 & 1996 & 0.69 & 18.25 & 58.4 & 50 & 51 & 79 & 743 & 915 & 91 & 0.02 & 29.18 \\
\hline 651 & 1996 & 0.703 & 18.04 & 58.3 & 50 & 51 & 78 & 745 & 925 & 90.9 & 0.03 & 29.17 \\
\hline 652 & 1996 & 0.352 & $9 \overline{5} . \overline{49}$ & 83 & 167 & 172 & 113 & $87 \overline{1}$ & 972 & 88.7 & 0.19 & 29.18 \\
\hline 653 & 1996 & 0.354 & 95.67 & 84.1 & 170 & 177 & 114 & 887 & 994 & 88.6 & 0.19 & 29.18 \\
\hline 654 & 1996 & 0.61 & 17.64 & 57.8 & 45 & 47 & 79 & 717 & 883 & 91 & 0.02 & 29.18 \\
\hline 655 & 1996 & 0.62 & 17.47 & 57.9 & 45 & 47 & 78 & 710 & 885 & 90.9 & 0.01 & 29.17 \\
\hline 656 & 1996 & 0.367 & 98.93 & 82.9 & 152 & 158 & 107 & 859 & 962 & 88.7 & 0.15 & 29.19 \\
\hline 657 & 1996 & 0.37 & 98.68 & 83.7 & 154 & 159 & 110 & 876 & 982 & 88.7 & 0.15 & 29.19 \\
\hline 658 & 1996 & 0.605 & 17.24 & 52.9 & 33 & 33 & 83 & 642 & 801 & 91.2 & 0.01 & 29.19 \\
\hline 659 & 1996 & 0.604 & 17.26 & 52.6 & 33 & 33 & 82 & 628 & 802 & 91.1 & 0.01 & 29.18 \\
\hline 660 & 1996 & 0.387 & 47.77 & 65.6 & 62 & 62 & 77 & 718 & 830 & 89.8 & 0.04 & 29.19 \\
\hline 661 & 1996 & 0.386 & 47.99 & 65.1 & 62 & 62 & 77 & 726 & 840 & 89.9 & 0.05 & 29.18 \\
\hline 662 & 1996 & 0.367 & 75.75 & 74.2 & 93 & 95 & 77 & 775 & 874 & 89.1 & 0.13 & 29.18 \\
\hline 663 & 1996 & 0.367 & 75.99 & 74.8 & 94 & 96 & 77 & 789 & 890 & 89.4 & 0.15 & 29.18 \\
\hline 664 & 1996 & 0.369 & 103.16 & 84.7 & 128 & 129 & 100 & 839 & 931 & 88.8 & 0.21 & 29.17 \\
\hline 665 & 1996 & 0.379 & 102.11 & 85.5 & 129 & 131 & 102 & 865 & 962 & 89 & 0.22 & 29.18 \\
\hline
\end{tabular}


Recorded Data

\begin{tabular}{|c|c|c|c|c|c|c|c|c|c|c|c|c|}
\hline $\begin{array}{c}\text { Run } \\
\text { Number }\end{array}$ & $\mathrm{RH}(\%)$ & Density & Stioch A/F & $\begin{array}{c}\text { GEM Eq } \\
\qquad a\end{array}$ & $\begin{array}{c}\text { GEM Vol } \\
\text { Eff }(\%)\end{array}$ & $\begin{array}{l}\text { Exh \#1 } \\
(\operatorname{deg} F)\end{array}$ & $\begin{array}{l}\text { Exh \#2 } \\
(\operatorname{deg} F)\end{array}$ & $\begin{array}{l}\text { Exh \#3 } \\
(\operatorname{deg} F)\end{array}$ & $\begin{array}{l}\text { Exh \#4 } \\
(\operatorname{deg} F)\end{array}$ & $\begin{array}{l}\text { Exh \#5 } \\
\text { (deg F) }\end{array}$ & $\begin{array}{l}\text { Exh \#6 } \\
(\operatorname{deg} F)\end{array}$ & $\begin{array}{l}\text { Water In } \\
\text { (deg F) }\end{array}$ \\
\hline 632 & 53.2 & 0.3 & 16.36 & 0.65 & 66.8 & 932 & 886 & 901 & 898 & 872 & 876 & 111 \\
\hline 633 & 53.22 & $0 . \overline{3}$ & 16.36 & 0.65 & 67 & 960 & 907 & 922 & 919 & 891 & 897 & 114 \\
\hline 634 & 49.52 & 0.303 & 16.33 & 0.64 & 66.2 & 961 & 911 & 925 & 922 & 902 & 905 & 127 \\
\hline 635 & 49.26 & 0.303 & 16.33 & 0.64 & 66.9 & 993 & 941 & $9 \overline{54}$ & 950 & 928 & 931 & 131 \\
\hline 636 & 51.67 & 0.302 & 16.33 & 0.64 & 77.2 & 964 & 932 & 941 & 943 & 921 & 917 & 141 \\
\hline 637 & 53.43 & 0.303 & 16.33 & 0.64 & 77.7 & 996 & 959 & 970 & 979 & 955 & 960 & 144 \\
\hline 638 & 5468 & 0.308 & 16.33 & 0.64 & 77.4 & 984 & 961 & 966 & 975 & 953 & 939 & 157 \\
\hline 639 & 58.19 & 0.309 & 16.33 & 0.64 & 77.5 & 1021 & 996 & 1001 & 1013 & 987 & 970 & 159 \\
\hline 640 & 59.51 & 0.309 & 16.33 & 0.64 & 80.6 & 1024 & 1009 & 1008 & 1018 & 997 & 974 & 164 \\
\hline 641 & 59.8 & 0.312 & 16.33 & 0.64 & 80.6 & 1062 & 1045 & 1045 & 1055 & 1029 & 1007 & 165 \\
\hline 642 & 61.68 & 0.313 & 16.33 & 0.64 & 82 & 1076 & 1066 & 1061 & 1064 & 1046 & 1020 & 169 \\
\hline 643 & 61.43 & 0.314 & 16.33 & 0.64 & 82.4 & 1126 & 1116 & 1111 & 1108 & 1090 & 1057 & 165 \\
\hline 644 & 60.34 & 0.332 & 16.33 & 0.69 & 65.3 & 926 & 852 & 863 & 869 & 837 & 856 & 103 \\
\hline 645 & 58.34 & 0.329 & 16.33 & 0.69 & 65.6 & 936 & 848 & 873 & 856 & 825 & 855 & 92 \\
\hline 646 & 56.08 & 0.315 & 16.33 & 0.64 & 71.4 & 911 & 866 & 881 & 878 & 853 & 852 & 109 \\
\hline 647 & 56.51 & 0.314 & 16.33 & 0.64 & 72.4 & 942 & 892 & 908 & 905 & $8 \overline{7} 7$ & 875 & 109 \\
\hline 648 & 57.93 & 0.31 & 16.33 & 0.64 & 79 & 920 & 891 & 898 & 902 & 876 & 867 & 118 \\
\hline 649 & 57.78 & 0.31 & 16.33 & 0.64 & 79 & 956 & 925 & 933 & 938 & 910 & 901 & 127 \\
\hline 650 & 56.33 & 0.314 & 16.34 & 0.8 & 75.6 & 901 & 818 & 852 & 841 & 810 & 834 & 84 \\
\hline 651 & 57.4 & 0.313 & 16.34 & 0.8 & 75.6 & 915 & 823 & 859 & 845 & 811 & 839 & 83 \\
\hline 652 & 60.93 & 0.304 & 16.34 & 0.7 & $80 . \overline{7}$ & 909 & 865 & 886 & 882 & 852 & 848 & 107 \\
\hline 653 & 59.16 & 0.303 & 16.34 & 0.7 & 80.7 & 932 & 885 & 905 & 901 & 873 & 869 & 109 \\
\hline 654 & 57.86 & 0.311 & 16.34 & 0.75 & 77.5 & 872 & 785 & 805 & 810 & 777 & 807 & 83 \\
\hline 655 & 57.23 & 0.31 & 16.34 & 0.75 & 77.5 & 878 & 781 & 811 & 802 & 770 & 805 & 80 \\
\hline 656 & 56.68 & 0.303 & 16.34 & 0.72 & 81.7 & 896 & 853 & 874 & 872 & 842 & 836 & 105 \\
\hline 657 & 58.51 & 0.302 & 16.34 & 0.72 & 81.7 & 919 & 874 & 892 & 890 & 861 & 854 & 109 \\
\hline 658 & 53.53 & 0.31 & 16.33 & 0.8 & 69.6 & 802 & 734 & 744 & 771 & 733 & 746 & 79 \\
\hline 659 & 50.68 & 0.31 & 16.33 & 0.8 & 69.6 & 816 & 733 & 765 & 770 & 733 & 742 & 74 \\
\hline 660 & 47.6 & 0.305 & 16.33 & 0.8 & 68.9 & 826 & 761 & 785 & 775 & 746 & 776 & 78 \\
\hline 661 & 48.06 & 0.305 & 16.33 & 0.8 & 68.9 & 842 & 773 & 799 & 787 & 759 & 788 & 79 \\
\hline 662 & 49.01 & 0.302 & 16.33 & 0.75 & 72.6 & 833 & 787 & 811 & 804 & 765 & 764 & 83 \\
\hline 663 & 47.27 & 0.302 & 16.33 & 0.75 & 72.6 & 849 & 800 & 825 & 818 & 779 & 776 & 87 \\
\hline $6 \overline{64}$ & 46.81 & 0.3 & 16.35 & 0.72 & 76.1 & 857 & 826 & 846 & 838 & 809 & 796 & 94 \\
\hline 665 & 46.56 & 0.301 & 16.35 & 0.72 & 76.1 & 888 & 853 & 872 & 868 & 836 & 816 & 96 \\
\hline
\end{tabular}




\begin{tabular}{|c|c|c|c|c|c|c|c|c|c|c|c|c|}
\hline $\begin{array}{c}\text { Run } \\
\text { Number }\end{array}$ & $\begin{array}{c}\text { Water Out } \\
\text { (deg F) }\end{array}$ & $\begin{array}{c}\text { Oil Sump } \\
\text { (deg F) }\end{array}$ & $\begin{array}{c}\text { Oil } \\
\text { Gallery } \\
\text { (deg F) }\end{array}$ & $\begin{array}{c}\text { Intake @ } \\
\text { LFE (deg } \\
\text { F) }\end{array}$ & $\begin{array}{c}\text { Oil Press } \\
\text { (psig) }\end{array}$ & \begin{tabular}{|c|} 
Fuel Flow \\
(@ RT \\
Meter) \\
\end{tabular} & $\begin{array}{c}\text { Inlet Rest } \\
\text { ("H2O) }\end{array}$ & $\mathrm{HC}(\mathrm{ppm})$ & $\mathrm{CO}(\mathrm{ppm})$ & $\mathrm{CO} 2(\%)$ & $\begin{array}{l}\text { NOX } \\
\text { (ppm) }\end{array}$ & $\mathrm{O} 2(\%)$ \\
\hline 632 & 181 & 203 & 87 & 54 & 45.9 & 0.62 & 2.21 & 1308 & 383.8 & 7.32 & 278.9 & 8.08 \\
\hline 633 & 181 & 204 & 89 & 53 & 45.8 & 0.6191 & 2.22 & 1303 & 386.3 & 7.32 & 136.9 & 8.09 \\
\hline 634 & 181 & 206 & 103 & 55 & 44.8 & 0.7185 & 2.84 & 1257 & 372.1 & 7.15 & 245.7 & 8.35 \\
\hline 635 & 181 & 207 & 102 & 57 & 44.7 & 0.7309 & 2.96 & 1285 & 377.5 & 7.13 & 118.5 & 8.34 \\
\hline 636 & 182 & 209 & 102 & 55 & 43 & 0.9331 & 3.9 & 1097 & 359.1 & 7.17 & 446 & 8.31 \\
\hline $63 \overline{7}$ & 182 & 210 & 102 & 53 & 43 & 0.932 & 3.89 & 1152 & 352.1 & 7.16 & 181.4 & 8.31 \\
\hline 638 & 183 & 213 & 102 & 55 & 41.8 & 1.0976 & 4.96 & 1026 & 351.9 & 7.18 & 509.7 & 8.33 \\
\hline 639 & 184 & 213 & 102 & 57 & 41.7 & 1.0936 & 5.05 & 1089 & 339.4 & 7.17 & 222 & 8.33 \\
\hline 640 & 185 & 217 & 104 & 54 & $40^{-}$ & 1.3871 & 7.22 & 1136 & 331.8 & 7.13 & 584 & 8.4 \\
\hline 641 & 185 & 218 & 103 & 56 & 39.9 & 1.3791 & 7.18 & 1196 & 317.7 & 7.12 & 256 & 8.42 \\
\hline 642 & 186 & 221 & 105 & 57 & 38.2 & 1712 & 9.77 & 1315 & 309.2 & 7.09 & 465.9 & 8.44 \\
\hline 643 & 184 & 221 & 104 & 57 & $38 . \overline{3}$ & 1.709 & 9.81 & 1339 & 289.1 & 7.1 & 192.4 & 8.45 \\
\hline 644 & $17 \overline{7}$ & 202 & 109 & 57 & 39.5 & 0.1882 & 0.64 & 3448 & 670.4 & 7.5 & 42.4 & 7.78 \\
\hline 645 & 177 & 197 & 109 & 56 & 40.3 & 0.1946 & 0.64 & 2492 & 685.3 & 7.73 & 35.4 & 7.42 \\
\hline 646 & 181 & 202 & 101 & 50 & 36.8 & 0.6047 & 2.3 & 1236 & 367.6 & 7.17 & 293.4 & 8.31 \\
\hline 647 & 181 & 202 & 101 & 51 & 36.7 & 0.6168 & 2.34 & 1276 & 362.9 & 7.17 & 130.7 & 8.3 \\
\hline 648 & 181 & 203 & 100 & 50 & 35.5 & 0.7678 & 3.09 & 1162 & 363.5 & 7.18 & 558.9 & 8.27 \\
\hline 649 & 181 & 205 & 100 & 50 & 35.8 & 0.7714 & 3.12 & 1228 & 350.5 & 7.14 & 213.8 & 8.34 \\
\hline 650 & 177 & 193 & 109 & 52 & 36 & 0.2197 & 0.55 & $14 \overline{6} 2$ & 580.6 & 9.02 & 417.4 & 5.23 \\
\hline 651 & 177 & 192 & 107 & 52 & 36.3 & 0.2223 & 0.55 & $13 \overline{61}$ & 560.7 & 9.03 & 223 & 5.21 \\
\hline 652 & 181 & 201 & 102 & 52 & 31.5 & 0.5989 & 1.94 & .770 & 354.4 & 7.98 & 1734.3 & 6.92 \\
\hline 653 & 181 & 203 & 102 & 50 & 31.3 & 0.6052 & 1.98 & 795 & 340.5 & 7.97 & 1003.8 & $\overline{6} . \overline{9}$ \\
\hline $65 \overline{4}$ & 177 & 195 & 108 & 51 & 30.8 & 0.1691 & 0.43 & 2312 & 581.2 & 8.53 & 172.6 & 6.13 \\
\hline 655 & 177 & $19 \overline{1}$ & $1 \overline{0} \overline{7}$ & 51 & 31.1 & 0.1687 & 0.42 & 1849 & 569.1 & 8.61 & 110.1 & 5.99 \\
\hline 656 & 181 & 200 & 98 & 52 & 28.3 & 0.5898 & 1.72 & 701 & 315.1 & 8.26 & 2547.9 & 6.43 \\
\hline $6 \overline{57}$ & $18 \overline{1}$ & 202 & 101 & 53 & 28 & 0.5942 & 1.75 & 726 & 310.2 & 8.26 & 1637.6 & 6.47 \\
\hline 658 & 177 & 192 & 117 & 55 & 23.6 & 0.1312 & 0.38 & 3163 & 568.2 & 8.99 & 313.1 & 5.3 \\
\hline 659 & 177 & 189 & 116 & 56 & 23.9 & 0.131 & 0.35 & 1969 & 568.8 & 9.11 & 192.5 & 5.11 \\
\hline 660 & 181 & 193 & 93 & 58 & 22.8 & 0.2351 & 0.53 & 1003 & 403.3 & 9.23 & 2313.2 & 4.71 \\
\hline 661 & 181 & 193 & 92 & 58 & 22.7 & 0.2361 & 0.6 & 1042 & 393.1 & 9.24 & 1391.7 & 4.8 \\
\hline 662 & 181 & 195 & 87 & 58 & 22.4 & 0.3601 & 0.71 & 779 & 345.2 & 8.67 & 2752.8 & 5.78 \\
\hline 663 & 180 & 196 & 86 & 58 & 22.4 & 0.3607 & 0.73 & 789 & 337.8 & 8.67 & 1869.1 & 5.8 \\
\hline 664 & 180 & 199 & 94 & 57 & 21.8 & 0.4948 & 1.34 & 745 & 304.9 & 8.27 & 2230.6 & 6.42 \\
\hline 665 & 181 & 199 & 97 & 57 & 21.7 & 0.5017 & 1.42 & 803 & 289.6 & 8.27 & 1347.8 & 6.47 \\
\hline
\end{tabular}


Recorded Data

\begin{tabular}{|c|c|c|c|c|c|c|c|c|c|c|c|c|}
\hline $\begin{array}{c}\text { Run } \\
\text { Number }\end{array}$ & $\begin{array}{c}\text { Methane } \\
(\%)\end{array}$ & $\begin{array}{c}\text { Ethane } \\
(\%)\end{array}$ & $\begin{array}{c}\text { Propane } \\
(\%)\end{array}$ & $\begin{array}{c}\text { Butane } \\
(\%)\end{array}$ & $\mathrm{CO} 2(\%)$ & N2 (\%) & $\begin{array}{c}\text { Air Flow } \\
\text { (Ib/hr) }\end{array}$ & $\begin{array}{l}\text { Power } \\
\text { (bhp) }\end{array}$ & $\begin{array}{c}\text { Humidity } \\
\text { (lb } \\
\text { H20/Air) }\end{array}$ & $\begin{array}{l}\text { NG Flow } \\
\text { (Ib/hr) }\end{array}$ & $\begin{array}{l}\text { Speed } \\
(\mathrm{ppm})\end{array}$ & $\begin{array}{c}\text { Torque (ft- } \\
\text { lb) }\end{array}$ \\
\hline 666 & 94.84 & 2.52 & 0.39 & 0.22 & 1.45 & 0.58 & 114.22 & 8.1 & 0.0028 & 6.49 & 654 & 65.1 \\
\hline 667 & 94.84 & 2.52 & 0.39 & 0.22 & 1.45 & 0.58 & 122.54 & 8.4 & 0.0028 & 6.93 & 657 & 67.2 \\
\hline 668 & 94.84 & 2.52 & 0.39 & 0.22 & 1.45 & 0.58 & 211.82 & 26.9 & 0.0027 & 10.8 & 642 & 220.2 \\
\hline 669 & 94.84 & 2.52 & 0.39 & $0.2 \overline{2}$ & 1.45 & 0.58 & 212.75 & 25.8 & 0.0028 & 10.9 & 644 & 210.8 \\
\hline 670 & 94.84 & 2.52 & 0.39 & $0.2 \overline{2}$ & 1.45 & 0.58 & 330.2 & 43.3 & 0.0029 & 15.61 & 650 & 350.2 \\
\hline 671 & 94.84 & 2.52 & 0.39 & 0.22 & 1.45 & 0.58 & 335.26 & 42.7 & 0.0028 & 15.9 & 651 & 344 \\
\hline 672 & 94.84 & $\overline{2} . \overline{5} \overline{2}$ & 0.39 & 0.22 & 1.45 & 0.58 & 475.37 & 62.6 & 0.0029 & 22.28 & 647 & 508 \\
\hline $67 \overline{3}$ & 94.84 & 2.52 & 0.39 & 0.22 & 1.45 & 0.58 & 481.44 & 62.4 & 0.0029 & 22.66 & 648 & 505.5 \\
\hline 674 & 93.79 & 2.89 & 0.81 & 0.61 & 1.31 & 0.58 & 2880.3 & 350.1 & 0.0072 & 114.89 & 1490 & 1233.9 \\
\hline 675 & 93.79 & 2.89 & 0.81 & 0.61 & 1.31 & 0.58 & 2909.2 & 3314 & $0.007 \overline{2}$ & 115.57 & 1503 & 1157.8 \\
\hline 676 & 93.79 & 2.89 & 0.81 & 0.61 & 1.31 & 0.58 & 1579 & 192 & 0.0074 & 64.72 & 1254 & 804.2 \\
\hline $67 \overline{7}$ & 93.79 & 2.89 & 0.81 & 0.61 & 1.31 & 0.58 & 1563.5 & 185.6 & 0.0074 & 64.08 & 1251 & 779.1 \\
\hline 678 & 93.79 & 2.89 & 0.81 & 061 & 1.31 & 0.58 & 2005 & 247.4 & 0.0075 & 80.08 & 1237 & 1050.6 \\
\hline 679 & 93.79 & 2.89 & 0.81 & $\overline{0} 61$ & 1.31 & 0.58 & 1988.9 & 237.8 & 0.0073 & 79.01 & 1252 & 997.7 \\
\hline $68 \overline{0}$ & 93.58 & 3.16 & 0.86 & 0.65 & 1.15 & 0.6 & 2376.9 & 298.3 & 0.0073 & 94.11 & 1247 & 1255.9 \\
\hline 681 & 93.58 & 3.16 & 0.86 & 0.65 & 1.15 & 0.6 & 2401.6 & 281.4 & 0.0072 & 95.04 & 1231 & 1200.2 \\
\hline 682 & 93.58 & 3.16 & 0.86 & 0.65 & 1.15 & 0.6 & 382.31 & 42.4 & 0.0073 & 18.27 & 1093 & 203.5 \\
\hline 683 & 93.58 & 3.16 & 0.86 & 0.65 & 1.15 & 0.6 & 381.01 & 41.9 & 0.007 & 18.66 & 1101 & 199.8 \\
\hline 684 & 93.58 & 3.16 & 0.86 & 0.65 & 1.15 & 0.6 & 576.35 & $73 . \overline{3}$ & 0.0069 & 27.1 & 1102 & 349.3 \\
\hline $68 \overline{5}$ & 93.58 & 3.16 & 0.86 & 0.65 & 1.15 & 0.6 & 581.93 & 73.5 & 0.007 & 27.43 & 1103 & 350.1 \\
\hline 686 & 93.58 & 3.16 & 0.86 & 0.65 & 1.15 & 0.6 & 1164.3 & 158.4 & 0.007 & 52.03 & $10 \overline{9}$ & 756.7 \\
\hline 687 & 93.58 & 3.16 & 0.86 & 0.65 & 1.15 & 0.6 & 1188.6 & 159 & 0.0071 & 53.02 & 1100 & 758.7 \\
\hline $68 \overline{8}$ & 93.58 & 3.16 & 0.86 & 0.65 & 1.15 & 0.6 & 1549.4 & 210.3 & 0.0068 & 67.64 & 1091 & 1012.3 \\
\hline $68 \overline{9}$ & 93.58 & 3.16 & 0.86 & 0.65 & 1.15 & 0.6 & 1556.9 & 209.9 & 0.0068 & 68.43 & 1100 & 1002 \\
\hline 690 & 93.58 & $\overline{3.16}$ & 0.86 & 0.65 & 1.15 & 0.6 & 1862.1 & 269.2 & 0.0066 & 84.14 & 1103 & 1281.4 \\
\hline 691 & 93.58 & 3.16 & $0 . \overline{86}$ & 0.65 & 1.15 & 0.6 & 1925.3 & 275.8 & 0.0065 & 86.74 & 1102 & 1314 \\
\hline 692 & 93.58 & 3.16 & 0.86 & 0.65 & 1.15 & 0.6 & 1936.4 & 269.3 & 0.0066 & 87.34 & 1093 & 1294.1 \\
\hline 693 & 93.58 & 3.16 & 0.86 & 0.65 & 1.15 & 0.6 & 1071.7 & 147.1 & 0.0064 & 48.75 & 1004 & 769.8 \\
\hline 694 & 93.58 & 3.16 & 0.86 & 0.65 & 1.15 & 0.6 & 1090.9 & 144.1 & 0.0063 & 49.48 & 994 & 761.4 \\
\hline 695 & 93.58 & 3.16 & 0.86 & 0.65 & 1.15 & 0.6 & 1242.8 & 184.2 & 0.0062 & 61.64 & 1000 & 967.2 \\
\hline 696 & 93.58 & 3.16 & 0.86 & 0.65 & 1.15 & 0.6 & 1248.4 & 176.7 & 0.0059 & 62.6 & 1001 & 927.2 \\
\hline 697 & 93.58 & 3.16 & 0.86 & 0.65 & 1.15 & 0.6 & 726.13 & 104.8 & 0.0061 & 37.39 & 802 & 686.1 \\
\hline 698 & 93.58 & 3.16 & 0.86 & 0.65 & 1.15 & 0.6 & 769.78 & 102.8 & 0.0061 & 38.19 & 801 & 673.4 \\
\hline $69 \overline{9}$ & 94.74 & 2.53 & 0.6 & 0.35 & 1.04 & 0.74 & 1319.6 & 133 & 0.0023 & 54.8 & 1949 & 358.5 \\
\hline
\end{tabular}


Recorded Data

\begin{tabular}{|c|c|c|c|c|c|c|c|c|c|c|c|c|}
\hline $\begin{array}{c}\text { Run } \\
\text { Number } \\
666\end{array}$ & Eq Ra & Equiv $\mathrm{O} 2$ & $\begin{array}{l}\text { Ign Tim } \\
\text { (deg) }\end{array}$ & BTE (\%) & $\begin{array}{l}\text { MAP } \\
\text { (psia) }\end{array}$ & $\begin{array}{l}\text { Boost AF } \\
\text { IC (deg F) }\end{array}$ & $\begin{array}{l}\text { Pre- } \\
\text { Turbine } \\
\text { (psig) }\end{array}$ & $\mid \begin{array}{c}\text { Comp Out } \\
\text { (psig) }\end{array}$ & $\begin{array}{l}\text { MAP } \\
\text { (psig) }\end{array}$ & 02 (volts) & $\begin{array}{l}\text { Time } \\
\text { (sec) }\end{array}$ & $\begin{array}{c}\text { Date } \\
\text { (julian) }\end{array}$ \\
\hline$-\frac{606}{667}$ & 0.925 & $\frac{0.787}{0.787}$ & 18 & 15.6 & 6.4 & 93 & 0.09 & 0.12 & -7.93 & 3.264 & 60639 & 32 \\
\hline 668 & $-\frac{0.925}{0.833}$ & $\frac{0.787}{0.782}$ & $1 \overline{3}$ & $1 \overline{5} .2$ & 6.65 & 92 & 0.09 & 0.12 & -7.68 & 3.264 & 60861 & 32 \\
\hline$\overline{6} \overline{6}$ & $\frac{0.833}{0.838}$ & $0.78 \frac{2}{3}$ & 17.5 & 312 & 9.41 & 86 & 0.2 & 0.18 & -4.93 & 3.27 & 61377 & 32 \\
\hline $67 \overline{0}$ & $\frac{0.838}{0.773}$ & $\frac{0.783}{0736}$ & 12.5 & 29.7 & 9.42 & 84 & 0.21 & 0.19 & -4.92 & 3.27 & 61572 & 32 \\
\hline $67 \overline{1}$ & $\begin{array}{l}0.773 \\
0.775\end{array}$ & 0.736 & 15 & 34.7 & 12.26 & 81 & 0.47 & 0.44 & -2.09 & 3.332 & 61992 & 32 \\
\hline 672 & $\begin{array}{l}0.775 \\
0.766\end{array}$ & $\begin{array}{l}0.732 \\
07 \overline{7}\end{array}$ & 10 & 336 & 12.39 & 79 & 0.51 & 0.49 & -1.95 & 3.338 & $622 \overline{7}$ & 32 \\
\hline $67 \overline{3}$ & $\frac{0.766}{0.769}$ & $\frac{0.702}{0.704}$ & 15 & 35.1 & 15.14 & 79 & 1.24 & 1.84 & 0.8 & 3.378 & 62468 & 32 \\
\hline $67 \overline{4}$ & $\frac{0.769}{0.653}$ & $\begin{array}{c}0.704 \\
06\end{array}$ & 10 & 344 & 15.25 & 82 & 1.3 & $1 . \overline{9}$ & 0.91 & 3.376 & 62733 & 32 \\
\hline 675 & $\begin{array}{l}0.653 \\
0.651\end{array}$ & $0 . \frac{0.6}{99}$ & 20 & 38 & 37.02 & 106 & 17.07 & 24.17 & 22.54 & 3.514 & 34745 & 37 \\
\hline$\overline{6} \overline{\overline{6}}$ & $\begin{array}{l}0.651 \\
0.671\end{array}$ & $\begin{array}{l}0.5999 \\
0.617\end{array}$ & 15 & 35.8 & 37.03 & 107 & 17.02 & 24.29 & 22.55 & 3.514 & 35137 & 37 \\
\hline $67 \overline{7}$ & 0.671 & 0.617 & 23 & 37 & 24.1 & 96 & 7.3 & 10.43 & 9.63 & 3.491 & 36008 & 37 \\
\hline 678 & 0.654 & 0.618 & 18 & 36.2 & 23.99 & 96 & 7.2 & 10.29 & 9.51 & 3.489 & 36577 & 37 \\
\hline$\overline{6} 7 \overline{9}$ & 0.651 & $\begin{array}{l}0.613 \\
0.612\end{array}$ & 21 & 386 & 30.63 & 102 & 10.93 & 17.2 & 16.15 & 3.497 & 37259 & 37 \\
\hline $68 \overline{0}$ & $\overline{0} . \overline{51}$ & $\frac{0.612}{0.61}$ & 16 & 376 & 30.06 & 99 & 10.54 & 16.62 & 15.59 & 3.497 & 37700 & 37 \\
\hline $6 \overline{81}$ & 0.65 & $\frac{0.61}{0.609}$ & 20 & 39.4 & 36 & 103 & $14 . \overline{2}$ & 22.7 & 21.52 & 3.5 & 38414 & 37 \\
\hline $6 \overrightarrow{82}$ & 0.785 & $\frac{0.609}{0.704}$ & 15 & 36.8 & 35.94 & 104 & 14.09 & 22.63 & 21.46 & 3.502 & 38851 & 37 \\
\hline 683 & 0.805 & $\frac{0}{0.704}$ & 29 & 28.8 & 9.09 & 93 & 0.43 & 0.28 & -5.39 & 3.376 & 39490 & 37 \\
\hline $6 \overline{84}$ & $\overline{0} . \overline{7} \overline{3}$ & 0.706 & 24 & 27.9 & 8.98 & 89 & 0.44 & 0.27 & -5.49 & 3.373 & 39939 & 37 \\
\hline 685 & 0.774 & $\begin{array}{c}0.7 \\
0.699\end{array}$ & 27 & 336 & 12.04 & 85 & 0.93 & 0.8 & -2.44 & 3.381 & 40348 & 37 \\
\hline$\overline{6} \overline{8} \overline{6}$ & 0.734 & $\frac{0.699}{0.678}$ & 22 & 33.3 & 12.07 & 83 & 0.96 & 0.88 & -2.41 & 3.382 & 40730 & 37 \\
\hline $68 \overline{7}$ & $0 . \overline{33}$ & 0.678 & 23 & 37.9 & 20.89 & 95 & 5.08 & 8.08 & 6.41 & 3.41 & 41445 & 37 \\
\hline 688 & 0.717 & $\begin{array}{l}0.679 \\
0.676\end{array}$ & 18 & 37.3 & 21.13 & 95 & 5.23 & 8.37 & 6.66 & 3.409 & 42066 & 37 \\
\hline 689 & $0.72 \overline{2}$ & $-\frac{0.676}{0.676}$ & 20 & 38.7 & 26.93 & 97 & 7.97 & 13.21 & 12.46 & 3.413 & 42812 & 37 \\
\hline 690 & 0.743 & $-\frac{0.676}{0.714}$ & 15 & 38.1 & 27.14 & 98 & 8.07 & 13.4 & 12.68 & 3.414 & 43152 & 37 \\
\hline 691 & 0.74 & $\begin{array}{l}0.714 \\
0.713\end{array}$ & 16 & 39.8 & 32.32 & 101 & 10.83 & 18.66 & 17.88 & 3.363 & 48626 & 37 \\
\hline$\overline{6} \overline{9}$ & 0.741 & $\frac{0.713}{0.713}$ & 14 & 39.5 & 33.24 & 102 & 11.37 & 19.61 & 18.81 & 3.363 & 49639 & 37 \\
\hline $6 \overline{93}$ & 0.747 & $\begin{array}{c}0.713 \\
0.7\end{array}$ & 11 & 38.4 & 33.25 & 102 & 11.35 & 19.61 & 18.82 & 3.363 & 49928 & 37 \\
\hline 694 & 0.745 & $\frac{0.7}{0.7}$ & 15 & 37.5 & 20.95 & 96 & 4.61 & 7.32 & 6.52 & 3.381 & 50725 & 37 \\
\hline 695 & 0.815 & $0 . \frac{0.7}{0.763}$ & 10 & 36.2 & 21.26 & 98 & 4.72 & 7.64 & 6.83 & 3.381 & 51589 & 37 \\
\hline 696 & 0.824 & $\frac{0.763}{0761}$ & 11 & 37.2 & 24.12 & 96 & 6.08 & 10.28 & 9.69 & 3.296 & 52816 & 37 \\
\hline 697 & $0 . \overline{846}$ & 0.761 & 6 & 35.1 & 24.13 & 96 & 6.05 & 10.29 & 9.7 & 3.299 & 53049 & 37 \\
\hline 698 & $\frac{0.846}{0.815}$ & 0.755 & 10 & 34.9 & 18.17 & 93 & 2.59 & 3.91 & 3.75 & 3.307 & 53715 & 37 \\
\hline 699 & $0.87 \frac{15}{0.684}$ & 0.745 & 5 & 33.5 & 18.78 & 94 & 2.89 & 4.56 & 4.36 & 3.321 & 53945 & 37 \\
\hline & & 6.544 & 30 & 30.2 & 15.1 & 108 & 6.36 & 9.28 & $0 . \overline{67}$ & 0.018 & 51596 & 44 \\
\hline
\end{tabular}




\begin{tabular}{|c|c|c|c|c|c|c|c|c|c|c|c|c|}
\hline $\begin{array}{c}\text { Run } \\
\text { Number }\end{array}$ & Year & $\begin{array}{c}\text { BSFC } \\
(\# / \mathrm{bhp}-\mathrm{hr}) \\
0.801 \\
\end{array}$ & $\begin{array}{c}\begin{array}{c}\text { BMEP } \\
\text { (psi) }\end{array} \\
13.49\end{array}$ & $\begin{array}{c}\begin{array}{c}\text { Vol Eff } \\
(\%)\end{array} \\
48.7\end{array}$ & $\begin{array}{c}\text { Intake Air } \\
\text { (acfm) }\end{array}$ & $\begin{array}{c}\text { Intake Air } \\
\text { (scfm) }\end{array}$ & $\begin{array}{c}\text { Boost B4 } \\
\text { IC (deg F) }\end{array}$ & $\begin{array}{c}\text { Stack } \\
\text { (deg F) }\end{array}$ & $\begin{array}{c}\text { Pre- } \\
\text { Turbine } \\
\text { (deg F) }\end{array}$ & $\begin{array}{l}\text { Fuel } \\
\text { Press } \\
\text { (psig) }\end{array}$ & EBP ("Hg) & Baro ("Hg) \\
\hline 667 & 1996 & 0.825 & 13.91 & $4 \frac{48}{4} .8$ & $\frac{25}{27}$ & $\frac{25}{27}$ & $\frac{86}{85}$ & 625 & 781 & 91.3 & 0.02 & 29.18 \\
\hline 668 & 1996 & 0.401 & 45.62 & 61.2 & $\frac{27}{46}$ & $\frac{27}{47}$ & 85 & 624 & 799 & 91.2 & 0.03 & 29.19 \\
\hline 669 & 1996 & 0.422 & 43.68 & 611 & $\frac{46}{46}$ & $\begin{array}{l}47 \\
47\end{array}$ & 79 & 670 & 783 & 90.5 & 0.08 & 29.18 \\
\hline 670 & 1996 & 0.36 & 72.54 & 71.2 & 71 & $\frac{47}{74}$ & 79 & 682 & 803 & 90.4 & 0.08 & 29.19 \\
\hline 671 & 1996 & $0 . \overline{373}$ & 71.27 & 71.2 & 73 & $\frac{74}{75}$ & 77 & 734 & 840 & 89.4 & 0.11 & 29.2 \\
\hline 672 & 1996 & 0.356 & 10524 & 83 & 103 & $\begin{array}{r}75 \\
106\end{array}$ & 78 & 762 & 872 & 89.4 & 0.11 & 29.2 \\
\hline $6 \overline{7} \overline{3}$ & 1996 & $0 . \overline{3} \overline{3}$ & 104.71 & 83.9 & 104 & 106 & 92 & 781 & 873 & 88.9 & 0.11 & 29.19 \\
\hline 674 & 1996 & 0.328 & 255.61 & $93 \overline{3}$ & $-\frac{104}{630}$ & 107 & 96 & 806 & 903 & 89 & 0.11 & 29.19 \\
\hline 675 & 1996 & 0.349 & 239.84 & 93.5 & $-\frac{6}{6} \frac{30}{4}$ & 641 & 285 & 958 & 1164 & 82.4 & 2.05 & 29.47 \\
\hline 676 & 1996 & 0.337 & 166.59 & 91.8 & $\frac{634}{34}$ & 648 & 285 & 1021 & 1221 & 823 & 2.19 & 29.47 \\
\hline 677 & 1996 & 0.345 & 161.4 & $91 . \overline{7}$ & $\frac{345}{34 \frac{1}{2}}$ & 351 & 179 & 913 & 1038 & 88.3 & 0.63 & 29.47 \\
\hline 678 & 1996 & 0.324 & 217.64 & 93.9 & & 348 & 178 & 951 & 1073 & 88.2 & 0.64 & 29.48 \\
\hline 679 & 1996 & 0.332 & 206.67 & $\overline{9} . \overline{3}$ & $\begin{array}{r}442 \\
-435\end{array}$ & 446 & 240 & 914 & 1080 & 86.3 & 0.98 & 29.48 \\
\hline 680 & 1996 & 0.316 & 260.17 & 94 & $\frac{43}{52} \frac{5}{3}$ & 443 & 233 & 957 & 1117 & 86.2 & 0.98 & 29.48 \\
\hline 681 & 1996 & 0.338 & 248.62 & 96.6 & $-\frac{52}{527}$ & 529 & 282 & 901 & 1100 & 84.4 & 1.35 & 29.48 \\
\hline 682 & 1996 & 0.431 & 42.16 & 68.1 & $-\frac{527}{85}$ & 535 & 280 & 952 & 1147 & 84.4 & 1.4 & 29.48 \\
\hline 683 & 1996 & 0.446 & 41.38 & 67.7 & 85 & 85 & 83 & 806 & 900 & 91 & 0.12 & 29.48 \\
\hline $6 \overline{8}$ & 1996 & 0.37 & 72.37 & 75.6 & $\frac{84}{128}$ & 85 & 78 & $79 \overline{5}$ & 899 & 90.8 & 0.12 & 29.47 \\
\hline 685 & 1996 & 0.373 & 72.52 & 75.7 & 128 & 128 & 82 & 840 & 932 & 90 & 0.16 & 29.47 \\
\hline 686 & $1 \overline{9} \overline{6}$ & 0.328 & 156.76 & 89.5 & 128 & 130 & 82 & 862 & 954 & 89.9 & 0.16 & 29.47 \\
\hline 687 & 1996 & 0.334 & 157.16 & $90 . \frac{5}{2}$ & 257 & 259 & 163 & 904 & 1025 & 88.2 & 0.33 & 29.47 \\
\hline 688 & 1996 & 0.322 & 209.7 & & 265 & 265 & 169 & 924 & 1050 & 87.7 & 0.35 & 29.46 \\
\hline $68 \overline{9}$ & 1996 & 0.326 & 207.56 & $-\frac{93}{92} \cdot \frac{2}{4}$ & 346 & 345 & 216 & 920 & 1071 & 86.4 & 0.56 & 29.45 \\
\hline 690 & 1996 & 0.313 & 265.44 & 93.4 & 349 & 347 & 218 & 952 & 1105 & 86.3 & 0.59 & 29.45 \\
\hline 691 & 1996 & 0.315 & 272.19 & 93.1 & 424 & 415 & 272 & 952 & 1137 & 85 & 0.83 & 29.4 \\
\hline 692 & 1996 & 0.324 & 268.07 & $-\frac{93.8}{953}$ & 441 & 429 & 282 & 969 & 1159 & 84.8 & 0.9 & 29.39 \\
\hline 693 & $19 \overline{9}$ & 0.331 & 159.46 & 95.3 & 443 & 431 & 281 & 998 & 1187 & 84.7 & 0.92 & 29.39 \\
\hline 694 & 1996 & 0.343 & 157.72 & -90.1 & 247 & 239 & 170 & 930 & 1053 & 89 & 0.32 & 29.38 \\
\hline 695 & 1996 & 0.335 & 200.36 & 91.6 & 251 & 243 & 174 & 969 & 1095 & 88.9 & 0.33 & 29.38 \\
\hline$\overline{6} \overline{9} \overline{6}$ & 1996 & 0.354 & 192.07 & 91.7 & 287 & 277 & 205 & 1000 & 1141 & 87.2 & 0.43 & 29.37 \\
\hline $6 \overline{97}$ & 1996 & 0.357 & 142.12 & 91.9 & 288 & 278 & 203 & 1047 & 1190 & 87.1 & 0.45 & 29.37 \\
\hline 698 & 1996 & 0.372 & $\begin{array}{l}142.12 \\
139.49\end{array}$ & 88.3 & 167 & 162 & 134 & 921 & 1033 & 89.5 & 0.25 & 29.37 \\
\hline $69 \overline{9}$ & 1996 & 0.412 & $\frac{139.49}{74.27}$ & 90.7 & 178 & 171 & 140 & 948 & 1068 & 89.1 & 0.26 & 29.37 \\
\hline & & & 74.27 & 80.1 & 299 & 294 & 183 & 953 & 1088 & 88.2 & 0.42 & 29.38 \\
\hline
\end{tabular}


Recorded Data

\begin{tabular}{|c|c|c|c|c|c|c|c|c|c|c|c|c|}
\hline $\begin{array}{c}\text { Run } \\
\text { Number }\end{array}$ & $\mathrm{RH}(\%)$ & Density & Stioch A/F & $\begin{array}{c}\text { GEM Eq } \\
\text { Ra }\end{array}$ & $\begin{array}{c}\text { GEM Vol } \\
\text { Eff }(\%)\end{array}$ & $\begin{array}{l}\text { Exh \#1 } \\
(\operatorname{deg} F)\end{array}$ & $\begin{array}{l}\text { Exh \#2 } \\
(\operatorname{deg} F)\end{array}$ & $\begin{array}{l}\text { Exh \#3 } \\
(\operatorname{deg} F)\end{array}$ & $\begin{array}{l}\text { Exh \#4 } \\
(\operatorname{deg} F)\end{array}$ & $\begin{array}{l}\text { Exh \#5 } \\
(\operatorname{deg} F)\end{array}$ & $\begin{array}{l}\text { Exh \#6 } \\
\text { (deg F) }\end{array}$ & $\begin{array}{l}\text { Water In } \\
\text { (deg F) }\end{array}$ \\
\hline $\begin{array}{l}666 \\
667\end{array}$ & 47.58 & 0.308 & 16.35 & 0.8 & 61.5 & 783 & 701 & 725 & 742 & 707 & 709 & 77 \\
\hline & 49.17 & 0.308 & $1 \overline{6} . \overline{35}$ & 0.8 & 62 & 803 & 714 & 742 & 744 & 711 & 716 & 75 \\
\hline 668 & 51.19 & 0.307 & 16.35 & 0.8 & 66.1 & 788 & 730 & 765 & 751 & 716 & 710 & 75 \\
\hline & 52.06 & $\mathbf{0} \mathbf{3 0} 7$ & 16.35 & 0.8 & 66.1 & 820 & 756 & 794 & 776 & 741 & 731 & 76 \\
\hline$\frac{670}{671}$. & 55.39 & 0.305 & 16.35 & 0.75 & 70.6 & 815 & 768 & 798 & 784 & 742 & 730 & 80 \\
\hline $\begin{array}{l}671 \\
672\end{array}$ & 54.64 & 0.304 & 16.35 & 0.75 & 70.6 & 843 & 792 & 824 & 811 & $7 \overrightarrow{6} 7$ & 754 & 81 \\
\hline$\frac{672}{673}$ & 55.19 & 0.303 & 16.35 & $0.7 \overline{2}$ & 76.8 & 802 & 776 & 794 & 787 & 747 & 733 & 83 \\
\hline $67 \frac{6}{6}$ & 56.47 & 0.303 & 16.35 & 0.72 & 76.8 & 826 & 800 & 819 & 814 & 773 & 757 & 86 \\
\hline 675 & $-\frac{6}{6} \cdot \frac{97}{17}$ & 0.311 & 16.38 & 0.65 & 90.5 & 1092 & 1087 & 1079 & 1085 & 1065 & 1034 & 168 \\
\hline 676 & $\begin{array}{l}67.17 \\
67.77\end{array}$ & 0.309 & 16.38 & 0.65 & 90.5 & 1154 & 1151 & 1144 & 1139 & 1122 & 1082 & 167 \\
\hline $6 \overline{7} \overline{7}$ & $\begin{array}{l}67.77 \\
66.11\end{array}$ & 0.322 & 16.38 & 0.65 & 83.5 & 952 & 946 & 937 & 950 & 927 & 900 & 143 \\
\hline 678 & $\frac{66.11}{64.4}$ & 0.32 & 16.38 & 0.65 & 83.5 & 987 & 976 & 969 & 981 & 958 & 926 & 140 \\
\hline 679 & $\begin{array}{c}64.4 \\
61.39\end{array}$ & 0.314 & 16.38 & 0.65 & 846 & $9 \overline{9} 6$ & 993 & 980 & 996 & $97 \overline{5}$ & $9 \overline{9} 9$ & $15 \overline{3}$ \\
\hline $68 \overline{0}$ & $\frac{61.39}{59.04}$ & 0.314 & 16.38 & 0.65 & 84.6 & 1029 & 1029 & 1019 & 1029 & 1007 & 969 & 155 \\
\hline$\overline{6} \overline{8} \overline{1}$ & 59.04 & 0.312 & 16.43 & 0.65 & 86.8 & 1023 & 1025 & 1015 & 1022 & 999 & $96 \overline{3}$ & $15 \overline{4}$ \\
\hline 682 & $\frac{58}{55} \cdot \frac{69}{23}$ & 0.312 & 16.43 & 0.65 & 86.8 & 1069 & 1075 & 1063 & 1063 & 1041 & 1002 & 154 \\
\hline 683 & $5 \frac{55}{52.63}$ & 0.329 & 16.43 & 0.72 & 68.7 & 886 & 824 & 845 & 833 & 813 & 814 & 92 \\
\hline 684 & $-\frac{52}{52} . \frac{68}{23}$ & -0.324 & 16.43 & 0.72 & 68.7 & 896 & 828 & 848 & 836 & 812 & 816 & 84 \\
\hline 685 & 53.94 & $-\frac{0}{0} \cdot \frac{318}{5}$ & 16.43 & 0.72 & 68.3 & 893 & 842 & 859 & 853 & 823 & 814 & 86 \\
\hline 686 & 55.49 & $\frac{0.315}{0.31}$ & 16.43 & 0.72 & 68.3 & 914 & 859 & 878 & 871 & 843 & 833 & 89 \\
\hline $6 \overline{8} \overline{7}$ & $5 \overline{3} . \overline{2}$ & $\frac{0.31}{0.309}$ & 16.43 & 0.7 & 86.1 & 933 & 918 & 922 & 931 & 903 & 882 & 119 \\
\hline 688 & $48.1 \overline{8}$ & $\frac{0.309}{0.308}$ & 16.43 & 0.7 & 86.5 & 954 & 942 & 941 & 955 & 925 & 904 & 124 \\
\hline 689 & 47.49 & $\frac{0}{0.308}$ & 16.43 & 0.7 & 85.4 & 971 & 969 & 960 & 978 & 951 & 921 & 141 \\
\hline 690 & 3965 & $\frac{0.309}{0.306}$ & 16.43 & 0.7 & 85.4 & 1002 & 1001 & 990 & 1012 & 983 & 949 & 142 \\
\hline 691 & 37.08 & $\frac{0.306}{0.307}$ & 16.43 & 0.74 & 85.6 & 1029 & 1039 & 1027 & 1046 & 1019 & 983 & 148 \\
\hline 692 & 37.95 & 0.307 & 16.43 & 0.74 & 86 & 1050 & 1060 & 1049 & 1066 & 1041 & 1003 & 149 \\
\hline 693 & 35.42 & 0.307 & 16.43 & 0.74 & 86 & 1072 & 1086 & 1074 & 1091 & 1065 & 1027 & 149 \\
\hline $6 \overline{9} \overline{4}$ & 34.76 & $=\frac{0.314}{0.311}$ & 16.43 & 0.72 & 86 & 947 & 937 & 941 & 955 & 925 & 900 & 126 \\
\hline 695 & 33.55 & 0.311 & 16.43 & 0.72 & 86.4 & 985 & 976 & 977 & 993 & 963 & 934 & 127 \\
\hline 696 & 32.46 & 0.306 & 16.43 & 0.78 & 86.1 & 1019 & 1021 & 1016 & 1038 & 1007 & 973 & 145 \\
\hline 697 & 33.03 & 0.306 & 16.43 & 0.78 & 86.1 & 1062 & 1066 & 1063 & 1083 & 1052 & 1012 & 145 \\
\hline 698 & 32.85 & 0.312 & 16.43 & 0.77 & 89.9 & 904 & 896 & 911 & 925 & 896 & 875 & 112 \\
\hline 699 & $\frac{32}{14.8}: \frac{8}{4}$ & 0.31 & 16.43 & 0.77 & 89.4 & 942 & 936 & 947 & 959 & 932 & 902 & 110 \\
\hline & & 0.303 & 16.47 & 0.66 & 71.2 & 1057 & 994 & 1008 & 993 & 984 & 990 & $15 \overline{5}$ \\
\hline
\end{tabular}


Recorded Data

\begin{tabular}{|c|c|c|c|c|c|c|c|c|c|c|c|c|}
\hline $\begin{array}{c}\begin{array}{c}\text { Run } \\
\text { Number }\end{array} \\
666\end{array}$ & $\begin{array}{c}\text { Water Out } \\
\text { (deg F) } \\
177\end{array}$ & $\begin{array}{l}\begin{array}{l}\text { Oil Sump } \\
\text { (deg F) }\end{array} \\
192\end{array}$ & $\begin{array}{c}\text { Oil } \\
\text { Gallery } \\
\text { (deg } F)\end{array}$ & $\begin{array}{c}\text { Intake @ } \\
\text { LFE (deg } \\
\text { F) }\end{array}$ & $\begin{array}{c}\text { Oil Press } \\
\text { (psig) }\end{array}$ & $\begin{array}{c}\text { Fuel Flow } \\
\text { (@ RT } \\
\text { Meter) }\end{array}$ & $\begin{array}{c}\text { Inlet Rest } \\
\text { ("H2O) }\end{array}$ & $H C(p p m)$ & $\mathrm{CO}(\mathrm{ppm})$ & $\mathrm{CO} 2(\%)$ & $\begin{array}{c}\text { NOx } \\
\text { (ppm) }\end{array}$ & $02(\%)$ \\
\hline$-\frac{666}{667}$ & $17 \frac{77}{18}$ & $-\frac{192}{188}$ & 122 & 57 & 18.7 & 0.112 & 0.28 & 2938 & 619.6 & 8.94 & 78.2 & 5.37 \\
\hline$\frac{667}{668}$ & $17 \frac{8}{18}$ & $\frac{188}{188}$ & 119 & 56 & 19.1 & 0.1196 & 0.34 & 2023 & 539.8 & 9.11 & 54.6 & 5.09 \\
\hline$-\frac{668}{669}$ & 181 & $\frac{188}{189}$ & 101 & 56 & 18.4 & 0.1869 & 0.54 & 1013 & 371 & 9.19 & 746.5 & 4.86 \\
\hline$-6 \frac{6}{6} \frac{9}{0}$ & $\frac{181}{180}$ & 189 & 99 & 56 & 18.3 & 0.1887 & 0.5 & 959 & 326.3 & 9.2 & 298.1 & 4.86 \\
\hline$-\frac{670}{671}$ & $18 \frac{180}{181}$ & 191 & 90 & 54 & 18.2 & 0.2724 & 0.73 & 809 & 334.9 & 8.66 & 1047.6 & 5.83 \\
\hline $\begin{array}{l}671 \\
672\end{array}$ & $\begin{array}{l}181 \\
181\end{array}$ & $\begin{array}{l}192 \\
194\end{array}$ & 89 & 54 & 18.1 & 0.2775 & 0.7 & 789 & . 298.9 & 8.64 & 524.4 & 5.87 \\
\hline$\frac{672}{673}$ & $\begin{array}{l}181 \\
182\end{array}$ & $\begin{array}{l}194 \\
196\end{array}$ & 88 & 54 & 17.6 & 0.3911 & 1.02 & 774 & 305.1 & 8.27 & 2477.2 & 6.44 \\
\hline$\frac{673}{674}$ & $\begin{array}{r}182 \\
187\end{array}$ & $\frac{196}{225}$ & 89 & 54 & 17.4 & 0.3975 & 1.05 & 783 & 286.2 & 8.27 & 1716.5 & 6.46 \\
\hline$\frac{674}{675}$ & $\begin{array}{l}187 \\
186\end{array}$ & $\frac{225}{2}$ & 102 & 57 & 37.3 & 1.9651 & 11.05 & 1150 & 303.4 & $\overline{7} . \overline{2} \overline{9}$ & 628.9 & 8.3 \\
\hline$\frac{675}{676}$ & 186 & 225 & 104 & 56 & 37.6 & 1.9844 & 11.1 & 1269 & 283.7 & 7.27 & 215.8 & 8.34 \\
\hline$\frac{676}{677}$ & 179 & 212 & 93 & 58 & 33.6 & 1.0684 & 4.73 & 1790 & 358.2 & 7.23 & $6 \overline{5} \overline{6}$ & 8.39 \\
\hline$\frac{677}{678}$ & 179 & 208 & 94 & 58 & 34.4 & 1.0635 & 4.74 & 1883 & 346.7 & 7.25 & 333 & 8.37 \\
\hline 678 & $18 \overline{4}$ & 214 & 98 & 60 & 32.4 & 1.353 & 6.48 & 1839 & 329.2 & 7.23 & 686.3 & 8.41 \\
\hline 679 & 184 & 215 & 96 & 58 & 32.5 & 1.3354 & 6.38 & 1952 & 317.1 & 7.23 & 317.2 & 8.36 \\
\hline 680 & $18 \overline{3}$ & 220 & 99 & 59 & 31.1 & 1.6001 & 8.33 & 1615 & 309.9 & 7.23 & 789 & 8.35 \\
\hline 681 & 183 & 218 & 100 & 58 & 31.4 & 1.616 & 8.31 & 1828 & 296.3 & 7.22 & 328.7 & 8.38 \\
\hline 682 & 178 & 201 & 96 & 62 & 32.6 & 0.2954 & 0.95 & 1423 & 457.9 & 8.29 & 477.3 & 6.64 \\
\hline 683 & 177 & 195 & 93 & 62 & 34 & 0.3057 & 0.93 & 1466 & 450.2 & 8.33 & 276.9 & 6.58 \\
\hline 684 & 181 & 197 & 89 & 62 & 32.8 & 0.4526 & 1.46 & 974 & 400.5 & 8.31 & 1416 & $6 . \overline{6}$ \\
\hline 685 & 181 & 199 & 88 & 61 & 32.5 & 0.462 & 1.46 & 1021 & 384.6 & 8.29 & 766.2 & 6.61 \\
\hline 686 & 182 & $20 \overline{7}$ & 94 & 61 & 29.5 & 0.8926 & 3.22 & 1170 & 277.2 & 8.05 & 2875.9 & 6.93 \\
\hline 687 & 182 & 210 & 94 & 63 & 29.1 & 0.9104 & 3.33 & 1422 & 279.8 & 8.01 & 1906.8 & 7.05 \\
\hline 688 & 183 & 214 & 95 & 64 & 27.8 & 1.1674 & 4.62 & 1782 & 226 & 7.95 & 2727.3 & 7.11 \\
\hline 689 & 183 & 215 & 95 & 65 & 27.8 & 1.1757 & 4.78 & 1840 & 237 & $7 . \overline{9}$ & 1662.8 & 7.17 \\
\hline 690 & 183 & 220 & 98 & 69 & 26.8 & 1.4616 & 6.09 & 1846 & 143.1 & 8.39 & 1695 & 6.3 \\
\hline 691 & $18 \overline{5}$ & 222 & 99 & 71 & 26.3 & 1.5021 & 6.42 & 1999 & 149.1 & 8.38 & 1490.2 & 6.33 \\
\hline 692 & 185 & 223 & 99 & 70 & 26.3 & 1.5127 & 6.43 & 1999 & 170.9 & 8.38 & 1166.5 & 6.36 \\
\hline 693 & $17 \overline{9}$ & 210 & 96 & 73 & 26.1 & 0.8248 & 3.05 & 1742 & 251.4 & 8.17 & 2255.3 & 6.71 \\
\hline 694 & 181 & 208 & 97 & 72 & 26.4 & 0.8455 & 3.12 & 1680 & 242.5 & 8.19 & 1349.7 & 6.7 \\
\hline 695 & 184 & 215 & 96 & 73 & 25.1 & 1.0702 & 3.69 & 1975 & 142.2 & 8.91 & 1684.1 & 5.35 \\
\hline 696 & $18 \overrightarrow{4}$ & 215 & 95 & 74 & 25.1 & 1.0866 & 3.72 & 1988 & 164.3 & 8.9 & 1161.8 & 5.42 \\
\hline 697 & 179 & 207 & 94 & 73 & 20.8 & 0.6371 & 1.86 & 1820 & 191.1 & 8.8 & 1435.4 & 5.56 \\
\hline 698 & 179 & 205 & 94 & 73 & 20.9 & 0.6548 & 2.01 & $20 \overline{94}$ & 196 & 8.64 & 2083.9 & 5.96 \\
\hline 699 & 181 & 218 & 106 & 68 & 57.8 & 0.9599 & $3 . \overline{84}$ & 1007 & 376.2 & 7.47 & 592.9 & 7.87 \\
\hline
\end{tabular}


Recorded Data

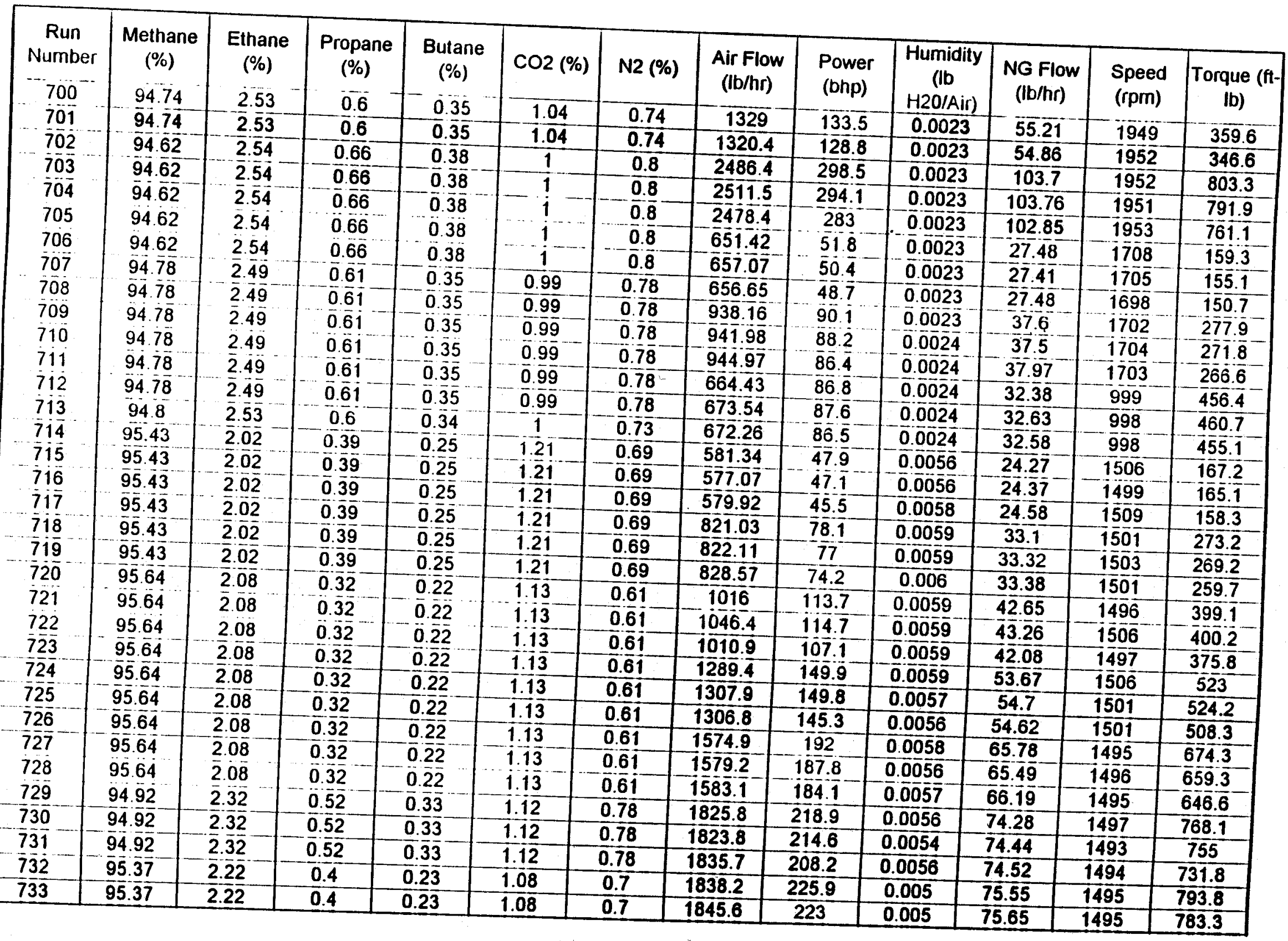


Recorded Data

\begin{tabular}{|c|c|c|c|c|c|c|c|c|c|c|c|c|}
\hline $\begin{array}{c}\text { Run } \\
\text { Number } \\
700\end{array}$ & Eq Ra & Equiv $\mathrm{O} 2$ & $\begin{array}{l}\begin{array}{l}\text { Ign Tim } \\
\text { (deg) }\end{array} \\
27\end{array}$ & BTE (\%) & $\begin{array}{l}\text { MAP } \\
\text { (psia) }\end{array}$ & $\begin{array}{c}\text { Boost AF } \\
\text { IC (deg F) }\end{array}$ & $\begin{array}{c}\text { Pre- } \\
\text { Turbine } \\
\text { (psig) }\end{array}$ & $\mid \begin{array}{c}\text { Comp Out } \\
\text { (psig) }\end{array}$ & $\begin{array}{l}\text { MAP } \\
\text { (psig) }\end{array}$ & $\mathrm{O} 2$ (volts) & $\begin{array}{l}\text { Time } \\
\text { (sec) }\end{array}$ & $\begin{array}{c}\text { Date } \\
\text { (julian) }\end{array}$ \\
\hline $\begin{array}{r}700 \\
701 \\
\end{array}$ & $\begin{array}{l}0.684 \\
0.684\end{array}$ & $\frac{6.51}{6.479}$ & 27 & 29.2 & 15.25 & 110 & 6.49 & 9.6 & 0.83 & 0.027 & 52013 & 44 \\
\hline 702 & $\frac{0.684}{0.687}$ & $-\frac{6.479}{6447}$ & $-\frac{24}{24}$ & 29.2 & 15.12 & 112 & 6.47 & 9.68 & 0.7 & 0.035 & 52232 & 44 \\
\hline 703 & $\begin{array}{l}0.687 \\
0.68\end{array}$ & $\begin{array}{l}6.447 \\
6.513\end{array}$ & 24 & 35.7 & 26.89 & 121 & 14.12 & 19.89 & 12.46 & 0.044 & 53341 & 44 \\
\hline 704 & $\begin{array}{r}0.68 \\
0.683\end{array}$ & $\frac{6.513}{6.472}$ & 21 & 35.2 & 26.87 & 120 & 14.08 & 19.82 & 12.45 & 0.026 & 53624 & 44 \\
\hline 705 & $\frac{0.683}{0.695}$ & $\begin{array}{l}6.472 \\
6.487\end{array}$ & 18 & 34.2 & 26.29 & 104 & 13.72 & 19.14 & 11.87 & 0.037 & 54049 & 44 \\
\hline 706 & $\begin{array}{l}0.695 \\
0.687\end{array}$ & $\begin{array}{l}6.487 \\
6.494\end{array}$ & 30 & 23.4 & 9.18 & 95 & 1.18 & 0.89 & -5.23 & 0.033 & 55590 & 44 \\
\hline 707 & 0.69 & $\frac{6.494}{6.52}$ & 27 & 228 & 9.2 & 93 & 1.19 & 0.93 & -5.22 & 0.031 & 55901 & 44 \\
\hline 708 & 0.66 & $\begin{array}{r}6.52 \\
6.493\end{array}$ & 24 & 22 & 9.23 & 93 & 1.2 & 0.95 & -5.19 & 0.024 & 56270 & 44 \\
\hline $70 \overline{9}$ & 0.656 & $\frac{6.493}{6.496}$ & 32 & 29.7 & 12.25 & 100 & 2.13 & 1.84 & -2.17 & 0.031 & 57171 & 44 \\
\hline 710 & 0.662 & $\frac{6}{6.43} \frac{49}{8}$ & 29 & 29.2 & 12.29 & 102 & 216 & 1.9 & -2.13 & 0.031 & 57621 & 44 \\
\hline $71 \overline{1}$ & 0.803 & $\frac{6.438}{6.487}$ & 26 & 28.3 & 12.35 & 102 & 2.2 & 199 & -2.06 & 0.046 & 58001 & 44 \\
\hline 712 & $0 . \overline{9} 8$ & $\begin{array}{l}6.487 \\
6.498\end{array}$ & 24 & 33.3 & 14.99 & 105 & 2 & 2.88 & 0.58 & 0.033 & 59188 & 44 \\
\hline 713 & 0.799 & $\begin{array}{l}6.498 \\
6508\end{array}$ & 21 & 33.3 & 15.06 & 105 & 2.05 & 2.98 & 0.65 & 0.03 & 59494 & 44 \\
\hline $71 \overline{4}$ & 0.686 & $\frac{6}{6.482}$ & 18 & $32 . \overline{9}$ & 15.01 & 104 & 2.06 & 3.05 & 0.61 & 0.028 & 59941 & 44 \\
\hline 715 & 0.694 & $\frac{6}{6.492}$ & 29.5 & 246 & 9.16 & 88 & $0 . \overline{91}$ & 0.68 & -5.2 & 0.034 & 34635 & 45 \\
\hline 716 & 0.696 & $\frac{6.492}{6.485}$ & 26.5 & 24.1 & 9.18 & 88 & 0.91 & 0.7 & -5.17 & 0.032 & 34881 & 45 \\
\hline 717 & 0.662 & $\begin{array}{l}6.485 \\
6.48\end{array}$ & 23.5 & 23 & 9.16 & 89 & 0.92 & 0.73 & -5.2 & 0.034 & 35390 & 45 \\
\hline 718 & 0.666 & $\begin{array}{r}6.48 \\
6.479\end{array}$ & 31 & 29.4 & 12.13 & $9 \overline{9}$ & 1.66 & 1.41 & -2.23 & 0.035 & 36431 & 45 \\
\hline 719 & 0.662 & $-\frac{6.479}{6.452}$ & 28 & 28.8 & 12.16 & 99 & 1.69 & 1.46 & -2.2 & 0.035 & 36694 & 45 \\
\hline 720 & 0.692 & $\begin{array}{r}6.452 \\
6.52\end{array}$ & 25 & 27.7 & 12.2 & 99 & 1.71 & 1.52 & -2.15 & 0.042 & 36999 & 45 \\
\hline 721 & 0.682 & $-\frac{6.52}{6.471}$ & 26.5 & 33.1 & 15.01 & 108 & 3.77 & 6.01 & 0.66 & $0.02 \overline{5}$ & 38045 & 45 \\
\hline 722 & 0.686 & $\begin{array}{r}6.471 \\
6.435\end{array}$ & 23.5 & 329 & 15.22 & 109 & 3.92 & 6.38 & 0.88 & 0.037 & 38508 & 45 \\
\hline $72 \overline{3}$ & 0.686 & $\frac{6}{6.43} \frac{43}{49}$ & 20.5 & 31.6 & 14.87 & 108 & 3.8 & 6.21 & 0.53 & 0.047 & 39040 & 45 \\
\hline 724 & 0.69 & $-\frac{6.439}{6.491}$ & 24 & 347 & 17.97 & 109 & 4.59 & 6.91 & 3.63 & $0 . \overline{0} \overline{6}$ & 39569 & 45 \\
\hline 725 & 0.689 & $\begin{array}{l}6.491 \\
6.523\end{array}$ & 21 & 34 & 18.12 & 103 & 4.6 & 6.97 & 3.77 & 0.032 & 39891 & 45 \\
\hline 726 & 0.689 & $\frac{6.523}{6.484}$ & 18 & 33 & 18.13 & 107 & 4.58 & 6.99 & 3.78 & 0.024 & 40205 & 45 \\
\hline 727 & 0.684 & $-\frac{6.484}{6.484}$ & 22 & 36.2 & 21.18 & 100 & 6.07 & 9.16 & 6.84 & 0.034 & 40803 & 45 \\
\hline 728 & 0.689 & $\frac{6.484}{6.486}$ & 19 & 35.6 & 21.2 & 104 & 6.07 & 9.21 & 6.86 & 0.034 & 41101 & 45 \\
\hline 729 & 0.668 & $\begin{array}{l}6.486 \\
6.446\end{array}$ & 16 & 34.5 & 21.23 & 105 & 6.07 & 9.22 & 6.89 & 0.033 & 41382 & 45 \\
\hline 730 & $0.67 \overline{1}$ & $\frac{6.446}{6.448}$ & 23 & 36.7 & 24.12 & 103 & 7.74 & 11.62 & 9.79 & 0.044 & 42060 & 45 \\
\hline 731 & 0.667 & $\begin{array}{l}6.448 \\
6.5\end{array}$ & 20 & 35.9 & 24.06 & 101 & 7.65 & 11.51 & 9.73 & 0.043 & 42437 & 45 \\
\hline 732 & 0.677 & $\frac{6.5}{6.434}$ & 17 & 34.8 & 24.06 & 102 & 7.61 & 11.59 & 9.72 & 0.03 & 42650 & 45 \\
\hline 733 & 0.676 & $\frac{6.434}{6.449}$ & 22 & 37.1 & 24.15 & 103 & 7.83 & 11.69 & 9.85 & 0.047 & 49818 & 45 \\
\hline
\end{tabular}


Recorded Data

\begin{tabular}{|c|c|c|c|c|c|c|c|c|c|c|c|c|}
\hline $\begin{array}{c}\begin{array}{c}\text { Run } \\
\text { Number }\end{array} \\
-700\end{array}$ & Year & $\frac{\begin{array}{c}\text { BSFC } \\
\text { (\#/bhp-hr) }\end{array}}{0.414}$ & $\begin{array}{c}\text { BMEP } \\
\text { (psi) } \\
7449\end{array}$ & $\begin{array}{c}\text { Vol Eff } \\
(\%)\end{array}$ & $\begin{array}{c}\text { Intake Air } \\
\text { (acfm) }\end{array}$ & $\begin{array}{c}\text { Intake Air } \\
\text { (scfm) }\end{array}$ & $\begin{array}{c}\text { Boost B4 } \\
\text { IC (deg F) }\end{array}$ & $\begin{array}{c}\text { Stack } \\
\text { (deg F) }\end{array}$ & $\begin{array}{c}\text { Pre- } \\
\text { Turbine } \\
\text { (deg F) }\end{array}$ & $\begin{array}{l}\text { Fuel } \\
\text { Press } \\
\text { (psig) }\end{array}$ & $\left|\mathrm{EBP}\left({ }^{\prime \prime} \mathrm{Hg}\right)\right|$ & Baro ("Hg) \\
\hline$-\frac{700}{70} \frac{1}{1}$ & $\frac{1996}{1996}$ & $\frac{0.414}{0.426}$ & $\frac{74.49}{71.79}$ & 80.2 & 301 & 296 & 189 & 966 & 1106 & 88.1 & 0.43 & 29.37 \\
\hline$\frac{701}{702}$ & $1 \frac{1996}{1996}$ & $\frac{0.426}{0.347}$ & $\frac{71.79}{166.41}$ & 80.4 & 299 & 294 & 187 & 979 & 1119 & 88 & 0.43 & 29.37 \\
\hline$\frac{702}{703}$ & 1996 & $\begin{array}{l}0.347 \\
0.353\end{array}$ & $\begin{array}{l}166.41 \\
164.05\end{array}$ & $86 . \frac{6}{3}$ & 564 & 553 & 270 & 1002 & 1196 & 82.9 & 1.56 & 29.37 \\
\hline 704 & 1996 & 0.353 & 164.05 & 87.3 & 570 & $5 \overline{59}$ & 270 & 1024 & 1219 & 82.9 & 1.59 & 29.37 \\
\hline 705 & 1996 & $\frac{0.363}{0.53}$ & 157.66 & $-\frac{8}{7} \frac{6}{6}$ & 563 & $5 \overline{52}$ & 264 & 1048 & 1239 & 82.9 & 1.61 & 29.36 \\
\hline 706 & 1996 & $\frac{0.53}{0.544}$ & 33 & 72.6 & 148 & 145 & 90 & 899 & - 991 & 89.8 & 0.11 & 29.36 \\
\hline 707 & 1996 & $-\frac{0.544}{0.564}$ & 32.14 & 72.9 & 150 & 146 & 91 & 912 & 1004 & 89.8 & 0.12 & 29.35 \\
\hline 708 & 1996 & $\frac{0.564}{0 . \overline{417}}$ & 31.21 & 72.9 & 150 & 146 & 92 & 927 & 1021 & 89.6 & 0.11 & 29.35 \\
\hline 709 & $1 \overline{9} 96$ & $-\frac{0.417}{0.425}$ & 57.57 & 79.1 & 213 & 209 & 100 & 916 & 997 & 89 & 0.21 & 29.35 \\
\hline 710 & $1 \overline{9} \overline{6}$ & $\begin{array}{l}0.425 \\
0.439\end{array}$ & 56.3 & 79.3 & 215 & 210 & 102 & 931 & 1012 & 89 & 0.22 & 29.35 \\
\hline $71 \overline{1}$ & 1996 & $\frac{0.439}{0.373}$ & 55.24 & 79.3 & 215 & 210 & 103 & 947 & 1030 & 89.1 & 0.22 & 29.34 \\
\hline 712 & 1996 & $0 . \frac{37}{0} \frac{3}{3}$ & 94.54 & 79.8 & 151 & 148 & 115 & 842 & 943 & 89.5 & 0.11 & 29.34 \\
\hline 713 & 1996 & 0.373 & 95.43 & 80.5 & 153 & 150 & 117 & 852 & 954 & 89.6 & 0.11 & 29.34 \\
\hline 714 & 1996 & $\frac{0.377}{0.506}$ & 94.28 & 80.5 & 153 & 150 & 118 & 866 & $9 \overrightarrow{6}$ & 89.5 & 0.11 & 29.33 \\
\hline 715 & 1996 & $\frac{0.506}{0.517}$ & 34.63 & 73.1 & 132 & 129 & 88 & 864 & 957 & 89.7 & 0.09 & 29.23 \\
\hline 716 & $19 \overline{6}$ & $\frac{0.517}{0.54}$ & 34.2 & 72.8 & 131 & 128 & 88 & 874 & 967 & 89.7 & 0.1 & 29.23 \\
\hline 717 & 1996 & $\frac{0.54}{0.424}$ & 32.79 & 73 & 132 & 129 & 90 & 893 & 988 & 89.7 & 0.1 & 29.23 \\
\hline 718 & 1996 & $\frac{0.424}{0.433}$ & 56.6 & 79.7 & 187 & 183 & 97 & 887 & 969 & 89.1 & 0.17 & 29.22 \\
\hline 719 & 1996 & $\frac{0.433}{0.45}$ & 55.76 & 79.4 & 187 & 183 & 98 & 902 & 986 & 89.2 & 0.17 & 29.22 \\
\hline 720 & 1996 & $\begin{array}{r}0.45 \\
0.375\end{array}$ & 53.8 & 79.8 & 189 & 184 & 99 & 920 & 1005 & 89.2 & 0.18 & 29.22 \\
\hline 721 & 1996 & 0.375 & 82.67 & 81.4 & 234 & 226 & 154 & 913 & 1030 & 88.4 & 0.25 & 29.22 \\
\hline 722 & $19 \overline{9} 6$ & $-\frac{0}{0}: \frac{377}{39} \frac{7}{3}$ & 82.9 & 82.1 & 240 & 233 & 158 & 929 & 1050 & 87.7 & 0.26 & 29.22 \\
\hline 723 & 1996 & $0.39 \frac{3}{0.358}$ & 77.85 & 81.6 & 233 & 225 & 160 & 950 & 1073 & 88 & 0.25 & 29.21 \\
\hline 724 & 1996 & $\frac{0.358}{0.365}$ & 108.33 & 85.8 & 299 & 287 & 164 & 942 & 1058 & 86.9 & 0.4 & 29.21 \\
\hline $72 \overrightarrow{5}$ & $19 \overline{9}$ & $\frac{0.365}{0.376}$ & 108.58 & 85.8 & 302 & 291 & 163 & 966 & 1080 & 86.9 & 0.42 & 29.21 \\
\hline 726 & 1996 & $\frac{0.376}{0.343}$ & 105.3 & 86.2 & 303 & 291 & 165 & 989 & 1104 & 86.9 & 0.42 & 29.21 \\
\hline 727 & $19 \overline{96}$ & $-\frac{0.343}{0.349}$ & 139.67 & 88.2 & 366 & 351 & 187 & 971 & 1097 & 85.9 & 0.6 & 29.2 \\
\hline 728 & 1996 & $\frac{0.349}{0.36}$ & 136.58 & 88.8 & 367 & 352 & 187 & 990 & 1118 & 85.8 & 0.61 & 29.2 \\
\hline 729 & 1996 & & 133.95 & 89.2 & 368 & 352 & 187 & 1014 & 1146 & 85.8 & 0.63 & 29.2 \\
\hline 730 & $19 \overline{96}$ & $\frac{0.339}{0.347}$ & 159.11 & 89.8 & 429 & 406 & 211 & 956 & 1096 & 85.1 & 0.79 & 29.19 \\
\hline $73 \overline{1}$ & 1996 & $\frac{0.347}{0.358}$ & 156.39 & 90 & 419 & 406 & 202 & 975 & 1114 & 85 & 0.81 & 29.19 \\
\hline $7 \overline{3} 2$ & $1 \overline{96}$ & $\frac{0.358}{0.334}$ & 151.6 & 90.6 & 421 & 409 & 202 & 1001 & 1145 & 85.2 & 0.83 & 29.19 \\
\hline 733 & 1996 & $\frac{0.334}{0.339}$ & 164.44 & 90.6 & 435 & 409 & 217 & 970 & 1115 & 84.8 & 0.8 & 29.12 \\
\hline & & 0.339 & 162.26 & 90.8 & 437 & 411 & 218 & 988 & 1134 & 84.9 & 0.81 & 29.11 \\
\hline
\end{tabular}


Recorded Data

\begin{tabular}{|c|c|c|c|c|c|c|c|c|c|c|c|c|}
\hline $\begin{array}{c}\text { Run } \\
\text { Number }\end{array}$ & RH (\%) & Density & Stioch AVF & $\underset{R_{a}}{\operatorname{GEM} E q}$ & $\begin{array}{c}\text { GEM Vol } \\
\text { Eff (\%) }\end{array}$ & $\begin{array}{l}\text { Exh \#1 } \\
(\operatorname{deg} F)\end{array}$ & $\begin{array}{l}\text { Exh \#2 } \\
\text { (deg F) }\end{array}$ & $\begin{array}{l}\text { Exh \#3 } \\
(\operatorname{deg} F)\end{array}$ & $\begin{array}{l}\text { Exh \#4 } \\
(\operatorname{deg} F)\end{array}$ & $\begin{array}{l}\text { Exh \#5 } \\
(\operatorname{deg} F)\end{array}$ & $\begin{array}{l}\text { Exh \#6 } \\
\text { (deg F) }\end{array}$ & $\begin{array}{l}\text { Water In } \\
(\operatorname{deg} F)\end{array}$ \\
\hline$\frac{700}{701}$ & $\frac{14.15}{14.23}$ & 0.303 & 16.47 & 0.66 & 71.2 & 1076 & 1012 & 1025 & 1009 & 1000 & 1003 & 156 \\
\hline $\begin{array}{l}\frac{701}{702} \\
702\end{array}$ & $\frac{14.23}{14}$ & 0.302 & 16.47 & $0.6 \overline{6}$ & 71.2 & 1090 & 1024 & 1038 & 1020 & 1013 & 1017 & 156 \\
\hline 703 & $-\frac{14}{13.94}$ & 0.294 & 16.47 & 0.66 & 80.6 & 1129 & 1104 & 1108 & 1111 & 1094 & 1076 & 161 \\
\hline $70 \overline{4}$ & $\frac{13.94}{13.9}$ & 0.296 & 16.47 & 0.66 & 80.6 & 1153 & 1126 & 1130 & 1132 & 1113 & 1096 & 161 \\
\hline 705 & $\begin{array}{r}13.9 \\
14.02\end{array}$ & 0.297 & 16.47 & 0.66 & 80.6 & 1171 & 1145 & 1148 & 1153 & 1133 & 1113 & 161 \\
\hline $70 \overrightarrow{6}$ & 14.02 & 0.306 & 16.47 & 0.65 & 69.5 & 986 & 910 & 926 & 909 & 890 & 902 & 112 \\
\hline$\overline{70} \overline{7}$ & 13.92 & 0.302 & 16.47 & 0.65 & 69.5 & 1004 & 927 & 944 & 922 & 901 & 917 & 111 \\
\hline 708 & 13.95 & 0.302 & 16.48 & 0.65 & 69.5 & 1026 & 943 & 954 & 938 & 915 & 933 & 110 \\
\hline $70 \overline{9}$ & $13 . \overline{9}$ & $\begin{array}{l}0.299 \\
0.299\end{array}$ & 16.48 & 0.63 & 70.1 & 957 & 897 & 915 & 909 & 885 & 893 & 120 \\
\hline 710 & 13.93 & $\begin{array}{l}0.299 \\
0.299\end{array}$ & $1 \overline{6} .48$ & 0.63 & 70.1 & 976 & 913 & 931 & 925 & 898 & 904 & 119 \\
\hline 7ij & 14.25 & $\frac{0.299}{0.301}$ & 16.48 & 0.63 & 70.1 & 993 & 931 & 947 & 940 & 911 & 918 & 120 \\
\hline 712 & 14.32 & 0.301 & 16.48 & 0.7 & 81.9 & 884 & 841 & 852 & 854 & 825 & 810 & 97 \\
\hline $71 \overline{3}$ & 14.42 & $\frac{0.301}{0.3}$ & 16.48 & 0.7 & 81.9 & 900 & 851 & 865 & 865 & 836 & 820 & 96 \\
\hline $71 \overline{4}$ & $47.5 \overline{3}$ & 0.3 & 16.49 & 0.7 & 81.9 & 914 & 865 & 882 & 879 & 850 & 833 & 98 \\
\hline 715 & 47.46 & & 16.43 & 0.66 & 67.3 & 947 & 884 & 901 & 885 & 861 & 871 & 101 \\
\hline 716 & 45.98 & $\begin{array}{l}0.299 \\
0.298\end{array}$ & 16.43 & 0.65 & 67.3 & 955 & 896 & 913 & 895 & 870 & 878 & 102 \\
\hline 717 & 43.71 & $\frac{0.298}{0.29}$ & 16.43 & 0.65 & 67.3 & 984 & 920 & 934 & 915 & 886 & 897 & 102 \\
\hline 718 & 43.8 & -0.295 & 16.43 & 0.63 & 6.8 & 920 & 876 & 893 & 887 & 861 & 856 & 113 \\
\hline 719 & 42.75 & 0.295 & 16.43 & 0.63 & 66.8 & 939 & 892 & 907 & $90 \overline{3}$ & 873 & 870 & 113 \\
\hline 720 & 40.99 & $\frac{0.29}{0.2} \frac{5}{9}$ & 16.43 & 0.63 & 66.8 & 960 & 911 & 924 & 920 & 887 & 884 & 112 \\
\hline 721 & 39.56 & $\frac{0.292}{0.291}$ & 16.49 & 0.66 & 66.8 & 976 & 928 & 941 & 939 & 916 & 915 & 133 \\
\hline $7 \overline{2} 2$ & 38.75 & $\frac{0.291}{0.293}$ & 16.49 & 0.66 & 67.1 & 994 & $94 \overline{6}$ & 959 & 957 & 935 & 933 & 136 \\
\hline $72 \overline{3}$ & 36.95 & $\frac{0.293}{0.29}$ & 16.49 & 0.66 & 67.1 & 1021 & 969 & 980 & 978 & 952 & 951 & 135 \\
\hline 724 & 35.64 & $\frac{0.29}{0.29}$ & 16.49 & 0.66 & 76.1 & 988 & 953 & 962 & 965 & 943 & 930 & 140 \\
\hline 725 & 35.72 & $\begin{array}{r}0.29 \\
0.29\end{array}$ & 16.49 & 0.66 & 77.1 & 1008 & 974 & 983 & 988 & 961 & 947 & 143 \\
\hline 726 & 35.22 & $\frac{0.29}{0.289}$ & 16.49 & 0.66 & 77.1 & 1032 & 999 & 1005 & 1012 & 983 & 967 & 146 \\
\hline 727 & 34.13 & $\frac{0.289}{0.29}$ & 16.49 & 0.66 & 77.6 & 1017 & 994 & 996 & 1007 & 985 & 958 & 157 \\
\hline 728 & 33.79 & $\begin{array}{c}0.29 \\
0.291\end{array}$ & 16.49 & 0.66 & 77.6 & 1033 & 1010 & 1012 & 1027 & 1005 & 976 & 158 \\
\hline 729 & 33.47 & $\begin{array}{l}0.291 \\
0.293\end{array}$ & 16.49 & 0.66 & 77.6 & 1057 & 1036 & 1038 & 1054 & 1029 & 996 & 159 \\
\hline 730 & 32.49 & $\frac{0.293}{0.293}$ & 16.43 & 0.64 & 81.2 & 1014 & 998 & 995 & 1008 & 988 & 956 & 160 \\
\hline 731 & 33.34 & & 16.43 & 0.64 & 81.2 & 1032 & 1017 & 1011 & 1026 & 1002 & 971 & 159 \\
\hline 732 & 23.91 & $\frac{0.294}{0.288}$ & 16.43 & 0.64 & 81.2 & 1060 & 1040 & 1034 & 1057 & 1032 & 993 & 159 \\
\hline 733 & 24.12 & $\frac{0.288}{0.288}$ & 16.48 & 0.66 & 81 & 1029 & 1012 & 1009 & 1025 & 1004 & 975 & 159 \\
\hline 733 & & 0.288 & 16.48 & 0.66 & 81 & 1046 & 1032 & 1028 & 1046 & 1023 & 891 & 159 \\
\hline
\end{tabular}


Recorded Data

离

\begin{tabular}{|c|c|c|c|c|c|c|c|c|c|c|c|c|}
\hline $\begin{array}{c}\begin{array}{c}\text { Run } \\
\text { Number }\end{array} \\
700\end{array}$ & $\mid \begin{array}{c}\text { Water Out } \\
\text { (deg F) } \\
182\end{array}$ & $\begin{array}{c}\text { Oil Sump } \\
\text { (deg F) }\end{array}$ & $\begin{array}{c}\begin{array}{c}\text { Oil } \\
\text { Gallery } \\
\text { (deg F) }\end{array} \\
108\end{array}$ & $\begin{array}{c}\text { Intake @ } \\
\text { LFE (deg } \\
\text { F) }\end{array}$ & $\begin{array}{c}\text { Oil Press } \\
\text { (psig) }\end{array}$ & \begin{tabular}{|c|} 
Fuel Flow \\
(@ RT \\
Meter) \\
\end{tabular} & $\begin{array}{c}\text { Inlet Rest } \\
\text { ("H2O) }\end{array}$ & $\mathrm{HC}(\mathrm{ppm})$ & $\mathrm{CO}(\mathrm{ppm})$ & $\mathrm{CO} 2(\%)$ & $\begin{array}{c}\text { NOx } \\
(\mathrm{ppm})\end{array}$ & $\mathrm{O} 2(\%)$ \\
\hline 701 & 182 & $\frac{218}{218}$ & $\frac{108}{107}$ & 68 & 57.8 & 0.9694 & 3.89 & 970 & 369.5 & 7.48 & 436.3 & 7.86 \\
\hline 702 & 184 & 230 & $\frac{107}{115}$ & 69 & 57.9 & 0.9649 & 3.89 & 983 & 365.7 & 7.49 & 277.6 & 7.86 \\
\hline 703 & 185 & 230 & $\begin{array}{l}115 \\
114\end{array}$ & 69 & 52.3 & 1.8731 & 9.06 & 453 & 313.4 & 7.64 & 1353.8 & 7.59 \\
\hline 704 & 184 & 229 & $\frac{114}{101}$ & 69 & 52.4 & 1.8631 & 9.23 & 462 & 312.3 & 7.63 & 853.8 & 7.62 \\
\hline 705 & 179 & 204 & $\frac{101}{100}$ & 69 & 53 & 1.8402 & 9.03 & 484 & 301.9 & 7.62 & 477.9 & 7.64 \\
\hline 706 & 179 & 203 & $\frac{100}{98}$ & 70 & 54.8 & 0.4766 & 1.71 & 1678 & 486.5 & 7.32 & 100.3 & 8.14 \\
\hline 707 & 180 & 204 & $\begin{array}{l}98 \\
97\end{array}$ & 70 & 54.8 & 0.478 & 1.73 & 1738 & 494.8 & 7.3 & 70.5 & 8.15 \\
\hline 708 & 181 & 208 & $-\frac{97}{102}$ & 71 & 54.6 & 0.483 & 1.73 & 1749 & 512.6 & 7.27 & 48.6 & 8.19 \\
\hline 709 & 181 & 207 & $\frac{102}{103}$ & 69 & 52.5 & 0.6688 & 2.59 & 1480 & 409.4 & 7 & 197.7 & 8.6 \\
\hline 710 & 181 & 207 & $\frac{103}{10} \frac{3}{4}$ & 70 & 52.7 & 0.6669 & 2.61 & 1565 & 424.6 & 6.98 & 122.3 & 8.61 \\
\hline 711 & 179 & 201 & $\begin{array}{l}104 \\
105\end{array}$ & 70 & 52.8 & 0.6746 & 2.61 & 1598 & 434.4 & 6.98 & 85.1 & 8.6 \\
\hline 712 & 179 & 201 & & 70 & 28.1 & 0.5726 & 1.76 & & & & & \\
\hline 713 & 180 & 201 & $\begin{array}{l}106 \\
105\end{array}$ & 70 & 28.1 & 0.5766 & 1.78 & 857 & 342.3 & 8 & 1416.2 & 6.91 \\
\hline 714 & $17 \overline{9}$ & 200 & 94 & 70 & 28.1 & 0.5762 & 1.77 & 849 & 338 & 8 & 972.7 & 6.92 \\
\hline 715 & 180 & 200 & 94 & $6 \overline{6}$ & 48.5 & 0.4299 & 1.47 & 1814 & 497.7 & 7.33 & 75.8 & 8.08 \\
\hline $71 \overline{6}$ & 180 & 201 & $\frac{94}{95}$ & 66 & $48 . \overline{3}$ & 0.4325 & 1.46 & 1880 & 508 & 7.32 & 57.2 & 8.1 \\
\hline 717 & 180 & 204 & $\frac{95}{102}$ & 68 & 48.6 & 0.4379 & 1.49 & 1892 & 503.3 & 7.37 & 44.7 & 8.02 \\
\hline 718 & 180 & 204 & $\frac{102}{102}$ & 68 & 47 & 0.5957 & 2.2 & 1720 & 458.9 & 6.91 & 107 & 8.73 \\
\hline 719 & 180 & 203 & & 68 & 47.2 & 0.5993 & 2.21 & 1812 & 477.4 & 6.9 & 69.2 & 8.73 \\
\hline 720 & 182 & 209 & $\frac{102}{108}$ & 69 & 47.3 & 0.6005 & 2.23 & 1892 & 504.5 & 6.88 & 47.6 & 8.75 \\
\hline 721 & $18 \overline{2}$ & 209 & $\begin{array}{l}108 \\
108\end{array}$ & 71 & 44.3 & 0.7749 & 2.88 & 980 & 348.9 & 7.98 & 1851.7 & 6.95 \\
\hline 722 & 181 & $20 \overline{8}$ & 108 & 70 & 44.7 & 0.7888 & 2.98 & 1138 & 362.8 & 7.51 & 310.6 & 7.78 \\
\hline 723 & 183 & 211 & 107 & 71 & 45.1 & 0.7634 & 2.84 & 1133 & 354.6 & 7.5 & 166.2 & 7.77 \\
\hline 724 & 183 & 211 & $\begin{array}{l}108 \\
104\end{array}$ & 73 & 43.7 & 0.9827 & 3.86 & 1011 & 363.5 & 7.43 & 527.4 & 7.88 \\
\hline 725 & 183 & 211 & $\begin{array}{l}104 \\
106\end{array}$ & 72 & 43.5 & 1.0009 & 3.91 & 904 & 321.3 & 7.54 & 321.6 & 7.68 \\
\hline 726 & 184 & 215 & $\frac{106}{101}$ & 73 & 43.6 & 1 & 3.93 & 950 & 338 & 7.44 & 207.6 & 7.87 \\
\hline 727 & 184 & 215 & $\frac{101}{103}$ & 74 & 41.5 & 1.2095 & 4.95 & 915 & 347.1 & 7.54 & 762.4 & 7.67 \\
\hline 728 & 184 & 215 & 103 & 73 & 41.5 & 1.1995 & 4.98 & 928 & 336.6 & 7.53 & 484.3 & 7.71 \\
\hline 729 & 184 & 216 & $\begin{array}{l}104 \\
101\end{array}$ & 73 & 41.7 & 1.2099 & 5.02 & 1049 & 434.3 & 7.79 & 1735.7 & 7.36 \\
\hline 730 & 184 & 216 & $\frac{101}{99}$ & 77 & 40.8 & 1.3463 & 6.12 & 1140 & 460.5 & 7.76 & 1115 & 7.43 \\
\hline 731 & 184 & 215 & $-\frac{99}{100}$ & 70 & 40.9 & 1.3477 & 6 & 1251 & 430.2 & 7.76 & 627.3 & 7.45 \\
\hline 732 & 185 & 218 & 100 & 69 & 41 & 1.3462 & 6.08 & 1249 & 423.2 & 8.07 & 540.9 & 6.98 \\
\hline 733 & 185 & $\frac{210}{218}$ & $\begin{array}{l}102 \\
102\end{array}$ & 78 & 40.2 & 1.3955 & 6.16 & 1349 & 423.1 & 7.79 & 1981 & 7.38 \\
\hline & & & 102 & 78 & 40.1 & 1.3953 & 6.2 & 1336 & 447.3 & 7.76 & 1387.8 & 7.46 \\
\hline
\end{tabular}


Recorded Data

\begin{tabular}{|c|c|c|c|c|c|c|c|c|c|c|c|c|}
\hline $\begin{array}{c}\begin{array}{c}\text { Run } \\
\text { Number }\end{array} \\
734\end{array}$ & \begin{tabular}{|c|}
$\begin{array}{c}\text { Methane } \\
(\%)\end{array}$ \\
95.37 \\
\end{tabular} & $\begin{array}{c}\begin{array}{c}\text { Ethane } \\
(\%)\end{array} \\
2.22\end{array}$ & $\begin{array}{c}\text { Propane } \\
(\%)\end{array}$ & $\begin{array}{c}\text { Butane } \\
(\%)\end{array}$ & $\mathrm{CO} 2(\%)$ & N2 (\%) & $\begin{array}{c}\text { Air Flow } \\
\text { (Ib/hr) }\end{array}$ & $\begin{array}{c}\text { Power } \\
\text { (bhp) }\end{array}$ & $\begin{array}{c}\text { Humidity } \\
\text { (1b } \\
\text { H20/Air) }\end{array}$ & $\begin{array}{c}\text { NG Flow } \\
\text { (1b/hr) }\end{array}$ & $\begin{array}{l}\text { Speed } \\
\text { (rpm) }\end{array}$ & $\begin{array}{c}\text { Torque (ft- } \\
\text { Ib) }\end{array}$ \\
\hline$-\frac{734}{735}$ & $\begin{array}{r}95.37 \\
95.37 \\
\end{array}$ & $\frac{2.22}{2.22}$ & 0.4 & 0.23 & 1.08 & 0.7 & 1845.5 & 220.1 & 0.0051 & 76.6 & 1497 & 772.2 \\
\hline$\frac{735}{736}$ & $\frac{95.37}{95.37}$ & $\frac{2.22}{2.22}$ & 0.4 & 0.23 & 1.08 & 0.7 & 2320.4 & 282.8 & 0.0051 & 93.78 & 1497 & 992.1 \\
\hline$\frac{736}{737}$ & $\frac{95.37}{95.37}$ & $\frac{2.22}{222}$ & 0.4 & 0.23 & 1.08 & 0.7 & 2336 & 278.2 & 0.005 & 94.16 & 1500 & 973.9 \\
\hline$\frac{737}{738}$ & 95.37 & 2.22 & 0.4 & 0.23 & 1.08 & 0.7 & 2360.9 & 271.8 & 0.0049 & 94.96 & 1504 & 949.2 \\
\hline$\frac{73}{7} \frac{8}{39}$ & 96.2 & 2.12 & 0.17 & 0.09 & 0.96 & 0.45 & 2319.7 & 291.6 & 0.005 & 95.46 & 1494 & 1025.1 \\
\hline $7 \frac{739}{40}$ & 96.2 & 2.12 & 0.17 & 0.09 & 0.96 & 0.45 & 2312 & 291.4 & 0.0047 & 94.54 & 1497 & 1022.3 \\
\hline $\begin{array}{r}740 \\
\frac{741}{1}\end{array}$ & 96.2 & 2.12 & 0.17 & 0.09 & 0.96 & 0.45 & 2278.6 & 279.3 & 0.0046 & 93.23 & 1493 & 982.6 \\
\hline$\frac{741}{742}$ & 96.37 & 2.14 & 0.12 & 0.05 & 0.92 & 0.41 & 2347.8 & 280.6 & 0.0046 & 96.81 & 1503 & 980.6 \\
\hline $\begin{array}{l}742 \\
743\end{array}$ & 96.37 & 2.14 & 0.12 & 0.05 & 0.92 & 0.41 & 847.91 & 101 & 0.0045 & 35.97 & 1251 & $42 \overline{3} . \overline{8}$ \\
\hline$-\frac{743}{744}$ & 96.37 & 2.14 & 0.12 & 0.05 & 0.92 & 0.41 & 862.35 & 101.1 & 0.0045 & 36.65 & 1250 & 424.8 \\
\hline$\frac{744}{745}$ & 96.37 & 2.14 & 0.12 & 0.05 & 0.92 & 0.41 & 877.47 & 100.6 & 0.0045 & 36.86 & 1250 & 422.5 \\
\hline$\frac{74}{746}$ & 96.37 & 2.14 & 0.12 & 0.05 & $0 . \overline{9}$ & 0.41 & 1102.6 & 137 & 0.0045 & 46.65 & 1257 & 572.4 \\
\hline$-\frac{746}{747}$ & 96.37 & 2.14 & 0.12 & 0.05 & 0.92 & 0.41 & 1134.4 & 139.4 & 0.0044 & 47.62 & 1255 & 583.6 \\
\hline $\begin{array}{r}747 \\
748\end{array}$ & 96.37 & 2.14 & 0.12 & 0.05 & 0.92 & 0.41 & 1115.8 & 134.4 & 0.0045 & 47.02 & $125 \overline{2}$ & 563.9 \\
\hline$\frac{748}{749}$ & 96.45 & 2.14 & 0.11 & 0.03 & 0.88 & 0.41 & 1766.9 & 216.1 & 0.0044 & 69.81 & 1253 & 906.3 \\
\hline 749 & 96.45 & 2.14 & 0.11 & 0.03 & 0.88 & 0.41 & 1775.4 & 212.4 & 0.0044 & 70.25 & 1247 & 894.4 \\
\hline 750 & 96.45 & 2.14 & 0.11 & 0.03 & 0.88 & 0.41 & 1776.5 & 207.9 & 0.0045 & 70.6 & 1253 & 871.3 \\
\hline 751 & 96.45 & 2.14 & 0.11 & 0.03 & 0.88 & 0.41 & 1956.4 & 232.9 & 0.0044 & 75.6 & 1238 & 988.2 \\
\hline 752 & 96.45 & 2.14 & 0.11 & 0.03 & 0.88 & 0.41 & 1957.8 & 235.1 & 0.0044 & 76.49 & 1246 & 990.9 \\
\hline 753 & 96.45 & 2.14 & 0.11 & 0.03 & 0.88 & 0.41 & 1981.6 & 231.7 & 0.0044 & 77.93 & 1248 & 974.8 \\
\hline 754 & 96.45 & 2.14 & 0.11 & 0.03 & 0.88 & 0.41 & 2213.3 & 260.3 & 0.0044 & 83.84 & 1250 & 1094.1 \\
\hline 755 & 96.45 & 2.14 & 0.11 & 0.03 & 0.88 & 0.41 & 2184.8 & 251.9 & 0.0044 & 82.51 & 1245 & 1063 \\
\hline 756 & 96.45 & 2.14 & 0.11 & 0.03 & 0.88 & 0.41 & 2179.8 & 241.7 & 0.0044 & 83.19 & 1233 & 1029.9 \\
\hline 757 & 96.45 & 2.14 & 0.11 & 0.03 & 0.88 & 0.41 & 2160.9 & 266.6 & 0.0044 & 84.69 & 1245 & 1124.1 \\
\hline 758 & 96.46 & 2.14 & 0.1 & 0.03 & 0.88 & 0.4 & 2159.8 & 259.3 & 0.0044 & 84.64 & 1232 & 1105.1 \\
\hline 759 & 96.46 & .2 .14 & 0.1 & 0.03 & 0.88 & 0.4 & 2158.8 & 255.5 & 0.0044 & 84.63 & 1244 & 1078.5 \\
\hline
\end{tabular}


Recorded Data

\begin{tabular}{|c|c|c|c|c|c|c|c|c|c|c|c|c|}
\hline $\begin{array}{c}\text { Run } \\
\text { Number }\end{array}$ & Eq Ra & Equiv 02 & $\begin{array}{c}\text { Ign Tim } \\
\text { (deg) }\end{array}$ & BTE (\%) & $\begin{array}{l}\text { MAP } \\
\text { (psia) }\end{array}$ & $\begin{array}{c}\text { Boost AF } \\
\text { IC (deg F) }\end{array}$ & $\begin{array}{l}\text { Pre- } \\
\text { Turbine } \\
\text { (psig) }\end{array}$ & $\begin{array}{c}\text { Comp Out } \\
\text { (psig) }\end{array}$ & $\begin{array}{l}\text { MAP } \\
\text { (psig) }\end{array}$ & $\mathrm{O} 2$ (volts) & $\begin{array}{l}\text { Time } \\
\text { (sec) }\end{array}$ & $\begin{array}{c}\text { Date } \\
\text { (julian) }\end{array}$ \\
\hline$\frac{734}{735}$ & 0.684 & 6.527 & 16 & 35.7 & 24.32 & 102 & 7.93 & 11.89 & 10.02 & 0.023 & $50 \overline{377}$ & 45 \\
\hline$\frac{73}{73} \frac{5}{6}$ & 0.666 & 6.523 & 22.5 & 37.4 & 30.31 & 107 & 11.9 & 17.67 & 16.02 & 0.024 & 51213 & 45 \\
\hline$\frac{73}{73} \frac{6}{7}$ & $\begin{array}{l}0.664 \\
0.663\end{array}$ & 6.469 & 19.5 & 36.7 & 30.41 & 108 & 11.98 & 17.72 & 16.11 & 0.038 & 51541 & 45 \\
\hline 737 & 0.663 & 6.472 & 16.5 & 35.5 & 30.44 & 108 & 12.03 & 17.81 & 16.14 & 0.037 & 51812 & 45 \\
\hline 738 & 0.684 & 6.509 & 21 & 37.6 & 30.24 & 106 & 11.85 & 17.53 & 15.95 & 0.027 & 52927 & 45 \\
\hline 739 & 0.679 & 6.5 & 21 & 38 & 30.03 & 104 & 11.83 & 17.35 & 15.75 & 0.03 & 55613 & 45 \\
\hline 740 & 0.68 & 6.463 & 18 & 36.9 & 29.71 & 105 & 11.59 & 16.9 & 15.43 & 0.039 & 55963 & 45 \\
\hline $7 \overrightarrow{4}$ & 0.687 & 6.466 & 15 & 356 & 30.54 & 107 & 12.23 & 17.95 & 16.26 & 0.039 & $56 \overline{314}$ & 45 \\
\hline $7 \overrightarrow{42}$ & 0.706 & 6.528 & 25.5 & 34.5 & 15.05 & 98 & 2.98 & 4.06 & 0.77 & 0.022 & 57248 & $4 \overline{5}$ \\
\hline 743 & 0.708 & 6.44 & 22.5 & 33.9 & 15.19 & 99 & 3.1 & 4.29 & 0.92 & 0.045 & 57651 & 45 \\
\hline $74 \overline{4}$ & 0.7 & 6.483 & $19 . \overline{5}$ & 33.5 & 15.38 & 98 & 3.23 & 4.54 & 1.1 & 0.034 & $57 \overline{90}$ & 45 \\
\hline $7 \overrightarrow{4}$ & 0.705 & 6.509 & 23.5 & 36.1 & 18.19 & 98 & 4.52 & 6.76 & 3.91 & 0.027 & $58 \overline{27}$ & $4 \overline{5}$ \\
\hline 746 & 0.699 & 6.495 & 20.5 & 36 & 18.54 & 95 & 4.71 & 7.19 & 4.26 & 0.031 & 58811 & 45 \\
\hline 747 & $\overrightarrow{0} 7 \overrightarrow{02}$ & $6.45 \overline{5}$ & 17.5 & 35.1 & 18.35 & 97 & 4.66 & 7.2 & 4.07 & 0.041 & 59178 & $4 \overline{5}$ \\
\hline 748 & 0.659 & 6.457 & 23 & 38 & 27.25 & 101 & 8.86 & 14.16 & 12.98 & 0.041 & 60165 & 45 \\
\hline 749 & 0.66 & 6.476 & 20 & 37.1 & 27.26 & 101 & 8.89 & 14.16 & 12.99 & 0.036 & 60474 & $4 \overline{5}$ \\
\hline 750 & 0.663 & 6.498 & 17 & 36.1 & 27.2 & 100 & 8.82 & 14.08 & 12.93 & 0.03 & 60769 & 45 \\
\hline 751 & 0.644 & 6.487 & 22 & 37.8 & 29.96 & 103 & 10.51 & 16.83 & 15.69 & 0.033 & 61338 & 45 \\
\hline 752 & 0.651 & 6.496 & 19 & 37.7 & 29.86 & 102 & 10.4 & 16.75 & 15.58 & 0.031 & 61585 & $4 \overline{5}$ \\
\hline 753 & 0.656 & 6.454 & 16 & 36.5 & 30.2 & 103 & 10.61 & 17.07 & 15.92 & 0.042 & 61925 & 45 \\
\hline 754 & 0.631 & 6.49 & 23 & 38.1 & 33.45 & 106 & 12.78 & 20.34 & 19.18 & 0.032 & 62380 & 45 \\
\hline 755 & 0.63 & 6.541 & 20 & 37.5 & 33.08 & 103 & 12.48 & 19.96 & 18.8 & 0.019 & 62586 & 45 \\
\hline 756 & 0.636 & 6.485 & 17 & 35.7 & 33.15 & 105 & 12.49 & 20.05 & 18.88 & 0.034 & 62786 & 45 \\
\hline 757 & 0.653 & 6.514 & 22 & 38.6 & 33.13 & 104 & 12.45 & 19.97 & 18.86 & 0.026 & 63033 & 45 \\
\hline 758 & 0.653 & .6 .484 & 19 & 37.6 & 33.05 & 104 & 12.34 & 19.97 & 18.77 & 0.034 & 63237 & 45 \\
\hline $75 \overrightarrow{9}$ & 0.654 & 6.538 & 16 & 37.1 & 32.97 & 104 & 12.24 & 19.88 & 18.69 & 0.02 & 63438 & 45 \\
\hline
\end{tabular}


Recorded Data

\begin{tabular}{|c|c|c|c|c|c|c|c|c|c|c|c|c|}
\hline $\begin{array}{c}\begin{array}{c}\text { Run } \\
\text { Number }\end{array} \\
\end{array}$ & Year & $\begin{array}{c}\text { BSFC } \\
\text { (\#/bhp-hr) }\end{array}$ & $\begin{array}{c}\text { BMEP } \\
\text { (psi) }\end{array}$ & $\begin{array}{c}\begin{array}{r}\text { Vol Eff } \\
(\%)\end{array} \\
\end{array}$ & $\begin{array}{c}\text { Intake Air } \\
\text { (acfm) }\end{array}$ & $\begin{array}{c}\text { Intake Air } \\
\text { (scfm) }\end{array}$ & $\mid \begin{array}{c}\text { Boost B4 } \\
\text { IC (deg F) }\end{array}$ & $\begin{array}{c}\text { Stack } \\
\text { (deg F) }\end{array}$ & $\begin{array}{c}\text { Pre- } \\
\text { Turbine } \\
\text { (deg F) }\end{array}$ & $\begin{array}{l}\text { Fuel } \\
\text { Press } \\
\text { (psig) }\end{array}$ & EBP $(" \mathrm{Hg})$ & Baro ("Hg) \\
\hline $7 \frac{734}{735}$ & 1996 & 0.348 & 159.96 & 90 & 437 & 411 & 218 & 1013 & 1162 & 84.7 & 0.85 & 29.11 \\
\hline$\frac{735}{736}$ & 1996 & 0.332 & 205.52 & 91.5 & 549 & 517 & 265 & 946 & 1125 & 83.4 & 1.29 & 29.1 \\
\hline 736 & 1996 & 0.338 & 201.75 & 91.7 & 551 & 520 & 264 & 968 & 1148 & 83.4 & 1.34 & 29.1 \\
\hline 737 & 1996 & 0.349 & 196.63 & 92.4 & 559 & 526 & 267 & 996 & 1175 & 83.4 & 1.39 & 29.1 \\
\hline 738 & 1996 & 0.327 & 212.35 & 91.8 & 551 & 516 & 266 & 968 & 1149 & 83 & 1.33 & 29.09 \\
\hline 739 & 1996 & 0.324 & 211.77 & 91.6 & 551 & 515 & 265 & 964 & 1143 & 83.3 & 1.3 & 29.08 \\
\hline 740 & 1996 & 0.334 & 203.54 & 91.6 & 544 & 507 & 263 & 985 & 1161 & 83.4 & 1.29 & 29.08 \\
\hline 741 & 1996 & 0.345 & 203.13 & 91.7 & 561 & 523 & 270 & 1020 & 1203 & 83 & 1.44 & 29.07 \\
\hline 742 & 1996 & 0.356 & 87.79 & 79.6 & 202 & 189 & 140 & 871 & 977 & 89 & 0.23 & 29.07 \\
\hline 743 & 1996 & 0.362 & 88 & 80.3 & 206 & 192 & 143 & 885 & 994 & 88.9 & 0.24 & 29.07 \\
\hline 744 & 1996 & 0.366 & 87.53 & 80.5 & 210 & 195 & 146 & $90 \overline{0}$ & 1014 & 88.8 & 0.24 & 29.07 \\
\hline 745 & 1996 & 0.341 & 118.57 & 85.1 & 263 & 245 & 171 & 896 & 1019 & 88.2 & 0.33 & 29.07 \\
\hline 746 & 1996 & 0.342 & 120.89 & 85.6 & 271 & 253 & 177 & 908 & 1034 & 88.2 & 0.35 & 29.07 \\
\hline 747 & 1996 & 0.35 & 116.8 & 85.6 & 267 & 248 & 178 & 925 & 1053 & 88.2 & 0.35 & 29.06 \\
\hline 748 & 1996 & 0.323 & 187.73 & 91.4 & 422 & 393 & 242 & 891 & 1048 & 85.3 & 0.76 & 29.06 \\
\hline 749 & 1996 & 0.331 & 185.28 & 92.3 & 424 & 395 & 242 & 910 & 1068 & 85.3 & 0.78 & 29.06 \\
\hline 750 & 1996 & 0.34 & 180.49 & 92 & 425 & 395 & 242 & 935 & 1092 & 85.5 & 0.8 & 29.06 \\
\hline $7 \overline{51}$ & 1996 & 0.325 & 204.7 & 93.5 & 467 & 436 & 264 & 890 & 1062 & 85.2 & 0.92 & 29.06 \\
\hline 752 & 1996 & 0.325 & 205.27 & 93.1 & 467 & 436 & 264 & 910 & 1083 & 84.9 & 0.93 & 29.06 \\
\hline 753 & 1996 & 0.336 & 201.94 & 93.3 & 473 & 441 & 267 & 940 & 1113 & 84.8 & 0.98 & 29.06 \\
\hline 754 & 1996 & 0.322 & 226.65 & 94.2 & 530 & 493 & 293 & 870 & 1063 & 84.1 & 1.14 & 29.06 \\
\hline 755 & 1996 & 0.327 & 220.2 & 93.9 & 524 & 486 & 292 & 890 & 1082 & 84.3 & 1.14 & 29.06 \\
\hline 756 & 1996 & 0.344 & 213.34 & 94.7 & 523 & 485 & 291 & 919 & 1108 & 84.2 & 1.17 & 29.06 \\
\hline 757 & 1996 & 0.318 & 232.86 & 93.1 & 518 & 481 & 292 & 888 & 1079 & 84.1 & 1.12 & 29.06 \\
\hline 758 & 1996 & 0.326 & 228.92 & 94.2 & 517 & 481 & 290 & 905 & 1097 & 84.1 & 1.13 & 29.06 \\
\hline 759 & 1996 & 0.331 & 223.42 & 93.5 & 515 & 481 & 289 & 933 & 1122 & 84.2 & 1.15 & 29.06 \\
\hline
\end{tabular}


Recorded Data

\begin{tabular}{|c|c|c|c|c|c|c|c|c|c|c|c|c|}
\hline $\begin{array}{c}\text { Run } \\
\text { Number }\end{array}$ & $\mathrm{RH}(\%)$ & Density & Stioch A/F & $\underset{\mathrm{Ra}}{\operatorname{GEM} \mathrm{Eq}}$ & $\begin{array}{c}\text { GEM Vol } \\
\text { Eff }(\%)\end{array}$ & $\begin{array}{l}\text { Exh \#1 } \\
(\operatorname{deg} F)\end{array}$ & $\begin{array}{l}\text { Exh \#2 } \\
(\operatorname{deg} F)\end{array}$ & $\begin{array}{l}\text { Exh \#3 } \\
(\operatorname{deg} F)\end{array}$ & $\begin{array}{l}\text { Exh \#4 } \\
(\operatorname{deg} F)\end{array}$ & $\begin{array}{l}\text { Exh \#5 } \\
(\operatorname{deg} F)\end{array}$ & $\begin{array}{l}\text { Exh \#6 } \\
(\operatorname{deg} F)\end{array}$ & $\begin{array}{l}\text { Water In } \\
\text { (deg F) }\end{array}$ \\
\hline 734 & 23.92 & 0.288 & 16.48 & 0.66 & 81 & 1072 & 1057 & 1053 & 1072 & 1048 & 1013 & 158 \\
\hline 735 & 23.03 & 0.287 & 16.48 & 0.64 & 82.6 & 1047 & 1040 & 1032 & 1043 & 1021 & 989 & 155 \\
\hline 736 & 22.45 & 0.288 & 16.48 & 0.64 & 82.6 & 1074 & 1063 & 1054 & 1066 & 1043 & 1007 & 155 \\
\hline 737 & 22.31 & 0.289 & 16.48 & 0.64 & 82.6 & 1104 & 1090 & 1083 & 1093 & 1068 & 1030 & 156 \\
\hline 738 & 21.85 & 0.284 & 16.62 & 0.66 & 82.6 & 1066 & 1060 & 1051 & 1067 & 1045 & 1011 & 157 \\
\hline 739 & 19.68 & 0.284 & 16.62 & 0.66 & 82.6 & 1063 & 1056 & 1050 & $\cdot 1060$ & 1038 & 1007 & 156 \\
\hline 740 & 18.83 & 0.284 & 16.62 & 0.66 & 82.6 & 1081 & 1072 & 1062 & 1076 & 1053 & 1020 & 156 \\
\hline $74 \overrightarrow{1}$ & 18.77 & 0.282 & 16.65 & 0.66 & 82.6 & 1121 & 1112 & 1102 & 1118 & 1093 & 1056 & 157 \\
\hline 742 & 18.53 & 0.293 & 16.65 & 0.66 & 71.8 & 922 & 877 & 890 & 892 & 866 & 864 & 115 \\
\hline $74 \overline{3}$ & 18.44 & 0.291 & 16.65 & 0.66 & 71.8 & 940 & 893 & 907 & 908 & $880^{\circ}$ & 874 & 114 \\
\hline $7 \overrightarrow{4}$ & 18.14 & 0.29 & 16.65 & 0.66 & 71.8 & 960 & $90 \overline{9}$ & 924 & $92 \overline{3}$ & 896 & 891 & 114 \\
\hline 745 & 18.33 & 0.287 & 16.65 & 0.66 & 78.9 & 944 & 916 & 923 & 928 & 903 & 890 & 124 \\
\hline 746 & 18.07 & 0.287 & 16.65 & 0.66 & 78.9 & 958 & 930 & 936 & 943 & 918 & 902 & 126 \\
\hline 747 & $1 \overline{8} .1 \overline{4}$ & -0.287 & 16.65 & 0.66 & 78.9 & 978 & 948 & 954 & 960 & 935 & 918 & 128 \\
\hline 748 & 18.16 & 0.283 & 16.67 & 0.63 & 82.8 & 966 & 956 & 948 & 965 & 941 & 910 & 139 \\
\hline 749 & 18.07 & 0.283 & 16.67 & 0.63 & 82.8 & 984 & 974 & 968 & 983 & 958 & 924 & 140 \\
\hline 750 & 18.13 & 0.283 & 16.67 & 0.63 & 82.8 & 1008 & 999 & 986 & 1008 & 980 & 945 & 141 \\
\hline 751 & 18.11 & 0.284 & 16.67 & 0.63 & 84.4 & 981 & 975 & 965 & 979 & 955 & 921 & 144 \\
\hline 752 & 18.22 & 0.284 & 16.67 & 0.63 & 84.4 & 1001 & 997 & 985 & 1000 & 976 & 937 & 146 \\
\hline 753 & 18.19 & 0.284 & 16.67 & 0.63 & 84.4 & 1028 & 1024 & 1016 & 1029 & 1005 & 963 & 147 \\
\hline 754 & 18.25 & 0.282 & 16.67 & 0.61 & 85.7 & 991 & 989 & 978 & 986 & 962 & 928 & 150 \\
\hline 755 & 18.22 & 0.283 & 16.67 & 0.61 & 85.7 & 1010 & 1007 & 998 & 1003 & 979 & 942 & $15 \overline{1}$ \\
\hline 756 & 17.97 & 0.282 & 16.67 & 0.61 & 85.7 & 1039 & 1034 & 1024 & 1028 & 1005 & 963 & 151 \\
\hline$\overline{7} 5 \overline{7}$ & 17.99 & 0.282 & 16.67 & 0.63 & 85.7 & 999 & 1001 & 990 & 1000 & 977 & 942 & 152 \\
\hline 758 & 18.03 & 0.282 & 16.67 & 0.63 & 85.7 & 1019 & 1017 & 1006 & 1016 & 994 & 956 & 153 \\
\hline 759 & 18.12 & 0.283 & 16.67 & 0.63 & 85.7 & 1042 & 1041 & 1031 & 1039 & 1017 & 974 & 153 \\
\hline
\end{tabular}


Recorded Data

\begin{tabular}{|c|c|c|c|c|c|c|c|c|c|c|c|c|}
\hline $\begin{array}{c}\text { Run } \\
\text { Number }\end{array}$ & $\begin{array}{c}\text { Water Out } \\
\text { (deg F) }\end{array}$ & $\begin{array}{c}\text { Oil Sump } \\
\text { (deg F) }\end{array}$ & $\begin{array}{c}\text { Oil } \\
\text { Gallery } \\
\text { (deg F) }\end{array}$ & $\begin{array}{c}\text { Intake @ } \\
\text { LFE (deg } \\
\text { F) }\end{array}$ & $\begin{array}{c}\begin{array}{c}\text { Oil Press } \\
\text { (psig) }\end{array} \\
\end{array}$ & $\begin{array}{c}\text { Fuel Flow } \\
\text { (@ RT } \\
\text { Meter) }\end{array}$ & $\begin{array}{c}\text { Inlet Rest } \\
\text { ('H2O) }\end{array}$ & HC (ppm) & $\mathrm{CO}(\mathrm{ppm})$ & $\mathrm{CO} 2(\%)$ & $\begin{array}{c}\text { NOx } \\
(\mathrm{ppm})\end{array}$ & $02(\%)$ \\
\hline 734 & 184 & 218 & 101 & 79 & 40.5 & 1.4121 & 6.28 & 1283 & 434.1 & 7.78 & 716.8 & 7.45 \\
\hline 735 & 183 & 220 & 105 & 78 & 39.2 & 1.7346 & 8.62 & 1206 & 417.4 & 8.27 & 641.9 & 6.65 \\
\hline 736 & $18 \overline{3}$ & 220 & 105 & 77 & 39.4 & 1.7356 & 8.74 & 1837 & 397.3 & 7.7 & 1671.6 & 7.54 \\
\hline 737 & $18 \overline{3}$ & 220 & 105 & 78 & 39.7 & 1.747 & 8.78 & 1647 & 418.8 & 7.72 & 1321.7 & 7.51 \\
\hline 738 & 185 & 223 & 103 & 79 & 38.5 & 1.7869 & 8.55 & 1234 & 404 & 8 & 1259.4 & 7.05 \\
\hline 739 & 182 & 221 & 102 & 80 & 39.1 & 1.767 & 8.56 & 1095 & 384.9 & 8.39 & 872.8 & 6.44 \\
\hline 740 & 183 & 220 & 102 & 81 & 39 & 1.7413 & 8.54 & 2236 & 374.5 & 7.64 & 1620 & 7.6 \\
\hline 741 & 183 & 220 & 104 & 81 & 39.5 & 1.8223 & 8.79 & 1945 & 352.2 & 7.97 & 1984.1 & 7.08 \\
\hline 742 & 179 & 205 & 100 & 81 & 36.5 & 0.6513 & 2.45 & $1 \overline{29}$ & 333.6 & 8.33 & 2205.2 & 6.48 \\
\hline 743 & $17 \overline{9}$ & 204 & 101 & 81 & 36.7 & 0.6692 & 2.48 & 991 & 342.9 & 8.64 & 1148.8 & 6 \\
\hline 744 & 180 & 203 & 100 & 81 & 36.9 & 0.6763 & 2.52 & 2264 & 203.6 & 8.53 & 3227.7 & 6.11 \\
\hline $7 \overline{4}$ & $18 \overline{2}$ & $20 \overline{7}$ & 99 & 81 & 35.4 & 0.8638 & 3.32 & 1850 & 194.9 & 8.73 & 3231.7 & 5.79 \\
\hline 746 & 182 & 208 & 98 & 81 & 35.1 & 0.882 & 3.41 & 832 & 226.1 & 8.84 & 2627.8 & 5.59 \\
\hline 747 & 182 & 208 & 99 & 82 & 35.2 & 0.87 & 3.38 & 650 & 258.6 & 9.21 & 1175 & 5.02 \\
\hline 748 & 182 & 213 & 100 & 81 & 33.1 & 1.3107 & 5.88 & 2466 & 140.7 & 8.74 & 3875.9 & 5.7 \\
\hline 749 & 182 & 214 & 100 & 81 & 33.2 & 1.3193 & 5.96 & 1715 & 149.4 & 8.9 & 3542.8 & 5.45 \\
\hline 750 & 183 & 213 & 100 & 81 & 33.3 & 1.3239 & 6.02 & 665 & 188.8 & 9.21 & 2559.8 & 4.93 \\
\hline 751 & 181 & 215 & 102 & 80 & 32.5 & 1.4123 & 6.86 & 603 & 226 & 9.62 & 1254.2 & 4.3 \\
\hline 752 & 182 & 215 & 100 & 80 & 32.5 & 1.4306 & 6.81 & 693 & 183.1 & 9.46 & 3343.1 & 4.54 \\
\hline 753 & 183 & 215 & 102 & 80 & 32.5 & 1.4597 & 7.01 & 713 & 213.3 & 9.66 & 2112.5 & 4.23 \\
\hline 754 & 184 & 216 & 104 & 81 & 32.2 & 1.5795 & 8.11 & 692 & 229.6 & 9.98 & 1003.9 & 3.63 \\
\hline 755 & 184 & 217 & 103 & 82 & 31.8 & 1.5503 & 7.98 & 730 & 249.9 & 10.22 & 418.9 & 3.2 \\
\hline 756 & 184 & 217 & 103 & 82 & 32 & 1.5654 & 7.99 & 1130 & 538.8 & 8.76 & 656.4 & 5.85 \\
\hline 757 & 185 & 218 & 103 & 81 & 31.7 & 1.5946 & 7.82 & 1055 & 475.1 & 8.68 & 446.6 & 5.97 \\
\hline 758 & 185 & 218 & 103 & 81 & 31.5 & 1.5932 & 7.9 & 1016 & 399.9 & 8.66 & 408.3 & 6 \\
\hline 759 & 185 & 219 & 103 & 80 & 31.4 & 1.5893 & 7.8 & 789 & 367 & 8.99 & 256.3 & 5.43 \\
\hline
\end{tabular}


APPENDIX C

FINAL CALIBRATION

325 bhp 
GEM Lean Burn Control System - Drive-By-Wire Parameters

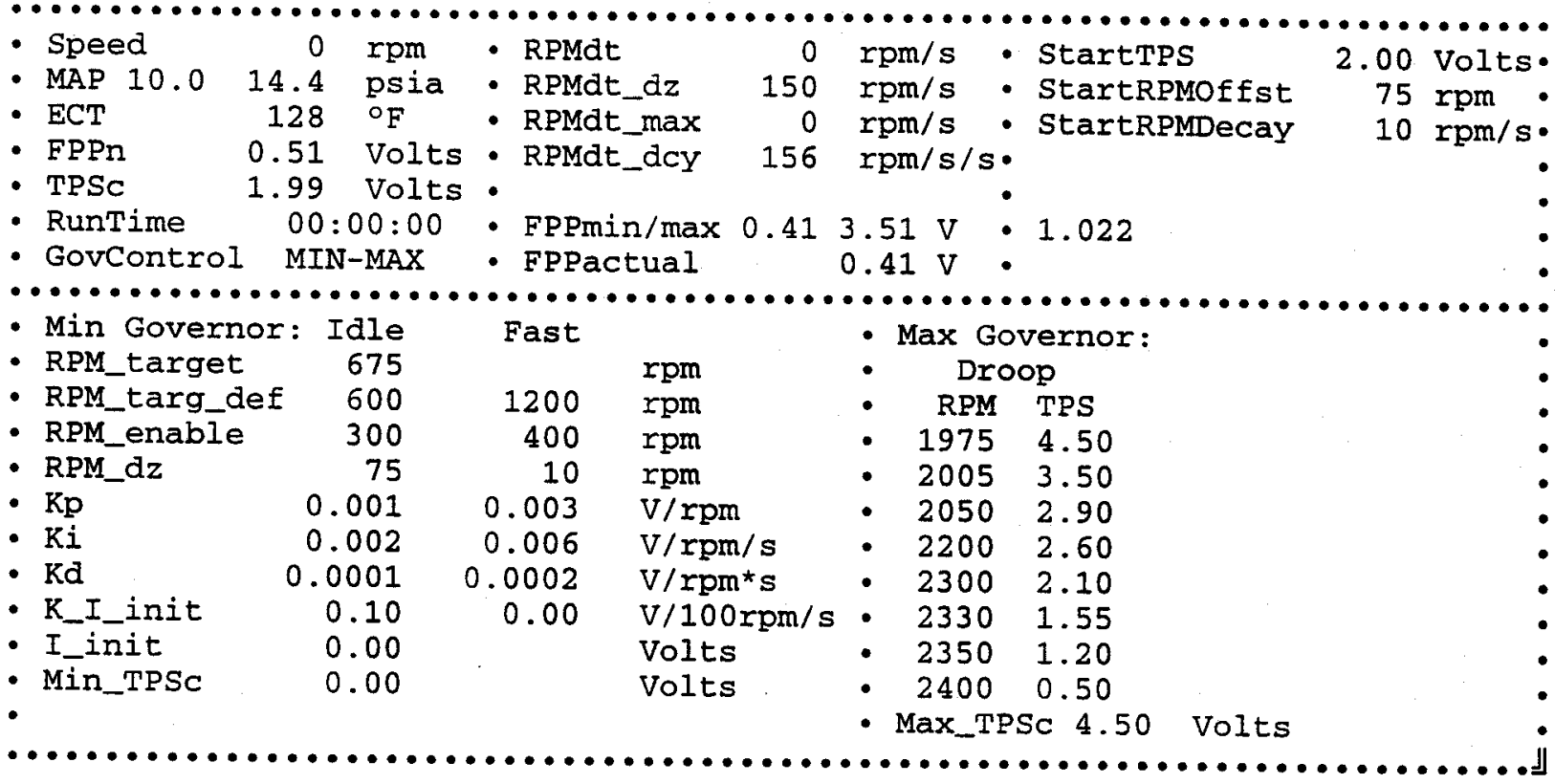


GEM Lean Burn Control System - ECT Based Modification Tables

\begin{tabular}{|c|c|c|c|c|c|c|c|}
\hline $\begin{array}{l}\text { - } \quad \text { ECT } \\
\text { - } \quad \text { OF })\end{array}$ & $\begin{array}{c}\text { Idle offset } \\
\text { (rpm) }\end{array}$ & $\begin{array}{l}\text { Adv Offset } \\
\text { (CAD BTDC) }\end{array}$ & $\begin{array}{c}\text { Max Boost } \\
\text { (psia) }\end{array}$ & $\begin{array}{c}\text { Phi Cold } \\
(q)\end{array}$ & - ECT & 128 & $\circ \mathrm{F}$ \\
\hline - -40 & 50 & +5.0 & 30.0 & 100 & - Speed & 0 & rpm \\
\hline-20 & 50 & +5.0 & 30.0 & 100 & - Idle & 675 & rpm \\
\hline 0 & 50 & +5.0 & 30.0 & 100 & - Idle_def & 600 & rpm \\
\hline 32 & 50 & +5.0 & 30.0 & 100 & - & & -20 \\
\hline 50 & 50 & +5.0 & 30.0 & 75 & - IgnAdv & 5.0 & ${ }^{\circ} \mathrm{BTDC}$ \\
\hline 70 & 50 & +3.0 & 30.0 & 75 & - Ignoffst & +0.0 & OBTDC \\
\hline 90 & 50 & +0.0 & 30.0 & 60 & - & & 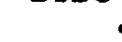 \\
\hline 110 & 50 & +0.0 & 32.1 & 50 & - MAP & 14.4 & psia \\
\hline 130 & 50 & +0.0 & 35.1 & 30 & - Max_boost & 15.1 & psia \\
\hline - $\quad 155$ & 0 & +0.0 & 35.1 & 30 & - Max_boost_s & 15.1 & psia \\
\hline 160 & 0 & +0.0 & 45.0 & 0 & - & & \\
\hline - 190 & 0 & +0.0 & 45.0 & 0 & - PhiECT & 32 & 8 \\
\hline - $\quad 200$ & 0 & +0.0 & 45.0 & 0 & - & & \\
\hline - $\quad 210$ & 0 & +0.0 & 45.0 & 0 & - & & \\
\hline - $\quad 220$ & 0 & +0.0 & 45.0 & 0 & - & & \\
\hline 230 & 0 & +0.0 & 45.0 & 0 & - & & \\
\hline
\end{tabular}


GEM Lean Burn Control System - Miscellaneous Calibrations

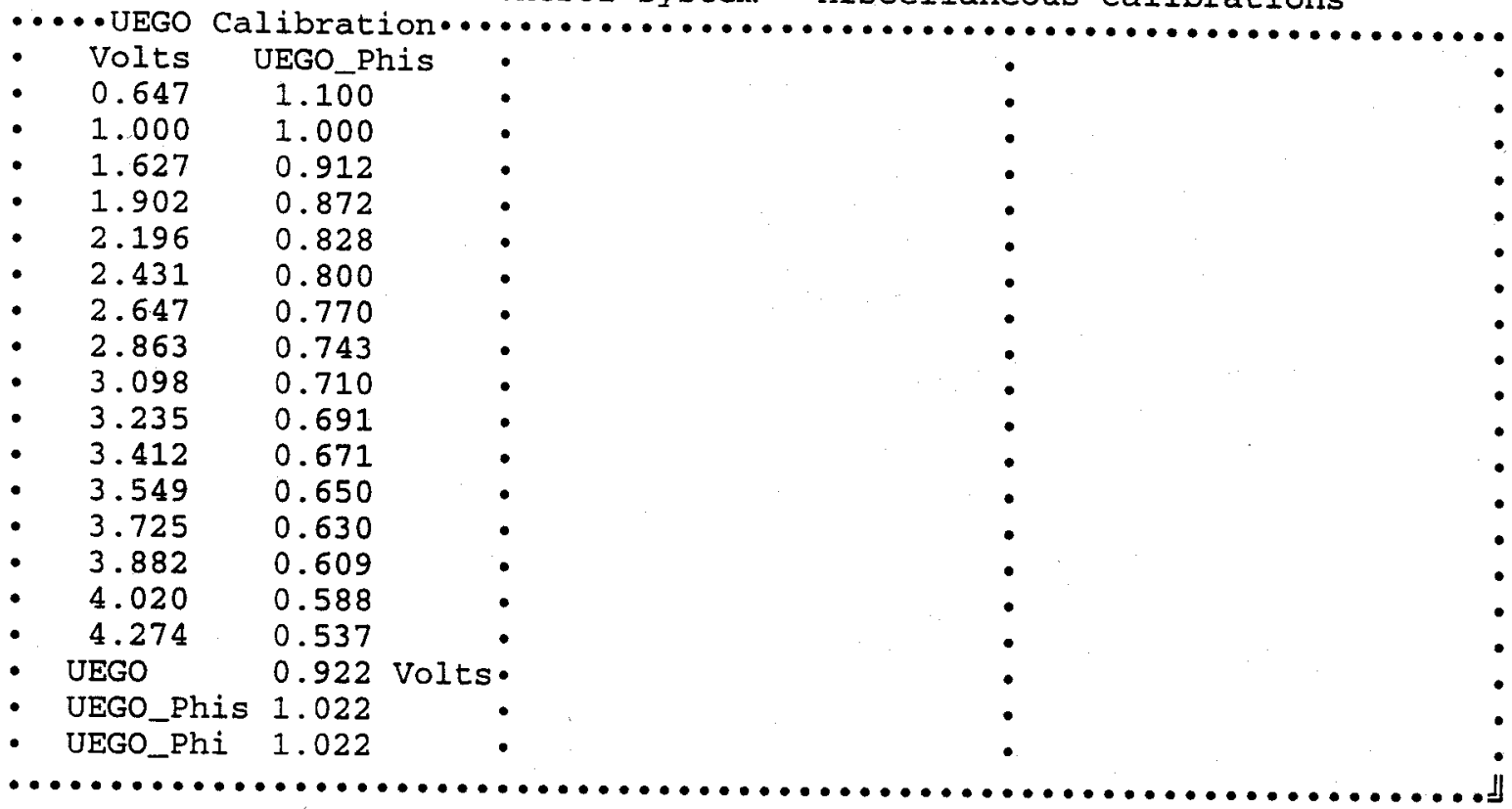


GEM Lean Burn Control System - Closed Loop Activation Map
MAP (psia) $\begin{array}{llllllllll}0.00 & & 5.96 & 11.92 & 18.05 & 24.01 & 29.97 & 35.23 & 42.06\end{array}$

\begin{tabular}{|c|c|c|c|c|c|c|c|c|c|c|c|c|c|c|c|c|}
\hline & & 4.0 & & 8.94 & & 14.72 & & 21.03 & & 26.99 & & 32.95 & & 38.03 & & 45.04 \\
\hline 0 & 0 & 0 & 0 & 0 & 0 & 0 & 0 & 0 & 0 & 0 & 0 & 0 & 0 & 0 & 0 & 0 \\
\hline 200 & 0 & 0 & 0 & 0 & 0 & 0 & 0 & 0 & 0 & 0 & 0 & 0 & 0 & 0 & 0 & 0 \\
\hline 400 & 0 & 0 & 1 & 1 & 1 & 1 & 1 & 1 & 1 & 1 & 1 & 1 & 1 & 1 & 0 & 0 \\
\hline 600 & 0 & 0 & 1 & 1 & 1 & 1 & 1 & 1 & 1 & 1 & 1 & 1 & 1 & 1 & 0 & 0 \\
\hline 800 & 0 & 0 & 1 & 1 & 1 & 1 & 1 & 1 & 1 & 1 & 1 & 1 & 1 & 1 & 0 & 0 \\
\hline 1000 & 0 & 0 & 1 & 1 & 1 & 1 & 1 & 1 & 1 & 1 & 1 & 1 & 1 & 1 & 0 & 0 \\
\hline 1100 & 0 & 0 & 1 & 1 & 1 & 1 & 1 & 1 & 1 & 1 & 1 & 1 & 1 & 1 & 0 & 0 \\
\hline 1250 & 0 & 0 & 1 & 1 & 1 & 1 & 1 & 1 & 1 & 1 & 1 & 1 & 1 & 1 & 0 & 0 \\
\hline 1500 & 0 & 0 & 1 & 1 & 1 & 1 & 1 & 1 & 1 & 1 & 1 & 1 & 1 & 1 & 0 & 0 \\
\hline 1700 & 0 & 0 & 1 & 1 & 1 & 1 & 1 & 1 & 1 & 1 & 1 & 1 & 1 & 1 & 0 & 0 \\
\hline 1950 & 0 & 0 & 1 & 1 & 1 & 1 & 1 & 1 & 1 & 1 & 1 & 1 & 1 & 1 & 0 & 0 \\
\hline 2000 & 0 & 0 & 1 & 1 & 1 & 1 & 1 & 1 & 1 & 1 & 1 & 1 & 1 & 1 & 0 & 0 \\
\hline
\end{tabular}

- Speed

$0 \mathrm{rpm} \cdot \mathrm{CL}$ _select

- MAP 14.

- FSO_on $1150 \mathrm{rpm}$

- $\mathrm{Phi}$

0 $(0-1) \cdot$ Controlmode

- UEGO_Phi

1.000

- FSO_TPS_min

1.022

- Cl_Mult

- Adpt Mult

OPEN LOOP

$850 \mathrm{rpm}$

$4.51 \mathrm{~V}$

- Block

$+0.00$

$q$
$z$
$z$


GEM Lean Burn Control System - Manifold Filling Calibration

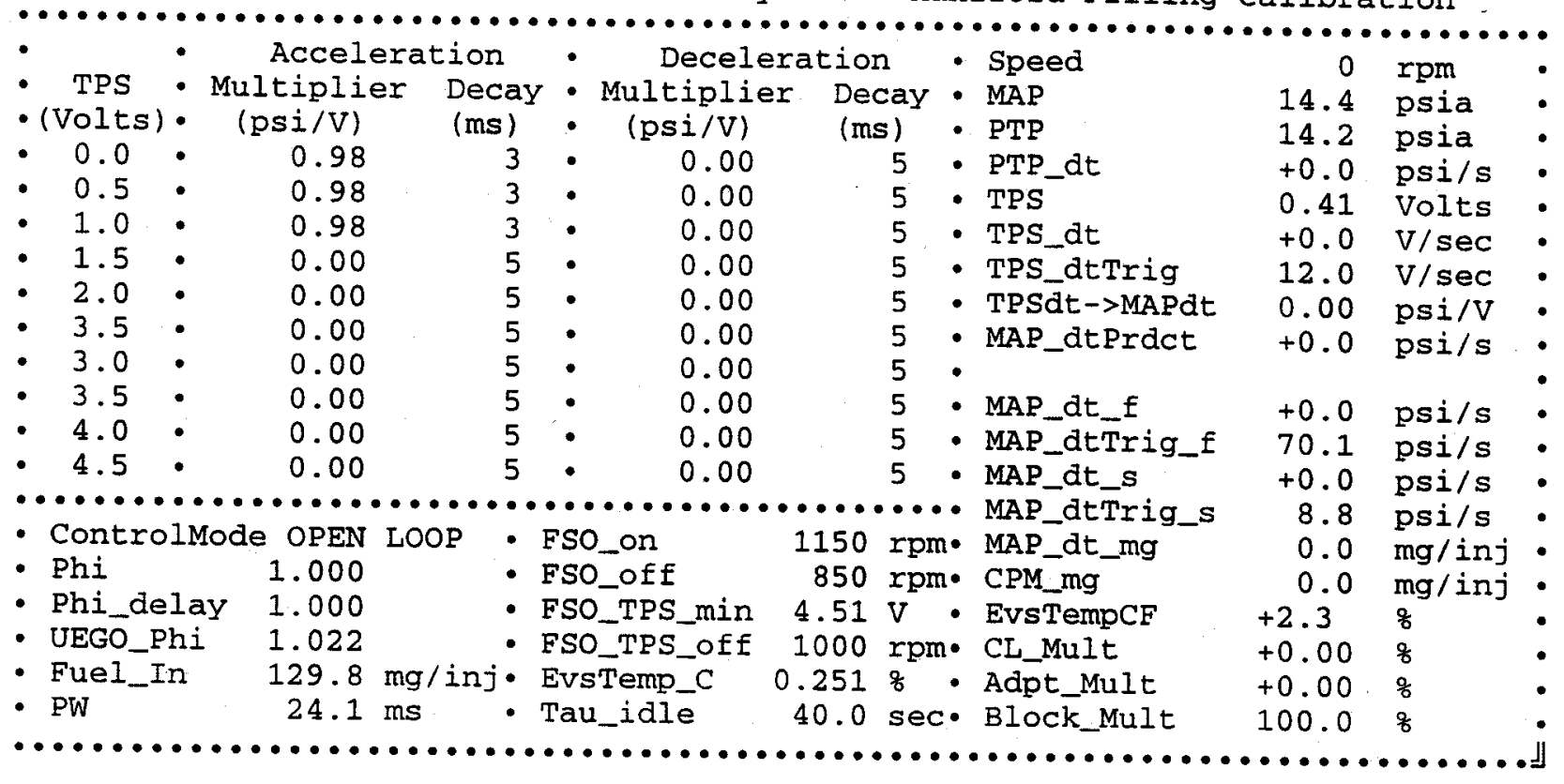




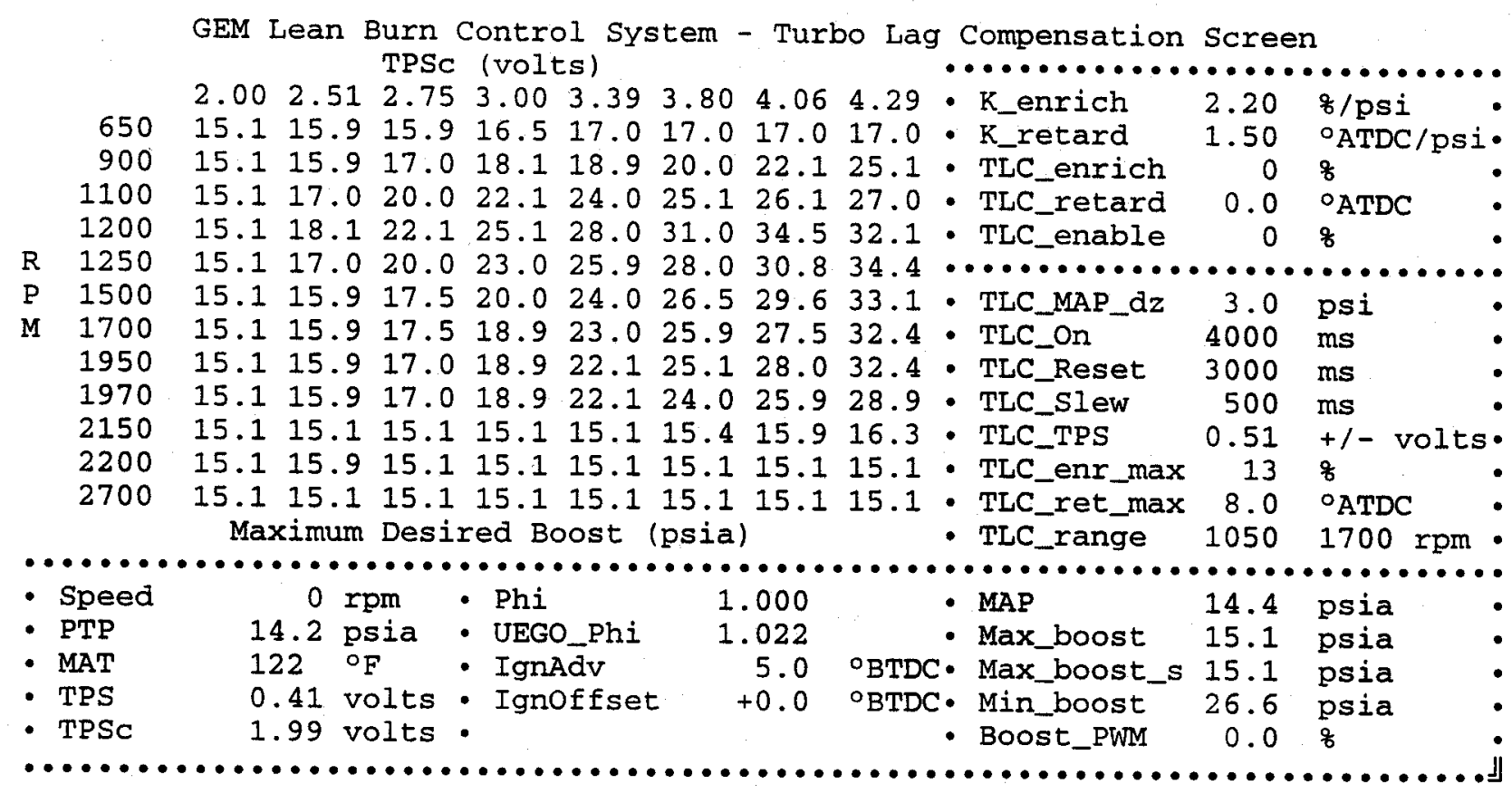




\begin{tabular}{|c|c|c|c|c|c|c|c|c|c|c|c|c|c|c|}
\hline & & & TPS & (volts & istem & - & $S E$ & $t$ & . & & $S$ & & & \\
\hline & .00 & 2.51 & 2.75 & 3.00 & 3.39 & 3.80 & 4.06 & 4.29 & - & $n$ & 1 & & $s / 0)$ & \\
\hline 650 & 15.1 & 15.9 & 15.9 & 16.5 & 17.0 & 17.0 & 17.0 & 17.0 & 15.1 & 80.0 & b & 1250 & 0.0 & \\
\hline & 1 & 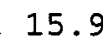 & 17.0 & 18.1 & 18.9 & 20.0 & 22.1 & 25.1 & .1 & 8 & b & 1700 & 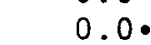 & \\
\hline 11 & & & 20.0 & 22.1 & 24.0 & 25.1 & 26.1 & 0 & 0 & & b & & 2.0 & \\
\hline & & & 22 & 25.1 & 28.0 & 31.0 & & 1 & & & b & $\angle 000$ & 2.0 & \\
\hline 12 & & & 20 & 23.0 & 25.9 & 28.0 & 30.8 & 4 & 9 & & •.. & $\mathrm{BP} \ldots$ & - Derate. & \\
\hline 15 & & & 17 & 20 & 24.0 & & 29 & & 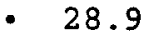 & & 0 & 10.0 & 5.0 & \\
\hline 1700 & & & 17.5 & 18.9 & 23.0 & 25.9 & 27 & 4 & 31.0 & 8 & 0 & 11.5 & 0.0 & \\
\hline 1950 & & & 17.0 & 18.9 & 22.1 & 25.1 & 28.0 & & 35.1 & 80 & & 0.0 & erat & \\
\hline $19^{\circ}$ & 1 & 15.9 & 17.0 & 18.9 & 22.1 & 24.0 & 25.9 & & $\bullet$ & & - & & & \\
\hline 2150 & 15.1 & 15.1 & 15.1 & 15.1 & 15.1 & 15.4 & 15.9 & 16.3 & - MAP & & 14.4 & $p s j$ & & \\
\hline 220 & & 15.9 & 15.1 & 15.1 & 15.1 & 15.1 & 15.1 & 15.1 & - Max_b & & 15.1 & si & & \\
\hline & 15 & 15.1 & 15.1 & 15.1 & 15.1 & 15.1 & 15.1 & 15.1 & - Max_b & t_s & 15.1 & si & & \\
\hline & $\mathbf{F}$ & & & & & & & & & & .6 & psi & & \\
\hline & & & & & 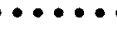 & & & & & & 0.8 & $\mathrm{dPr}$ & $\mathrm{ai} / \mathrm{d}$ & \\
\hline eed & & & & - Phi & & & .0 & & Kp_MA & & 12.00 & $8 / 5$ & osi & \\
\hline ?P & & & ia & - UEGO & _Phi & & .022 & & - Ki_MA & & 6.00 & $8 / \mathrm{k}$ & osi/se & \\
\hline MAT & & 122 & & - IgnA & $d v$ & & 5.0 & ${ }^{\circ} \mathrm{BTDC}$ & - Kd_MA. & & 2.00 & $8 / \bar{x}$ & osi*se & \\
\hline & & & ts & - Igno & ffs & & +0 & ${ }^{\circ} \mathrm{BTDC}$ & - Kd_PT & & 0.00 & $8 / 5$ & osi * & \\
\hline & & & & & & & & sia & ost & & 0.0 & $q$ & & \\
\hline
\end{tabular}


GEM Lean Burn Control System - COLD Phi Calibration Screen

MAP (psia)

$$
\begin{array}{llllllll}
0.00 & 5.96 & 11.92 & 18.05 & 24.01 & 29.97 & 35.23 & 42.06
\end{array}
$$

$\begin{array}{llllllll}4.03 & 8.94 & 14.72 & 21.03 & 26.99 & 32.95 & 38.03 & 45.04\end{array}$

$\begin{array}{lllllllllllllllll}0 & 100 & 100 & 100 & 100 & 100 & 100 & 100 & 100 & 100 & 100 & 100 & 100 & 100 & 100 & 100 & 100\end{array}$

$200 \quad 100100100100100100100100100 \quad 100100 \quad 100 \quad 100100100100$

$\begin{array}{lllllllllllllllll}400 & 95 & 95 & 95 & 95 & 95 & 90 & 83 & 83 & 83 & 83 & 83 & 83 & 83 & 83 & 83 & 80\end{array}$

$\begin{array}{lllllllllllllllll}600 & 95 & 95 & 95 & 95 & 95 & 90 & 80 & 79 & 78 & 78 & 78 & 78 & 78 & 78 & 78 & 70\end{array}$

$\begin{array}{llllllllllllllllll}\mathrm{R} & 800 & 95 & 95 & 95 & 95 & 95 & 90 & 80 & 79 & 78 & 78 & 73 & 73 & 71 & 71 & 71 & 68\end{array}$

$\begin{array}{llllllllllllllllll}\mathrm{P} & 1000 & 95 & 95 & 95 & 95 & 90 & 85 & 80 & 80 & 80 & 77 & 71 & 71 & 71 & 71 & 71 & 64\end{array}$

$\begin{array}{llllllllllllllllll}\text { M } & 1100 & 93 & 82 & 82 & 80 & 80 & 80 & 80 & 80 & 80 & 80 & 80 & 77 & 73 & 69 & 69 & 64\end{array}$

$\begin{array}{lllllllllllllllll}1250 & 93 & 82 & 77 & 74 & 72 & 69 & 67 & 67 & 67 & 67 & 67 & 67 & 67 & 67 & 67 & 64\end{array}$

$\begin{array}{lllllllllllllllll}1500 & 93 & 82 & 77 & 74 & 72 & 69 & 67 & 67 & 67 & 67 & 67 & 67 & 67 & 67 & 67 & 64\end{array}$

$\begin{array}{lllllllllllllllll}1700 & 93 & 82 & 77 & 74 & 72 & 69 & 67 & 67 & 67 & 67 & 67 & 67 & 67 & 67 & 67 & 64\end{array}$

$\begin{array}{lllllllllllllllll}1950 & 93 & 82 & 77 & 74 & 72 & 69 & 67 & 67 & 67 & 67 & 67 & 67 & 67 & 67 & 67 & 64\end{array}$

$\begin{array}{lllllllllllllllll}2000 & 93 & 82 & 77 & 74 & 72 & 69 & 67 & 67 & 67 & 67 & 67 & 67 & 67 & 67 & 67 & 64\end{array}$

COLD Equivalence Ratio (Phi*100)

- Speed

- MAP

$0 \mathrm{rpm} \cdot \mathrm{Phi}$

1.000

- IgnAdv

14.4 psia * UEGO_Phi 1.006

$5.0{ }^{\circ} \mathrm{BTDC} \cdot$ PhIECT $32 \%$

- ControlMode OPEN LOOP

- Ignoffset +0.0 BTDC - PhiMAP_C $0.8 \mathrm{dP} / \mathrm{dMAP}$

- CL_Mult 
GEM Lean Burn Control System - BASE Phi Calibration Screen MAP (psia)

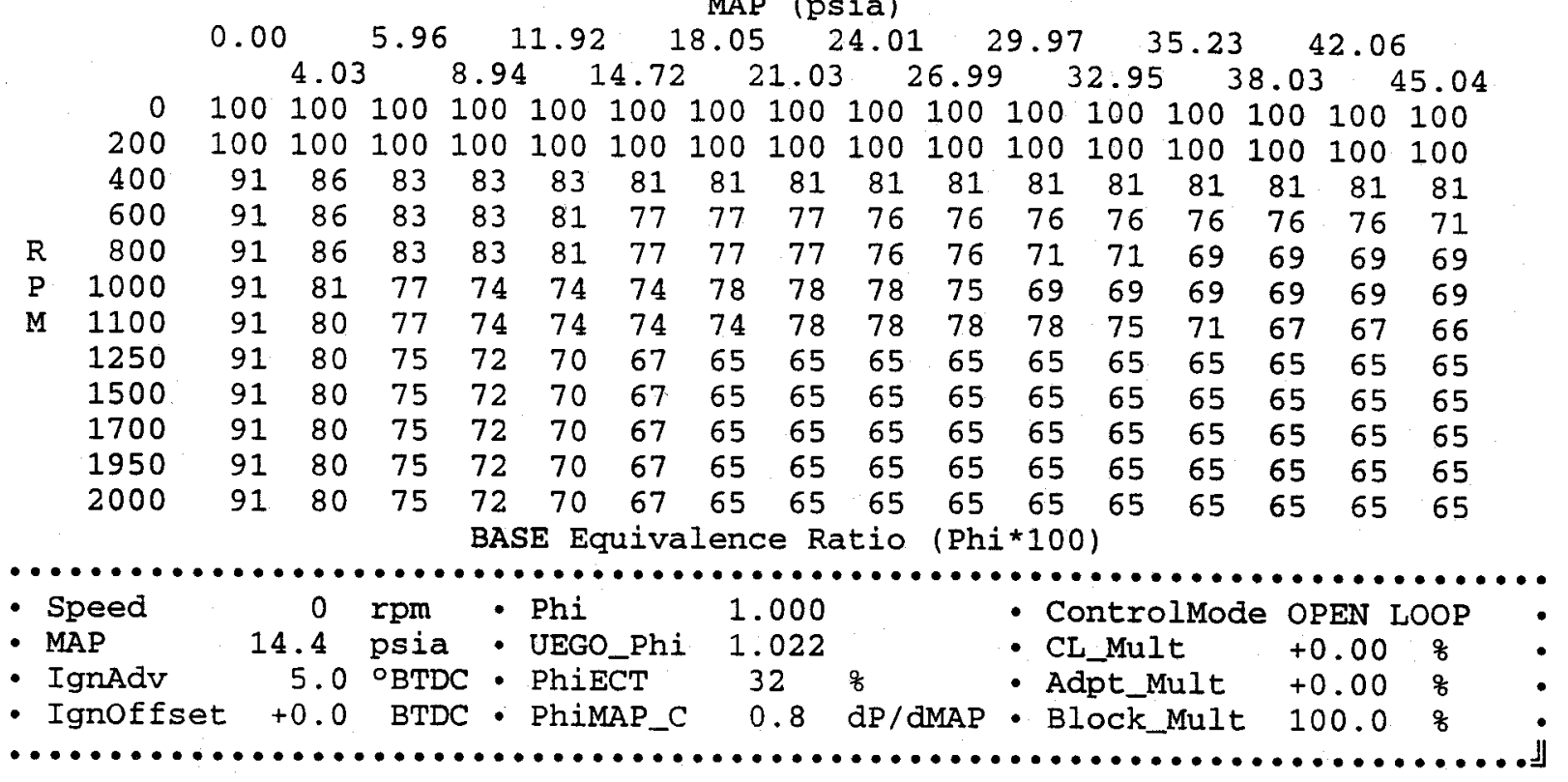


GEM Lean Burn Control System - Ignition Advance Calibration Screen MAP (psia)

\begin{tabular}{|c|c|c|c|c|c|c|c|c|c|c|c|c|c|c|c|c|}
\hline & 0.0 & & 5.96 & & 1.92 & & 8.05 & & 4.01 & & 9.97 & & 5.93 & & 2.06 & \\
\hline & & 4.03 & & 8.94 & & 4.72 & & 1.03 & & 6.99 & & 2.95 & & 8.03 & & 5.04 \\
\hline 270 & 5 & 5 & 5 & 5 & 5 & 5 & 5 & 5 & 5 & 5 & 5 & 5 & 5 & 5 & 5 & 5 \\
\hline 300 & 5 & 5 & 5 & 5 & 5 & 5 & 5 & 5 & 5 & 5 & 5 & 5 & 5 & 5 & 5 & 5 \\
\hline 400 & 13 & 13 & 13 & 13 & 13 & 13 & 12 & 12 & 12 & 12 & 12 & 12 & 12 & 12 & 12 & 5 \\
\hline 600 & 13 & 13 & 13 & 13 & 13 & 13 & 12 & 11 & 10 & 10 & 10 & 10 & 10 & 10 & 10 & 5 \\
\hline 800 & 16 & 16 & 16 & 15 & 16 & 15 & 15 & 13 & 12 & 12 & 12 & 10 & 10 & 10 & 10 & 5 \\
\hline 1000 & 26 & 26 & 26 & 20 & 20 & 18 & 14 & 14 & 13 & 13 & 13 & 12 & 10 & 10 & 10 & 5 \\
\hline 1100 & 27 & 27 & 27 & 24 & 21 & 18 & 16 & 15 & 14 & 13 & 13 & 13 & 10 & 10 & 10 & \\
\hline 1250 & 26 & 26 & 26 & 24 & 21 & 18 & 17 & 17 & 16 & 14 & 14 & 13 & 13 & 13 & 10 & 5 \\
\hline 1500 & 26 & 26 & 26 & 24 & 21 & 18 & 17 & 17 & 17 & 16 & 16 & 16 & 16 & 14 & 10 & 5 \\
\hline 1700 & 27 & 27 & 27 & 24 & 21 & 18 & 17 & 17 & 17 & 16 & 16 & 16 & 16 & 16 & 10 & 5 \\
\hline 1950 & 27 & 27 & 27 & 24 & 21 & 18 & 17 & 17 & 17 & 16 & 16 & 16 & 16 & 16 & 10 & \\
\hline 2100 & 27 & 27 & 27 & 24 & 21 & 18 & 17 & 17 & 17 & 16 & 16 & 16 & 16 & 16 & 10 & o \\
\hline
\end{tabular}

- Speed

- MAP

- PTP

- UEGO_Phi
14.4
14.2

rpm - IgnAdv

psia - Ignoffset

psia - TLC_retard

- $\mathrm{Phi}$
5.0 BTDC - ControlMode OPEN LOOP +0.0 OBTDC - CL_Mult

0.0

OATDC
$+0.00 \%$ +0.00 \&

100.0 \% 


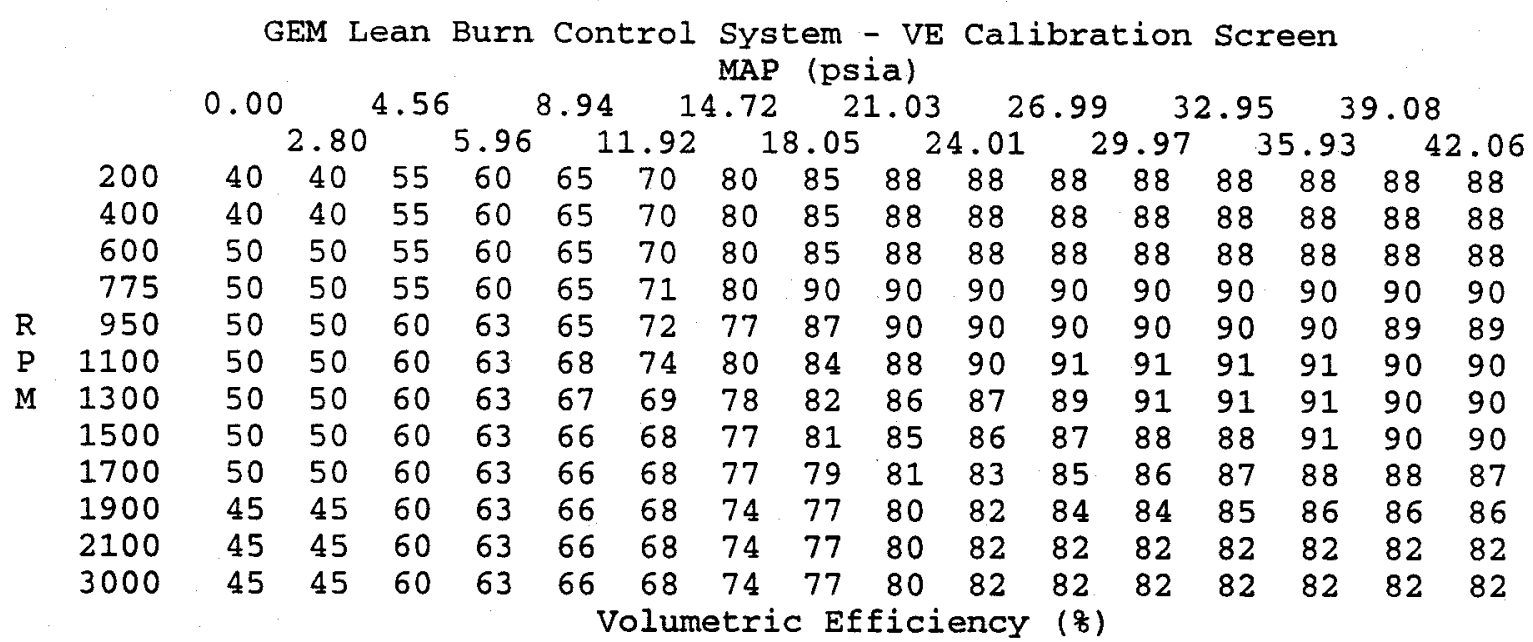

- Speed

- MAP

$0 \mathrm{rpm}$

- VE_std

$78.7 \%$

- PTP

14.4 psia

- VE_mod

$100.3 \%$

14.2 psia $\cdot V_{1}$ act

80.8 \%

- UEGO_Phi

1.017

- $\mathrm{Ph} i$

1.000

- Controlmode OPEN LOOP

- CL_Mult +0.00

- Adpt_mult +0.00 \&

- Block_Mult 100.0 \& 
GEM Lean Burn Control System - Adaptive Parameter Table

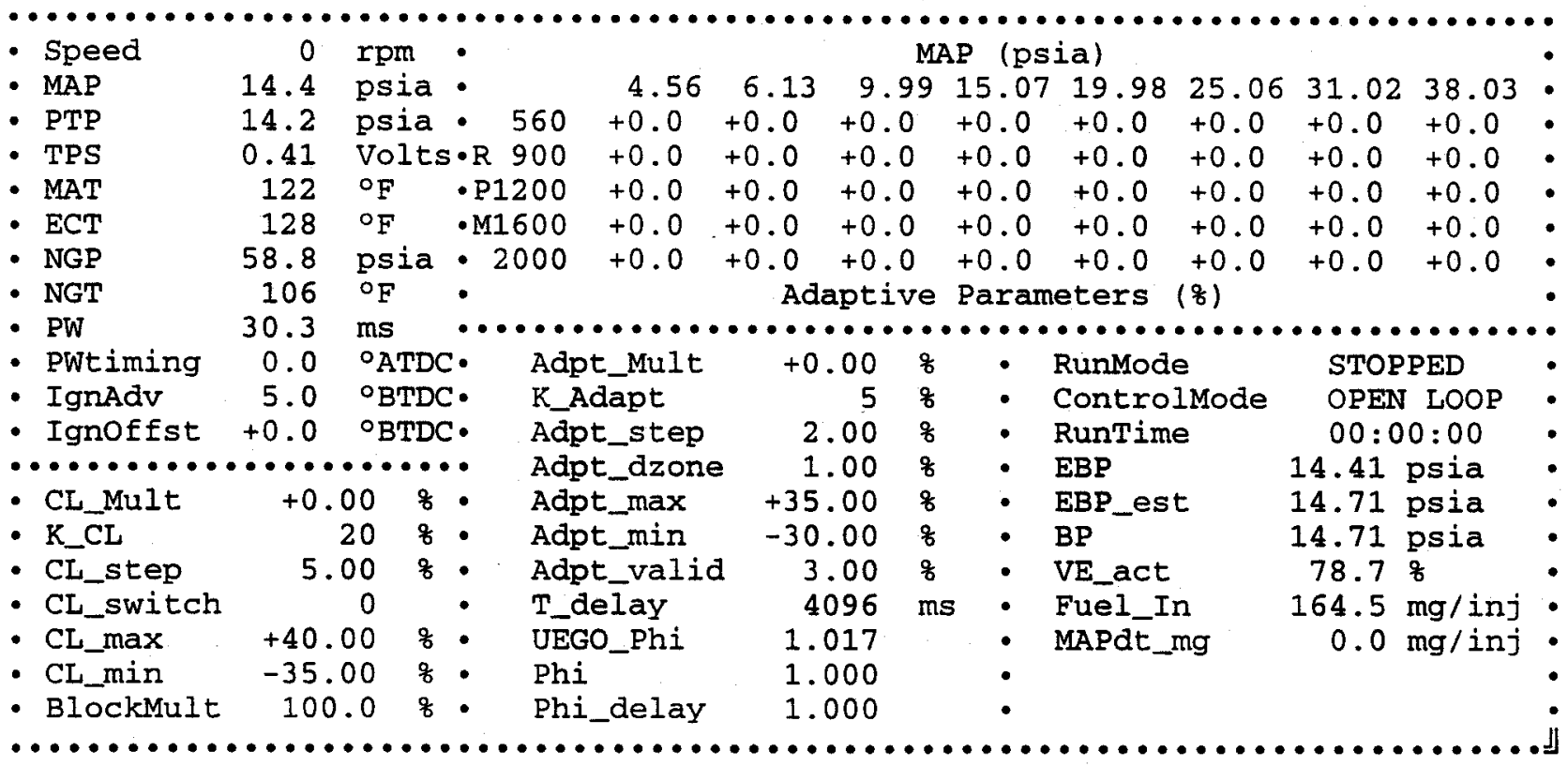


GEM Lean Burn Contro1 System Faults PROM ID: 2570-008- 09/11/98

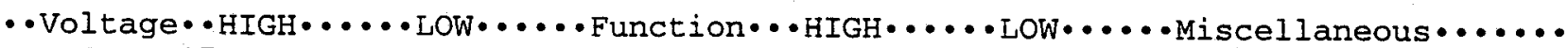

- EBP 17:OK 18:OK

- FPP 21:OK $22:$ OK

- EBP Exp 19:OK

$29:$ OK

- Trigger

$42: \mathrm{OK}$

- TPS EXP $57:$ OK

$81: \mathrm{OK}$

- TPS Cal

$82: O K$

- TPS $54:$ OK

$56:$ OK

- MAT

$23: \mathrm{OK}$

$25:$ OK

- MAP

$33: \mathrm{OK}$

- NGP Exp 41:OK

$43:$ OK

- BP Exp 35:OK

$36:$ OK

$34:$ OK

- BoostExp 71:OK

- J1708 Rx 83:OK

- PTP

$48:$ OK

- CL Limit 44:OK

$72:$ OK

- ECT

26:OK

$27:$ OK

- NGT

$26:$ OK

- Al Limit 46:OK

$45:$ OK

$15: \mathrm{OK}$

- NGP 38:OK

$39:$ OK

- NGTT

$64:$ OK

-

$65:$ OK

- NGTP

$66:$ OK

$67:$ OK

- UEGOP 63:OK

$62:$ OK

- UEGOH 58:OK

$59:$ OK

- UEGOR 68:OK

$69:$ OK

- Batt 53:OK

$52:$ OK

- J1708 Tx 84:OK

- ECT High Exp 28:OK

- MAT High Exp 24:OK

- NGTP High Exp 83:OK

- PTP Active 49:OK

- OverBoost $73:$ OK

- UEGOS Fail 13:OK

- Oil Pressure 74:OK

- Inj DutyCycle 75:OK

-

$\cdot$

-

$\bullet$

-ECU Internal .......

- COP 55:OK

- Interrupt 55:OK

- Analog 55:OK

- Execution 55:OK

- Clocks 55:OK

- Stack $55:$ OK

- RAM $51:$ OK

-

$\bullet$ 
GEM Lean Burn Control System Diagnostics

\begin{tabular}{|c|c|c|c|c|c|c|c|c|c|c|}
\hline - $\mathrm{FPP}$ & $21: \mathrm{OK}$ & $22: 0 K$ & - UEGOS Fail & $13: \mathrm{OK}$ & - Speed & 0 & rpm & & & \\
\hline - TPS & $16: O K$ & $17: \mathrm{OK}$ & - Spark Input & $42: \mathrm{OK}$ & - MAP & 14.4 & psia & 15.1 & Des & \\
\hline - TPSExp & $18: \mathrm{OK}$ & $19: \mathrm{OK}$ & - TPS_Cal & $29: \mathrm{OK}$ & - $\mathrm{PTP}$ & 14.2 & psia & 1.63 & V & • \\
\hline - MAT & $23:$ OK & $25:$ OK & - MAT_HiExp & $24:$ OK & - MAT & 122 & ${ }^{\circ} \mathrm{F}$ & 1.37 & V & \\
\hline - MAP & $33:$ OK & $34:$ OK & - OverBoost & $73: O K$ & - ECT & 128 & ${ }^{\circ} \mathrm{F}$ & 1.43 & V & \\
\hline - PTP & $35:$ OK & $36: \mathrm{OK}$ & - PTP_Active & $37: O K$ & - NGP & 93.1 & - psia & 2.37 & $\mathrm{~V}$ & - \\
\hline - ECT & $26:$ OK & $27:$ OK & - ECT_HiExp & $28:$ OK & - NGT & 106 & ${ }^{\circ} F$ & 1.96 & $\mathrm{~V}$ & \\
\hline - NGT & $14: \mathrm{OK}$ & $15: O K$ & - Oil_Press & $74: \mathrm{OK}$ & - FPP & 0.41 & $\mathrm{~V}$ & 0.41 & $\operatorname{Mir}$ & \\
\hline - NGP & $38:$ OK & $39:$ OK & - Inj_DutyCyc & $75:$ OK & - TPS & 1.99 & VCmd & 0.02 & $\mathrm{FB}$ & . \\
\hline - NGPExp & $41: \mathrm{OK}$ & $43:$ OK & - EBP_Hi & : OK & - EBP & 14.41 & psia & 2.41 & V & . \\
\hline - BPExp & $35:$ OK & $34:$ OK & - EBP_Lo & : OK & - $\mathrm{Phi}$ & 1.022 & Act & 1.000 & Des & \\
\hline - NGTT & $64:$ OK & $65:$ OK & - EBP_HiExp & :OK & - NGTP & 0 & psig & 0.02 & $\mathrm{~V}$ & • \\
\hline - NGTP & $66: \mathrm{OK}$ & $67: O K$ & - EBP_LOExp & :OK & - NGTT & -50 & ${ }^{\circ} \mathrm{F}$ & 5.00 & V & $\bullet$ \\
\hline - UEGOP & $63:$ OK & $62:$ OK & - Interrupts & $55:$ OK & - Batt & 14.2 & $\mathrm{~V}$ & & & \\
\hline - UEGOH & $58:$ OK & $59:$ OK & - $\mathrm{COP}$ & $55:$ OK & - IgnAdv & & 5.0 & ${ }^{\circ} \mathrm{BTDC}$ & & 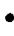 \\
\hline - UEGOR & $68:$ OK & $69:$ OK & - Execution & $55: O K$ & - $\mathrm{PW}$ & & 19.5 & ms & & 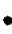 \\
\hline - Volts & $53:$ OK & $52: \mathrm{OK}$ & - Clocks & $55: \mathrm{OK}$ & - CL_MuI & & +0.00 & $\frac{q}{8}$ & & $\bullet$ \\
\hline - Boost & $71:$ OK & $72:$ OK & - Stack & $55:$ OK & - AL_Mul & & +0.00 & $\%$ & & - \\
\hline ALlim & $46: O K$ & $47: O K$ & - Analog & $55: \mathrm{OK}$ & - Contro & & PEN LC & DOP & & 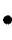 \\
\hline CLlim & $44:$ OK & $45: \mathrm{OK}$ & - RAM & $51:$ OK & - RunTin & & $0: 00:$ & & & \\
\hline
\end{tabular}




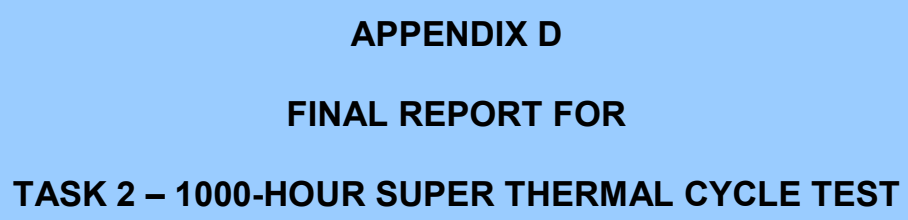

SwRI PROJECT NO. 03-7501-002 


\title{
400-HOUR VALVE PERFORMANCE, 1500-HOUR DURABILITY, AND BREAK-IN AND PERFORMANCE MAPPING OF MACK E-7 NATURAL GAS ENGINES
}

\author{
FINAL REPORT \\ TASK 2 - 1000-HOUR SUPER THERMAL CYCLE TEST
}

SwRI Project No. 03-7501-002

Prepared for:

Mack Trucks, Inc. 13302 Pennsylvania Ave.

Hagerstown, MD 21742

Prepared by:

James P. Chiu

November 1996

Approved:

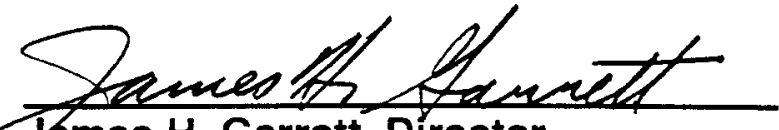




\section{EXECUTIVE SUMMARY}

The original goal of this task was to perform two 1500-hour durability tests. Due to budget constraints, the two 1500-hour durability tests were replaced with one 1000-hour super thermal cycle durability test. The super thermal cycle consisted of two minutes at idle (unloaded stage) and 10 minutes at high speed and load (loaded stage). The engine was thermally "shocked" with $38^{\circ} \mathrm{C}$ $\left(100^{\circ} \mathrm{F}\right)$ coolant at the unloaded stage and $94^{\circ} \mathrm{C}\left(200^{\circ} \mathrm{F}\right)$ coolant at the loaded stage to produce a $56^{\circ} \mathrm{C}\left(100^{\circ} \mathrm{F}\right)$ temperature difference between the stages.

The 1000-hour super thermal cycle durability test was completed. Basic engine performance data were recorded every hour during the test, and more detailed performance data were recorded every 25 hours. Oil consumption was recorded every 50 hours. Valve stem heights, valve lash, spark plug gaps, cylinder compression, and cylinder leak down were recorded every 250 hours.

Oil consumption was consistent for the first 750 hours. During the last 250 hours the engine had high oil consumption in part due to a failed blowby filter, which allowed oil to enter the engine directly through the intake system. Platinum-nickel tipped spark plugs showed a gap life of approximately 410 hours, whereas platinum-iridium tipped spark plugs showed a gap life of approximately 540 hours, although some of the platinum-iridium spark plugs appeared to be failing at the crimp before 540 hours. Cylinder pressure checks showed no loss of compression during the test, and valve recession was low.

Major problems encountered during the tests were spark plug failures, the blowby filter failure, and, as reported by Mack Trucks, piston cracking. Further work should include an investigation of the spark plug failure, a redesign of the blowby filter for a longer life, a redesign of the piston to prevent the cracking, and another 1000-hour test to verify the durability of the pistons. 
TABLE OF CONTENTS

Page

LIST OF ILLUSTRATIONS $\ldots \ldots \ldots \ldots \ldots \ldots \ldots \ldots \ldots \ldots \ldots \ldots \ldots$ vii

LIST OF TABLES

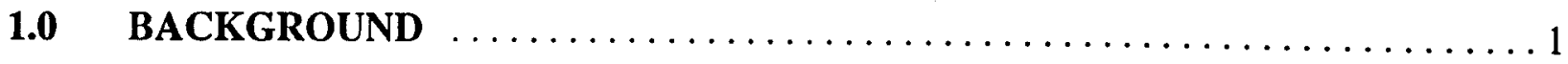

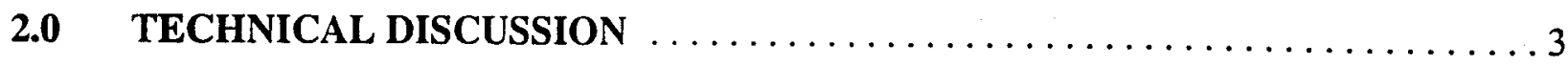

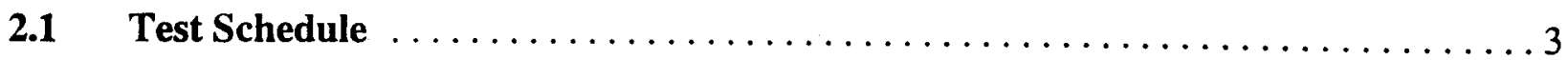

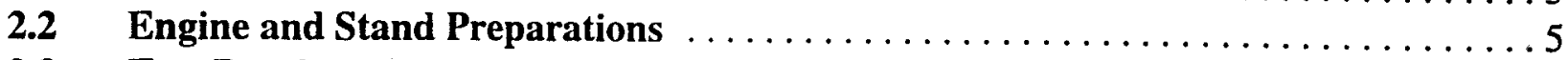

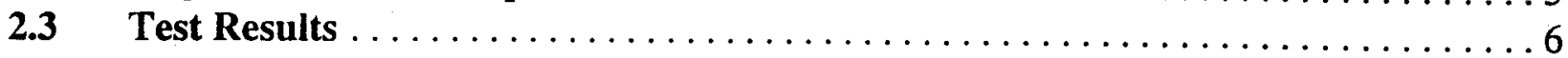

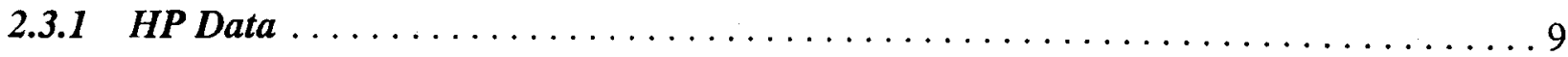

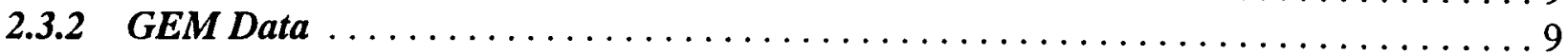

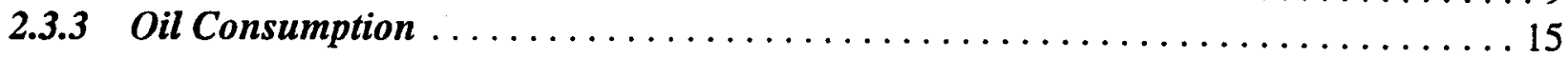

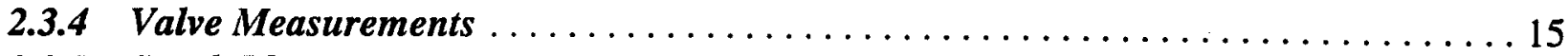

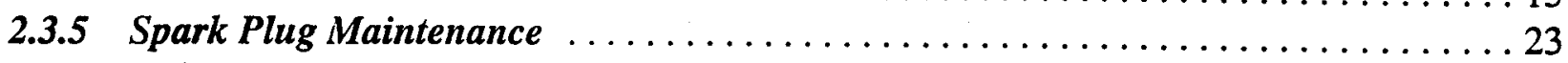

2.3.6 Cylinder Compression and Leak Down Measurements ................. 25

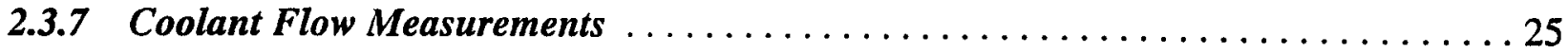

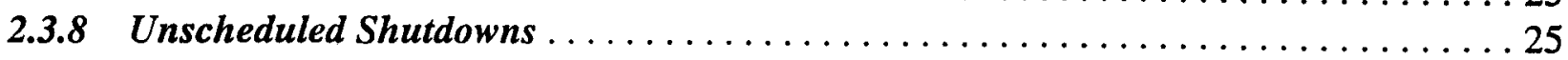

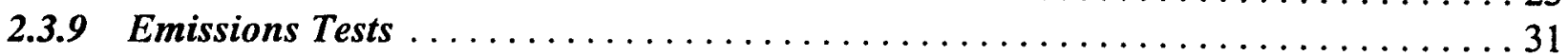

3.0 CONCLUSIONS \& RECOMMENDATIONS $\ldots \ldots \ldots \ldots \ldots \ldots \ldots \ldots \ldots$

\section{APPENDICES}

A LISTING OF RUN NUMBERS

B GEM TRANSIENT DATA

C VALVE STEM HEIGHT MEASUREMENTS

D LETTERS SENT TO CHAMPION SPARK PLUGS

E LOW SPEED DATA FOR EMISSIONS TEST AT 0 HOURS ON THE STC TEST

F EMISSIONS RESULTS FOR TESTS AT 0 HOURS ON THE STC TEST

$G$ LOW SPEED DATA FOR EMISSIONS TEST AT 500 HOURS ON THE STC TEST

H EMISSIONS RESULTS FOR TESTS AT 500 HOURS ON THE STC TEST 
LIST OF ILLUSTRATIONS

Figure

Page

1 SUPER THERMAL CYCLE TEST STAND

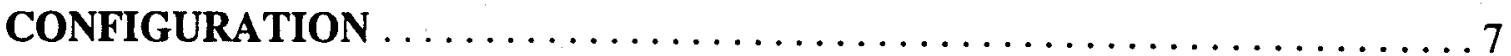

2 FUEL FILTER SIZE AND LOCATION FOR THE STC TEST $\ldots \ldots \ldots \ldots \ldots 8$

3 AVERAGE OF 40 READINGS RECORDED BETWEEN

1 AND 1000 HOURS FROM THE GEM SYSTEM

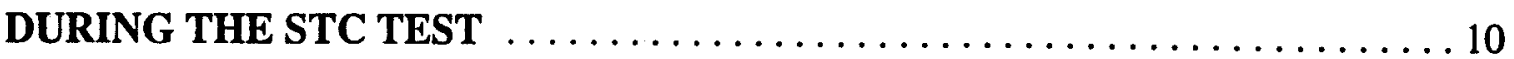

4 AVERAGE OF 40 READINGS AND \pm ONE STANDARD

DEVIATION FOR VE RECORDED BETWEEN 1 AND 1000

HOURS FROM THE GEM SYSTEM DURING THE STC TEST 11

5 AVERAGE OF 40 READINGS AND \pm ONE STANDARD

DEVIATION FOR ENGINE SPEED RECORDED BETWEEN

1 AND 1000 HOURS FROM THE GEM SYSTEM DURING

THE STC TEST

6 AVERAGE OF 40 READINGS AND \pm ONE STANDARD

DEVIATION FOR MAP RECORDED BETWEEN

1 AND 1000 HOURS FROM THE GEM SYSTEM

DURING THE STC TEST

7 AVERAGE OF 40 READINGS AND \pm ONE STANDARD

DEVIATION FOR ECT RECORDED BETWEEN

1 AND 1000 HOURS FROM THE GEM SYSTEM

DURING THE STC TEST

8 DATA FROM THE INJECTOR BLOCK LEAK DOWN

BEFORE THE START OF THE STC TEST

9 DATA FROM THE INJECTOR BLOCK LEAK DOWN

AT THE END OF THE STC TEST

10 INJECTOR BLOCK LEAK DOWN RATE DURING THE STC TEST $\ldots \ldots \ldots 18$

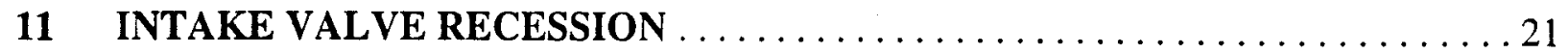

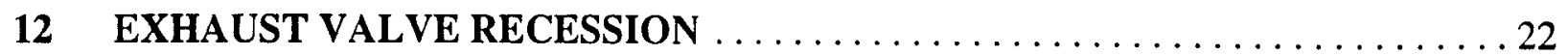

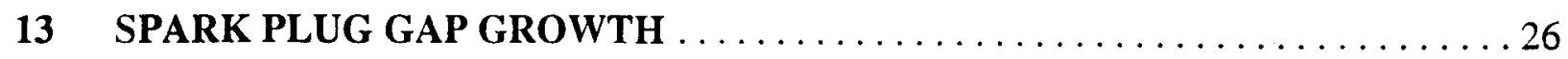




\section{LIST OF TABLES}

Table

Page

1 TEST SCHEDULE 3

2 SUPER THERMAL CYCLE .4

3 SCHEDULED DATA RECORDING AND MAINTENANCE $\ldots \ldots \ldots \ldots \ldots 4$

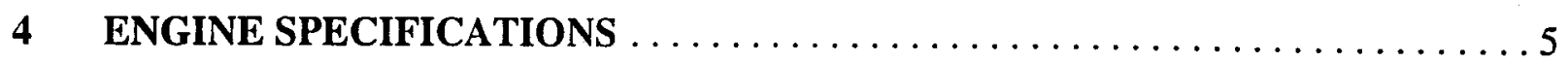

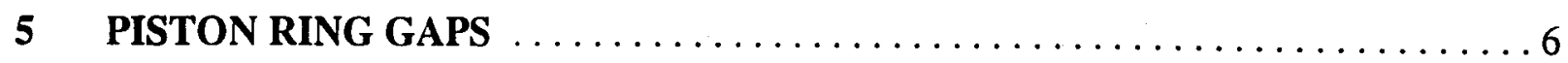

6 RUNTIME HISTORY OF ENGINE SINCE REBUILD ..............9

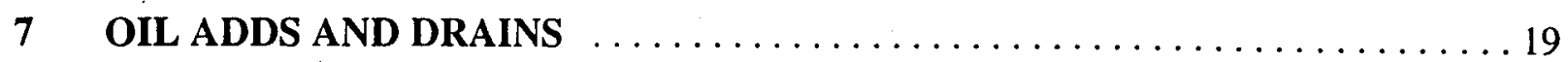

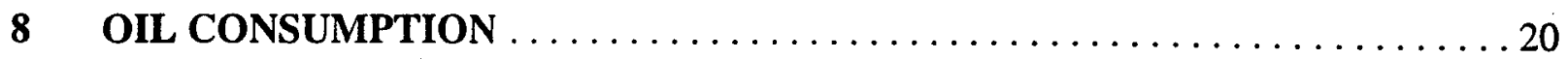

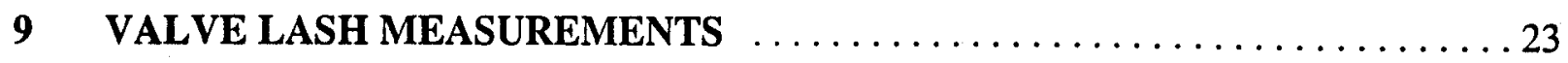

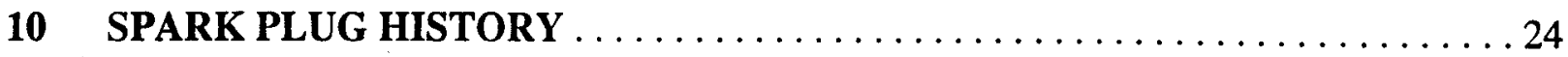

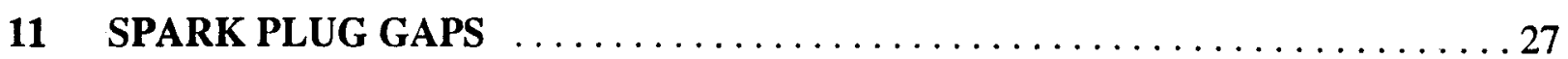

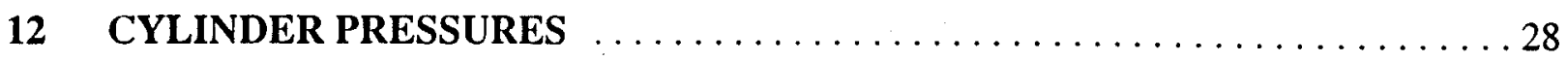

13 CYLINDER PRESSURE LEAK DOWN $\ldots \ldots \ldots \ldots \ldots \ldots \ldots \ldots \ldots \ldots \ldots \ldots$

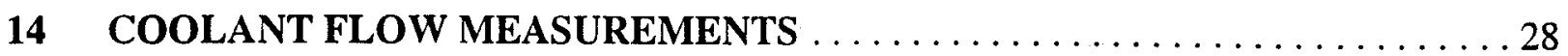

15 UNSCHEDULED SHUTDOWNS ON THE STC TEST $\ldots \ldots \ldots \ldots \ldots \ldots$

16 RESULTS OF THE 13-MODE EUROPEAN EMISSIONS TEST AT 0 HOURS ON THE STC TEST

17 RESULTS OF THE 13-MODE EUROPEAN EMISSIONS TEST AT 500 HOURS ON THE STC TEST

18 EMISSIONS SUMMARY WITH THE FINAL RVI CALIBRATION . . . . . 33

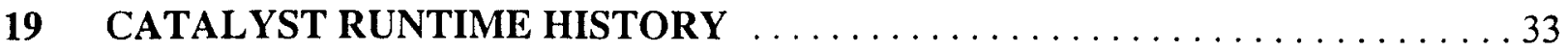




\subsection{BACKGROUND}

The goal of this project was to aid Mack Trucks, Inc. in expediting the development of a natural gas-fueled Mack E-7 engine. The project was originally broken down into three individual tasks. The purpose of the first task, "Four 100-Hour Valve Wear Tests," was to verify a valve seat/valve combination that exhibits the greatest resistance to wear. The purpose of the second task, "Two 1500-Hour Durability Tests," was to verify the durability of the recommended valve seat/valve combination and other natural gas-related hardware, including the engine control system. The purpose of the final task, "Ten Engine Break-In and Performance Mapping," was to verify through break-in procedures and performance mapping that fresh preproduction engines are assembled properly and that control system components function as required. A fourth task was added to the project for unscheduled maintenance, to account for changes in the original scope of work as directed by Mack Trucks, Inc.

The second task, "Two 1500-Hour Durability Tests," was replaced with, "One 1000-Hour Durability Test," using the super thermal cycle (STC) as provided by Mack Trucks. This durability test was completed and is reported herein.

Funding for this task was provided by this project and by the "Mack LNG Vehicle Development" project conducted by SwRI for Mack Trucks. 


\subsection{TECHNICAL DISCUSSION}

A 1000-hour super thermal cycle (STC) durability test was conducted. The STC test cycle (as provided by Mack Trucks) consisted of two minutes at idle (unloaded stage) and ten minutes at high speed and load (loaded stage) with a coolant out temperature difference of $56^{\circ} \mathrm{C}\left(100^{\circ} \mathrm{F}\right)$ between the loaded and unloaded stages. The following sections describe the test schedule, engine and stand preparations, and test results.

\section{$2.1 \quad$ Test Schedule}

The test schedule for the durability test engine is shown in Table 1 . The engine was rebuilt for this test schedule as described in the next section. Total engine hours, as described in this report, refers to the hours on the engine since the rebuild. STC test hours refers to the hours of actual test time on the STC cycle.

TABLE 1. TEST SCHEDULE

\begin{tabular}{|l|c|c|c||}
\hline \multicolumn{1}{|c|}{ Test } & $\begin{array}{c}\text { Time } \\
\text { (hours) }\end{array}$ & $\begin{array}{c}\text { Engine Speed } \\
(\mathbf{r p m})\end{array}$ & $\begin{array}{c}\text { Power } \\
(\mathbf{k W})\end{array}$ \\
\hline Break-In & 12 & 1950 & 121 \\
\hline Break-In & 12 & 1950 & 242 \\
\hline Valve Recession & 100 & 1950 & 266 \\
\hline Emissions & As needed & 13 Mode Points & 13 Mode Points \\
\hline STC & 500 & STC Cycle & STC Cycle \\
\hline Emissions & As needed & 13 Mode Points & 13 Mode Points \\
\hline STC & 500 & STC Cycle & STC Cycle \\
\hline
\end{tabular}

The cycle for the STC test is shown in Table 2. The unloaded stage is at idle speed with minimum load. The loaded stage was selected by Mack Trucks as $1700 \mathrm{rpm}$ and $110 \%$ load based on a power curve as provided by Renualt V.I. for an engine rated at $261 \mathrm{~kW}(350 \mathrm{hp})$ at $1900 \mathrm{rpm}$ to reach $290 \mathrm{~kW}$ at $1700 \mathrm{rpm}$. At the loaded stage, the equivalence ratio was increased from 0.65 to 0.67 and the ignition timing was set to MBT. Due to the high ambient temperature in San Antonio at the time of the year this test was performed, the specified coolant out temperature for the unloaded stage (idle) of $32^{\circ} \mathrm{C}\left(90^{\circ} \mathrm{F}\right)$ could not be obtained. Therefore, a coolant out temperature of $38^{\circ} \mathrm{C}$ $\left(100^{\circ} \mathrm{F}\right)$ was specified for the unloaded stage and $94^{\circ} \mathrm{C}\left(200^{\circ} \mathrm{F}\right)$ for the loaded stage. These temperatures maintain the $56^{\circ} \mathrm{C}\left(100^{\circ} \mathrm{F}\right)$ differential between the loaded and unloaded stages.

The schedule for data recording and maintenance during the 1000 -hour durability test is shown in Table 3. Shutdowns were scheduled every 25 hours. 
TABLE 2. SUPER THERMAL CYCLE

\begin{tabular}{|l|c|c||}
\hline \multicolumn{1}{|c|}{ Parameter } & Unloaded & Loaded \\
\hline \hline Engine Speed $(\mathrm{rpm})$ & 650 & 1700 \\
\hline Power $(\mathrm{kW})$ & 4 & 290 \\
\hline Time (minutes) & 2 & 10 \\
\hline $\begin{array}{l}\text { Coolant Out Temperature } \\
\left({ }^{\circ} \mathrm{C} \text { at end of cycle) }\right.\end{array}$ & 38 & 94 \\
\hline
\end{tabular}

TABLE 3. SCHEDULED DATA RECORDING AND MAINTENANCE

\begin{tabular}{||l|l||}
\hline \multicolumn{1}{|c|}{ Description } & \multicolumn{1}{c|}{ Schedule } \\
\hline \hline Oil Sample & At one hour after oil change \\
\hline HP Hourly Data & Every hour \\
\hline HP Snapshot Data & Every 25 hours \\
\hline GEM Transient Data & Every 25 hours \\
\hline GEM NGP Data & Every 25 hours \\
\hline Oil Level Check (fill as needed) & Every 25 hours \\
\hline Oil Sample & Every 50 hours \\
\hline Oil Fill (record oil consumption) & Every 50 hours \\
\hline Drain Blowby Filter Housing & Every 50 hours \\
\hline Drain Gas Filter Housings & Every 50 hours \\
\hline Add Adsorption Filter to Gas Line & At 250 hours \\
\hline Replace Adsorption Filter Element & Every 250 hours \\
\hline Measure Valve Stem Heights & Every 250 hours \\
\hline Measure Valve Lash & Every 250 hours \\
\hline Measure Spark Plug Gaps & Every 250 hours \\
\hline Measure Cylinder Compression & Every 250 hours \\
\hline Measure Cylinder Leak Down & Every 250 hours \\
\hline Remove Catalyst & At 500 hours \\
\hline Change Oil and Filters & At 500 hours \\
\hline Replace Coalescing Fuel Filters & Every 500 hours \\
\hline Measure Coolant Flow & Every 500 hours \\
\hline \hline
\end{tabular}




\subsection{Engine and Stand Preparations}

The engine ( $\mathrm{S} / \mathrm{N}$ 9E-0002) used for the Task 1 valve wear tests was rebuilt for the 1000-hour durability test. There were a total of 653.4 hours on the engine block at the time of the rebuild which included 415.5 hours for the valve tests and 237.9 hours of miscellaneous time.

The engine was assembled with new spark plugs, pistons, piston rings, liners, and cylinder heads (provided by Mack Trucks). The pistons were modified by Mack Trucks to include a groove for the oil control ring groove which is closer to the piston top. The second rings were also modified to a Napier style ring. Engine specifications are shown in Table 4. The compression ring gaps were measured, and the results are shown in Table 5. Valve lash was set to $0.41 \mathrm{~mm}(0.016 ")$ for intake valves and $0.71 \mathrm{~mm}(0.028 ")$ for exhaust valves, and spark plug gaps were set to $0.25 \mathrm{~mm}(0.010 ")$.

\section{TABLE 4. ENGINE SPECIFICATION}

\begin{tabular}{||l|l||}
\hline Engine Model & E7G-325 \\
\hline Engine Serial Number & 9 E0002 \\
\hline Engine Displacement (L) & 12 \\
\hline Bore and Stroke (mm) & $123.8 \times 165.1$ \\
\hline Piston & Modified Zolliner Aluminum H Ring \\
\hline First Ring & 1 st Design \\
\hline Second Ring & Modified to a Napier Style \\
\hline Oil Ring & 2 nd Design \\
\hline Camshaft Timing & $8.57^{\circ}$ CA Advanced \\
\hline Ignition System Controller Supplier & MESA \\
\hline Ignition System Coil Supplier & Nippondenso \\
\hline Ignition System Type & Inductive \\
\hline Ignition System Timing Sensor & Electro, K Coil \\
\hline Spark Plug Supplier & Champion \\
\hline Mixer Type & Fabricated Annular with One 90 Bend \\
\hline Number of Holes in Mixing Ring & 9 \\
\hline Fuel Metering System Supplier & MESA \\
\hline Fuel Injector Type & Servojet SP021S1 \\
\hline Number of Injectors & 8 \\
\hline Throttle Supplier & Woodward \\
\hline Throttle Model & FLOWTECH. 68mm \\
\hline Throttle Type & Electronic, Digital \\
\hline UEGO Sensor & NGK Universal \\
\hline
\end{tabular}


TABLE 5. PISTON RING GAPS

\begin{tabular}{|c|c|c|c|c||}
\hline \hline Cylinder & $\begin{array}{c}\text { 1st Ring Gap } \\
\text { (mm) }\end{array}$ & $\begin{array}{c}\text { 1st Ring Gap } \\
\text { (in.) }\end{array}$ & $\begin{array}{c}\text { 2nd Ring Gap } \\
\text { (mm) }\end{array}$ & $\begin{array}{c}\text { 2nd Ring Gap } \\
\text { (in) }\end{array}$ \\
\hline \hline 1 & 0.56 & 0.022 & 0.71 & 0.028 \\
\hline 2 & 0.53 & 0.021 & 0.69 & 0.027 \\
\hline 3 & 0.56 & 0.022 & 0.69 & 0.027 \\
\hline 4 & 0.53 & 0.021 & 0.69 & 0.027 \\
\hline 5 & 0.56 & 0.022 & 0.71 & 0.028 \\
\hline 6 & 0.56 & 0.022 & 0.69 & 0.027 \\
\hline
\end{tabular}

After the break-in, 100-hour valve recession test, and emissions tests were performed, the test stand was modified to thermally "shock" the engine for the durability test. Two coolant tanks were installed in the test cell to provide either hot coolant to the engine during the loaded stage, or cold coolant during the unloaded stage. The hot tank was maintained at approximately $93^{\circ} \mathrm{C}$ $\left(200^{\circ} \mathrm{F}\right)$ at the beginning of the loaded stage and the cold tank was maintained at approximately $38^{\circ} \mathrm{C}\left(100^{\circ} \mathrm{F}\right)$ at the beginning of the unloaded stage. A schematic of the coolant system for the test is shown in Figure 1. A mixture of $\mathrm{Nalcool}^{\mathrm{TM}} 2000$ and demineralized water at a ratio of $489 \mathrm{ml}$ (16 ounces) of $\mathrm{Nalcool}^{\mathrm{TM}}$ to 15.21 (4 gallons) of water was added to the cooling system. The test stand was also equipped with an external electric motor to operate the water pump on the engine at a constant speed that was equivalent to the speed of the water pump at $2000 \mathrm{rpm}$ when the engine is in its normal configuration.

The oil and filters were changed. A catalyst was installed for Renault V.I. at the beginning of the STC test for aging. At the request of Renault V.I., it was removed at 500 test hours and will be returned to Mack Trucks. A closed crankcase ventilation system was installed, which included a blowby filter and canister provided by Mack Trucks and an in line blowby flow meter. The blowby was routed to the filter, the inline flow meter, and then to the inlet air for the engine between the laminar flow element and the turbocharger. New coalescing natural gas filters were installed. New style fuel injectors (part number SP-021S1) designed for LNG use were received from BKM Inc. and installed. An adsorption filter was added to the natural gas fuel line at 250 test hours on the STC. The first 250 hours were conducted without the adsorption filter in the fuel line. Figure 2 shows the schematic of the fuel filtering system with the adsorption filter installed.

\subsection{Test Results}

There were a total of 1147.6 hours on the engine since the rebuild for the durability test. A runtime history is shown in Table 6. The valve recession test was performed for Task 1 of this project, and therefore the results are not reported herein. Results of the HP data, GEM data, oil consumption, valve measurements, spark plug maintenance, cylinder con ipression and leak down measurements, coolant flow measurements, unscheduled shutdowns, and emissions tests are presented in the following sections. 


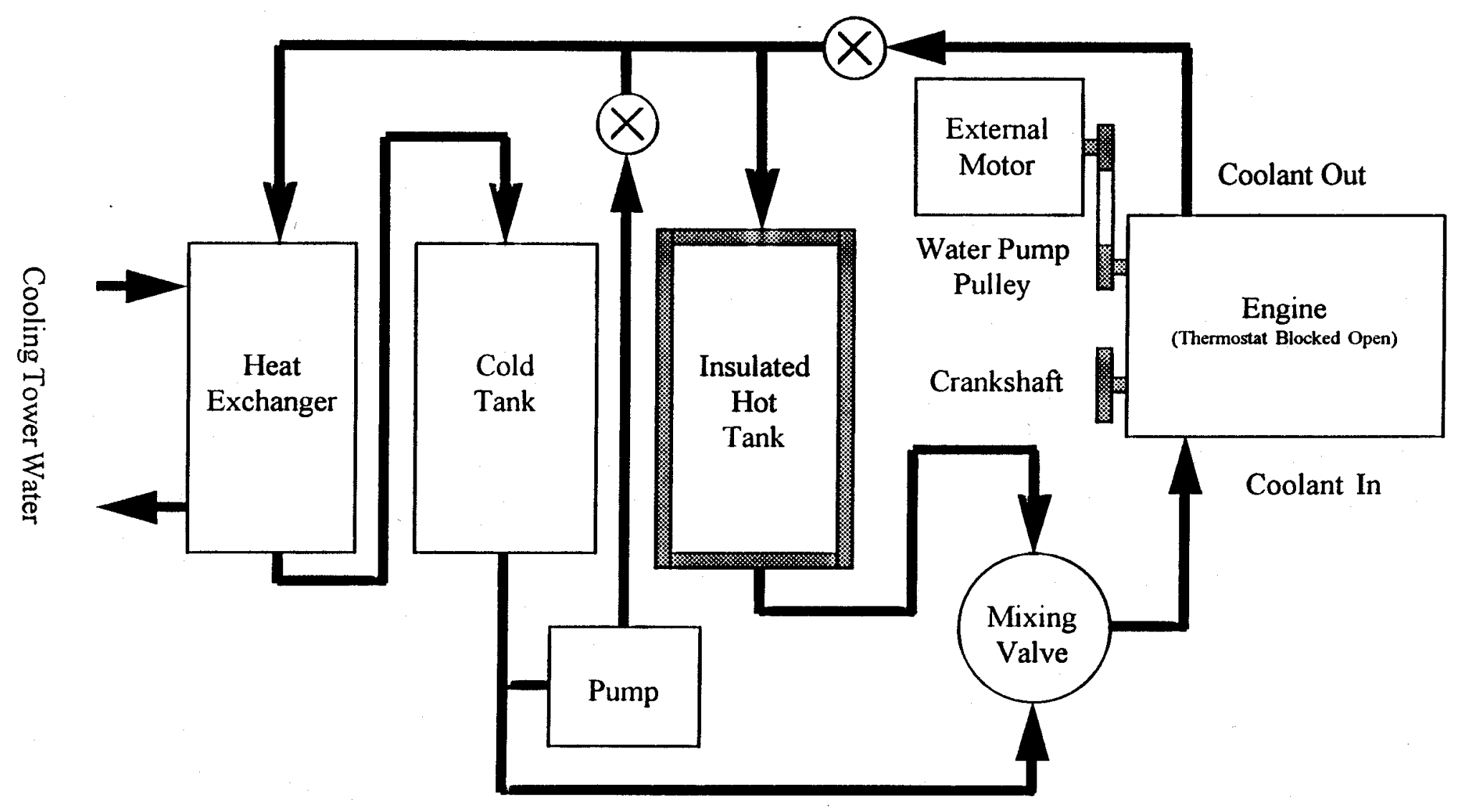

FIGURE 1. SUPER THREMAL CYCLE TEST STAND CONFIGURATION 
From

High Pressure

Natural Gas

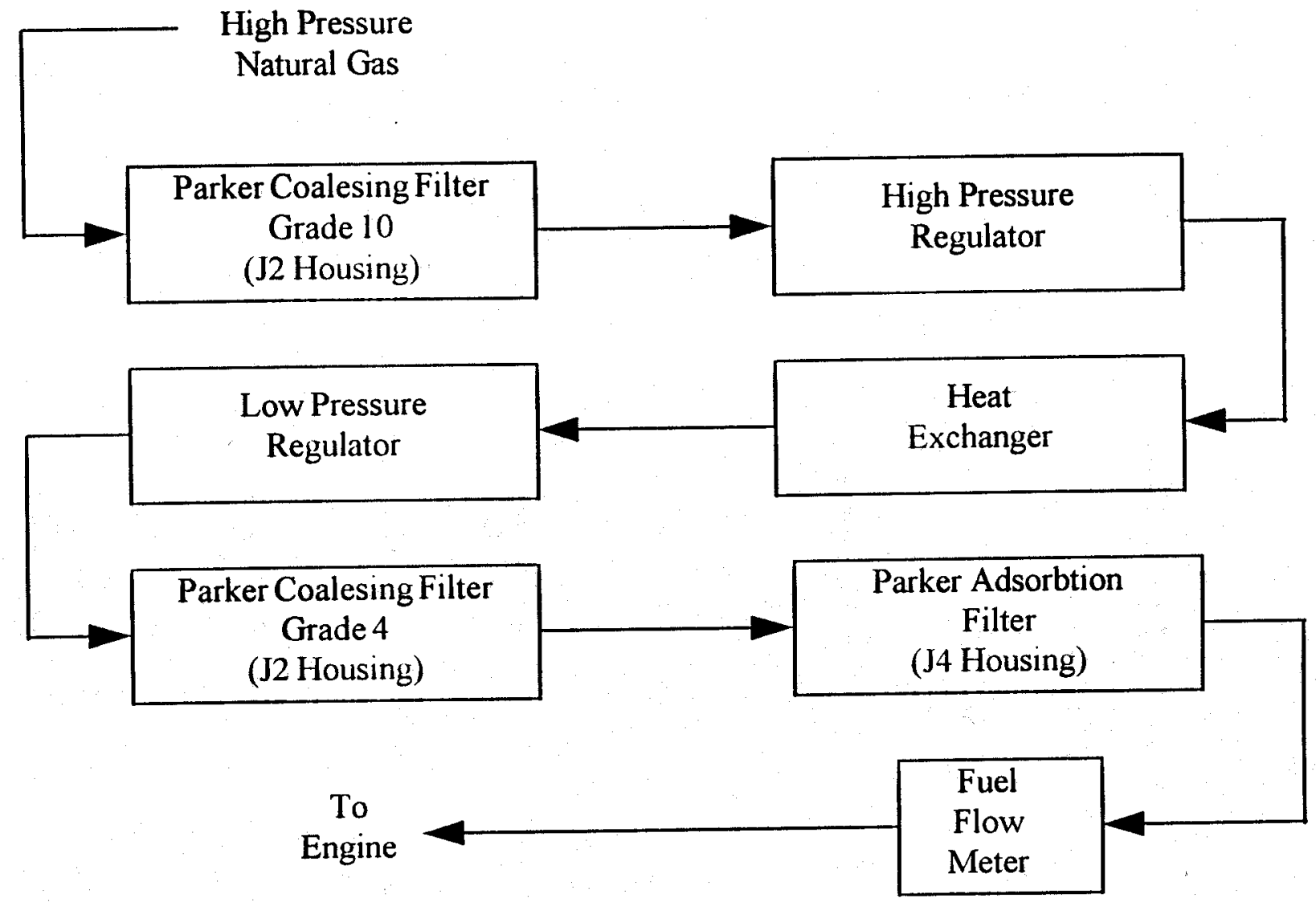

FIGURE 2. FUEL FILTER SIZE AND LOCATION FOR THE STC TEST 
TABLE 6. RUNTIME HISTORY OF ENGINE SINCE THE REBUILD

\begin{tabular}{||c|c|c|c||}
\hline Test Type & $\begin{array}{c}\text { Test Type } \\
\text { Time (Hours) }\end{array}$ & $\begin{array}{c}\text { Miscellaneous } \\
\text { Time (Hours) }\end{array}$ & $\begin{array}{c}\text { Total Engine } \\
\text { Time (Hours) }\end{array}$ \\
\hline \hline Break-In* & 24.0 & 0.8 & 24.8 \\
\hline Valve Recession & 100.0 & 4.5 & 129.3 \\
\hline STC & 250.0 & $7.6 * *$ & 386.9 \\
\hline STC & 500.0 & 3.7 & 640.6 \\
\hline STC & 750.0 & $4.9 * *$ & 895.5 \\
\hline STC & 1000.0 & 2.1 & 1147.6 \\
\hline $\begin{array}{l}* \\
\text { Includes Both 12-Hour Break-In Tests } \\
* * \text { Includes Emissions Tests }\end{array}$
\end{tabular}

\subsubsection{HP Data}

Hourly and snapshot data were recorded by the HP data acquisition system during the test. Hourly data were recorded by an average of two points within one minute of the end of each stage. Snapshot data were recorded for one full cycle in approximately 15 second intervals as a snapshot. There were a total of 1974 hourly readings and 2295 snapshot readings during the 1000-hour STC test. The hourly readings were not recorded correctly at 290 test hours due to unknown reasons, at 752 test hours due to an unscheduled shutdown during the data recording, at 836 test hours due to unknown reasons, and at 914 test hours due to an unscheduled shutdown during the data recording. A listing of all run numbers recorded during the STC test with corresponding test hours and data type is included in Appendix A.

\subsubsection{GEM Data}

Data from the gas engine management (GEM) system were recorded every 25 hours. Transient data were recorded for one full cycle in one second intervals for engine speed, manifold air pressure (MAP), volumetric efficiency (VE), and engine coolant temperature (ECT). Figure 3 shows the average readings from 1 to 1000 hours from the transient data. There were 40 sets of data recorded. Data at 275 and 300 were not recorded correctly due to operator error, and additional data at 309 hours were recorded. Figures 4-7 show the average for each parameter along with a band of one standard deviation. Figures for individual reading are included in Appendix B.

Data from the GEM system for injector block leak down were recorded every 25 hours. Data were recorded for two minutes at one second intervals by starting the data acquisition with the ignition key turned off, then turning the ignition key on (without starting the engine) within five seconds after starting the data acquisition. Turning the key on energizes the solenoid in the injector block to allow fuel to pressurize the injector block. When the GEM system does not receive a signal 


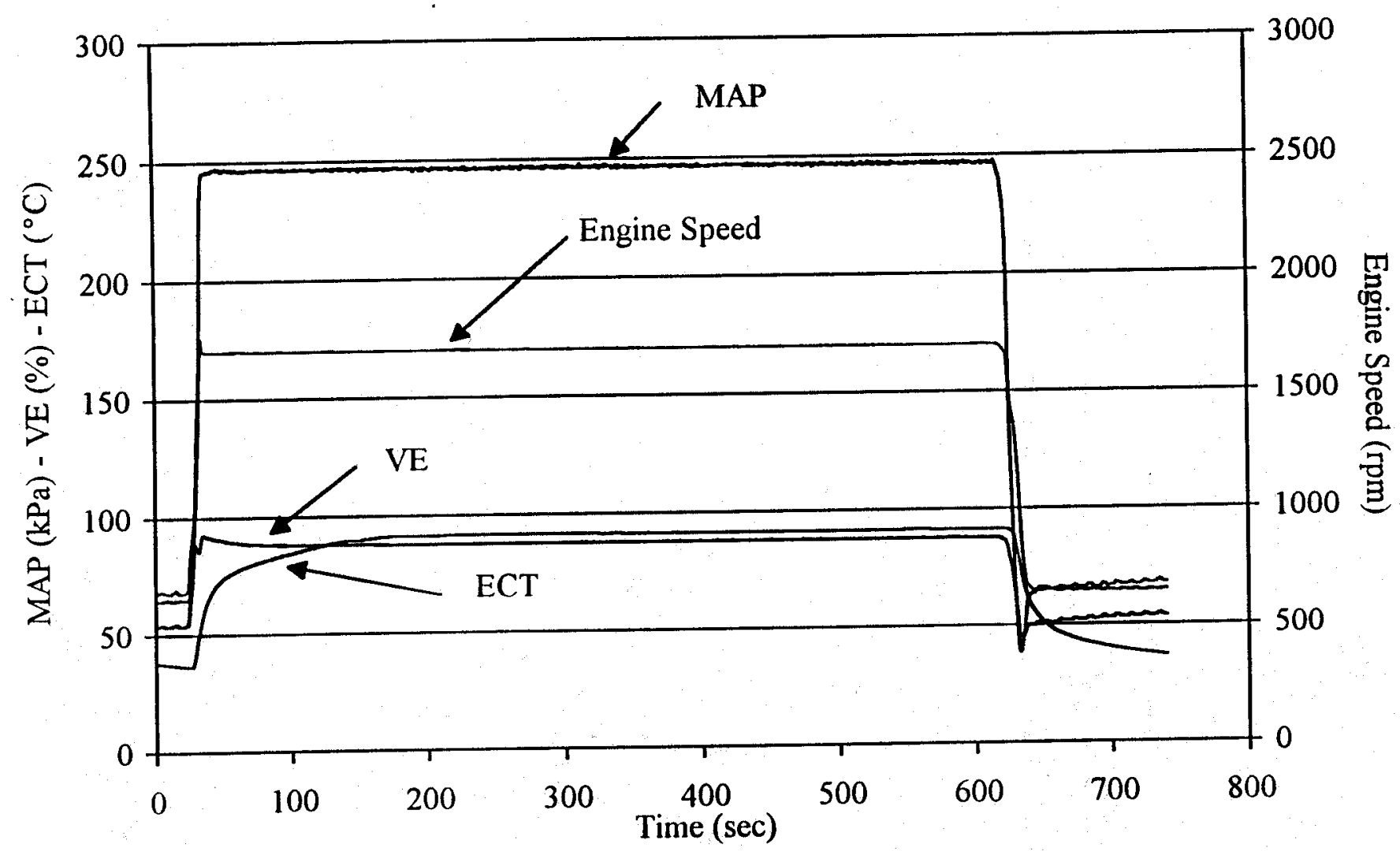

FIGURE 3. A : I I GE OF 40 READINGS RECORDED BETWEEN 1 AND 1000 HOURS FROM THE GEM SYSTEM DURING THE STC TEST. 


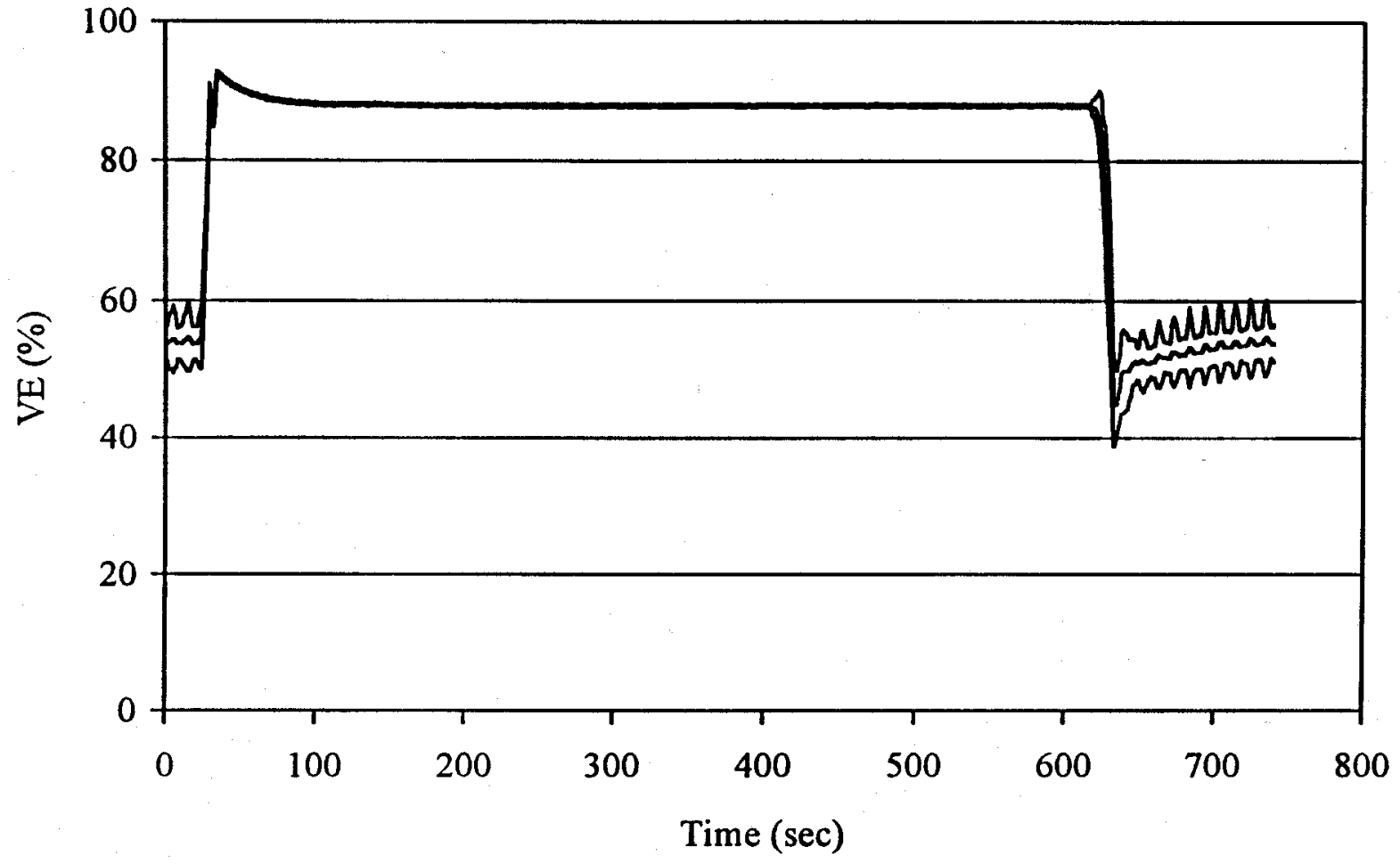

FIGURE 4. AVERAGE OF 40 READINGS AND \pm ONE STANDARD DEVIATION FOR VE RECORDED BETWEEN 1 AND 1000 HOURS FROM THE GEM SYSTEM DURING THE STCTEST. 


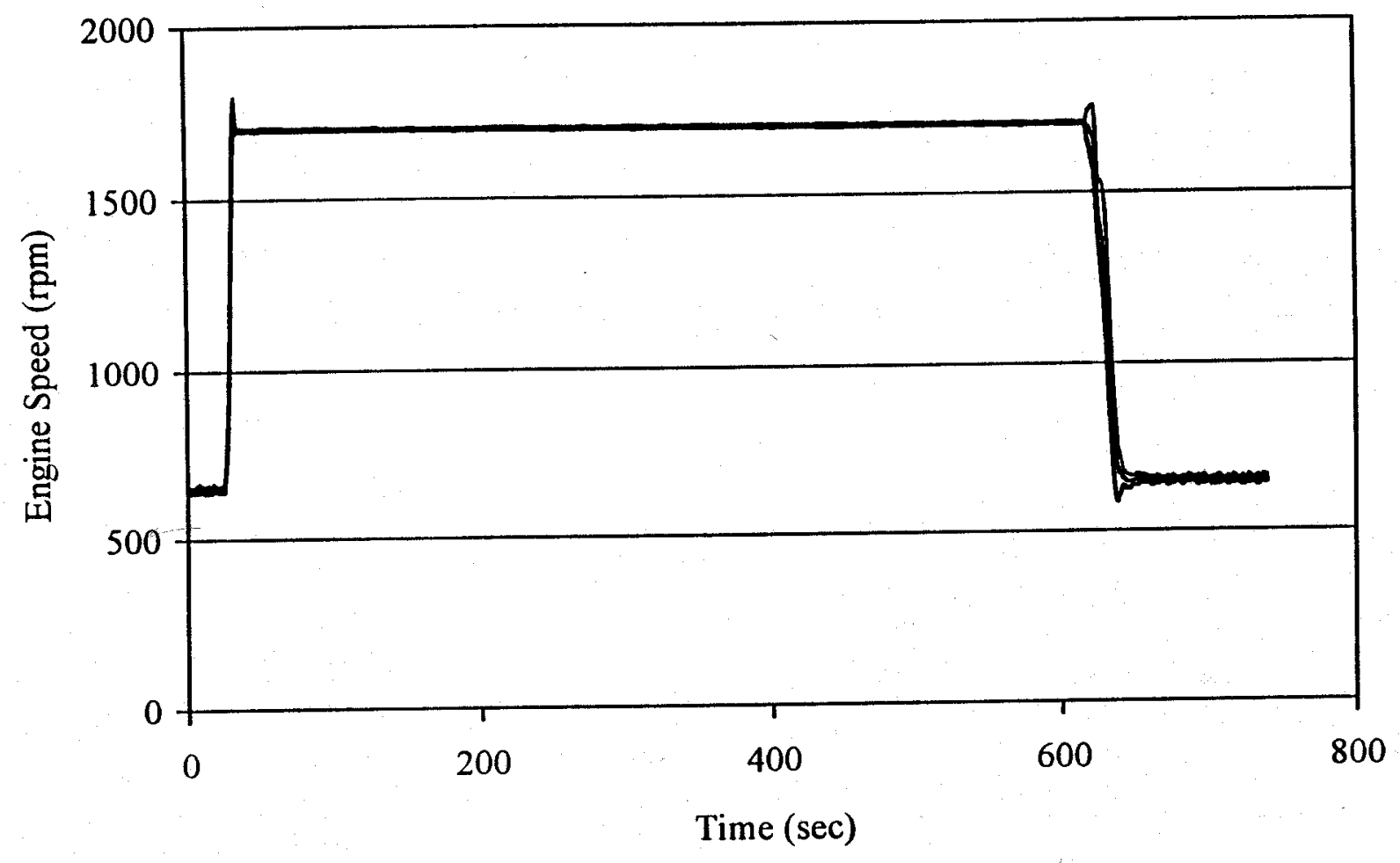

FIGURE 5. AVERAGE OF 40 READINGS AND \pm ONE STANDARD DEVIATION FOR ENGINE SPEED RECORDED BETWEEN 1 AND 1000 HOURS FROM THE GEM SYSTEM DURING THE STC TEST. 


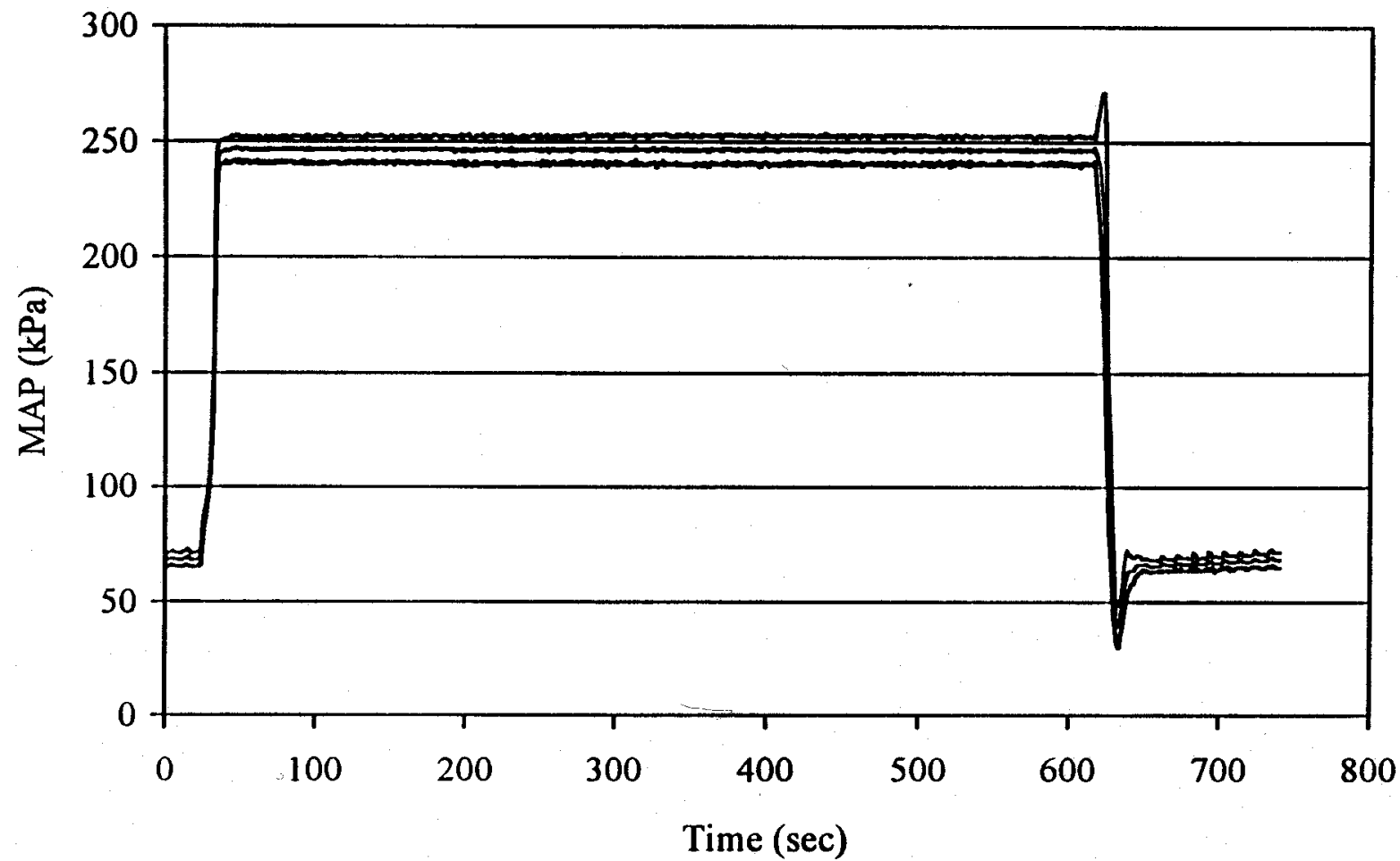

FIGURE 6. AVERAGE OF 40 READINGS AND \pm ONE STANDARD DEVIATION FOR MAP RECORDED BETWEEN 1 AND 1000 HOURS FROM THE GEM SYSTEM DURING THE STC TEST. 


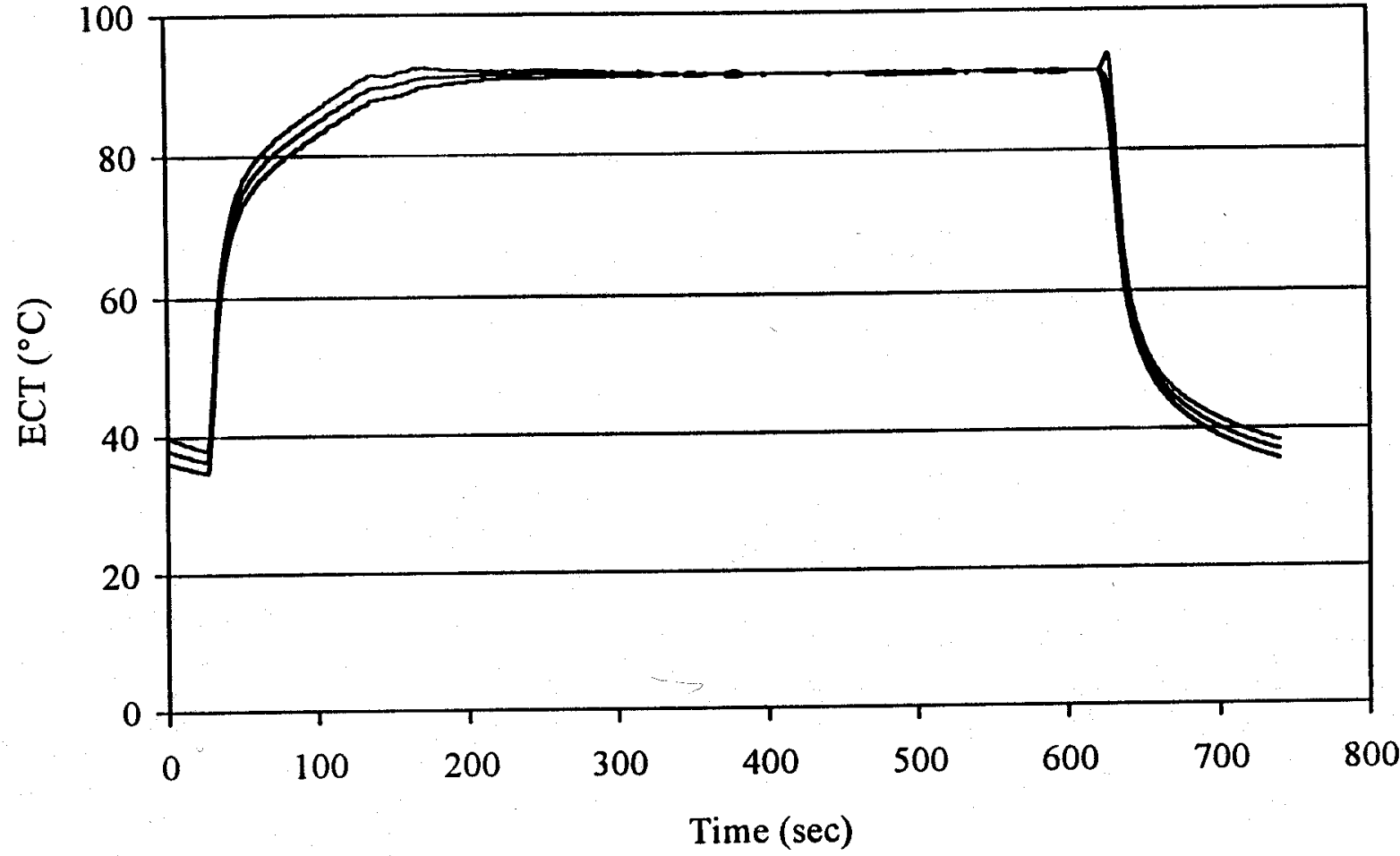

FIGURE 7. AVERAGE OF 40 READINGS AND \pm ONE STANDARD DEVIATION FOR ECT RECORDED BETWEEN 1 AND 1000 HOURS FROM THE GEM SYSTEM DURING THE STC TEST. 
that the engine has started, it de-energizes the solenoid, and the GEM system records the pressure in the injector block as it leaks down. Figures 8 and 9 show the data from the injector block leak down at the beginning and end of the test, respectively. All other data from the injector block leak down showed similar results. The static pressure to the injector block (setting of the low pressure regulator) is set to maintain at least $620 \mathrm{kPa}(90 \mathrm{psia})$ when the engine is in the loaded stage. The regulator was reset at 250 hours to account for the pressure loss associated with the addition of the adsorption filter, therefore the static pressure in the injector block at the end of the test was greater than at the beginning. Figure 10 shows the leak down rate during the STC test. Each of the injectors operated for approximately 68 million cycles at the end of the test.

\subsubsection{Oil Consumption}

Dryden Experimental natural gas oil was used for the durability test. Oil samples were taken and sent to Dryden Oil for analysis. Results of the analysis were sent to Mack Trucks.

Oil was added and oil consumption was recorded every 50 hours during the STC test: In addition, the level was checked at 25 hours after each oil add, and oil was added as needed. The procedure used for recording oil consumption was as follows:

(1) While engine is idling, take a small discard sample from the engine and record weight.

(2) After taking discard sample, take oil sample from engine and record weight.

(3) After engine is stopped, drain oil from blowby filter housing and record weight.

(4) After engine is stopped, record weight of oil leakage.

(5) After the engine has soaked for 10 minutes, fill with oil until level is at the full mark on the dip stick and record weight.

(6) Calculate oil consumption from the amount of oil added in step 5 plus the amount of oil added at the 25 hour oil level check (if any), minus the amount of oil drained in steps 1-4 minus any other oil drains during the 50 hours.

The estimated accuracy of the oil adds is approximately \pm 100 grams. Oil adds and drains are shown in Table 7 , and oil consumption is shown in Table 8 along with average blowby for each stage. At 150 hours, the drain from the blowby filter was not recorded correctly, therefore the oil consumption calculation at 150 hours in Table 8 includes the oil drained from the blowby filter housing.

\subsubsection{Valve Measurements}

Valve lash and valve stem heights were measured before, during, and after the STC test. Results of the intake and exhaust valve stem height measurements are shown in Figures 11 and 12, respectively. Data for the valve stem height measurements are included in Appendix C. Valve lash measurements are shown in Table 9. 


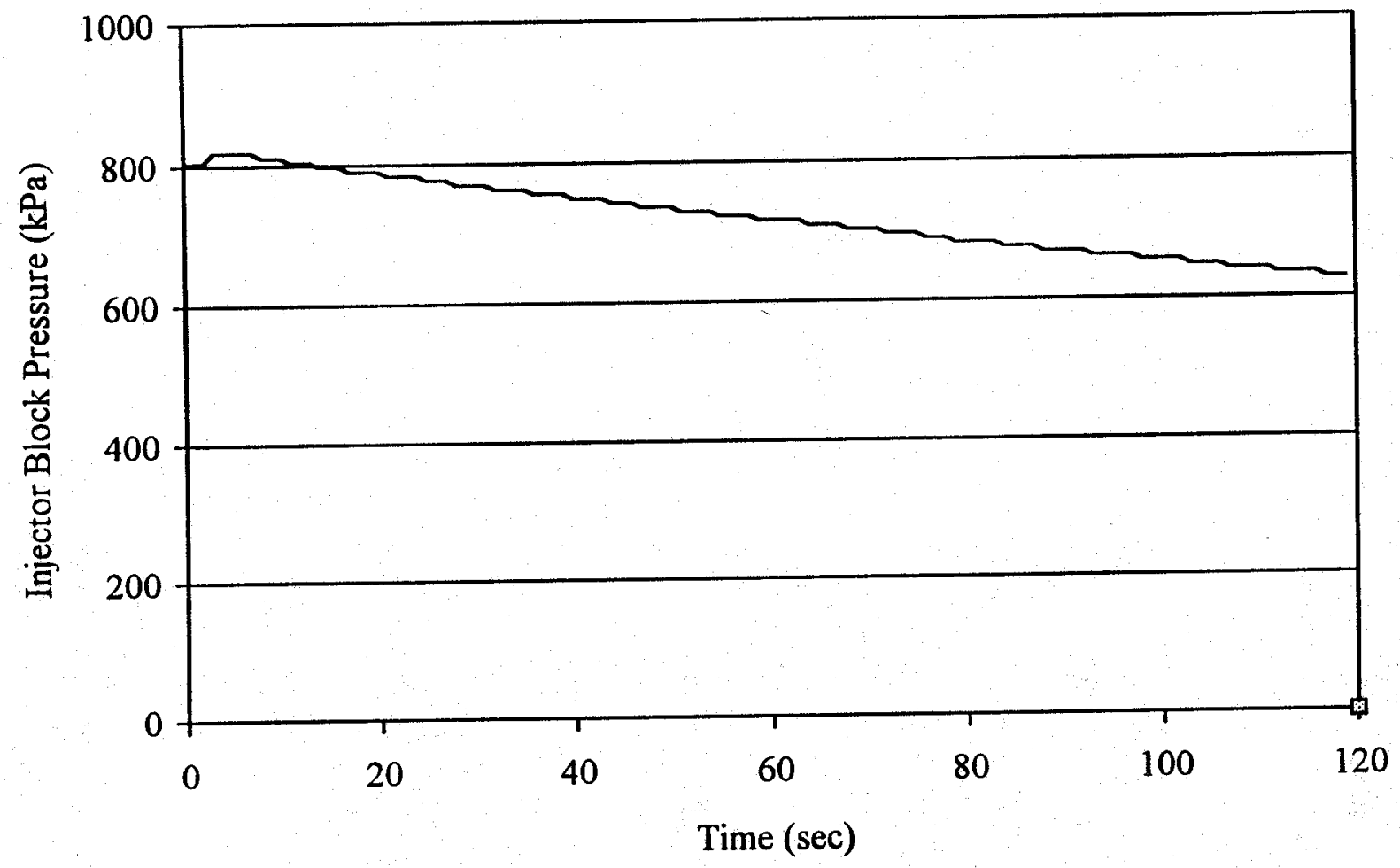

FIGURE 8. DATA FROM THE INJECTOR BLOCK LEAK DOWN BEFORE THE START OF STC TEST. 


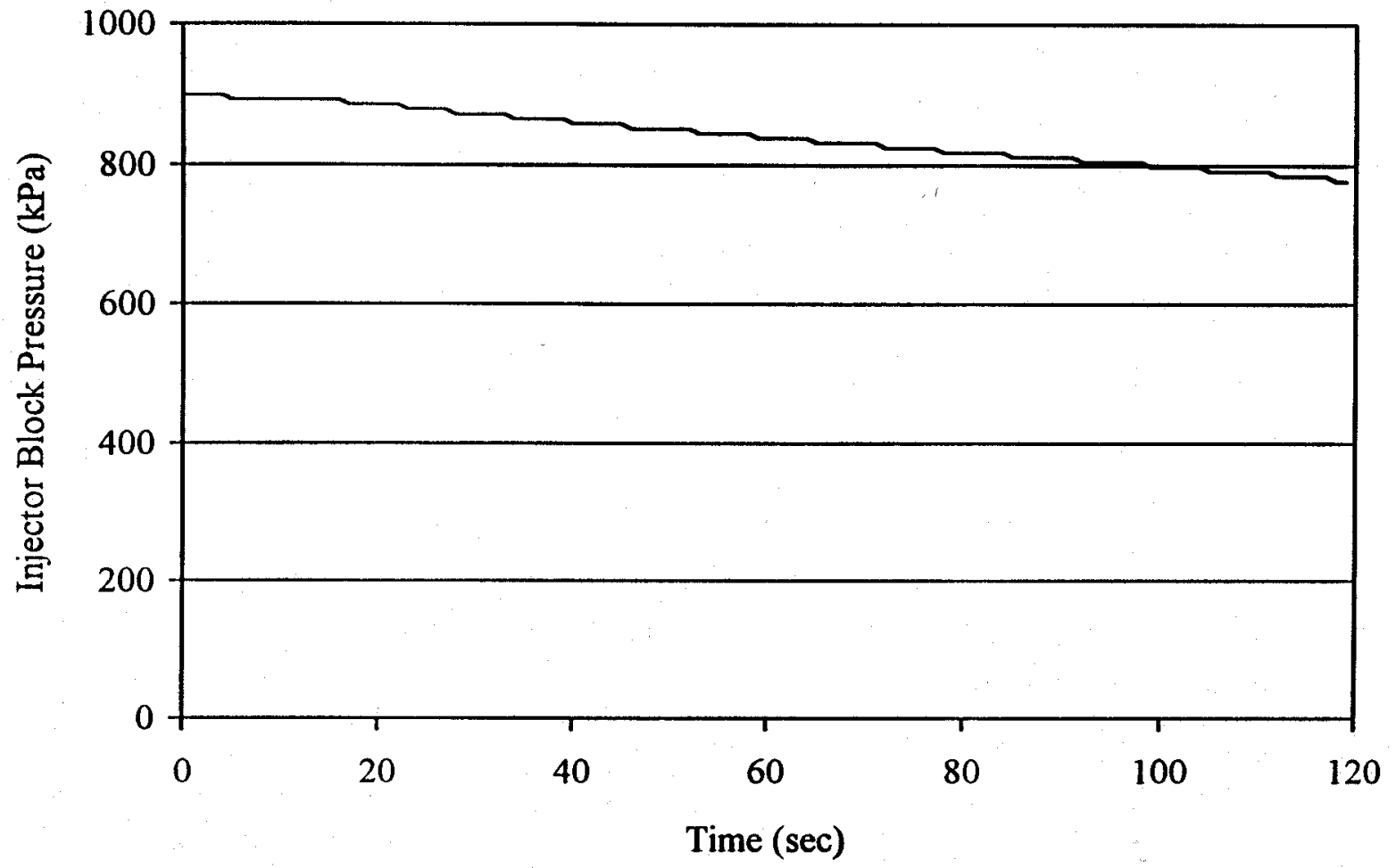

FIGURE 9. DATA FROM THE INJECTOR BLOCK LEAK DOWN AT THE END OF STC TEST. 


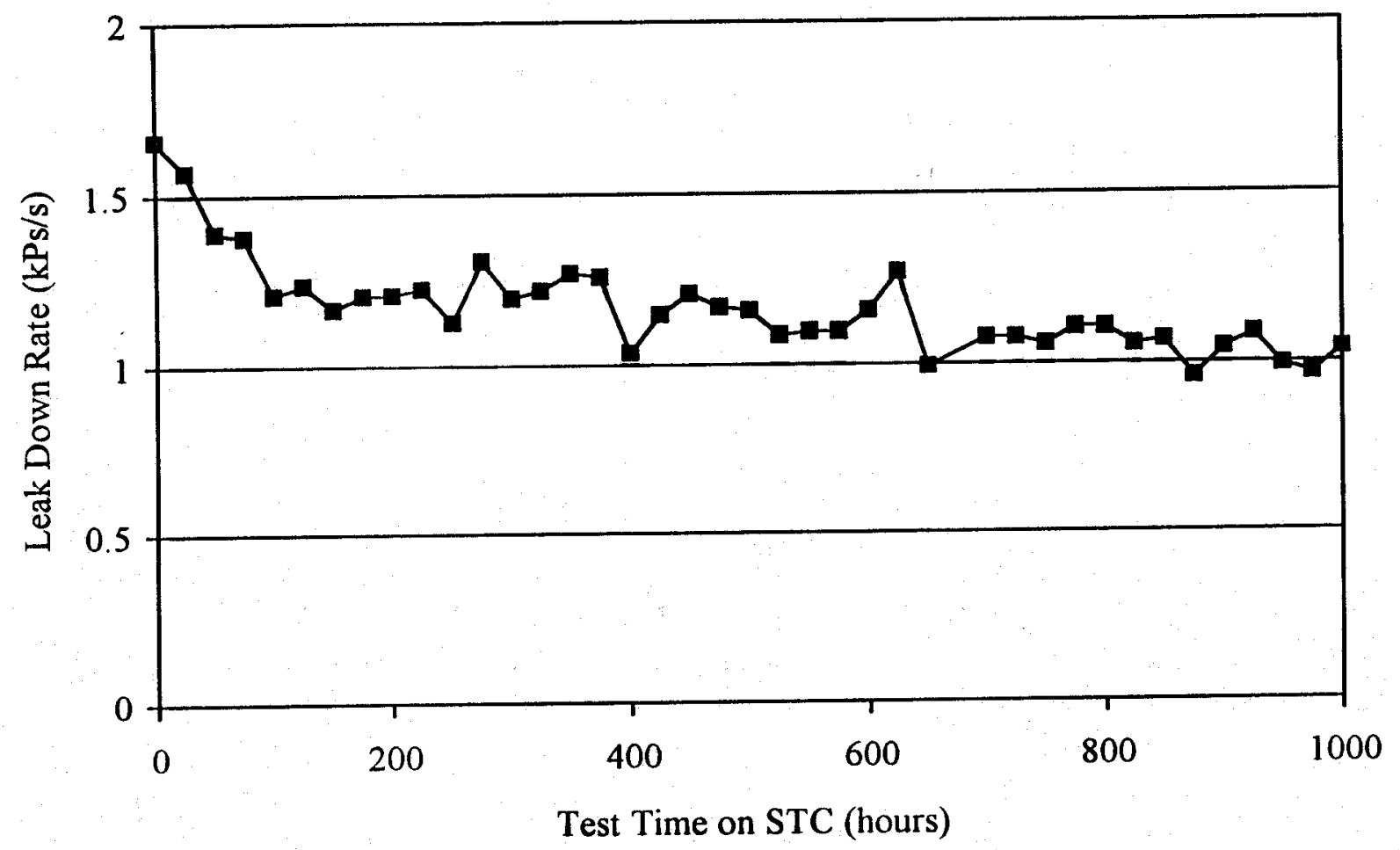

FIGURE 10. INJECTOR BLOCK LEAK DOWN RATE DURING THE STC TEST 
TABLE 7. OIL ADDS AND DRAINS

\begin{tabular}{|c|c|c|c|c|c||}
\hline $\begin{array}{c}\text { Test } \\
\text { Hour }\end{array}$ & $\begin{array}{c}\text { Oil } \\
\text { Add } \\
\text { (grams) }\end{array}$ & $\begin{array}{c}\text { Oil Drained } \\
\text { for Discard } \\
\text { (grams) }\end{array}$ & $\begin{array}{c}\text { Oil Drained } \\
\text { for Sample } \\
\text { (grams) }\end{array}$ & $\begin{array}{c}\text { Oil Drained } \\
\text { from Leakage } \\
\text { (grams) }\end{array}$ & $\begin{array}{c}\text { Oil Drained } \\
\text { from Blowby } \\
\text { (grams) }\end{array}$ \\
\hline \hline 1 & & 28 & 150 & & \\
\hline 50 & 2433 & 25 & 148 & 7 & 8 \\
\hline 100 & 1788 & 33 & 121 & 14 & 17 \\
\hline 150 & 1287 & 28 & 137 & 9 & \\
\hline 200 & 1956 & 28 & 138 & 6 & 33 \\
\hline 250 & 2827 & 24 & 144 & 7 & 39 \\
\hline 300 & 1949 & 40 & 148 & 25 & 57 \\
\hline 350 & 2345 & 24 & 141 & 28 & 28 \\
\hline 400 & 2440 & 18 & 134 & 21 & 77 \\
\hline 450 & 3047 & 12 & 136 & 18 & 114 \\
\hline 500 & 3168 & 23 & 151 & 21 & 111 \\
\hline 501 & & 19 & 144 & & 149 \\
\hline 550 & 2145 & 28 & 145 & 3 & 149 \\
\hline 600 & 3022 & 24 & 138 & 1 & 131 \\
\hline 650 & 2801 & 18 & 143 & 2 & 110 \\
\hline 700 & 2900 & 5 & 144 & 14 & 118 \\
\hline 750 & 2641 & 15 & 145 & 1 & 137 \\
\hline 775 & 2359 & & & & \\
\hline 800 & 1841 & 40 & 131 & 4 & 137 \\
\hline 850 & 3978 & 24 & 146 & 33 & 127 \\
\hline 870 & 1870 & & & & \\
\hline 900 & 3159 & 26 & 128 & 49 & 30 \\
\hline 925 & 1617 & & & & \\
\hline 950 & 3208 & 22 & 144 & 130 & \\
\hline 975 & 1632 & & & & \\
\hline 1000 & 3181 & 28 & 131 & & 12 \\
\hline
\end{tabular}




\section{TABLE 8. OIL CONSUMPTION}

\begin{tabular}{|c|c|c|c|c||}
\hline $\begin{array}{c}\text { Test } \\
\text { Hour }\end{array}$ & $\begin{array}{c}\text { Oil } \\
\text { Consumption } \\
\text { (grams) }\end{array}$ & $\begin{array}{c}\text { Oil } \\
\text { Consumption } \\
\text { (g/kw-hr) }\end{array}$ & $\begin{array}{c}\text { Average Blowby for } \\
\text { 650 rpm Operation } \\
\text { (//min) }\end{array}$ & $\begin{array}{c}\text { Average Blowby for } \\
\mathbf{1 7 0 0} \text { rpm Operation } \\
\text { (//min) }\end{array}$ \\
\hline \hline $0-50$ & 2,067 & 0.174 & 7.5 & 106.5 \\
\hline $50-100$ & 1,593 & 0.133 & 6.6 & 108.2 \\
\hline $100-150$ & 1,113 & 0.094 & 7.0 & 109.6 \\
\hline $150-200$ & 1,751 & 0.148 & 7.6 & 111.6 \\
\hline $200-250$ & 2,613 & 0.221 & 8.0 & 114.0 \\
\hline $250-300$ & 1,679 & 0.143 & 9.0 & 119.4 \\
\hline $300-350$ & 2,124 & 0.182 & 9.3 & 123.0 \\
\hline $350-400$ & 2,190 & 0.185 & 9.1 & 124.9 \\
\hline $400-450$ & 2,767 & 0.229 & 10.5 & 130.9 \\
\hline $450-500$ & 2,862 & 0.239 & 10.3 & 131.4 \\
\hline $500-550$ & 1,647 & 0.137 & 10.1 & 134.6 \\
\hline $550-600$ & 2,728 & 0.229 & 10.3 & 129.7 \\
\hline $600-650$ & 2,528 & 0.209 & 9.6 & 126.7 \\
\hline $650-700$ & 2,619 & 0.219 & 9.4 & 133.2 \\
\hline $700-750$ & 2,343 & 0.196 & 9.7 & 136.3 \\
\hline $750-800$ & 3,880 & 0.328 & 9.8 & 137.5 \\
\hline $800-850$ & 3,621 & 0.307 & 10.1 & 141.8 \\
\hline $850-900$ & 4,789 & 0.406 & 10.4 & 150.3 \\
\hline $900-950$ & 4,517 & 0.380 & 10.6 & 146.3 \\
\hline $950-1000$ & 4,497 & 0.371 & 9.3 & 128.2 \\
\hline $0-1000$ & 53,928 & 0.227 & & \\
\hline & & & & \\
\hline
\end{tabular}




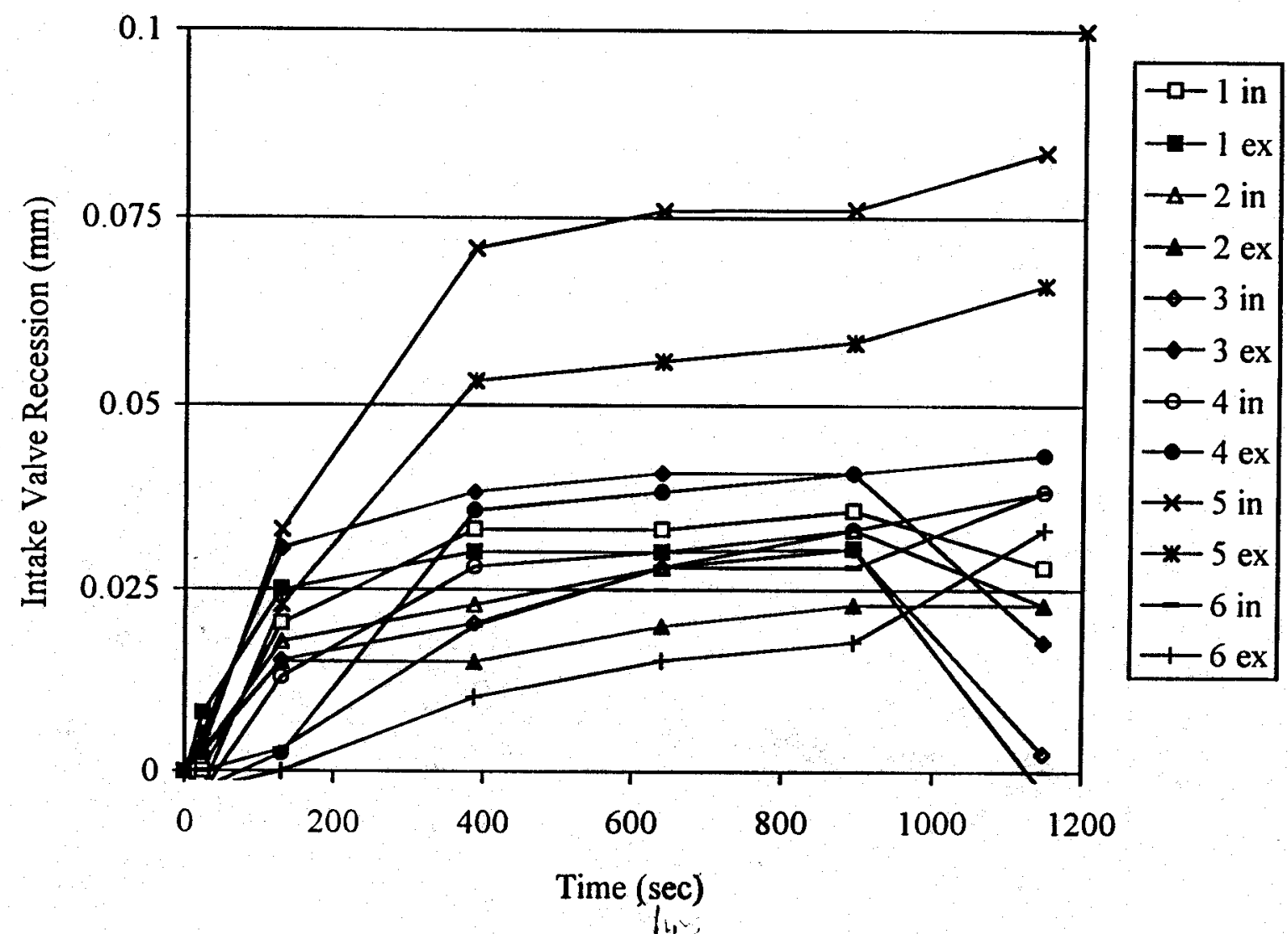

FIGURE 11. INTAKE VALVE RECESSION 


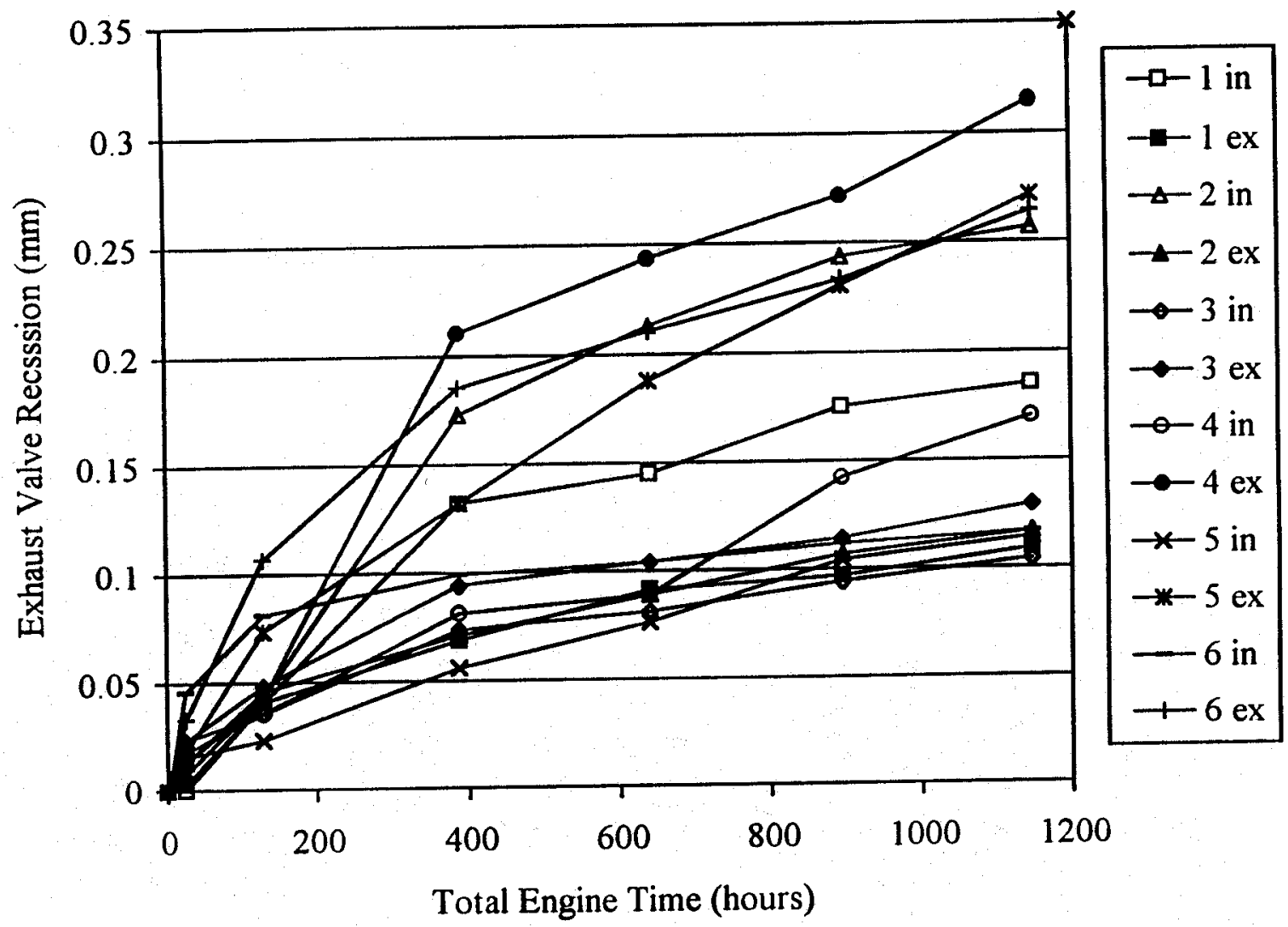

FIGURE 12. EXHAUST VALVE RECESSION 
TABLE 9. VALVE LASH MEASUREMENTS

\begin{tabular}{|c|c|c|c|c|c|c|c|c||}
\hline \multirow{2}{*}{ Cylinder } & \multirow{2}{*}{ Valve } & \multicolumn{7}{|c|}{ Lash (mm) @ Total Engine Hour } \\
\cline { 3 - 9 } & & $\mathbf{0 . 0}$ & $\mathbf{2 4 . 8}$ & $\mathbf{1 2 9 . 3}$ & $\mathbf{3 8 6 . 9}$ & $\mathbf{6 4 0 . 6}$ & $\mathbf{8 9 5 . 5}$ & $\mathbf{1 1 4 7 . 8}$ \\
\hline \hline 1 & in. & 0.41 & 0.33 & 0.30 & 0.28 & 0.28 & 0.28 & 0.28 \\
\hline 2 & in. & 0.41 & 0.36 & 0.30 & 0.28 & 0.25 & 0.25 & 0.25 \\
\hline 3 & in. & 0.41 & 0.36 & 0.33 & 0.30 & 0.28 & 0.28 & 0.30 \\
\hline 4 & in. & 0.41 & 0.38 & 0.33 & 0.28 & 0.28 & 0.25 & 0.28 \\
\hline 5 & in. & 0.41 & 0.38 & 0.36 & 0.36 & 0.30 & 0.30 & 0.30 \\
\hline 6 & in. & 0.41 & 0.38 & 0.33 & 0.30 & 0.28 & 0.28 & 0.25 \\
\hline 1 & ex. & 0.71 & 0.66 & 0.61 & 0.53 & 0.51 & 0.51 & 0.46 \\
\hline 2 & ex. & 0.71 & 0.69 & 0.61 & 0.56 & 0.51 & 0.51 & 0.48 \\
\hline 3 & ex. & 0.71 & 0.66 & 0.61 & 0.53 & 0.56 & 0.56 & 0.56 \\
\hline 4 & ex. & 0.71 & 0.66 & 0.64 & 0.56 & 0.53 & 0.51 & 0.46 \\
\hline 5 & ex. & 0.71 & 0.66 & 0.61 & 0.53 & 0.51 & 0.51 & 0.51 \\
\hline 6 & ex. & 0.71 & 0.69 & 0.64 & 0.58 & 0.53 & 0.53 & 0.53 \\
\hline
\end{tabular}

\subsubsection{Spark Plugs Maintenance}

Champion spark plugs were used for the durability test. New spark plugs were installed in the engine before the break-in. All new spark plugs installed in the engine were initially gapped to $0.25 \mathrm{~mm}\left(0.010^{\prime \prime}\right)$. The history of spark plug maintenance is shown in Table 10.

The initial set of RX501PYP/1E5 spark plugs were platinum-nickel tipped. At 410.6 total engine hours, the No. 4 cylinder started misfiring due to a large gap and the spark plug was replaced. At 416.8 total engine hours, the No. 5 cylinder started misfiring due to a large gap and the spark plug was replaced. At 427.5 total engine hours, spark plug Nos. 1,2,3, \& 6 were replaced. All replacements were with RX501PYP/1E5 spark plugs (platinum-nickel tipped). All six of the original spark plugs were sent to Champion for inspection.

At 565.1 total engine hours, the RX501PYP/1E5 spark plugs (platinum-nickel tipped) were replaced with RC550PYP/4F6 spark plugs (platinum-iridium tipped) for short-term use until the platinum-iridium tipped RX501PYP spark plugs were available. Since these spark plugs have a $19 \mathrm{~mm}$ reach $\left(0.75^{\prime \prime}\right)$ and the engine requires a $13 \mathrm{~mm}(0.50 ")$ reach. they were modified to fit this engine by adding a thick copper washer. 
TABLE 10. SPARK PLUG HISTORY

\begin{tabular}{|c|c|c|}
\hline $\begin{array}{c}\begin{array}{c}\text { Total Engine } \\
\text { Hours }\end{array} \\
\end{array}$ & $\begin{array}{l}\text { Test Hours } \\
\text { on the STC } \\
\end{array}$ & Description \\
\hline 0.0 & -- & $\begin{array}{l}\text { Installed new Champion RX501PYP/1E5 (platinum-nickel) } \\
\text { spark plugs, gapped to } 0.25 \mathrm{~mm}(0.010 ") \text {. }\end{array}$ \\
\hline 129.3 & 0.0 & Checked gaps, start of STC test. \\
\hline 386.9 & 250.0 & Checked gaps. \\
\hline 410.6 & 273.5 & $\begin{array}{l}\text { Misfire on cylinder No. } 4 \text {, replaced with new Champion } \\
\text { RX501PYP/1E5, gapped to } 0.25 \mathrm{~mm}\left(0.010^{\prime \prime}\right) \text {. }\end{array}$ \\
\hline 416.8 & 278.9 & $\begin{array}{l}\text { Misfire on cylinder No. 5, replaced with new Champion } \\
\text { RX501PYP/1E5, gapped to } 0.25 \mathrm{~mm}\left(0.010^{\prime \prime}\right) \text {. }\end{array}$ \\
\hline 427.5 & 289.5 & $\begin{array}{l}\text { Replaced spark plugs in cylinders Nos. } 1,2,3 \text {, and } 6 \text { with new } \\
\text { Champion RX501PYP/1E5, gapped to } 0.25 \mathrm{~mm}\left(0.010^{\prime \prime}\right) \text {. }\end{array}$ \\
\hline 565.1 & 425.0 & $\begin{array}{l}\text { Replaced spark plugs in all cylinders with new Champion } \\
\text { RC550PYP/4F6 (platinum-iridium) spark plugs, gapped to } \\
0.25 \mathrm{~mm}(0.010 ") \text {. (Spark plugs were modified with } \\
\text { additional copper gasket to provide the correct depth) }\end{array}$ \\
\hline 640.6 & 500.0 & $\begin{array}{l}\text { Replaced spark plugs in all cylinders with new Champion } \\
\text { RX501PYP/2H6 (platinum-iridium) spark plugs, gapped to } \\
0.25 \mathrm{~mm}\left(0.010^{\prime \prime}\right) \text {. }\end{array}$ \\
\hline 895.5 & 750.0 & Checked Gaps. \\
\hline 897.8 & 752.0 & $\begin{array}{l}\text { Spark plug in the No. } 2 \text { cylinder failed (ceramic insulator } \\
\text { broke into small pieces), replaced with new Champion } \\
\text { RX501PYP/2H6 gapped to } 0.25 \mathrm{~mm}\left(0.010^{\prime \prime}\right) \text {. Also replace } \\
\text { spark plug boot. }\end{array}$ \\
\hline 1060.8 & 914.1 & $\begin{array}{l}\text { Spark plug in the No. } 4 \text { cylinder failed (ceramic insulator } \\
\text { separated from metal shell), replaced with new Champion } \\
\text { RX501PYP/1E5 gapped to } 0.25 \mathrm{~mm}\left(0.010^{\prime \prime}\right) \text {. Also replaced } \\
\text { spark plug boot. }\end{array}$ \\
\hline 1060.8 & 914.1 & $\begin{array}{l}\text { Spark Plug in the No. } 1 \text { cylinder showed possible signs of } \\
\text { leakage between the ceramic insulator and the metal shell, } \\
\text { replaced with new Champion RX501PYP/2H6, gapped to } \\
0.25 \mathrm{~mm}(0.010 ") \text {. }\end{array}$ \\
\hline 1060.8 & 914.1 & Checked gaps while engine was down. \\
\hline 1079.8 & 932.7 & $\begin{array}{l}\text { Misfire on cylinder No. 6, replaced with new Champion } \\
\text { RX501PYP/1E5, gapped to } 0.25 \mathrm{~mm}\left(0.010^{\prime \prime}\right) \text {. }\end{array}$ \\
\hline 1147.6 & 1000.0 & Checked gaps. \\
\hline
\end{tabular}


At 640.6 total engine hours, the RC550PYP/4F6 spark plugs (platinum-iridium tipped) were replaced with RX501PYP/2H6 spark plugs (platinum-iridium tipped). The gaps on these spark plugs were checked at 895.5 total engine hours ( 254.9 hours on the spark plugs), as scheduled. At 897.7 total engine hours (257.1 hours on the spark plug), the spark plug in the No. 2 cylinder broke apart and was replaced with a RX501PYP/2H6 spark plug (platinum-iridium tipped). At 1060.8 total engine hours ( 420.2 hours on the spark plug), the spark plug in the No. 4 cylinder broke apart and was replaced with a RX501PYP/1E5 spark plug (platinum-nickel tipped), and the spark plug in the No. 1 cylinder showed signs of leakage and was replaced with a RX50IPYP/2H6 spark plug (platinum-iridium tipped). These three spark plugs were sent to Champion for inspection. At 1079.8 total engine hours ( 439.2 hours on the spark plug), the No. 6 cylinder started misfiring due to a large gap, and the spark plug was replaced with a RX501PYP/1E5 spark plug (platinum-nickel tipped).

Copies of the letters sent to Champion Spark Plugs concerning this test are included in Appendix D. The letters included a detailed discussion of the failure of the platinum-iridium spark plugs. According to Champion Spark Plugs, the failure was a result of over-torquing the spark plugs during installation. However, further experiments conducted by SwRI indicate that the spark plugs were not over-torqued. Figure 13 shows the spark plug gap growth and Table 11 shows the data.

\subsubsection{Cylinder Compression and Leak Down Measurements}

Cylinder compression and leak down measurements were recorded every 250 test hours on the STC cycle. The results are shown in Tables 12 and 13 respectively. Cylinder leak down was performed by regulating air upstream of a $1.02 \mathrm{~mm}\left(0.040^{\prime \prime}\right)$ orifice at $690 \mathrm{kPa}_{\text {gage }}(100 \mathrm{psig})$, and measuring pressure downstream of the orifice (cylinder pressure). The percent leak down is the difference between the upstream and downstream pressure normalized by the upstream pressure.

\subsubsection{Coolant Flow Measurements}

Coolant flow was measured every 500 hours on the STC cycle. A turbine flow meter was installed in the coolant line between the outlet of the engine and the gate valve for these tests. Coolant flow was measured at a typical valve position during the unloaded and loaded stages. The results are shown in Table 14.

\subsubsection{Unscheduled Shutdowns}

Shutdowns for the engine are scheduled for every 25 hours. Unscheduled shutdowns are shown in Table 15. 


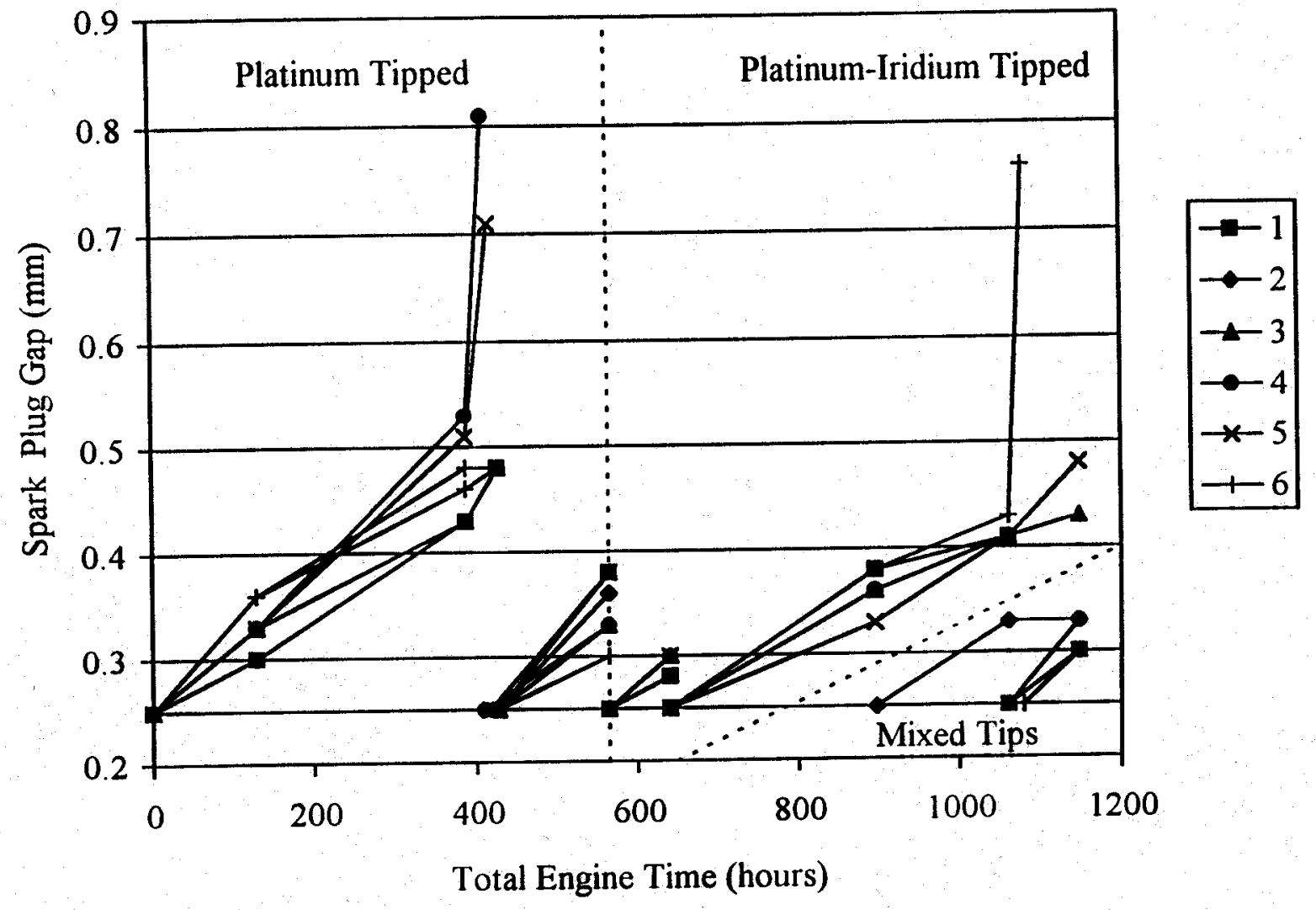

FIGURE 13. SPARK PLUG GAP GROWTH 
TABLE 11. SPARK PLUG GAPS

\begin{tabular}{|c|c|c|c|c|c|c|c|}
\hline \multirow{2}{*}{$\begin{array}{l}\text { Total } \\
\text { Engine } \\
\text { Hours }\end{array}$} & \multirow{2}{*}{$\begin{array}{c}\text { STC } \\
\text { Test } \\
\text { Hours }\end{array}$} & \multicolumn{6}{|c|}{ Gap for Cylinder (mm) } \\
\hline & & 1 & 2 & 3 & 4 & 5 & 6 \\
\hline 0.0 & 0.0 & 0.25 & 0.25 & 0.25 & 0.25 & 0.25 & 0.25 \\
\hline 129.3 & 0.0 & 0.30 & 0.30 & 0.33 & 0.33 & 0.33 & 0.36 \\
\hline 386.9 & 250.0 & 0.43 & 0.43 & 0.43 & 0.53 & 0.51 & 0.46 \\
\hline \multirow{3}{*}{410.6} & \multirow{3}{*}{273.5} & \multirow{3}{*}{\multicolumn{3}{|c|}{ 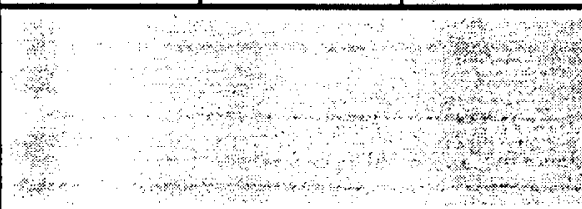 }} & 0.81 & \multirow{3}{*}{\multicolumn{2}{|c|}{ 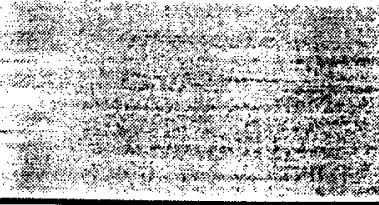 }} \\
\hline & & & & & Replaced & & \\
\hline & & & & & 0.25 & & \\
\hline \multirow{3}{*}{416.8} & \multirow{3}{*}{278.9} & \multirow{3}{*}{\multicolumn{2}{|c|}{ th }} & \multirow{3}{*}{\multicolumn{2}{|c|}{ hatwoty }} & 0.71 & 4. rem \\
\hline & & & & & & Replaced & \\
\hline & & & & & & 0.25 & $3+2 y$ \\
\hline \multirow{3}{*}{427.5} & \multirow{3}{*}{289.5} & 0.48 & 0.48 & 0.48 & \multirow{2}{*}{\multicolumn{2}{|c|}{ 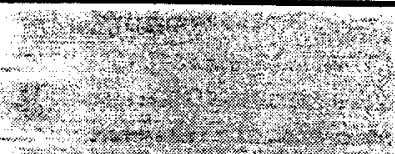 }} & 0.48 \\
\hline & & & Replaced & & & & Replaced \\
\hline & & 0.25 & 0.25 & 0.25 & 3y & $y^{2}-5 y+2$ & 0.25 \\
\hline \multirow{3}{*}{565.1} & \multirow{3}{*}{425.0} & 0.38 & 0.36 & 0.33 & 0.33 & 0.38 & 0.30 \\
\hline & & & & & aced & & \\
\hline & & 0.25 & 0.25 & 0.25 & 0.25 & 0.25 & 0.25 \\
\hline \multirow{3}{*}{640.6} & \multirow{3}{*}{500.0} & 0.28 & 0.30 & 0.25 & 0.28 & 0.30 & 0.30 \\
\hline & & \multicolumn{6}{|c|}{ Replaced } \\
\hline & & 0.25 & 0.25 & 0.25 & 0.25 & 0.25 & 0.25 \\
\hline 895.5 & 750.0 & 0.38 & 0.38 & 0.36 & 0.36 & 0.33 & 0.38 \\
\hline \multirow{2}{*}{897.8} & \multirow{2}{*}{752.0} & & Replaced & & & & \\
\hline & & & 0.25 & & & & \\
\hline \multirow{3}{*}{1060.8} & \multirow{3}{*}{914.1} & 0.41 & \multirow{3}{*}{0.33} & \multirow{3}{*}{0.41} & Replaced & \multirow{3}{*}{0.41} & \multirow{3}{*}{0.43} \\
\hline & & Replaced & & & 025 & & \\
\hline & & 0.25 & & & $0.2 \mathrm{~J}$ & & \\
\hline \multirow{3}{*}{1079.8} & \multirow{3}{*}{932.7} & & & & & & 0.76 \\
\hline & & & & & & & Replaced \\
\hline & & & & & & & 0.25 \\
\hline 1147.6 & 1000.0 & 0.30 & 0.33 & 0.43 & 0.33 & 0.48 & 0.30 \\
\hline
\end{tabular}


TABLE 12. CYLINDER PRESSURES

\begin{tabular}{||l||c|c|c|c|c||}
\hline \multicolumn{7}{|c|}{ Compression @ 200 rpm $\left(\mathbf{K P a}_{\text {aqars }}\right)$} \\
\hline \hline Total Engine Hours & $\mathbf{1 2 9 . 3}$ & $\mathbf{3 8 6 . 9}$ & $\mathbf{6 4 0 . 6}$ & $\mathbf{8 9 5 . 5}$ & $\mathbf{1 1 4 7 . 6}$ \\
\hline Hours on STC Cycle & $\mathbf{0 . 0}$ & $\mathbf{2 5 0 . 0}$ & $\mathbf{5 0 0 . 0}$ & $\mathbf{7 5 0 . 0}$ & $\mathbf{1 0 0 0 . 0}$ \\
\hline Cylinder No. 1 & 1469 & 1517 & 1586 & 1660 & 1590 \\
\hline Cylinder No. 2 & 1276 & 1448 & 1517 & 1520 & 1480 \\
\hline Cylinder No. 3 & 1531 & 1689 & 1724 & 1690 & 1690 \\
\hline Cylinder No. 4 & 1531 & 1620 & 1586 & 1660 & 1620 \\
\hline Cylinder No. 5 & 1572 & 1565 & 1586 & 1720 & 1690 \\
\hline Cylinder No. 6 & 1538 & 1517 & 1655 & 1690 & 1690 \\
\hline
\end{tabular}

TABLE 13. CYLINDER PRESSURE LEAK DOWN

\begin{tabular}{|l||c|c|c|c|c||}
\hline \multicolumn{7}{|c|}{ Leak Down (\%) } \\
\hline Total Engine Hours & $\mathbf{1 2 9 . 3}$ & $\mathbf{3 8 6 . 9}$ & $\mathbf{6 4 0 . 6}$ & $\mathbf{8 9 5 . 5}$ & $\mathbf{1 1 4 7 . 6}$ \\
\hline Hours on STC Cycle & $\mathbf{0 . 0}$ & $\mathbf{2 5 0 . 0}$ & $\mathbf{5 0 0 . 0}$ & $\mathbf{7 5 0 . 0}$ & $\mathbf{1 0 0 0 . 0}$ \\
\hline \hline Cylinder No. 1 & 78 & 80 & 80 & 76 & 74 \\
\hline Cylinder No. 2 & 80 & 88 & 87 & 88 & 82 \\
\hline Cylinder No. 3 & 36 & 36 & 41 & 48 & 50 \\
\hline Cylinder No. 4 & 74 & 47 & 78 & 56 & 75 \\
\hline Cylinder No. 5 & 49 & 43 & 47 & 57 & 52 \\
\hline Cylinder No. 6 & 18 & 30 & 17 & 21 & 22 \\
\hline
\end{tabular}

TABLE 14. COOLANT FLOW MEASUREMENTS

\begin{tabular}{|c|c|c|}
\hline $\begin{array}{c}\text { Hours on STC } \\
\text { Cycle }\end{array}$ & $\begin{array}{c}\text { Coolant Flow at } \\
\text { Unloaded Stage } \\
(\mathbf{l} / \mathbf{m})\end{array}$ & $\begin{array}{c}\text { Coolant Flow at } \\
\text { Loaded Stage } \\
(\mathbf{V} / \mathbf{m})\end{array}$ \\
\hline 0.0 & 232 & 314 \\
\hline 500.0 & 303 & 336 \\
\hline 1000.0 & 295 & 329 \\
\hline
\end{tabular}


TABLE 15. UNSCHEDULED SHUTDOWNS ON THE STC TEST

\begin{tabular}{|c|c|c|c|}
\hline Date & $\begin{array}{c}\text { Total } \\
\text { Engine } \\
\text { Time } \\
\text { (Hours) } \\
\end{array}$ & $\begin{array}{c}\text { STC } \\
\text { Test } \\
\text { Time } \\
\text { (Hours) } \\
\end{array}$ & Description \\
\hline $8 / 1 / 96$ & 160.4 & 26.1 & $\begin{array}{l}\text { "ALlim" high fault on GEM. Adjusted VE table, set } \\
\text { K-Adapt to } 0 \% \text {. Stopped engine and restarted. }\end{array}$ \\
\hline $8 / 1 / 96$ & 165.8 & 32.1 & $\begin{array}{l}\text { "CLlim" low fault on GEM. Stopped engine and } \\
\text { restarted. }\end{array}$ \\
\hline $8 / 2 / 96$ & 176.0 & 42.0 & $\begin{array}{l}\text { Air in cooling system. Stopped engine. Pressurized } \\
\text { coolant system with regulated shop air. Restarted engine. }\end{array}$ \\
\hline $8 / 2 / 96$ & 184.1 & 50.0 & $\begin{array}{l}\text { Engine would not start. Provided more fuel to start. } \\
\text { Restarted engine. }\end{array}$ \\
\hline $8 / 3 / 96$ & 205.3 & 71.1 & $\begin{array}{l}\text { Low water pressure to building. Stopped engine. Water } \\
\text { pressure to building restored. Restarted engine. }\end{array}$ \\
\hline $8 / 5 / 96$ & 236.2 & 100.4 & $\begin{array}{l}\text { Engine would not start. Modified VE table to provide } \\
\text { more fuel at idle. Restarted engine. }\end{array}$ \\
\hline $8 / 7 / 96$ & 294.9 & 158.9 & $\begin{array}{l}\text { "UEGOS fail" fault on GEM. Stopped engine and } \\
\text { restarted. }\end{array}$ \\
\hline $8 / 7 / 96$ & 297.0 & 160.9 & $\begin{array}{l}\text { "UEGOS fail" fault on GEM. Stopped engine. Replaced } \\
\text { UEGO sensor. Restarted engine. }\end{array}$ \\
\hline $8 / 10 / 96$ & 350.4 & 213.8 & $\begin{array}{l}\text { "UEGOS fail" fault on GEM. Stopped engine and } \\
\text { restarted. }\end{array}$ \\
\hline $8 / 10 / 96$ & 351.1 & 214.4 & $\begin{array}{l}\text { "UEGOS fail" fault on GEM. Stopped and restarted } \\
\text { engine. }\end{array}$ \\
\hline $8 / 13 / 96$ & 410.6 & 273.5 & $\begin{array}{l}\text { Cylinder No. } 4 \text { misfiring. Stopped engine. Replaced } \\
\text { spark plug. Restarted engine. }\end{array}$ \\
\hline $8 / 13 / 96$ & 412.6 & 275.2 & $\begin{array}{l}\text { "CLlim" low fault on GEM. Stopped engine and } \\
\text { restarted. }\end{array}$ \\
\hline $8 / 13 / 96$ & 416.8 & 278.9 & $\begin{array}{l}\text { Cylinder No. } 5 \text { misfiring. Stopped engine. Replaced } \\
\text { spark plug. Restarted engine. }\end{array}$ \\
\hline $8 / 14 / 96$ & 427.6 & 289.5 & $\begin{array}{l}\text { Stopped engine. Replaced spark plugs in cylinders Nos. } \\
1,2,3 \text {, and } 6 \text {. Restarted engine. }\end{array}$ \\
\hline $8 / 15 / 96$ & 445.4 & 307.0 & $\begin{array}{l}\text { Engine mounting bolt broke. Stopped engine. Replaced } \\
\text { bolt. Restarted engine. }\end{array}$ \\
\hline $8 / 17 / 96$ & 505.2 & 366.3 & $\begin{array}{l}\text { "UEGOS fail" fault on GEM. Stopped and restarted } \\
\text { engine. }\end{array}$ \\
\hline $8 / 19 / 96$ & 548.7 & 409.3 & Lab power failure. Engine stopped. Restarted engine. \\
\hline
\end{tabular}




\section{TABLE 15. UNSCHEDULED SHUTDOWNS ON THE STC TEST (CONT'D)}

\begin{tabular}{||l|c|c|l||}
\hline Date & $\begin{array}{c}\text { Total } \\
\text { Engine } \\
\text { Time } \\
\text { (Hours) }\end{array}$ & $\begin{array}{c}\text { STC } \\
\text { Test } \\
\text { Time } \\
\text { (Hours) }\end{array}$ & \multicolumn{1}{|c||}{ Description } \\
\hline $8 / 19 / 96$ & 522.2 & 412.3 & $\begin{array}{l}\text { Lost fuel pressure. Engine stopped. Fuel supply restored. } \\
\text { Restarted engine. }\end{array}$ \\
\hline $8 / 28 / 96$ & 658.5 & 515.3 & $\begin{array}{l}\text { Loose driveshaft coupling flange . Stopped engine. } \\
\text { Replaced flange and coupling. Restarted engine. }\end{array}$ \\
\hline $9 / 3 / 96$ & 821.0 & 577.0 & $\begin{array}{l}\text { Gas compressor failure. Engine stopped. Gas compressor } \\
\text { restarted. Restarted engine. }\end{array}$ \\
\hline $9 / 8 / 96$ & 845.2 & 700.3 & $\begin{array}{l}\text { Coolant leak at inlet ot turbocharger. Coolant line on } \\
\text { engine was supplied by SwRI. Stopped engine. Replaced } \\
\text { coolant line. Restarted engine. }\end{array}$ \\
\hline $9 / 9 / 96$ & 851.1 & 705.8 & $\begin{array}{l}\text { Engine mounting bolt broke. Stopped engine. Replaced } \\
\text { bolt. Restarted engine. }\end{array}$ \\
\hline $9 / 11 / 96$ & 895.0 & 749.6 & $\begin{array}{l}\text { Boost hose at turbocharger outlet came loose. Stopped } \\
\text { engine. Tightened clamps on boost hose. Restarted } \\
\text { engine. }\end{array}$ \\
\hline $9 / 21 / 96$ & 1079.8 & 932.7 & $\begin{array}{l}\text { Cylinder No. 6 misfiring. Stopped engine. Replaced } \\
\text { spark plug. Restarted engine. }\end{array}$ \\
\hline $9 / 12 / 96$ & 897.8 & 752.0 & $\begin{array}{l}\text { Spark plug in the No. 2 cylinder failed. Stopped engine. } \\
\text { Replaced spark plug and spark plug boot. Also replaced } \\
\text { exhaust stack thermocouple Restarted engine. }\end{array}$ \\
\hline $9 / 20 / 96$ & 1060.8 & 914.1 & $\begin{array}{l}\text { Spark plug in the No. 4 cylinder failed. Stopped engine. } \\
\text { Measured gap on all spark plugs. Replaced spark plug } \\
\text { and spark plug boot on No. 4 cylinder, replaced spark plug } \\
\text { on No. 1 cylinder. Restarted engine. }\end{array}$ \\
\hline
\end{tabular}




\subsubsection{Emissions Tests}

Exhaust emissions for the European emissions test (ECE R49, December 1993), both before and after the catalyst, were recorded at 0 and 500 test hours on the STC test. The engine was returned to its original cooling sytem configuration for these tests, and the calibration from the "Mack LNG Engine Development for Long-Haul Truck" project for Renault V.I. (RVI) conducted at SwRI was used. The test stand used for the "Mack LNG Engine Development for a Long Haul Truck" (cell 04) was different from the one used for the emissions tests at 0 and 500 hours reported here (cell 05). Due to the differences in the test stands, the exhaust system was lengthened by 41 $\mathrm{cm}(16 ")$ between the engine and the catalyst.

Results of the 13 mode tests at 0 test hours are shown in Table 16. Additional tests were conducted with the exhaust pipe insulated between the turbocharger and the catalyst for Mode 6 . Low speed data (run numbers 890-891, 894-915) are included in Appendix E. Further details of the emissions results are included in Appendix F.

\section{TABLE 16. RESULTS OF THE 13-MODE EUROPEAN EMISSIONS TEST AT 0 HOURS ON THE STC TEST}

\begin{tabular}{|c|c|c|c|c|c|c|c||}
\hline \multirow{2}{*}{ Mode } & \multirow{2}{*}{$\begin{array}{c}\text { Catalyst } \\
\text { THC Eff }\end{array}$} & \multicolumn{2}{|c|}{ BSTHC } & \multicolumn{2}{c|}{ BSNO $_{\mathbf{s}}$ (corrected) } & \multicolumn{3}{c||}{ BSCO } \\
\cline { 3 - 8 } & (g/kw-hr) & g/bhp-hr) & (g/kw-hr) & g/bhp-hr) & (g/kw-hr) & g/bhp-hr) \\
\hline $1,7,13$ & $15 \%$ & 13.87 & 10.37 & 0.71 & 0.53 & 0.00 & 0.00 \\
\hline 2 & $1 \%$ & 11.75 & 8.78 & 0.72 & 0.54 & 0.00 & 0.00 \\
\hline 3 & $32 \%$ & 3.18 & 2.38 & 1.54 & 1.15 & 0.00 & 0.00 \\
\hline 4 & $70 \%$ & .092 & 0.69 & 3.23 & 2.41 & 0.00 & 0.00 \\
\hline 5 & $72 \%$ & 1.50 & 1.12 & 3.78 & 2.83 & 0.00 & 0.00 \\
\hline 6 & $78 \%$ & 1.43 & 1.07 & 2.60 & 1.95 & 0.00 & 0.00 \\
\hline 8 & $83 \%$ & 0.40 & 0.30 & 2.22 & 1.66 & 0.00 & 0.00 \\
\hline 9 & $91 \%$ & 0.31 & 0.20 & 1.76 & 1.32 & 0.00 & 0.00 \\
\hline 10 & $96 \%$ & 0.32 & 0.20 & 1.01 & 0.75 & 0.00 & 0.00 \\
\hline 11 & $88 \%$ & 1.20 & 0.89 & 0.93 & 0.69 & 0.00 & 0.00 \\
\hline 12 & $84 \%$ & 3.40 & 2.54 & 0.91 & 0.68 & 0.00 & 0.00 \\
\hline 13 -mode & --- & 1.66 & 1.24 & 2.48 & 1.85 & 0.00 & 0.00 \\
\hline $6 *$ & $77 \%$ & 1.41 & 1.06 & 3.18 & 2.37 & 0.00 & 0.00 \\
\hline $13-$ mode* & --- & 1.65 & 1.23 & 2.71 & 2.02 & 0.00 & 0.00 \\
\hline$*$ Exhaust pipe insulated on mode 6. & & & & & \\
\hline \hline
\end{tabular}


Results of the 13 mode tests at 500 test hours are shown in Table 17. Additional tests were conducted with the exhaust pipe insulated between the turbocharger and the catalyst for Mode 6 . Low speed data (run numbers 3269-3292) are included in Appendix G. Further details of the emissions results are included in Appendix $\mathrm{H}$.

\section{TABLE 17. RESULTS OF THE 13-MODE EUROPEAN EMISSIONS TEST AT 500 HOURS ON THE STC TEST}

\begin{tabular}{||l|c|c|c|c|c|c|c||}
\hline \multirow{2}{*}{ Mode } & \multirow{2}{*}{$\begin{array}{c}\text { Catalyst } \\
\text { THC } \\
\text { Eff. }\end{array}$} & \multicolumn{2}{|c|}{ BSTHC } & BSNO (corrected) & \multicolumn{3}{c|}{ BSCO } \\
\cline { 3 - 8 } & (g/kw-hr) & g/bhp-hr) & (g/kw-hr) & g/bhp-hr) & (g/kw-hr) & g/bhp-hr) \\
\hline \hline $1,7,13$ & $1 \%$ & 18.06 & 13.47 & 0.64 & 0.48 & 0.00 & 0.00 \\
\hline 2 & $8 \%$ & 18.18 & 13.56 & 0.54 & 0.40 & 0.00 & 0.00 \\
\hline 3 & $14 \%$ & 5.03 & 3.75 & 0.90 & 0.67 & 0.00 & 0.00 \\
\hline 4 & $25 \%$ & 2.14 & 1.60 & 1.77 & 1.32 & 0.00 & 0.00 \\
\hline 5 & $24 \%$ & 4.04 & 3.01 & 1.87 & 1.40 & 0.00 & 0.00 \\
\hline 6 & $27 \%$ & 4.40 & 3.28 & 1.57 & 1.17 & 0.00 & 0.00 \\
\hline 8 & $33 \%$ & 7.41 & 5.53 & 0.98 & 0.73 & 0.00 & 0.00 \\
\hline 9 & $28 \%$ & 5.87 & 4.38 & 0.96 & 0.72 & 0.00 & 0.00 \\
\hline 10 & $44 \%$ & 8.62 & 6.43 & 0.64 & 0.48 & 0.00 & 0.00 \\
\hline 11 & $24 \%$ & 12.34 & 9.20 & 0.70 & 0.52 & 0.00 & 0.00 \\
\hline 12 & $24 \%$ & 27.49 & 20.50 & 0.74 & 0.56 & 0.00 & 0.00 \\
\hline 13 -mode & $26 \%$ & 5.86 & 4.37 & 1.35 & 1.01 & 0.00 & 0.00 \\
\hline $6 *$ & $63 \%$ & 2.06 & 1.53 & 1.70 & 1.27 & 0.00 & 0.00 \\
\hline 13 -mode* & $36 \%$ & 4.91 & 3.66 & 1.41 & 1.05 & 0.00 & 0.00 \\
\hline$*$ Exhaust pipe insulated on mode 6. & & & & & & \\
\hline
\end{tabular}

At 500 hours on the STC test, the catalyst was removed from the exhaust system at the request of RVI. Table 18 shows a summary of all the emissions results using the final calibration from the Mack LNG Engine Development for a Long Haul Truck. A total runtime history of the catalyst is shown in Table 19. 
TABLE 18. EMISSIONS SUMMARY WITH THE FINAL RVI CALIBRATION

\begin{tabular}{|l|c|c|c|c||}
\hline \multicolumn{1}{|c|}{ Test } & $\begin{array}{c}\text { Catalyst } \\
\text { THC Eff. }\end{array}$ & $\begin{array}{c}\text { BSHC } \\
(\mathbf{g} / \mathbf{k w}-\mathbf{h r})\end{array}$ & $\begin{array}{c}\text { BSNO } \\
(\mathrm{g} / \mathbf{k w}-\mathbf{h r})\end{array}$ & $\begin{array}{c}\text { BSCO } \\
(\mathrm{g} / \mathbf{k w}-\mathbf{h r})\end{array}$ \\
\hline \hline Original & $80 \%$ & 0.84 & 3.25 & 0.00 \\
\hline Before STC Test & $65 \%$ & 1.66 & 2.53 & 0.00 \\
\hline Before STC Test * & $68 \%$ & 1.65 & 2.71 & 0.00 \\
\hline At 500 hours on the STC Test & $26 \%$ & 5.86 & 1.35 & 0.00 \\
\hline At 500 hours on the STC Test * & $36 \%$ & 4.91 & 1.41 & 0.00 \\
\hline Target & --- & 1.0 & 3.5 & 3.0 \\
\hline Euro II & $-\cdots$ & 1.1 & 7.0 & 4.0 \\
\hline * Exhuast pipe insulated on mode 6. & & & \\
\hline
\end{tabular}

TABLE 19. CATALYST RUNTIME HISTORY

\begin{tabular}{||l|c|c||}
\hline \multicolumn{1}{|c|}{ Purpose } & Hours & $\begin{array}{c}\text { Total } \\
\text { Hours }\end{array}$ \\
\hline \hline Break-In & 21.9 & 21.9 \\
\hline Emissions Tests & 47.3 & 69.2 \\
\hline Reat Rejection Tests & 2.4 & 71.6 \\
\hline Demonstration to RVI & 4.7 & 76.3 \\
\hline Emissions Tests & 2.8 & 79.1 \\
\hline STC Test & 511.3 & 590.4 \\
\hline Emissions Tests & 2.6 & 593.0 \\
\hline
\end{tabular}




\subsection{CONCLUSIONS \& RECOMMENDATIONS}

A 1000-hour durability test was successfully completed. Several conclusions regarding the technical content of the program were made.

(1) Oil consumption increased significantly during the last 250 hours of the STC test. The high oil consumption can be attributed in part to a failed blowby filter, which allowed oil to directly enter the engine through the intake system. The filter had broken apart and had plugged the drain on the blowby canister as shown by the low blowby drains during the last 250 hours on the STC cycle. Heavy oil deposits were observed on the inlet to the turbocharger.

(2) The valve/valve seat materials selected for this test showed low recession. As reported by Mack Trucks, half of the valves had rotators installed, and the other half did not. The rotators did not appear to affect the valve recession significantly.

(3) The platinum-iridium tipped spark plugs showed a lower gap growth than the platinumnickel tipped spark plugs.

(4) After the test, the engine was shipped to Mack Trucks for disassembly. Mack Trucks reported that one of the pistons had a crack in it, and that the valves with rotators installed showed wear from the rotation of the valves.

(5) The catalyst showed significant loss in hydrocarbon conversion efficiency when aged for 500 hours.

Recommendations for future work include:

(1) Improved design of the blowby filter for longer life;

(2) Further research into the cause of the platinum-iridium tipped spark plug failures, which did not occur with the platinum-nickel tipped spark plugs;

(3) Use of platinum-iridium tipped spark plugs with a recommended regap maintenance interval to extend the life of the spark plugs (Note: Due to the high BMEP during this test, spark plug regap maintenance interval may be extended to a period longer than shown here);

(4) Redesign of the piston and another 1000-hour STC test to verify the durability of the new piston design. 
APPENDIX A

LISTING OF RUN NUMBERS 
STC Test Runs

\begin{tabular}{|c|c|c|c|c|c|c|}
\hline $\begin{array}{l}\text { Run \# } \\
\text { From }\end{array}$ & $\begin{array}{c}\text { Run \# } \\
\text { To }\end{array}$ & $\begin{array}{c}\text { Test Hours } \\
\text { From }\end{array}$ & $\begin{array}{c}\text { Test Hours } \\
\text { To }\end{array}$ & Hourly & Snapshot & Comments \\
\hline 978 & 979 & 0 & 0 & $x$ & & \\
\hline 980 & 1035 & 0 & 0 & & $x$ & \\
\hline 1036 & 1083 & 1 & 24 & $x$ & & \\
\hline 1084 & 1139 & 25 & 25 & & $x$ & \\
\hline 1140 & 1141 & 25 & 25 & $\bar{x}$ & & \\
\hline 1142 & 1189 & 26 & 49 & $\mathrm{x}$ & & \\
\hline 1190 & 1245 & 50 & 50 & & $x$ & \\
\hline 1258 & 1258 & 50 & 50 & $x$ & & $650 \mathrm{rpm}$ reading \\
\hline 1298 & 1298 & 50 & 50 & $\bar{x}$ & & $1700 \mathrm{rpm}$ reading \\
\hline 1302 & 1349 & 51 & 74 & $x$ & & \\
\hline 1350 & 1405 & 75 & 75 & & $x$ & \\
\hline 1406 & 1407 & 75 & 75 & $x$ & & \\
\hline 1408 & 1455 & 76 & 99 & $x$ & & \\
\hline $1+56$ & 1511 & 100 & 100 & & $x$ & \\
\hline 1512 & 1513 & 100 & 100 & $x$ & & \\
\hline 1514 & 1561 & 101 & 124 & $x$ & & \\
\hline 1562 & 1617 & 125 & 125 & & $x$ & \\
\hline 1618 & 1619 & 125 & 125 & $\bar{x}$ & & \\
\hline 1620 & 1623 & 126 & 127 & $x$ & & \\
\hline 1626 & 1669 & 128 & 149 & $x$ & & \\
\hline 1726 & 1727 & 150 & 150 & $x$ & & \\
\hline 1728 & 1783 & 150 & 150 & & $x$ & \\
\hline 1784 & 1831 & 151 & 174 & $x$ & & \\
\hline 1832 & 1887 & 175 & 175 & & $x$ & \\
\hline 1888 & 1889 & 175 & 175 & $x$ & & \\
\hline 1890 & 1937 & 176 & 199 & $\mathbf{x}$ & & \\
\hline 1938 & 1993 & 200 & 200 & & $x$ & \\
\hline 1994 & 1995 & 200 & 200 & $x$ & & \\
\hline 1996 & 2043 & 201 & 224 & $x$ & & \\
\hline 2044 & 2099 & 225 & 225 & & $x$ & \\
\hline 2100 & 2101 & 225 & 225 & $x$ & & \\
\hline 2102 & 2149 & 226 & 249 & $x$ & & \\
\hline 2150 & 2205 & 250 & 250 & & $\mathrm{x}$ & \\
\hline 2206 & 2207 & 250 & 250 & $x$ & & \\
\hline 2208 & 2255 & 251 & 274 & $x$ & & \\
\hline 2256 & 2311 & 275 & 275 & & $x$ & \\
\hline 2312 & 2313 & 275 & 275 & $\bar{x}$ & & \\
\hline 2314 & 2341 & 276 & 289 & $x$ & & \\
\hline & & 290 & 290 & $x$ & & Missed Reading \\
\hline 2344 & 2361 & 291 & 299 & $x$ & & \\
\hline 2362 & 2417 & 300 & 300 & & $x$ & \\
\hline 2418 & 2419 & 300 & 300 & $x$ & & \\
\hline $2+20$ & $2+31$ & 301 & 306 & $x$ & & \\
\hline 2433 & $2+68$ & 307 & 324 & $\mathrm{x}$ & & \\
\hline 2469 & 2523 & 325 & 325 & & $\mathrm{x}$ & \\
\hline 2524 & 2525 & 325 & 325 & $x$ & & \\
\hline 2526 & 2573 & 326 & 349 & $x$ & & \\
\hline 2574 & 2629 & 350 & 350 & & $x$ & \\
\hline 2630 & 2631 & 350 & 350 & $x$ & & \\
\hline
\end{tabular}


STC Test Runs

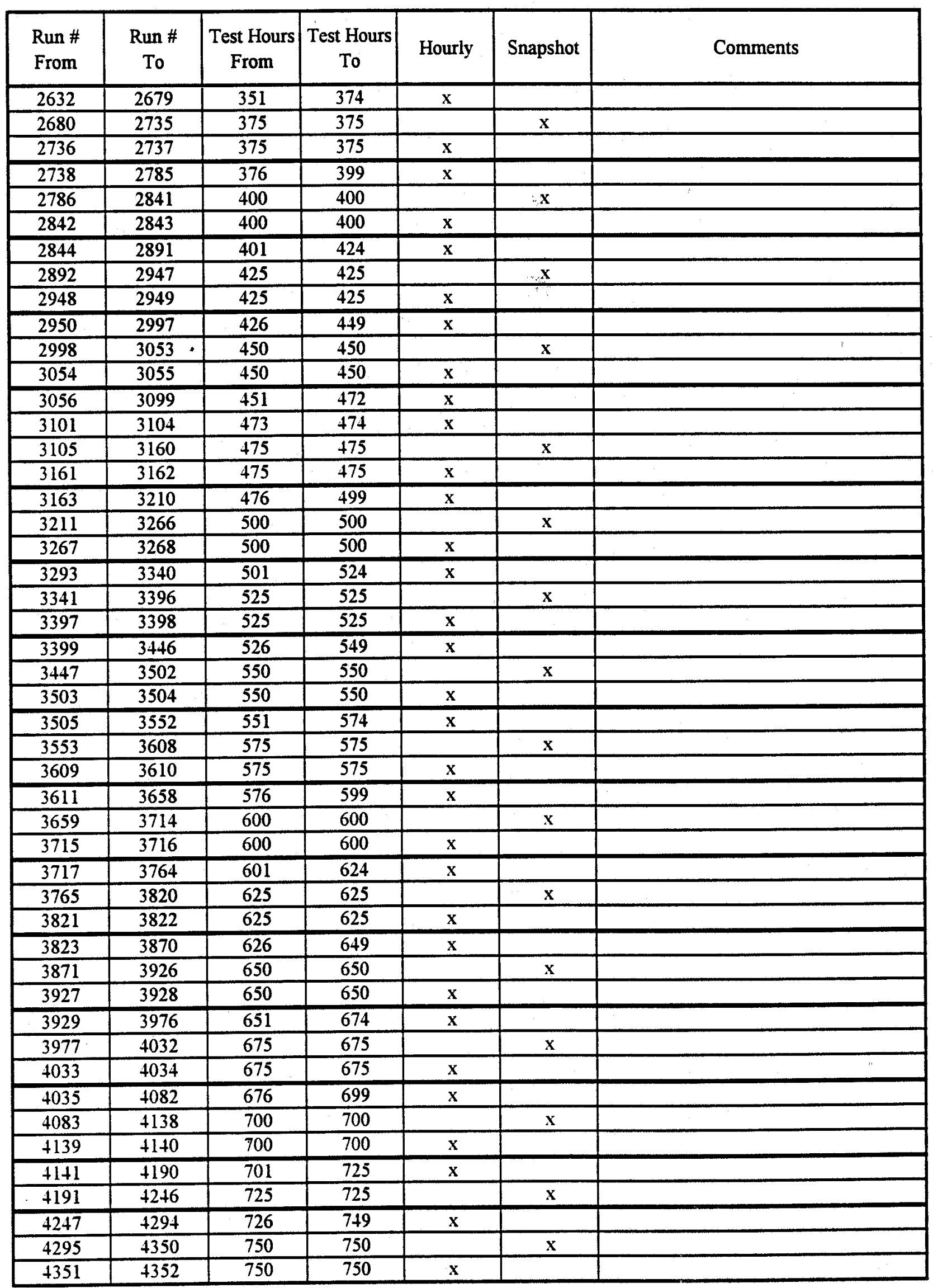


STC Test Runs

\begin{tabular}{|c|c|c|c|c|c|c|}
\hline $\begin{array}{l}\text { Run \# } \\
\text { From }\end{array}$ & $\begin{array}{c}\text { Run \# } \\
\text { To }\end{array}$ & $\begin{array}{c}\text { Test Hours } \\
\text { From }\end{array}$ & $\begin{array}{c}\text { Test Hours } \\
\text { To }\end{array}$ & Hourly & Snapshot & Comments \\
\hline \multirow[t]{2}{*}{4353} & 4354 & 751 & 751 & $\bar{x}$ & & \\
\hline & & 752 & 752 & $\bar{x}$ & & Missed Reading \\
\hline 4356 & 4399 & 753 & 774 & $\mathrm{x}$ & & \\
\hline 4400 & 4455 & 775 & 775 & & $x$ & \\
\hline 4456 & 4457 & 775 & 775 & $x$ & & \\
\hline 4458 & 4505 & 776 & 799 & $x$ & & \\
\hline 4506 & 4561 & 800 & 800 & & $x$ & \\
\hline 4562 & 4563 & 800 & 800 & $x$ & & \\
\hline 4564 & 4611 & 801 & 824 & $x$ & & \\
\hline 4612 & 4667 & 825 & 825 & & $x$ & \\
\hline 4668 & 4669 & 825 & 825 & $\bar{x}$ & & \\
\hline \multirow[t]{2}{*}{4670} & 4689 & 826 & 835 & $x$ & & \\
\hline & & 836 & 836 & & & Missed reading \\
\hline 4690 & +715 & 837 & 849 & $x$ & & \\
\hline 4716 & 4771 & 850 & 850 & & $\bar{x}$ & \\
\hline 4772 & 4773 & 850 & 850 & $\bar{x}$ & & \\
\hline 4774 & 4821 & 851 & 874 & $x$ & & \\
\hline 4822 & 4877 & 875 & 875 & & $x$ & \\
\hline 4878 & 4879 & 875 & 875 & $x$ & & \\
\hline 4880 & 4927 & 876 & 899 & $x$ & & \\
\hline 4928 & 4983 & 900 & 900 & & $x$ & \\
\hline 4984 & 4985 & 900 & 900 & $\bar{x}$ & & \\
\hline \multirow[t]{2}{*}{4986} & 5011 & 901 & 913 & $x$ & & \\
\hline & & 914 & 914 & & & Missed Reading \\
\hline 5013 & 5032 & 915 & 924 & $x$ & & \\
\hline 5033 & 5088 & 925 & 925 & & $x$ & \\
\hline 5089 & 5090 & 925 & 925 & $x$ & & \\
\hline 5091 & 5102 & 926 & 931 & $\mathrm{x}$ & & \\
\hline 5105 & 5140 & 932 & 949 & $x$ & & \\
\hline 5141 & 5196 & 950 & 950 & & $x$ & \\
\hline 5197 & 5198 & 950 & 950 & $x$ & & \\
\hline 5199 & 5246 & 951 & 974 & $\bar{x}$ & & \\
\hline 5247 & 5302 & 975 & 975 & & $x$ & \\
\hline 5303 & 5304 & 975 & 975 & $x$ & & \\
\hline 5305 & 5352 & 976 & 999 & $x$ & & \\
\hline 5353 & 5408 & 1000 & 1000 & & $x$ & \\
\hline 5409 & 5410 & 1000 & 1000 & $\bar{x}$ & & \\
\hline
\end{tabular}


APPENDIX B

GEM TRANSIENT DATA 


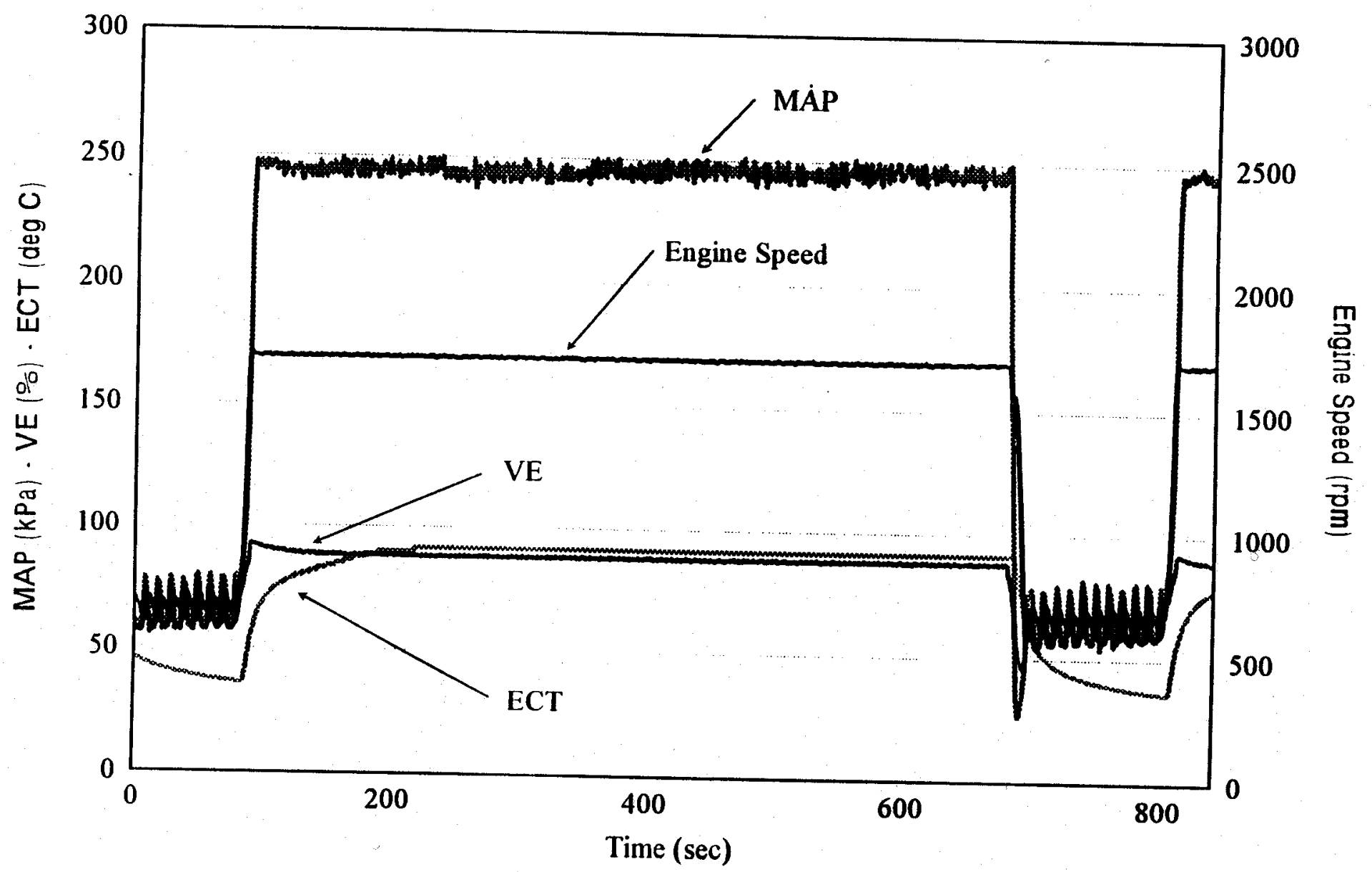

TRANSIENT DATA RECORDED FROM THE GEM SYSTEM FOR ONE FULL CYCLE AT I HOUR 


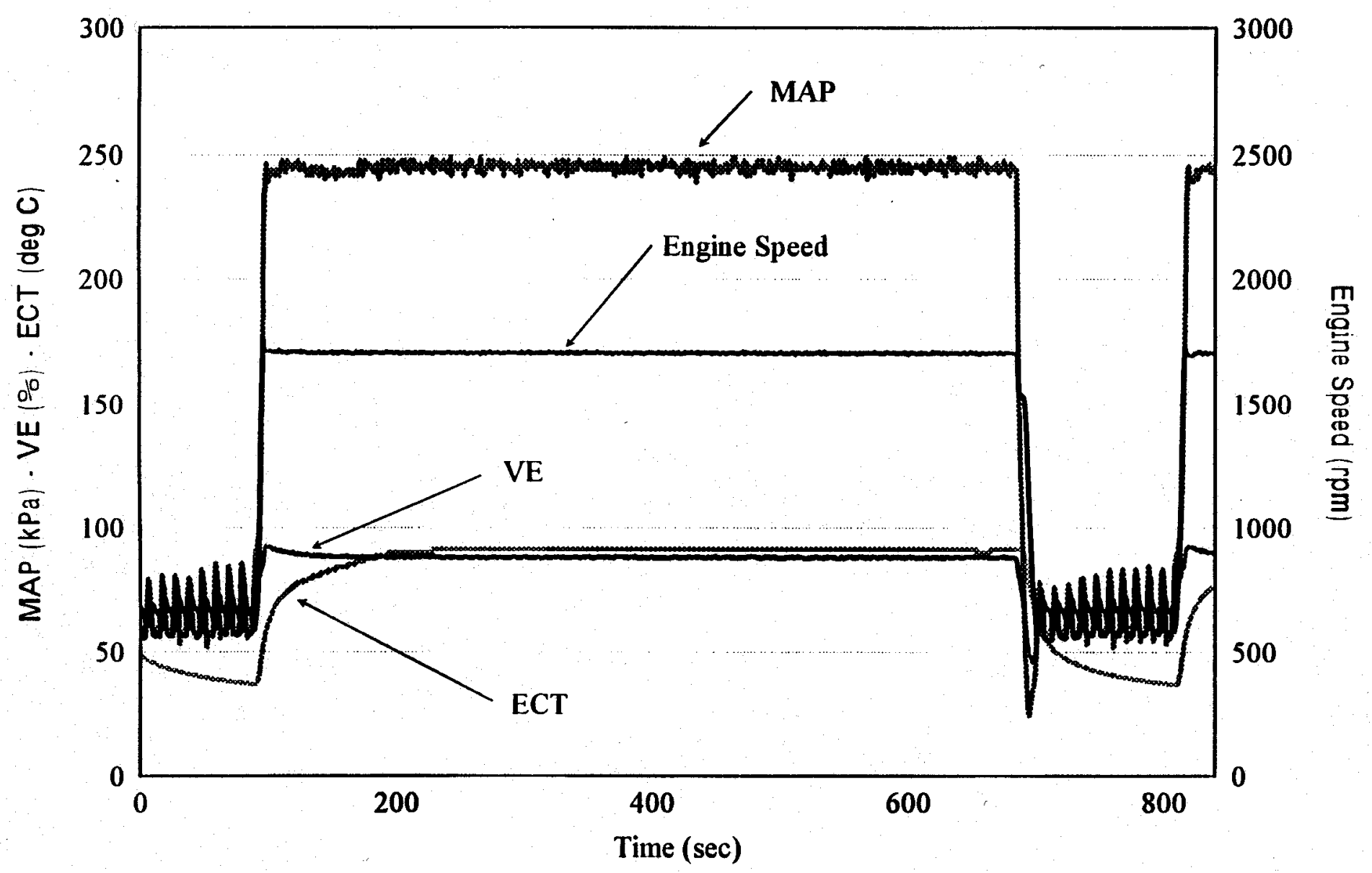

TRANSIENT DATA RECORDED FROM THE GEM SYSTEM FOR ONE FULL CYCLE AT 25 HOUR 


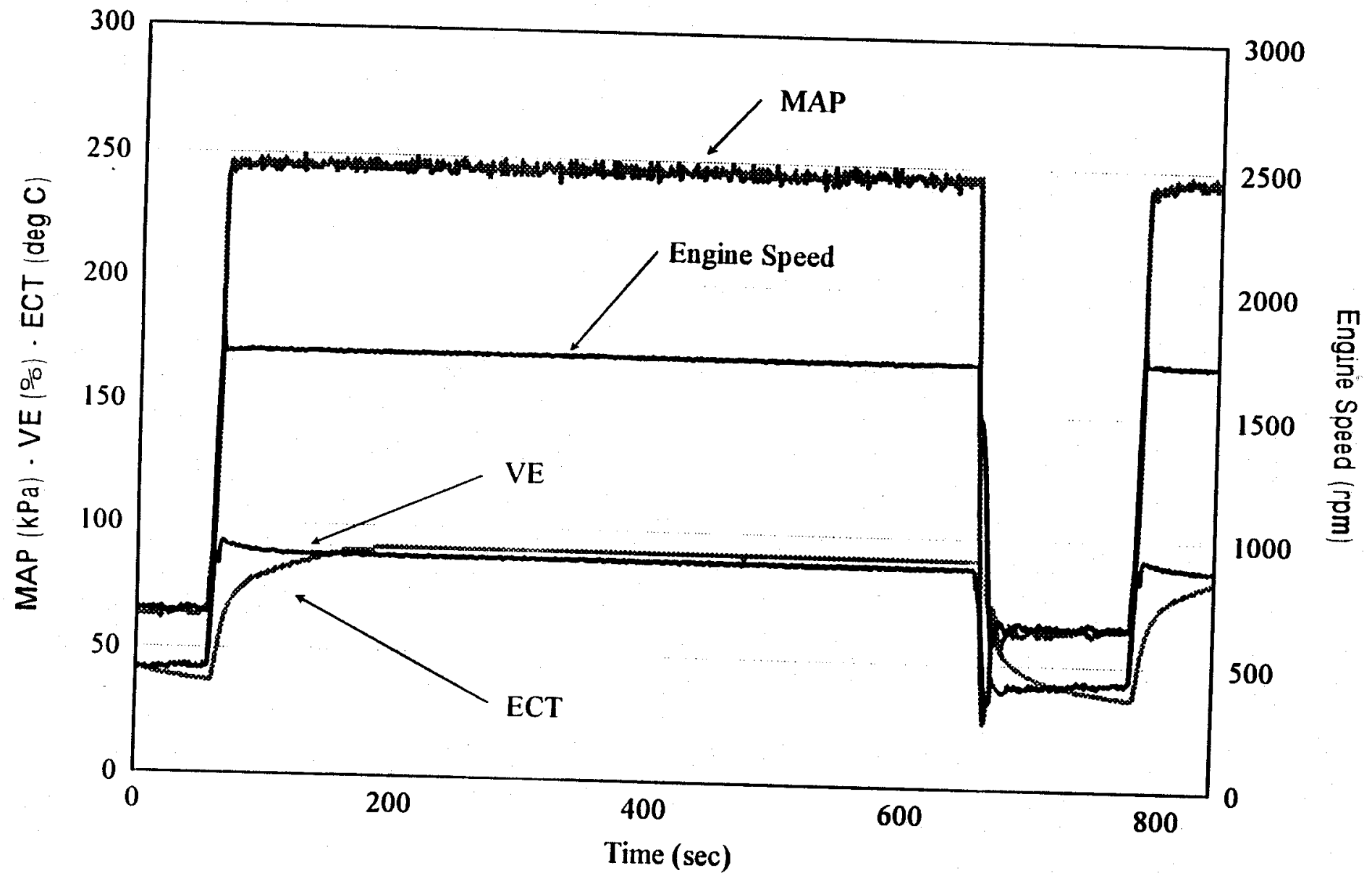

TRANSIENT DATA RECORDED FROM THE GEM SYSTEM FOR ONE FULL CYCLE AT 50 HOUR 


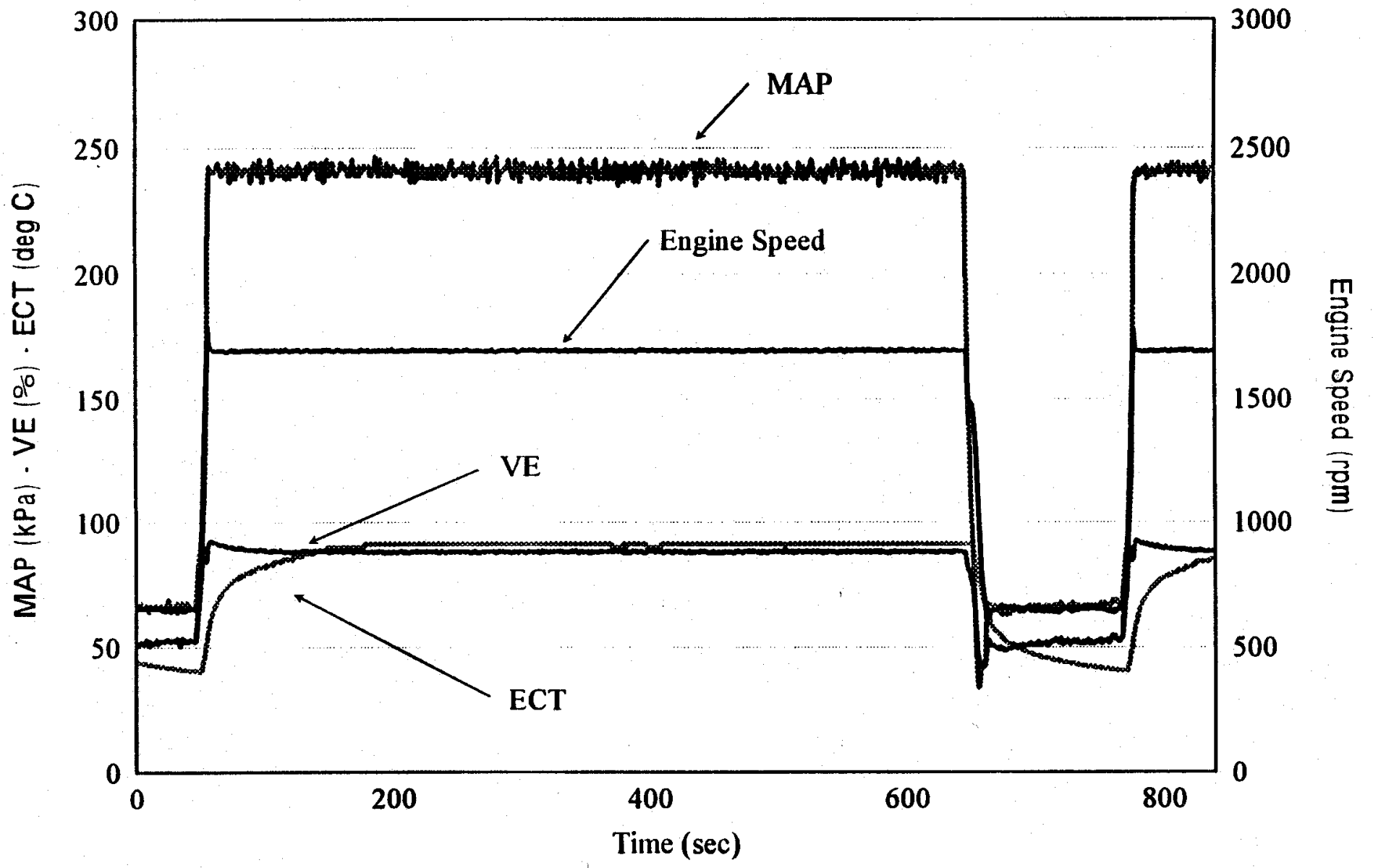

TRANSIENT DATA RECORDED FROM THE GEM SYSTEM FOR ONE FULL CYCLE AT 75 HOUR 


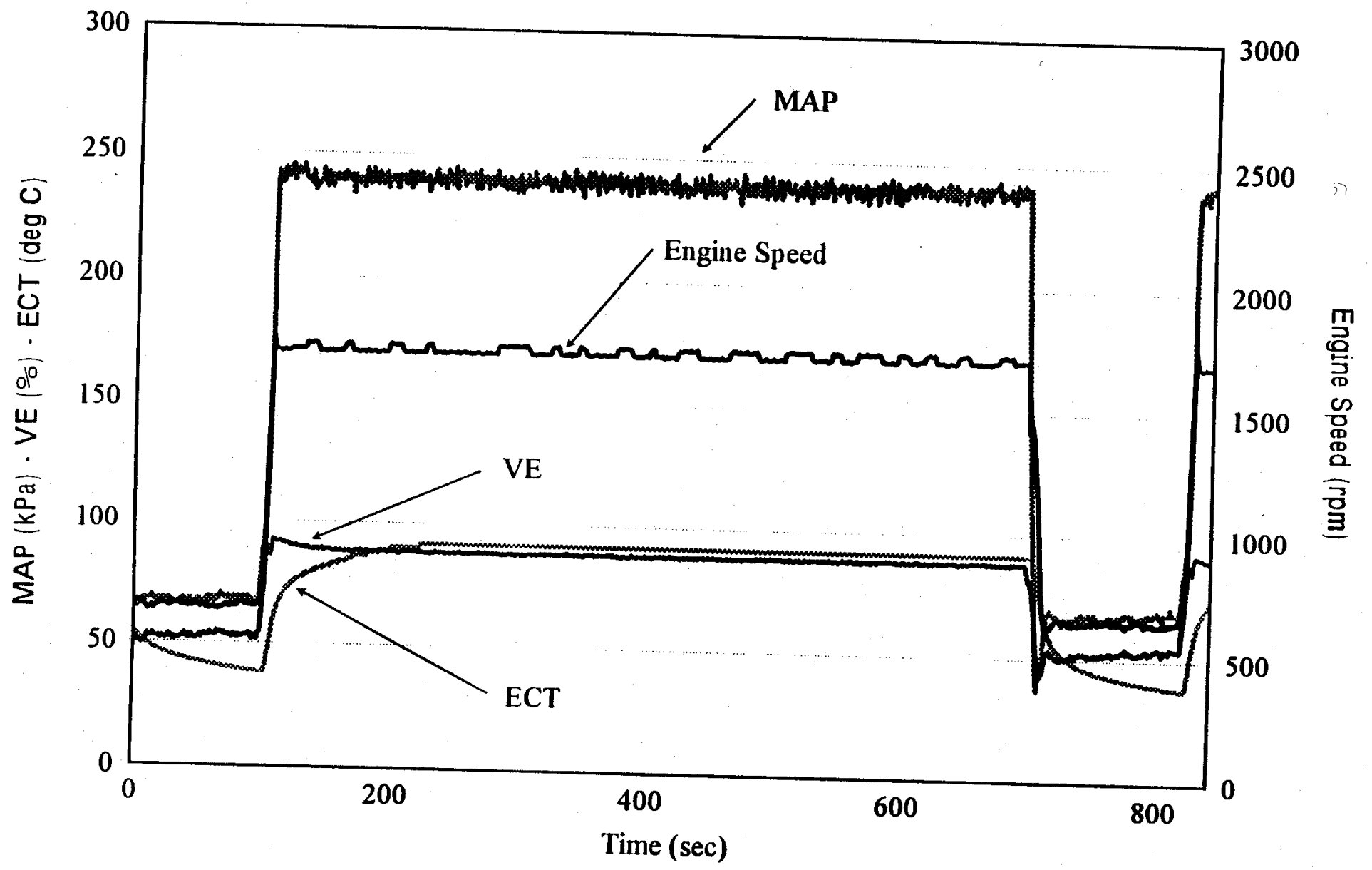

TRANSIENT DATA RECORDED FROM THE GEM SYSTEM FOR ONE FULL CYCLE AT 100 HOUR 


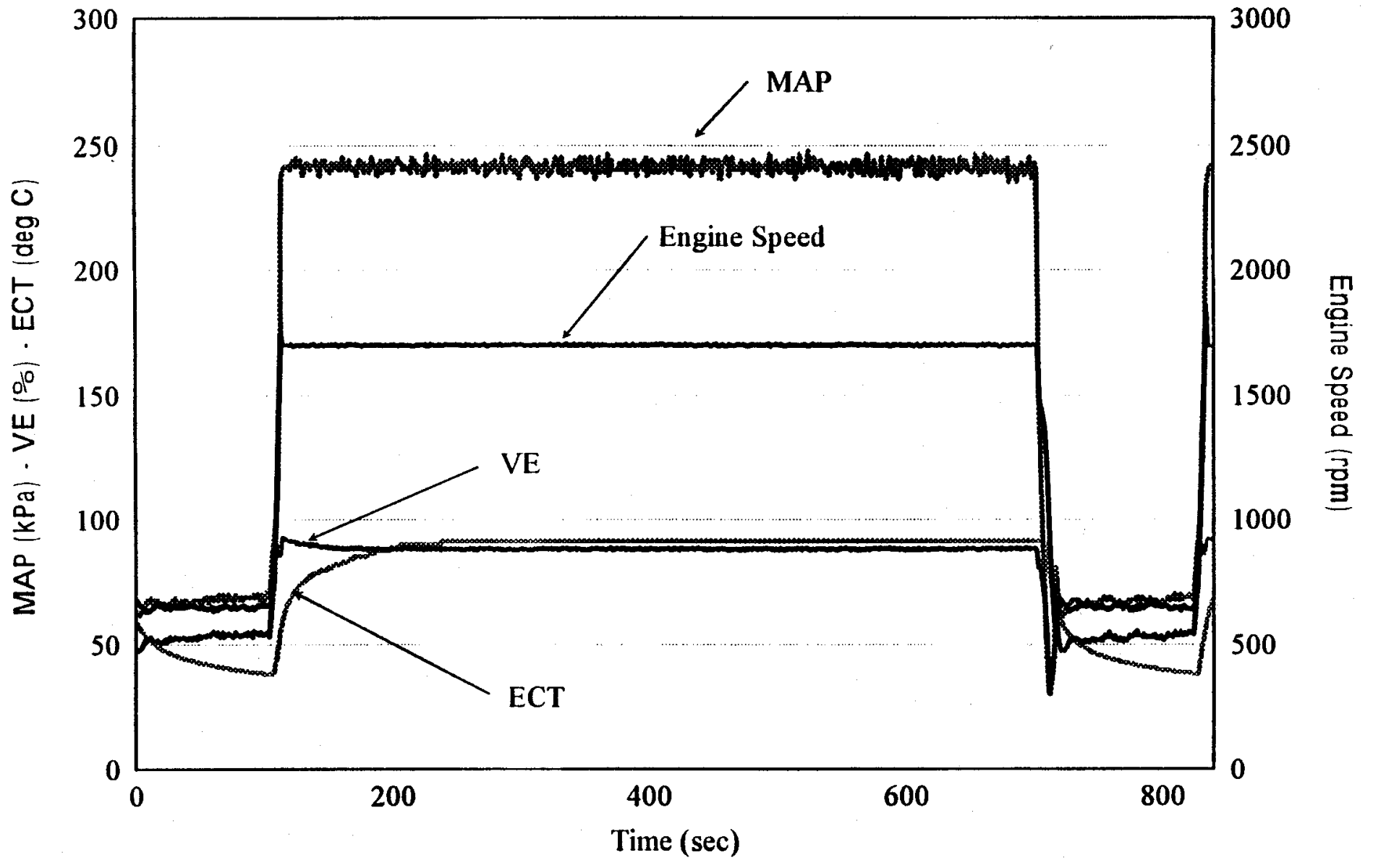

TRANSIENT DATA RECORDED FROM THE GEM SYSTEM FOR ONE FULL CYCLE AT 125 HOUR 


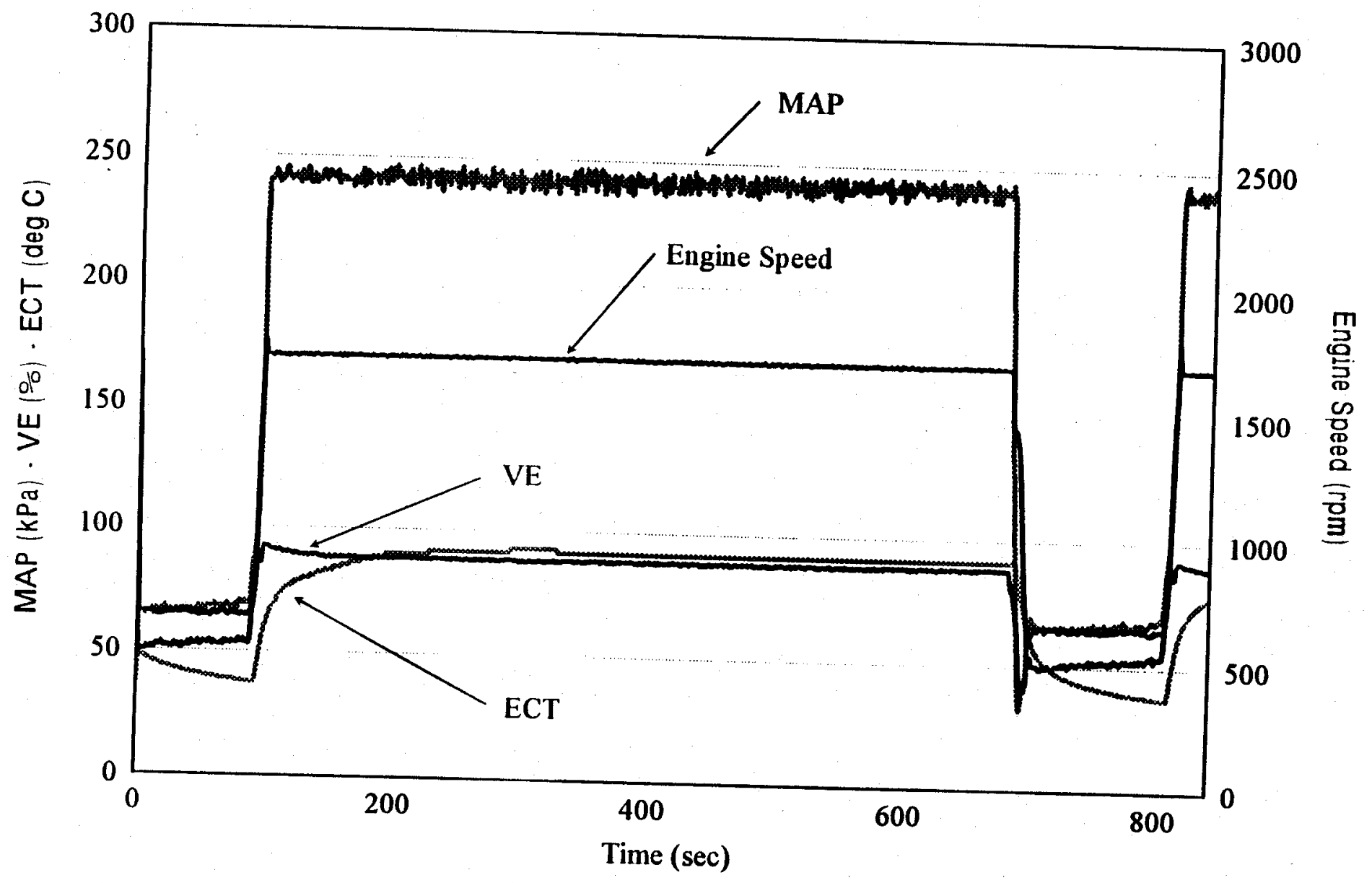

TRANSIENT DATA RECORDED FROM THE GEM SYSTEM FOR ONE FULL CYCLE AT 150 HOUR 


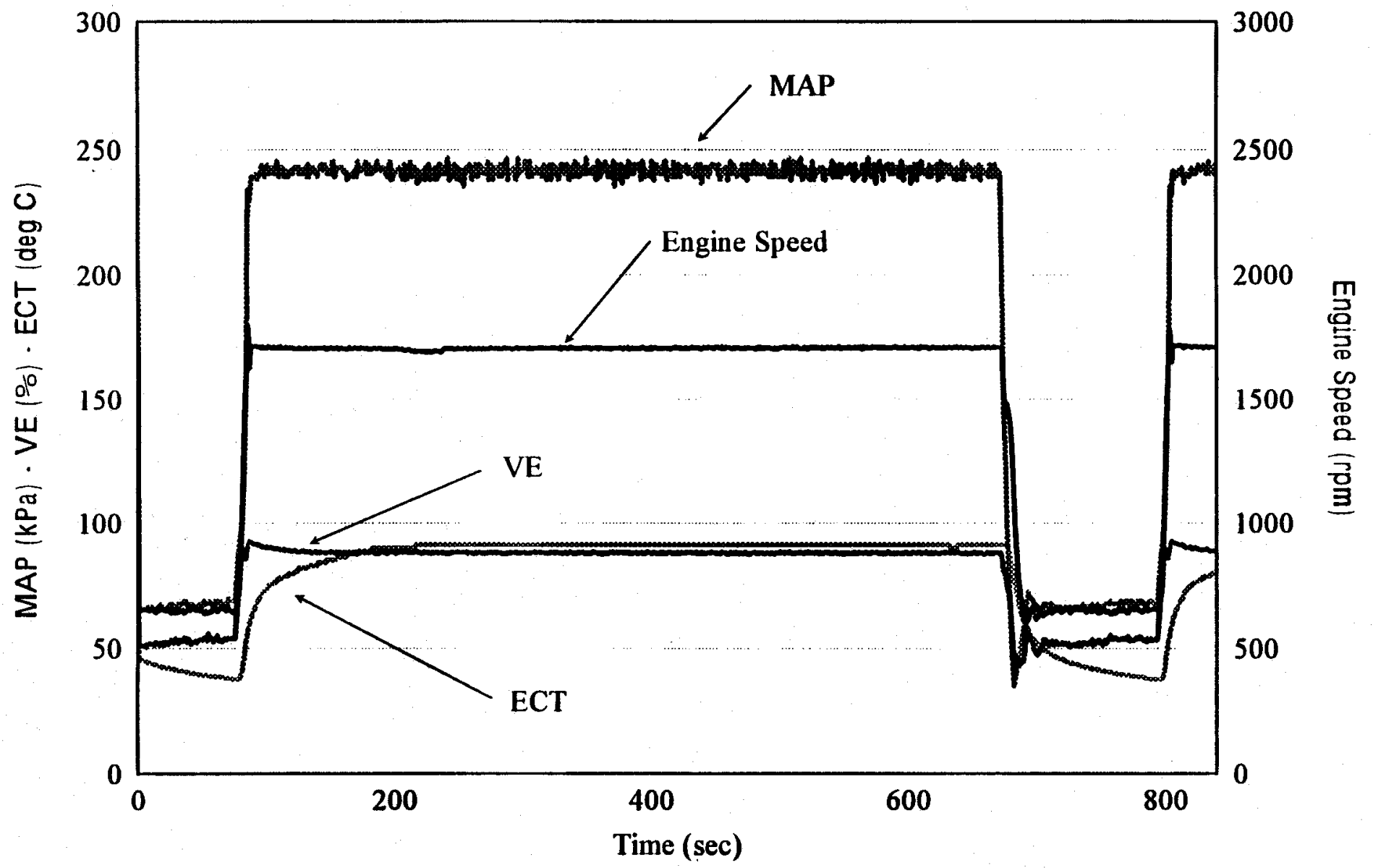

TRANSIENT DATA RECORDED FROM THE GEM SYSTEM FOR ONE FULL CYCLE AT 175 HOUR 


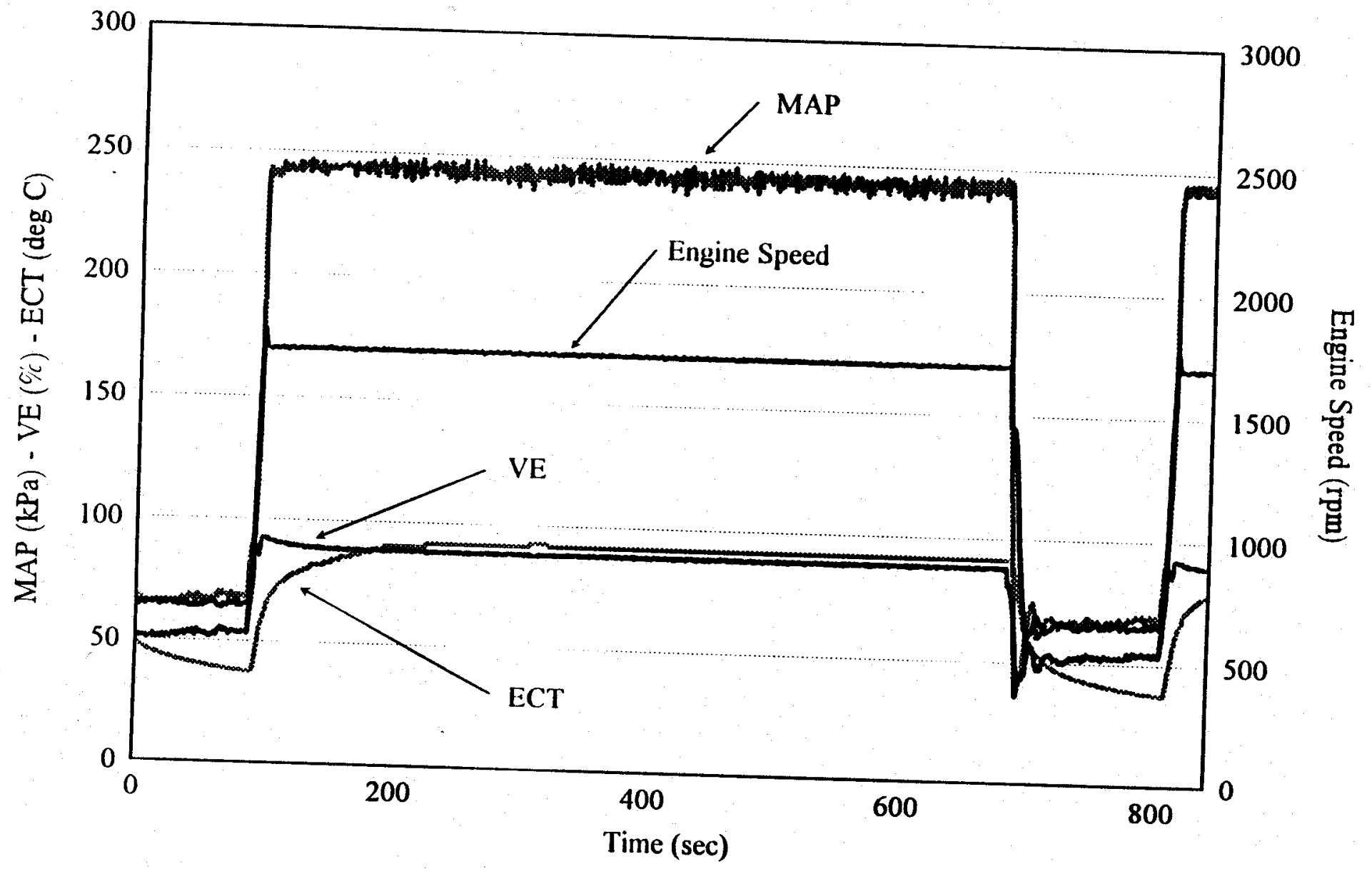

TRANSIENT DATA RECORDED FROM THE GEM SYSTEM FOR ONE FULL CYCLE AT 200 HOUR 


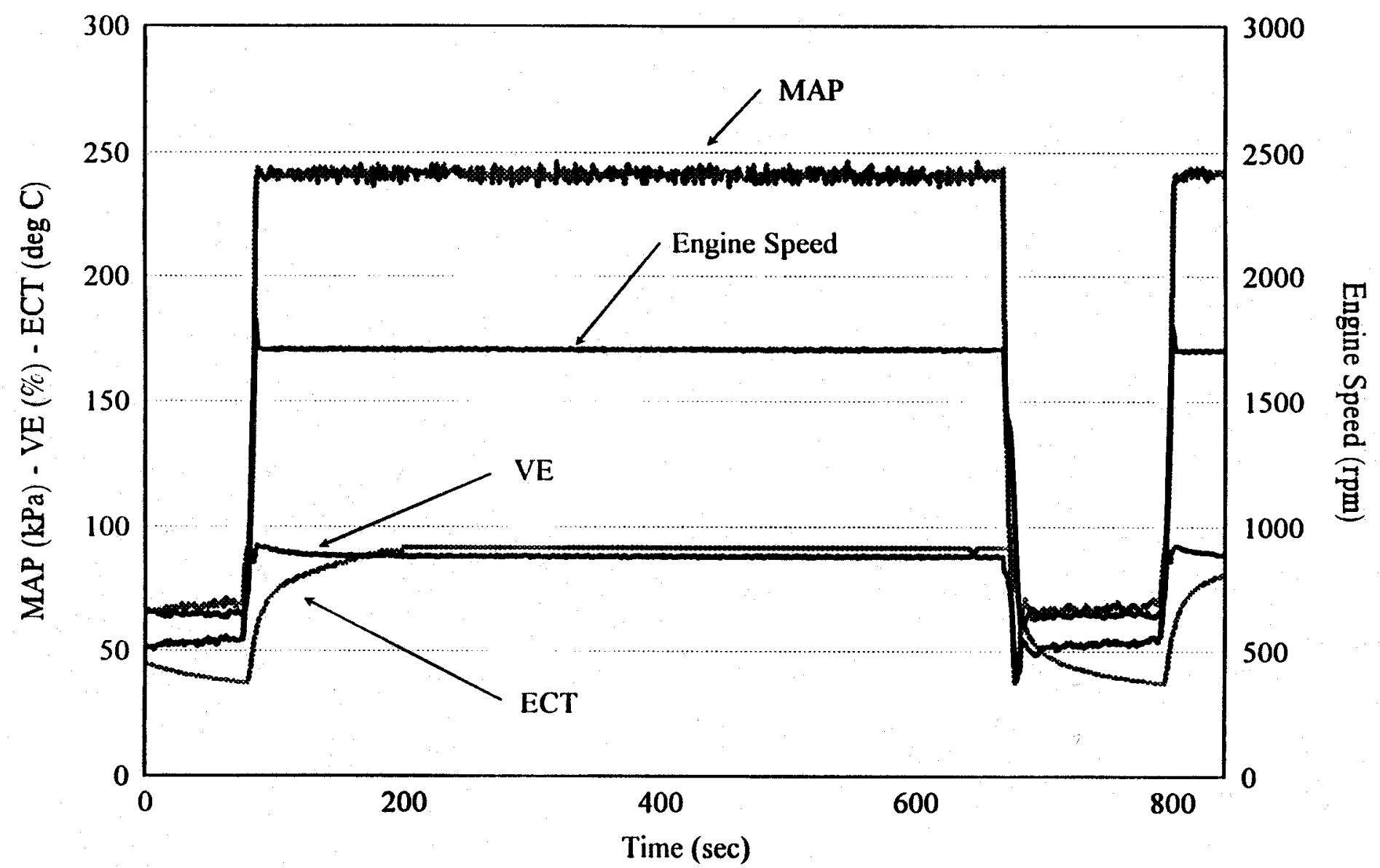

TRANSIENT DATA RECORDED FROM THE GEM SYSTEM FOR ONE FULL CYCLE AT 225 HOUR 


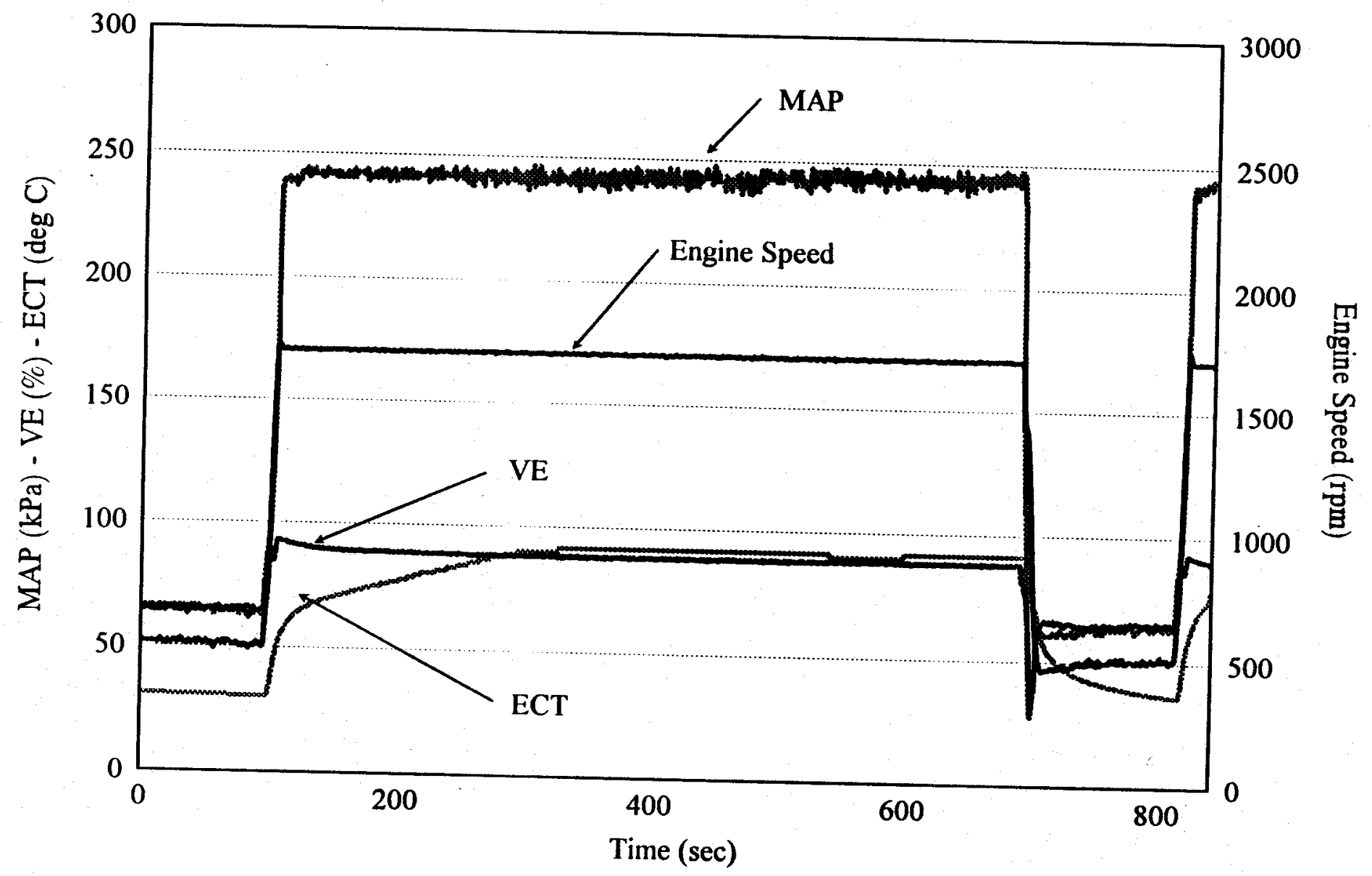

TRANSIENT DATA RECORDED FROM THE GEM SYSTEM FOR ONE FULL CYCLE AT 250 HOUR 


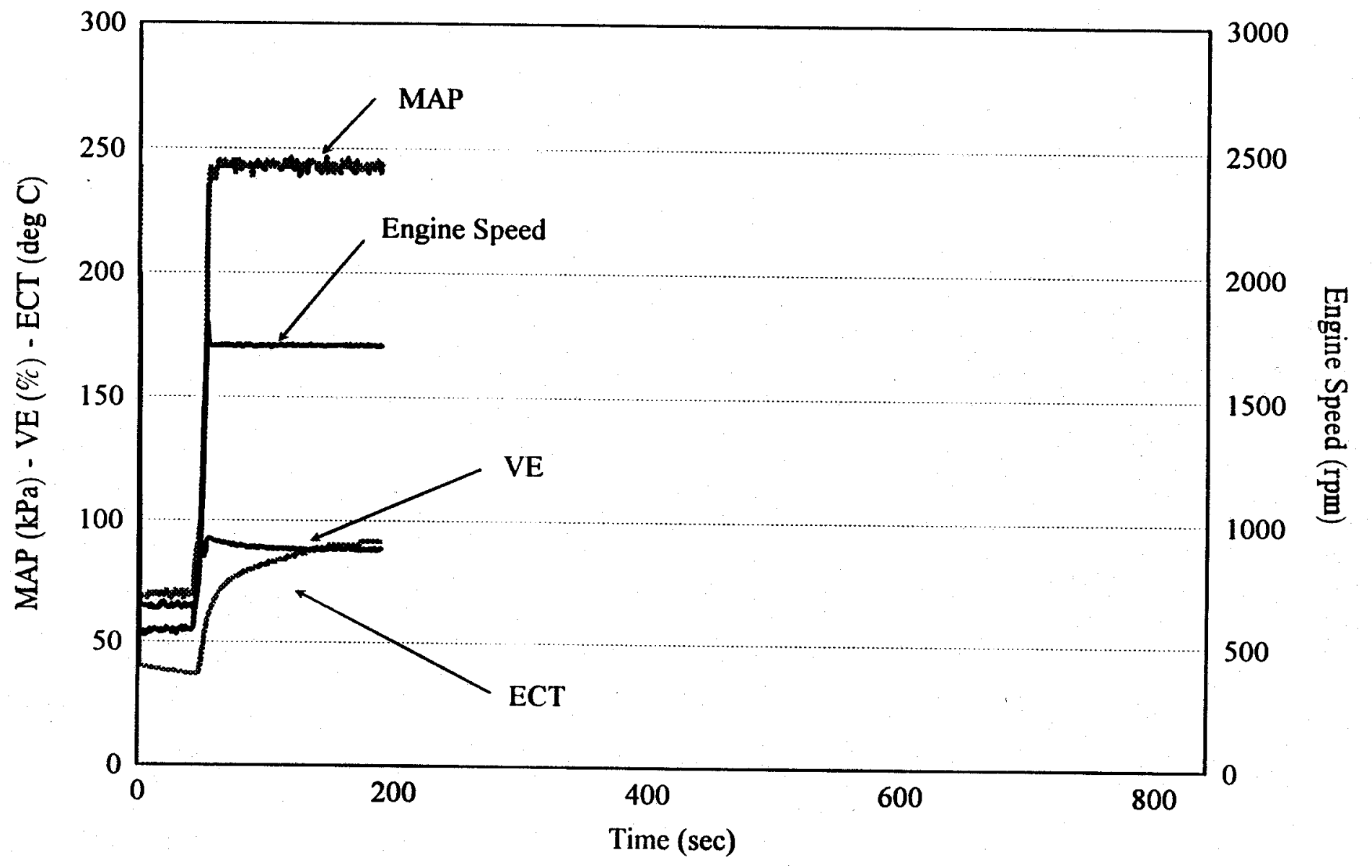

TRANSIENT DATA RECORDED FROM THE GEM SYSTEM FOR ONE FULL CYCLE AT 275 HOUR 


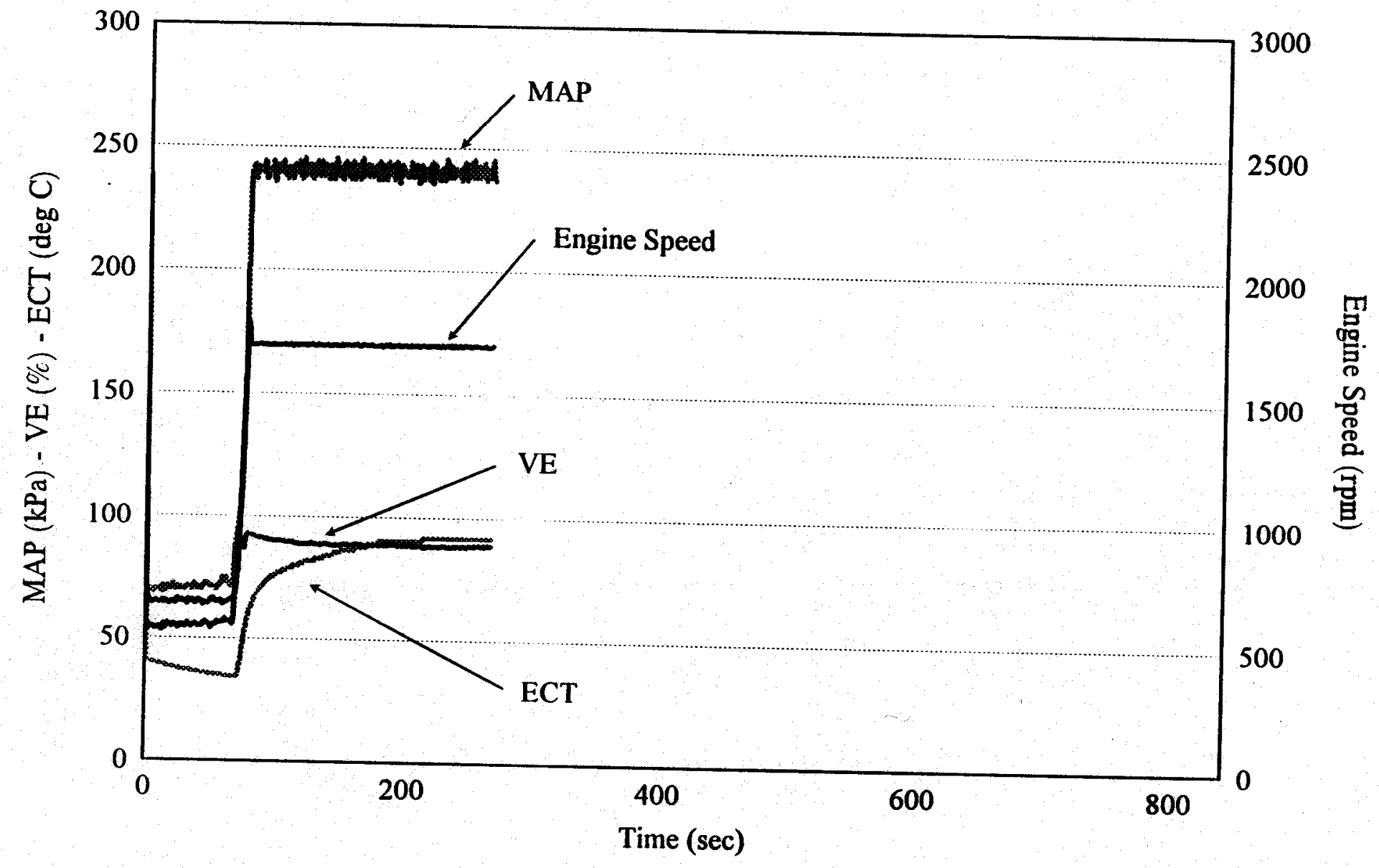

TRANSIENT DATA RECORDED FROM THE GEM SYSTEM FOR ONE FULL CYCLE AT 300 HOUR 


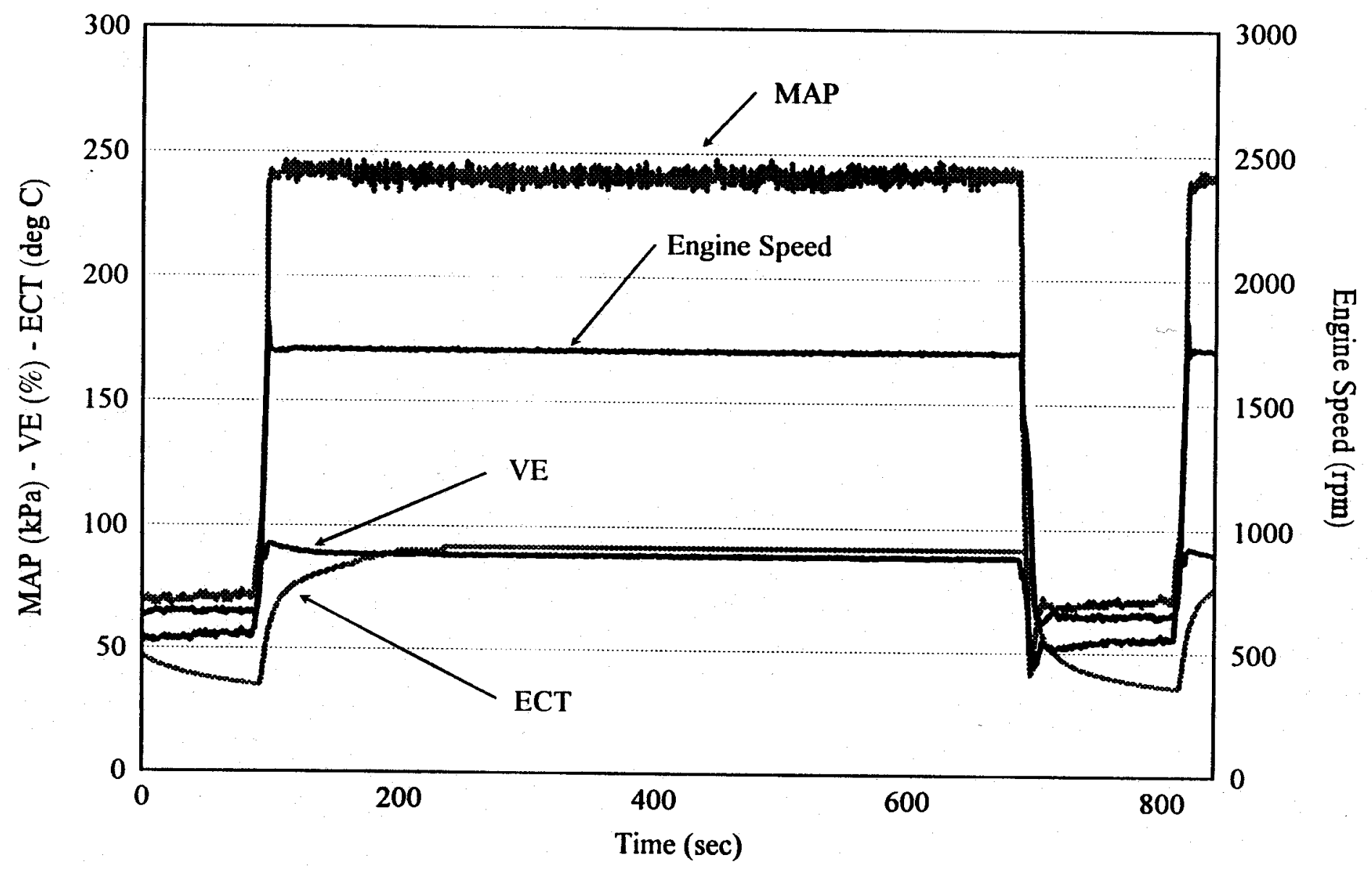

TRANSIENT DATA RECORDED FROM THE GEM SYSTEM FOR ONE FULL CYCLE AT 309 HOUR 


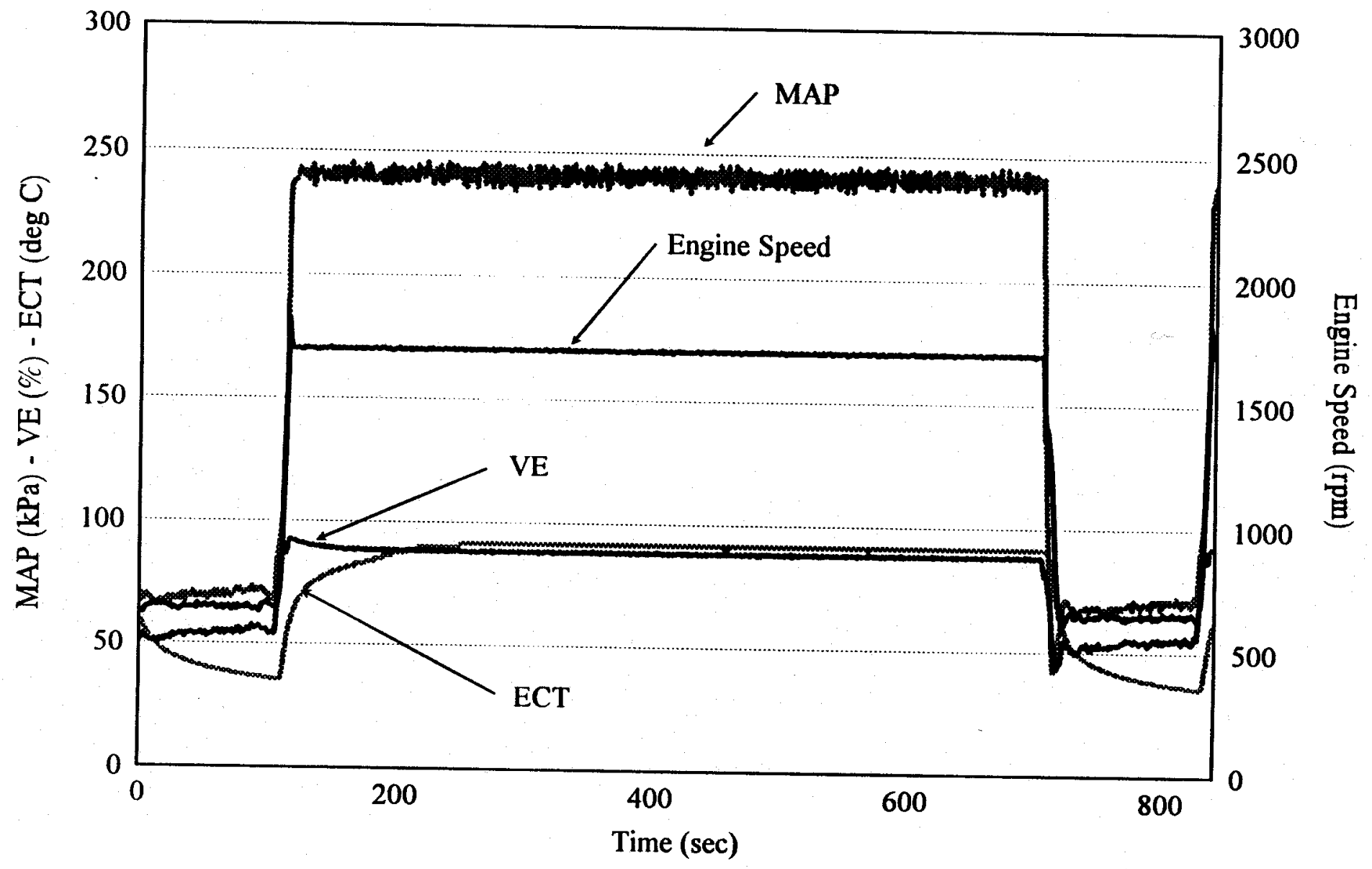

TRANSIENT DATA RECORDED FROM THE GEM SYSTEM FOR ONE FULL CYCLE AT 325 HOUR 


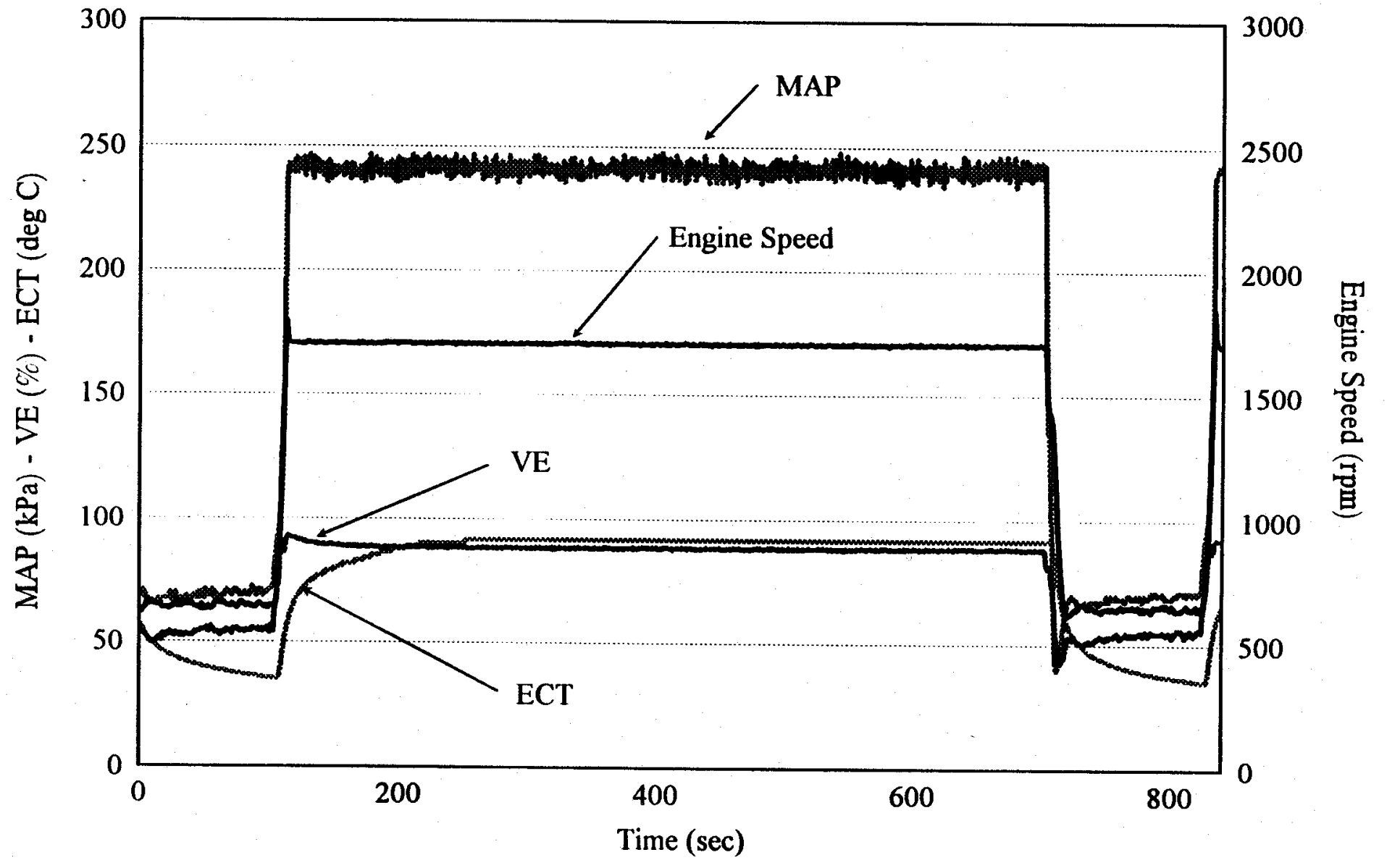

TRANSIENT DATA RECORDED FROM THE GEM SYSTEM FOR ONE FULL CYCLE AT 350 HOUR 


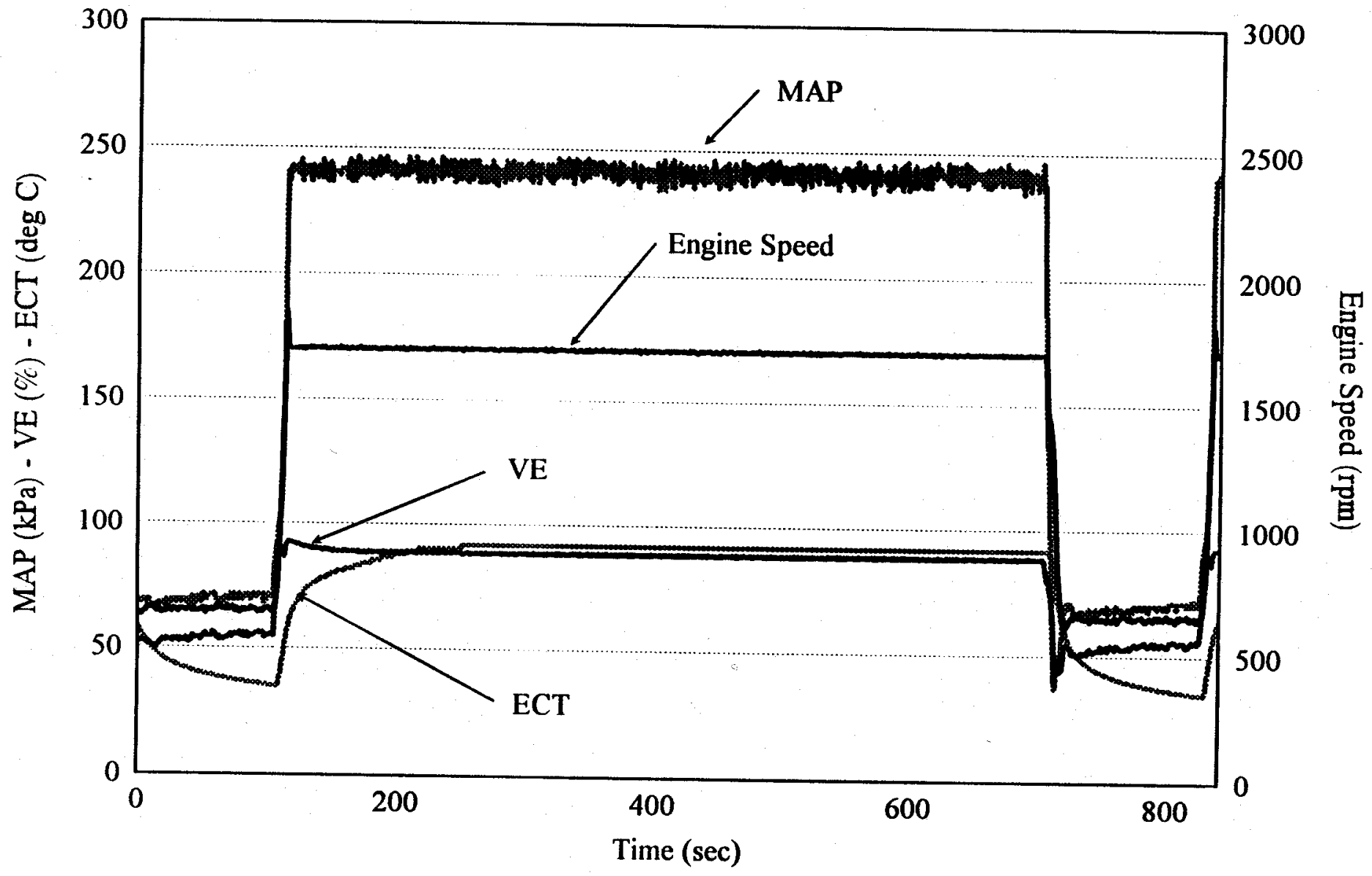

TRANSIENT DATA RECORDED FROM THE GEM SYSTEM FOR ONE FULL CYCLE AT 375 HOUR 


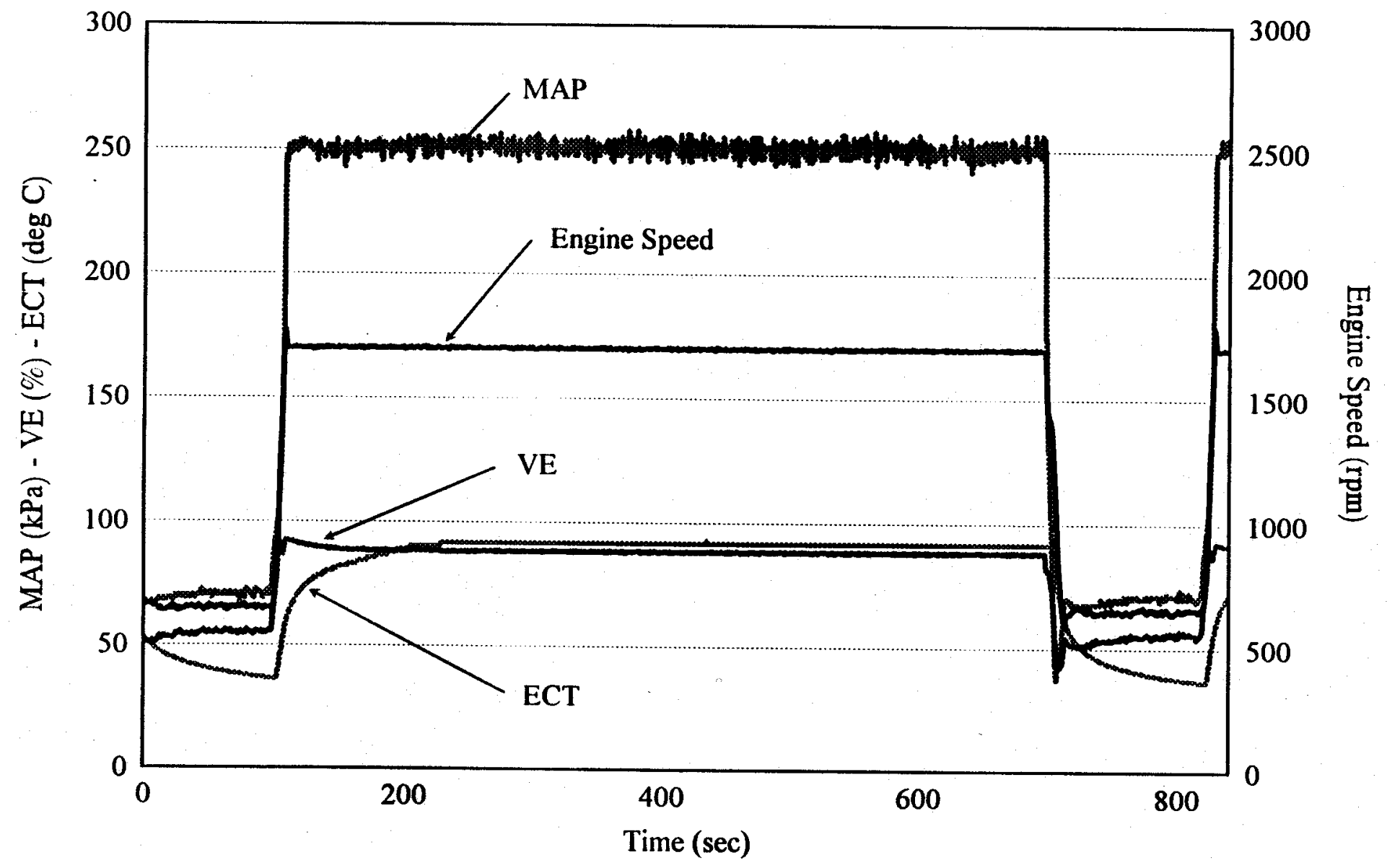

TRANSIENT DATA RECORDED FROM THE GEM SYSTEM FOR ONE FULL CYCLE AT 400 HOUR 


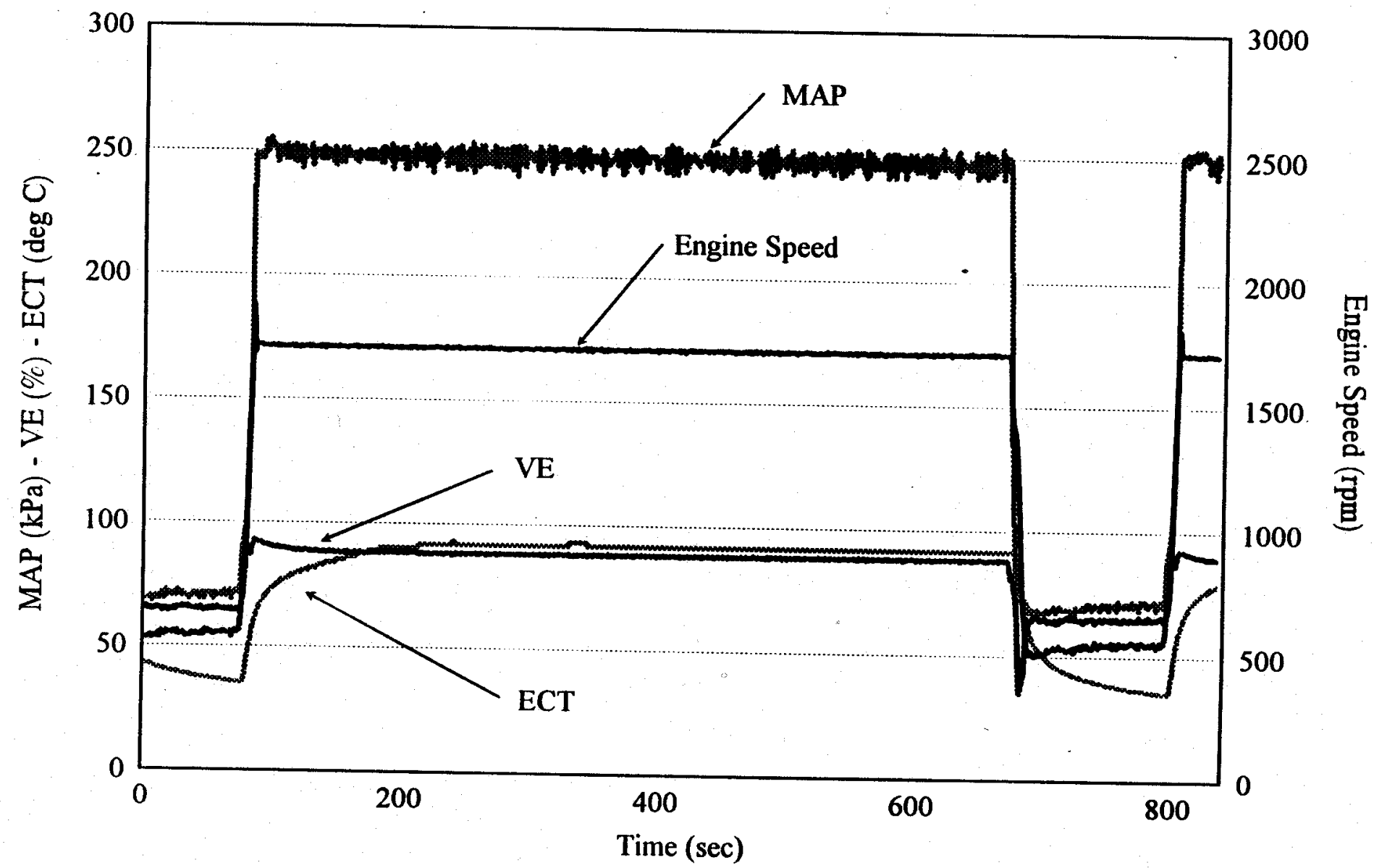

TRANSIENT DATA RECORDED FROM THE GEM SYSTEM FOR ONE FULL CYCLE AT 425 HOUR 


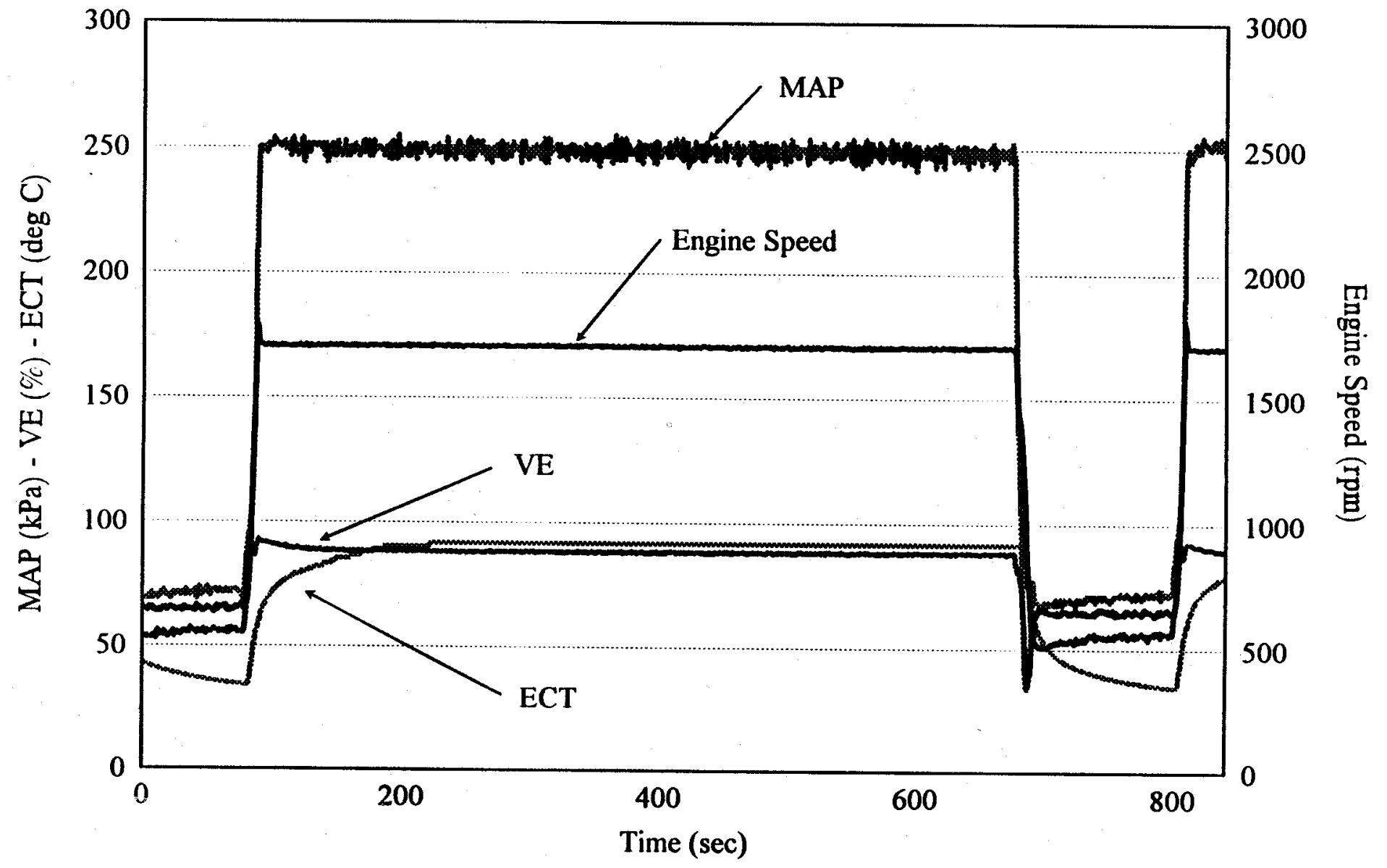

TRANSIENT DATA RECORDED FROM THE GEM SYSTEM FOR ONE FULL CYCLE AT 450 HOUR 


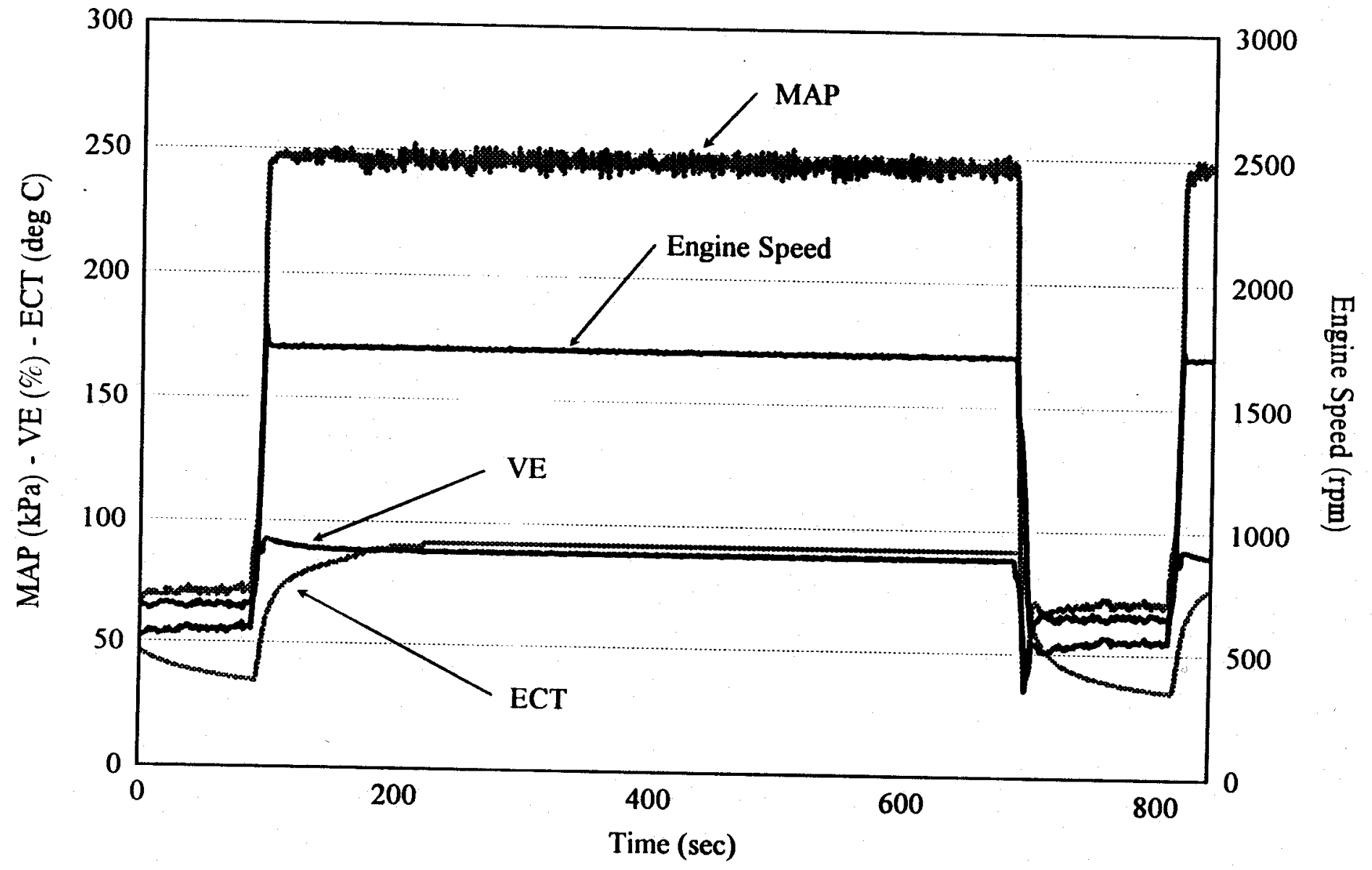

TRANSIENT DATA RECORDED FROM THE GEM SYSTEM FOR ONE FULL CYCLE AT 475 HOUR 


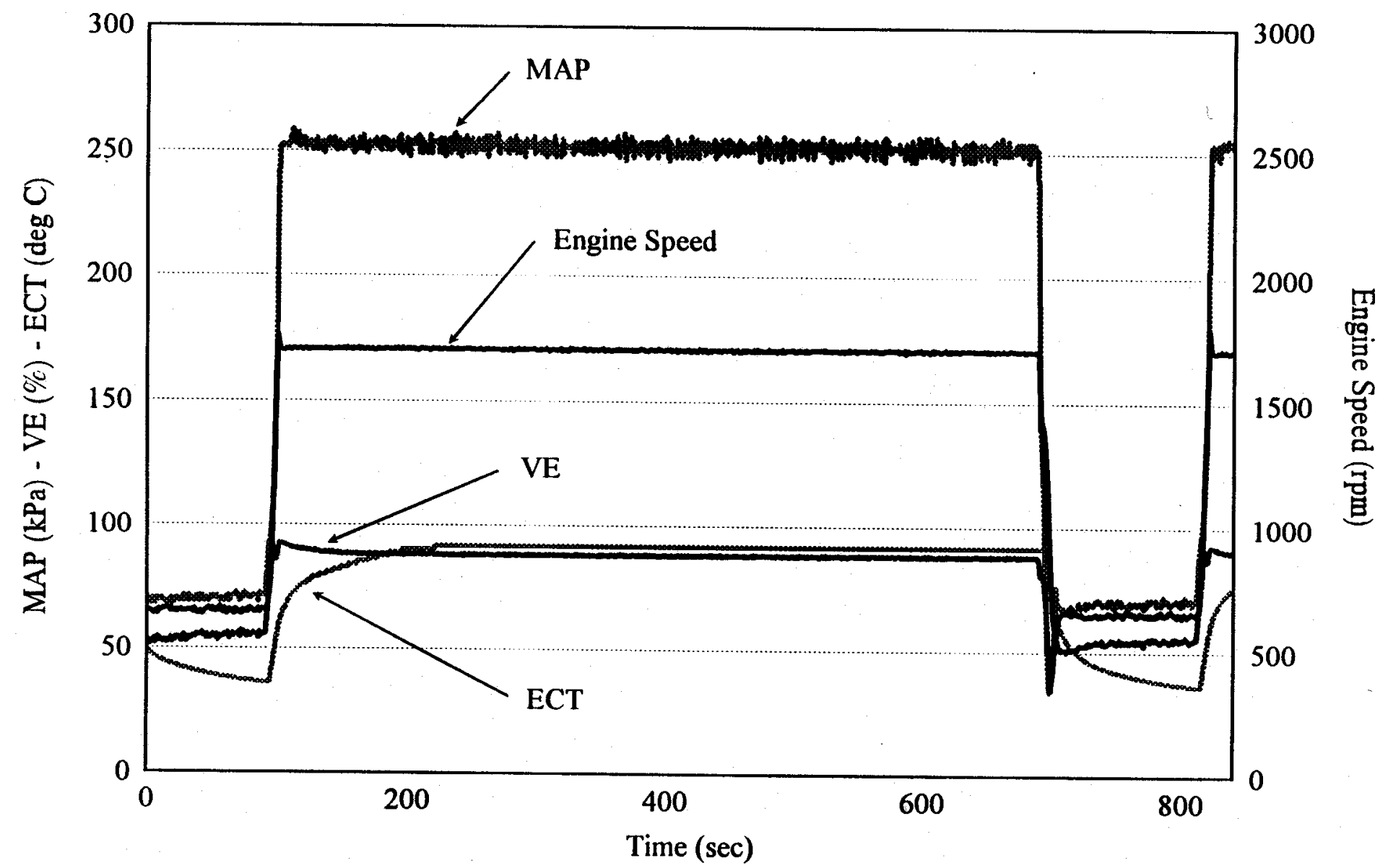

TRANSIENT DATA RECORDED FROM THE GEM SYSTEM FOR ONE FULL CYCLE AT 500 HOUR 


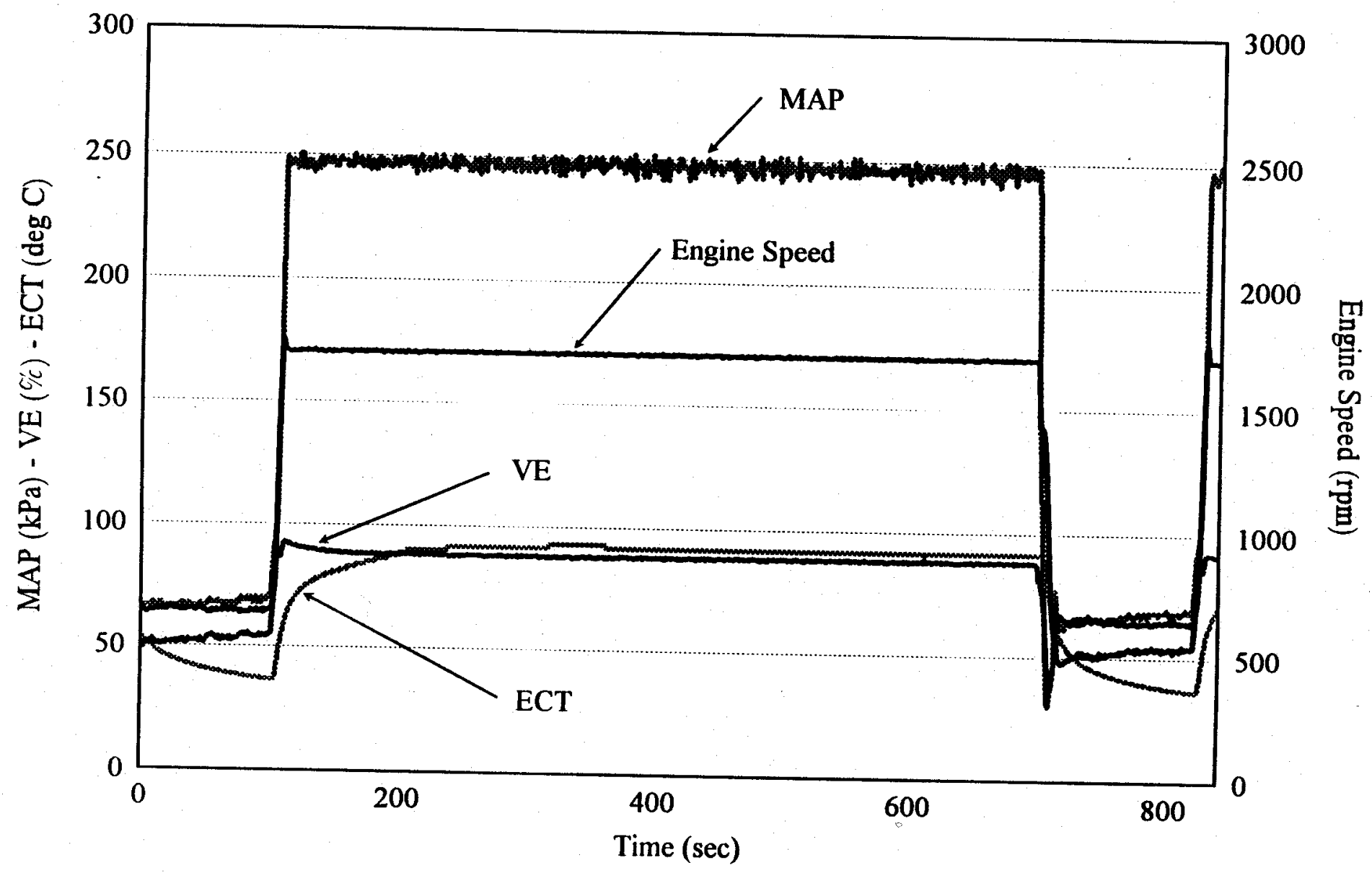

TRANSIENT DATA RECORDED FROM THE GEM SYSTEM FOR ONE FULL CYCLE AT 525 HOUR 


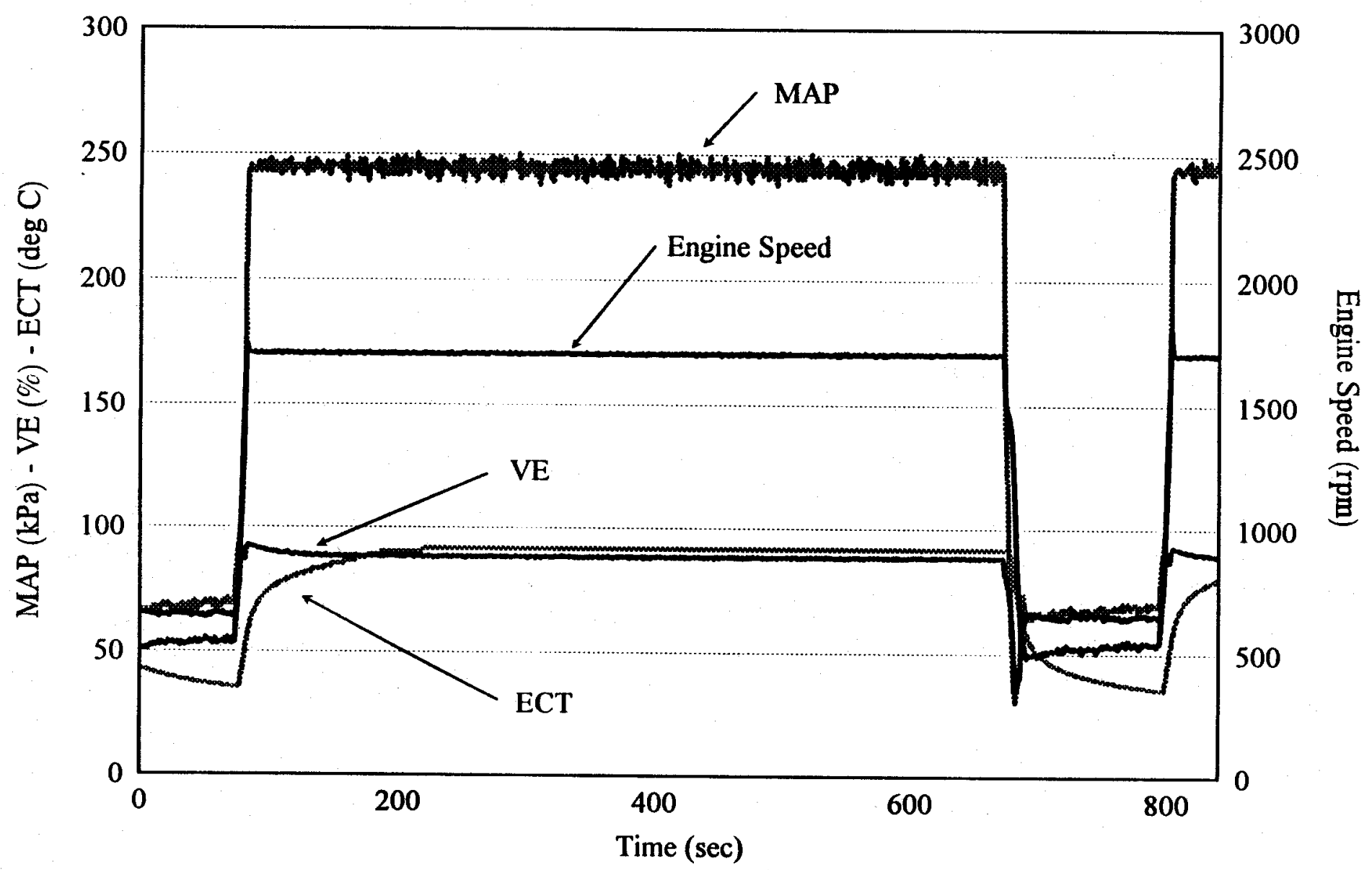

TRANSIENT DATA RECORDED FROM THE GEM SYSTEM FOR ONE FULL CYCLE AT 550 HOUR 


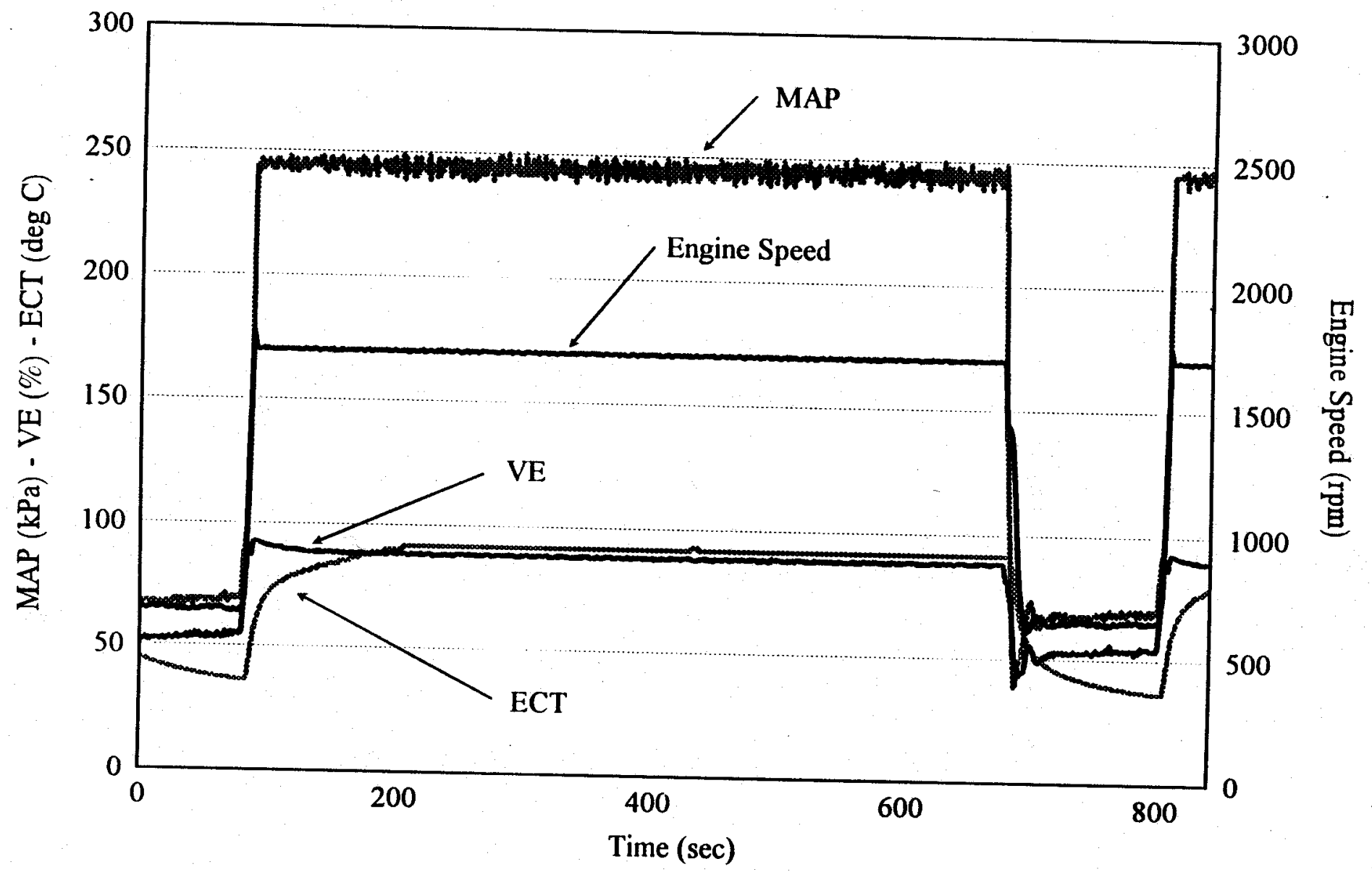

TRANSIENT DATA RECORDED FROM THE GEM SYSTEM FOR ONE FULL CYCLE AT 575 HOUR 


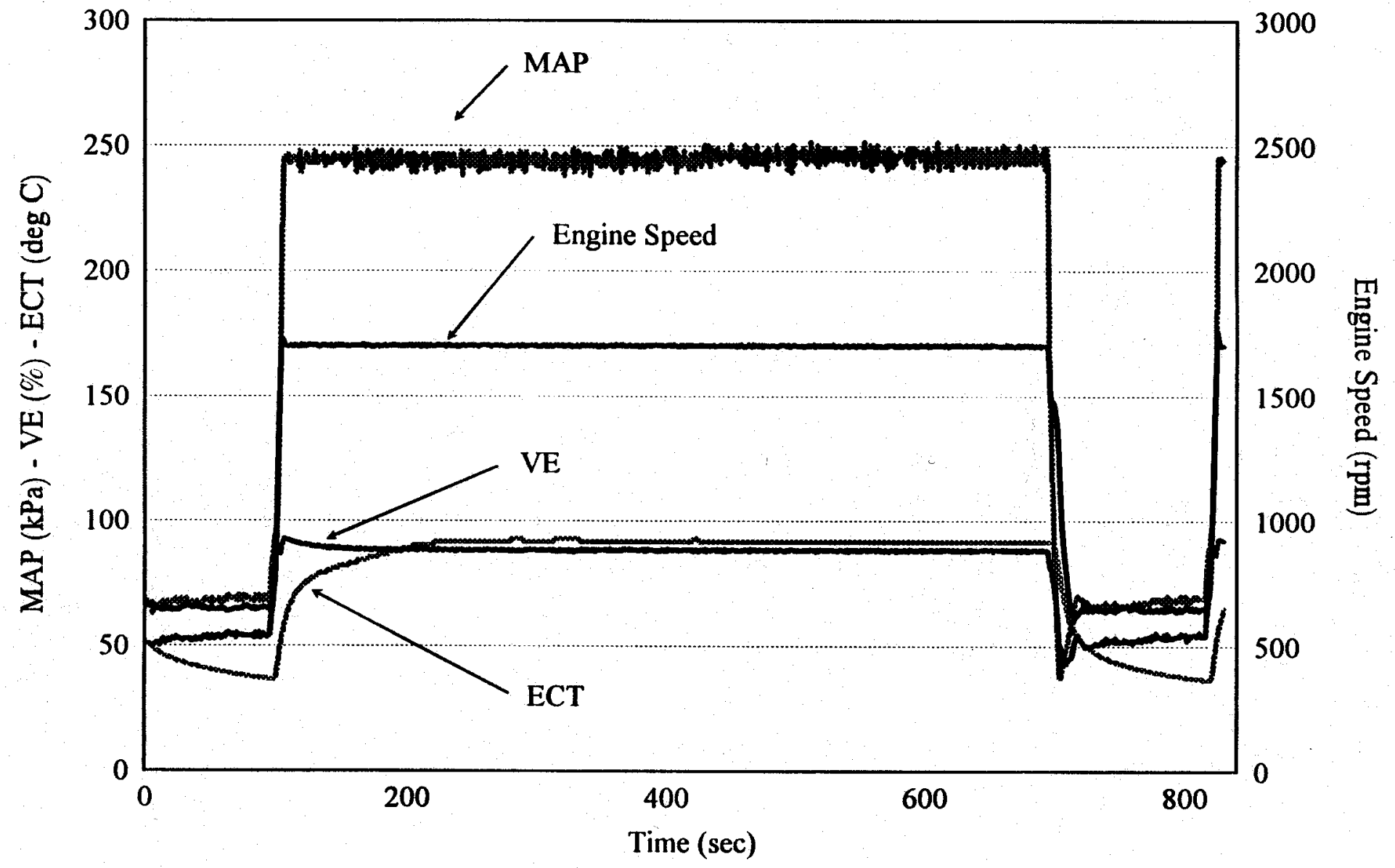

TRANSIENT DATA RECORDED FROM THE GEM SYSTEM FOR ONE FULL CYCLE AT 600 HOUR 


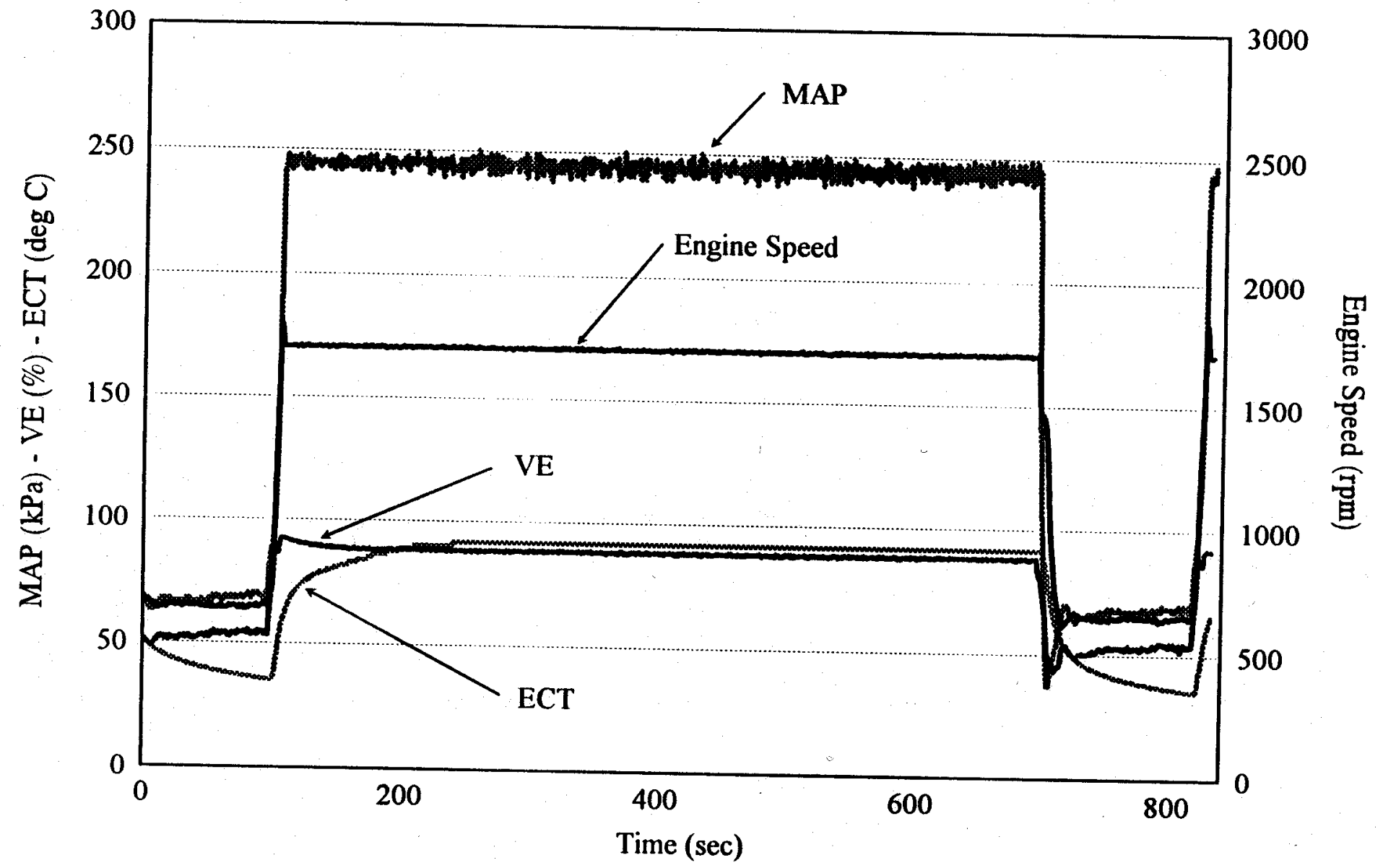

TRANSIENT DATA RECORDED FROM THE GEM SYSTEM FOR ONE FULL CYCLE AT 625 HOUR 


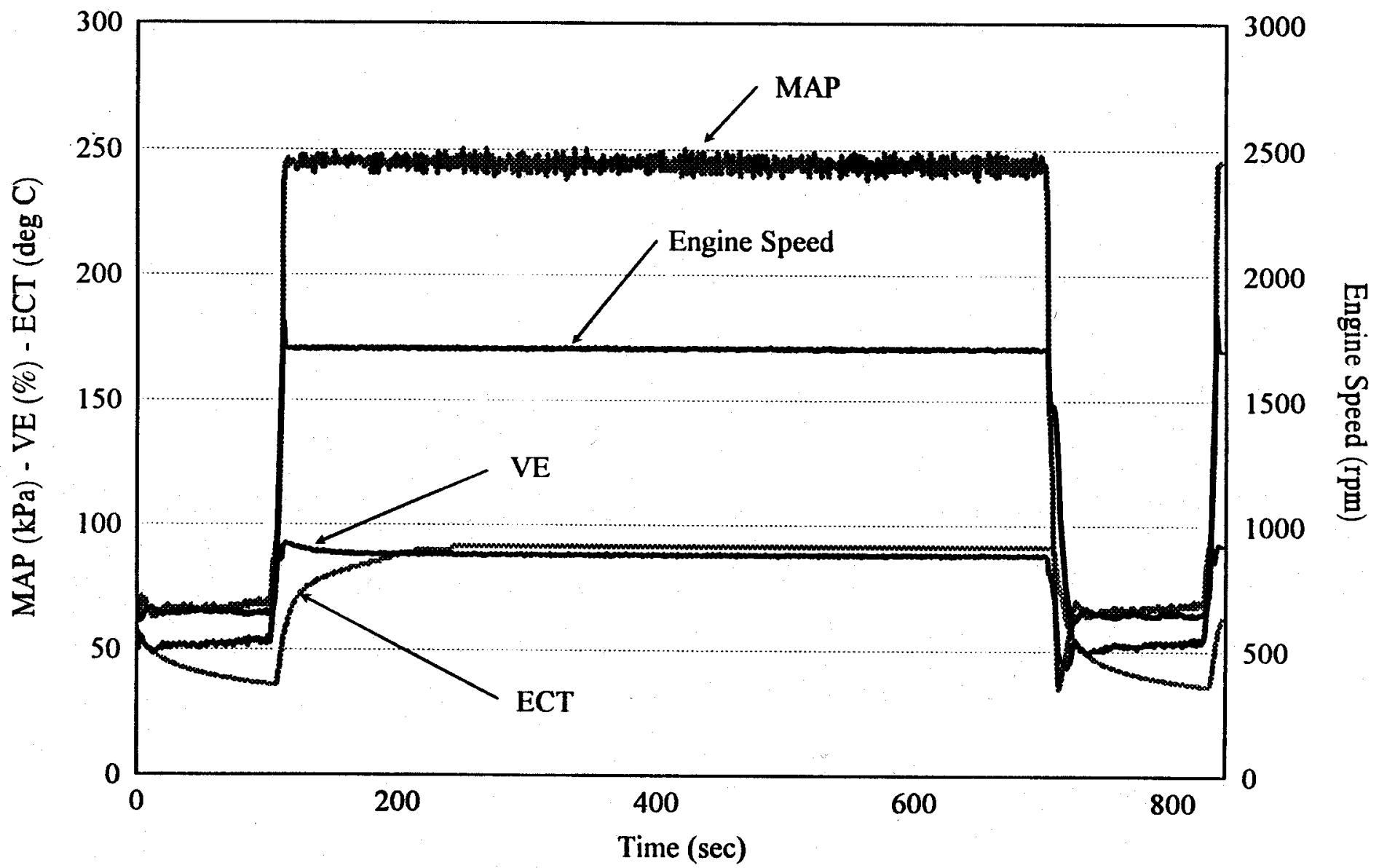

TRANSIENT DATA RECORDED FROM THE GEM SYSTEM FOR ONE FULL CYCLE AT 650 HOUR 


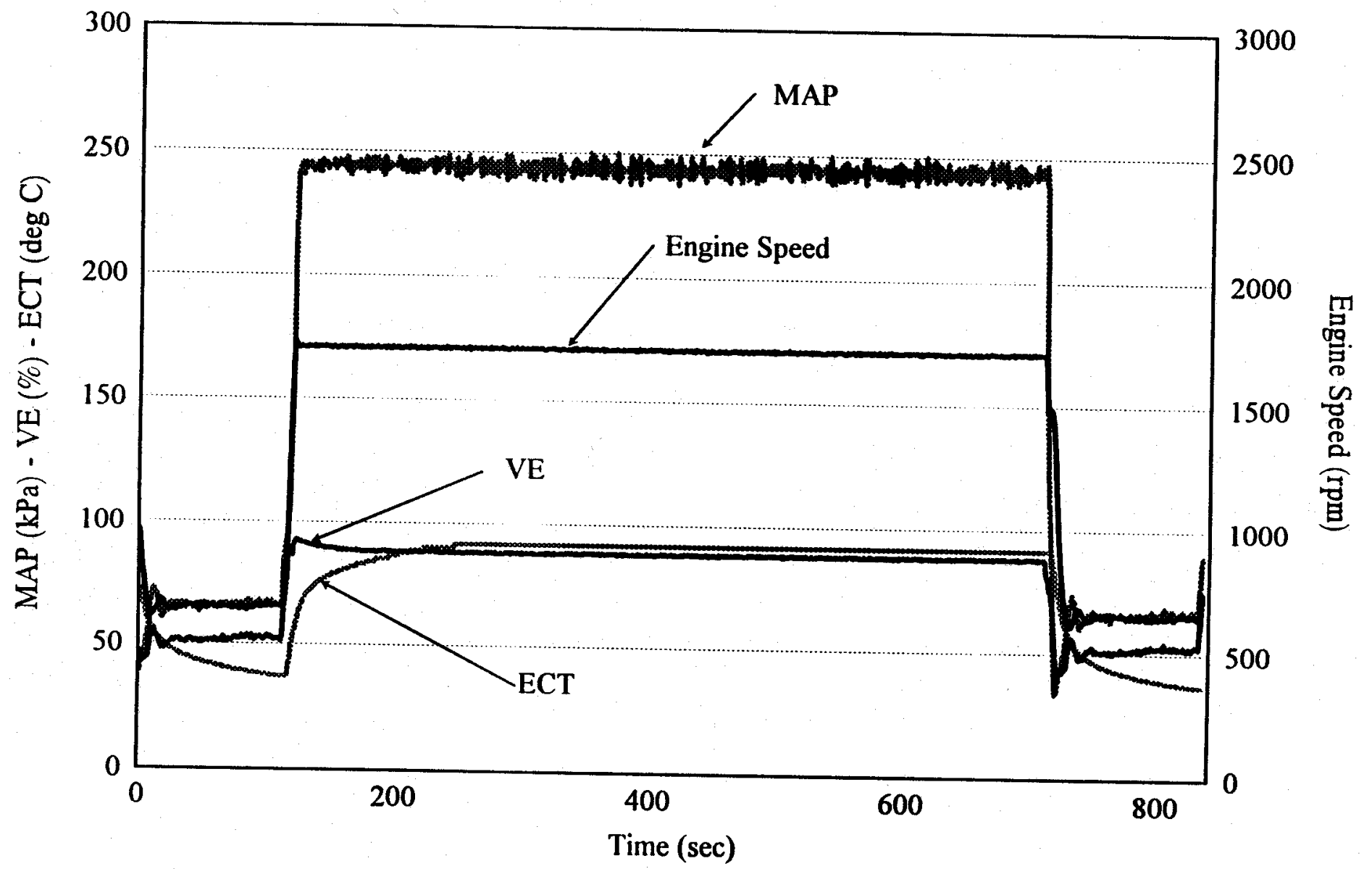

TRANSIENT DATA RECORDED FROM THE GEM SYSTEM FOR ONE FULL CYCLE AT 675 HOUR 


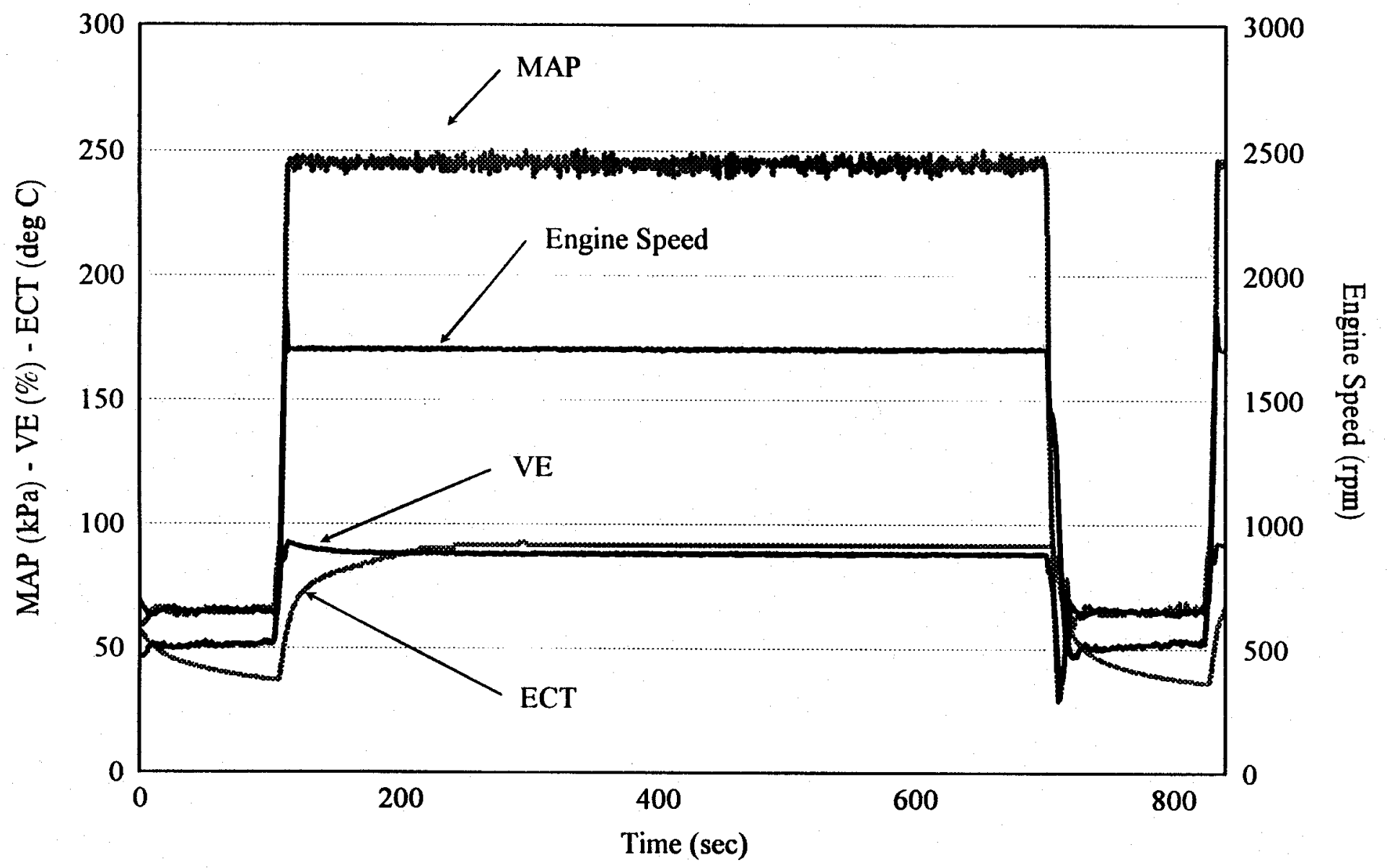

TRANSIENT DATA RECORDED FROM THE GEM SYSTEM FOR ONE FULL CYCLE AT 700 HOUR 


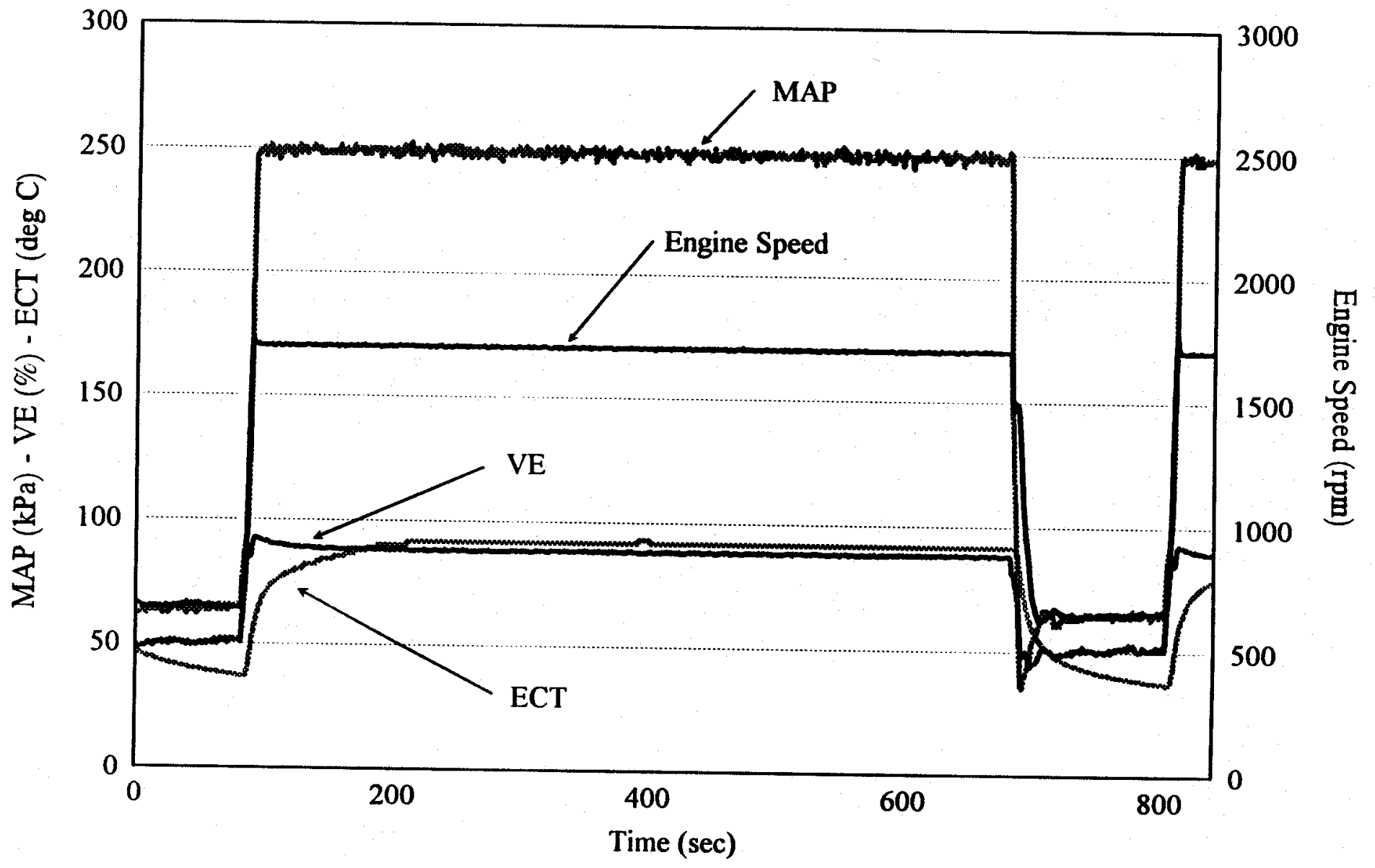

TRANSIENT DATA RECORDED FROM THE GEM SYSTEM FOR ONE FULL CYCLE AT 725 HOUR 


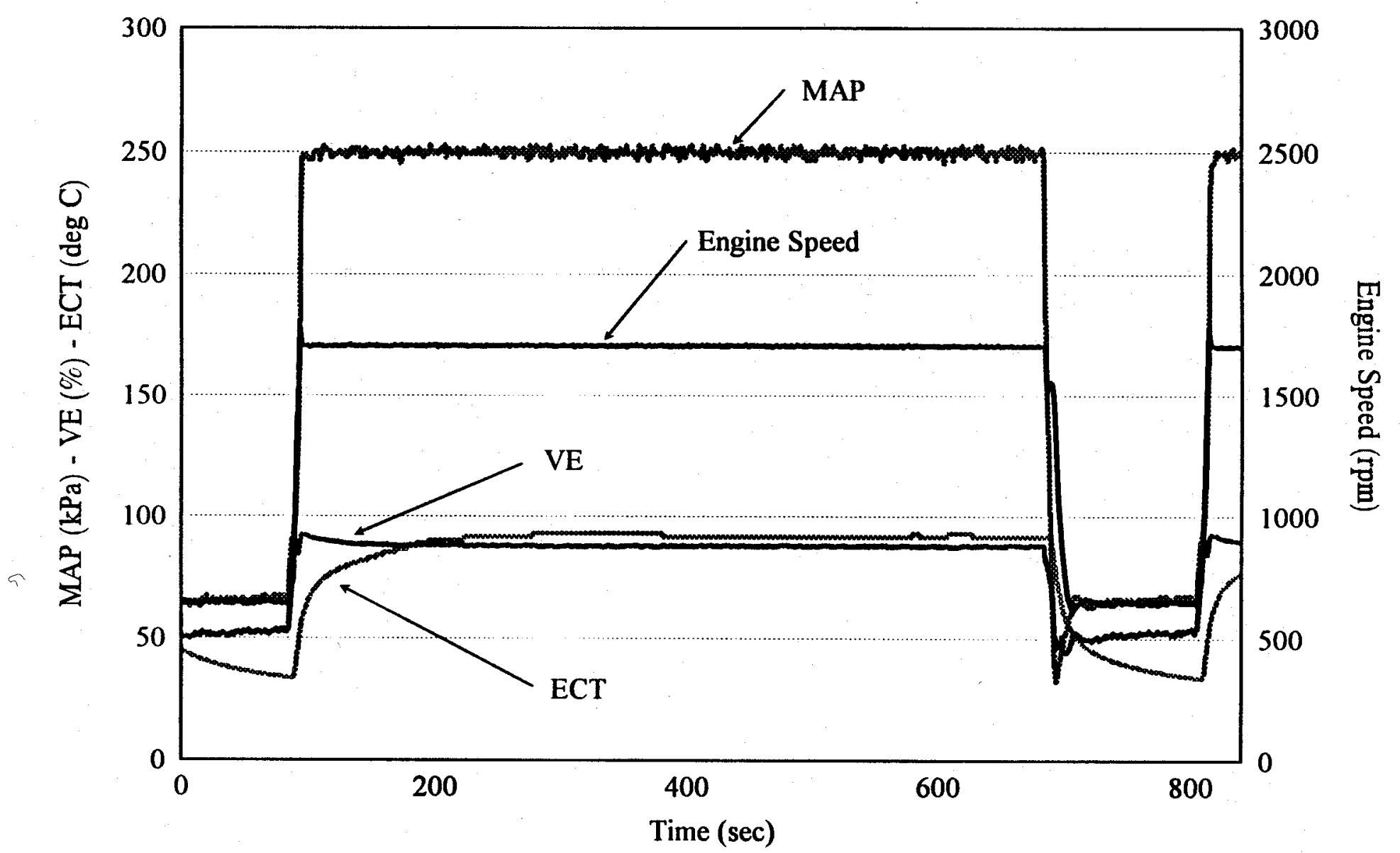

TRANSIENT DATA RECORDED FROM THE GEM SYSTEM FOR ONE FULL CYCLE AT 750 HOUR 


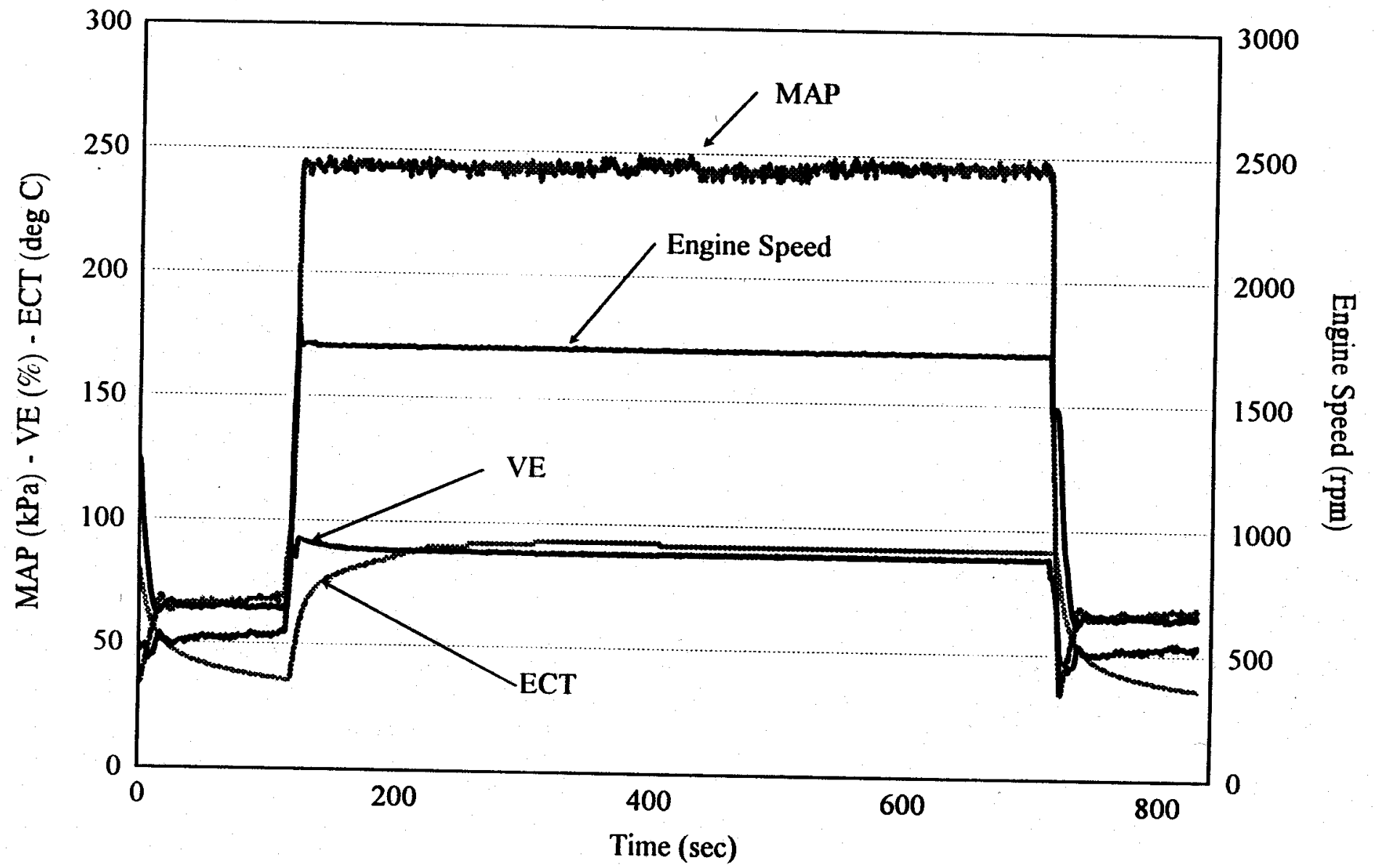

TRANSIENT DATA RECORDED FROM THE GEM SYSTEM FOR ONE FULL CYCLE AT 775 HOUR 


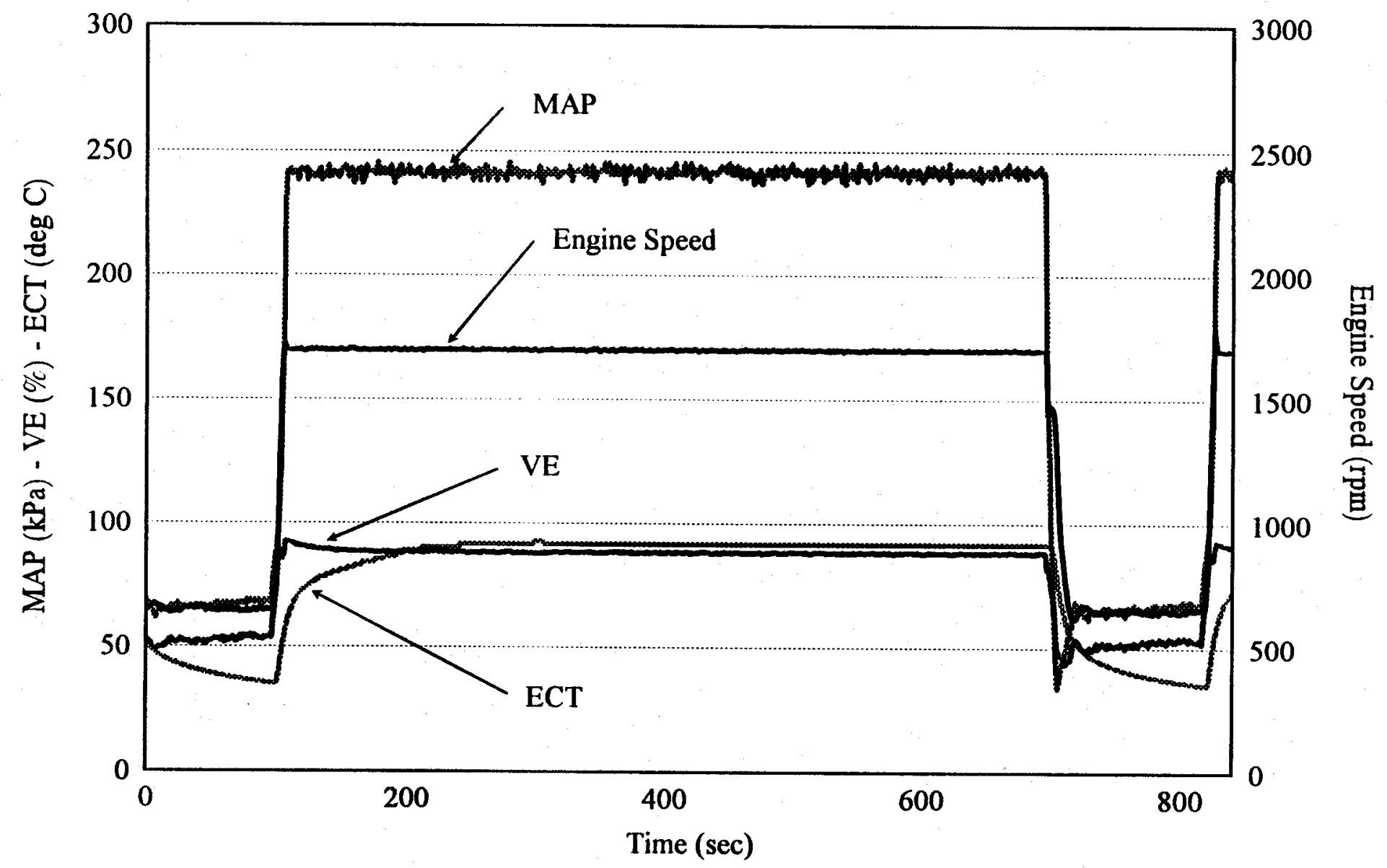

TRANSIENT DATA RECORDED FROM THE GEM SYSTEM FOR ONE FULL CYCLE AT 800 HOUR 
APPENDIX C

VALVE STEM HEIGHT MEASUREMENTS 
Valve Recession

\begin{tabular}{|c|c|c|c|c|c|c|c|c|c|}
\hline Total Hours & 0 & 24.8 & 24.8 & 129.3 & 129.3 & 104.5 & 386.9 & 386.9 & 257.6 \\
\hline STC Hours & & & & 0 & & & 250 & & \\
\hline Intake & Reading & Reading & Recession & Reading & Recession & Recession & Reading & Recession & Recession \\
\hline 1 in & 0.5409 & 0.5409 & 0.0000 & 0.5417 & 0.0008 & 0.0008 & 0.5422 & 0.0013 & 0.0005 \\
\hline $1 \mathrm{ex}$ & 0.5380 & 0.5383 & 0.0003 & 0.5390 & 0.0010 & 0.0007 & 0.5392 & 0.0012 & 0.0002 \\
\hline 2 in & 0.5385 & 0.5386 & 0.0001 & 0.5392 & 0.0007 & 0.0006 & 0.5394 & 0.0009 & 0.0002 \\
\hline $2 \mathrm{ex}$ & 0.5366 & 0.5367 & 0.0001 & 0.5372 & 0.0006 & 0.0005 & 0.5372 & 0.0006 & 0.0000 \\
\hline 3 in & 0.5369 & 0.5370 & 0.0001 & 0.5375 & 0.0006 & 0.0005 & 0.5377 & 0.0008 & 0.0002 \\
\hline $3 \mathrm{ex}$ & 0.5364 & 0.5366 & 0.0002 & 0.5376 & 0.0012 & 0.0010 & 0.5379 & 0.0015 & 0.0003 \\
\hline 4 in & 0.5415 & 0.5414 & -0.0001 & 0.5420 & 0.0005 & 0.0006 & 0.5426 & 0.0011 & 0.0006 \\
\hline $4 \mathrm{ex}$ & 0.5375 & 0.5374 & -0.0001 & 0.5376 & 0.0001 & 0.0002 & 0.5 & 0.0014 & 0.0013 \\
\hline 5 in & 0.5371 & 0.5372 & 0.0001 & 0.5384 & 0.0013 & 0.0012 & 0.5399 & 28 & 0.0015 \\
\hline $5 \mathrm{ex}$ & 0.5366 & 0.5365 & -0.0001 & 0.5375 & 0.0009 & 0.0010 & 0.5387 & 0.0021 & 012 \\
\hline 6 in & 0.5385 & 0.5385 & 0.0000 & 0.5386 & 0.0001 & 0.0001 & 0.5393 & 0.0008 & 0.0007 \\
\hline $6 \mathrm{ex}$ & 0.5369 & 0.5368 & -0.0001 & 0.5369 & 0.0000 & 0.0001 & 0.5373 & 0.0004 & 0.0004 \\
\hline Average & 0.5380 & 0.5380 & 0.0000 & 0.5386 & 0.0006 & 0.0006 & 0.5392 & 0.0012 & 0.0006 \\
\hline Exhaust & Reading & Reading & Recession & Reading & Recession & Recession & Reading & Recession & Recession \\
\hline 1 in & 0.5148 & 0.5148 & 0.0000 & 0.5164 & 0.0016 & 0.0016 & 0.5200 & 0.0052 & 0.0036 \\
\hline $1 \mathrm{ex}$ & 0.5136 & 0.5137 & 0.0001 & 0.5152 & 0.0016 & 0.0015 & 0.5163 & 0.0027 & 0.0011 \\
\hline 2 in & 0.5162 & 0.5165 & 0.0003 & 0.5179 & 0.0017 & 0.0014 & 0.5230 & 0.0068 & 0.0051 \\
\hline $2 \mathrm{ex}$ & 0.5112 & 0.5117 & 0.0005 & 0.5130 & 0.0018 & 0.0013 & 0.5140 & 0.0028 & 0.0010 \\
\hline 3 in & 0.5159 & 0.5168 & 0.0009 & 0.5173 & 0.0014 & 0.0005 & 0.5188 & 0.0029 & 0.0015 \\
\hline $3 \mathrm{ex}$ & 0.5151 & 0.5160 & 0.0009 & 0.5170 & 0.0019 & 0.0010 & 0.5188 & 0.0037 & 0.0018 \\
\hline 4 in & 0.5148 & 0.5155 & 0.0007 & 0.5162 & 0.0014 & 0.0007 & 0.5180 & 0.0032 & 0.0018 \\
\hline $4 \mathrm{ex}$ & 0.5130 & 0.5135 & 0.0005 & 0.5147 & 0.0017 & 0.0012 & 0.5213 & 0.0083 & 0.0066 \\
\hline 5 in & 0.5142 & 0.5148 & 0.0006 & 0.5151 & 0.0009 & 0.0003 & 0.5164 & 0.0022 & 0.0013 \\
\hline $5 \mathrm{ex}$ & 0.5128 & 0.5135 & 0.0007 & 0.5157 & 0.0029 & 0.0022 & 0.5180 & 0.0052 & 0.0023 \\
\hline 6 in & 0.5139 & 0.5157 & 0.0018 & 0.5171 & 0.0032 & 0.0014 & 0.5178 & 0.0039 & 0.0007 \\
\hline $6 \mathrm{ex}$ & 0.5139 & 0.5152 & 0.0013 & 0.5181 & 0.0042 & 0.0029 & 0.5212 & 0.0073 & 0.0031 \\
\hline Average & 0.5141 & 0.5148 & 0.0007 & 0.5161 & 0.0020 & 0.0013 & 0.5186 & 0.0045 & 0.0025 \\
\hline
\end{tabular}


Valve Recession

\begin{tabular}{|c|c|c|c|c|c|c|c|c|c|}
\hline Total Hours & 640.6 & 640.6 & 253.7 & 895.5 & 895.5 & 254.9 & 1174.6 & 1174.6 & 279.1 \\
\hline STC Hours & 500 & & & 750 & & & 1000 & & \\
\hline Intake & Reading & Recession & Recession & Reading & Recession & Recession & Reading & Recession & Recession \\
\hline 1 in & 0.5422 & 0.0013 & 0.0000 & 0.5423 & 0.0014 & 0.0001 & 0.5420 & 0.0011 & -0.0003 \\
\hline $1 \mathrm{ex}$ & 0.5392 & 0.0012 & 0.0000 & 0.5392 & 0.0012 & 0.0000 & 0.5379 & -0.0001 & -0.0013 \\
\hline 2 in & 0.5396 & 0.0011 & 0.0002 & 0.5398 & 0.0013 & 0.0002 & 0.5394 & 0.0009 & -0.0004 \\
\hline $2 \mathrm{ex}$ & 0.5374 & 0.0008 & 0.0002 & 0.5375 & 0.0009 & 0.0001 & 0.5375 & 0.0009 & 0.0000 \\
\hline 3 in & 0.5380 & 0.0011 & 0.0003 & 0.5381 & 0.0012 & 0.0001 & 0.5370 & 0.0001 & -0.0011 \\
\hline 3 ex & 0.5380 & 0.0016 & 0.0001 & 0.5380 & 0.0016 & 0.0000 & 0.5371 & 0.0007 & -0.0009 \\
\hline 4 in & 0.5427 & 0.0012 & 0.0001 & 0.5428 & 0.0013 & 0.0001 & 0.5430 & 0.0015 & 0.0002 \\
\hline $4 \mathrm{ex}$ & 0.5390 & 0.0015 & 0.0001 & 0.5391 & 0.0016 & 0.0001 & 0.5392 & 0.0017 & 0.0001 \\
\hline 5 in & 0.5401 & 0.0030 & 0.0002 & 0.5401 & 0.0030 & 0.0000 & 0.5404 & 0.0033 & 0.0003 \\
\hline $5 \mathrm{ex}$ & 0.5388 & 0.0022 & 0.0001 & 0.5389 & 0.0023 & 0.0001 & 0.5392 & 0.0026 & 0.0003 \\
\hline 6 in & 0.5396 & 0.0011 & 0.0003 & 0.5396 & 0.0011 & 0.0000 & 0.5400 & 0.0015 & 0.0004 \\
\hline $6 \mathrm{ex}$ & 0.5375 & 0.0006 & 0.0002 & 0.5376 & 0.0007 & 0.0001 & 0.5382 & 0.0013 & 0.0006 \\
\hline Average & 0.5393 & 0.0014 & 0.0001 & 0.5394 & 0.0015 & 0.0001 & 0.5392 & 0.0013 & -0.0002 \\
\hline Exhaust & Reading & Recession & Recession & Reading & Recession & Recession & Reading & Recession & Recession \\
\hline 1 in & 0.5205 & 0.0057 & 0.0005 & 0.5217 & 0.0069 & 0.0012 & 0.5221 & 0.0073 & 0.0004 \\
\hline $1 \mathrm{ex}$ & 0.5172 & 0.0036 & 0.0009 & 0.5174 & 0.0038 & 0.0002 & 0.5179 & 0.0043 & 0.0005 \\
\hline 2 in & 0.5246 & 0.0084 & 0.0016 & 0.5258 & 0.0096 & 0.0012 & 0.5263 & 0.0101 & 0.0005 \\
\hline $2 \mathrm{ex}$ & 0.5147 & 0.0035 & 0.0007 & 0.5154 & 0.0042 & 0.0007 & 0.5158 & 0.0046 & 0.0004 \\
\hline $3 \mathrm{in}$ & 0.5191 & 0.0032 & 0.0003 & 0.5196 & 0.0037 & 0.0005 & 0.5200 & 0.0041 & 0.0004 \\
\hline $3 \mathrm{ex}$ & 0.5192 & 0.0041 & 0.0004 & 0.5196 & 0.0045 & 0.0004 & 0.5202 & 0.0051 & 0.0006 \\
\hline 4 in & 0.5183 & 0.0035 & 0.0003 & 0.5204 & 0.0056 & 0.0021 & 0.5215 & 0.0067 & 0.0011 \\
\hline $4 \mathrm{ex}$ & 0.5226 & 0.0096 & 0.0013 & 0.5237 & 0.0107 & 0.0011 & 0.5254 & 0.0124 & 0.0017 \\
\hline 5 in & 0.5172 & 0.0030 & 0.0008 & 0.5183 & 0.0041 & 0.0011 & 0.5187 & 0.0045 & 0.0004 \\
\hline $5 \mathrm{ex}$ & 0.5202 & 0.0074 & 0.0022 & 0.5219 & 0.0091 & 0.0017 & 0.5235 & 0.0107 & 0.0016 \\
\hline $6 \mathrm{in}$ & 0.5180 & 0.0041 & 0.0002 & 0.5183 & 0.0044 & 0.0003 & 0.5185 & 0.0046 & 0.0002 \\
\hline $6 \mathrm{ex}$ & 0.5222 & 0.0083 & 0.0010 & 0.5231 & 0.0092 & 0.0009 & 0.5243 & 0.0104 & 0.0012 \\
\hline Average & 0.5195 & 0.0054 & 0.0008 & 0.5204 & 0.0063 & 0.0010 & 0.5212 & 0.0071 & 0.0008 \\
\hline
\end{tabular}


APPENDIX D

LETTERS SENT TO CHAMPION SPARK PLUGS 


\section{SOUTHWEST RESEARCH INSTITUTE}

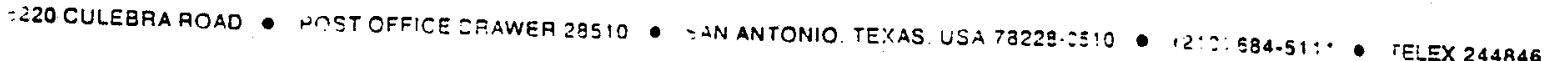
ENOINE ANO VEHICLE RESEARCH DIVISION FAX: (210) 522-2019

August 14. 1996

Mr. Bob Freeman

Staff Production Engineer

Cooper Automotive

Champion Spark Plug Division

P.O. Box 910

Toledo, $\mathrm{OH}$ 43661-0001

Dear Mr. Freeman:

Mr. John Bartel of Mack Trucks. Inc. requested that I send you this set of used spark plugs from a Mack E7 narural gas-rueled engine.

The enclosed spark plugs are marked with the cylinder number. The run time history of the spark plugs is shown in the table below.

\begin{tabular}{|c|c|c|c|c|}
\hline Description & $\begin{array}{l}\text { Engine } \\
\text { Speed } \\
\text { (rpm) } \\
\end{array}$ & $\begin{array}{l}\text { Power } \\
\text { (bhp) } \\
\end{array}$ & $\begin{array}{c}\begin{array}{c}\text { Time } \\
\text { (hours) }\end{array} \\
\end{array}$ & $\begin{array}{c}\text { Total } \\
\text { Time } \\
\text { (hours) } \\
\end{array}$ \\
\hline Steady State Test Time & 1950 & 163 & 12.0 & 12.0 \\
\hline Steady State Test Time & 1950 & 325 & 12.0 & 24.0 \\
\hline Steady State Test Iime & 1950 & 357 & 100.0 & 124.0 \\
\hline Cycle Test Time * & $650 / 1700$ & $5 / 389$ & 273.5 & 397.5 \\
\hline \multicolumn{3}{|c|}{ Other Time (warm up, cool down, troubleshooting) } & 13.1 & 410.6 \\
\hline \multicolumn{5}{|c|}{ Repiaced no. 4 spark plug due to misfires } \\
\hline Cycle Test Time * & $650 / 1700$ & $5 / 389$ & 5.4 & 416.0 \\
\hline \multicolumn{3}{|c|}{ Other Time (warm up, cool down. troubleshooting) } & 0.8 & 416.8 \\
\hline \multicolumn{5}{|c|}{ Replaced no. 5 spark plug due to misfires } \\
\hline Cycle Test Time * & $650 / 1700$ & $5 / 389$ & 10.6 & 427.4 \\
\hline \multicolumn{3}{|c|}{ Other Time (warm up, cool down. troubleshooting) } & 0.1 & 427.5 \\
\hline
\end{tabular}


Mr. Bub Freeman

August 14. 1996

Page 2

Cylinder no. 4 began to misfire first at 410.6 hours. The no. 4 spark plug was repiaced and the misfires stopped. Cylinder no. 5 began to misfire at 416.8 hours. The no. 5 spark piug was repiaced and the misfires stopped. The spark plugs in cylinder nos. 1,2,3, and 6 were not misfiring, but were replaced at the request of John Bartel. Spark plug gaps are shown below.

\begin{tabular}{|c|c|c|c|c|c|c||}
\hline Cylinder & $\begin{array}{c}0.0 \\
\text { Total } \\
\text { Engine } \\
\text { Hours }\end{array}$ & $\begin{array}{c}129.3 \\
\text { Total } \\
\text { Engine } \\
\text { Hours }\end{array}$ & $\begin{array}{c}386.9 \\
\text { Total } \\
\text { Engine } \\
\text { Hours }\end{array}$ & $\begin{array}{c}410.6 \\
\text { Total } \\
\text { Engine } \\
\text { hours }\end{array}$ & $\begin{array}{c}416.8 \\
\text { Total } \\
\text { Engine } \\
\text { Hours }\end{array}$ & $\begin{array}{c}427.5 \\
\text { Total } \\
\text { Engine } \\
\text { hours }\end{array}$ \\
\hline \hline 1 & $0.010^{\prime \prime}$ & $0.012^{\prime \prime}$ & $0.017^{\prime \prime}$ & & & $0.019^{\prime \prime}$ \\
\hline 2 & $0.010^{\prime \prime}$ & $0.012^{\prime \prime}$ & $0.017^{\prime \prime}$ & & & $0.019^{\prime \prime}$ \\
\hline 3 & $0.010^{\prime \prime}$ & $0.013^{\prime \prime}$ & $0.017^{\prime \prime}$ & & & $0.019^{\prime \prime}$ \\
\hline 4 & $0.010^{\prime \prime}$ & $0.013^{\prime \prime}$ & $0.021^{\prime \prime}$ & $0.032^{\prime \prime}$ & & \\
\hline 5 & $0.010^{\prime \prime}$ & $0.013^{\prime \prime}$ & $0.020^{\prime \prime}$ & & $0.028 "$ & \\
\hline 6 & $0.010^{\prime \prime}$ & $0.014^{\prime \prime}$ & $0.018^{\prime \prime}$ & & & $0.019^{\prime \prime}$ \\
\hline
\end{tabular}

If you have any questions. please feel free to contact me. I can be reached by telephone at (210) $522-2570$ or by fax at 1210) 522-2019.

Sincerely,

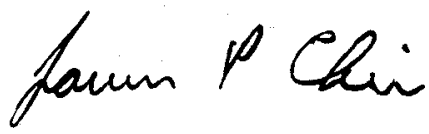

James P. Chiu, P.E.

Senior Research Engineer

Gas and Industrial Engine Deveiopment

Department of Engine Research

$/ \mathrm{lmm}$

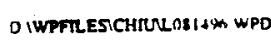

Enclosures 


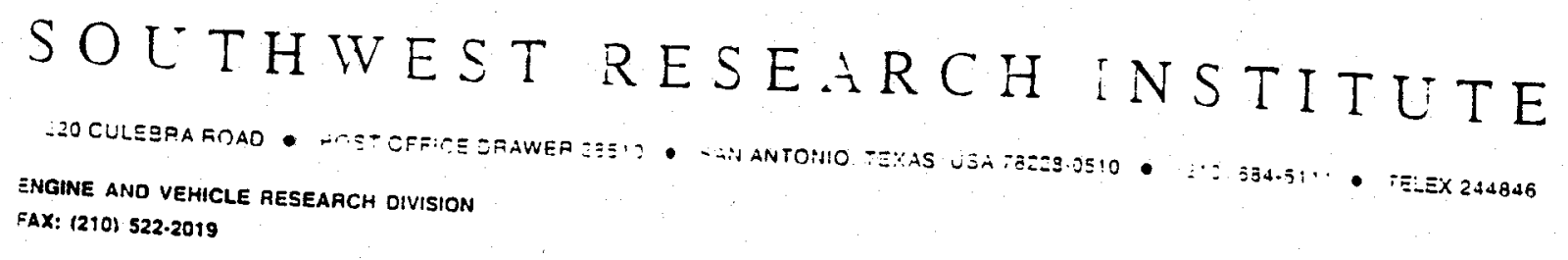

September $1: 1996$

Mr. Bob Freeman

Staff Product Engineer

Cooper Automotive

Champion Spark Plug Division

P.O. Box 910

Toledo. $\mathrm{OH}$ 43661-0001

Dear Mr. Freeman: Enclosed are the parts or a spark plug from the durability test we are conducting tor John
Bartel at Mack Trucks.

The spark plug is a RX501PYP/2H6. and has 257.2 hours on it. The 257.2 hours include 252.0 hours of super thermai cycle (STC) test time and 5.2 hours of miscellaneous test time. The STC consists of 10 minutes at $1700 \mathrm{rpm}$ and $389 \mathrm{bhp}$, and two(2) minutes at $650 \mathrm{rpm}$ and six (6) bhp. The initial gap of the spark plug was 0.010 ". At 254.9 hours. the spark plug was removed, and the gap was $0.015 "$. It was reinstalled. and at 257.2 hours it broke apart.

If you have any questions. please feel free to contact me. I can be reached by telephone at (210) $522-2570$ or by fax at (210) $522-2019$.

Sincerely.

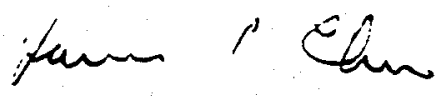

James P. Chiu. P.E.

Senior Research Engineer

Gas and Industrial Engine Development

Department of Engine Research

$/ \mathrm{Imm}$

D WPFRES CHIULLO911\% WPD

Enciosures 


\title{
SOLTHVESTRESEARCH INSTITUTE :DO CULEJAA FOAO ENGINE AND VEHICLE RESEARCH DIVISION FAX: $(210) 522-2019$
}

September 20.1996

\author{
Mr. Bill Squire
}

Senior Account Manager

OEM Sales \& Marketing

Cooper Automotive

Champion Spark Plug Division

900 Upton Avenue

Toledo. OH 43607

Dear Mr. Squire:

Enclosed are two spark piugs rrom a durability test we are conducting for John Bartei at Mack Trucks. Mr. Bartel spoke to you today about these spark plugs and he requested that I send them to you.

The engine for the durability test operates on a cycle for two minutes at $650 \mathrm{rpm}$. minimum load. $100^{\circ} \mathrm{F}$ coolant out temperature and 10 minutes at $1700 \mathrm{rpm}, 1202 \mathrm{lb}-\mathrm{ft}, 200^{\circ} \mathrm{F}$ coolant out temperature. The spark plugs enclosed (RX501PYP/2H6) were installed at 500 test hours. They were removed at 750 test hours for gap measurements and reinstalled. At 914.1 test hours, the spark plug on the no. 4 cylinder failed. and the spark piug from the no. 1 cylinder shows signs of combustion products between the ceramic and the shell next to the crimp. Both of these spark plugs are enclosed for you to examination. Yone of the spark plugs required high torques to remove them. In addition to the 414.1 hours of test time on the spark plugs. there were 6.1 hours of misceilaneous time rwarm-ups and cooi-downs).

Our procedure for installing spark plugs is to coat the threads with an anti-seize compound and insert the plug into the head until it is finger tight. The spark plug is then turned an additional 1/4 turn for new spark plugs or 1/8 turn for used spark plugs with a used gasket. An experiment. performed after this spark plug failure. showed:

(1) The torque required for installing a used spark plug (RX501PYP/1E5) at $1 / 8$ turn after it is finger tight is $10 \mathrm{ft}-\mathrm{lb}$.

(2) To torque a new spark plug (RX501PYP//ES or IES) to $20 \mathrm{ft}-1$ bs requires a $1 / 2-34$ turn arter it is finger tight.

(3) To torque a used spark plug (RX501PYP/2H6) with a used gasket to $20 \mathrm{ft}-\mathrm{lbs}$ requires a $1 / 8-1 / 4$ turn atter it is finger tight. 
Mrr. Bill Squire

Septemiver 20. 1996

Page 2 follows:

The gasket thickness of a spark plug (RX501PYP/1E5) for a given tightening torque are as

$\begin{array}{ll}\text { New } & 0.080^{\prime \prime} \\ 20 \mathrm{ft}-\mathrm{lbs} & 0.050^{\prime \prime} \\ 30 \mathrm{ft}-\mathrm{lbs} & 0.043^{\prime \prime} \\ 40 \mathrm{ft}-\mathrm{lbs} & 0.043^{\prime \prime}\end{array}$

A torque of $40 \mathrm{ft}-\mathrm{lbs}$ was the highest we felt comfortable with. in order to avoid damaging the engine.

If you have any questions. piease teei free to contact me. I san be reached by telephone at (210) 522-2570 or by fax at (210) 522-2019.

Sincereiy,

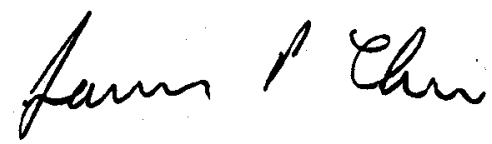

James P. Chiu, P.E.

Senior Research Engineer

Gas and Industrial Engine Development

Department of Engine Research

Imm

Enciosures

cc: John Bartel. Mack Trucks (w/o enclosures) 


\section{SOLTHWEST RESEARCH NSTITLTE

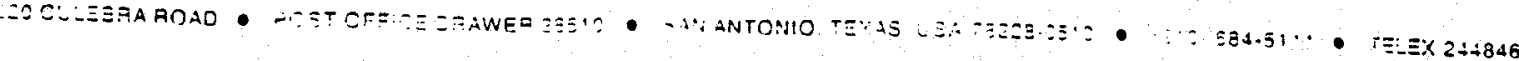 \\ ENGINE ANO VEHICLE RESEARCH DIVISION \\ $=A X:(210) 522.2019$}

September 30.1996

Mr. Bob Freeman

Staff Product Engineer

Cooper Automotive

Champion Spark Plug Division

P.O. Box 910

Toledo. OH 43661-0001

Dear Mr. Freeman:

This letter is a follow-up to the letter I sent to Mr. Bill Squire dated September 20.1996 regarding spark plugs trom a durability test we are conducting for John Bartel at. Mack Trucks.

In the previous letter. the gasket thickness of a spark plug for a given tightening torque was given. The spark plug was torqued into the head. but was never operated in an engine. Further experiments on gasket thicknesses were performed on spark plugs that have been operated in an engine. Two new spark plugs with different date codes were torqued to $20 \mathrm{ft}$-lbs. The spark plugs were operated for 86.8 hours. and the gasket thickness (after the 86.8 hours) for the RX501PYP/IES was 0.048 " and for the RX501PYP/2H6
was 0.044 ".

The resuits indicate that either the gasket thickness after running in an engine may not be a good indicator of initial tightening torque. or that the spark plug gasket thicknesses are different for spark plugs with different date codes and the same tightening torque. The gasket thickness experiment on the from the previous letter ivas performed on a RX50IPYP/IES. The $0.048^{\prime \prime}$ gasket thickness of the used RX501PYP/IE5 is closest to the 0.050 " resuits for $20 \mathrm{ft}$-lbs of torque on a new spark plug. where as the 0.044 " gasket thickness of the used RXS0IPYP/2H6 is closest to the $0.043^{\prime \prime}$ resuit for $30-40 \mathrm{ft}$-lbs of torque on a new RX501PYP/IE spark plug. Since I do not have any more new RX50 IPYP/2I16 spark plugs. I currentiy cannot pertorm a new gasket thickness experiment on this spark plug.

If you have any questions. please teel free to contact me. I can be reached by telephone at (210) $522-$ 2570 or oy fax at (210) 522-2019.

Sincereiy,

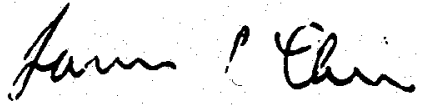

James P. Chiu. P.E.

Senior Research Engineer

Gas and Industrial Engine Deveiopment

Department of Engine Researcin

Imm

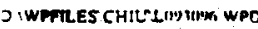

cc: John Bartel. . Wack Trucks

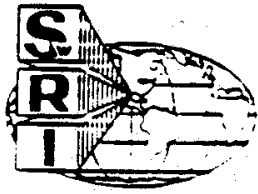


APPENDIX E

LOW SPEED DATA FOR EMISSIONS TEST AT O HOURS ON THE STC TEST 


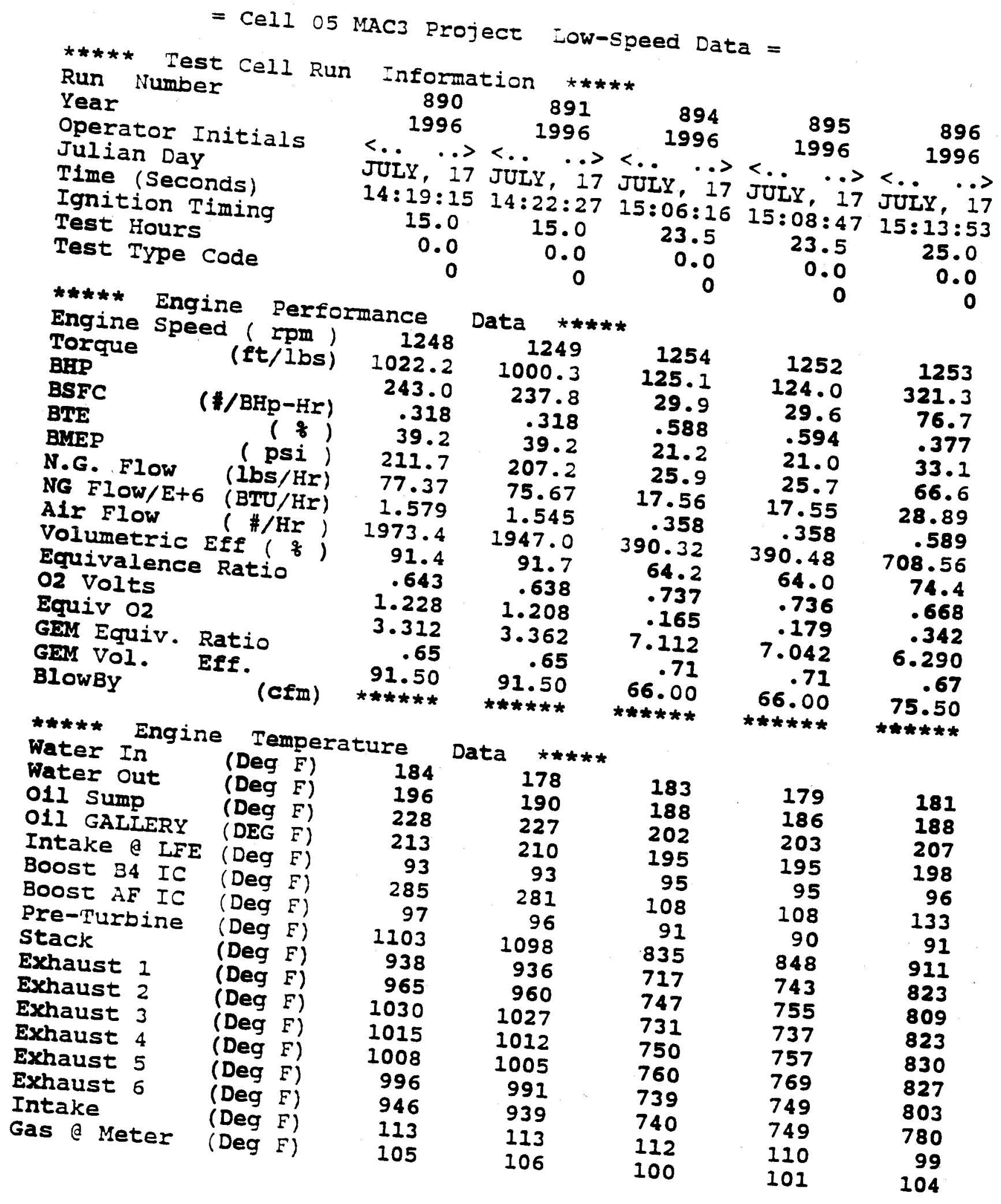


= Cell o5 MAC3 Project Low-Speed Data = (concinuea)

Run Number

890

891

894

895

896

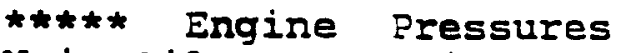

Main oil Pres (psig)

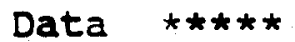

Fuel Pressure (psig)

41.5

42.1

52.4

94.6

99.4

52.3

100.2

49.3

Compress out (psig)

16.41

.14

Inlet Manifold (psig)

16.97

15.63

Inlet Manifold(psia)

30.50

$-5.80$

29.96

Pre-Turbine

(psig)

10.38

Exhaust Bk Pr ("HG)

10.06

LFE Filter Pr ("H2O)

2.05

LFE Delta Pr ("H2O)

.87

2.00

8.53

.74

.16

3.94

H

(1bH20/IbAir)

.009

RH (out)

Barometer

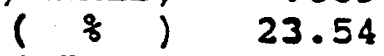

( "Hg) 29.18

.85

.14

.76

.010

.009

24.22

21.52

29.18

29.17

.15

$-5.76$

2.18

8.56

.74

.16

.14

.76

.009

21.22

29.17

$-1.05$

13.28

1.62

.37

.26

1.39

.009

21.15

29.17

***** Natural Gas Composition

NG : Methane

NG : Ethane

NG \% Propane

NG \& $\mathrm{CO} 2$

NG \% Nitrogen

Butane Actual (\%)

94.71

2.55

.49

1.09

.92

.24

94.71
2.55

94.56

2.56

94.56

94.56

2.56

.52

.49
1.09

1.08

.52

2.56

1.08

1.00

1.00

.92

.28

.28

17.05

.24

17.09

17.09

20412 .

20412 .

20392 .

20392 .

1.08

1.00

.28

17.09

20392 . 


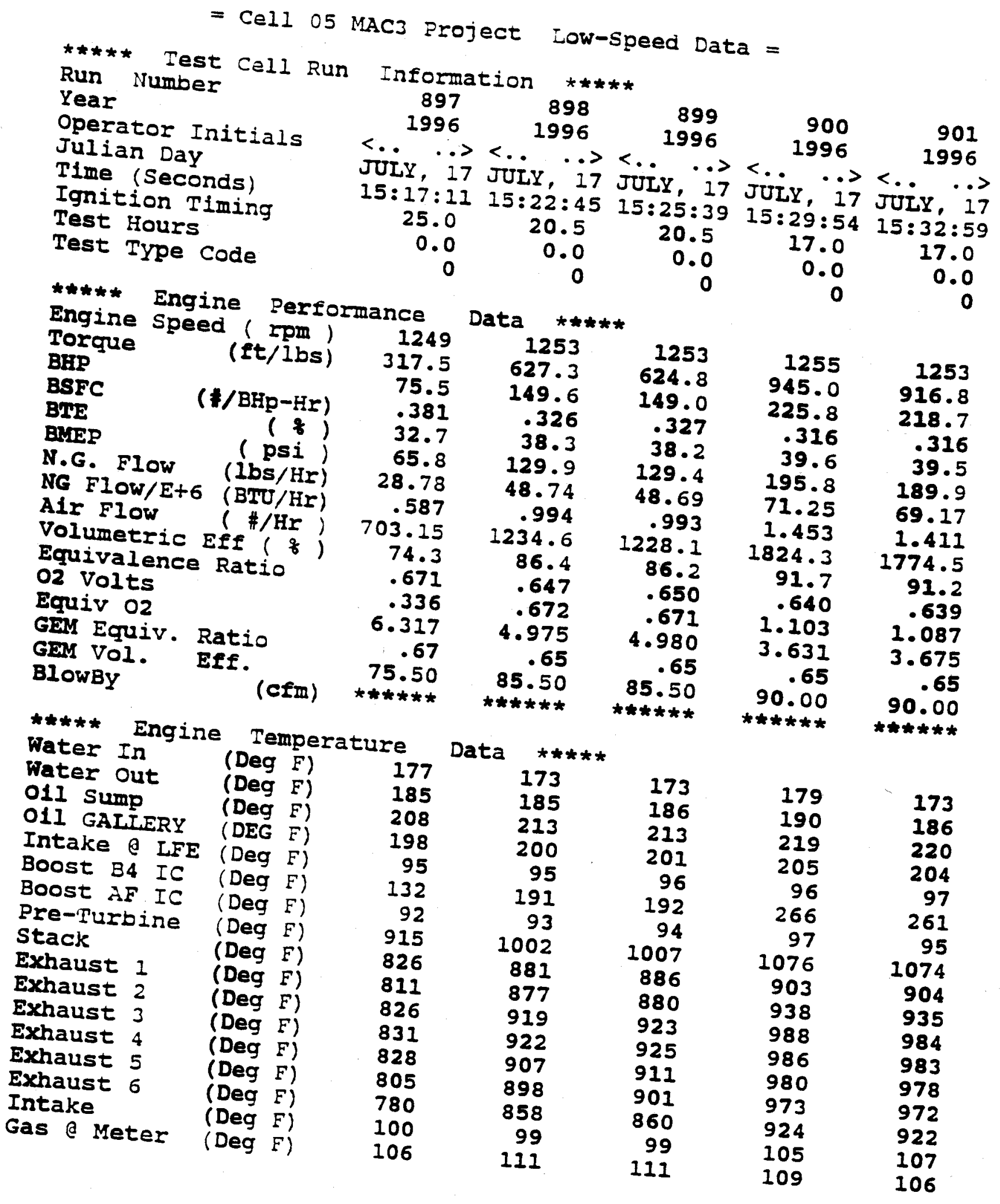




$$
\begin{gathered}
\text { Cell } 05 \text { MAC3 Project Low-Speed Data = } \\
\text { (continued) }
\end{gathered}
$$

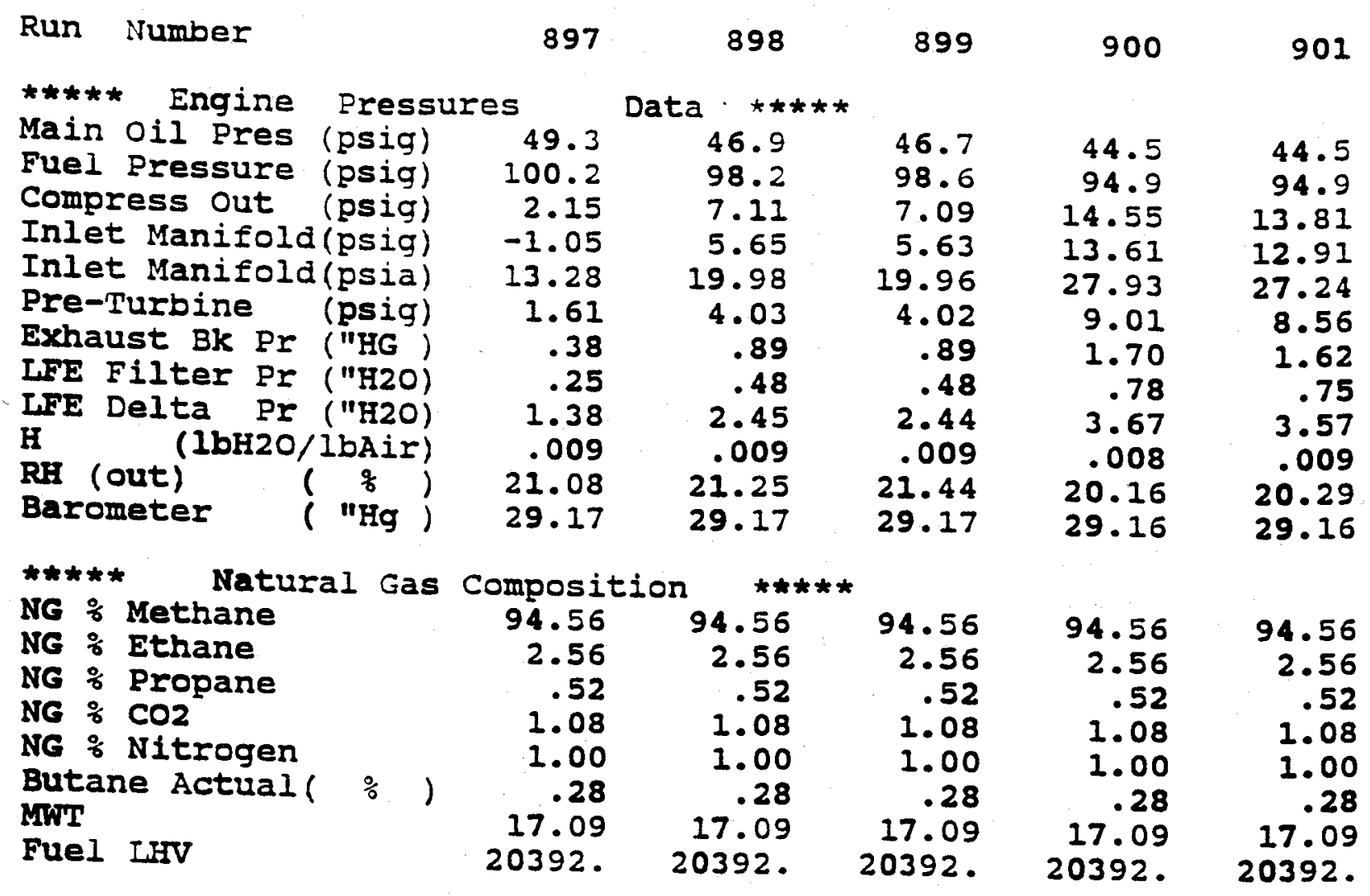




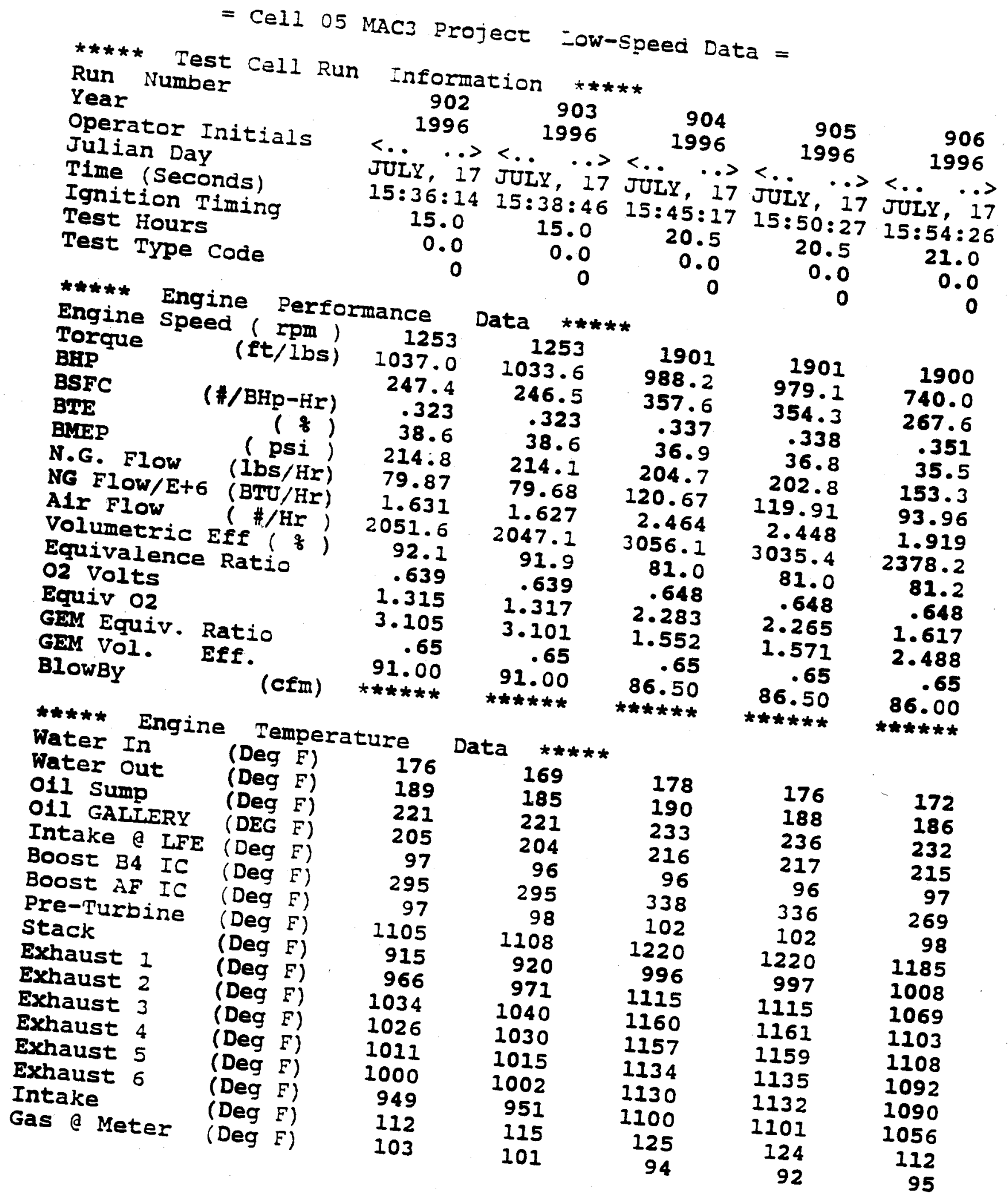




$$
\text { = Cell } 05 \text { MAC3 Project Low-speed Data = }
$$

$\begin{array}{llllll}\text { Run Number } & 902 & 903 & 904 & 905 & 906\end{array}$

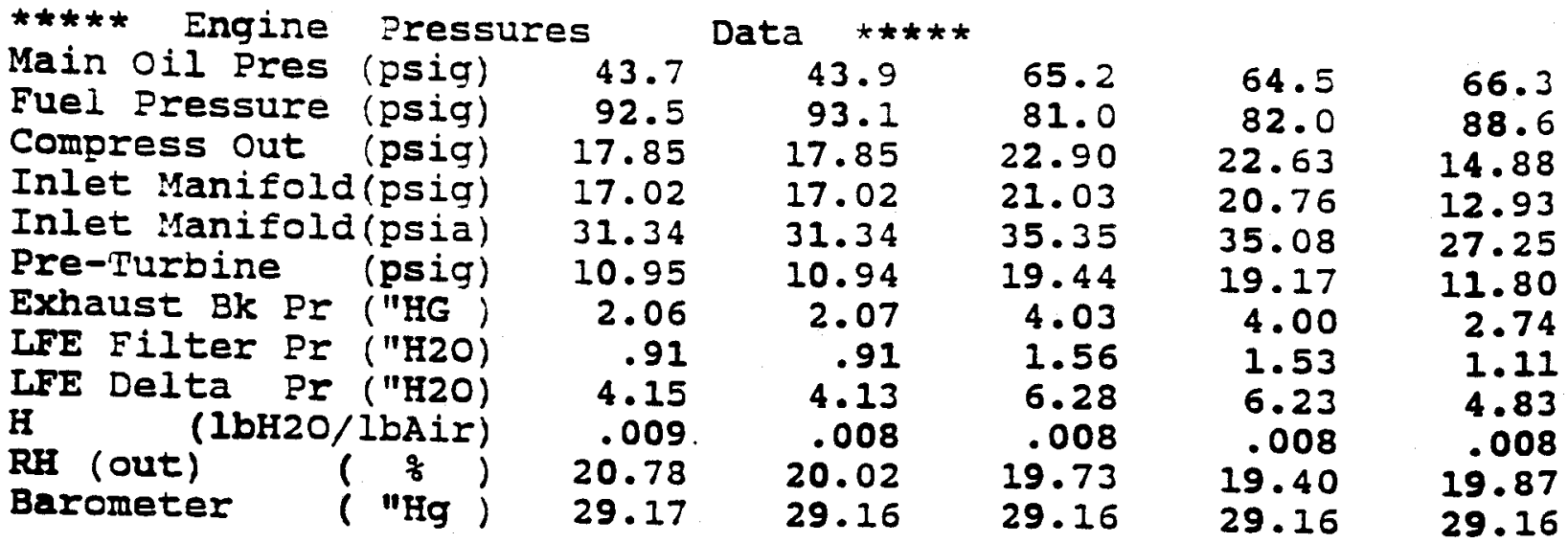

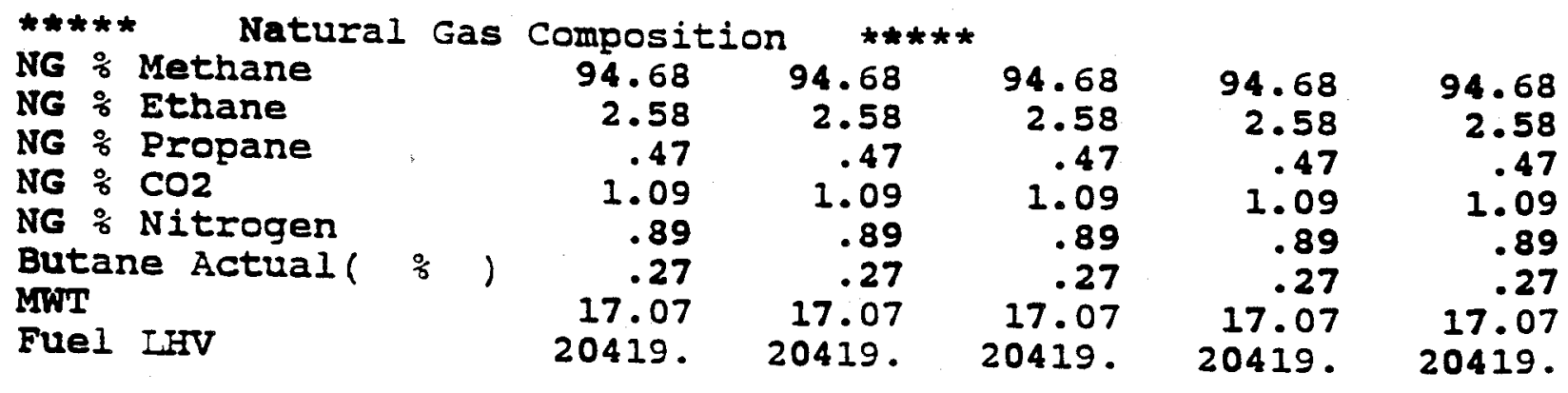




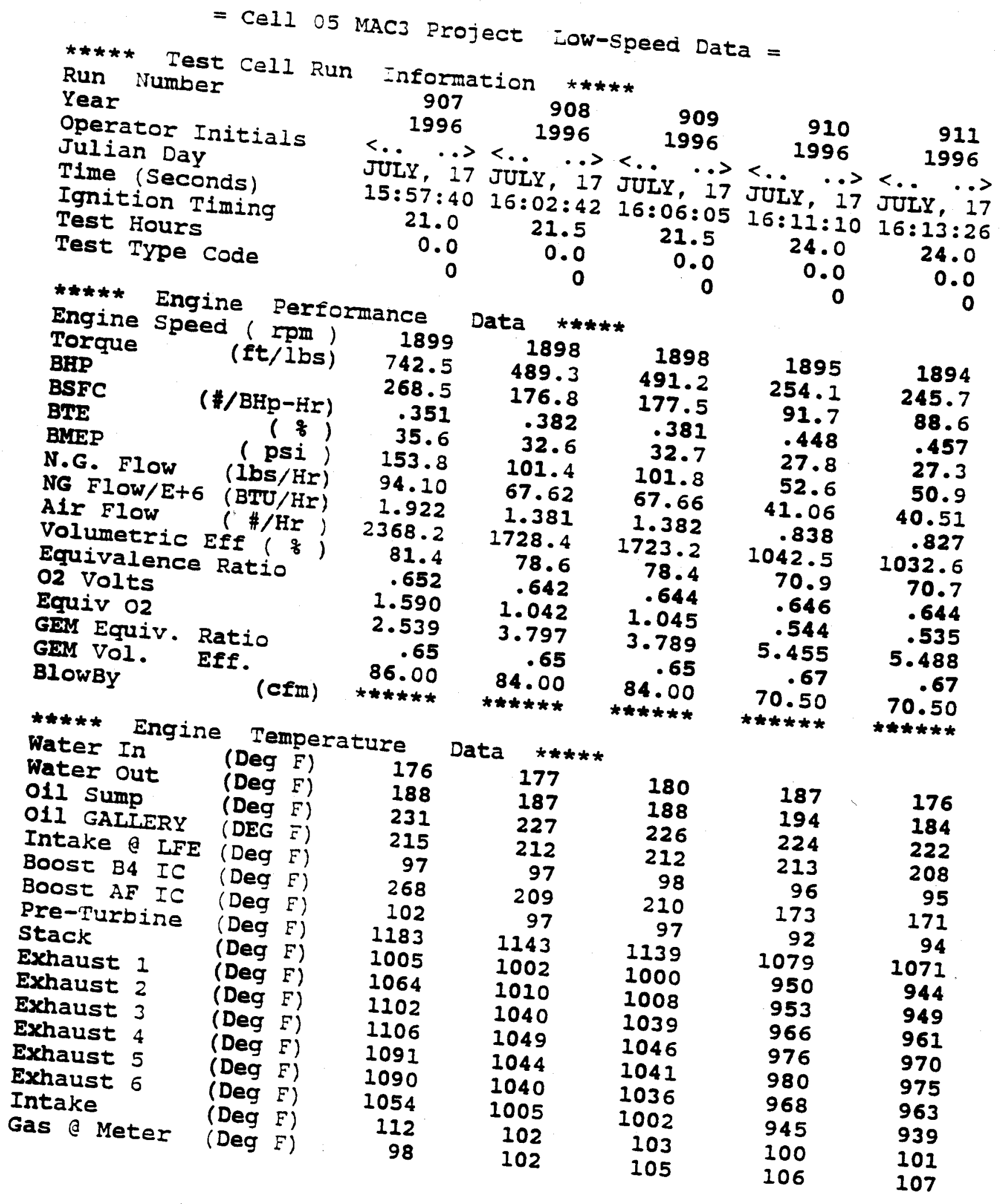




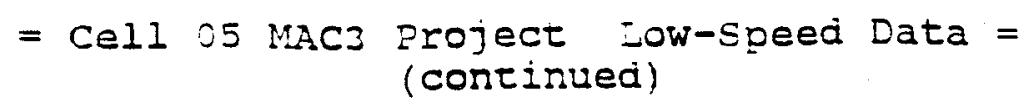

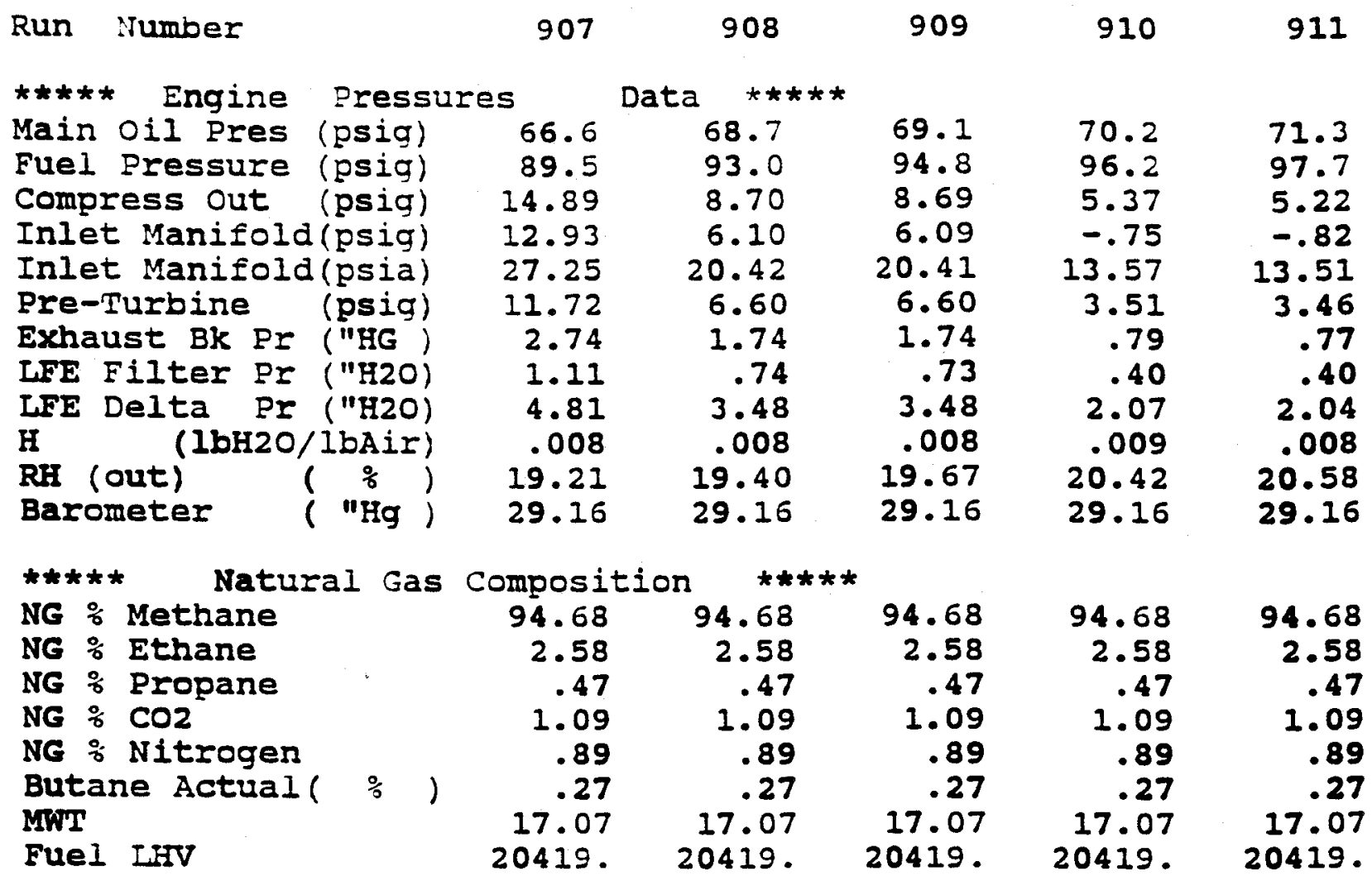




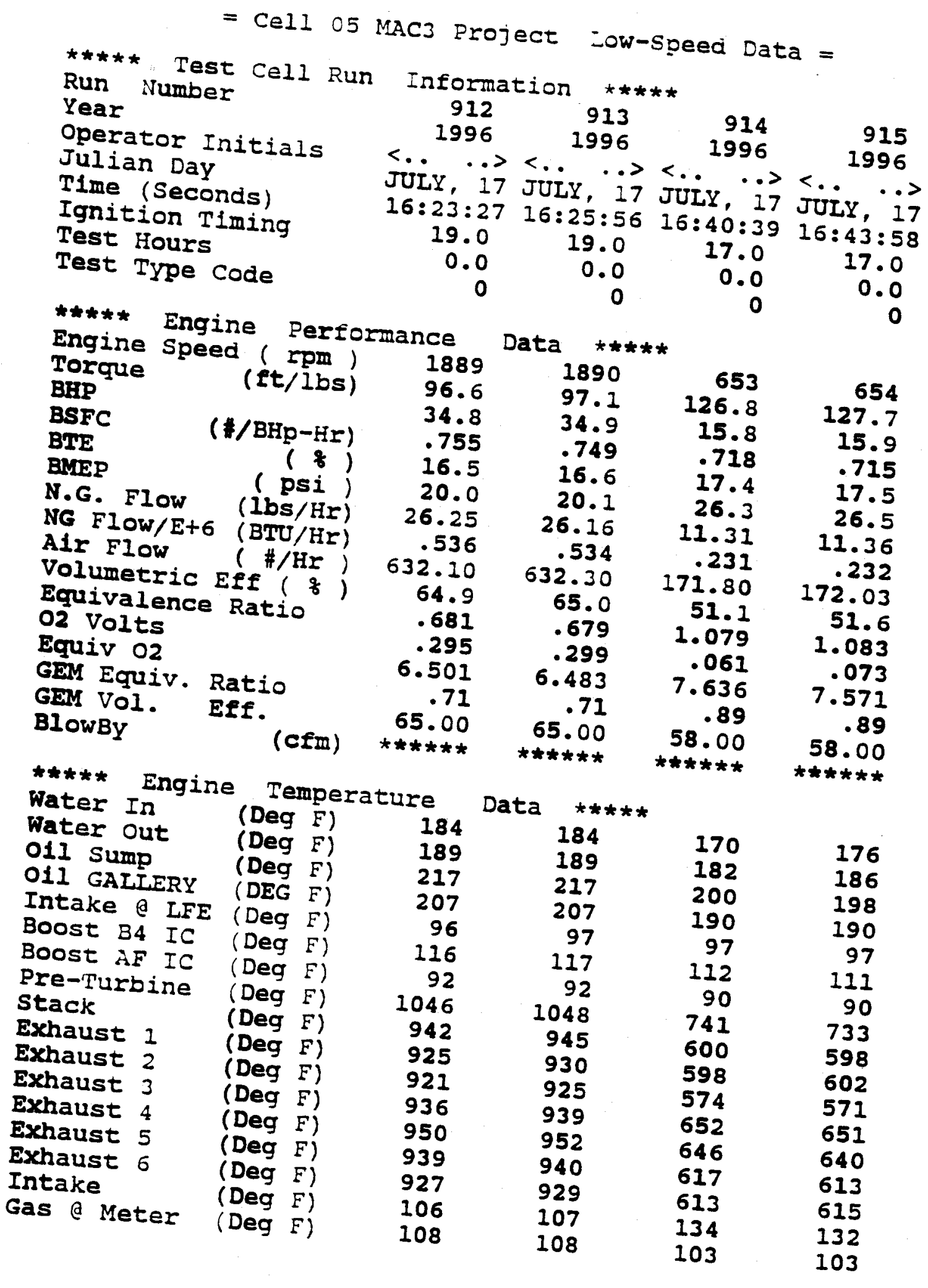




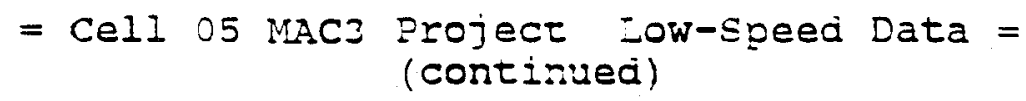

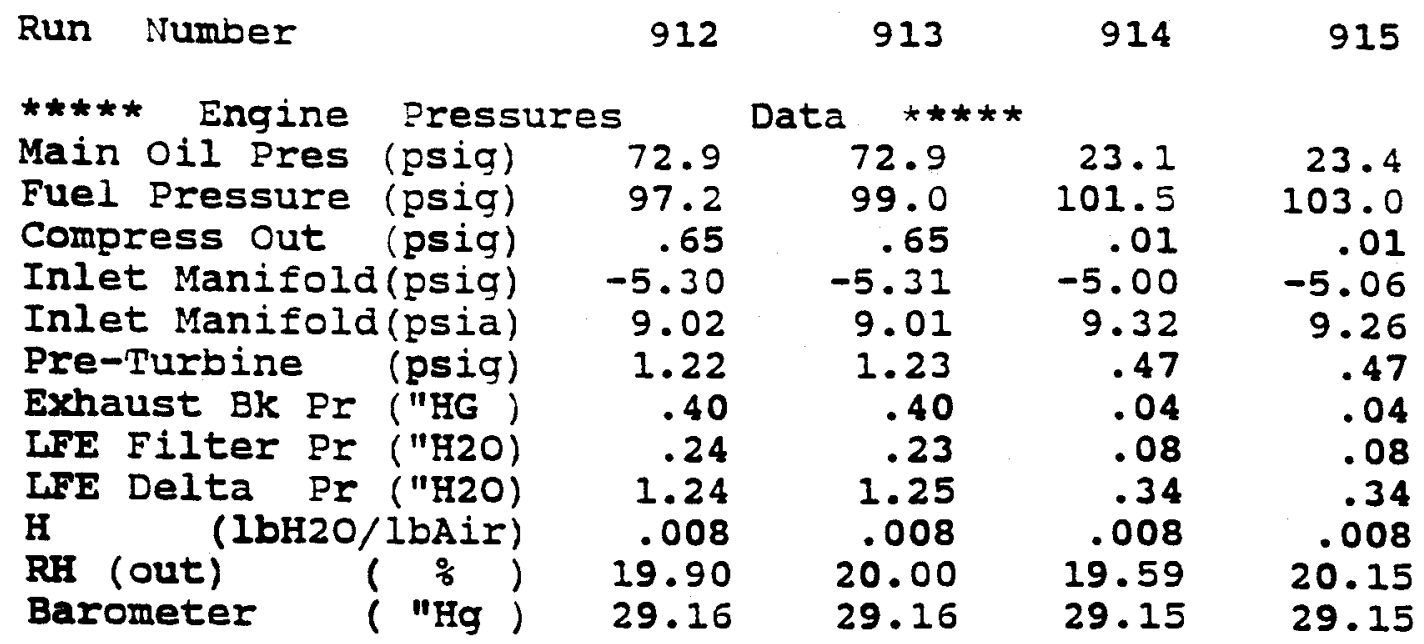

$\begin{array}{lrrrr}\star \star * * * * \text { Natural Gas Composition } * * * * * & & \\ \text { NG \% Methane } & 94.68 & 94.68 & 94.43 & 94.43 \\ \text { NG \% Ethane } & 2.58 & 2.58 & 2.61 & 2.61 \\ \text { NG \% Propane } & .47 & .47 & .58 & .58 \\ \text { NG \% CO2 } & 1.09 & 1.09 & 1.11 & 1.11 \\ \text { NG \% Nitrogen } & .89 & .89 & .93 & .93 \\ \text { Butane Actual ( } \% \text { ) } & .27 & .27 & .33 & .33 \\ \text { MWT } & 17.07 & 17.07 & 17.13 & 17.13 \\ \text { Fuel LFV } & 20419 . & 20419 . & 20395 . & 20395 .\end{array}$




\section{APPENDIX F}

EMISSIONS RESULTS FOR TESTS AT 0 HOURS ON THE STC TEST 


\begin{tabular}{|c|c|c|c|c|c|c|c|c|c|c|c|c|c|c|c|c|c|c|}
\hline RUN & Mode & Cal & $\begin{array}{l}\text { Engine } \\
\text { Speed } \\
\text { (rpm) }\end{array}$ & $\begin{array}{l}\text { MAP } \\
\text { (KPa) }\end{array}$ & Load & $\begin{array}{l}\text { Equiv } \\
\text { Ratio }\end{array}$ & $\begin{array}{l}\text { Ignition } \\
\text { Timing } \\
\text { (deg } \\
\text { ETDC) }\end{array}$ & $\begin{array}{c}\text { Degrees } \\
\text { Irom MBT }\end{array}$ & $\begin{array}{c}\text { BSTHC } \\
(g / k w-h)\end{array}$ & $\begin{array}{c}\text { BSCO } \\
(\theta / \mathbf{k} w h)\end{array}$ & $\begin{array}{l}\text { BSNOX } \\
\text { (OAWW-hr) }\end{array}$ & BTE (\$) & $\begin{array}{c}\text { Power } \\
\text { (Nw) }\end{array}$ & HC eft & CO eff & $\begin{array}{c}\text { Stack } \\
\text { Temp } \\
\text { (deg C) }\end{array}$ & $\left|\begin{array}{c}\text { B4 Cat } \\
\text { Temp } \\
(\operatorname{deg} C)\end{array}\right|$ & $\begin{array}{c}\text { AF Cat } \\
\text { Tenp } \\
(\operatorname{deg} C\end{array}$ \\
\hline 890 & $6^{\circ}$ & Before & 1248 & 270 & $100 \%$ & 0.653 & 150 & -4 & 6.08 & 1.09 & 3.07 & 38.5 & 181.2 & & & 919 & 914 & 956 \\
\hline 891 & $6^{\circ}$ & After & 1249 & 270 & $100 \%$ & 0.648 & 150 & -4 & 1.42 & 0.00 & 3.18 & 38.4 & 177.3 & $77 \%$ & $100 \%$ & 917 & 911 & 953 \\
\hline 894 & 2 & Before & $1 \overline{254}$ & 146 & $10 \%$ & 0.716 & 235 & 0 & 11.89 & 328 & 0.54 & 218 & 22.3 & & & 698 & 626 & 544 \\
\hline 895 & 2 & After & 1252 & 145 & $10 \%$ & 0.709 & 235 & 0 & 1178 & 000 & 0.72 & 218 & 221 & $1 \%$ & $100 \%$ & 724 & 651 & 586 \\
\hline 896 & 3 & Before & 1253 & 228 & $25 \%$ & 0.673 & 250 & 0 & 4.72 & 1.60 & 1.43 & 32.8 & 57.2 & & & 804 & 757 & 728 \\
\hline 897 & 3 & After & 1249 & 225 & $25 \%$ & 0.667 & 250 & 0 & 3.19 & 0.00 & 1.54 & 32.8 & 56.3 & $32 \%$ & $100 \%$ & 807 & 760 & 739 \\
\hline 898 & 4 & Before & 1253 & 264 & $50 \%$ & 0.656 & 205 & 0 & 3.03 & 1.25 & 3.14 & 37.6 & 111.6 & & & 862 & 828 & 824 \\
\hline 899 & 4 & After & 1253 & 263 & $50 \%$ & 0.653 & 205 & 0 & 0.92 & 0.00 & 3.23 & 379 & 111.1 & $70 \%$ & $101 \%$ & 847 & 830 & 829 \\
\hline 900 & 5 & Before & 1255 & 273 & $75 \%$ & 0.653 & 17.0 & -3 & 5.31 & 1.12 & 3.48 & 38.6 & 168.4 & & & 884 & 859 & 888 \\
\hline 901 & 5 & After & 1253 & 272 & $75 \%$ & 0.649 & 17.0 & -3 & 150 & 0.00 & 3.79 & 38.7 & 163.1 & $72 \%$ & $101 \%$ & 885 & 859 & $88 \overline{7}$ \\
\hline 902 & 6 & Before & 1253 & 266 & $100 \%$ & 0.653 & 150 & -4 & 6.55 & 1.12 & 240 & 37.6 & 184.5 & & & 896 & 873 & 908 \\
\hline 903 & 6 & After & 1253 & 266 & $100 \%$ & 0.647 & 150 & -4 & 1.44 & 0.00 & 2.61 & 37.9 & 183.8 & $78 \%$ & $101 \%$ & 901 & 878 & 918 \\
\hline 904 & 8 & Before & 1901 & 254 & $100 \%$ & 0.653 & 205 & -3 & 2.28 & 1.56 & 2.13 & 36.5 & 266.7 & & & 977 & 955 & 960 \\
\hline 905 & 8 & After & 1901 & 254 & $100 \%$ & 0.648 & 205 & -3 & 0.40 & 0.00 & 2.22 & 36.7 & 264.2 & $83 \%$ & $101 \%$ & 978 & 958 & 963 \\
\hline 906 & 9 & Before & 1900 & 245 & $75 \%$ & 0.655 & 210 & -3 & 3.47 & 1.67 & 1.47 & 35.0 & 199.5 & & & 989 & 964 & $\overrightarrow{978}$ \\
\hline 907 & 9 & After & 1899 & 245 & $75 \%$ & 0.651 & 210 & -3 & 0.32 & 0.00 & 1.77 & 355 & 200.2 & $91 \%$ & $101 \%$ & 986 & 964 & 978 \\
\hline $5 \sqrt{3}$ & 63 & Before & 1898 & 225 & $50 \%$ & 0.655 & 21.5 & -3 & 7.01 & 2.07 & 0.85 & 31.8 & 131.8 & & & 983 & 955 & 993 \\
\hline$\underline{909}$ & 10 & After & 1898 & 225 & $50 \%$ & 0.650 & 21.5 & -3 & 0.31 & 0.00 & 1.01 & 32.3 & 132.4 & $96 \%$ & $101 \%$ & 981 & 954 & 991 \\
\hline 910 & 11 & Before & 1895 & 192 & $25 \%$ & 0.674 & 24.0 & -3 & 9.95 & 260 & 0.75 & 26.6 & 68.4 & & & 931 & 886 & 935 \\
\hline 911 & 11 & After & 1894 & 188 & $25 \%$ & 0.669 & 240 & -3 & 1.20 & 0.00 & 0.93 & 26.2 & 66.1 & $88 \%$ & $100 \%$ & 925 & 886 & 35 \\
\hline 312 & 12 & Before & 1889 & 114 & $10 \%$ & 0.713 & 190 & -3 & 21.47 & 5.68 & 0.68 & 15.7 & 26.0 & & & 923 & 859 & 901 \\
\hline 913 & 12 & After & 1890 & 114 & $10 \%$ & 0.706 & 190 & -3 & 3.41 & 0.00 & 0.91 & 15.9 & 26.0 & $84 \%$ & $100 \%$ & 926 & 859 & 901 \\
\hline 914 & 1 & Beforo & 653 & 120 & & 0.875 & 170 & 0 & 16.40 & 290 & 0.62 & 214 & 11.8 & & & 581 & 510 & 476 \\
\hline$\underline{9} 9$ & 1 & After & 654 & 121 & & $0 \overline{833}$ & 170 & 0 & 13.91 & 0.00 & 0.71 & 226 & 119 & $15 \%$ & $101 \%$ & 579 & 509 & 457 \\
\hline
\end{tabular}

- Insulated exhaust pipe 


\begin{tabular}{|c|c|c|c|c|c|c|c|c|c|c|c|c|c|c|c|c|c|c|}
\hline RUN & Mode & Cal & $\begin{array}{l}\text { Engine } \\
\text { Speed } \\
\text { (rpm) }\end{array}$ & $\begin{array}{l}\text { MAP } \\
\text { (pola) }\end{array}$ & Load & $\begin{array}{l}\text { Equiv } \\
\text { Ratio }\end{array}$ & $\begin{array}{l}\text { Ignition } \\
\text { Timing } \\
\text { (deg } \\
\text { ETDC) }\end{array}$ & $\begin{array}{c}\text { Degrees } \\
\text { Irom MBT }\end{array}$ & $\begin{array}{c}\text { BSTHC } \\
\text { (o/hp-hr) }\end{array}$ & $\begin{array}{c}\text { BSCO } \\
(\mathrm{g} / \mathrm{h} p-\mathrm{hr})\end{array}$ & $\begin{array}{c}\text { BSNOx } \\
\text { (othp-hy) }\end{array}$ & BTE (\%) & $\begin{array}{l}\text { Power } \\
\text { (ohp) }\end{array}$ & HC eft & CO elf & $\begin{array}{c}\text { Stack } \\
\text { Temp } \\
(\operatorname{deg} F)\end{array}$ & $\begin{array}{c}\text { B4 Cat } \\
\text { Temp } \\
(\text { deg F) }\end{array}$ & $\begin{array}{c}\text { AF Cat } \\
\text { Tonp } \\
\text { (deg F })\end{array}$ \\
\hline 890 & $\sigma^{\circ}$ & Before & 1248 & 39.2 & $100 \%$ & 0.653 & 150 & -4 & 4.53 & 0.81 & 2.29 & 38.5 & 243.0 & & & 938 & 933 & 975 \\
\hline 891 & $6^{\circ}$ & After & 1249 & 392 & $100 \%$ & $0 . \overline{648}$ & 150 & -4 & 1.06 & 0.00 & 2.37 & 38.4 & 237.8 & $77 \%$ & $100 \%$ & 936 & 930 & 972 \\
\hline 894 & 2 & Before & 1254 & 21.2 & $10 \%$ & 0.716 & 235 & 0 & 8.87 & 244 & 0.41 & 21.8 & 29.9 & & & 717 & 645 & 563 \\
\hline 895 & 2 & After & 1252 & 21 & $10 \%$ & 0.709 & 235 & 0 & 8.78 & 0.00 & 0.54 & 21.8 & 29.6 & $1 \%$ & $100 \%$ & 743 & 670 & 605 \\
\hline 896 & 3 & Before & 1253 & 33.1 & $25 \%$ & 0.673 & 250 & 0 & 3.52 & 1.19 & 1.07 & 32.8 & 76.7 & & & 823 & 776 & 747 \\
\hline 897 & 3 & After & 1249 & 327 & $25 \%$ & 0.667 & 250 & 0 & 238 & 0.00 & 1.15 & 328 & 75.5 & $32 \%$ & $100 \%$ & 826 & 779 & 758 \\
\hline 898 & 4 & Before & 1253 & 38.3 & $50 \%$ & 0.656 & 205 & 0 & 2.26 & 0.93 & 234 & 37.6 & 149.6 & & & 881 & 847 & 843 \\
\hline 899 & 4 & After & 1253 & 38.2 & $50 \%$ & 0.653 & 205 & 0 & 0.69 & 0.00 & 241 & 37.9 & 149.0 & $70 \%$ & $101 \%$ & 866 & 849 & 848 \\
\hline 900 & 5 & Before & 1255 & 39.6 & $75 \%$ & 0.653 & 170 & -3 & 3.96 & 0.83 & 260 & 38.6 & 225.8 & & & 903 & 878 & 907 \\
\hline 901 & 5 & After & 1253 & 39.5 & $75 \%$ & 0.649 & 17.0 & -3 & 1.12 & 0.00 & 2.83 & 38.7 & 218.7 & $72 \%$ & $101 \%$ & 904 & 878 & 906 \\
\hline 902 & 6 & Before & 1253 & 38.6 & $100 \%$ & 0.653 & 150 & -4 & 4.89 & 0.63 & 1.79 & 37.6 & 247.4 & & & 915 & 892 & 927 \\
\hline 903 & 6 & After & 1253 & 38.6 & $100 \%$ & 0.647 & 150 & -4 & 1.07 & 0.00 & 1.94 & 37.9 & 246.5 & $78 \%$ & $101 \%$ & 920 & 897 & 937 \\
\hline 904 & 8 & Before & 1901 & 36.9 & $100 \%$ & 0.653 & 205 & -3 & 1.70 & 1.16 & 1.59 & 36.5 & 357.6 & & & 996 & 974 & 979 \\
\hline 905 & 8 & After & 1901 & 36.8 & $100 \%$ & 0.648 & 205 & -3 & 0.30 & 0.00 & 1.66 & 36.7 & 354.3 & $83 \%$ & $101 \%$ & 997 & 977 & 982 \\
\hline 906 & 9 & Before & 1900 & 35.5 & $75 \%$ & 0.655 & 210 & -3 & 2.59 & 1.25 & 1.09 & 35.0 & 267.6 & & & 1008 & 983 & 997 \\
\hline 907 & 9 & After & 1899 & 35.6 & $75 \%$ & 0.651 & 210 & -3 & 0.24 & 0.00 & 1.32 & 35.5 & 268.5 & $91 \%$ & $101 \%$ & 1005 & 983 & 997 \\
\hline 908 & 10 & Before & 1898 & 32.6 & $50 \%$ & 0.655 & 21.5 & -3 & 5.23 & 1.54 & 0.63 & 31.8 & 176.8 & & & 1002 & 974 & 1012 \\
\hline 909 & 10 & After & 1898 & 32.7 & $50 \%$ & 0.650 & 21.5 & -3 & 0.23 & 000 & 0.75 & 32.3 & 177.5 & $96 \%$ & $101 \%$ & 1000 & 973 & 1010 \\
\hline 910 & 11 & \begin{tabular}{|l|} 
Before \\
\end{tabular} & 1895 & 27.8 & $25 \%$ & 0.674 & 24.0 & -3 & 7.42 & 194 & 0.56 & 26.6 & 91.7 & & & 950 & 905 & 954 \\
\hline 911. & 11 & After & 1894 & 27.3 & $25 \%$ & 0.669 & 24.0 & -3 & 0.89 & 0.00 & 0.69 & 26.2 & 88.6 & $88 \%$ & $100 \%$ & 944 & 905 & 54 \\
\hline 912 & 12 & Before & 1889 & 16.5 & $10 \%$ & 0.713 & 190 & -3 & 16.01 & $4 . \overline{23}$ & 0.51 & 15.7 & 34.8 & & & 942 & 878 & 920 \\
\hline 913 & 12 & After & 1890 & 16.6 & $10 \%$ & 0.706 & 19.0 & -3 & 2.54 & 0.00 & 0.68 & 15.9 & 34.9 & $84 \%$ & $100 \%$ & 945 & $87 \overline{8}$ & 920 \\
\hline 914 & 1 & Before & 653 & 17.4 & & 0.875 & 170 & 0 & 12.23 & 2.16 & $0 . \overline{46}$ & 21.4 & 15.8 & & & 600 & 529 & 495 \\
\hline 915 & 1 & After & 654 & 17.5 & & 0.833 & 170 & 0 & 10.37 & 0.00 & 0.53 & 22.6 & 15.9 & $15 \%$ & $101 \%$ & 598 & 528 & 476 \\
\hline
\end{tabular}

- Insulated exhaust pipe 


\begin{tabular}{|c|c|c|c|c|c|c|c|c|c|c|c|c|c|c|c|c|c|c|}
\hline RUN & Mode & Cat & $\begin{array}{l}\text { Engine } \\
\text { Speed } \\
\text { (rpm) }\end{array}$ & $\begin{array}{c}\text { MAP } \\
\text { (psia) }\end{array}$ & Load & $\begin{array}{l}\text { Equiv } \\
\text { Ratio }\end{array}$ & $\begin{array}{l}\text { Ignition } \\
\text { Timing } \\
\text { (deg } \\
\text { BTDC) }\end{array}$ & $\begin{array}{l}\text { Degrees } \\
\text { from MBT }\end{array}$ & $\begin{array}{c}\text { BSTHC } \\
\text { (g/bhp-hr) }\end{array}$ & $\begin{array}{c}\text { BSCO } \\
\text { (g/ohp-hr) }\end{array}$ & $\begin{array}{c}\text { BSNOx } \\
\text { (g/bhp-hr) }\end{array}$ & BTE $(\%)$ & $\begin{array}{l}\text { Power } \\
\text { (bhp) }\end{array}$ & $\mathrm{HC}$ eff & Co eff & $\begin{array}{c}\text { Stack } \\
\text { Temp } \\
\text { (deg F) }\end{array}$ & $\begin{array}{c}\text { B4 Cat } \\
\text { Temp } \\
(\operatorname{deg} F)\end{array}$ & $\begin{array}{c}\text { AF Cat } \\
\text { Tenp } \\
(\operatorname{deg} F)\end{array}$ \\
\hline 3269 & $6^{*}$ & Before & 1245 & 35.8 & $100 \%$ & 0.654 & 150 & -4 & 4.10 & 0.97 & 1.00 & 37.0 & 238.0 & & & 961 & 954 & 975 \\
\hline 3270 & $6^{*}$ & After & 1245 & 35.6 & $100 \%$ & 0.648 & 150 & -4 & 1.53 & 0.00 & 1.27 & 37.1 & 234.0 & $63 \%$ & $100 \%$ & $\overline{961}$ & 953 & 972 \\
\hline 3271 & 2 & Before & 1245 & 15.4 & $10 \%$ & 0706 & 235 & 0 & 14.75 & 3.27 & 0.25 & 21.1 & 29.7 & & & 786 & 722 & 698 \\
\hline 3272 & 2 & After & 1245 & 15.5 & $10 \%$ & 0.704 & 235 & 0 & 13.55 & -0.01 & 0.40 & 21.3 & 30.0 & $8 \%$ & $100 \%$ & 777 & 711 & 678 \\
\hline 3273 & 3 & Before & 1246 & 27.6 & $25 \%$ & 0.668 & 250 & 0 & 4.36 & 1.59 & 048 & 32.2 & 760 & & & 828 & 780 & 750 \\
\hline 3274 & 3 & After & 1247 & 27.7 & $25 \%$ & 0.666 & 250 & 0 & 3.75 & -0.01 & 0.67 & 32.4 & 76.1 & $14 \%$ & $100 \%$ & 831 & 785 & 760 \\
\hline 3275 & 4 & Before & 1248 & 33.7 & $50 \%$ & 0.649 & 205 & 0 & 2.12 & 1.10 & 1.22 & 37.3 & 149.8 & & & 889 & 854 & 834 \\
\hline 3276 & 4 & After & 1248 & 33.7 & $50 \%$ & 0.645 & 205 & 0 & 1.60 & -0.01 & 1.32 & 37.3 & 149.4 & $25 \%$ & $100 \%$ & 894 & 859 & 842 \\
\hline 3277 & 5 & Before & 1248 & 35.9 & $75 \%$ & 0.646 & 170 & -3 & 3.96 & 0.99 & 121 & 37.5 & 220.3 & & & 919 & 892 & 888 \\
\hline 3278 & 5 & After & 1248 & 35.8 & $75 \%$ & 0.643 & 170 & -3 & 3.01 & -0.01 & 1.40 & 37.6 & 219.9 & $24 \%$ & $101 \%$ & 920 & 893 & 890 \\
\hline 3279 & 6 & Before & 1248 & 35.2 & $100 \%$ & 0.647 & 15.0 & -4 & 4.50 & 1.00 & 0.90 & 36.7 & 2290 & & & 937 & 911 & 908 \\
\hline 3280 & 6 & After & 1247 & 35.3 & $100 \%$ & 0.644 & 150 & -4 & 3.28 & 000 & 1.17 & 369 & 229.6 & $27 \%$ & $100 \%$ & 937 & 910 & 908 \\
\hline 3281 & 8 & Before & 1896 & 31.9 & $100 \%$ & 0.643 & 20.5 & -3 & 8.22 & 1.95 & 0.58 & 32.7 & 326.4 & & & 991 & 967 & 987 \\
\hline 3282 & 8 & After & 1892 & 32.2 & $100 \%$ & 0.637 & 20.5 & -3 & 5.53 & 0.00 & 0.73 & 33.3 & 330.9 & $33 \%$ & $100 \%$ & 991 & 969 & 986 \\
\hline 3283 & 9 & Before & 1889 & 31.8 & $75 \%$ & 0.647 & 210 & -3 & 6.11 & 1.74 & 0.62 & 33.0 & 265.8 & & & 1012 & 986 & 999 \\
\hline 3284 & 9 & After & 1888 & 31.8 & $75 \%$ & 0.641 & 21.0 & -3 & 4.38 & -0.01 & 072 & 33.3 & 264.7 & $28 \%$ & $100 \%$ & 1008 & 983 & $\overline{994}$ \\
\hline 3285 & 10 & Before & 1884 & 28.6 & $50 \%$ & 0.646 & 21.5 & -3 & 11.47 & 2.25 & 0.38 & 30.0 & 177.1 & & & 1000 & 974 & 1003 \\
\hline 3286 & 10 & After & 1885 & 28.9 & $50 \%$ & 0.639 & 21.5 & -3 & 6.43 & -0.01 & 0.47 & 30.7 & 179.9 & $44 \%$ & $100 \%$ & 1000 & 974 & 999 \\
\hline 3287 & 11 & Before & 1896 & 23.2 & $25 \%$ & 0.667 & 240 & -3 & 12.13 & 2.65 & 0.39 & 25.3 & 90.3 & & & 952 & 910 & 920 \\
\hline 3288 & 11 & After & 1895 & 23.2 & $25 \%$ & 0.662 & 240 & -3 & 9.20 & -0.01 & 0.52 & 25.5 & 90.6 & $24 \%$ & $100 \%$ & 944 & 904 & 907 \\
\hline 3289 & 12 & Before & 1891 & 13.1 & $10 \%$ & 0.700 & 19.0 & -3 & 26.80 & 5.32 & 0.41 & 14.9 & 34.2 & & & 944 & 884 & 872 \\
\hline 3290 & 12 & After & 1892 & 13 & $10 \%$ & 0.696 & 190 & -3 & 20.50 & -0.02 & 0.55 & 15.1 & 34.4 & $24 \%$ & $100 \%$ & 935 & 870 & 855 \\
\hline 3291 & 1 & Before & 654 & 12.5 & & 0.873 & 17.0 & 0 & 13.64 & 2.68 & 0.30 & 209 & 16.0 & & & 644 & 595 & \\
\hline 3292 & 1 & After & 649 & 124 & & 0.869 & 170 & 0 & 13.47 & -0.01 & 0.48 & 21.1 & 15.9 & $1 \%$ & $101 \%$ & 634 & 568 & 563 \\
\hline
\end{tabular}

- Insulated exhaust pipe 


\begin{tabular}{|c|c|c|c|c|c|c|c|c|c|c|c|c|c|c|c|c|c|c|}
\hline RUN & Mode & Before & $\begin{array}{c}\text { Engine } \\
\text { Speed } \\
(\text { rpm) }\end{array}$ & $\begin{array}{l}\text { MAP } \\
\text { (KPa) }\end{array}$ & Load & $\begin{array}{l}\text { Equiv } \\
\text { Ratio }\end{array}$ & $\begin{array}{l}\text { Ignition } \\
\text { Timing } \\
\text { (deg } \\
\text { BTDC) }\end{array}$ & $\begin{array}{c}\text { Degrees } \\
\text { from MBT }\end{array}$ & $\begin{array}{c}\text { BSTHC } \\
(g / k w-h r)\end{array}$ & $\begin{array}{c}\text { BSCO } \\
(g / k w-h r)\end{array}$ & $\begin{array}{c}\text { BSNOx } \\
(\mathbf{g} / \mathbf{k} w-\mathbf{h r})\end{array}$ & BTE (\%) & $\begin{array}{c}\text { Power } \\
\text { (kw) }\end{array}$ & $\mathrm{HC}$ eff & $\mathrm{CO}$ eft & $\begin{array}{c}\text { Slack } \\
\text { Temp } \\
(\operatorname{deg} C)\end{array}$ & $\left|\begin{array}{c}\text { B4 Cat } \\
\text { Temp } \\
\text { (deg C) }\end{array}\right|$ & $\left|\begin{array}{c}\text { AF Cat } \\
\text { Tenp } \\
(\text { deg } C\end{array}\right|$ \\
\hline 3270 & $\frac{6^{*}}{6^{*}}$ & $\frac{\text { Before }}{\text { After }}$ & $\frac{1245}{1245}$ & $\frac{247}{245}$ & $100 \%$ & \begin{tabular}{|l|}
0.654 \\
\end{tabular} & 150 & -4 & 5.49 & 1.30 & 1.35 & 37.0 & 177.5 & & & 942 & 935 & 956 \\
\hline 3271 & 2 & $\frac{\text { After }}{\text { Before }}$ & $\frac{1245}{1245}$ & $\frac{245}{106}$ & $100 \%$ & 0.648 & 150 & -4 & 2.06 & 0.00 & 1.70 & 37.1 & 174.5 & $63 \%$ & $100 \%$ & 942 & 934 & $\overline{953}$ \\
\hline 3272 & 2 & After & $\frac{1245}{1245}$ & $\frac{106}{107}$ & $10 \%$ & 0706 & 235 & 0 & 19.78 & 4.38 & 0.34 & 21.1 & 22.1 & & & 767 & 703 & $\overline{679}$ \\
\hline 3273 & 3 & Before & $\frac{1245}{1246}$ & $\frac{107}{190}$ & $10 \%$ & 0.704 & 235 & 0 & 18.18 & -0.01 & 0.54 & 21.3 & 22.4 & $8 \%$ & $100 \%$ & 758 & 692 & 659 \\
\hline 3274 & 3 & After & 1247 & $\frac{190}{191}$ & $25 \%$ & 0.668 & 250 & 0 & 5.85 & 2.13 & 064 & 32.2 & 56.7 & & & 809 & 761 & 731 \\
\hline 3275 & 4 & Before & 1248 & $\frac{191}{232}$ & $25 \%$ & 0.666 & 250 & 0 & 5.03 & -0.01 & 0.90 & 324 & 567 & $14 \%$ & $100 \%$ & 812 & 766 & 741 \\
\hline 3276 & 4 & After & 1248 & $\frac{232}{232}$ & $50 \%$ & 0.649 & 205 & 0 & 285 & 1.48 & 1.63 & 37.3 & 111.7 & & & 870 & 835 & 815 \\
\hline 3277 & 5 & Before & 1248 & $\frac{232}{248}$ & $50 \%$ & 0.645 & 205 & 0 & 2.14 & -0.01 & 177 & 37.3 & 111.4 & $25 \%$ & $100 \%$ & 875 & 840 & 823 \\
\hline 3278 & 5 & After & 1248 & $\frac{248}{247}$ & $75 \%$ & 0.646 & 170 & -3 & 5.31 & 1.33 & 163 & 37.5 & 164.3 & & & 900 & 873 & 869 \\
\hline 3279 & 6 & Before & 1248 & $\frac{247}{243}$ & \begin{tabular}{|c|}
$75 \%$ \\
$100 \%$
\end{tabular} & 0.643 & 170 & -3 & 4.04 & -0.01 & 1.87 & 37.6 & 164.0 & $24 \%$ & $101 \%$ & 901 & $\overline{874}$ & 871 \\
\hline 3280 & 6 & After & 1247 & $\frac{243}{243}$ & $\frac{100 \%}{100 \%}$ & \begin{tabular}{|l|}
0.647 \\
\end{tabular} & 150 & -4 & 6.03 & 1.34 & 1.21 & 36.7 & 170.8 & & & 918 & 892 & 889 \\
\hline 3281 & 8 & Before & 1896 & $\frac{243}{220}$ & \begin{tabular}{|l|}
$100 \%$ \\
$100 \%$
\end{tabular} & 0.644 & 15.0 & -4 & 440 & -0.01 & 1.57 & 36.9 & 171.2 & $27 \%$ & $100 \%$ & 918 & 891 & 889 \\
\hline 3282 & 8 & After & 1892 & $\frac{220}{222}$ & $\frac{100 \%}{100 \%}$ & 0.643 & 205 & -3 & 11.03 & 261 & 0.78 & 32.7 & 243.4 & & & 972 & 948 & 968 \\
\hline 3283 & 9 & Before & 1889 & 219 & $\frac{100 \%}{75 \%}$ & 0637 & 205 & -3 & 7.41 & 0.00 & 0.98 & 33.3 & 246.8 & $33 \%$ & $100 \%$ & 972 & 950 & 967 \\
\hline 3284 & 9 & After & 1888 & 219 & $\frac{75 \%}{75 \%}$ & 0.647 & 21.0 & -3 & 8.19 & 2.33 & 0.83 & 33.0 & 198.2 & & & 993 & 967 & 980 \\
\hline 3285 & 10 & Before & 1884 & 197 & $\frac{75 \%}{50 \%}$ & 0.641 & 21.0 & -3 & 5.87 & -0.01 & 0.96 & 33.3 & 197.4 & $28 \%$ & $100 \%$ & 989 & 964 & 975 \\
\hline 3286 & 10 & After & 1885 & 199 & $\frac{50 \%}{50 \%}$ & 0.646 & 21.5 & -3 & 15.38 & 3.01 & 0.51 & 30.0 & 132.1 & & & 981 & 955 & 984 \\
\hline 3287 & 11 & Before & 1896 & $\frac{159}{160}$ & $50 \%$ & 0.639 & 21.5 & -3 & 8.62 & -0.01 & 0.64 & 30.7 & 134.2 & $44 \%$ & $100 \%$ & 981 & 955 & 980 \\
\hline 3288 & 11 & After & 1895 & $\frac{160}{160}$ & $\frac{25 \%}{25 \%}$ & 0.667 & 24.0 & -3 & 16.27 & 3.56 & 0.52 & 25.3 & 67.3 & & & 933 & 891 & 901 \\
\hline 3289 & 12 & Before & 1891 & 90 & $\frac{25 \%}{10 \%}$ & 0.662 & 24.0 & -3 & 12.34 & -0.02 & 0.70 & 25.5 & 67.6 & $24 \%$ & $100 \%$ & 925 & 885 & 888 \\
\hline 3290 & 12 & After & 1892 & $\frac{50}{90}$ & $\frac{10 \%}{10 \%}$ & 0.700 & 19.0 & -3 & 35.94 & 7.14 & 0.55 & 14.9 & 25.5 & & & 925 & 865 & 853 \\
\hline 3291 & 1 & Before & 654 & 86 & $10 \%$ & 0.696 & 19.0 & -3 & 27.49 & -0.03 & 0.74 & 15.1 & 25.7 & $24 \%$ & $100 \%$ & 916 & 851 & 836 \\
\hline 3292 & 1 & After & 649 & $\frac{86}{85}$ & $\frac{0}{0}$ & 0.873 & 17.0 & 0 & 18.29 & 3.59 & 0.40 & 20.9 & 11.9 & & & 625 & 576 & -19 \\
\hline & & & & & 0 & 0.869 & 17.0 & 0 & 18.06 & -0.02 & 0.64 & 21.1 & 11.9 & $1 \%$ & $101 \%$ & 615 & 549 & 544 \\
\hline
\end{tabular}


APPENDIX G

LOW SPEED DATA FOR EMISSIONS TEST AT 500 HOURS ON THE STC TEST 


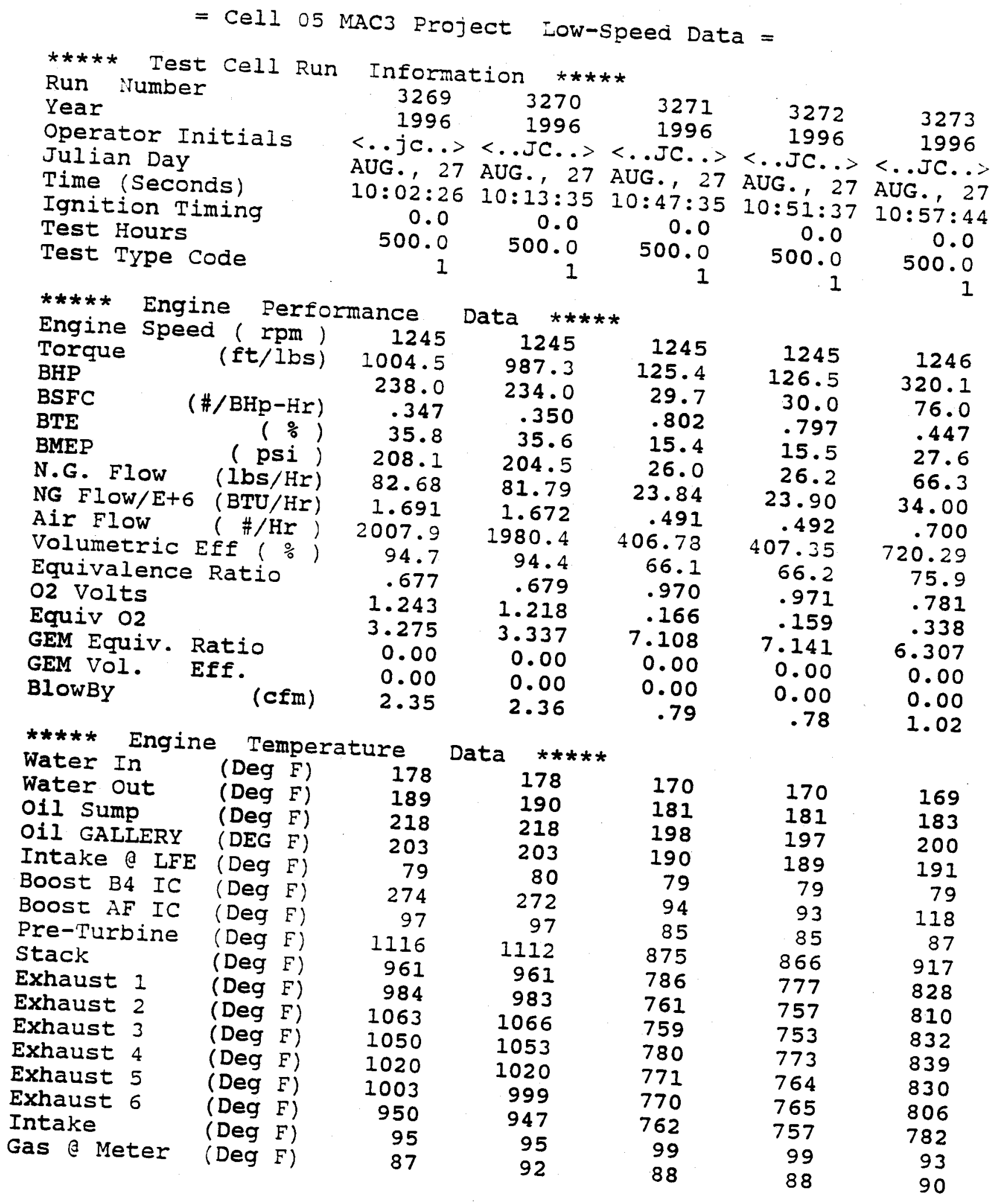


Cell 05 MAC3 Project Low-speed Data =
(continued)

Run Number

3269

3270

3271

3272

3273

$* * * * *$ Engine

Main Oil Pressures

Fuel Pressure (psig)

44.7

Data $* * * * *$

Compress out (psig)

98.5

44.4

98.6

54.1

109.1

.16

Inlet Manifold(psig)

17.10

16.79

15.99

30.61

30.32

Pre-Turbine

(psig)

10.72

10.55

2.56

.91

LFE Filter Pr ("H2O)

2.59

LFE Delta Pr ("H2O)

.93

3.87

$\mathrm{H}$

(IbH2O/IbAir)

.020

3.83

.020

$\mathrm{RH}$ (out)

Barometer

$\left(\begin{array}{c}\% \\ (" \mathrm{Hg})\end{array}\right.$

87.46

29.18

83.03

29.18

$-5.40$

8.94

.84

.27

.15

.76

.020

83.09

29.18

Natural Gas Composition

NG \% Methane
NG \% Ethane
NG \% Propane
NG $\%$ CO2

NG \% Nitrogen

Butane Actual (\%)

MWT

Fuel LHV
95.01

2.47

.42

.77

.20

17.00

20447 .
1.13

95.01
2.47
.42
1.13
.77
.20

17.00 20447.
95.43
2.62
.25
.98
.59
.12

16.88 20595 .
54.3
108.4
.16
-5.39
8.94
.84
.26
.15
.76
.020
81.71
29.18

95.43

2.62

.25

.98

.59

.12

16.88

20595 .
51.6 108.7

2.29

$-.76$

13.57

2.00 .46 .27

1.36

.020

83.05

95.43

2.62

.25

.98

.59

.12

16.88

20595.
29.18 
Cell 05 MAC2 Project Low-Speed Data =

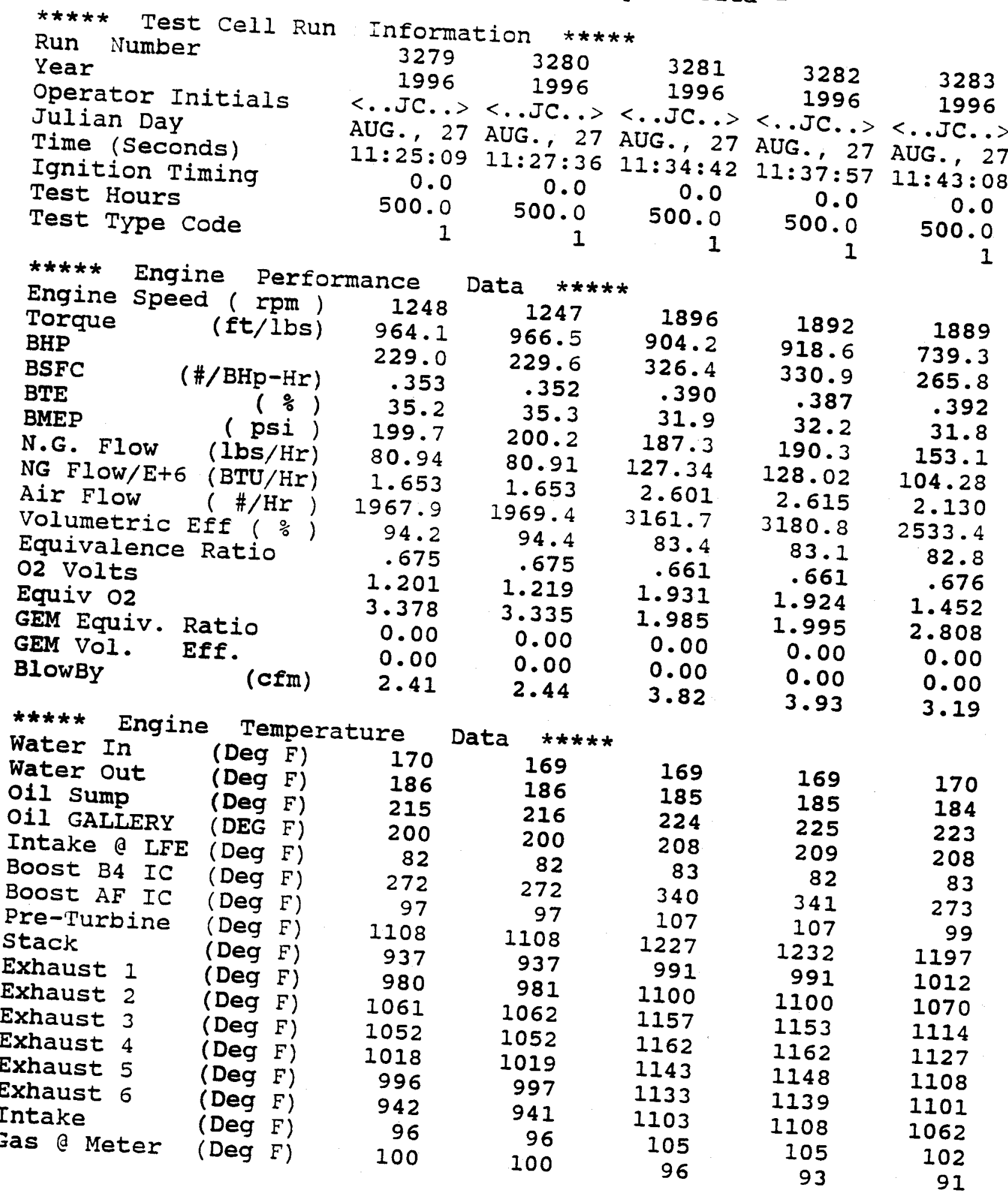




$$
\begin{gathered}
\text { Cell } 05 \text { MaC3 Project Jow-speed Data = } \\
\text { (continued) }
\end{gathered}
$$

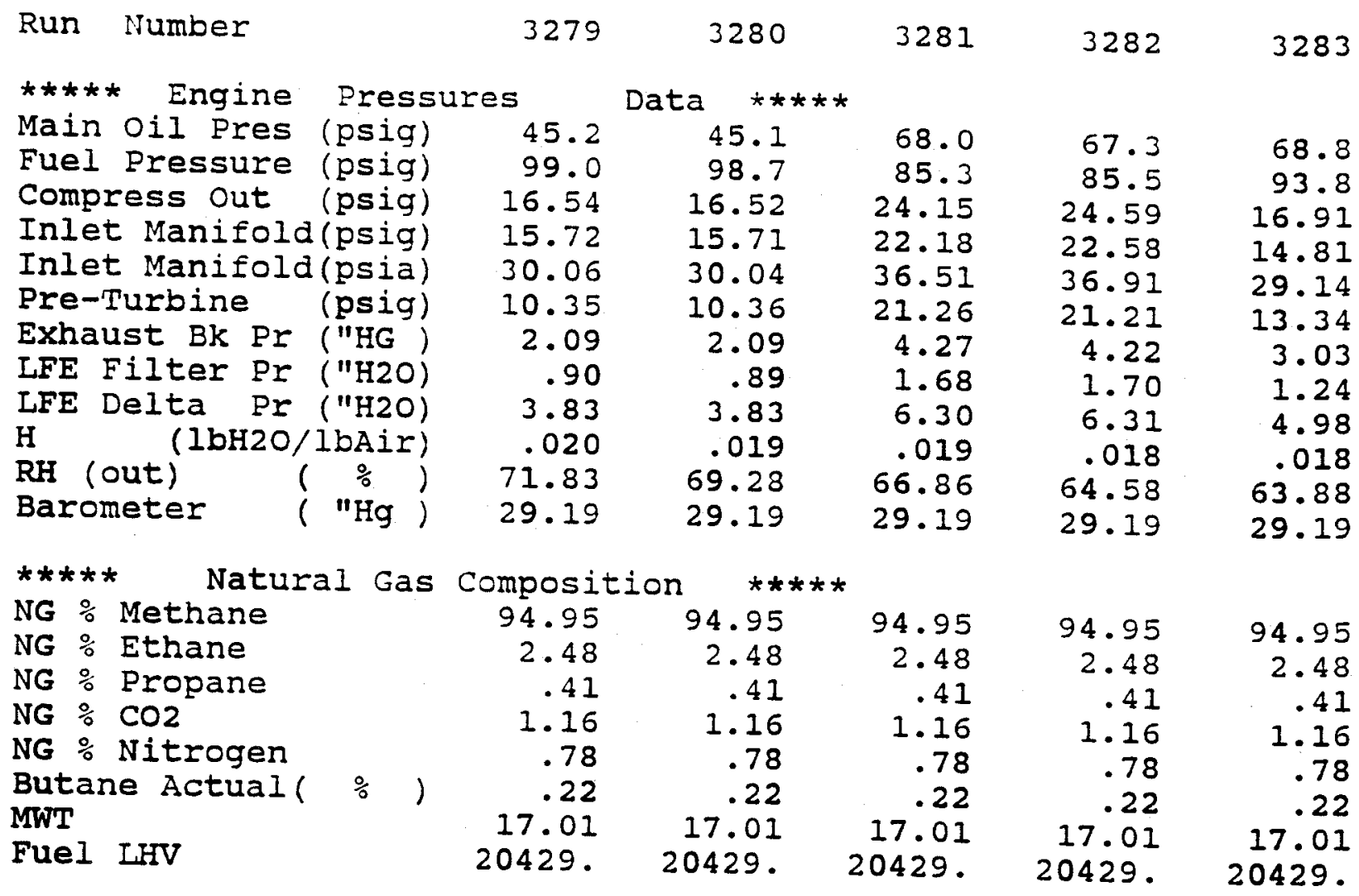




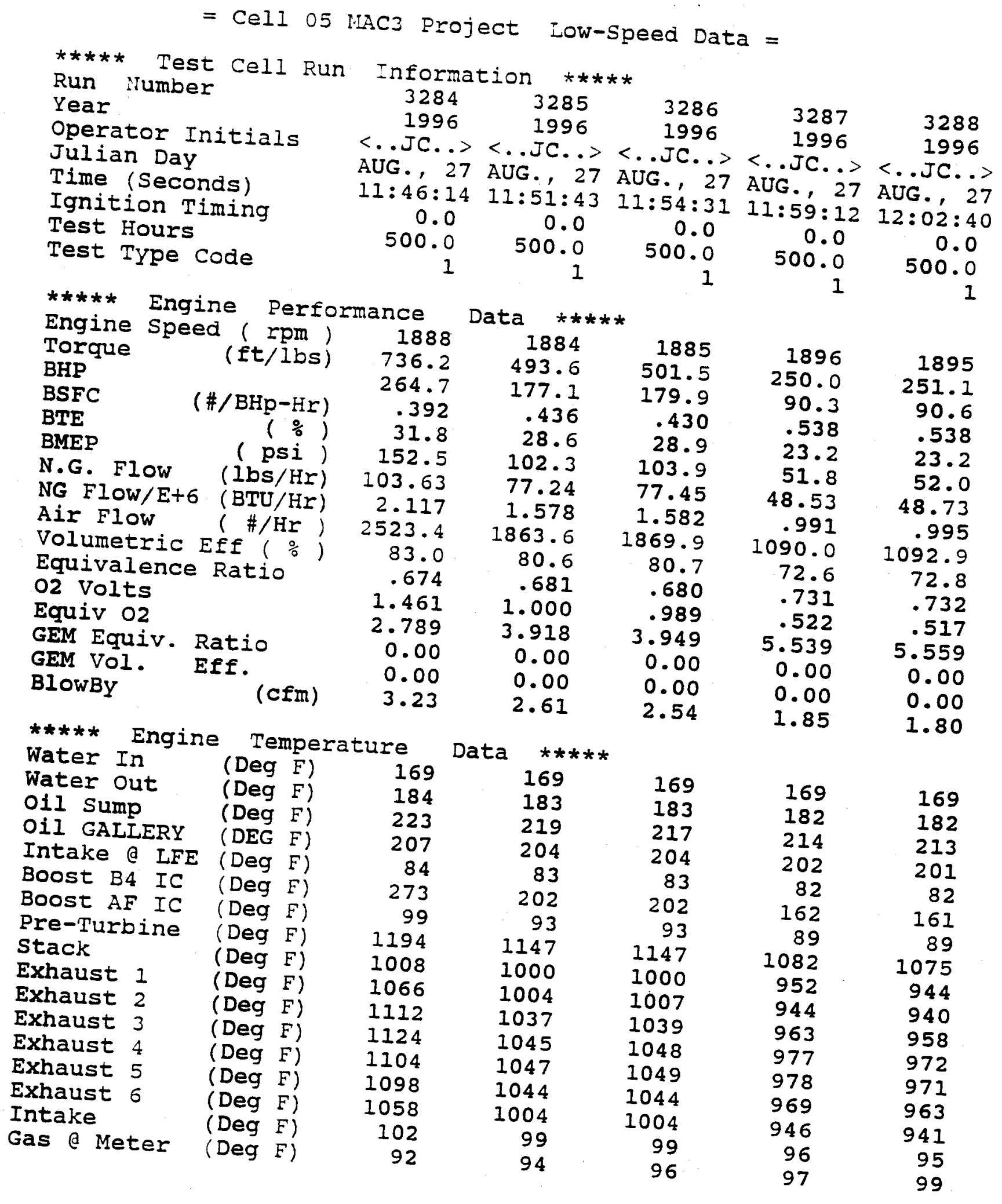



Cell 05 MAC3 Project Low-Speed Data =
(continued)

$$
3284
$$

$\star \star \star \star \star$
Main Oil Pres
Fuel Pressure Pressures

(psig)

(psig)

(psig)

Inlet Manifold(psig)

Inlet Manifold(psia)

Pre-Turbine

(psig)

Exhaust Bk Pr ("HG)

LFE Filter Pr ("H2O)

LFE Delta Pr ("H2O)

$\mathrm{H}$

$\mathrm{RH}$ (out)

Barometer

92.6

16.75

14.68

29.02

13.11

3.01

1.24

4.98

.018

64.46

29.19
3285

3286

3287

3288

Data $* * * * *$

69.071 .1

73.0

105.6

5.50

$-.28$

14.05

3.81

1.15

.43

2.09

.018

63.61
73.2

104.4

5.54

$-.26$

14.07

3.83

1.15

.43

2.09

.018

65.21

29.18
101.2

9.36

7.52

21.86

7.20

1.92

.82

3.62

.018

64.23

29.19
71.4

99.8

9.40

7.56

21.89

7.25

1.92

.82

3.63

.017

62.36

29.19
29.18

\section{***** Natural Gas Composition}

NG \% Methane

NG \% Ethane

NG \% Propane

NG \% $\mathrm{CO} 2$

NG \% Nitrogen

Butane Actual( \%)

MWT

Fuel LHV
94.95

2.48

.41

1.16

.78

.22

17.01

20429 .

94.95
2.48
.41
1.16
.78
.22

17.01

20429 . $\begin{array}{rr}94.95 & 94.95 \\ 2.48 & 2.48 \\ .41 & .41 \\ 1.16 & 1.16 \\ .78 & .78 \\ .22 & .22\end{array}$

$17.01 \quad 17.01$ 20429 .
20429 . 
= Cel1 05 MAC3 Project Low-Speed Data =

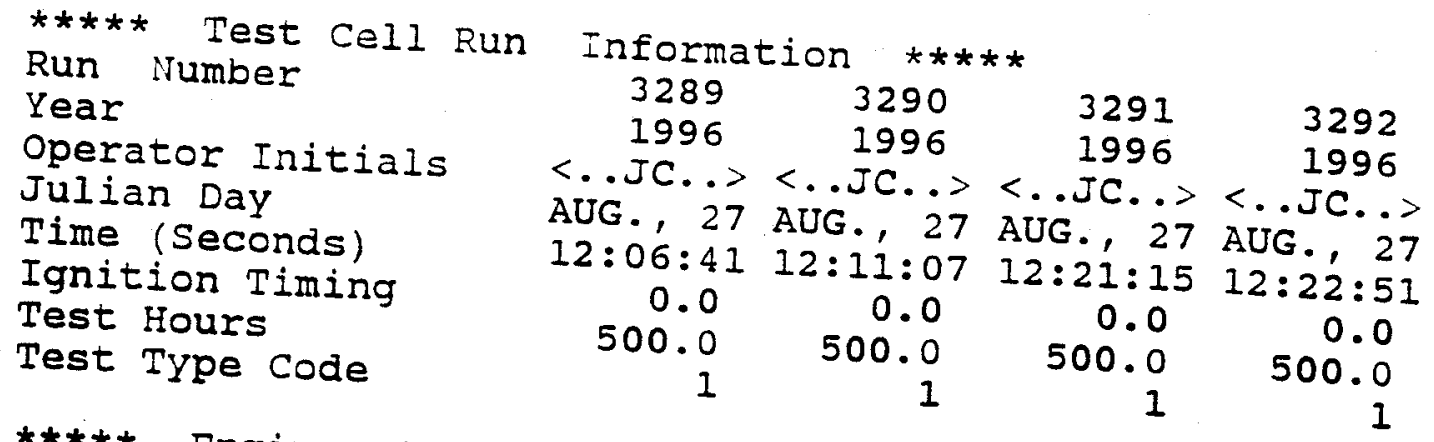

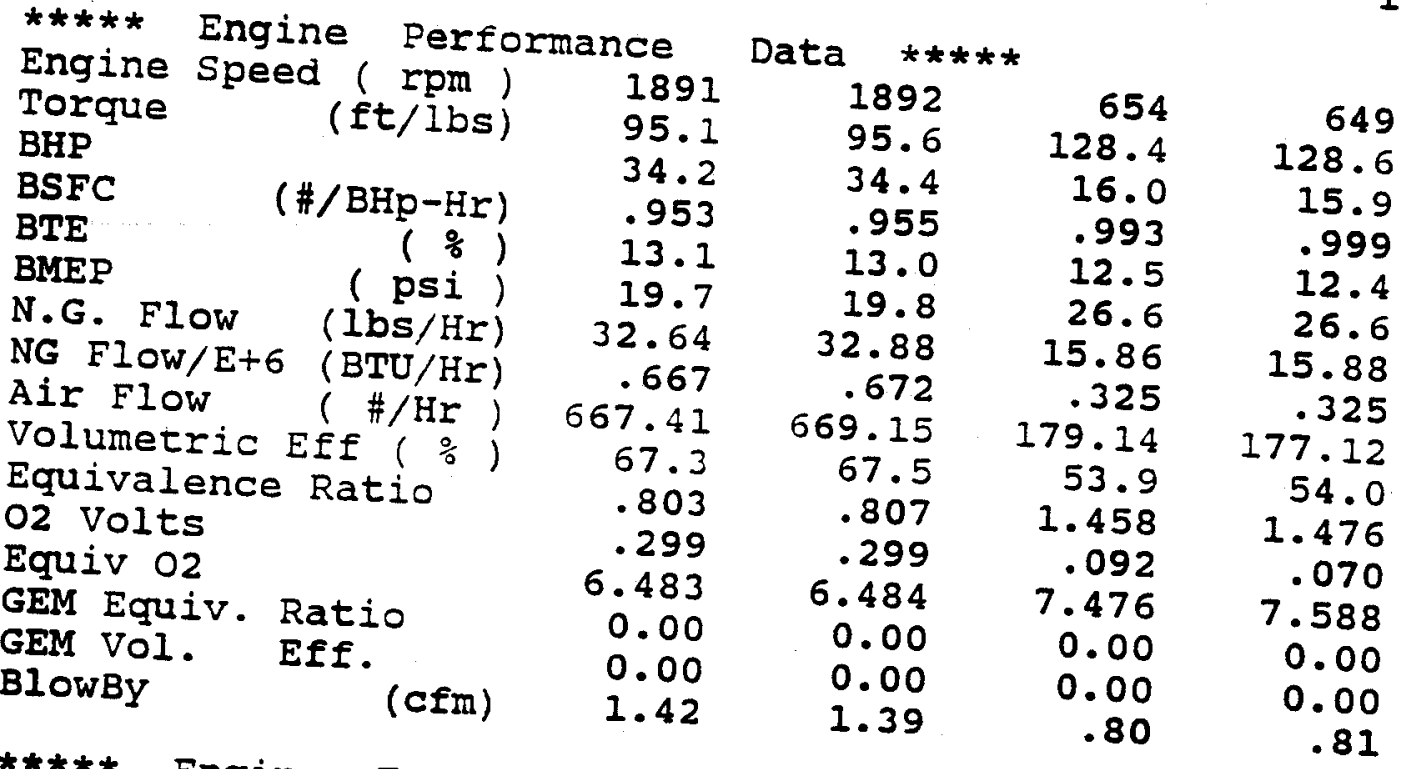

$\begin{array}{llrrrr}* \star * * * \text { Engine } & \text { Temperature } & \text { Data } * * * * * & & \\ \text { Water In } & \text { (Deg F) } & 170 & 169 & 160 & \\ \text { Water Out } & \text { (Deg F) } & 181 & 180 & 180 & 161 \\ \text { Oil Sump } & \text { (Deg F) } & 210 & 208 & 195 & 180 \\ \text { Oil GALIERY } & \text { (DEG F) } & 199 & 198 & 185 & 194 \\ \text { Intake @ LFE } & \text { (Deg F) } & 83 & 83 & 84 & 185 \\ \text { Boost B4 IC } & \text { (Deg F) } & 106 & 104 & 101 & 100 \\ \text { Boost AF IC } & \text { (Deg F) } & 88 & 87 & 85 & 84 \\ \text { Pre-Turbine } & \text { (Deg F) } & 1052 & 1042 & 759 & 751 \\ \text { Stack } & \text { (Deg F) } & 944 & 935 & 644 & 634 \\ \text { Exhaust 1 } & \text { (Deg F) } & 907 & 900 & 596 & 591 \\ \text { Exhaust 2 } & \text { (Deg F) } & 911 & 904 & 619 & 613 \\ \text { Exhaust 3 } & \text { (Deg F) } & 934 & 929 & 667 & 662 \\ \text { Exhaust 4 } & \text { (Deg F) } & 944 & 936 & 645 & 640 \\ \text { Exhaust 5 } & \text { (Deg F) } & 941 & 933 & 648 & 641 \\ \text { Exhaust 6 } & \text { (Deg F) } & 928 & 922 & 633 & 630 \\ \text { Intake } & \text { (Deg F) } & 98 & 98 & 123 & 124 \\ \text { Gas E Meter } & \text { (Deg F) } & 99 & 99 & 97 & 97\end{array}$


= Cell 05 MAC3 Project Low-Speed Data = (continued)

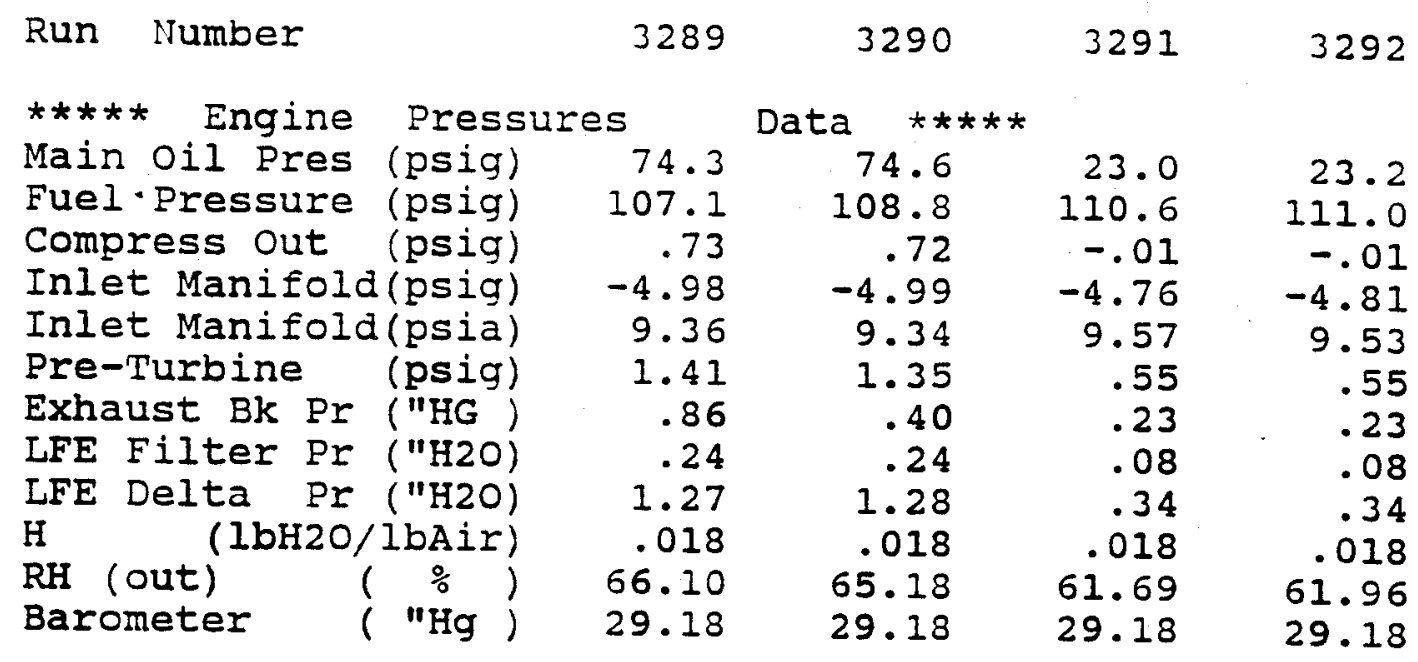

$\begin{array}{lrrrr}\star * * * * \text { Natural Gas Composition } * * * * * & \\ \text { NG \% Methane } & 94.95 & 94.95 & 95.21 & 95.21 \\ \text { NG \% Ethane } & 2.48 & 2.48 & 2.49 & 2.49 \\ \text { NG \% Propane } & .41 & .41 & .33 & .33 \\ \text { NG \% CO2 } & 1.16 & 1.16 & 1.14 & 1.14 \\ \text { NG \% Nitrogen } & .78 & .78 & .66 & .66 \\ \text { Butane Actual( } \% \text { ) } & .22 & .22 & .17 & .17 \\ \text { MWT } & 17.01 & 17.01 & 16.96 & 16.96 \\ \text { Fuel LHV } & 20429 . & 20429 . & 20482 . & 20482 .\end{array}$


= Cell 05 ilAC3 Project Low-Speed Data =

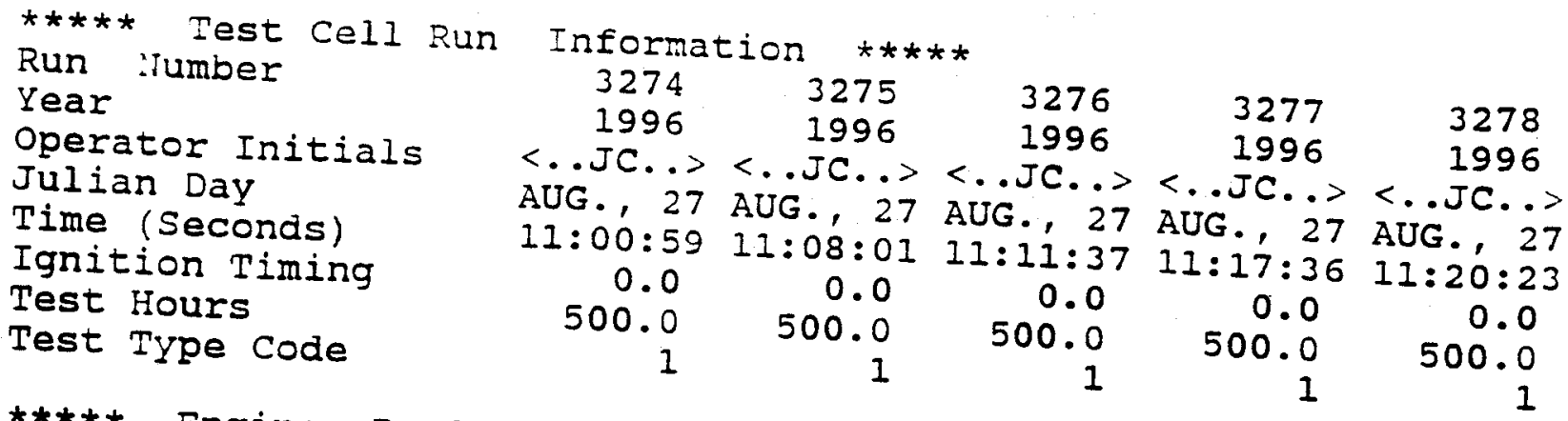

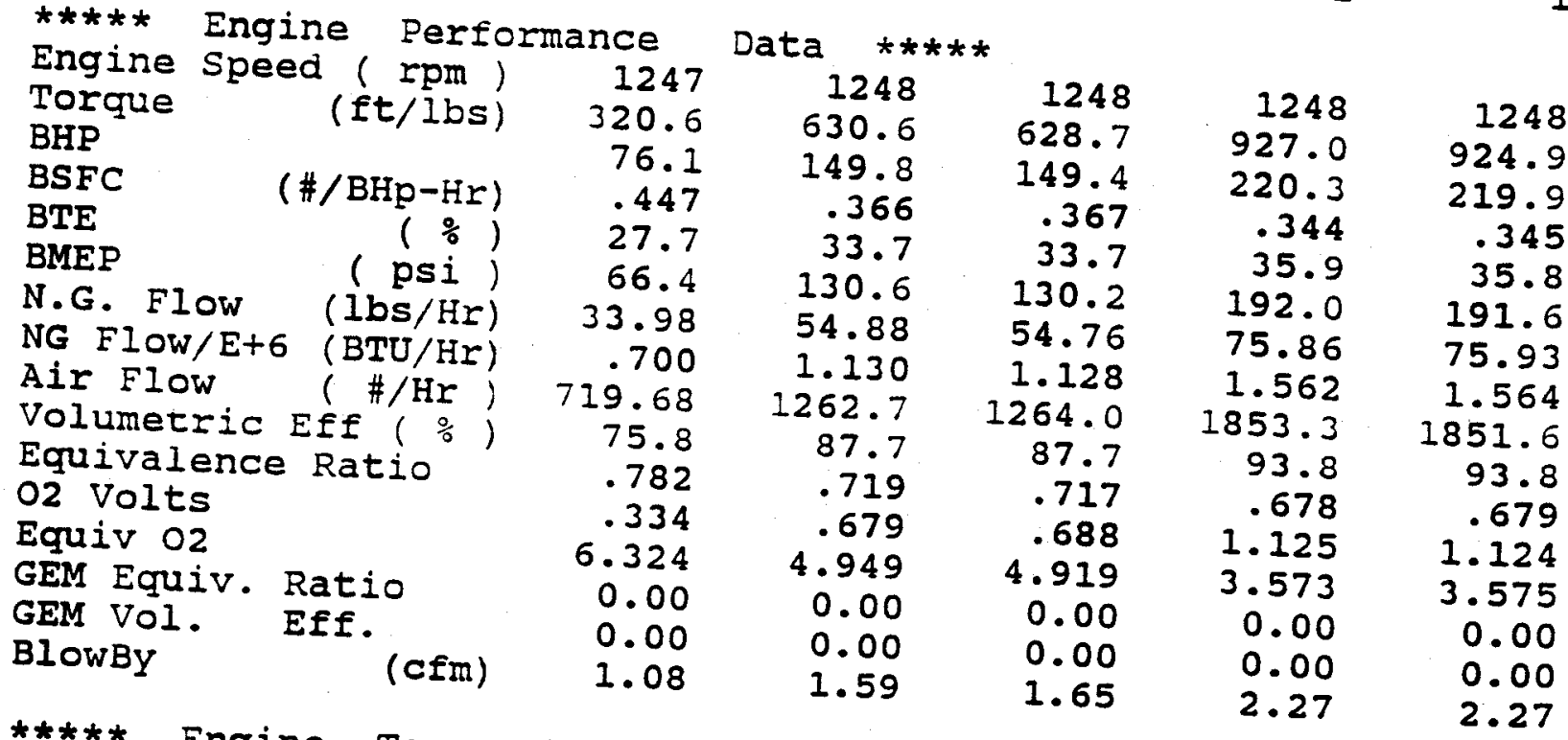

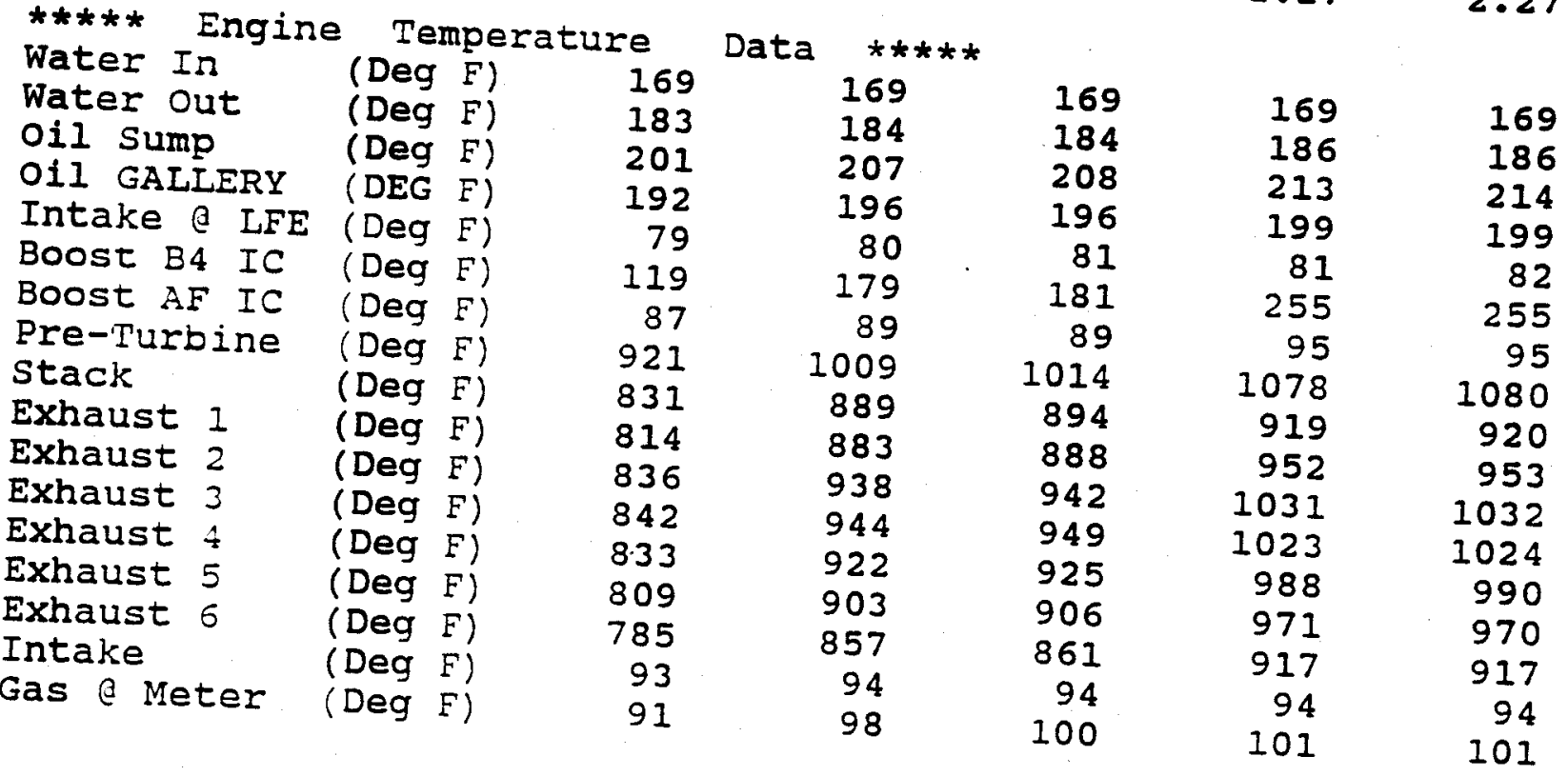




$$
\begin{gathered}
\text { cell } 05 \text { IIAC3 Project Low-Speed Data = } \\
\text { (continued) }
\end{gathered}
$$

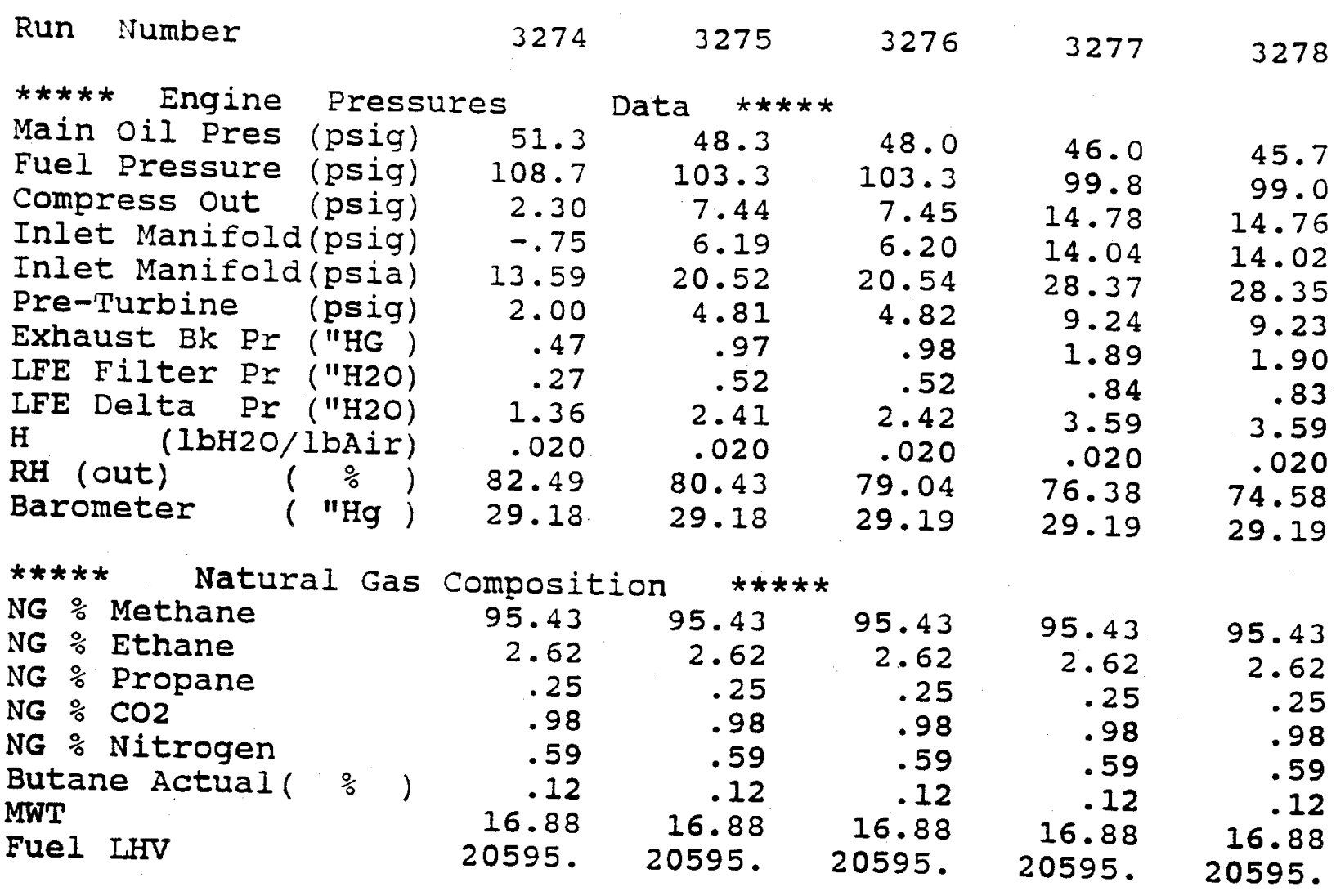


APPENDIX H

EMISSIONS RESULTS FOR TESTS AT 500 HOURS ON THE STC TEST 


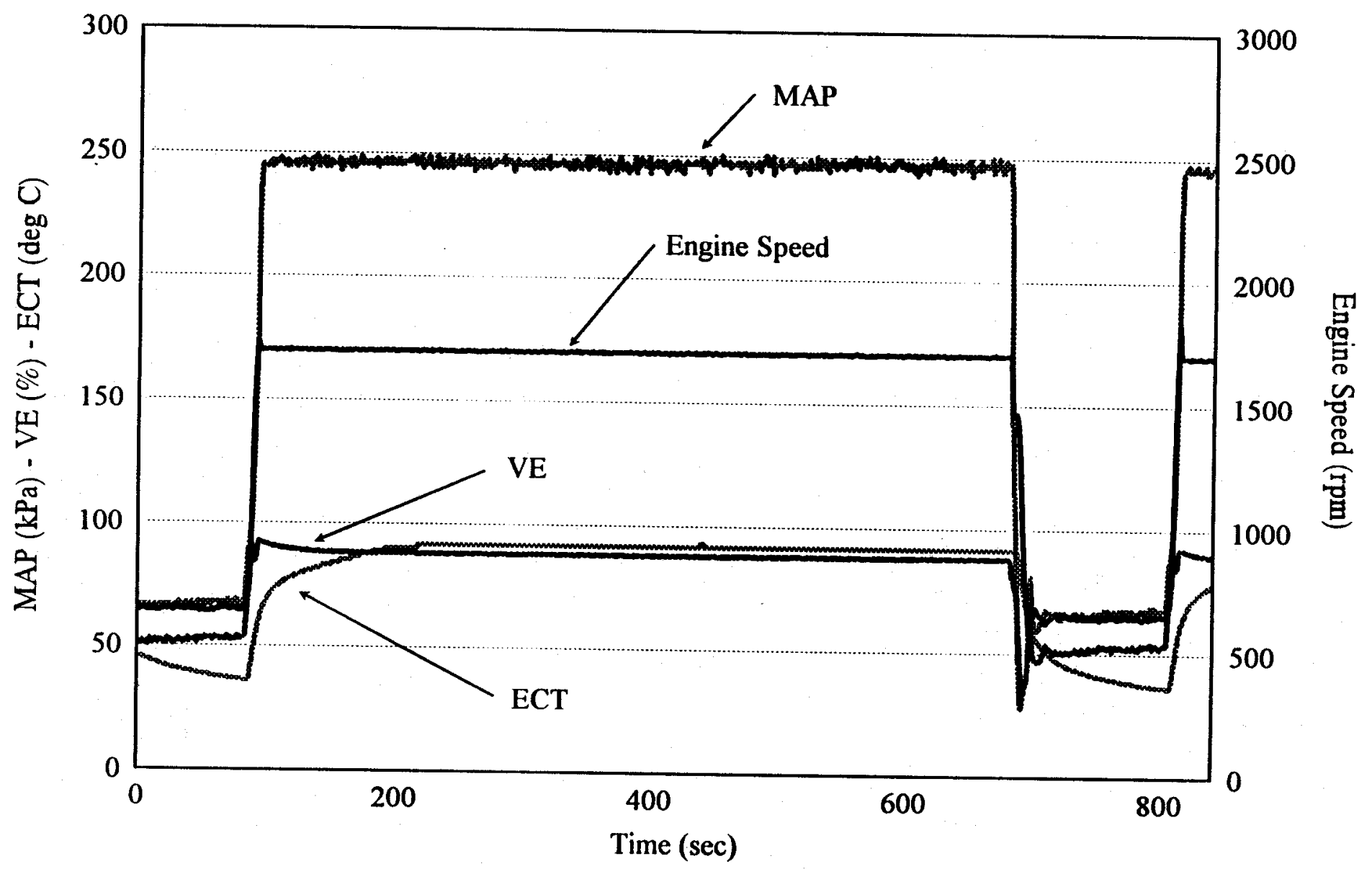

TRANSIENT DATA RECORDED FROM THE GEM SYSTEM FOR ONE FULL CYCLE AT 825 HOUR 


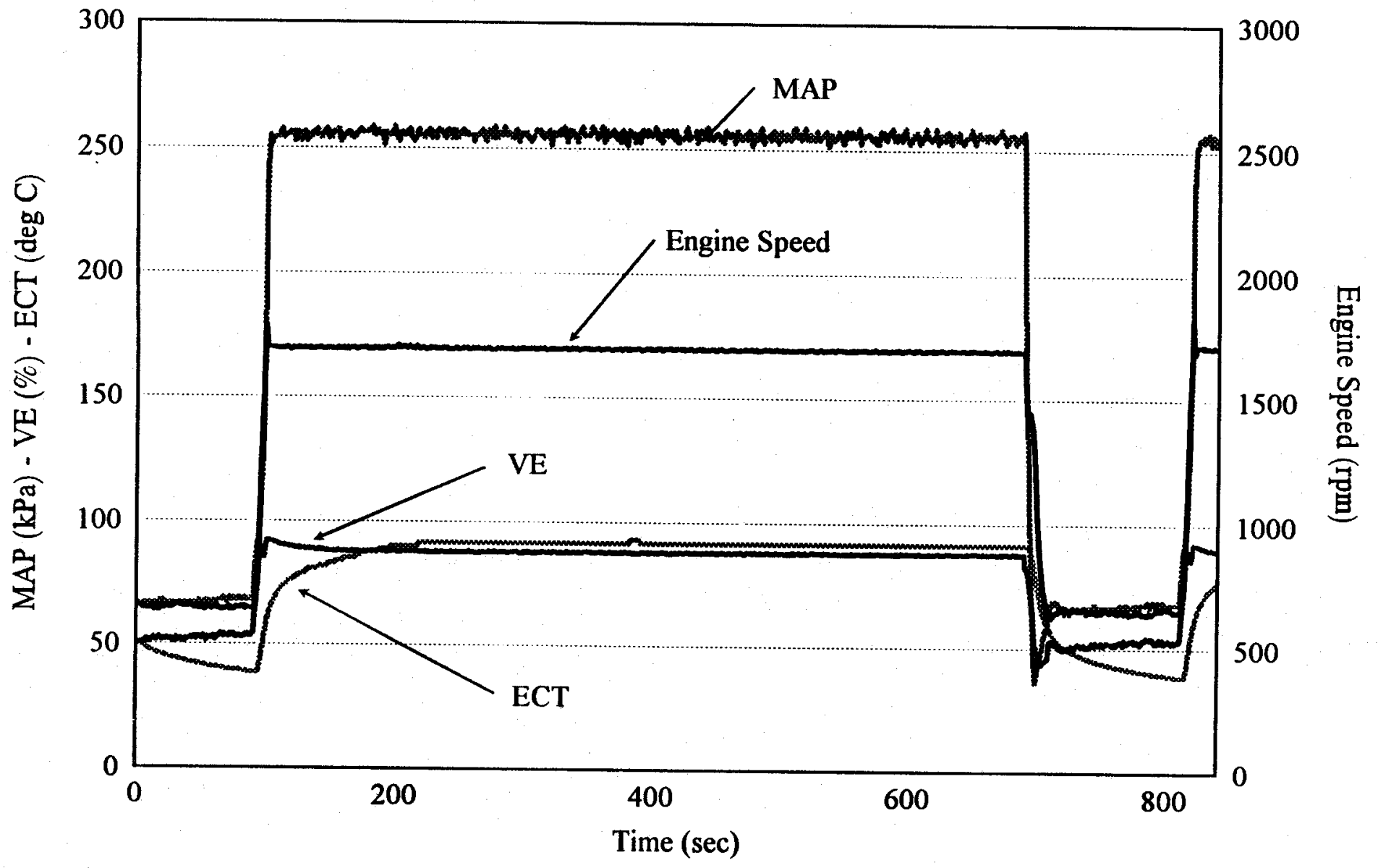

TRANSIENT DATA RECORDED FROM THE GEM SYSTEM FOR ONE FULL CYCLE AT 850 HOUR 


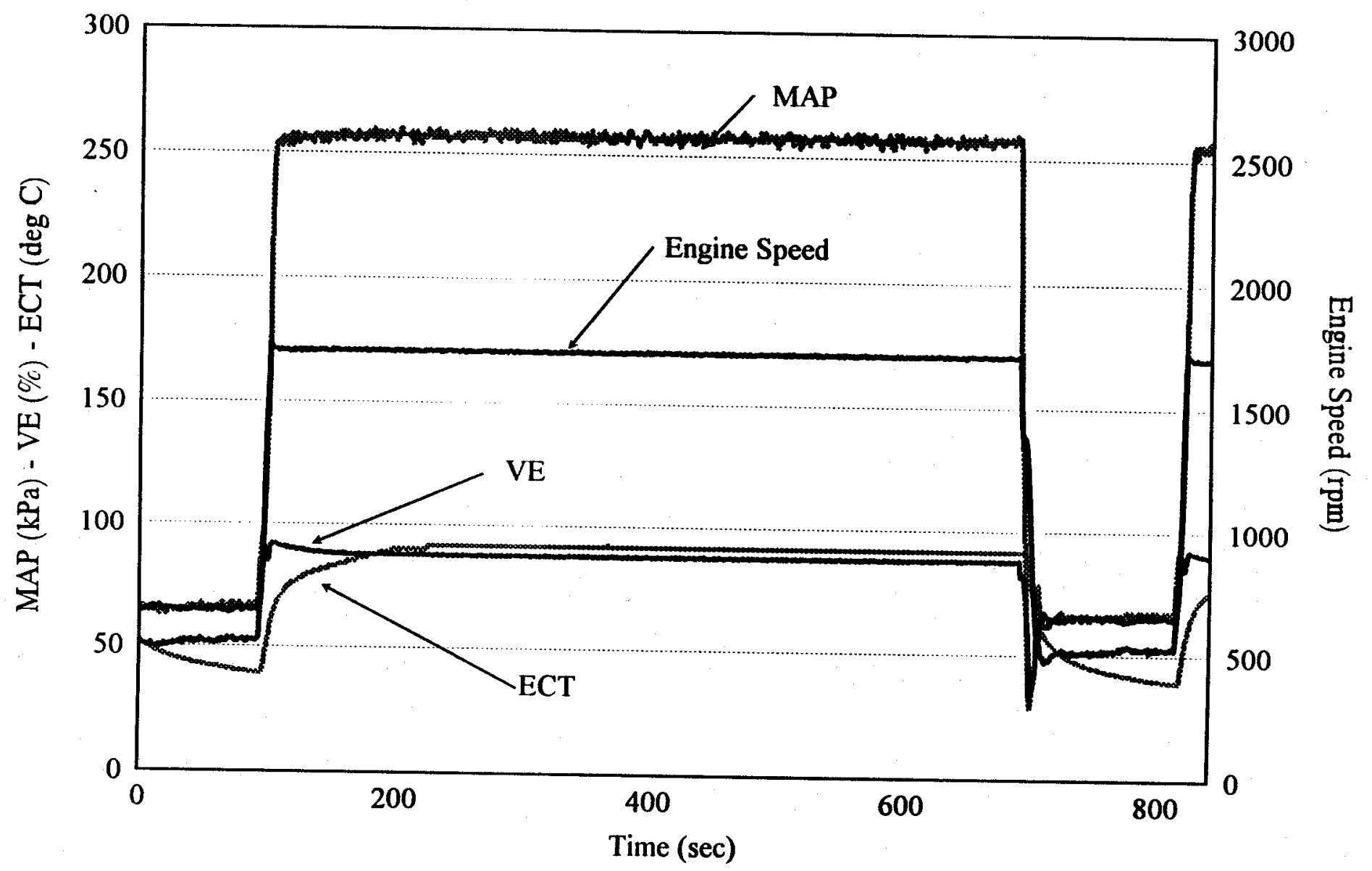

TRANSIENT DATA RECORDED FROM THE GEM SYSTEM FOR ONE FULL CYCLE AT 875 HOUR 


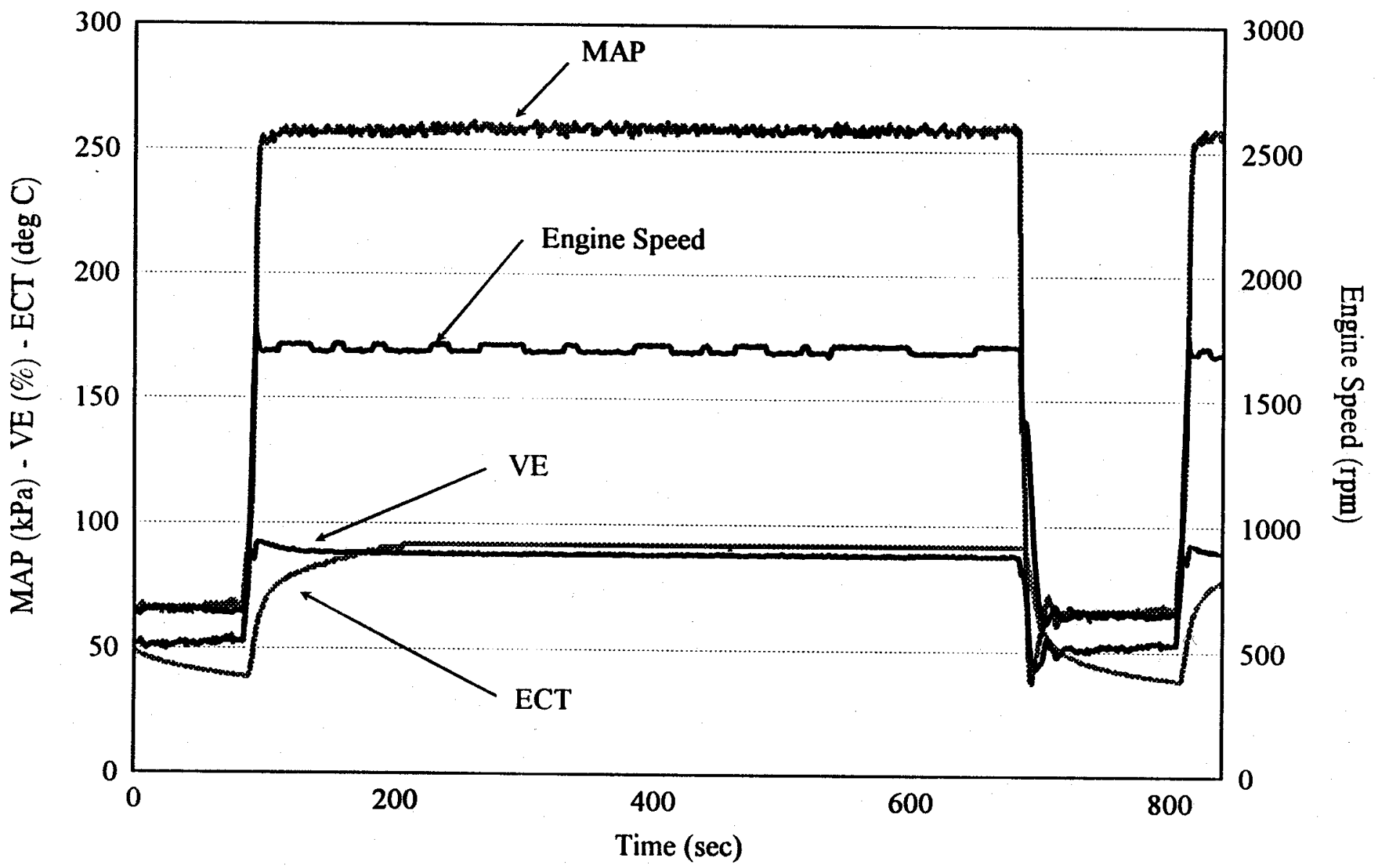

TRANSIENT DATA RECORDED FROM THE GEM SYSTEM FOR ONE FULL CYCLE AT 900 HOUR 


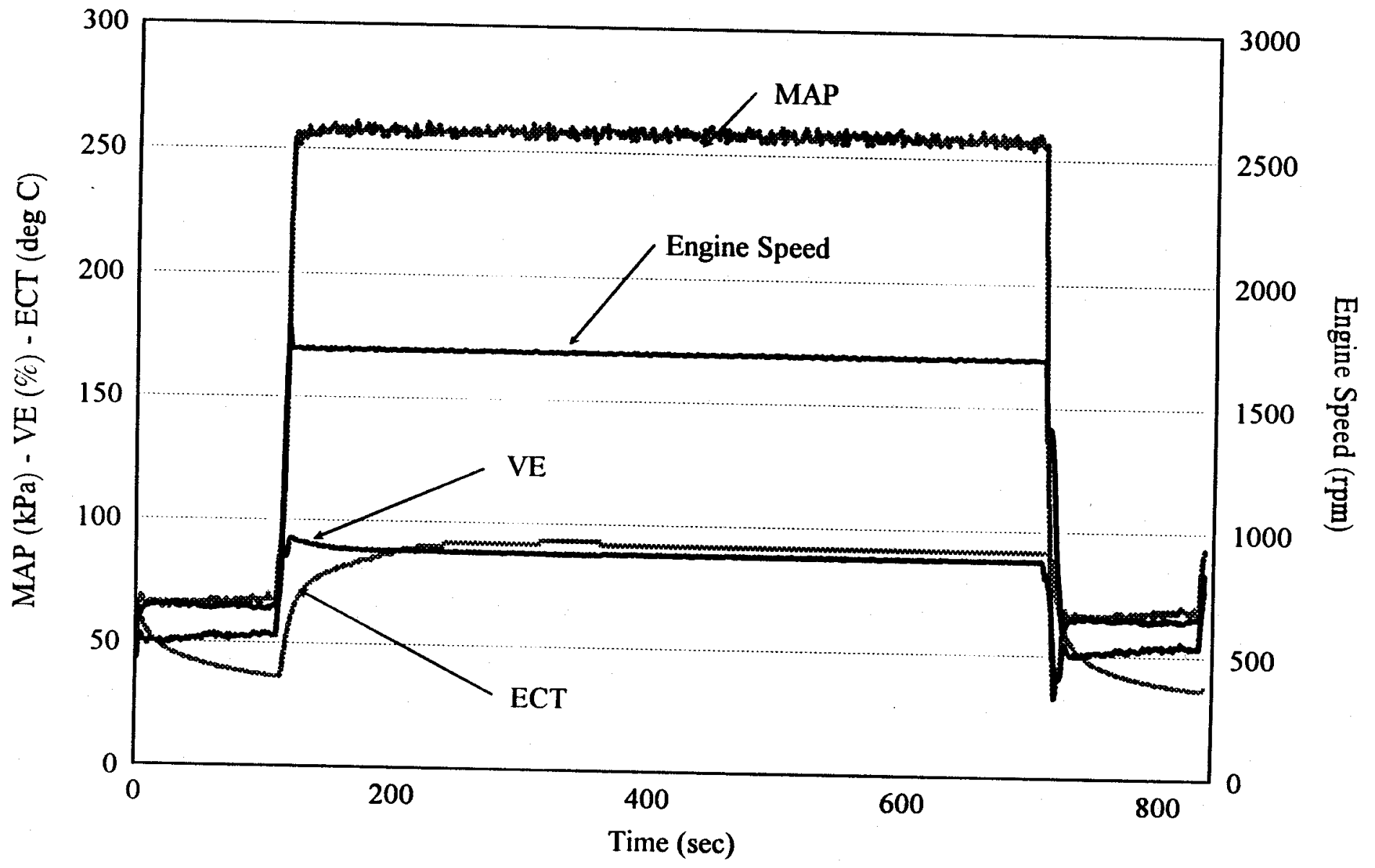

TRANSIENT DATA RECORDED FROM THE GEM SYSTEM FOR ONE FULL CYCLE AT 925 HOUR 


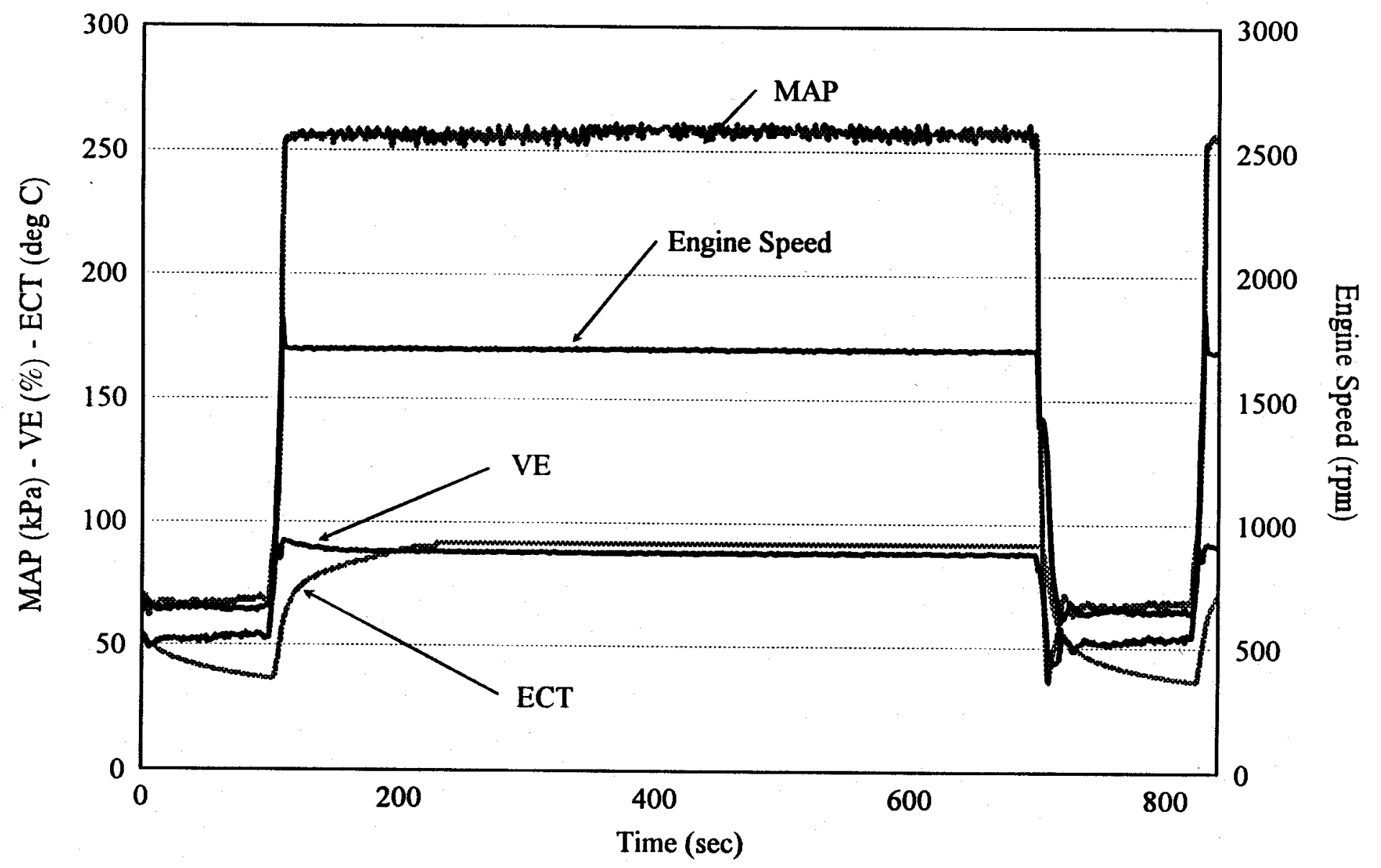

TRANSIENT DATA RECORDED FROM THE GEM SYSTEM FOR ONE FULL CYCLE AT 950 HOUR 


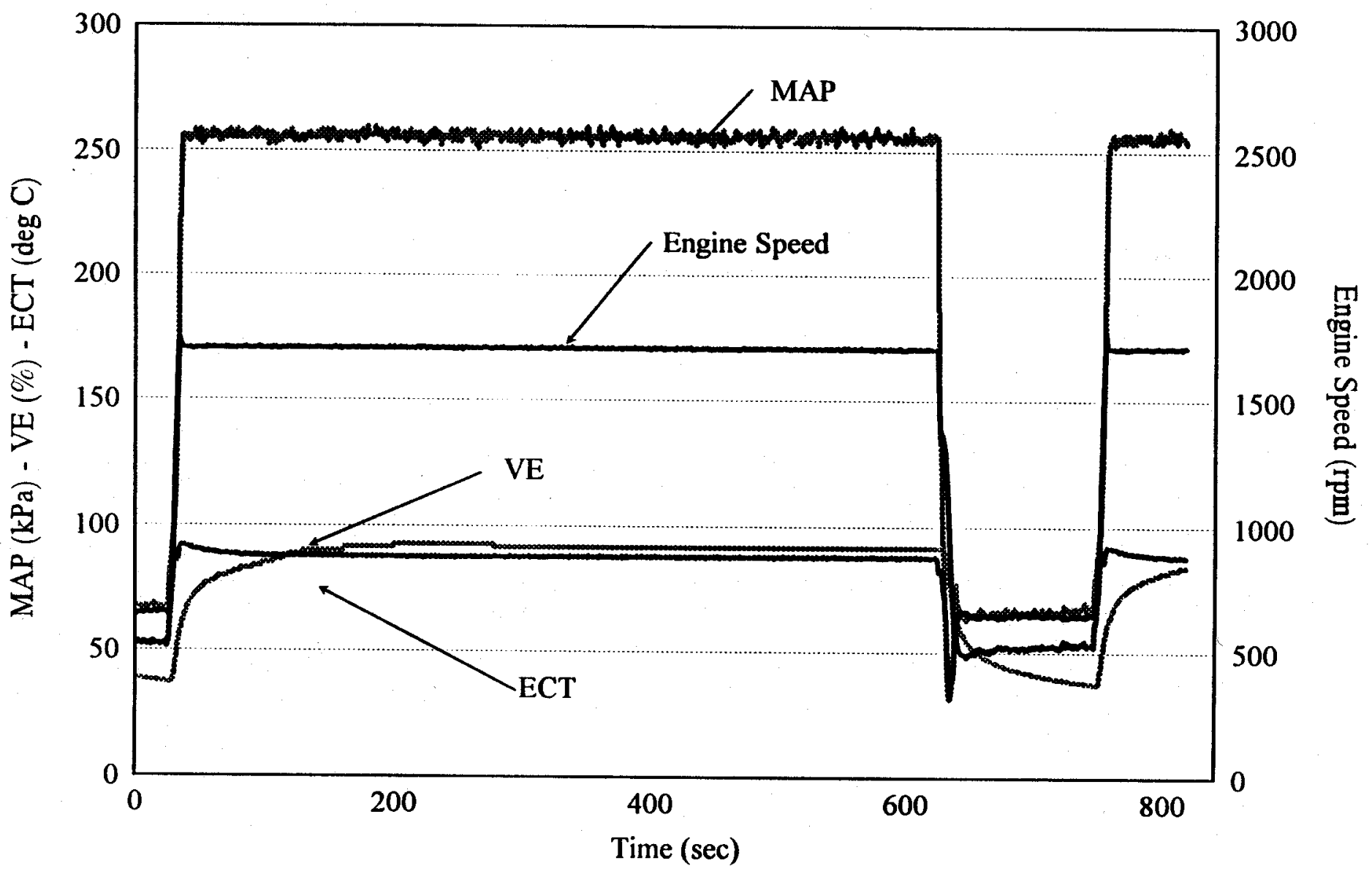

TRANSIENT DATA RECORDED FROM THE GEM SYSTEM FOR ONE FULL CYCLE AT 975 HOUR 


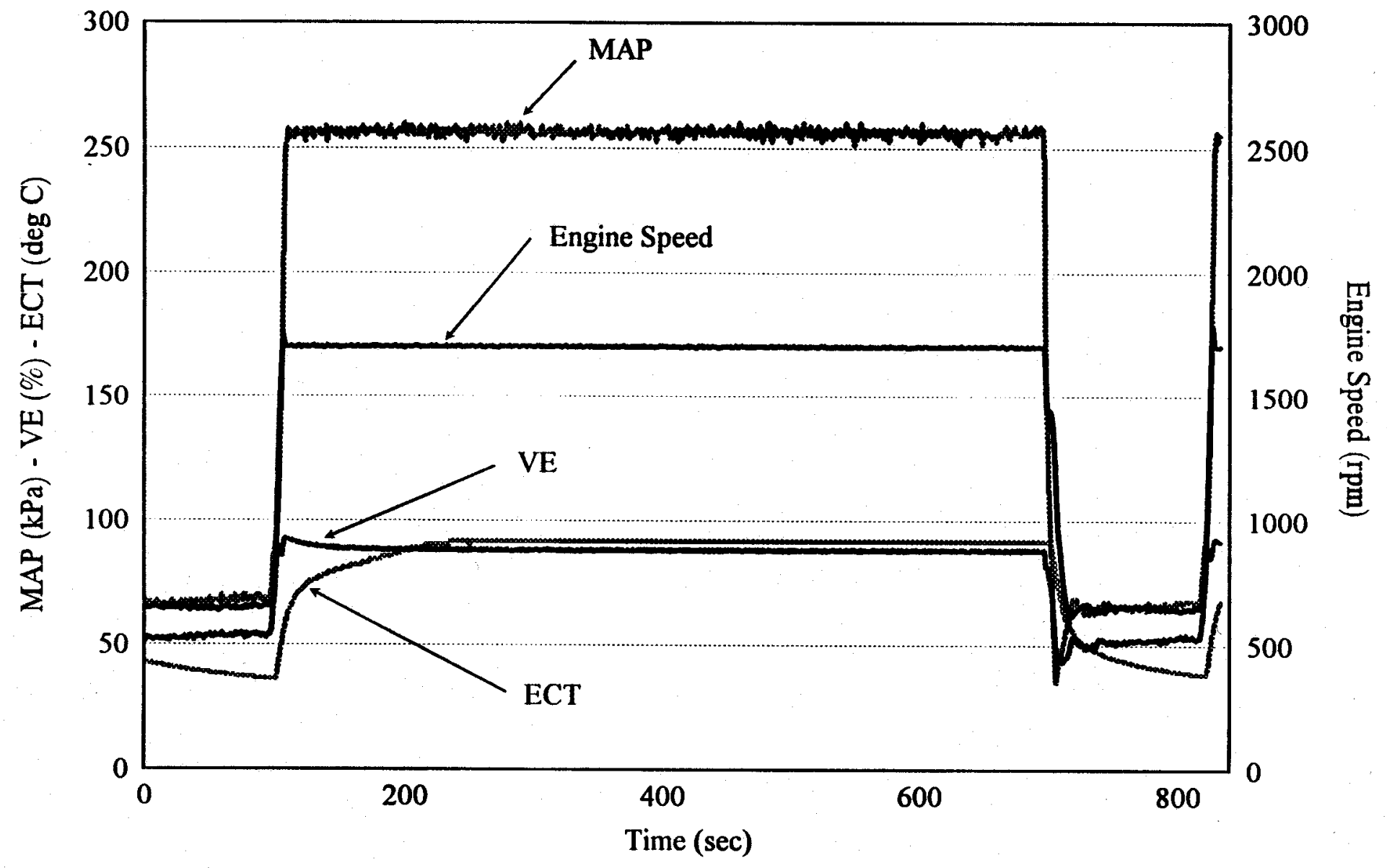

TRANSIENT DATA RECORDED FROM THE GEM SYSTEM FOR ONE FULL CYCLE AT 1000 HOUR 
APPENDIX E

FINAL REPORT FOR

1000-HOUR SUPER THERMAL CYCLE TEST ON A

MACK E-7 NATURAL GAS FUELED ENGINE

SwRI PROJECT NO. 03-1248 


\title{
1000-HOUR SUPER THERMAL CYCLE DURABILITY TEST ON A MACK E-7 NATURAL GAS FUELED ENGINE
}

\author{
FINAL REPORT
}

SwRI Project No. 03-1248

Prepared for:

Mack Trucks, Inc.

13302 Pennsylvania Ave.

Hagerstown, MD 21742

Contract No. EXP144683

Prepared by:

James P. Chiu

Richard J. Branecky

December 1998

\section{Approved:}

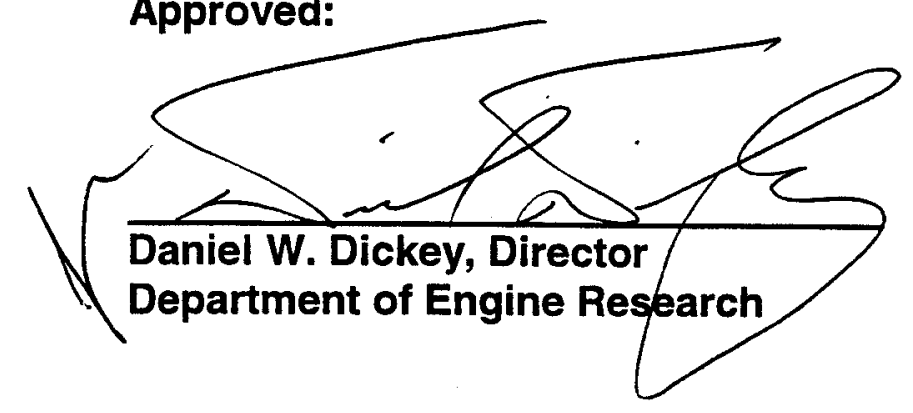




\section{EXECUTIVE SUMMARY}

The goal of this task was to perform a 1000-hour Super Thermal Cycle (STC) Durability Test. The super thermal cycle consisted of two minutes at idle (unloaded stage) and 10 minutes at high speed and load (loaded stage). The engine was thermally "shocked" with $43^{\circ} \mathrm{C}\left(110^{\circ} \mathrm{F}\right)$ coolant at the unloaded stage and $99^{\circ} \mathrm{C}\left(210^{\circ} \mathrm{F}\right)$ coolant at the loaded stage to produce a $56^{\circ} \mathrm{C}\left(100^{\circ} \mathrm{F}\right)$ temperature difference. This was the second super thermal cycle test performed on this engine model. The first test had problems with a blowby filter failure and a piston cracking. Both of these items were redesigned for this test.

The 1000-hour Super Thermal Cycle Durability Test was successfully completed. Basic engine performance data were recorded every hour during the test, and more detailed performance data were recorded every 25 hours. Oil consumption was recorded every 50 hours. Valve stem heights, valve lash, spark plug gaps, cylinder compression, and cylinder leak down were recorded every 250 hours.

A borescope inspection of the pistons at 500,750, and 1000 hours showed no signs of piston cracking. The blowby filter was still functioning properly at 1000 hours. The spark plugs were able to operate for 500 hours without maintenance during this test.

Major maintenance items before the start of the STC test included a rebuild of the front cylinder head, replacement of the UEGO sensor, and replacement of all injectors. Major maintenance items during the STC test included a misfire problem caused by noise on the mag pickup signal, replacement of the throttle, and replacement of two injectors. 
LIST OF ILLUSTRATIONS $\ldots \ldots \ldots \ldots \ldots \ldots \ldots \ldots \ldots \ldots \ldots \ldots \ldots \ldots \ldots \ldots$, vii

LIST OF TABLES $\ldots \ldots \ldots \ldots \ldots \ldots \ldots \ldots \ldots \ldots \ldots \ldots \ldots \ldots \ldots \ldots, \quad$ ix

1.0 BACKGROUND $\ldots \ldots \ldots \ldots \ldots \ldots \ldots \ldots \ldots \ldots \ldots \ldots \ldots \ldots \ldots \ldots \ldots, 1$

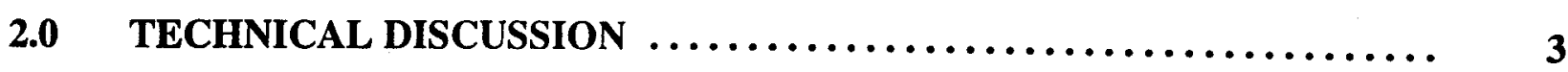

2.1 Test Schedule $\ldots \ldots \ldots \ldots \ldots \ldots \ldots \ldots \ldots \ldots \ldots \ldots \ldots \ldots \ldots \ldots \ldots \ldots \ldots, 3$

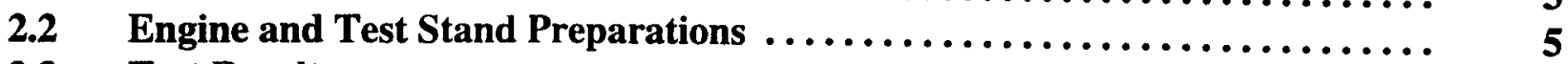

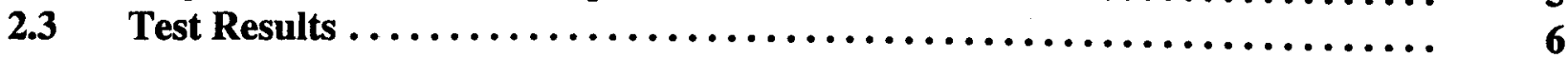

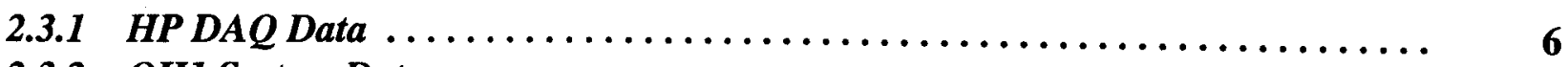

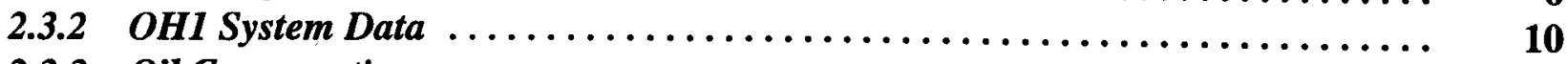

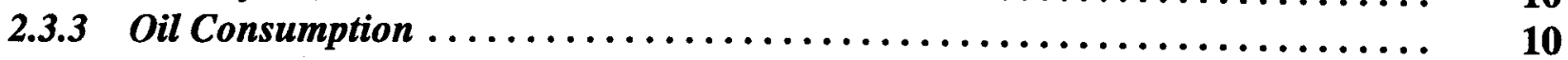

2.3.4 Valve Measurements ..................................... 18

2.3.5 Spark Plug Maintenance ................................ 22

2.3.6 Cylinder Compression and Leak Down Measurements ............... 22

2.3.7 Coolant Flow Measurements ............................. 24

2.3.8 Unscheduled Shutdowns/Maintenance ....................... 25

$3.0 \quad$ CONCLUSIONS \& RECOMMENDATIONS $\ldots \ldots \ldots \ldots \ldots \ldots \ldots \ldots . . \ldots$

\section{APPENDICES}

A LISTING OF HP DAQ RECORDS

B OHI TRANSIENT DATA

C SAMPLE "HOURLY" HP DAQ DATA

D SAMPLE "SNAPSHOT" HP DAQ DATA

E ETHYL OIL ANALYSIS RESULTS

F VALVE STEM HEIGHT MEASUREMENT \& RECESSION DATA 


\section{LIST OF ILLUSTRATIONS}

Figure

Page

1 Super Thermal Cycle STC Test Stand

Configuration

2 Fuel Filter Size and Location for

the STC Test Stand

3 Average of $\mathbf{4 0}$ Readings Recorded Between

1 and 1000 Test Hours from the Woodward OH1

During the STC Test

4 Average of $\mathbf{4 0}$ Readings and \pm One Standard Deviation

for VE Recorded Between 1 and 1000 Test Hours

from the Woodward OH1 During the STC Test..$\ldots \ldots \ldots \ldots \ldots \ldots \ldots$

5 Average of 40 Readings And \pm One Standard Deviation

For Engine Speed Recorded Between 1 And 1000 Test

Hours From The Woodward OH1 During the STC Test ...

6 Average of $\mathbf{4 0}$ Readings And \pm One Standard Deviation

For MAP Recorded Between 1 And 1000 Test Hours

From The Woodward OH1 During the STC Test

7 Average of 40 Readings And \pm One Standard Deviation

For ECT Recorded Between 1 And 1000 Test Hours

From The Woodward OH1 During the STC Test

8 Injector Block Leak Down Rates

During the STC Test $\ldots \ldots \ldots \ldots \ldots \ldots \ldots \ldots \ldots \ldots \ldots \ldots \ldots \ldots, 16$

9 Oil Consumption Trend $\ldots \ldots \ldots \ldots \ldots \ldots \ldots \ldots \ldots \ldots \ldots \ldots \ldots, 19$

10 Intake Valve Recession $\ldots \ldots \ldots \ldots \ldots \ldots \ldots \ldots \ldots \ldots \ldots \ldots \ldots \ldots, 20$

11 Exhaust Valve Recession $\ldots \ldots \ldots \ldots \ldots \ldots \ldots \ldots \ldots \ldots \ldots \ldots \ldots, 21$

12 Spark Plug Gap Growth $\ldots \ldots \ldots \ldots \ldots \ldots \ldots \ldots \ldots \ldots \ldots \ldots \ldots, 23$ 


\section{LIST OF TABLES}

Table

Page

$1 \quad$ Test Schedule $\ldots \ldots \ldots \ldots \ldots \ldots \ldots \ldots \ldots \ldots \ldots \ldots \ldots \ldots \ldots \ldots \ldots \ldots \ldots \ldots \ldots, \ldots$

$2 \quad$ Super Thermal Cycle $\ldots \ldots \ldots \ldots \ldots \ldots \ldots \ldots \ldots \ldots \ldots \ldots \ldots \ldots \ldots \ldots \ldots \ldots \ldots$

3 Scheduled Data Recording and Maintenance .................... 4

$4 \quad$ Engine Specifications $\ldots \ldots \ldots \ldots \ldots \ldots \ldots \ldots \ldots \ldots \ldots \ldots \ldots \ldots \ldots \ldots \ldots \ldots \ldots$

$5 \quad$ Runtime History of Engine $\ldots \ldots \ldots \ldots \ldots \ldots \ldots \ldots \ldots \ldots \ldots \ldots \ldots \ldots \ldots \ldots$

6 Maintenance History Prior to STC Test $\ldots \ldots \ldots \ldots \ldots \ldots \ldots \ldots \ldots \ldots \ldots$

$7 \quad$ Oil Adds and Drains $\ldots \ldots \ldots \ldots \ldots \ldots \ldots \ldots \ldots \ldots \ldots \ldots \ldots \ldots \ldots \ldots$

$8 \quad$ Oil Consumption $\ldots \ldots \ldots \ldots \ldots \ldots \ldots \ldots \ldots \ldots \ldots \ldots \ldots \ldots \ldots \ldots \ldots \ldots \ldots \ldots . \ldots$

$9 \quad$ Valve Lash Measurements $\ldots \ldots \ldots \ldots \ldots \ldots \ldots \ldots \ldots \ldots \ldots \ldots \ldots \ldots \ldots \ldots \ldots . \ldots 22$

10 Cylinder Compression Pressures $\ldots \ldots \ldots \ldots \ldots \ldots \ldots \ldots \ldots \ldots \ldots \ldots \ldots \ldots \ldots$

11 Cylinder Pressure Leak down $\ldots \ldots \ldots \ldots \ldots \ldots \ldots \ldots \ldots \ldots \ldots \ldots \ldots \ldots$

12 Coolant Flow Measurements $\ldots \ldots \ldots \ldots \ldots \ldots \ldots \ldots \ldots \ldots \ldots \ldots \ldots \ldots \ldots \ldots$

13 Unscheduled Shutdowns/maintenance

During the STC Test $\ldots \ldots \ldots \ldots \ldots \ldots \ldots \ldots \ldots \ldots \ldots \ldots \ldots \ldots \ldots \ldots \ldots \ldots$ 


\subsection{BACKGROUND}

The goal of this project was to aid Mack Trucks, Inc. in expediting the development of a natural gas-fueled Mack E-7 engine and to perform part of the tests to determine an emissions deterioration factor for the engine. This report covers the second of two 1000-hour durability tests. The purpose of the first " 1000 -Hour Durability Test" was to verify the durability of the natural gasrelated hardware, including the valve/valve seat combination and to verify acceptability of the engine control system. Based on the results from this test, it was recommended that additional testing be conducted to verify the durability of the suspect components, including the blowby filter element, the platinum-iridium tipped spark plugs, and the pistons used for the Mack E-7 natural gas-fueled engine. (SwRI Final Report, entitled "Task 2 - 1000-Hour Super Thermal Cycle Test", SwRI Project No. 03-7501-002, dated November 1996). An improved blowby filter design, a spark plug maintenance (regap) interval, and a redesigned piston were incorporated into this second test.

Funding for the testing, completed prior to the STC test, was provided by the "Mack LNG Vehicle Development" project conducted by SwRI for Mack Trucks. The STC durability test was conducted at SwRI's Engine and Vehicle Research Division, Department of Engine Research, Automotive Research Laboratory (443 Avenue C, Bldg. 69) and the results are reported herein. 


\subsection{TECHNICAL DISCUSSION}

A 1000-hour Super Thermal Cycle (STC) Durability Test was conducted on a Mack E-7 natural gas fueled engine. The STC test cycle (as provided by Mack Trucks) consisted of two stages, inciuding two minutes at idle (unloaded stage) and ten minutes at high speed and load (loaded stage), for a total cycle time of twelve minutes. The operating condition for engine coolant out temperature differed by a minimum of $56^{\circ} \mathrm{C}\left(100^{\circ} \mathrm{F}\right)$ between the loaded and unloaded stages. The following sections describe the test schedule, engine and test stand preparations, and test results.

\section{$2.1 \quad$ Test Schedule}

The test schedule for the STC durability test engine is shown in Table 1. The engine was used for previous work, including emissions and performance testing, prior to running the durability test. Total engine hours, as described in this report, refers to the total hours on the engine since the start of all testing. STC test hours refers to the hours of actual test time on the STC test.

Table 1. Test Schedule

\begin{tabular}{||l|c|c|c||}
\hline \multicolumn{1}{|c|}{ Test } & $\begin{array}{c}\text { Time } \\
\text { (hours) }\end{array}$ & $\begin{array}{c}\text { Engine Speed } \\
(\mathbf{r p m})\end{array}$ & $\begin{array}{c}\text { Power } \\
(\mathbf{k W})\end{array}$ \\
\hline Break-In & 125 & 1950 & 242 \\
\hline $\begin{array}{l}\text { Emissions (12-mode) } \\
\text { \& Performance Tests }\end{array}$ & As needed & Various & Various \\
\hline Miscellaneous testing & As needed & Various & Various \\
\hline Transient Emissions & As needed & FTP Cycle & FTP Cycle \\
\hline STC Durability Test & 1000 & STC Cycle & STC Cycle \\
\hline Valve Rotation & As needed & $1700 / 600$ & Minimum \\
\hline
\end{tabular}

The cycle for the STC test is shown in Table 2. On the unloaded stage, the engine is operated at idle speed with minimum load. The operating conditions for the loaded stage were selected by Mack Trucks as $1700 \mathrm{rpm}$ and $110 \%$ load, based on a power curve provided by Renualt V.I. for an engine rated at $261 \mathrm{~kW}(350 \mathrm{hp})$ at $1900 \mathrm{rpm}$, to reach $290 \mathrm{~kW}$ at $1700 \mathrm{rpm}$. At the loaded stage, the equivalence ratio was at 0.65 and the ignition timing was set to MBT. Due to the high ambient temperature typical to San Antonio at the time of the year this test was performed, the specified coolant out temperature for the unloaded stage (idle) of $32^{\circ} \mathrm{C}\left(90^{\circ} \mathrm{F}\right)$ could not be obtained. Therefore, a coolant out temperature of $38-43^{\circ} \mathrm{C}\left(100-110^{\circ} \mathrm{F}\right)$ was specified for the unloaded stage and $99^{\circ} \mathrm{C}\left(210^{\circ} \mathrm{F}\right)$ for the loaded stage. These temperatures maintained a minimum of $56^{\circ} \mathrm{C}\left(100^{\circ} \mathrm{F}\right)$ differential between the loaded and unloaded stages during the test.

The schedule for engine data recording and engine maintenance, during the 1000-hour durability test, is shown in Table 3. Shutdowns were scheduled every 25 test hours. 
Table 2. Super Thermal Cycle

\begin{tabular}{||l|c|c||}
\hline \multicolumn{1}{|c|}{ Parameter } & Unloaded & Loaded \\
\hline Engine Speed (rpm) & 600 & 1700 \\
\hline Power $(\mathrm{kW})$ & 4 & 290 \\
\hline Time (minutes) & 2 & 10 \\
\hline $\begin{array}{l}\text { Coolant Out Temperature } \\
\left({ }^{\circ} \mathrm{C} \text { at end of cycle) }\right.\end{array}$ & $38-43$ & 99 \\
\hline
\end{tabular}

Table 3. Scheduled Data Recording And Maintenance

\begin{tabular}{|l|l||}
\hline \multicolumn{1}{|c|}{ Description } & \multicolumn{1}{c|}{ Schedule } \\
\hline Oil Sample & At one hour after oil change \\
\hline HP DAQ Hourly Data & Every hour \\
\hline HP DAQ Snapshot Data & Every 25 hours \\
\hline OH1 Transient Data & Every 25 hours \\
\hline OH1 NGP Data & Every 25 hours \\
\hline Oil Level Check (fill as needed) & Every 25 hours \\
\hline Oil Sample & Every 50 hours \\
\hline Oil Fill (record oil consumption) & Every 50 hours \\
\hline Drain Blowby Filter Housing & Every 50 hours \\
\hline Drain Gas Filter Housings & Every 50 hours \\
\hline Replace Adsorption Filter Element & As needed \\
\hline Measure Valve Stem Heights & Every 250 hours \\
\hline Measure Valve Lash & Every 250 hours \\
\hline Measure Spark Plug Gaps & Every 250 hours \\
\hline Measure Cylinder Compression & Every 250 hours \\
\hline Measure Cylinder Leak Down & Every 250 hours \\
\hline Change Oil and Filters & At 500 hours \\
\hline Replace Coalescing Fuel Filters & Every 500 hours \\
\hline Measure Coolant Flow & Every 500 hours \\
\hline
\end{tabular}




\subsection{Engine and Test Stand Preparations}

The Mack E-7G engine ( $\mathrm{S} / \mathrm{N} 5 \mathrm{~N}-1311$ ), used for previous work including emissions (both steady-state and transient) and performance testing, was also used for the 1000-hour STC durability test. There were a total of 304.5 hours on the engine prior to the start of the durability test, including 160.5 hours for the break-in, steady-state emissions, and performance testing, 111.8 hours for the transient emissions tests, and 32.2 hours of miscellaneous time.

The pistons in this engine were redesigned and modified for this test, by Mack Trucks, based on the results of the first STC durability test (see Conclusions and Recommendations in SwRIReport 03-7501-002, dated November 1996). A redesigned blowby filter was also incorporated for this test. The engine specifications are shown in Table 4. Valve lash was reset to $0.41 \mathrm{~mm}\left(0.016^{\prime \prime}\right)$ for intake valves and $0.71 \mathrm{~mm}\left(0.028^{\prime \prime}\right)$ for exhaust valves at the beginning of the STC test. The spark plugs were replaced with new plugs, with a date code of $2 \mathrm{H} 6$, and gaps were set to $0.25 \mathrm{~mm}\left(0.010^{\prime \prime}\right)$.

Table 4. Engine Specification

\begin{tabular}{||l|l||}
\hline Engine Model & E7G-325 \\
\hline Engine Serial Number & $5 \mathrm{~N} 1311$ \\
\hline Engine Displacement (L) & 12 \\
\hline Bore and Stroke (mm) & 123.8 x 165.1 \\
\hline Piston & Modified Zollner Aluminum H Ring* \\
\hline First Ring & PNN 349GC3105 \\
\hline Second Ring & PNN 349GC3106 \\
\hline Oil Rail & P/N 350GC342 \\
\hline Oil Expander & P/N 350GC341 \\
\hline Camshaft Timing & Standard gas timing \\
\hline Ignition System Controller Supplier & Woodward \\
\hline Ignition System Coil Supplier & Nippondenso \\
\hline Ignition System Type & Inductive \\
\hline Ignition System Timing Sensor & Bosch \\
\hline Spark Plug Supplier & Champion \\
\hline Mixer Type & Cast Annular with One 90 Bend \\
\hline Number of Holes in Mixing Ring & 9 \\
\hline Fuel Metering System Supplier & Woodward \\
\hline Fuel Injector Type & Servojet SP021S1 \\
\hline Number of Injectors & 8 \\
\hline Throttle Supplier & Woodward \\
\hline Throttle Model & FLOWTECH, 68mm \\
\hline Throttle Type & Electronic, Analog \\
\hline UEGO Sensor & Honda \\
\hline * Pistons were modified for the second 1000-Hour Durability Test, per the Conclusions and \\
Recommendations of the first SwRI 1000-Hour Test Report, dated November 1996 \\
\hline \hline
\end{tabular}


After the break-in, steady-state emissions, and performance tests were conducted, the test stand cooling system was modified for the thermal "shock" of the engine on the STC durability test. Two coolant tanks were installed in the test cell to provide either hot coolant to the engine during the loaded stage or cold coolant during the unloaded stage. The hot tank was maintained at approximately $99^{\circ} \mathrm{C}\left(210^{\circ} \mathrm{F}\right)$ at the beginning of the loaded stage and the cold tank was controlled to approximately $38-43^{\circ} \mathrm{C}\left(100-110^{\circ} \mathrm{F}\right)$ near the end of the unloaded stage. A schematic of the coolant system for the STC test is shown in Figure 1. A mixture of Nalcool ${ }^{\mathrm{TM}} 2000$ and demineralized water, at a ratio of $489 \mathrm{ml}$ (16 ounces) of $\mathrm{Nalcool}^{\mathrm{TM}}$ to 15.21 ( 4 gallons) of water, was added to the engine cooling system. The test stand was also equipped with an external electric motor to operate the engine water pump at a constant speed that was equivalent to the speed of the water pump at $2000 \mathrm{rpm}$ (when the engine is in its normal configuration).

The oil and filters were changed prior to the start of the test. A closed crankcase ventilation system was installed, which included a blowby filter and canister provided by Mack Trucks and an in line blowby flow meter. The blowby was routed to the filter, then the in line flow meter, and finally to the inlet air system for the engine (between the laminar flow element and the turbocharger). New coalescing natural gas filters were installed. An adsorption filter and a low pressure coalescing filter (provided by Clean Air Partners, model number 616190-1) were added to the natural gas fuel supply line for the STC test stand. Figure 2 shows the schematic of the fuel filtering system with the two new filters installed.

\section{$2.3 \quad$ Test Results}

A total of 1326.0 hours were put on the engine since the beginning of all test work. A runtime history for the engine is shown in Table 5. Maintenance performed on the engine prior to the STC test is shown in Table 6. Results of the STC test program including the HP DAQ data, Woodward $\mathrm{OH} 1$ data, oil consumption, valve lash and recession measurements, spark plug maintenance, cylinder compression and leak down measurements, coolant flow measurements, and unscheduled shutdowns/maintenance are presented in the following sections.

\subsubsection{HP DAQ Data}

Hourly and snapshot engine data were recorded by the HP Data Acquisition System (DAQ) during the STC test. Hourly data were recorded, by an average of two points, with a target of recording data within 30 seconds of the end of each stage. Snapshot data were recorded for one full 12-minute cycle, in approximately 15 second intervals, every 25 test hours. There were a total of 2000 hourly readings and 2296 snapshot readings recorded during the course of the 1000-hour STC test. The hourly readings at 238 and 761 test hours were inadvertently missed by the engine operator. The hourly readings at 657 and 668 test hours were recorded incorrectly (during engine ramp) due to an HP DAQ system problem. Extra readings were recorded at 709 test hours due an engine operator error. A listing of all HP DAQ run numbers recorded during the STC test, with corresponding test hours and data type, is included in Appendix A. 


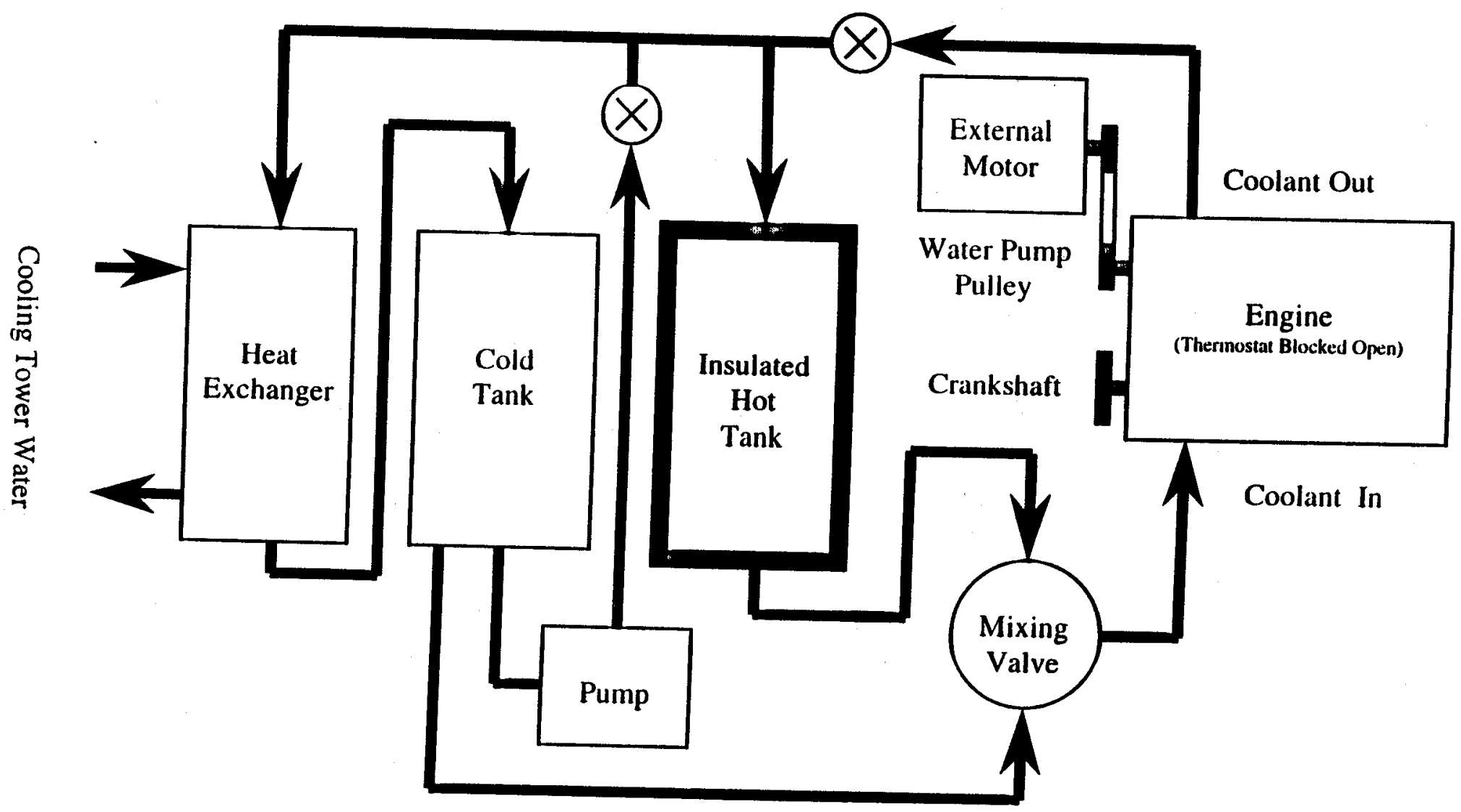

Figure 1. Super Thermal Cycle (STC) Test Stand Configuration 
From

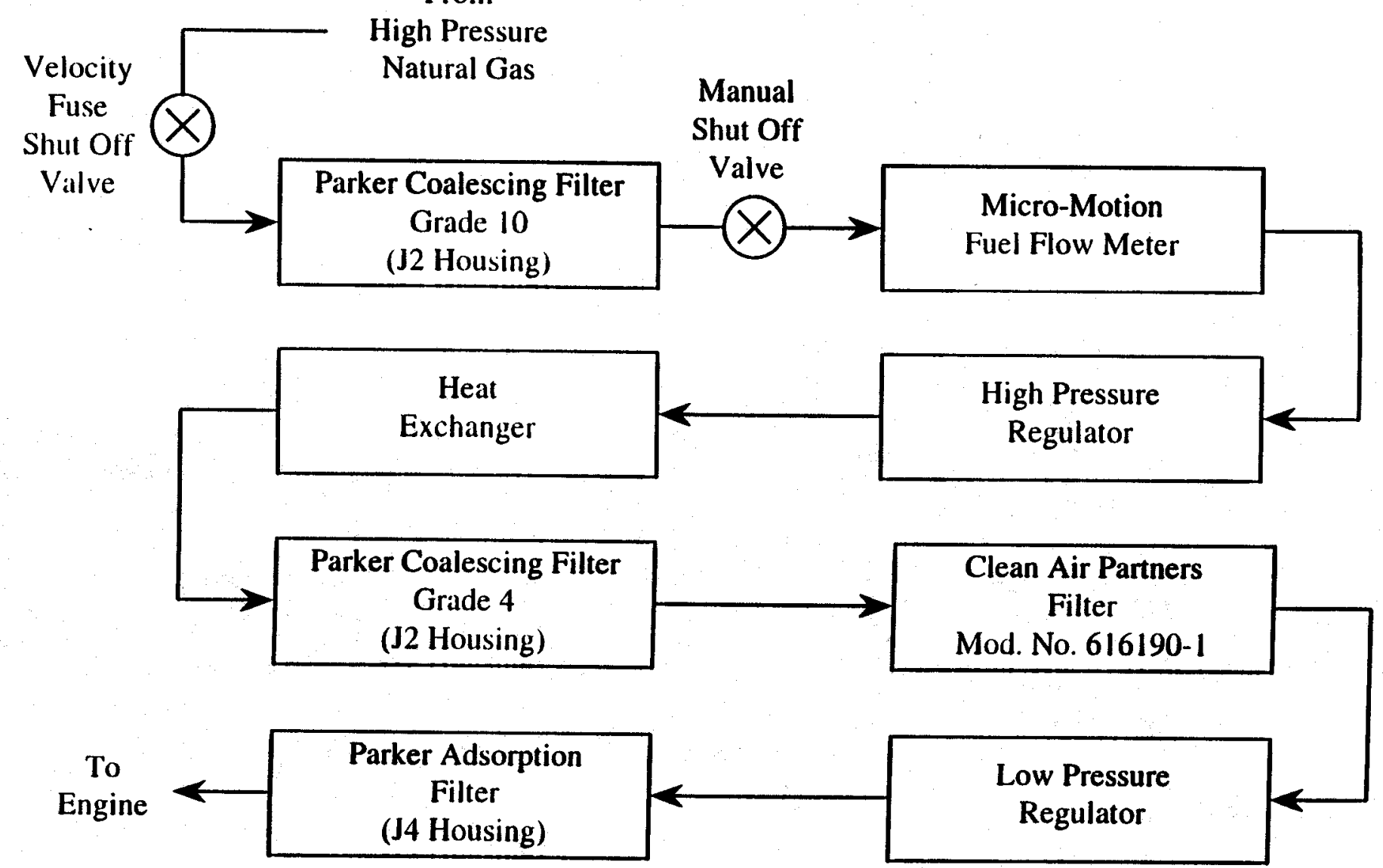

Figure 2. Fuel Filter Size and Location for the STC Test Stand 
Table 5. Runtime History of Engine

\begin{tabular}{||c|c|c|c||}
\hline Test Type & $\begin{array}{c}\text { Test Time } \\
\text { (Hours) }\end{array}$ & $\begin{array}{c}\text { Miscellaneous } \\
\text { Time (Hours) }\end{array}$ & $\begin{array}{c}\text { Total Engine } \\
\text { Time (Hours) }\end{array}$ \\
\hline \hline Break-In & 125.0 & 35.5 & 160.5 \\
\hline Miscellaneous Tests & -- & 15.8 & 176.3 \\
\hline Transient Emissions & -- & 111.8 & 288.1 \\
\hline STC Test & 0.0 & 16.4 & 304.5 \\
\hline STC Test & 250.0 & 5.8 & 560.3 \\
\hline STC Test & 500.0 & 3.4 & 813.7 \\
\hline STC Test & 750.0 & 3.2 & 1066.9 \\
\hline STC Test & 1000.0 & 5.5 & 1322.4 \\
\hline Valve Rotation Tests & -- & 3.6 & 1326.0 \\
\hline
\end{tabular}

Table 6. Maintenance History Prior to STC Test

\begin{tabular}{||c|c|l||}
\hline Date & $\begin{array}{c}\text { Total } \\
\text { Engine } \\
\text { Time (Hours) }\end{array}$ & \multicolumn{1}{|c||}{ Description } \\
\hline \hline $12 / 2 / 97$ & 0.1 & Replaced one bad Injector. \\
\hline $12 / 15 / 97$ & 51.8 & Replaced NGP Sensor. \\
\hline $12 / 16 / 97$ & 64.4 & Replaced UEGO $\left(\mathrm{O}_{2}\right)$ Sensor. \\
\hline $12 / 19 / 97$ & 64.7 & Replaced Air Compressor. \\
\hline $12 / 19 / 97$ & 117.6 & Replaced Ignition Control Box. \\
\hline $12 / 23 / 97$ & 161.4 & $\begin{array}{l}\text { Rebuilt No. 3 Cyl. Head (replaced exhaust valve on exhaust } \\
\text { manifold side) and replaced Piston. Replaced Air Compressor. }\end{array}$ \\
\hline $1 / 26 / 98$ & 163.7 & Installed new ICM (from Woodward). \\
\hline $6 / 9 / 98$ & 232.2 & Replaced all 8 Fuel Injectors. \\
\hline $7 / 2 / 98$ & 288.1 & $\begin{array}{l}\text { Installed a gasket between Turbocharger and Exhaust Manifold } \\
\text { Flanges (gasket was missing). }\end{array}$ \\
\hline $7 / 20 / 98$ & 294.2 & $\begin{array}{l}\text { Replaced all Spark Plugs and set gaps to 0.010 inches (used } \\
\text { Champion RX501PYP/2H6 Spark Plugs). }\end{array}$ \\
\hline \hline
\end{tabular}




\subsubsection{OH1 System Data}

Data from the Woodward OH1 gas engine management system were recorded every 25 hours. Transient data were recorded for one full cycle in one second intervals for engine speed, manifold air pressure (MAP), volumetric efficiency (VE), and engine coolant temperature (ECT). Figure 3 shows the average readings from 1 to 1000 hours from the transient data. There were 40 sets of data recorded. Data at 275 hours were recorded but inadvertently erased and the ECT data at 825 hours were not recorded due to operator error. Figures 4 through 7 show the average for each parameter along with a band of one standard deviation. Figures for individual reading are included in Appendix B.

Data from the OH1 system for injector block leak down were recorded every 25 hours. Data were recorded for two minutes at one second intervals by starting the data acquisition with the ignition key turned off, then turning the ignition key on (without starting the engine) within five seconds after starting the data acquisition. Turning the key on energizes the solenoid in the injector block to allow fuel to pressurize the injector block. When the $\mathrm{OH} 1$ system does not receive a signal that the engine has started, it de-energizes the solenoid, and the $\mathrm{OH} 1$ system records the pressure in the injector block as it leaks down. The static pressure to the injector block (setting of the low pressure regulator) is set to maintain at least $690 \mathrm{kPa}$ (100 psia) when the engine is in the loaded stage. Figure 8 shows the leak down rates calculated during the STC test. There were several leak down rates of zero $\mathrm{kPa} / \mathrm{s}$. A manual shut off valve was closed to verify that the solenoid valve on the injector block was fully closed. There was no indication that the pressure sensor was reading incorrectly. Almost all of the injectors operated for approximately 71 million cycles by the end of the test. However, two injectors were replaced during the course of the test, one at 555 test hours (approx. 615 hours of service, 41 million cycles) and another at 693 test hours (approx. 755 hours of service, 51 million cycles). Further details can be found in the unscheduled maintenance section of this report.

\subsubsection{Oil Consumption}

Dryden experimental natural gas oil was used for the first 500 hours of the STC durability test. Oil samples were taken every 50 test hours and sent directly to Dryden Oil for analysis. Ethyl experimental natural gas oil was used for the second 500 hours of the test. Oil samples were taken, again every 50 test hours, and the analysis was conducted at SwRI (by the Dept. Of Petroleum Products Research, Automotive Products and Emissions Research Division). The results were forwarded to Ethyl and can also be found in Appendix E.

Oil was added and oil consumption was recorded every 50 hours during the STC test. In addition, the level was checked at 25 hour intervals (after each oil add), and oil was added only as needed. The procedure used for recording oil consumption was as follows:

1. While engine is idling, take a small discard sample from the engine and record weight.

2. After taking discard sample, take an oil sample from engine and record weight.

3. After engine is stopped, drain oil from blowby filter housing and record weight.

4. After engine is stopped, record weight of all oil leakage.

5. After the engine has soaked for 10 minutes, fill with oil until level is at the full mark on the dip stick and record weight of oil added. 


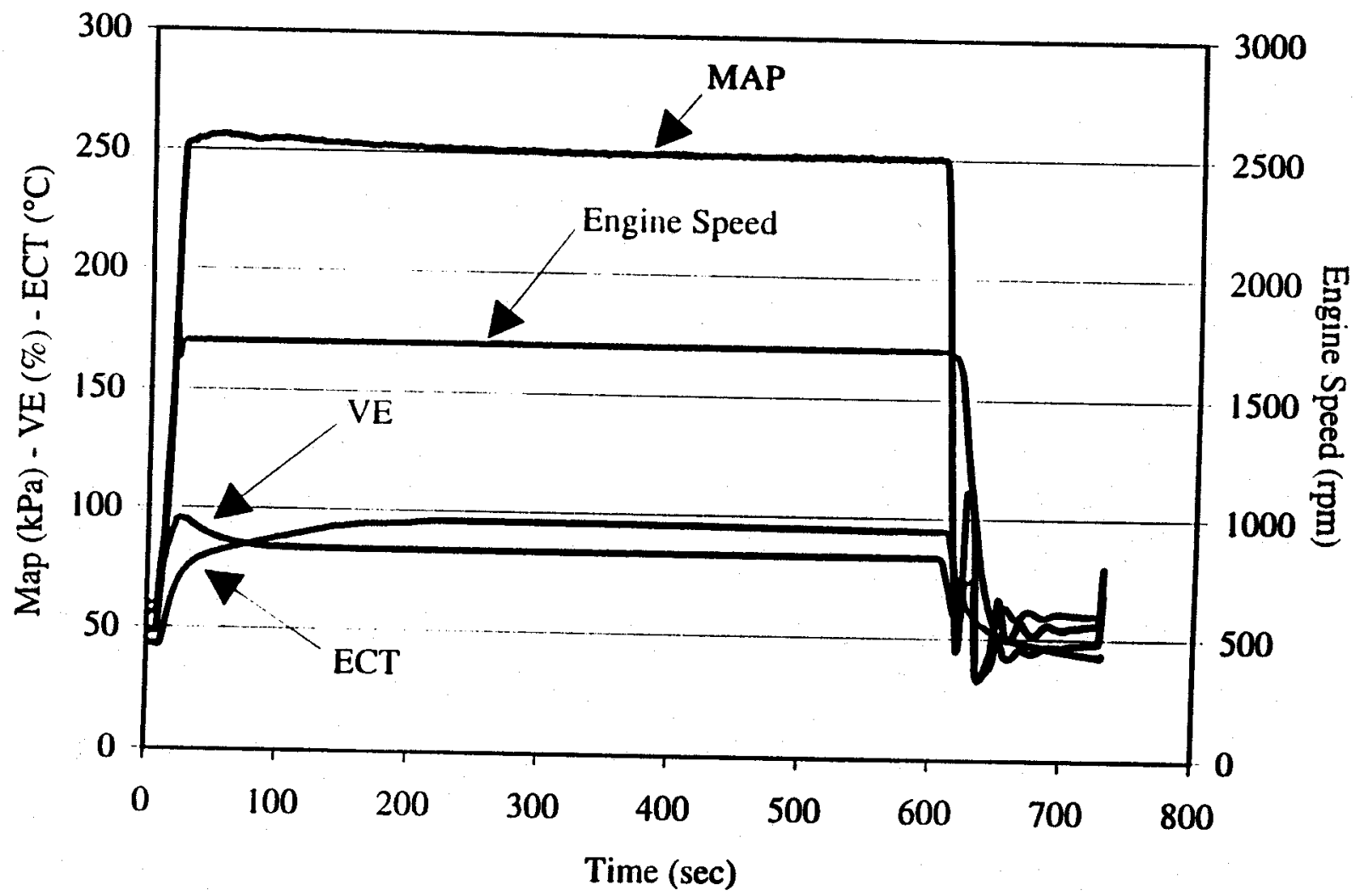

Figure 3. Average of 40 Readings Recorded Between 1 and 1000 Test Hours From the Woodward OH1 During the STC Test 


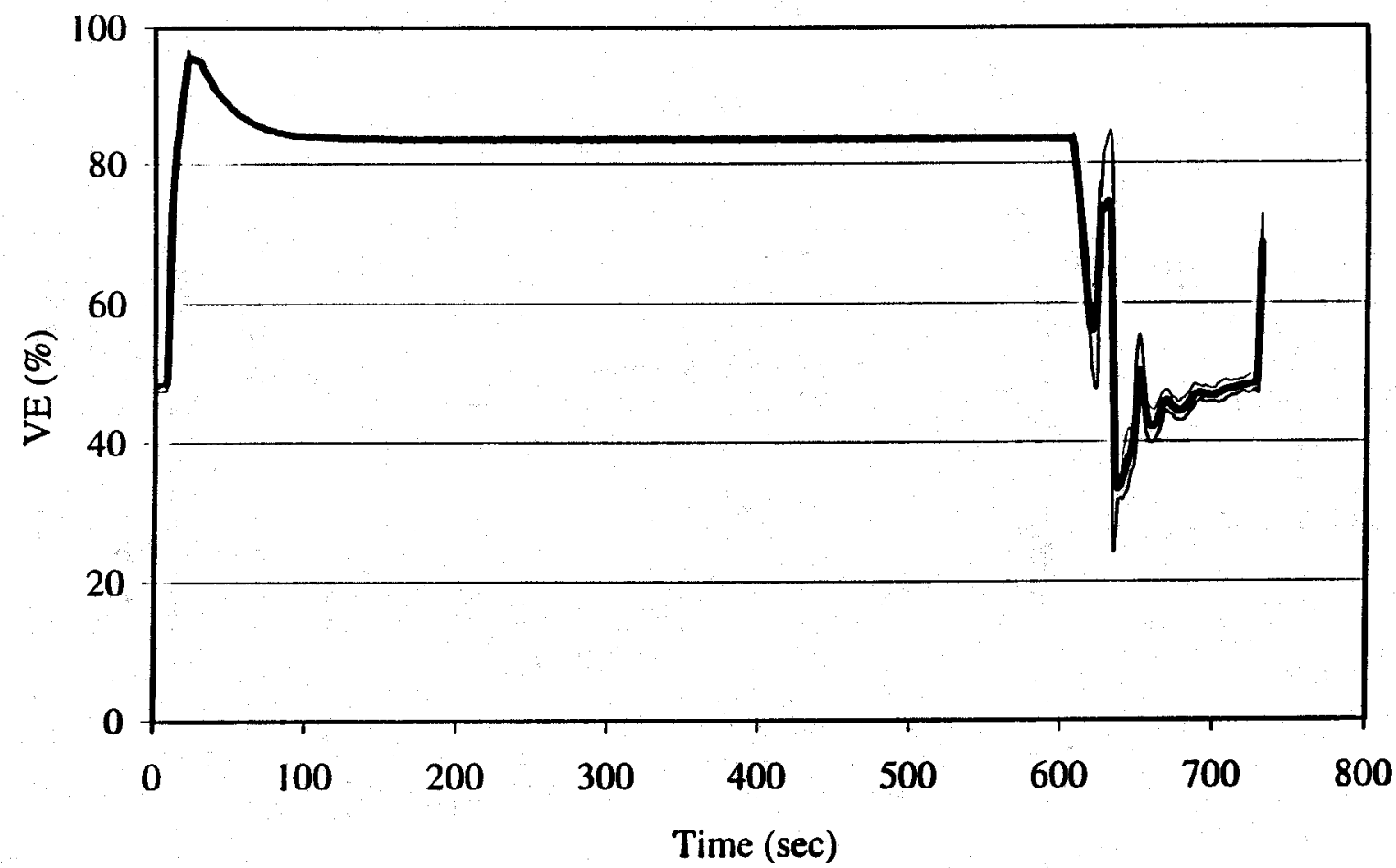

Figure 4. Average of 40 Readings and +/. One Standard Deviation For VE Recorded Between 1 and 1000 Test Hours From the Woodward OH1 During the STC Test 


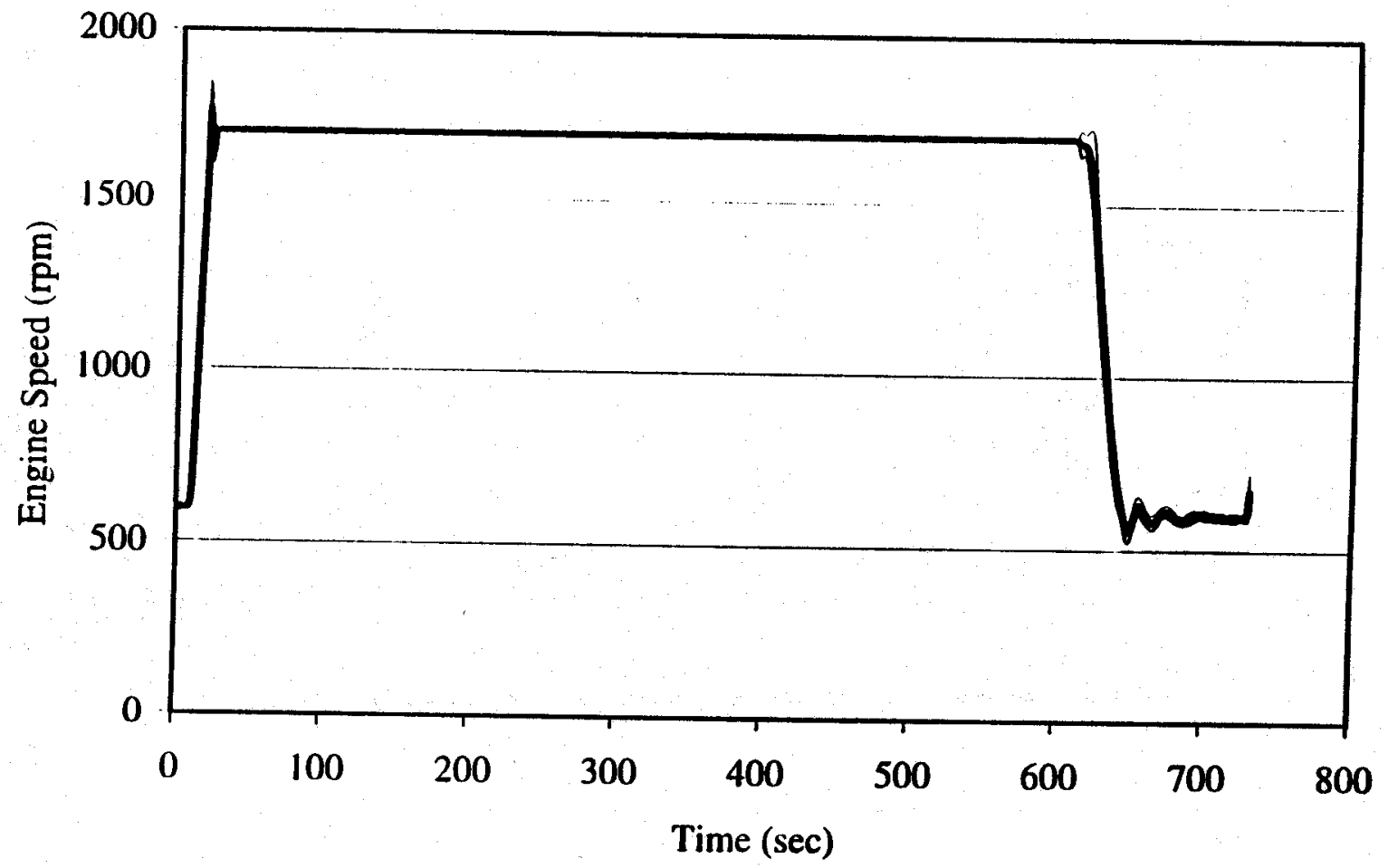

Figure 5. Average of $\mathbf{4 0}$ Readings and +/- One Standard Deviation For Engine Speed Recorded Between 1 and 1000 Test Hours From the Woodward OH1 During the STC Test 


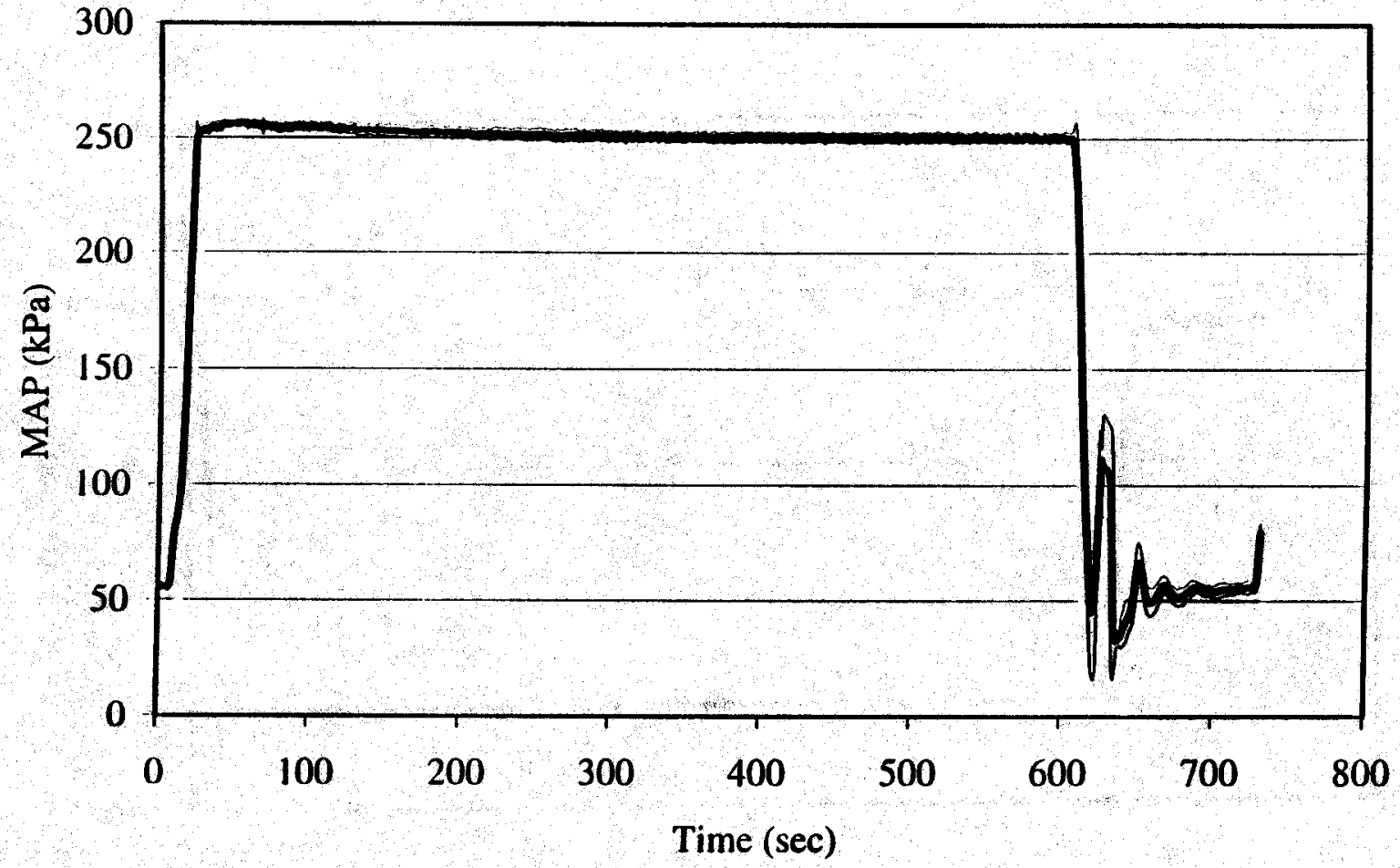

Figure 6. Average of 40 Readings and +/. One Standard Deviation For MAP Recorded Between 1 and 1000 Test Hours From the Woodward OH1 During the STC Test 


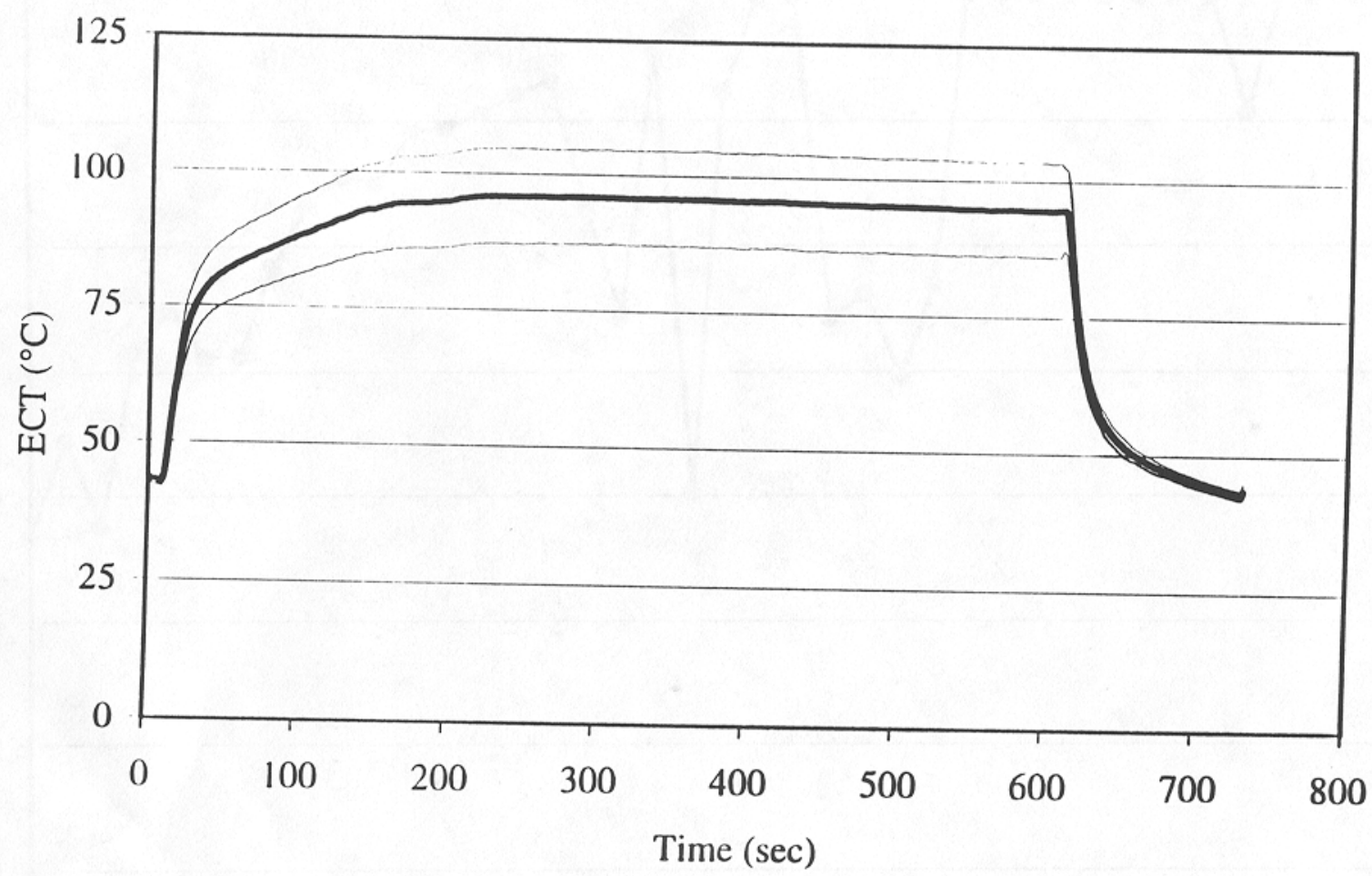

Figure 7. Average of 40 Readings and +/- One Standard Deviation For ECT Recorded Between 1 and 1000 Test Hours From the Woodward OH1 During the STC Test 


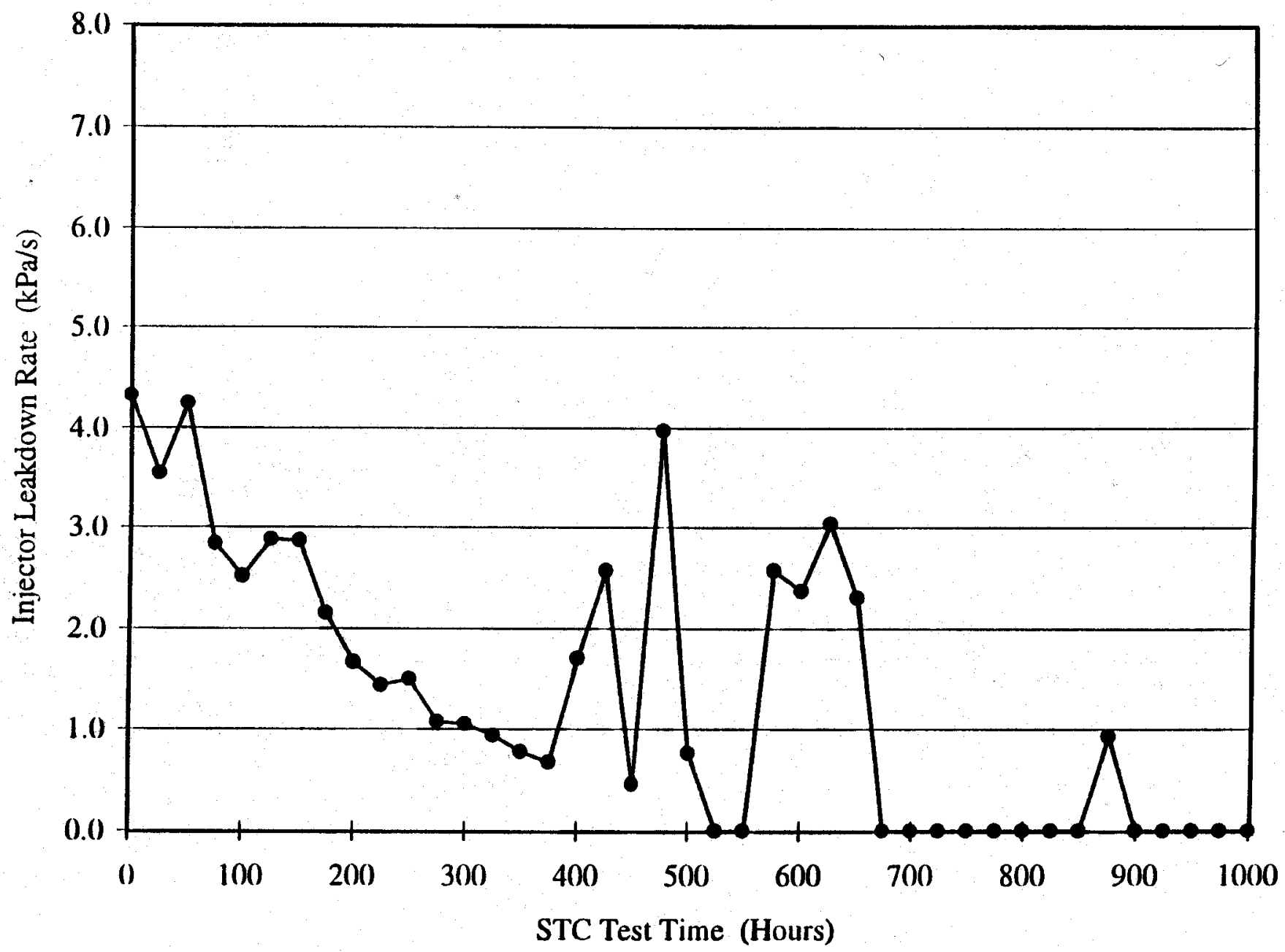

Figure 8. Injector Block Leak Down Rate During the STC Test 
6. Calculate oil consumption from the amount of oil added in step 5, plus the amount of oil added at the 25 hour oil level check (if any), minus the amount of oil drained in steps $1-4$, minus any other oil drains during the 50 hours.

The estimated accuracy of the oil adds is approximately \pm 100 grams. Oil adds and drains are shown in Table 7, and the calculated oil consumption are shown in Table 8 along with the average blowby for each interval. At 100 test hours, the oil added appears abnormally high and may not have been recorded correctly. Therefore, the oil consumption calculation at 100 test hours is probably incorrect. A graph of the oil consumption trend from the STC durability test is shown in Figure 9.

Table 7. Oil Adds and Drains

\begin{tabular}{|c|c|c|c|c|c|}
\hline $\begin{array}{l}\text { Test } \\
\text { Hour }\end{array}$ & $\begin{array}{c}\begin{array}{c}\text { Oil } \\
\text { Add } \\
\text { (grams) }\end{array} \\
\end{array}$ & $\begin{array}{c}\begin{array}{c}\text { Oil Drained } \\
\text { for Discard } \\
\text { (grams) } \\
\end{array} \\
\end{array}$ & $\begin{array}{c}\text { Oil Drained } \\
\text { for Sample } \\
\text { (grams) } \\
\end{array}$ & $\begin{array}{c}\text { Oil Drained } \\
\text { from Leakage } \\
\text { (grams) }\end{array}$ & $\begin{array}{l}\text { Oil Drained from } \\
\text { Blowby } \\
\text { (grams) }\end{array}$ \\
\hline 1 & - & 19 & 142 & - & -- \\
\hline 50 & 1583 & 39 & 149 & 5 & 61 \\
\hline 100 & $(3181)$ & 46 & 141 & 2 & 64 \\
\hline 150 & 1213 & 32 & 142 & 0 & 49 \\
\hline 200 & 1089 & 91 & 146 & 4 & 63 \\
\hline 250 & 1780 & 35 & 144 & 7 & 52 \\
\hline 300 & 1487 & 34 & 150 & 4 & 54 \\
\hline 350 & 930 & 26 & 149 & 6 & 56 \\
\hline 400 & 1020 & 28 & 141 & 4 & 61 \\
\hline 450 & 1493 & 34 & 143 & 5 & 62 \\
\hline 500 & 1425 & 34 & 145 & 8 & 54 \\
\hline 501 & -- & 58 & 214 & - & -- \\
\hline 550 & 2137 & 34 & 207 & 4 & 70 \\
\hline 600 & 1683 & 68 & 210 & 4 & 75 \\
\hline 650 & 1686 & 58 & 217 & 5 & 77 \\
\hline 700 & 1057 & 25 & 213 & 3 & 75 \\
\hline 750 & 2390 & 61 & 209 & 2 & 74 \\
\hline 800 & 1315 & 24 & 217 & 4 & 76 \\
\hline 850 & 1231 & 19 & 214 & 6 & 105 \\
\hline 900 & 2528 & 42 & 228 & 5 & 62 \\
\hline 950 & 1350 & 25 & 216 & 9 & 84 \\
\hline 1000 & 1827 & 24 & 216 & 7 & 53 \\
\hline
\end{tabular}


Table 8. Oil Consumption

\begin{tabular}{|c|c|c|c|c||}
\hline $\begin{array}{c}\text { Test } \\
\text { Hour }\end{array}$ & $\begin{array}{c}\text { Oil } \\
\text { Consumption } \\
\text { (grams) }\end{array}$ & $\begin{array}{c}\text { Oonsumption } \\
\text { (g/kW-hr) }\end{array}$ & $\begin{array}{c}\text { Average Blowby for } \\
\mathbf{6 0 0} \text { rpm Operation } \\
(\text { //min) }\end{array}$ & $\begin{array}{c}\text { Average Blowby for } \\
\text { 1700 rpm Operation } \\
(\text { (/min) }\end{array}$ \\
\hline $0-50$ & 1168 & 0.099 & 5.35 & 147.7 \\
\hline $50-100$ & 2928 & 0.249 & 5.17 & 143.4 \\
\hline $100-150$ & 990 & 0.084 & 5.66 & 142.4 \\
\hline $150-200$ & 785 & 0.067 & 6.01 & 141.8 \\
\hline $200-250$ & 1542 & 0.131 & 6.20 & 144.1 \\
\hline $250-300$ & 1245 & 0.107 & 6.29 & 146.1 \\
\hline $300-350$ & 693 & 0.060 & 6.68 & 146.4 \\
\hline $350-400$ & 786 & 0.068 & 6.61 & 148.2 \\
\hline $400-450$ & 1249 & 0.106 & 7.39 & 145.0 \\
\hline $450-500$ & 1184 & 0.101 & 8.61 & 142.3 \\
\hline $500-550$ & 1550 & 0.133 & 7.50 & 149.8 \\
\hline $550-600$ & 1326 & 0.114 & 6.80 & 150.1 \\
\hline $600-650$ & 1329 & 0.116 & 6.54 & 151.9 \\
\hline $650-700$ & 741 & 0.064 & 5.62 & 153.9 \\
\hline $700-750$ & 2044 & 0.172 & 5.53 & 148.1 \\
\hline $750-800$ & 994 & 0.084 & 6.74 & 154.1 \\
\hline $800-850$ & 887 & 0.075 & 6.59 & 158.6 \\
\hline $850-900$ & 2191 & 0.186 & 7.61 & 161.2 \\
\hline $900-950$ & 1016 & 0.087 & 8.42 & 156.9 \\
\hline $950-1000$ & 1527 & 0.133 & 8.43 & 163.1 \\
\hline \hline $0-1000$ & 26,175 & 0.112 & 6.69 & \\
\hline & & & & \\
\hline
\end{tabular}

\subsubsection{Valve Measurements}

Valve lash and valve stem heights were measured before, during, and after the STC durability test. Results of the intake and exhaust valve stem height measurements or valve recession data are shown in Figures 10 and 11 , respectively. Data for the valve stem height measurements are also included in Appendix F. Valve lash measurements are shown in Table 9. 


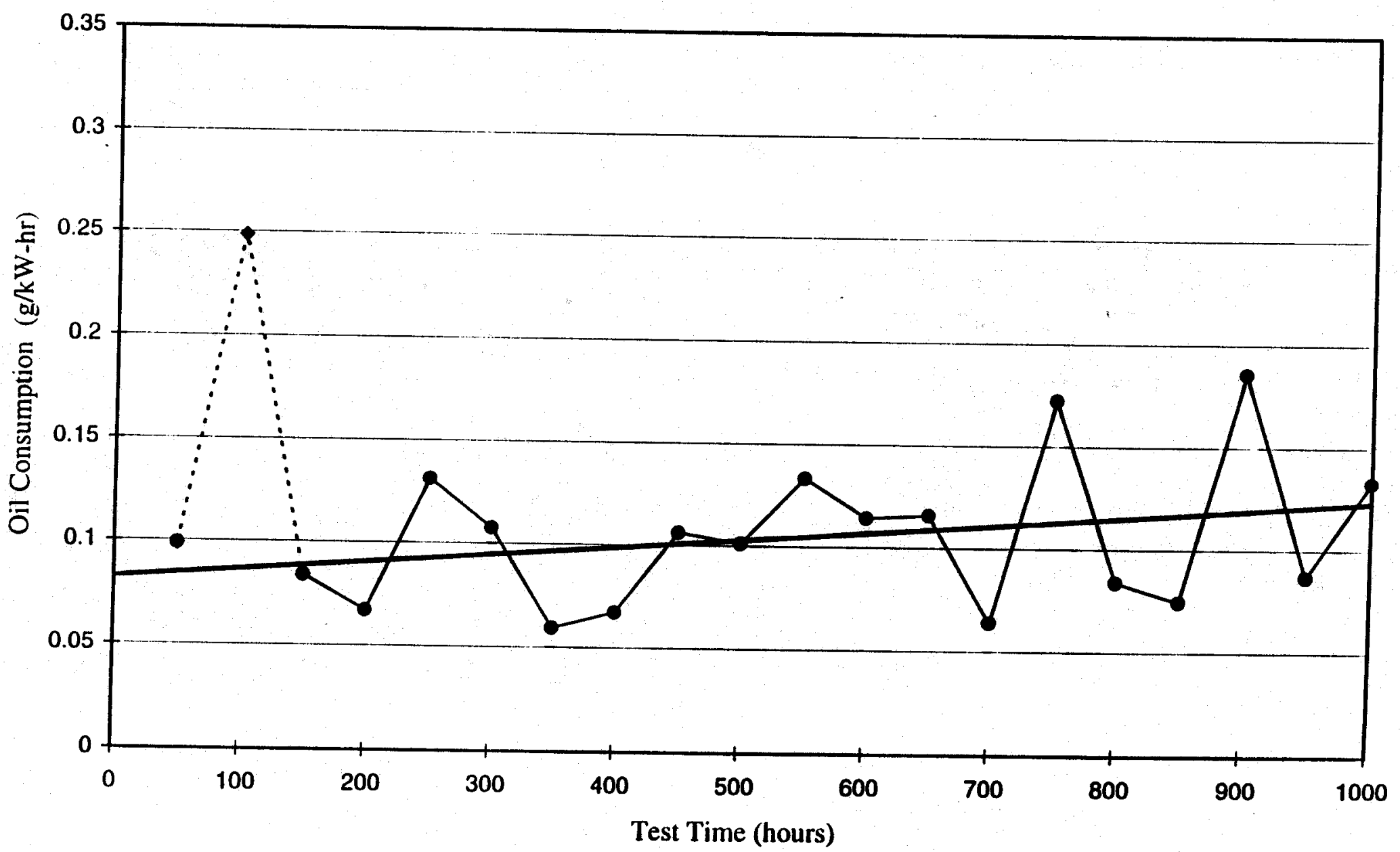

Figure 9. Oil Consumption Trend 


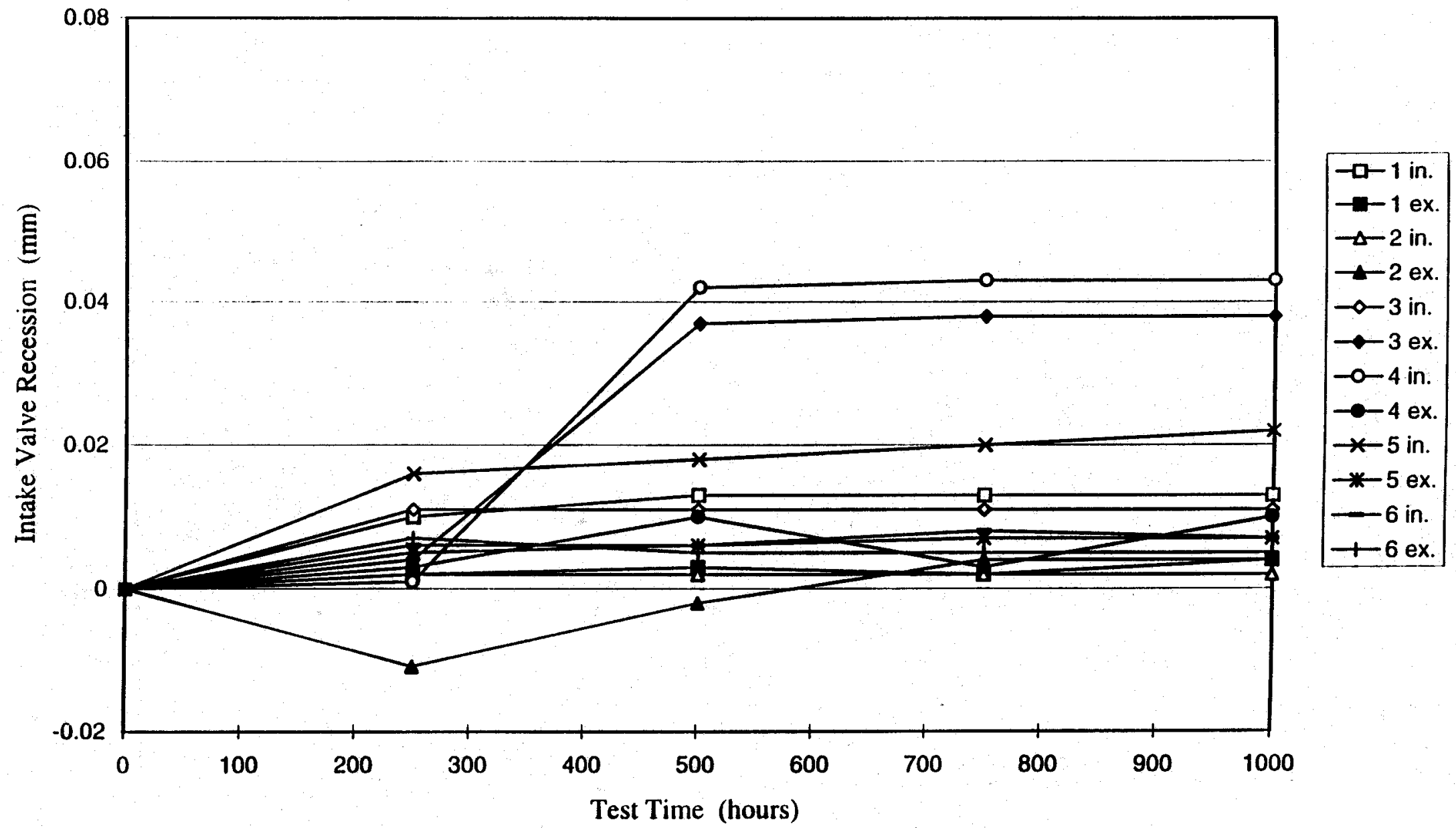

Figure 10. Intake Valve Recession 


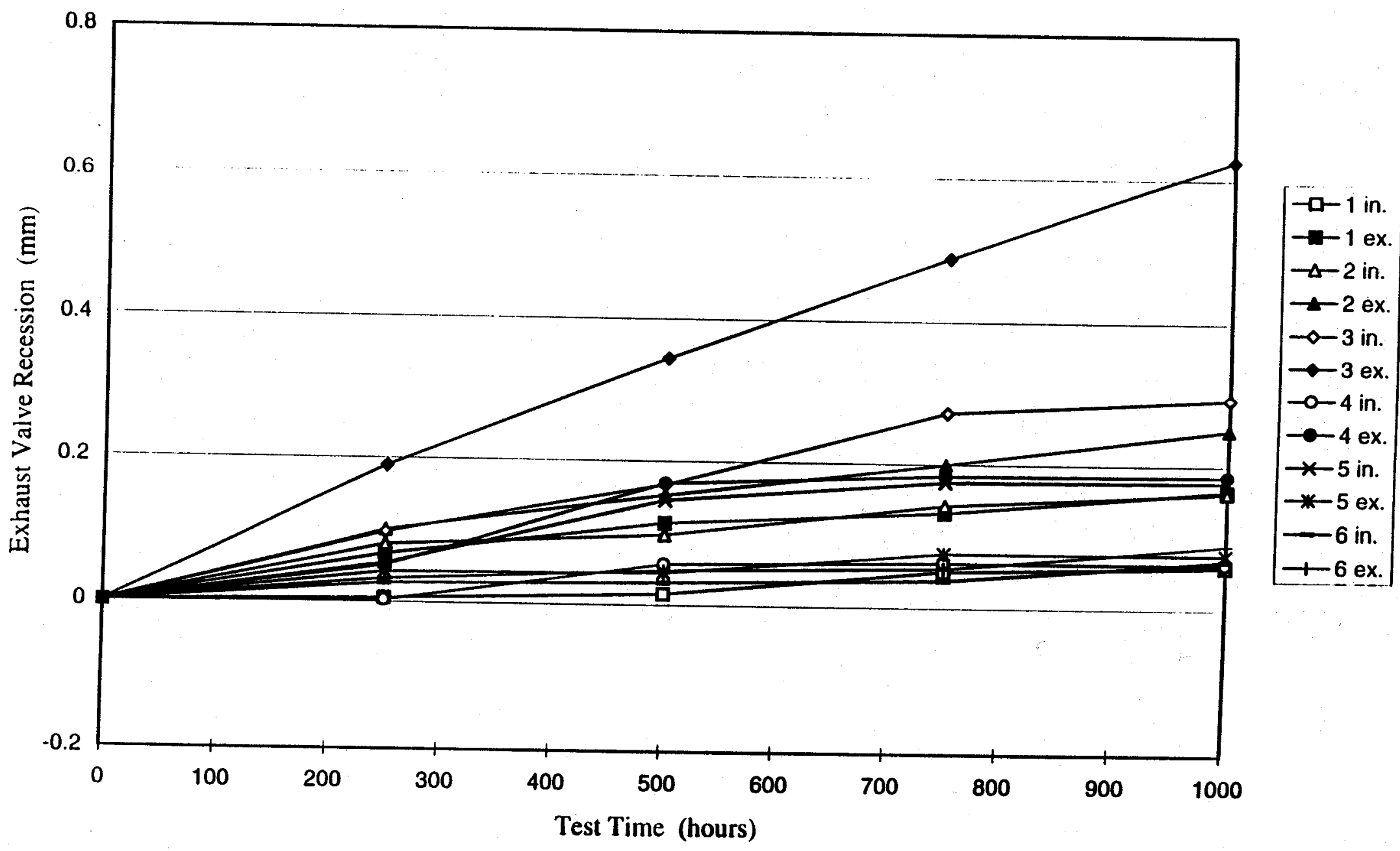

Figure 11. Exhaust Valve Recession 
Table 9. Valve Lash Measurements

\begin{tabular}{|c|c|c|c|c|c|c||}
\hline \multirow{2}{*}{ Cylinder } & \multirow{2}{*}{ Valve } & \multicolumn{5}{|c|}{ Valve Lash (mm) @ STC Test Hours } \\
\cline { 3 - 7 } & & 0.0 & 250.0 & 500.0 & 750.0 & $\mathbf{1 0 0 0 . 0}$ \\
\hline 1 & in. & 0.41 & 0.38 & 0.38 & 0.38 & 0.41 \\
\hline 2 & in. & 0.41 & 0.41 & 0.41 & 0.41 & 0.41 \\
\hline 3 & in. & 0.41 & 0.38 & 0.38 & 0.38 & 0.38 \\
\hline 4 & in. & 0.41 & 0.41 & 0.41 & 0.41 & 0.38 \\
\hline 5 & in. & 0.41 & 0.41 & 0.43 & 0.41 & 0.43 \\
\hline 6 & in. & 0.41 & 0.43 & 0.43 & 0.43 & 0.43 \\
\hline 1 & ex. & 0.71 & 0.66 & 0.64 & 0.64 & 0.61 \\
\hline 2 & ex. & 0.71 & 0.61 & 0.58 & 0.53 & 0.51 \\
\hline 3 & ex. & 0.71 & 0.58 & $0.48 *$ & 0.61 & 0.48 \\
\hline 4 & ex. & 0.71 & 0.69 & 0.64 & 0.61 & 0.61 \\
\hline 5 & ex. & 0.71 & 0.69 & 0.66 & 0.64 & 0.61 \\
\hline 6 & ex. & 0.71 & 0.66 & 0.66 & 0.66 & 0.64 \\
\hline$*$ Reset Exhaust Valve Lash to Specifications (0.71 mm) \\
\hline
\end{tabular}

\subsubsection{Spark Plugs Maintenance}

Champion spark plugs were used for the STC durability test. New spark plugs were installed in the engine before the start of the test. All new spark plugs installed in the engine were initially gapped to $0.25 \mathrm{~mm}\left(0.010^{\prime \prime}\right)$. The spark plug gaps were measured every 250 test hours and the gap growth rate can be seen in Figure 12. The gaps were reset to $0.25 \mathrm{~mm}\left(0.010^{\prime \prime}\right)$ at 500 test hours.

\subsubsection{Cylinder Compression and Leak Down Measurements}

Cylinder compression and leak down measurements were recorded every 250 test hours on the STC cycle. The results are shown in Tables 10 and 11, respectively. Cylinder leak down was performed by regulating air upstream of a $1.02 \mathrm{~mm}(0.040$ ") orifice at $690 \mathrm{kPa}$ gage $(100 \mathrm{psig})$ and measuring the pressure downstream of the orifice (cylinder pressure). The percent leak down is the difference between the upstream and downstream pressure normalized by the upstream pressure. 


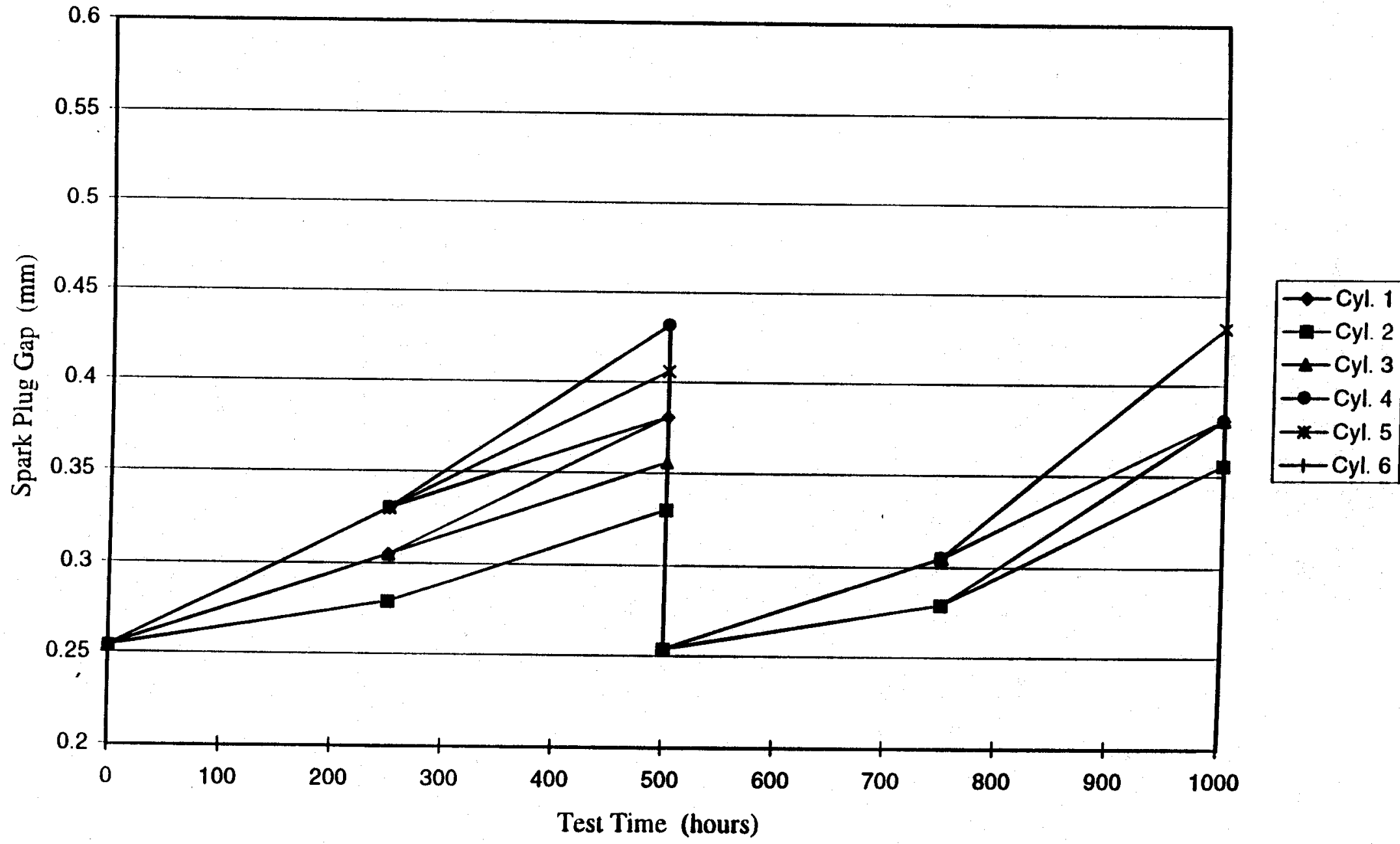

Figure 12. Spark Plug Gap Growth 
Table 10. Cylinder Compression Pressures

\begin{tabular}{||c||c|c|c|c|c||}
\hline \multicolumn{7}{|c|}{ Compression @ 250 rpm (KPa } \\
\hline \hline Total Engine Hours & 291.5 & $\mathbf{5 6 0 . 8}$ & $\mathbf{8 1 4 . 5}$ & $\mathbf{1 0 6 7 . 4}$ & $\mathbf{1 3 2 2 . 4}$ \\
\hline Hours on STC Test & $\mathbf{0 . 0}$ & $\mathbf{2 5 0 . 0}$ & $\mathbf{5 0 0 . 0}$ & $\mathbf{7 5 0 . 0}$ & $\mathbf{1 0 0 0 . 0}$ \\
\hline Cylinder No. 1 & 1987 & 1918 & 1945 & 1904 & 1891 \\
\hline Cylinder No. 2 & 1960 & 1987 & 2001 & 1960 & 1946 \\
\hline Cylinder No. 3 & 1973 & 2015 & 2015 & 1953 & 1967 \\
\hline Cylinder No. 4 & 1987 & 2036 & 2036 & 1973 & 1973 \\
\hline Cylinder No. 5 & 1973 & 2042 & 2029 & 1987 & 1980 \\
\hline Cylinder No. 6 & 2001 & 2029 & 2029 & 1987 & 1967 \\
\hline
\end{tabular}

Table 11. Cylinder Pressure Leak down

\begin{tabular}{||c||c|c|c|c|c||}
\hline \multicolumn{7}{|c|}{ Leak Down (\%) } \\
\hline Total Engine Hours & $\mathbf{2 9 1 . 5}$ & $\mathbf{5 6 0 . 8}$ & $\mathbf{8 1 4 . 5}$ & $\mathbf{1 0 6 7 . 4}$ & $\mathbf{1 3 2 2 . 4}$ \\
\hline Hours on STC Test & $\mathbf{0 . 0}$ & $\mathbf{2 5 0 . 0}$ & $\mathbf{5 0 0 . 0}$ & $\mathbf{7 5 0 . 0}$ & $\mathbf{1 0 0 0 . 0}$ \\
\hline Cylinder No. 1 & 48 & 52 & 56 & 35 & 57 \\
\hline Cylinder No. 2 & 27 & 10 & 12 & 7 & 8 \\
\hline Cylinder No. 3 & 5 & 14 & 8 & 4 & 4 \\
\hline Cylinder No. 4 & 33 & 20 & 17 & 13 & 22 \\
\hline Cylinder No. 5 & 20 & 10 & 18 & 7 & 6 \\
\hline Cylinder No. 6 & 20 & 8 & 5 & 4 & 8 \\
\hline
\end{tabular}

\subsubsection{Coolant Flow Measurements}

Coolant flow was measured every 500 hours on the STC cycle. A turbine flow meter was installed in the coolant line between the outlet of the engine and the gate valve for the thermal "shock test setup. Coolant flow was measured at a typical valve position during the unloaded and loaded stages of the cycle. The results are shown in Table 12. 
Table 12. Coolant Flow Measurements

\begin{tabular}{|c|c|c|}
\hline $\begin{array}{c}\text { Hours on } \\
\text { STC Test }\end{array}$ & $\begin{array}{c}\text { Coolant Flow at } \\
\text { Unloaded Stage } \\
(\mathbf{l} / \mathbf{m})\end{array}$ & $\begin{array}{c}\text { Coolant Flow at } \\
\text { Loaded Stage } \\
(\mathbf{l} / \mathbf{m})\end{array}$ \\
\hline \hline 0.0 & 321 & 362 \\
\hline 500.0 & 314 & 365 \\
\hline 1000.0 & 313 & 366 \\
\hline
\end{tabular}

\subsubsection{Maintenance/ Unscheduled Shutdowns}

Major maintenance items before the start of the STC test included a rebuild of the front cylinder head, at 161.4 total engine hours, due to an exhaust valve failure on the number three cylinder. Also included were the replacement of the UEGO sensor, at 64.4 total engine hours, due to an incorrect equivalence ratio readings and the replacement of the injectors, at 232.2 total engine hours, due to slow reacting injectors.

Major maintenance items during the STC test included a misfire problem caused by noise on the mag pickup signal. The mag pickup was shimmed out by 0.020 inches. The throttle was replaced at 871.8 total engine hours due to a "sticking" problem. Two injectors were replaced at 871 and 1010 total engine hours due to "sticking".

Shutdowns for the engine, during the STC test were scheduled for every 25 test hours. Unscheduled and/or extended shutdowns, along with unscheduled maintenance events, are shown in Table 13.

Table 13. Unscheduled Shutdowns/Maintenance During STC Test

\begin{tabular}{||l|c|c|l||}
\hline Date & $\begin{array}{c}\text { Total } \\
\text { Engine } \\
\text { Time } \\
\text { (Hours) }\end{array}$ & $\begin{array}{c}\text { STC } \\
\text { Test } \\
\text { Time } \\
\text { (Hours) }\end{array}$ & \multicolumn{1}{|c|}{ Description } \\
\hline \hline $7 / 22 / 98$ & 311.7 & 7.2 & “AL_Lim" low fault on OH1. Noted occasional misfire. \\
\hline $7 / 23 / 98$ & 322.4 & 17.9 & $\begin{array}{l}\text { Engine running erratic. Stopped engine and found broken } \\
\text { wire to fuel metering block shut-off valve. Repaired wire } \\
\text { and restarted engine. }\end{array}$ \\
\hline $7 / 24 / 98$ & 332.6 & 27.2 & $\begin{array}{l}\text { Idle speed high at unloaded stage }(\sim 1100 \text { rpm). Stopped } \\
\text { engine. No faults or problems found. Restarted engine. }\end{array}$ \\
\hline $7 / 25 / 98$ & 335.3 & 39.4 & $\begin{array}{l}\text { Idle speed erratic. No faults found. Stopped and restarted } \\
\text { engine. Idle speed still erratic. }\end{array}$ \\
\hline
\end{tabular}


Table 13. Unscheduled Shutdowns/Maintenance During STC Test - Cont'd

\begin{tabular}{||l|c|c|l||}
\hline \hline Date & $\begin{array}{c}\text { Total } \\
\text { Engine } \\
\text { Time } \\
\text { (Hours) }\end{array}$ & $\begin{array}{c}\text { STC } \\
\text { Test } \\
\text { Time } \\
\text { (Hours) }\end{array}$ & \multicolumn{1}{|c||}{ Description } \\
\hline $7 / 25 / 98$ & 350.9 & 45.0 & $\begin{array}{l}\text { Idle speed erratic. Found pinched and/or shorted wire } \\
\text { (RPM_targ_def). Repaired wire (w/engine running). }\end{array}$ \\
\hline $7 / 25 / 98$ & 354.9 & 49.0 & $\begin{array}{l}\text { Engine over-speeds/surges during ramp to loaded stage. } \\
\text { "Inj DutyCycle" fault noted. Also occasional "NGP Exp" } \\
\text { low fault noted. Cleared faults and continued test. }\end{array}$ \\
\hline $7 / 26 / 98$ & 382.4 & 76.0 & $\begin{array}{l}\text { Idle speed high ( 700 rpm). Reset by turning ignition key } \\
\text { off and back on at idle stage. }\end{array}$ \\
\hline $7 / 27 / 98$ & 392.9 & 86.5 & $\begin{array}{l}\text { Multiple faults noted including "NGP Exp" low, "Boost } \\
\text { Exp" low, "PTP Active", and Inj DutyCycle. Also an } \\
\text { occasional "Control Closed Loop". Reset and continue. }\end{array}$ \\
\hline $7 / 27 / 98$ & 400.5 & 94.1 & $\begin{array}{l}\text { Stopped engine to repair broken mounting bolt. Adjust } \\
\text { ramp rates due to over-speed condition. Also adjust OH1 } \\
\text { System controller due to continuous faults. Restarted } \\
\text { engine. }\end{array}$ \\
\hline $7 / 27 / 98$ & 413.2 & 106.2 & $\begin{array}{l}\text { Boost hose failure (Lab). Stopped engine and replaced } \\
\text { boost hose. Restarted engine. }\end{array}$ \\
\hline $7 / 28 / 98$ & 427.4 & 120.1 & $\begin{array}{l}\text { Stopped engine to repair water pump/drive motor belt } \\
\text { guard (Lab). Restarted engine. }\end{array}$ \\
\hline $7 / 28 / 98$ & 428.3 & 120.7 & $\begin{array}{l}\text { Engine stopped. "NGP Exp" low fauit noted. Repaired } \\
\text { broken wire to injector block solenoin. Restarted engine. }\end{array}$ \\
\hline $7 / 29 / 98$ & 440.2 & 132.0 & $\begin{array}{l}\text { Idle speed High ( 1000 rpm). Reset oy turning ignition } \\
\text { key off and back on at idle stage. }\end{array}$ \\
\hline & 458.4 & 150.0 & $\begin{array}{l}\text { Scheduled shutdown - Found broken mounting bolt. } \\
\text { Replaced all mount boits, repaired engine mount, and } \\
\text { realigned w/dyno. Also replaced wire harness w/new one. }\end{array}$ \\
\hline & 461.6 & 152.7 & $\begin{array}{l}\text { Intermittent misfires. Stopped engine to troubleshoot. } \\
\text { Replaced ignition harness and changed ignition timing to } \\
\text { MBT at 22 deg BTDC (3 deg retard). Restarted engine. }\end{array}$ \\
\hline
\end{tabular}


Table 13. Unscheduled Shutdowns/Maintenance During STC Test - Cont'd

\begin{tabular}{|c|c|c|c|}
\hline Date & $\begin{array}{l}\text { Total } \\
\text { Engine } \\
\text { Time } \\
\text { (Hours) } \\
\end{array}$ & $\begin{array}{c}\text { STC } \\
\text { Test } \\
\text { Time } \\
\text { (Hours) } \\
\end{array}$ & Description \\
\hline 7/31/98 & 484.5 & 175.0 & $\begin{array}{l}\text { Scheduled shutdown - Changed to new Blowby cannister } \\
\text { assembly (supplied by Mack). Reinstalled original } \\
\text { ignition harness. Inserted } 0.020^{\prime \prime} \text { shims under cam gear } \\
\text { speed sensor due to false pulses causing misfires. }\end{array}$ \\
\hline $8 / 3 / 98$ & 543.3 & 233.2 & $\begin{array}{l}\text { Boost hose leaking (Lab). Stopped engine and replaced } \\
\text { boost hose. Restarted engine. }\end{array}$ \\
\hline $8 / 3 / 98$ & 546.2 & 236.0 & $\begin{array}{l}\text { Idle speed high }(\sim 750 \mathrm{rpm}) . \text { Reset by turning ignition key } \\
\text { off and back on at idle stage. }\end{array}$ \\
\hline $8 / 4 / 98$ & 560.8 & 250.0 & $\begin{array}{l}\text { Scheduled shutdown - Replaced CNG metering block } \\
\text { shut-off solenoid. Also replaced turbocharger wastegate } \\
\text { actuator due to leaking diaphragm. }\end{array}$ \\
\hline $8 / 10 / 98$ & 701.7 & 390.0 & $\begin{array}{l}\text { Stopped engine to investigate misfire problem (per } \\
\text { Mack's request). Removed shims under cam sensor and } \\
\text { observe engine. Misfires occurred w/o shims. Reinstall } \\
\text { shims and restarted engine. }\end{array}$ \\
\hline $8 / 13 / 98$ & 766.4 & 453.1 & Stopped engine to run engine on adjacent test stand. \\
\hline $8 / 14 / 98$ & 788.4 & 475.0 & $\begin{array}{l}\text { Scheduled shutdown - Left engine shutdown to run engine } \\
\text { on adjacent test stand. }\end{array}$ \\
\hline $8 / 17 / 98$ & 813.7 & 500.0 & $\begin{array}{l}\text { Scheduled shutdown - Replaced rear head valve cover } \\
\text { gasket due to leak (discolored, burnt, brittle from heat). } \\
\text { Reset spark plug gaps to } 0.010^{\prime \prime} \text { and reset Cyl. No. } 3 \\
\text { exhaust lash from 0.019" to } 0.028 " \text {. Also inspected } \\
\text { pistons for cracks (per Mack's request) using a bore scope } \\
\text { with video camera to document piston conditions. }\end{array}$ \\
\hline $8 / 19 / 98$ & 839.6 & 525.0 & $\begin{array}{l}\text { Scheduled shutdown - Installed valve before } \mathrm{CNG} \\
\text { metering block to verify injector leak down rate data. }\end{array}$ \\
\hline $8 / 20 / 98$ & 859.9 & 545.1 & $\begin{array}{l}\text { Engine did not ramp up to loaded condition. "TPS Exp" } \\
\text { low and "Boost Exp" low faults were noted. Reset by } \\
\text { turning ignition key off and back on at idle. Resumed test. } \\
\text { Faults continued intermittently. }\end{array}$ \\
\hline $8 / 20 / 98$ & 864.8 & 550.0 & $\begin{array}{l}\text { Scheduled shutdown - Removed throttle unit for } \\
\text { inspection due to possible binding/sticking. Cleaned and } \\
\text { reinstalled unit. Restarted engine. }\end{array}$ \\
\hline
\end{tabular}


Table 13. Unscheduled Shutdowns/Maintenance During STC Test - Cont'd

\begin{tabular}{|c|c|c|c|}
\hline Date & $\begin{array}{l}\text { Total } \\
\text { Engine } \\
\text { Time } \\
\text { (Hours) }\end{array}$ & $\begin{array}{c}\text { STC } \\
\text { Test } \\
\text { Time } \\
\text { (Hours) }\end{array}$ & Description \\
\hline $8 / 21 / 98$ & 870.8 & 555.4 & $\begin{array}{l}\text { Engine stopped. Found bad injector (stuck open). } \\
\text { Replaced w/new injector (m/n SP021S1). Restarted } \\
\text { engine. }\end{array}$ \\
\hline $8 / 21 / 98$ & 871.8 & 556.1 & $\begin{array}{l}\text { Engine not ramping down/hanging up. Stopped engine. } \\
\text { Found throttle unit sticking again. Replaced w/new } \\
\text { throttle unit (p/n 8535-124). Restarted engine. }\end{array}$ \\
\hline $8 / 27 / 98$ & 1009.9 & 693.4 & $\begin{array}{l}\text { Engine erratic w/misfires. Stopped engine. Found bad } \\
\text { injector (slow response). Replaced w/new injector }(\mathrm{m} / \mathrm{n} \\
\text { SP021S1). Restarted engine. }\end{array}$ \\
\hline $8 / 31 / 98$ & 1066.9 & 750.0 & $\begin{array}{l}\text { Scheduled shutdown - Changed adsorption filter element } \\
\text { on fuel system due to oil/water drained. Also inspect } \\
\text { pistons for cracks using bore scope with video camera. }\end{array}$ \\
\hline $9 / 4 / 98$ & 1153.1 & 835.3 & $\begin{array}{l}\text { Lab off-line due to power failure. Stopped engine. Reset } \\
\text { hardware and restarted engine. }\end{array}$ \\
\hline $9 / 4 / 98$ & 1167.8 & 850.0 & $\begin{array}{l}\text { Scheduled shutdown - Left engine down for Holiday } \\
\text { weekend. Restated engine on } 9 / 8 / 98 \text {. }\end{array}$ \\
\hline 9/9/98 & 1204.1 & 885.7 & $\begin{array}{l}\text { Dyno case temperature safety tripped (Lab). Reset and } \\
\text { brought engine back up to loaded stage. Resumed test. }\end{array}$ \\
\hline $9 / 11 / 98$ & 1231.5 & 912.8 & $\begin{array}{l}\text { Stopped engine due to operator shift change arrived late. } \\
\text { Restarted engine. }\end{array}$ \\
\hline $9 / 11 / 98$ & 1254.1 & 935.0 & $\begin{array}{l}\text { Stopped engine for Lab weekend shutdown. Restarted } \\
\text { engine on } 9 / 14 / 98 \text {. }\end{array}$ \\
\hline $9 / 14 / 98$ & 1272.0 & 950.6 & $\begin{array}{l}\text { Engine not ramping up to loaded condition. "Boost Exp" } \\
\text { low fault. Turned ignition key off and on. Resumed test. }\end{array}$ \\
\hline $9 / 14 / 98$ & 1276.7 & 955.3 & $\begin{array}{l}\text { Engine not ramping up to loaded condition. "Boost Exp" } \\
\text { low fault. Turned ignition key off and on. Resumed test. }\end{array}$ \\
\hline $9 / 15 / 98$ & 1300.5 & 979.0 & $\begin{array}{l}\text { Idle speed high ( } 750 \mathrm{rpm}) \text {. "FPP" fault noted. Turned } \\
\text { ignition key off and on to reset. Resumed test. }\end{array}$ \\
\hline
\end{tabular}




\subsection{CONCLUSIONS \& RECOMMENDATIONS}

A 1000 hour durability test was successfully completed. Several conclusions regarding the technical content of the program were made.

1. The number three exhaust valve on the exhaust side showed high valve recession. This may be due to a problem caused by the rebuild of the cylinder heads when this exhaust valve was bent.

2. Injector block leak down rates were measured at zero $\mathrm{kPa} / \mathrm{sec}$ during the test. Tests were conducted which confirmed the zero leak down rate. A zero leak down rate indicates no leakage through the injectors. Normally, there is a small amount of leakage through the injectors when they are closed.

3. Two injectors were replaced during the STC test. The injectors were "sticking" where the injectors were not fully closing. Further investigation into this problem should be conducted to avoid problems in the field.

4. The spark plugs gap growth showed that a regap interval of 500 hours was possible during the STC test.

5. A borescope was used to inspect the pistons for cracks at 500, 750, and 1000 test hours. No indications of piston cracking were noted.

6. The blowby filter showed no signs of degradation during the 1000 hour STC test.

7. An investigation of the high exhaust valve recession on the number three cylinder on the exhaust side should be conducted. 
APPENDIX A

LISTING OF HP DAQ RECORDS 


\section{HP DAQ RECORDS FROM STC DURABILITY TEST}

\begin{tabular}{|c|c|c|c|c|c|c|}
\hline $\begin{array}{l}\text { Run \# } \\
\text { From }\end{array}$ & $\begin{array}{c}\text { Run \# } \\
\text { To }\end{array}$ & $\begin{array}{c}\text { Test Hours } \\
\text { From }\end{array}$ & $\mid \begin{array}{c}\text { Test Hours } \\
\text { To }\end{array}$ & $\begin{array}{l}\text { Hourly } \\
\text { Data }\end{array}$ & $\begin{array}{c}\text { Snapshot } \\
\text { Data }\end{array}$ & Comments \\
\hline 392 & 393 & () & 0 & $x$ & & \\
\hline 394 & 449 & $\overline{0}$ & 0 & & $x$ & \\
\hline 450 & 483 & 1 & 17 & $x$ & & \\
\hline 484 & 484 & 18 & 18 & $x$ & & Engine stopped, reading aborted \\
\hline 485 & 498 & 18 & 24 & $x$ & & \\
\hline 499 & 554 & 25 & 25 & & $x$ & \\
\hline 555 & 556 & 25 & 25 & $x$ & & \\
\hline 557 & 604 & 26 & 49 & $x$ & & \\
\hline 605 & 660 & 50 & 50 & & $x$ & \\
\hline 661 & 662 & 50 & 50 & $x$ & & \\
\hline 663 & 710 & 51 & 74 & $x$ & & \\
\hline 711 & 766 & 75 & 75 & & $x$ & \\
\hline 767 & 768 & 75 & 75 & $x$ & & \\
\hline 769 & 816 & 76 & 99 & $x$ & & \\
\hline 817 & 872 & 100 & 100 & & $x$ & \\
\hline 873 & 874 & 100 & 100 & $x$ & & \\
\hline 875 & 922 & 101 & 124 & $x$ & & \\
\hline 923 & 978 & 125 & 125 & & $x$ & \\
\hline 979 & 980 & 125 & 125 & $x$ & & \\
\hline 981 & 1028 & 126 & 149 & $x$ & & \\
\hline 1029 & 1084 & 150 & 150 & & $x$ & \\
\hline 1085 & 1086 & 150 & 150 & $x$ & & \\
\hline 1087 & 1134 & 151 & 174 & $x$ & & \\
\hline 1135 & 1190 & 175 & 175 & & $x$ & \\
\hline 1191 & 1192 & 175 & 175 & $x$ & & \\
\hline 1193 & 1240 & 176 & 199 & $x$ & & \\
\hline 1241 & 1296 & 200 & 200 & & $x$ & \\
\hline 1297 & 1298 & 200 & 200 & $x$ & & \\
\hline 1299 & 1346 & 201 & 224 & $x$ & & \\
\hline 1347 & 1402 & 225 & 225 & & $x$ & \\
\hline 1403 & 1404 & 225 & 225 & $x$ & & \\
\hline \multirow[t]{2}{*}{1405} & 1428 & 226 & 237 & $x$ & & \\
\hline & & 238 & 238 & $x$ & & Missed reading \\
\hline 1429 & 1450 & 239 & 249 & $x$ & & \\
\hline 1451 & 1506 & 250 & 250 & & $x$ & \\
\hline 1507 & 1508 & 250 & 250 & $x$ & & \\
\hline 1509 & 1556 & 251 & 274 & $x$ & & \\
\hline 1557 & 1612 & 275 & 275 & & $x$ & \\
\hline 1613 & 1614 & 275 & 275 & $x$ & & \\
\hline 1615 & 1662 & 276 & 299 & $x$ & & \\
\hline 1663 & 1718 & 300 & 300 & & $x$ & \\
\hline 1719 & 1720 & 300 & 300 & $x$ & & \\
\hline 1721 & 1768 & 301 & 324 & $x$ & & \\
\hline 1769 & 1824 & 325 & 325 & & $x$ & \\
\hline 1825 & 1826 & 325 & 325 & $x$ & & \\
\hline 1827 & 1874 & 326 & 349 & $x$ & & \\
\hline 1875 & 1930 & 350 & 350 & & $x$ & \\
\hline 1931 & 1932 & 350 & 350 & $x$ & & \\
\hline
\end{tabular}


HP DAQ RECORDS FROM STC DURABILITY TEST

\begin{tabular}{|c|c|c|c|c|c|c|}
\hline $\begin{array}{l}\text { Run \# } \\
\text { From }\end{array}$ & $\begin{array}{l}\text { Run \# } \\
\text { To }\end{array}$ & $\begin{array}{c}\text { Test Hours } \\
\text { From }\end{array}$ & $\begin{array}{c}\text { Test Hours } \\
\text { To }\end{array}$ & $\begin{array}{l}\text { Hourly } \\
\text { Data }\end{array}$ & $\begin{array}{l}\text { Snapshot } \\
\text { Data }\end{array}$ & Comments \\
\hline 1933 & 1980 & 351 & 374 & $x$ & & \\
\hline 1981 & 2036 & 375 & 375 & & $x$ & \\
\hline 2037 & 2038 & 375 & 375 & $\mathrm{x}$ & & \\
\hline 2039 & 2086 & 376 & 399 & $x$ & & \\
\hline 2087 & 2142 & 400 & 400 & & $x$ & \\
\hline 2143 & 2144 & 400 & 400 & $x$ & & \\
\hline 2145 & 2192 & 401 & 424 & $\mathrm{x}$ & & \\
\hline 2193 & 2248 & 425 & 425 & & $x$ & \\
\hline 2249 & 2250 & 425 & 425 & $x$ & & \\
\hline 2251 & 2298 & 426 & 449 & $x$ & & \\
\hline 2299 & 2354 & 450 & 450 & & $x$ & \\
\hline 2355 & 2356 & 450 & 450 & $x$ & & \\
\hline 2357 & 2404 & 451 & 474 & $\mathrm{x}$ & & \\
\hline 2405 & 2460 & 475 & 475 & & $\mathrm{x}$ & \\
\hline 2461 & 2462 & 475 & 475 & $x$ & & \\
\hline 2463 & 2510 & 476 & 499 & $x$ & & \\
\hline 2511 & 2566 & 500 & 500 & & $x$ & \\
\hline 2567 & 2568 & 500 & 500 & $x$ & & \\
\hline 2569 & 2616 & 501 & 524 & $x$ & & \\
\hline 2617 & 2672 & 525 & 525 & & $x$ & \\
\hline 2673 & 2674 & 525 & 525 & $\mathrm{x}$ & & \\
\hline 2675 & 2722 & 526 & 549 & $x$ & & \\
\hline 2723 & 2778 & 550 & 550 & & $\bar{x}$ & \\
\hline 2779 & 2780 & 550 & 550 & $x$ & & \\
\hline 2781 & 2828 & 551 & 574 & $\mathrm{x}$ & & \\
\hline 2829 & 2884 & 575 & 575 & & $x$ & \\
\hline 2885 & 2886 & 575 & 575 & $\mathrm{x}$ & & \\
\hline 2887 & 2934 & 576 & 599 & $x$ & & \\
\hline 2935 & 2990 & 600 & 600 & & $x$ & \\
\hline 2991 & 2992 & 600 & 600 & $x$ & & \\
\hline 2993 & 3040 & 601 & 624 & $x$ & & \\
\hline 3041 & 3096 & 625 & 625 & & $x$ & \\
\hline 3097 & 3098 & 625 & 625 & $\mathrm{x}$ & & \\
\hline 3099 & 3146 & 626 & 649 & $\mathrm{x}$ & & \\
\hline 3147 & 3202 & 650 & 650 & & $x$ & \\
\hline 3203 & 3204 & 650 & 650 & $x$ & & \\
\hline 3205 & 3216 & 651 & 656 & $\mathrm{x}$ & & \\
\hline 3217 & 3218 & 657 & 657 & $\mathrm{x}$ & & Bad reading. recorded incorrectly \\
\hline 3219 & 3238 & 658 & 667 & $x$ & & \\
\hline 3239 & 3240 & 668 & 668 & $x$ & & Bad reading. recorded incorrectly \\
\hline 3241 & 3252 & 669 & 674 & $\mathrm{x}$ & & \\
\hline 3253 & 3308 & 675 & 675 & & $x$ & \\
\hline 3309 & 3310 & 675 & 675 & $x$ & & \\
\hline 3311 & 3358 & 676 & 699 & $x$ & & \\
\hline 3359 & 3414 & 700 & 700 & & $x$ & \\
\hline 3415 & 3416 & 700 & 700 & $x$ & & \\
\hline 3417 & 3434 & 7() 1 & 709 & $x$ & & \\
\hline $3+35$ & 3436 & 709 & 709 & $x$ & & Extra reading unas water \\
\hline
\end{tabular}

Page 2 of 3 


\section{HP DAQ RECORDS FROM STC DURABILITY TEST}

\begin{tabular}{|c|c|c|c|c|c|c|}
\hline $\begin{array}{l}\text { Run \# } \\
\text { From }\end{array}$ & $\begin{array}{c}\text { Run \# } \\
\text { To }\end{array}$ & $\begin{array}{c}\text { Test Hours } \\
\text { From }\end{array}$ & $\left|\begin{array}{c}\text { Test Hours } \\
\text { To }\end{array}\right|$ & $\begin{array}{l}\text { Hourly } \\
\text { Data }\end{array}$ & $\begin{array}{c}\text { Snapshot } \\
\text { Data }\end{array}$ & Comments \\
\hline 3437 & 3466 & 710 & 724 & $x$ & & \\
\hline 3467 & 3522 & 725 & 725 & & $x$ & \\
\hline 3523 & 3524 & 725 & 725 & $x$ & & \\
\hline 3525 & 3572 & 726 & 749 & $x$ & & \\
\hline 3573 & 3628 & 750 & 750 & & $x$ & \\
\hline 3629 & 3630 & 750 & 750 & $x$ & & \\
\hline 3631 & 3650 & 751 & 760 & $x$ & & \\
\hline & & 761 & 761 & $\mathrm{x}$ & & Missed reading \\
\hline 3651 & 3676 & 762 & 774 & $\mathrm{x}$ & & \\
\hline 3677 & 3732 & 775 & 775 & & $x$ & \\
\hline 3733 & 3734 & 775 & 775 & $x$ & & \\
\hline 3735 & 3782 & 776 & 799 & $x$ & & \\
\hline 3783 & 3838 & 800 & 800 & & $x$ & \\
\hline 3839 & 3940 & 800 & 800 & $x$ & & \\
\hline 3841 & 3888 & 801 & 824 & $x$ & & \\
\hline 3889 & 3944 & 825 & 825 & & $x$ & \\
\hline 3945 & 3946 & 825 & 825 & $x$ & & \\
\hline 3947 & 3994 & 826 & 849 & $x$ & & \\
\hline 3995 & 4050 & 850 & 850 & & $x$ & \\
\hline 4051 & 4052 & 850 & 850 & $x$ & & \\
\hline 4053 & 4100 & 851 & 874 & $\mathrm{x}$ & & \\
\hline 4101 & 4156 & 875 & 875 & & $x$ & \\
\hline 4157 & 4158 & 875 & 875 & $x$ & & \\
\hline 4159 & 4206 & 876 & 899 & $x$ & & \\
\hline 4207 & 4262 & 900 & 900 & & $x$ & \\
\hline 4263 & 4264 & 900 & 900 & $x$ & & \\
\hline 4265 & 4312 & 901 & 924 & $x$ & & \\
\hline 4313 & 4368 & 925 & 925 & & $x$ & \\
\hline 4369 & 4370 & 925 & 925 & $x$ & & \\
\hline 4371 & 4418 & 926 & 949 & $x$ & & \\
\hline 4419 & 4474 & 950 & 950 & & $x$ & \\
\hline 4475 & 4476 & 950 & 950 & $x$ & & \\
\hline 4477 & 4524 & 951 & 974 & $\mathrm{x}$ & & \\
\hline 4525 & 4580 & 975 & 975 & & $x$ & \\
\hline 4581 & 4582 & 975 & 975 & $x$ & & \\
\hline 4583 & 4630 & 976 & 999 & $x$ & & \\
\hline 4631 & 4686 & 1000 & 1000 & & $x$ & \\
\hline 4687 & 4688 & 1000 & 1000 & $x$ & & \\
\hline
\end{tabular}


APPENDIX B

OH1 TRANSIENT DATA 
TRANSIENT DATA RECORDED FROM GEM SYSTEM FOR ONE FULL CYCLE AT O TEST HOURS

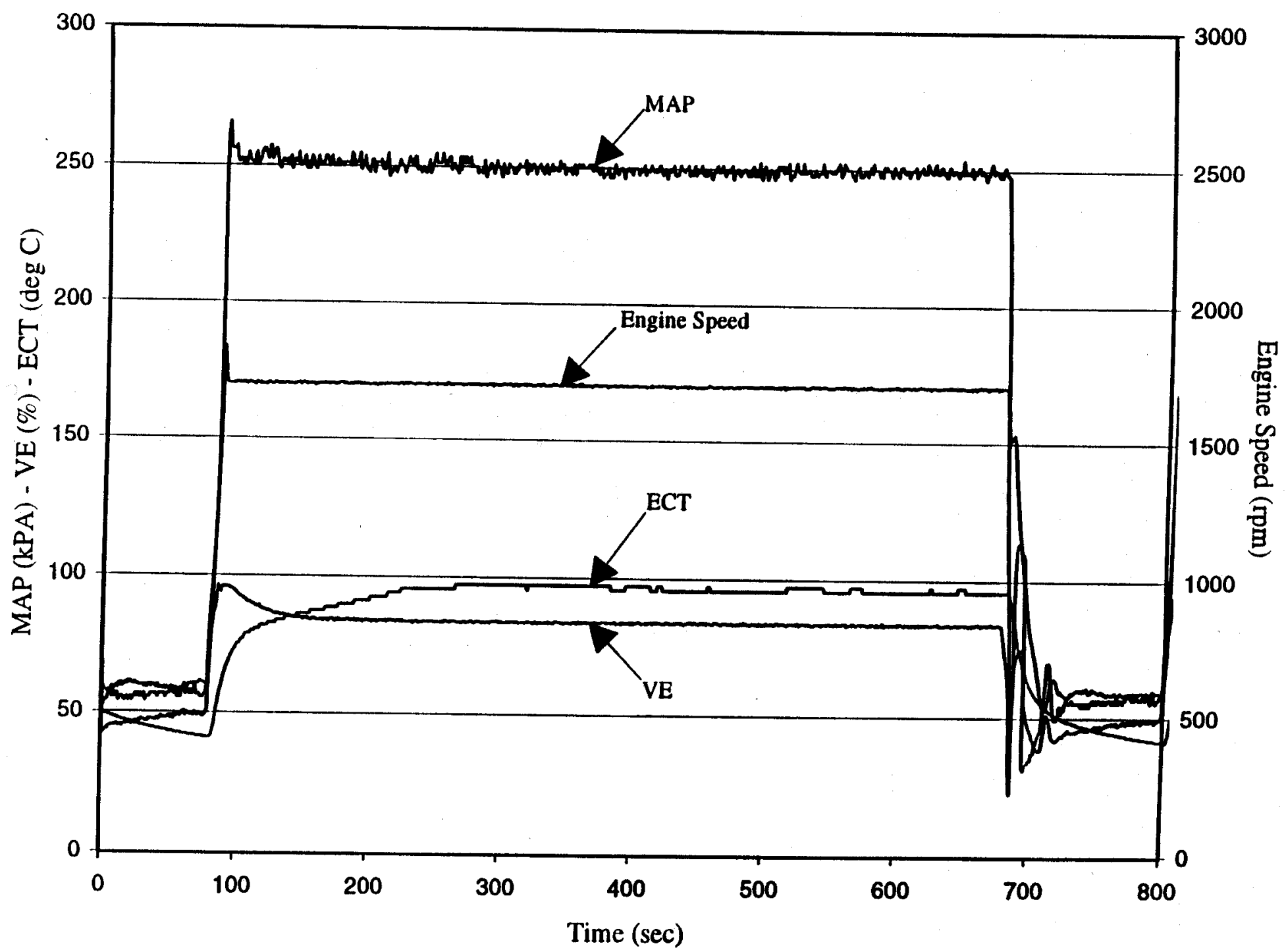


TRANSIENT DATA RECORDED FROM GEM SYSTEM FOR ONE FULL CYCLE AT 25 TEST HOURS

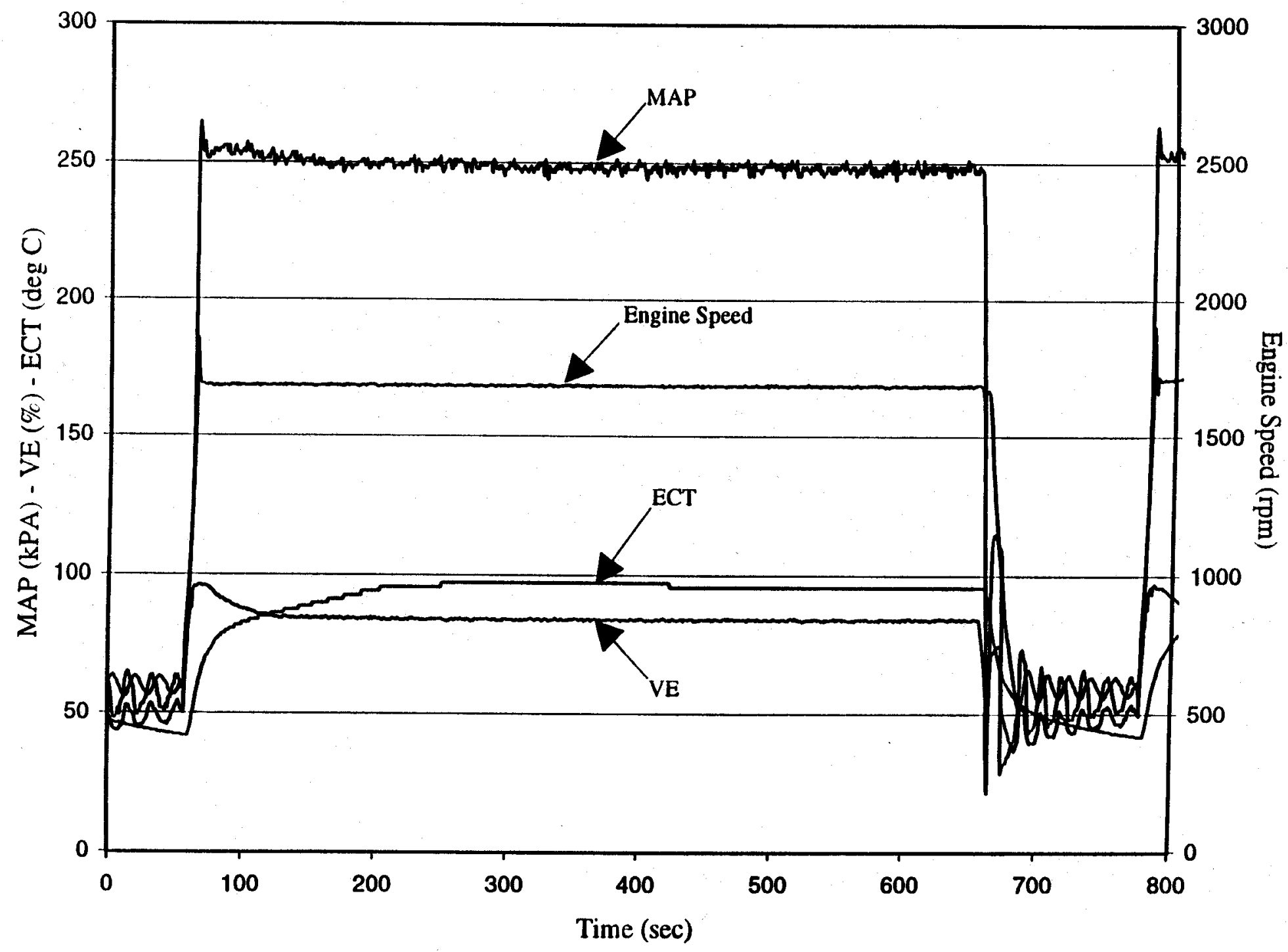


TRANSIENT DATA RECORDED FROM GEM SYSTEM FOR ONE FULL CYCLE AT 50 TEST HOURS

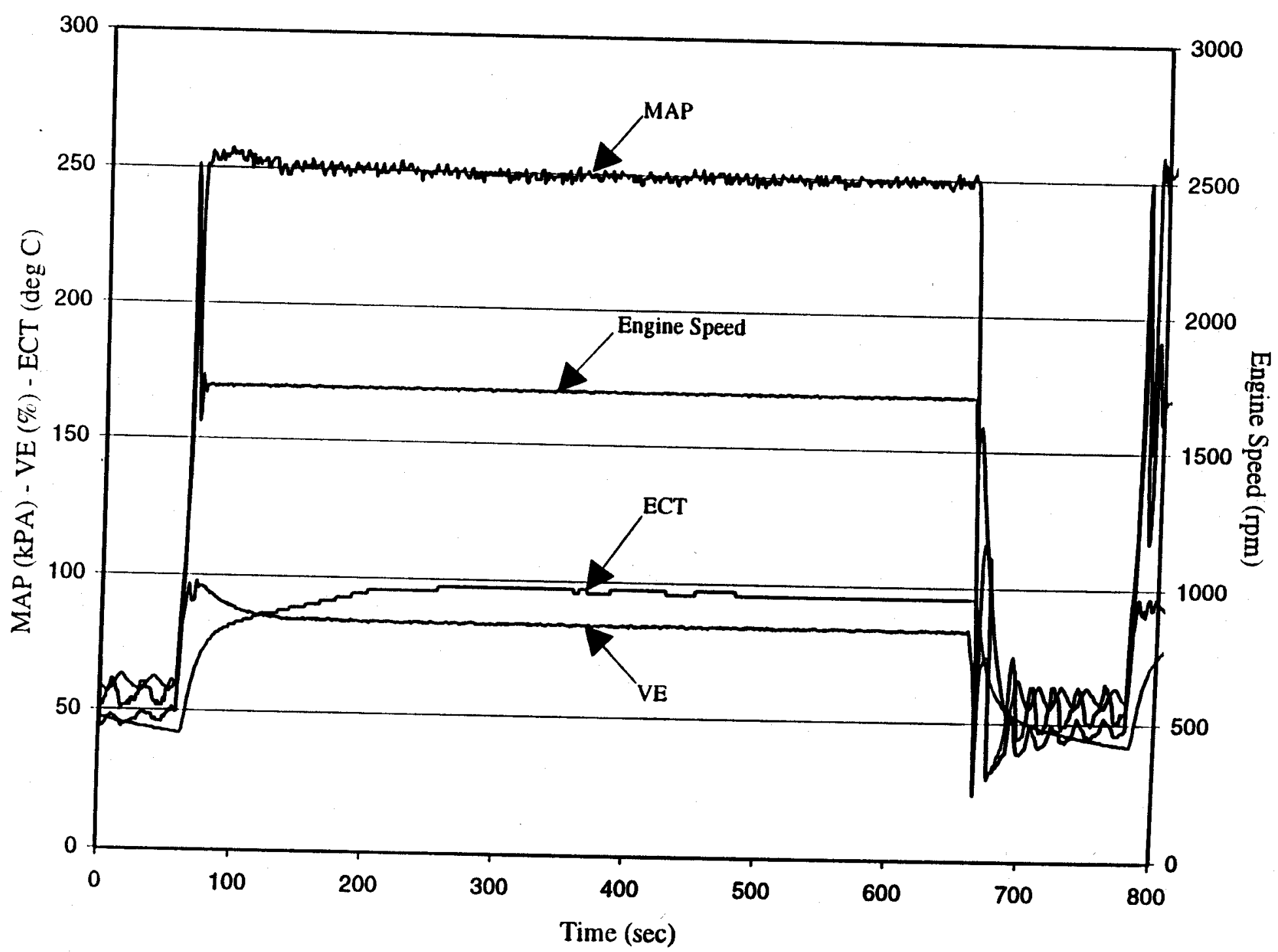


TRANSIENT DATA RECORDED FROM GEM SYSTEM FOR ONE FULL CYCLE AT 75 TEST HOURS

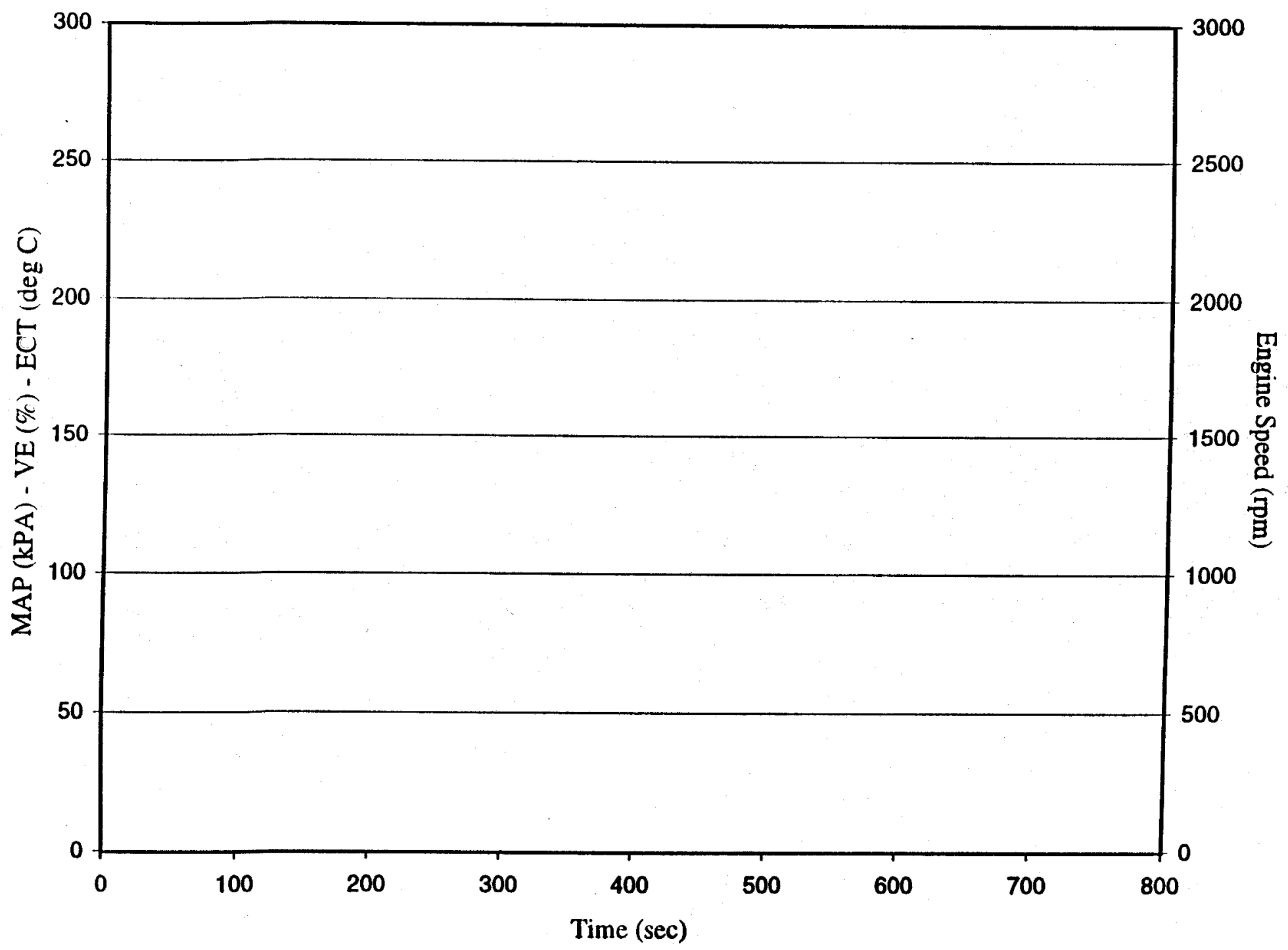


TRANSIENT DATA RECORDED FROM GEM SYSTEM FOR ONE FULL CYCLE AT 100 TEST HOURS

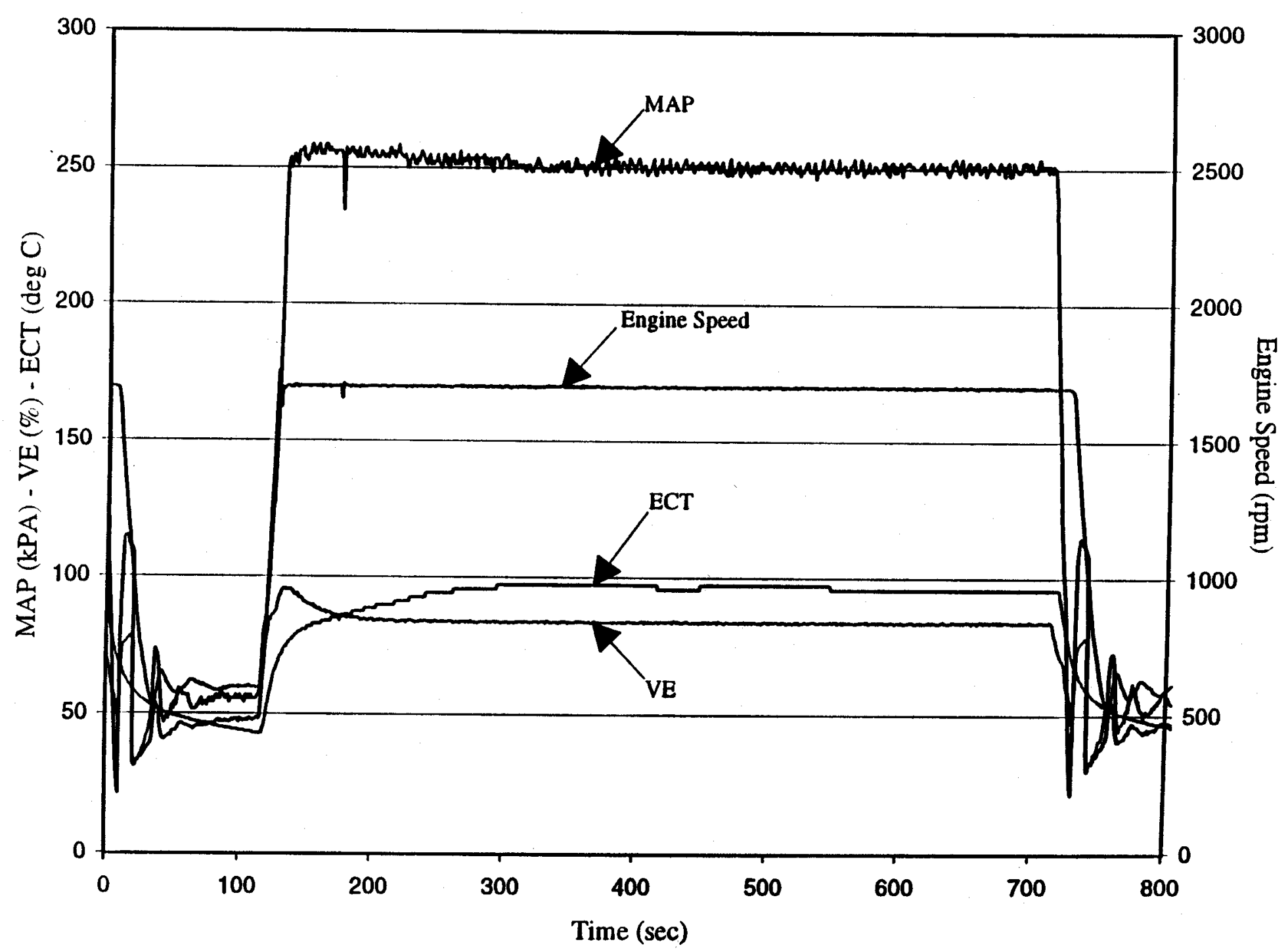

C:IExcelMack_datal100.dat 
TRANSIENT DATA RECORDED FROM GEM SYSTEM FOR ONE FULL CYCLE AT 125 TEST HOURS

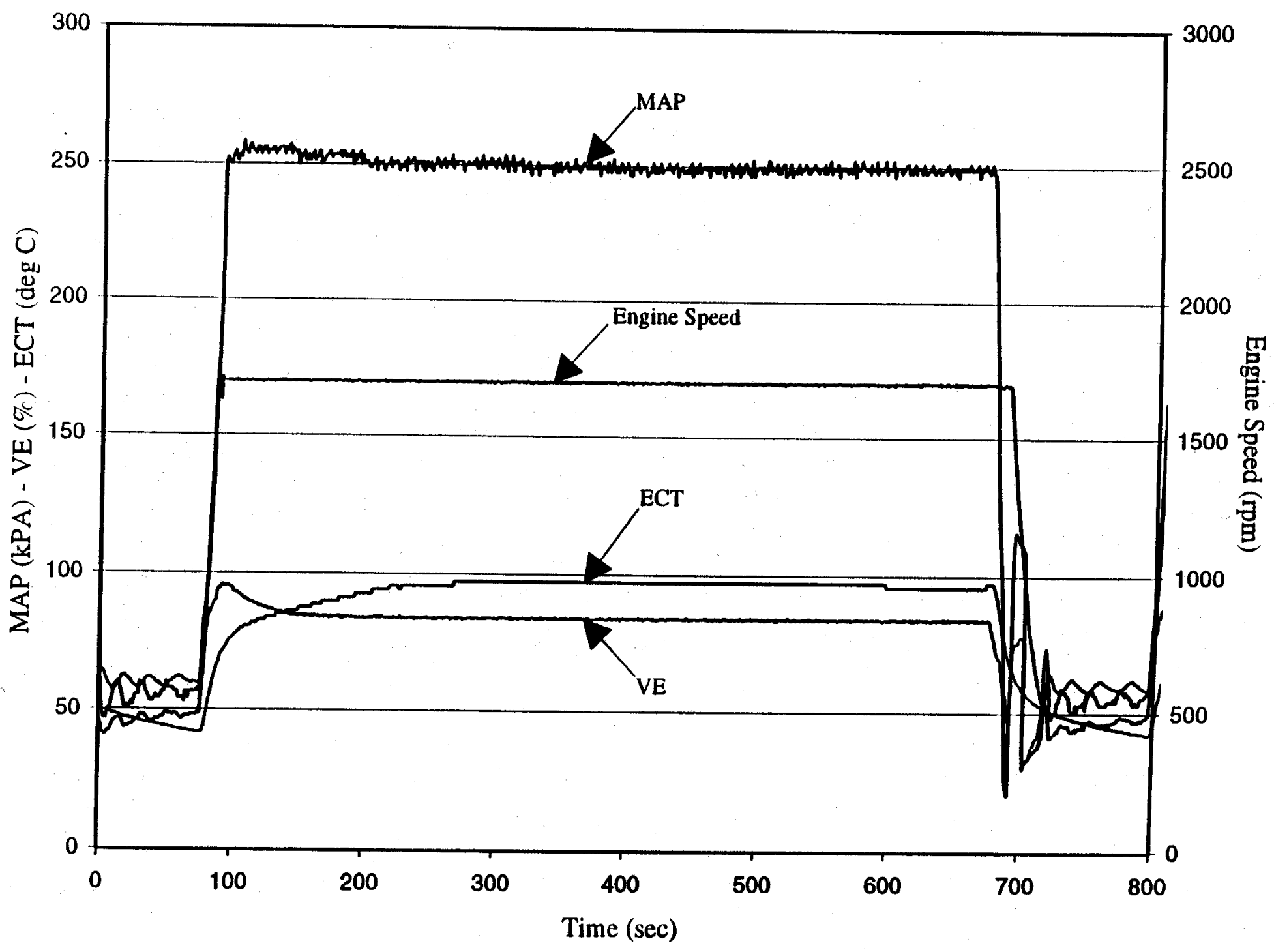


TRANSIENT DATA RECORDED FROM GEM SYSTEM FOR ONE FULL CYCLE AT 150 TEST HOURS

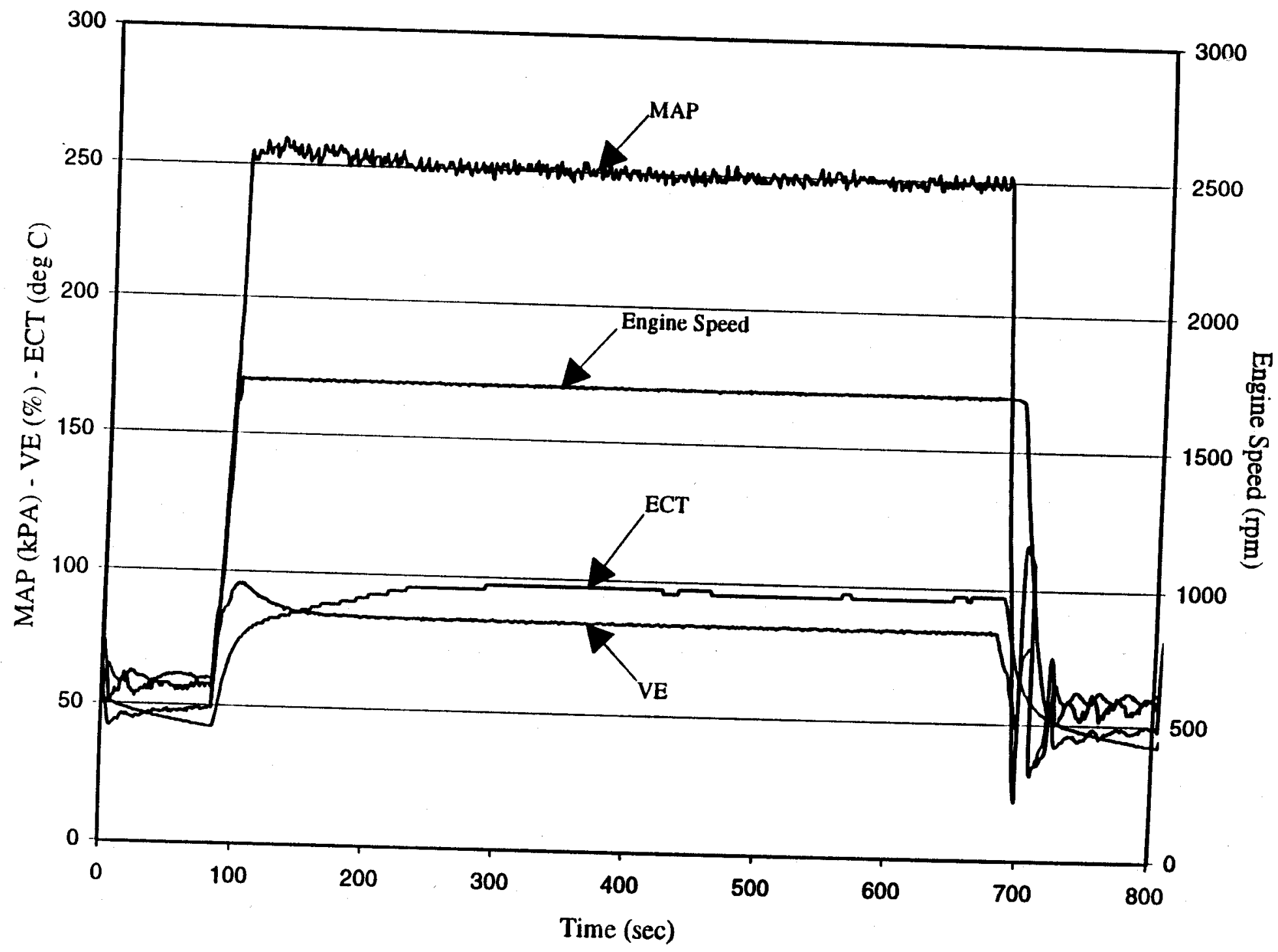

C:IExcelMack_datal1 50.dat 
TRANSIENT DATA RECORDED FROM GEM SYSTEM FOR ONE FULL CYCLE AT 175 TEST HOURS

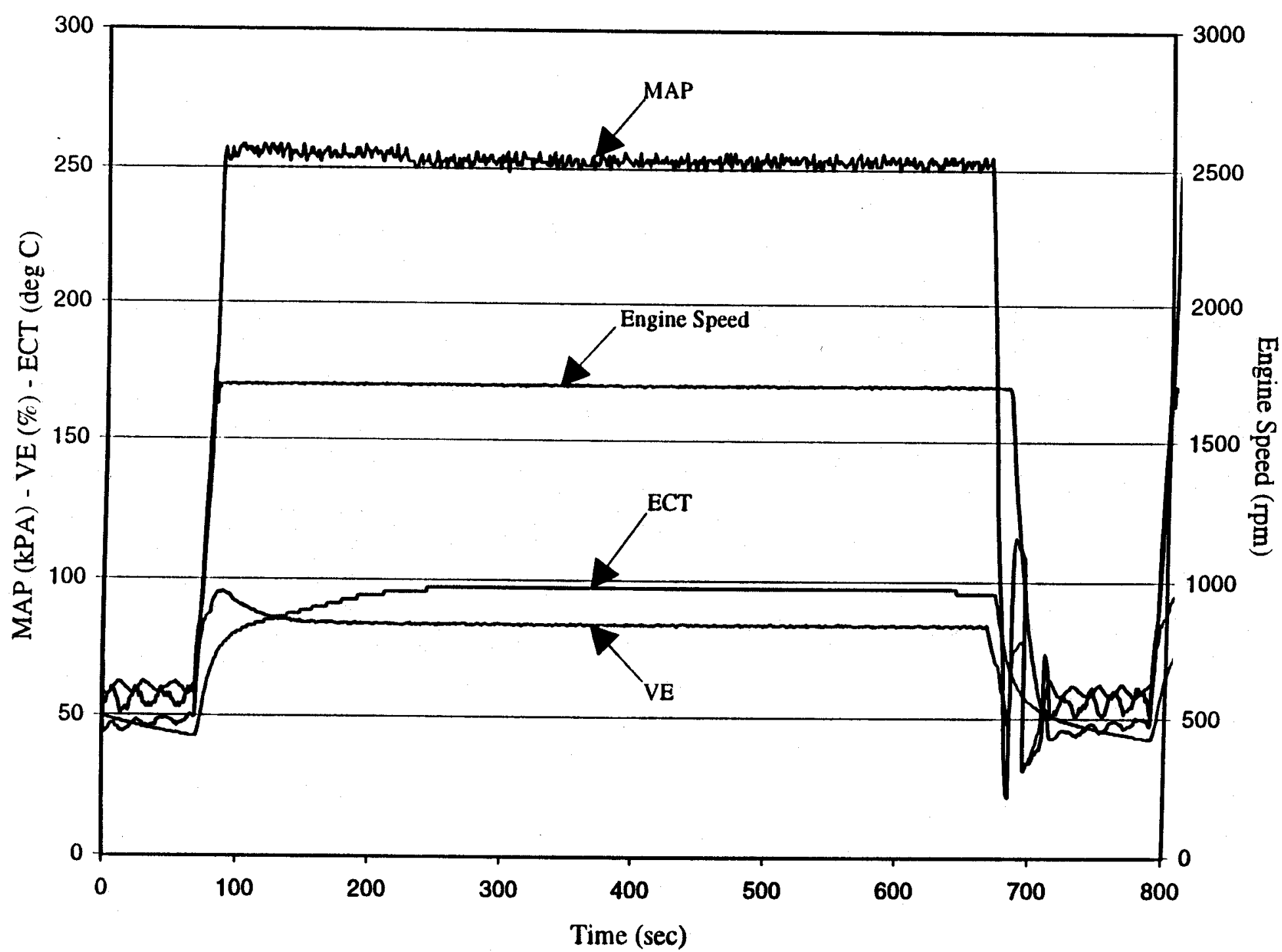


TRANSIENT DATA RECORDED FROM GEM SYSTEM FOR ONE FULL CYCLE AT 200 TEST HOURS

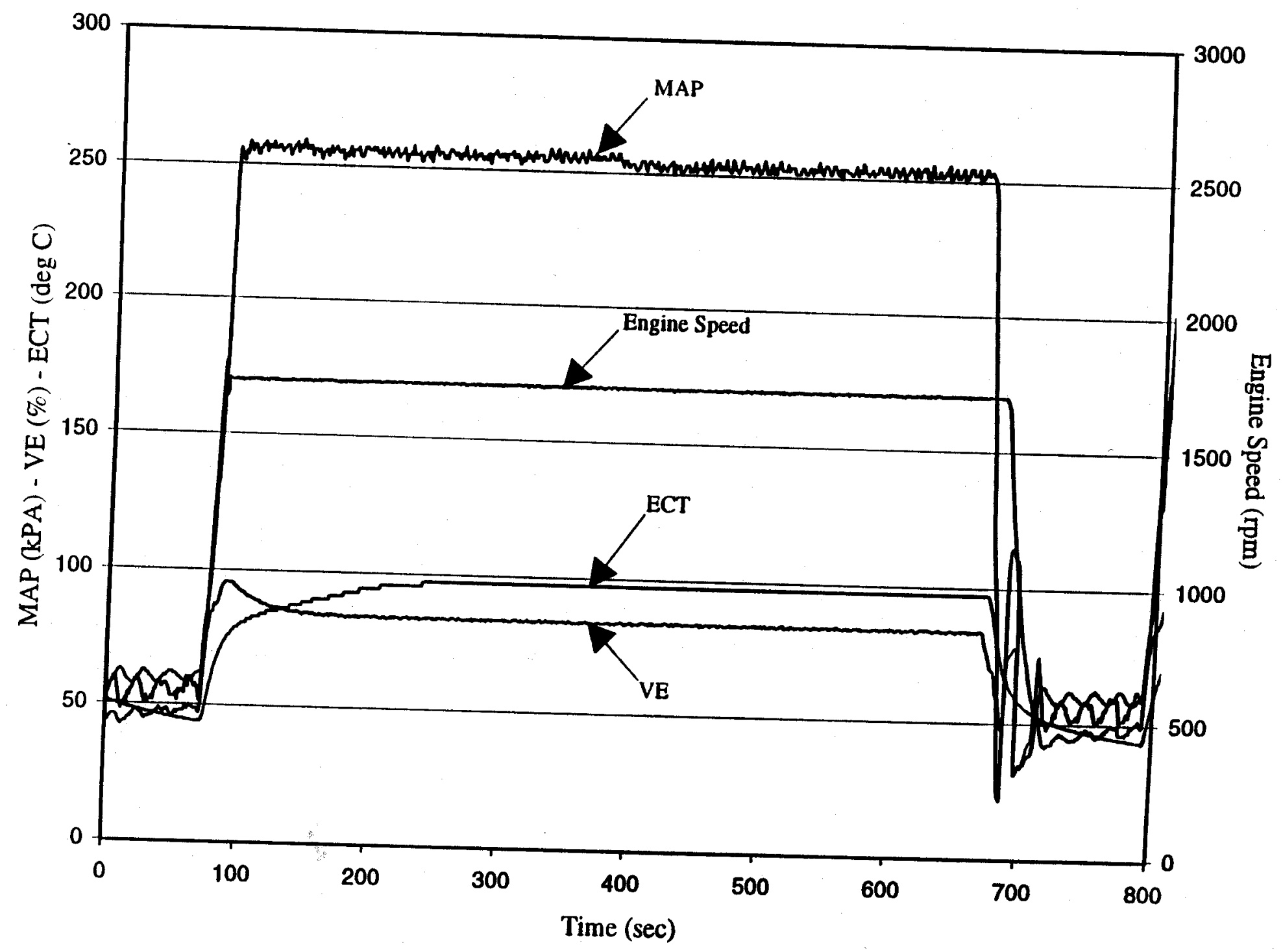


TRANSIENT DATA RECORDED FROM GEM SYSTEM FOR ONE FULL CYCLE AT 225 TEST HOURS

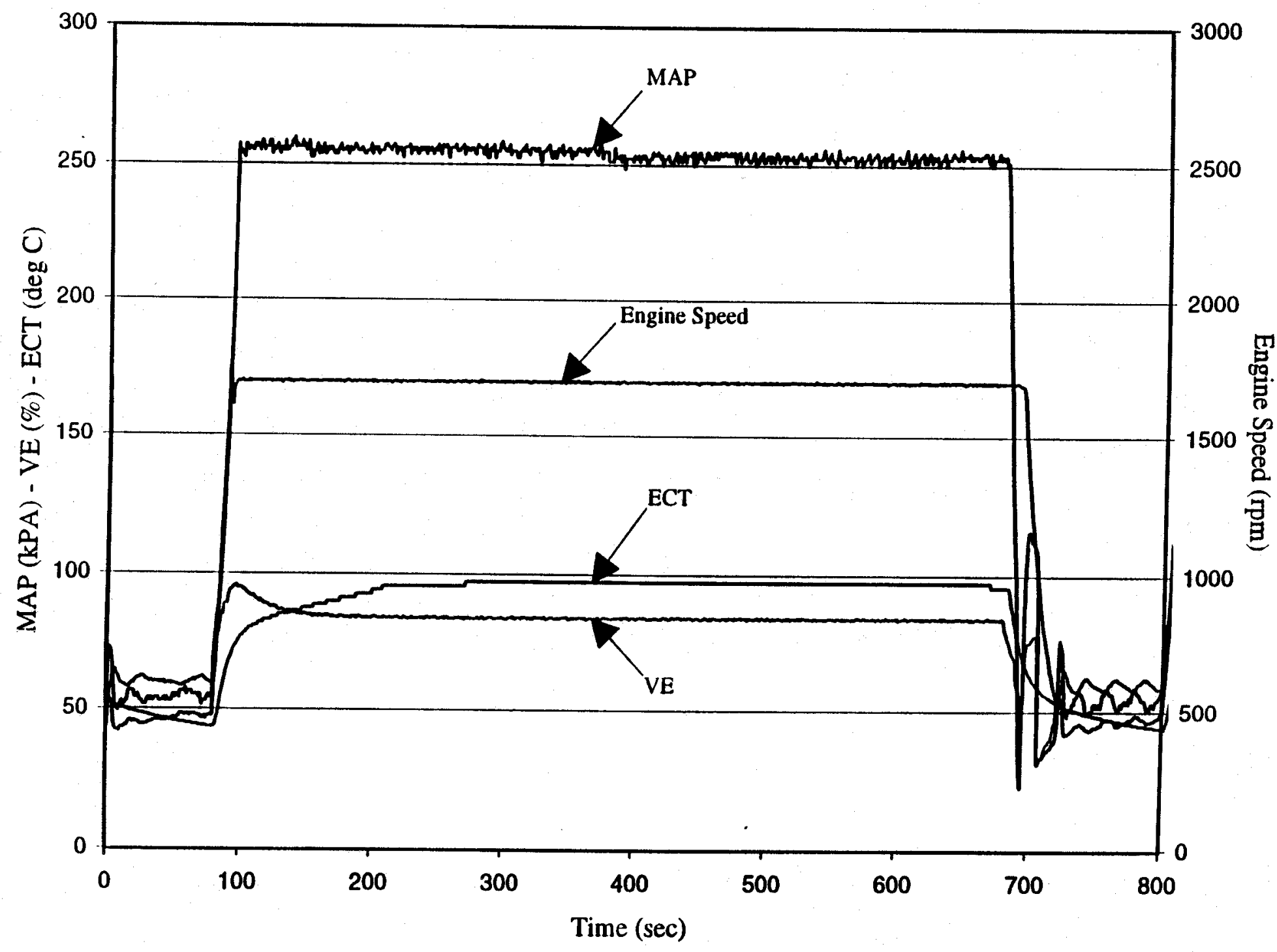


TRANSIENT DATA RECORDED FROM GEM SYSTEM FOR ONE FULL CYCLE AT 250 TEST HOURS

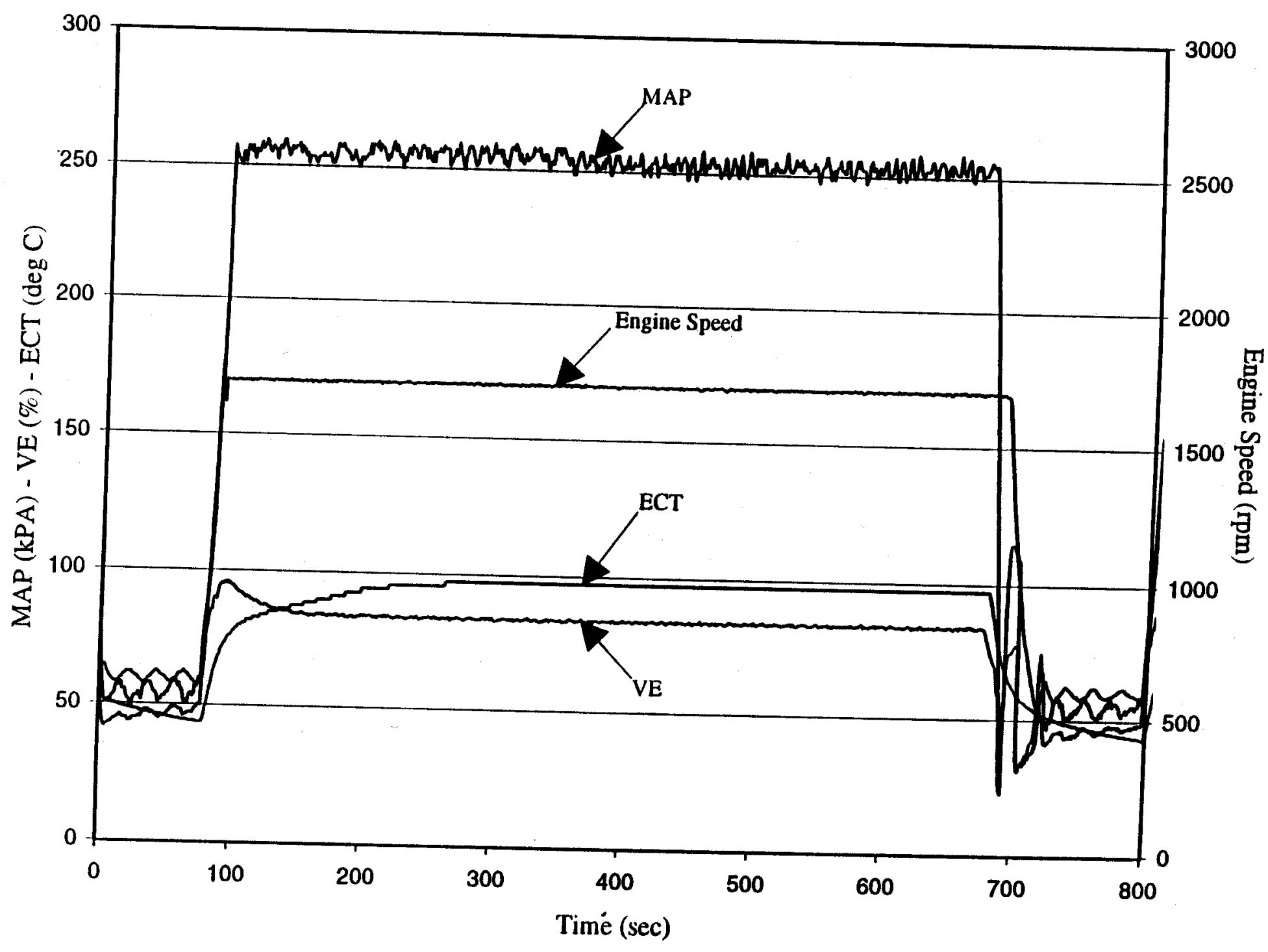


TRANSIENT DATA RECORDED FROM GEM SYSTEM FOR ONE FULL CYCLE AT 250 TEST HOURS

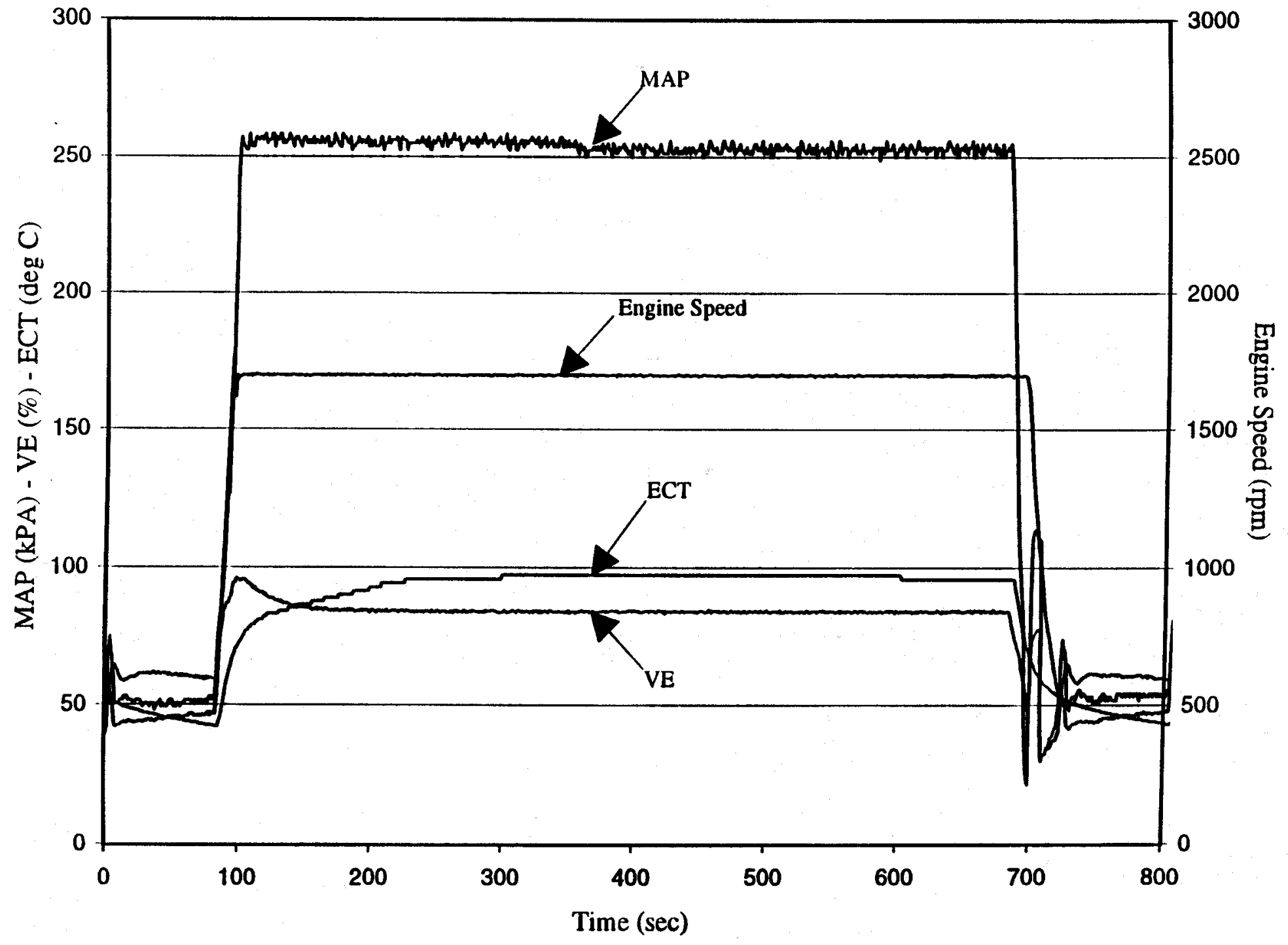

C:LExcellMack_data1250B.dat 
TRANSIENT DATA RECORDED FROM GEM SYSTEM FOR ONE FULL CYCLE AT 275 TEST HOURS

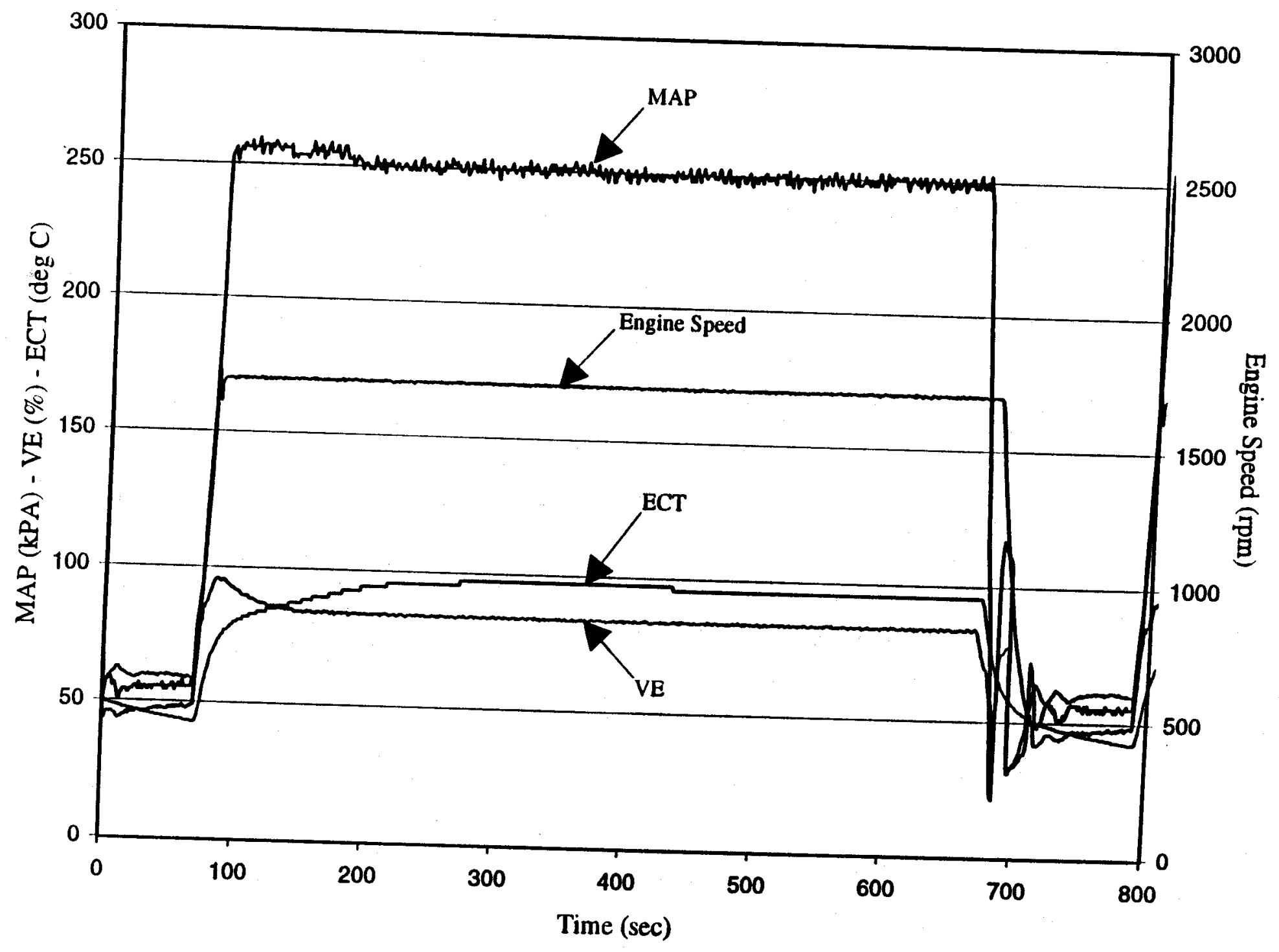


TRANSIENT DATA RECORDED FROM GEM SYSTEM FOR ONE FULL CYCLE AT 300 TEST HOURS

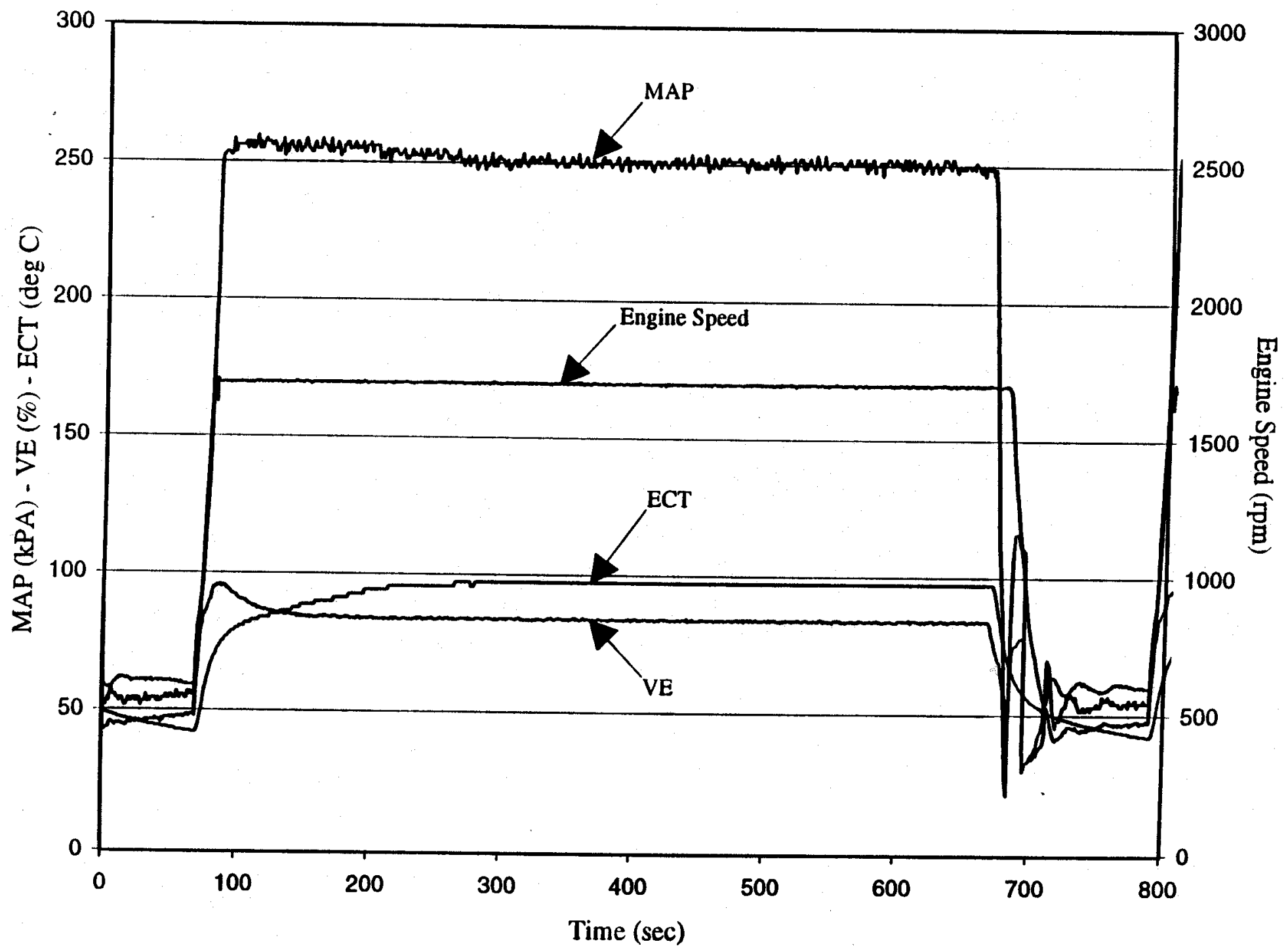


TRANSIENT DATA RECORDED FROM GEM SYSTEM FOR ONE FULL CYCLE AT 325 TEST HOURS

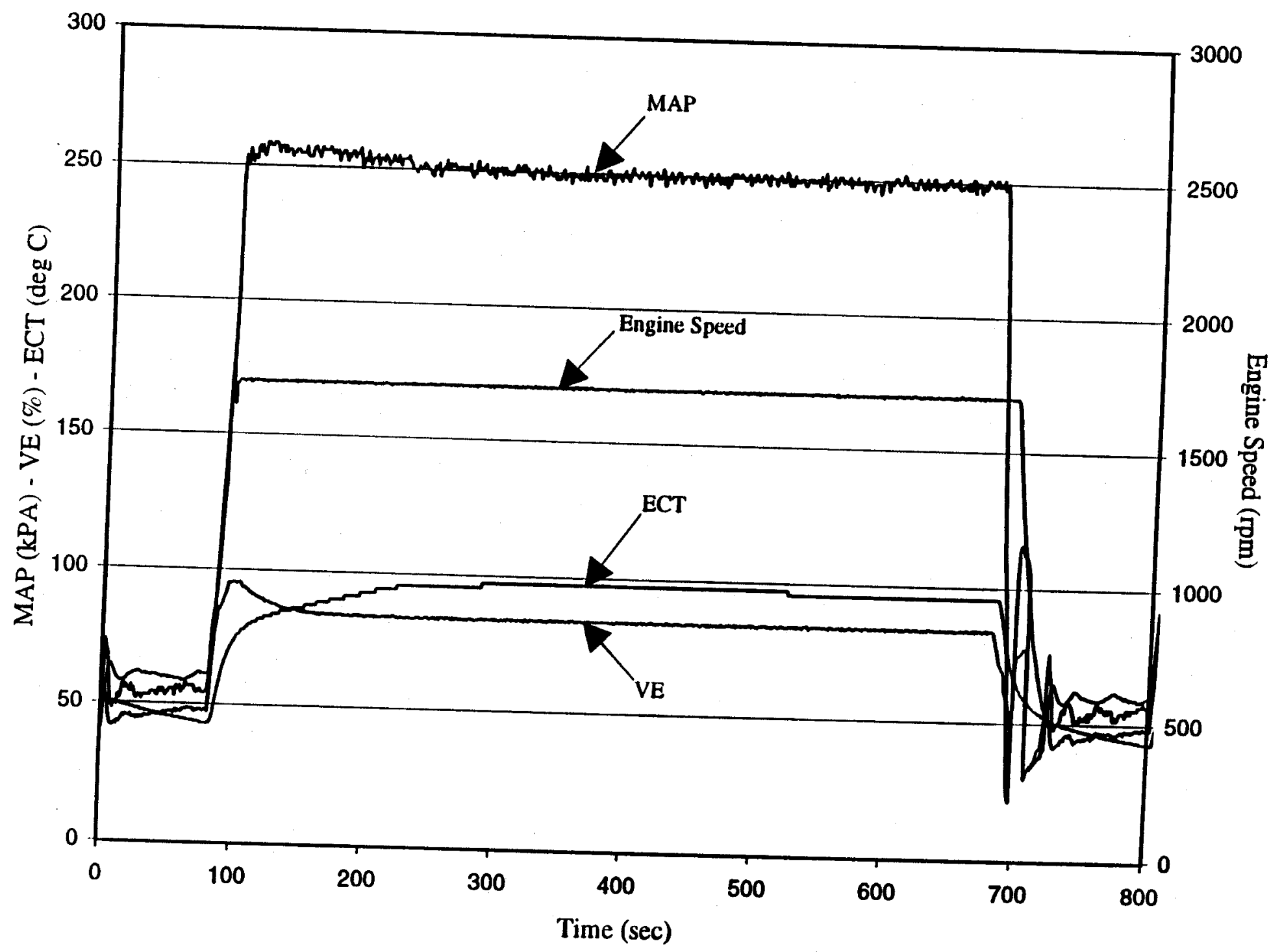


TRANSIENT DATA RECORDED FROM GEM SYSTEM FOR ONE FULL CYCLE AT 350 TEST HOURS

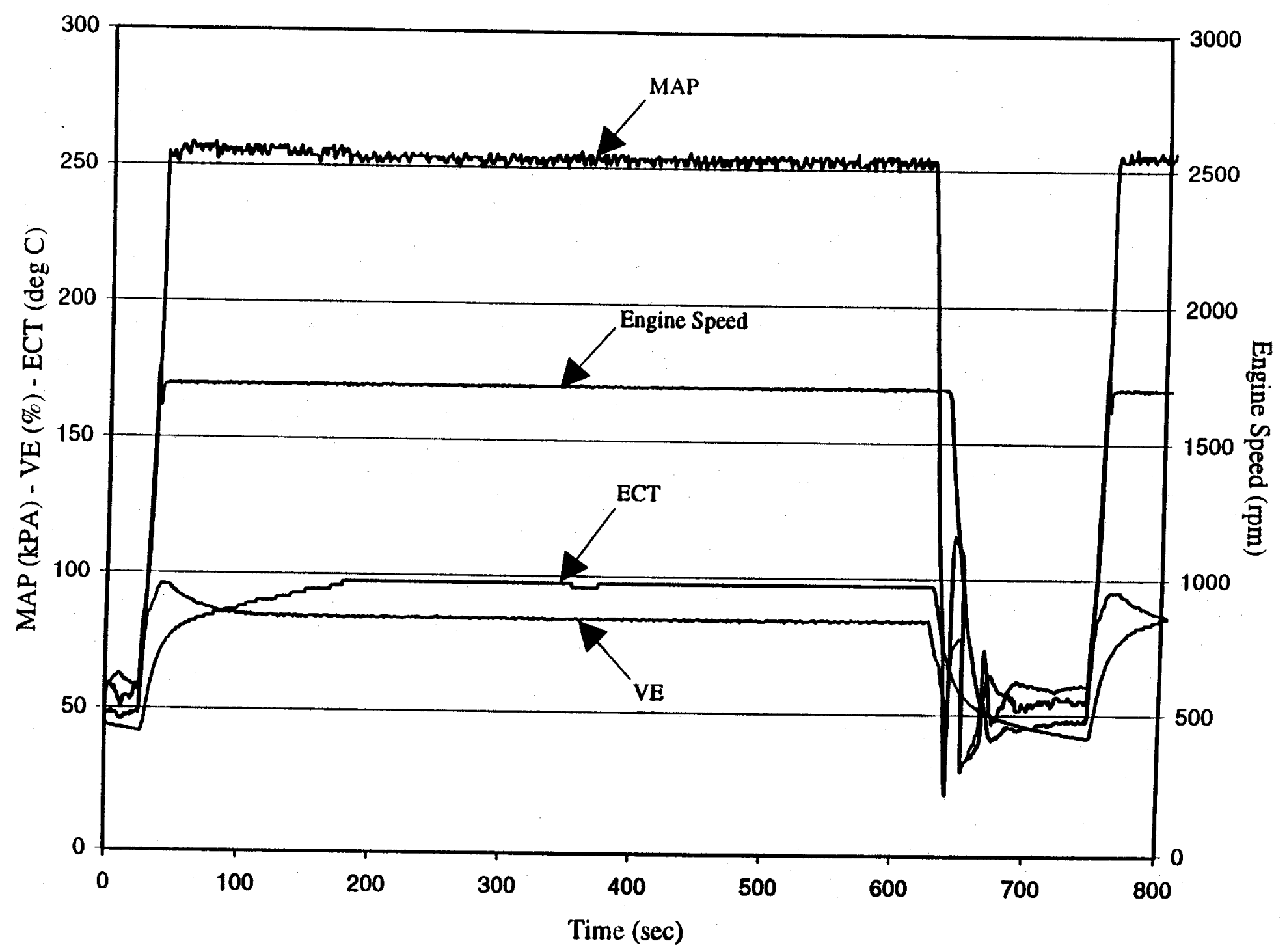


TRANSIENT DATA RECORDED FROM GEM SYSTEM FOR ONE FULL CYCLE AT 375 TEST HOURS

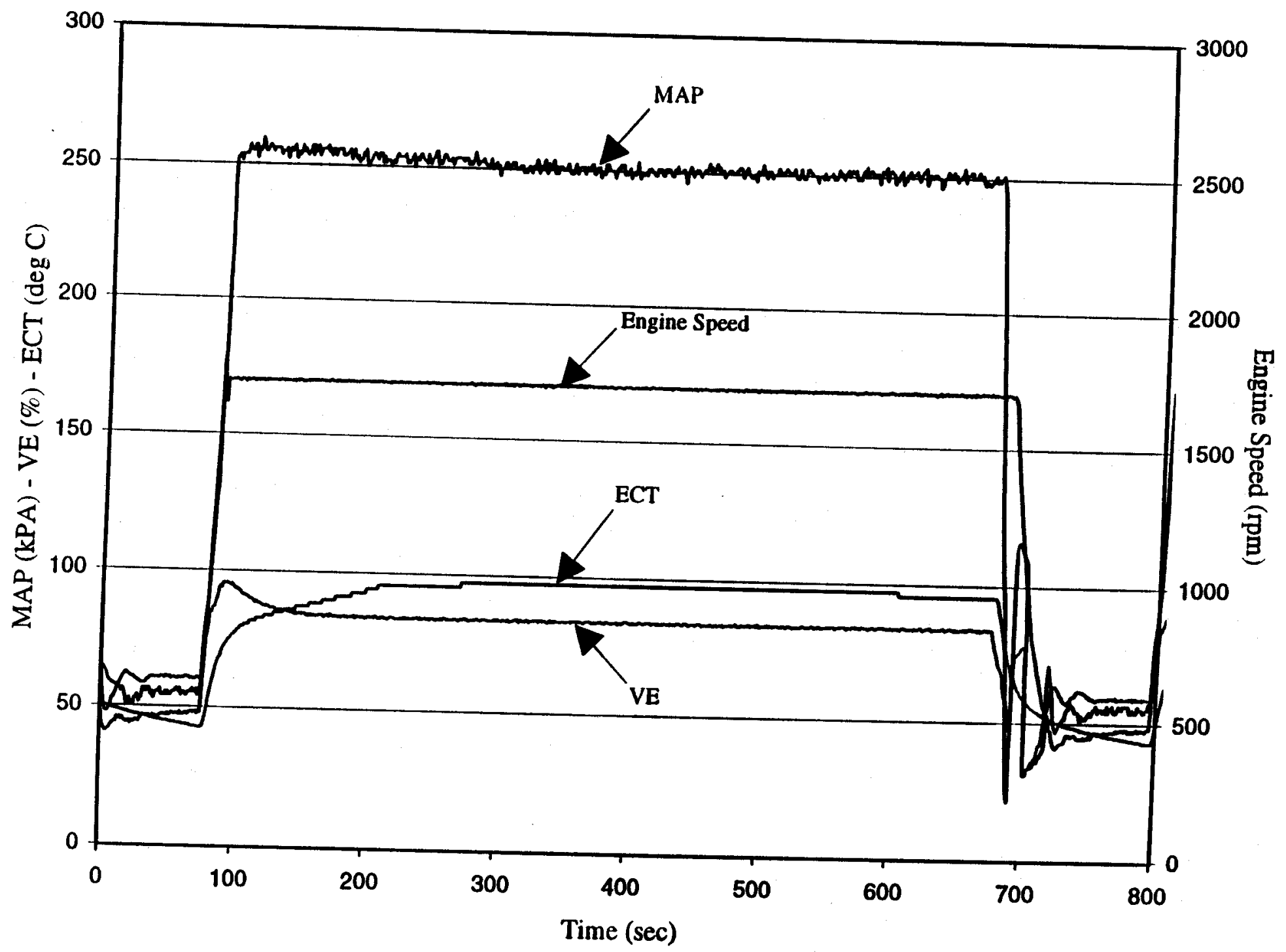


TRANSIENT DATA RECORDED FROM GEM SYSTEM FOR ONE FULL CYCLE AT 400 TEST HOURS

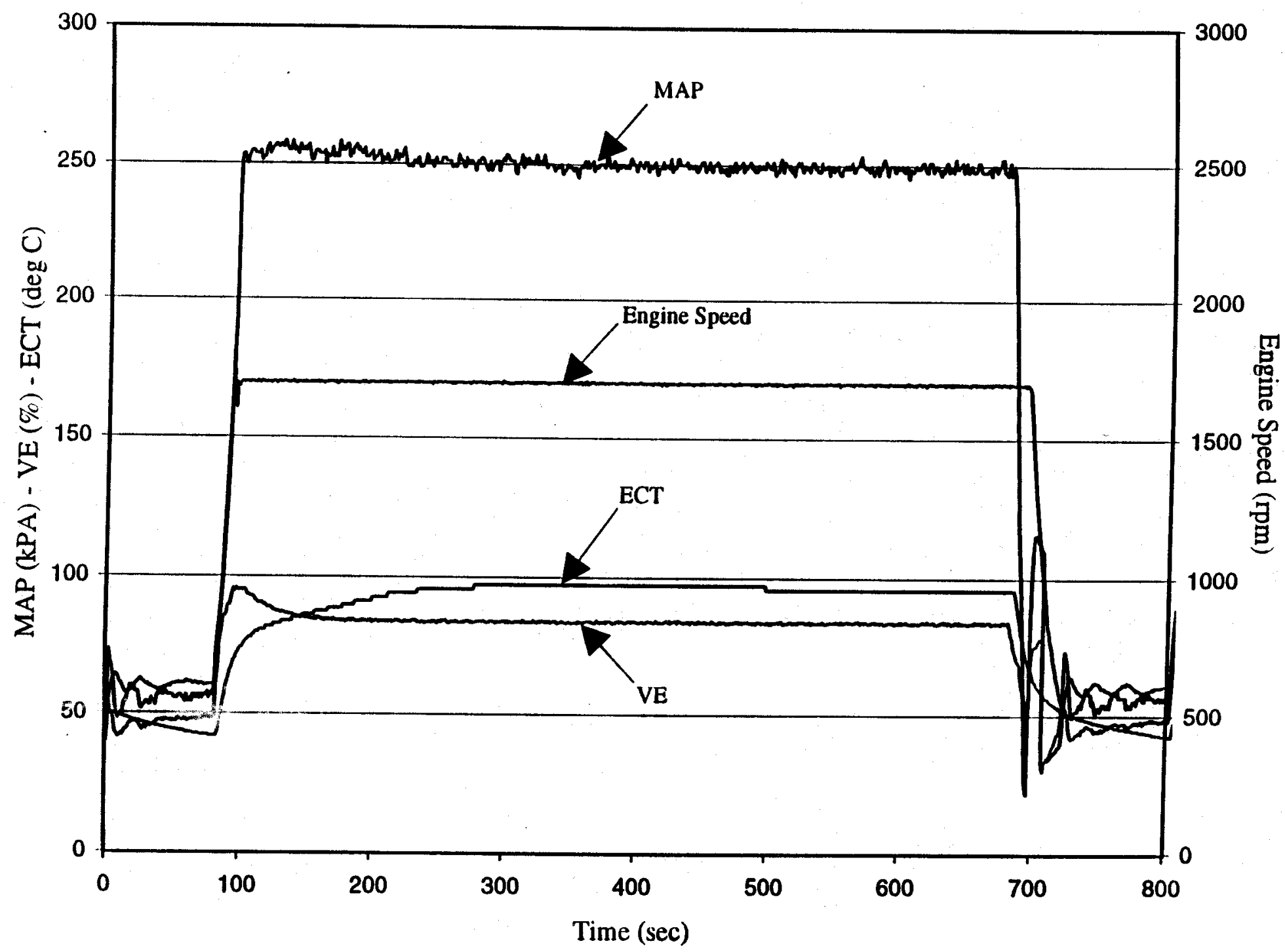


TRANSIENT DATA RECORDED FROM GEM SYSTEM FOR ONE FULL CYCLE AT 425 TEST HOURS

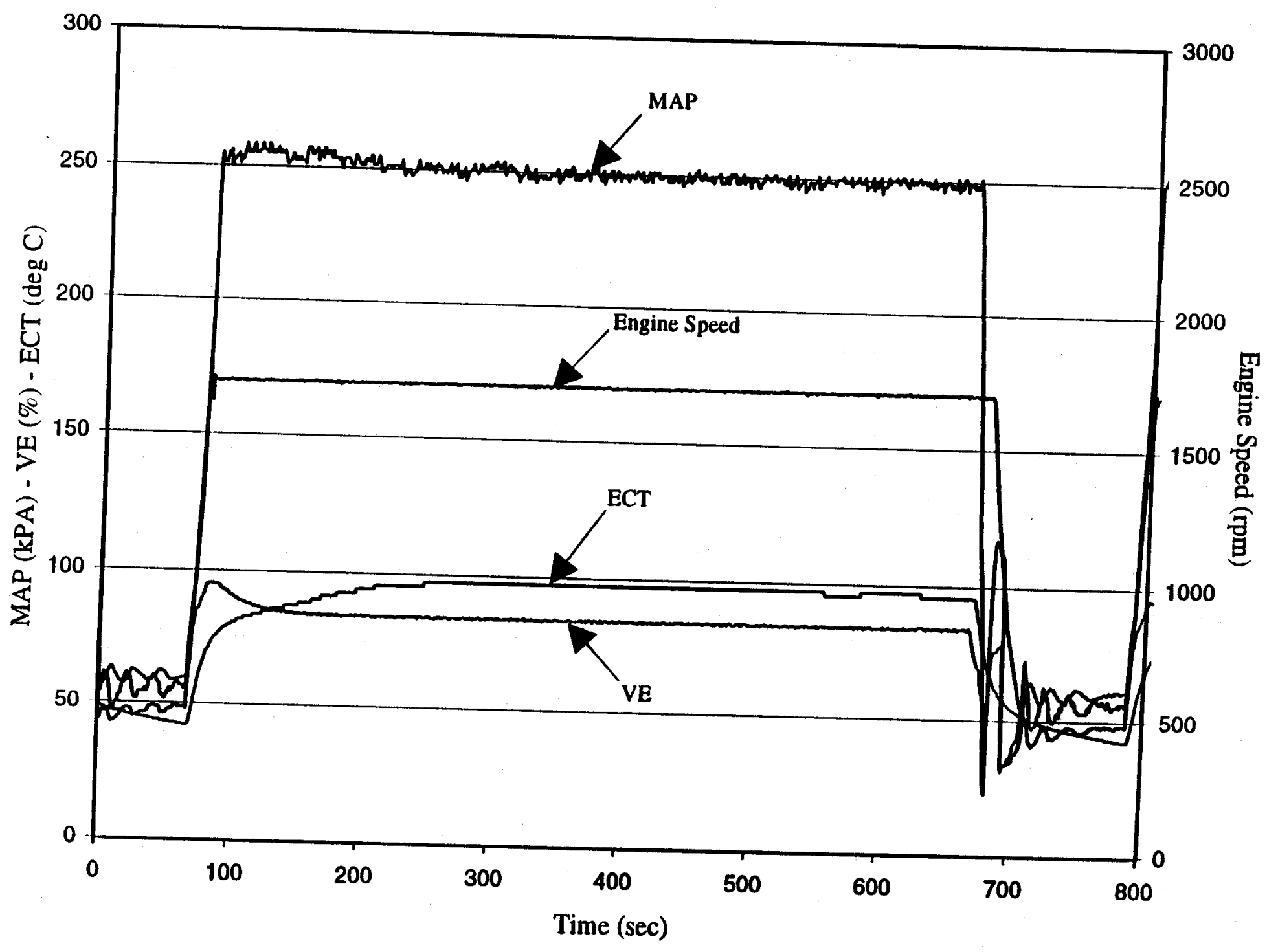


TRANSIENT DATA RECORDED FROM GEM SYSTEM FOR ONE FULL CYCLE AT 450 TEST HOURS

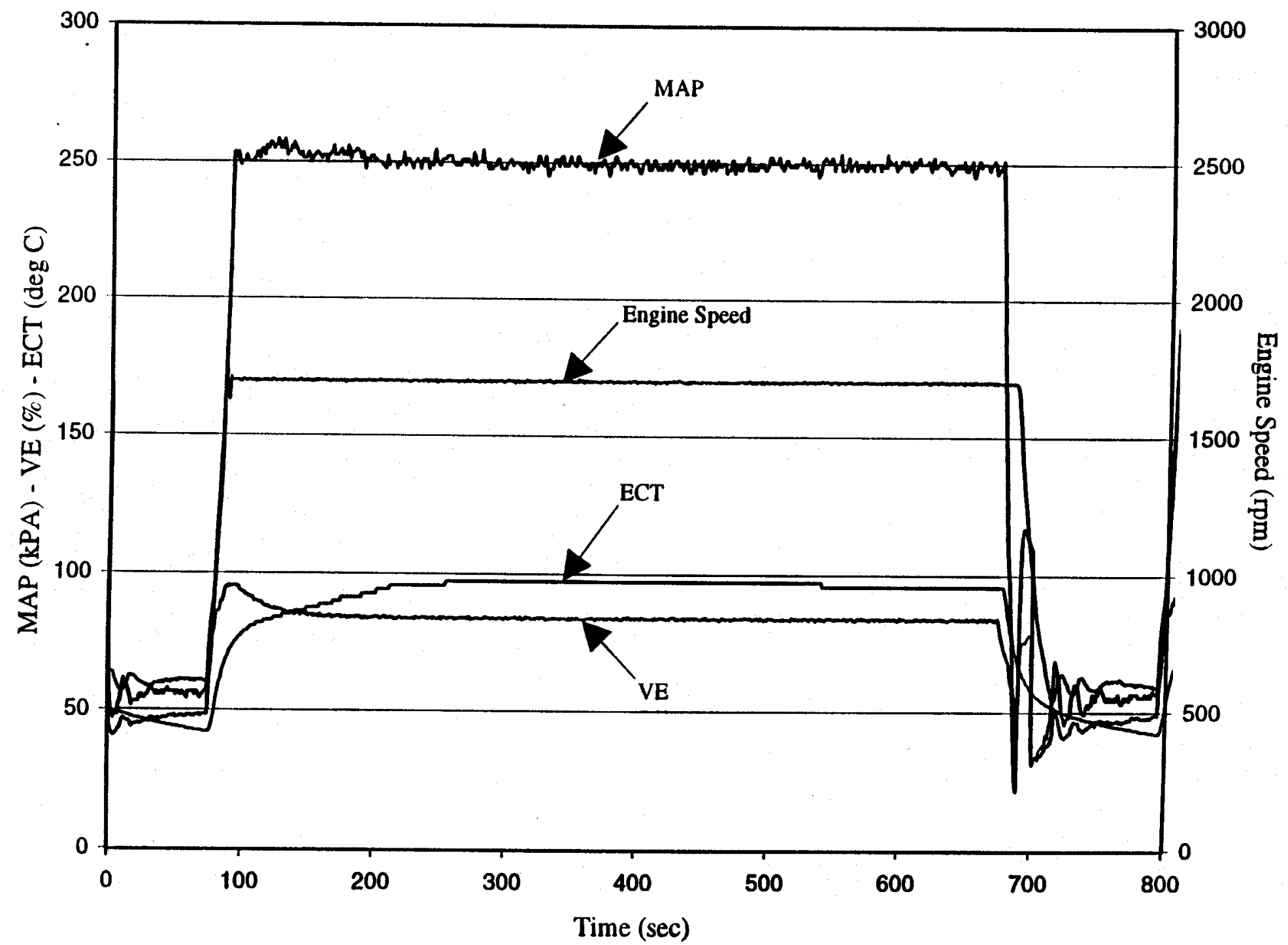


TRANSIENT DATA RECORDED FROM GEM SYSTEM FOR ONE FULL CYCLE AT 475 TEST HOURS

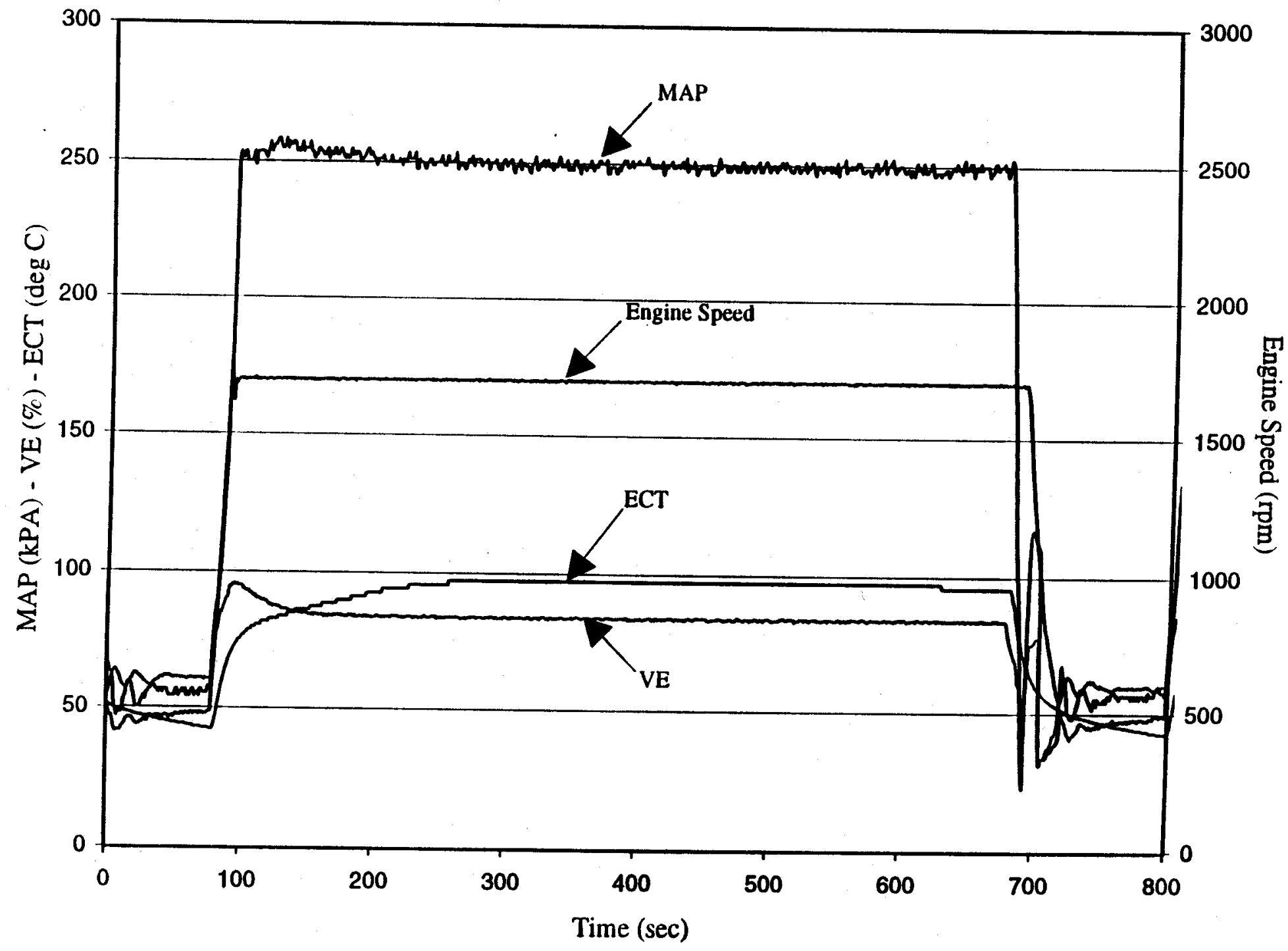

C:ExcelMack_datal475.dat 
TRANSIENT DATA RECORDED FROM GEM SYSTEM FOR ONE FULL CYCLE AT 500 TEST HOURS

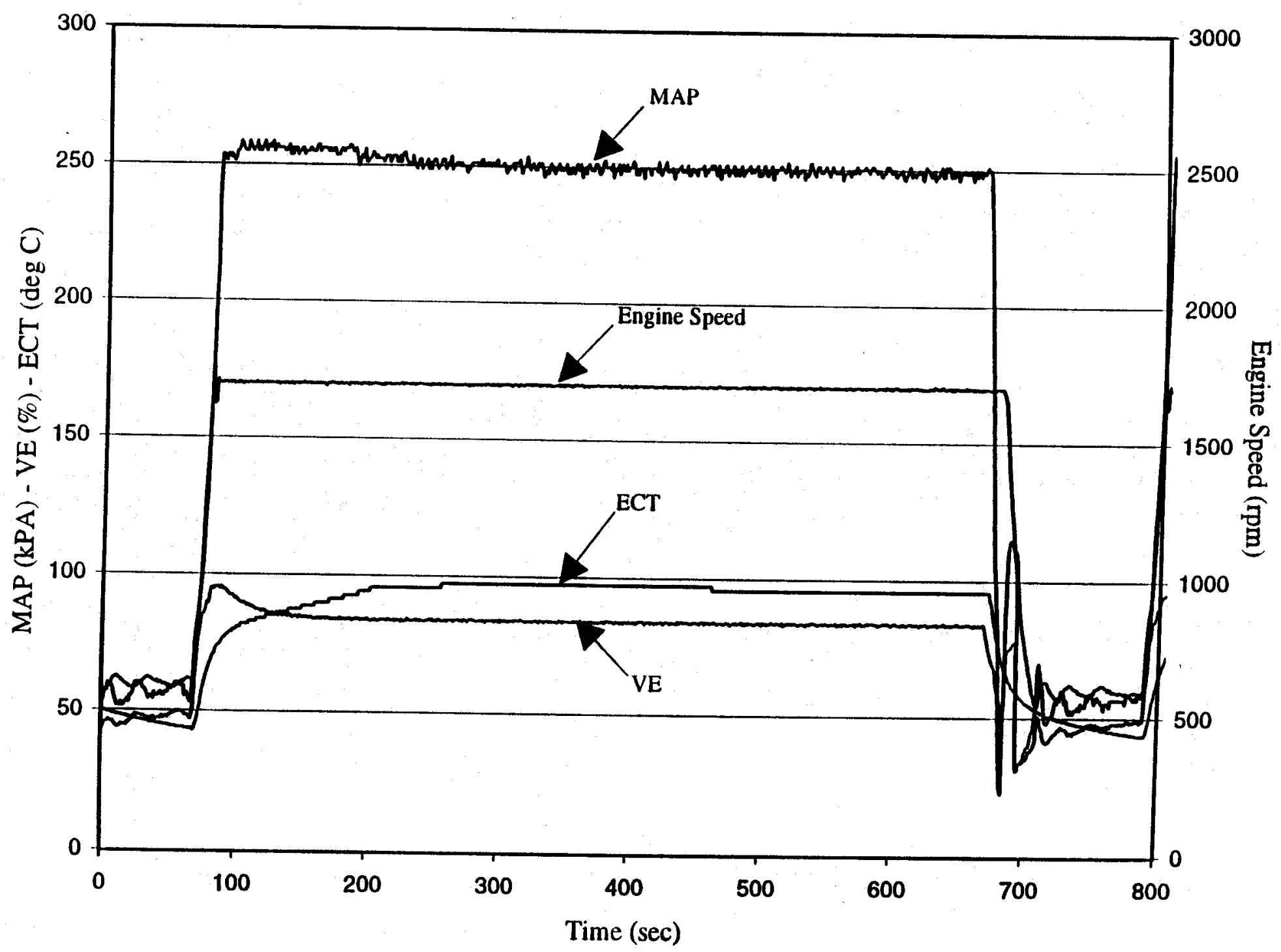


TRANSIENT DATA RECORDED FROM GEM SYSTEM FOR ONE FULL CYCLE AT 525 TEST HOURS

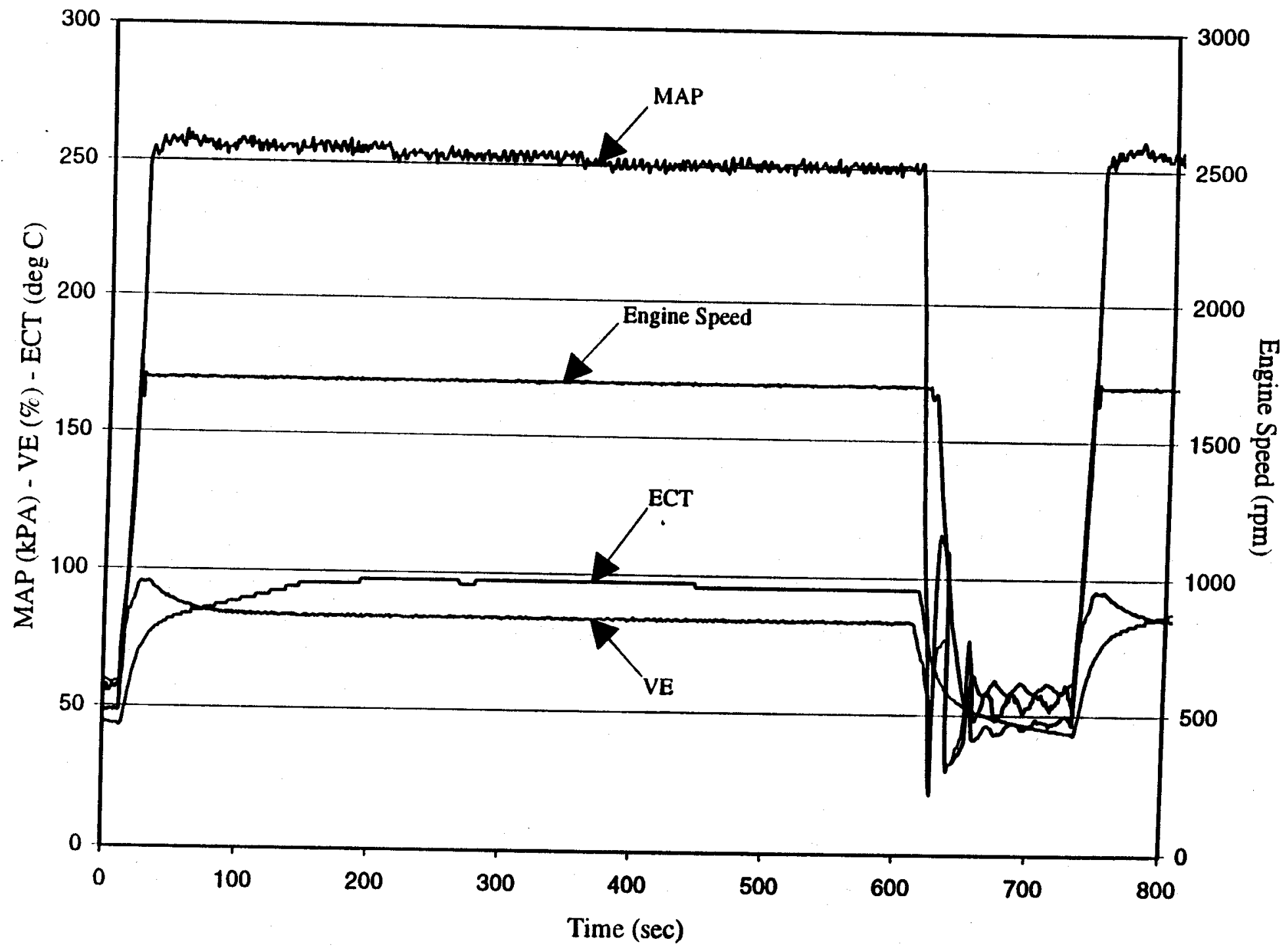


TRANSIENT DATA RECORDED FROM GEM SYSTEM FOR ONE FULL CYCLE AT 550 TEST HOURS

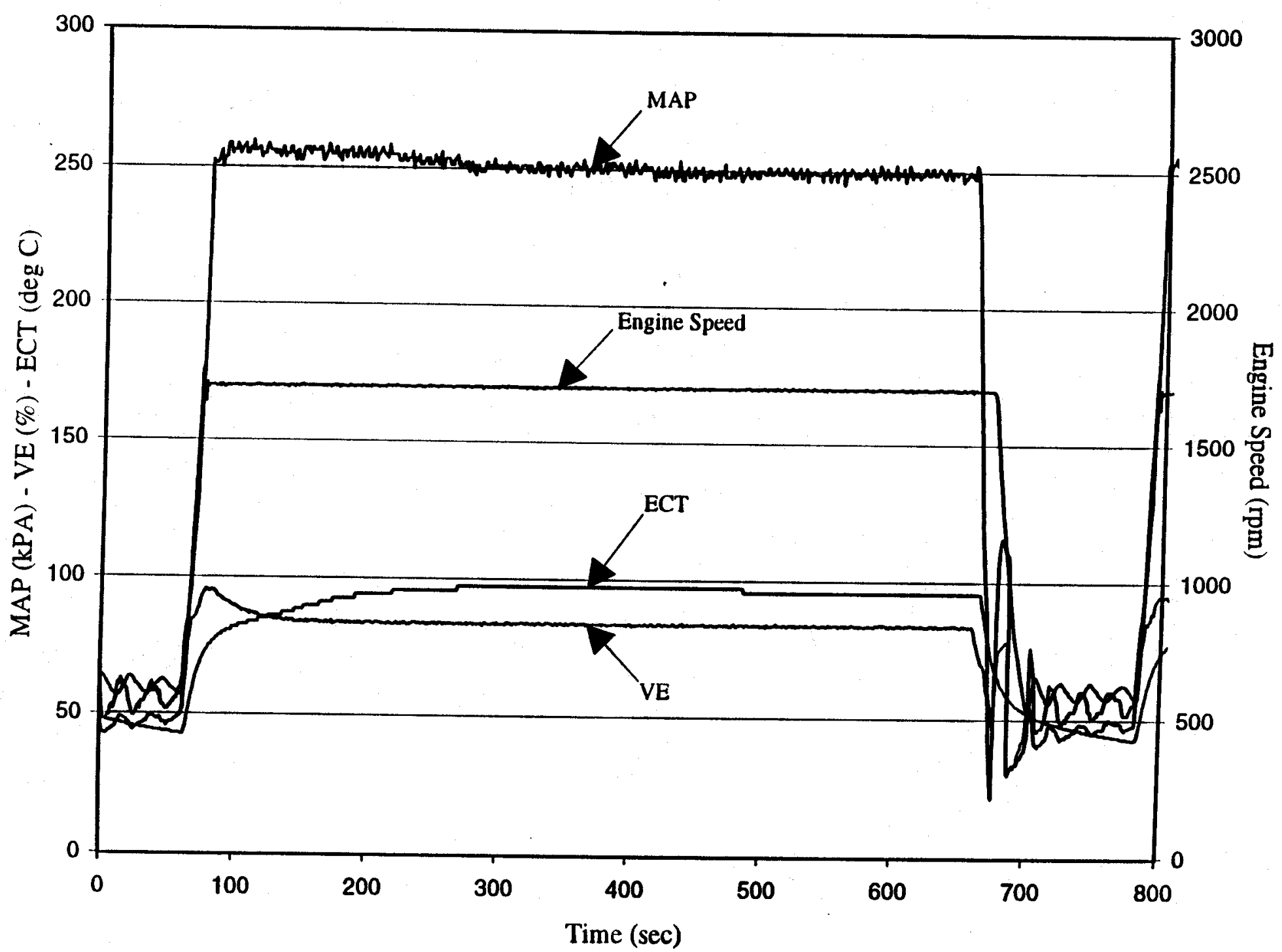


TRANSIENT DATA RECORDED FROM GEM SYSTEM FOR ONE FULL CYCLE AT 575 TEST HOURS

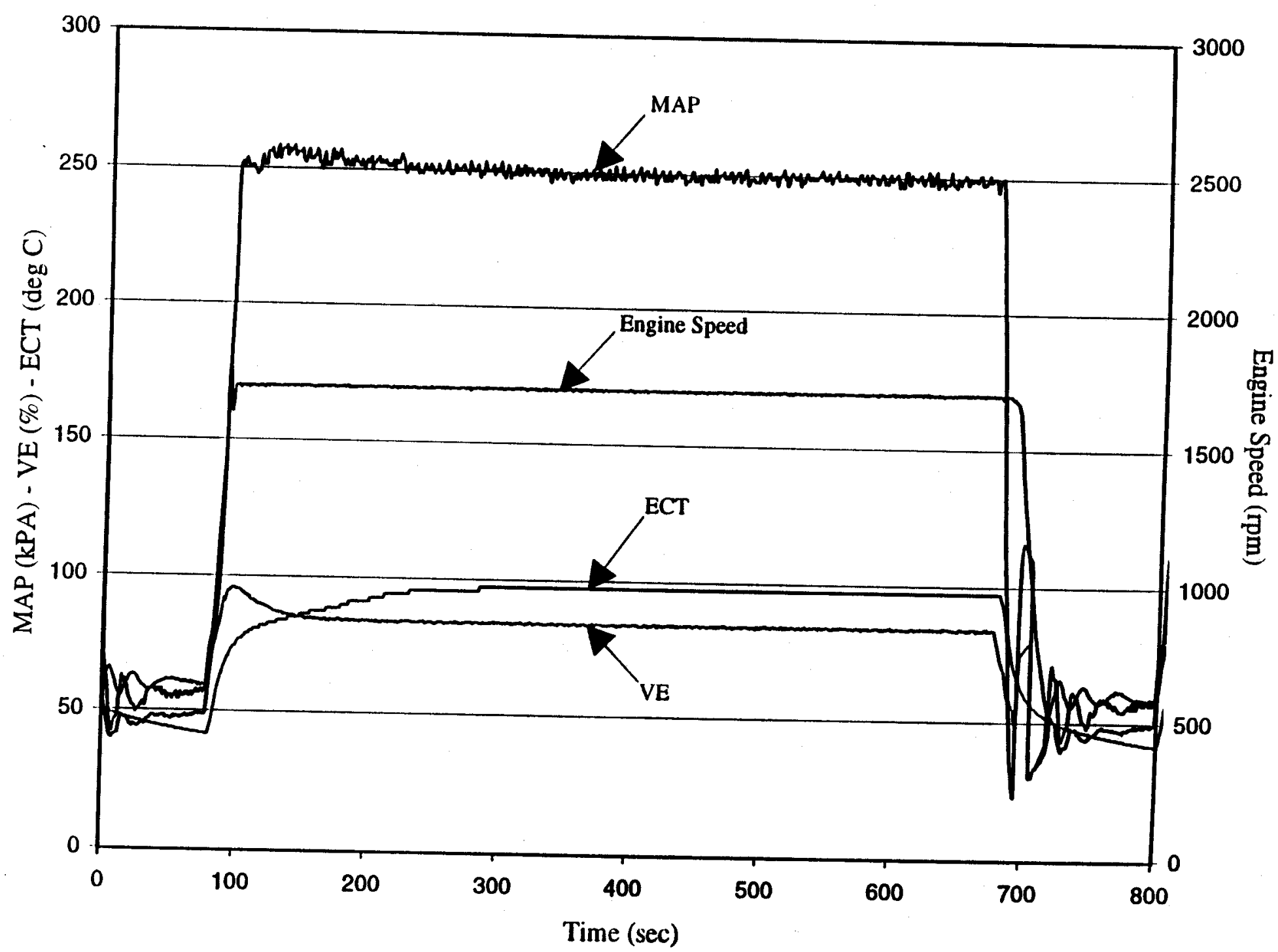

C:LxcellMack_datal575.dat 
TRANSIENT DATA RECORDED FROM GEM SYSTEM FOR ONE FULL CYCLE AT 600 TEST HOURS

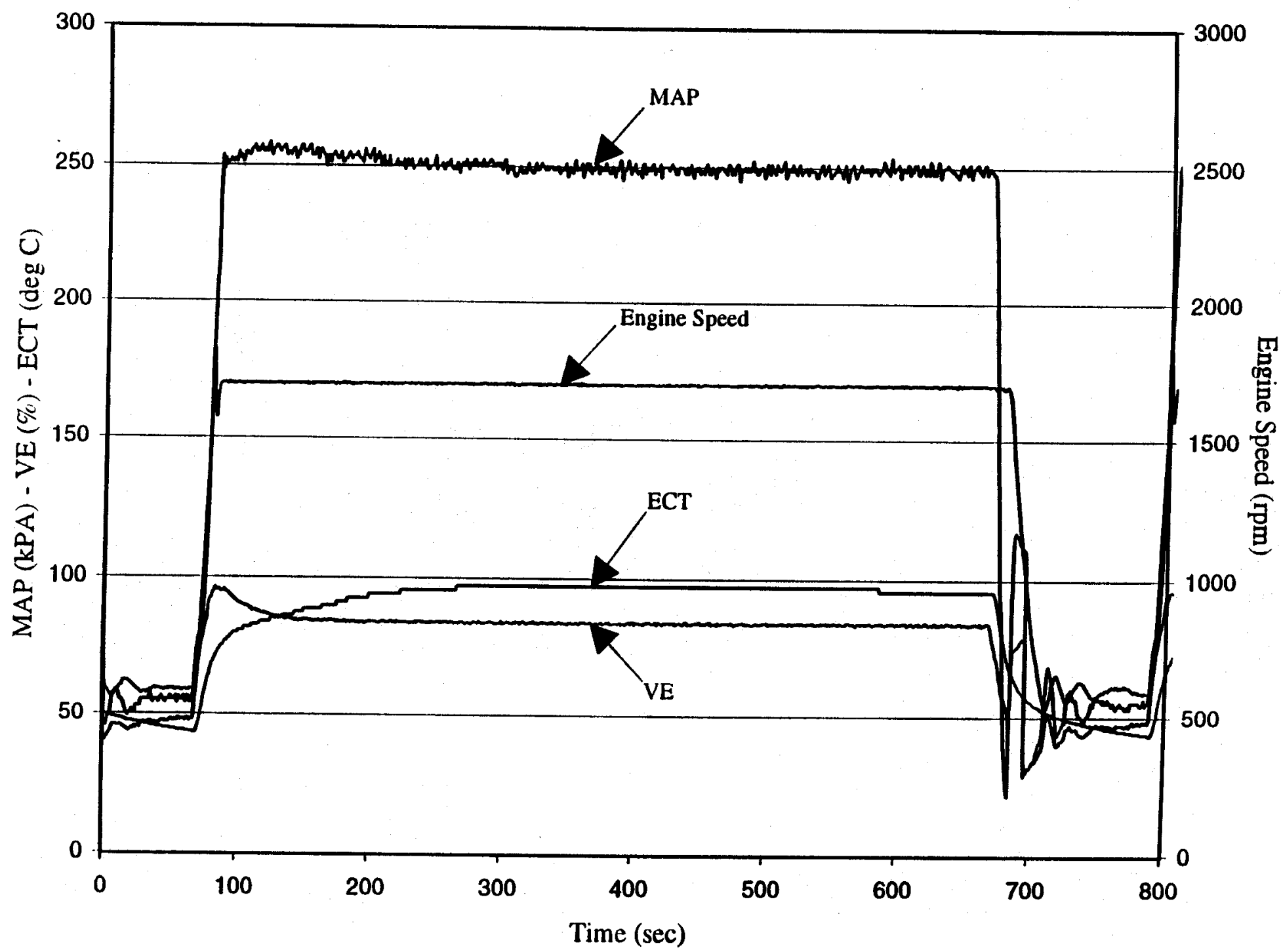


TRANSIENT DATA RECORDED FROM GEM SYSTEM FOR ONE FULL CYCLE AT 625 TEST HOURS

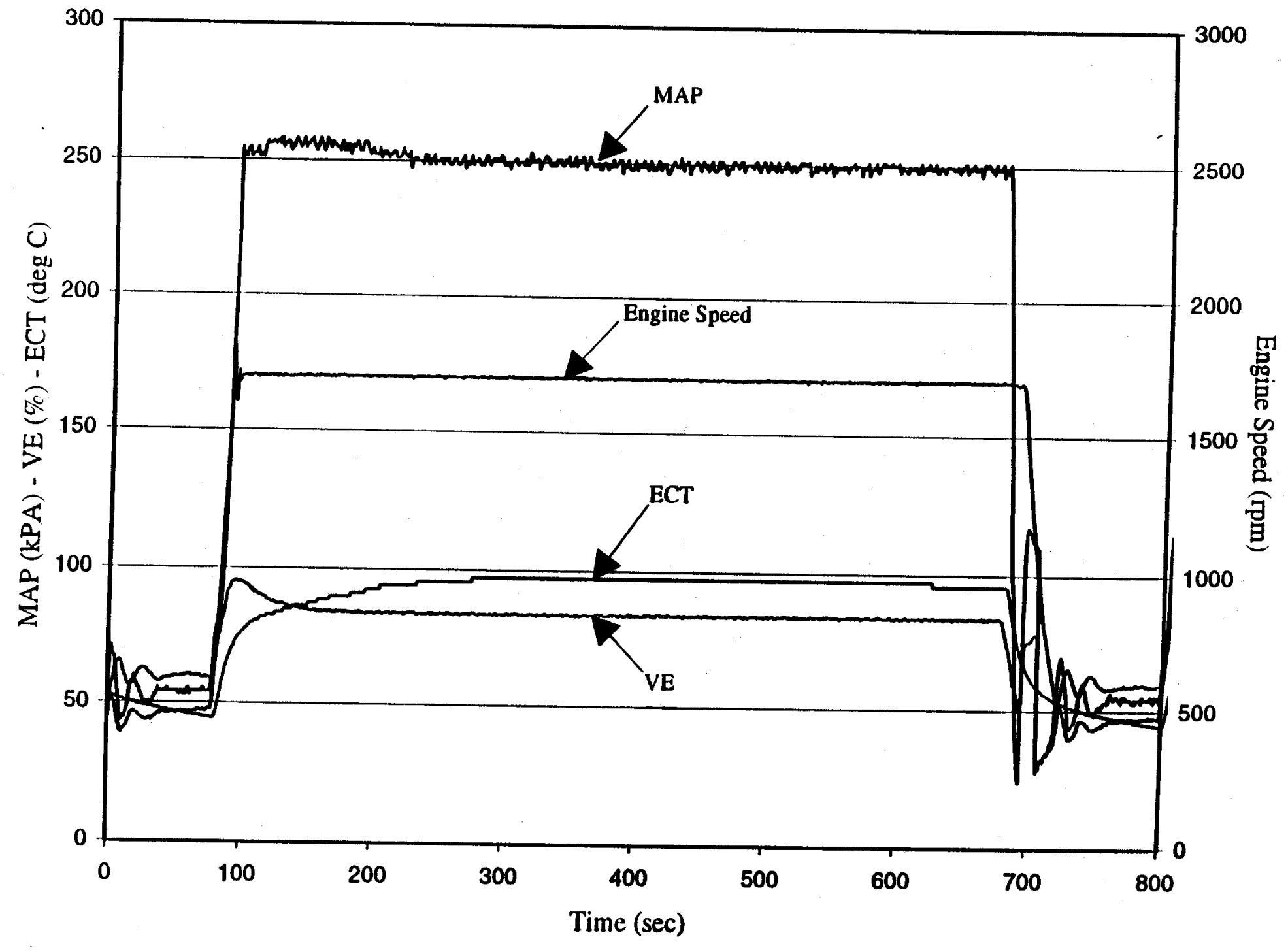


TRANSIENT DATA RECORDED FROM GEM SYSTEM FOR ONE FULL CYCLE AT 650 TEST HOURS

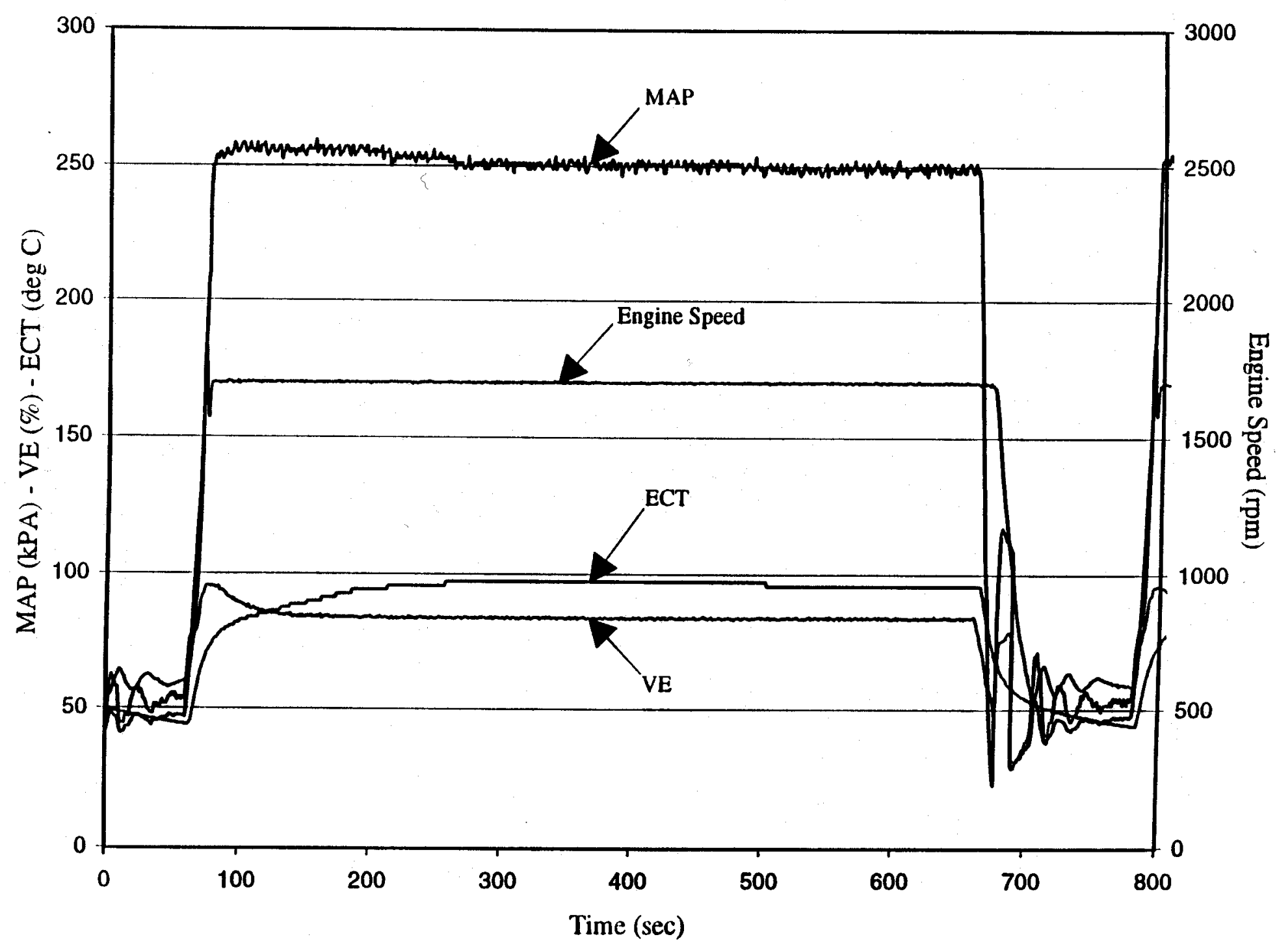


TRANSIENT DATA RECORDED FROM GEM SYSTEM FOR ONE FULL CYCLE AT 675 TEST HOURS

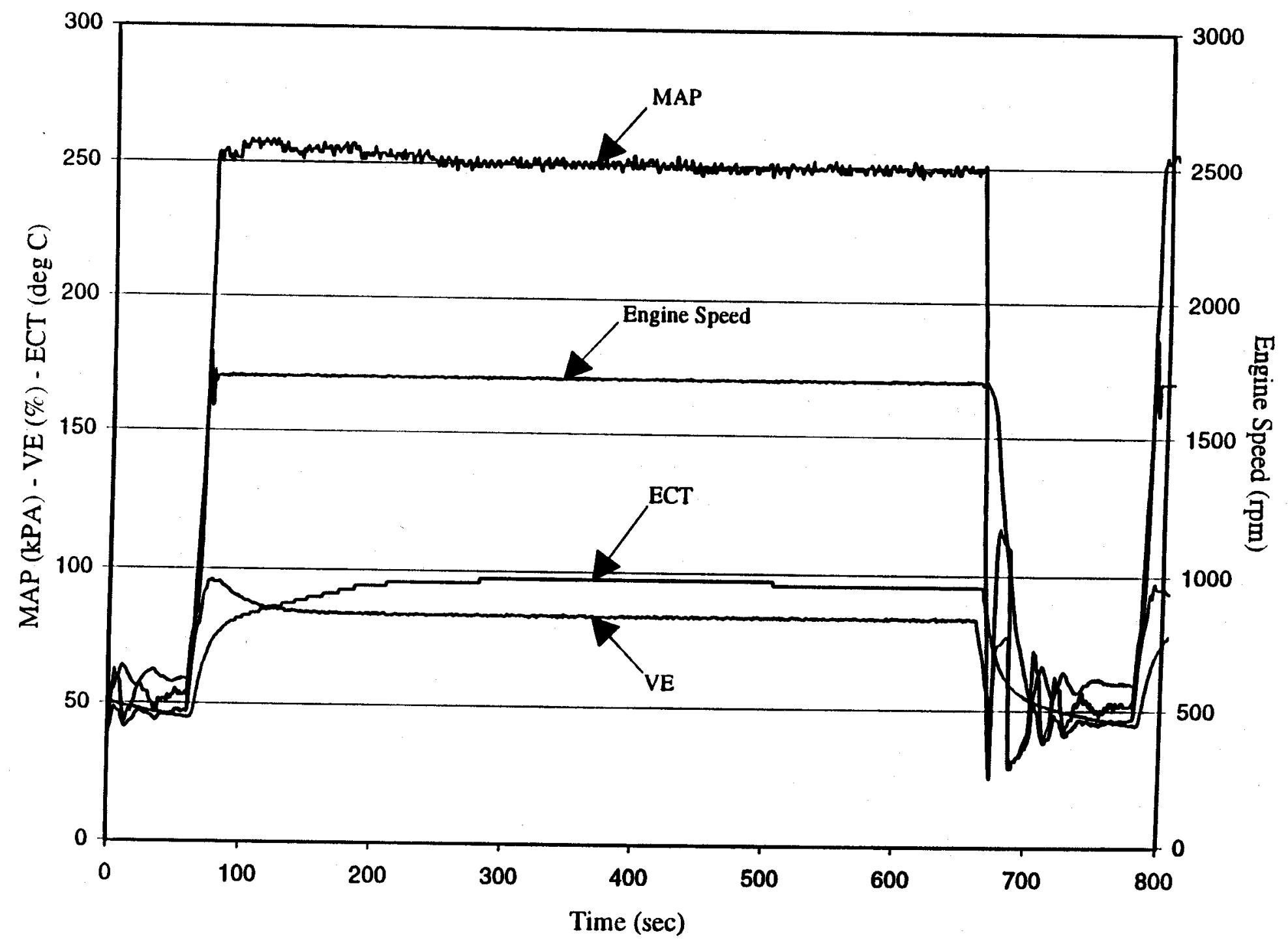


TRANSIENT DATA RECORDED FROM GEM SYSTEM FOR ONE FULL CYCLE AT 700 TEST HOURS

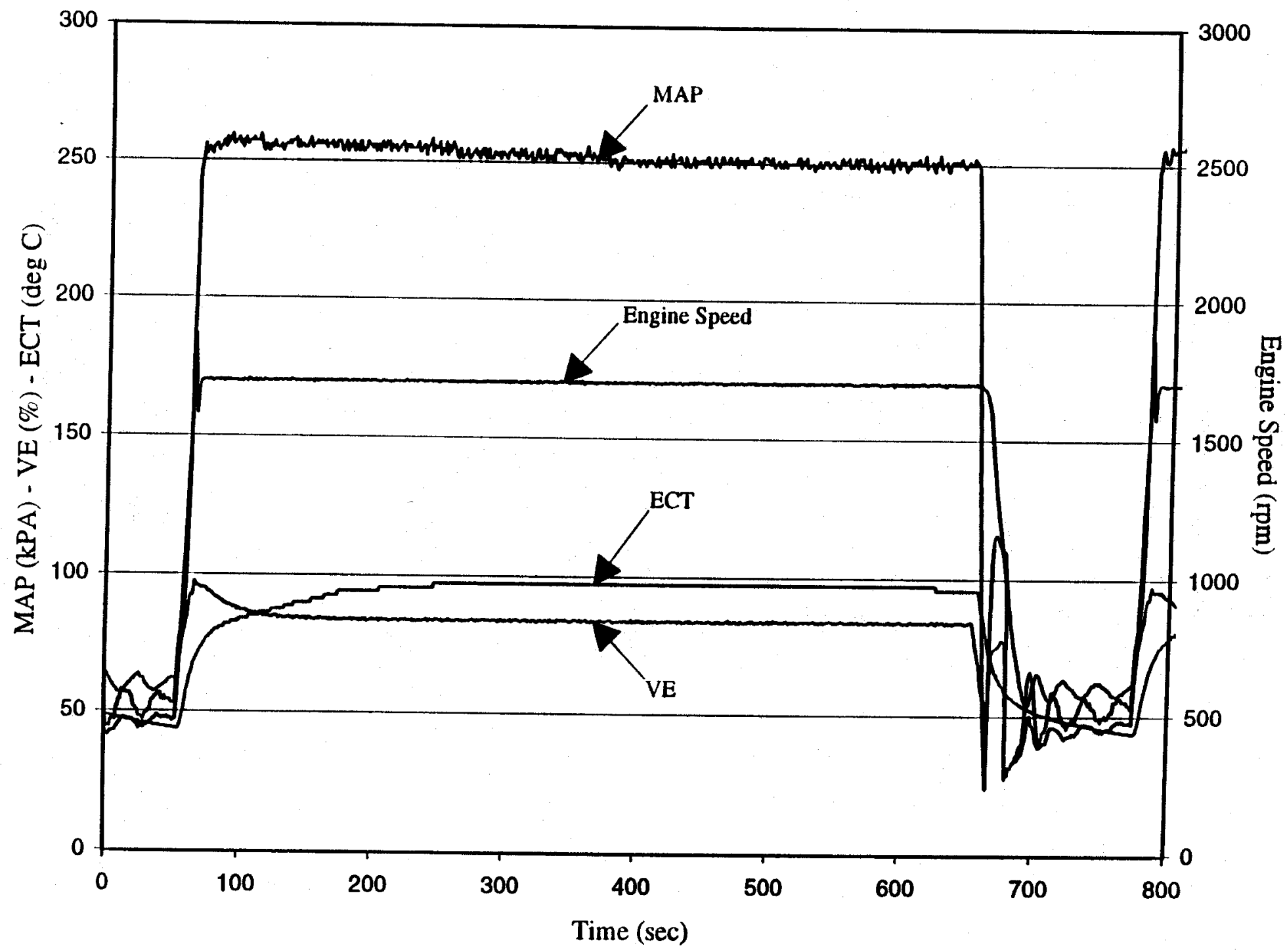


TRANSIENT DATA RECORDED FROM GEM SYSTEM FOR ONE FULL CYCLE AT 725 TEST HOURS

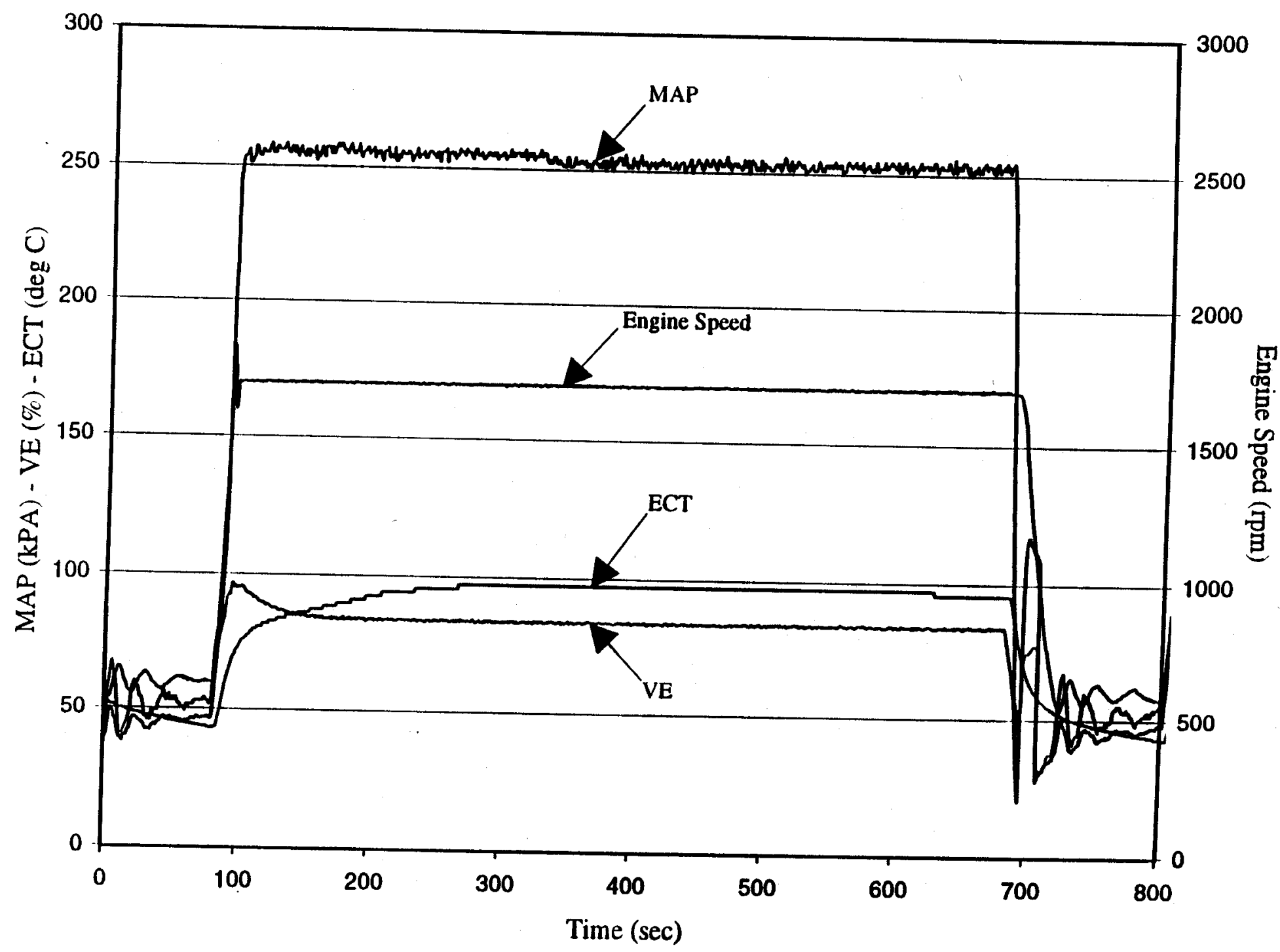


TRANSIENT DATA RECORDED FROM GEM SYSTEM FOR ONE FULL CYCLE AT 750 TEST HOURS

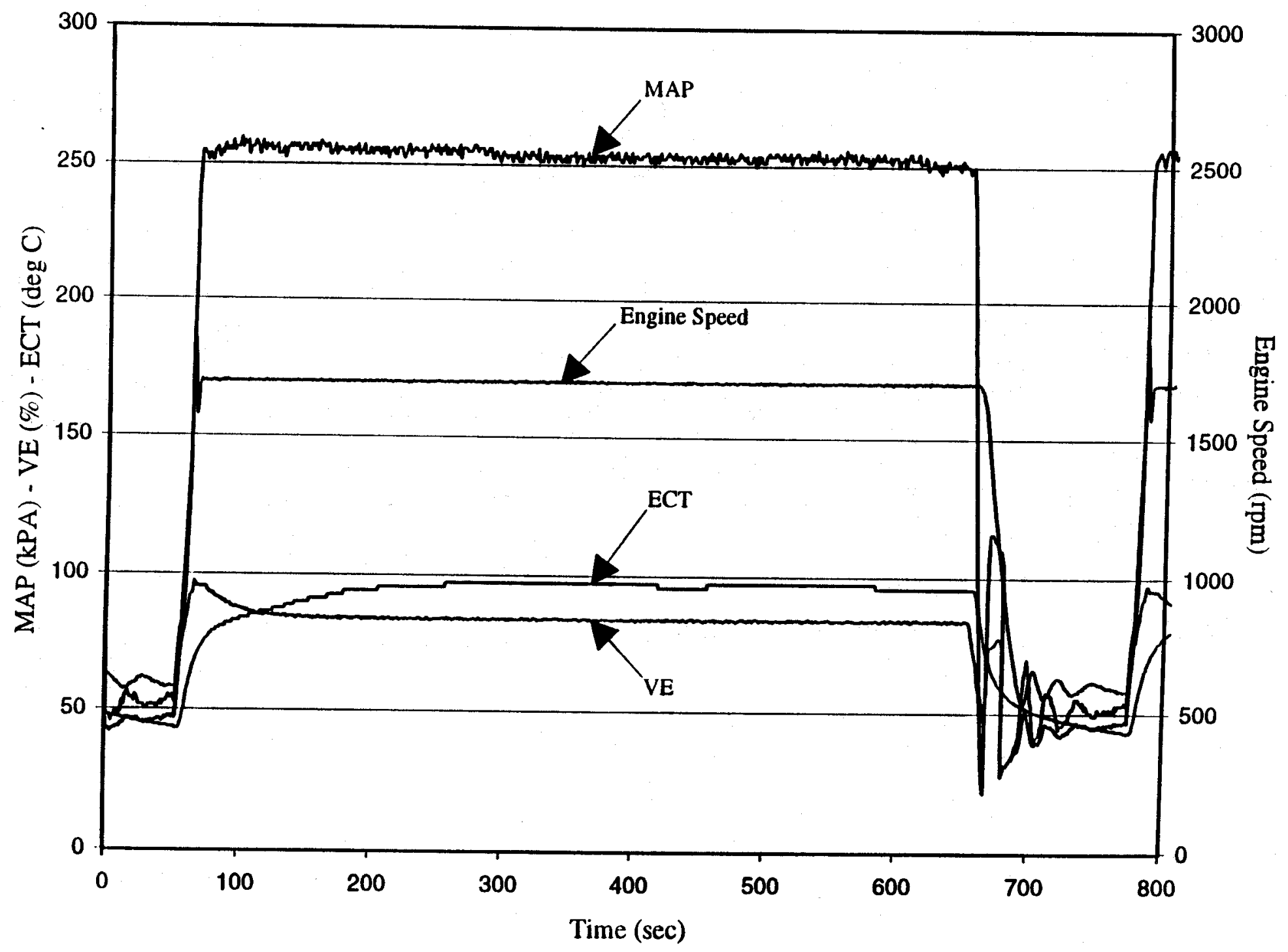


TRANSIENT DATA RECORDED FROM GEM SYSTEM FOR ONE FULL CYCLE AT 775 TEST HOURS

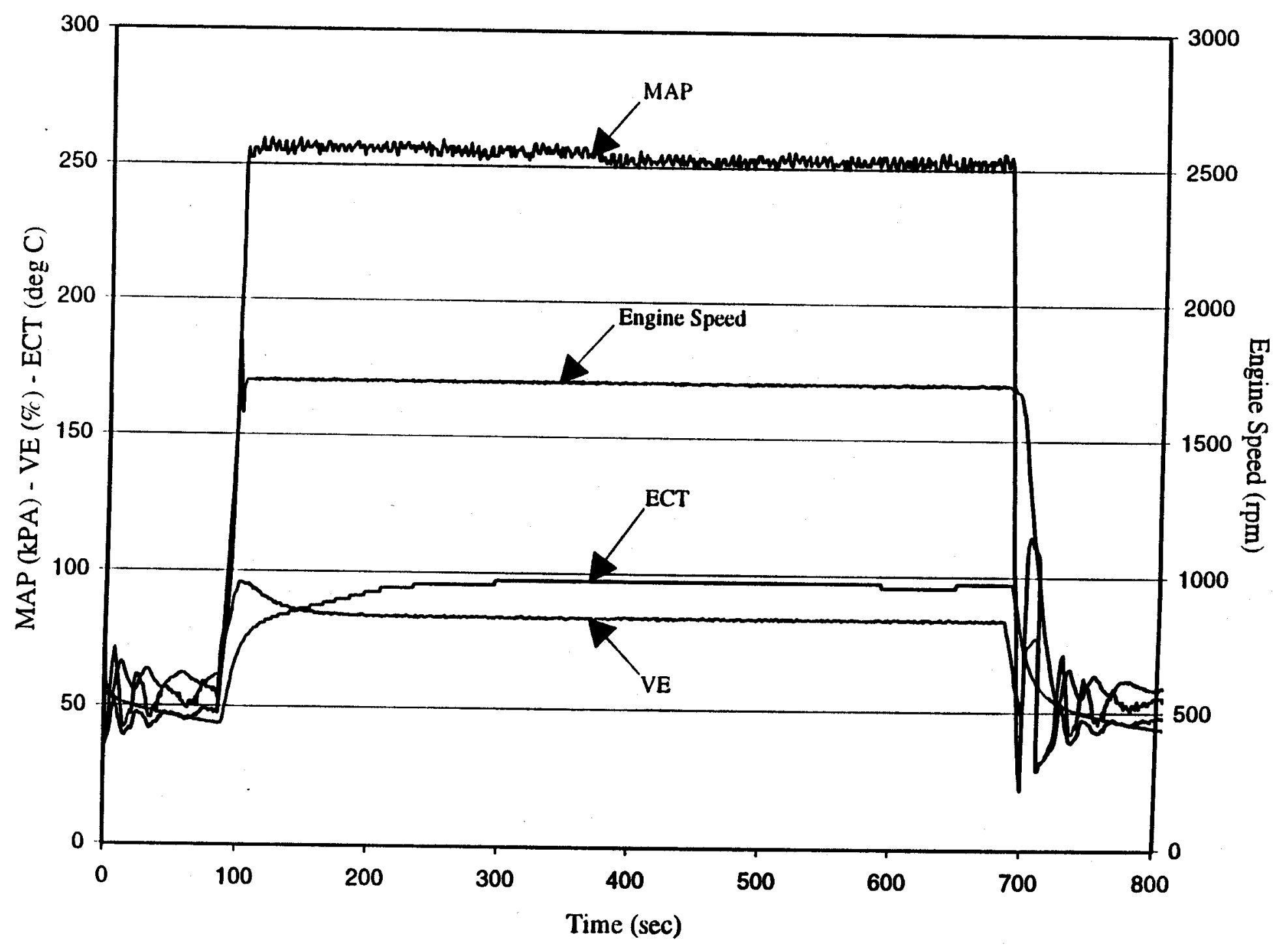


TRANSIENT DATA RECORDED FROM GEM SYSTEM FOR ONE FULL CYCLE AT 800 TEST HOURS

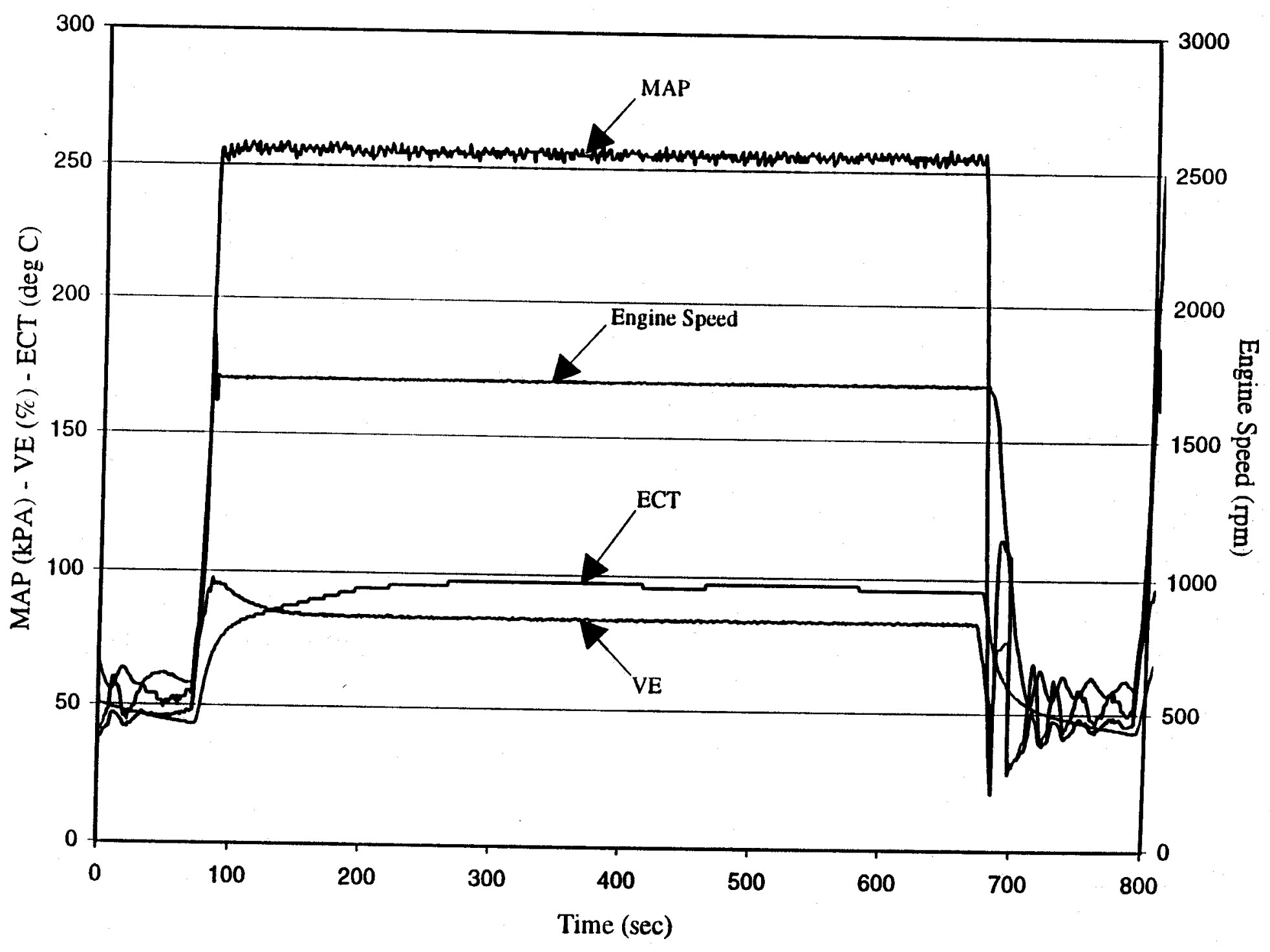


TRANSIENT DATA RECORDED FROM GEM SYSTEM FOR ONE FULL CYCLE AT 825 TEST HOURS

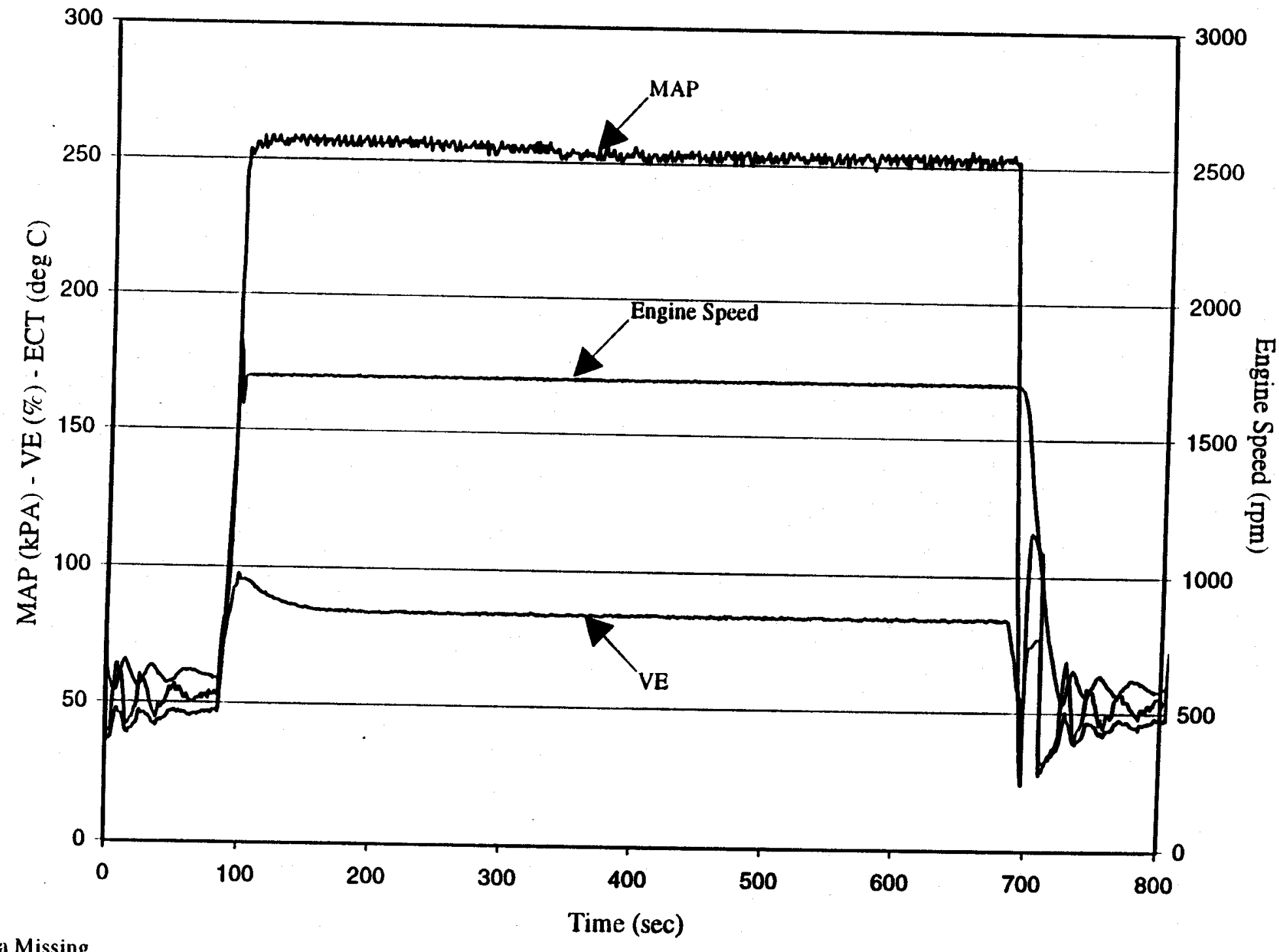


TRANSIENT DATA RECORDED FROM GEM SYSTEM FOR ONE FULL CYCLE AT 850 TEST HOURS

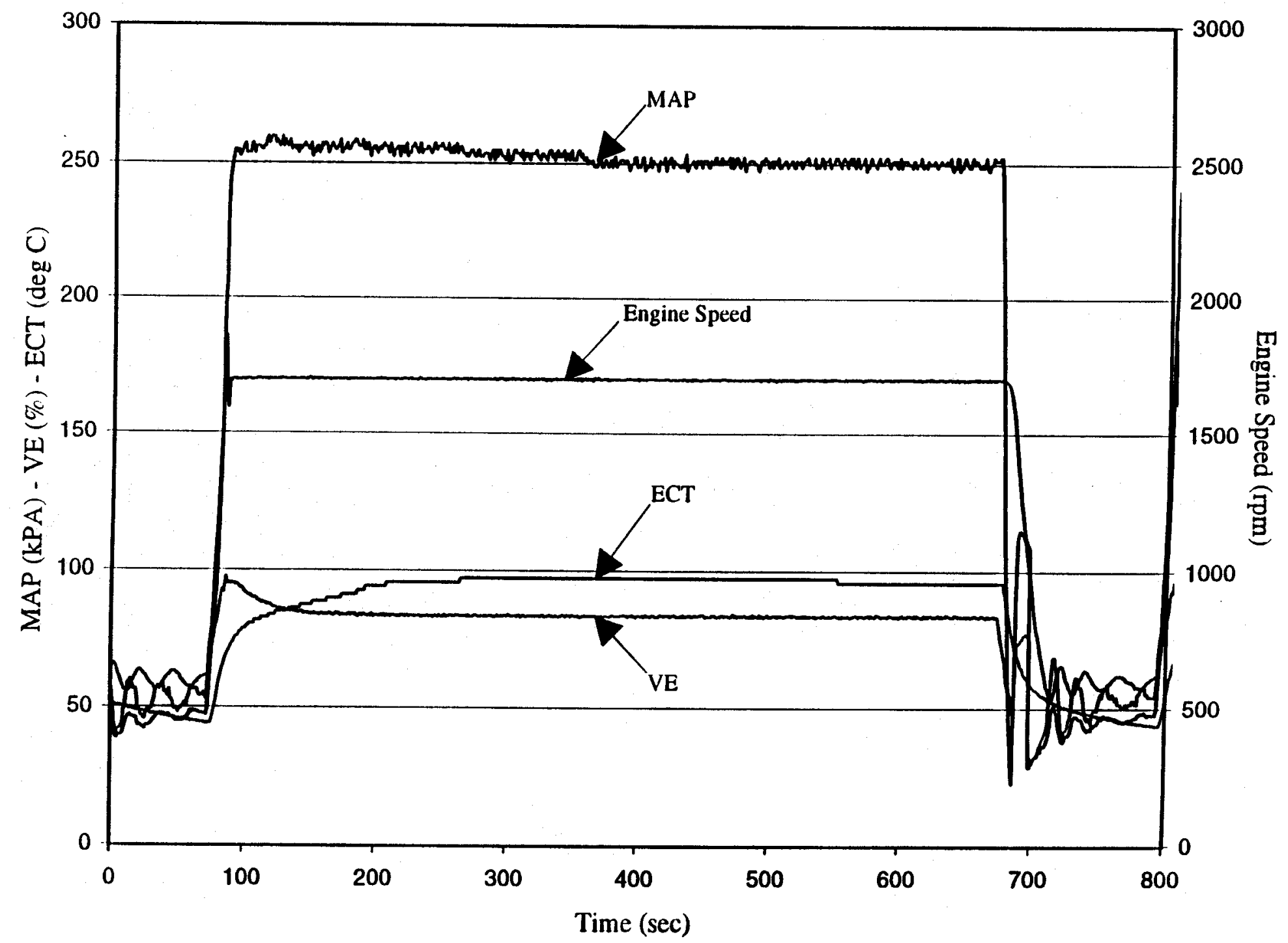


TRANSIENT DATA RECORDED FROM GEM SYSTEM FOR ONE FULL CYCLE AT 875 TEST HOURS

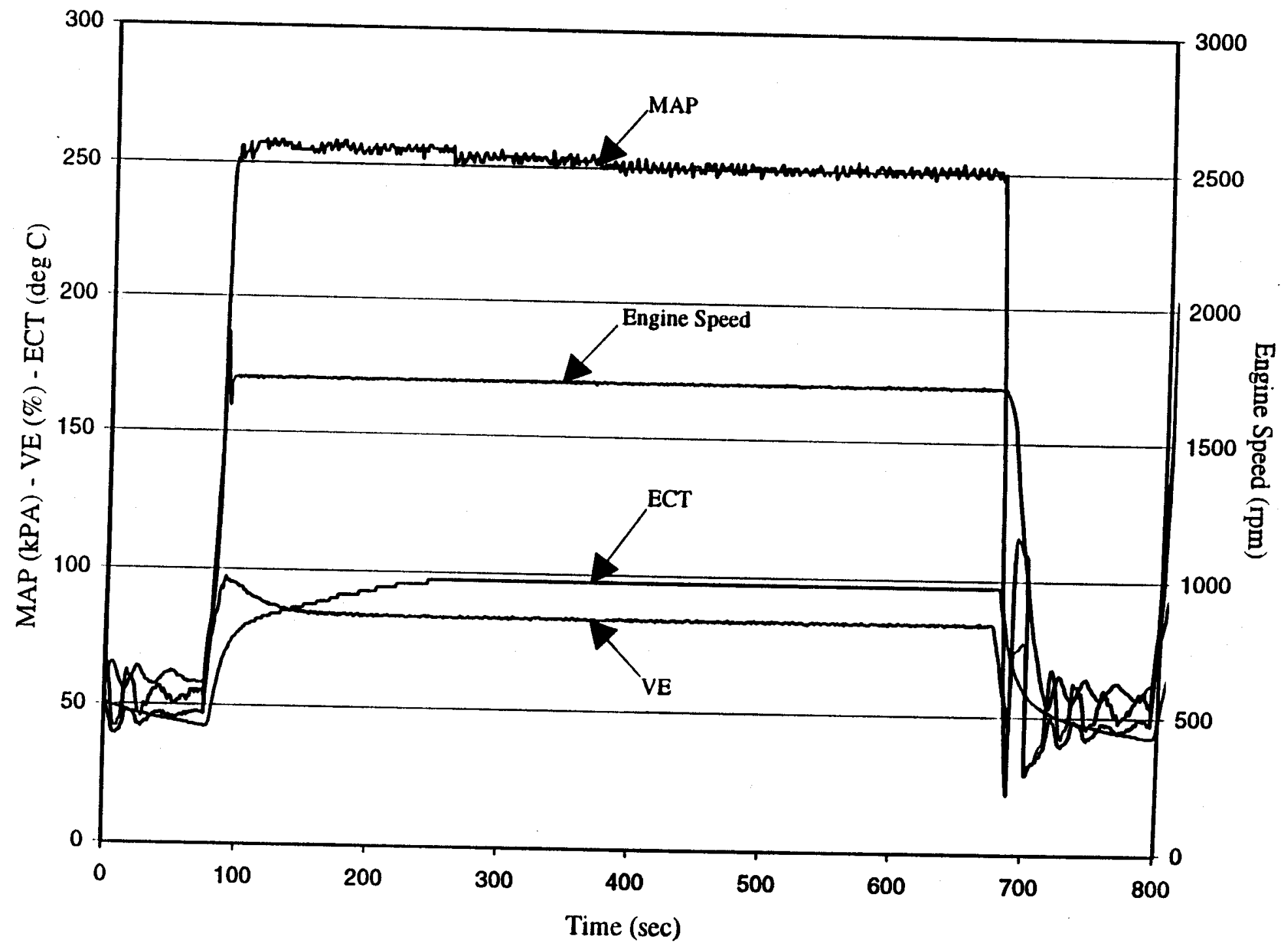

C:ExcelMack_datal875.dat 


\section{TRANSIENT DATA RECORDED FROM GEM SYSTEM FOR ONE FULL CYCLE AT 900 TEST HOURS}

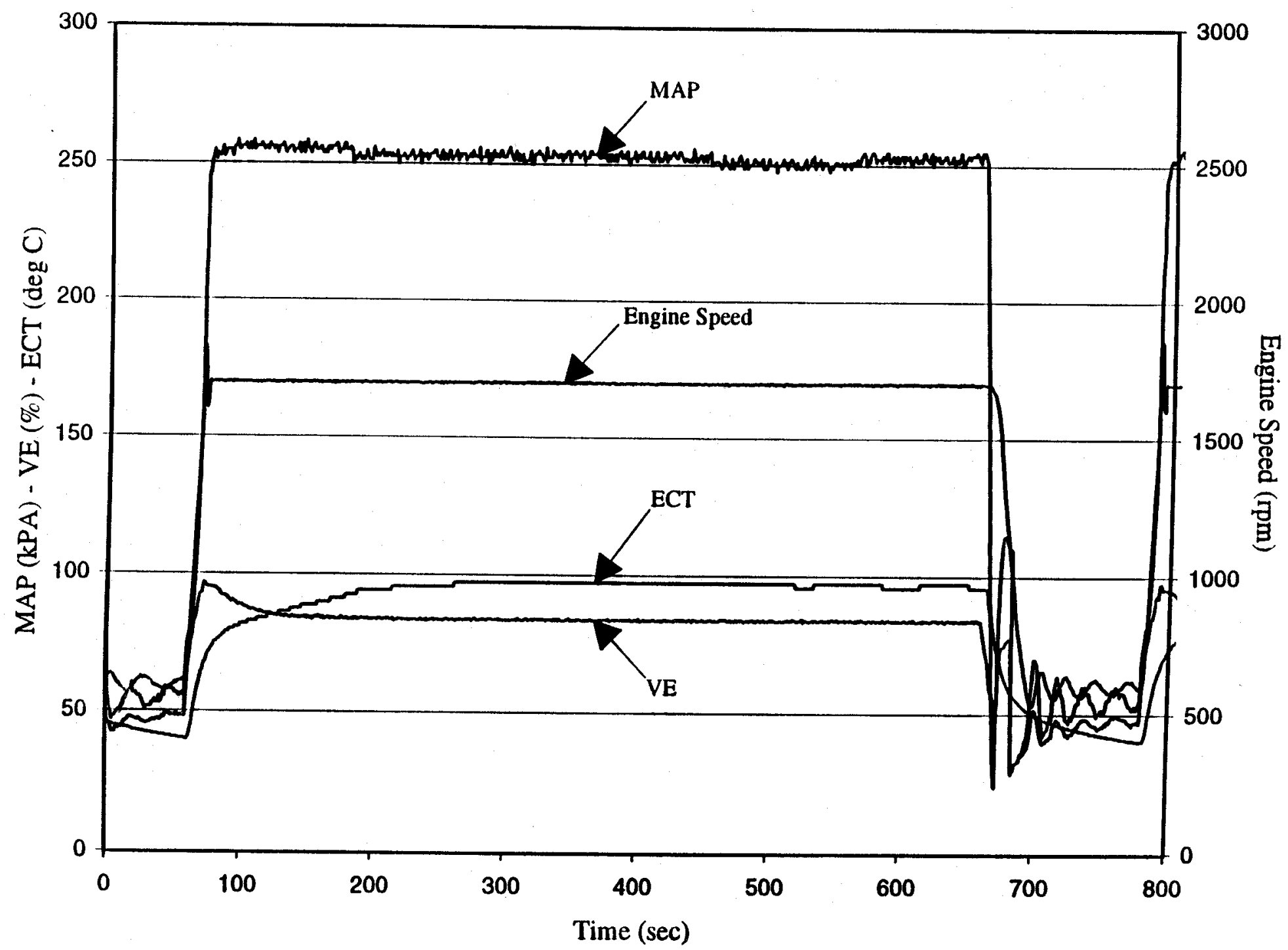


TRANSIENT DATA RECORDED FROM GEM SYSTEM FOR ONE FULL CYCLE AT 928 TEST HOURS

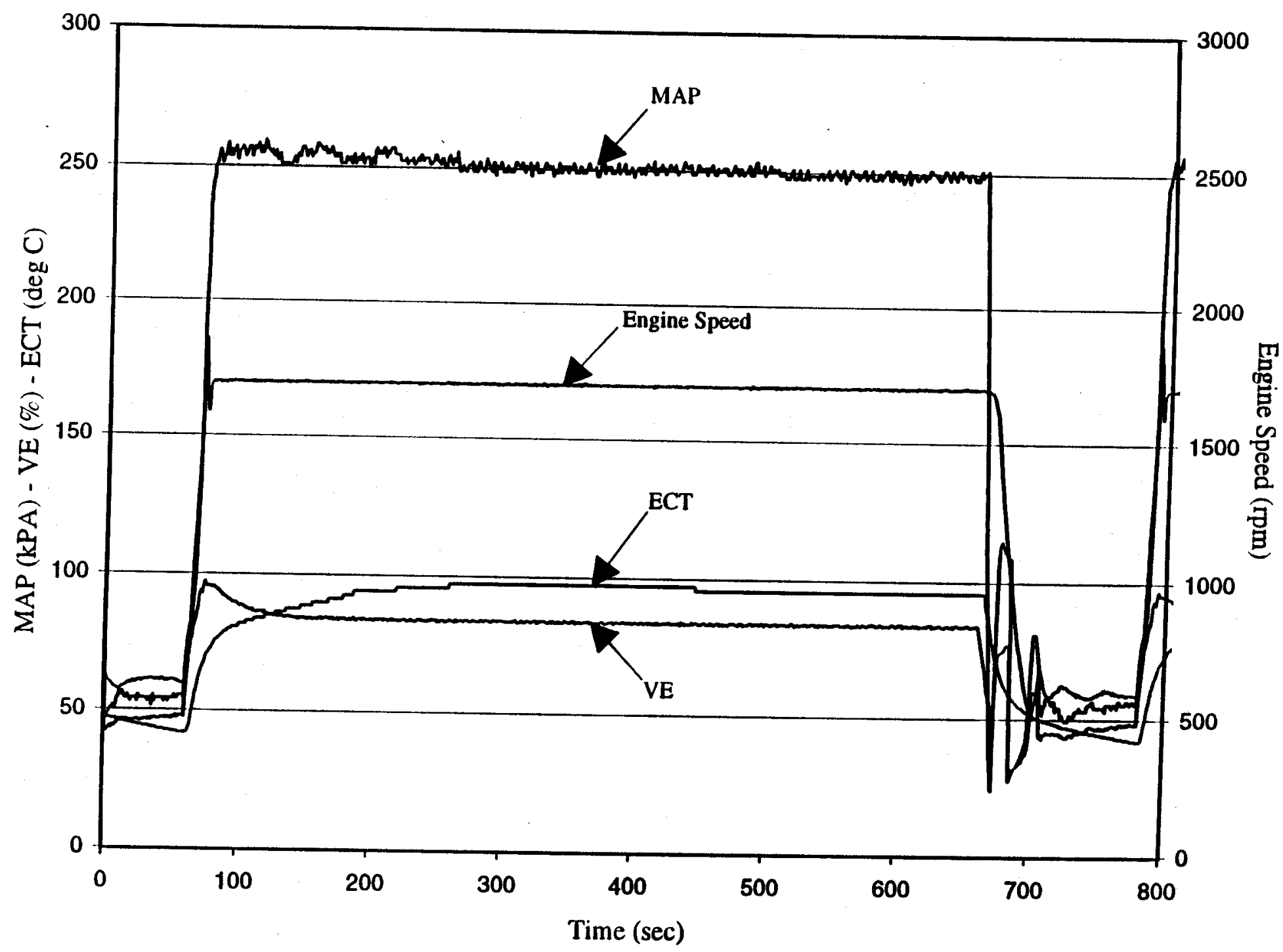


TRANSIENT DATA RECORDED FROM GEM SYSTEM FOR ONE FULL CYCLE AT 950 TEST HOURS

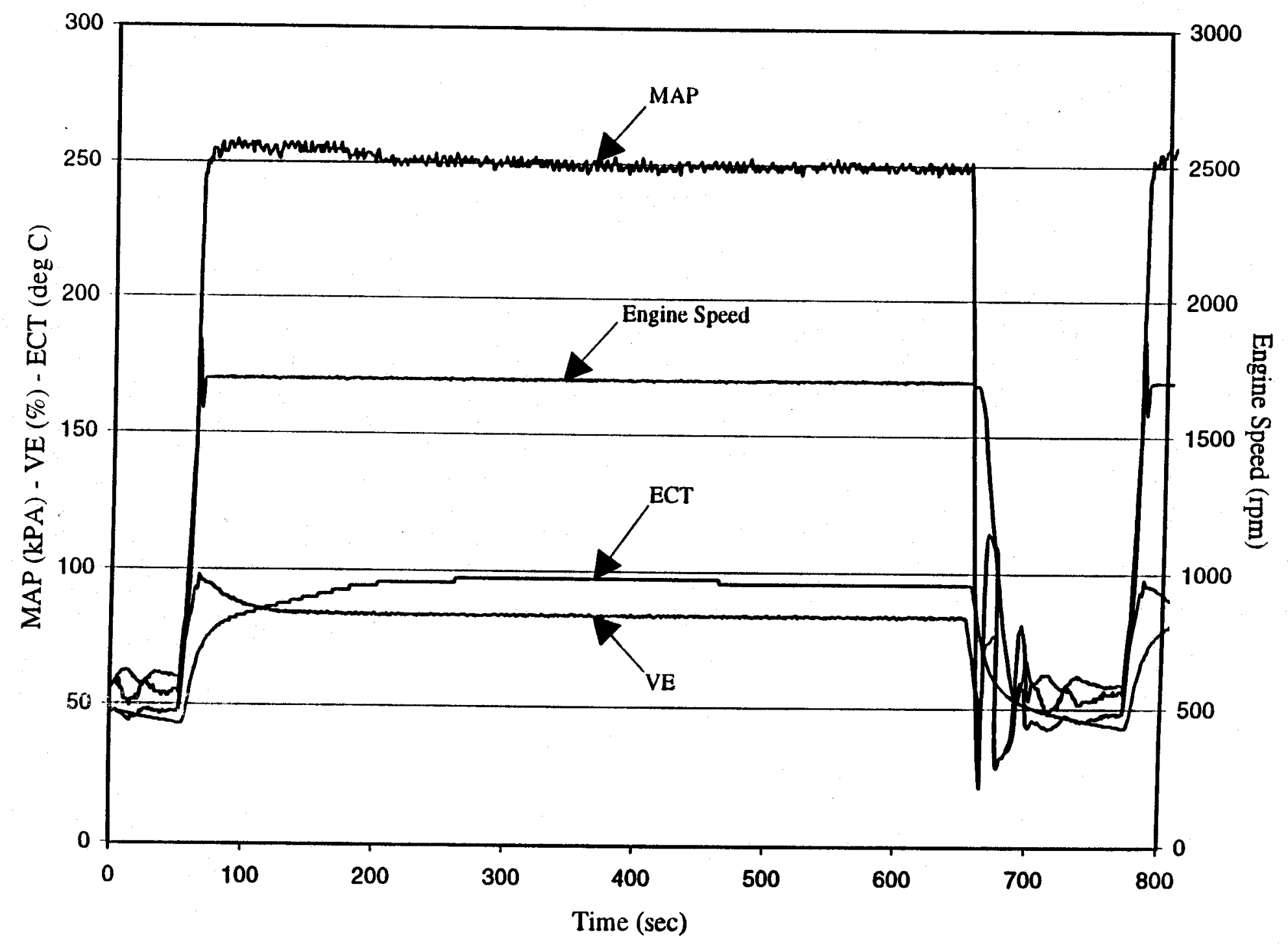


TRANSIENT DATA RECORDED FROM GEM SYSTEM FOR ONE FULL CYCLE AT 975 TEST HOURS

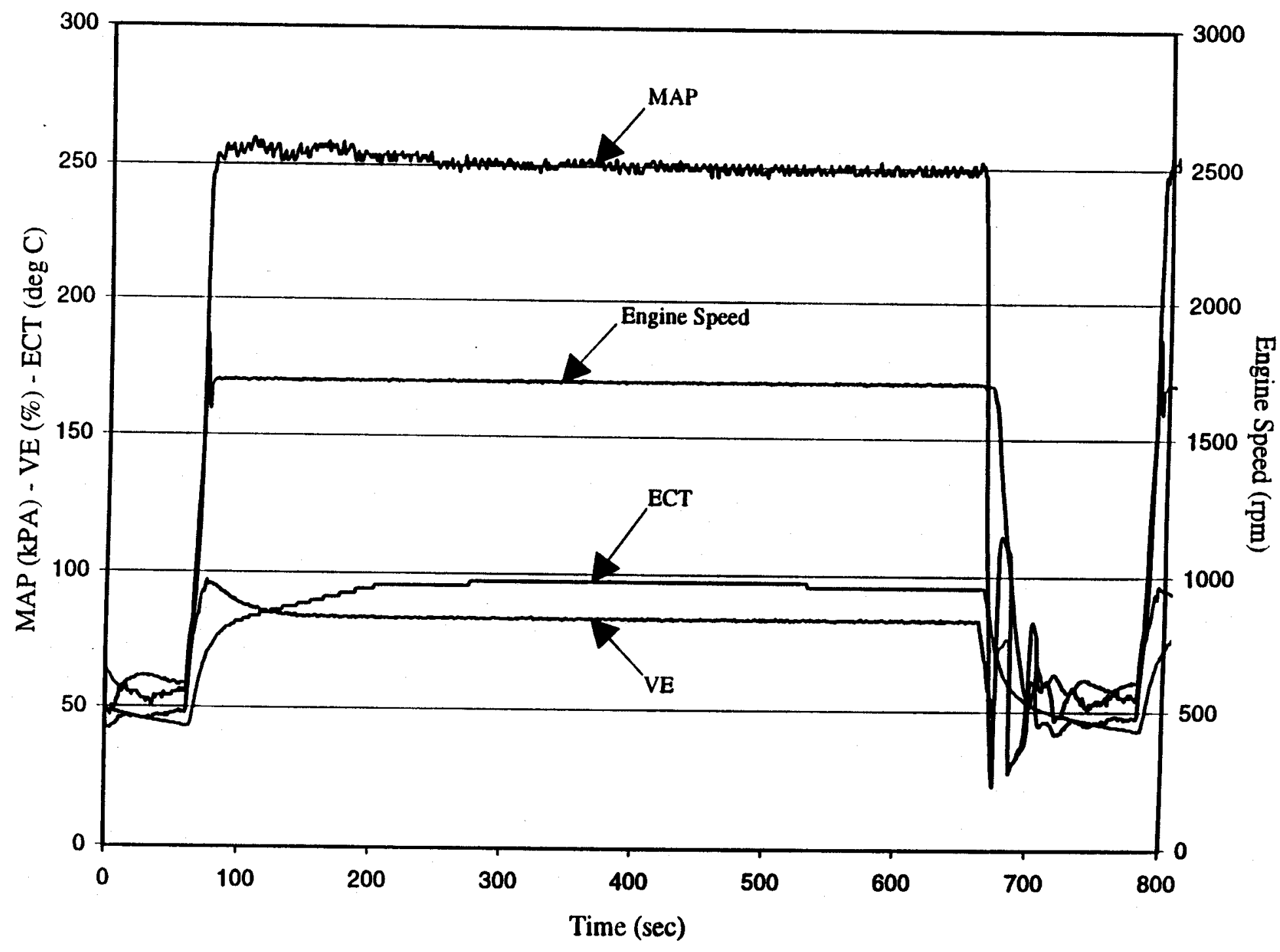


TRANSIENT DATA RECORDED FROM GEM SYSTEM FOR ONE FULL CYCLE AT 1000 TEST HOURS

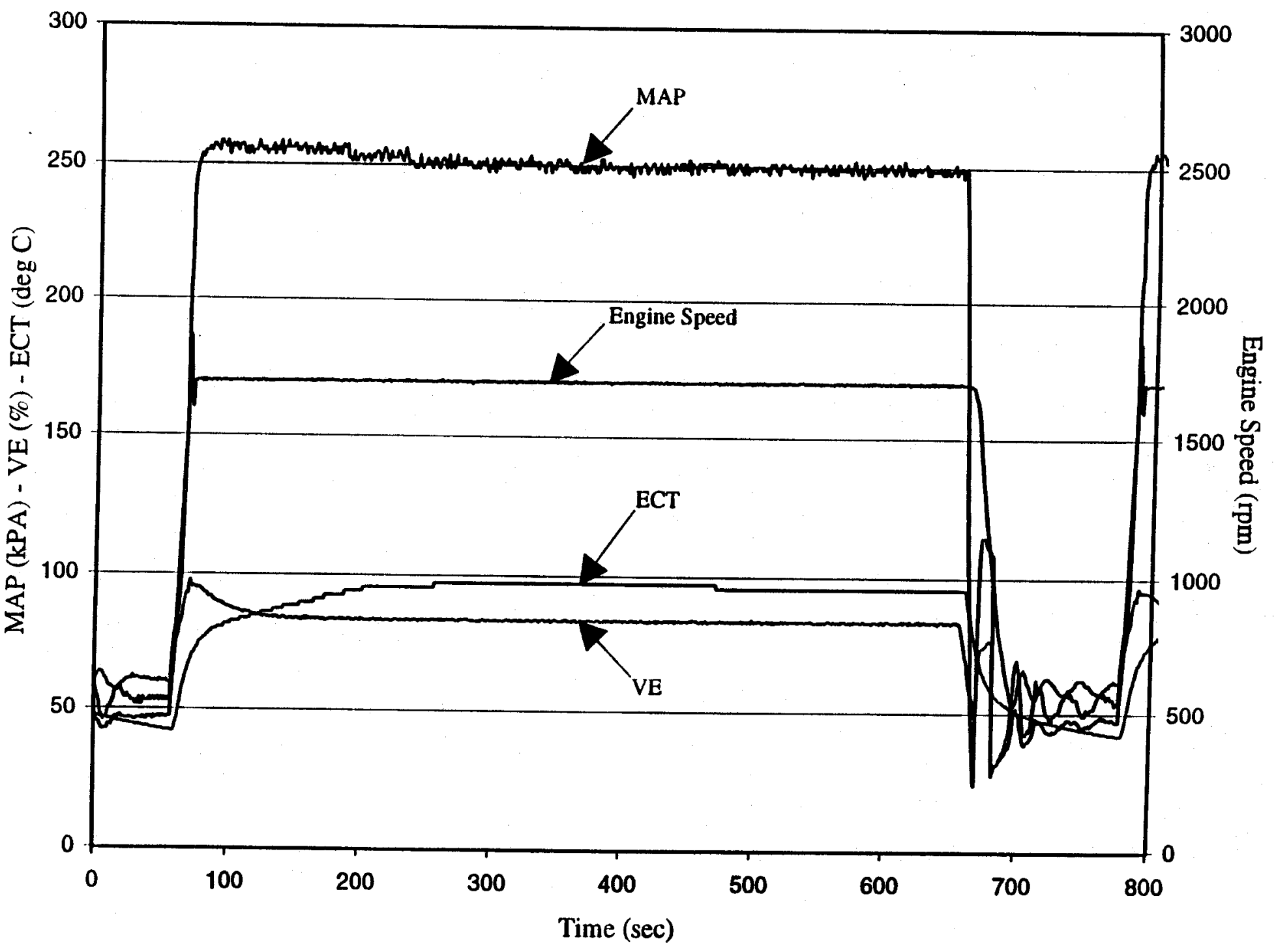


APPENDIX C

SAMPLE HOURLY HP DAQ DATA 


$$
\text { = Cell } 05 \text { MAC4 Project Low-Speed Data = }
$$

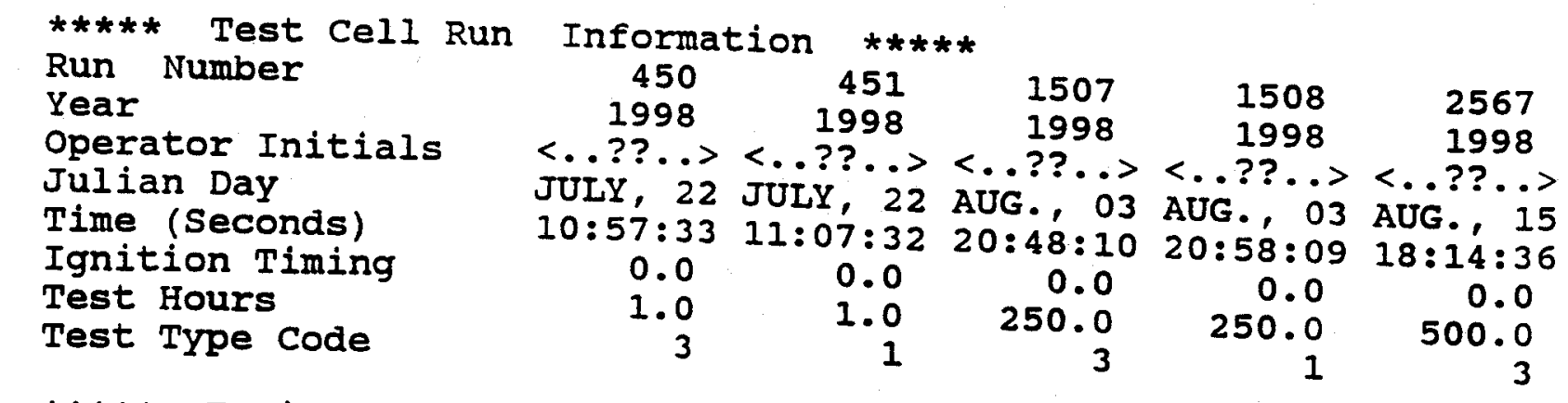

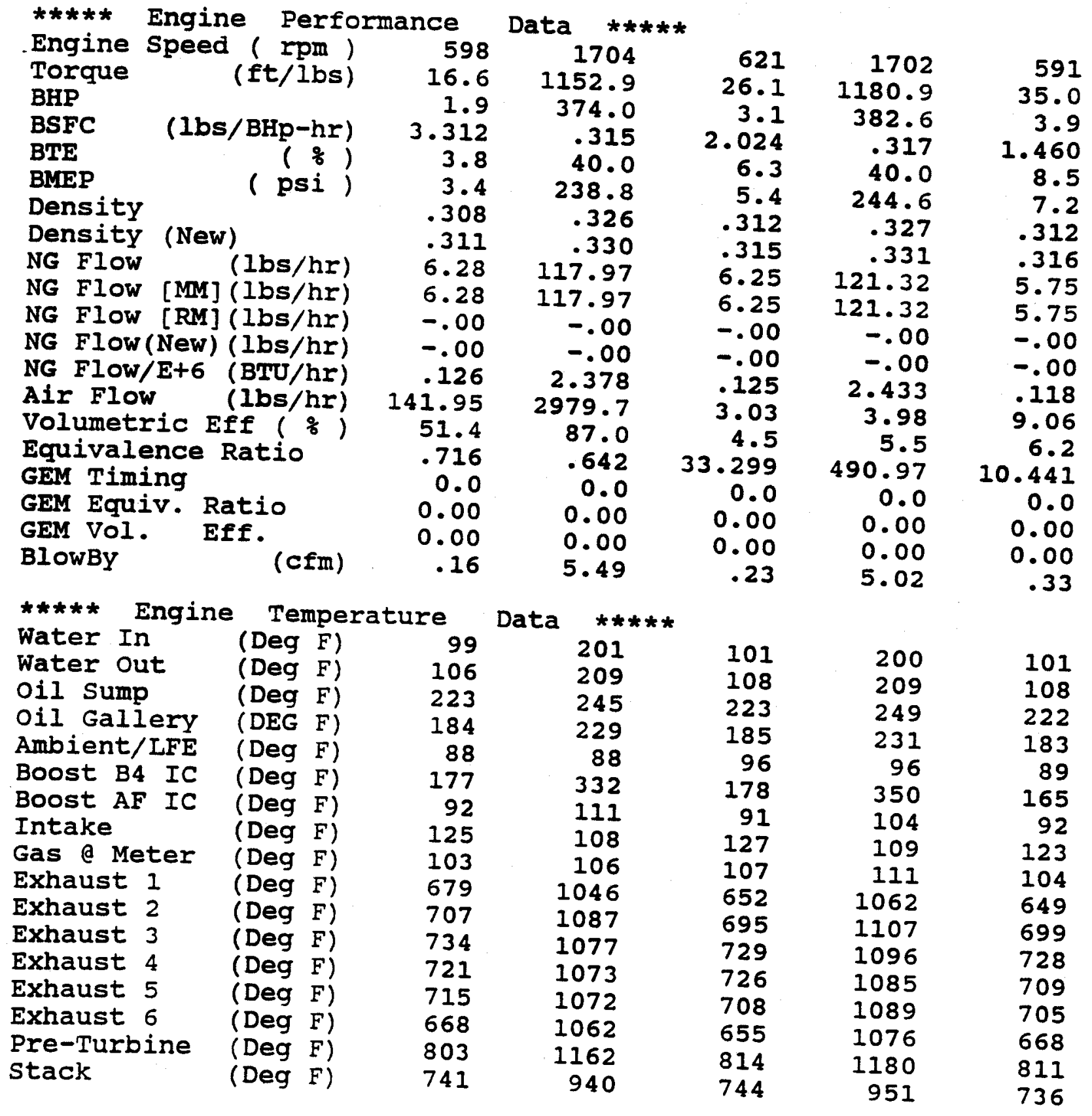




$$
=\text { Cell } 05 \text { MAC4 } \begin{gathered}
\text { Project Low-Speed Data = } \\
\text { (continued) }
\end{gathered}
$$

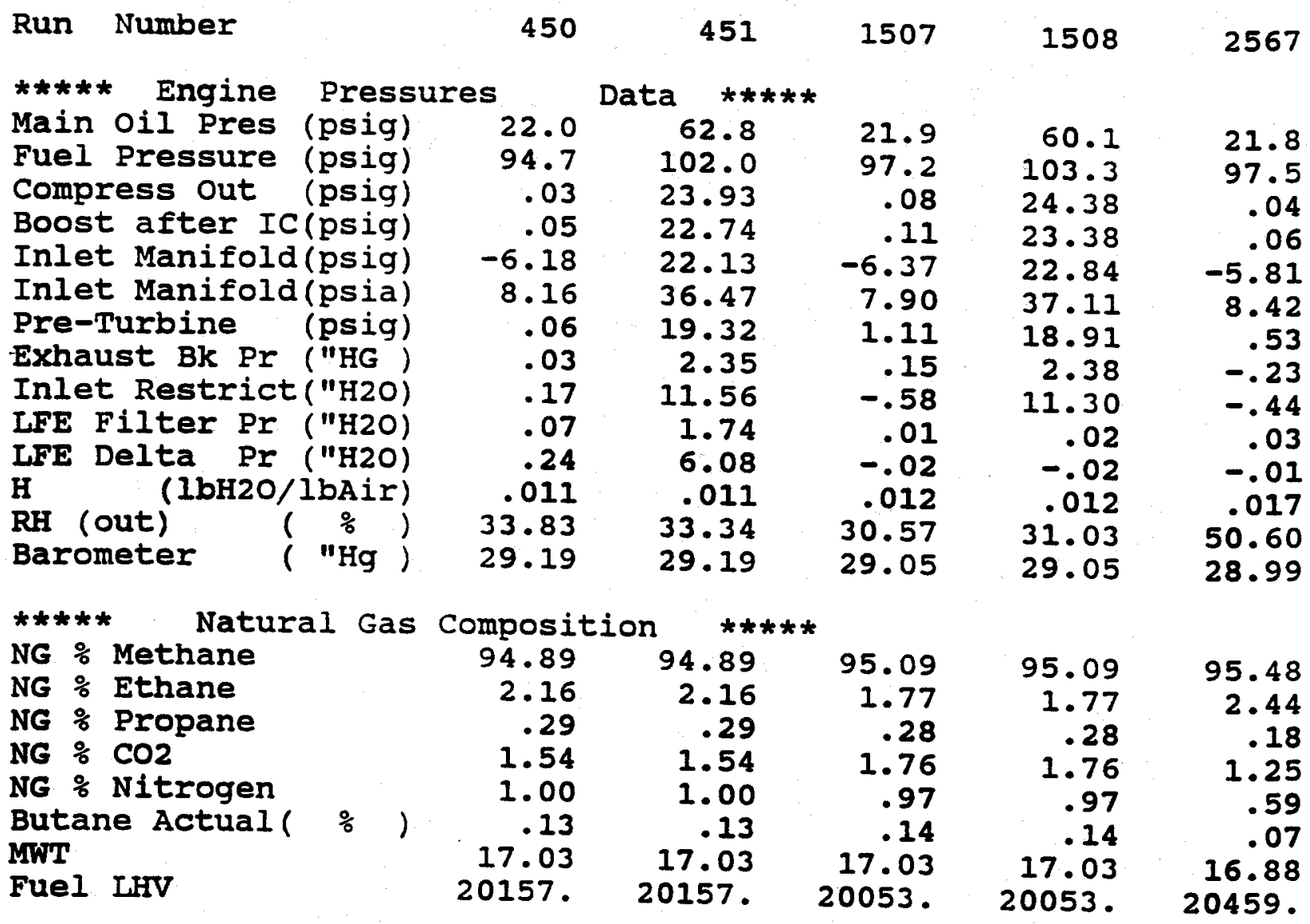


= Cell 05 MAC4 Project Low-Speed Data =

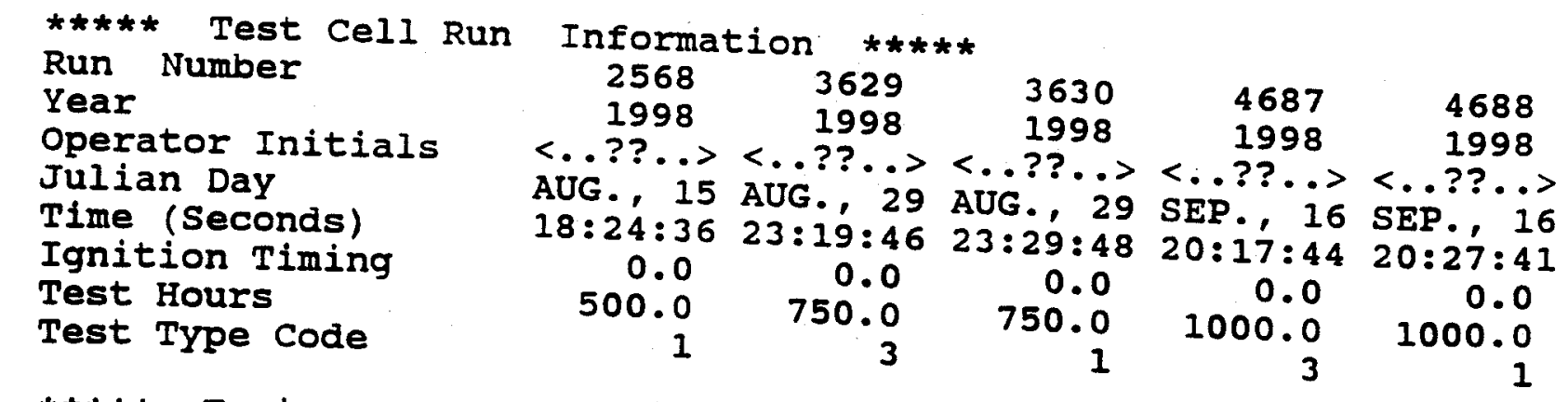

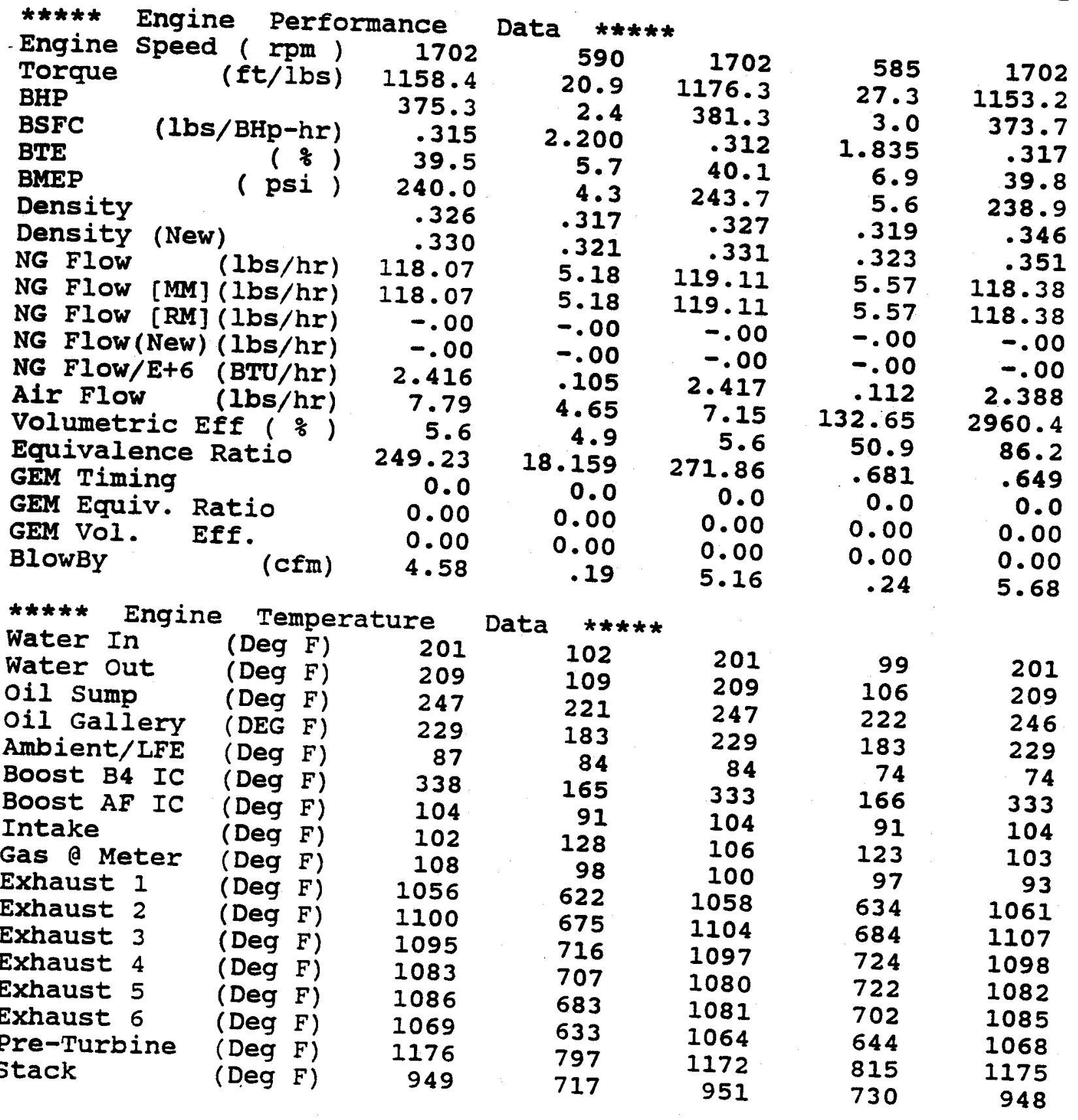




$$
\begin{aligned}
\text { Cell } 05 \text { MAC4 } & \begin{array}{l}
\text { Project Low-Speed Data = } \\
\text { (continued) }
\end{array}
\end{aligned}
$$

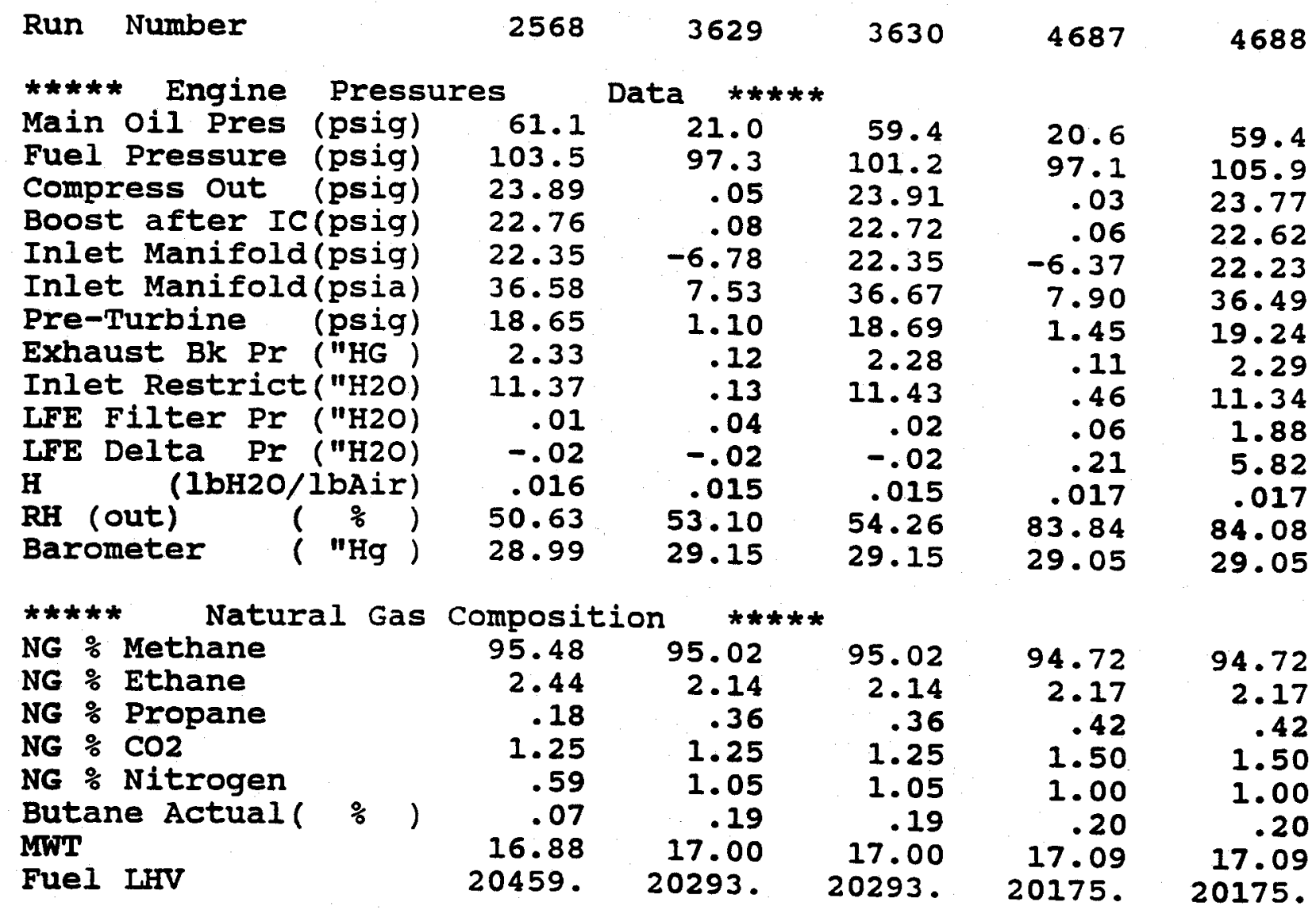


= Cell 05 MAC4 Project Low-Speed Data =

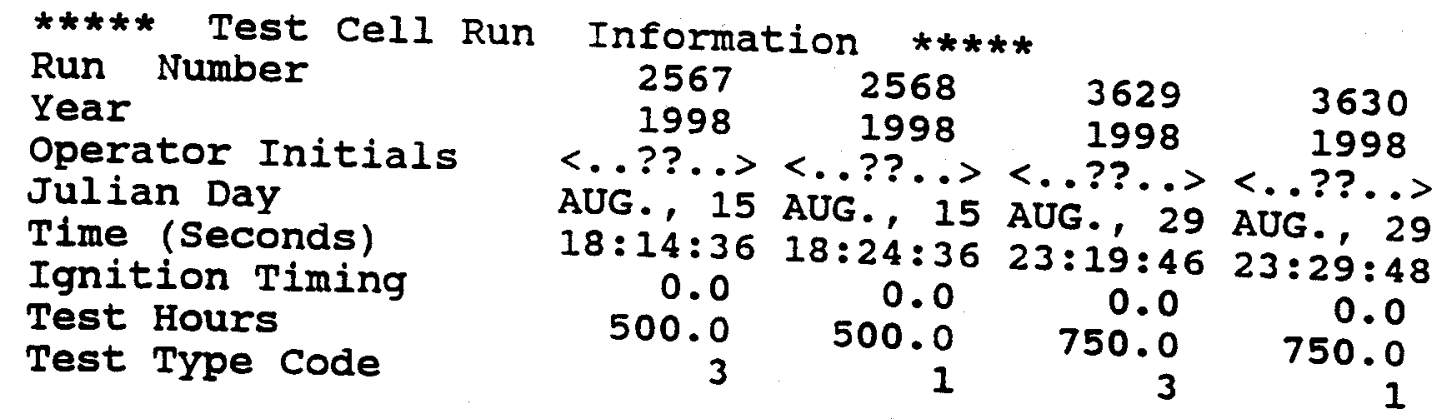

***** Engine Performance Data $* * * * *$

Engine Speed ( rpm

Torque

BHP
BSFC

BTE

BMEP

Density

Density (New)

NG Flow

NG Flow [MM] (lbs/hr)

NG Flow [RM] (lbs/hr)

NG Flow (New) (lbs/hr)

NG Flow/E+6 (BTU/hr)

Air Flow (lbs/hr)

Volumetric Eff ( $\frac{\circ}{6}$ )

GEM Timing

GEM Equiv. Ratio

GEM VOl. Eff.

BlowBY

(cfm)
Equivalence Ratio

\section{1}

35.0

3.9

1.460

8.5

7.2

.312

.316

5.75

5.75

$-.00$

$-.00$

.118

9.06

6.2

10.441

0.0

0.00

0.00

.33

$\star \star \star \star *$ Engine Temperature

Water In

Water out

Oil Sump

Oil Gallery

Ambient/LFE

Boost B4 IC

Boost AF IC

Intake

Gas @ Meter

Exhaust 1

Exhaust 2

Exhaust 3

Exhaust 4

Exhaust 5

Exhaust 6

Pre-Turbine

stack

$\begin{array}{lr}\text { (Deg F) } & 101 \\ \text { (Deg F) } & 108 \\ \text { (Deg F) } & 222 \\ \text { (DEG F) } & 183 \\ \text { (Deg F) } & 89 \\ \text { (Deg F) } & 165 \\ \text { (Deg F) } & 92 \\ \text { (Deg F) } & 123 \\ \text { (Deg F) } & 104 \\ \text { (Deg F) } & 649 \\ \text { (Deg F) } & 699 \\ \text { (Deg F) } & 728 \\ \text { (Deg F) } & 709 \\ \text { (Deg F) } & 705 \\ \text { (Deg F) } & 668 \\ \text { (Deg F) } & 811 \\ \text { (Deg F) } & 736\end{array}$

$$
\begin{array}{r}
1702 \\
1158.4 \\
375.3 \\
.315 \\
39.5 \\
240.0 \\
.326 \\
.330 \\
118.07 \\
118.07 \\
-.00 \\
-.00 \\
2.416 \\
7.79 \\
5.6 \\
249.23 \\
0.0 \\
0.00 \\
0.00 \\
4.58
\end{array}
$$

Data

$* * * * *$
201
209
247
229

87

338

104

102

108

1056

1100

1095

1083

1086

1069

1176

949

590
20.9
2.4
2.200
5.7
4.3
.317
.321
5.18
5.18
-.00
-.00
.105
4.65
4.9

1702

1176.3

381.3

.312

40.1

243.7

.327

.331

119.11

119.11

$-.00$

$-.00$

2.417

7.15

5.6

$18.159 \quad 271.86$

$0.0 \quad 0.0$

$0.00 \quad 0.00$

0.00

.19

0.00

5.16

$\begin{array}{rr}102 & 201 \\ 109 & 209 \\ 221 & 247 \\ 183 & 229 \\ 84 & 84 \\ 165 & 333 \\ 91 & 104 \\ 128 & 106 \\ 98 & 100 \\ 622 & 1058 \\ 675 & 1104 \\ 716 & 1097 \\ 707 & 1080 \\ 683 & 1081 \\ 633 & 1064 \\ 797 & 1172 \\ 717 & 951\end{array}$


= Cell 05 MAC4 Project Low-Speed Data = (continued)

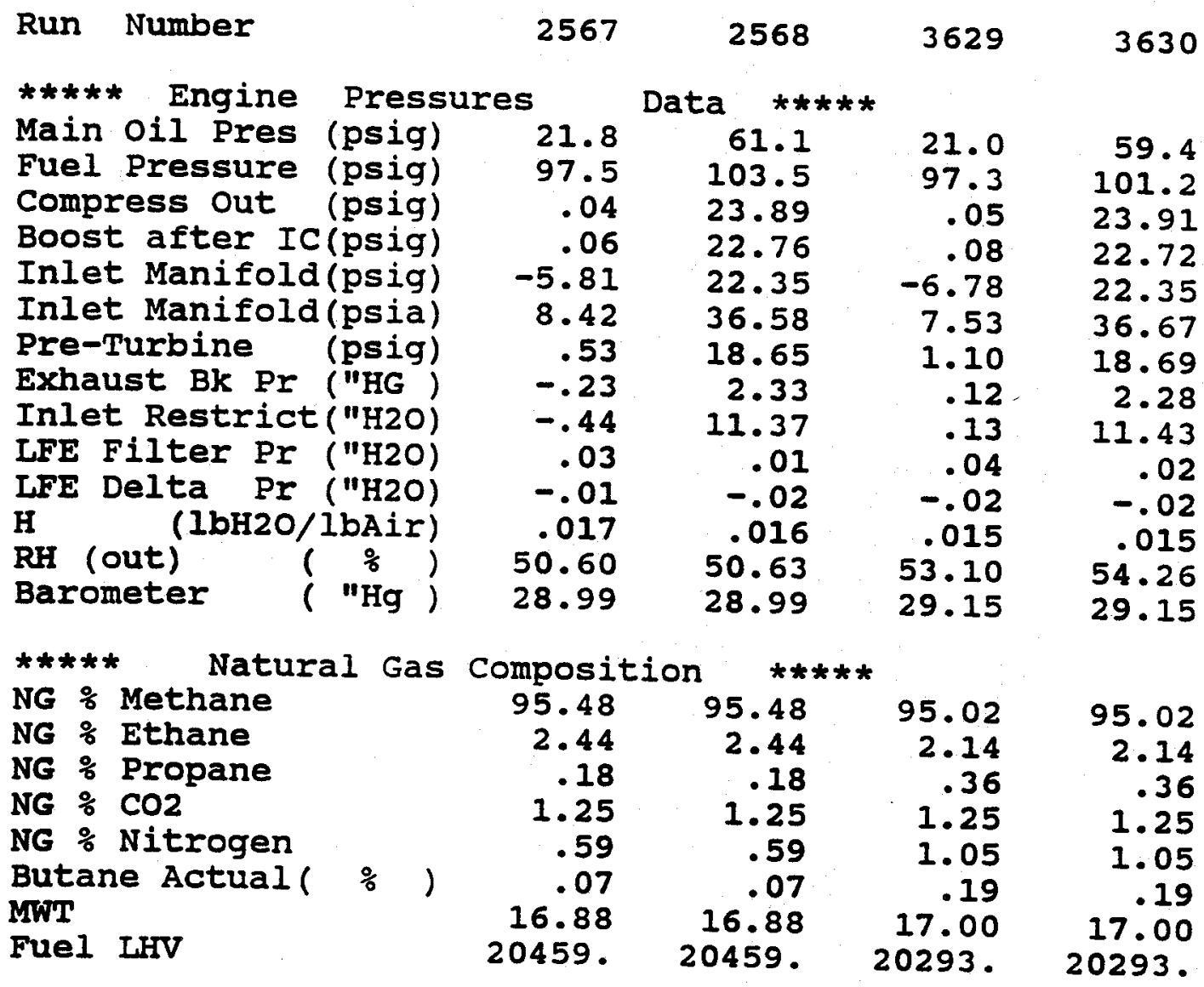


= Cell 05 MAC4 Project Low-Speed Data =

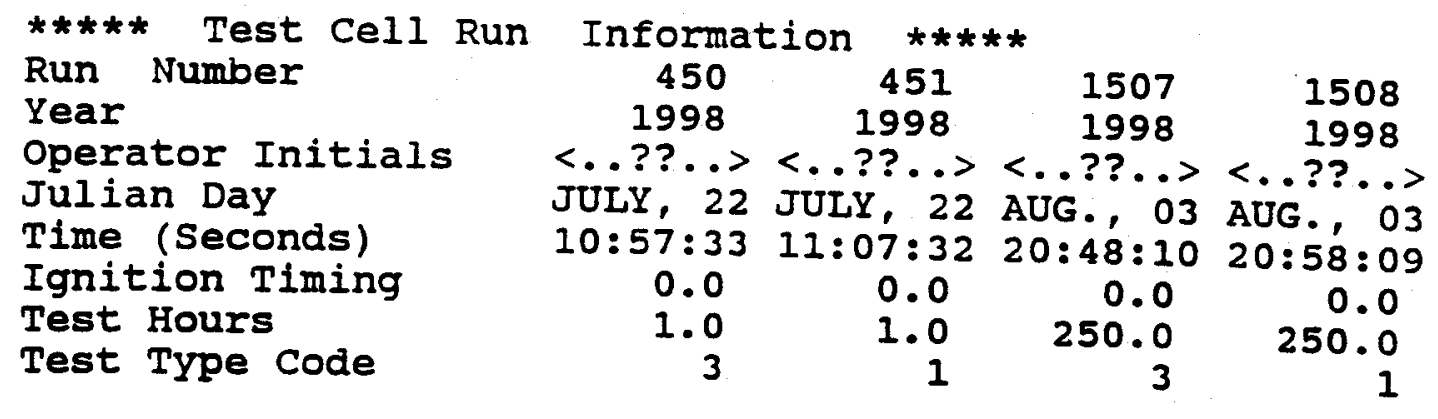

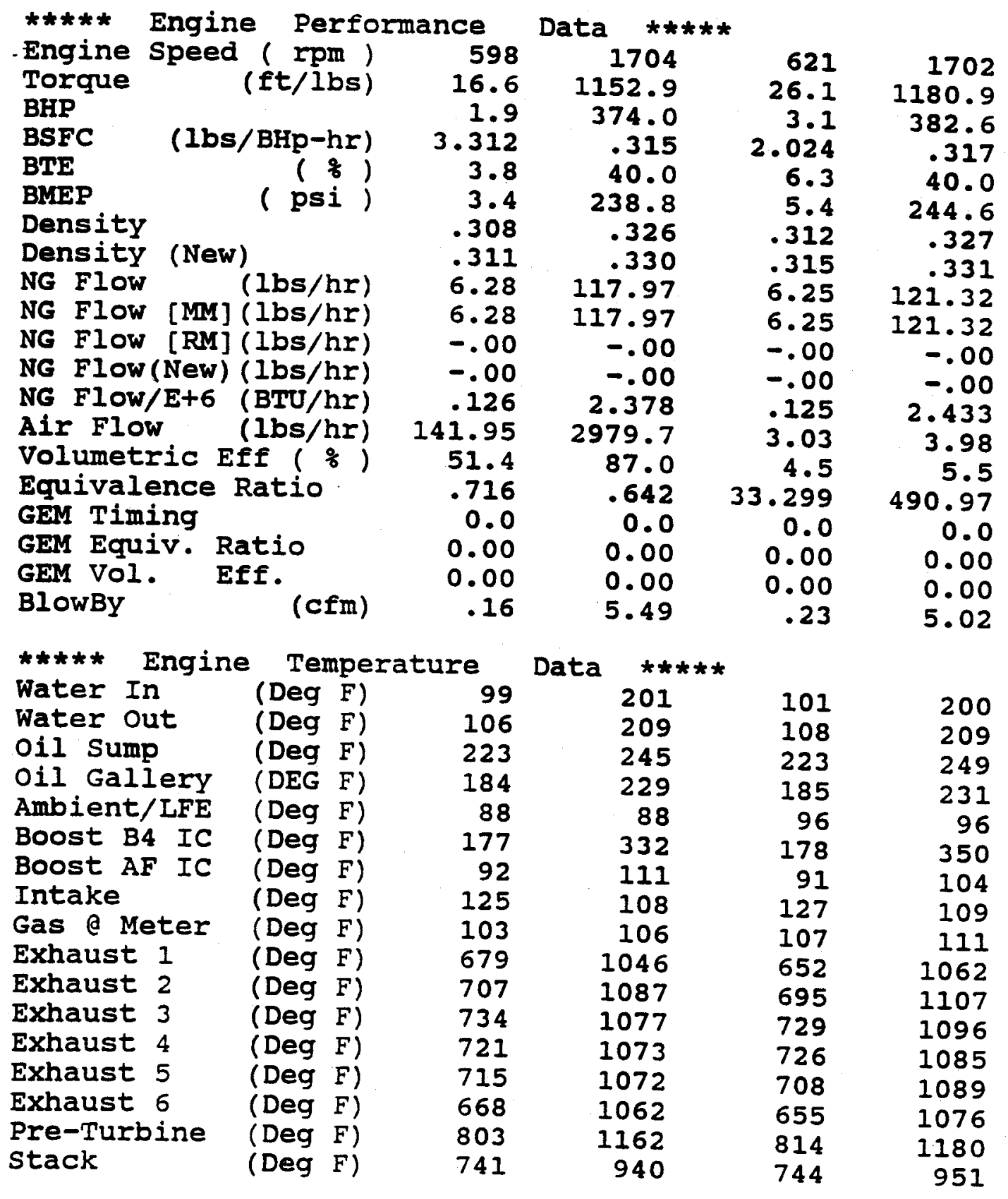




$$
\text { = Cell } 05 \text { MAC4 } \begin{aligned}
& \text { Project Low-Speed Data = } \\
& \text { (continued) }
\end{aligned}
$$

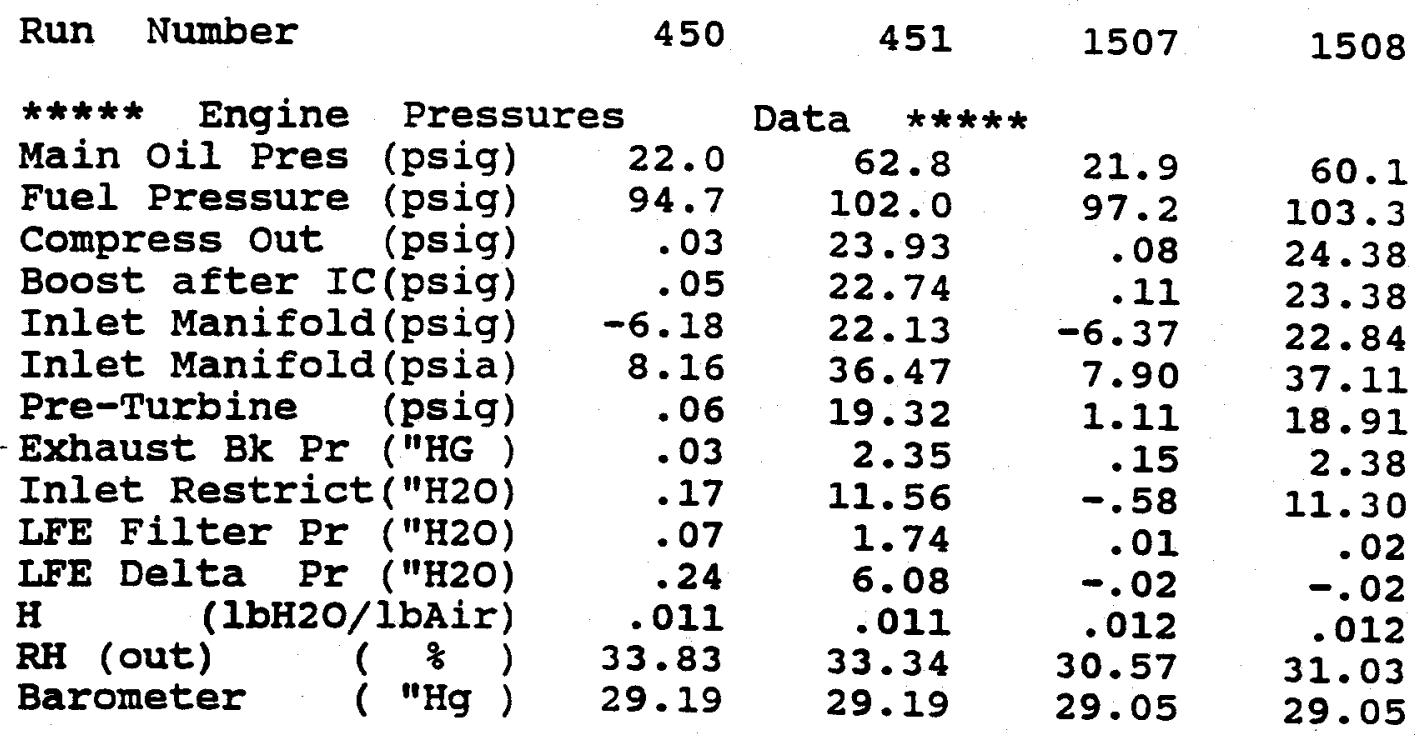

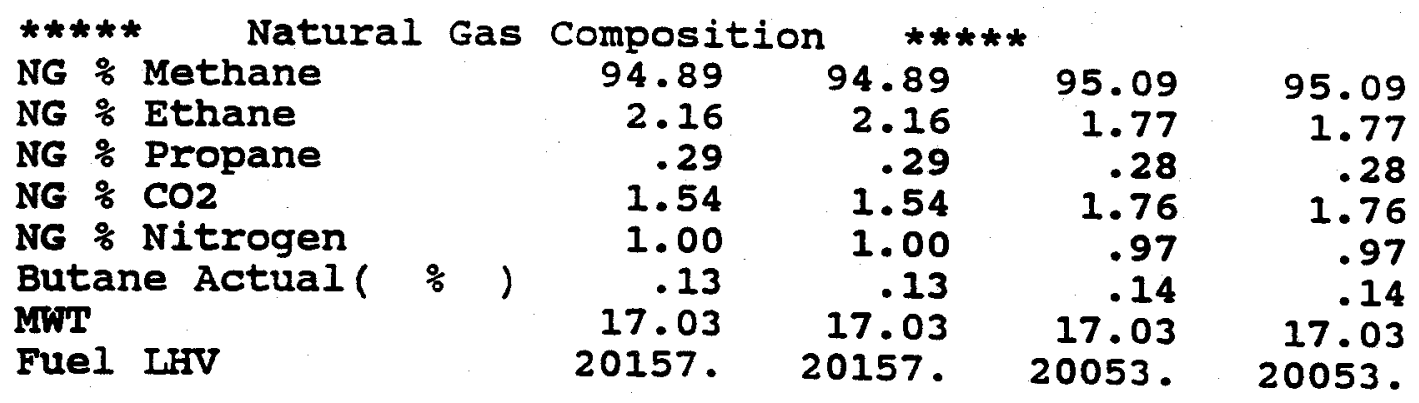


= Cell 05 MAC4 Project Low-speed Data =

\begin{tabular}{lcc}
$* \star * * *$ Test Cell Run & \multicolumn{2}{c}{ Information $* * * * *$} \\
Run Number & 4687 & 4688 \\
Year & 1998 & 1998 \\
Operator Initials & $<. . ? ? .>$ & $<. . ? ? . .>$ \\
Julian Day & SEP. 16 & SEP. 16 \\
Time (Seconds) & $20: 17: 44$ & $20: 27: 41$ \\
Ignition Timing & 0.0 & 0.0 \\
Test Hours & 1000.0 & 1000.0 \\
Test Type Code & 3 & 1
\end{tabular}

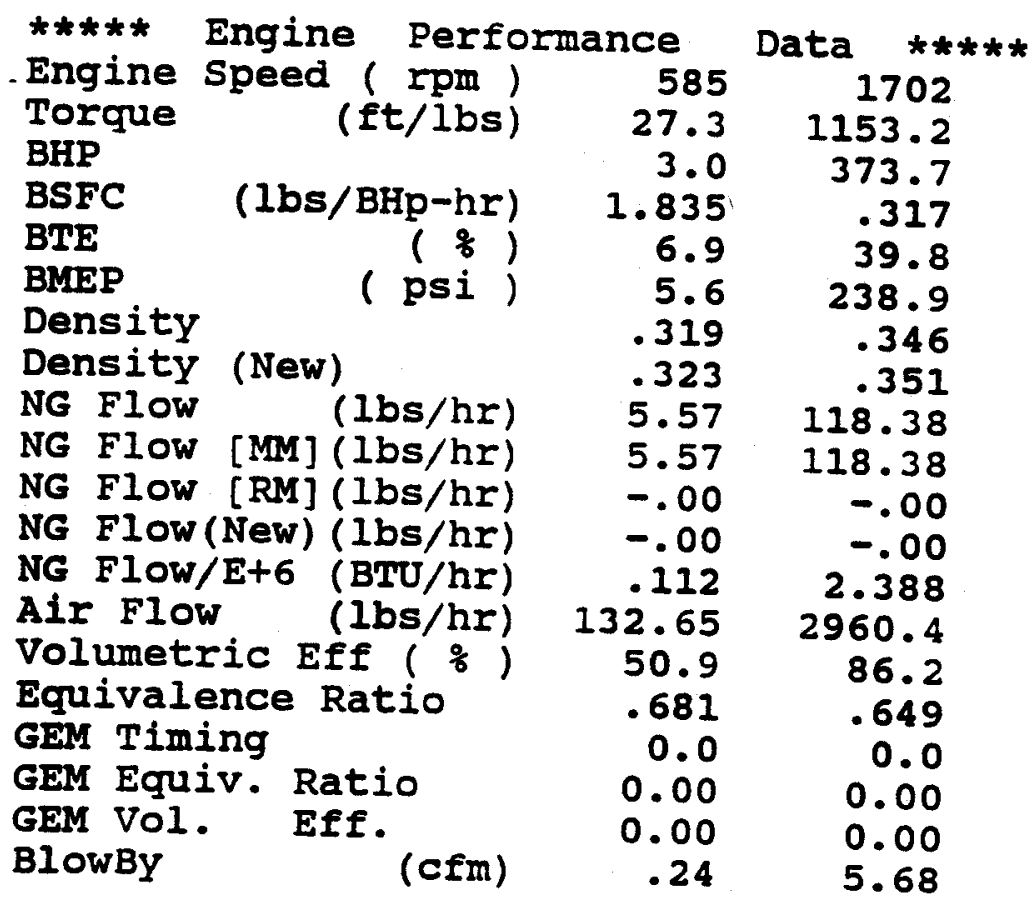

\begin{tabular}{llrr}
$* * * * *$ Engine & \multicolumn{2}{c}{ Temperature } & Data \\
Water In & (Deg F) & 99 & 201 \\
Water Out & (Deg F) & 106 & 209 \\
Oil Sump & (Deg F) & 222 & 246 \\
Oil Gallery & (DEG F) & 183 & 229 \\
Ambient/IFE & (Deg F) & 74 & 74 \\
Boost B4 IC & (Deg F) & 166 & 333 \\
Boost AF IC & (Deg F) & 91 & 104 \\
Intake & (Deg F) & 123 & 103 \\
Gas @ Meter & (Deg F) & 97 & 93 \\
Exhaust 1 & (Deg F) & 634 & 1061 \\
Exhaust 2 & (Deg F) & 684 & 1107 \\
Exhaust 3 & (Deg F) & 724 & 1098 \\
Exhaust 4 & (Deg F) & 722 & 1082 \\
Exhaust 5 & (Deg F) & 702 & 1085 \\
Exhaust 6 & (Deg F) & 644 & 1068 \\
Pre-Turbine & (Deg F) & 815 & 1175 \\
Stack & (Deg F) & 730 & 948
\end{tabular}




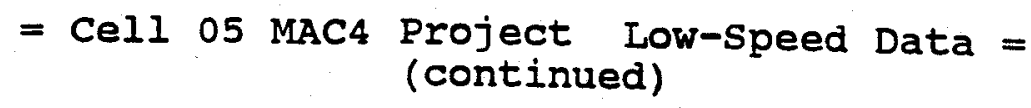

\begin{tabular}{|c|c|c|c|}
\hline Number & & 4687 & 4688 \\
\hline Engine & Press & & 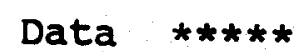 \\
\hline Main oil Pres & (psig) & 20.6 & 59.4 \\
\hline Fuel Pressure & (psig) & 97.1 & 105.9 \\
\hline Compress Out & (psig) & .03 & 23.77 \\
\hline Boost after IC & (psig) & .06 & 22.62 \\
\hline Inlet Manifold & (psig) & -6.37 & 22.23 \\
\hline Inlet Manifold & (psia) & 7.90 & 36.49 \\
\hline Pre-Turbine & (psig) & 1.45 & 19.24 \\
\hline $\begin{array}{l}\text { Exhaust Bk Pr } \\
\text { Inlet Restrict }\end{array}$ & $\begin{array}{l}\text { ("HG) } \\
\text { ("H2O) }\end{array}$ & $\begin{array}{l}.11 \\
.46\end{array}$ & $\begin{array}{r}2.29 \\
11.34\end{array}$ \\
\hline LFE Filter Pr & ("H2O) & .06 & 1.88 \\
\hline $\begin{array}{l}\text { LFE Delta }{ }_{\text {H }}^{\text {Pr }} \\
\text { (1bH2O/ }\end{array}$ & $\begin{array}{l}\text { ("H2O) } \\
\text { IbAir) }\end{array}$ & $\begin{array}{r}.21 \\
.017\end{array}$ & $\begin{array}{r}5.82 \\
.017\end{array}$ \\
\hline RH (out) & $\div$ & 83.84 & 84.08 \\
\hline Barometer & "Hg ) & 29.05 & 29.05 \\
\hline
\end{tabular}

$\begin{array}{lrr}* * * * * \quad \text { Natural Gas Composition } & * * * * \\ \text { NG \% Methane } & 94.72 & 94.72 \\ \text { NG \% Ethane } & 2.17 & 2.17 \\ \text { NG \% Propane } & .42 & .42 \\ \text { NG \% CO2 } & 1.50 & 1.50 \\ \text { NG \% Nitrogen } & 1.00 & 1.00 \\ \text { Butane Actual ( } \% \text { ) } & .20 & .20 \\ \text { MWT } & 17.09 & 17.09 \\ \text { Fuel IHV } & 20175 . & 20175 .\end{array}$




\section{APPENDIX D}

\section{SAMPLE SNAPSHOT HP DAQ DATA}


= Cell 05 MAC4 Project Low-Speed Data =

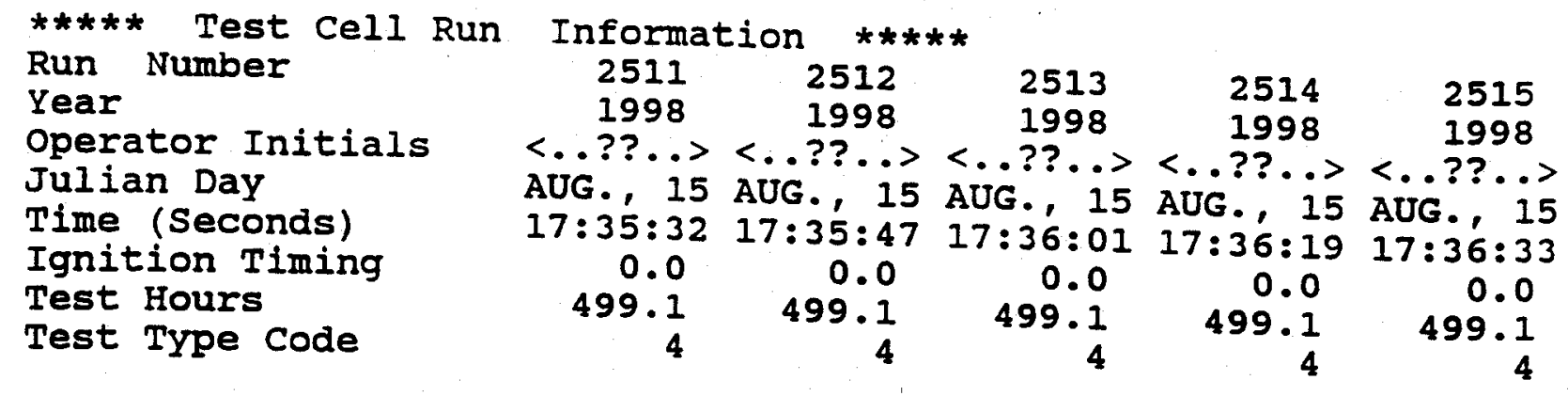

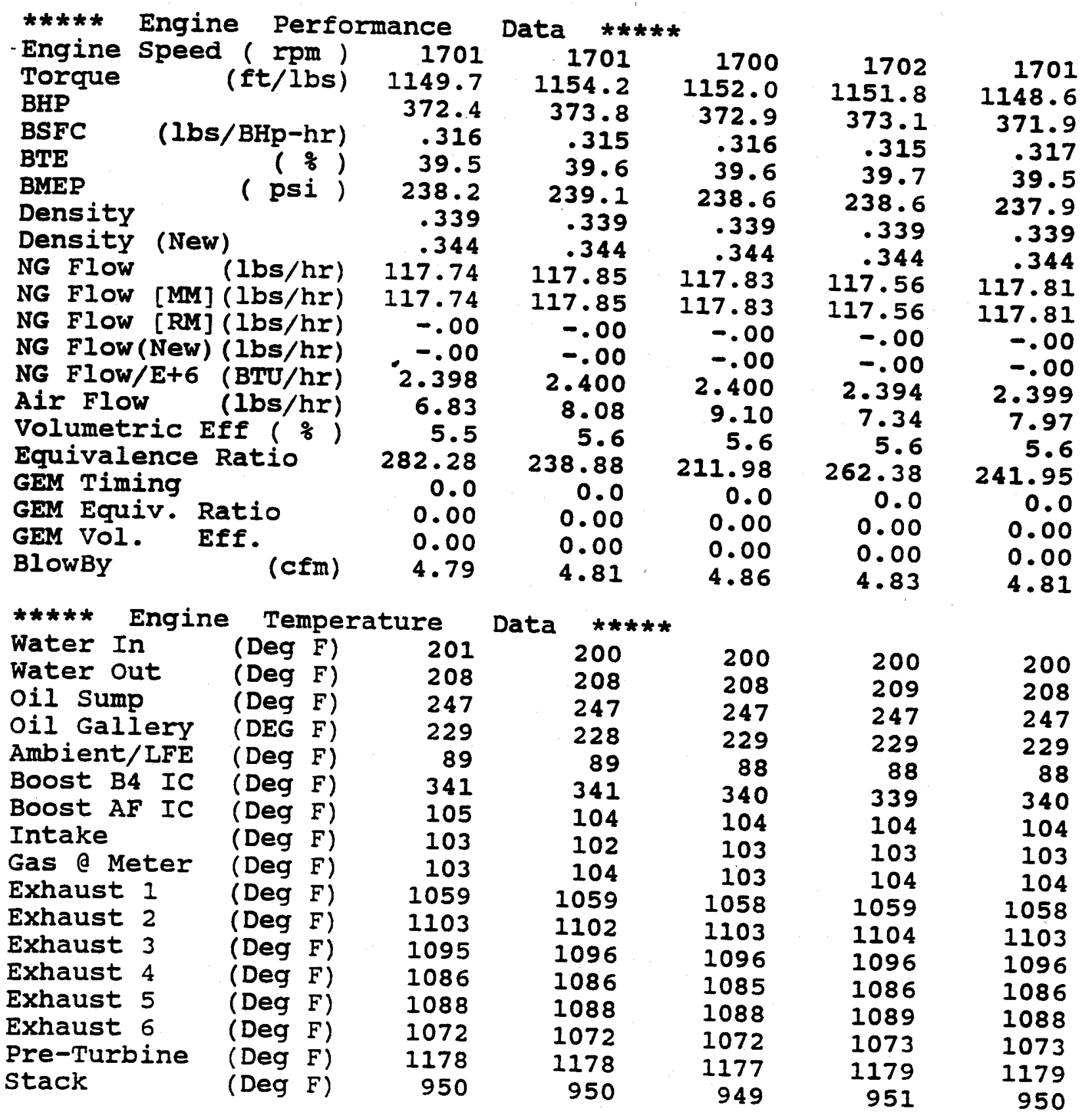




$$
\text { = Cell } 05 \text { MAC4 Project Low-Speed Data = }
$$

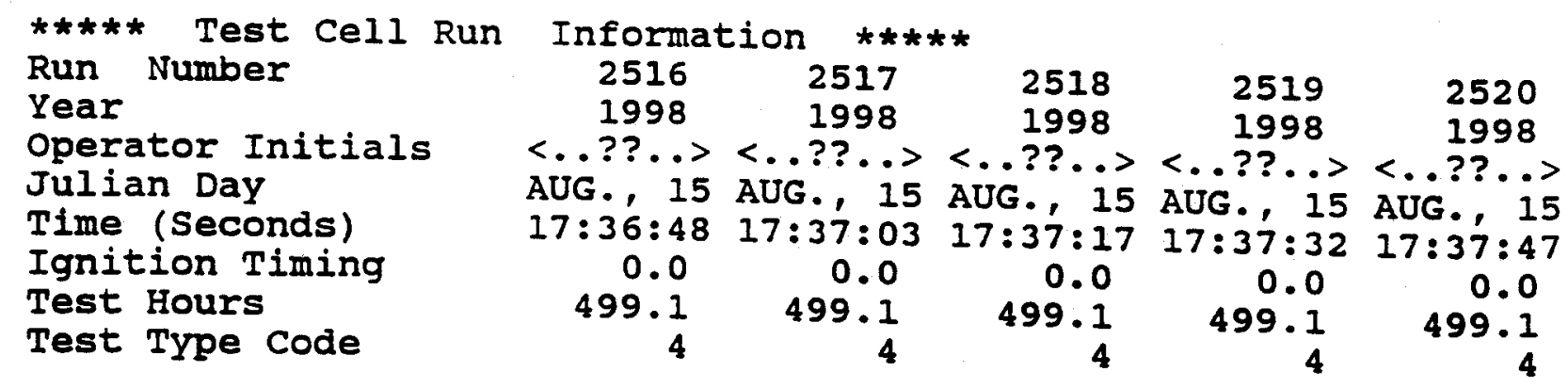

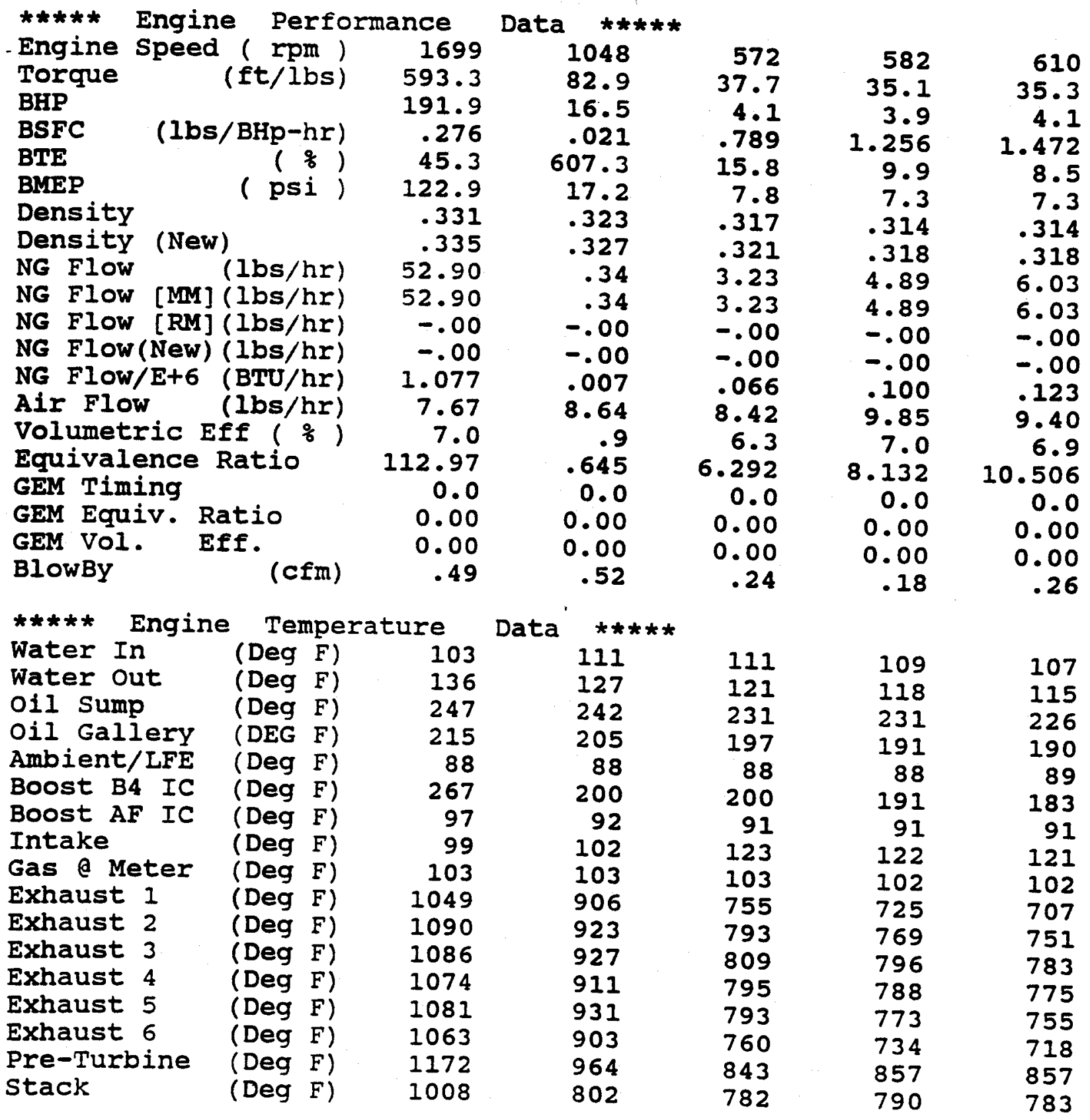


$=$ Cell 05 MAC4 Project Low-Speed Data $=$ (continued)

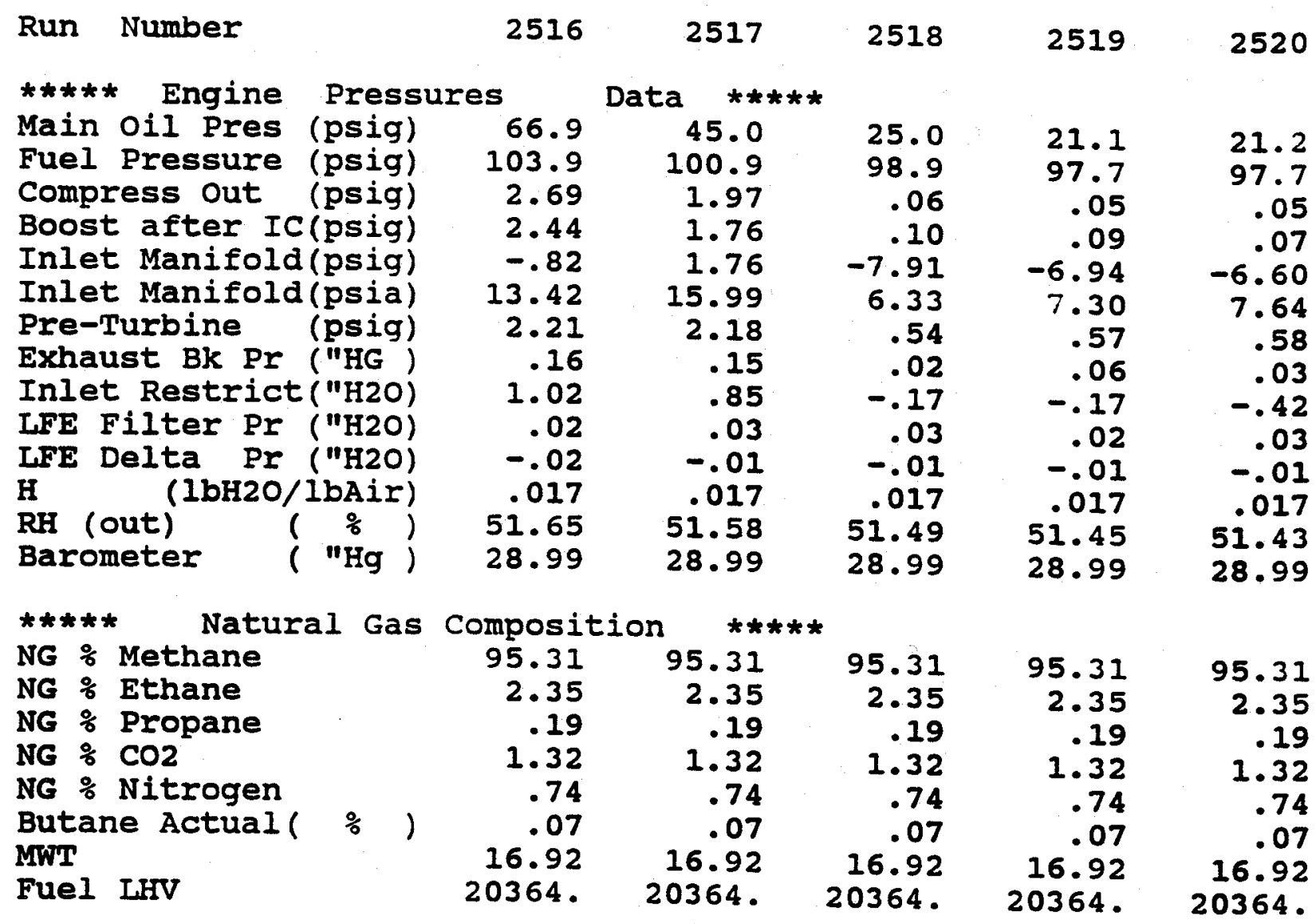


= Cell 05 MAC4 Project Low-Speed Data =

\begin{tabular}{|c|c|c|c|c|c|}
\hline $\begin{array}{l}\star \star \star \star \star \text { Test Cell Rur } \\
\text { Run Number } \\
\text { Year } \\
\text { Operator Initials } \\
\text { Julian Day } \\
\text { Time (Seconds) } \\
\text { Ignition Timing } \\
\text { Test Hours } \\
\text { Test Type Code }\end{array}$ & $\begin{array}{r}\text { Informa } \\
2521 \\
1998 \\
<\ldots ? ? . .> \\
\text { AUG. } 15 \\
17: 38: 01 \\
0.0 \\
499.1 \\
4\end{array}$ & $\begin{array}{r}2522 \\
1998 \\
<\ldots ? ? .> \\
\text { AUG. } 15 \\
17: 38: 16 \\
0.0 \\
499.1 \\
4\end{array}$ & $\begin{array}{c}2523 \\
1998 \\
<\ldots ? ? . .> \\
\text { AUGG. 15 } \\
17: 38: 35 \\
0.0 \\
499.1 \\
4\end{array}$ & $\begin{array}{c}2524 \\
1998 \\
<\ldots ? . .> \\
\text { AUG. } 15 \\
17: 38: 46 \\
0.0 \\
499.1 \\
4\end{array}$ & $\begin{array}{r}2525 \\
1998 \\
<\ldots ? . .> \\
\text { AUGG. } 15 \\
17: 39: 04 \\
0.0 \\
499.1 \\
4\end{array}$ \\
\hline
\end{tabular}

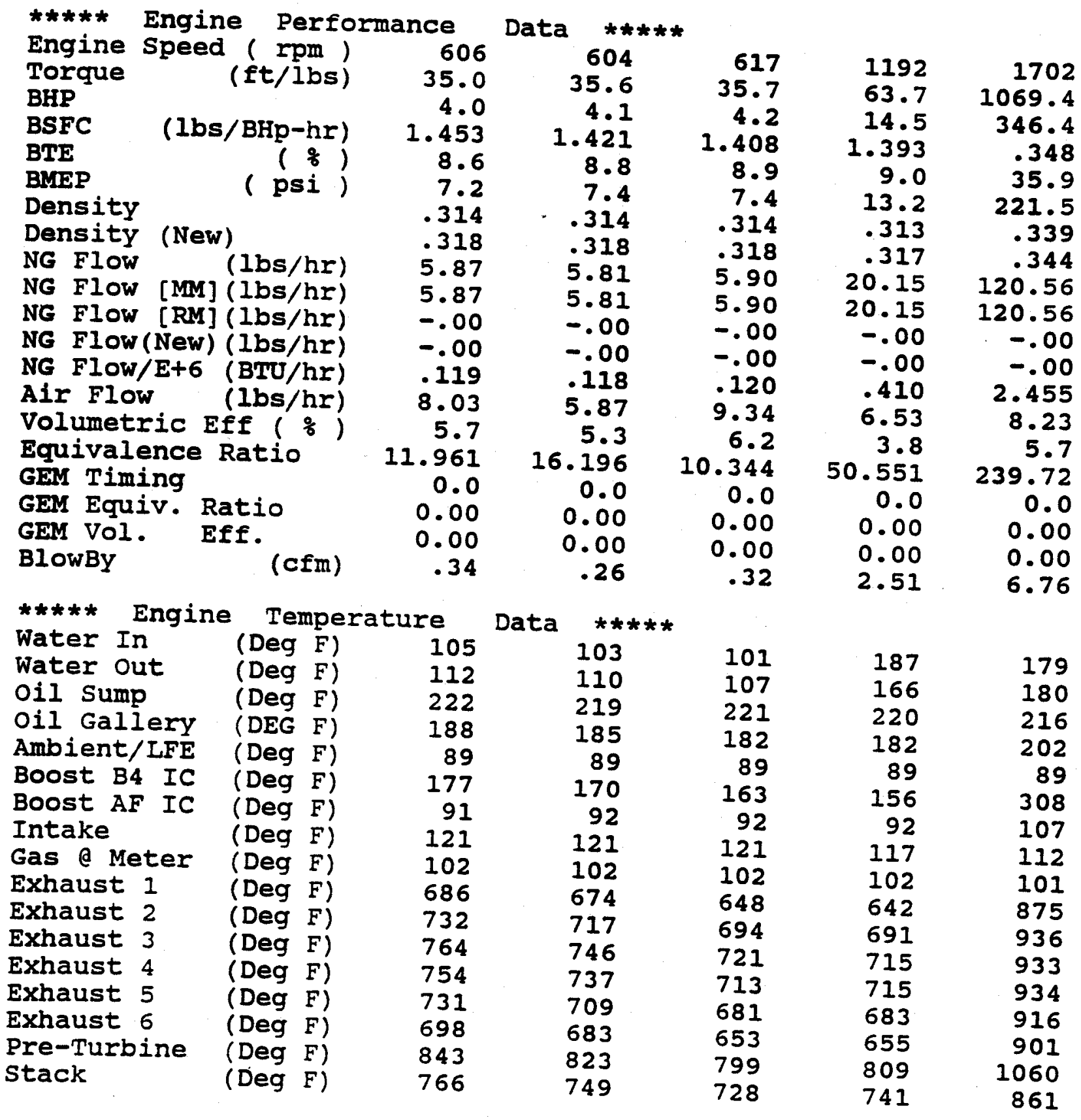


= Cell 05 MAC4 Project Low-Speed Data = (continued)

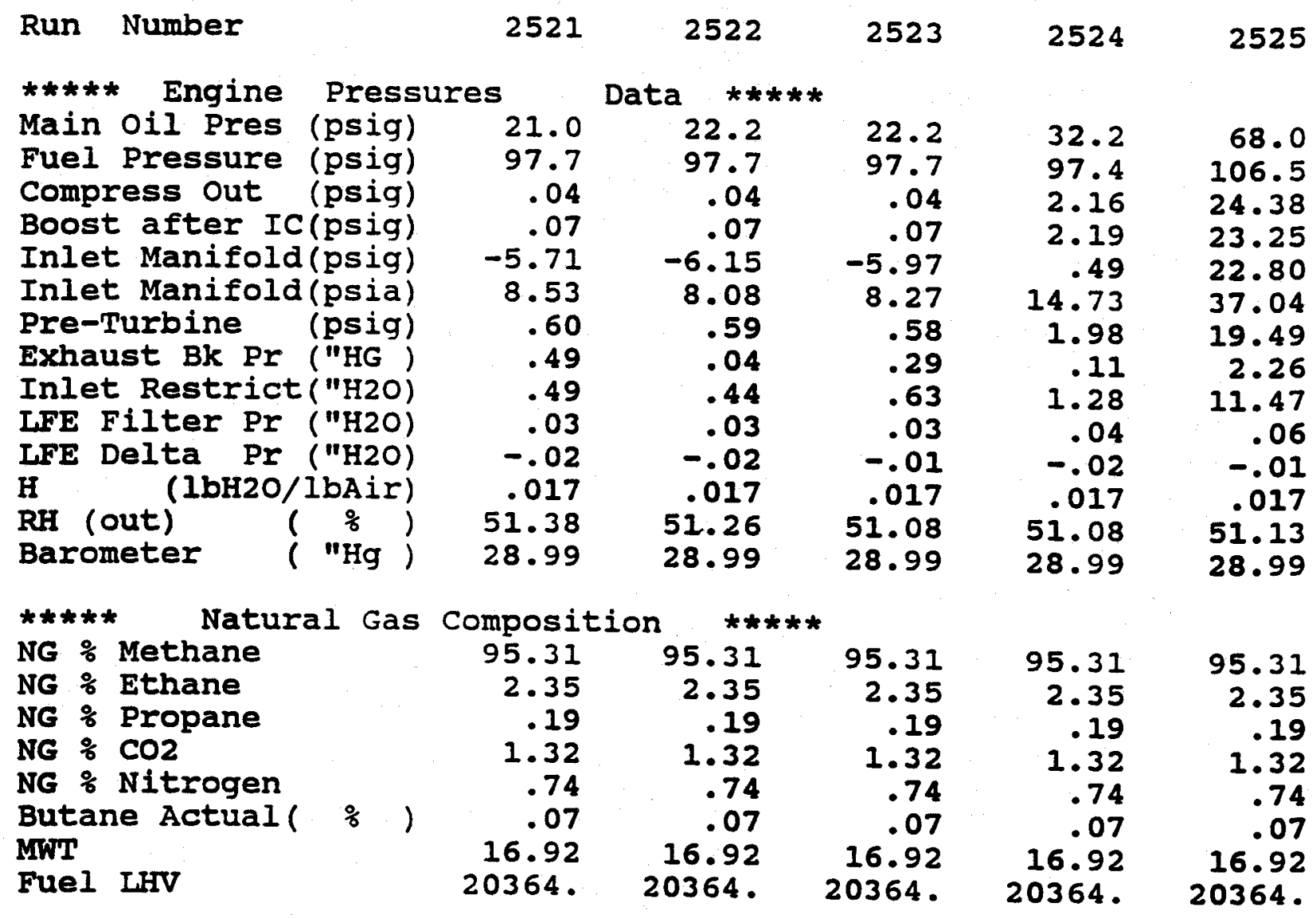


= Cell 05 MAC4 Project Low-Speed Data =

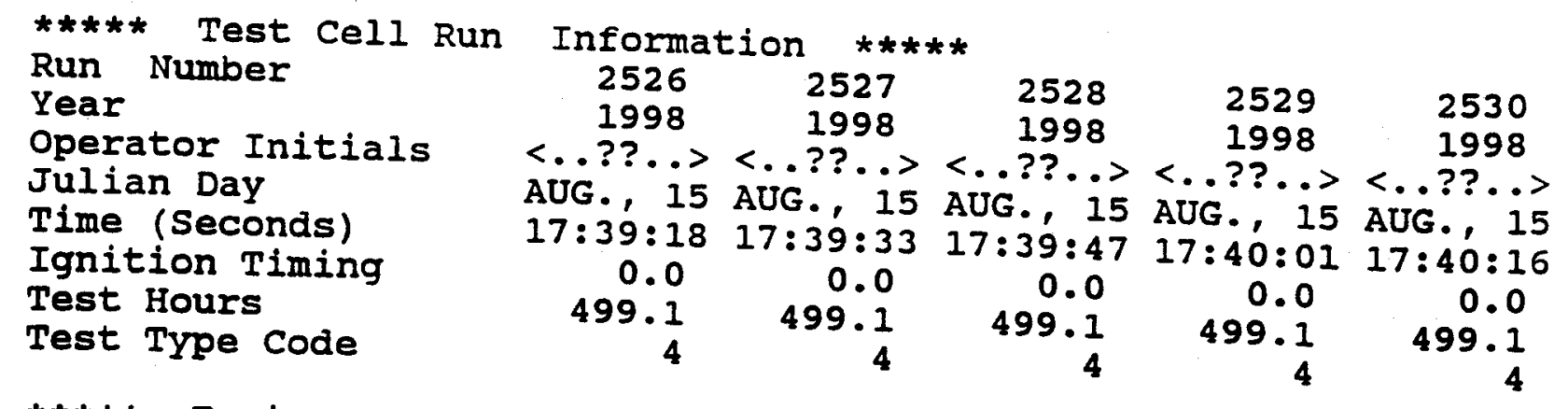

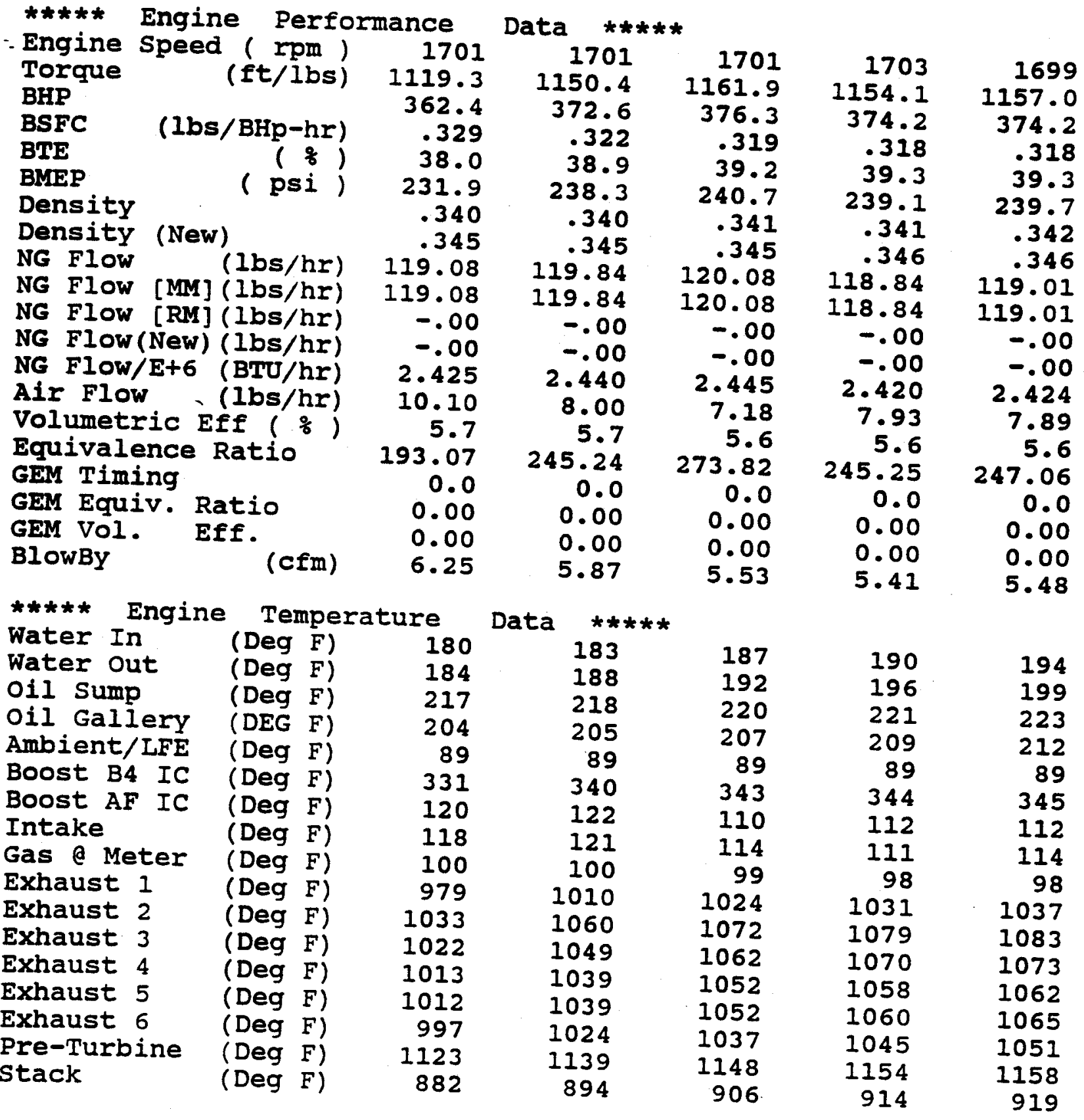




$$
\begin{gathered}
\text { Cell } 05 \text { MAC4 Project Low-speed Data = } \\
\text { (continued) }
\end{gathered}
$$

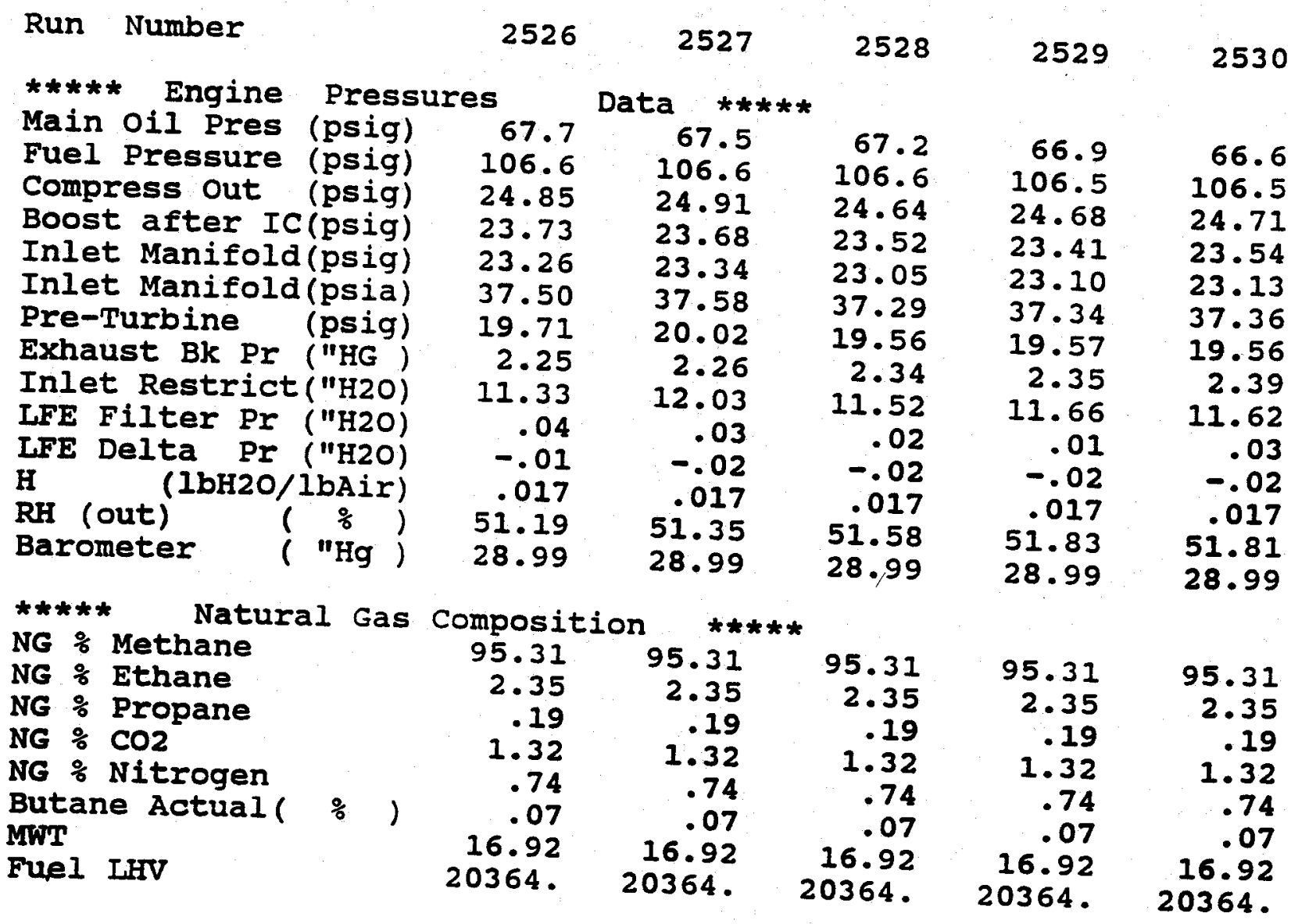


= Cell 05 MAC4 Project Low-Speed Data =

\begin{tabular}{|c|c|c|c|c|c|}
\hline$* * * * *$ Test Cell Ru & I & ** & & & \\
\hline $\begin{array}{l}\text { Run Number } \\
\text { Year }\end{array}$ & 2531 & 2532 & 2533 & 2534 & 2535 \\
\hline Operator Initials & $\begin{array}{c}1998 \\
<\ldots ? ? . .>\end{array}$ & 1998 & 1998 & 1998 & 1998 \\
\hline Julian Day & AUG., 15 & AUG , 15 & $\begin{array}{l}<\ldots ? ? . .> \\
\text { AUG., } 15\end{array}$ & $<\ldots ? ? \ldots>$ & $<\ldots ? ? \ldots>$ \\
\hline Time (Seconds) & $17: 40: 34$ & $17: 40: 48$ & $17: 41: 03$ & $\begin{array}{l}\text { AUG. } 115 \\
17: 41: 17\end{array}$ & AUG . , 15 \\
\hline Ignition Timing & 0.0 & 0.0 & 0.0 & $17: 41: 17$ & $17: 41: 31$ \\
\hline Test Hours & 499.1 & 499.1 & $9 y$ & 0.0 & 0.0 \\
\hline Test Type Cod & 4 & 4 & 4 & $\begin{array}{r}499 \cdot 1 \\
4\end{array}$ & 499.1 \\
\hline
\end{tabular}

\begin{tabular}{|c|c|c|c|c|c|c|}
\hline$\star * * * *$ & Perfor & lance & ata & & & \\
\hline Engine Speed & (rpm ) & 1701 & 1703 & 1701 & 1702 & 1701 \\
\hline Torque & (ft/lbs) & 1163.1 & 1159.4 & 1159.6 & 1158.4 & 1151.5 \\
\hline BHP & & 376.6 & 375.9 & 375.6 & 375.4 & 372.8 \\
\hline BSFC & /BHp-hr) & .317 & .315 & .316 & .314 & .315 \\
\hline BTE & $(\%)$ & 39.5 & 39.7 & 39.5 & 39.8 & 39.7 \\
\hline BMEP & ( psi ) & 240.9 & 240.2 & 240.2 & 240.0 & 238.5 \\
\hline Density & & .340 & .340 & .340 & .340 & .340 \\
\hline Density (New) & & .345 & .344 & .344 & .344 & .345 \\
\hline NG Flow & (Ibs/hr) & 119.27 & 118.41 & 118.77 & 118.00 & 117.29 \\
\hline NG Flow [MM] & (lbs/hr) & 119.27 & 118.41 & 118.77 & 118.00 & 117.29 \\
\hline NG Flow [RM] & ] (lbs/hr) & -.00 & -.00 & -.00 & -.00 & -.00 \\
\hline NG Flow(New) & ) (Ibs/hr) & -.00 & -.00 & -.00 & -.00 & -.00 \\
\hline NG Flow/E+6 & (BTU/hr) & 2.429 & 2.411 & 2.419 & 2.403 & 2.389 \\
\hline Air Flow & (1bs/hr) & 10.38 & 7.13 & 9.17 & 9.57 & 9.95 \\
\hline Volumetric E & Eff $(\%)$ & 5.7 & 5.6 & 5.6 & 5.7 & 5.6 \\
\hline Equivalence & Ratio & 188.15 & 271.89 & 212.09 & 201.91 & 192.93 \\
\hline GEM Timing & & 0.0 & 0.0 & 0.0 & 0.0 & 0.0 \\
\hline GEM Equiv. R & Ratio & 0.00 & 0.00 & 0.00 & 0.00 & 0.00 \\
\hline GEM Vol. & Eff. & 0.00 & 0.00 & 0.00 & 0.00 & 0.00 \\
\hline BlowBy & (cfm) & 5.18 & 5.21 & 5.13 & 5.05 & 4.97 \\
\hline$\star \star \star * * *$ & Temper & ure & Data & & & \\
\hline Water In & $(\operatorname{Deg} F)$ & 199 & 202 & 202 & 203 & 203 \\
\hline Water out & (Deg F) & 204 & 207 & 208 & 209 & 210 \\
\hline Oil Sump & (Deg F) & 225 & 226 & 229 & 231 & 233 \\
\hline Oil Gallery & (DEG F) & 214 & 216 & 217 & 219 & 220 \\
\hline Ambient/LFE & (Deg F) & 89 & 89 & 89 & 89 & 89 \\
\hline Boost B4 IC & (Deg F) & 347 & 344 & 345 & 344 & 341 \\
\hline Boost AF IC & (Deg F) & 111 & 109 & 109 & 108 & 107 \\
\hline Intake & (Deg $F$ ) & 111 & 109 & 108 & 107 & 106 \\
\hline Gas @ Meter & (Deg F) & 97 & 97 & 97 & 97 & 97 \\
\hline Exhaust 1 & (Deg F) & 1043 & 1043 & 1046 & 1048 & 1049 \\
\hline Exhaust 2 & $(\operatorname{Deg} F)$ & 1090 & 1091 & 1092 & 1093 & 1096 \\
\hline Exhaust 3 & $(\operatorname{Deg} F)$ & 1078 & 1080 & 1081 & 1084 & 1085 \\
\hline Exhaust 4 & $\left(\begin{array}{ll}\operatorname{Deg} & F\end{array}\right)$ & 1067 & 1069 & 1072 & 1074 & 1074 \\
\hline Exhaust 5 & $(\operatorname{Deg} F)$ & 1070 & 1073 & 1074 & 1076 & 1078 \\
\hline Exhaust 6 & (Deg F) & 1056 & 1058 & 1059 & 1063 & 1061 \\
\hline Pre-Turbine & $(\operatorname{Deg} F)$ & 1163 & 1165 & 1167 & 1170 & 1169 \\
\hline stack & $(\operatorname{Deg} F)$ & 926 & 930 & 933 & 937 & 939 \\
\hline
\end{tabular}




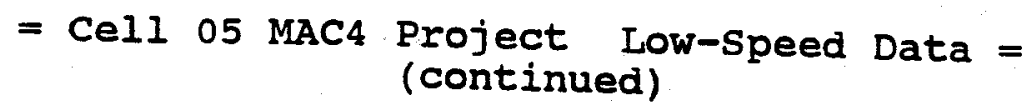

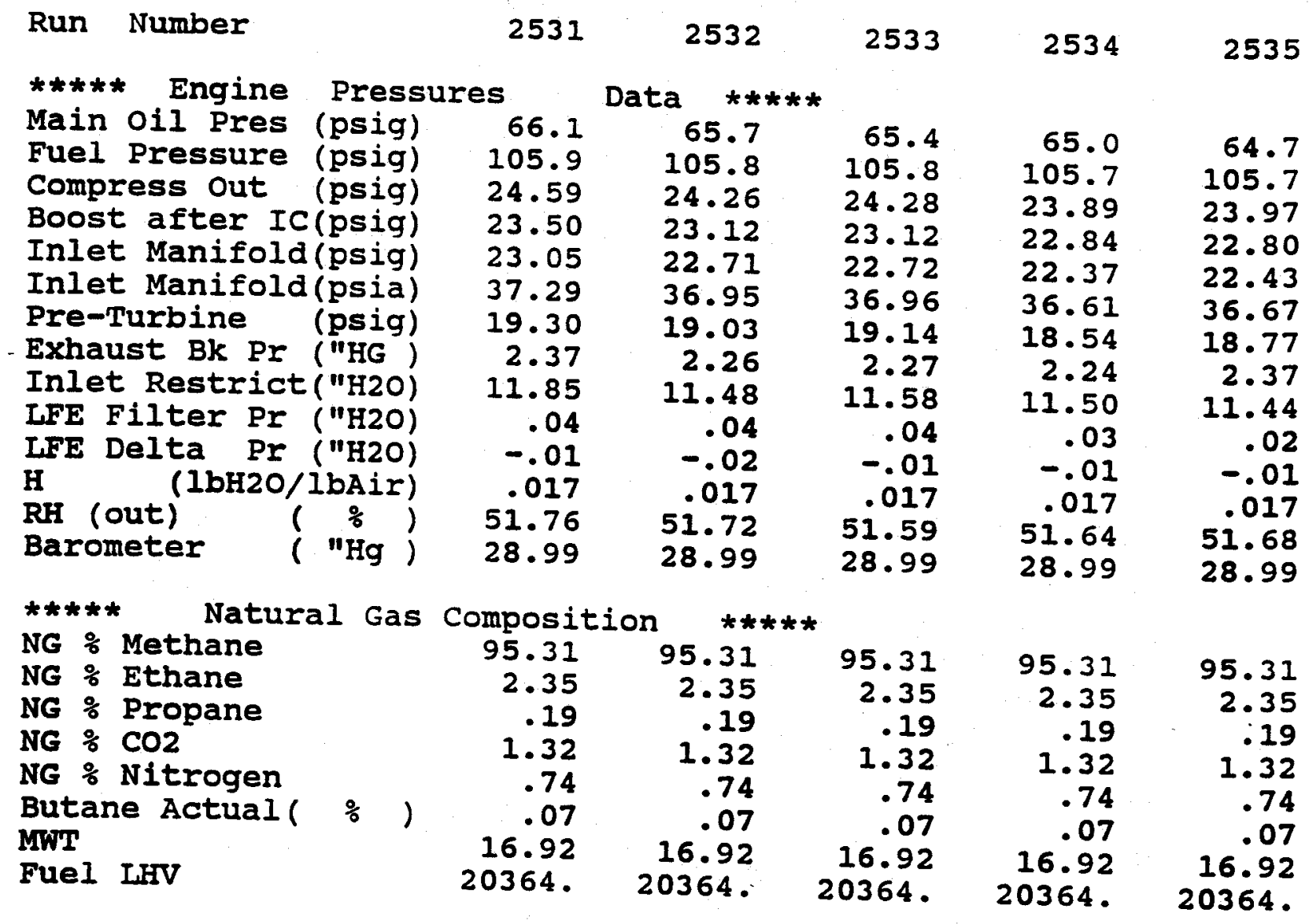


= Cell 05 MAC4 Project Low-Speed Data =

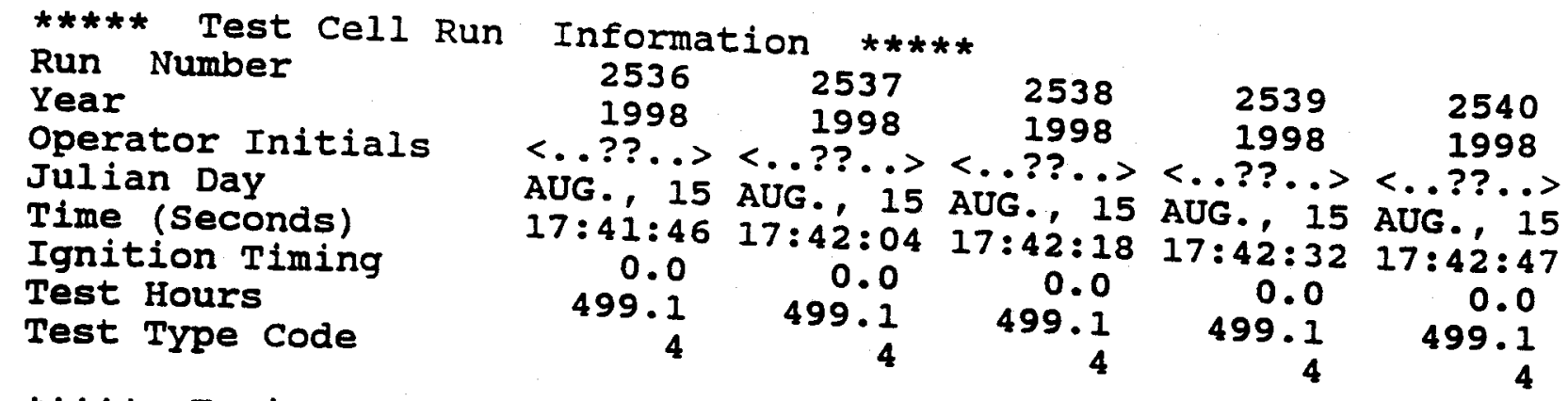

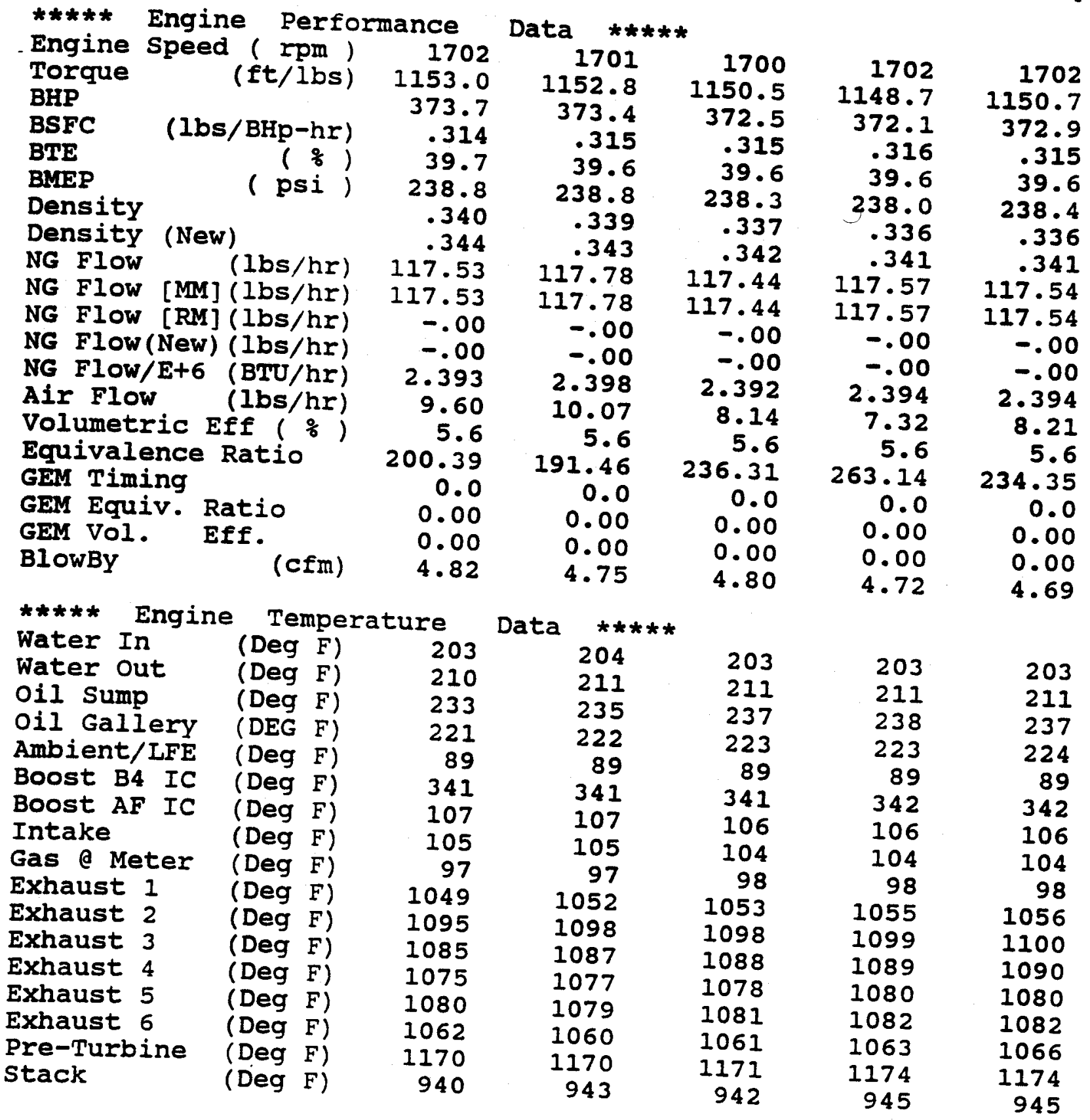


= Cell 05 MAC4 Project Low-Speed Data = (continued)

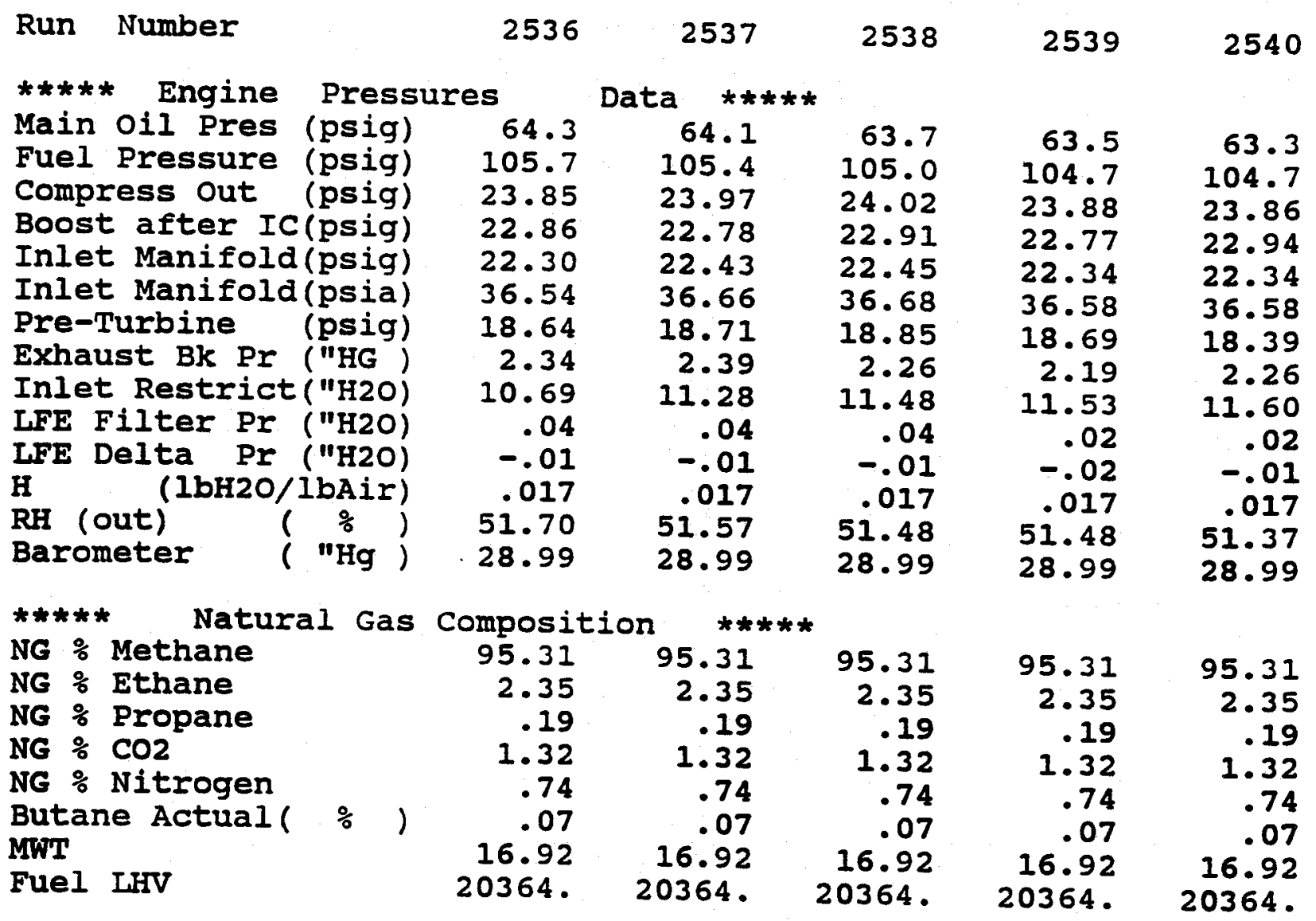


= Cell 05 MAC4 Project Low-Speed Data =

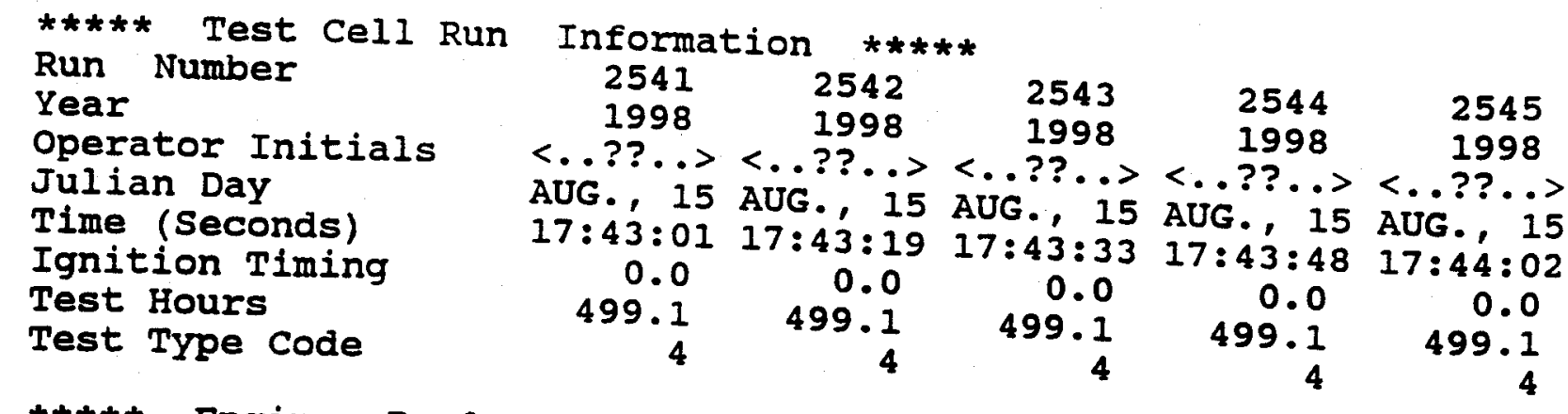

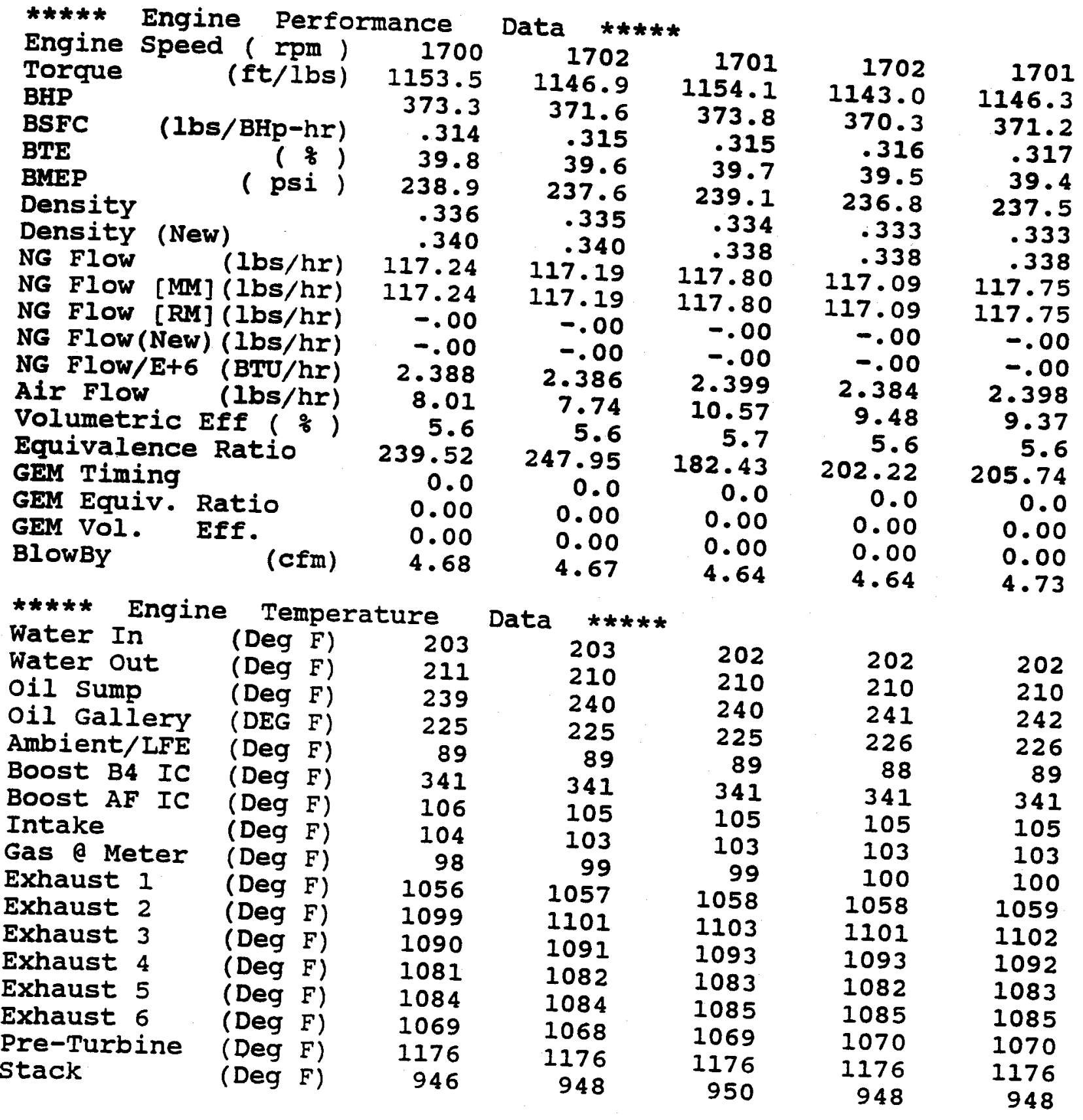


= Cell 05 MAC4 Project Low-Speed Data = (continued)

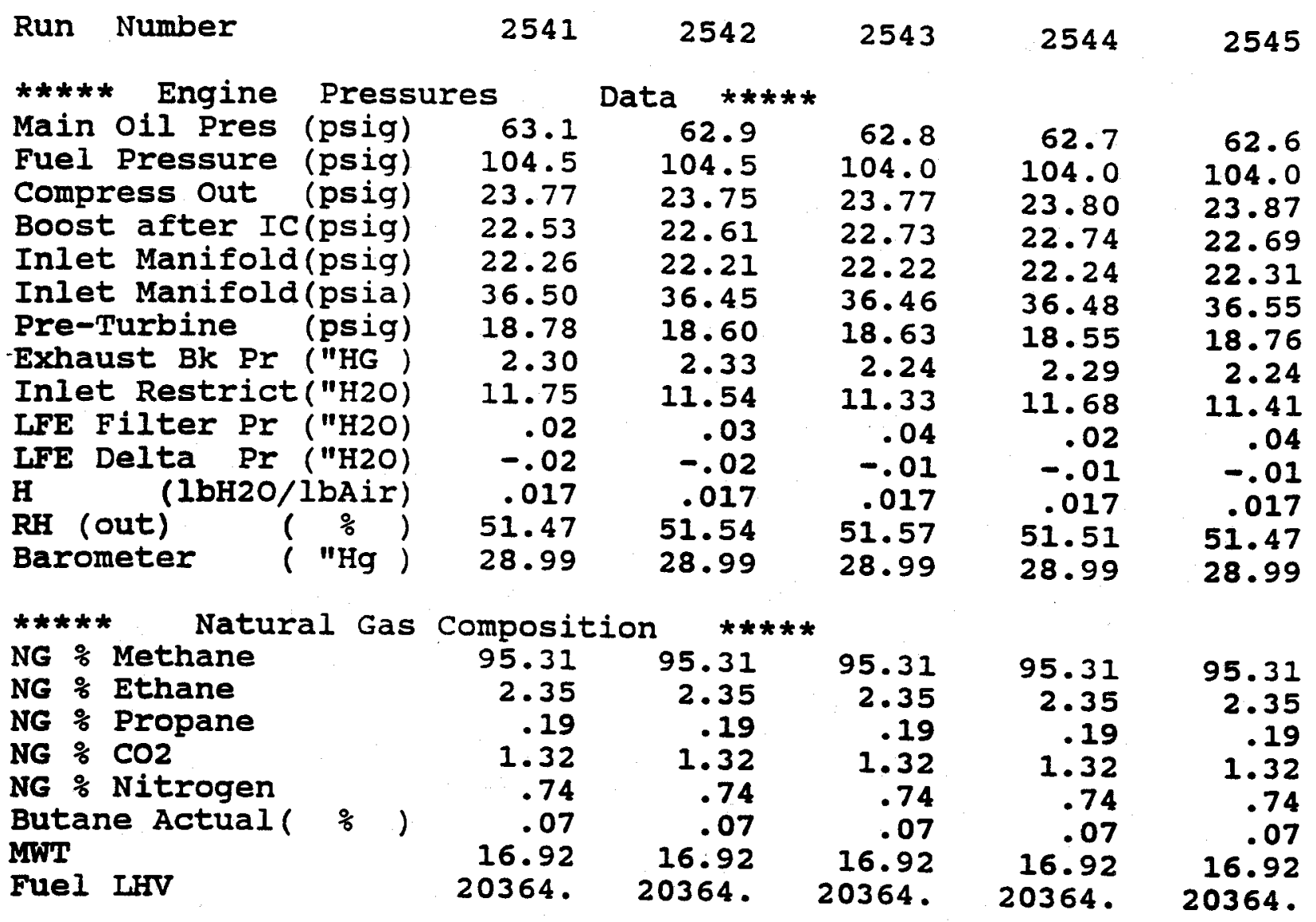


= Cell 05 MAC4 Project Low-speed Data =

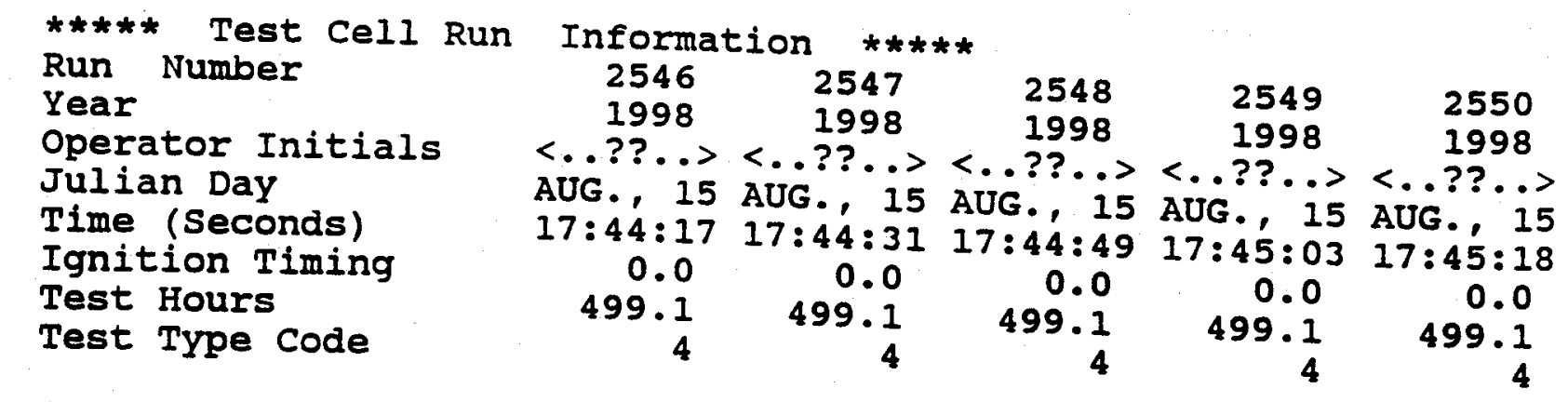

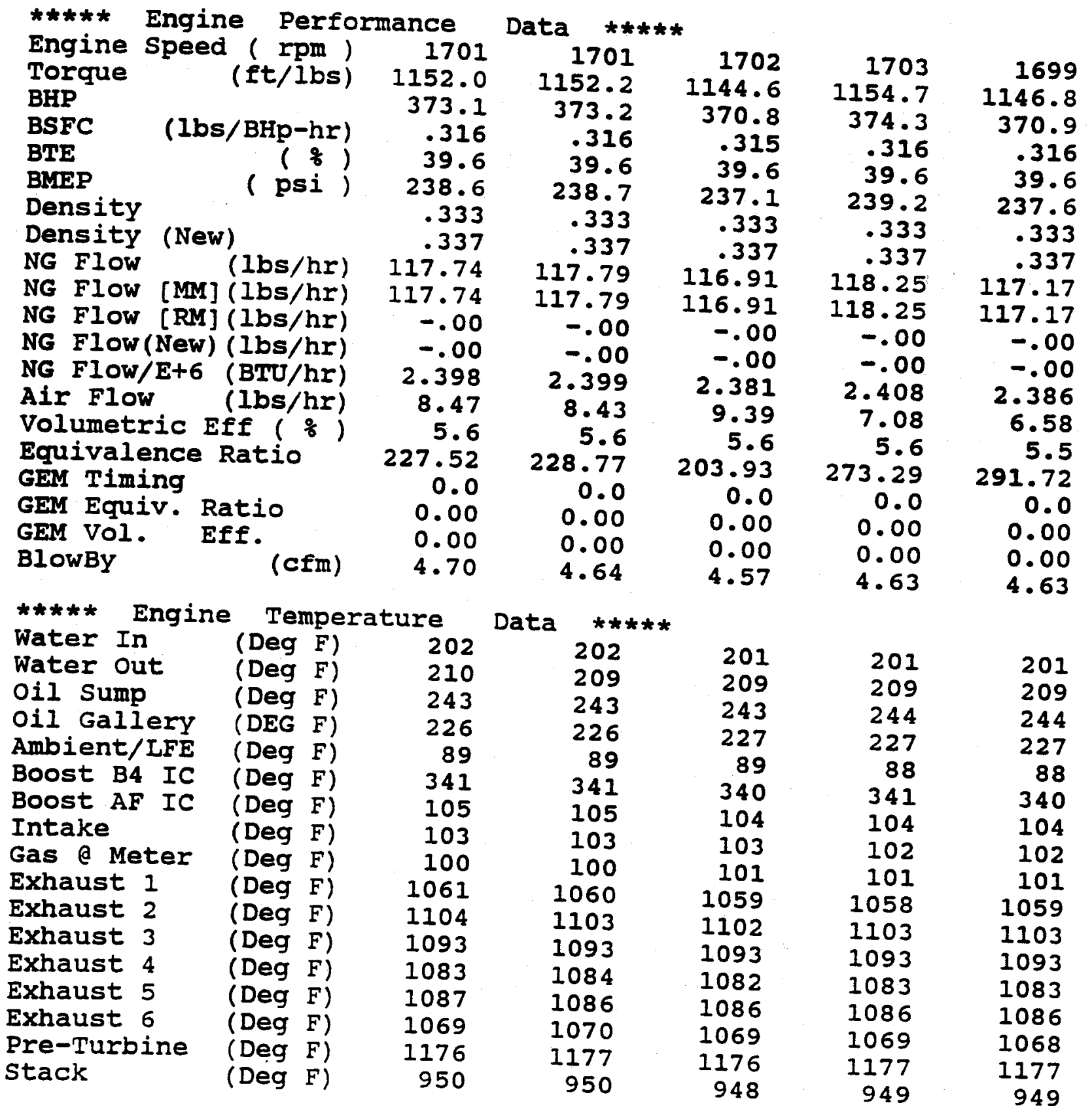




$$
\text { = cell } 05 \text { MAC4 } \begin{aligned}
& \text { Project Low-Speed Data = } \\
& \text { (continued) }
\end{aligned}
$$

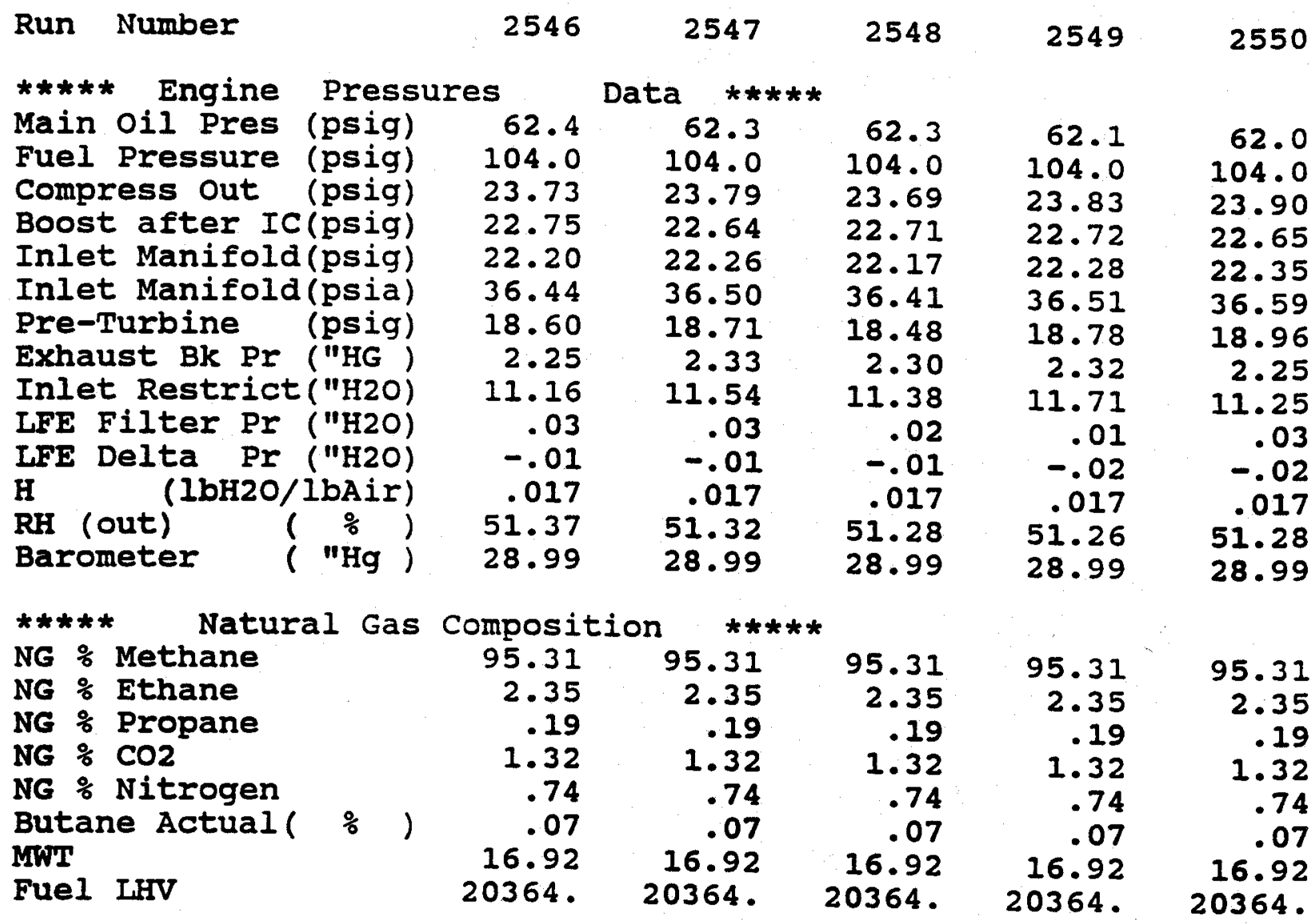


= Cell 05 MAC4 Project Low-speed Data =

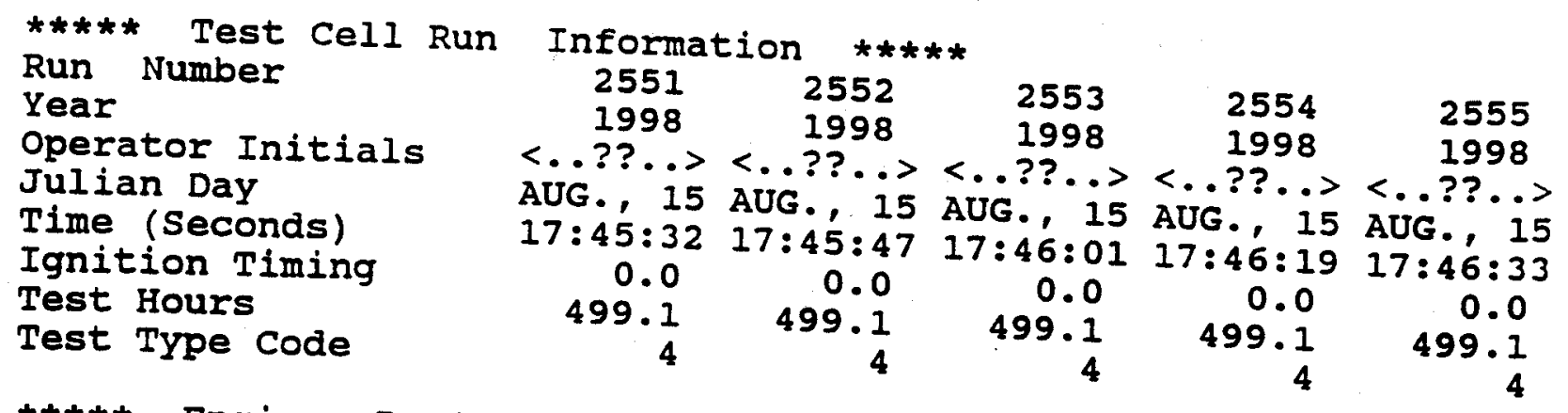

$\star \star \star * *$ Engine Performance Data *****

Engine Speed ( $r p m$ ) 1701

Torque (ft/lbs) 1147.4

BHP

BSFC

BTE

BMEP

Density

Density

NG Flow

NG Flow

(lbs/BHp-hr)

371.6

.316

39.6

( psi)

237.7

.332

(New)

.337

(1bs/hr)

[MM] (lbs/hr)

NG Flow [RM] (lbs/hr)
NG Flow (New) (lbs/hr)

NG Flow/E+6 (BTU/hr)

Air Flow (lbs/hr)

Volumetric Eff ( $\%$ )

Equivalence Ratio

GEM Timing

GEM Equiv. Ratio

GEM Vol. Eff.

BIOWBY

(cfm)

117.26

117.26

$-.00$

$-.00$

2.388

7.13

5.6

269.25

0.0

0.00

0.00

4.67

1701
1138.4
368.7
.317
39.4
235.8
.332
.337
116.94
116.94
-.00
-.00
2.381
10.76
5.6
177.95
0.0
0.00
0.00
4.64

***** Engine Temperature

Water In

Water out

Oil sump

Oil Gallery

Ambient/LFE

Boost B4 IC

Boost AF IC

Intake

Gas @ Meter

Exhaust 1

Exhaust 2

Exhaust 3

Exhaust 4

Exhaust 5

Exhaust 6

Pre-Turbine

Stack

$\begin{array}{lr}\text { (Deg F) } & 201 \\ \text { (Deg F) } & 209 \\ \text { (Deg F) } & 244 \\ \text { (DEG F) } & 227 \\ \text { (Deg F) } & 89 \\ \text { (Deg F) } & 340 \\ \text { (Deg F) } & 104 \\ \text { (Deg F) } & 102 \\ \text { (Deg F) } & 101 \\ \text { (Deg F) } & 1059 \\ \text { (Deg F) } & 1103 \\ \text { (Deg F) } & 1094 \\ \text { (Deg F) } & 1084 \\ \text { (Deg F) } & 1087 \\ \text { (Deg F) } & 1071 \\ \text { (Deg F) } & 1177 \\ \text { (Deg F) } & 949\end{array}$

Data

$\star \star \star * \star$

\section{1}

209

244

228

90

340

105

103

101

1059

1103

1095

1085

1088

1072

1178

950

$$
\begin{array}{r}
1700 \\
1152.2 \\
373.0 \\
.316 \\
39.5 \\
238.7 \\
.332 \\
.336 \\
117.95 \\
117.95 \\
-.00 \\
-.00 \\
2.402 \\
4.46 \\
5.5 \\
432.96 \\
0.0 \\
0.00 \\
0.00 \\
4.66
\end{array}
$$

201

209

245

228

88

340

105

103

101

1058

1103

1095

1086

1087

1071

1178

950

$$
\begin{array}{r}
1700 \\
1148.1 \\
371.6 \\
.316 \\
39.6 \\
237.8 \\
.332 \\
.336 \\
117.31 \\
117.31 \\
-.00 \\
-.00 \\
2.389 \\
10.06 \\
5.6 \\
190.98 \\
0.0 \\
0.00 \\
0.00 \\
4.63
\end{array}
$$

201

209

245

228

88

340

104

103

102

1059

1103

1094

1085

1088

1073

1178

950
1702

1149.9

372.5

.316

39.6

238.2

.332

.336

117.64

117.64

$-.00$

$-.00$

2.396

8.06

5.6

238.99

0.0

0.00

0.00

4.62

201

209

245

228

88

340

104

102

102

1060

1103

1094

1085

1087

1073

1179

950 
= Cell 05 MAC4 Project Low-Speed Data =

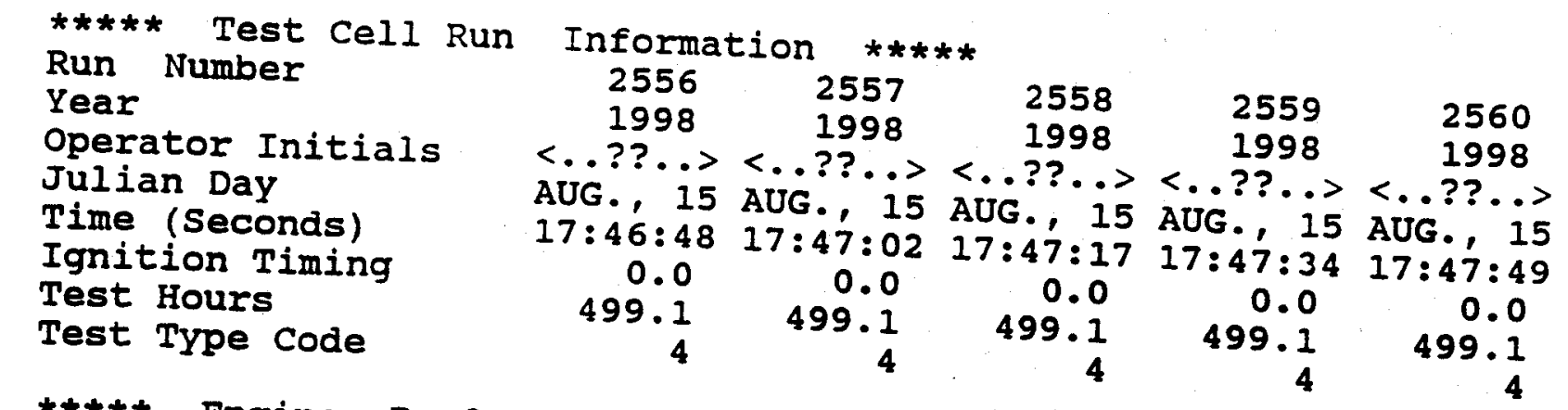

\begin{tabular}{|c|c|c|c|c|c|c|}
\hline $\begin{array}{ll}\star \star \star * * & \text { En } \\
\text { ngine } & S p\end{array}$ & $\begin{array}{l}\text { Engine } \\
\text { Speed }\end{array}$ & & Data & * & & \\
\hline Torque & $\begin{array}{r}\text { Speed }(\mathrm{rpm}) \\
\text { (ft/lbs) }\end{array}$ & $\begin{array}{r}1702 \\
1145.9\end{array}$ & $\begin{array}{r}1701 \\
1148.0\end{array}$ & 1702 & 1701 & 1701 \\
\hline $\begin{array}{l}\text { BHP } \\
\text { BSFC }\end{array}$ & & 371.3 & $\begin{array}{r}371.9 \\
371.0\end{array}$ & $\begin{array}{r}1152.7 \\
373.5\end{array}$ & 1151.7 & 1153 \\
\hline $\begin{array}{l}\text { BSFC } \\
\text { BTE }\end{array}$ & (lbs/BHp-hr) & .315 & .316 & .3 & $\begin{array}{r}373.0 \\
.316\end{array}$ & $\begin{array}{r}373.5 \\
.316\end{array}$ \\
\hline BMEP & ( psi) & $\begin{array}{r}39.7 \\
237.4\end{array}$ & $\begin{array}{r}39 . \\
237 .\end{array}$ & $\begin{array}{r}39 \\
238\end{array}$ & 39. & 39.5 \\
\hline Density & & .332 & $\begin{array}{r}.332 \\
.38\end{array}$ & $\begin{array}{r}238 \\
.3\end{array}$ & 238 & 238.9 \\
\hline Density & (New) & .336 & .336 & .33 & .3 & .332 \\
\hline $\begin{array}{l}\text { NG Flow } \\
\text { NG Flow }\end{array}$ & (Ibs/hr) & 116.91 & .17 .60 & 117.5 & $\dot{7}^{3}$ & \\
\hline $\begin{array}{l}\text { NG Flow } \\
\text { NG Flow }\end{array}$ & $\begin{array}{l}{[\mathrm{MM}](1 \mathrm{bs} / \mathrm{hr})} \\
{[\mathrm{RM}](1 \mathrm{bs} / \mathrm{hr})}\end{array}$ & 116.91 & 17.60 & 117.5 & $\begin{array}{l}117.80 \\
117.80\end{array}$ & 118.0 \\
\hline NG Flow(1 & (New) $(1 \mathrm{bs} / \mathrm{hr})$ & $\begin{array}{l}-.00 \\
-.00\end{array}$ & -.00 & -.00 & -.00 & -.0 \\
\hline NG Flow/E & $\mathrm{E}+6(\mathrm{BTU} / \mathrm{hr})$ & 2.381 & $\begin{array}{r}-.00 \\
2.395\end{array}$ & $\begin{array}{r}-.00 \\
2.394\end{array}$ & $\begin{array}{r}-.00 \\
2.300\end{array}$ & -.0 \\
\hline $\begin{array}{l}\text { Air Flow } \\
\text { Volumetr: }\end{array}$ & :ic $\operatorname{Eff}\left(\begin{array}{l}\text { (lbs } / \mathrm{hr}) \\
\left.\frac{\mathrm{q}}{6}\right)\end{array}\right.$ & $\begin{array}{r}6.33 \\
5.5\end{array}$ & 8.55 & $\begin{array}{r}2.394 \\
7.82\end{array}$ & $\begin{array}{r}2.399 \\
7.56\end{array}$ & $\begin{array}{r}2.404 \\
9.25\end{array}$ \\
\hline $\begin{array}{l}\text { Equivale } \\
\text { GEM Timi }\end{array}$ & nce Ratio & 302.47 & $\begin{array}{r}5.6 \\
225.19\end{array}$ & $\begin{array}{r}5.6 \\
246.05\end{array}$ & $\begin{array}{r}5.6 \\
55.06\end{array}$ & 5.6 \\
\hline $\begin{array}{l}\text { GEM Timil } \\
\text { GEM Equi }\end{array}$ & & 0.0 & 0.0 & $\begin{array}{r}246.05 \\
0.0\end{array}$ & $\begin{array}{r}55.06 \\
0.0\end{array}$ & $\begin{array}{r}208.94 \\
0.0\end{array}$ \\
\hline & Eff. & $\begin{array}{l}0.00 \\
0.00\end{array}$ & 0.00 & 0.00 & $\begin{array}{l}0.0 \\
0.00\end{array}$ & $\begin{array}{l}0.0 \\
0.00\end{array}$ \\
\hline$-2.1 x_{1}$ & & 4.63 & 4.68 & $\begin{array}{l}0.00 \\
4.60\end{array}$ & $\begin{array}{l}0.00 \\
4.59\end{array}$ & $\begin{array}{l}0.00 \\
4.58\end{array}$ \\
\hline
\end{tabular}

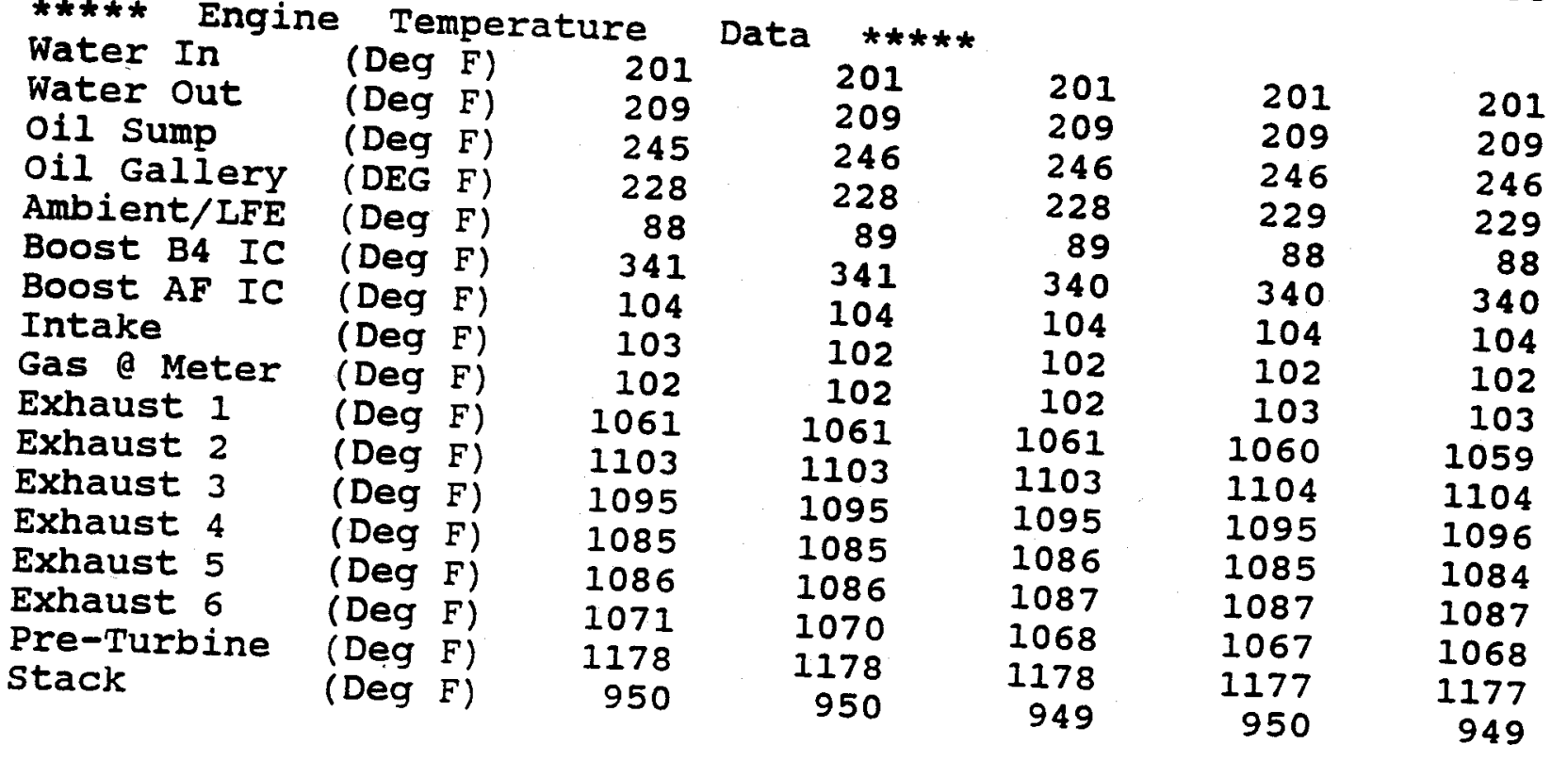


= Cell 05 MAC4 Project Low-speed Data = (continued)

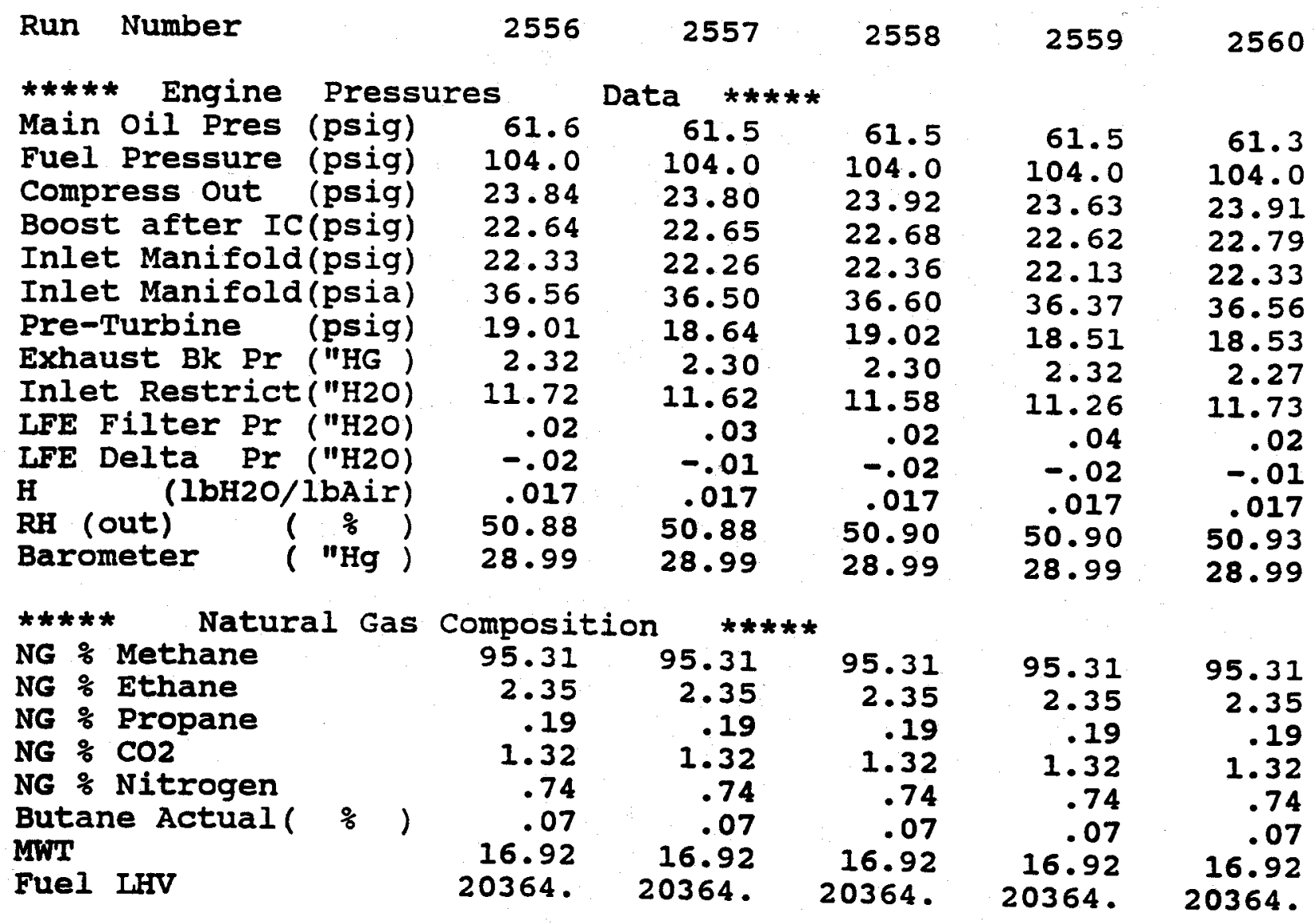


= Cell 05 MAC4 Project Low-Speed Data =

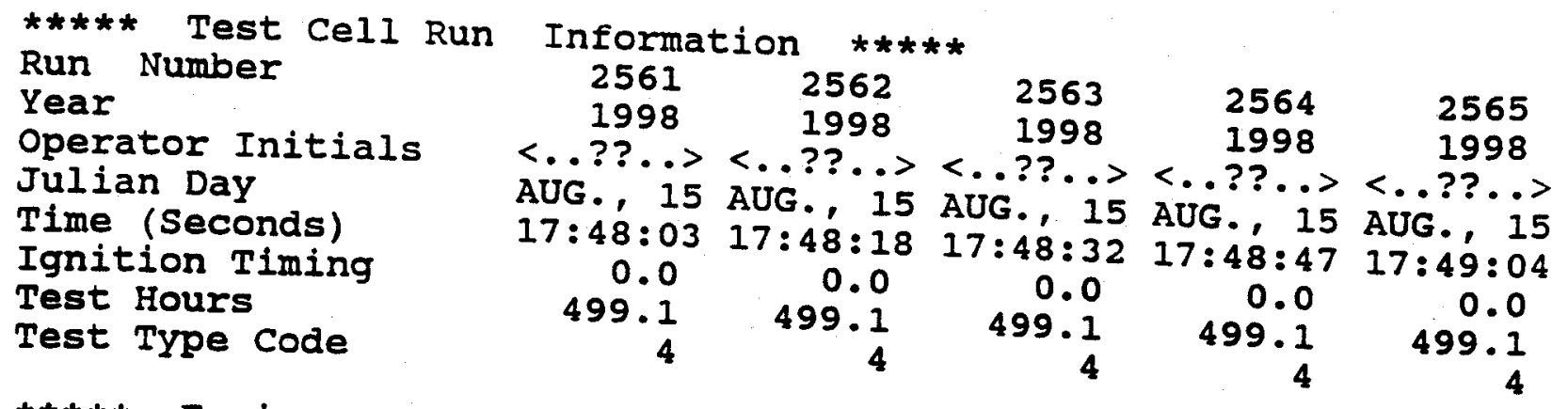

***** Engine Performance Data $* * * * *$

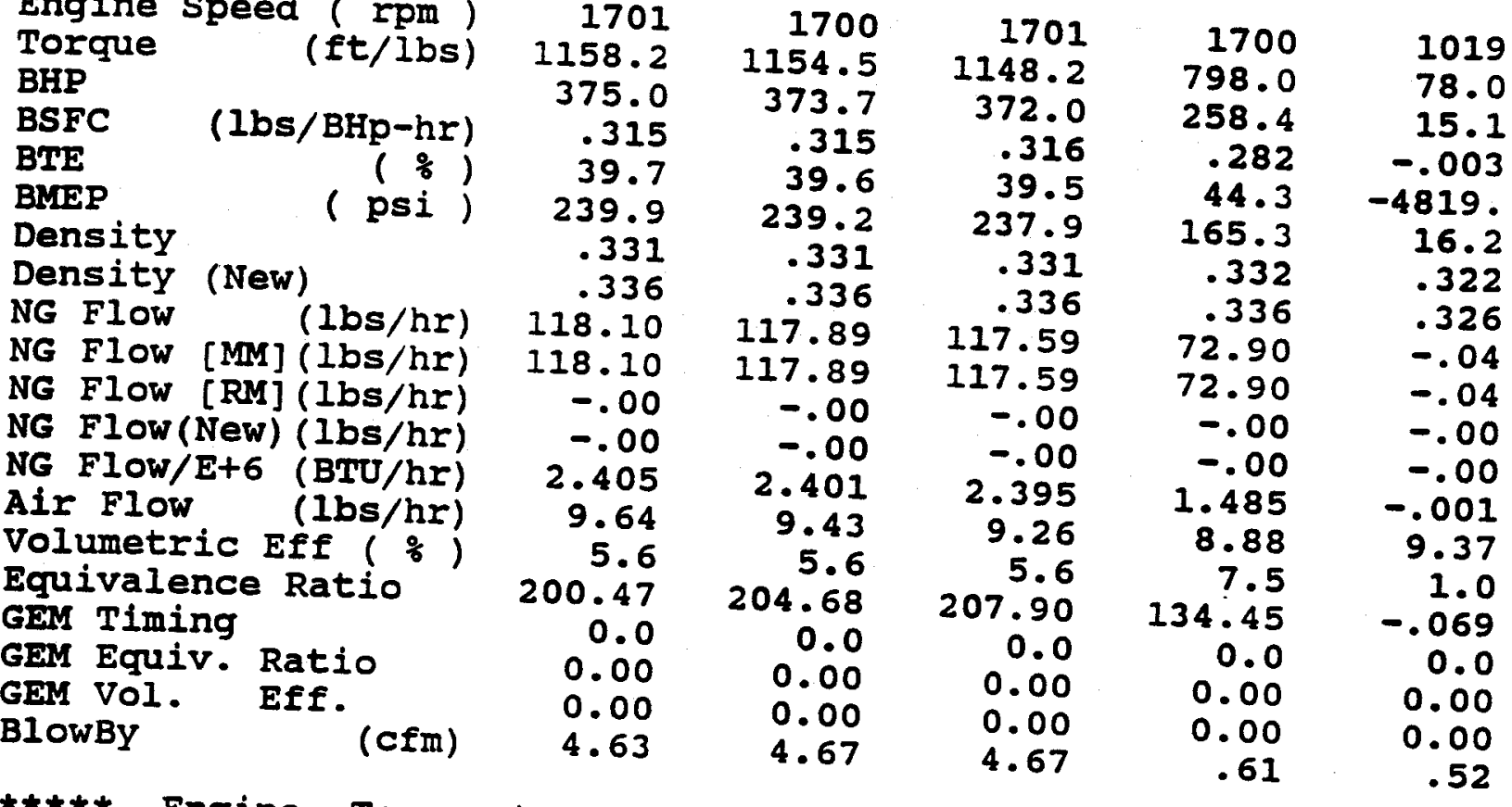

\begin{tabular}{|c|c|c|c|c|c|c|}
\hline ***** Engin & 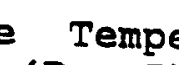 & & Data & & & \\
\hline $\begin{array}{l}\text { Water In } \\
\text { Water out }\end{array}$ & (Deg F) & 201 & 201 & 201 & 102 & 111 \\
\hline oil Sump & 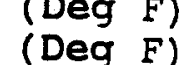 & $\begin{array}{l}209 \\
247\end{array}$ & 209 & 209 & 151 & 127 \\
\hline Oil Gallery & (DEG F) & $\begin{array}{l}247 \\
229\end{array}$ & 247 & 247 & 247 & 241 \\
\hline Ambient/LFE & $(\operatorname{Deg} F)$ & $\begin{array}{r}229 \\
88\end{array}$ & 229 & 229 & 222 & 205 \\
\hline Boost B4 IC & $(\operatorname{Deg} F)$ & 339 & $\begin{array}{r}88 \\
340\end{array}$ & 89 & 89 & 89 \\
\hline Boost AF IC & $(\operatorname{Deg} F)$ & 104 & $\begin{array}{l}340 \\
105\end{array}$ & 340 & 298 & 199 \\
\hline Intake & (Deg F) & 102 & $\begin{array}{l}105 \\
102\end{array}$ & 105 & 100 & 93 \\
\hline Gas @ Meter & (Deg F) & 103 & 103 & $\begin{array}{l}103 \\
103\end{array}$ & 100 & 102 \\
\hline Exhaust 1 & (Deg F) & 1057 & 1057 & $\begin{array}{r}103 \\
1057\end{array}$ & 103 & 103 \\
\hline Exhaust 2 & (Deg F) & 1103 & 1103 & $\begin{array}{l}1057 \\
1104\end{array}$ & 1054 & 894 \\
\hline Exhaust 3 & (Deg F) & 1095 & 1095 & 1104 & 1097 & 913 \\
\hline Exhaust 4 & (Deg F) & 1086 & 1087 & 1094 & 1092 & 915 \\
\hline Exhaust 5 & (Deg F) & 1089 & & 1087 & 1080 & 898 \\
\hline Exhaust 6 & (Deg F) & 1069 & 1071 & 1088 & 1085 & 918 \\
\hline Pre-Turbine & (Deg F) & 1178 & 1179 & $\begin{array}{l}1072 \\
1170\end{array}$ & 1066 & 891 \\
\hline & (Deg F) & 949 & 951 & $\begin{array}{r}1179 \\
950\end{array}$ & $\begin{array}{r}1175 \\
987\end{array}$ & 946 \\
\hline
\end{tabular}



= Cell 05 MAC4 Project Low-speed Data =
(continued)

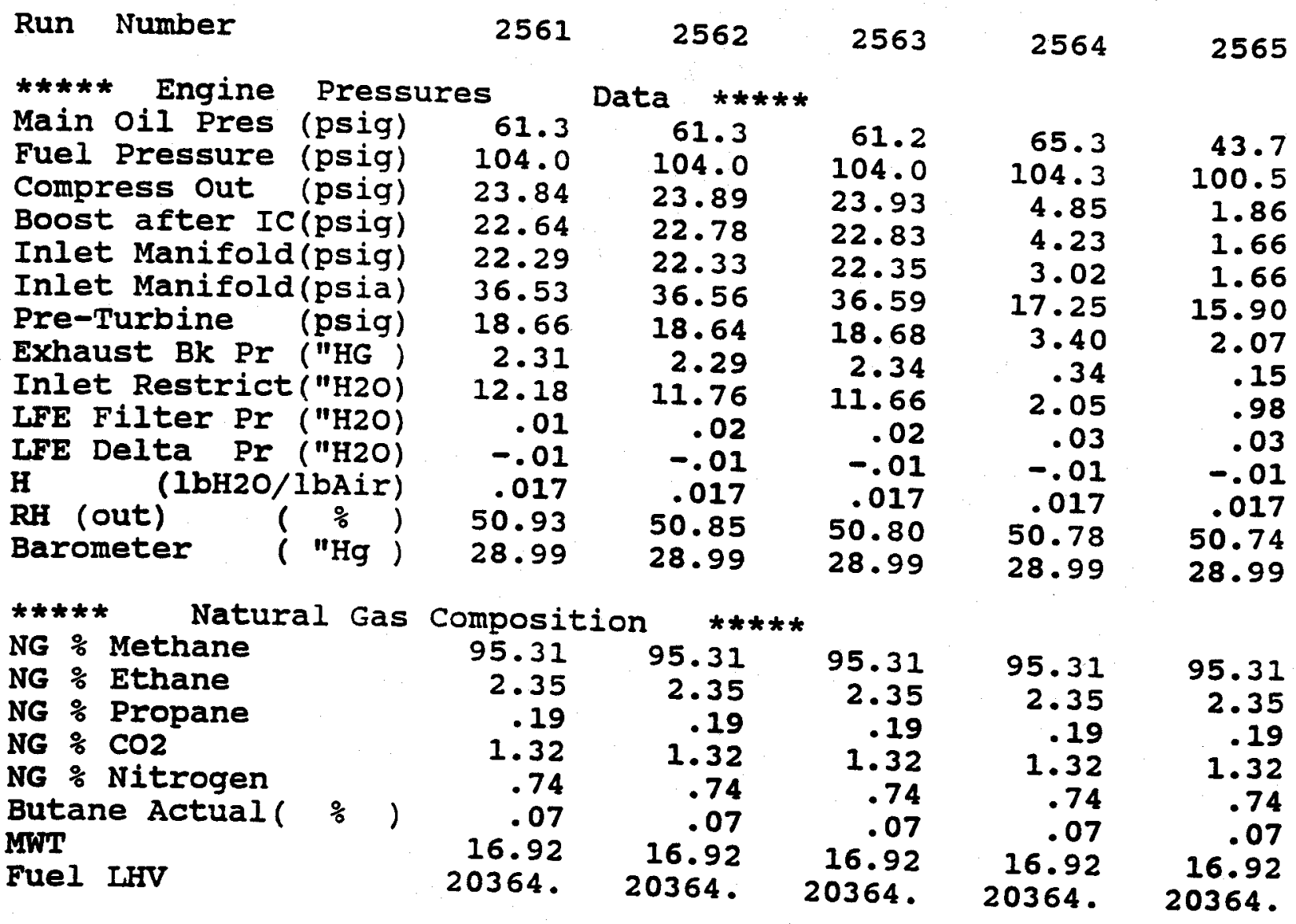


SOUTHWEST RESEARCH INSTITUTE

Automotive Products and Emissions Research Analytical Laboratory

MACK E7 G

$\mathrm{S} / \mathrm{N} 5 \mathrm{~N} 1311$

Ethyl Oil for natural gas testing

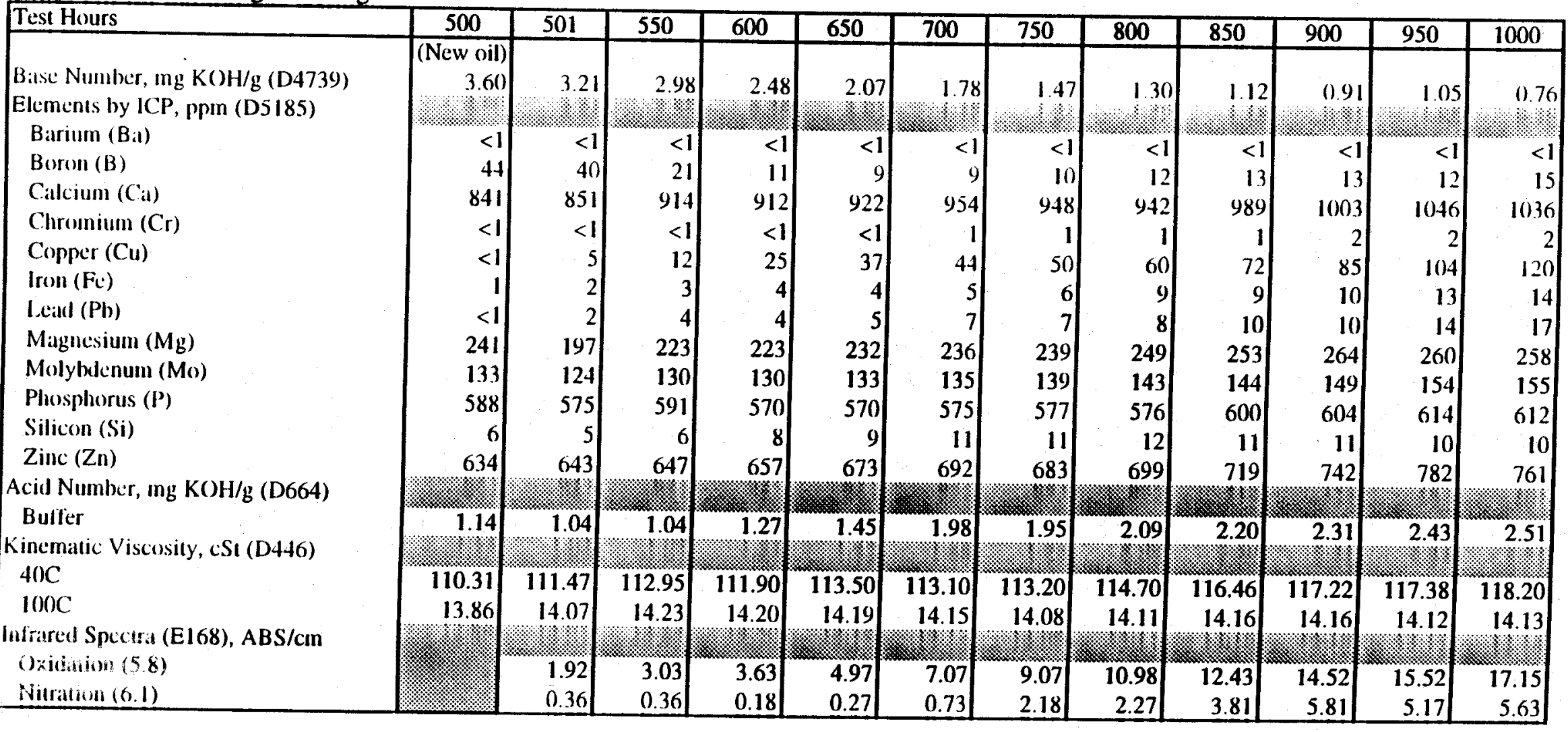


APPENDIX E

ETHYL OIL ANALYSIS RESULTS 


\section{VALVE RECESSION DATA}

\section{Exhaust Valve Stem Height Measurements $(\mathrm{mm})$}

\begin{tabular}{|c|c|c|c|c|c|c|c|c|c|c|c|c|c|}
\hline 0 & 288.1 & 13.088 & 13.133 & 13.088 & 13.118 & 13,105 & $3 \mathrm{ex}$. & 4 in. & 4 ex. & 5 in. & $5 \mathrm{ex}$. & 6 in. & $6 \mathrm{ex}$ \\
\hline 250 & 560.3 & 13.094 & 13.201 & 13.169 & & $\frac{13.105}{12002}$ & 13.277 & 13.122 & 13.162 & 13.058 & 13.152 & 13.096 & 13.215 \\
\hline 500 & 813.7 & 13.104 & 13.247 & 13.186 & $13 .<19$ & 13.203 & 13.467 & 13.125 & 13.214 & 13.113 & 13.195 & 13.123 & 13.248 \\
\hline 750 & 1066.9 & 13.14 & 13.265 & 13.23 & $\frac{13.27}{13.318}$ & 13.272 & 13.622 & 13.18 & 13.331 & 13.203 & 13.198 & 13.128 & 13.264 \\
\hline 1000 & 1321.6 & 13.148 & 13.298 & 13.25 & $\frac{13.318}{13368}$ & 13.377 & 13.765 & 13.186 & 13.346 & 13.233 & 13.229 & 13.135 & 13.271 \\
\hline \multirow{2}{*}{\multicolumn{14}{|c|}{ 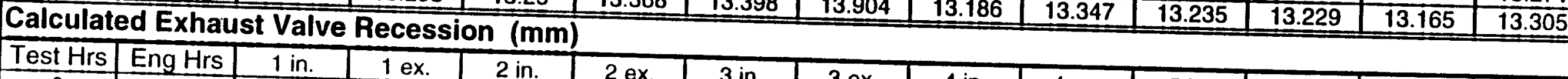 }} \\
\hline Test Hrs & Eng Hrs & $1 \mathrm{in}$. & & & & & & & & & & & \\
\hline 0 & 288.1 & 0 & $\frac{10 x}{0}$ & 2 in. & $2 \mathrm{ex}$. & 3 in. & 3 ex. & 4 in. & 4 ex. & 5 in. & $5 \mathrm{ex}$. & 6 in. & $6 \mathrm{ex}$. \\
\hline 250 & 560.3 & 0.006 & 0.068 & $\frac{0}{0081}$ & 0 & 0 & 0 & 0 & 0 & 0 & 0 & 0 & 0 \\
\hline 500 & 813.7 & 0.016 & 0.114 & $\frac{0.081}{0.098}$ & 0.101 & 0.098 & 0.19 & 0.003 & 0.052 & 0.055 & 0.043 & 0.027 & 0.033 \\
\hline 750 & 1066.9 & 0.052 & 0.132 & $\frac{0.098}{0.142}$ & 0.152 & 0.167 & 0.345 & 0.058 & 0.169 & 0.145 & 0.046 & 0.032 & 0.049 \\
\hline 1000 & 1321.6 & 0.06 & 0.165 & $\frac{0.142}{0.162}$ & 0.2 & 0.272 & 0.488 & 0.064 & 0.184 & 0.175 & 0.077 & 0.039 & 0.056 \\
\hline OTF & & & & 0.162 & 0.25 & 0.293 & 0.627 & 0.064 & 0.185 & 0.177 & 0.077 & 0.069 & 0.09 \\
\hline
\end{tabular}

\section{Intake Valve Stem Height Measurements (mm)}

\begin{tabular}{|c|c|c|c|c|c|c|c|c|c|c|c|c|c|}
\hline Test Hrs & Eng Hrs & 1 in. & $1 \mathrm{ex}$. & & & 3 in & & & & & & & \\
\hline 0 & 288.1 & 14.139 & 14.011 & 14.002 & $\frac{c}{13.908}$ & 14.137 & $3 \mathrm{ex}$ & $4 \mathrm{in}$. & 4 ex. & 5 in. & 5 ex. & 6 in. & $6 \mathrm{ex}$. \\
\hline 250 & 560.3 & 14.149 & 14.013 & 14.004 & 13.897 & & 13.964 & 14.075 & 14.062 & 13.949 & 13.95 & 14.017 & 13.958 \\
\hline 500 & 813.7 & 14.152 & 14.014 & 14.004 & 13.906 & $\frac{14.148}{14.148}$ & 13.968 & 14.076 & 14.065 & 13.965 & 13.955 & 14.023 & 13.965 \\
\hline 750 & 1066.9 & 14.152 & 14.013 & 14.004 & 13.912 & $\frac{14.148}{14.148}$ & 14.001 & 14.117 & 14.072 & 13.967 & 13.956 & 14.023 & 13.963 \\
\hline 1000 & 1321.6 & 14.152 & 14.015 & 14.004 & 13.912 & $\frac{14.148}{14.148}$ & 14.002 & 14.118 & 14.065 & 13.969 & 13.957 & 14.025 & 13.963 \\
\hline \multirow{2}{*}{\multicolumn{14}{|c|}{ 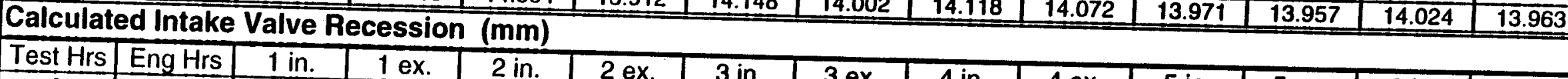 }} \\
\hline Test Hrs & Eng Hrs & $1 \mathrm{in}$. & $1 \mathrm{ex}$. & & & & & & & & & & \\
\hline 0 & 288.1 & 0 & 0 & 2 in. & $2 \mathrm{ex}$. & 3 in. & $3 \mathrm{ex}$. & 4 in. & $4 \mathrm{ex}$. & 5 in. & 5 ex. & 6 in. & $6 \mathrm{ex}$. \\
\hline 250 & 560.3 & 0.01 & & 0 & 0 & 0 & 0 & 0 & 0 & 0 & 0 & 0 & 0 \\
\hline 500 & 813.7 & 0.013 & $\frac{0.002}{0.003}$ & 0.002 & -0.011 & 0.011 & 0.004 & 0.001 & 0.003 & 0.016 & 0.005 & 0.006 & 0.007 \\
\hline 750 & 1066.9 & 0.013 & $\frac{0.003}{0.002}$ & 0.002 & -0.002 & 0.011 & 0.037 & 0.042 & 0.01 & 0.018 & 0.006 & 0.006 & 0.005 \\
\hline 1000 & 1321.6 & 0.013 & $\frac{0.002}{0.004}$ & 0.002 & 0.004 & 0.011 & 0.038 & 0.043 & 0.003 & 0.02 & 0.007 & 0.008 & 0.005 \\
\hline \multicolumn{10}{|c|}{ 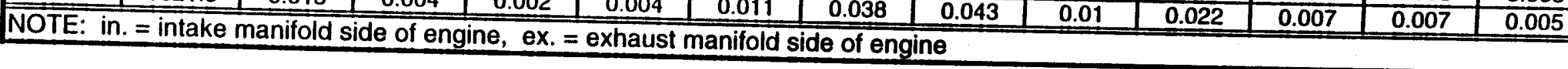 } & & & & \\
\hline
\end{tabular}


APPENDIX F

VALVE STEM HEIGHT MEASUREMENT AND RECESSION DATA 
= Cell 05 MAC4 Project Low-Speed Data =

$\begin{array}{lc}\star \star * * * \text { Test Cell Run } & \begin{array}{c}\text { Information } \\ 256 * * *\end{array} \\ \text { Run Number } & 1998 \\ \text { Year } & <. . ? ? . .> \\ \text { Operator Initials } & \text { AUG. } 15 \\ \text { Julian Day } & 17: 49: 19 \\ \text { Time (Seconds) } & 0.0 \\ \text { Ignition Timing } & 499.1 \\ \text { Test Hours } & 1\end{array}$

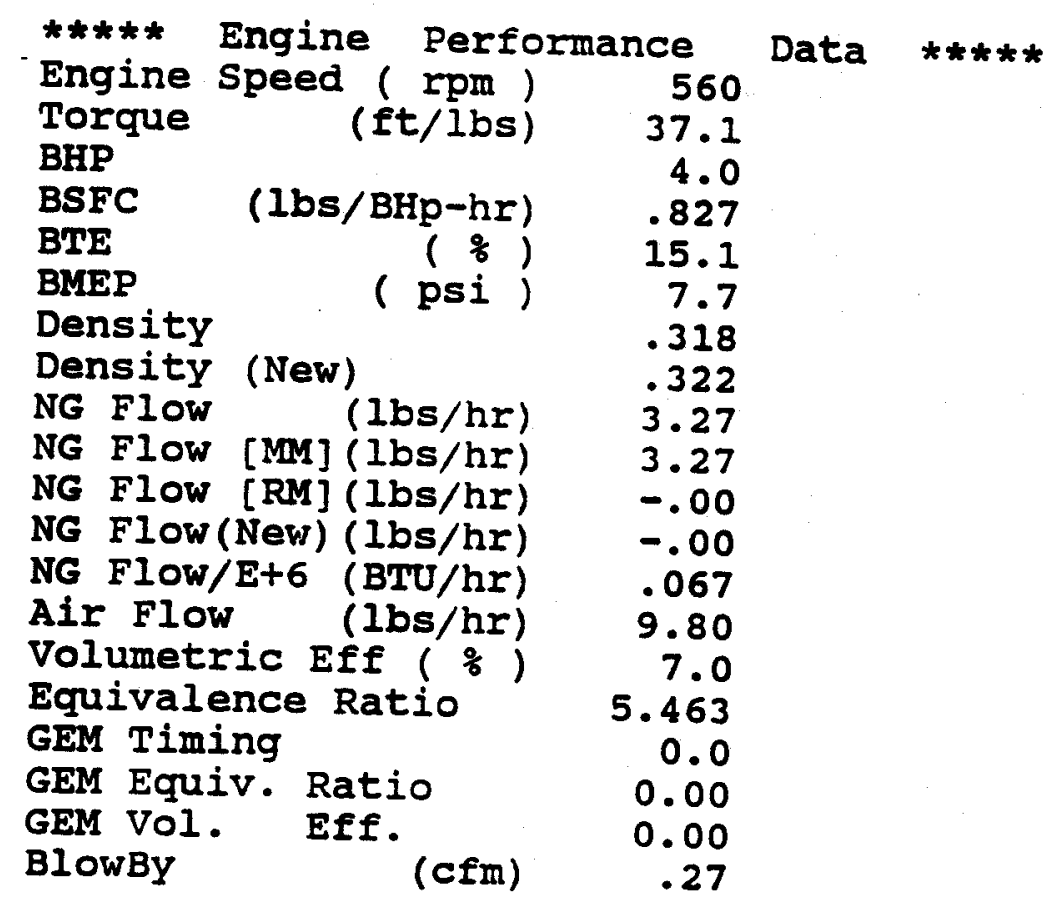

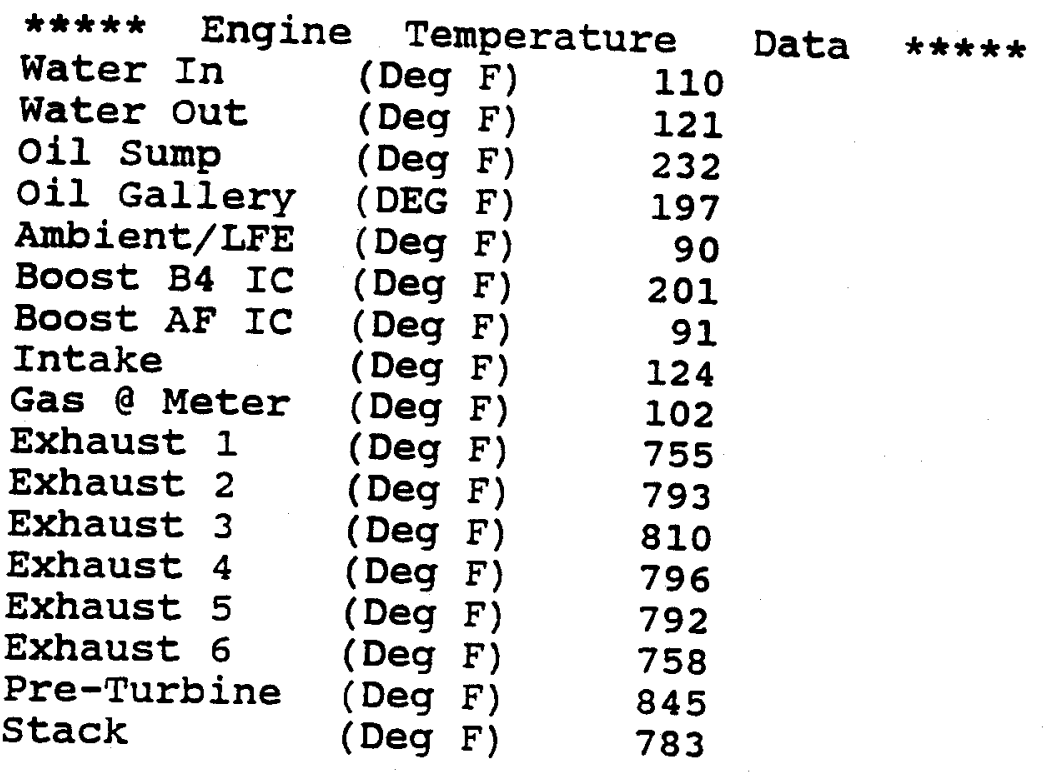


= Cell 05 MAC4 Project Low-Speed Data = (continued)

Run Number $\quad 2566$

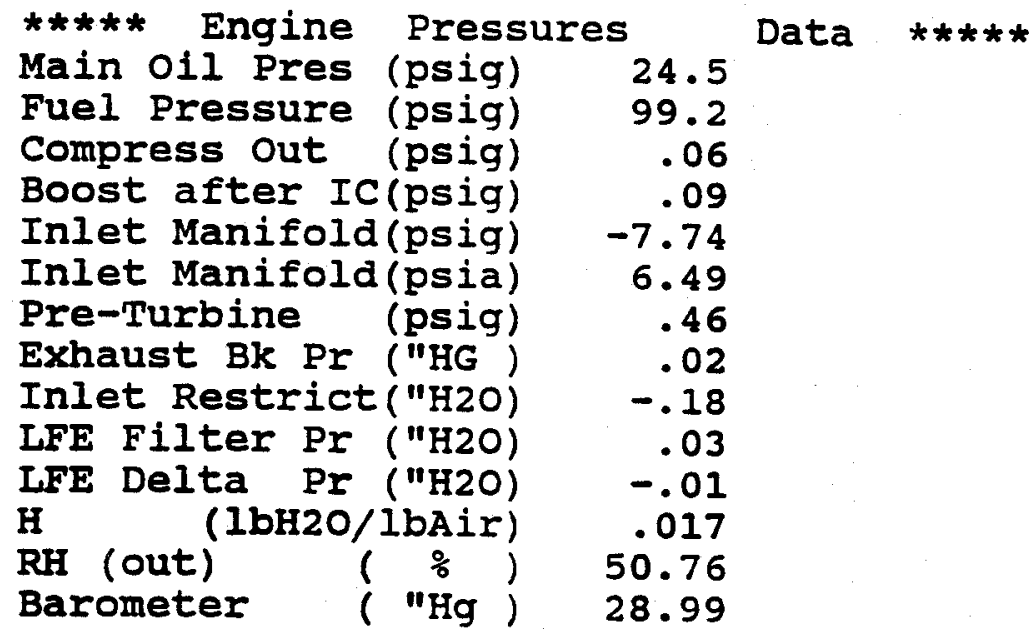

***** Natural Gas Composition

NG : Methane

NG \% Ethane

95.31

NG \% Propane

2.35

NG \% $\mathrm{CO} 2$

NG \% Nitrogen

Butane Actual ( $\%$ )

MWT

Fuel LHV

.19

1.32

.74

.07

16.92

20364 . 
APPENDIX F

HISTOGRAMS FROM THE DATA LOGGER 


\section{MACK MR LNG Truck}

\section{Histogram}
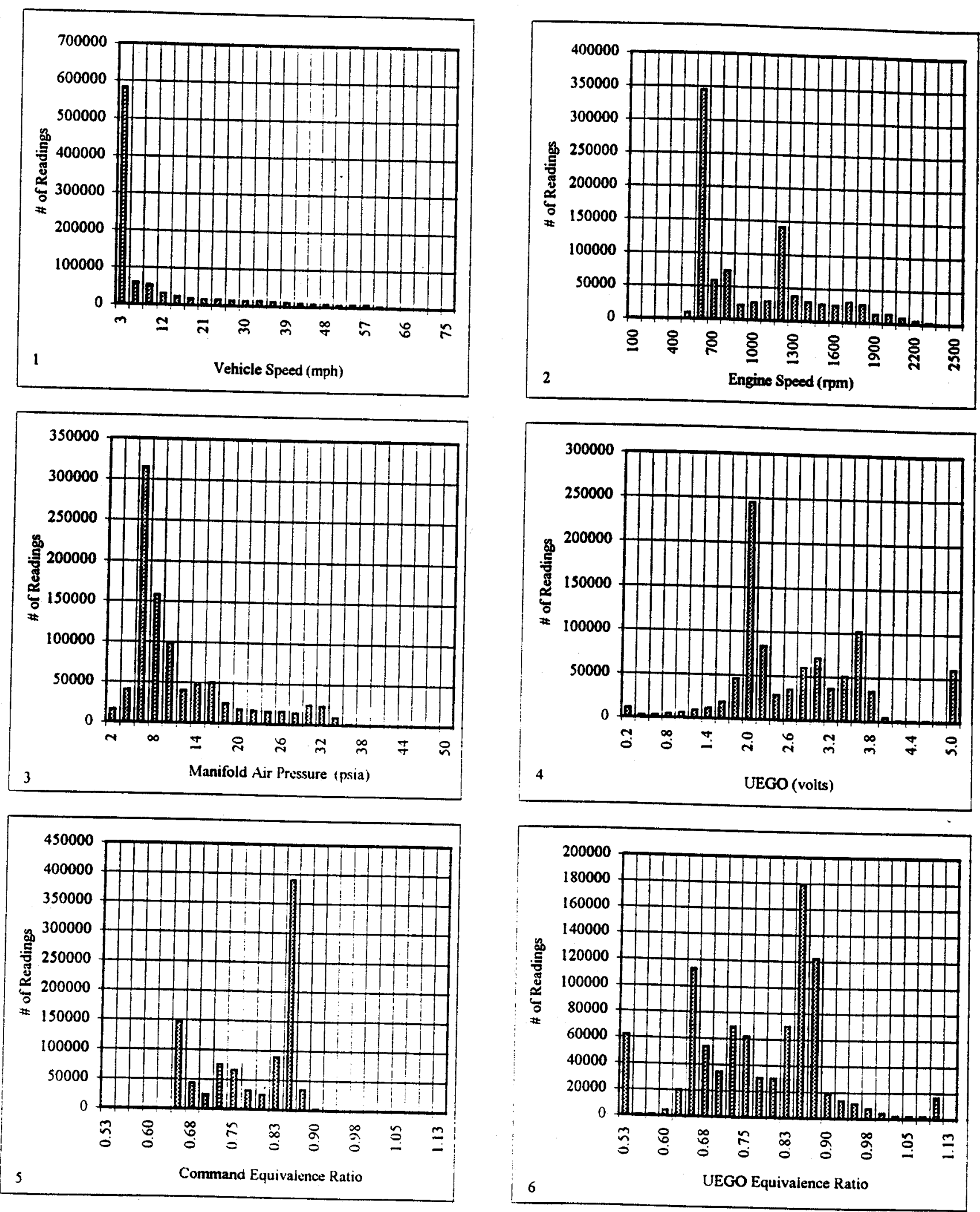
MACK MR LNG Truck

Histogram
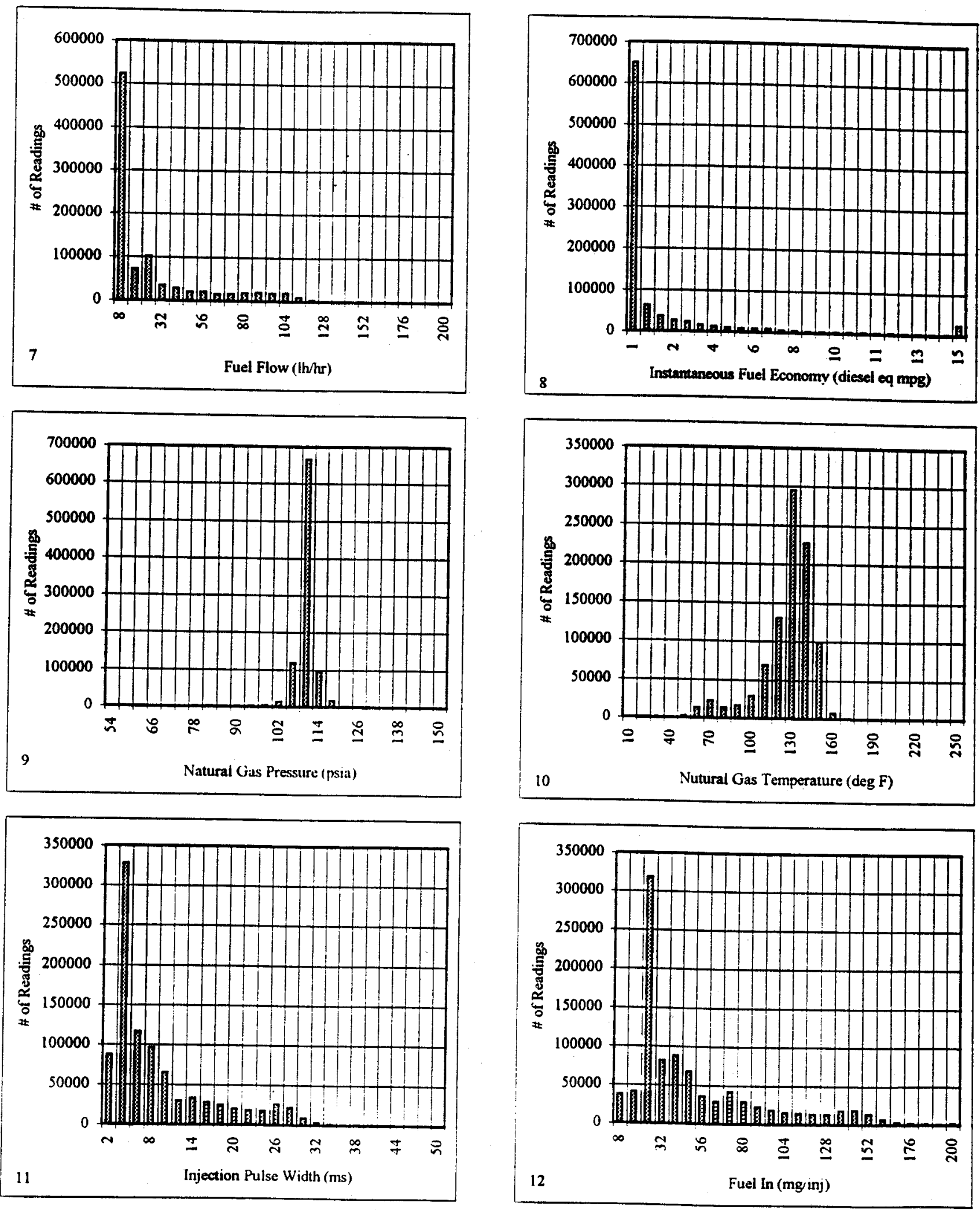
MACK MR LNG Truck

Histogram
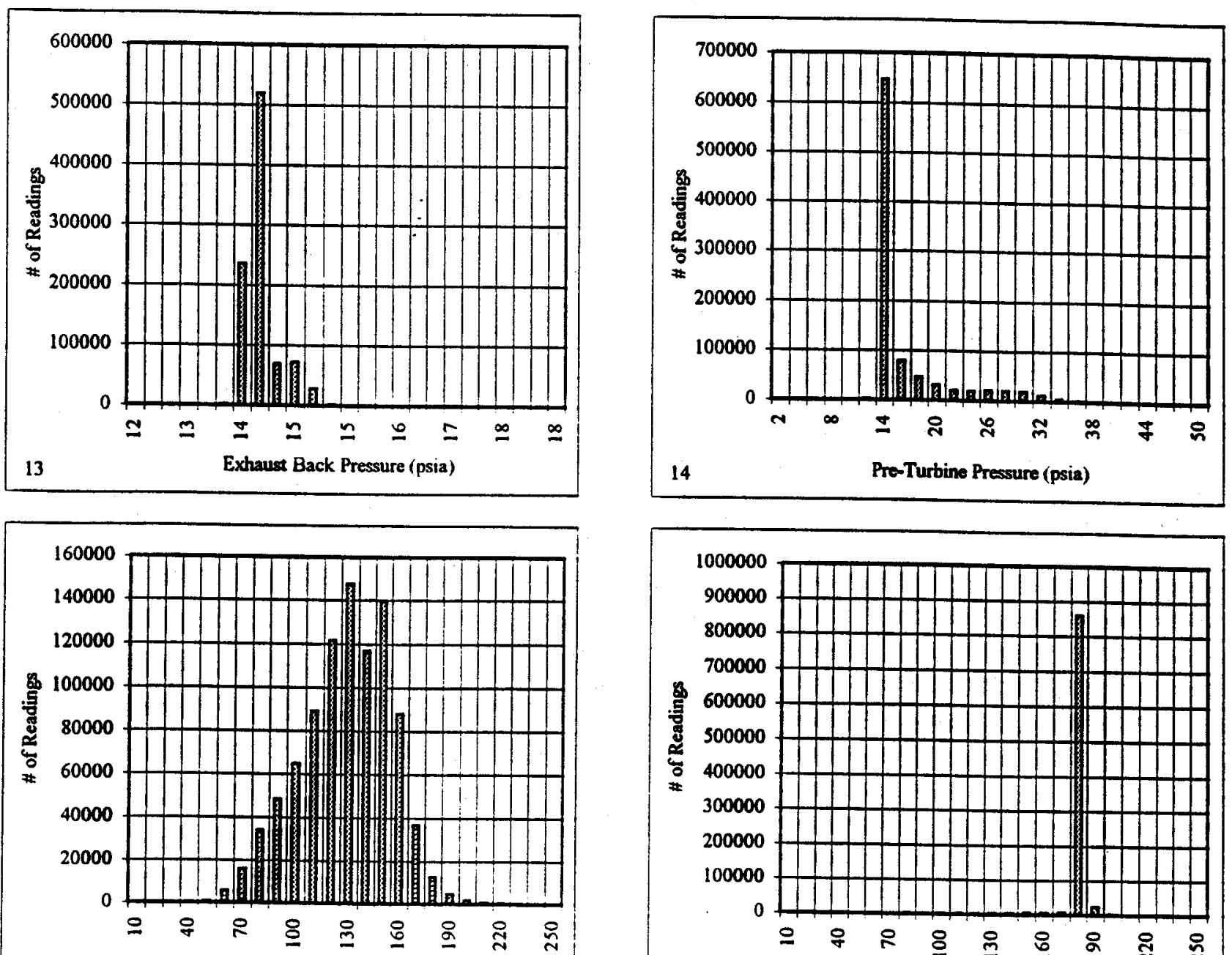

Manifold Air Temperature (deg F)
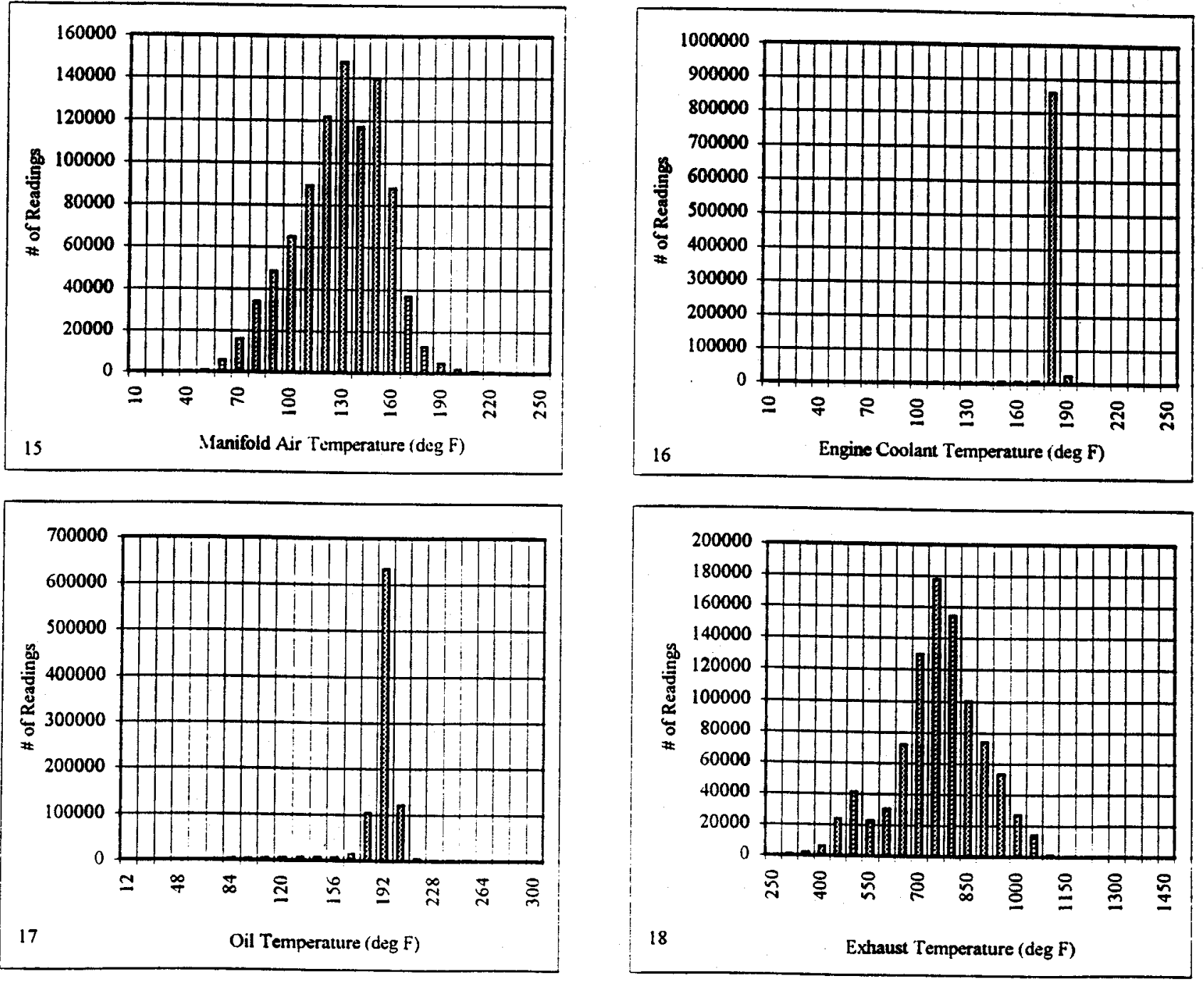
MACK MR LNG Truck

Histogram
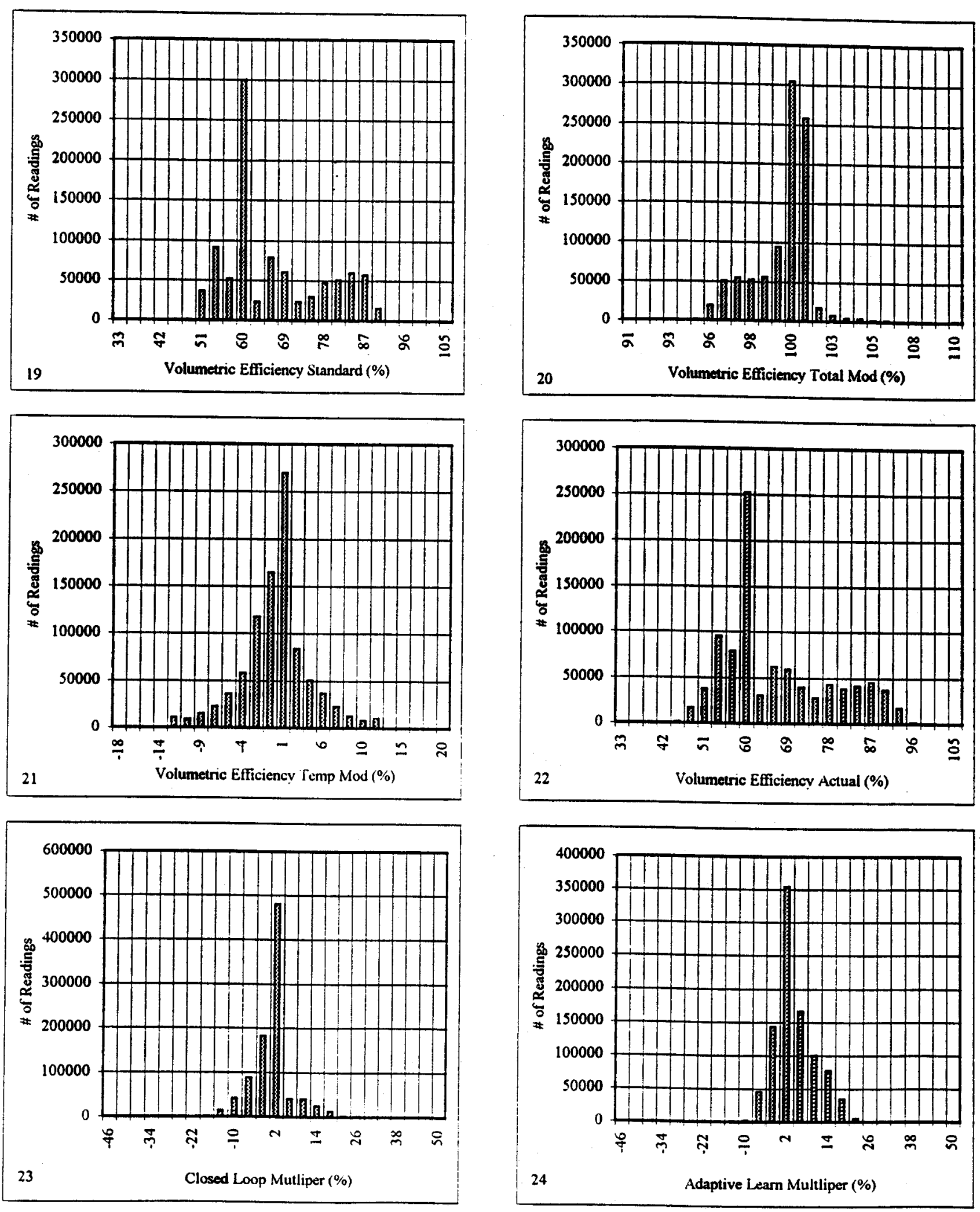
MACK MR LNG Truck

Histogram
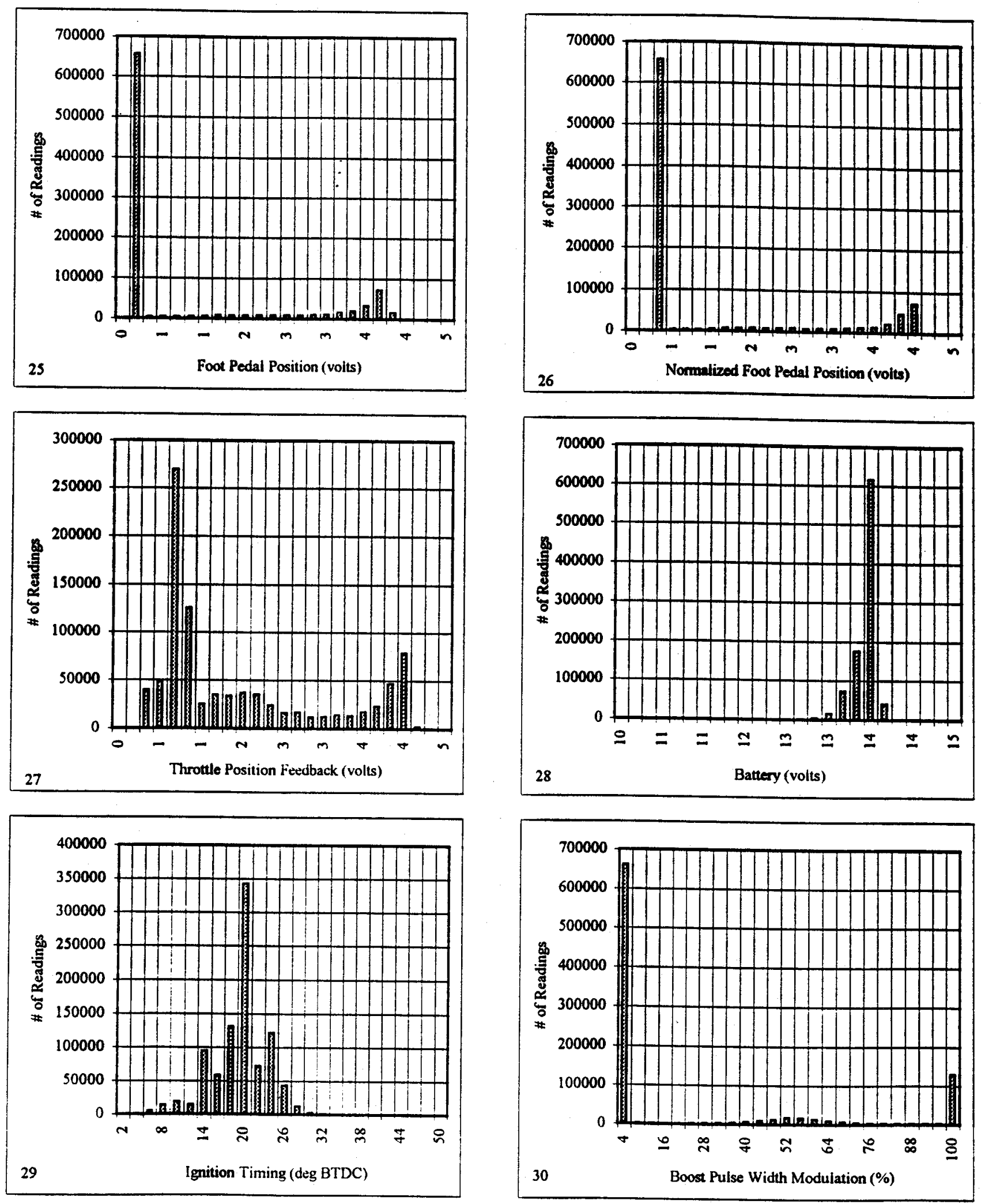
MACK MR LNG Truck

Histogram
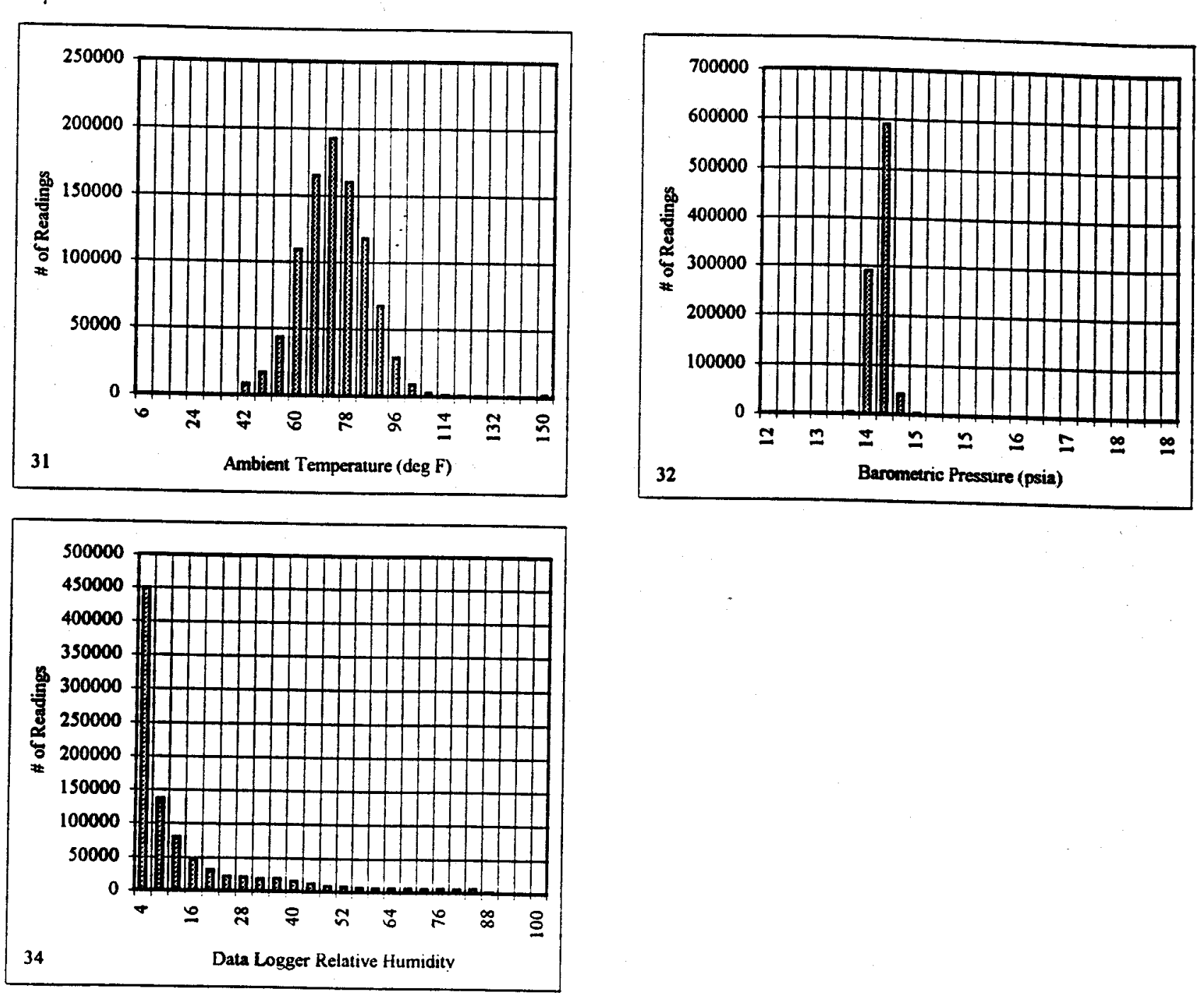


\section{APPENDIX G \\ FINAL REPORT FOR \\ TRANSIENT EMISSIONS TESTING ON A MACK E7G ENGINE \\ SwRI PROJECT NO. 03-2377}




\section{SOUTH HEST RE \\ 6220 CULEBAA ROAD - POST OFFICE ORAWEA 28510 ENGINE AND VEHICLE RESEARCH DIVISION FAX: (210) 522-2019}

April 15, 1999

Mr. John Bartel

Mack Trucks, Inc.

13302 Pennsylvania Avenue

Hagerstown, MD 21742

Subject: Southwest Research Institute Project No. 03-2377, Final Report, "Transient Emissions Testing on a Mack E7G Engine"

\subsection{BACKGROUND}

The goal of this project was to perform transient emissions tests after a 1000-hour durability test. This report covers one " 0 " hour transient emissions test and one " 0 " hour idle CO test in addition to two "1000" hour transient emissions tests (with the same calibration as the " 0 " hour test), two 325 bhp transient emissions test (with a revised calibration), and two 350 bhp transient emissions test (with a revised calibration).

Funding for the break-in and 0 hour transient emissions tests was provided by the "Mack LNG Vehicle Development" project (SwRI Project No. 03-7276) conducted by SwRI for Mack Trucks. Funding for the 1000 -hour durability tests was provided by the "1000-Hour Super Trhermal Cycle Durability Test on a Mack E-7 Natural Gas Fueled Engine" project (SwRI Project No. 03-1248) conducted by SwRI for Mack Trucks. The transient emissions tests were conducted in SwRI's Automotive Products and Emissions Research Division, Department of

\subsection{TECHNICAL DISCUSSION}

A Mack E-7 natural gas fueled engine (S/N 5N1311) was received at SwRI for a 125hour break-in, a "0" hour transient emissions and idle CO test, a 1000-hour durability test. and
"1000" hour transient emissions tests.

The test schedule for the engine is shown in Table 1. The break-in consisted of 125 hours at rated power $(1950 \mathrm{rpm}, 242 \mathrm{~kW})$. Miscellaneous tests were conducted to ensure proper operation over the entire range of engine conditions. Transient emissions tests were conducted to obtain a " 0 " hour test for determination of a deterioration factor. A 1000-hour durability test was conducted on the Mack super thermal cycle (STC). Details of the durability test. inciuding maintenance before and during the tests, are included in the final report for the "1000-Hour Super Thermal Cycle Durability Test on a Mack E-7 Natural Gas Fueled Engine". SwRI Project No. 03-1248. December 1998. Transient emissions testing was conducted following the 1000-
hour durability test. 
Mr. John Bartel

April 15, 1999

Page 2

Table 1. Runtime History of the Engine

\begin{tabular}{|c|c|c|c|}
\hline Test & $\begin{array}{c}\text { Test Time } \\
\text { (hours) }\end{array}$ & $\begin{array}{c}\text { Miscellaneous } \\
\text { Time (hours) }\end{array}$ & $\begin{array}{c}\text { Total Engine } \\
\text { Time (hours) }\end{array}$ \\
\hline Break-In & 125.0 & 35.5 & 160.5 \\
\hline Miscellaneous Tests & -- & 15.8 & 176.3 \\
\hline Transient Emissions & -- & 111.8 & 288.1 \\
\hline STC Test & 1000 & 37.9 & 1326.0 \\
\hline Transient Emissions & -- & 65.9 & 1391.9 \\
\hline
\end{tabular}

The transient emissions tests were conducted on the engine as shown in Table 2. An idle CO test was conducted at " 0 " test hours with the deterioration factor calibration and the Garrett turbocharger. The calibration at idle is identical for the deterioration, 235 BHP, and 350 BHP calibrations. All tests were conducted on natural gas, which meets the CARB specification for emissions testing. All equipment and procedures used for testing and calculations are in accordance with 40 CFR Part 86 Subpart N. A test facilities description, test results, and natural gas composition are included in Appendices A-G for the transient emissions tests. Idle CO emissions tests results are included in Appendix $\mathrm{H}$.

Table 2. Transient Emissions Testing

\begin{tabular}{|c|c|c|c|}
\hline $\begin{array}{c}\text { Test } \\
\text { Numbers } \\
\end{array}$ & Test & Calibration & Notes \\
\hline $\begin{array}{l}612-\mathrm{Cl} \\
612-\mathrm{H} 1 \\
\end{array}$ & "0" Hour Test & $\begin{array}{l}\text { Deterioration } \\
\text { Factor } \\
\end{array}$ & $\begin{array}{l}\text { Transient test performed } \\
\text { after the } 125 \text { hour break-in. }\end{array}$ \\
\hline $\begin{array}{l}218-\mathrm{Cl} 1 \\
218-\mathrm{H} 1 \\
\end{array}$ & "1000" Hour Test & $\begin{array}{l}\text { Deterioration } \\
\text { Factor } \\
\end{array}$ & $\begin{array}{l}\text { No Maintenance after the } \\
\text { 1000-hour durability test }\end{array}$ \\
\hline $\begin{array}{l}219-\mathrm{Cl} \\
219-\mathrm{H} 1 \\
\end{array}$ & $\begin{array}{l}\text { Test at } 325 \text { bhp with } \\
\text { Garrett turbocharger } \\
\end{array}$ & $\begin{array}{l}325 \mathrm{BHP} \\
\text { Certification } \\
\end{array}$ & \begin{tabular}{|l|} 
Maximum Turbo Lag \\
Enrichment set to $8 \%$ \\
\end{tabular} \\
\hline $\begin{array}{l}222-\mathrm{Cl} \\
222-\mathrm{H} 1 \\
\end{array}$ & "1000" Hour Test & $\begin{array}{l}\text { Deterioration } \\
\text { Factor } \\
\end{array}$ & Regapped spark plugs \\
\hline $\begin{array}{l}224-\mathrm{Cl} \\
224-\mathrm{H} 1 \\
\end{array}$ & $\begin{array}{l}\text { Test at } 325 \text { bhp with } \\
\text { Schwitzer turbocharger }\end{array}$ & $\begin{array}{l}325 \mathrm{BHP} \\
\text { Certification } \\
\end{array}$ & \begin{tabular}{|l|} 
Maximum Turbo Lag \\
Enrichment set to $13 \%$ \\
\end{tabular} \\
\hline $\begin{array}{l}225-\mathrm{Cl} \\
225-\mathrm{H} 1 \\
\end{array}$ & $\begin{array}{l}\text { Test at } 350 \text { bhp with } \\
\text { Schwitzer turbocharger }\end{array}$ & $\begin{array}{l}350 \text { BHP } \\
\text { Certification } \\
\end{array}$ & \begin{tabular}{|l|} 
Maximum Turbo Lag \\
Enrichment set to $10 \%$ \\
\end{tabular} \\
\hline $\begin{array}{l}225-\mathrm{C} 2 \\
225-\mathrm{H} 2 \\
\end{array}$ & $\begin{array}{l}\text { Test at } 350 \text { bhp with } \\
\text { Schwitzer turbocharger }\end{array}$ & $\begin{array}{l}350 \text { BHP } \\
\text { Certification }\end{array}$ & $\begin{array}{l}\text { Maximum Turbo Lag } \\
\text { Enrichment set to } 8 \% \\
\end{array}$ \\
\hline
\end{tabular}


Mr. John Bartel

April 15, 1999

Page 3

The tests for the deterioration factors are shown in Table 3. The "1000" hour test is with regapped spark plugs. The projection to 290,000 miles was calculated as shown in Table 4 .

Table 3. Deterioration Factor for the Mack E7G Engine

\begin{tabular}{|l|c|c|c|c|}
\hline & $\begin{array}{c}\text { "0" } \\
\text { Hour Test } \\
\text { (g/bhp-hr) }\end{array}$ & $\begin{array}{c}\text { "1000" } \\
\text { Hour Test } \\
\text { (g/bhp-hr) }\end{array}$ & $\begin{array}{c}\text { Deterioration } \\
\text { Factor } \\
\text { 0-290k miles }\end{array}$ & $\begin{array}{c}\text { Deterioration } \\
\text { Factor } \\
\mathbf{7 6 . 5 5 1 - 2 9 0 k} \text { miles }\end{array}$ \\
\hline THC & 5.271 & 3.927 & 0.000 & 0.000 \\
\hline NMHC & 0.957 & 0.506 & 0.000 & 0.000 \\
\hline NMHC+NO & 2.606 & 2.202 & 0.178 & 0.131 \\
\hline NO $_{\mathrm{x}}$ & 1.649 & 1.696 & 0.178 & 0.131 \\
\hline $\mathrm{CO}$ & 1.641 & 1.607 & 0.000 & 0.000 \\
\hline Particulate & 0.042 & 0.030 & 0.000 & 0.000 \\
\hline Formaldehyde & 0.161 & 0.145 & 0.000 & 0.000 \\
\hline
\end{tabular}

Table 4. Deterioration Factor Calculation

\begin{tabular}{|l|c|}
\hline Distance (miles) & 290,000 \\
\hline Fuel Economy of the Diesel Truck (mpg) & 5.7 \\
\hline Diesel Equivalent Fuel Economy of the Natural Gas Truck (mpg) & 4.8 \\
\hline Actual LNG Fuel Economy (mpg) & 2.97 \\
\hline Fuel Usage for 290,000 miles (gal) & 97667 \\
\hline LNG Fuel Density (lbs/gal) & 3.84 \\
\hline Fuel Usage for 290,000 miles (lbs) & 375,042 \\
\hline Durability Test Time (hours) & 1000 \\
\hline Durability Test Fuel Flow (lb/gal) & 99 \\
\hline Durability Test Fuel Usage (lb) & 99,000 \\
\hline Equivalent Durability Distance (miles) & 76,551 \\
\hline Time to 290.000 Miles (hours) & 3,788 \\
\hline
\end{tabular}


Mr. John Bartel

April 15, 1999

Page 4

Official test results for the Schwitzer turbocharger at 325 bhp and the Schwitzer turbocharger at $350 \mathrm{bhp}$ (maximum turbo lag enrichment set to 10\%) are shown in Table 5. The certification levels are shown in Table 6 . Certification levels were calculated using the 77.551-
$290 \mathrm{k}$ deterioration factor.

Table 5. Official Test Results

\begin{tabular}{|l|c|c|}
\hline & 325 BHP & 350 BHP \\
\hline HC/OMHCE (g/bhp-hr) & 3.075 & 2.905 \\
\hline NMHC/OMNMHCE (g/bhp-hr) & 0.324 & 0.276 \\
\hline HC+NOx (g/bhp-hr) & 2.399 & 2.468 \\
\hline CARBON MONOXIDE (g/bhp-hr) & 1.385 & 1.319 \\
\hline OXIDE OF NITROGEN (g/bhp-hr) & 2.075 & 2.192 \\
\hline PARTICULATE (g/bhp-hr) & 0.029 & 0.026 \\
\hline FORMALDEHYDE (g/bhp-hr) & 0.135537 & 0.116236 \\
\hline IDLE CO (\%) & 0.11 & 0.11 \\
\hline CO2 (g/bhp-hr) & 462.4 & 448.9 \\
\hline
\end{tabular}

Table 6. Certification Levels

\begin{tabular}{|l|c|c|}
\hline & 325 BHP & 350 BHP \\
\hline HC/OMHCE (g/bhp-hr) & 3.1 & 2.9 \\
\hline NMHC/OMNMHCE )g/bhp-hr) & 0.324 & 0.276 \\
\hline HC+NOx (g/bhp-hr) & 2.53 & 2.60 \\
\hline CARBON MONOXIDE (g/bhp-hr) & 1.4 & 1.3 \\
\hline OXIDE OF NITROGEN (g/bhp-hr) & 2.2 & 2.3 \\
\hline PARTICULATE (g/bhp-hr) & 0.03 & 0.03 \\
\hline FORMALDEHYDE (g/bhp-hr) & 0.136 & 0.116 \\
\hline
\end{tabular}


Mr. John Bartel

April 15, 1999

Page 5

\section{CLOSURE}

If you have any questions or comments, please contact me by telephone at (210) 52-2570, by fax at (210) 522-2019, or by e-mail at jchiu@ swri.edu.

Sincerely,

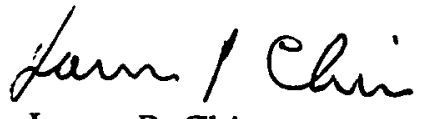

James P. Chiu

Senior Research Engineer

Gas and Industrial Engine Development

Department of Engine Research

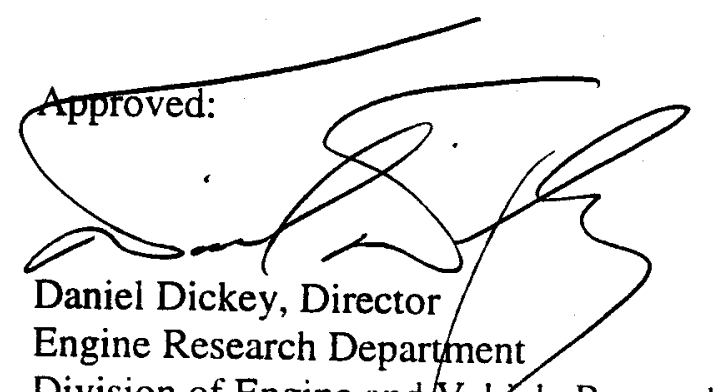

This report must be reproduced in full, unless SwRI approves a summary

or abridgement

Division of Engine and Vehrcle Research 
Appendix A

Test Numbers 612-C1 and 612-H2

“0” Hour Transient Emissions Test

Deterioration Factor Calibration

Garrett Turbocharger 


\section{TEST FACILITIES DESCRIPTION}

Emission certification testing was conducted at:

Southwest Research institute 6220 Culebra Road

P.O. Drawer 28510

San Antonio, TX 78228-0510

Certification activities at Southwest Research Institute were in test cell 3. Cell 3 uses a General absorption ability of dyno in combination with a Mid-West inductor dyno for a combined power measurement. Air t5 hp. A Lebow (rotary transformer) in line torque meter is used for torque conditioned for temp air intercooling is simulated as required per SAE 1937. Engine inlet air is throttle control and a Compudas comidity. A LabVIEW -based computer system is used for engine and acquire data. The data is then prec system is used to control the engine and dynamometer Gaseous emissions are measured dilute utilizing the full-flow dilution method and particulate sampling makes use of the double dilution method. Both primary and secondary dilution air is

The main dilution tunnel uses a positive displacement pump driven at a constant speed in combination with a heat exchanger. The particulate measurement system uses gas meters to on Pallflex $90 \mathrm{~mm}$ filters.

All equipment and procedures used for testing and calculations are in accordance with 40 CFR Part
86 Subpart N. 


\section{Southwest Research Institute - Department of Emissions Research \\ Composite Transient Emission Test Results \\ Project No. 03-7276-004}

Engine Model: 98 Mack E7 CNG

Engine Desc.: $\quad 0.0 \mathrm{~L}(0 \mathrm{CID}) 6$

Engine Cycle: Diesel

Engine S/N:
Date: 06/12/1998 Time: 09:16

Program HDT: $4.03-\mathrm{C}$

Cell: 3 Bag Cart: 1
NAT GAS CNG, CARB-CNG

HCR: 3.838

FID Resp: 1.00

$\mathrm{H}=0.230 \mathrm{C}=0.714 \mathrm{O}=0.000 \mathrm{X}=0.056$

Engine Oil:

Test Numbers

Cold: 612-C1 Hot: 612-H1

\section{Brake-Specific Emission Results}

$\begin{array}{lrlrl}\text { BSTHC (Cell) } & 5.271 & \mathrm{~g} / \mathrm{hp}-\mathrm{hr} & 7.069 & \mathrm{~g} / \mathrm{kW}-\mathrm{hr} \\ \text { CO } & 1.641 & \mathrm{~g} / \mathrm{hp}-\mathrm{hr} & 2.200 \mathrm{~g} / \mathrm{kW}-\mathrm{hr} \\ \text { NOx (Cell) } & 1.649 & \mathrm{~g} / \mathrm{hp}-\mathrm{hr} & 2.211 & \mathrm{~g} / \mathrm{kW}-\mathrm{hr} \\ \text { Particulate } & 0.042 & \mathrm{~g} / \mathrm{hp}-\mathrm{hr} & 0.056 \mathrm{~g} / \mathrm{kW}-\mathrm{hr} \\ \text { CO2 } & 499.4 & \mathrm{~g} / \mathrm{hp}-\mathrm{hr} & 669.72 & \mathrm{~g} / \mathrm{kW}-\mathrm{hr} \\ \text { BSFC } & 0.436 & \mathrm{lb} / \mathrm{hp}-\mathrm{hr} & 0.584 & \mathrm{~kg} / \mathrm{kW}-\mathrm{hr} \\ \text { NMHC } & 0.957 & \mathrm{~g} / \mathrm{hp}-\mathrm{hr} & 1.283 & \mathrm{~g} / \mathrm{kW}-\mathrm{hr} \\ \text { CH4 } & 4.314 & \mathrm{~g} / \mathrm{hp}-\mathrm{hr} & 5.786 \mathrm{~g} / \mathrm{kW}-\mathrm{hr} \\ & & & & \\ \text { Work: } & 23.84 & \mathrm{hp}-\mathrm{hr} & 17.78 \mathrm{~kW}-\mathrm{hr} \\ \text { Reference Work: } & 24.94 & \mathrm{hp}-\mathrm{hr} & 18.60 \mathrm{~kW}-\mathrm{hr}\end{array}$




\section{Southwest Research Institute - Department of Emissions Research EPA Cold Transient Emission Test Results \\ Project No. 03-7276-004}

Engine Model: 98 Mack E7 CNG Engine Desc.: $\quad 0.0 \mathrm{~L}(\mathrm{O} \mathrm{CID}) 6$ Engine Cycle: Diesel Engine S/N:
Test No.: $612 \cdot \mathrm{C1}$

Date: 06/12/1998 Time:09:16

Program HDT: $4.03-\mathrm{C}$

Cell: 3 Bag Cart: 1
NAT GAS CNG, CARB-CNG

HCR: $\quad 3.838$ FID Resp: 1.00

$H=0.230 \mathrm{C}=0.714 \mathrm{O}=0.000 \mathrm{X}=0.056$

Engine Oil:
Ambient/Test Cell Conditions

$\begin{array}{llll}\text { Barometer: } & 29.08 & \text { in } \mathrm{Hg} & 98.5 \mathrm{kPa} \\ \begin{array}{l}\text { Engine Inlet Air } \\ \text { Temperature: }\end{array} & 79.0 & { }^{\circ} \mathrm{F} & 26.1^{\circ} \mathrm{C} \\ \text { Dew Point: } & 59.8 & { }^{\circ} \mathrm{F} & 15.4^{\circ} \mathrm{C} \\ \text { Abs. Humidity: } & 79.3 & \mathrm{gr} / \mathrm{lb} & 11.3 \mathrm{~g} / \mathrm{kg} \\ \begin{array}{l}\text { Rel. Humidity: } \\ \text { Dilution Air: }\end{array} & 52 & \% & \\ \text { Temperature: } & 78.0 & { }^{\circ} \mathrm{F} & 25.6{ }^{\circ} \mathrm{C} \\ \text { Abs. Humidity } & 100.3 & \mathrm{gr} / \mathrm{lb} & 14.3 \mathrm{~g} / \mathrm{kg} \\ \text { Rel. Humidity: } & 67 & \% & \end{array}$

Measured Gaseous Data

HC Sample

HC Bckgrd

CO Sample

CO Bckgrd

NOx Sample

NOx Bckgrd

CO2 Sample

CO2 Bckgrd

$\mathrm{CH} 4$ Sample

$\mathrm{CH} 4$ Bckgrd

Meter Range Concentration

$\begin{array}{lr}\mathrm{n} / \mathrm{a} & 169.47 \mathrm{ppm} \\ \mathrm{n} / \mathrm{a} & 6.68 \mathrm{ppm}\end{array}$

$\begin{array}{rrr}30.7 & 2 & 29.86 \mathrm{ppm}\end{array}$

$\begin{array}{lll}0.5 & 2 & 0.47 \mathrm{ppm}\end{array}$

$\mathrm{n} / \mathrm{a} \quad 19.87 \mathrm{ppm}$

$\begin{array}{lll}0.1 & 2 & 0.10 \mathrm{ppm}\end{array}$

$\begin{array}{lll}71.2 & 1 & 0.6107 \%\end{array}$

$\begin{array}{lll}8.7 & 1 & 0.0583 \%\end{array}$

n/a $\mathrm{n} / \mathrm{a} \quad 133.47 \mathrm{ppm}(1.00)$

n/a n/a $\quad 2.84 \mathrm{ppm}$

\section{Corrected Concentrations}

$\mathrm{HC}$

$\mathrm{CC}$

NOX

$\mathrm{CO} 2$

$\mathrm{CH} 4$

$\mathrm{RHC}$

$\mathrm{HC}$

$\mathrm{CO}$

NOx

Particulate

$\mathrm{CO} 2$

$\mathrm{CH} 4$

$\mathrm{RHC}$

Fuel

$\begin{aligned} 163.22 & \text { ppm } \\ 28.25 & \text { ppm } \\ 19.10 & \text { ppm } \\ 0.5562 & \% \\ 130.81 & \text { ppm } \\ 32.41 & \text { ppm }\end{aligned}$

Mass Emissions

$\begin{array}{rl}129.371 & \text { grams } \\ 39.660 & \text { grams } \\ 44.539 & \text { grams } \\ 1.074 & \text { grams } \\ 12.271 & \mathrm{~kg} \\ 105.173 & \text { grams } \\ 24.199 & \text { grams } \\ 10.70 \mathrm{lb} & 4.85 \mathrm{~kg}\end{array}$

\begin{tabular}{crr} 
& \multicolumn{2}{c}{ Sample Flows } \\
scfm & scmm \\
Blower 1 Rate: & $2,117.1$ & 59.96 \\
Blower 2 Rate: & 0.0 & 0.00 \\
90 mm System: & & \\
Gas Meter 1: & 1.49 & 0.04 \\
Gas Meter 2: & 2.40 & 0.07 \\
Sample Rate: & 0.91 & 0.03 \\
Chemistry Sample Rate: & 2.78 & 0.08 \\
Total Flow Rate: & $2,120.77$ & 60.06
\end{tabular}

Filter Number: $\quad \begin{array}{r}\text { Particulate Data } \\ \text { 4693.0-78 (pair) }\end{array}$

Weight Gain, mg: $\quad 0.462$

Sample Multiplier: $\quad 2.324$

NOx Humidity CF:

Correction Factors

Dry-to-Wet CF, Sample:

1.011

0.966

Dry-to-Wet CF, Bckgrd:

Dilution Factor:

0.977

15.42

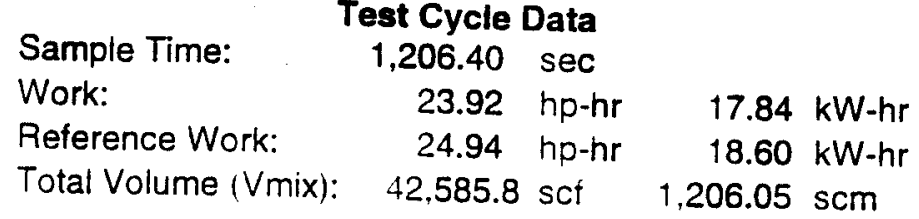

Brake-Specific Emission Results

$\begin{array}{llll}\text { BSTHC (Cell) } & 5.409 \mathrm{~g} / \mathrm{hp}-\mathrm{hr} & 7.253 \mathrm{~g} / \mathrm{kW}-\mathrm{hr} \\ \text { CO } & 1.658 \mathrm{~g} / \mathrm{hp}-\mathrm{hr} & 2.223 \mathrm{~g} / \mathrm{kW}-\mathrm{hr} \\ \text { NOx (Cell) } & 1.862 \mathrm{~g} / \mathrm{hp}-\mathrm{hr} & 2.497 \mathrm{~g} / \mathrm{kW}-\mathrm{hr} \\ \text { Particulate } & 0.045 \mathrm{~g} / \mathrm{hp}-\mathrm{hr} & 0.060 \mathrm{~g} / \mathrm{kW}-\mathrm{hr} \\ \text { CO2 } & 513.0 \mathrm{~g} / \mathrm{hp}-\mathrm{hr} & 687.97 \mathrm{~g} / \mathrm{kW}-\mathrm{hr} \\ \text { BSFC } & 0.447 \mathrm{lb} / \mathrm{hp}-\mathrm{hr} & 0.272 \mathrm{~kg} / \mathrm{kW}-\mathrm{hr} \\ \text { NMHC } & 1.012 \mathrm{~g} / \mathrm{hp}-\mathrm{hr} & 1.357 \mathrm{~g} / \mathrm{kW}-\mathrm{hr} \\ \text { CH4 } & 4.397 \mathrm{~g} / \mathrm{hp}-\mathrm{hr} & 5.896 \mathrm{~g} / \mathrm{kW}-\mathrm{hr}\end{array}$




\section{Southwest Research Institute - Department of Emissions Research EPA Cold Transient Emission Test Results \\ Project No. 03-7276-004}

Engine Model: 98 Mack E7 CNG Engine Desc.: $0.0 \mathrm{~L}(0 \mathrm{CID}) 6$ Engine Cycle: Diesel Engine S/N:

\section{Formaidehyde} Concentration Mass

Acetaldehyde Concentration Mass

Acrolein Concentration Mass

Acetone

Concentration Mass

Propionaldehude Concentration Mass

Crotonaidehyde Concentration Mass

Isobutyr + MEK

Concentration Mass

Benzaldehyde Concentration Mass

Hexanaldehyde Concentration Mass
Test No.: $612 \cdot \mathrm{C} 1$

Date: 06/12/1998 Time:09:16

Program HDT: $\quad 4.03-\mathrm{C}$

Cell: 3 Bag Cart: 1
NAT GAS CNG, CARB-CNG

HCR: $\quad 3.838$ FID Resp: 1.00

$\mathrm{H}=0.230 \mathrm{C}=0.714 \mathrm{O}=0.000 \mathrm{X}=0.056$

\begin{tabular}{|c|c|c|c|}
\hline Samp & & Backgro & und \\
\hline $\begin{array}{r}2.844 \\
, 127.64\end{array}$ & $\begin{array}{l}\mathrm{ppm} \\
\mathrm{mg}\end{array}$ & 0.007 & $\mathrm{ppm}$ \\
\hline $\begin{array}{r}0.099 \\
208.69\end{array}$ & $\begin{array}{l}\text { ppm } \\
\mathrm{mg}\end{array}$ & 0.001 & $\mathrm{ppm}$ \\
\hline $\begin{array}{l}0.020 \\
55.13\end{array}$ & $\begin{array}{l}\mathrm{ppm} \\
\mathrm{mg}\end{array}$ & 0.000 & $\mathrm{ppm}$ \\
\hline $\begin{array}{l}0.022 \\
52.59\end{array}$ & $\begin{array}{l}\mathrm{ppm} \\
\mathrm{mg}\end{array}$ & 0.004 & $\mathrm{ppm}$ \\
\hline $\begin{array}{l}0.016 \\
43.93\end{array}$ & $\begin{array}{l}\mathrm{ppm} \\
\mathrm{mg}\end{array}$ & 0.000 & ppm \\
\hline $\begin{array}{l}0.007 \\
23.93\end{array}$ & $\begin{array}{l}\mathrm{ppm} \\
\mathrm{mg}\end{array}$ & 0.000 & ppm \\
\hline $\begin{array}{r}0.000 \\
0.00\end{array}$ & $\begin{array}{l}\mathrm{ppm} \\
\mathrm{mg}\end{array}$ & 0.000 & $\mathrm{ppm}$ \\
\hline $\begin{array}{r}0.000 \\
0.00\end{array}$ & $\begin{array}{l}\mathrm{ppm} \\
\mathrm{mg}\end{array}$ & 0.000 & $\mathrm{ppm}$ \\
\hline $\begin{array}{r}0.001 \\
2.81\end{array}$ & $\begin{array}{l}\mathrm{ppm} \\
\mathrm{mg}\end{array}$ & 0.000 & $\mathrm{ppm}$ \\
\hline
\end{tabular}

\section{Brake-Specific Results}

Formaidehyde

Acetaldehyde

Acrolein

Acetone

Propionaldehyde
$172.560 \mathrm{mg} / \mathrm{hp}-\mathrm{hr}$

$8.725 \mathrm{mg} / \mathrm{hp}-\mathrm{hr}$

$2.305 \mathrm{mg} / \mathrm{hp}-\mathrm{hr}$

$2.199 \mathrm{mg} / \mathrm{hp}-\mathrm{hr}$

$1.837 \mathrm{mg} / \mathrm{hp}-\mathrm{hr}$
Crotonaldehyde

Isobutyr + MEK

Benzaldehyde

Hexanaidehyde
$1.000 \mathrm{mg} / \mathrm{hp}-\mathrm{hr}$

$0.000 \mathrm{mg} / \mathrm{hp}-\mathrm{hr}$

$0.000 \mathrm{mg} / \mathrm{hp}-\mathrm{hr}$

$0.118 \mathrm{mg} / \mathrm{hp}-\mathrm{hr}$ 
Southwest Research Institute - Department of Emissions Research

EPA Hot Transient Emission Test Resuits

Project No. 03-7276-004

Engine Model: 98 Mack E7 CNG

Engine Desc.: $0.0 \mathrm{~L}$ (0 CID) 6

Engine Cycle: Diesel

Engine S/N:
Test No.: $612-\mathrm{H1}$

Date: 06/12/1998 Time:09:56

Program HDT: $4.03-\mathrm{C}$

Cell: 3 Bag Cart:
NAT GAS CNG, CARB-CNG

HCR: 3.838 FID Resp: 1.00

$H=0.230 \mathrm{C}=0.714 \mathrm{O}=0.000 \mathrm{X}=0.056$

\section{Ambient/Test Cell Conditions}

Barometer:

Engine inlet Air

29.09 in $\mathrm{Hg} \quad 98.5 \mathrm{kPa}$

Temperature:

Dew Point:

$80.0 \quad{ }^{\circ} \mathrm{F}$

$26.7^{\circ} \mathrm{C}$

Abs. Humidity:

$60.0 \quad{ }^{\circ} \mathrm{F} \quad 15.6^{\circ} \mathrm{C}$

Rel. Humidity:

$79.8 \quad \mathrm{gr} / \mathrm{lb} \quad 11.4 \mathrm{~g} / \mathrm{kg}$

Dilution Air:

$51 \%$

\begin{tabular}{lrr} 
& \multicolumn{3}{c}{ Sample Flows } \\
scfm & \multicolumn{1}{c}{ scmm } \\
Blower 1 Rate: & $2,117.4$ & 59.97 \\
Blower 2 Rate: & 0.0 & 0.00 \\
90 mm System: & & \\
Gas Meter 1: & 1.49 & 0.04 \\
Gas Meter 2: & 2.39 & 0.07 \\
Sample Rate: & 0.90 & 0.03 \\
Chemistry Sample Rate: & 2.75 & 0.08 \\
Total Flow Rate: & $2,121.04$ & 60.07
\end{tabular}

Temperature:

Abs. Humidity

Rel. Humidity:

Measured Gaseous Data
HC Sample

HC Bckgrd

CO Sample

CO Bckgrd

NOx Sample

NOx Bckgrd

CO2 Sample

$\mathrm{CO} 2$ Bckgrd

CH4 Sample

$\mathrm{CH} 4$ Bckgrd

$\begin{array}{lcc}79.0 & { }^{\circ} \mathrm{F} & 26.1^{\circ} \mathrm{C} \\ 104.3 & \mathrm{gr} / \mathrm{lb} & 14.9 \mathrm{~g} / \mathrm{kg} \\ 68 & \% & \end{array}$

Meter Range Concentration

$\begin{array}{rrrl}\mathrm{n} / \mathrm{a} & & 163.66 & \mathrm{ppm} \\ \mathrm{n} / \mathrm{a} & & 6.39 & \mathrm{ppm} \\ 30.2 & 2 & 29.37 & \mathrm{ppm} \\ 0.5 & 2 & 0.47 & \mathrm{ppm} \\ \mathrm{n} / \mathrm{a} & & 17.14 & \mathrm{ppm}(\text { Dry }) \\ 0.1 & 2 & 0.10 & \mathrm{ppm} \\ 69.6 & 1 & 0.5908 & \% \\ 8.6 & 1 & 0.0576 & \% \\ \mathrm{n} / \mathrm{a} & \mathrm{n} / \mathrm{a} & 130.29 & \mathrm{ppm}(1.00) \\ \mathrm{n} / \mathrm{a} & \mathrm{n} / \mathrm{a} & 3.05 & \mathrm{ppm}\end{array}$

\section{Corrected Concentrations}

$\begin{array}{lrl}\mathrm{HC} & 157.67 & \mathrm{ppm} \\ \mathrm{CO} & 27.79 & \mathrm{ppm} \\ \mathrm{NOx} & 16.45 & \% \\ \mathrm{CO} 2 & 0.5368 & \% \\ \mathrm{CH} 4 & 127.43 & \mathrm{ppm} \\ \mathrm{RHC} & 30.24 & \mathrm{ppm}\end{array}$

$\mathrm{HC}$

Mass Emissions

$\mathrm{CO}$

NOx

Particulate

$\mathrm{CO} 2$

$\mathrm{CH} 4$

$\mathrm{RHC}$

Fuel grams

$\begin{array}{ll}39.026 & \text { grams } \\ 38.433 & \text { grams }\end{array}$

0.987 grams

$11.847 \mathrm{~kg}$

102.482 grams

22.578 grams

$10.33 \mathrm{lb} \quad 4.69 \mathrm{~kg}$
Particulate Data

Filter Number: $\quad 4695.0-80$ (pair)

Weight Gain, mg: $\quad 0.421$

Sample Multiplier: $\quad 2.345$

NOx Humidity CF:

\section{Correction Factors}

Dry-to-Wet CF, Sample:

Dry-to-Wet CF, Bckgrd:

Dilution Factor:

1.013

0.965

0.977

15.94

\begin{tabular}{lrr}
\multicolumn{5}{c}{ Test Cycle Data } & \\
Sample Time: & $1,206.50 \mathrm{sec}$ & \\
Work: & $23.83 \mathrm{hp}-\mathrm{hr}$ & $17.77 \mathrm{~kW}-\mathrm{hr}$ \\
Reference Work: & $24.94 \mathrm{hp}-\mathrm{hr}$ & $18.60 \mathrm{~kW}-\mathrm{hr}$ \\
Total Volume (Vmix): & $42.595 .2 \mathrm{scf}$ & $1,206.32 \mathrm{scm}$
\end{tabular}

Brake-Specific Emission Resuits

$\begin{array}{llll}\text { BSTHC (Cell) } & 5.248 \mathrm{~g} / \mathrm{hp}-\mathrm{hr} & 7.038 \mathrm{~g} / \mathrm{kW}-\mathrm{hr} \\ \text { CO } & 1.638 \mathrm{~g} / \mathrm{hp}-\mathrm{hr} & 2.196 \mathrm{~g} / \mathrm{kW}-\mathrm{hr} \\ \text { NOx (Cell) } & 1.613 \mathrm{~g} / \mathrm{hp}-\mathrm{hr} & 2.163 \mathrm{~g} / \mathrm{kW}-\mathrm{hr} \\ \text { Particulate } & 0.041 \mathrm{~g} / \mathrm{hp}-\mathrm{hr} & 0.056 \mathrm{~g} / \mathrm{kW}-\mathrm{hr} \\ \text { CO2 } & 497.1 \mathrm{~g} / \mathrm{hp}-\mathrm{hr} & 666.67 \mathrm{~g} / \mathrm{kW}-\mathrm{hr} \\ \text { BSFC } & 0.434 \mathrm{lb} / \mathrm{hp}-\mathrm{hr} & 0.264 \mathrm{~kg} / \mathrm{kW}-\mathrm{hr} \\ \text { NMHC } & 0.947 \mathrm{~g} / \mathrm{hp}-\mathrm{hr} & 1.271 \mathrm{~g} / \mathrm{kW}-\mathrm{hr} \\ \text { CH4 } & 4.301 \mathrm{~g} / \mathrm{hp}-\mathrm{hr} & 5.767 \mathrm{~g} / \mathrm{kW}-\mathrm{hr}\end{array}$




\section{Southwest Research Institute - Department of Emissions Research EPA Hot Transient Emission Test Resuits \\ Project No. 03-7276-004}

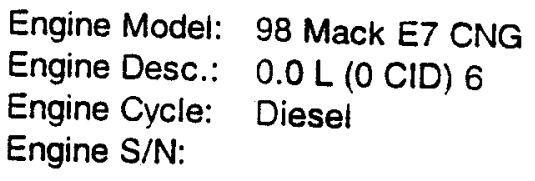

Formaldehyde

Concentration Mass

Acetaldehyde Concentration Mass

Acrolein

Concentration Mass

Acetone

Concentration Mass

Propionaldehyde

Concentration Mass

Crotonaldehyde

Concentration Mass

Isobutyr + MEK

Concentration Mass

Benzaldehyde Concentration Mass

Hexanaldehyde Concentration Mass
Test No: $612-\mathrm{H} 1$

Date: 06/12/1998 Time: 09:56

Program HDT: $4.03-\mathrm{C}$

Cell: 3 Bag Cart:
NAT GAS CNG, CARB-CNG

HCR: $\quad 3.838$ FID Resp: 1.00

$\mathrm{H}=0.230 \mathrm{C}=0.714 \mathrm{O}=0.000 \mathrm{X}=0.056$

\begin{tabular}{|c|c|c|c|}
\hline \multicolumn{2}{|c|}{ Sample } & \multicolumn{2}{|c|}{ Background } \\
\hline $\begin{array}{r}2.610 \\
3,785.62\end{array}$ & $\begin{array}{l}\mathrm{ppm} \\
\mathrm{mg}\end{array}$ & 0.007 & ppm \\
\hline $\begin{array}{r}0.095 \\
200.62\end{array}$ & $\begin{array}{l}\text { ppm } \\
\text { mg }\end{array}$ & 0.001 & $\mathrm{ppm}$ \\
\hline $\begin{array}{l}0.018 \\
49.97\end{array}$ & $\begin{array}{l}\text { ppm } \\
\text { mg }\end{array}$ & 0.000 & ppm \\
\hline $\begin{array}{l}0.018 \\
39.73\end{array}$ & $\begin{array}{l}\mathrm{ppm} \\
\mathrm{mg}\end{array}$ & 0.004 & $\mathrm{ppm}$ \\
\hline $\begin{array}{l}0.006 \\
16.67\end{array}$ & $\begin{array}{l}\mathrm{ppm} \\
\mathrm{mg}\end{array}$ & 0.000 & ppm \\
\hline $\begin{array}{l}0.006 \\
19.10\end{array}$ & $\begin{array}{l}\mathrm{ppm} \\
\mathrm{mg}\end{array}$ & 0.000 & ppm \\
\hline $\begin{array}{r}0.000 \\
0.00\end{array}$ & $\begin{array}{l}\mathrm{ppm} \\
\mathrm{mg}\end{array}$ & 0.000 & ppm \\
\hline $\begin{array}{r}0.000 \\
0.00\end{array}$ & $\begin{array}{l}\mathrm{ppm} \\
\mathrm{mg}\end{array}$ & 0.000 & ppm \\
\hline $\begin{array}{r}0.000 \\
0.00\end{array}$ & $\begin{array}{l}\mathrm{ppm} \\
\mathrm{mg}\end{array}$ & 0.000 & ppm \\
\hline
\end{tabular}

\section{Brake-Specific Results}

Formaldehyde

Acetaldehyde

Acrolein

Acetone

Propionaldehyde
$158.859 \mathrm{mg} / \mathrm{hp}-\mathrm{hr}$

$8.419 \mathrm{mg} / \mathrm{hp}-\mathrm{hr}$

$2.097 \mathrm{mg} / \mathrm{hp}-\mathrm{hr}$

$1.667 \mathrm{mg} / \mathrm{hp}-\mathrm{hr}$

$0.700 \mathrm{mg} / \mathrm{hp}-\mathrm{hr}$
Crotonaidehyde

Isobutyr + MEK

Benzaldehyde

Hexanaldehyde
$0.802 \mathrm{mg} / \mathrm{hp}-\mathrm{hr}$

$0.000 \mathrm{mg} / \mathrm{hp}-\mathrm{hr}$

$0.000 \mathrm{mg} / \mathrm{hp}-\mathrm{hr}$

$0.000 \mathrm{mg} / \mathrm{hp}-\mathrm{hr}$ 
Source:

Station \#:

Station Name:

Field:

Report Date: 06/05/1998 Sample Date: Flowing Pressure: 0

Flowing Temp.: $0 \quad \frac{0}{F}$

Method: $c: \backslash e z c h r o m \backslash m e t h o d s \backslash s c o t t$.

File: c:lezchrom \chrom $\backslash 037276 \mathrm{eg} .3$

Gas Analysis by Chromatograph

Name

NITROGEN

METHANE

$\mathrm{CO} 2$

ETHANE

$\mathrm{H} 2 \mathrm{~S}$

PROPANE

ISO-BUTANE

JUTANE

ISO-PENTANE

PENTANE

HEXANES

HEPTANES

OCTANES

I SONONANES

Mole \% BTU SG GPM

$\begin{array}{rrr}3.661 & 0.000 & 0.035 \\ 90.603 & 915.090 & 0.502 \\ 0.000 & 0.000 & 0.000 \\ 3.672 & 64.980 & 0.038 \\ 0.000 & 0.000 & 0.000 \\ 2.064 & 51.032 & 0.031 \\ 0.000 & 0.000 & 0.000 \\ 0.000 & 0.000 & 0.000 \\ 0.000 & 0.000 & 0.000 \\ 0.000 & 0.000 & 0.000 \\ 0.000 & 0.000 & 0.000 \\ 0.000 & 0.000 & 0.000 \\ 0.000 & 0.000 & 0.000 \\ 0.000 & 0.000 & 0.000\end{array}$

Ideal Total

100.000

$1032.002 \quad 0.607$

0.567

Gross BTU/Real Cu. Ft. (@60 deg $\mathrm{F}, 0.000$ )

Dry $=1034.286$

Sat. $=1017.456$

Actual $=1034.286$

0.000 1bs. Water MMCF)

Gasoline Content Propane GPM $=0.567$

Butane GPM $=0.000$

Gaboline GPM $=0.000$

26\# Gasoline GPM $=0.000$

Total GPM $=0.567$

Real Speciftc Gravity Calculated $=0.6079$

On-Site Specific Gravity $=0.0000$

Gas Compressablilty $=0.9978$

marks: 
Appendix B

Test Numbers 218-C1 and 218-H1

"1000" Hour Transient Emissions Test

Deterioration Factor Calibration

Garrett Turbocharger

No Engine Maintenance 


\section{TEST FACILITIES DESCRIPTION}

Emission certification testing was conducted at:

Southwest Research Institute

6220 Culebra Road

P.O. Drawer 28510

San Antonio, TX 78228-0510

Certification activities at Southwest Research Institute were in test cell 4. Cell 4 uses a General absorption ability of dyno in combination with a Mid-West inductor dyno for a combined power measurement. Air to $400 \mathrm{hp}$. A Lebow (rotary transformer) in line torque meter is used for torque conditioned for temperature and hung is simulated as required per SAE 1937. Engine inlet air is the engine and dynamometer and acquire A LabVIEW -based computer system is used to control code.

Gaseous emissions are measured dilute utilizing the full-flow dilution method and particulate filtered and temperature controlled.

The main dilution tunnel uses a positive dispiacement pump driven at a constant speed in combination with a heat exchanger. The particulate measurement system uses gas meters to measure the secondary dilution air supply and total sample volumes, and particulate is collected
on Pallflex $90 \mathrm{~mm}$ filters. All equipment and procedures used for testing and calculations are in accordance with 40 CFR Part
86 Subpart N. 


\section{Southwest Research Institute - Department of Emissions Research}

Composite Transient Emission Test Resuits

Project No. 03-7276-004

Engine Model: 98 Mack E7 CNG

Engine Desc.: $\quad 0.0 \mathrm{~L}(0 \mathrm{CID}) 6$

Engine Cycle: Diese!

Engine S/N:
Date: 02/18/1999 Time: 11:20

Program HDT: 4.04-C

Cell: 4 Bag Cart: 2
NAT GAS CNG, CARB-CNG

HCR: 3.865

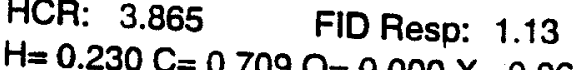

Engine Oil:
$O=0.000 X=0.061$

Test Numbers

Cold: 0218-C1 Hot: 0218-H1

Brake-Specific Emission Results

BSTHC (Cell)

CO

$3.863 \mathrm{~g} / \mathrm{hp}-\mathrm{hr}$

$1.490 \mathrm{~g} / \mathrm{hp}-\mathrm{hr}$

$1.763 \mathrm{~g} / \mathrm{hp}-\mathrm{hr}$

$0.029 \mathrm{~g} / \mathrm{hp}-\mathrm{hr}$

$482.6 \mathrm{~g} / \mathrm{hp}-\mathrm{hr}$

$0.421 \mathrm{lb} / \mathrm{hp}-\mathrm{hr}$

$0.451 \mathrm{~g} / \mathrm{hp}-\mathrm{hr}$

$5.180 \mathrm{~g} / \mathrm{kW}-\mathrm{hr}$

NOx (Cell)

$3.412 \mathrm{~g} / \mathrm{hp}-\mathrm{hr}$

$1.998 \mathrm{~g} / \mathrm{kW}-\mathrm{hr}$

Particulate

$\mathrm{CO} 2$

BSFC

NMHC

$\mathrm{CH}_{4}$

$24.92 \mathrm{hp}-\mathrm{hr}$

$24.41 \mathrm{hp}-\mathrm{hr}$

$2.365 \mathrm{~g} / \mathrm{kW}-\mathrm{hr}$

$0.039 \mathrm{~g} / \mathrm{kW}-\mathrm{hr}$

$647.17 \mathrm{~g} / \mathrm{kW}-\mathrm{hr}$

$0.564 \mathrm{~kg} / \mathrm{kW}-\mathrm{hr}$

$0.604 \mathrm{~g} / \mathrm{kW}-\mathrm{hr}$

Work:

Reference Work:

24.41 hphr




\section{Southwest Research Institute - Department of Emissions Research EPA Cold Transient Emission Test Resuits \\ Project No. 03-7276-004}

Engine Model: 98 Mack E7 CNG Engine Desc.: $\quad 0.0 \mathrm{~L}(0 \mathrm{CID}) 6$ Engine Cycle: Diesel

Engine S/N:
Test No.: 0218-C1

Date: 02/18/1999 Time: 11:20

Program HDT: $4.04-\mathrm{C}$

Cell: 4 Bag Cart: 2
NAT GAS CNG, CARB-CNG

HCR: 3.865 FID Resp: 1.13

$\mathrm{H}=0.230 \mathrm{C}=0.709 \mathrm{O}=0.000 \mathrm{X}=0.061$

\section{Ambient/Test Cell Conditions}

$\begin{array}{llll}\text { Barometer: } & 29.00 & \text { in } \mathrm{Hg} & 98.2 \mathrm{kPa} \\ \text { Engine Inlet Air } & & & \\ \text { Temperature: } & 73.0 & { }^{\circ} \mathrm{F} & 22.8^{\circ} \mathrm{C} \\ \text { Dew Point: } & 60.2 & { }^{\circ} \mathrm{F} & 15.7^{\circ} \mathrm{C} \\ \text { Abs. Humidity: } & 80.7 & \mathrm{gr} / \mathrm{lb} & 11.5 \mathrm{~g} / \mathrm{kg} \\ \quad \text { Rel. Humidity: } & 64 & \% & \\ \text { Dilution Air: } & & & \\ \text { Temperature: } & 74.0 & { }^{\circ} \mathrm{F} & 23.3^{\circ} \mathrm{C} \\ \text { Abs. Humidity } & 52.1 & \mathrm{gr} / \mathrm{lb} & 7.4 \mathrm{~g} / \mathrm{kg} \\ \text { Rel. Humidity: } & 40 & \% & \end{array}$

\begin{tabular}{lrrrl}
\multicolumn{5}{c}{ Measured Gaseous Data } \\
Heter & Range & Concentration \\
HC Sample & $\mathrm{n} / \mathrm{a}$ & & $146.31 \mathrm{ppm}$ \\
HC Bckgrd & $\mathrm{n} / \mathrm{a}$ & & $3.80 \mathrm{ppm}$ \\
CO Sample & 29.2 & 2 & $28.34 \mathrm{ppm}$ \\
CO Bckgrd & 0.4 & 2 & $0.39 \mathrm{ppm}$ \\
NOx Sample & $\mathrm{n} / \mathrm{a}$ & & $22.41 \mathrm{ppm}$ (Dry) \\
NOx Bckgrd & 0.1 & 2 & $0.10 \mathrm{ppm}$ \\
CO2 Sample & 62.1 & 1 & 0.5959 & $\%$ \\
CO2 Bckgrd & 5.6 & 1 & 0.0509 & $\%$ \\
CH4 Sample & $\mathrm{n} / \mathrm{a}$ & $\mathrm{n} / \mathrm{a}$ & $112.75 \mathrm{ppm}(1.13)$ \\
CH4 Bckgrd & $\mathrm{n} / \mathrm{a}$ & $\mathrm{n} / \mathrm{a}$ & $1.86 \mathrm{ppm}$ \\
& & & &
\end{tabular}

Particulate Data

1103.0-1 (pair)

Weight Gain, mg: $\quad 0.340$

Sample Multiplier: $\quad 2.416$

NOx Humidity CF:

Correction Factors

Dry-to-Wet CF $\quad 1.015$

0.977

Dilution Factor:

0.988

15.83

Corrected Concentrations

$\begin{array}{lrl}\text { HC } & \text { Corrected Concentrations } \\ \mathrm{CO} & 142.75 & \mathrm{ppm} \\ \mathrm{NOx} & 27.11 & \mathrm{ppm} \\ \mathrm{CO} 2 & 21.79 & \mathrm{ppm} \\ \mathrm{CH} 4 & 0.5482 & \% \\ \mathrm{RHC} & 111.01 & \mathrm{ppm} \\ & 17.31 & \mathrm{ppm}\end{array}$

HC

$\mathrm{CO}$

NOx

Particulate

$\mathrm{CO} 2$

$\mathrm{CH} 4$

RHC

Fuel

\section{Mass Emissions}

$\begin{array}{rl}104.362 & \text { grams } \\ 38.885 & \text { grams } \\ 52.106 & \text { grams } \\ 0.821 & \text { grams } \\ 12.355 & \mathrm{~kg} \\ 91.163 & \text { grams } \\ 13.199 & \text { grams } \\ \text { ib } & 4.89 \mathrm{~kg}\end{array}$

\begin{tabular}{|c|c|c|c|c|}
\hline \multicolumn{5}{|c|}{ Test Cycle Data } \\
\hline Sample Time: & $1,207.90$ & sec & & \\
\hline Work: & 24.86 & hp-hr & 18.54 & kW-hr \\
\hline Reference Work: & 24.41 & hp-hr & 18.20 & $k W-h r$ \\
\hline Total Volume (Vmix): & $43,497.1$ & sct & $1,231.86$ & $\mathrm{scm}$ \\
\hline
\end{tabular}

Brake-Specific Emission Results

$\begin{array}{llll}\text { BSTHC (Cell) } & 4.198 \mathrm{~g} / \mathrm{hp}-\mathrm{hr} & 5.630 \mathrm{~g} / \mathrm{kW}-\mathrm{hr} \\ \text { CO } & 1.564 \mathrm{~g} / \mathrm{hp}-\mathrm{hr} & 2.098 \mathrm{~g} / \mathrm{kW}-\mathrm{hr} \\ \text { NOx (Cell) } & 2.096 \mathrm{~g} / \mathrm{hp}-\mathrm{hr} & 2.811 \mathrm{~g} / \mathrm{kW}-\mathrm{hr} \\ \text { Particulate } & 0.033 \mathrm{~g} / \mathrm{hp}-\mathrm{hr} & 0.044 \mathrm{~g} / \mathrm{kW}-\mathrm{hr} \\ \text { CO2 } & 497.0 \mathrm{~g} / \mathrm{hp}-\mathrm{hr} & 666.44 \mathrm{~g} / \mathrm{kW}-\mathrm{hr} \\ \text { BSFC } & 0.434 \mathrm{lb} / \mathrm{hp}-\mathrm{hr} & 0.264 \mathrm{~kg} / \mathrm{kW}-\mathrm{hr} \\ \text { NMHC } & 0.531 \mathrm{~g} / \mathrm{hp}-\mathrm{hr} & 0.712 \mathrm{~g} / \mathrm{kW}-\mathrm{hr} \\ \text { CH4 } & 3.667 \mathrm{~g} / \mathrm{hp}-\mathrm{hr} & 4.918 \mathrm{~g} / \mathrm{kW}-\mathrm{hr}\end{array}$




\section{Southwest Research institute - Department of Emissions Research EPA Cold Transient Emission Test Results Project No. 03-7276-004}

Engine Model: 98 Mack E7 CNG Engine Desc.: $\quad 0.0 \mathrm{~L}(0 \mathrm{CID}) 6$ Engine Cycle: Diesel Engine S/N:

\section{Formaidehyde Concentration Mass \\ Acetaldehyde Concentration Mass \\ Acrolein Concentration Mass \\ Acetone \\ Concentration Mass \\ Propionaldehyde Concentration Mass \\ Crotonaldehyde Concentration Mass \\ Isobutyr + MEK \\ Concentration Mass \\ Benzaldehyde Concentration Mass \\ Hexanaldehyde Concentration Mass}

Test No.: 0218-C1

Date: 02/18/1999 Time: $11: 20$

Program HDT: $4.04-\mathrm{C}$

Cell: 4 Bag Cart: 2
NAT GAS CNG, CARB-CNG

HCR: 3.865 FID Resp: 1.13

$\mathrm{H}=0.230 \mathrm{C}=0.709 \mathrm{O}=0.000 \mathrm{X}=0.061$

Engine Oil:

\section{Sampie}$$
2.402 \mathrm{ppm}
$$$$
3,604.01 \mathrm{mg}
$$

$0.073 \mathrm{ppm}$

$158.98 \mathrm{mg}$

$0.014 \mathrm{ppm}$

$40.54 \mathrm{mg}$

$0.004 \mathrm{ppm}$

$6.35 \mathrm{mg}$

$0.015 \mathrm{ppm}$

$43.25 \mathrm{mg}$

$0.003 \mathrm{ppm}$

$10.62 \mathrm{mg}$

$0.001 \mathrm{ppm}$

$5.34 \mathrm{mg}$

$0.000 \mathrm{ppm}$

$1.79 \mathrm{mg}$

$0.000 \mathrm{ppm}$

$0.00 \mathrm{mg}$

\section{Background}

$0.003 \mathrm{ppm}$

$0.001 \mathrm{ppm}$

0.000 ppm

$0.002 \mathrm{ppm}$

$0.000 \mathrm{ppm}$

$0.000 \mathrm{ppm}$

$0.000 \mathrm{ppm}$

$0.000 \mathrm{ppm}$

$0.000 \mathrm{ppm}$

\section{Brake-Specific Results}

\section{Formaldehyde}

Acetaldehyde

Acrolein

Acetone

Propionaldehyde
$144.972 \mathrm{mg} / \mathrm{hp}-\mathrm{hr}$

$6.395 \mathrm{mg} / \mathrm{hp}-\mathrm{hr}$

$1.631 \mathrm{mg} / \mathrm{hp}-\mathrm{hr}$

$0.255 \mathrm{mg} / \mathrm{hp}-\mathrm{hr}$

$1.740 \mathrm{mg} / \mathrm{hp}-\mathrm{hr}$
Crotonaldehyde

Isobutyr + MEK

Benzaldehyde

Hexanaldehyde
$0.427 \mathrm{mg} / \mathrm{hp}-\mathrm{hr}$

$0.215 \mathrm{mg} / \mathrm{hp}-\mathrm{hr}$

$0.072 \mathrm{mg} / \mathrm{hp}-\mathrm{hr}$

$0.000 \mathrm{mg} / \mathrm{hp}-\mathrm{hr}$ 


\section{Southwest Research Institute - Department of Emissions Research \\ EPA Hot Transient Emission Test Results \\ Project No. 03-7276-004}

Engine Model: 98 Mack E7 CNG

Engine Desc.: $0.0 \mathrm{~L}$ (0 CID) 6

Engine Cycle: Diesel

Engine S/N:
Test No.: 0218-H1

Date: 02/18/1999 Time: 12:00

Program HDT: $4.04-\mathrm{C}$

Cell: 4 Bag Cart: 2
NAT GAS CNG, CARB-CNG

HCR: 3.865 FID Resp: 1.13

$H=0.230 \mathrm{C}=0.709 \mathrm{O}=0.000 \mathrm{X}=0.061$

\section{Ambient/Test Cell Conditions}

Barometer:

Engine Inlet Air

$$
28.99 \text { in } \mathrm{Hg} 98.2 \mathrm{kPa}
$$

Temperature:

Dew Point:

Abs. Humidity:

Rel. Humidity:

Dilution Air:

Temperature:

Abs. Humidity

Rel. Humidity:

$75.0 \quad{ }^{\circ} \mathrm{F} \quad 23.9^{\circ} \mathrm{C}$

$58.8 \quad{ }^{\circ} \mathrm{F} \quad 14.9^{\circ} \mathrm{C}$

$76.7 \quad \mathrm{gr} / \mathrm{lb} \quad 11.0 \mathrm{~g} / \mathrm{kg}$

$57 \%$

$74.0 \quad{ }^{\circ} \mathrm{F} \quad 23.3^{\circ} \mathrm{C}$

$56.6 \quad \mathrm{gr} / \mathrm{bb} \quad 8.1 \mathrm{~g} / \mathrm{kg}$

$44 \%$

\section{Measured Gaseous Data}

HC Sample

HC Bckgrd

CO Sample

CO Bckgrd

NOx Sample

NOx Bckgrd

$\mathrm{CO} 2$ Sample

CO2 Bckgrd

$\mathrm{CH} 4$ Bckgrd

Meter Range Concentration
$\mathrm{CH} 4$ Sample

$\begin{array}{rrrl}\mathrm{n} / \mathrm{a} & & 134.10 & \mathrm{ppm} \\ \mathrm{n} / \mathrm{a} & & 4.50 & \mathrm{ppm} \\ 27.5 & 2 & 26.68 & \mathrm{ppm} \\ 0.2 & 2 & 0.19 & \mathrm{ppm} \\ \mathrm{n} / \mathrm{a} & & 18.53 & \mathrm{ppm} \text { (Dry) } \\ 0.1 & 2 & 0.10 & \mathrm{ppm} \\ 60.0 & 1 & 0.5747 & \% \\ 5.1 & 1 & 0.0464 & \% \\ \mathrm{n} / \mathrm{a} & \mathrm{n} / \mathrm{a} & 103.95 & \mathrm{ppm}(1.13) \\ \mathrm{n} / \mathrm{a} & \mathrm{n} / \mathrm{a} & 1.78 \mathrm{ppm}\end{array}$

\section{Corrected Concentrations}

$\mathrm{HC}$

NO

$\mathrm{CO} 2$

$\mathrm{CH} 4$

$\mathrm{RHC}$

$\mathrm{HC}$

$\mathrm{CO}$

NOx

Particulate

$\mathrm{CO} 2$

$\mathrm{CH} 4$

$\mathrm{RHC}$

Fuel

$\begin{aligned} 129.87 & \text { ppm } \\ 25.68 & \text { ppm } \\ 17.99 & \text { ppm } \\ 0.5311 & \% \\ 102.28 & \text { ppm } \\ 14.29 & \text { ppm }\end{aligned}$

Mass Emissions

C

94.914

36.830

grams

0.707 grams

$11.972 \mathrm{~kg}$

84.013 grams

10.902 grams

$10.43 \mathrm{lb} \quad 4.73 \mathrm{~kg}$

\begin{tabular}{lrr} 
& \multicolumn{3}{c}{ Sample Flows } \\
sctm & \multicolumn{1}{c}{ scmm } \\
Blower 1 Rate: & $2,160.1$ & 61.18 \\
Blower 2 Rate: & 0.0 & 0.00 \\
90 mm System: & & \\
Gas Meter 1: & 1.48 & 0.04 \\
Gas Meter 2: & 2.38 & 0.07 \\
Sample Rate: & 0.90 & 0.03 \\
Chemistry Sample Rate: & 2.47 & 0.07 \\
Total Flow Rate: & $2,163.51$ & 61.27
\end{tabular}

Filter Number: Particulate Data

Weight Gain, mg: $119.0-2$ (pair)

Sample Multiplier: $\quad 2.397$

NOx Humidity CF:

Dry-to-Wet CF, Sample:

1.004

0.976

$\begin{array}{ll}\text { Dry-to-Wet CF, Bckgrd: } & 0.987 \\ \text { Dilution Factor: } & 16.43\end{array}$

0.04

0.03

61.27

\section{Test Cycle Data \\ $1,207.90 \mathrm{sec}$ \\ Work: \\ Reference Work: \\ $24.93 \mathrm{hp}-\mathrm{hr}$ \\ $\begin{array}{lrr}\text { Reference Work: } & 24.41 \mathrm{hp}-\mathrm{hr} & 18.20 \mathrm{~kW} \text {-hr } \\ \text { Total Volume (Vmix): } & 43,505.2 \mathrm{scf} & 1,232.09 \mathrm{scm}\end{array}$ \\ $18.59 \mathrm{~kW}-\mathrm{hr}$ \\ $1,232.09 \mathrm{scm}$}

\section{Brake-Specific Emission Results}

$\begin{array}{lll}\text { BSTHC (Cell) } & 3.807 \mathrm{~g} / \mathrm{hp}-\mathrm{hr} & 5.106 \mathrm{~g} / \mathrm{kW}-\mathrm{hr} \\ \text { CO } & 1.477 \mathrm{~g} / \mathrm{hp}-\mathrm{hr} & 1.981 \mathrm{~g} / \mathrm{kW}-\mathrm{hr} \\ \text { NOx (Cell) } & 1.708 \mathrm{~g} / \mathrm{hp}-\mathrm{hr} & 2.290 \mathrm{~g} / \mathrm{kW}-\mathrm{hr} \\ \text { Particulate } & 0.028 \mathrm{~g} / \mathrm{hp}-\mathrm{hr} & 0.038 \mathrm{~g} / \mathrm{kW}-\mathrm{hr} \\ \text { CO2 } & 480.2 \mathrm{~g} / \mathrm{hp}-\mathrm{hr} & 643.97 \mathrm{~g} / \mathrm{kW}-\mathrm{hr} \\ \text { BSFC } & 0.419 \mathrm{lb} / \mathrm{hp}-\mathrm{hr} & 0.255 \mathrm{~kg} / \mathrm{kW}-\mathrm{hr} \\ \text { NMHC } & 0.437 \mathrm{~g} / \mathrm{hp}-\mathrm{hr} & 0.586 \mathrm{~g} / \mathrm{kW}-\mathrm{hr} \\ \text { CH4 } & 3.370 \mathrm{~g} / \mathrm{hp}-\mathrm{hr} & 4.519 \mathrm{~g} / \mathrm{kW}-\mathrm{hr}\end{array}$




\section{Southwest Research Institute - Department of Emissions Research \\ EPA Hot Transient Emission Test Resuits \\ Project No. 03-7276-004}

Engine Model: 98 Mack E7 CNG Engine Desc.: $0.0 \mathrm{~L}(0 \mathrm{CID}) 6$ Engine Cycle: Diesel

Engine $S / N$ :

Formaldehyde
Concentration
Mass
Acetaldehyde
Concentration
Mass
Acrolein
Concentration
Mass
Acetone
Concentration
Mass
Propionaldehyde
Concentration
Mass
Crotonaidehyde
Concentration
Mass
Isobutyr + MEK
Concentration
Mass
Benzaddehyde
Concentration
Mass
Hexanaldehyde
Concentration
Mass

Test No.: 0218-H1

Date: 02/18/1999 Time: 12:00

Program HDT: $4.04-\mathrm{C}$

Cell: 4 Bag Cart: 2
NAT GAS CNG, CARB-CNG HCR: $\quad 3.865$ FID Resp: 1.13 $\mathrm{H}=0.230 \mathrm{C}=0.709 \mathrm{O}=0.000 \mathrm{X}=0.061$ Engine Oil:

\begin{tabular}{|c|c|c|c|}
\hline \multicolumn{2}{|c|}{ Sample } & \multicolumn{2}{|c|}{ Background } \\
\hline $\begin{array}{r}2.171 \\
, 256.01\end{array}$ & $\begin{array}{l}\text { ppm } \\
\text { mg }\end{array}$ & 0.003 & ppm \\
\hline $\begin{array}{r}0.065 \\
140.76\end{array}$ & $\begin{array}{l}\mathrm{ppm} \\
\mathrm{mg}\end{array}$ & 0.001 & ppm \\
\hline $\begin{array}{l}0.013 \\
35.29\end{array}$ & $\begin{array}{l}\text { ppm } \\
\text { mg }\end{array}$ & 0.000 & ppm \\
\hline $\begin{array}{r}0.001 \\
0.00\end{array}$ & $\begin{array}{l}\text { ppm } \\
\mathrm{mg}\end{array}$ & 0.002 & ppm \\
\hline $\begin{array}{l}0.014 \\
40.67\end{array}$ & $\begin{array}{l}\mathrm{ppm} \\
\mathrm{mg}\end{array}$ & 0.000 & ppm \\
\hline $\begin{array}{r}0.003 \\
9.11\end{array}$ & $\begin{array}{l}\mathrm{ppm} \\
\mathrm{mg}\end{array}$ & 0.000 & ppm \\
\hline $\begin{array}{r}0.001 \\
2.20\end{array}$ & $\begin{array}{l}\mathrm{ppm} \\
\mathrm{mg}\end{array}$ & 0.000 & ppm \\
\hline $\begin{array}{r}0.000 \\
2.15\end{array}$ & $\begin{array}{l}\mathrm{ppm} \\
\mathrm{mg}\end{array}$ & 0.000 & ppm \\
\hline $\begin{array}{r}0.000 \\
0.00\end{array}$ & $\begin{array}{l}\mathrm{ppm} \\
\mathrm{mg}\end{array}$ & 0.000 & ppr \\
\hline
\end{tabular}

\section{Brake-Specific Results}

\section{Formaldehyde}

Acetaldehyde

Acrolein

Acetone

Propionaldehyde

$$
\begin{array}{rl}
130.606 & \mathrm{mg} / \mathrm{hp}-\mathrm{hr} \\
5.646 & \mathrm{mg} / \mathrm{hp}-\mathrm{hr} \\
1.416 \mathrm{mg} / \mathrm{hp}-\mathrm{hr} \\
0.000 \mathrm{mg} / \mathrm{hp}-\mathrm{hr} \\
1.631 \mathrm{mg} / \mathrm{hp}-\mathrm{hr}
\end{array}
$$

Crotonaldehyde

Isobutyr + MEK

Benzaldehyde

Hexanaldehyde
$0.365 \mathrm{mg} / \mathrm{hp}-\mathrm{hr}$

$0.088 \mathrm{mg} / \mathrm{hp}-\mathrm{hr}$

$0.086 \mathrm{mg} / \mathrm{hp}-\mathrm{hr}$

$0.000 \mathrm{mg} / \mathrm{hp}-\mathrm{hr}$ 
Source:

Station \#: Station Name:

Field:
Report Date: 02/08/1999 Sample Date: Flowing Pressure: 0 Flowing Temp.: 0 psig

Method: c: \ezchrom \methods\scott:

File: c: lezchrom \chrom $\backslash 032377$ jbo. 3

Name

NITROGEN

METHANE

$\mathrm{CO} 2$

ETHANE

$\mathrm{H} 2 \mathrm{~S}$

PROPANE

ISO-BUTANE

TANE

J-PENTANE

PENTANE

HEXANES

HEPTANES

OCTANES

ISONONANES

Ges Analysis by Chromatograph

Mole \%

3.929

90.470

0.000

3.650

0.000

1.951

0.000

0.000

0.000

0.000

0.000

0.000

0.000

0.000
BTU

SG

$\begin{array}{rr}0.000 & 0.038 \\ 913.747 & 0.501 \\ 0.000 & 0.000\end{array}$

64.590

0.000

49.089

0.000

0.000

0.000

0.000

0.000

0.000

0.000

0.000

0.038

0.000

0.030

0.000

0.000

0.000

0.000

0.000

0.000

0.000

0.000

0.536

Ideal Total

100.000

$1027.427 \quad 0.607$

0.536

GFM

Gross BTU/Real Cu. Et.

(@60 deg F, 0.000 )

Dry $=1029.683$

Sat. $=1012.930$

Actua.1 $=1029.683$

0.000 1bs. water, MMCF)

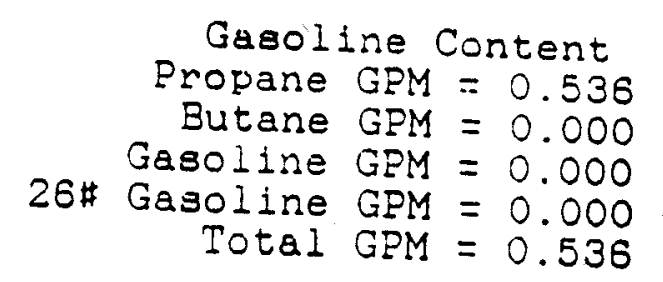

Real Specific Gravity Calculated $=0.6078$

On-Site Specifte Gravity $=0.0000$

Gas Compressablitty $=0.9978$

narks: 
Appendix C

Test Numbers 219-C1 and 219-H1

"1000" Hour Transient Emissions Test 325 BHP Calibration

Maximum Turbo Lag Enrichment $=8 \%$

Garrett Turbocharger

No Engine Maintenance 


\section{TEST FACILITIES DESCRIPTION}

Emission certification testing was conducted at:

Southwest Research Institute 6220 Culebra Road

P.O. Drawer 28510

San Antonio, TK 78228-0510

Certification activities at Southwest Research Institute were in test cell 4. Cell 4 uses a General absorption ability of $400 \mathrm{hp}$. A Lebow (rotary a Mid-West inductor dyno for a combined power measurement. Air to air intercooling is simulated tormer) in line torque meter is used for torque conditioned for temperature and humidity. A LabVIEW required per SAE 1937. Engine inlet air is the engine and dynamometer and acquire data. code.

Gaseous emissions are measured dilute utilizing the full-flow dilution method and particulate filtered and temperature controlled. measure the secondary dilution air supply and totate measurement system uses gas meters to on Pallflex $90 \mathrm{~mm}$ filters.

All equipment and procedures used for testing and calculations are in accordance with 40 CFR Part
86 Subpart N. 


\section{Southwest Research Institute - Department of Emissions Research Composite Transient Emission Test Results \\ Project No. 03-7276-004}

Engine Model: 98 Mack E7 CNG Engine Desc.: $\quad 0.0 \mathrm{~L}(0 \mathrm{CID}) 6$ Engine Cycle: Diesel Engine S/N:
Date: 02/19/1999 Time: 09:15 Program HDT: $4.04-\mathrm{C}$

Cell: 4 Bag Cart: 2
NAT GAS CNG, CARB-CNG

HCR: 3.865 FID Resp: 1.13

$H=0.230 \mathrm{C}=0.709 \mathrm{O}=0.000 \mathrm{X}=0.061$

Engine Oil:

Test Numbers

Cold: 0219-C1 Hot: 0219-H1

\section{Brake-Specific Emission Resuits}

$\begin{array}{lrlrl}\text { BSTHC (Cell) } & 3.235 & \mathrm{~g} / \mathrm{hp}-\mathrm{hr} & 4.339 & \mathrm{~g} / \mathrm{kW}-\mathrm{hr} \\ \text { CO } & 1.385 & \mathrm{~g} / \mathrm{hp}-\mathrm{hr} & 1.858 \mathrm{~g} / \mathrm{kW}-\mathrm{hr} \\ \text { NOx (Cell) } & 2.150 & \mathrm{~g} / \mathrm{hp}-\mathrm{hr} & 2.883 & \mathrm{~g} / \mathrm{kW}-\mathrm{hr} \\ \text { Particulate } & 0.027 & \mathrm{~g} / \mathrm{hp}-\mathrm{hr} & 0.036 & \mathrm{~g} / \mathrm{kW}-\mathrm{hr} \\ \text { CO2 } & 456.9 & \mathrm{~g} / \mathrm{hp}-\mathrm{hr} & 612.69 & \mathrm{~g} / \mathrm{kW}-\mathrm{hr} \\ \text { BSFC } & 0.397 & \mathrm{lb} / \mathrm{hp}-\mathrm{hr} & 0.533 & \mathrm{~kg} / \mathrm{kW}-\mathrm{hr} \\ \text { NMHC } & 0.389 & \mathrm{~g} / \mathrm{hp}-\mathrm{hr} & 0.521 & \mathrm{~g} / \mathrm{kW}-\mathrm{hr} \\ \text { CH4 } & 2.847 & \mathrm{~g} / \mathrm{hp}-\mathrm{hr} & 3.817 \mathrm{~g} / \mathrm{kW}-\mathrm{hr}\end{array}$

Work:

Reference Work:

$25.17 \mathrm{hp}-\mathrm{hr}$

$24.59 \mathrm{hp}-\mathrm{hr}$

$18.77 \mathrm{~kW}$-hr

$18.34 \mathrm{~kW}-\mathrm{hr}$ 


\section{Southwest Research Institute - Department of Emissions Research \\ EPA Cold Transient Emission Test Results \\ Project No. 03-7276-004}

Engine Model: 98 Mack E7 CNG

Engine Desc.: $\quad 0.0 \mathrm{~L}$ (0 CID) 6

Engine Cycle: Diesel

Engine S/N:
Test No.: 0219-C1

Date: 02/19/1999 Time:09:15

Program HDT: $4.04-\mathrm{C}$

Cell: 4 Bag Cart: 2
NAT GAS CNG, CARB-CNG

HCR: 3.865 FID Resp: 1.13

$\mathrm{H}=0.230 \mathrm{C}=0.709 \mathrm{O}=0.000 \mathrm{X}=0.061$

Engine Oil:

\section{Ambient/Test Cell Conditions}

Barometer:

Engine Inlet Air

Temperature:

Dew Point:

Abs. Humidity:

Rel. Humidity:

Dilution Air:

Temperature:

Abs. Humidity

Rel. Humidity:
29.17 in $\mathrm{Hg} 98.8 \mathrm{kPa}$

$\begin{array}{lcc}76.0 & { }^{\circ} \mathrm{F} & 24.4^{\circ} \mathrm{C} \\ 58.2 & { }^{\circ} \mathrm{F} & 14.6{ }^{\circ} \mathrm{C} \\ 74.5 & \mathrm{gr} / \mathrm{lb} & 10.6 \mathrm{~g} / \mathrm{kg} \\ 54 & \% & \end{array}$

73.0 ${ }^{\circ} \mathrm{F} \quad 22.8^{\circ} \mathrm{C}$

$24.7 \quad \mathrm{gr} / \mathrm{bb} \quad 3.5 \mathrm{~g} / \mathrm{kg}$ $20 \%$

Measured Gaseous Data

HC Sample

HC Bckgrd

CO Sample

CO Bckgrd

NOx Sample

NOx Bckgrd

CO2 Sample

$\mathrm{CO} 2$ Bckgrd

$\mathrm{CH} 4$ Sample

$\mathrm{CH} 4$ Bckgrd

Meter Range Concentration

$\begin{array}{rrrl}\mathrm{n} / \mathrm{a} & & 121.08 & \mathrm{ppm} \\ \mathrm{n} / \mathrm{a} & & 3.80 & \mathrm{ppm} \\ 27.8 & 2 & 26.97 & \mathrm{ppm} \\ 0.3 & 2 & 0.29 & \mathrm{ppm} \\ \mathrm{n} / \mathrm{a} & & 26.96 & \mathrm{ppm} \text { (Dry) } \\ 0.3 & 2 & 0.30 & \mathrm{ppm} \\ 59.8 & 1 & 0.5727 & \% \\ 4.9 & 1 & 0.0445 & \% \\ \mathrm{n} / \mathrm{a} & \mathrm{n} / \mathrm{a} & 94.82 & \mathrm{ppm}(1.13) \\ \mathrm{n} / \mathrm{a} & \mathrm{n} / \mathrm{a} & 1.89 & \mathrm{ppm}\end{array}$

Corrected Concentrations

$\begin{array}{lrl}\text { HC } & 117.51 & \text { ppm } \\ \mathrm{CO} & 26.07 & \mathrm{ppm} \\ \mathrm{NOx} & 26.23 & \mathrm{ppm} \\ \mathrm{CO} 2 & 0.5309 & \% \\ \mathrm{CH} 4 & 93.04 & \mathrm{ppm} \\ \mathrm{RHC} & 12.37 & \mathrm{ppm}\end{array}$

$\mathrm{HC}$

Mass Emissions

$\mathrm{CO}$

NOx

Particulate

$\mathrm{CO} 2$

$\mathrm{CH} 4$

$\mathrm{RHC}$

Fuel

\begin{tabular}{rl}
86.504 & grams \\
37.678 & . grams \\
62.191 & grams \\
0.633 & grams \\
12.056 & $\mathrm{~kg}$ \\
76.999 & grams \\
9.505 & grams \\
\hline & $4.76 \mathrm{~kg}$
\end{tabular}

Sample Flows

Blower 1 Rate:

Blower 2 Rate:

$90 \mathrm{~mm}$ System:

Gas Meter 1:

Gas Meter 2:

Sample Rate:

Chemistry Sampie Rate:

Total Flow Rate:

scfm

$2,177.3$

0.0

61.66

0.00

1.46

2.35

0.04

0.07

0.89

0.03

2.46

$2,180.66$

0.07

61.76
Particulate Data

Weight Gain, mg: $120.0-3$ (pair)

Sample Multiplier: $\quad 2.444$

NOx Humidity CF:

Dry-to-Wet CF. Sample:

Dry-to-Wet CF, Bckgrd:

Dilution Factor:

\section{Correction Factors}

\begin{tabular}{lrlrl}
\multicolumn{5}{c}{ Test Cycle Data } \\
Sample Time: & $1,207.40$ & $\mathrm{sec}$ & & \\
Work: & 25.17 & $\mathrm{hp}-\mathrm{hr}$ & $18.77 \mathrm{~kW}-\mathrm{hr}$ \\
Reference Work: & 24.59 & $\mathrm{hp}-\mathrm{hr}$ & $18.34 \mathrm{~kW}-\mathrm{hr}$ \\
Total Volume $\left(V_{\text {mix }}\right):$ & $43,832.5 \mathrm{scf}$ & $1,241.36 \mathrm{scm}$
\end{tabular}

0.999

0.983

0.994

16.52

\section{Brake-Specific Emission Results}

$\begin{array}{llrl}\text { BSTHC (Cell) } & 3.437 \mathrm{~g} / \mathrm{hp}-\mathrm{hr} & 4.609 \mathrm{~g} / \mathrm{kW}-\mathrm{hr} \\ \text { CO } & 1.497 \mathrm{~g} / \mathrm{hp}-\mathrm{hr} & 2.007 \mathrm{~g} / \mathrm{kW}-\mathrm{hr} \\ \text { NOx (Cell) } & 2.471 \mathrm{~g} / \mathrm{hp}-\mathrm{hr} & 3.313 \mathrm{~g} / \mathrm{kW}-\mathrm{hr} \\ \text { Particulate } & 0.025 \mathrm{~g} / \mathrm{hp}-\mathrm{hr} & 0.034 \mathrm{~g} / \mathrm{kW}-\mathrm{hr} \\ \text { CO2 } & 479.0 \mathrm{~g} / \mathrm{hp}-\mathrm{hr} & 642.35 \mathrm{~g} / \mathrm{kW}-\mathrm{hr} \\ \text { BSFC } & 0.417 \mathrm{lb} / \mathrm{hp}-\mathrm{hr} & 0.253 \mathrm{~kg} / \mathrm{kW}-\mathrm{hr} \\ \text { NMHC } & 0.378 \mathrm{~g} / \mathrm{hp}-\mathrm{hr} & 0.506 \mathrm{~g} / \mathrm{kW}-\mathrm{hr} \\ \text { CH4 } & 3.059 \mathrm{~g} / \mathrm{hp}-\mathrm{hr} & 4.102 \mathrm{~g} / \mathrm{kW}-\mathrm{hr}\end{array}$




\section{Southwest Research Institute - Department of Emissions Research \\ EPA Cold Transient Emission Test Results \\ Project No. 03-7276-004}

Engine Model: 98 Mack E7 CNG Engine Desc.: $0.0 \mathrm{~L}$ (0 CID) 6 Engine Cycle: Diesel

Engine $\mathrm{S} / \mathrm{N}$ :

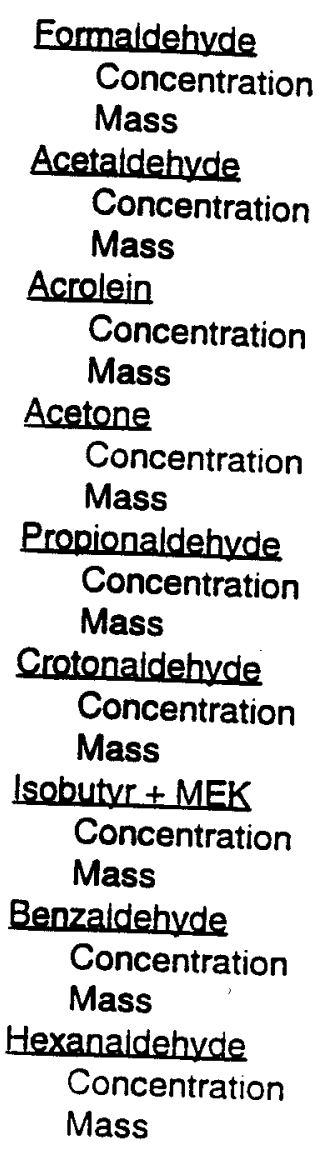

Test No.: 0219-C1

Date: 02/19/1999 Time: 09:15

Program HDT: $4.04-\mathrm{C}$

Cell: 4 Bag Cart: 2
NAT GAS CNG, CARB-CNG

HCR: 3.865 FID Resp: 1.13

$H=0.230 \mathrm{C}=0.709 \mathrm{O}=0.000 \mathrm{X}=0.061$

$\begin{array}{rl}\text { Sample } & \text { Background } \\ 2.004 \mathrm{ppm} & 0.002 \mathrm{ppm} \\ 3.051 .93 \mathrm{mg} & \\ & \\ 0.062 \mathrm{ppm} & 0.002 \mathrm{ppm} \\ 135.07 \mathrm{mg} & \\ 0.010 \mathrm{ppm} & 0.000 \mathrm{ppm} \\ 27.11 \mathrm{mg} & \\ 0.004 \mathrm{ppm} & 0.002 \mathrm{ppm} \\ 6.04 \mathrm{mg} & \\ 0.013 \mathrm{ppm} & 0.001 \mathrm{ppm} \\ 35.29 \mathrm{mg} & \\ 0.005 \mathrm{ppm} & 0.001 \mathrm{ppm} \\ 12.68 \mathrm{mg} & \\ 0.002 \mathrm{ppm} & 0.000 \mathrm{ppm} \\ 5.64 \mathrm{mg} & \\ 0.000 \mathrm{ppm} & 0.000 \mathrm{ppm} \\ 0.00 \mathrm{mg} & \\ 0.000 \mathrm{ppm} & 0.000 \mathrm{ppm} \\ 0.00 \mathrm{mg} & \\ \end{array}$

\section{Brake-Specific Results}

Formaidehyde

Acetaldehyde

Acrolein

Acetone

Propionaldehyde

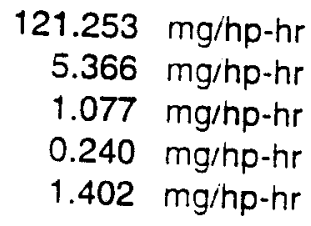

Crotonaldehyde

Isobutyr + MEK

Benzaldehyde

Hexanaidehyde $\begin{array}{ll}0.504 & \mathrm{mg} / \mathrm{hp}-\mathrm{hr} \\ 0.224 & \mathrm{mg} / \mathrm{hp}-\mathrm{hr} \\ 0.000 & \mathrm{mg} / \mathrm{hp}-\mathrm{hr} \\ 0.000 & \mathrm{mg} / \mathrm{hp}-\mathrm{hr}\end{array}$ 


\section{Southwest Research Institute - Department of Emissions Research EPA Hot Transient Emission Test Results \\ Project No. 03-7276-004}

Engine Model: 98 Mack E7 CNG Engine Desc.: $\quad 0.0 \mathrm{~L}$ (0 CID) 6 Engine Cycle: Diesel Engine $\mathrm{S} / \mathrm{N}$ :
Test No.: 0219-H1

Date: 02/19/1999 Time:09:55

Program HDT: $\quad 4.04-\mathrm{C}$

Cell: 4 Bag Cart: 2
NAT GAS CNG, CARB-CNG

HCR: 3.865 FID Resp: 1.13

$H=0.230 \mathrm{C}=0.709 \mathrm{O}=0.000 \mathrm{X}=0.061$
Ambient/Test Cell Conditions

Barometer: Engine Inlet Air

Temperature:

Dew Point:

Abs. Humidity:

Rel. Humidity:

Dilution Air:

Temperature:

Abs. Humidity

Rel. Humidity:

$$
29.17 \text { in } \mathrm{Hg} 98.8 \mathrm{kPa}
$$

$\begin{array}{lcl}78.0 & { }^{\circ} \mathrm{F} & 25.6{ }^{\circ} \mathrm{C} \\ 58.2 & { }^{\circ} \mathrm{F} & 14.6{ }^{\circ} \mathrm{C} \\ 74.5 & \mathrm{gr} / \mathrm{lb} & 10.6 \mathrm{~g} / \mathrm{kg} \\ 51 & \% & \end{array}$

\section{Measured Gaseous Data}

HC Sample

HC Bckgrd

CO Sample

CO Bckgrd

NOx Sample

Meter Range Concentration

$\begin{array}{lcc}73.0 & { }^{\circ} \mathrm{F} & 22.8^{\circ} \mathrm{C} \\ 24.7 & \mathrm{gr} / \mathrm{b} & 3.5 \mathrm{~g} / \mathrm{kg} \\ 20 & \% & \end{array}$

NOx Bckgrd

CO2 Sample

$\mathrm{CO} 2 \mathrm{Bckgrd}$

$\mathrm{CH} 4$ Sample

$\mathrm{CH} 4$ Bckgrd

$\begin{array}{rr}n / a & \\ n / a & \\ 25.2 & 2 \\ 0.1 & 2 \\ n / a & \\ 0.1 & 2 \\ 56.7 & 1 \\ 4.6 & 1 \\ n / a & n / a \\ n / a & n / a\end{array}$

$112.69 \mathrm{ppm}$

$3.50 \mathrm{ppm}$

$24.44 \mathrm{ppm}$

$0.10 \mathrm{ppm}$

22.71 ppm (Dry)

$0.10 \mathrm{ppm}$

$0.5416 \%$

$0.0418 \%$

87.20 ppm (1.13)

$1.83 \mathrm{ppm}$

\section{Corrected Concentrations}

$\begin{array}{lrl}\mathrm{HC} & 109.39 & \mathrm{ppm} \\ \mathrm{CO} & 23.80 & \mathrm{ppm} \\ \mathrm{NOx} & 22.25 & \mathrm{ppm} \\ \mathrm{CO} 2 & 0.5022 & \% \\ \mathrm{CH} 4 & 85.48 & \mathrm{ppm} \\ \mathrm{RHC} & 12.79 & \mathrm{ppm}\end{array}$

$\mathrm{HC}$

Mass Emissions

CO

NOx

Particulate

$\mathrm{CO} 2$

$\mathrm{CH} 4$

$\mathrm{RHC}$

Fuel

$\begin{array}{rr}80.589 & \text { grams } \\ 34.402 & \text {. grams } \\ 52.770 & \text { grams } \\ 0.691 & \text { grams } \\ 11.407 & \mathrm{~kg} \\ 70.757 & \text { grams } \\ 9.832 & \text { grams } \\ & 4.50 \mathrm{~kg}\end{array}$

\section{Sample Flows}

scfm

Blower 1 Rate:

Blower 2 Rate:

$90 \mathrm{~mm}$ System:

Gas Meter 1:

Gas Meter 2:

Sample Rate:

$2,177.2$

0.0

61.66

0.00

1.47

0.04

2.38

0.90

0.07

0.03

Total Flow Rate:

$2,180.63$

0.07

61.76
Particulate Dato

Filter Number: $\quad 1121.0-4$ (pair)

Weight Gain, mg: $\quad 0.287$

Sample Multiplier: $\quad 2.408$

NOx Humidity CF:

Dry-to-Wet CF, Sample:

Dry-to-Wet CF, Bckgrd:

Dilution Factor:

0.994

17.48

\begin{tabular}{lrrr}
\multicolumn{5}{c}{ Test Cycle Data } & \\
Sample Time: & $1,207.70$ & $\mathrm{sec}$ & \\
Work: & $25.17 \mathrm{hp}-\mathrm{hr}$ & $18.77 \mathrm{~kW}-\mathrm{hr}$ \\
Reference Work: & $24.59 \mathrm{hp}-\mathrm{hr}$ & $18.34 \mathrm{~kW}-\mathrm{hr}$ \\
Total Volume (Vmix): & $43,841.3 \mathrm{scf}$ & $1,241.61 \mathrm{scm}$
\end{tabular}

\section{Brake-Specific Emission Results}

$\begin{array}{llll}\text { BSTHC (Cell) } & 3.202 \mathrm{~g} / \mathrm{hp}-\mathrm{hr} & 4.294 \mathrm{~g} / \mathrm{kW}-\mathrm{hr} \\ \text { CO } & 1.367 \mathrm{~g} / \mathrm{hp}-\mathrm{hr} & 1.833 \mathrm{~g} / \mathrm{kW}-\mathrm{hr} \\ \text { NOx (Cell) } & 2.097 \mathrm{~g} / \mathrm{hp}-\mathrm{hr} & 2.811 \mathrm{~g} / \mathrm{kW}-\mathrm{hr} \\ \text { Particulate } & 0.027 \mathrm{~g} / \mathrm{hp}-\mathrm{hr} & 0.037 \mathrm{~g} / \mathrm{kW}-\mathrm{hr} \\ \text { CO2 } & 453.2 \mathrm{~g} / \mathrm{hp}-\mathrm{hr} & 607.74 \mathrm{~g} / \mathrm{kW}-\mathrm{hr} \\ \text { BSFC } & 0.394 \mathrm{lb} / \mathrm{hp}-\mathrm{hr} & 0.240 \mathrm{~kg} / \mathrm{kW}-\mathrm{hr} \\ \text { NMHC } & 0.391 \mathrm{~g} / \mathrm{hp}-\mathrm{hr} & 0.524 \mathrm{~g} / \mathrm{kW}-\mathrm{hr} \\ \text { CH4 } & 2.811 \mathrm{~g} / \mathrm{hp}-\mathrm{hr} & 3.770 \mathrm{~g} / \mathrm{kW}-\mathrm{hr}\end{array}$




\section{Southwest Research Institute - Department of Emissions Research \\ EPA Hot Transient Emission Test Resuits}

Engine Model: 98 Mack E7 CNG Engine Desc.: $\quad 0.0 \mathrm{~L}$ (0 CID) 6 Engine Cycle: Diesel Engine S/N:
Test No.: 0219-H1

Date: 02/19/1999 Time:09:55

Program HDT: $\quad 4.04-\mathrm{C}$

Cell: 4 Bag Cart: 2
NAT GAS CNG, CARB-CNG

HCR: 3.865 FID Resp: 1.13

$\mathrm{H}=0.230 \mathrm{C}=0.709 \mathrm{O}=0.000 \mathrm{X}=0.061$

\section{Formaldehyde \\ Concentration \\ Mass \\ Acetaldehyde \\ Concentration \\ Mass \\ Acrolein \\ Concentration \\ Mass \\ Acetone \\ Concentration \\ Mass \\ Propionaldehyde Concentration \\ Mass \\ Crotonaldehyde \\ Concentration \\ Mass \\ Isobutyr + MEK \\ Concentration \\ Mass \\ Benzaldehyde \\ Concentration \\ Mass \\ Hexanaidehyde \\ Concentration \\ Mass}

\begin{tabular}{rl} 
Sample & Background \\
$2.023 \mathrm{ppm}$ & $0.002 \mathrm{ppm}$ \\
$3,084.26 \mathrm{mg}$ & \\
$0.061 \mathrm{ppm}$ & $0.002 \mathrm{ppm}$ \\
$131.84 \mathrm{mg}$ & \\
$0.008 \mathrm{ppm}$ & $0.000 \mathrm{ppm}$ \\
$22.89 \mathrm{mg}$ & \\
$0.004 \mathrm{ppm}$ & $0.002 \mathrm{ppm}$ \\
$7.02 \mathrm{mg}$ & \\
$0.015 \mathrm{ppm}$ & $0.001 \mathrm{ppm}$ \\
$41.84 \mathrm{mg}$ & \\
$0.007 \mathrm{ppm}$ & $0.001 \mathrm{ppm}$ \\
$21.89 \mathrm{mg}$ & \\
$0.002 \mathrm{ppm}$ & $0.000 \mathrm{ppm}$ \\
$6.47 \mathrm{mg}$ & \\
$0.000 \mathrm{ppm}$ & $0.000 \mathrm{ppm}$ \\
$1.39 \mathrm{mg}$ & \\
$0.000 \mathrm{ppm}$ & $0.000 \mathrm{ppm}$ \\
$0.00 \mathrm{mg}$ & \\
\hline
\end{tabular}

\section{Brake-Specific Results}

Formaldehyde

Acetaldehyde

Acrolein

Acetone

Propionaidehyde

$$
\begin{array}{rl}
122.537 & \mathrm{mg} / \mathrm{hp}-\mathrm{hr} \\
5.238 & \mathrm{mg} / \mathrm{hp}-\mathrm{hr} \\
0.909 & \mathrm{mg} / \mathrm{hp}-\mathrm{hr} \\
0.279 & \mathrm{mg} / \mathrm{hp}-\mathrm{hr} \\
1.662 \mathrm{mg} / \mathrm{hp}-\mathrm{hr}
\end{array}
$$

Crotonaidehyde

Isobutyr + MEK

Benzaldehyde

Hexanaldehyde
$0.870 \mathrm{mg} / \mathrm{hp}-\mathrm{hr}$

$0.257 \mathrm{mg} / \mathrm{hp}-\mathrm{hr}$

$0.055 \mathrm{mg} / \mathrm{hp}-\mathrm{hr}$

$0.000 \mathrm{mg} / \mathrm{hp}-\mathrm{hr}$ 
Sourca:

Station \#:

Station Name:

Field:

Report Date: 02/06/1999

Sample Date:

Flowing Pressure: 0

Elowing Temp.: 0

poig

Method: c: \ezchrom \methods \scott:

File: c:lezchrom lch:sm $032377 \mathrm{jbo} .3$

Gas Analysis by Chromatograph

Name

NITROGEN

METHANE

$\mathrm{CO} 2$

ETHANE

$\mathrm{H} 2 \mathrm{~S}$

PROPANE

ISO-BUTANE

TANE

JO-PENTANE

ZENTANE

IEXANES

HEPTANES

OCTANES

ISONONANES

Mole \% BTU SG GPM

$\begin{array}{llll}3.929 & 0.000 & 0.038\end{array}$

90.470

0.000

3.650

0.000

1.951

0.000

0.000

0.000

0.000

0.000

0.000

0.000

0.000

$\begin{array}{rr}0.000 & 0.038 \\ 913.747 & 0.501\end{array}$

$0.000 \quad 0.000$

$\begin{array}{ll}64.590 & 0.038\end{array}$

$0.000 \quad 0.000$

$\begin{array}{ll}49.089 & 0.030\end{array}$

$0.000 \quad 0.000$

$0.000 \quad 0.000$

$0.000 \quad 0.000$

$\begin{array}{lll}0.000 & 0.000\end{array}$

$0.000 \quad 0.000$

$0.000 \quad 0.000$

$0.000 \quad 0.000$

$0.000 \quad 0.000$

GPM

ideal Total

100.000

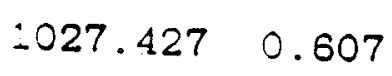

0.536

Gross BTU/Real Cu. Ft.

(@60 deg $F, \quad 0.000$ )

Dry $=1029.683$

Sat. $=1012.930$

Actual $=1029.683$

0.000 1bs. Water, MMCF)

Gasoline Content

Propane GPM $=0.536$

Butane GPM $=0.000$

Gasoline GPM $=0.000$

26\# Gasoline GPM $=0.000$

Total GPM $=0.536$

Real Specific Gravity Calculated $=0.6078$

On-Site Specifle Gravity $=0.0000$

Gas Compressability $=0.9978$

harks: 
Appendix D

Test Numbers 222-C1 and 222-H1

"1000" Hour Transient Emissions Test

Deterioration Factor Calibration

Garrett Turbocharger

Spark Plugs Regapped 


\section{TEST FACILITIES DESCRIPTION}

Emission certification testing was conducted at:

Southwest Research Institute

6220 Culebra Road

P.O. Drawer 28510

San Antonio. TX 78228-0510

Certification activities at Southwest Research Institute were in test cell 4. Cell 4 uses a General Electric direct current dyno in combination with a Mid-West inductor dyno for a combined power measurement. Air to air intercolow (rotary transformer) in line torque meter is used for torque conditioned for temperature and humidity. A L the engine and dynamometer and acquire dabV -based computer system is used to control code.

Gaseous emissions are measured dilute utilizing the full-flow dilution method and particulate sampling makes use of the double dilution method. Both primary and secondary dilution air is
filtered and temperature controlled.

The main dilution turinel uses a positive displacement pump driven at a constant speed in combination with a heat exchanger. The particulate measurement system uses gas meters to measure the secondary dilution air supply and total sample volumes, and particulate is collected
on Paliflex $90 \mathrm{~mm}$ filters.

All equipment and procedures used for testing and calculations are in accordance with 40 CFR Part
86 Subpart N. 


\section{Southwest Research Institute - Department of Emissions Research \\ Composite Transient Emission Test Results \\ Project No. 03-7276-004}

Engine Model: 98 Mack E7 CNG

Engine Desc.: $\quad 0.0 \mathrm{~L}(0 \mathrm{CID}) 6$

Engine Cycle: Diesel

Engine S/N:
Date: 02/22/1999 Time: 09:50

Program HDT: $4.04-\mathrm{C}$

Cell: 4 Bag Cart: 2
NAT GAS CNG, CARB-CNG

HCR: 3.865

FID Resp: 1.13

$H=0.230 \mathrm{C}=0.709 \mathrm{O}=0.000 \mathrm{X}=0.061$

Engine Oil:

\section{Test Numbers}

\section{Cold: 0222-C1 Hot: 0222-H1}

\section{Brake-Specific Emission Results}

BSTHC (Cell)

CO

NOx (Cell)

Particulate

$\mathrm{CO} 2$

BSFC

NMHC

$\mathrm{CH}_{4}$

Work:

Reference Work:
$3.927 \mathrm{~g} / \mathrm{hp}-\mathrm{hr}$

$1.607 \mathrm{~g} / \mathrm{hp}-\mathrm{hr}$

$1.696 \mathrm{~g} / \mathrm{hp}-\mathrm{hr}$

$0.030 \mathrm{~g} / \mathrm{hp}-\mathrm{hr}$

$481.1 \mathrm{~g} / \mathrm{hp}-\mathrm{hr}$

$0.420 \mathrm{lb} / \mathrm{hp}-\mathrm{hr}$

$0.506 \mathrm{~g} / \mathrm{hp}-\mathrm{hr}$

$3.421 \mathrm{~g} / \mathrm{hp}-\mathrm{hr}$

$25.17 \mathrm{hp}-\mathrm{hr}$

$24.69 \mathrm{hp}-\mathrm{hr}$
$5.267 \mathrm{~g} / \mathrm{kW}-\mathrm{hr}$

$2.155 \mathrm{~g} / \mathrm{kW}-\mathrm{hr}$

$2.274 \mathrm{~g} / \mathrm{kW}-\mathrm{hr}$

$0.040 \mathrm{~g} / \mathrm{kW}$ - $\mathrm{hr}$

$645.13 \mathrm{~g} / \mathrm{kW}-\mathrm{hr}$

$0.563 \mathrm{~kg} / \mathrm{kW}$ - $\mathrm{hr}$

$0.679 \mathrm{~g} / \mathrm{kW}-\mathrm{hr}$

$4.588 \mathrm{~g} / \mathrm{kW}-\mathrm{hr}$

$18.77 \mathrm{~kW}-\mathrm{hr}$

$18.41 \mathrm{~kW}-\mathrm{hr}$ 


\section{Southwest Research Institute - Department of Emissions Research EPA Cold Transient Emission Test Results Project No. 03-7276-004}

Engine Model: 98 Mack E7 CNG Engine Desc.: $\quad 0.0 \mathrm{~L}(0 \mathrm{CID}) 6$ Engine Cycle: Diesel Engine S/N:
Test No.: 0222-C1

Date: 02/22/1999 Time:09:50

Program HDT: $4.04-\mathrm{C}$

Cell: 4 Bag Cart: 2
NAT GAS CNG, CARB-CNG HCR: $\quad 3.865$ FID Resp: 1.13 $\mathrm{H}=0.230 \mathrm{C}=0.709 \mathrm{O}=0.000 \mathrm{X}=0.061$

\section{Ambient/Test Cell Conditions}

Barometer:

Engine Inlet Air

Temperature:

Dew Point:

Abs. Humidity:

Rel. Humidity:

Dilution Air:

Temperature:

Abs. Humidity

Rel. Humidity:

$\begin{array}{lcc}74.0 & { }^{\circ} \mathrm{F} & 23.3^{\circ} \mathrm{C} \\ 38.4 & \mathrm{gr} / \mathrm{bb} & 5.5 \mathrm{~g} / \mathrm{kg} \\ 30 & \% & \end{array}$

Measured Gaseous Data

HC Sample

HC Bckgrd

CO Sample

CO Bckgrd

NOx Sample

NOx Bckgrd

CO2 Sample

$\mathrm{CO} 2$ Bckgrd

$\mathrm{CH} 4$ Sample

CH4 Bckgrd

29.35 in $\mathrm{Hg} \quad 99.4 \mathrm{kPa}$

$\begin{array}{lcl}74.0 & { }^{\circ} \mathrm{F} & 23.3^{\circ} \mathrm{C} \\ 57.4 & { }^{\circ} \mathrm{F} & 14.1{ }^{\circ} \mathrm{C} \\ 71.9 & \mathrm{gr} / \mathrm{lb} & 10.3 \mathrm{~g} / \mathrm{kg}\end{array}$

$56 \%$

Meter Range Concentration

$\begin{array}{rrrl}\mathrm{n} / \mathrm{a} & & 143.89 & \mathrm{ppm} \\ \mathrm{n} / \mathrm{a} & & 4.70 & \mathrm{ppm} \\ 31.5 & 2 & 30.58 \mathrm{ppm} \\ 0.5 & 2 & 0.49 & \mathrm{ppm} \\ \mathrm{n} / \mathrm{a} & & 23.55 & \mathrm{ppm} \text { (Dry) } \\ 0.5 & 2 & 0.50 \mathrm{ppm} \\ 62.3 & 1 & 0.5980 & \% \\ 5.4 & 1 & 0.0491 & \% \\ \mathrm{n} / \mathrm{a} & \mathrm{n} / \mathrm{a} & 112.34 & \mathrm{ppm}(1.13) \\ \mathrm{n} / \mathrm{a} & \mathrm{n} / \mathrm{a} & 2.69 \mathrm{ppm}\end{array}$

\section{Corrected Concentrations}

$\begin{array}{lrl}\text { HC } & 139.49 & \mathrm{ppm} \\ \mathrm{CO} & 29.29 & \mathrm{ppm} \\ \mathrm{NOx} & 22.60 & \mathrm{ppm} \\ \mathrm{CO} 2 & 0.5520 & \% \\ \mathrm{CH} 4 & 109.83 & \mathrm{ppm} \\ \mathrm{RHC} & 15.38 & \mathrm{ppm}\end{array}$

$\mathrm{HC}$

\section{Mass Emissions}

$\mathrm{CO}$

103.217

NOx

Particulate

$\mathrm{CO} 2$

$\mathrm{CH} 4$

$\mathrm{RHC}$

Fuel
42.543 grams

53.505 grams

0.722 grams

$12.599 \mathrm{~kg}$

91.347 grams

11.871 grams

$10.99 \mathrm{lb} \quad 4.99 \mathrm{~kg}$

\section{Sample Flows}

sctm

Blower 1 Rate:

Blower 2 Rate:

$90 \mathrm{~mm}$ System:

Gas Meter 1:

Gas Meter 2:

Sample Rate:

Chemistry Sample Rate:

Total Flow Rate:

$2,188.1$

0.0

scmm

61.97

0.00

0.04

1.46

0.07

2.34

0.02

2.54

$2,191.52$

0.07

62.07
Filter Number: Particulate Data

Weight Gain, mg: $\quad$ 133.0-5 (pair)

Sample Multiplier: $\quad 2.480$

NOx Humidity CF:

Dry-to-Wet CF, Sample:

Dry-to-Wet CF, Bckgrd:

Dilution Factor:

\section{Correction Factors}

\begin{tabular}{lrrr}
\multicolumn{5}{c}{ Test Cycle Data } & & \\
Sample Time: & $1,207.50$ & $\mathrm{sec}$ & \\
Work: & $25.03 \mathrm{hp}-\mathrm{hr}$ & $18.66 \mathrm{~kW}-\mathrm{hr}$ \\
Reference Work: & 24.69 & $\mathrm{hp}-\mathrm{hr}$ & $18.41 \mathrm{~kW}-\mathrm{hr}$ \\
Total Volume (Vmix): & $44,053.1 \mathrm{scf}$ & $i .247 .61 \mathrm{scm}$
\end{tabular}

0.992

0.980

0.991

15.77

\section{Brake-Specific Emission Results}

$\begin{array}{llll}\text { BSTHC (Cell) } & 4.124 \mathrm{~g} / \mathrm{hp}-\mathrm{hr} & 5.530 \mathrm{~g} / \mathrm{kW}-\mathrm{hr} \\ \text { CO } & 1.700 \mathrm{~g} / \mathrm{hp}-\mathrm{hr} & 2.279 \mathrm{~g} / \mathrm{kW}-\mathrm{hr} \\ \text { NOx (Cell) } & 2.138 \mathrm{~g} / \mathrm{hp}-\mathrm{hr} & 2.867 \mathrm{~g} / \mathrm{kW}-\mathrm{hr} \\ \text { Particulate } & 0.029 \mathrm{~g} / \mathrm{hp}-\mathrm{hr} & 0.039 \mathrm{~g} / \mathrm{kW}-\mathrm{hr} \\ \text { CO2 } & 503.4 \mathrm{~g} / \mathrm{hp}-\mathrm{hr} & 675.02 \mathrm{~g} / \mathrm{kW}-\mathrm{hr} \\ \text { BSFC } & 0.439 \mathrm{lb} / \mathrm{hp}-\mathrm{hr} & 0.267 \mathrm{~kg} / \mathrm{kW}-\mathrm{hr} \\ \text { NMHC } & 0.474 \mathrm{~g} / \mathrm{hp}-\mathrm{hr} & 0.636 \mathrm{~g} / \mathrm{kW}-\mathrm{hr} \\ \mathrm{CH} 4 & 3.649 \mathrm{~g} / \mathrm{hp}-\mathrm{hr} & 4.894 \mathrm{~g} / \mathrm{kW}-\mathrm{hr}\end{array}$




\section{Southwest Research institute - Department of Emissions Research \\ EPA Cold Transient Emission Test Resuits \\ Project No. 03-7276-004}

Engine Model: 98 Mack E7 CNG Engine Desc.: $\quad 0.0 \mathrm{~L}$ (O CID) 6 Engine Cycle: Diesel

Engine S/N:
Test No.: 0222-C1

Date: 02/22/1999 Time:09:50

Program HDT: $\quad 4.04-\mathrm{C}$

Cell: 4 Bag Cart: 2
NAT GAS CNG, CARB-CNG

HCR: 3.865 FID Resp: 1.13

$\mathrm{H}=0.230 \mathrm{C}=0.709 \mathrm{O}=0.000 \mathrm{X}=0.061$

\section{Eormaldehyde \\ Concentration \\ Mass \\ Acetaldehyde \\ Concentration Mass \\ Acrolein \\ Concentration \\ Mass \\ Acetone \\ Concentration \\ Mass \\ Propionaldehyde \\ Concentration \\ Mass \\ Crotonaldehyde \\ Concentration \\ Mass \\ Isobutyr + MEK \\ Concentration \\ Mass \\ Benzaldehyde \\ Concentration \\ Mass \\ Hexanaidehyde \\ Concentration \\ Mass}

\begin{tabular}{rr}
\multicolumn{1}{c}{ Sample } & Background \\
$2.597 \mathrm{ppm}$ & $0.004 \mathrm{ppm}$ \\
$3,958.28 \mathrm{mg}$ & \\
& \\
$0.079 \mathrm{ppm}$ & $0.003 \mathrm{ppm}$ \\
$172.00 \mathrm{mg}$ & \\
$0.006 \mathrm{ppm}$ & $0.000 \mathrm{ppm}$ \\
$15.12 \mathrm{mg}$ & \\
$0.019 \mathrm{ppm}$ & $0.005 \mathrm{ppm}$ \\
$43.27 \mathrm{mg}$ & \\
$0.008 \mathrm{ppm}$ & $0.000 \mathrm{ppm}$ \\
$24.16 \mathrm{mg}$ & \\
$0.000 \mathrm{ppm}$ & $0.001 \mathrm{ppm}$ \\
$0.00 \mathrm{mg}$ & \\
$0.003 \mathrm{ppm}$ & $0.001 \mathrm{ppm}$ \\
$5.45 \mathrm{mg}$ & \\
$0.000 \mathrm{ppm}$ & $0.000 \mathrm{ppm}$ \\
$0.00 \mathrm{mg}$ & \\
$0.000 \mathrm{ppm}$ & $0.000 \mathrm{ppm}$ \\
$0.00 \mathrm{mg}$ &
\end{tabular}

\section{Brake-Specific Results}

Formaidehyde

Acetaldehyde

Acrolein

Acetone

Propionaldehyde

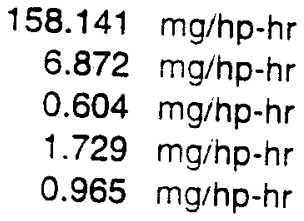

Crotonaidehyde

Isobutyr + MEK

Benzaldehyde

Hexanaldehyde
$0.000 \mathrm{mg} / \mathrm{hp}-\mathrm{hr}$

$0.218 \mathrm{mg} / \mathrm{hp}-\mathrm{hr}$

$0.000 \mathrm{mg} / \mathrm{hp}-\mathrm{hr}$

$0.000 \mathrm{mg} / \mathrm{hp}-\mathrm{hr}$ 


\section{Southwest Research Institute - Department of Emissions Research EPA Hot Transient Emission Test Results Project No. 03-7276-004}

Engine Model: 98 Mack E7 CNG Engine Desc.: $0.0 \mathrm{~L}(0 \mathrm{CID}) 6$ Engine Cycle: Diesel Engine S/N:
Test No.: $0222 \cdot \mathrm{H} 1$

Date: 02/22/1999 Time: 12:24

Program HDT: $\quad 4.04-\mathrm{C}$

Cell: 4 Bag Cart: 2
NAT GAS CNG, CARB-CNG

HCR: $\quad 3.865$ FID Resp: 1.13

$\mathrm{H}=0.230 \mathrm{C}=0.709 \mathrm{O}=0.000 \mathrm{X}=0.061$

\section{Ambient/Test Cell Conditions}

\section{Barometer:}

Engine Inlet Air

Temperature:

Dew Point:

Abs. Humidity:

Dilution Air:

Temperature:

Abs. Humidity

Rel. Humidity:
Rel. Humidity:

29.29 in $\mathrm{Hg} \quad 99.2 \mathrm{kPa}$

$$
\begin{array}{lcl}
77.0 & { }^{\circ} \mathrm{F} & 25.0^{\circ} \mathrm{C} \\
60.3 & { }^{\circ} \mathrm{F} & 15.7{ }^{\circ} \mathrm{C} \\
80.1 & \text { gr/lb } & 11.4 \mathrm{~g} / \mathrm{kg} \\
56 & \% &
\end{array}
$$

$$
\begin{array}{lcc}
76.0 & { }^{\circ} \mathrm{F} & 24.4{ }^{\circ} \mathrm{C} \\
43.7 & \mathrm{gr} / \mathrm{lb} & 6.2 \mathrm{~g} / \mathrm{kg} \\
32 & \% &
\end{array}
$$

Measured Gaseous Data

HC Sample
HC Bckgrd

CO Sample

CO Bckgrd

NOx Sample

NOx Bckgrd

CO2 Sample

$\mathrm{CO} 2$ Bckgrd

$\mathrm{CH} 4$ Sample

$\mathrm{CH} 4$ Bckgrd
Meter Range Concentration

$137.07 \mathrm{ppm}$

$4.60 \mathrm{ppm}$

$0.10 \mathrm{ppm}$

$17.49 \mathrm{ppm}$ (Dry)

$0.20 \mathrm{ppm}$

$0.5757 \%$

$0.0509 \%$

$104.60 \mathrm{ppm}(1.13)$

$2.04 \mathrm{ppm}$

$\begin{array}{rr}n / a & \\ n / a & \\ 29.4 & 2 \\ 0.1 & 2 \\ n / a & \\ 0.2 & 2 \\ 60.1 & 1 \\ 5.6 & 1 \\ n / a & n / a \\ n / a & n / a\end{array}$

$28.53 \mathrm{ppm}$

\section{Corrected Concentrations}

$\begin{array}{lrl}\text { HC } & 132.75 & \text { ppm } \\ \text { CO } & 27.66 & \text { ppm } \\ \text { NOx } & 16.93 & \text { ppm } \\ \mathrm{CO} 2 & 0.5279 & \% \\ \mathrm{CH} 4 & 102.68 & \mathrm{ppm} \\ \mathrm{RHC} & 16.72 & \mathrm{ppm}\end{array}$

Mass Emissions

$\mathrm{HC}$

$\mathrm{CO}$

NOx

Particulate

$\mathrm{CO} 2$

$\mathrm{CH} 4$

$\mathrm{RHC}$

Fuel

$\begin{array}{rl}98.113 & \text { grams } \\ 40.096 & \text { grams } \\ 40.869 & \text { grams } \\ 0.755 & \text { grams } \\ 12.025 & \mathrm{~kg} \\ 85.236 & \text { grams } \\ 12.877 & \text { grams } \\ \mathrm{lb} & 4.76 \mathrm{~kg}\end{array}$

\section{Sample Flows}

scfm

Blower 1 Rate:

Blower 2 Rate:

$90 \mathrm{~mm}$ System:

Gas Meter 1:

Gas Meter 2:

Sample Rate:

$2,183.4$

0.0

1.48

2.39

0.91

Chemistry Sample Rate:

2.54

Total Flow Rate:

$2,186.85$

scmm

61.84

0.00

0.04

0.07

0.03

0.07

61.93
Filter Number: $\quad \begin{gathered}\text { Particulate Data } \\ 1136.0-8 \text { (pair) }\end{gathered}$

Weight Gain, mg:

Sample Multiplier:

0.313

2.412

NOx Humidity CF:

Dry-to-Wet CF, Sample:

Dry-to-Wet CF, Bckgrd:

Dilution Factor:

1.014

0.979

0.990

16.39

\begin{tabular}{lrrr}
\multicolumn{5}{c}{ Test Cycle Data } \\
Sample Time: & $1,207.70 \mathrm{sec}$ & & \\
Work: & $25.19 \mathrm{hp}-\mathrm{hr}$ & $18.78 \mathrm{~kW}-\mathrm{hr}$ \\
Reference Work: & $24.69 \mathrm{hp}-\mathrm{hr}$ & $18.41 \mathrm{~kW}-\mathrm{hr}$ \\
Total Volume (Vmix): & $43,966.5 \mathrm{scf}$ & $1,245.16 \mathrm{scm}$
\end{tabular}

\section{Brake-Specific Emission Results}

$\begin{array}{llll}\text { BSTHC (Cell) } & 3.895 \mathrm{~g} / \mathrm{hp}-\mathrm{hr} & 5.223 \mathrm{~g} / \mathrm{kW}-\mathrm{hr} \\ \text { CO } & 1.592 \mathrm{~g} / \mathrm{hp}-\mathrm{hr} & 2.135 \mathrm{~g} / \mathrm{kW}-\mathrm{hr} \\ \text { NOx (Cell) } & 1.622 \mathrm{~g} / \mathrm{hp}-\mathrm{hr} & 2.176 \mathrm{~g} / \mathrm{kW}-\mathrm{hr} \\ \text { Particulate } & 0.030 \mathrm{~g} / \mathrm{hp}-\mathrm{hr} & 0.040 \mathrm{~g} / \mathrm{kW}-\mathrm{hr} \\ \text { CO2 } & 477.4 \mathrm{~g} / \mathrm{hp}-\mathrm{hr} & 640.18 \mathrm{~g} / \mathrm{kW}-\mathrm{hr} \\ \text { BSFC } & 0.417 \mathrm{lb} / \mathrm{hp}-\mathrm{hr} & 0.253 \mathrm{~kg} / \mathrm{kW}-\mathrm{hr} \\ \text { NMHC } & 0.511 \mathrm{~g} / \mathrm{hp}-\mathrm{hr} & 0.686 \mathrm{~g} / \mathrm{kW}-\mathrm{hr} \\ \mathrm{CH} 4 & 3.384 \mathrm{~g} / \mathrm{hp}-\mathrm{hr} & 4.538 \mathrm{~g} / \mathrm{kW}-\mathrm{hr}\end{array}$




\section{Southwest Research Institute - Department of Emissions Research \\ EPA Hot Transient Emission Test Results \\ Project No. 03-7276-004}

Engine Model: 98 Mack E7 CNG Engine Desc.: $0.0 \mathrm{~L}(0 \mathrm{CID}) 6$ Engine Cycle: Diesel Engine S/N:

\section{Formaldehyde} Concentration Mass

Acetaldehyde Concentration Mass

Acrolein Concentration Mass

Acetone

Concentration Mass

Propionaldehyde Concentration Mass

Crotonaldehyde Concentration Mass

Isobutyr + MEK

Concentration Mass

Benzaldehyde Concentration Mass

Hexanaidehyde Concentration Mass
Test No: : $0222 \cdot \mathrm{H} 1$

Date: 02/22/1999 Time: 12:24

Program HDT: $4.04-\mathrm{C}$

Cell: 4 Bag Cart: 2
NAT GAS CNG, CARB-CNG HCR: 3.865 FID Resp: 1.13 $H=0.230 \mathrm{C}=0.709 \mathrm{O}=0.000 \mathrm{X}=0.061$

\section{Sample}

$2.363 \mathrm{ppm}$

$3,591.99 \mathrm{mg}$

$0.072 \mathrm{ppm}$

$153.91 \mathrm{mg}$

$0.006 \mathrm{ppm}$

$16.11 \mathrm{mg}$

$0.000 \mathrm{ppm}$

$0.00 \mathrm{mg}$

$0.005 \mathrm{ppm}$

$14.36 \mathrm{mg}$

$0.002 \mathrm{ppm}$

$5.29 \mathrm{mg}$

$0.003 \mathrm{ppm}$

$5.98 \mathrm{mg}$

$0.000 \mathrm{ppm}$

$0.00 \mathrm{mg}$

$0.000 \mathrm{ppm}$

$0.00 \mathrm{mg}$
Background

$0.004 \mathrm{ppm}$

$0.003 \mathrm{ppm}$

$0.000 \mathrm{ppm}$

0.005 ppm

$0.000 \mathrm{ppm}$

0.001 ppm

0.001 ppm

$0.000 \mathrm{ppm}$

$0.000 \mathrm{ppm}$

\section{Brake-Specific Results}

Formaldehyde

Acetaldehyde

Acrolein

Acetone

Propionaldehyde

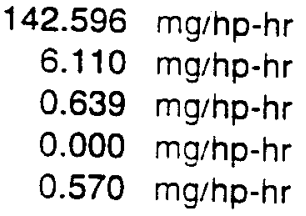

Crotonaldehyde

Isobutyr + MEK

Benzaidehyde

Hexanaldehyde
$0.210 \mathrm{mg} / \mathrm{hp}-\mathrm{hr}$

$0.237 \mathrm{mg} / \mathrm{hp}-\mathrm{hr}$

$0.000 \mathrm{mg} / \mathrm{hp}-\mathrm{hr}$

$0.000 \mathrm{mg} / \mathrm{hp}-\mathrm{hr}$ 


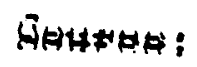

Station \#:

Station Namo:

\section{Field:}

Method:

File:

c: \ezchrom \methods \acott.

c: \ezchrom \chrom $\backslash 031732 \mathrm{jc} .1$
Report Date: 02/15/1999 Flowing Pressure:

Gas Analysis by Chromatograph

Name

NITROGEN

METHANE

$\mathrm{CO} 2$

ETHANE

H2S

PROPANE

ISO-BUTANE

TANE

-SO-PENTANE

PENTANB

HEXANES

HEPTANES

OCTANES

ISONONANES
Mole \%

3.824

90.768

0.000

3.623

0.000

1.785

0.000

0.000

0.000

0.000

0.000

0.000

0.000

0.000
BTU

SG

$\begin{array}{rr}0.000 & 0.037 \\ 916.757 & 0.503 \\ 0.000 & 0.000 \\ 64.113 & 0.038\end{array}$

0.000

44.912

0.000

0.000

0.000

0.000

0.000

0.000

0.000

0.000

0.000

0.027

0.000

0.000

0.000

0.000

0.000

0.000

0.000

0.000

GPM

poler

Ideal Total

Grose BTU/Real Cu. Ft.

(ब 60 deg $F, 0.000$ )

$100.000 \quad 1025.782 \quad 0.605$

0.491

0.491

Dry $=1028.022$

Sat. $=1011.297$

Actual $=1028.022$

0.000 Ibs. Water/MMCE)

$$
\begin{aligned}
& \text { Gasoline Content } \\
& \text { Propane GPM }=0.491 \\
& \text { Butane GPM }=0.000 \\
& \text { Gasoline GPM }=0.000 \\
& \text { 26\# Gasoline GPM }=0.000 \\
& \text { Total GPM }=0.491
\end{aligned}
$$

Real Specific Gravity Calculated $=0.6056$

On-Site Specific Gravity $=0.0000$

Gas Coupressability $=0.9978$

lariks: 
Appendix E

Test Numbers 224-C1 and 224-H1

"1000" Hour Transient Emissions Test 325 BHP Calibration

Maximum Turbo Lag Enrichment $=13 \%$

Schwitzer Turbocharger

Spark Plugs Regapped 


\section{Southwest Research Institute - Department of Emissions Research EPA Hot Transient Emission Test Results \\ Project No. 03-7276-004}

Engine Model: 98 Mack E7 CNG Engine Desc.: $\quad 0.0 \mathrm{~L}(0 \mathrm{CID}) 6$ Engine Cycle: Diesel

Engine S/N:
Test No.: 0224-H1

Date: 02/24/1999 Time: 09:38

Program HDT: $4.04-\mathrm{C}$

Cell: 4 Bag Cart: 2
NAT GAS CNG, CARB-CNG

HCR: 3.849 FID Resp: 1.13

$\mathrm{H}=0.230 \mathrm{C}=0.712 \mathrm{O}=0.000 \mathrm{X}=0.057$

\begin{tabular}{llll}
\multicolumn{5}{c}{ Ambient/Test Cell Conditions } \\
$\begin{array}{l}\text { Barometer: } \\
\text { Engine Inlet Air }\end{array}$ & 29.37 & in $\mathrm{Hg}$ & $99.4 \mathrm{kPa}$ \\
Temperature: & 77.0 & ${ }^{\circ} \mathrm{F}$ & $25.0^{\circ} \mathrm{C}$ \\
Dew Point: & 59.6 & ${ }^{\circ} \mathrm{F}$ & $15.3^{\circ} \mathrm{C}$ \\
Abs. Humidity: & 77.9 & $\mathrm{gr} / \mathrm{lb}$ & $11.1 \mathrm{~g} / \mathrm{kg}$ \\
Rel. Humidity: & 55 & $\%$ & \\
Dilution Air: & & & \\
Temperature: & 75.0 & ${ }^{\circ} \mathrm{F}$ & $23.9{ }^{\circ} \mathrm{C}$ \\
Abs. Humidity & 67.8 & $\mathrm{gr} / \mathrm{lb}$ & $9.7 \mathrm{~g} / \mathrm{kg}$ \\
Rel. Humidity: & 51 & $\%$ &
\end{tabular}

Measured Gaseous Data

HC Sample

HC Bckgrd

CO Sample

CO Bckgrd

NOx Sample

NOx Bckgrd

CO2 Sampie

$\mathrm{CO} 2$ Bckgrd

$\mathrm{CH} 4$ Sample

CH4 Bckgrd

$\begin{array}{rrrl}\text { Meter } & \text { Range } & \text { Concentration } \\ n / a & & 108.21 & \mathrm{ppm} \\ \mathrm{n} / \mathrm{a} & & 4.80 \mathrm{ppm} \\ 25.4 & 2 & 24.63 \mathrm{ppm} \\ \mathrm{n} / \mathrm{a} & 2 & 0.00 \mathrm{ppm} \\ \mathrm{n} / \mathrm{a} & & 22.56 \mathrm{ppm} \text { (Dry) } \\ 3.3 & 1 & 0.85 & \mathrm{ppm} \\ 59.3 & 1 & 0.5677 & \% \\ 7.0 & 1 & 0.0638 & \% \\ \mathrm{n} / \mathrm{a} & \mathrm{n} / \mathrm{a} & 84.46 & \mathrm{ppm}(1.13) \\ \mathrm{n} / \mathrm{a} & \mathrm{n} / \mathrm{a} & 2.10 \mathrm{ppm} & \end{array}$

\section{Corrected Concentrations}

$\begin{array}{lrl}\text { HC } & 103.70 & \text { ppm } \\ \mathrm{CO} & 23.81 & \mathrm{ppm} \\ \mathrm{NOx} & 21.17 & \mathrm{ppm} \\ \mathrm{CO} 2 & 0.5077 & \% \\ \mathrm{CH} 4 & 82.49 & \mathrm{ppm} \\ \mathrm{RHC} & 10.49 & \mathrm{ppm}\end{array}$

$\mathrm{HC}$

Mass Emissions

$\mathrm{CO}$

NOx

Particulate

$\mathrm{CO} 2$

$\mathrm{CH}_{4}$

$\mathrm{RHC}$

Fuel

76.782

34.624

50.945

0.728

11.601

68.681

8.10 grams

grams

grams

grams

$\mathrm{kg}$

grams

grams

NOx Humidity CF:

Dry-to-Wet CF, Sample:

Dry-to-Wet CF, Bckgrd:

Sample Flows

Blower 1 Rate:

Blower 2 Rate:

$90 \mathrm{~mm}$ System:

Gas Meter 1:

Gas Meter 2:

Sample Rate:

Chemistry Sample Rate:

Total Flow Rate:

Filter Number: Particulate Data

Weight Gain, mg:

Sample Multiplier:

Dilution Factor:

$$
\text { scfm }
$$

$2,189.7$

0.0

scmm

62.01

0.00

\subsection{6}

2.38

0.92

2.52

$2,193.15$

1202.0- (pair)

(pair)
0.306

2.379

\begin{tabular}{llrl}
\multicolumn{5}{c}{ Test Cycle Data } & \\
Sample Time: & $1,207.90 \mathrm{sec}$ & \\
Work: & $25.31 \mathrm{hp}-\mathrm{hr}$ & $18.87 \mathrm{~kW}-\mathrm{hr}$ \\
Reference Work: & $24.90 \mathrm{hp}-\mathrm{hr}$ & $18.57 \mathrm{~kW}-\mathrm{hr}$ \\
Total Volume (Vmix): & $44,100.9 \mathrm{scf}$ & $1,248.96 \mathrm{scm}$
\end{tabular}

BSTHC (Cell)

\section{Brake-Specific Emission Results}

CO

NOx (Cell)

$3.034 \mathrm{~g} / \mathrm{hp}-\mathrm{hr}$

Particulate

$\mathrm{CO} 2$

BSFC

NMHC

$\mathrm{CH} 4$
$1.368 \mathrm{~g} / \mathrm{hp}-\mathrm{hr}$

$2.013 \mathrm{~g} / \mathrm{hp}-\mathrm{hr}$

$0.029 \mathrm{~g} / \mathrm{hp}-\mathrm{hr}$

$458.3 \mathrm{~g} / \mathrm{hp}-\mathrm{hr}$

$0.396 \mathrm{lb} / \mathrm{hp}-\mathrm{hr}$

$0.320 \mathrm{~g} / \mathrm{hp}-\mathrm{hr}$

$2.714 \mathrm{~g} / \mathrm{hp}-\mathrm{hr}$
$4.068 \mathrm{~g} / \mathrm{kW}-\mathrm{hr}$

$1.835 \mathrm{~g} / \mathrm{kW}-\mathrm{hr}$

$2.699 \mathrm{~g} / \mathrm{kW}-\mathrm{hr}$

$0.039 \mathrm{~g} / \mathrm{kW}$-hr

$614.64 \mathrm{~g} / \mathrm{kW}-\mathrm{hr}$

$0.241 \mathrm{~kg} / \mathrm{kW}-\mathrm{hr}$

$0.429 \mathrm{~g} / \mathrm{kW}-\mathrm{hr}$

$3.639 \mathrm{~g} / \mathrm{kW}-\mathrm{hr}$ 


\section{Southwest Research Institute - Department of Emissions Research EPA Hot Transient Emission Test Results \\ Project No. 03-7276-004}

Engine Model: 98 Mack E7 CNG Engine Desc.: $\quad 0.0 \mathrm{~L}$ (0 CID) 6 Engine Cycle: Diesel Engine S/N
Test No.: 0224-H1

Date: 02/24/1999 Time:09:38

Program HDT: $\quad 4.04-\mathrm{C}$

Cell: 4 Bag Cart: 2
NAT GAS CNG, CARB-CNG HCR: 3.849 FID Resp: 1.13 $\mathrm{H}=0.230 \mathrm{C}=0.712 \mathrm{O}=0.000 \mathrm{X}=0.057$

\section{Eormaldehyde Concentration Mass \\ Acetaldehyde Concentration Mass \\ Acrolein \\ Concentration Mass \\ Acetone \\ Concentration Mass \\ Propionaldehyde Concentration Mass \\ Crotonaldehyde Concentration Mass \\ Isobutyr + MEK Concentration Mass \\ Benzaldehyde Concentration Mass \\ Hexanaidehyde Concentration Mass}

\begin{tabular}{rl}
\multicolumn{1}{c}{ Sample } & Background \\
$2.247 \mathrm{ppm}$ & $0.005 \mathrm{ppm}$ \\
$3,405.64 \mathrm{mg}$ & \\
$0.067 \mathrm{ppm}$ & $0.002 \mathrm{ppm}$ \\
$144.26 \mathrm{mg}$ & \\
$0.004 \mathrm{ppm}$ & $0.000 \mathrm{ppm}$ \\
$11.86 \mathrm{mg}$ & \\
$0.003 \mathrm{ppm}$ & $0.003 \mathrm{ppm}$ \\
$0.45 \mathrm{mg}$ & \\
$0.003 \mathrm{ppm}$ & $0.000 \mathrm{ppm}$ \\
$9.74 \mathrm{mg}$ & \\
$0.000 \mathrm{ppm}$ & $0.000 \mathrm{ppm}$ \\
$0.00 \mathrm{mg}$ & \\
$0.001 \mathrm{ppm}$ & $0.000 \mathrm{ppm}$ \\
$3.78 \mathrm{mg}$ & \\
$0.000 \mathrm{ppm}$ & $0.000 \mathrm{ppm}$ \\
$0.00 \mathrm{mg}$ & \\
$0.001 \mathrm{ppm}$ & $0.000 \mathrm{ppm}$ \\
$4.77 \mathrm{mg}$ & \\
&
\end{tabular}

Background Engine Oil:

\section{Brake-Specific Results}

\section{Formaldehyde} Acetaldehyde Acrolein Acetone Propionaldehyde

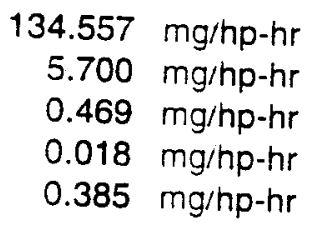

Crotonaldehyde

Isobutyr + MEK

Benzaldehyde

Hexanaldehyde
$0.000 \mathrm{mg} / \mathrm{hp}-\mathrm{hr}$

$0.149 \mathrm{mg} / \mathrm{hp}-\mathrm{hr}$

$0.000 \mathrm{mg} / \mathrm{hp}-\mathrm{hr}$

$0.188 \mathrm{mg} / \mathrm{hp}-\mathrm{hr}$ 
Source:

Station \#:

Station Name:

Fleid:

File:

iv

Report Date: $02 / 22 / 1999$
Sample Date:

lowling Pressure: 0

Flowing Temp.: 0

psig

c: \ezchrom \methods $\backslash$ scott:

F

Name

NITROGEN

METHANE

$\mathrm{CO} 2$

ETHANE

25

PROPANE

SO-BUTANE

TANE

JO-PENTANE

DENTANE

IEXANES

IEPTANES

CTANES

SONONANES

Gas Analysis by Chrometograph

Mole \% BTU SG GPM

$\begin{array}{rrr}3.592 & 0.000 & 0.035 \\ 90.867 & 917.757 & 0.503 \\ 0.000 & 0.000 & 0.000 \\ 3.660 & 64.767 & 0.038 \\ 0.000 & 0.000 & 0.000 \\ 1.881 & 47.328 & 0.029 \\ 0.000 & 0.000 & 0.000 \\ 0.000 & 0.000 & 0.000 \\ 0.000 & 0.000 & 0.000 \\ 0.000 & 0.000 & 0.000 \\ 0.000 & 0.000 & 0.000 \\ 0.000 & 0.000 & 0.000 \\ 0.000 & 0.000 & 0.000 \\ 0.000 & 0.000 & 0.000\end{array}$

Jaal Total

Gross BTU/Real Cu. Ft.

$$
\begin{array}{llll}
100.000 & 1029.852 & 0.605 & 0.517
\end{array}
$$

(@ 60 deg F, 0.000 )

Dry $=1032.117$

Sat. $=1015.323$

ctual $=1032.117$

0.000 1bs. water MMCF)

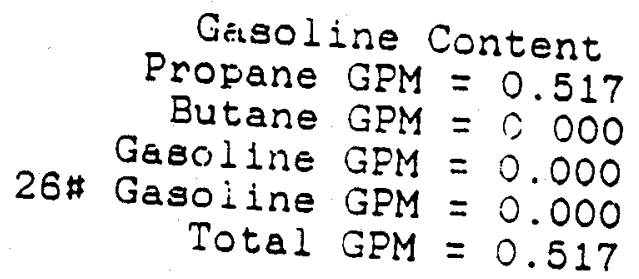

Real Specifte Gravity Calculated $=0.6058$

On-Site Specific Gravity $=0.0058$
Compre

Gas Compressab111ty $=0.9978$

sks: 


\author{
Appendix F \\ Test Numbers 225-C1 and 225-H1 \\ "1000" Hour Transient Emissions Test \\ 350 BHP Calibration \\ Maximum Turbo Lag Enrichment $=10 \%$ \\ Schwitzer Turbocharger \\ Spark Plugs Regapped
}




\section{TEST FACILITIES DESCRIPTION}

Emission certification testing was conducted at:

Southwest Research Institute

6220 Culebra Road

P.O. Drawer 28510

San Antonio, TX 78228-0510

Certification activities at Southwest Research Institute were in test cell 4. Cell 4 uses a General Electric direct current dyno in combination with a Mid-West inductor dyno for a combined power measurement. Air to air intercooling is (rotary transformer) in line torque meter is used for torque conditioned for temperature and humidity. A Lated as required per SAE 1937. Engine inlet air is the engine and dynamometer and acqu. A LabVIEW -based computer system is used to control code. sampling makes use of the double dilution method. Both primary and secondary dilution air is
filtered and temperature controlled.

The main dilution tunnel uses a positive displacement pump driven at a constant speed in combination with a heat exchanger. The particulate measurement system uses gas meters to on Pallflex $90 \mathrm{~mm}$ filters.

All equipment and procedures used for testing and calculations are in accordance with 40 CFR Part
86 Subpart $N$. 


\section{Southwest Research Institute - Department of Emissions Research \\ Composite Transient Emission Test Results \\ Project No. 03-7276-004}

Engine Model: 98 Mack E7 CNG

Engine Desc.: $\quad 0.0 \mathrm{~L}$ (0 CID) 6

Engine Cycle: Diesel

Engine S/N:
Date: 02/25/1999 Time: 08:55

Program HDT: $4.04-\mathrm{C}$

Cell: 4 Bag Cart: 2
NAT GAS CNG, CARB-CNG

HCR: 3.849

FID Resp: 1.13

$H=0.230 C=0.712 O=0.000 X=0.057$ Engine Oil:

Test Numbers

Cold: $0225-\mathrm{C} 1$ Hot: $0225-\mathrm{H} 1$

\section{Brake-Specific Emission Results}

$\begin{array}{lrlrl}\text { BSTHC (Cell) } & 2.905 & \mathrm{~g} / \mathrm{hp}-\mathrm{hr} & 3.895 & \mathrm{~g} / \mathrm{kW}-\mathrm{hr} \\ \text { CO } & 1.319 & \mathrm{~g} / \mathrm{hp}-\mathrm{hr} & 1.769 & \mathrm{~g} / \mathrm{kW}-\mathrm{hr} \\ \text { NOx (Cell) } & 2.192 & \mathrm{~g} / \mathrm{hp}-\mathrm{hr} & 2.940 & \mathrm{~g} / \mathrm{kW}-\mathrm{hr} \\ \text { Particulate } & 0.026 & \mathrm{~g} / \mathrm{hp}-\mathrm{hr} & 0.035 & \mathrm{~g} / \mathrm{kW}-\mathrm{hr} \\ \text { CO2 } & 448.9 & \mathrm{~g} / \mathrm{hp}-\mathrm{hr} & 602.01 & \mathrm{~g} / \mathrm{kW}-\mathrm{hr} \\ \text { BSFC } & 0.388 & \mathrm{lb} / \mathrm{hp}-\mathrm{hr} & 0.520 & \mathrm{~kg} / \mathrm{kW}-\mathrm{hr} \\ \text { NMHC } & 0.276 & \mathrm{~g} / \mathrm{hp}-\mathrm{hr} & 0.370 & \mathrm{~g} / \mathrm{kW}-\mathrm{hr} \\ \text { CH4 } & 2.629 & \mathrm{~g} / \mathrm{hp}-\mathrm{hr} & 3.525 \mathrm{~g} / \mathrm{kW}-\mathrm{hr} \\ \text { Work: } & & & & \\ \text { Reference Work: } & 25.23 & \mathrm{hp}-\mathrm{hr} & 18.82 \mathrm{~kW}-\mathrm{hr} \\ & 25.64 & \mathrm{hp}-\mathrm{hr} & 19.12 \mathrm{~kW}-\mathrm{hr}\end{array}$




\section{Southwest Research Institute - Department of Emissions Research EPA Cold Transient Emission Test Results \\ Project No. 03-7276-004}

Engine Model: 98 Mack E7 CNG Engine Desc.: $\quad 0.0 \mathrm{~L}$ (0 CID) 6 Engine Cycle: Diesel Engine S/N:
Test No.: 0225-C1

Date: 02/25/1999 Time:08:55

Program HDT: $4.04-\mathrm{C}$

Cell: 4 Bag Cart: 2
NAT GAS CNG, CARB-CNG

HCR: $\quad 3.849$ FID Resp: 1.13

$\mathrm{H}=0.230 \mathrm{C}=0.712 \mathrm{O}=0.000 \mathrm{X}=0.057$ Engine Oil:

\begin{tabular}{llll}
\multicolumn{5}{c}{ Ambient/Test Cell Conditions } \\
$\begin{array}{l}\text { Barometer: } \\
\text { Engine inlet Air }\end{array}$ & 29.23 & in $\mathrm{Hg}$ & $99.0 \mathrm{kPa}$ \\
Temperature: & 75.0 & ${ }^{\circ} \mathrm{F}$ & $23.9^{\circ} \mathrm{C}$ \\
Dew Point: & 58.2 & ${ }^{\circ} \mathrm{F}$ & $14.6^{\circ} \mathrm{C}$ \\
Abs. Humidity: & 74.4 & $\mathrm{gr} / \mathrm{lb}$ & $10.6 \mathrm{~g} / \mathrm{kg}$ \\
Rel. Humidity: & 56 & $\%$ & \\
$\begin{array}{l}\text { Dilution Air: } \\
\text { Temperature: }\end{array}$ & 74.0 & ${ }^{\circ} \mathrm{F}$ & $23.3^{\circ} \mathrm{C}$ \\
Abs. Humidity & 74.8 & $\mathrm{gr} / \mathrm{lb}$ & $10.7 \mathrm{~g} / \mathrm{kg}$ \\
Rel. Humidity: & 58 & $\%$ &
\end{tabular}

HC Sample

Measured Gaseous Data

HC Bckgrd

CO Sample

CO Bckgrd

NOx Sample

NOx Bckgrd

CO2 Sample

$\mathrm{CO} 2$ Bckgrd

$\mathrm{CH} 4$ Sample

$\mathrm{CH} 4$ Bckgrd
Meter Range Concentration

$\begin{array}{lll}25.7 & 2 & 24.92 \mathrm{ppm}\end{array}$

$\mathrm{n} / \mathrm{a} \quad 2 \quad 0.00 \mathrm{ppm}$

n/a 28.43 ppm (Dry)

$59.6 \quad 1$

$5.6 \quad 1$

$n / a \quad n / a$

$n / a \quad n / a$
$0.31 \mathrm{ppm}$

$0.5707 \%$

$0.0509 \%$

$84.10 \mathrm{ppm}(1.13)$

$2.07 \mathrm{ppm}$

$\begin{array}{rr}\mathrm{n} / \mathrm{a} & 105.78 \mathrm{ppm} \\ \mathrm{n} / \mathrm{a} & 4.90 \mathrm{ppm}\end{array}$

1.21

Corrected Concentrations

$\begin{array}{lrl}\text { HC } & 101.17 & \text { ppm } \\ \mathrm{CO} & 24.04 & \mathrm{ppm} \\ \mathrm{NOx} & 27.35 & \mathrm{ppm} \\ \mathrm{CO} 2 & 0.5229 & \% \\ \mathrm{CH} 4 & 82.16 & \mathrm{ppm} \\ \mathrm{RHC} & 8.34 & \mathrm{ppm}\end{array}$

$\mathrm{HC}$

$\mathrm{CO}$

NOx

Particulate

$\mathrm{CO} 2$

$\mathrm{CH} 4$

$\mathrm{RHC}$

Fuel

\section{Mass Emissions}

$$
\begin{aligned}
& 74.460 \text { grams } \\
& 34.768 \text {. grams } \\
& 64.876 \text { grams } \\
& 0.709 \text { grams } \\
& 11.885 \mathrm{~kg} \\
& 68.051 \text { grams } \\
& 6.409 \text { grams }
\end{aligned}
$$

$$
\begin{array}{ll}
10.27 \mathrm{lb} & 4.66 \mathrm{~kg}
\end{array}
$$

\section{Sample Flows}

Blower 1 Rate:

Blower 2 Rate:

$90 \mathrm{~mm}$ System:

Gas Meter 1:

Gas Meter 2:

Sample Rate:

Chemistry Sample Rate:

Total Flow Rate:

scfm

$2,178.7$

0.0

61.70

0.00

1.47

0.04

2.39

0.92

0.07

0.03

0.07

61.80 $\begin{array}{lc} & \text { Particulate Data } \\ \text { Filter Number: } & 1203.0-11 \text { (pair) } \\ \text { Weight Gain, mg: } & 0.299 \\ \text { Sample Multiplier: } & 2.372\end{array}$

NOx Humidity CF:

Dry-to-Wet CF, Sample:

Dry-to-Wet CF, Bckgrd:

Dilution Factor:

\begin{tabular}{lrr}
\multicolumn{5}{c}{ Test Cycle Data } \\
Sample Time: & $1,207.70 \mathrm{sec}$ & \\
Work: & $25.24 \mathrm{hp}-\mathrm{hr}$ & $18.82 \mathrm{~kW}-\mathrm{hr}$ \\
Reference Work: & $25.64 \mathrm{hp}-\mathrm{hr}$ & $19.12 \mathrm{~kW}-\mathrm{hr}$ \\
Total Volume (Vmix): & $43,872.8 \mathrm{scf}$ & $1,242.50 \mathrm{scm}$
\end{tabular}

0.998

0.972

0.983

16.66

$\begin{array}{llll}\text { BSTHC (Cell) } & 2.950 \mathrm{~g} / \mathrm{hp}-\mathrm{hr} & 3.956 \mathrm{~g} / \mathrm{kW}-\mathrm{hr} \\ \text { CO } & 1.378 \mathrm{~g} / \mathrm{hp}-\mathrm{hr} & 1.847 \mathrm{~g} / \mathrm{kW}-\mathrm{hr} \\ \text { NOx (Cell) } & 2.570 \mathrm{~g} / \mathrm{hp}-\mathrm{hr} & 3.447 \mathrm{~g} / \mathrm{kW}-\mathrm{hr} \\ \text { Particulate } & 0.028 \mathrm{~g} / \mathrm{hp}-\mathrm{hr} & 0.038 \mathrm{~g} / \mathrm{kW}-\mathrm{hr} \\ \text { CO2 } & 470.9 \mathrm{~g} / \mathrm{hp}-\mathrm{hr} & 631.45 \mathrm{~g} / \mathrm{kW}-\mathrm{hr} \\ \text { BSFC } & 0.407 \mathrm{lb} / \mathrm{hp}-\mathrm{hr} & 0.247 \mathrm{~kg} / \mathrm{kW}-\mathrm{hr} \\ \text { NMHC } & 0.254 \mathrm{~g} / \mathrm{hp}-\mathrm{hr} & 0.341 \mathrm{~g} / \mathrm{kW}-\mathrm{hr} \\ \mathrm{CH} 4 & 2.696 \mathrm{~g} / \mathrm{hp}-\mathrm{hr} & 3.616 \mathrm{~g} / \mathrm{kW}-\mathrm{hr}\end{array}$




\section{Southwest Research Institute - Department of Emissions Research EPA Cold Transient Emission Test Resuits \\ Project No. 03-7276-004}

Engine Model: 98 Mack E7 CNG

Engine Desc.: $\quad 0.0 \mathrm{~L}$ (0 CID) 6

Engine Cycle: Diesel

Engine $\mathrm{S} / \mathrm{N}$ :
Test No.: $0225-\mathrm{C} 1$

Date: 02/25/1999 Time:08:55

Program HDT: $4.04-\mathrm{C}$

Cell: 4 Bag Cart: 2
NAT GAS CNG, CARB-CNG

HCR: $\quad 3.849$ FID Resp: 1.13

$\mathrm{H}=0.230 \mathrm{C}=0.712 \mathrm{O}=0.000 \mathrm{X}=0.057$

\begin{tabular}{|c|c|c|c|c|}
\hline Formaldehyde & Sampl & & Backgrou & und \\
\hline $\begin{array}{l}\text { Concentration } \\
\text { Mass } \\
\text { Acetaldehyde }\end{array}$ & $\begin{array}{r}2.046 \\
3,080.11\end{array}$ & $\begin{array}{l}\mathrm{ppm} \\
\mathrm{mg}\end{array}$ & 0.005 & $\mathrm{ppm}$ \\
\hline $\begin{array}{l}\text { Concentration } \\
\text { Mass } \\
\text { Acrolein }\end{array}$ & $\begin{array}{r}0.066 \\
141.11\end{array}$ & $\begin{array}{l}\mathrm{ppm} \\
\mathrm{mg}\end{array}$ & 0.002 & $\mathrm{ppm}$ \\
\hline $\begin{array}{l}\text { Concentration } \\
\text { Mass } \\
\text { Acetone }\end{array}$ & $\begin{array}{l}0.011 \\
31.34\end{array}$ & $\begin{array}{l}\mathrm{ppm} \\
\mathrm{mg}\end{array}$ & 0.000 & ppm \\
\hline $\begin{array}{l}\text { Concentration } \\
\text { Mass } \\
\text { Propionaldehyde }\end{array}$ & $\begin{array}{l}0.009 \\
13.58\end{array}$ & $\begin{array}{l}\mathrm{ppm} \\
\mathrm{mg}\end{array}$ & 0.004 & $\mathrm{ppm}$ \\
\hline $\begin{array}{l}\text { Concentration } \\
\text { Mass } \\
\text { Crotonaldehyde }\end{array}$ & $\begin{array}{l}0.014 \\
40.09\end{array}$ & $\begin{array}{l}\text { ppm } \\
\mathrm{mg}\end{array}$ & 0.000 & $\mathrm{ppm}$ \\
\hline $\begin{array}{l}\text { Concentration } \\
\text { Mass } \\
\text { Isobutyr + MEK }\end{array}$ & $\begin{array}{l}0.006 \\
20.36\end{array}$ & $\begin{array}{l}\mathrm{ppm} \\
\mathrm{mg}\end{array}$ & 0.001 & ppm \\
\hline $\begin{array}{l}\text { Concentration } \\
\text { Mass } \\
\text { Benzaldehyde }\end{array}$ & $\begin{array}{r}0.001 \\
0.77\end{array}$ & $\begin{array}{l}\text { ppm } \\
\mathrm{mg}\end{array}$ & 0.001 & $\mathrm{ppm}$ \\
\hline $\begin{array}{l}\text { Concentration } \\
\text { Mass } \\
\text { Hexanaldehyde }\end{array}$ & $\begin{array}{r}0.001 \\
3.91\end{array}$ & $\begin{array}{l}\text { ppm } \\
\text { mg }\end{array}$ & 0.000 & ppm \\
\hline $\begin{array}{l}\text { Concentration } \\
\text { Mass }\end{array}$ & $\begin{array}{r}0.000 \\
0.00\end{array}$ & $\begin{array}{l}\mathrm{ppm} \\
\mathrm{mg}\end{array}$ & 0.000 & $\mathrm{ppm}$ \\
\hline
\end{tabular}

\section{Brake-Specific Results}

Formaldehyde

Acetaldehyde

Acroiein

Acetone

Propionaldehyde

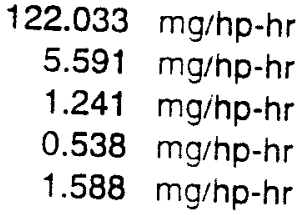

Crotonaldehyde Isobutyr + MEK Benzaldehyde Hexanaldehyde $\begin{array}{ll}0.807 & \mathrm{mg} / \mathrm{hp}-\mathrm{hr} \\ 0.030 & \mathrm{mg} / \mathrm{hp}-\mathrm{hr} \\ 0.155 & \mathrm{mg} / \mathrm{hp}-\mathrm{hr} \\ 0.000 \mathrm{mg} / \mathrm{hp}-\mathrm{hr}\end{array}$ 


\section{Southwest Research Institute - Department of Emissions Research \\ EPA Hot Transient Emission Test Results \\ Project No. 03-7276-004}

Engine Model: 98 Mack ET CNG

Engine Desc.: $\quad 0.0 \mathrm{~L}(0 \mathrm{CID}) 6$

Engine Cycle: Diesel

Engine S/N:
Test No.: 0225-H1

Date: 02/25/1999 Time:09:35

Program HDT: $\quad 4.04-\mathrm{C}$

Cell: 4 Bag Cart: 2
NAT GAS CNG, CARB-CNG

HCR: $\quad 3.849$ FID Resp: 1.13

$\mathrm{H}=0.230 \mathrm{C}=0.712 \mathrm{O}=0.000 \mathrm{X}=0.057$

\section{Ambient/Test Cell Conditions \\ Barometer: \\ Engine Inlet Air \\ Temperature: \\ Dew Point: \\ Abs. Humidity: \\ Rel. Humidity: \\ Dilution Air: \\ Temperature: \\ Abs. Humidity \\ Rel. Humidity: \\ 29.23 in $\mathrm{Hg} \quad 99.0 \mathrm{kPa}$

$\begin{array}{lcl}76.0 & { }^{\circ} \mathrm{F} & 24.4^{\circ} \mathrm{C} \\ 59.9 & { }^{\circ} \mathrm{F} & 15.5^{\circ} \mathrm{C} \\ 79.1 & \text { gr/lb } & 11.3 \mathrm{~g} / \mathrm{kg} \\ 57 & \% & \end{array}$ \\ 74.0 $\quad{ }^{\circ} \mathrm{F} \quad 23.3^{\circ} \mathrm{C}$ \\ $79.8 \quad \mathrm{gr} / \mathrm{lb} \quad 11.4 \mathrm{~g} / \mathrm{kg}$ \\ $62 \%$}

\section{Measured Gaseous Data}

HC Sample

HC Bckgrd

CO Sample

CO Bckgrd

NOx Sample

NOx Bckgrd

CO2 Sample

$\mathrm{CO} 2$ Bckgrd

$\mathrm{CH} 4$ Sample

$\mathrm{CH} 4$ Bckgrd

Meter Range $C$

$\begin{array}{rrrl}\mathrm{n} / \mathrm{a} & & 103.28 & \mathrm{ppm} \\ \mathrm{n} / \mathrm{a} & & 4.40 & \mathrm{ppm} \\ 24.4 & 2 & 23.66 & \mathrm{ppm} \\ \mathrm{n} / \mathrm{a} & 2 & 0.00 & \mathrm{ppm} \\ \mathrm{n} / \mathrm{a} & & 23.26 & \mathrm{ppm}(\text { Dry }) \\ 1.1 & 1 & 0.28 & \mathrm{ppm} \\ 57.3 & 1 & 0.5476 & \% \\ 6.3 & 1 & 0.0574 & \% \\ \mathrm{n} / \mathrm{a} & \mathrm{n} / \mathrm{a} & 81.50 & \mathrm{ppm}(1.13) \\ \mathrm{n} / \mathrm{a} & \mathrm{n} / \mathrm{a} & 2.00 & \mathrm{ppm}\end{array}$

Corrected Concentrations

$\begin{array}{lrl}\mathrm{HC} & 99.13 & \mathrm{ppm} \\ \mathrm{CO} & 22.81 & \mathrm{ppm} \\ \mathrm{NOx} & 22.33 & \mathrm{ppm} \\ \mathrm{CO} 2 & 0.4935 & \% \\ \mathrm{CH} 4 & 79.61 & \mathrm{ppm} \\ \mathrm{RHC} & 9.17 & \mathrm{ppm}\end{array}$

$\mathrm{HC}$

Mass Emissions

$\mathrm{CO}$

NOx

Particulate

$\mathrm{CO} 2$

$\mathrm{CH} 4$

$\mathrm{RHC}$

Fuel

$\begin{array}{rl}73.099 & \text { grams } \\ 33.039 & \text { grams } \\ 53.720 & \text { grams } \\ 0.640 & \text { grams } \\ 11.234 & \mathrm{~kg} \\ 66.039 & \text { grams } \\ 7.060 & \text { grams } \\ \text { 1b } & 4.40 \mathrm{~kg}\end{array}$

Sample Flows

\begin{tabular}{lrr} 
Blower 1 Rate: & scfm & \multicolumn{1}{c}{ scmm } \\
Blower 2 Rate: & $2,181.9$ & 61.79 \\
$90 \mathrm{~mm}$ System: & 0.0 & 0.00
\end{tabular}

$90 \mathrm{~mm}$ System:

Gas Meter 1:

Gas Meter 2:

Sample Rate:

Chemistry Sample Rate:

Total Flow Rate:

\subsection{7}

2.41

0.94

2.54

$2,185.34$

\subsection{4}

0.07

0.03

0.07

61.89
Filter Number: Particulate Data

Weight Gain, mg: $1204.0-12$ (pair)

Sample Multiplier: $\quad 2.327$

NOx Humidity CF:

Dry-to-Wet CF, Sample:

Dry-to-Wet CF, Bckgrd:

Dilution Factor:

\section{Correction Factors}

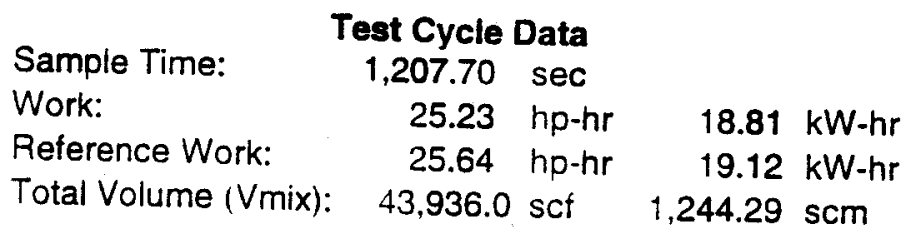

1.011

0.971

0.982

17.36 


\section{Southwest Research Institute - Department of Emissions Research \\ EPA Hot Transient Emission Test Resuits \\ Project No. 03-7276-004}

Engine Model: 98 Mack E7 CNG Engine Desc.: $\quad 0.0 \mathrm{~L}$ (0 CID) 6 Engine Cycle: Diesel Engine S/N:
Test No.: 0225-H1

Date: 02/25/1999 Time: 09:35

Program HDT: $4.04-\mathrm{C}$

Cell: 4 Bag Cart: 2
NAT GAS CNG, CARB-CNG

HCR: 3.849 FID Resp: 1.13

$H=0.230 \mathrm{C}=0.712 \mathrm{O}=0.000 \mathrm{X}=0.057$

Engine Oil:

\begin{tabular}{l} 
Formaldehyde \\
Concentration \\
Mass \\
Acetaldehyde \\
Concentration \\
Mass \\
Acrolein \\
\hline Concentration \\
Mass \\
Acetone \\
Concentration \\
Mass \\
Propionaldehyde \\
Concentration \\
Mass \\
Crotonaldehyde \\
Concentration \\
Mass \\
Isobutyr + MEK \\
Concentration \\
Mass \\
Benzaldehyde \\
Concentration \\
Mass \\
Hexanaldehyde \\
Concentration \\
Mass
\end{tabular}

\begin{tabular}{|c|c|c|c|}
\hline \multicolumn{2}{|c|}{ Sample } & \multicolumn{2}{|c|}{ Background } \\
\hline $\begin{array}{r}1.931 \\
2,908.25\end{array}$ & $\begin{array}{l}\text { ppm } \\
\mathrm{mg}\end{array}$ & 0.005 & ppm \\
\hline $\begin{array}{r}0.063 \\
133.71\end{array}$ & $\begin{array}{l}\text { ppm } \\
\text { mg }\end{array}$ & 0.002 & ppm \\
\hline $\begin{array}{l}0.010 \\
28.43\end{array}$ & $\begin{array}{l}\mathrm{ppm} \\
\mathrm{mg}\end{array}$ & 0.000 & ppm \\
\hline $\begin{array}{r}0.007 \\
6.67\end{array}$ & $\begin{array}{l}\mathrm{ppm} \\
\mathrm{mg}\end{array}$ & 0.004 & ppm \\
\hline $\begin{array}{l}0.013 \\
37.96\end{array}$ & $\begin{array}{l}\mathrm{ppm} \\
\mathrm{mg}\end{array}$ & 0.000 & $\mathrm{ppm}$ \\
\hline $\begin{array}{l}0.006 \\
19.31\end{array}$ & $\begin{array}{l}\mathrm{ppm} \\
\mathrm{mg}\end{array}$ & 0.001 & ppm \\
\hline $\begin{array}{r}0.002 \\
3.11\end{array}$ & $\begin{array}{l}\mathrm{ppm} \\
\mathrm{mg}\end{array}$ & 0.001 & ppm \\
\hline $\begin{array}{r}0.001 \\
4.98\end{array}$ & $\begin{array}{l}\mathrm{ppm} \\
\mathrm{mg}\end{array}$ & 0.000 & ppm \\
\hline $\begin{array}{r}0.000 \\
0.00\end{array}$ & $\begin{array}{l}\mathrm{ppm} \\
\mathrm{mg}\end{array}$ & 0.000 & ppm \\
\hline
\end{tabular}

Background

$0.005 \mathrm{ppm}$

$\mathrm{pm}$

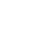

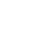




\section{Source:}

Station \#:

Station Name:

Fleld:

Method: c: \ezchrom $\backslash$ methods $\backslash$ scott:

Report Date: 02/22/1999 Flowing Pressure: 0

Flowing Teinp.: $0 \quad$ poig

File: c: lezchrom

Gas Analysis by Chrometograph

vame

ITROGEN

AETHANE

02

ITHANE

$2 S$

ROPANE

30-BUTANE

TANE

-O-PENTANE

ENTANE

EXANES

EPTANES

CTANES

SONONANES

$\begin{array}{rrc}\text { Mole \% } & \text { BTU } & \text { SG } \\ & & \\ 3.592 & 0.000 & 0.035 \\ 90.867 & 917.757 & 0.503 \\ 0.000 & 0.000 & 0.000 \\ 3.660 & 64.767 & 0.038 \\ 0.000 & 0.000 & 0.000 \\ 1.881 & 47.328 & 0.029 \\ 0.000 & 0.000 & 0.000 \\ 0.000 & 0.000 & 0.000 \\ 0.000 & 0.000 & 0.000 \\ 0.000 & 0.000 & 0.000 \\ 0.000 & 0.000 & 0.000 \\ 0.000 & 0.000 & 0.000 \\ 0.000 & 0.000 & 0.000 \\ 0.000 & 0.000 & 0.000\end{array}$

GPM

deal Total

Grose BTU/Real Cu. Ft.

(@ 60 deg $F, 0.000$ )

$100.000 \quad 1029.852 \quad 0.605 \quad 0.51 \%$

Dry $=1032.117$

Sat. $=1015.323$

ctual $=1032.117$

0.000 lbs. water/MMCF)

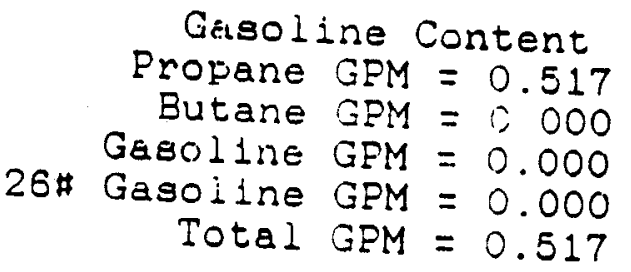

Real Specific Gravity Calculated $=0.6058$

On-Site Specific Gravity $=0.0000$

Gas Compressab111ty $=0.9978$

arks: 
Appendix G

Test Numbers 225-C2 and 225-H2

"1000" Hour Transient Emissions Test 350 BHP Calibration

Maximum Turbo Lag Enrichment $=8 \%$

Schwitzer Turbocharger

Spark Plugs Regapped 


\section{TEST FACILITIES DESCRIPTION}

Emission certification testing was conducted at:

Southwest Research Institute

6220 Culebra Road

P.O. Drawer 28510

San Antonio, TX 78228-0510

Certification activities at Southwest Research Institute were in test cell 4. Cell 4 uses a General absorption ability of $400 \mathrm{hp}$. A Lebow (rotary a Mid-West inductor dyno for a combined power measurement. Air to air intercooling is simulated ss required line torque meter is used for torque conditioned for temperature and humidity. A Lated as required per SAE 1937. Engine inlet air is the engine and dynamometer and acquire dabVIEW -based computer system is used to controf code.

Gaseous emissions are measured dilute utilizing the full-flow dilution method and particulate sampling makes use of the double dilution method. Both primary and secondary dilution air is
filtered and temperature controlled.

The main dilution tunnel uses a positive displacement pump driven at a constant speed in measure the secondary dilutionger. The particulate measurement system uses gas meters to on Pallflex $90 \mathrm{~mm}$ filters. All equipment and procedures used for testing and calculations are in accordance with 40 CFR Part
86 Subpart $N$. 
Southwest Research Institute - Department of Emissions Research
Composite Transient Emission Test Results

Project No. 03-7276-004

Engine Model: 98 Mack E7 CNG

Engine Desc.: $\quad 0.0 \mathrm{~L}$ (O CID) 6

Engine Cycle: Diesel

Engine $\mathrm{S} / \mathrm{N}$ :
Date: 02/25/1999 Time: 02:40

Program HDT: $4.04-\mathrm{C}$

Cell: 4 Bag Cart: 2
NAT GAS CNG, CARB-CNG

HCR: 3.849

FID Resp: 1.13

$\mathrm{H}=0.230 \mathrm{C}=0.712 \mathrm{O}=0.000 \mathrm{X}=0.057$

Engine Oil:

\section{Test Numbers}

Cold: $0225-\mathrm{C} 2$ Hot: $0225-\mathrm{H} 2$

\section{Brake-Specific Emission Results}

BSTHC (Cell)

CO

NOx (Cell)

Particulate

$\mathrm{CO} 2$

BSFC

NMHC

$\mathrm{CH}_{4}$

Work:

Reference Work:
$2.858 \mathrm{~g} / \mathrm{hp}-\mathrm{hr}$

$1.341 \mathrm{~g} / \mathrm{hp}-\mathrm{hr}$

$2.197 \mathrm{~g} / \mathrm{hp}-\mathrm{hr}$

$0.028 \mathrm{~g} / \mathrm{hp}-\mathrm{hr}$

$452.4 \mathrm{~g} / \mathrm{hp}-\mathrm{hr}$

$0.391 \mathrm{lb} / \mathrm{hp}-\mathrm{hr}$

$0.210 \mathrm{~g} / \mathrm{hp}-\mathrm{hr}$

$2.649 \mathrm{~g} / \mathrm{hp}-\mathrm{hr}$

25.14 hp-hr

$25.64 \mathrm{hp}-\mathrm{hr}$
$3.833 \mathrm{~g} / \mathrm{kW}-\mathrm{hr}$

$1.798 \mathrm{~g} / \mathrm{kW}-\mathrm{hr}$

$2.947 \mathrm{~g} / \mathrm{kW}-\mathrm{hr}$

$0.038 \mathrm{~g} / \mathrm{kW}-\mathrm{hr}$

$606.64 \mathrm{~g} / \mathrm{kW}-\mathrm{hr}$

$0.524 \mathrm{~kg} / \mathrm{kW}-\mathrm{hr}$

$0.281 \mathrm{~g} / \mathrm{kW}-\mathrm{hr}$

$3.552 \mathrm{~g} / \mathrm{kW}$-hr

$18.75 \mathrm{~kW}-\mathrm{hr}$

$19.12 \mathrm{~kW}-\mathrm{hr}$ 


\section{Southwest Research Institute - Department of Emissions Research}

EPA Cold Transient Emission Test Resuits

Engine Model: 98 Mack E7 CNG Engine Desc.: $\quad 0.0 \mathrm{~L}$ (0 CID) 6 Engine Cycle: Diesel Engine S/N:
Project No. 03-7276-004
Test No.: 0225-C2

Date: 02/25/1999 Time: 02:40

Program HDT: $\quad 4.04-\mathrm{C}$

Cell: 4 Bag Cart: 2
NAT GAS CNG, CARB-CNG

HCR: $\quad 3.849$ FID Resp: 1.13
$\mathrm{H}=0.230 \mathrm{C}=0.712 \mathrm{O}=0.000 \mathrm{X}=0.057$

\section{Barometer: \\ Engine Inlet Air \\ Temperature: \\ Dew Point: \\ Abs. Humidity: \\ Rel. Humidity: \\ Dilution Air: \\ Temperature: \\ Abs. Humidity \\ Rel. Humidity: \\ 29.11 in $\mathrm{Hg} \quad 98.6 \mathrm{kPa}$ \\ $74.0 \quad{ }^{\circ} \mathrm{F}$ \\ $58.8 \quad{ }^{\circ} \mathrm{F}$ \\ $76.4 \quad \mathrm{gr} / \mathrm{ib} \quad 10.9 \mathrm{~g} / \mathrm{kg}$ \\ $59 \%$

$$
\begin{array}{lcc}
79.0 & { }^{\circ} \mathrm{F} & 26.1{ }^{\circ} \mathrm{C} \\
76.9 & \text { gr/lb } & 11.0 \mathrm{~g} / \mathrm{kg} \\
50 & \% &
\end{array}
$$

Measured Gaseous Data

Meter Range Concentration

HC Sample

HC Bckgrd

CO Sample

CO Bckgrd

NOx Sample

NOx Bckgrd

CO2 Sample

$\mathrm{CO} 2$ Bckgrd

$\mathrm{CH} 4$ Sample

$\mathrm{CH} 4$ Bckgrd

\begin{tabular}{|c|c|}
\hline רcentra & \\
\hline 110.86 & ppm \\
\hline 5.50 & ppm \\
\hline 25.61 & $\mathrm{ppm}$ \\
\hline 0.00 & $\mathrm{ppm}$ \\
\hline 26.99 & ppm (Dry) \\
\hline 0.50 & $\mathrm{ppm}$ \\
\hline 0.5838 & $\%$ \\
\hline 0.0675 & $\%$ \\
\hline 88.12 & ppm (1.13) \\
\hline 2.05 & ppm \\
\hline
\end{tabular}

$\begin{array}{rr}n / a & \\ n / a & \\ 26.4 & 2 \\ n / a & 2 \\ n / a & \\ 0.5 & 2 \\ 60.9 & 1 \\ 7.4 & 1 \\ n / a & n / a \\ n / a & n / a\end{array}$

\begin{tabular}{lrl} 
& \multicolumn{3}{c}{ Corrected Concentrations } \\
$\mathrm{HC}$ & 105.70 & $\mathrm{ppm}$ \\
$\mathrm{CO}$ & 24.76 & $\mathrm{ppm}$ \\
$\mathrm{NOx}$ & 25.75 & $\mathrm{ppm}$ \\
$\mathrm{CO} 2$ & 0.5204 & $\%$ \\
$\mathrm{CH} 4$ & 86.20 & $\mathrm{ppm}$ \\
$\mathrm{RHC}$ & 8.29 & $\mathrm{ppm}$
\end{tabular}

$\mathrm{HC}$

CO

NOx

Particulate

$\mathrm{CO} 2$

$\mathrm{CH} 4$

$\mathrm{RHC}$

Fuel
Mass Emissions

\section{Corrected Concentrations}

$$
77.318
$$

35.600

61.041

0.701

11.761

70.981

6.338 grams

grams

grams

grams

$\mathrm{kg}$

grams

grams

$\begin{array}{ll}\text { NOx Humidity CF: } & 1.004 \\ \text { Dry-to-Wet CF, Sample: } & 0.971 \\ \text { Dry-to-Wet CF, Bckgrd: } & 0.983 \\ \text { Dilution Factor: } & 16.28\end{array}$

Blower 1 Rate:

Blower 2 Rate:

$90 \mathrm{~mm}$ System:

Gas Meter 1:

Gas Meter 2:

Sample Rate:

Chemistry Sample Rate:

Total Flow Rate:

Sample Flows

sctm

$2,166.2$

0.0

scmm

61.35

0.00

1.47

2.38

0.91

2.49

$2,169.55$

0.04

0.07

0.03

0.07

61.44

Filter Number: $\quad \begin{gathered}\text { Particulate Data } \\ \text { 1229.0-13 (pair) }\end{gathered}$

Weight Gain, mg: $\quad 0.294$

Sample Multiplier:

2.383

\begin{tabular}{lrr}
\multicolumn{5}{c}{ Test Cycle Data } \\
Sample Time: & $1,207.60 \mathrm{sec}$ & \\
Work: & $25.25 \mathrm{hp}-\mathrm{hr}$ & $18.83 \mathrm{~kW}-\mathrm{hr}$ \\
Reference Work: & $25.64 \mathrm{hp}-\mathrm{hr}$ & $19.12 \mathrm{~kW}-\mathrm{hr}$ \\
Total Volume (Vmix): & $43,615.7 \mathrm{scf}$ & $1,235.22 \mathrm{scm}$
\end{tabular}

Brake-Specific Emission Resuits
BSTHC (Cell) $3.062 \mathrm{~g} / \mathrm{hp}-\mathrm{hr}$

CO $\quad \begin{array}{lll}3.062 \mathrm{~g} / \mathrm{hp}-\mathrm{hr} & 4.106 \mathrm{~g} / \mathrm{kW}-\mathrm{hr}\end{array}$

NOx (Cell) $2.417 \mathrm{~g} / \mathrm{hp}-\mathrm{hr} \quad 1.891 \mathrm{~g} / \mathrm{kW}-\mathrm{hr}$

Particulate $\quad 0.417 \mathrm{~g} / \mathrm{hp}-\mathrm{hr} \quad 3.242 \mathrm{~g} / \mathrm{kW}-\mathrm{hr}$

$\mathrm{CO} 2$

$0.037 \mathrm{~g} / \mathrm{kW}$-hr

BSFC

$0.028 \mathrm{~g} / \mathrm{hp}-\mathrm{hr}$

$465.8 \mathrm{~g} / \mathrm{hp}-\mathrm{hr}$

$\mathrm{NMHC}$

$0.403 \mathrm{lb} / \mathrm{hp}-\mathrm{hr}$

$624.61 \mathrm{~g} / \mathrm{kW}-\mathrm{hr}$

$\mathrm{CH} 4$

$0.251 \mathrm{~g} / \mathrm{hp}-\mathrm{hr}$

$0.245 \mathrm{~kg} / \mathrm{kW}-\mathrm{hr}$

$0.337 \mathrm{~g} / \mathrm{kW}-\mathrm{hr}$

$3.770 \mathrm{~g} / \mathrm{kW}-\mathrm{hr}$ 
Engine Model: 98 Mack E7 CNG Engine Desc.: $\quad 0.0 \mathrm{~L}$ (0 CID) 6 Engine Cycle: Diese Engine S/N:

\section{Southwest Research Institute - Department of Emissions Research \\ EPA Cold Transient Emission Test Resuits \\ Project No. 03-7276-004}

Test No.: 0225-C2

Date: 02/25/1999 Time:02:40

Program HDT: $\quad 4.04-\mathrm{C}$

Cell: 4 Bag Cart: 2
NAT GAS CNG, CARB-CNG HCR: $\quad 3.849$ FID Resp: 1.13 $H=0.230 \mathrm{C}=0.712 \mathrm{O}=0.000 \mathrm{X}=0.057$

\section{Formaldehyde}

Concentration

Mass

Acetaldehyde

Concentration

Mass

Acrolein

Concentration

Mass

Acetone

Concentration

Mass

Propionaldehyde

Concentration

Mass

Crotonaidehyde

Concentration

Mass

Isobutyr + MEK

Concentration

Mass

Benzaldehyde

Concentration

Mass

Hexanaidehyde

Concentration

Mass
Sample

$1.942 \mathrm{ppm}$

$2,900.67 \mathrm{mg}$

$0.063 \mathrm{ppm}$

$133.11 \mathrm{mg}$

$0.010 \mathrm{ppm}$

$28.12 \mathrm{mg}$

$0.013 \mathrm{ppm}$

$21.60 \mathrm{mg}$

$0.011 \mathrm{ppm}$

$30.80 \mathrm{mg}$

$0.008 \mathrm{ppm}$

$22.79 \mathrm{mg}$

$\begin{array}{rl}0.001 & \mathrm{ppm} \\ 1.82 \mathrm{mg}\end{array}$

$0.001 \mathrm{ppm}$

$3.12 \mathrm{mg}$

$0.000 \mathrm{ppm}$

$0.00 \mathrm{mg}$
Background

0.007 ppm

0.003 ppm

0.000 ppm

$0.005 \mathrm{ppm}$

$0.000 \mathrm{ppm}$

$0.001 \mathrm{ppm}$

$0.000 \mathrm{ppm}$

$0.000 \mathrm{ppm}$

$0.000 \mathrm{ppm}$

\section{Brake-Specific Results}

\section{Formaldehyde}

Acetaldehyde

Acrolein

Acetone

Propionaldehyde

\author{
$114.878 \mathrm{mg} / \mathrm{hp}-\mathrm{hr}$ \\ $5.272 \mathrm{mg} / \mathrm{hp}-\mathrm{hr}$ \\ $1.114 \mathrm{mg} / \mathrm{hp}-\mathrm{hr}$ \\ $0.855 \mathrm{mg} / \mathrm{hp}-\mathrm{hr}$ \\ $1.220 \mathrm{mg} / \mathrm{hp}-\mathrm{hr}$
}

Crotonaldehyde

Isobutyr + MEK

Benzaidehyde

Hexanaldehyde
$0.902 \mathrm{mg} / \mathrm{hp}-\mathrm{hr}$

$0.072 \mathrm{mg} / \mathrm{hp}-\mathrm{hr}$

$0.124 \mathrm{mg} / \mathrm{hp}-\mathrm{hr}$

$0.000 \mathrm{mg} / \mathrm{hp}-\mathrm{hr}$ 


\section{Southwest Research Institute - Department of Emissions Research EPA Hot Transient Emission Test Results \\ Project No. 03-7276-004}

Engine Model: 98 Mack E7 CNG Engine Desc.: $\quad 0.0 \mathrm{~L}(0 \mathrm{CID}) 6$ Engine Cycle: Diesel Engine S/N:
Test No.: 0225-H2

Date: 02/25/1999 Time:03:20

Program HDT: $4.04-\mathrm{C}$
Cell: 4 Bag Cart: 2
NAT GAS CNG, CARB-CNG

HCR: $\quad 3.849$ FID Resp: 1.13

$H=0.230 \mathrm{C}=0.712 \mathrm{O}=0.000 \mathrm{X}=0.057$

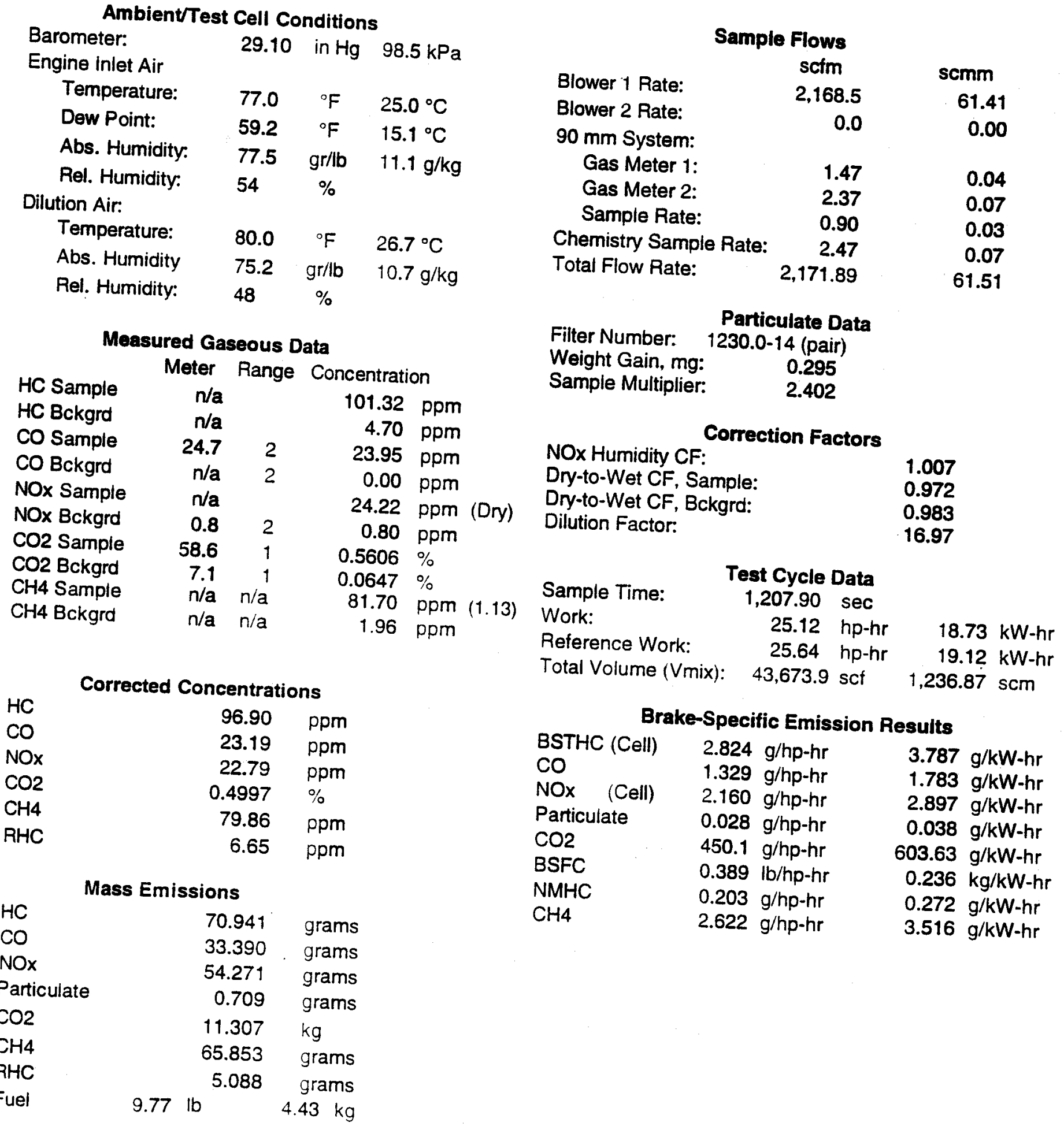




\section{Southwest Research Institute - Department of Emissions Research EPA Hot Transient Emission Test Results \\ Project No. 03-7276-004}

Engine Model: 98 Mack E7 CNG Engine Desc.: $0.0 \mathrm{~L}(0 \mathrm{CID}) 6$ Engine Cycle: Diesel Engine S/N:

Formaldehyde
Concentration
Mass
Acetaldehyde
Concentration
Mass
Acrolein
Concentration
Mass
Acetone
Concentration
Mass
Propionaldehyde
Concentration
Mass
Crotonaldehyde
Concentration
Mass
Isobutyr + MEK
Concentration
Mass
Benzaldehyde
Concentration
Mass
Hexanaldehyde
Concentration
Mass

\author{
Test No.: 0225- $\mathrm{H} 2$ \\ Date: 02/25/1999 Time: 03:20 \\ Program HDT: $4.04-\mathrm{C}$ \\ Cell: 4 Bag Cart: 2
}

\author{
NAT GAS CNG, CARB-CNG \\ HCR: 3.849 FID Resp: 1.13 \\ $H=0.230 C=0.712 O=0.000 X=0.057$ \\ Engine Oil:
}

\begin{tabular}{rl}
\multicolumn{1}{c}{ Sample } & Background \\
$1.904 \mathrm{ppm}$ & $0.007 \mathrm{ppm}$ \\
$2,850.43 \mathrm{mg}$ & \\
& \\
$0.063 \mathrm{ppm}$ & $0.003 \mathrm{ppm}$ \\
$133.52 \mathrm{mg}$ & \\
$0.010 \mathrm{ppm}$ & $0.000 \mathrm{ppm}$ \\
$29.23 \mathrm{mg}$ & \\
$0.010 \mathrm{ppm}$ & $0.005 \mathrm{ppm}$ \\
$14.97 \mathrm{mg}$ & \\
$0.011 \mathrm{ppm}$ & $0.000 \mathrm{ppm}$ \\
$32.45 \mathrm{mg}$ & \\
$0.006 \mathrm{ppm}$ & $0.001 \mathrm{ppm}$ \\
$18.70 \mathrm{mg}$ & \\
$0.001 \mathrm{ppm}$ & $0.000 \mathrm{ppm}$ \\
$3.64 \mathrm{mg}$ & \\
$0.000 \mathrm{ppm}$ & $0.000 \mathrm{ppm}$ \\
$0.00 \mathrm{mg}$ & \\
$0.000 \mathrm{ppm}$ & $0.000 \mathrm{ppm}$ \\
$0.00 \mathrm{mg}$ & \\
\hline
\end{tabular}

\section{Brake-Specific Resuits}

Formaldehyde

Acetaldehyde

Acrolein

Acetone

Propionaldehyde

\footnotetext{
$113.473 \mathrm{mg} / \mathrm{hp}-\mathrm{hr}$

$5.315 \mathrm{mg} / \mathrm{hp}-\mathrm{hr}$

$1.164 \mathrm{mg} / \mathrm{hp}-\mathrm{hr}$

$0.596 \mathrm{mg} / \mathrm{hp}-\mathrm{hr}$

$1.292 \mathrm{mg} / \mathrm{hp}-\mathrm{hr}$
}
Crotonaidehyde Isobutyr + MEK Benzaldehyde Hexanaldehyde

$\begin{array}{ll}0.744 & \mathrm{mg} / \mathrm{hp}-\mathrm{hr} \\ 0.145 \mathrm{mg} / \mathrm{hp}-\mathrm{hr} \\ 0.000 \mathrm{mg} / \mathrm{hp}-\mathrm{hr} \\ 0.000 \mathrm{mg} / \mathrm{hp}-\mathrm{hr}\end{array}$ 
Source:

Station \#:

Station Name:

Fleld:

Report Date: 02/22/1999 Flowilugale Date:

Flowing Temp. 0

Method: c: \ezchrom $\backslash$ methods $\backslash$ scott:

File: c:lezchrom lahrom\031732Ch1u. 2

Gas Analysis by Chrometograph

Name

NITROGEN

METHANE

$\mathrm{CO} 2$

ETHANE

$\mathrm{H} 2 \mathrm{~S}$

PROPANE

ISO-BUTANE

TANE

JO-PENTANE

PENTANE

HEXANES

HEPTANES

DCTANES

ISONONANES

Mole \% BTU SG GPM

$\begin{array}{rrr}3.592 & 0.000 & 0.035 \\ 90.867 & 917.757 & 0.503 \\ 0.000 & 0.000 & 0.000 \\ 3.660 & 64.767 & 0.038 \\ 0.000 & 0.000 & 0.000 \\ 1.881 & 47.328 & 0.029 \\ 0.000 & 0.000 & 0.000 \\ 0.000 & 0.000 & 0.000 \\ 0.000 & 0.000 & 0.000 \\ 0.000 & 0.000 & 0.000 \\ 0.000 & 0.000 & 0.000 \\ 0.000 & 0.000 & 0.000 \\ 0.000 & 0.000 & 0.000 \\ 0.000 & 0.000 & 0.000\end{array}$

Igal Total

$$
100.000 \quad 1029.852 \quad 0.605 \quad 0.51 \%
$$

Gross BTU/Real Cu. Ft.

(@60 deg F, 0.000 )

Dry $=1032.117$

Sat. $=1015.323$

Actual = 1032.117

0.000 (ba. water/MMCF)

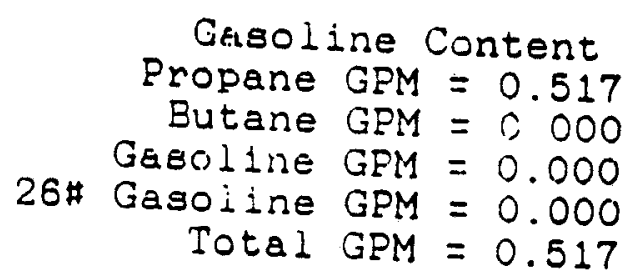

Real Specifio Gravity Calculated $=0.6058$

On-Site Specific Gravity $=0.0000$

Gas Compreesability $=0.9978$ 
Appendix H

Test Number C061698-MACK03

"0" Hour Idle CO Test

Deterioration Factor Calibration

Garrett Turbocharger 


\section{TEST FACILITIES DESCRIPTION}

Emission certification testing was conducted at:

Southwest Research Institute

6220 Culebra Road

P.O. Drawer 28510

San Antonio, TX 78228-0510

Certification activities at Southwest Research Institute were in test cell 3. Cell 3 uses a General Electric direct current dyno in combination with a Mid-West inductor dyno for a combined power measurement. Air to air intercooling is simy transformer) in line torque meter is used for torque conditioned for temperature and hum is simulated as required per SAE 1937. Engine inlet air is throttle control and a Compudas computer A LabVIEW -based computer system is used for engine and acquire data. The data is then processed via SwRI Fortran code.

Gaseous emissions are measured dilute uniling the fullflow diltin sampling makes use of the double dilutio utilizing the full-flow dilution method and particulate filtered and temperature controlled.

The main dilution tunnel uses a positive displacement pump driven at a constant speed in combination with a heat exchanger. The particulate measurement system uses gas meters to on Pallflex $90 \mathrm{~mm}$ filters.

All equipment and procedures used for testing and calculations are in accordance with 40 CFR Part
86 Subpart $N$. 
$\frac{03-7276-004}{\text { Project Number }}$

\section{HEAVY-DUTY OTTO-CYCLE ENGINE
USING RAW EMISSION SAMPLING}

CO61698-mACKO3

Test Number

\begin{tabular}{|c|c|c|c|}
\hline Date: $6-16-98$ Time: $\mathrm{PM}$ & Engine Model: & $M A C K$ & \\
\hline Cell No.: 3 & Serial No. or ID: 5 & $5 N 1311$ & \\
\hline Engine Operator: J. ELIZONDO & Curb Idle Speed: 6 & $600 \pm 5 \mathrm{pm}$ & Project Leader: M. STARR \\
\hline CO ANALYZER & Analyzer 10: & 000210 & Range Used: 3,000 PPM \\
\hline Cart Operator: W. SHACKELFORD & Calibration Date: 5 & $5-25-98$ & Curve Date: $5 \cdot 25-98$ \\
\hline Uf Replace Flip-Top Filier & If Calibrate Temper: & rature & eak Chock Sampling System \\
\hline Pre-Test Zero: $\varnothing$ md & & Pre-Test Span: & $89.4 \mathrm{md} / 2,682.96$ \\
\hline Post-Test Zero: $-0.4 \mathrm{md}$ & & Post-Test Span: & $89.6 \mathrm{md}$ \\
\hline$-0.4 \mathrm{md}$ & (Limit = 2 MO) & Difference: & $+0.2 \mathrm{md}$ \\
\hline
\end{tabular}

1. WARM- UP ENGINE: $\varangle$-Transient Test $\square$-Power Map

2. OPERATE ENGINE AT 200000 RPM FOR 60 SECONDS:

(<1 minune prior to Slep 2)

2.a. Barometric Pressure:

2.b. Engine Intake Air Dewpoint: $\frac{28.88}{58.0}{ }^{\circ} \mathrm{F}$

2.c. Engine Intake Air Temperature:

2.d. Time at $250000 \mathrm{rmm}: 60$

90 ' ${ }^{\circ} \mathrm{F}$

3. CUAB IDLE SPEED FOR 30 SECONDS (zero torque \& CO to Sample) : 30 Seconds $( \pm 5) 600 \mathrm{rm}$

4. CO TEST SAMPLING AT IDLE FOR $60 \rightarrow 64$ SECONDS:

4.a. CO Analyzer Iniet Pressure:

4.b. CO Analyzer Sample Gas Temperature: $\frac{3}{71.8} " \mathrm{H}_{2} \mathrm{O}$ (Start of Sample)

4.c. CO Analyzer Intet Pressure

4.c. CO Analyzer Inlet Pressure: $\quad 3 \quad \mathrm{H}_{2} \mathrm{O}$ (End of Sample)

4.d. CO Test Sampling Period: 60 Seconds $(60 \rightarrow 64)$

5. IMMEDIATE POST-SAMPLING DATA AT IDLE:

5.a. Barometric Pressure:

5.b. Engine Intake Air Dewpoint: $\frac{28.86}{58.6}{ }^{\circ} \mathrm{Hg}$

5.c. Engine intake Air Temperature: $90{ }^{\circ} \mathrm{F}$

of No Comment

a Comments Provided on Test Comment Form

NCAR Submitted

CO VALUE: Average $C O$.over 60 second sampling period in Step 4. (Use 10 meter aivision readings at 6-second intervals) $0.1 *(\underline{50.0}+\underline{49.0}+48.3+48.3+\underline{49.0}+\underline{47.7}+\underline{48.4}+\underline{47.4}+\underline{48.2}+47.5)=48.4$ IDLE CO: $0.11 \%$ (On a Dry Basis - convert as necessary.) 
Source:

Station \#:

Station Name: iv

Field:

Report Date: 06/05/1998 Flowing Preseure:

Flowing Temp.: 0

p818

Method: c: \ezchrom $\backslash$ methods $\backslash$ scott.

Flle: c: lezchrom \chrom $\backslash 037276$ eg. 3

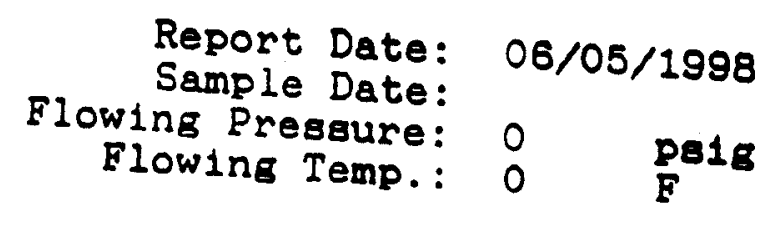

ilame

Gas Analysis by Chromatograph

NITROGEN

METHANB

$\mathrm{CO} 2$

ETHANB

$\mathrm{H} 2 \mathrm{~S}$

PROPANE

ISO-BUTANE

UTANE

ISO-PENTANE

PENTANE

HEXANBS

HBPTANES

DCTANES

I SONONANES

Mole \%

BTU SG

GPM

3.661

90.603

0.000

3.672

0.000

2.064

0.000

0.000

0.000

0.000

0.000

0.000

0.000

0.000

$0.000 \quad 0.035$

$915.090 \quad 0.502$

$0.000 \quad 0.000$

$64.980 \quad 0.038$

$0.000 \quad 0.000$

$51.932 \quad 0.031$

$0.000 \quad 0.000$

0.567

$0.000 \quad 0.000$

$0.000 \quad 0.000$

$0.000 \quad 0.000$

$0.000 \quad 0.000$

$0.000 \quad 0.000$

$0.000 \quad 0.000$

$0.000 \quad 0.000$

[deal Total

Grose BTU/Real Cu. Ft.

$\begin{array}{llll}100.000 & 1032.002 & 0.607 & 0.567\end{array}$

(@60 deg $F, 0.000$ )

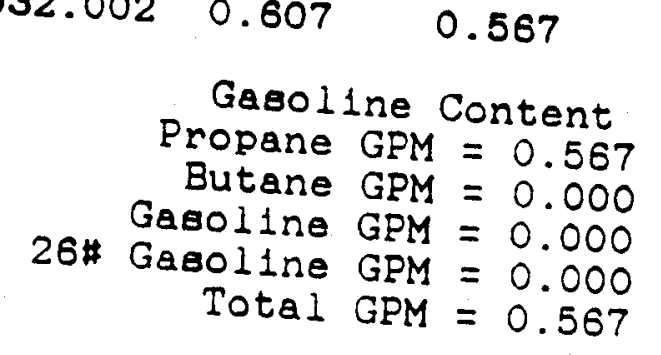

Real Specific Gravity Calculated $=0.6079$

$$
\begin{aligned}
\text { On-Site Specific Gravity }=0.0000
\end{aligned}
$$

Gas Compressab111ty $=0.9978$

nerks: 
APPENDIX H

FINAL CALIBRATION

$350 \mathrm{bhp}$ 
GEM Lean Burn Control System Monitor

PROM ID: 2570-008- 09/11/98

\begin{tabular}{|c|c|c|c|c|c|c|}
\hline $\begin{array}{l}\text { Speed } \\
\text { MAP }\end{array}$ & $\begin{array}{r}0 \\
14.4\end{array}$ & $\begin{array}{l}\text { rpm } \\
\text { psia }\end{array}$ & $\begin{array}{l}\text { - Raw Voltages } \\
\text { - MAP raw }\end{array}$ & & & - Diagnostics: \\
\hline ECT & $\begin{array}{r}14.4 \\
128\end{array}$ & ${ }_{F}$ & - ECT raw & $\begin{array}{l}1.57 \\
1.43\end{array}$ & $\begin{array}{l}\text { volts } \\
\text { volts }\end{array}$ & - NORMAL \\
\hline MAT & 122 & ${ }^{\circ} \mathrm{F}$ & - MAT raw & 1.37 & volts & - Spark: \\
\hline PTP & 14.2 & psia & - PTP raw & 1.63 & volts & - (firing order) \\
\hline EBP & 14.41 & psia & - EBP raw & 2.41 & volts & - 1 - ENABLED \\
\hline NGP & 57.8 & psia & - NGP raw & 1.67 & volts & - 2 - ENABLED \\
\hline NGT & 106 & ${ }^{\circ} \mathrm{F}$ & - NGT raw & 1.96 & volts & - 3 - ENABLED \\
\hline NGTP & 0 & psig & - NGTP raw & 0.02 & volts & - 4 - ENABLED \\
\hline NGTT & -50 & ${ }^{\circ} \mathrm{F}$. & - NGTT raw & 5.00 & volts & - 5 - ENABLED \\
\hline UEGO & 1.006 & phi & - UEGO raw & 0.94 & volts & - 6 - ENABLED \\
\hline $\begin{array}{l}\text { FPP } \\
\text { TPS Command }\end{array}$ & 0.51 & $\frac{8}{8}$ & - FPP raw & 0.41 & volts & - 7 - ENABLED \\
\hline $\begin{array}{l}\text { TPS_Command } \\
\text { TPS_Feedbck }\end{array}$ & 1.99 & $\begin{array}{l}\text { volts } \\
\text { volts }\end{array}$ & & $\cdots \cdots$ & $\ddot{m} \ddot{0}$ & $\because 8$ - ENABLED \\
\hline $\begin{array}{l}\text { TPS_Feedbck } \\
\text { Baro }\end{array}$ & $\begin{array}{r}0.02 \\
14.71\end{array}$ & $\begin{array}{l}\text { volts } \\
\text { psia }\end{array}$ & - Controlmode & 00: & $\begin{array}{l}00: 00 \\
L O O P\end{array}$ & \\
\hline Battery & $\begin{array}{r}14.2 \\
14.2\end{array}$ & volts & - RunMode & STOI & PPED & - 1 - ENABLED \\
\hline SparkAdv & 5.0 & ${ }^{\circ} \mathrm{BTDC}$ & - & & & - 2 - ENABLED \\
\hline PW & 30.7 & $\mathrm{~ms}$ & - & & & - 3 - ENABLED \\
\hline CL_Mult & +0.00 & \% & $\therefore$ FPP Minimum & 0.41 & volts & - 4 - ENABLED \\
\hline AL_Mult & +0.00 & 8 & lelGauge & 0 & 8 & - \\
\hline
\end{tabular}




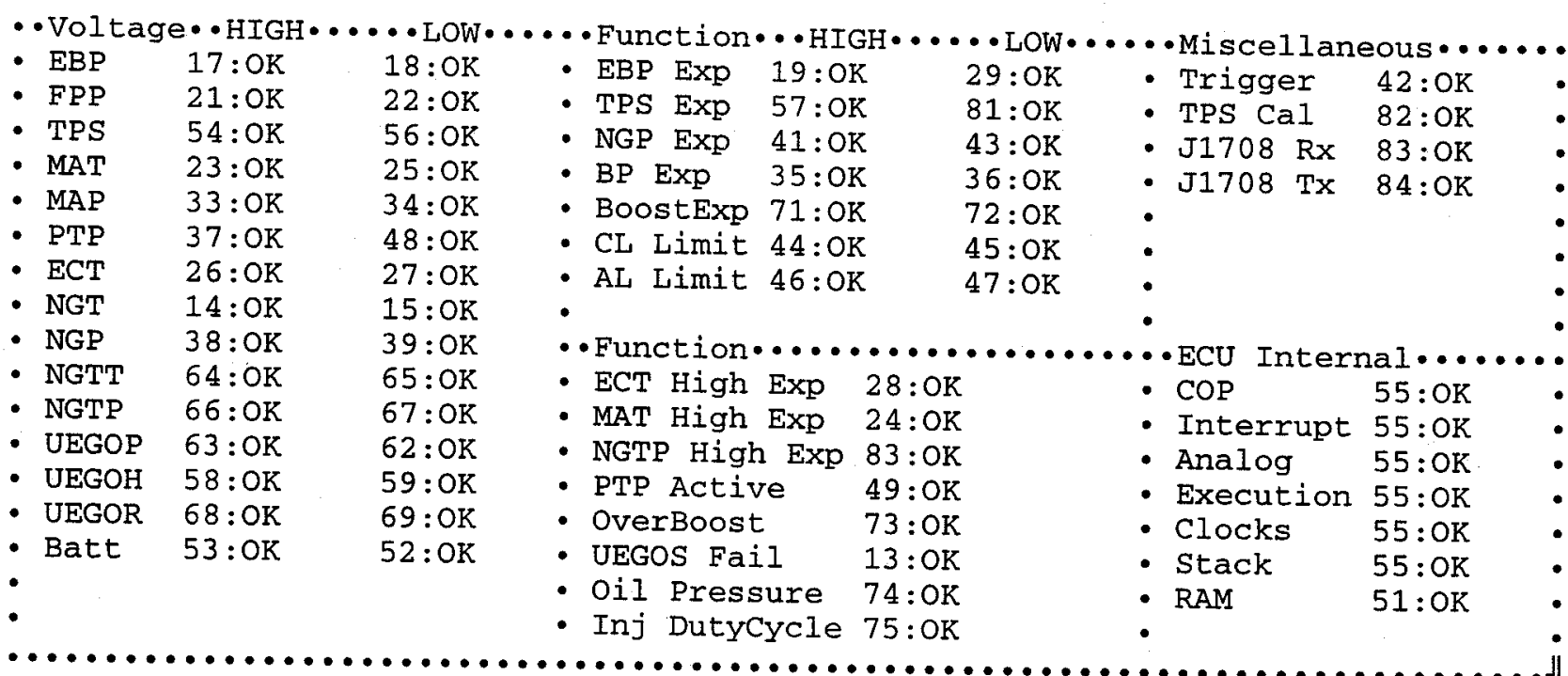


GEM Lean Burn Control System Diagnostics

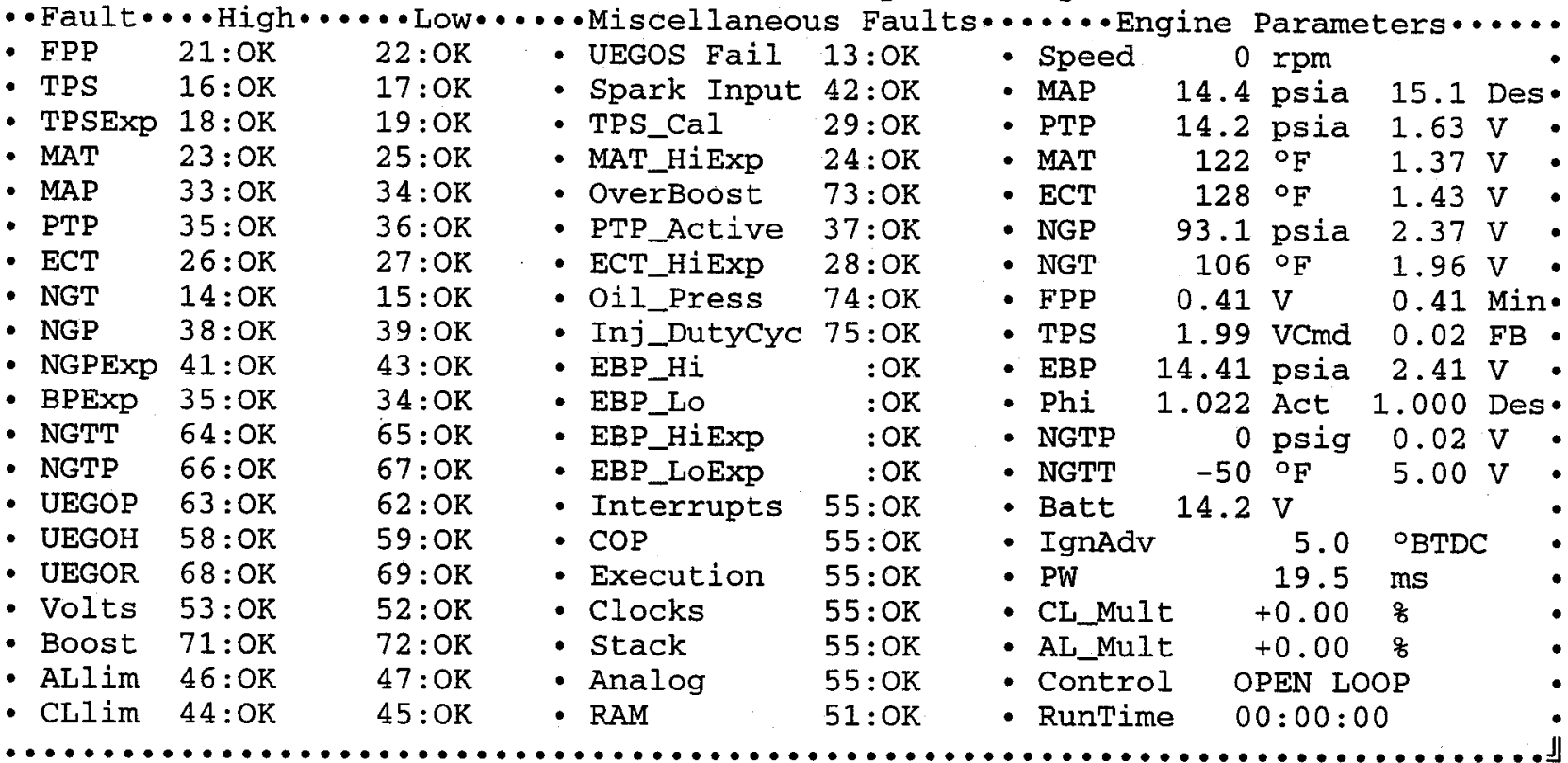


GEM Lean Burn Control System - Drive-By-Wire Parameters

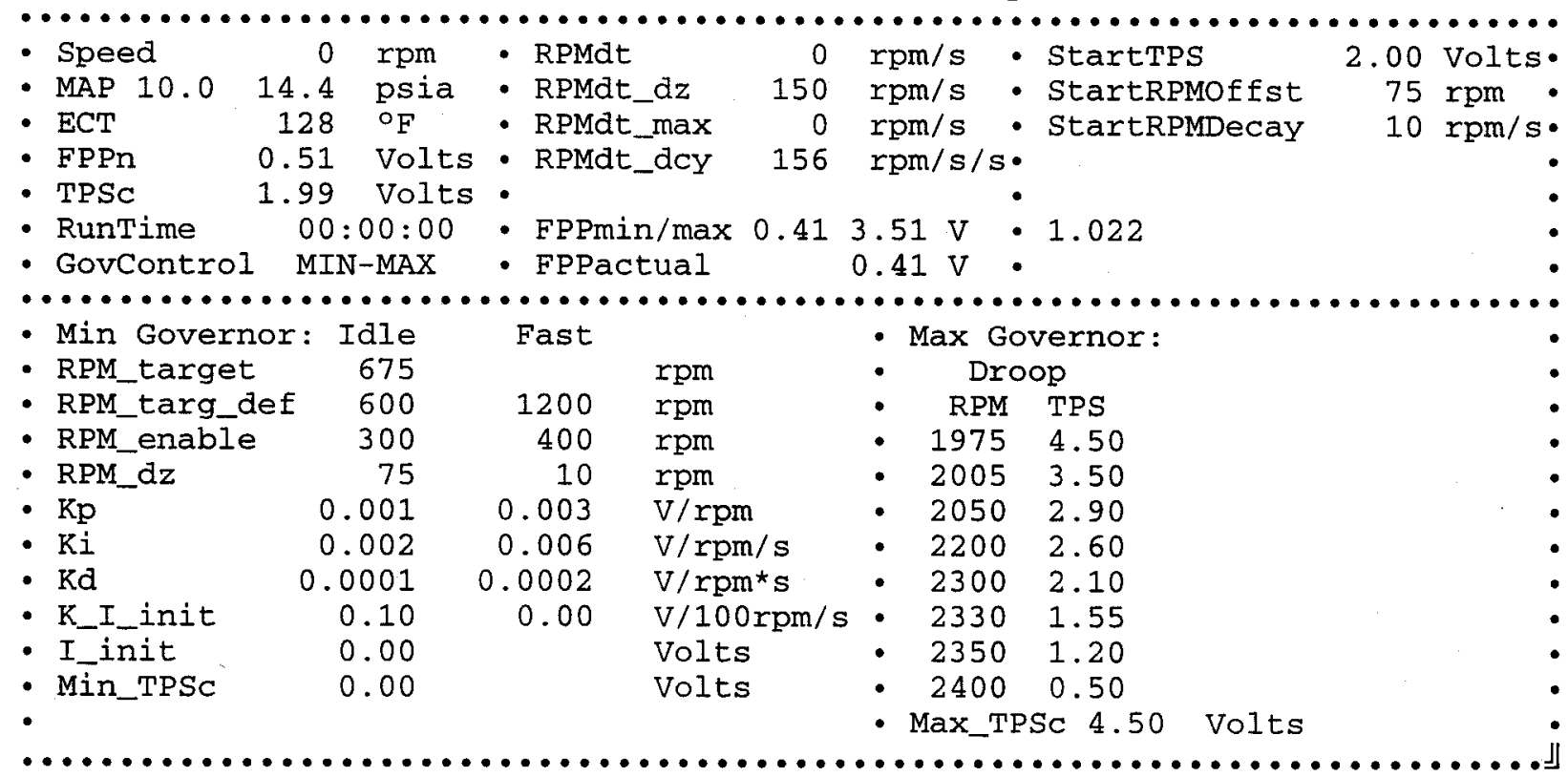


GEM Lean Burn Control System - ECT Based Modification Tables

\begin{tabular}{|c|c|c|c|c|c|c|c|}
\hline $\begin{array}{ll}\text { - } & \text { ECT } \\
\text { - } & \left.{ }^{\circ} \mathrm{F}\right)\end{array}$ & $\begin{array}{c}\text { Idle Offset } \\
\text { (rpm) }\end{array}$ & $\begin{array}{l}\text { Adv Offset } \\
\text { (CAD BTDC) }\end{array}$ & $\begin{array}{c}\text { Max Boost } \\
\text { (psia) }\end{array}$ & $\begin{array}{l}\text { Phi Cold } \\
(z)\end{array}$ & - ECT & 128 & ${ }^{\circ} \mathrm{F}$ \\
\hline - -40 & 50 & +5.0 & 30.0 & 100 & - Speed & 0 & $\mathrm{rpm}$ \\
\hline-20 & 50 & +5.0 & 30.0 & 100 & - Idle & 675 & rpm \\
\hline 0 & 50 & +5.0 & 30.0 & 100 & - Idle_def & 600 & $\mathrm{rpm}$ \\
\hline 32 & 50 & +5.0 & 30.0 & 100 & - & & 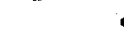 \\
\hline 50 & 50 & +5.0 & 30.0 & 75 & - IgnAdv & 5.0 & ${ }^{\circ} \mathrm{BTDC}$ \\
\hline 70 & 50 & +3.0 & 30.0 & 75 & - Ignoffst & +0.0 & ${ }^{\circ} \mathrm{BTDC}$ \\
\hline 90 & 50 & +0.0 & 30.0 & 60 & - & & s \\
\hline 110 & 50 & +0.0 & 32.1 & 50 & - MAP & 14.4 & psia \\
\hline 130 & 50 & +0.0 & 35.1 & 30 & - Max_boost & 15.1 & psia \\
\hline 155 & 0 & +0.0 & 35.1 & 30 & - Max_boost_s & 15.1 & psia \\
\hline 160 & 0 & +0.0 & 45.0 & 0 & - & & Fin \\
\hline 190 & 0 & +0.0 & 45.0 & 0 & - PhiECT & 32 & $\%$ \\
\hline 200 & 0 & +0.0 & 45.0 & 0 & - & & \\
\hline 210 & 0 & +0.0 & 45.0 & 0 & - & & s \\
\hline 220 & 0 & +0.0 & 45.0 & 0 & - & & \\
\hline 230 & 0 & +0.0 & 45.0 & 0 & - & & \\
\hline
\end{tabular}


GEM Lean Burn Control System - Miscellaneous Calibrations

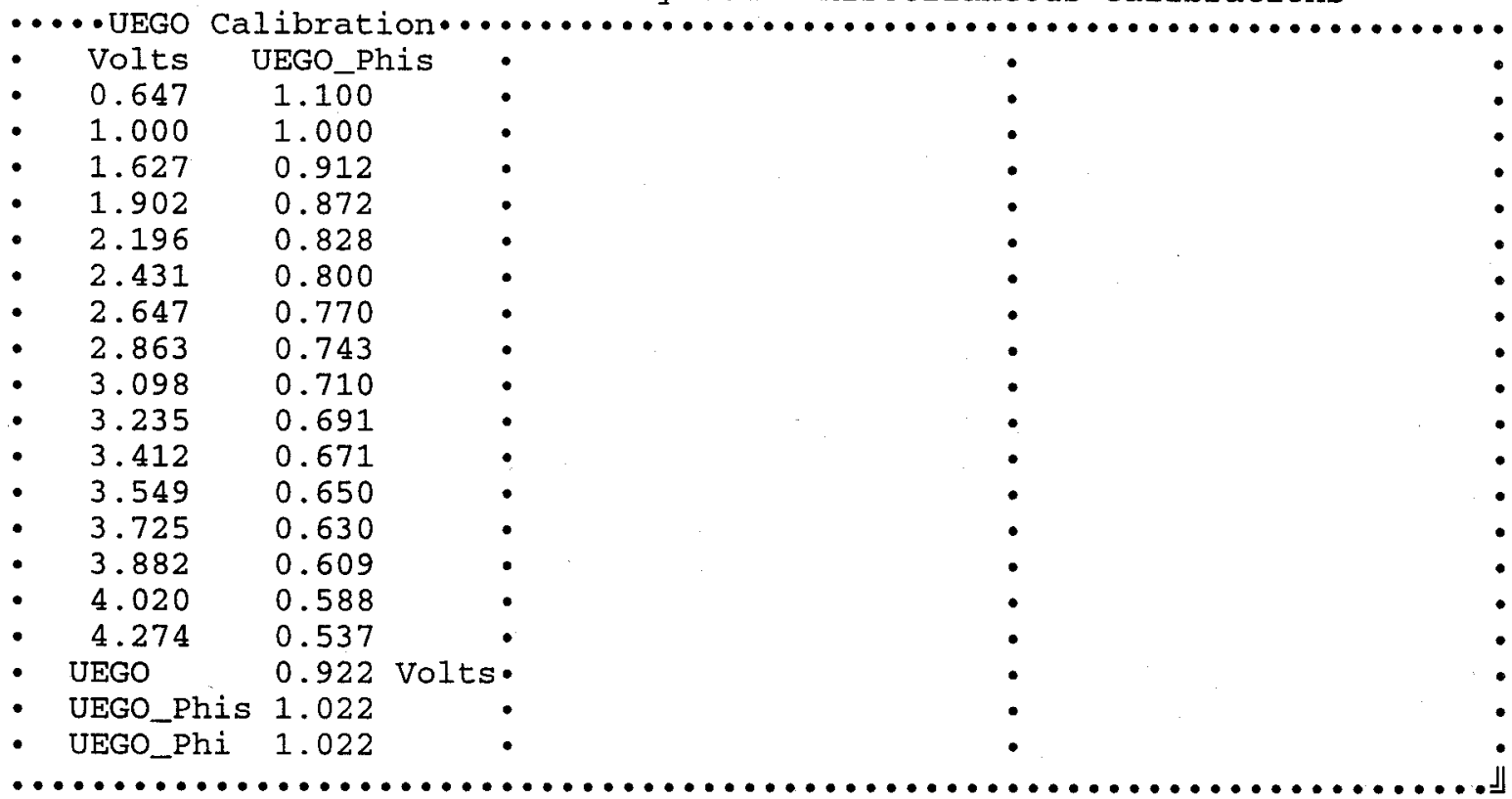


GEM Lean Burn Control System - Closed Loop Activation Map $0.00 \quad$ MAP (psia)

\begin{tabular}{|c|c|c|c|c|c|c|c|c|c|c|c|c|c|c|c|c|}
\hline & 0.0 & & 5.96 & & 11.9 & & 8.0 & 2 & 4.0 & & 9.9 & & 35.2 & & 12.0 & \\
\hline & & 4. & & 8.94 & & 14.72 & & 21.03 & & 26.99 & & 32.95 & & 38.03 & & 45.04 \\
\hline 0 & 0 & 0 & 0 & 0 & 0 & 0 & 0 & 0 & 0 & 0 & 0 & 0 & 0 & 0 & 0 & 0 \\
\hline 200 & 0 & 0 & 0 & 0 & 0 & 0 & 0 & 0 & 0 & 0 & 0 & 0 & 0 & 0 & 0 & 0 \\
\hline 400 & 0 & 0 & 1 & 1 & 1 & 1 & 1 & 1 & 1 & 1 & 1 & 1 & 1 & 1 & 0 & 0 \\
\hline 600 & 0 & 0 & 1 & 1 & 1 & 1 & 1 & 1 & 1 & 1 & 1 & 1 & 1 & 1 & 0 & 0 \\
\hline 800 & 0 & 0 & 1 & 1 & 1 & 1 & 1 & 1 & 1 & 1 & 1 & 1 & 1 & 1 & 0 & 0 \\
\hline 1000 & 0 & 0 & 1 & 1 & 1 & 1 & 1 & 1 & 1 & 1 & 1 & 1 & 1 & 1 & 0 & 0 \\
\hline 1100 & 0 & 0 & 1 & 1 & 1 & 1 & 1 & 1 & 1 & 1 & 1 & 1 & 1 & 1 & 0 & 0 \\
\hline 1250 & 0 & 0 & 1 & 1 & 1 & 1 & 1 & 1 & 1 & 1 & 1 & 1 & 1 & 1 & 0 & 0 \\
\hline 1500 & 0 & 0 & 1 & 1 & 1 & 1 & 1 & 1 & 1 & 1 & 1 & 1 & 1 & 1 & 0 & 0 \\
\hline 1700 & 0 & 0 & 1 & 1 & 1 & 1 & 1 & $\overrightarrow{1}$ & 1 & 1 & 1 & 1 & 1 & 1 & 0 & 0 \\
\hline 1950 & 0 & 0 & 1 & 1 & 1 & 1 & 1 & 1 & 1 & 1 & 1 & 1 & 1 & 1 & 0 & 0 \\
\hline 2000 & 0 & 0 & 1 & 1 & 1 & 1 & 1 & 1 & 1 & 1 & 1 & 1 & 1 & 1 & 0 & 0 \\
\hline
\end{tabular}

- Speed

- MAP

$0 \mathrm{rpm}$

- CL_select

- FSO_on

14.4 psia

$1150 \mathrm{rpm}$

- Phi

0

- UEGO_Phi

1.000

$(0-1)$. ControlMode OPEN LOOP

- FSO_off

$850 \mathrm{rpm}$

- FSO_TPS_min

1.022

- CL_Mult

$+0.00$

- Adpt_Mult

$+0.00$

- Block_Mult 100.0

8
8
8
8


GEM Lean Burn Control System - Manifold Filling Calibration

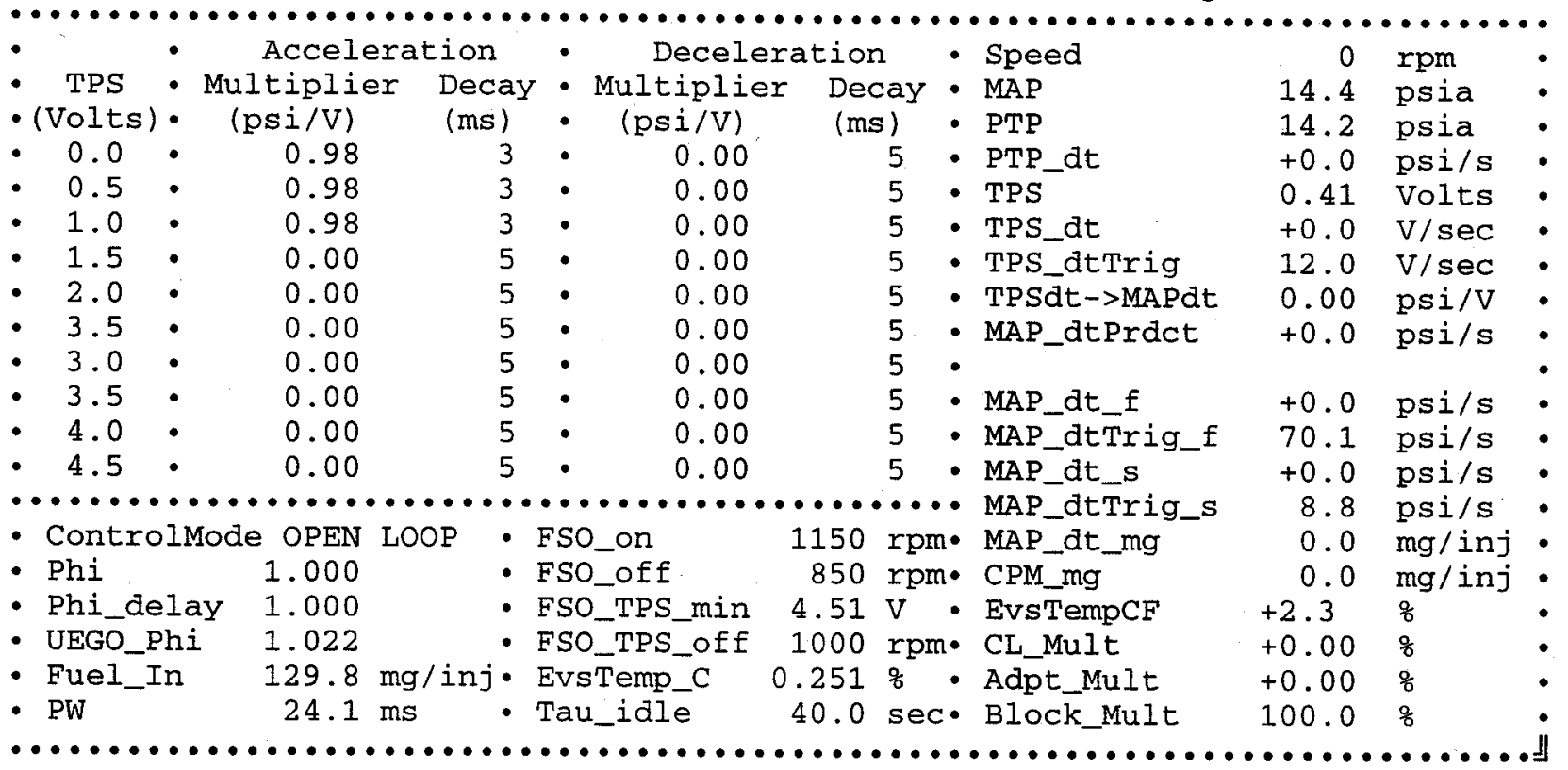


GEM Lean Burn Control System - Turbo Lag Compensation Screen TPSC (volts) 


\begin{tabular}{|c|c|c|c|c|c|c|c|c|c|c|c|c|c|}
\hline GE & ean & 3urn & $\begin{array}{l}\text { Cont } \\
\text { TPS }\end{array}$ & $\begin{array}{l}\text { rol s } \\
\text { (volt }\end{array}$ & $\begin{array}{l}\text { ystem } \\
\text { s) }\end{array}$ & $-B$ & $\because \mathrm{BC}$ & st & $\begin{array}{l}\text { ntrol C } \\
\text { - Boost }\end{array}$ & $\begin{array}{l}b r \\
x I\end{array}$ & $\begin{array}{l}\text { tion } \\
t \cdots\end{array}$ & $\begin{array}{l}\text { reer } \\
\text { ed.I }\end{array}$ & Kd_PTP. \\
\hline & 2.00 & 2.51 & 2.75 & 3.00 & 3.39 & 3.80 & 4.06 & 4.29 & - (psia) & (q) & - & pm) & $\left.\frac{q}{\partial s} / p\right) \cdot$ \\
\hline 650 & 15.1 & 15.9 & 15.9 & 16.5 & 17.0 & 17.0 & 17.0 & 17.0 & 15.1 & 80.0 & 0. & 50 & 0.0. \\
\hline 900 & & 15.9 & 17.0 & 18.1 & 18.9 & 20.0 & 22.1 & 24.0 & 18.1 & 80.0 & 0 & 00 & 0.0. \\
\hline 1100 & 15 & 17.0 & 20.0 & 22.1 & 24.0 & 25.1 & 26.1 & 27.5 & 20.0 & 80.0 & 0 & 800 & 2.0 \\
\hline 1200 & & 18.1 & 22.1 & 25.1 & 28.0 & 31.0 & 34.5 & 35.2 & 22.1 & 80.0 & 0 . & 000 & 2.0 \\
\hline 1250 & & 17.0 & 20.0 & 23.0 & 25.9 & 28.0 & 30.8 & 33.8 & 25.9 & 80.0 & $\cdots \cdot \mathrm{B}$ & $P \bullet \cdot I$ & Derate \\
\hline 1500 & 15 & 15.9 & 17.5 & 20.0 & 24.0 & 26.5 & 29.6 & 32.4 & 9 & 80.0 & 0 & 10.0 & 5.0 \\
\hline 1700 & & 15.9 & 17.5 & 18.9 & 23.0 & 25.9 & 27.5 & .2 & .0 & 80.0 & 0 & 11.5 & 0.0 \\
\hline 1950 & & 15.9 & 17.0 & 8.9 & 22.1 & 25.1 & 28.0 & 31.0 & 35.1 & 80.0 & 0. & .0 & sDerate \\
\hline 1970 & 15.1 & 15.9 & 17.0 & 18.9 & 22.1 & 24.0 & 25.9 & 28.9 & 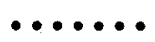 & $\ldots$ & .... & $\ldots$ & $\ldots \ldots$ \\
\hline 2150 & 15 & 15.1 & 15.1 & 15.1 & 15.1 & 15.4 & 15.9 & 16.3 & - MAP & & 14.4 & psic & \\
\hline 2200 & 15.1 & 15.9 & 15.1 & 15.1 & 15.1 & 15.1 & 15.1 & 15.1 & - Max_b & $t$ & 15.1 & psi. & \\
\hline 2700 & 1 & 15.1 & 15.1 & 15.1 & 15.1 & 15.1 & 15.1 & 15.1 & - Max_b & t_s & 15.1 & psi & \\
\hline & & Maxir & ת & & & & sia) & & - Min_b & st & 26.6 & psi & \\
\hline & & $\cdots$ & $\bullet \bullet$ & $\cdots$ & • & $\cdots$ & 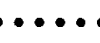 & & - $\mathrm{Pr}$ & & 0.8 & $\mathrm{dPh}$ & $2 i / \mathrm{dMAP}$ \\
\hline Speed & & 0 & & - Phi & & & .000 & & - Kp_MA & & 12.00 & $q / p$ & $s i$ \\
\hline - PTP & & 4.2 & sia & - UEG & D_Phi & & .022 & & - Ki_MA & & 6.00 & $\frac{\circ}{6} / \mathrm{p}$ & si/sec \\
\hline MAT & & 22 & & - Ign & Adv & & 5.0 & ${ }^{\circ} \mathrm{BTD}$ & $\therefore$ Kd_MA & & 2.00 & $\% / p$ & $\operatorname{si*} \sec$ \\
\hline FPPn & & 51 & Lts & - Ign & ffset & & -0.0 & ${ }^{\circ} \mathrm{BTD}$ & $\therefore$ Kd_PT & & 0.00 & $\% / p$ & si*sec \\
\hline 1500 & & & LS & - EBP & & & .41 & psia & - Boost & & 0.0 & q & \\
\hline
\end{tabular}




\begin{tabular}{|c|c|c|c|c|c|c|c|c|c|c|c|c|c|c|c|c|c|}
\hline & & $\mathrm{M} \mathrm{L} \in$ & an $\mathrm{F}$ & Burn & Cont & trol & Syst & tem & $\begin{array}{l}\text { COI } \\
\text { (ps }\end{array}$ & $\begin{array}{l}\text { LD Pl } \\
\text { sia) }\end{array}$ & $\mathrm{hi} C$ & alibr & atio & on $S$ & zreen & & \\
\hline & & 0.00 & & 5.96 & & 11.92 & & 18.05 & & 24.01 & & 29.97 & & 35.2 & & 2.06 & \\
\hline & & & 4.03 & & 8.94 & & 14.72 & & 21.03 & & 26.9 & & 2.95 & & 38.03 & & 15.04 \\
\hline & 0 & 100 & 100 & 100 & 100 & 100 & 100 & 100 & 100 & 100 & 100 & 100 & 100 & 100 & 100 & 100 & 100 \\
\hline & 200 & 100 & 100 & 100 & 100 & 100 & 100 & 100 & 100 & 100 & 100 & 100 & 100 & 100 & 100 & 100 & 100 \\
\hline & 400 & 95 & 95 & 95 & 95 & 95 & 90 & 83 & 83 & 83 & 83 & 83 & 83 & 83 & 83 & 83 & 80 \\
\hline & 600 & 95 & 95 & 95 & 95 & 95 & 90 & 80 & 79 & 78 & 78 & 78 & 78 & 78 & 78 & 78 & 70 \\
\hline $\mathrm{R}$ & 800 & 95 & 95 & 95 & 95 & 95 & 90 & 80 & 79 & 78 & 78 & 73 & 73 & 71 & 71 & 71 & 68 \\
\hline$P$ & 1000 & 95 & 95 & 95 & 95 & 90 & 85 & 80 & 80 & 80 & 77 & 71 & 71 & 71 & 71 & 71 & 64 \\
\hline M & 1100 & 93 & 82 & 82 & 80 & 80 & 80 & 80 & 80 & 80 & 80 & 80 & 77 & 73 & 69 & 69 & 64 \\
\hline & 1250 & 93 & 82 & 77 & 74 & 72 & 69 & 67 & 67 & 67 & 67 & 67 & 67 & 67 & 67 & 67 & 64 \\
\hline & 1500 & 93 & 82 & 77 & 74 & 72 & 69 & 67 & 67 & 67 & 67 & 67 & 67 & 67 & 67 & 67 & 64 \\
\hline & 1700 & 93 & 82 & 77 & 74 & 72 & 69 & 67 & 67 & 67 & 67 & 67 & 67 & 67 & 67 & 67 & 64 \\
\hline & 1950 & 93 & 82 & 77 & 74 & 72 & 69 & 67 & 67 & 67 & 67 & 67 & 67 & 67 & 67 & 67 & 64 \\
\hline & 2000 & 93 & 82 & 77 & 74 & 72 & 69 & 67 & 67 & 67 & 67 & 67 & 67 & 67 & 67 & 67 & 64 \\
\hline & & & & & & COLD & 7 & $\mathrm{rale}$ & ce & Rat & & $\mathrm{Phi}$ * & 0) & & & & \\
\hline & & & & & & $\cdots$ & & & 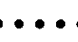 & 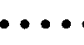 & & & & & & & \\
\hline & beed & & & $\mathrm{rpm}$ & & Phi & & & .000 & & & - $\mathrm{Co}$ & ontro & LlMo & e OP & PEN I & LOOP \\
\hline - 1 & & & .4 & psia & • & UEGC & O_Phi & & .006 & & & - $\mathrm{CL}$ & _Mu & & & 0.00 & 8 \\
\hline & InAdV & & 5.0 & O BTD & $\mathrm{C}$ & Phi & $\mathrm{ECT}$ & & 3 & 8 & & - Ad & apt 1 & Mult & & 0.00 & $q$ \\
\hline & $10 \mathrm{ffs}$ & & & BTDC & $\mathrm{C} \cdot$ & Phil & MAP & & .8 & $\mathrm{dP}$ & IAP & - $\mathrm{B}$ & 109 & Mor & & 00.0 & 8 \\
\hline
\end{tabular}


GEM Lean Burn Control System - BASE Phi Calibration screen $\begin{array}{llllllllll}0.00 & 5.96 & 11.92 & 18.05 & 24.01 & 29.97 & 35.23 & 42.06\end{array}$

$\begin{array}{lllllllllllllllll} & 100 & 100 & 100 & 100 & 100 & 14.72 & 21.03 & 26.99 & 32.95 & 38.03 & 45.04\end{array}$

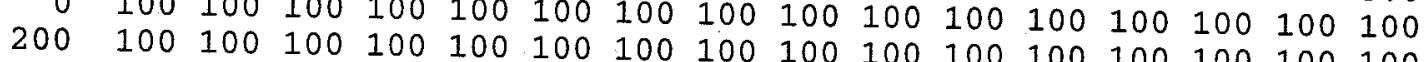

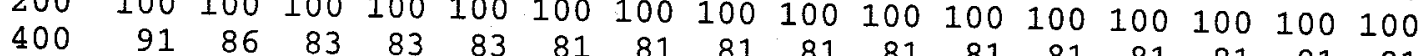

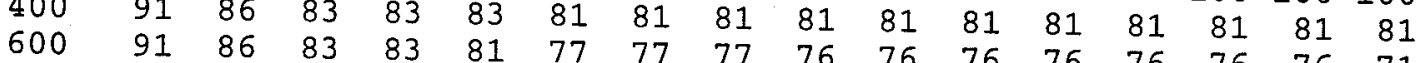

800 $\begin{array}{llll}91 & 86 & 83 & 83 \\ 91 & 86 & 83\end{array}$

$\begin{array}{llllllllll}1000 & 91 & 81 & 77 & 74 & 74 & 74 & 78 & 78 & 78\end{array}$

1100

1250

1500

1700

1950

2000

9180

$91 \cdot 80$

9180

918

9180

$77 \quad 74$

$74 \quad 7$

747

$74 \quad 74$

6565

$\begin{array}{lll}75 & 72 & 70 \\ 75 & 72 & 70\end{array}$

67
67

67
67

918

$\begin{array}{lll}75 & 72 & 70\end{array}$

67
67

$65 \quad 65$

$\begin{array}{lll}65 & 65 & 65\end{array}$

7270

BASE Equivalence Ratio

$\begin{array}{llllllll}76 & 76 & 76 & 76 & 76 & 76 & 76 & 71\end{array}$

Speed

- MAP

$0 \mathrm{rpm}$ - $\mathrm{Phi}$

1.000

- Ignadv 14.4 psia

- UEGO

5.0 BTDC - PhIECT

1.022

32

$\begin{array}{ll}32 & \circ \\ 0.8 & \mathrm{aP}\end{array}$

$76 \quad 76$

$\begin{array}{lll}71 & 71 & 69\end{array}$

$\begin{array}{lll}69 & 69 & 69\end{array}$

- Ignoffset

+0.0 BTDC - PhIMAP_C

- Controlmode

- CL_Mult

- Adpt_Mult

OPEN LOOP

$+0.00 \%$

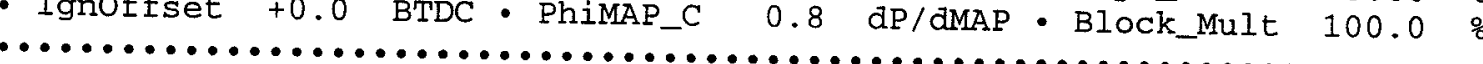


GEM Lean Burn Control System - Ignition Advance Calibration Screen MAP (psia)

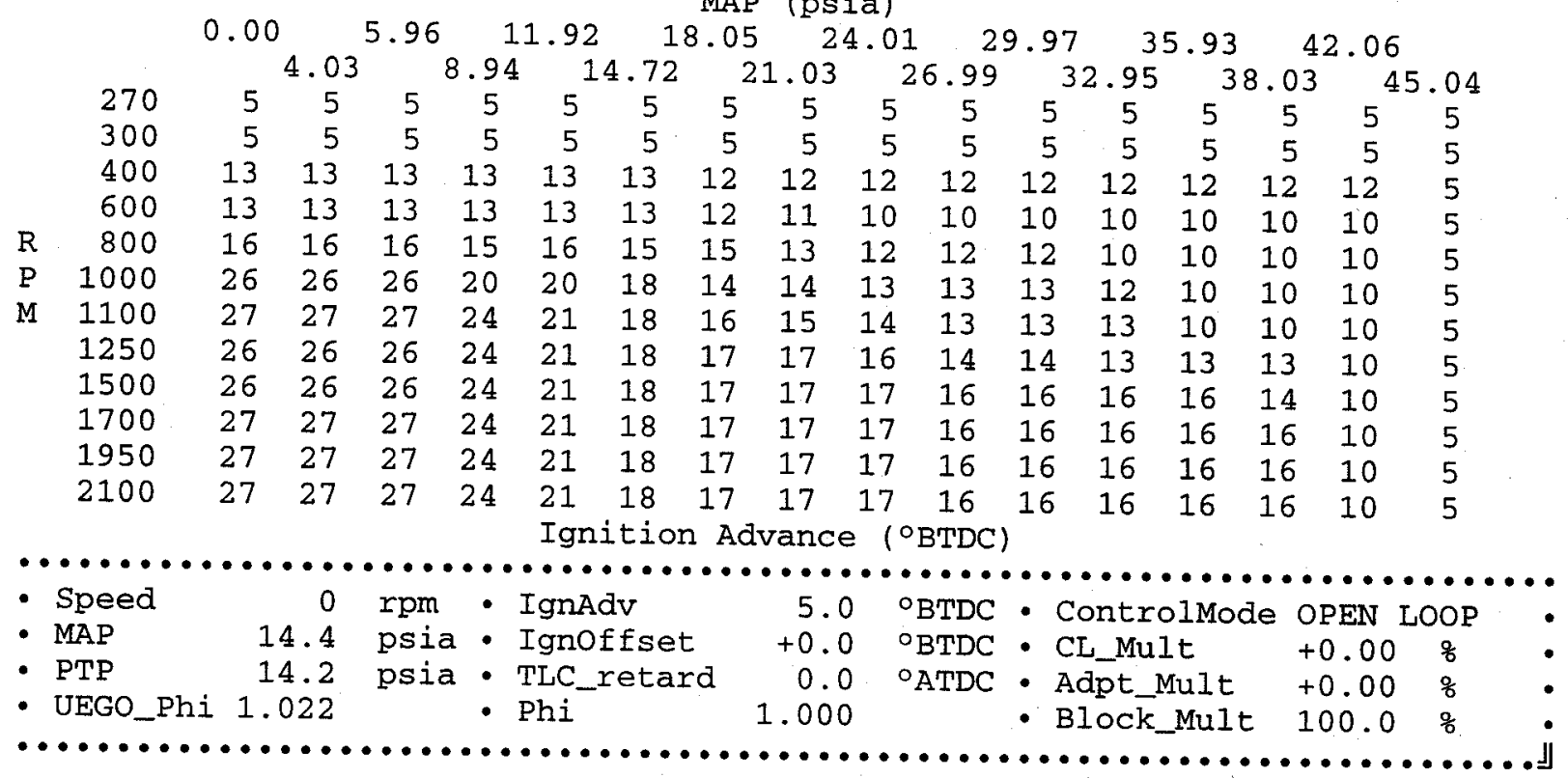


GEM Lean Burn Control System - VE Calibration Screen

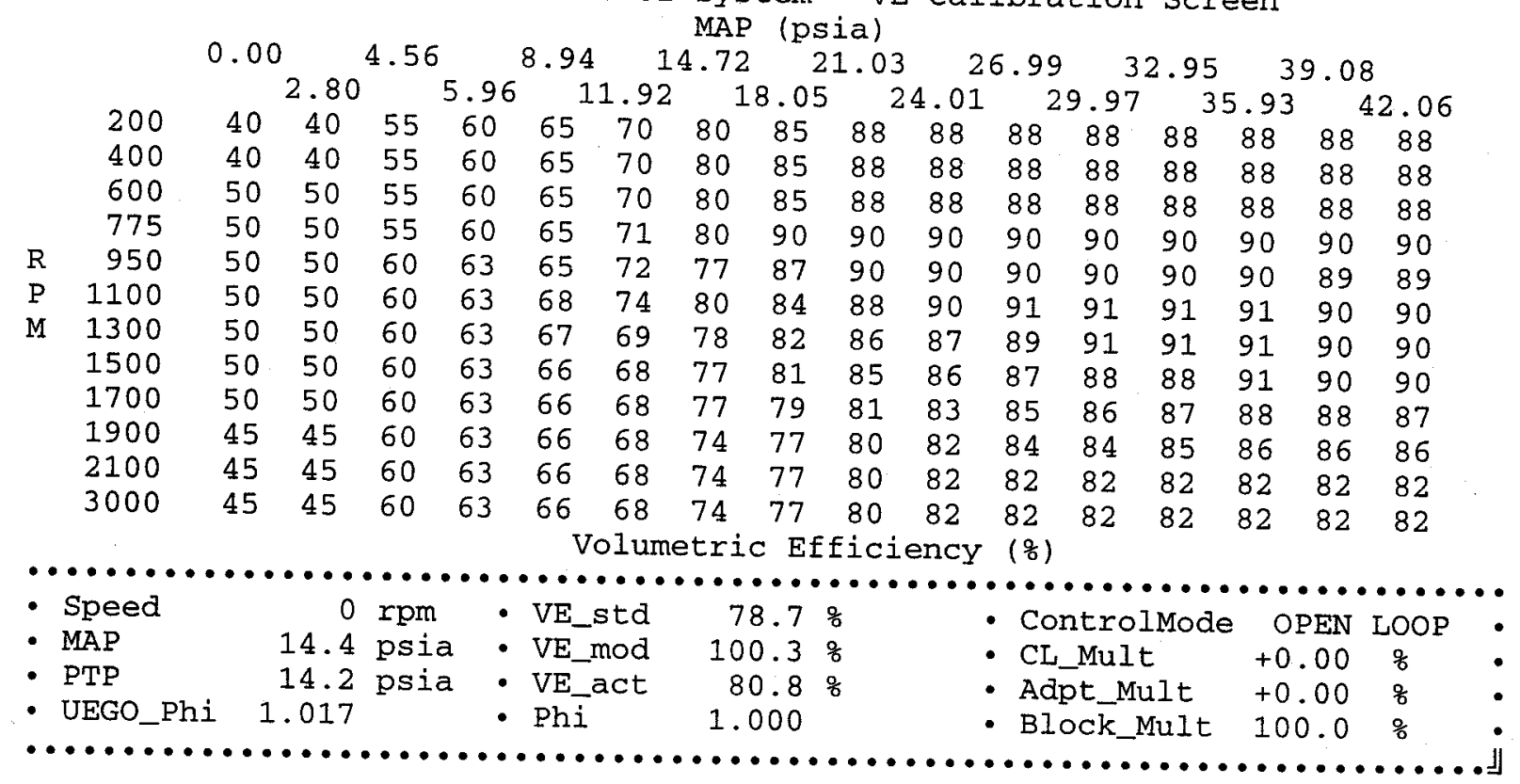


GEM Lean Burn Control System - Adaptive Parameter Table

\begin{tabular}{|c|c|c|c|c|c|c|c|c|c|c|c|c|}
\hline Speed & 0 & $\mathrm{rpm}$ & - & & & & MA & $P$ (ps: & ia) & & & \\
\hline - MAP & 14.4 & psia & - & 4.56 & 6.13 & 9.5 & 99 & 15.07 & 19.98 & 25.06 & 31.02 & 38.03 \\
\hline $\begin{array}{l}\text { PTP } \\
\text { TPS }\end{array}$ & 14.2 & psia & 560 & +0.0 & +0.0 & +0. & & +0.0 & +0.0 & +0.0 & +0.0 & +0.0 \\
\hline & 0.41 & Volts & - R 900 & +0.0 & +0.0 & +0. & & +0.0 & +0.0 & +0.0 & +0.0 & +0.0 \\
\hline MAT & 122 & ${ }^{\circ} \mathrm{F}$ & -P1200 & +0.0 & +0.0 & +0. & & +0.0 & +0.0 & +0.0 & +0.0 & +0.0 \\
\hline ECT & 128 & ${ }^{\circ} \mathrm{F}$ & -M1600 & +0.0 & +0.0 & +0. & & +0.0 & +0.0 & +0.0 & +0.0 & +0.0 \\
\hline $\begin{array}{l}\text { NGP } \\
\text { NGT }\end{array}$ & $\begin{array}{r}58.8 \\
106\end{array}$ & $\begin{array}{l}\text { psia } \\
{ }^{\circ} \mathrm{F}\end{array}$ & : 2000 & +0.0 & +0.0 & $\begin{array}{l}+0 . \\
\text { tpti }\end{array}$ & & $\begin{array}{l}+0.0 \\
\text { Parame }\end{array}$ & $\begin{array}{r}+0.0 \\
\text { eters }\end{array}$ & $\begin{array}{l}+0.0 \\
\left(\frac{8}{8}\right)\end{array}$ & +0.0 & +0.0 \\
\hline PW & 30.3 & $\mathrm{~ms}$ & $\cdots \cdots$ & & $\cdots$ & $\cdots$ & & & & & & \\
\hline PWtiming & 0.0 & ${ }^{\circ}$ ATDC & Adp & $t$ t_Mult & +0.0 & 00 & 8 & - & RunMode & & STOPI & PED \\
\hline IgnAdv & 5.0 & ${ }^{\circ} \mathrm{BTDC}$ & K_A & dapt & & 5 & 8 & - & Control & IMode & OPEN & LOOP \\
\hline Ignoffst & +0.0 & ${ }^{\circ} \mathrm{BTDC}$ & Adp & t_step & 2. & 00 & $\frac{8}{8}$ & - & RunTime & & $00: 0$ & $0: 00$ \\
\hline C Mult & $\cdots \cdots$ & $\cdots$ & Adp & E_dzone & & 00 & $\%$ & E & EBP & & 14.41 & psia \\
\hline CL_MuIt & +0.00 & 0 & Adp & E_max & +35.0 & 00 & \% & - $\mathrm{F}$ & EBP_est & & 14.71 & psia \\
\hline K_CL & 20 & 0 & Adp & E_min & -30.0 & 00 & $\%$ & - & BP & & 14.71 & psia \\
\hline CL_step & 5.00 & 0 & Adp & E_valid & 3.0 & 00 & $\%$ & - & VE_act & & 78.7 & \\
\hline $\begin{array}{l}\text { CL_switch } \\
C L \text { max }\end{array}$ & +4000 & 0 & T_d & elay. & 409 & 96 & $\mathrm{~ms}$ & - & Fuel_In & & $164.5 \mathrm{n}$ & $\mathrm{mg} / \mathrm{inj}$ \\
\hline CL_min & -35.00 & 0 & Phi & D_Phi & $\begin{array}{l}1.01 \\
1.00\end{array}$ & & & $\bullet$ & MAPdt_m & & $0.0 \mathrm{n}$ & jز \\
\hline ockMult & 100.0 & 0 & Phi & _delay & 1.00 & & & . & & & & \\
\hline
\end{tabular}


GEM Lean Burn Control System Monitor

PROM ID: 2570-008- 09/11/98

- Speed

- MAP

- ECT

- MAT

- PTP

- EBP

- NGP

- NGT

- NGTP

- NGTT

- UEgO

- FPP

$\begin{aligned} 0 & \text { rpm } \\ 14.4 & \text { psia }\end{aligned}$

128

122

14.2

14.41

57.8 106

0

$-50$

1.006

0.51

- TPS_Command 1.99

- TPS_Feedbck 0.02

- Baro

- Battery

- SparkAdv

- $\mathrm{PW}$

- CL_Mult

- AL_Mult
14.71

14.2

5.0 30.7

$+0.00$

$+0.00$
${ }^{\circ} \mathrm{F}$

${ }^{\circ} \mathrm{F}$

psia

psia

psia

${ }^{\circ} \mathrm{F}$

psig

phi

o

volts

volts

psia RunTime

- Controlmode

volts - RunMode

${ }^{\circ} \mathrm{BTDC}$.

ms

$\%$

$\frac{7}{0}$
- ECT raw

- MAT raw

- PTP raw

- EBP raw

- NGP raw

- NGT raw

- NGTP raw

- NGTT raw

- UEgo raw

- FPP raw

- FPP Minimum

- FuelGauge

\subsection{7}

1.43

1.37

1.63

2.41

1.67

1.96

0.02

5.00

0.94

0.41

volts: Diagnostics:

volts.

volts - Spark:

volts - (firing order)

volts - 1 - ENABLED

volts - 2 - ENABLED

volts - 3 - ENABLED

volts - 4 - ENABLED

volts - 5 - ENABLED

volts - 6 - ENABLED

volts -7 - ENABLED

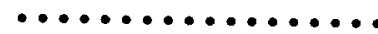

$00: 00: 00$

OPEN LOOP

STOPPED

- Injector:

- 1 - ENABLED

- 2 - ENABLED

- 3 - ENABLED

0.41 volts $: 4$ - ENABLED

$0 \%$ 


\section{REPORT DOCUMENTATION PAGE}

Form Approved OMB NO. 0704-0188

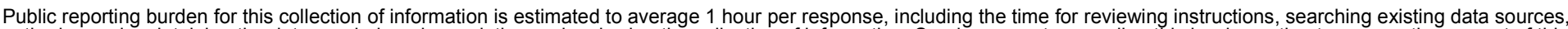

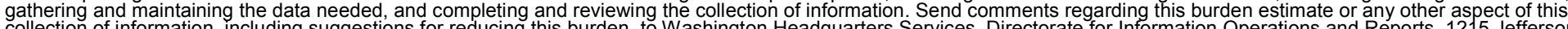

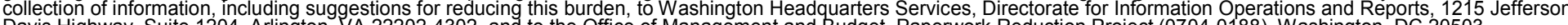

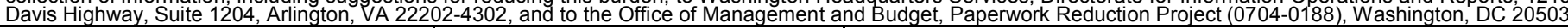

\begin{tabular}{|l|l|l}
\hline 1. AGENCY USE ONLY (Leave blank) & $\begin{array}{l}\text { 2. REPORT DATE } \\
\text { January } 2000\end{array}$ & $\begin{array}{l}\text { 3. REPORT TYPE AND DATES COVERED } \\
\text { Subcontractor report }\end{array}$ \\
\hline
\end{tabular}

4. TITLE AND SUBTITLE Subcontractor report

Mack LNG Vehicle Development

6. AUTHOR(S)

(C) AAC-5-15249-01

(TA) FU913310

Southwest Research Institute

7. PERFORMING ORGANIZATION NAME(S) AND ADDRESS(ES)

Southwest Research Institute

6220 Culebra Road

San Antonio, 78228-0510

9. SPONSORING/MONITORING AGENCY NAME(S) AND ADDRESS(ES)

National Renewable Energy Laboratory

10. SPONSORING/MONITORING

1617 Cole Blvd.

Golden, CO 80401

\section{SUPPLEMENTARY NOTES}

\section{2a. DISTRIBUTION/AVAILABILITY STATEMENT}

12b. DISTRIBUTION CODE

National Technical Information Service

U.S. Department of Commerce

5285 Port Royal Road

Springfield, VA 22161

13. ABSTRACT (Maximum 200 words) The goal of this project was to install a production-ready, state-of-the-art engine control system on the Mack E7G natural gas engine to improve efficiency and lower exhaust emissions. In addition, the power rating was increased from 300 brake horsepower (bhp) to 325 bhp. The emissions targets were oxides of nitrogen plus nonmethane hydrocarbons of less than $2.5 \mathrm{~g} / \mathrm{bhp}$-hr and particulate matter of less than $0.05 \mathrm{~g} / \mathrm{bhp}$-hr on $99 \%$ methane. Vehicle durability and field testing were also conducted. Further development of this engine should include efficiency improvements and oxides of nitrogen reductions.

\section{SUBJECT TERMS}

Mack trucks, liquefied natural gas, alternative fuels, emissions, alternative fuel vehicles

15. NUMBER OF PAGES

16. PRICE CODE

\begin{tabular}{l|l}
$\begin{array}{l}\text { 17. SECURITY CLASSIFICATION } \\
\text { OF REPORT }\end{array}$ & $\begin{array}{l}\text { 18. SECURITY CLASSIFICATION } \\
\text { OF THIS PAGE } \\
\text { Unclassified }\end{array}$ \\
\hline
\end{tabular}

19. SECURITY CLASSIFICATION OF ABSTRACT Unclassified
8. PERFORMING ORGANIZATION
REPORT NUMBER

NREL/SR-540-26935

NSN 7540-01-280-5500

Standard Form 298 (Rev. 2-89) Prescribed by ANSI Std. Z39-18 